\title{
Cleanup Verification Package for the 118-B-1, 105-B Solid Waste Burial Ground
}

\author{
Prepared for the U.S. Department of Energy \\ by Washington Closure Hanford
}





\section{EXECUTIVE SUMMARY}

This cleanup verification package documents completion of remedial action, sampling activities, and compliance with cleanup criteria for the 118-B-1, 105-B Solid Waste Burial Ground. The 118-B-1 Burial Ground, part of the 100-BC-2 Operable Unit, is located in the 100-B/C Area of the Hanford Site in southeastern Washington State. This waste site was the primary burial ground for general wastes from the operation of the 105-B Reactor and P-10 Tritium Separation Project and also received waste from the 105-N Reactor. The burial ground was in operation between 1944 and 1973 and received reactor hardware, process piping and tubing, fuel spacers, glassware, electrical components, tritium process wastes, soft wastes and other miscellaneous debris.

Remediation of the 118-B-1 Burial Ground began on February 2, 2004, and was completed on June 7, 2007. Remedial action activities involved removing and stockpiling the uncontaminated overburden, and removing the buried waste material and the underlying contaminated soil for disposal. All contaminated solid waste materials were disposed at the Environmental Restoration Disposal Facility, with the exception of spent nuclear fuel, which will be transferred to the $100-\mathrm{K}$ Basins for interim storage prior to final packaging and disposal.

Results of cleanup verification sampling, laboratory analyses, and data evaluations for the 118-B-1 site (which includes the remediation footprint, the overburden and below cleanup level soil stockpiles, and the waste staging pile area) indicate that residual tritium concentrations in vadose zone soils below the remediation footprint exceed remedial action objectives for the protection of groundwater. An explanation of significant difference to the Record of Decision for the 100-BC-1, 100-BC-2, 100-DR-1, 100-DR-2, 100-FR-2, 100-HR-2, and 100-KR-2 Operable Units, Hanford Site (100 Area Burial Grounds), Benton County, Washington (Burial Grounds ROD) assessed balancing factors, and concluded that tritium-contaminated soil may remain in-place, with institutional controls that prohibit future irrigation to minimize further mobilization of 
residual tritium contamination to groundwater and the Columbia River. All remedial action objectives and goals for direct exposure, protection of groundwater, and protection of the Columbia River for contaminants other than tritium have been met (see Table ES-1).

Soil cleanup levels were established in the Burial Ground ROD based on a limited ecological risk assessment. Although not required by the Burial Ground ROD, a comparison against ecological risk screening levels has been made for the 118-B-1 waste site contaminants of concern and contaminants of potential concern. Screening levels were not exceeded for the site constituents, with the exception of barium, boron, cadmium, lead, mercury, molybdenum, selenium, and vanadium. Exceedance of screening values does not necessarily indicate the existence of risk to ecological receptors. Concentrations of cadmium, vanadium, and selenium are within the range of Hanford Site background levels, and molybdenum concentrations are consistent with those seen elsewhere at the Hanford Site (no established background value is available for molybdenum). Exceedances for barium, boron, lead, and mercury will be evaluated in the context of additional lines of evidence as part of the baseline risk assessment. A more complete quantitative ecological risk assessment will be presented in the baseline risk assessment for the river corridor portion of the Hanford Site and will be used to support the final closeout decision for the 118-B-1 waste site.

This evaluation supports a reclassification of the 118-B-1 waste site to Interim Closed Out, in accordance with the Hanford Federal Facility Agreement and Consent Order (Ecology et al. 1989) and the Tri-Party Agreement Handbook Management Procedures, Waste Site Reclassification Guideline TPA-MP-14 (DOE-RL 2007). A copy of the waste site reclassification form is included as Attachment ES-1. 
CVP-2007-00006

Rev. 0

Table ES-1. Summary of Remedial Action Goals for the 118-B-1 Burial Ground. (2 Pages)

\begin{tabular}{|c|c|c|c|c|}
\hline $\begin{array}{l}\text { Regulatory } \\
\text { Requirement }\end{array}$ & Remedial Action Goals & Results & $\begin{array}{l}\text { Remedial } \\
\text { Action } \\
\text { Objectives } \\
\text { Attained? }\end{array}$ & Ref. \\
\hline $\begin{array}{l}\text { Direct Exposure - } \\
\text { Radionuclides }\end{array}$ & $\begin{array}{l}\text { Attain } 15 \mathrm{mrem} / \mathrm{yr} \text { dose rate } \\
\text { above background over } \\
1,000 \text { years. }\end{array}$ & $\begin{array}{l}\text { The maximum predicted dose rates for } \\
\text { the } 118-\mathrm{B}-1 \text { Burial Ground are less than } \\
15 \mathrm{mrem} / \mathrm{yr} \text {. Carbon- } 14 \text { and strontium- } \\
90 \text { activities were not included in the } \\
\text { plant ingestion pathway for the } \\
\text { remediation footprint, because residual } \\
\text { activities are below the root penetration } \\
\text { zone. }\end{array}$ & Yes & $a, b$ \\
\hline $\begin{array}{l}\text { Direct Exposure - } \\
\text { Nonradionuclides }\end{array}$ & $\begin{array}{l}\text { Attain individual COC/COPC } \\
\text { RAGs. }\end{array}$ & $\begin{array}{l}\text { All individual COC/COPC concentrations } \\
\text { are below the direct exposure criteria. }\end{array}$ & Yes & $\mathrm{C}$ \\
\hline \multirow[t]{4}{*}{$\begin{array}{l}\text { Nonradionuclide } \\
\text { Risk Requirements }\end{array}$} & $\begin{array}{l}\text { Attain hazard quotient of less } \\
\text { than } 1 \text { for all individual } \\
\text { noncarcinogens. }\end{array}$ & $\begin{array}{l}\text { All hazard quotients for individual } \\
\text { nonradionuclide COCs/COPCs are less } \\
\text { than } 1 .\end{array}$ & \multirow{4}{*}{ Yes } & $d$ \\
\hline & $\begin{array}{l}\text { Attain a cumulative hazard } \\
\text { quotient of less than } 1 \text { for } \\
\text { noncarcinogens. }\end{array}$ & $\begin{array}{l}\text { The cumulative hazard quotient for all } \\
\text { areas and focused samples }\left(8.8 \times 10^{-1}\right) \\
\text { is less than } 1 .\end{array}$ & & d \\
\hline & $\begin{array}{l}\text { Attain an excess cancer risk of } \\
\text { less than } 1 \times 10^{-6} \text { for individual } \\
\text { carcinogens. }\end{array}$ & $\begin{array}{l}\text { All excess cancer risk values for } \\
\text { individual nonradionuclide } \\
\text { COCs/COPCs are less than } 1 \times 10^{-6} \text {. }\end{array}$ & & $d$ \\
\hline & $\begin{array}{l}\text { Attain a total excess cancer } \\
\text { risk of less than } 1 \times 10^{-5} \text { for } \\
\text { carcinogens. }\end{array}$ & $\begin{array}{l}\text { The total excess carcinogenic risk for all } \\
\text { areas and focused samples }\left(8.0 \times 10^{-7}\right) \\
\text { is less than } 1 \times 10^{-5} \text {. }\end{array}$ & & d \\
\hline \multirow[t]{2}{*}{$\begin{array}{l}\text { Groundwater/River } \\
\text { Protection - } \\
\text { Radionuclides }\end{array}$} & $\begin{array}{l}\text { Attain single COC/COPC } \\
\text { groundwater and river } \\
\text { protection RAGS. }\end{array}$ & $\begin{array}{l}\text { Among the radionuclide COCs/COPCs, } \\
\text { only cesium-137, cobalt-60, } \\
\text { strontium-90, and tritium are predicted to } \\
\text { reach groundwater, at concentrations } \\
\text { significantly below the RAGs. Tritium } \\
\text { was not included in the evaluation for } \\
\text { the remediation footprint, as an } \\
\text { assessment of balancing factors } \\
\text { concluded that residual tritium } \\
\text { contamination should be left in-place } \\
\text { with institutional controls to minimize } \\
\text { further mobilization of residual tritium to } \\
\text { groundwater. }\end{array}$ & \multirow[t]{2}{*}{ Yes } & $a, b$ \\
\hline & $\begin{array}{l}\text { Attain national primary } \\
\text { drinking water regulations: } \\
\text { 4-mrem/yr (beta/gamma) dose } \\
\text { rate to target receptor/organs. }\end{array}$ & $\begin{array}{l}\text { All organ specific doses are below the 4- } \\
\mathrm{mrem} / \mathrm{yr} \text { dose standard. }\end{array}$ & & $\mathrm{g}$ \\
\hline
\end{tabular}


CVP-2007-00006

Rev. 0

Table ES-1. Summary of Remedial Action Goals for the 118-B-1 Burial Ground. (2 Pages)

\begin{tabular}{|c|c|c|c|c|}
\hline $\begin{array}{l}\text { Regulatory } \\
\text { Requirement }\end{array}$ & Remedial Action Goals & Results & $\begin{array}{l}\text { Remedial } \\
\text { Action } \\
\text { Objectives } \\
\text { Attained? }\end{array}$ & Ref. \\
\hline & $\begin{array}{l}\text { Meet drinking water standards } \\
\text { for alpha emitters: the more } \\
\text { stringent of } 15 \mathrm{pCi} / \mathrm{L} \text { MCL or } \\
1 / 25^{\mathrm{th}} \text { of the derived } \\
\text { concentration guide for DOE } \\
\text { Order } 5400.5 .^{\mathrm{h}}\end{array}$ & $\begin{array}{l}\text { No alpha-emitting radionuclide COC/COPCs } \\
\text { are predicted to impact groundwater. }\end{array}$ & Yes & $a, b$ \\
\hline & $\begin{array}{l}\text { Meet total uranium standard of } \\
21.2 \mathrm{pCi} / \mathrm{L} .\end{array}$ & $\begin{array}{l}\text { Isotopic uranium concentrations are } \\
\text { below background. }\end{array}$ & Yes & c \\
\hline $\begin{array}{l}\text { Groundwater/River } \\
\text { Protection - } \\
\text { Nonradionuclides }\end{array}$ & $\begin{array}{l}\text { Attain individual } \\
\text { nonradionuclide groundwater } \\
\text { and river cleanup } \\
\text { requirements. }\end{array}$ & $\begin{array}{l}\text { Residual concentrations of multiple } \\
\text { metals and organic compounds } \\
\text { exceeded soil RAGs for the protection of } \\
\text { groundwater and/or the Columbia River. } \\
\text { However, none of these are predicted to } \\
\text { migrate to groundwater (and thus the } \\
\text { Columbia River) at concentrations } \\
\text { exceeding groundwater or river criteria } \\
\text { within } 1,000 \text { years, with the exception of } \\
\text { beta-BHC. However, modeling of the } \\
\text { hydraulic conductivity and gradient data } \\
\text { for the } 100-B / C \text { Area shows that there is } \\
\text { a soil-to-groundwater dilution factor of } \\
\text { approximately } 6 \text {, indicating that beta- } \\
\text { BHC will not leach to groundwater or the } \\
\text { Columbia River at concentrations } \\
\text { exceeding cleanup criteria. Therefore, } \\
\text { residual contaminant concentrations } \\
\text { achieve the remedial action objectives } \\
\text { for groundwater and river protection. }\end{array}$ & Yes & c \\
\hline $\begin{array}{l}\text { Other Supporting } \\
\text { Information }\end{array}$ & \multicolumn{3}{|l|}{ Sample design calculation brief. } & k \\
\hline \multicolumn{5}{|c|}{ 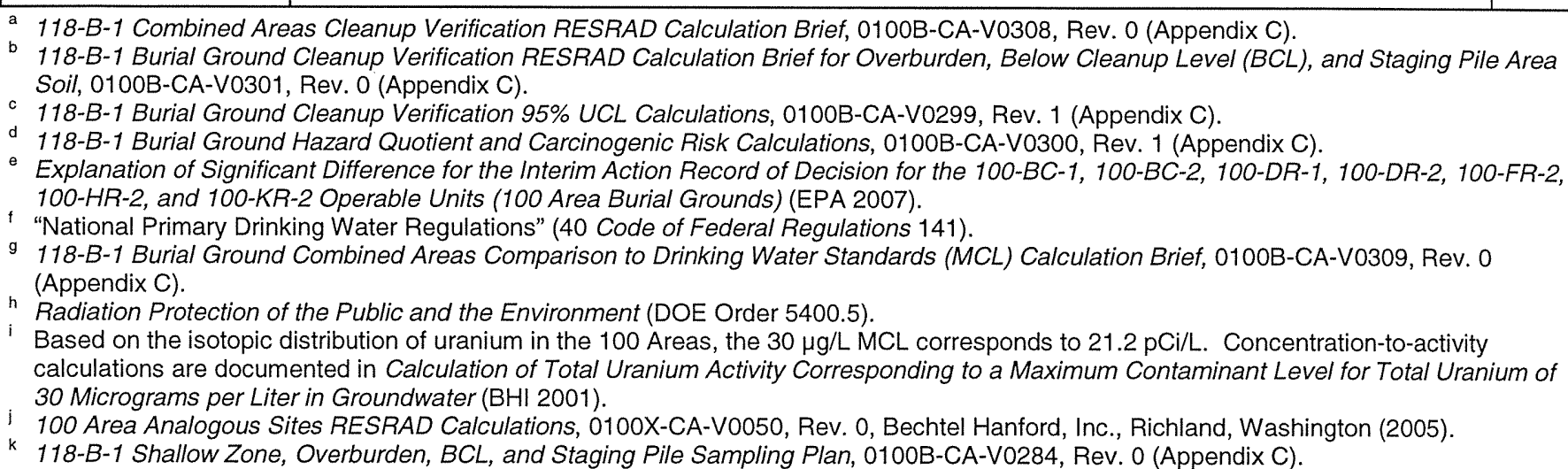 } \\
\hline \multicolumn{5}{|c|}{$\begin{array}{ll}\mathrm{COC} & =\text { contaminant of concern } \\
\mathrm{COPC} & =\text { contaminant of potential concern } \\
\mathrm{MCL} & =\text { maximum contaminant level (drinking water standard) }\end{array}$} \\
\hline
\end{tabular}


Attachment ES-1. Waste Site Reclassification Form.

\begin{tabular}{|c|c|c|}
\hline \multirow{2}{*}{ Date Submitted: $\quad 12 / 12 / 07$} & WASTE SITE RECLASSIFICATION FORM & \multirow{5}{*}{ Control Number: $2007-032$} \\
\hline & Operable Unit(s): $\quad 100-B C-2$ & \\
\hline Originator: $\quad$ L.M.Dittmer & Waste Site Code: $\quad 118-\mathrm{B}-1$ & \\
\hline Phone: $\quad 372-9227$ & Type of Reclassification Action: & \\
\hline & $\begin{array}{l}\text { Closed Out } \square \quad \text { Interim Closed Out } \square \text { No Action } \square \\
\text { RCRA Postclosure } \square \quad \text { Rejected } \square \quad \text { Consolidated } \square\end{array}$ & \\
\hline
\end{tabular}

This form documents agreement among parties listed authorizing classification of the subject unit as Closed Out, Interim Closed Out, No Action, RCRA Postclosure, Rejected, or Consolidated. This form also authorizes backfill of the waste management unit, if appropriate, for Closed Out and Interim Closed Out units. Final removal from the NPL of No Action and Closed Out waste management units will occur at a future date.

Description of current waste site condition:

The 118-B-1 Burial Ground was the primary burial ground for general wastes from the operation of the 105-B Reactor and P-10 Tritium Separation Project. Remediation, verification sampling, and RESidual RADioactivity (RESRAD) modeling have been performed in accordance with remedial action objectives and goals established by the Record of Decision for the 100-BC-1, 100-BC-2, 100-DR-1, 100-DR-2, 100-FR-2, 100-HR-2, and 100-KR-2 Operable Units, Hanford Site (100 Area Burial Grounds), Benton County, Washington (Burial Ground ROD), U.S. Environmental Protection Agency, Region 10, Seattle, Washington and the October 2007 Explanation of Significant Difference for the Interim Action Record of Decision for the 100-BC-1, 100-BC-2, 100-DR-1, 100-DR-2, 100-FR-2, 100-HR-2, and 100-KR-2 Operable Units (100 Area Burial Grounds) (118-B-1 ESD), U.S. Environmental Protection Agency, Region 10, Seattle, Washington. The selected remedy involved (1) excavating the site to the extent required to meet specified soil cleanup levels, (2) disposing of contaminated excavation materials at the Environmental Restoration Disposal Facility at the 200 Area of the.Hanford Site or appropriate treatment, storage, and disposal facilities, (3) demonstrating through verification sampling and RESRAD modeling that cleanup goals have been achieved, and (4) proposing the site for reclassification as Interim Closed Out.

\section{Basis for reclassification:}

In accordance with this evaluation, the verification sampling and modeling results support a reclassification of this site to Interim Closed Out. The current site conditions achieve the remedial action objectives established in the Burial Ground ROD, as amended by the 118-B-1 ESD. The results show that residual tritium concentrations in the vadose zone beneath the burial ground exceed remedial action objectives for the protection of groundwater. The 118-B-1 ESD assessed balancing factors and concluded that residual tritium contamination should be left in-place with institutional controls to prohibit irrigation, minimizing further mobilization of residual tritium. The results of verification sampling and RESRAD modeling for contaminant concentrations other than tritium do not preclude any future uses (as bounded by the rural-residential scenario). With institutional controls to prevent irrigation, the results also demonstrate that residual contaminant concentrations are protective of groundwater and the Columbia River. The acceptability of direct exposure to residual tritium contamination in the deep vadose zone has not been demonstrated; therefore, institutional controls to prevent uncontrolled drilling or excavation into the deep zone are also required. The basis for reclassification is described in detail in the Cleanup Verification Package for the 118-B-1, 105-B Solid Waste Burial Ground (CVP-2007-00006), Washington Closure Hanford, Richland, Washington.

Waste Site Controls:

Engineered Controls: Yes $\square$ No $\square \quad$ Institutional Controls: Yes $\square$ No $\square \quad$ O\&M requirements: Yes $\square$ No $\square$ Deed restrictions to prohibit irrigation and prevent uncontrolled drilling or excavation into the deep zone (greater than $4.6 \mathrm{~m}$ $[15 \mathrm{ft}]$ below ground surface).

S. L. Charboneau

DOE Federal Project Director (printed)

N.A

Ecology Project Manager (printed)

L. Buelow

EPA Project Manager (printed)

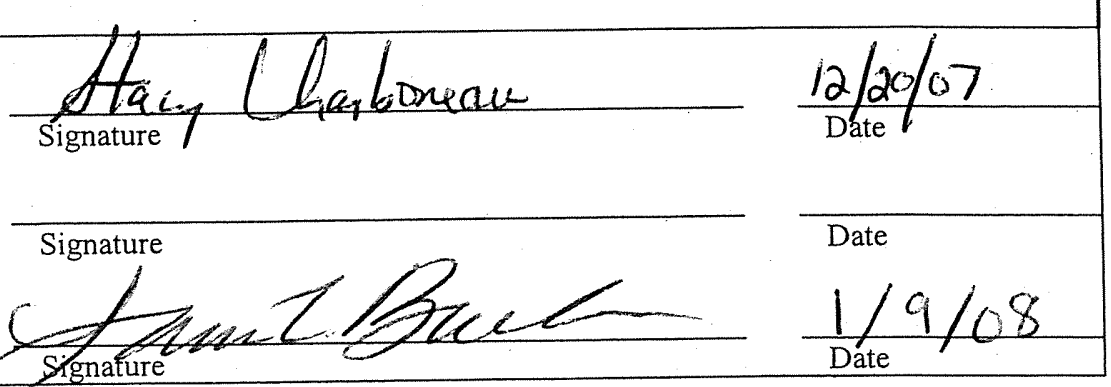


. 


\section{CONTENTS}

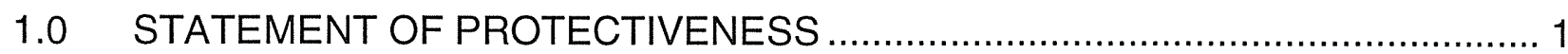

2.0 BACKGROUND AND GENERAL SITE INFORMATION ................................... 2

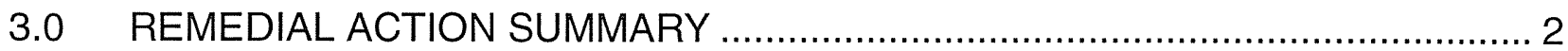

3.1 EXCAVATION AND DISPOSAL INFORMATION …............................ 4

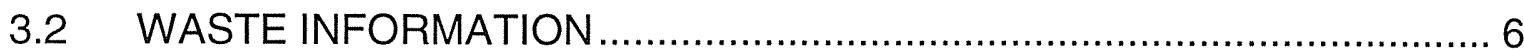

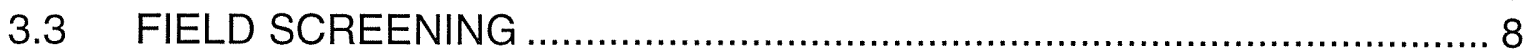

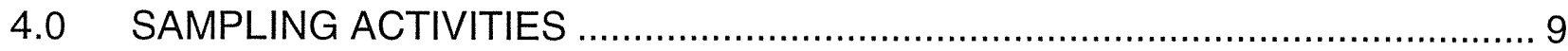

4.1 VERIFICATION SAMPLING DESIGN SELECTION AND BASIS ............ 9

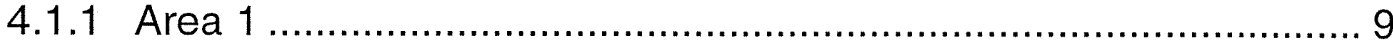

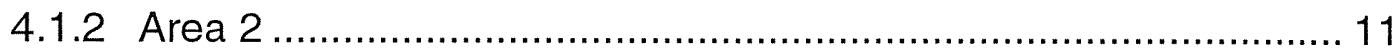

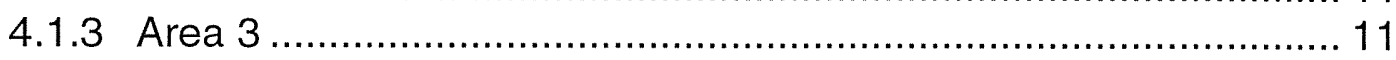

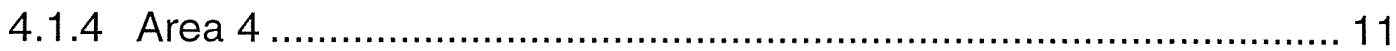

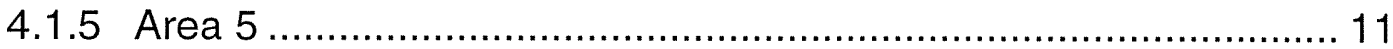

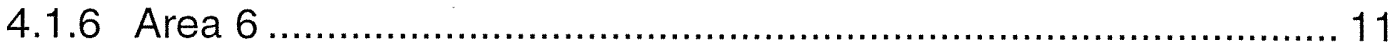

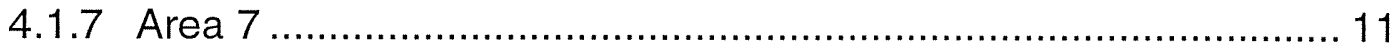

4.1.8 Overburden Stockpiles............................................................... 11

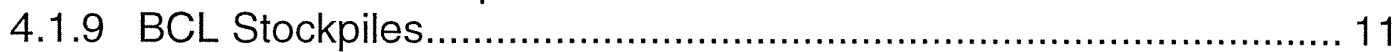

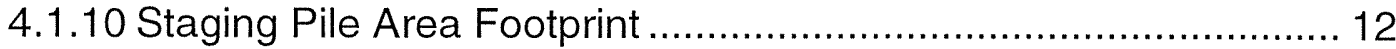

4.1.11 Statistical Sampling Design.................................................... 12

4.1.12 Focused Sampling …......................................................... 12

4.1.13 Tritium Plume Characterization Sampling ................................... 13

4.2 CONTAMINANTS OF CONCERN AND CONTAMINANTS OF POTENTIAL CONCERN FOR VERIFICATION SAMPLING ................... 13

4.2.1 Statistical Sampling............................................................ 15

4.2.2 Focused Sampling ........................................................... 16

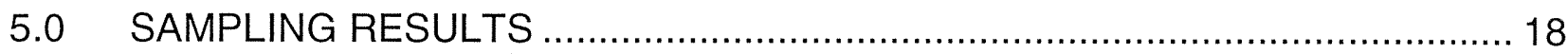

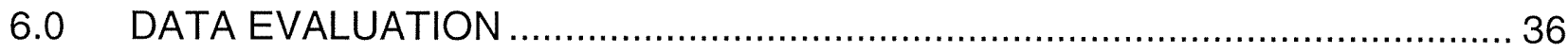

6.1 COMPARISON OF SAMPLE DATA TO RAGS …............................. 37

6.2 COMPARISON OF SAMPLE DATA TO ECOLOGICAL RISK SCREENING LEVELS .............................................................. 37

6.3 HUMAN HEALTH RISK EVALUATION FOR NONRADIONUCLIDES..... 38

6.4 WAC THREE-PART TEST FOR NONRADIONUCLIDES ..................... 38

6.5 RESRAD MODELING FOR RADIONUCLIDES .................................39

6.5.1 Attainment of Radionuclide Direct Exposure RAG ...................... 39

6.5.2 Attainment of Radionuclide Excess Lifetime Cancer Risk Goal .... 39

6.5.3 Attainment of Radionuclide Groundwater and River Protection RAGS ................................................................................ 40

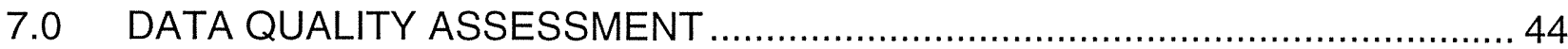


8.0 SUMMARY FOR WASTE SITE RECLASSIFICATION ................................ 45

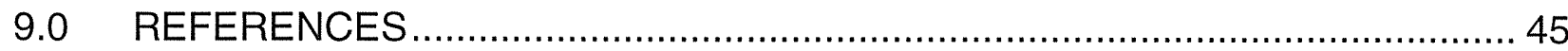

\section{APPENDICES}

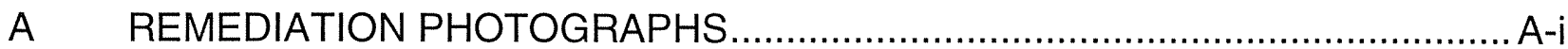

B SYSTEMATIC RADIOLOGICAL SURVEYS ............................................. B

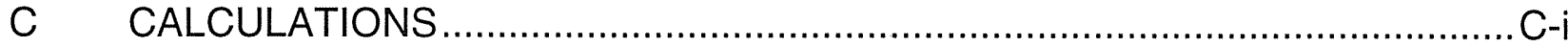

C1 118-B-1 Shallow Zone, Overburden and Staging Pile Sampling Plan, 0100B-CA-V0284..........................................................................

C2 118-B-1 Burial Ground Cleanup Verification 95\% UCL Calculations,

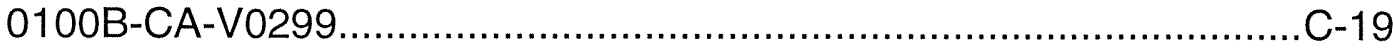

C3 118-B-1 Burial Ground Hazard Quotient and Carcinogenic Risk Calculations, 0100B-CA-V0300 .................................................... 195

C4 118-B-1 Burial Ground Cleanup Verification RESRAD Calculation Brief for Overburden, Below Cleanup Level (BCL), and Staging Pile Area Soil, 0100B-CA-V0301 ........................................-200

C5 118-B-1 Combined Areas Cleanup Verification RESRAD Calculation Brief, 0100B-CA-V0308.

C6 118-B-1 Burial Ground Combined Areas Comparison to Drinking Water Standards (MCL) Calculation Brief, 0100B-CA-V0309 ..............-454

D TRITIUM PLUME CHARACTERIZATION SAMPLING RESULTS ................... D-i

E DATA QUALITY ASSESSMENT ................................................................

\section{FIGURES}

1. Location of the 118-B-1 Burial Ground................................................................. 3

2. Historical Aerial Photograph of the 100-B/C Area, Including the 118-B-1 Burial Ground.

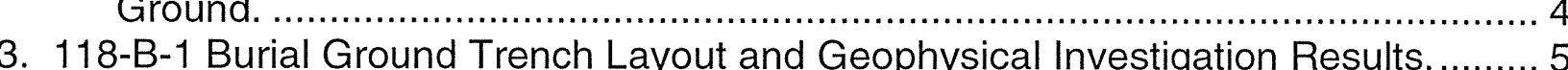

4. 118-B-1 Burial Ground Post-Excavation Topographic Survey. ................................ 7

5. 118-B-1 Burial Ground After Major Excavation Activities (taken November 2006).... 8

6. 118-B-1 Area Boundaries for Verification Sampling............................................ 10

7. Location of Focused Verification Soil Samples at the .......................................... 14

8. RESRAD Analysis for the Combined Areas in the Remediation Footprint-All

Radionuclides, All-Pathways Dose Rate Estimate. ....................................... 40

9. RESRAD Analysis for the Overburden Stockpiles- All Radionuclides, All-Pathways

Dose Rate Estimate. ................................................................................. 40

10. RESRAD Analysis for the BCL Stockpiles- All Radionuclides, All-Pathways Dose

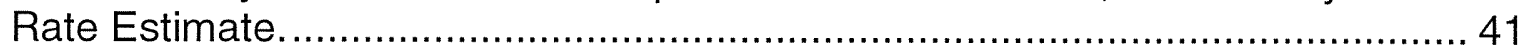

11. RESRAD Analysis for the SPA Footprint-All Radionuclides, All-Pathways Dose Rate Estimate. 
12. RESRAD Analysis - Radionuclide Risk, All Pathways - Combined Areas in the Remediation Footprint.

13. RESRAD Analysis - Radionuclide Risk, All Pathways - Overburden Stockpiles... 42

14. RESRAD Analysis - Radionuclide Risk, All Pathways - BCL Stockpiles............... 43

15. RESRAD Analysis - Radionuclide Risk, All Pathways - SPA Footprint................. 43

16. Dose Rate to Organs from Groundwater........................................................ 44

\section{TABLES}

1. Number of Statistical Verification Samples for the 118-B-1 Burial Ground................ 12

2. Summary of the 118-B-1 Waste Site COCs/COPCs for ......................................... 15

3. 118-B-1 Focused Verification Sample Summary................................................. 16

4. Comparison of Statistical Contaminant Concentrations to Action Levels for the

118-B-1 Area 1 Verification Sampling Event. ................................................. 19

5. Comparison of Statistical Contaminant Concentrations to Action Levels for the

118-B-1 Area 2 Verification Sampling Event. ............................................... 20

6. Comparison of Statistical Contaminant Concentrations to Action Levels for the

118-B-1 Area 3 Verification Sampling Event. .............................................. 21

7. Comparison of Statistical Contaminant Concentrations to Action Levels for the

118-B-1 Area 4 Verification Sampling Event. .................................................. 23

8. Comparison of Statistical Contaminant Concentrations to Action Levels for the

118-B-1 Area 5 Verification Sampling Event. ............................................... 24

9. Comparison of Statistical Contaminant Concentrations to Action Levels for the

118-B-1 Area 6 Verification Sampling Event.................................................. 25

10. Comparison of Statistical Contaminant Concentrations to Action Levels for the

118-B-1 Area 7 Verification Sampling Event. ................................................ 27

11. Comparison of Statistical Contaminant Concentrations to Action Levels for the

118-B-1 Overburden Verification Sampling Event........................................ 28

12. Comparison of Statistical Contaminant Concentrations to Action Levels for the

118-B-1 BCL Material Stockpiles Verification Sampling Event.......................... 30

13. Comparison of Statistical Contaminant Concentrations to Action Levels for the

118-B-1 Staging Pile Area Footprint Verification Sampling Event. ..................... 32

14. Comparison of Maximum Contaminant Concentrations to Action Levels for the 118-B-1 Focused Verification Samples. ....................................................... 34

15. Estimated Peak Radionuclide Groundwater Concentrations for the 118-B-1

Burial Ground. 


\section{ACRONYMS AND ABBREVIATIONS}

$\begin{array}{ll}\text { BCL } & \text { below cleanup level } \\ \text { BCM } & \text { bank cubic meters } \\ \text { BCY } & \text { bank cubic yards } \\ \text { CFR } & \text { Code of Federal Regulations } \\ \text { COC } & \text { contaminant of concern } \\ \text { COPC } & \text { contaminant of potential concern } \\ \text { DOE-RL } & \text { U.S. Department of Energy Richland Operations Office } \\ \text { DQA } & \text { data quality assessment } \\ \text { EPA } & \text { U.S. Environmental Protection Agency } \\ \text { ERDF } & \text { Environmental Restoration Disposal Facility } \\ \text { ESD } & \text { Explanation of Significant Difference } \\ \text { ICP } & \text { inductively coupled plasma } \\ \text { MCL } & \text { maximum contaminant level (drinking water standard) } \\ \text { PCB } & \text { polychlorinated biphenyl } \\ \text { RAG } & \text { remedial action goal } \\ \text { RDR/RAWP } & \text { remedial design report/remedial action work plan } \\ \text { RESRAD } & \text { RESidual RADioactivity dose assessment model } \\ \text { ROD } & \text { Record of Decision } \\ \text { SAP } & \text { sampling and analysis plan } \\ \text { SPA } & \text { staging pile area } \\ \text { SSI } & \text { site-specific instruction } \\ \text { SNF } & \text { spent nuclear fuel } \\ \text { TPH } & \text { total petroleum hydrocarbons } \\ \text { TSD } & \text { treatment, storage, and disposal } \\ \text { UCL } & \text { upper confidence limit } \\ \text { WAC } & \text { Washington Administrative Code }\end{array}$


CVP-2007-00006

Rev. 0

\subsection{STATEMENT OF PROTECTIVENESS}

This report demonstrates that the 118-B-1 Burial Ground was remediated in accordance with the Record of Decision for the 100-BC-1, 100-BC-2, 100-DR-1, 100-DR-2, 100-FR-2, 100-HR-2, and 100-KR-2 Operable Units, Hanford Site (100 Area Burial Grounds), Benton County, Washington (Burial Grounds ROD) (EPA 2000) and the Explanation of Significant Difference for the Interim Action Record of Decision for the 100-BC-1, 100-BC-2, 100-DR-1, 100-DR-2, 100-FR-2, 100-HR-2, and 100-KR-2 Operable Units (100 Area Burial Grounds) (118-B-1 ESD) (EPA 2007), and meets the objectives and goals for interim closure as established in the Burial Ground ROD and the Remedial Design Report/Remedial Action Work Plan for the 100 Area (RDR/RAWP) (DOE-RL 2005).

The preferred remedy specified in the ROD (EPA 2000) and conducted for the 118-B-1 site included (1) excavating the site to the extent required to meet specified soil cleanup levels, (2) disposing of contaminated excavation materials at the Environmental Restoration Disposal Facility (ERDF) at the 200 Area of the Hanford Site or appropriate treatment, storage, and disposal (TSD) facilities, (3) backfilling the site with overburden and clean soil to average adjacent grade elevation, and (4) planting native vegetation species across the site. Excavation was driven by remedial action objectives for direct exposure, protection of groundwater, and protection of the Columbia River.

The results of verification sampling indicated that vadose zone soils beneath the burial ground contained residual tritium contamination in excess of remedial action objectives for the protection of groundwater. The 118-B-1 ESD (EPA 2007) was approved by the Tri-Parties to leave residual tritium-contaminated soil in-place based on consideration of balancing factors, and includes institutional controls to prohibit future irrigation at the 118-B-1 waste site. The results of verification sampling show that residual concentrations of contaminants other than tritium do not preclude any other future uses (as bounded by the rural-residential scenario) of shallow zone soils (i.e., surface to

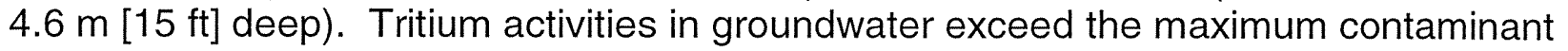
level (MCL) of 20,000 pCi/L. With institutional controls to prevent irrigation, further mobilization of residual vadose zone tritium-contamination is predicted to be prevented to the extent that the MCL will not be exceeded for Columbia River water (EPA 2007). Further, removing this driving force will allow for natural decay of a larger portion of the residual vadose zone tritium inventory before reaching groundwater. Residual concentrations of non-tritium contaminants are protective of groundwater and the Columbia River. The acceptability of direct exposure to residual tritium contamination in the deep vadose zone has not been demonstrated; therefore, institutional controls to prevent uncontrolled drilling or excavation into the deep zone are also required.

Soil cleanup levels were established in the Burial Ground ROD (EPA 2000) based on a limited ecological risk assessment. Although not required by the Burial Ground ROD, a comparison against ecological risk screening levels has been made for the 118-B-1 waste site contaminants of concern (COCs)/contaminants of potential concern (COPCs). Screening levels were not exceeded for the site constituents, with the exception of barium, boron, cadmium, lead, mercury, molybdenum, selenium, and 
vanadium. Exceedance of screening values does not necessarily indicate the existence of risk to ecological receptors. Concentrations of cadmium, vanadium, and selenium are within the range of Hanford Site background levels, and molybdenum concentrations are consistent with those seen elsewhere at the Hanford Site (no established background value is available for molybdenum). Exceedances for barium, boron, lead, and mercury will be evaluated in the context of additional lines of evidence as part of the baseline risk assessment. A more complete quantitative ecological risk assessment will be presented in the baseline risk assessment for the river corridor portion of the Hanford Site and will be used to support the final closeout decision for this waste site.

\subsection{BACKGROUND AND GENERAL SITE INFORMATION}

The 118-B-1 Burial Ground is located in the 100-BC-2 Operable Unit of the Hanford Site

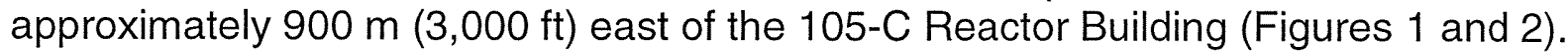
This waste site operated from 1944 to 1973, receiving general radioactive and nonradioactive wastes from the 105-B and 105-N Reactors, construction wastes from modification of the 105-B Reactor Building, and process wastes from the P-10 Tritium Separation Project (Carpenter 1994).

The original burial ground consisted of six to eight trenches oriented in an east-west direction, but was expanded over its operational lifetime to 21 east-west trenches and 2 north-south trenches on the west side of the burial ground, according to historical documentation (Carpenter 1994) (Figure 3). During remediation of the site, it was discovered that 2 of the 23 trenches had not been used for waste disposal. The burial ground also consisted of several spline silos constructed from 3- to 3.7-m (10- to 12-ft) diameter metal culvert piping and 3 spacer pits shored with railroad ties. The overall lateral footprint of the burial ground prior to remediation was approximately $225 \mathrm{~m}$ by 90 $\mathrm{m}(740 \mathrm{ft}$ by $300 \mathrm{ft})$.

A geophysical survey was performed over the 118-B-1 Burial Ground in 1993 (WHC 1993) to locate and map subsurface anomalies, and was validated by limited exploratory excavation in March 2002 (Ludowise 2002). Multiple subsurface anomalous zones were observed, generally consistent with the historical trench layout (Figure 3).

\subsection{REMEDIAL ACTION SUMMARY}

This section contains specific information about the excavation and disposal activities for the 118-B-1 Burial Ground. It also contains information about the types of wastes encountered in the burial ground and the field screening conducted. 


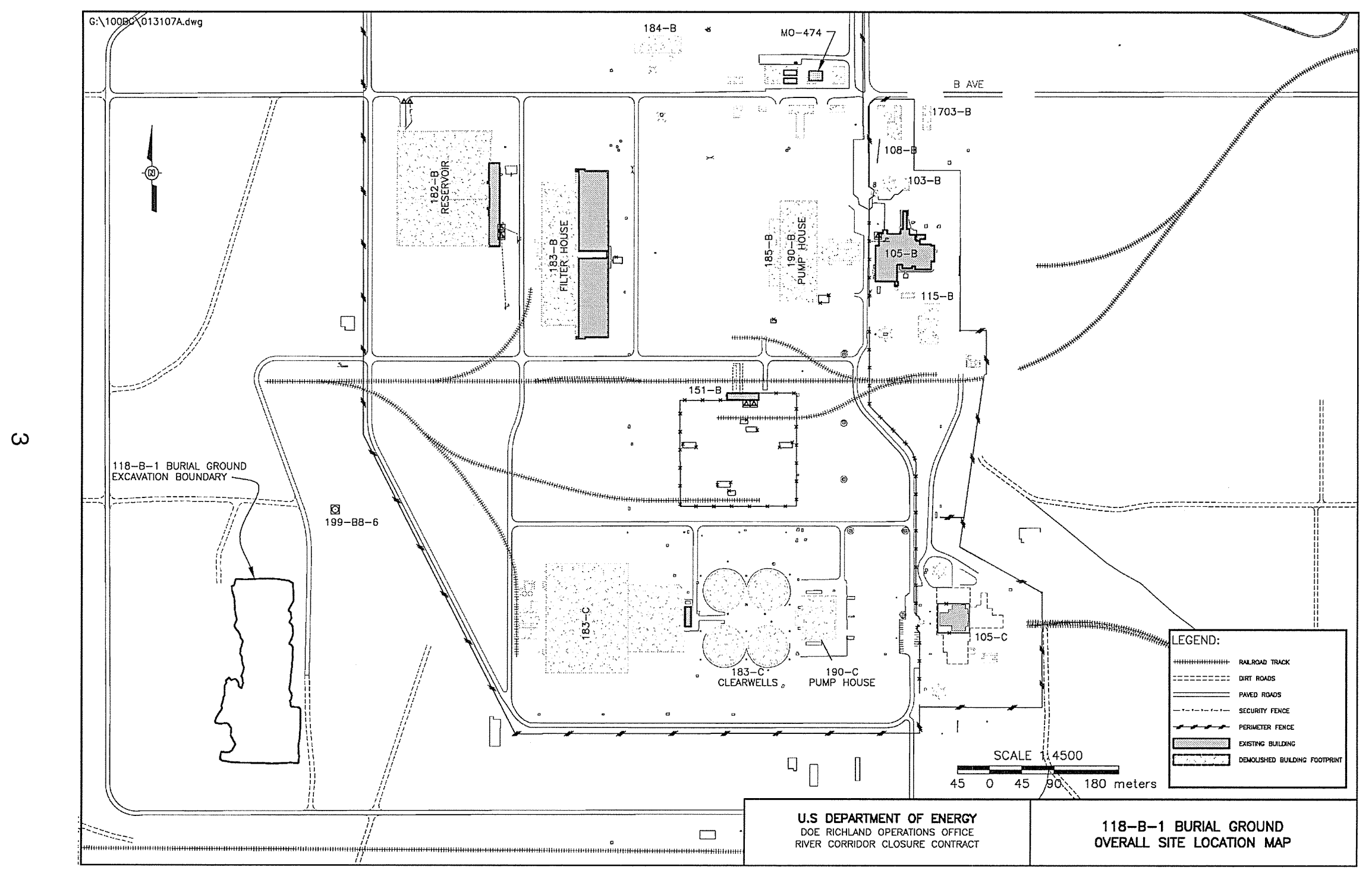

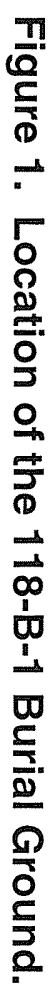


Figure 2. Historical Aerial Photograph of the 100-B/C Area, Including the 118-B-1 Burial Ground.

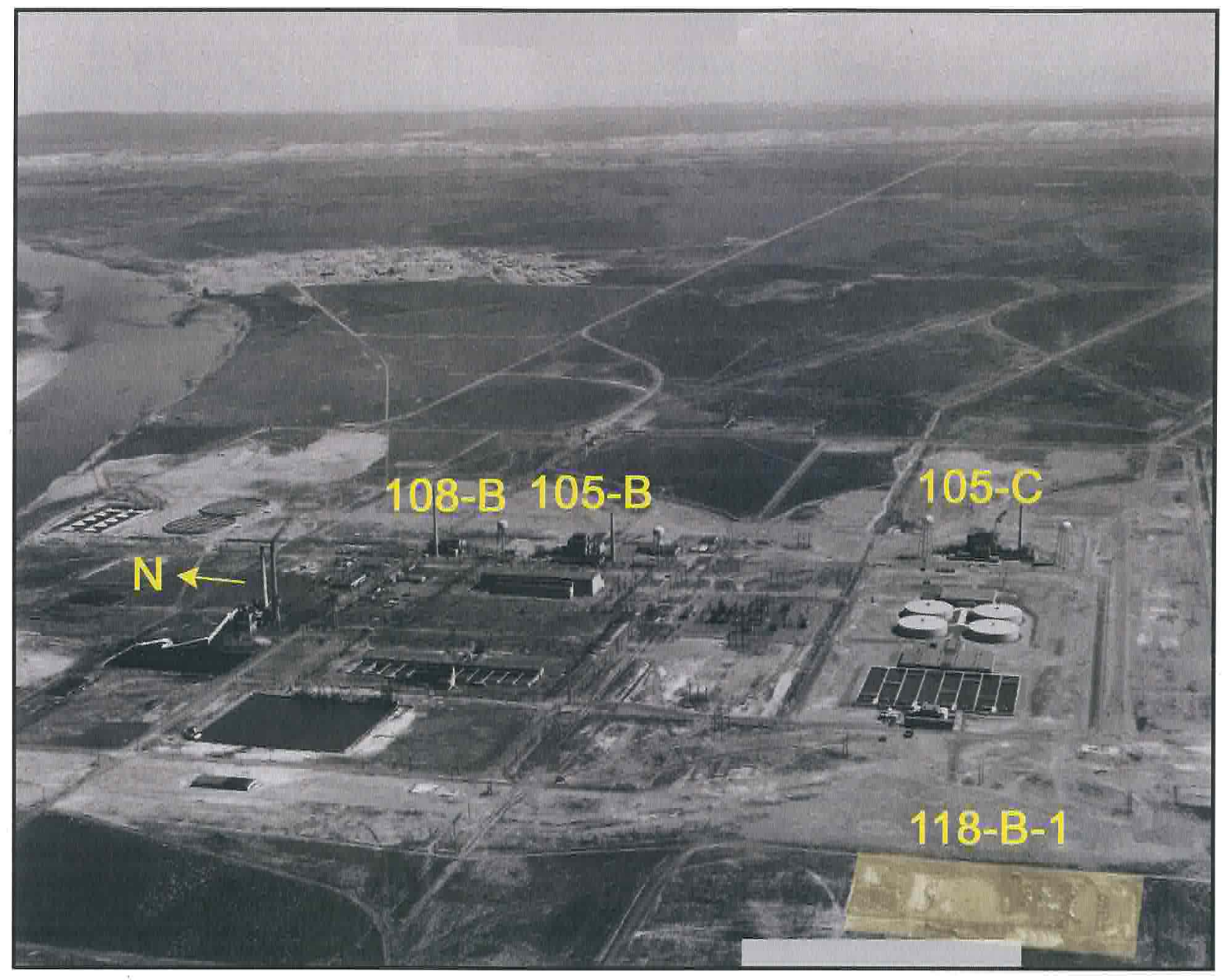

\subsection{EXCAVATION AND DISPOSAL INFORMATION}

Remedial action of the 118-B-1 Burial Ground began on February 2, 2004, with overburden removal. Approximately $20,000 \mathrm{BCM}(26,200 \mathrm{BCY})$ of overburden material was removed before beginning excavation and sorting of the burial ground on March 16, 2004. Suspect spent nuclear fuel (SNF) was discovered on September 15, 2004, and remedial activities suspended until the Authorization Basis could be assessed and revised to address SNF. Load-out operations resumed on April 11, 2005, for previously sorted and segregated material. All remedial activities (excavation, sorting, and loadout) resumed on August 24, 2005, and were completed on June 7, 2007, with the exception of characterization and dispositioning of SNF and approximately 100 remaining anomalous containers. 


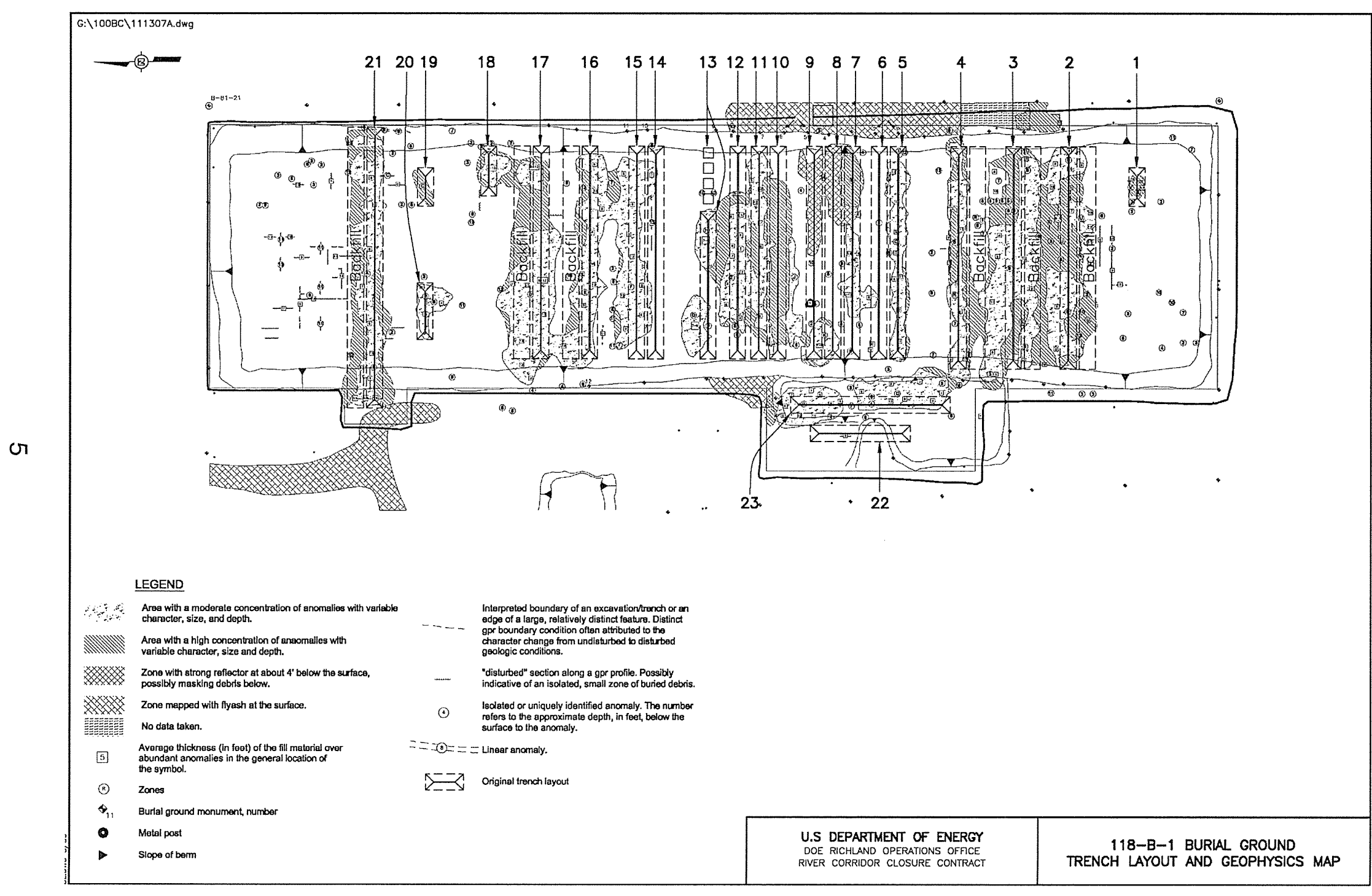

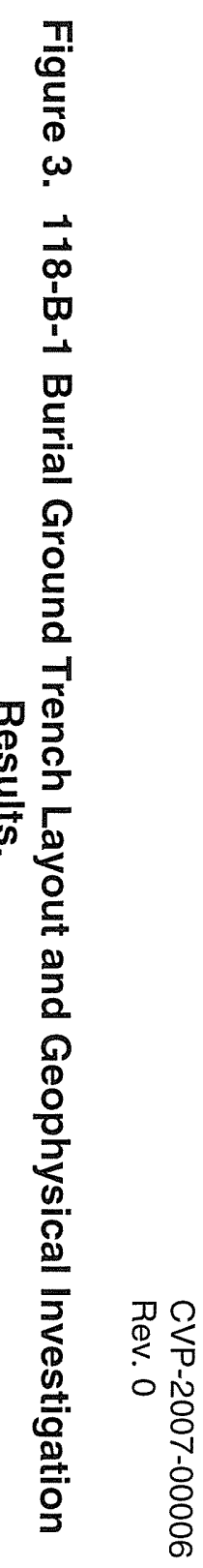


Over 132,300 tons $(120,000$ metric tons) of debris and contaminated soil from the 118-B-1 Burial Ground was removed and disposed at ERDF. The post-excavation topographic survey for the burial ground is shown in Figure 4. At the conclusion of remediation activities, the excavated area was approximately $10 \mathrm{~m}(33 \mathrm{ft})$ at its deepest, with a lateral footprint of approximately $21,600 \mathrm{~m}^{2}$ (5.3 acres). Approximately $136,000 \mathrm{~m}^{2}$ (33.6 acres) in total at the site were disturbed, including stockpiles and waste sorting and staging areas. An aerial photograph taken near the completion of excavation activities is shown in Figure 5.

\subsection{WASTE INFORMATION}

Twenty-three burial trenches were anticipated in the 118-B-1 Burial Ground, based on historical information; geophysical investigations indicated debris in 22 of those trenches. Exploratory excavation was performed at Trench 22, where no indications of buried debris were observed by geophysical instrumentation, and no debris or other waste forms were identified. Excavation at Trench 1 revealed only a large piece of metal, and no further waste forms were encountered. Waste forms encountered in the other 21 trenches included several thousand perforated and non-perforated spacers, spline cases, piping and tubing, miscellaneous metal, tritium furnaces, wax, lead items, mineral oil, reactor parts and hardware, SNF, hydraulic hoses and parts, degraded personal protective equipment, glassware, compressed gas cylinders, friable and nonfriable asbestos containing material, gaskets, metal lathe turnings, dried paints, tar, electrical components, suspect pipe joint compound, and other miscellaneous debris. Photographs of selected waste items are provided in Appendix A.

Additionally, approximately 1,500 anomalous items were discovered during excavation and sorting operations. Approximately 1,000 of these were tubes used for packaging and disposal of tritium gas, approximately 350 were barium desiccant tubes, 81 were tubes used for packaging and disposal of mercury, approximately 50 were glass/plastic bottles, and approximately 70 were unique anomalous containers or solids.

Suspect SNF discovered during remediation activities was staged in two bunkers within the 118-B-1 waste staging pile area. Six pieces of confirmed SNF and two fractional pieces were identified following characterization. This material was transferred to the $100-\mathrm{K}$ fuel storage basins for interim storage prior to final packaging and disposal. 
Figure 4. 118-B-1 Burial Ground Post-Excavation Topographic Survey.

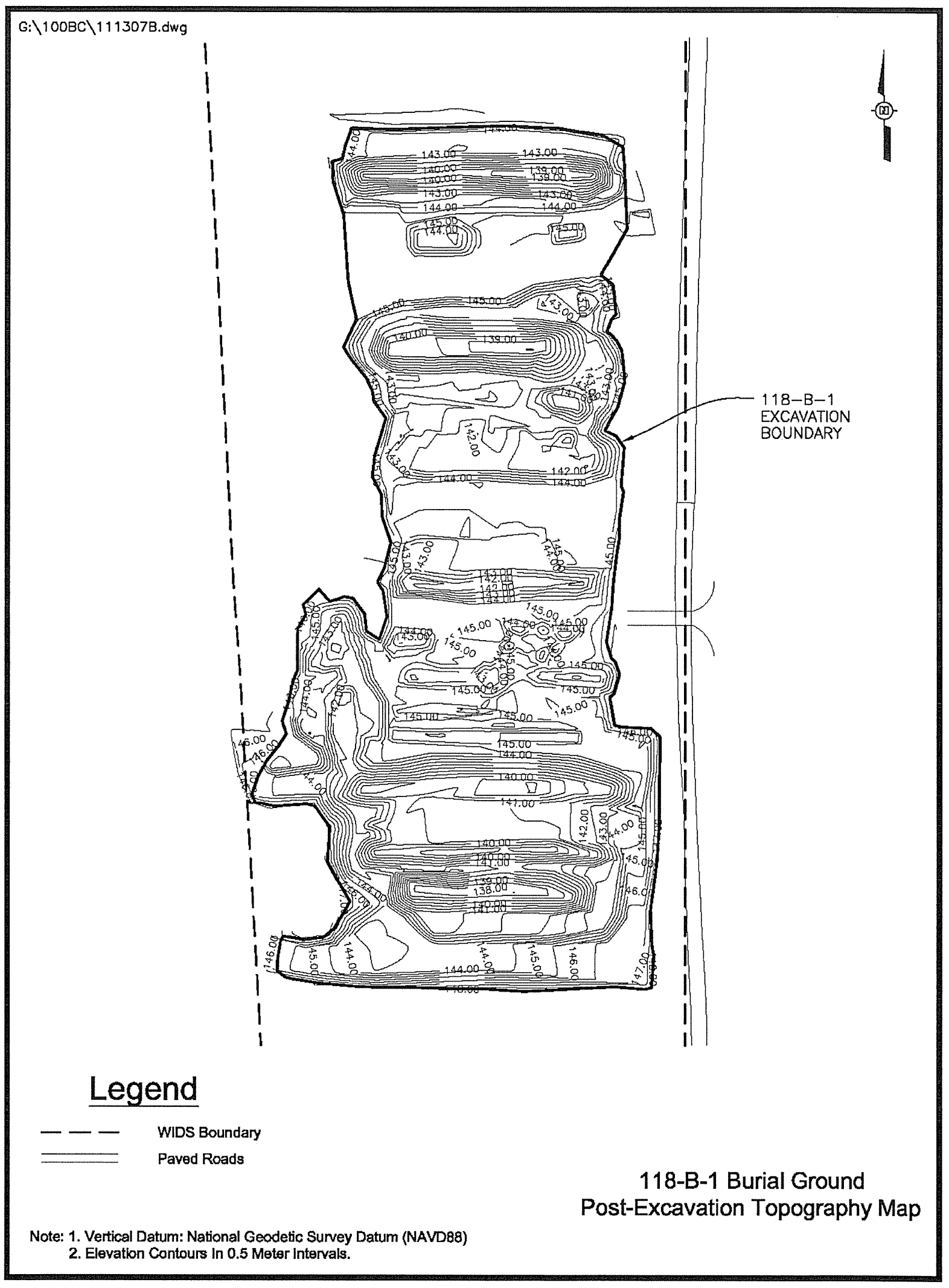


Figure 5. 118-B-1 Burial Ground After Major Excavation Activities (taken November 2006).

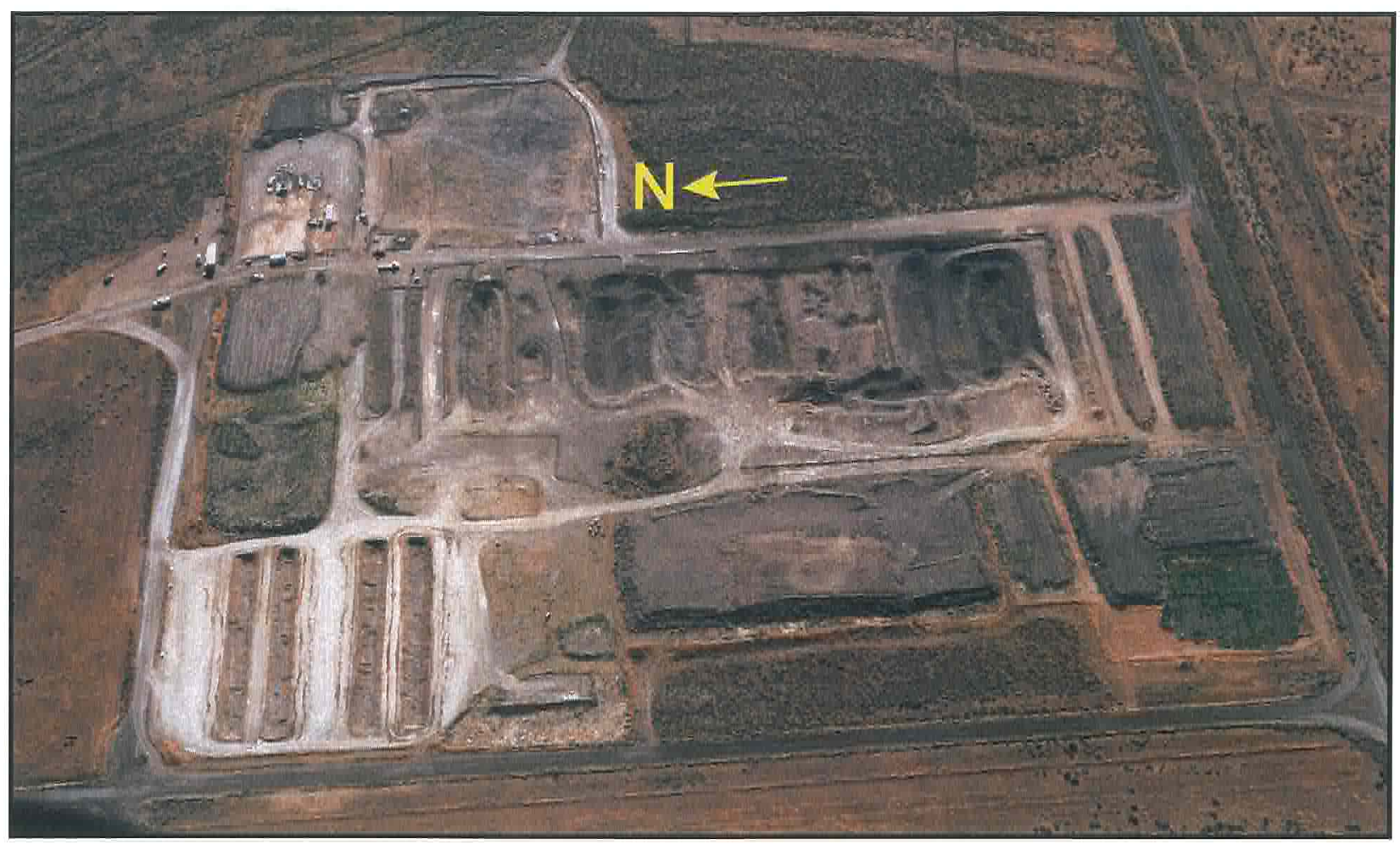

\subsection{FIELD SCREENING}

Radiological field screening was conducted during and after the site remedial actions as specified in the 100 Area Burial Grounds Remedial Action Sampling and Analysis Plan (Burial Ground SAP) (DOE-RL 2001a). Field screening was used to guide the excavation to quickly assess the presence and level of contamination. Field screening at the site included using systematic global positioning radiological data mapping surveys in the excavation, on stockpiled material, and in interstitial areas with instrumentation specific to the detection of radiation associated with gamma and betaemitting radionuclides.

The radiological surveys are provided in Appendix B. Multiple areas of elevated radiological activity requiring additional remediation were identified by the surveys. Additional material was selectively removed from these areas, and follow-up surveys performed using systematic global positioning surveys or hand-held instrumentation to confirm the adequacy of removal. 
CVP-2007-00006

Rev. 0

\subsection{SAMPLING ACTIVITIES}

Following remediation of the 118-B-1 Burial Ground, verification sampling was conducted in July 2006 and October through December 2006. Additional focused verification samples were collected from March to June 2007. Verification sampling is performed to collect data to determine if the remedial action goals (RAGs) have been met. RAGs are the specific numeric goals against which the verification data are evaluated to demonstrate attainment of the remedial action objectives as established in the Burial Ground ROD (EPA 2000) and the RDR/RAWP (DOE-RL 2005). The following subsections provide additional discussion of the information used to develop the COCs and COPCs for verification and focused sampling, as well as the sampling design selection and basis.

\subsection{VERIFICATION SAMPLING DESIGN SELECTION AND BASIS}

This section describes the basis for selection of an appropriate sampling design and the determination of the number of verification samples to collect at the 118-B-1 Burial Ground. Due to the complexity of the 118-B-1 Burial Ground, the standard sampling strategy identified in the Burial Ground SAP (DOE-RL 2001a) was deemed insufficient, and a more robust sampling design was developed, as described in the Site Specific Instruction for Close-out Approach for 118-B-1 (WCH 2006).

For statistical verification sampling of the 118-B-1 Burial Ground remediation footprint, the footprints of the 21 trenches containing waste and surrounding land were grouped into one shallow zone decision unit with seven separate areas based on similarity of the waste forms observed during remediation, as described in the following subsections. In addition to these seven areas, the 118-B-1 waste site included one decision unit for the overburden stockpiles, divided into one area for the true overburden material stockpiles and one area for the below cleanup level $(\mathrm{BCL})$ material stockpiles, and one decision unit/area for the waste staging and sorting area footprints (Figure 6). All ten areas were verified to meet the more restrictive shallow zone cleanup criteria, although the

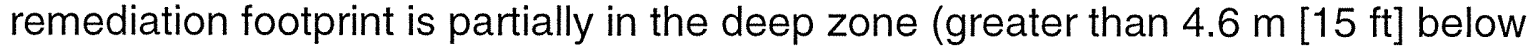
ground surface).

\subsubsection{Area 1}

Area 1 is composed of the footprints of Trenches 2 through 4 . The waste stream observed within these trenches included general debris, perforated and non-perforated spacers, sandblasting material, gearboxes, compressed gas cylinders, small glass containers, mercury tubes, tritium tubes, asbestos, barium desiccant tubes, splines, and SNF. 
Figure 6. 118-B-1 Area Boundaries for Verification Sampling.

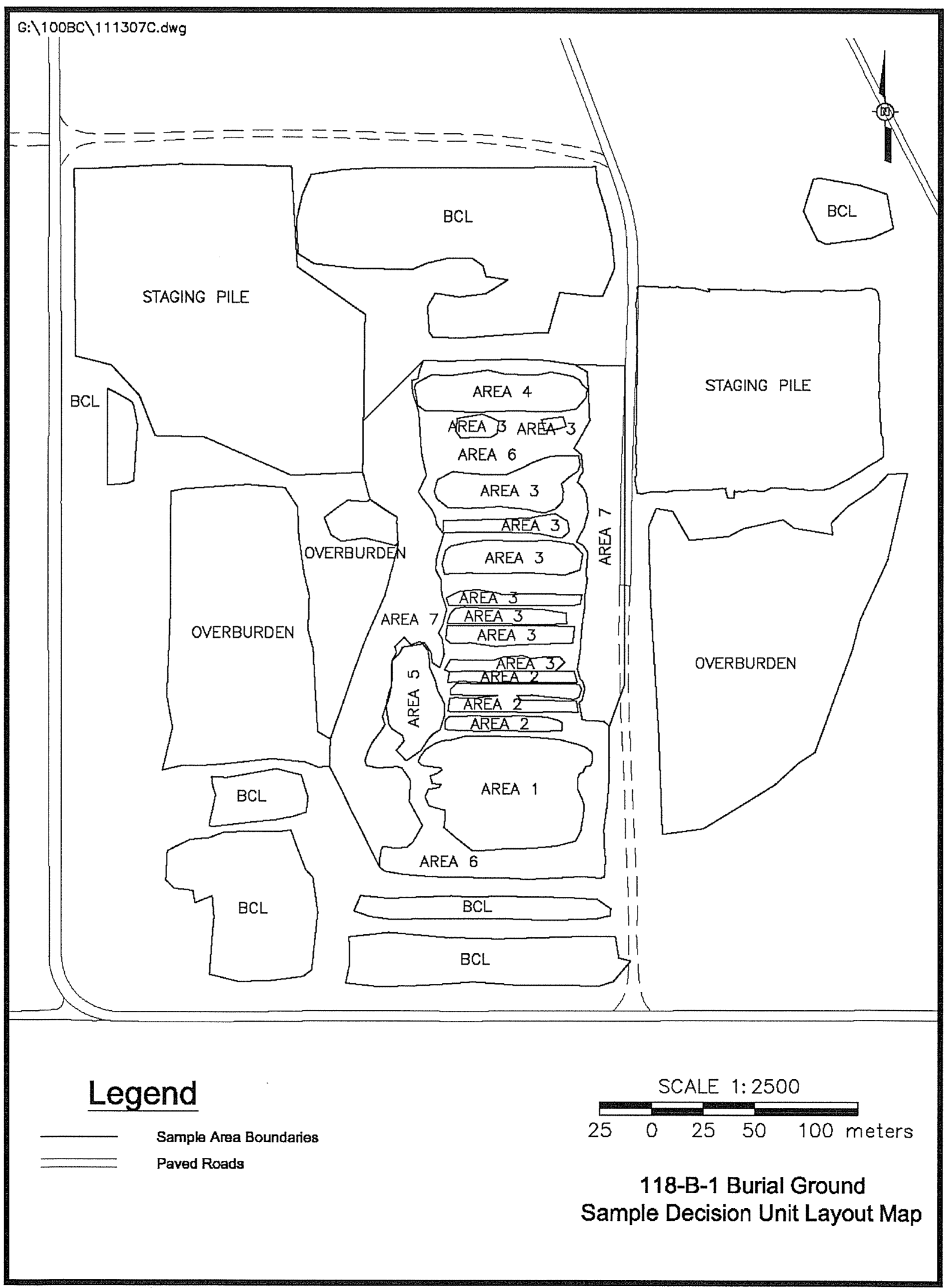




\subsubsection{Area 2}

Area 2 is composed of the footprints of Trenches 5 through 8 . The waste stream observed within these trenches included general debris, perforated and non-perforated spacers, tritium pots, and tritium furnaces.

\subsubsection{Area 3}

Area 3 is composed of the footprints of Trenches 9 through 20. The waste stream observed within these trenches included general debris, perforated and non-perforated spacers, tritium pots, tritium furnaces, small glass containers, mercury tubes, tritium tubes, wax, metal shavings, and a lead-lined cask.

\subsubsection{Area 4}

Area 4 is composed of the footprint of Trench 21. The waste stream observed within this trench included general debris, perforated and non-perforated spacers, ink, small glass containers, gearboxes, suspect herbicides, barium desiccant tubes, and silver slag.

\subsubsection{Area 5}

Area 5 is composed of the footprint of Trench 23. The waste stream observed within this trench included steel railing, a decayed drum containing oil, and elemental mercury.

\subsubsection{Area 6}

Area 6 is composed of the areas between trenches that were not included in Sampling Areas 1 through 5.

\subsubsection{Area 7}

Area 7 is composed of the haul roads between the burial ground and the waste staging and sorting areas.

\subsubsection{Overburden Stockpiles}

The overburden area consists of overburden removed from the 118-B-1. Burial Ground at the beginning of remedial operations as well as the historic spoils stockpiled onsite during excavation of the burial trenches.

\subsubsection{BCL Stockpiles}

The BCL stockpiles area consists of BCL material excavated in the layback between trenches. 


\subsubsection{Staging Pile Area Footprint}

The staging pile area (SPA) decision unit consists of the footprints of two waste staging and sorting areas used during remedial activities.

\subsubsection{Statistical Sampling Design}

The number of sampling areas within each area was determined by the overall footprint size of the area, per the Burial Ground SAP (DOE-RL 2001a). The default number of verification samples for each decision subunit is four (4) composite samples. The required number of verification samples for each of the areas associated with the 118-B-1 waste site is listed in Table 1.

Each statistical verification sample was a composite formed by combining soil collected at four randomly selected nodes within each sampling area. The verification samples were submitted to offsite laboratories for analysis using approved Environmental Protection Agency (EPA) analytical methods, as required by the SAP (DOE-RL 2001a). The sample design methodology and sample location figures are presented in the calculation brief for verification sample design in Appendix C.

Table 1. Number of Statistical Verification Samples for the 118-B-1 Burial Ground.

\begin{tabular}{|c|c|c|c|c|}
\hline Area & Footprint $\left(\mathrm{ft}^{2}\right)$ & $\begin{array}{c}\text { Size Classification } \\
\left(\mathrm{ft}^{2}\right)\end{array}$ & $\begin{array}{c}\text { Decision } \\
\text { Subunits }\end{array}$ & $\begin{array}{c}\text { Verification } \\
\text { Samples }\end{array}$ \\
\hline Area 1 & 40,600 & Small $(<100,000)$ & 1 & 4 composites \\
\hline Area 2 & 16,300 & Small $(<100,000)$ & 1 & 4 composites \\
\hline Area 3 & 50,000 & Small $(<100,000)$ & 1 & 4 composites \\
\hline Area 4 & 15,600 & Small $(<100,000)$ & 1 & 4 composites \\
\hline Area 5 & 11,700 & Small $(<100,000)$ & 1 & 4 composites \\
\hline Area 6 & 99,700 & Small $(<100,000)$ & 1 & 4 composites \\
\hline Area 7 & 99,000 & Small $(<100,000)$ & 1 & 4 composites \\
\hline Overburden Stockpiles & 247,900 & $\begin{array}{c}\text { Medium } \\
(100,000 \text { to } \\
400,000)\end{array}$ & 4 & 16 composites \\
\hline BCL Stockpiles & 215,500 & $\begin{array}{c}\text { Medium } \\
(100,000 \text { to } \\
400,000)\end{array}$ & 4 & 16 composites \\
\hline Staging Pile Area Footprint & 300,100 & $\begin{array}{c}\text { Medium } \\
(100,000 \text { to } \\
400,000)\end{array}$ & 4 & 16 composites \\
\hline
\end{tabular}

$\mathrm{BCL}=$ below cleanup level

\subsubsection{Focused Sampling}

Focused samples were collected to provide confidence for the absence of contamination in residual soil beneath locations exhibiting visual soil stains, buried liquid 
wastes or equipment, or large inventories of dangerous/hazardous wastes (e.g., lead debris and mercury-containing piping). Additionally, focused samples may be collected from locations where process knowledge indicates the potential for elevated concentrations of alpha- or beta-emitter contamination, or where waste characterization sampling results indicate potential elevated concentrations of chemical contaminants above RAGs.

Nineteen focused soil verification samples were collected, in addition to the statistical cleanup verification samples. Eleven of the focused samples were collected within the footprints of anomalous waste items or where high in-process sampling results occurred within the remediation footprint (Figure 7). Two of the focused samples were collected from soil underlying the SNF bunkers located in the SPA. The remaining six samples were collected at the locations of special waste staging areas. Additional description of the selection of focused samples is provided with the discussion of the development of COCs/COPCs, in Section 4.2.2.

\subsubsection{Tritium Plume Characterization Sampling}

In addition to the statistical and focused verification samples collected, eleven potholes were excavated in the remediation footprint and sampled at approximately $1-\mathrm{m}$ (3-ft) intervals to characterize the extent of tritium contamination in the vadose zone underlying the 118-B-1 Burial Ground. One characterization borehole was also completed, including vadose zone and groundwater sampling, at the location of the highest detections of residual tritium. The sampling locations and results of these characterization activities are provided in Appendix D, and were used in the assessment of balancing factors for the 118-B-1 ESD (EPA 2007).

\subsection{CONTAMINANTS OF CONCERN AND CONTAMINANTS OF POTENTIAL CONCERN FOR VERIFICATION SAMPLING}

The SAP (DOE-RL 2001a) identifies the COCs for burial grounds based on waste forms encountered. Additional waste site COCs/COPCs were identified for the 118-B-1 Burial Ground based on process knowledge and results of in-process sampling. Based on the observed waste forms found during remediation activities and the results of waste characterization sampling, COCs/COPCs were identified for each decision unit in the Site Specific Instruction for Close-Out Approach for 118-B-1 (118-B-1 SSI) (WCH 2006). The 118-B-1 SSI documents the agreements between the Department of Energy Richland Operations Office (DOE-RL) and the EPA regarding interim waste site closeout for the 118-B-1 Burial Ground. The COCs/COPCs identified in the 118-B-1 SSI (WCH 2006) include americium-241, carbon-14, cesium-137, cobalt-60, europium152, europium-154, europium-155, nickel-63, plutonium-238, plutonium-239/240, plutonium-241, silver-108m, strontium-90, 
Figure 7. Location of Focused Verification Soil Samples at the 118-B-1 Burial Ground.

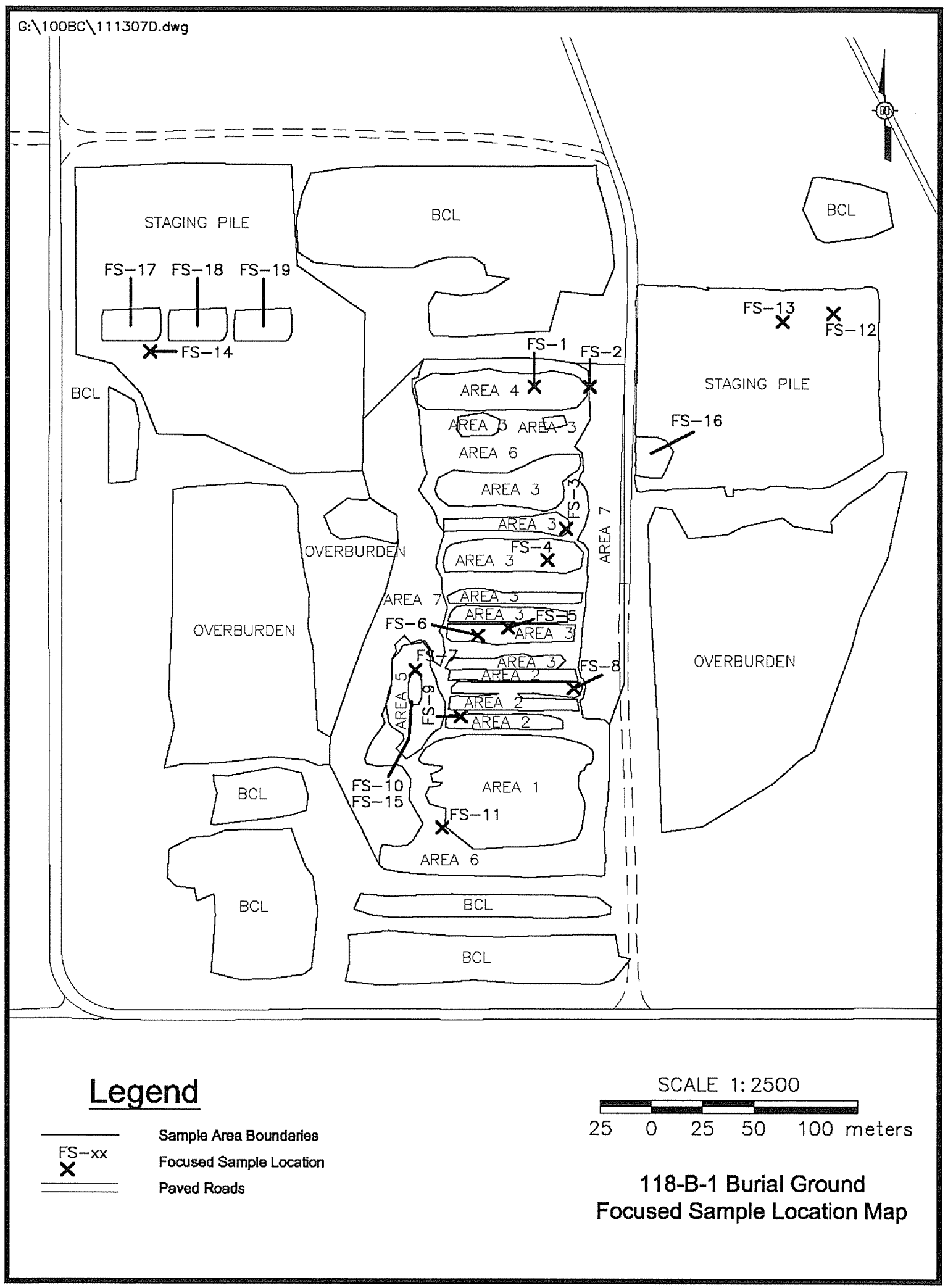


tritium, uranium-233/234, uranium-235, uranium-238, the expanded list of inductively coupled plasma (ICP) metals (arsenic, antimony, barium, beryllium, boron, cadmium, total chromium, cobalt, copper, lead, manganese, molybdenum, nickel, selenium, silver, vanadium, and zinc), hexavalent chromium, mercury, polychlorinated biphenyls (PCBs), total petroleum hydrocarbons (TPH), semi-volatile organic compounds, volatile organic compounds, herbicides, pesticides, and asbestos. The COCs/COPCs detected at the 118-B-1 Burial Ground and their respective points of compliance, the remedial action goals (RAGs), are summarized in Section 5.0 of this cleanup verification package.

\subsubsection{Statistical Sampling}

The remediation footprint, stockpiles, and SPA footprint were divided into ten areas, based on use and the similarity of waste forms observed during remediation, as described in Section 4.1. The COCs/COPCs for the five areas associated with the remediation footprints for the burial trenches were identified based on the waste forms observed (WCH 2006). The COCs/COPCs for the remaining five areas (interstitial areas, haul roads, overburden stockpiles, BCL stockpiles, and the SPA footprint) are inclusive of all COCs/COPCs for the remediation footprint. The COCs/COPCs for each area are listed in Table 2.

Table 2. Summary of the 118-B-1 Waste Site COCs/COPCs for Statistical Verification Sampling. (2 Pages)

\begin{tabular}{|c|c|c|c|c|c|c|}
\hline cOCs/COPCs & Area 1 & Area 2 & Area 3 & Area 4 & Area 5 & $\begin{array}{c}\text { Areas } 6 \& 7, \\
\text { Overburden } \\
\text { Stockpiles, BCL } \\
\text { Stockpiles, and SPA }\end{array}$ \\
\hline \multicolumn{7}{|c|}{ Radionuclides } \\
\hline Americium-241 & $x$ & $\mathrm{X}^{\mathrm{a}}$ & $x^{2}$ & & & $x$ \\
\hline Carbon-14 & $x$ & $x$ & $x$ & $x$ & $x$ & $x$ \\
\hline Gamma-emitters $^{b}$ & $x$ & $x$ & $x$ & $x$ & $x$ & $x$ \\
\hline Nickel-63 & $x$ & $x$ & $x$ & $x$ & $x$ & $x$ \\
\hline Plutonium-238 & $x$ & $X^{a}$ & $x^{a}$ & & & $x$ \\
\hline Plutonium-239/240 & $x$ & $X^{a}$ & $x^{a}$ & & & $x$ \\
\hline Plutonium-241 & $x$ & $x^{a}$ & $x^{a}$ & & & $x$ \\
\hline Strontium-90 & $x$ & $x$ & $x$ & $x$ & $x$ & $x$ \\
\hline Silver-108m & $x$ & $x$ & $x$ & $x$ & $x$ & $x$ \\
\hline Tritium & $x$ & $x$ & $x$ & $x$ & $x$ & $x$ \\
\hline Uranium-233/234 & $x$ & & & & & $x$ \\
\hline Uranium-235 & $x$ & & & & & $x$ \\
\hline Uranium-238 & $x$ & & & & & $x$ \\
\hline
\end{tabular}


Table 2. Summary of the 118-B-1 Waste Site COCs/COPCs for Statistical Verification Sampling. (2 Pages)

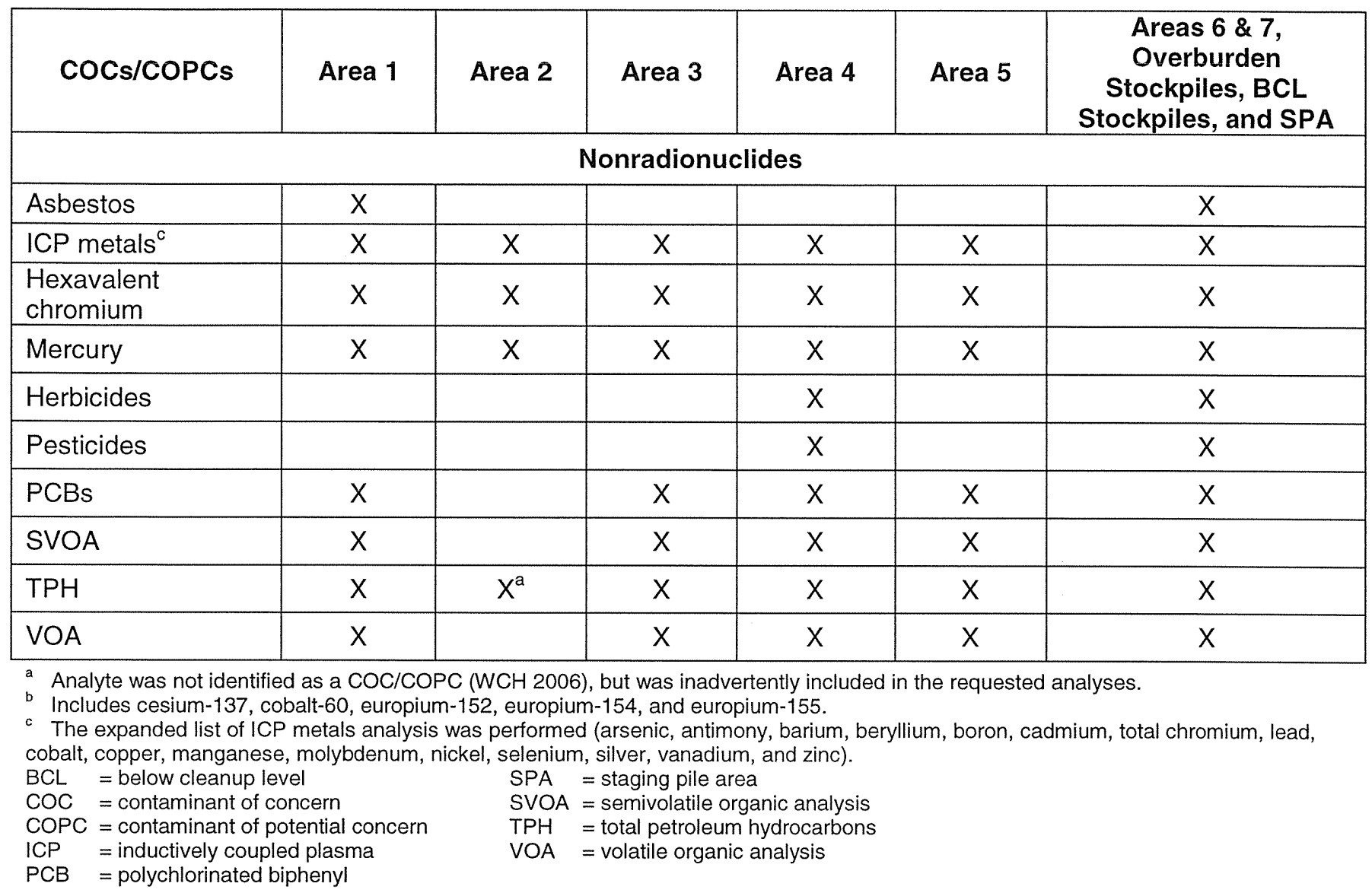

\subsubsection{Focused Sampling}

The COCs/COPCs for focused samples were identified based on waste forms encountered, in-process sampling, and process knowledge (WCH 2006), and are listed in Table 3.

Table 3. 118-B-1 Focused Verification Sample Summary. (3 Pages)

\begin{tabular}{|c|c|c|c|c|}
\hline $\begin{array}{l}\text { Sample } \\
\text { Location }\end{array}$ & $\begin{array}{l}\text { Sample } \\
\text { Number }\end{array}$ & Associated Anomaly & $\begin{array}{l}\text { Coordinate } \\
\text { Locations }^{a}\end{array}$ & COCs/COPCs \\
\hline 1 & J12R24 & $\begin{array}{l}\text { Pesticide/herbicide odor in } \\
\text { Trench } 21\end{array}$ & $\begin{array}{l}\text { N } 144048.4 \\
\text { E 564414.0 }\end{array}$ & $\begin{array}{l}\text { Herbicides, pesticides, PCBs, } \\
\text { SVOA, TPH, and VOA }\end{array}$ \\
\hline 2 & J12R25 & $\begin{array}{l}\text { Cache of barium desiccant } \\
\text { tubes in Trench } 21\end{array}$ & $\begin{array}{l}N 144048.3 \\
\text { E 564441.1 }\end{array}$ & ICP metals ${ }^{b}$ \\
\hline 3 & J12R26 & $\begin{array}{l}\text { Cache of tritium tubes in } \\
\text { Trench } 16\end{array}$ & $\begin{array}{l}N 143978.3 \\
\text { E } 564429.5 \\
\end{array}$ & Tritium and ICP metals ${ }^{b}$ \\
\hline 4 & J12R27 & $\begin{array}{l}\text { Cache of tritium tubes in } \\
\text { Trench } 15\end{array}$ & $\begin{array}{l}\text { N } 143962.9 \\
\text { E } 564420.6\end{array}$ & Tritium \\
\hline 5 & J12R28 & $\begin{array}{l}\text { Cache of mercury tubes in } \\
\text { Trench } 12\end{array}$ & $\begin{array}{l}\text { N } 143929.4 \\
\text { E 564401.6 }\end{array}$ & ICP metals ${ }^{b}$ and mercury \\
\hline
\end{tabular}


CVP-2007-00006

Rev. 0

Table 3. 118-B-1 Focused Verification Sample Summary. (3 Pages)

\begin{tabular}{|c|c|c|c|c|}
\hline $\begin{array}{l}\text { Sample } \\
\text { Location }\end{array}$ & $\begin{array}{l}\text { Sample } \\
\text { Number }\end{array}$ & Associated Anomaly & $\begin{array}{l}\text { Coordinate } \\
\text { Locations }^{a}\end{array}$ & COCs/COPCs \\
\hline 6 & J12R29 & $\begin{array}{l}\text { Cache of lead debris in } \\
\text { Trench } 10\end{array}$ & $\begin{array}{l}N 143925.8 \\
E 564387.2\end{array}$ & ICP metals ${ }^{b}$ \\
\hline 7 & J13P85 & $\begin{array}{l}\text { Mercury-contaminated soil } \\
\text { in Trench } 23\end{array}$ & $\begin{array}{l}N 143908.7 \\
E 564357.0\end{array}$ & ICP metals ${ }^{b}$ and mercury \\
\hline 8 & J12R31 & $\begin{array}{l}\text { Cache of lead debris in } \\
\text { Trench } 7\end{array}$ & $\begin{array}{l}\text { N } 143899.7 \\
\text { E } 564433.3\end{array}$ & ICP metals ${ }^{b}$ \\
\hline 9 & J12R32 & $\begin{array}{l}\text { Cache of lead debris in } \\
\text { Trench } 5\end{array}$ & $\begin{array}{l}\text { N } 143885.7 \\
\text { E } 564378.8\end{array}$ & ICP metals ${ }^{b}$ \\
\hline 10 & J12R33 & $\begin{array}{l}\text { Deteriorated drum } \\
\text { containing mineral oil in } \\
\text { Trench } 23\end{array}$ & $N A^{c}$ & PCBs, SVOA, TPH, and VOA \\
\hline 11 & J12R34 & $\begin{array}{l}\text { Cache of tritium and } \\
\text { mercury tubes in Trench } 2\end{array}$ & $\begin{array}{l}\text { N } 143831.1 \\
\text { E } 564370.1\end{array}$ & Tritium, ICP metals ${ }^{b}$, and mercury \\
\hline 12 & $\mathrm{~J} 14 \mathrm{Y} 75$ & SNF Bunker 1 & $\begin{array}{l}\text { N } 144084.4 \\
\text { E } 564559.6\end{array}$ & $\begin{array}{l}\text { Americium-241, gamma-emitting } \\
\text { radionuclides, plutonium-238, } \\
\text { plutonium-239/240, plutonium-241, } \\
\text { uranium-233/234, uranium-235, and } \\
\text { uranium-238 }\end{array}$ \\
\hline 13 & J14Y76 & SNF Bunker 2 & $\begin{array}{l}N 144080.2 \\
E 564535.2\end{array}$ & $\begin{array}{l}\text { Americium-241, gamma-emitting } \\
\text { radionuclides, plutonium-238, } \\
\text { plutonium-239/240, plutonium-241, } \\
\text { uranium-233/234, uranium-235, and } \\
\text { uranium-238 }\end{array}$ \\
\hline 14 & J12XB3 & $\begin{array}{l}\text { Initial process cell dumping } \\
\text { area }\end{array}$ & $\begin{array}{l}\text { N } 144065.2 \\
\text { E } 564227.9\end{array}$ & $\begin{array}{l}\text { Gamma-emitting radionuclides, ICP } \\
\text { metals }{ }^{\mathrm{b}} \text {, and mercury }\end{array}$ \\
\hline 15 & J13NX4 & $\begin{array}{l}\text { Mercury-contaminated soil } \\
\text { staging area in Trench } 23\end{array}$ & $N A^{d}$ & ICP metals ${ }^{b}$ and mercury \\
\hline 16 & J13P84 & $\begin{array}{l}\text { Mercury-contaminated soil } \\
\text { staging area in waste } \\
\text { staging pile area }\end{array}$ & $N A^{e}$ & ICP metals ${ }^{b}$ and mercury \\
\hline 17 & $\begin{array}{l}\mathrm{J} 14 \mathrm{JJ} 3 / \\
\mathrm{J} 152 \mathrm{M} 1^{\dagger}\end{array}$ & $\begin{array}{l}\text { Mercury-contaminated soil } \\
\text { staging area in west end of } \\
\text { Process Cell } 4\end{array}$ & $N A^{g}$ & $\begin{array}{l}\text { Gamma-emitting radionuclides, } \\
\text { tritium, ICP metals }{ }^{b} \text {, and mercury }\end{array}$ \\
\hline 18 & J14L38 & $\begin{array}{l}\text { Mercury-contaminated soil } \\
\text { staging area in middle of } \\
\text { Process Cell } 4\end{array}$ & $N A^{g}$ & $\begin{array}{l}\text { Gamma-emitting radionuclides, } \\
\text { tritium, ICP metals } \mathrm{s}^{\mathrm{b}} \text {, and mercury }\end{array}$ \\
\hline 19 & J14L39 & $\begin{array}{l}\text { Mercury-contaminated soil } \\
\text { staging area in east end of } \\
\text { Process Cell } 4\end{array}$ & $N A^{g}$ & $\begin{array}{l}\text { Gamma-emitting radionuclides, } \\
\text { tritium, ICP metals }{ }^{b} \text {, and mercury }\end{array}$ \\
\hline
\end{tabular}

Sources: Field logbooks EFL-1173-8, EFL-1173-10, EFL-1173-11, EFL-1173-12, and EFL-1173-13 (WCH 2007a, 2007b, 2007c, 2007d, 2007e)

a Washington State Plane, South Zone meters.

b Samples were analyzed for the expanded ICP metal list including antimony, arsenic, barium, beryllium, boron, cadmium, chromium, cobalt, copper, lead, manganese, molybdenum, nickel, selenium, vanadium, silver, and zinc.

c Sample location adjusted from that listed in WCH (2006), due to significant topography change following loadout of mercurycontaminated soils. Sample was collected by combining multiple aliquots from the base of the trench excavation in the area into one sample.

d Sample was collected by combining multiple aliquots from the excavated staging area into one sample. 
Table 3. 118-B-1 Focused Verification Sample Summary. (3 Pages)

\footnotetext{
e Sample was collected by combining multiple aliquots from areas with elevated field XRF mercury measurements into one sample.

$f$ Results for Sample J14JJ3 indicated residual mercury contamination in excess of cleanup criteria. Additional material was removed and replacement Sample J152M1 was collected and analyzed for tritium, ICP metals, and mercury.

g Samples collected beneath staged mercury-contaminated soil in Process Cell 4 were collected by combining multiple aliquots from areas with elevated field XRF mercury measurements into one sample.

$\mathrm{COC}=$ contaminant of concern

$\mathrm{COPC}=$ contaminant of potential concern

$\mathrm{ICP}=$ inductively coupled plasma

NA $=$ not applicable

PCB = polychlorinated biphenyl

SNF = spent nuclear fuel

SVOA = semivolatile organic analysis

$\mathrm{TPH}=$ total petroleum hydrocarbons

$\mathrm{VOA}=$ volatile organic analysis

$\mathrm{XRF}=\mathrm{x}$-ray fluorescence
}

\subsection{SAMPLING RESULTS}

All verification samples were submitted to offsite laboratories for analysis using approved U.S. Environmental Protection Agency (EPA) analytical methods, as required per the Burial Ground SAP (DOE-RL 2001a). The laboratory-reported data results from the verification sampling were used in the statistical calculations and are included with the $95 \%$ upper confidence limit (UCL) calculations in Appendix C. The laboratoryreported data for the focused samples are included with the supporting data attached to the $95 \%$ UCL calculations.

The primary statistical calculation to evaluate compliance with cleanup standards is the $95 \%$ UCL on the arithmetic mean of the data. The $95 \%$ UCL values for each COC/COPC are computed for each of the 118-B-1 Burial Ground areas as specified by the RDR/RAWP (DOE-RL 2005), with calculations provided in Appendix C. Prior to calculating the $95 \% \cup C L$, the individual sample results are reviewed and, as appropriate, adjusted per the SAP (DOE-RL 2001a) and RDR/RAWP (DOE-RL 2005). When a nonradionuclide COC was detected in fewer than $50 \%$ of the verification samples collected, the maximum detected value was used for comparison against the RAGs. Statistical evaluations were not performed on data sets with no reported detections. Evaluation of the verification data from the focused samples was performed by direct comparison of the maximum sample result from all focused samples for each COC/COPC against cleanup criteria.

Comparisons of the statistical results for site COCs/COPCs with the RAGs (cleanup criteria) for each of the 118-B-1 Burial Ground areas are listed in Tables 4 through 13. Comparison of the maximum results for COCs/COPCs detected in focused verification samples with the RAGs are listed in Table 14. Calculated cleanup levels are not presented in the Cleanup Levels and Risk Calculations (CLARC) Database (Ecology 2005) under the Washington Administrative Code (WAC) 173-340-740(3) for aluminum, calcium, iron, magnesium, potassium, silicon, and sodium; therefore, these constituents are not considered site COCs/COPCs. Potassium-40, radium-226, radium-228, 
thorium-228, and thorium-232 were detected in samples collected at the site, but are not considered within the statistical calculations. These isotopes are naturally occurring, not related to the operational history of the site, and/or were detected below background levels. All individual cleanup verification sample results are included with the $95 \% \mathrm{UCL}$ calculation in Appendix C.

Initial data results from Area 5 and the SPA footprint indicated the presence of cesium137 and tritium, respectively, at concentrations exceeding the cleanup criteria in one sampling area of each decision unit. Consequently, additional soil was removed from these areas and the areas re-sampled and analyzed for gamma-emitting radionuclides and tritium, respectively. The second analyses for cesium-137 and tritium were used to perform the 95\% UCL calculations for Area 5 and the SPA, respectively, the results of which are presented in Tables 8 and 13.

Initial focused sample data results from the western end of the mercury-contaminated soil staging area in Process Cell 4 (Focused Sample 17) indicated the presence of residual mercury at a concentration exceeding the cleanup criteria. Consequently, additional soil was removed from this area and the area re-sampled and analyzed for tritium, ICP metals, and mercury. The second analysis for mercury was used in evaluation of the focused sample results, and the maximum results for tritium and ICP metals from each sampling event were used in the evaluation, the results of which are presented in Table 14.

A portion of the verification data for the herbicide 2,4-DB in the SPA footprint were rejected due to data quality deficiencies. The maximum detected accepted value was used for evaluation purposes in the SPA footprint, with additional discussion provided in the data quality assessment (DQA) (Appendix E).

Table 4. Comparison of Statistical Contaminant Concentrations to Action Levels for the 118-B-1 Area 1 Verification Sampling Event.. ${ }^{*}$ (2 Pages)

\begin{tabular}{|c|c|c|c|c|c|c|}
\hline \multirow[b]{2}{*}{ COC/COPC } & \multirow[b]{2}{*}{$\begin{array}{c}\text { Statistical } \\
\text { Result } \\
(\mathrm{pCi} / \mathrm{g})\end{array}$} & \multicolumn{3}{|c|}{ 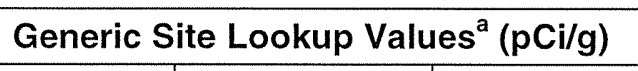 } & \multirow{2}{*}{$\begin{array}{l}\text { Does the } \\
\text { Statistical } \\
\text { Result } \\
\text { Exceed } \\
\text { Lookup } \\
\text { Values? }\end{array}$} & \multirow[b]{2}{*}{$\begin{array}{l}\text { Does the } \\
\text { Statistical } \\
\text { Result Pass } \\
\text { RESRAD } \\
\text { Modeling? }\end{array}$} \\
\hline & & $\begin{array}{l}\text { Shallow } \\
\text { Zone } \\
\text { Lookup } \\
\text { Value }^{b}\end{array}$ & $\begin{array}{l}\text { Groundwater } \\
\text { Protection } \\
\text { Lookup } \\
\text { Value }\end{array}$ & $\begin{array}{l}\text { River } \\
\text { Protection } \\
\text { Lookup } \\
\text { Value }\end{array}$ & & \\
\hline Carbon-14 & 1.93 & 5.16 & $--^{c}$ & $-^{c}$ & No & \multirow{5}{*}{ Yes $^{e}$} \\
\hline Cesium-137 & 0.201 & 6.2 & $1,465^{\mathrm{d}}$ & $1,465^{d}$ & No & \\
\hline Cobalt-60 & 0.039 & 1.4 & $13,900^{d}$ & $13,900^{d}$ & No & \\
\hline Europium-152 & 0.695 & 3.3 & $--^{c}$ & $--^{c}$ & No & \\
\hline Strontium-90 & 0.125 & 4.5 & $27.6^{d}$ & $27.6^{d}$ & No & \\
\hline Tritium & 158 & $459^{d}$ & $12.6^{d}$ & $12.6^{d}$ & Yes & $-n^{f}$ \\
\hline Uranium-233/234 & $0.566(<B G)$ & $1.1^{\mathrm{g}}$ & $1.1^{\mathrm{g}}$ & $1.1^{\mathrm{g}}$ & No & - \\
\hline Uranium-235 & $0.051(<B G)$ & $0.61^{c}$ & $0.5^{h}$ & $0.5^{h}$ & No & -- \\
\hline Uranium-238 & $0.518(<B G)$ & $1.1^{g}$ & $1.1^{9}$ & $1.1^{9}$ & No & -- \\
\hline
\end{tabular}


Table 4. Comparison of Statistical Contaminant Concentrations to Action Levels for the 118-B-1 Area 1 Verification Sampling Event. ${ }^{*}$ (2 Pages)

\begin{tabular}{|c|c|c|c|c|c|c|}
\hline \multirow[b]{2}{*}{ COC/COPC } & \multirow[b]{2}{*}{$\begin{array}{c}\text { Statistical } \\
\text { Result } \\
\text { (mg/kg) }\end{array}$} & \multicolumn{3}{|c|}{ Remedial Action Goals ${ }^{\mathrm{a}}(\mathrm{mg} / \mathrm{kg}$ ) } & \multirow[b]{2}{*}{$\begin{array}{l}\text { Does the } \\
\text { Statistical } \\
\text { Data Set } \\
\text { Exceed } \\
\text { RAGs? }\end{array}$} & \multirow[b]{2}{*}{$\begin{array}{c}\text { Does the } \\
\text { Statistical } \\
\text { Result Pass } \\
\text { RESRAD } \\
\text { Modeling? }\end{array}$} \\
\hline & & $\begin{array}{c}\text { Direct } \\
\text { Exposure }\end{array}$ & $\begin{array}{l}\text { Soil Cleanup } \\
\text { Level for } \\
\text { Groundwater } \\
\text { Protection }\end{array}$ & $\begin{array}{c}\text { Soil } \\
\text { Cleanup } \\
\text { Level for } \\
\text { River } \\
\text { Protection }\end{array}$ & & \\
\hline Arsenic & $3.2(<B G)$ & 20 & 20 & 20 & No & -- \\
\hline Barium & $60.4(<B G)$ & $5,600^{i}$ & $132^{\mathrm{i}, \mathrm{j}}$ & $224^{i}$ & No & -- \\
\hline Beryllium & $0.60(<B G)$ & $10.4^{k}$ & $1.51^{j}$ & $1.51^{i}$ & No & -- \\
\hline Boron' & 1.4 & 16,000 & 320 & $--^{m}$ & No & $-\cdots$ \\
\hline Chromium (total) & $7.1(<\mathrm{BG})$ & $80,000^{i}$ & $18.5^{j}$ & $18.5^{\mathrm{j}}$ & No & -- \\
\hline Cobalt & $10.2(<B G)$ & 1,600 & 32 & $--^{m}$ & No & -- \\
\hline Copper & $17.7(<\mathrm{BG})$ & 2,960 & 59.2 & $22.0^{i}$ & No & - \\
\hline Lead & $4.8(<B G)$ & 353 & $10.2^{j}$ & $10.2^{j}$ & No & -- \\
\hline Manganese & $381(<B G)$ & 11,200 & $512^{i}$ & $512^{j}$ & No & -- \\
\hline Mercury & 1.3 & 24 & $0.33^{i}$ & $0.33^{i}$ & Yes & Yes $^{n}$ \\
\hline Nickel & $11.2(<\mathrm{BG})$ & 1,600 & $19.1^{j}$ & 27.4 & No & - \\
\hline Vanadium & $52.0(<B G)$ & 560 & $85.1^{j}$ & $-\mathrm{-m}^{\mathrm{m}}$ & No & -- \\
\hline Zinc & $45.8(<\mathrm{BG})$ & 24,000 & 480 & $67.8^{j}$ & No & -- \\
\hline bis(2-Ethylhexyl)phthalate & 0.19 & 71.4 & 0.625 & 0.36 & No & -- \\
\hline Di-n-butylphthalate & 0.12 & 8,000 & 160 & 540 & No & -- \\
\hline 4-Methyl-2-pentanone & 0.006 & 6,400 & 64 & $--^{m}$ & No & -- \\
\hline Acetone & 0.014 & 72,000 & 720 & $--^{m}$ & No & -- \\
\hline Methylene chloride & 0.019 & 133 & 0.5 & 0.94 & No & -- \\
\hline
\end{tabular}

${ }^{*}$ All footnotes are provided following Table 14.

= not applicable

BG = background (obtained from DOE-RL 1996 and DOE-RL 2001b, unless otherwise noted)

$\mathrm{COC}=$ contaminant of concern

COPC = contaminant of potential concern

RAG = remedial action goal

RESRAD = RESidual RADioactivity (dose-assessment model)

Table 5. Comparison of Statistical Contaminant Concentrations to Action Levels for the 118-B-1 Area 2 Verification Sampling Event. ${ }^{*}$ (2 Pages)

\begin{tabular}{|c|c|c|c|c|c|c|}
\hline \multirow[b]{2}{*}{ COC/COPC } & \multirow[b]{2}{*}{$\begin{array}{c}\text { Statistical } \\
\text { Result } \\
(\mathrm{pCi} / \mathrm{g})\end{array}$} & \multicolumn{3}{|c|}{ Generic Site Lookup Values $^{a}(\mathrm{pCi} / \mathrm{g})$} & \multirow{2}{*}{$\begin{array}{l}\text { Does the } \\
\text { Statistical } \\
\text { Result } \\
\text { Exceed } \\
\text { Lookup } \\
\text { Values? }\end{array}$} & \multirow[b]{2}{*}{$\begin{array}{l}\text { Does the } \\
\text { Statistical } \\
\text { Result Pass } \\
\text { RESRAD } \\
\text { Modeling? }\end{array}$} \\
\hline & & $\begin{array}{l}\text { Shallow } \\
\text { Zone } \\
\text { Lookup } \\
\text { Value }^{b}\end{array}$ & $\begin{array}{l}\text { Groundwater } \\
\text { Protection } \\
\text { Lookup } \\
\text { Value }\end{array}$ & $\begin{array}{l}\text { River } \\
\text { Protection } \\
\text { Lookup } \\
\text { Value }\end{array}$ & & \\
\hline Cesium-137 & 0.163 & 6.2 & $1,465^{d}$ & $1,465^{d}$ & No & \multirow{2}{*}{$Y_{e s}^{e}$} \\
\hline Strontium-90 & 0.172 & 4.5 & $27.6^{d}$ & $27.6^{d}$ & No & \\
\hline Tritium & 39.4 & $459^{d}$ & $12.6^{d}$ & $12.6^{d}$ & Yes & $-a^{f}$ \\
\hline
\end{tabular}


Table 5. Comparison of Statistical Contaminant Concentrations to Action Levels for the 118-B-1 Area 2 Verification Sampling Event.. ${ }^{*}$ (2 Pages)

\begin{tabular}{|c|c|c|c|c|c|c|}
\hline \multirow[b]{2}{*}{ COC/COPC } & \multirow[b]{2}{*}{$\begin{array}{c}\text { Statistical } \\
\text { Result } \\
\text { (mg/kg) }\end{array}$} & \multicolumn{3}{|c|}{ Remedial Action Goals ${ }^{\mathrm{a}}(\mathrm{mg} / \mathrm{kg})$} & \multirow[b]{2}{*}{$\begin{array}{l}\text { Does the } \\
\text { Statistical } \\
\text { Data Set } \\
\text { Exceed } \\
\text { RAGs? }\end{array}$} & \multirow[b]{2}{*}{$\begin{array}{l}\text { Does the } \\
\text { Statistical } \\
\text { Result Pass } \\
\text { RESRAD } \\
\text { Modeling? }\end{array}$} \\
\hline & & $\begin{array}{c}\text { Direct } \\
\text { Exposure }\end{array}$ & $\begin{array}{l}\text { Soil Cleanup } \\
\text { Level for } \\
\text { Groundwater } \\
\text { Protection }\end{array}$ & $\begin{array}{l}\text { Soil } \\
\text { Cleanup } \\
\text { Level for } \\
\text { River } \\
\text { Protection }\end{array}$ & & \\
\hline Arsenic & $2.9(<B G)$ & 20 & 20 & 20 & No & $-\cdots$ \\
\hline Barium & $58.1(<B G)$ & $5,600^{i}$ & $132^{i, j}$ & $224^{i}$ & No & -- \\
\hline Beryllium & $0.64(<B G)$ & $10.4^{\mathrm{k}}$ & $1.51^{j}$ & $1.51^{\mathrm{j}}$ & No & -- \\
\hline Boron' & 4.7 & 16,000 & 320 & $--^{m}$ & No & -- \\
\hline Chromium (total) & $6.7(<\mathrm{BG})$ & $80,000^{i}$ & $18.5^{\mathrm{j}}$ & $18.5^{\mathrm{i}}$ & No & -- \\
\hline Chromium (hexavalent) & 0.29 & 2.1 & $4.8^{\circ}$ & 2 & No & -- \\
\hline Cobalt & $8.5(<B G)$ & 1,600 & 32 & $--^{m}$ & No & -- \\
\hline Copper & $16.6(<B G)$ & 2,960 & 59.2 & $22.0^{\mathrm{j}}$ & No & -- \\
\hline Lead & $4.8(<B G)$ & 353 & $10.2^{\mathrm{j}}$ & $10.2^{\mathrm{i}}$ & No & -- \\
\hline Manganese & $321(<B G)$ & 11,200 & $512^{i}$ & $512^{j}$ & No & -- \\
\hline Nickel & $10.5(<B G)$ & 1,600 & $19.1^{\mathrm{i}}$ & 27.4 & No & -- \\
\hline Vanadium & $54.5(<B G)$ & 560 & $85.1^{j}$ & $-{ }^{m}$ & No & - \\
\hline Zinc & $43.2(<B G)$ & 24,000 & 480 & $67.8^{j}$ & No & -- \\
\hline
\end{tabular}

${ }^{*}$ All footnotes are provided following Table 14.

= not applicable

BG = background (obtained from DOE-RL 1996 and DOE-RL 2001b, unless otherwise noted)

$\mathrm{COC}=$ contaminant of concern

COPC = contaminant of potential concern

$\mathrm{RAG} \quad=$ remedial action goal

RESRAD = RESidual RADioactivity (dose-assessment model)

Table 6. Comparison of Statistical Contaminant Concentrations to Action Levels for the 118-B-1 Area 3 Verification Sampling Event.. ${ }^{*}$ (2 Pages)

\begin{tabular}{|c|c|c|c|c|c|c|}
\hline \multirow[b]{2}{*}{$\mathrm{COC} / \mathrm{COPC}$} & \multirow[b]{2}{*}{$\begin{array}{c}\text { Statistical } \\
\text { Result } \\
\text { (pCi/g) }\end{array}$} & \multicolumn{3}{|c|}{ 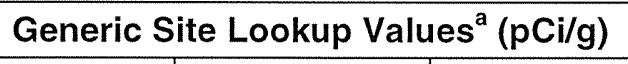 } & \multirow{2}{*}{$\begin{array}{l}\text { Does the } \\
\text { Statistical } \\
\text { Result } \\
\text { Exceed } \\
\text { Lookup } \\
\text { Values? }\end{array}$} & \multirow[b]{2}{*}{$\begin{array}{l}\text { Does the } \\
\text { Statistical } \\
\text { Result Pass } \\
\text { RESRAD } \\
\text { Modeling? }\end{array}$} \\
\hline & & $\begin{array}{l}\text { Shallow } \\
\text { Zone } \\
\text { Lookup } \\
\text { Value }^{b}\end{array}$ & $\begin{array}{l}\text { Groundwater } \\
\text { Protection } \\
\text { Lookup } \\
\text { Value }\end{array}$ & $\begin{array}{l}\text { River } \\
\text { Protection } \\
\text { Lookup } \\
\text { Value }\end{array}$ & & \\
\hline Cesium-137 & 0.776 & 6.2 & $1,465^{d}$ & $1,465^{d}$ & No & \multirow{2}{*}{ Yes $^{e}$} \\
\hline Strontium-90 & 2.56 & 4.5 & $27.6^{d}$ & $27.6^{d}$ & No & \\
\hline Tritium & 12.8 & $459^{d}$ & $12.6^{d}$ & $12.6^{d}$ & Yes & $--^{f}$ \\
\hline
\end{tabular}


Rev. 0

Table 6. Comparison of Statistical Contaminant Concentrations to Action Levels for the 118-B-1 Area 3 Verification Sampling Event.* (2 Pages)

\begin{tabular}{|c|c|c|c|c|c|c|}
\hline \multirow[b]{2}{*}{ COC/COPC } & \multirow[b]{2}{*}{$\begin{array}{c}\text { Statistical } \\
\text { Result } \\
(\mathrm{mg} / \mathrm{kg})\end{array}$} & \multicolumn{3}{|c|}{ Remedial Action Goals ${ }^{\mathrm{a}}$ (mg/kg) } & \multirow[b]{2}{*}{$\begin{array}{l}\text { Does the } \\
\text { Statistical } \\
\text { Data Set } \\
\text { Exceed } \\
\text { RAGs? }\end{array}$} & \multirow[b]{2}{*}{$\begin{array}{c}\text { Does the } \\
\text { Statistical } \\
\text { Result Pass } \\
\text { RESRAD } \\
\text { Modeling? }\end{array}$} \\
\hline & & $\begin{array}{c}\text { Direct } \\
\text { Exposure }\end{array}$ & $\begin{array}{l}\text { Soil Cleanup } \\
\text { Level for } \\
\text { Groundwater } \\
\text { Protection }\end{array}$ & $\begin{array}{l}\text { Soil } \\
\text { Cleanup } \\
\text { Level for } \\
\text { River } \\
\text { Protection }\end{array}$ & & \\
\hline Arsenic & $3.7(<\mathrm{BG})$ & 20 & 20 & 20 & No & -- \\
\hline Barium & $62.6(<B G)$ & $5,600^{i}$ & $132^{i, j}$ & $224^{i}$ & No & -- \\
\hline Beryllium & $0.63(<B G)$ & $10.4^{k}$ & $1.51^{j}$ & $1.51^{\mathrm{j}}$ & No & -- \\
\hline Boron' & 6.5 & 16,000 & 320 & $--^{m}$ & No & - \\
\hline Chromium (total) & $7.7(<B G)$ & $80,000^{i}$ & $18.5^{\mathrm{j}}$ & $18.5^{\mathrm{j}}$ & No & -- \\
\hline Chromium (hexavalent) & 0.21 & 2.1 & $4.8^{\circ}$ & 2 & No & -- \\
\hline Cobalt & $9.1(<B G)$ & 1,600 & 32 & $--^{m}$ & No & - \\
\hline Copper & $17.1(<B G)$ & 2,960 & 59.2 & $22.0^{\mathrm{j}}$ & No & -- \\
\hline Lead & $5.7(<B G)$ & 353 & $10.2^{j}$ & $10.2^{j}$ & No & -- \\
\hline Manganese & $367(<B G)$ & 11,200 & $512^{j}$ & $512^{j}$ & No & -- \\
\hline Mercury & $0.21(<B G)$ & 24 & $0.33^{j}$ & $0.33^{\mathrm{j}}$ & No & -- \\
\hline Nickel & $15.9(<B G)$ & 1,600 & $19.1^{j}$ & 27.4 & No & -- \\
\hline Vanadium & $47.5(<B G)$ & 560 & $85.1^{j}$ & $--^{m}$ & No & -- \\
\hline Zinc & $43.7(<B G)$ & 24,000 & 480 & $67.8^{\mathrm{i}}$ & No & - \\
\hline bis(2-Ethylhexyl)phthalate & 0.14 & 71.4 & 0.625 & 0.36 & No & -- \\
\hline Di-n-butylphthalate & 0.16 & 8,000 & 160 & 540 & No & -- \\
\hline 4-Methyl-2-pentanone & 0.006 & 6,400 & 64 & $--^{m}$ & No & -- \\
\hline Acetone & 0.015 & 72,000 & 720 & $--^{m}$ & No & -- \\
\hline Carbon tetrachloride & 0.009 & 7.7 & 0.034 & 0.05 & No & -- \\
\hline Methylene chloride & 0.019 & 133 & 0.5 & 0.94 & No & - \\
\hline
\end{tabular}

${ }^{*}$ All footnotes are provided following Table 14.

= not applicable

BG = background (obtained from DOE-RL 1996 and DOE-RL 2001b, unless otherwise noted)

$\mathrm{COC}=$ contaminant of concern

COPC = contaminant of potential concern

$\mathrm{RAG} \quad=$ remedial action goal

RESRAD = RESidual RADioactivity (dose-assessment model) 
Table 7. Comparison of Statistical Contaminant Concentrations to Action Levels for the 118-B-1 Area 4 Verification Sampling Event.* (2 Pages)

\begin{tabular}{|c|c|c|c|c|c|c|}
\hline \multirow[b]{2}{*}{ COC/COPC } & \multirow[b]{2}{*}{$\begin{array}{c}\text { Statistical } \\
\text { Result } \\
(\mathrm{pCi} / \mathrm{g})\end{array}$} & \multicolumn{3}{|c|}{ Generic Site Lookup Values $^{a}(\mathrm{pCi} / \mathrm{g})$} & \multirow{2}{*}{$\begin{array}{l}\text { Does the } \\
\text { Statistical } \\
\text { Result } \\
\text { Exceed } \\
\text { Lookup } \\
\text { Values? }\end{array}$} & \multirow[b]{2}{*}{$\begin{array}{c}\text { Does the } \\
\text { Statistical } \\
\text { Result Pass } \\
\text { RESRAD } \\
\text { Modeling? }\end{array}$} \\
\hline & & $\begin{array}{l}\text { Shallow } \\
\text { Zone } \\
\text { Lookup } \\
\text { Value }^{b}\end{array}$ & $\begin{array}{l}\text { Groundwater } \\
\text { Protection } \\
\text { Lookup } \\
\text { Value }\end{array}$ & $\begin{array}{l}\text { River } \\
\text { Protection } \\
\text { Lookup } \\
\text { Value } \\
\end{array}$ & & \\
\hline Cesium-137 & 0.345 & 6.2 & $1,465^{d}$ & $1,465^{d}$ & No & $Y_{e s}{ }^{e}$ \\
\hline \multirow[b]{2}{*}{ COC/COPC } & \multirow[b]{2}{*}{$\begin{array}{c}\text { Statistical } \\
\text { Result } \\
(\mathrm{mg} / \mathrm{kg})\end{array}$} & \multicolumn{3}{|c|}{ Remedial Action Goals ${ }^{a}$ (mg/kg) } & \multirow[b]{2}{*}{$\begin{array}{l}\text { Does the } \\
\text { Statistical } \\
\text { Data Set } \\
\text { Exceed } \\
\text { RAGs? }\end{array}$} & \multirow[b]{2}{*}{$\begin{array}{c}\text { Does the } \\
\text { Statistical } \\
\text { Result Pass } \\
\text { RESRAD } \\
\text { Modeling? }\end{array}$} \\
\hline & & $\begin{array}{c}\text { Direct } \\
\text { Exposure }\end{array}$ & $\begin{array}{l}\text { Soil Cleanup } \\
\text { Level for } \\
\text { Groundwater } \\
\text { Protection }\end{array}$ & $\begin{array}{l}\text { Soil } \\
\text { Cleanup } \\
\text { Level for } \\
\text { River } \\
\text { Protection }\end{array}$ & & \\
\hline Arsenic & $3.5(<B G)$ & 20 & 20 & 20 & No & -- \\
\hline Barium & $48.6(<B G)$ & $5,600^{i}$ & $132^{\mathrm{i}, \mathrm{j}}$ & $224^{i}$ & No & -- \\
\hline Beryllium & $0.40(<B G)$ & $10.4^{k}$ & $1.51^{\mathrm{j}}$ & $1.51^{\mathrm{j}}$ & No & -- \\
\hline Boron' & 3.9 & 16,000 & 320 & $-m^{m}$ & No & -- \\
\hline Chromium (total) & $6.6(<B G)$ & $80,000^{i}$ & $18.5^{\mathrm{j}}$ & $18.5^{j}$ & No & -- \\
\hline Chromium (hexavalent) & 0.22 & 2.1 & $4.8^{\circ}$ & 2 & No & -- \\
\hline Cobalt & $8.6(<B G)$ & 1,600 & 32 & $--^{m}$ & No & -- \\
\hline Copper & $16.2(<B G)$ & 2,960 & 59.2 & $22.0^{i}$ & No & -- \\
\hline Lead & $4.9(<B G)$ & 353 & $10.2^{\mathrm{j}}$ & $10.2^{\mathrm{i}}$ & No & -- \\
\hline Manganese & $334(<\mathrm{BG})$ & 11,200 & $512^{j}$ & $512^{j}$ & No & -- \\
\hline Nickel & $10.6(<B G)$ & 1,600 & $19.1^{j}$ & 27.4 & No & -- \\
\hline Vanadium & $49.9(<B G)$ & 560 & $85.1^{j}$ & $--^{m}$ & No & -- \\
\hline Zinc & $42.1(<B G)$ & 24,000 & 480 & $67.8^{j}$ & No & -- \\
\hline Aroclor-1254 & 0.080 & 0.5 & $0.017^{p}$ & $0.017^{p}$ & Yes & Yes $^{n}$ \\
\hline bis(2-Ethylhexyl)phthalate & 0.41 & 71.4 & 0.625 & 0.36 & Yes & $Y_{e s}{ }^{n}$ \\
\hline Di-n-butylphthalate & 0.063 & 8,000 & 160 & 540 & No & -- \\
\hline Diethylphthalate & 0.017 & 64,000 & 1,280 & 4,600 & No & -- \\
\hline Phenol & 0.17 & 24,000 & 480 & 4,200 & No & -- \\
\hline Acetone & 0.012 & 72,000 & 720 & $--^{m}$ & No & -- \\
\hline Carbon tetrachloride & 0.016 & 7.7 & 0.034 & 0.05 & No & -- \\
\hline Methylene chloride & 0.010 & 133 & 0.5 & 0.94 & No & -- \\
\hline beta-BHC & 0.0078 & 0.556 & 0.00486 & 0.00554 & Yes & Yes $^{q}$ \\
\hline 4,4'-DDE & 0.016 & 2.94 & 0.0257 & $0.005^{p}$ & Yes & Yes $^{n}$ \\
\hline Dieldrin & 0.0038 & 0.0625 & $0.003^{p}$ & $0.003^{p}$ & Yes & $Y_{e s^{n}}$ \\
\hline Endosulfan sulfate & 0.0022 & 480 & 9.6 & 0.0112 & No & -- \\
\hline Endrin & 0.0015 & 24 & 0.2 & 0.039 & No & -- \\
\hline Endrin aldehyde & 0.0055 & 24 & 0.2 & 0.039 & No & -- \\
\hline gamma-Chlordane & 0.0025 & $0.769^{r}$ & $0.0165^{p, r}$ & $0.0165^{p}$ & No & -- \\
\hline
\end{tabular}


Table 7. Comparison of Statistical Contaminant Concentrations to Action Levels for the 118-B-1 Area 4 Verification Sampling Event.* (2 Pages)

\begin{tabular}{|c|c|c|c|c|c|c|}
\hline \multirow[b]{2}{*}{ COC/COPC } & \multirow[b]{2}{*}{$\begin{array}{c}\text { Statistical } \\
\text { Result } \\
(\mathrm{mg} / \mathrm{kg})\end{array}$} & \multicolumn{3}{|c|}{ Remedial Action Goals ${ }^{\mathrm{a}}$ (mg/kg) } & \multirow[b]{2}{*}{$\begin{array}{l}\text { Does the } \\
\text { Statistical } \\
\text { Data Set } \\
\text { Exceed } \\
\text { RAGs? }\end{array}$} & \multirow[b]{2}{*}{$\begin{array}{l}\text { Does the } \\
\text { Statistical } \\
\text { Result Pass } \\
\text { RESRAD } \\
\text { Modeling? }\end{array}$} \\
\hline & & $\begin{array}{c}\text { Direct } \\
\text { Exposure }\end{array}$ & $\begin{array}{l}\text { Soil Cleanup } \\
\text { Level for } \\
\text { Groundwater } \\
\text { Protection }\end{array}$ & $\begin{array}{c}\text { Soil } \\
\text { Cleanup } \\
\text { Level for } \\
\text { River } \\
\text { Protection }\end{array}$ & & \\
\hline 2,4-D & 0.013 & 800 & 7 & $--^{m}$ & No & -- \\
\hline 2,4-DB & 0.016 & 640 & 12.8 & $-m^{m}$ & No & -- \\
\hline Dalapon & 0.037 & 2,400 & 20 & $-\mathrm{m}^{m}$ & No & -- \\
\hline Dichloroprop $^{\mathrm{s}}$ & 0.14 & 800 & 7 & $-\mathrm{m}^{\mathrm{m}}$ & No & -- \\
\hline Pentachlorophenol & 0.014 & 8.33 & 0.073 & 0.056 & No & -- \\
\hline
\end{tabular}

${ }^{*}$ All footnotes are provided following Table 14.

-- $\quad$ not applicable

BG = background (obtained from DOE-RL 1996 and DOE-RL 2001b, unless otherwise noted)

$\mathrm{COC}=$ contaminant of concern

$\mathrm{COPC}=$ contaminant of potential concern

$\mathrm{RAG} \quad=$ remedial action goal

RESRAD = RESidual RADioactivity (dose-assessment model)

Table 8. Comparison of Statistical Contaminant Concentrations to Action Levels for the 118-B-1 Area 5 Verification Sampling Event.* (2 Pages)

\begin{tabular}{|c|c|c|c|c|c|c|}
\hline \multirow[b]{2}{*}{ COC/COPC } & \multirow[b]{2}{*}{$\begin{array}{c}\text { Statistical } \\
\text { Result } \\
(\mathrm{pCi} / \mathrm{g})\end{array}$} & \multicolumn{3}{|c|}{ 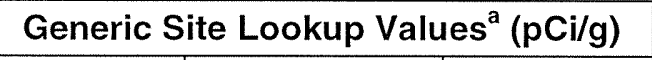 } & \multirow{2}{*}{$\begin{array}{l}\text { Does the } \\
\text { Statistical } \\
\text { Result } \\
\text { Exceed } \\
\text { Lookup } \\
\text { Values? }\end{array}$} & \multirow[b]{2}{*}{$\begin{array}{c}\text { Does the } \\
\text { Statistical } \\
\text { Result Pass } \\
\text { RESRAD } \\
\text { Modeling? }\end{array}$} \\
\hline & & $\begin{array}{l}\text { Shallow } \\
\text { Zone } \\
\text { Lookup } \\
\text { Value }^{b}\end{array}$ & $\begin{array}{l}\text { Groundwater } \\
\text { Protection } \\
\text { Lookup } \\
\text { Value }\end{array}$ & $\begin{array}{l}\text { River } \\
\text { Protection } \\
\text { Lookup } \\
\text { Value }\end{array}$ & & \\
\hline Cesium-137 & 2.40 & 6.2 & $1,465^{d}$ & $1,465^{d}$ & No & \multirow{2}{*}{$\mathrm{Yes}^{\mathrm{e}}$} \\
\hline Strontium-90 & 1.89 & 4.5 & $27.6^{d}$ & $27.6^{d}$ & No & \\
\hline \multirow[b]{2}{*}{ COC/COPC } & \multirow[b]{2}{*}{$\begin{array}{c}\text { Statistical } \\
\text { Result } \\
(\mathrm{mg} / \mathrm{kg})\end{array}$} & \multicolumn{3}{|c|}{ Remedial Action Goals ${ }^{\mathrm{a}}$ (mg/kg) } & \multirow[b]{2}{*}{$\begin{array}{l}\text { Does the } \\
\text { Statistical } \\
\text { Data Set } \\
\text { Exceed } \\
\text { RAGs? }\end{array}$} & \multirow[b]{2}{*}{$\begin{array}{c}\text { Does the } \\
\text { Statistical } \\
\text { Result Pass } \\
\text { RESRAD } \\
\text { Modeling? }\end{array}$} \\
\hline & & $\begin{array}{c}\text { Direct } \\
\text { Exposure }\end{array}$ & $\begin{array}{l}\text { Soil Cleanup } \\
\text { Level for } \\
\text { Groundwater } \\
\text { Protection }\end{array}$ & $\begin{array}{l}\text { Soil } \\
\text { Cleanup } \\
\text { Level for } \\
\text { River } \\
\text { Protection }\end{array}$ & & \\
\hline Arsenic & $2.8(<B G)$ & 20 & 20 & 20 & No & -- \\
\hline Barium & $46.7(<B G)$ & $5,600^{i}$ & $132^{\mathrm{i}, \mathrm{j}}$ & $224^{i}$ & No & -- \\
\hline Beryllium & $0.1(<B G)$ & $10.4^{k}$ & $1.51^{\mathrm{j}}$ & $1.51^{\mathrm{j}}$ & No & -- \\
\hline Boron' & 1.1 & 16,000 & 320 & $-m^{m}$ & No & -- \\
\hline Cadmium & $0.20(<B G)$ & 13.9 & $0.81^{j}$ & $0.81^{j}$ & No & -- \\
\hline Chromium (total) & $5.0(<B G)$ & $80,000^{i}$ & $18.5^{\mathrm{j}}$ & $18.5^{\mathrm{j}}$ & No & -- \\
\hline Chromium (hexavalent) & 0.26 & 2.1 & $4.8^{\circ}$ & 2 & No & -- \\
\hline Cobalt & $7.2(<B G)$ & 1,600 & 32 & $-m^{m}$ & No & -- \\
\hline Copper & $14.8(<B G)$ & 2,960 & 59.2 & $22.0^{j}$ & No & -- \\
\hline
\end{tabular}


Table 8. Comparison of Statistical Contaminant Concentrations to Action Levels for the 118-B-1 Area 5 Verification Sampling Event.* (2 Pages)

\begin{tabular}{|c|c|c|c|c|c|c|}
\hline \multirow[b]{2}{*}{ COC/COPC } & \multirow[b]{2}{*}{$\begin{array}{c}\text { Statistical } \\
\text { Result } \\
\text { (mg/kg) }\end{array}$} & \multicolumn{3}{|c|}{ Remedial Action Goals ${ }^{\mathrm{a}}$ (mg/kg) } & \multirow[b]{2}{*}{$\begin{array}{l}\text { Does the } \\
\text { Statistical } \\
\text { Data Set } \\
\text { Exceed } \\
\text { RAGs? }\end{array}$} & \multirow[b]{2}{*}{$\begin{array}{c}\text { Does the } \\
\text { Statistical } \\
\text { Result Pass } \\
\text { RESRAD } \\
\text { Modeling? }\end{array}$} \\
\hline & & $\begin{array}{c}\text { Direct } \\
\text { Exposure }\end{array}$ & $\begin{array}{l}\text { Soil Cleanup } \\
\text { Level for } \\
\text { Groundwater } \\
\text { Protection }\end{array}$ & $\begin{array}{l}\text { Soil } \\
\text { Cleanup } \\
\text { Level for } \\
\text { River } \\
\text { Protection }\end{array}$ & & \\
\hline Lead & $3.8(<B G)$ & 353 & $10.2^{\mathrm{i}}$ & $10.2^{\mathrm{j}}$ & No & -- \\
\hline Manganese & $290(<B G)$ & 11,200 & $512^{j}$ & $512^{i}$ & No & -- \\
\hline Mercury & $0.03(<B G)$ & 24 & $0.33^{j}$ & $0.33^{j}$ & No & -- \\
\hline Nickel & $7.4(<B G)$ & 1,600 & $19.1^{\mathrm{j}}$ & 27.4 & No & -- \\
\hline Vanadium & $37.4(<B G)$ & 560 & $85.1^{j}$ & $--^{m}$ & No & - \\
\hline Zinc & $36.8(<B G)$ & 24,000 & 480 & $67.8^{\mathrm{j}}$ & No & - \\
\hline bis(2-Ethylhexyl)phthalate & 0.17 & 71.4 & 0.625 & 0.36 & No & -- \\
\hline Acetone & 0.017 & 72,000 & 720 & $--^{m}$ & No & -- \\
\hline Methylene chloride & 0.013 & 133 & 0.5 & 0.94 & No & $\ldots$ \\
\hline
\end{tabular}

*All footnotes are provided following Table 14.

-. = not applicable

BG = background (obtained from DOE-RL 1996 and DOE-RL 2001b, unless otherwise noted)

$\mathrm{COC}=$ contaminant of concern

COPC = contaminant of potential concern

RAG = remedial action goal

RESRAD = RESidual RADioactivity (dose-assessment model)

Table 9. Comparison of Statistical Contaminant Concentrations to Action Levels for the 118-B-1 Area 6 Verification Sampling Event.* (2 Pages)

\begin{tabular}{|c|c|c|c|c|c|c|}
\hline \multirow[b]{2}{*}{ COC/COPC } & \multirow[b]{2}{*}{$\begin{array}{c}\text { Statistical } \\
\text { Result } \\
\text { (pci/g) }\end{array}$} & \multicolumn{3}{|c|}{ Generic Site Lookup Values $^{a}(\mathrm{pCi} / \mathrm{g})$} & \multirow{2}{*}{$\begin{array}{l}\text { Does the } \\
\text { Statistical } \\
\text { Result } \\
\text { Exceed } \\
\text { Lookup } \\
\text { Values? }\end{array}$} & \multirow[b]{2}{*}{$\begin{array}{l}\text { Does the } \\
\text { Statistical } \\
\text { Result Pass } \\
\text { RESRAD } \\
\text { Modeling? }\end{array}$} \\
\hline & & $\begin{array}{l}\text { Shallow } \\
\text { Zone } \\
\text { Lookup } \\
\text { Value }^{\text {b }}\end{array}$ & $\begin{array}{c}\text { Groundwater } \\
\text { Protection } \\
\text { Lookup } \\
\text { Value }\end{array}$ & $\begin{array}{l}\text { River } \\
\text { Protection } \\
\text { Lookup } \\
\text { Value }\end{array}$ & & \\
\hline Cesium-137 & 0.150 & 6.2 & $1,465^{\mathrm{d}}$ & $1,465^{d}$ & No & Yes $^{e}$ \\
\hline Tritium & 3.19 & $459^{d}$ & $12.6^{d}$ & $12.6^{d}$ & No & $-{ }^{f}$ \\
\hline Uranium-233/234 & $0.550(<B G)$ & $1.1^{\mathrm{g}}$ & $1.1^{\mathrm{g}}$ & $1.1^{g}$ & No & -- \\
\hline Uranium-238 & $0.690(<B G)$ & $1.1^{\mathrm{g}}$ & $1.1^{\mathrm{g}}$ & $1.1^{\mathrm{g}}$ & No & -- \\
\hline \multirow[b]{2}{*}{ COC/COPC } & \multirow[b]{2}{*}{$\begin{array}{c}\text { Statistical } \\
\text { Result } \\
\text { (mg/kg) }\end{array}$} & \multicolumn{3}{|c|}{ Remedial Action Goals ${ }^{\mathrm{a}}(\mathrm{mg} / \mathrm{kg})$} & \multirow[b]{2}{*}{$\begin{array}{l}\text { Does the } \\
\text { Statistical } \\
\text { Data Set } \\
\text { Exceed } \\
\text { RAGs? }\end{array}$} & \multirow[b]{2}{*}{$\begin{array}{c}\text { Does the } \\
\text { Statistical } \\
\text { Result Pass } \\
\text { RESRAD } \\
\text { Modeling? }\end{array}$} \\
\hline & & $\begin{array}{c}\text { Direct } \\
\text { Exposure }\end{array}$ & $\begin{array}{c}\text { Soil Cleanup } \\
\text { Level for } \\
\text { Groundwater } \\
\text { Protection }\end{array}$ & $\begin{array}{l}\text { Soil } \\
\text { Cleanup } \\
\text { Level for } \\
\text { River } \\
\text { Protection }\end{array}$ & & \\
\hline Arsenic & $4.1(<B G)$ & 20 & 20 & 20 & No & -- \\
\hline Barium & $80.1(<B G)$ & $5,600^{i}$ & $132^{\mathrm{i}, \mathrm{j}}$ & $224^{i}$ & No & -- \\
\hline Beryllium & $0.36(<B G)$ & $10.4^{k}$ & $1.51^{\mathrm{j}}$ & $1.51^{\mathrm{j}}$ & No & - \\
\hline
\end{tabular}


Table 9. Comparison of Statistical Contaminant Concentrations to Action Levels for the 118-B-1 Area 6 Verification Sampling Event.* (2 Pages)

\begin{tabular}{|c|c|c|c|c|c|c|}
\hline \multirow[b]{2}{*}{ COC/COPC } & \multirow[b]{2}{*}{$\begin{array}{c}\text { Statistical } \\
\text { Result } \\
\text { (mg/kg) }\end{array}$} & \multicolumn{3}{|c|}{ Remedial Action Goals ${ }^{a}(\mathrm{mg} / \mathrm{kg})$} & \multirow[b]{2}{*}{$\begin{array}{c}\text { Does the } \\
\text { Statistical } \\
\text { Data Set } \\
\text { Exceed } \\
\text { RAGs? }\end{array}$} & \multirow[b]{2}{*}{$\begin{array}{l}\text { Does the } \\
\text { Statistical } \\
\text { Result Pass } \\
\text { RESRAD } \\
\text { Modeling? }\end{array}$} \\
\hline & & $\begin{array}{c}\text { Direct } \\
\text { Exposure }\end{array}$ & $\begin{array}{l}\text { Soil Cleanup } \\
\text { Level for } \\
\text { Groundwater } \\
\text { Protection }\end{array}$ & $\begin{array}{c}\text { Soil } \\
\text { Cleanup } \\
\text { Level for } \\
\text { River } \\
\text { Protection }\end{array}$ & & \\
\hline Boron' & 6.1 & 16,000 & 320 & $--^{m}$ & No & -- \\
\hline Chromium (total) & $7.4(<B G)$ & $80,000^{i}$ & $18.5^{\mathrm{j}}$ & $18.5^{\mathrm{j}}$ & No & -- \\
\hline Chromium (hexavalent) & 0.27 & 2.1 & $4.8^{\circ}$ & 2 & No & -- \\
\hline Cobalt & $8.5(<B G)$ & 1,600 & 32 & $--^{m}$ & No & -- \\
\hline Copper & $16.6(<B G)$ & 2,960 & 59.2 & $22.0^{j}$ & No & -- \\
\hline Lead & $5.0(<B G)$ & 353 & $10.2^{\mathrm{j}}$ & $10.2^{\mathrm{j}}$ & No & -- \\
\hline Manganese & $323(<B G)$ & 11,200 & $512^{i}$ & $512^{j}$ & No & -- \\
\hline Mercury & $0.02(<B G)$ & 24 & $0.33^{\mathrm{j}}$ & $0.33^{\mathrm{j}}$ & No & -- \\
\hline Nickel & $11.4(<B G)$ & 1,600 & $19.1^{\mathrm{j}}$ & 27.4 & No & -- \\
\hline Vanadium & $49.0(<B G)$ & 560 & $85.1^{\mathrm{j}}$ & $-m$ & No & -- \\
\hline Zinc & $41.4(<B G)$ & 24,000 & 480 & $67.8^{\mathrm{j}}$ & No & -- \\
\hline bis(2-Ethylhexyl)phthalate & 0.32 & 71.4 & 0.625 & 0.36 & $Y_{e s}{ }^{u}$ & Yes $^{n}$ \\
\hline Butylbenzylphthalate & 0.024 & 16,000 & 320 & 250 & No & -- \\
\hline Di-n-butylphthalate & 0.079 & 8,000 & 160 & 540 & No & -- \\
\hline Phenol & 0.023 & 24,000 & 480 & 4,200 & No & -- \\
\hline Acetone & 0.008 & 72,000 & 720 & $-m^{m}$ & No & -- \\
\hline Carbon tetrachloride & 0.007 & 7.7 & 0.034 & 0.05 & No & -- \\
\hline Methylene chloride & 0.008 & 133 & 0.5 & 0.94 & No & - \\
\hline Aldrin & 0.00050 & 0.0588 & $0.00165^{p}$ & $0.00165^{p}$ & No & -- \\
\hline $2,4-D$ & 0.062 & 800 & 7 & $--^{m}$ & No & -- \\
\hline 2,4-DB & 0.043 & 640 & 12.8 & $--^{m}$ & No & -- \\
\hline 2,4,5-TP & 0.012 & 640 & 5 & $--^{m}$ & No & -- \\
\hline Dalapon & 0.036 & 2,400 & 20 & $--^{m}$ & No & -- \\
\hline Dichloroprops & 0.10 & 800 & 7 & $--^{m}$ & No & -- \\
\hline
\end{tabular}

*All footnotes are provided following Table 14.

-- $\quad=$ not applicable

BG = background (obtained from DOE-RL 1996 and DOE-RL 2001b, unless otherwise noted)

$\mathrm{COC}=$ contaminant of concern

COPC = contaminant of potential concern

RAG = remedial action goal

RESRAD = RESidual RADioactivity (dose-assessment model) 
Table 10. Comparison of Statistical Contaminant Concentrations to Action Levels for the 118-B-1 Area 7 Verification Sampling Event.* (2 Pages)

\begin{tabular}{|c|c|c|c|c|c|c|}
\hline \multirow[b]{2}{*}{ COC/COPC } & \multirow[b]{2}{*}{$\begin{array}{c}\text { Statistical } \\
\text { Result } \\
(p C i / g)\end{array}$} & \multicolumn{3}{|c|}{ Generic Site Lookup Values $^{a}(\mathrm{pCi} / \mathrm{g})$} & \multirow{2}{*}{$\begin{array}{l}\text { Does the } \\
\text { Statistical } \\
\text { Result } \\
\text { Exceed } \\
\text { Lookup } \\
\text { Values? }\end{array}$} & \multirow[b]{2}{*}{$\begin{array}{c}\text { Does the } \\
\text { Statistical } \\
\text { Result Pass } \\
\text { RESRAD } \\
\text { Modeling? }\end{array}$} \\
\hline & & $\begin{array}{l}\text { Shallow } \\
\text { Zone } \\
\text { Lookup } \\
\text { Value }^{b}\end{array}$ & $\begin{array}{c}\text { Groundwater } \\
\text { Protection } \\
\text { Lookup } \\
\text { Value }\end{array}$ & $\begin{array}{l}\text { River } \\
\text { Protection } \\
\text { Lookup } \\
\text { Value }\end{array}$ & & \\
\hline Cesium-137 & 0.517 & 6.2 & $1,465^{\mathrm{d}}$ & $1,465^{d}$ & No & \multirow{2}{*}{ Yes $^{e}$} \\
\hline Europium-152 & 0.098 & 3.3 & $--^{c}$ & $--^{c}$ & No & \\
\hline Uranium-233/234 & $0.671(<B G)$ & $1.1^{\mathrm{g}}$ & $1.1^{\mathrm{g}}$ & $1.1^{g}$ & No & -- \\
\hline Uranium-238 & $0.725(<B G)$ & $1.1^{\mathrm{g}}$ & $1.1^{\mathrm{g}}$ & $1.1^{\mathrm{g}}$ & No & -- \\
\hline \multirow[b]{2}{*}{ COC/COPC } & \multirow[b]{2}{*}{$\begin{array}{l}\text { Statistical } \\
\text { Result } \\
(\mathrm{mg} / \mathrm{kg})\end{array}$} & \multicolumn{3}{|c|}{ Remedial Action Goals ${ }^{\mathrm{a}}(\mathrm{mg} / \mathrm{kg})$} & \multirow[b]{2}{*}{$\begin{array}{l}\text { Does the } \\
\text { Statistical } \\
\text { Data Set } \\
\text { Exceed } \\
\text { RAGs? }\end{array}$} & \multirow[b]{2}{*}{$\begin{array}{c}\text { Does the } \\
\text { Statistical } \\
\text { Result Pass } \\
\text { RESRAD } \\
\text { Modeling? }\end{array}$} \\
\hline & & $\begin{array}{c}\text { Direct } \\
\text { Exposure }\end{array}$ & $\begin{array}{c}\text { Soil Cleanup } \\
\text { Level for } \\
\text { Groundwater } \\
\text { Protection }\end{array}$ & $\begin{array}{c}\text { Soil } \\
\text { Cleanup } \\
\text { Level for } \\
\text { River } \\
\text { Protection } \\
\end{array}$ & & \\
\hline Arsenic & $3.5(<B G)$ & 20 & 20 & 20 & No & - \\
\hline Barium & 132 & $5,600^{i}$ & $132^{\mathrm{i}, \mathrm{i}}$ & $224^{i}$ & Yes & Yes $^{n}$ \\
\hline Beryllium & $0.31(<\mathrm{BG})$ & $10.4^{k}$ & $1.51^{\mathrm{j}}$ & $1.51^{\mathrm{i}}$ & No & -- \\
\hline Boron' & 13.7 & 16,000 & 320 & $--^{m}$ & No & -- \\
\hline Chromium (total) & $8.9(<B G)$ & $80,000^{i}$ & $18.5^{\mathrm{j}}$ & $18.5^{\mathrm{j}}$ & No & -- \\
\hline Chromium (hexavalent) & 0.28 & 2.1 & $4.8^{\circ}$ & 2 & No & -- \\
\hline Cobalt & $7.5(<B G)$ & 1,600 & 32 & $--^{m}$ & No & -- \\
\hline Copper & 22.2 & 2,960 & 59.2 & $22.0^{\mathrm{i}}$ & Yes & Yes $^{n}$ \\
\hline Lead & 11.0 & 353 & $10.2^{j}$ & $10.2^{i}$ & Yes & Yes $^{n}$ \\
\hline Manganese & $329(<B G)$ & 11,200 & $512^{j}$ & $512^{j}$ & No & -- \\
\hline Mercury & $0.02(<B G)$ & 24 & $0.33^{j}$ & $0.33^{\mathrm{i}}$ & No & -- \\
\hline Molybdenum' & 2.1 & 400 & 8 & $--^{m}$ & No & $-\cdots$ \\
\hline Nickel & $10.6(<B G)$ & 1,600 & $19.1^{j}$ & 27.4 & No & -- \\
\hline Vanadium & $39.8(<B G)$ & 560 & $85.1^{\mathrm{j}}$ & $--^{m}$ & No & - \\
\hline Zinc & $47.5(<B G)$ & 24,000 & 480 & $67.8^{\mathrm{i}}$ & No & -- \\
\hline 2-Methylnaphthalene & 0.026 & 320 & 3.2 & $-^{m}$ & No & -- \\
\hline Diethylphthalate & 0.025 & 64,000 & 1,280 & 4,600 & No & -- \\
\hline Naphthalene & 0.019 & 1,600 & 16.0 & 988 & No & -- \\
\hline Acetone & 0.009 & 72,000 & 720 & $--^{m}$ & No & -- \\
\hline Carbon tetrachloride & 0.011 & 7.7 & 0.034 & 0.05 & No & -- \\
\hline Methylene chloride & 0.008 & 133 & 0.5 & 0.94 & No & -- \\
\hline alpha-Chlordane & 0.012 & $0.769^{r}$ & $0.0165^{p, r}$ & $0.0165^{p}$ & No & -- \\
\hline 4,4'-DDE & 0.015 & 2.94 & 0.0257 & $0.005^{p}$ & Yes & $Y_{e s}{ }^{n}$ \\
\hline Dieldrin & 0.002 & 0.0625 & $0.003^{p}$ & $0.003^{p}$ & No & -- \\
\hline Endrin ketone & 0.0078 & 24 & 0.2 & 0.039 & No & -- \\
\hline
\end{tabular}


Table 10. Comparison of Statistical Contaminant Concentrations to Action Levels for the 118-B-1 Area 7 Verification Sampling Event.* (2 Pages)

\begin{tabular}{|c|c|c|c|c|c|c|}
\hline \multirow[b]{2}{*}{$\mathrm{COC} / \mathrm{COPC}$} & \multirow[b]{2}{*}{$\begin{array}{c}\text { Statistical } \\
\text { Result } \\
\text { (mg/kg) }\end{array}$} & \multicolumn{3}{|c|}{ Remedial Action Goals ${ }^{\mathrm{a}}(\mathrm{mg} / \mathrm{kg})$} & \multirow[b]{2}{*}{$\begin{array}{c}\text { Does the } \\
\text { Statistical } \\
\text { Data Set } \\
\text { Exceed } \\
\text { RAGs? }\end{array}$} & \multirow[b]{2}{*}{$\begin{array}{l}\text { Does the } \\
\text { Statistical } \\
\text { Result Pass } \\
\text { RESRAD } \\
\text { Modeling? }\end{array}$} \\
\hline & & $\begin{array}{c}\text { Direct } \\
\text { Exposure }\end{array}$ & $\begin{array}{l}\text { Soil Cleanup } \\
\text { Level for } \\
\text { Groundwater } \\
\text { Protection }\end{array}$ & $\begin{array}{c}\text { Soil } \\
\text { Cleanup } \\
\text { Level for } \\
\text { River } \\
\text { Protection }\end{array}$ & & \\
\hline Methoxychlor & 0.0091 & 400 & 4 & 1.67 & No & -- \\
\hline $2,4-D$ & 0.023 & 800 & 7 & $--^{m}$ & No & -- \\
\hline $2,4-D B$ & 0.027 & 640 & 12.8 & $-m^{m}$ & No & -- \\
\hline Dalapon & 0.027 & 2,400 & 20 & $-{ }^{m}$ & No & -- \\
\hline Dicamba & 0.013 & 2,400 & 48 & $-{ }^{m}$ & No & - \\
\hline Dichloroprop $^{s}$ & 0.050 & 800 & 7 & $-m^{m}$ & No & -- \\
\hline Picloram & 0.014 & 5,600 & 50 & $-m^{m}$ & No & -- \\
\hline
\end{tabular}

${ }^{*}$ All footnotes are provided following Table 14.

-- $\quad=$ not applicable

BG = background (obtained from DOE-RL 1996 and DOE-RL 2001b, unless otherwise noted)

$\mathrm{COC}=$ contaminant of concern

COPC = contaminant of potential concern

RAG = remedial action goal

RESRAD = RESidual RADioactivity (dose-assessment model)

Table 11. Comparison of Statistical Contaminant Concentrations to Action Levels for the 118-B-1 Overburden Verification Sampling Event.* (3 Pages)

\begin{tabular}{|c|c|c|c|c|c|c|}
\hline \multirow[b]{2}{*}{ COC/COPC } & \multirow[b]{2}{*}{$\begin{array}{l}\text { Statistical } \\
\text { Result } \\
\text { (pCi/g) }\end{array}$} & \multicolumn{3}{|c|}{ Generic Site Lookup Values ${ }^{a}(\mathrm{pCi} / \mathrm{g})$} & \multirow{2}{*}{$\begin{array}{l}\text { Does the } \\
\text { Statistical } \\
\text { Result } \\
\text { Exceed } \\
\text { Lookup } \\
\text { Values? }\end{array}$} & \multirow[b]{2}{*}{$\begin{array}{l}\text { Does the } \\
\text { Statistical } \\
\text { Result Pass } \\
\text { RESRAD } \\
\text { Modeling? }\end{array}$} \\
\hline & & $\begin{array}{l}\text { Shallow } \\
\text { Zone } \\
\text { Lookup } \\
\text { Value }^{b}\end{array}$ & $\begin{array}{c}\text { Groundwater } \\
\text { Protection } \\
\text { Lookup } \\
\text { Value }\end{array}$ & $\begin{array}{l}\text { River } \\
\text { Protection } \\
\text { Lookup } \\
\text { Value }\end{array}$ & & \\
\hline Cesium-137 & $0.225(<B G)$ & 6.2 & $1,465^{d}$ & $1,465^{d}$ & No & -- \\
\hline Europium-152 & 0.07 & 3.3 & $--^{c}$ & $--^{c}$ & No & Yes $^{v}$ \\
\hline Strontium-90 & $0.175(<B G)$ & 4.5 & $27.6^{d}$ & $27.6^{d}$ & No & -- \\
\hline Uranium-233/234 & $0.542(<B G)$ & $1.1^{\mathrm{g}}$ & $1.1^{\mathrm{g}}$ & $1.1^{g}$ & No & -- \\
\hline Uranium-235 & $0.033(<B G)$ & $0.61^{c}$ & $0.5^{h}$ & $0.5^{\mathrm{h}}$ & No & -- \\
\hline Uranium-238 & $0.556(<B G)$ & $1.1^{\mathrm{g}}$ & $1.1^{\mathrm{g}}$ & $1.1^{g}$ & No & - \\
\hline \multirow[b]{2}{*}{ COC/COPC } & \multirow[b]{2}{*}{$\begin{array}{c}\text { Statistical } \\
\text { Result } \\
\text { (mg/kg) }\end{array}$} & \multicolumn{3}{|c|}{ Remedial Action Goals ${ }^{\mathrm{a}}(\mathrm{mg} / \mathrm{kg})$} & \multirow[b]{2}{*}{$\begin{array}{l}\text { Does the } \\
\text { Statistical } \\
\text { Data Set } \\
\text { Exceed } \\
\text { RAGs? }\end{array}$} & \multirow[b]{2}{*}{$\begin{array}{c}\text { Does the } \\
\text { Statistical } \\
\text { Result Pass } \\
\text { RESRAD } \\
\text { Modeling? }\end{array}$} \\
\hline & & $\begin{array}{c}\text { Direct } \\
\text { Exposure }\end{array}$ & $\begin{array}{c}\text { Soil Cleanup } \\
\text { Level for } \\
\text { Groundwater } \\
\text { Protection }\end{array}$ & $\begin{array}{c}\text { Soil } \\
\text { Cleanup } \\
\text { Level for } \\
\text { River } \\
\text { Protection }\end{array}$ & & \\
\hline Arsenic & $3.1(<B G)$ & 20 & 20 & 20 & No & -- \\
\hline Barium & $59.3(<B G)$ & $5,600^{i}$ & $132^{i, j}$ & $224^{i}$ & No & -- \\
\hline Beryllium & $0.48(<B G)$ & $10.4^{\mathrm{k}}$ & $1.51^{j}$ & $1.51^{i}$ & No & -- \\
\hline
\end{tabular}


Table 11. Comparison of Statistical Contaminant Concentrations to Action Levels for the 118-B-1 Overburden Verification Sampling Event.* (3 Pages)

\begin{tabular}{|c|c|c|c|c|c|c|}
\hline \multirow[b]{2}{*}{ COC/COPC } & \multirow[b]{2}{*}{$\begin{array}{c}\text { Statistical } \\
\text { Result } \\
\text { (mg/kg) }\end{array}$} & \multicolumn{3}{|c|}{ Remedial Action Goals ${ }^{\mathrm{a}}(\mathrm{mg} / \mathrm{kg})$} & \multirow[b]{2}{*}{$\begin{array}{l}\text { Does the } \\
\text { Statistical } \\
\text { Data Set } \\
\text { Exceed } \\
\text { RAGs? }\end{array}$} & \multirow[b]{2}{*}{$\begin{array}{c}\text { Does the } \\
\text { Statistical } \\
\text { Result Pass } \\
\text { RESRAD } \\
\text { Modeling? }\end{array}$} \\
\hline & & $\begin{array}{c}\text { Direct } \\
\text { Exposure }\end{array}$ & $\begin{array}{l}\text { Soil Cleanup } \\
\text { Level for } \\
\text { Groundwater } \\
\text { Protection }\end{array}$ & $\begin{array}{c}\text { Soil } \\
\text { Cleanup } \\
\text { Level for } \\
\text { River } \\
\text { Protection }\end{array}$ & & \\
\hline Boron' & 2.0 & 16,000 & 320 & $--^{m}$ & No & -- \\
\hline Cadmium $^{t}$ & $0.43(<B G)$ & 13.9 & $0.81^{j}$ & $0.81^{\mathrm{i}}$ & No & -- \\
\hline Chromium (total) & $7.2(<B G)$ & $80,000^{i}$ & $18.5^{j}$ & $18.5^{\mathrm{i}}$ & No & -- \\
\hline Chromium (hexavalent) & 0.23 & 2.1 & $4.8^{\circ}$ & 2 & No & -- \\
\hline Cobalt & $8.7(<B G)$ & 1,600 & 32 & $-\mathrm{n}^{m}$ & No & -- \\
\hline Copper & $16.0(<B G)$ & 2,960 & 59.2 & $22.0^{j}$ & No & -- \\
\hline Lead & $5.1(<B G)$ & 353 & $10.2^{\mathrm{j}}$ & $10.2^{j}$ & No & -- \\
\hline Manganese & $346(<B G)$ & 11,200 & $512^{j}$ & $512^{i}$ & No & -- \\
\hline Mercury & $0.05(<B G)$ & 24 & $0.33^{j}$ & $0.33^{\mathrm{i}}$ & No & -- \\
\hline Molybdenum' & 0.83 & 400 & 8 & $-\mathrm{m}^{\mathrm{m}}$ & No & -- \\
\hline Nickel & $10.1(<B G)$ & 1,600 & $19.1^{j}$ & 27.4 & No & -- \\
\hline Selenium ${ }^{t}$ & $0.67(<B G)$ & 400 & 5 & 1 & No & -- \\
\hline Vanadium & $46.6(<B G)$ & 560 & $85.1^{\mathrm{j}}$ & $-\mathrm{m}^{\mathrm{m}}$ & No & -- \\
\hline Zinc & $40.0(<B G)$ & 24,000 & 480 & $67.8^{j}$ & No & -- \\
\hline Aroclor-1254 & 0.038 & 0.5 & $0.017^{\mathrm{P}}$ & $0.017^{p}$ & Yes & Yes $^{n}$ \\
\hline bis(2-Ethylhexyl)phthalate & 0.06 & 71.4 & 0.625 & 0.36 & No & -- \\
\hline Di-n-butylphthalate & 0.12 & 8,000 & 160 & 540 & No & -- \\
\hline Phenol & 0.022 & 24,000 & 480 & 4,200 & No & - \\
\hline 4-Methyl-2-pentanone & 0.011 & 6,400 & 64 & $--^{m}$ & No & -- \\
\hline Acetone & 0.039 & 72,000 & 720 & $--^{m}$ & No & -- \\
\hline Carbon tetrachloride & 0.011 & 7.7 & 0.034 & 0.05 & No & - \\
\hline Methylene chloride & 0.016 & 133 & 0.5 & 0.94 & No & -- \\
\hline Toluene & 0.002 & 6,400 & 64 & 1,360 & No & -- \\
\hline Xylenes (total) & 0.001 & 16,000 & 160 & $-m^{m}$ & No & -- \\
\hline beta-BHC & 0.00064 & 0.556 & 0.00486 & 0.00554 & No & -- \\
\hline 4,4'-DDD & 0.0019 & 4.17 & 0.0365 & $0.005^{p}$ & No & -- \\
\hline $4,4^{\prime}-\mathrm{DDE}$ & 0.0013 & 2.94 & 0.0257 & $0.005^{p}$ & No & -- \\
\hline $4,4^{\prime}$-DDT & 0.0011 & 2.94 & 0.0257 & $0.005^{p}$ & No & -- \\
\hline Endosulfan I & 0.0028 & 480 & 9.6 & 0.0112 & No & - \\
\hline Endosulfan II & 0.00060 & 480 & 9.6 & 0.0112 & No & -- \\
\hline Endosulfan sulfate & 0.00087 & 480 & 9.6 & 0.0112 & No & -- \\
\hline Endrin aldehyde & 0.0019 & 24 & 0.2 & 0.039 & No & -- \\
\hline gamma-Chlordane & 0.0025 & $0.769^{r}$ & $0.0165^{p, r}$ & $0.0165^{p}$ & No & -- \\
\hline
\end{tabular}


Table 11. Comparison of Statistical Contaminant Concentrations to Action Levels for the 118-B-1 Overburden Verification Sampling Event.* (3 Pages)

\begin{tabular}{|c|c|c|c|c|c|c|}
\hline \multirow[b]{2}{*}{ COC/COPC } & \multirow[b]{2}{*}{$\begin{array}{c}\text { Statistical } \\
\text { Result } \\
(\mathrm{mg} / \mathrm{kg})\end{array}$} & \multicolumn{3}{|c|}{ Remedial Action Goals ${ }^{\mathrm{a}}$ (mg/kg) } & \multirow[b]{2}{*}{$\begin{array}{c}\text { Does the } \\
\text { Statistical } \\
\text { Data Set } \\
\text { Exceed } \\
\text { RAGs? }\end{array}$} & \multirow[b]{2}{*}{$\begin{array}{l}\text { Does the } \\
\text { Statistical } \\
\text { Result Pass } \\
\text { RESRAD } \\
\text { Modeling? }\end{array}$} \\
\hline & & $\begin{array}{c}\text { Direct } \\
\text { Exposure }\end{array}$ & $\begin{array}{l}\text { Soil Cleanup } \\
\text { Level for } \\
\text { Groundwater } \\
\text { Protection }\end{array}$ & $\begin{array}{c}\text { Soil } \\
\text { Cleanup } \\
\text { Level for } \\
\text { River } \\
\text { Protection }\end{array}$ & & \\
\hline Methoxychlor & 0.0067 & 400 & 4 & 1.67 & No & -- \\
\hline $2,4-D$ & 0.026 & 800 & 7 & $--^{m}$ & No & -- \\
\hline 2,4-DB & 0.087 & 640 & 12.8 & $--^{m}$ & No & -- \\
\hline $2,4,5-\mathrm{T}$ & 0.0039 & 800 & 16 & $-\mathrm{m}^{\mathrm{m}}$ & No & -- \\
\hline 2,4,5-TP & 0.0096 & 640 & 5 & $--^{m}$ & No & -- \\
\hline Dalapon & 0.023 & 2,400 & 20 & $--^{m}$ & No & -- \\
\hline Dicamba & 0.016 & 2,400 & 48 & $--^{m}$ & No & -- \\
\hline Dichloroprop ${ }^{\mathrm{s}}$ & 0.11 & 800 & 7 & $--^{m}$ & No & -- \\
\hline Pentachlorophenol & 0.016 & 8.33 & 0.073 & 0.056 & No & -- \\
\hline
\end{tabular}

*All footnotes are provided following Table 14.

-- $\quad=$ not applicable

BG = background (obtained from DOE-RL 1996 and DOE-RL 2001b, unless otherwise noted)

$\mathrm{COC}=$ contaminant of concern

COPC = contaminant of potential concern

RAG = remedial action goal

RESRAD = RESidual RADioactivity (dose-assessment model)

Table 12. Comparison of Statistical Contaminant Concentrations to Action Levels for the 118-B-1 BCL Material Stockpiles Verification Sampling Event.* (3 Pages)

\begin{tabular}{|c|c|c|c|c|c|c|}
\hline \multirow[b]{2}{*}{ COC/COPC } & \multirow[b]{2}{*}{$\begin{array}{c}\text { Statistical } \\
\text { Result } \\
(\mathrm{pCi} / \mathrm{g})\end{array}$} & \multicolumn{3}{|c|}{ Generic Site Lookup Values $^{\mathrm{a}}(\mathrm{pCi} / \mathrm{g}$ ) } & \multirow{2}{*}{$\begin{array}{c}\text { Does the } \\
\text { Statistical } \\
\text { Result } \\
\text { Exceed } \\
\text { Lookup } \\
\text { Values? }\end{array}$} & \multirow[b]{2}{*}{$\begin{array}{c}\text { Does the } \\
\text { Statistical } \\
\text { Result Pass } \\
\text { RESRAD } \\
\text { Modeling? }\end{array}$} \\
\hline & & $\begin{array}{l}\text { Shallow } \\
\text { Zone } \\
\text { Lookup } \\
\text { Value }^{b}\end{array}$ & $\begin{array}{c}\text { Groundwater } \\
\text { Protection } \\
\text { Lookup } \\
\text { Value }\end{array}$ & $\begin{array}{l}\text { River } \\
\text { Protection } \\
\text { Lookup } \\
\text { Value }\end{array}$ & & \\
\hline Cesium-137 & 0.159 & 6.2 & $1,465^{d}$ & $1,465^{d}$ & No & \multirow{4}{*}{ Yes $^{v}$} \\
\hline Cobalt-60 & 0.05 & 1.4 & $13,900^{d}$ & $13,900^{d}$ & No & \\
\hline Europium-152 & 0.068 & 3.3 & $-a^{c}$ & $--^{c}$ & No & \\
\hline Strontium-90 & 0.120 & 4.5 & $27.6^{d}$ & $27.6^{d}$ & No & \\
\hline Uranium-233/234 & $0.564(<B G)$ & $1.1^{g}$ & $1.1^{\mathrm{g}}$ & $1.1^{\mathrm{g}}$ & No & -- \\
\hline Uranium-235 & $0.039(<B G)$ & $0.61^{\mathrm{c}}$ & $0.5^{\mathrm{h}}$ & $0.5^{\mathrm{h}}$ & No & -- \\
\hline Uranium-238 & $0.587(<B G)$ & $1.1^{\mathrm{g}}$ & $1.1^{\mathrm{g}}$ & $1.1^{\mathrm{g}}$ & No & -- \\
\hline
\end{tabular}


Table 12. Comparison of Statistical Contaminant Concentrations to Action Levels for the 118-B-1 BCL Material Stockpiles Verification Sampling Event.* (3 Pages)

\begin{tabular}{|c|c|c|c|c|c|c|}
\hline \multirow[b]{2}{*}{ COC/COPC } & \multirow[b]{2}{*}{$\begin{array}{c}\text { Statistical } \\
\text { Result } \\
(\mathrm{mg} / \mathrm{kg})\end{array}$} & \multicolumn{3}{|c|}{ Remedial Action Goals ${ }^{a}$ (mg/kg) } & \multirow[b]{2}{*}{$\begin{array}{c}\text { Does the } \\
\text { Statistical } \\
\text { Data Set } \\
\text { Exceed } \\
\text { RAGs? }\end{array}$} & \multirow[b]{2}{*}{$\begin{array}{l}\text { Does the } \\
\text { Statistical } \\
\text { Result Pass } \\
\text { RESRAD } \\
\text { Modeling? }\end{array}$} \\
\hline & & $\begin{array}{c}\text { Direct } \\
\text { Exposure }\end{array}$ & $\begin{array}{l}\text { Soil Cleanup } \\
\text { Level for } \\
\text { Groundwater } \\
\text { Protection }\end{array}$ & $\begin{array}{l}\text { Soil } \\
\text { Cleanup } \\
\text { Level for } \\
\text { River } \\
\text { Protection }\end{array}$ & & \\
\hline Arsenic & $3.7(<\mathrm{BG})$ & 20 & 20 & 20 & No & -- \\
\hline Barium & $60.8(<B G)$ & $5,600^{i}$ & $132^{i, j}$ & $224^{i}$ & No & -- \\
\hline Beryllium & $0.56(<B G)$ & $10.4^{k}$ & $1.51^{\mathrm{i}}$ & $1.51^{\mathrm{j}}$ & No & -- \\
\hline Boron' & 1.8 & 16,000 & 320 & $--^{m}$ & No & -- \\
\hline Chromium (total) & $8.1(<B G)$ & $80,000^{i}$ & $18.5^{\mathrm{j}}$ & $18.5^{\mathrm{j}}$ & No & -- \\
\hline Chromium (hexavalent) & 0.24 & 2.1 & $4.8^{\circ}$ & 2 & No & -- \\
\hline Cobalt & $9.2(<B G)$ & 1,600 & 32 & $-m^{m}$ & No & -- \\
\hline Copper & $15.9(<B G)$ & 2,960 & 59.2 & $22.0^{j}$ & No & - \\
\hline Lead & $5.1(<B G)$ & 353 & $10.2^{\mathrm{j}}$ & $10.2^{\mathrm{j}}$ & No & -- \\
\hline Manganese & $366(<B G)$ & 11,200 & $512^{j}$ & $512^{i}$ & No & -- \\
\hline Mercury & $0.03(<B G)$ & 24 & $0.33^{j}$ & $0.33^{i}$ & No & -- \\
\hline Molybdenum' & 0.43 & 400 & 8 & $-\mathrm{m}^{\mathrm{m}}$ & No & -- \\
\hline Nickel & $11.1(<B G)$ & 1,600 & $19.1^{j}$ & 27.4 & No & -- \\
\hline Selenium $^{t}$ & 1.0 & 400 & 5 & 1 & No & -- \\
\hline Vanadium & $48.8(<B G)$ & 560 & $85.1^{j}$ & $--^{m}$ & No & -- \\
\hline Zinc & $41.4(<B G)$ & 24,000 & 480 & $67.8^{\mathrm{j}}$ & No & -- \\
\hline bis(2-Ethylhexyl)phthalate & 0.08 & 71.4 & 0.625 & 0.36 & No & -- \\
\hline Di-n-butylphthalate & 0.13 & 8,000 & 160 & 540 & No & -- \\
\hline Diethylphthalate & 0.019 & 64,000 & 1,280 & 4,600 & No & -- \\
\hline Phenol & 0.034 & 24,000 & 480 & 4,200 & No & -- \\
\hline 4-Methyl-2-pentanone & 0.007 & 6,400 & 64 & $--^{m}$ & No & -- \\
\hline Acetone & 0.020 & 72,000 & 720 & $--^{m}$ & No & -- \\
\hline Carbon tetrachloride & 0.011 & 7.7 & 0.034 & 0.05 & No & -- \\
\hline Methylene chloride & 0.014 & 133 & 0.5 & 0.94 & No & -- \\
\hline Toluene & 0.001 & 6,400 & 64 & 1,360 & No & - \\
\hline Xylenes (total) & 0.001 & 16,000 & 160 & $--^{m}$ & No & - \\
\hline beta-BHC & 0.0022 & 0.556 & 0.00486 & 0.00554 & No & -- \\
\hline 4,4'-DDT & 0.0016 & 2.94 & 0.0257 & $0.005^{p}$ & No & -- \\
\hline Endosulfan I & 0.0049 & 480 & 9.6 & 0.0112 & No & -- \\
\hline Endosulfan sulfate & 0.00084 & 480 & 9.6 & 0.0112 & No & -- \\
\hline Endrin aldehyde & 0.0015 & 24 & 0.2 & 0.039 & No & -- \\
\hline Endrin ketone & 0.0014 & 24 & 0.2 & 0.039 & No & -- \\
\hline Heptachlor & 0.00043 & 0.222 & 0.002 & $0.002^{p}$ & No & -- \\
\hline
\end{tabular}


Table 12. Comparison of Statistical Contaminant Concentrations to Action Levels for the 118-B-1 BCL Material Stockpiles Verification Sampling Event.* (3 Pages)

\begin{tabular}{|c|c|c|c|c|c|c|}
\hline \multirow[b]{2}{*}{ COC/COPC } & \multirow[b]{2}{*}{$\begin{array}{c}\text { Statistical } \\
\text { Result } \\
\text { (mg/kg) }\end{array}$} & \multicolumn{3}{|c|}{ Remedial Action Goals ${ }^{a}(\mathrm{mg} / \mathrm{kg})$} & \multirow[b]{2}{*}{$\begin{array}{l}\text { Does the } \\
\text { Statistical } \\
\text { Data Set } \\
\text { Exceed } \\
\text { RAGs? }\end{array}$} & \multirow[b]{2}{*}{$\begin{array}{l}\text { Does the } \\
\text { Statistical } \\
\text { Result Pass } \\
\text { RESRAD } \\
\text { Modeling? }\end{array}$} \\
\hline & & $\begin{array}{c}\text { Direct } \\
\text { Exposure }\end{array}$ & $\begin{array}{l}\text { Soil Cleanup } \\
\text { Level for } \\
\text { Groundwater } \\
\text { Protection }\end{array}$ & $\begin{array}{l}\text { Soil } \\
\text { Cleanup } \\
\text { Level for } \\
\text { River } \\
\text { Protection }\end{array}$ & & \\
\hline Methoxychlor & 0.0053 & 400 & 4 & 1.67 & No & -- \\
\hline 2,4-DB & 0.0098 & 640 & 12.8 & $--^{m}$ & No & -- \\
\hline Dalapon & 0.048 & 2,400 & 20 & $--^{m}$ & No & -- \\
\hline Dicamba & 0.013 & 2,400 & 48 & $-{ }_{-m}^{m}$ & No & -- \\
\hline Dichloroprop ${ }^{\mathrm{s}}$ & 0.21 & 800 & 7 & $-{ }^{m}$ & No & -- \\
\hline
\end{tabular}

*All footnotes are provided following Table 14.

-- $\quad=$ not applicable

$\mathrm{BCL} \quad=$ below cleanup level

$\mathrm{BG} \quad=$ background (obtained from DOE-RL 1996 and DOE-RL 2001b, unless otherwise noted)

$\mathrm{COC}=$ contaminant of concern

COPC = contaminant of potential concern

RAG = remedial action goal

RESRAD = RESidual RADioactivity (dose-assessment model)

Table 13. Comparison of Statistical Contaminant Concentrations to Action Levels for the 118-B-1 Staging Pile Area Footprint Verification Sampling Event.* (3 Pages)

\begin{tabular}{|c|c|c|c|c|c|c|}
\hline \multirow[b]{2}{*}{ COC/COPC } & \multirow[b]{2}{*}{$\begin{array}{l}\text { Statistical } \\
\text { Result } \\
\text { (pCi/g) }\end{array}$} & \multicolumn{3}{|c|}{ Generic Site Lookup Values $^{a}(\mathrm{pCi} / \mathrm{g}$ ) } & \multirow{2}{*}{$\begin{array}{c}\text { Does the } \\
\text { Statistical } \\
\text { Result } \\
\text { Exceed } \\
\text { Lookup } \\
\text { Values? }\end{array}$} & \multirow[b]{2}{*}{$\begin{array}{l}\text { Does the } \\
\text { Statistical } \\
\text { Result Pass } \\
\text { RESRAD } \\
\text { Modeling? }\end{array}$} \\
\hline & & $\begin{array}{l}\text { Shallow } \\
\text { Zone } \\
\text { Lookup } \\
\text { Value }^{b}\end{array}$ & $\begin{array}{l}\text { Groundwater } \\
\text { Protection } \\
\text { Lookup } \\
\text { Value }\end{array}$ & $\begin{array}{l}\text { River } \\
\text { Protection } \\
\text { Lookup } \\
\text { Value }\end{array}$ & & \\
\hline Americium-241 & 0.061 & 31.1 & $--^{c}$ & $--^{c}$ & No & \multirow{9}{*}{ Yes $^{v}$} \\
\hline Carbon-14 & 1.6 & 5.16 & $\mathrm{O}^{\mathrm{c}}$ & $--^{c}$ & No & \\
\hline Cesium-137 & 0.57 & 6.2 & $1,465^{d}$ & $1,465^{d}$ & No & \\
\hline Cobalt- 60 & 0.028 & 1.4 & $13,900^{d}$ & $13,900^{d}$ & No & \\
\hline Europium-152 & 0.20 & 3.3 & $--^{c}$ & $--^{c}$ & No & \\
\hline Nickel-63 & 5.05 & $4,013^{d}$ & $83^{d}$ & $83^{d}$ & No & \\
\hline Plutonium-239/240 & 0.125 & 33.9 & $--^{c}$ & $\mathrm{C}^{\mathrm{c}}$ & No & \\
\hline Strontium-90 & 0.236 & 4.5 & $27.6^{d}$ & $27.6^{d}$ & No & \\
\hline Tritium & 14.6 & $459^{d}$ & $12.6^{d}$ & $12.6^{d}$ & Yes & \\
\hline Uranium-233/234 & $0.625(<B G)$ & $1.1^{\mathrm{g}}$ & $1.1^{\mathrm{g}}$ & $1.1^{g}$ & No & -- \\
\hline Uranium-235 & $0.042(<B G)$ & $0.61^{c}$ & $0.5^{h}$ & $0.5^{\mathrm{h}}$ & No & -- \\
\hline Uranium-238 & $0.562(<B G)$ & $1.1^{g}$ & $1.1^{g}$ & $1.1^{\mathrm{g}}$ & No & -- \\
\hline
\end{tabular}


Table 13. Comparison of Statistical Contaminant Concentrations to Action Levels for the 118-B-1 Staging Pile Area Footprint Verification Sampling Event.* (3 Pages)

\begin{tabular}{|c|c|c|c|c|c|c|}
\hline \multirow[b]{2}{*}{ COC/COPC } & \multirow[b]{2}{*}{$\begin{array}{c}\text { Statistical } \\
\text { Result } \\
\text { (mg/kg) }\end{array}$} & \multicolumn{3}{|c|}{ Remedial Action Goals ${ }^{\mathrm{a}}(\mathrm{mg} / \mathrm{kg})$} & \multirow[b]{2}{*}{$\begin{array}{c}\text { Does the } \\
\text { Statistical } \\
\text { Data Set } \\
\text { Exceed } \\
\text { RAGs? }\end{array}$} & \multirow[b]{2}{*}{$\begin{array}{l}\text { Does the } \\
\text { Statistical } \\
\text { Result Pass } \\
\text { RESRAD } \\
\text { Modeling? }\end{array}$} \\
\hline & & $\begin{array}{c}\text { Direct } \\
\text { Exposure }\end{array}$ & $\begin{array}{l}\text { Soil Cleanup } \\
\text { Level for } \\
\text { Groundwater } \\
\text { Protection }\end{array}$ & $\begin{array}{c}\text { Soil } \\
\text { Cleanup } \\
\text { Level for } \\
\text { River } \\
\text { Protection }\end{array}$ & & \\
\hline Arsenic & $2.3(<\mathrm{BG})$ & 20 & 20 & 20 & No & -- \\
\hline Barium & $72.5(<B G)$ & $5,600^{i}$ & $132^{\mathrm{i}, \mathrm{j}}$ & $224^{i}$ & No & -- \\
\hline Beryllium & $0.36(<B G)$ & $10.4^{k}$ & $1.51^{\mathrm{j}}$ & $1.51^{\mathrm{j}}$ & No & -- \\
\hline Boron' & 1.9 & 16,000 & 320 & $--^{m}$ & No & -- \\
\hline Cadmium $^{t}$ & $0.53(<B G)$ & 13.9 & $0.81^{\mathrm{j}}$ & $0.81^{j}$ & No & - \\
\hline Chromium (total) & $8.3(<\mathrm{BG})$ & $80,000^{i}$ & $18.5^{\mathrm{j}}$ & $18.5^{\mathrm{j}}$ & No & -- \\
\hline Chromium (hexavalent) & 0.27 & 2.1 & $4.8^{\circ}$ & 2 & No & -- \\
\hline Cobalt & $7.7(<B G)$ & 1,600 & 32 & $--^{m}$ & No & -- \\
\hline Copper & $14.8(<B G)$ & 2,960 & 59.2 & $22.0^{j}$ & No & -- \\
\hline Lead & $4.7(<B G)$ & 353 & $10.2^{\mathrm{j}}$ & $10.2^{\mathrm{j}}$ & No & -- \\
\hline Manganese & $330(<B G)$ & 11,200 & $512^{j}$ & $512^{j}$ & No & -- \\
\hline Mercury & $0.13(<B G)$ & 24 & $0.33^{j}$ & $0.33^{j}$ & No & -- \\
\hline Molybdenum $^{n}$ & 0.45 & 400 & 8 & $-\mathrm{m}^{\mathrm{m}}$ & No & -- \\
\hline Nickel & $10.8(<B G)$ & 1,600 & $19.1^{\mathrm{i}}$ & 27.4 & No & -- \\
\hline Vanadium & $46.4(<B G)$ & 560 & $85.1^{j}$ & $--^{m}$ & No & -- \\
\hline Zinc & $38.8(<\mathrm{BG})$ & 24,000 & 480 & $67.8^{j}$ & No & -- \\
\hline Aroclor-1242 & 0.047 & 0.5 & $0.017^{p}$ & $0.017^{\mathrm{p}}$ & Yes & $Y_{e s}{ }^{n}$ \\
\hline Aroclor-1254 & 0.17 & 0.5 & $0.017^{p}$ & $0.017^{\mathrm{p}}$ & Yes & $Y_{e s}{ }^{n}$ \\
\hline Aroclor-1260 & 0.056 & 0.5 & $0.017^{p}$ & $0.017^{\mathrm{P}}$ & Yes & Yes $^{n}$ \\
\hline bis(2-Ethylhexyl)phthalate & 0.09 & 71.4 & 0.625 & 0.36 & No & -- \\
\hline Di-n-butylphthalate & 0.11 & 8,000 & 160 & 540 & No & -- \\
\hline Methylene chloride & 0.014 & 133 & 0.5 & 0.94 & No & -- \\
\hline Trichloroethene & 0.002 & 2.5 & 0.01 & 0.31 & No & -- \\
\hline Aldrin & 0.0010 & 0.0588 & $0.00165^{p}$ & $0.00165^{p}$ & No & -- \\
\hline alpha-Chlordane & 0.00080 & $0.769^{r}$ & $0.0165^{p, r}$ & $0.0165^{p}$ & No & -- \\
\hline beta-BHC & 0.00082 & 0.556 & 0.00486 & 0.00554 & No & -- \\
\hline 4,4'-DDE & 0.0065 & 2.94 & 0.0257 & $0.005^{r}$ & Yes & Yes $^{n}$ \\
\hline Endosulfan sulfate & 0.0019 & 480 & 9.6 & 0.0112 & No & -- \\
\hline gamma-Chlordane & 0.0065 & $0.769^{r}$ & $0.0165^{p, r}$ & $0.0165^{p}$ & No & -- \\
\hline Methoxychlor & 0.0017 & 400 & 4 & 1.67 & No & -- \\
\hline $2,4-D$ & 0.048 & 800 & 7 & $--^{m}$ & No & -- \\
\hline $2,4-D B$ & $0.028^{w}$ & 640 & 12.8 & $--^{m}$ & No & -- \\
\hline $2,4,5-T$ & 0.013 & 800 & 16 & $--^{m}$ & No & - \\
\hline
\end{tabular}


Table 13. Comparison of Statistical Contaminant Concentrations to Action Levels for the 118-B-1 Staging Pile Area Footprint Verification Sampling Event.* (3 Pages)

\begin{tabular}{|c|c|c|c|c|c|c|}
\hline \multirow[b]{2}{*}{ COC/COPC } & \multirow[b]{2}{*}{$\begin{array}{c}\text { Statistical } \\
\text { Result } \\
(\mathrm{mg} / \mathrm{kg})\end{array}$} & \multicolumn{3}{|c|}{ Remedial Action Goals ${ }^{\mathrm{a}}$ (mg/kg) } & \multirow[b]{2}{*}{$\begin{array}{c}\text { Does the } \\
\text { Statistical } \\
\text { Data Set } \\
\text { Exceed } \\
\text { RAGs? }\end{array}$} & \multirow[b]{2}{*}{$\begin{array}{l}\text { Does the } \\
\text { Statistical } \\
\text { Result Pass } \\
\text { RESRAD } \\
\text { Modeling? }\end{array}$} \\
\hline & & $\begin{array}{c}\text { Direct } \\
\text { Exposure }\end{array}$ & $\begin{array}{l}\text { Soil Cleanup } \\
\text { Level for } \\
\text { Groundwater } \\
\text { Protection }\end{array}$ & $\begin{array}{c}\text { Soil } \\
\text { Cleanup } \\
\text { Level for } \\
\text { River } \\
\text { Protection }\end{array}$ & & \\
\hline $2,4,5-\mathrm{TP}$ & 0.043 & 640 & 5 & $--^{m}$ & No & -- \\
\hline Dalapon & 0.043 & 2,400 & 20 & $--^{m}$ & No & -- \\
\hline Dicamba & 0.023 & 2,400 & 48 & $--^{m}$ & No & - \\
\hline Dichloroprops & 0.049 & 800 & 7 & $-^{m}$ & No & -- \\
\hline Pentachlorophenol & 0.0040 & 8.33 & 0.073 & 0.056 & No & -- \\
\hline
\end{tabular}

${ }^{*}$ All footnotes are provided following Table 14.

= not applicable

BG = background (obtained from DOE-RL 1996 and DOE-RL 2001b, unless otherwise noted)

$\mathrm{COC}=$ contaminant of concern

COPC = contaminant of potential concern

RAG = remedial action goal

RESRAD = RESidual RADioactivity (dose-assessment model)

Table 14. Comparison of Maximum Contaminant Concentrations to Action Levels for the 118-B-1 Focused Verification Samples. ${ }^{*}$ (2 Pages)

\begin{tabular}{|c|c|c|c|c|c|c|}
\hline \multirow[b]{2}{*}{ COC/COPC } & \multirow[b]{2}{*}{$\begin{array}{l}\text { Maximum } \\
\text { Result } \\
\text { (pCi/g) }\end{array}$} & \multicolumn{3}{|c|}{ Generic Site Lookup Values ${ }^{a}(\mathrm{pCi} / \mathrm{g})$} & \multirow{2}{*}{$\begin{array}{l}\text { Does the } \\
\text { Maximum } \\
\text { Result } \\
\text { Exceed } \\
\text { Lookup } \\
\text { Values? }\end{array}$} & \multirow[b]{2}{*}{$\begin{array}{c}\text { Does the } \\
\text { Maximum } \\
\text { Result Pass } \\
\text { RESRAD } \\
\text { Modeling? }\end{array}$} \\
\hline & & $\begin{array}{l}\text { Shallow } \\
\text { Zone } \\
\text { Lookup } \\
\text { Value }^{b}\end{array}$ & $\begin{array}{l}\text { Groundwater } \\
\text { Protection } \\
\text { Lookup } \\
\text { Value }\end{array}$ & $\begin{array}{l}\text { River } \\
\text { Protection } \\
\text { Lookup } \\
\text { Value }\end{array}$ & & \\
\hline Cesium-137 & 0.353 & 6.2 & $1,465^{\mathrm{d}}$ & $1,465^{d}$ & No & \multirow{2}{*}{ Yes $^{e}$} \\
\hline Cobalt-60 & 0.155 & 1.4 & $13,900^{d}$ & $13,900^{d}$ & No & \\
\hline Tritium & 137 & $459^{d}$ & $12.6^{d}$ & $12.6^{d}$ & Yes & $-{ }^{f}$ \\
\hline Uranium-233/234 & $0.587(<B G)$ & $1.1^{\mathrm{g}}$ & $1.1^{\mathrm{g}}$ & $1.1^{\mathrm{g}}$ & No & -- \\
\hline Uranium-238 & $0.564(<B G)$ & $1.1^{\mathrm{g}}$ & $1.1^{\mathrm{g}}$ & $1.1^{g}$ & No & -- \\
\hline \multirow[b]{2}{*}{ COC/COPC } & \multirow[b]{2}{*}{$\begin{array}{l}\text { Maximum } \\
\text { Result } \\
(\mathrm{mg} / \mathrm{kg})\end{array}$} & \multicolumn{3}{|c|}{ Remedial Action Goals ${ }^{\mathrm{a}}(\mathrm{mg} / \mathrm{kg})$} & \multirow[b]{2}{*}{$\begin{array}{l}\text { Does the } \\
\text { Maximum } \\
\text { Result } \\
\text { Exceed } \\
\text { Lookup } \\
\text { Values? }\end{array}$} & \multirow[b]{2}{*}{$\begin{array}{l}\text { Does the } \\
\text { Maximum } \\
\text { Result Pass } \\
\text { RESRAD } \\
\text { Modeling? }\end{array}$} \\
\hline & & $\begin{array}{c}\text { Direct } \\
\text { Exposure }\end{array}$ & $\begin{array}{l}\text { Soil Cleanup } \\
\text { Level for } \\
\text { Groundwater } \\
\text { Protection }\end{array}$ & $\begin{array}{c}\text { Soil } \\
\text { Cleanup } \\
\text { Level for } \\
\text { River } \\
\text { Protection } \\
\end{array}$ & & \\
\hline Arsenic & 7.0 & 20 & 20 & 20 & No & - \\
\hline Barium & 261 & $5,600^{i}$ & $132^{\mathrm{i}, \mathrm{j}}$ & $224^{i}$ & Yes & $Y_{e s}{ }^{n}$ \\
\hline Beryllium & $0.58(<B G)$ & $10.4^{k}$ & $1.51^{\mathrm{j}}$ & $1.51^{\mathrm{j}}$ & No & -- \\
\hline Boron' & 24.5 & 16,000 & 320 & $-\mathrm{m}^{\mathrm{m}}$ & No & -- \\
\hline Cadmium $^{t}$ & $0.28(<\mathrm{BG})$ & 13.9 & $0.81^{j}$ & $0.81^{\mathrm{j}}$ & No & -- \\
\hline Chromium (total) & $16.4(<B G)$ & $80,000^{i}$ & $18.5^{\mathrm{j}}$ & $18.5^{\mathrm{j}}$ & No & -- \\
\hline
\end{tabular}


Table 14. Comparison of Maximum Contaminant Concentrations to Action Levels for the 118-B-1 Focused Verification Samples.* (2 Pages)

\begin{tabular}{|c|c|c|c|c|c|c|}
\hline \multirow[b]{2}{*}{ COC/COPC } & \multirow[b]{2}{*}{$\begin{array}{c}\text { Maximum } \\
\text { Result } \\
(\mathrm{mg} / \mathrm{kg})\end{array}$} & \multicolumn{3}{|c|}{ Remedial Action Goals ${ }^{a}(\mathrm{mg} / \mathrm{kg})$} & \multirow{2}{*}{$\begin{array}{l}\text { Does the } \\
\text { Maximum } \\
\text { Result } \\
\text { Exceed } \\
\text { Lookup } \\
\text { Values? }\end{array}$} & \multirow[b]{2}{*}{$\begin{array}{c}\text { Does the } \\
\text { Maximum } \\
\text { Result Pass } \\
\text { RESRAD } \\
\text { Modeling? }\end{array}$} \\
\hline & & $\begin{array}{c}\text { Direct } \\
\text { Exposure }\end{array}$ & $\begin{array}{l}\text { Soil Cleanup } \\
\text { Level for } \\
\text { Groundwater } \\
\text { Protection }\end{array}$ & $\begin{array}{c}\text { Soil } \\
\text { Cleanup } \\
\text { Level for } \\
\text { River } \\
\text { Protection }\end{array}$ & & \\
\hline Cobalt & $11.3(<B G)$ & 1,600 & 32 & $-\mathrm{m}^{\mathrm{m}}$ & No & $-\cdots$ \\
\hline Copper & 28.0 & 2,960 & 59.2 & $22.0^{j}$ & Yes & Yes $^{n}$ \\
\hline Lead & 34.3 & 353 & $10.2^{\mathrm{j}}$ & $10.2^{\mathrm{j}}$ & Yes & $Y_{e s}{ }^{n}$ \\
\hline Manganese & $421(<B G)$ & 11,200 & $512^{j}$ & $512^{j}$ & No & -- \\
\hline Mercury & 14.5 & 24 & $0.33^{j}$ & $0.33^{j}$ & Yes & Yes $^{n}$ \\
\hline Molybdenum' & 0.81 & 400 & 8 & $-\mathrm{-m}^{\mathrm{m}}$ & No & -- \\
\hline Nickel & $15.2(<B G)$ & 1,600 & $19.1^{\mathrm{i}}$ & 27.4 & No & -- \\
\hline Vanadium & $62.8(<B G)$ & 560 & $85.1^{j}$ & $--^{m}$ & No & -- \\
\hline Zinc & $65.1(<B G)$ & 24,000 & 480 & $67.8^{j}$ & No & -- \\
\hline bis(2-Ethylhexyl)phthalate & 0.038 & 71.4 & 0.625 & 0.36 & No & -- \\
\hline Acetone & 0.099 & 72,000 & 720 & $--^{m}$ & No & -- \\
\hline Methylene chloride & 0.016 & 133 & 0.5 & 0.94 & No & -- \\
\hline Methoxychlor & 0.0047 & 400 & 4 & 1.67 & No & -- \\
\hline Dicamba & 0.0091 & 2,400 & 48 & $--^{m}$ & No & -- \\
\hline
\end{tabular}

*All footnotes are provided following Table 14.

$--\quad=$ not applicable

BG = background (obtained from DOE-RL 1996 and DOE-RL 2001b, unless otherwise noted)

$\mathrm{COC}=$ contaminant of concern

$\mathrm{COPC}=$ contaminant of potential concern

NA $\quad=$ not applicable

RAG = remedial action goal

RESRAD = RESidual RADioactivity (dose-assessment model)

\section{Verification Sample Summary Table Footnotes}

a Lookup values and RAGs obtained from the RDR/RAWP (DOE-RL 2005) or calculated per WAC 173340-720, 730, and 740, Method B, 1996, unless otherwise noted.

b Activity corresponding to a single-radionuclide $15 \mathrm{mrem} / \mathrm{yr}$ exposure as calculated using a generic RESidual RADioactivity (RESRAD) model (DOE-RL 2005).

c No value-RESRAD modeling predicts the contaminant will not reach groundwater within 1,000 years.

' Lookup value calculated per 100 Area Radionuclide and Nonradionuclide Lookup Values for the 1995 Interim Remedial Action Record of Decision (BHI 2004).

e 118-B-1 Combined Areas Cleanup Verification RESRAD Calculation Brief, Appendix C.

$f$ The 118-B-1 ESD provides for controls to minimize further mobilization of residual tritium contamination in the deep vadose zone and allow interim site closure without further excavation based on evaluation of balancing factors.

9 The calculated lookup value is below the Hanford-specific statistical soil background activity. The value presented is the Hanford-specific statistical soil background activity.

${ }^{n}$ The lookup value is below the required minimum detectable activity (MDA). The value presented is the required MDA. 
i Noncarcinogenic cleanup levels calculated from WAC 173-340, 1996 (Method B), as presented in the RDR/RAWP (DOE-RL 2005).

i Where cleanup levels are less than background, cleanup levels default to background (WAC 173-340700[4][d]) (1996).

${ }^{k}$ Carcinogenic cleanup level calculated based on the inhalation exposure pathway per WAC 173-340750[3], 1996 (Method B for air quality) and an airborne particulate mass loading rate of $0.0001 \mathrm{~g} / \mathrm{m}^{3}$ (WDOH 1997).

1 No Hanford Site-specific or Washington State background value available.

$\mathrm{m}$ No parameters (bioconcentration factors or ambient water quality criteria values) are available from the Ecology Cleanup Levels and Risk Calculations database (Ecology 2005) or other databases to calculate cleanup levels (WAC 173-340-730(3)(a)(iii), 1996 [Method B for surface waters]).

${ }^{n}$ Based on the 100 Area Analogous Sites RESRAD Calculations (BHI 2005) and contaminant-specific soil portioning coefficient $\left(\mathrm{K}_{\mathrm{d}}\right)$ value, contaminant will not migrate vertically more than $3 \mathrm{~m}(10 \mathrm{ft})$ in

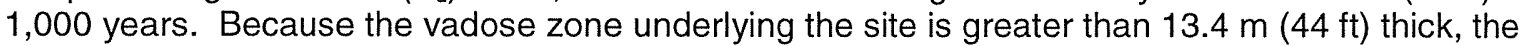
contaminant will not reach groundwater (and thus the Columbia River) in 1,000 years.

- Calculated cleanup level (per WAC 173-340-720(3), 1996 [Method B for groundwater] and WAC 173340-740(3)(a)(ii)(A), 1996 ["100 times rule"]) presented is lower than that presented in the RDR/RAWP (DOE-RL 2005), based on updated oral reference dose value (as provided in the EPA's Integrated Risk Information System available on the internet at http://www.epa.gov/iris).

${ }^{\mathrm{p}}$ Where cleanup levels are less than the required detection limit (RDL), cleanup levels default to the RDL (WAC 173-340-707[2], 1996 and DOE-RL 2005).

q The soil-to-groundwater dilution factor modeled for the 100-B/C Area indicates that the small residual mass of beta-BHC will not be sufficient to migrate to groundwater or the river and result in a concentration exceeding the WAC 173-340-720(3), 1996 (Method B for groundwater) carcinogenic groundwater cleanup level $(0.0486 \mu \mathrm{g} / \mathrm{L}$ ) or the WAC 173-340-730(3), 1996 (Method B for surface waters) carcinogenic surface water cleanup level $(0.0277 \mu \mathrm{g} / \mathrm{L})$.

$r$ Carcinogenic cleanup level calculated from WAC 173-340, 1996 (Method B), as presented in the RDR/RAWP (DOE-RL 2005).

$s$ Toxicity data for this chemical are not available. RAGs for dichloroprop are based on the surrogate chemical 2,4-D.

t Hanford Site-specific background value is not available; not evaluated during background study. Value used is from Natural Background Soil Metals Concentrations in Washington State (Ecology 1994).

$u$ The statistical verification data set fails the third component of the WAC 173-340-740(7)(e) 3-part test in comparison against soil RAG for river protection (i.e., more than $10 \%$ of the data set exceeds the soil RAG value).

$\checkmark$ 118-B-1 Burial Ground Cleanup Verification RESRAD Calculation Brief for Overburden, Below Cleanup Level (BCL), and Staging Pile Area Soil, Appendix C.

${ }^{w}$ A portion of the 2,4-DB data for the staging pile area was rejected based on data quality deficiencies; the value presented is the maximum detected accepted value.

\subsection{DATA EVALUATION}

This section demonstrates that remedial actions at the 118-B-1 Burial Ground have achieved the applicable RAGs and comply with applicable nonradionuclide risk requirements and WAC 173-340-740(7)(e) three-part test criteria. This section also describes the RESidual RADioactivity (RESRAD) modeling performed that predicts the dose rate, the excess lifetime cancer risk, and the impact on groundwater and the river from residual radionuclide $\mathrm{COC/COPC}$ concentrations. This section also presents a comparison against ecological soil screening levels. 


\subsection{COMPARISON OF SAMPLE DATA TO RAGS}

Tables 4 through 14 compare the cleanup verification statistical and focused sample values to the soil RAGs and lookup values for direct exposure, protection of groundwater, and protection of the Columbia River. Barium, copper, lead, mercury, aroclor-1242, aroclor-1254, aroclor-1260, bis(2-ethylhexyl)phthalate, 4,4'-DDE, and dieldrin were quantified at concentrations exceeding soil protection RAGs for groundwater and/or river protection in one or more areas. Data were not collected on the vertical extent of these contaminants, but given the lowest soil-partitioning coefficient for these constituents ( $22 \mathrm{~mL} / \mathrm{g}$ for copper), none would be expected to migrate more than $3 \mathrm{~m}$ (10 ft) vertically in 1,000 years (BHI 2005). The vadose zone underlying the 118-B-1 Burial Ground excavation is greater than $13.4 \mathrm{~m}(44 \mathrm{ft})$ thick; therefore, residual concentrations of these contaminants are predicted to be protective of groundwater. The only pathway for contaminant migration to the Columbia River is via groundwater; therefore, residual concentrations of these contaminants are also predicted to be protective of the Columbia River.

The pesticide beta-BHC was also quantified slightly above the soil RAGs for groundwater and river protection in one sample from Area 4. The low soil-partitioning coefficient associated with this contaminant $(2.14 \mathrm{~mL} / \mathrm{g})$ prevents the use of conservative migration modeling to demonstrate protectiveness. However, modeling of the hydraulic conductivity and gradient data for the 100-B/C Area shows that there is a soil-to-groundwater dilution factor of approximately 6 in the soils underlying the 118-B-1 site, which indicates that beta-BHC will not leach to groundwater at concentrations exceeding the most restrictive WAC 173-340-720 cleanup level for groundwater $(0.049 \mu \mathrm{g} / \mathrm{L})$. Further, the fact that the only detection of beta-BHC in this area was a trace residual concentration detected in one soil sample suggests that there is insufficient residual mass of beta-BHC present to migrate through $13.4 \mathrm{~m}(44 \mathrm{ft})$ of underlying vadose zone soil to reach groundwater (and thus the Columbia River) and result in concentrations exceeding groundwater or surface water RAGs. Therefore, the remedial action objectives for protection of groundwater and the Columbia River are considered to have been achieved.

Tritium was also quantified above lookup values for the protection of groundwater and the Columbia River in Areas 1,2, and 3, as well as the SPA footprint, and in focused samples associated with those areas. These exceedances were addressed by sitespecific assessment and RESRAD modeling, described in Section 6.5 of this document.

\subsection{COMPARISON OF SAMPLE DATA TO ECOLOGICAL RISK SCREENING LEVELS}

Soil cleanup levels were established in the Burial Ground ROD (EPA 2000) based on a limited ecological risk assessment. Although not required by the Burial Ground ROD, a comparison against ecological risk screening levels has been made for the 118-B-1 waste site COCs/COPCs. Screening levels were not exceeded for the site constituents, with the exception of barium, boron, cadmium, lead, mercury, molybdenum, selenium, and vanadium. Exceedance of screening values does not necessarily indicate the 
existence of risk to ecological receptors. Concentrations of cadmium, vanadium, and selenium are within the range of Hanford Site background levels, and molybdenum concentrations are consistent with those seen elsewhere at the Hanford Site (no established background value is available for molybdenum). Exceedances for barium, boron, lead, and mercury will be evaluated in the context of additional lines of evidence as part of the baseline risk assessment. A more complete quantitative ecological risk assessment will be presented in the baseline risk assessment for the river corridor portion of the Hanford Site and will be used to support the final closeout decision for this waste site.

\subsection{HUMAN HEALTH RISK EVALUATION FOR NONRADIONUCLIDES}

The human health risk evaluations are calculated for nonradionuclide COCs/COPCs, per WAC 173-340-740(3)(a)(iii)(A) and (B). The requirements include a hazard quotient of less than 1.0 for all individual noncarcinogens, a cumulative hazard quotient of less than 1.0, an individual contaminant carcinogenic risk of less than $1 \times 10^{-6}$, and a cumulative excess carcinogenic risk of less than $1 \times 10^{-5}$. The details of the hazard quotient calculations are provided in Appendix C.

Hazard quotient and excess carcinogenic risk calculations were conservatively performed for the 118-B-1 Burial Ground using the highest of the focused and statistical values from all areas. Risk values were not calculated for constituents that were not detected or were detected at concentrations below Hanford Site or Washington State background values. All individual hazard quotients are below 1.0, and all individual excess carcinogenic risk values are below $1 \times 10^{-6}$. The cumulative hazard quotient for the 118-B-1 waste site is $8.8 \times 10^{-1}$ and the cumulative excess carcinogenic risk value is $8.0 \times 10^{-7}$, satisfying the criteria of less than 1.0 and less than $1 \times 10^{-5}$, respectively. Therefore, nonradionuclide risk requirements are met.

\subsection{WAC THREE-PART TEST FOR NONRADIONUCLIDES}

When using a statistical sampling approach, a RAG requirement for nonradionuclides is the WAC 173-340-740(7)(e) three-part test. The WAC 173-340 three-part test consists of the following criteria: (1) the cleanup verification $95 \% \mathrm{UCL}$ value must be less than the cleanup level, (2) no single detection can exceed two times the cleanup criteria, and (3) the percentage of samples exceeding the cleanup criteria must be less than $10 \%$ of the data set.

The application of the three-part test for the 118-B-1 Burial Ground is included in the 95\% UCL calculation (Appendix C). The results of this evaluation indicate that all residual COC/COPC concentrations pass the three-part test in comparison against applicable RAGs, except for barium, copper, lead, mercury, bis(2-ethylhexyl)phthalate, and 4,4'-DDE in comparison against soil RAGs for protection of groundwater and/or the Columbia River in one or more sampling areas. However, based on RESRAD modeling (BHI 2005), as described in Section 6.1, none of these contaminants are predicted to 
migrate to groundwater or the Columbia River in 1,000 years. Therefore, the requirements of the three-part test are met.

\subsection{RESRAD MODELING FOR RADIONUCLIDES}

For radionuclide contaminants, site-specific RESRAD (ANL 2005) modeling was used to determine contaminant migration and to predict the dose rate, the excess lifetime cancer risk, and the impact on groundwater and the Columbia River from residual radionuclide COC/COPC concentrations (DOE-RL 2005). For the 118-B-1 Burial Ground, separate evaluations were performed for the overburden stockpiles, BCL material stockpiles, and staging pile footprint. The maximum value for each radionuclide analyte for all remediation footprint areas (Areas 1 through 7 ) and focused samples were conservatively included in one combined evaluation. Residual tritium concentrations within the remediation footprint were not included in the RESRAD evaluations for groundwater and river protection, because institutional controls implemented per the 118-B-1 ESD (EPA 2007) will allow attenuation of residual vadose zone tritium contamination. Residual concentrations of carbon-14 and strontium-90 were not included in the plant ingestion pathway for the remediation footprint, as this would lead to a predicted exceedance of direct exposure criteria, and residual concentrations of these contaminants are below the root penetration zone.

The individual radionuclide cleanup statistical values from Tables 4 through 14 were entered into the RESRAD computer code with the results included in the RESRAD calculations in Appendix $C$. The RESRAD-derived groundwater radionuclide concentrations as compared to groundwater RAGs are provided in the RESRAD calculations and the Comparison to Drinking Water Standards Calculation in Appendix C. Specific results from the calculations are summarized in the following subsections.

\subsubsection{Attainment of Radionuclide Direct Exposure RAG}

The direct exposure RAG for radionuclide COCs is expressed in terms of an allowable radiation dose rate above background, which is $15 \mathrm{mrem} / \mathrm{yr}$ over 1,000 years. The results of the RESRAD dose rate predictions for the 118-B-1 Burial Ground are summarized in Figures 8 through 11. The maximum dose rates for all areas occurs in the present year (2007), with predicted values of $13.0 \mathrm{mrem} / \mathrm{yr}, 0.309 \mathrm{mrem} / \mathrm{yr}$, $1.70 \mathrm{mrem} / \mathrm{yr}$, and $6.96 \mathrm{mrem} / \mathrm{yr}$ for the combined remediation footprint, overburden stockpiles, BCL material stockpiles, and SPA footprint, respectively. All dose rate predictions are less than the $15 \mathrm{mrem} / \mathrm{yr}$ RAG over 1,000 years.

\subsubsection{Attainment of Radionuclide Excess Lifetime Cancer Risk Goal}

The "National Oil and Hazardous Substances Pollution Contingency Plan" (40 Code of Federal Regulations [CFR] 300) presents a target range for residual radionuclide risk of $10^{-4}$ to $10^{-6}$. The RESRAD model calculates the excess lifetime cancer risk associated 
Figure 8. RESRAD Analysis for the Combined Areas in the Remediation Footprint- All Radionuclides, All-Pathways Dose Rate Estimate.

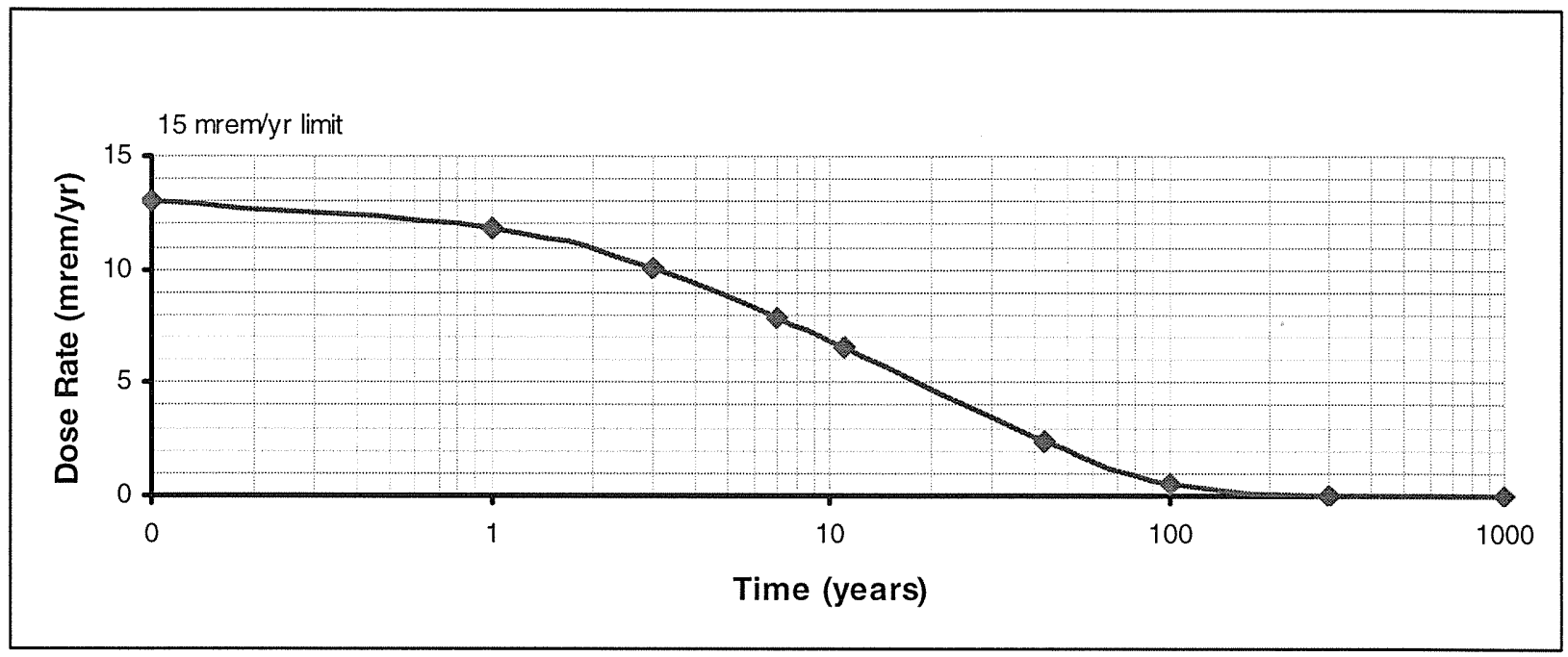

Figure 9. RESRAD Analysis for the Overburden Stockpiles- All Radionuclides, All-Pathways Dose Rate Estimate.

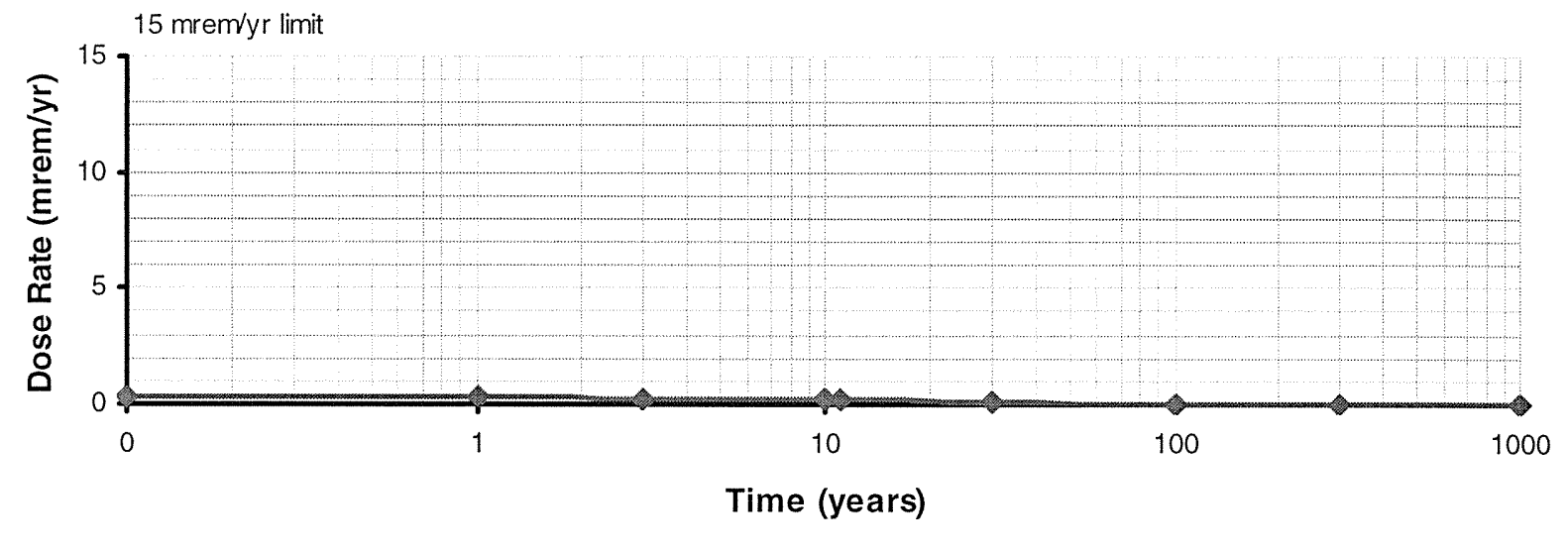

with the estimated radiation dose rates, with results summarized in Figures 12 through 15. For the 118-B-1 Burial Ground, the maximum excess lifetime cancer risk occurs at the present year (2007) for all areas, with predicted values of $1.38 \times 10^{-4}, 3.65 \times 10^{-6}$, $1.98 \times 10^{-5}$, and $5.82 \times 10^{-5}$ for the combined remediation footprint, overburden stockpiles, BCL material stockpiles, and SPA footprint, respectively.

\subsubsection{Attainment of Radionuclide Groundwater and River Protection RAGS}

The attainment of groundwater and river protection RAGs for radionuclides is determined by four criteria: (1) attain single COC/COPC groundwater and river RAGs, (2) attain the National Primary Drinking Water Regulations (40 CFR 141) $4 \mathrm{mrem} / \mathrm{yr}$ 
Figure 10. RESRAD Analysis for the BCL Stockpiles- All Radionuclides, All-Pathways Dose Rate Estimate.

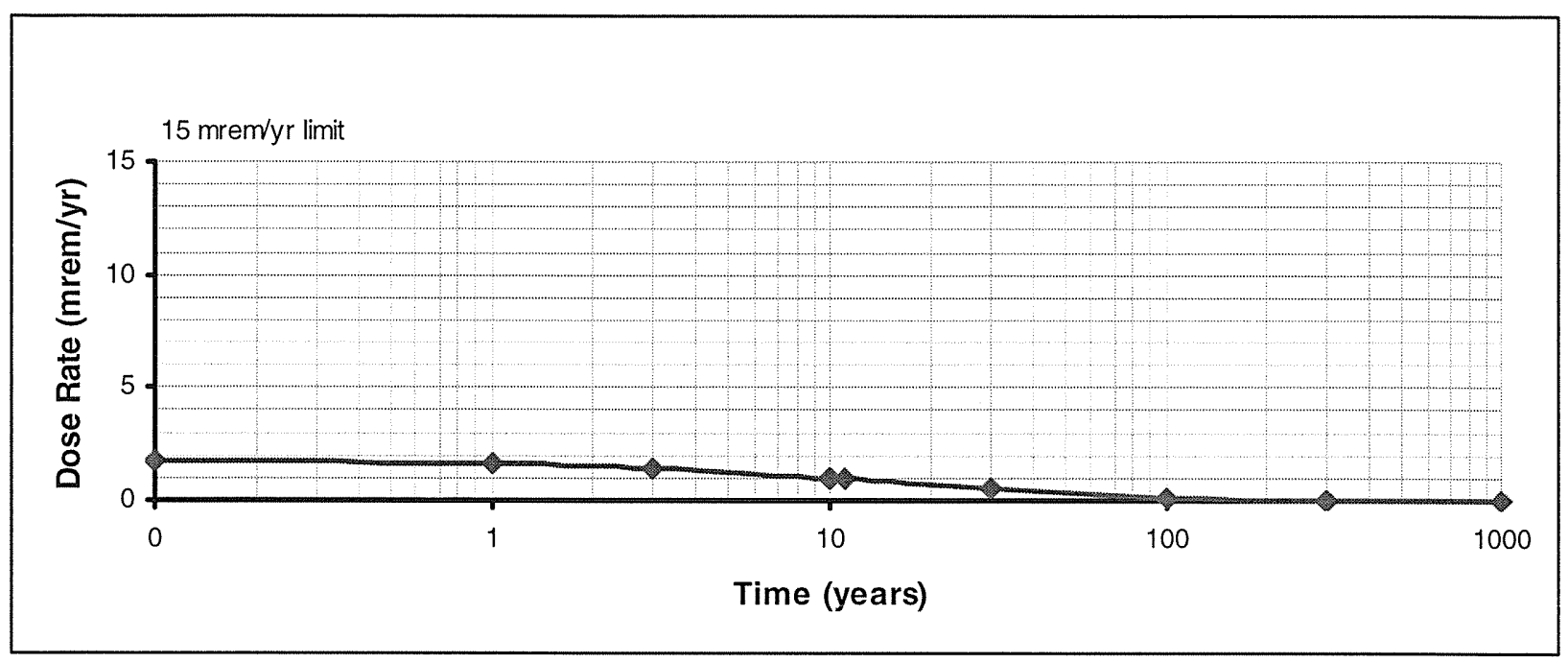

Figure 11. RESRAD Analysis for the SPA Footprint- All Radionuclides, All-Pathways Dose Rate Estimate.

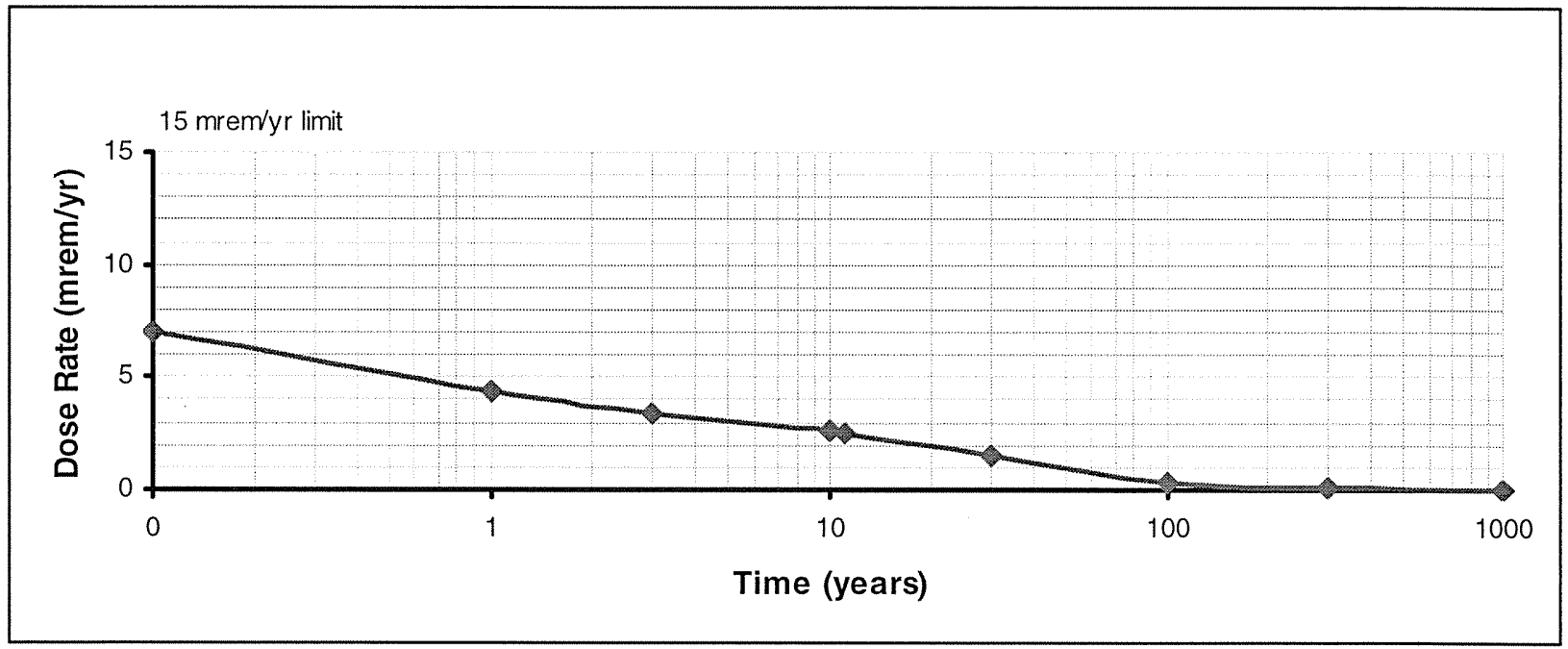

(beta/gamma) dose standard to target receptor/organ, and (3) meet drinking water standards for non-uranium alpha-emitters (the more stringent of $15 \mathrm{pCi} / \mathrm{L} \mathrm{MCL}$ or $1 / 25$ th of the derived concentration guide [DOE Order 5400.5]); and 4) meet total uranium standard of $21.2 \mathrm{pCi} / \mathrm{L}$.

The first criterion is determined by comparing peak concentrations of radionuclide COCs/COPCs to the RAGs. The peak concentration is the maximum predicted value from all areas evaluated, as shown in the RESRAD calculation briefs in Appendix C. Of the radionuclide COCs/COPCs for the 118-B-1 Burial Ground, only cesium-137, cobalt-60, strontium-90, and tritium are predicted to reach groundwater in 1,000 years. 
Figure 12. RESRAD Analysis - Radionuclide Risk, All Pathways - Combined Areas in the Remediation Footprint.

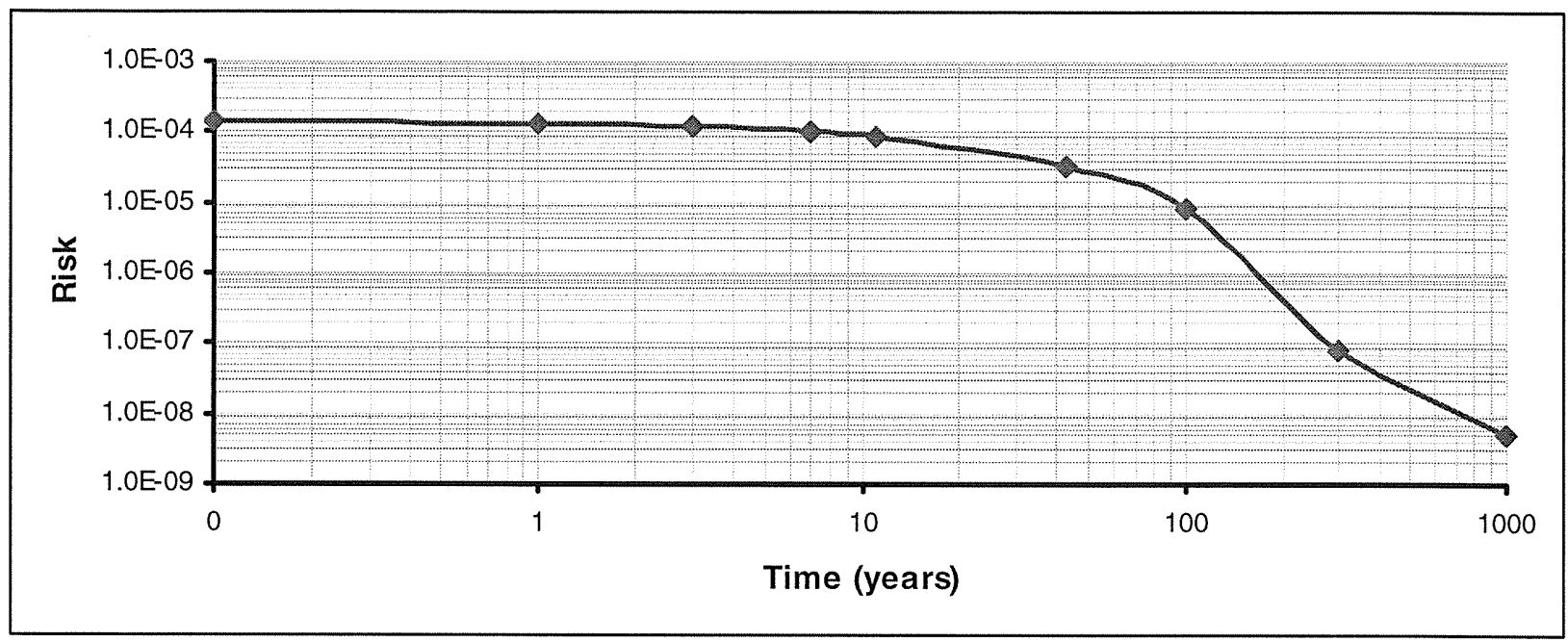

Figure 13. RESRAD Analysis - Radionuclide Risk, All Pathways Overburden Stockpiles.

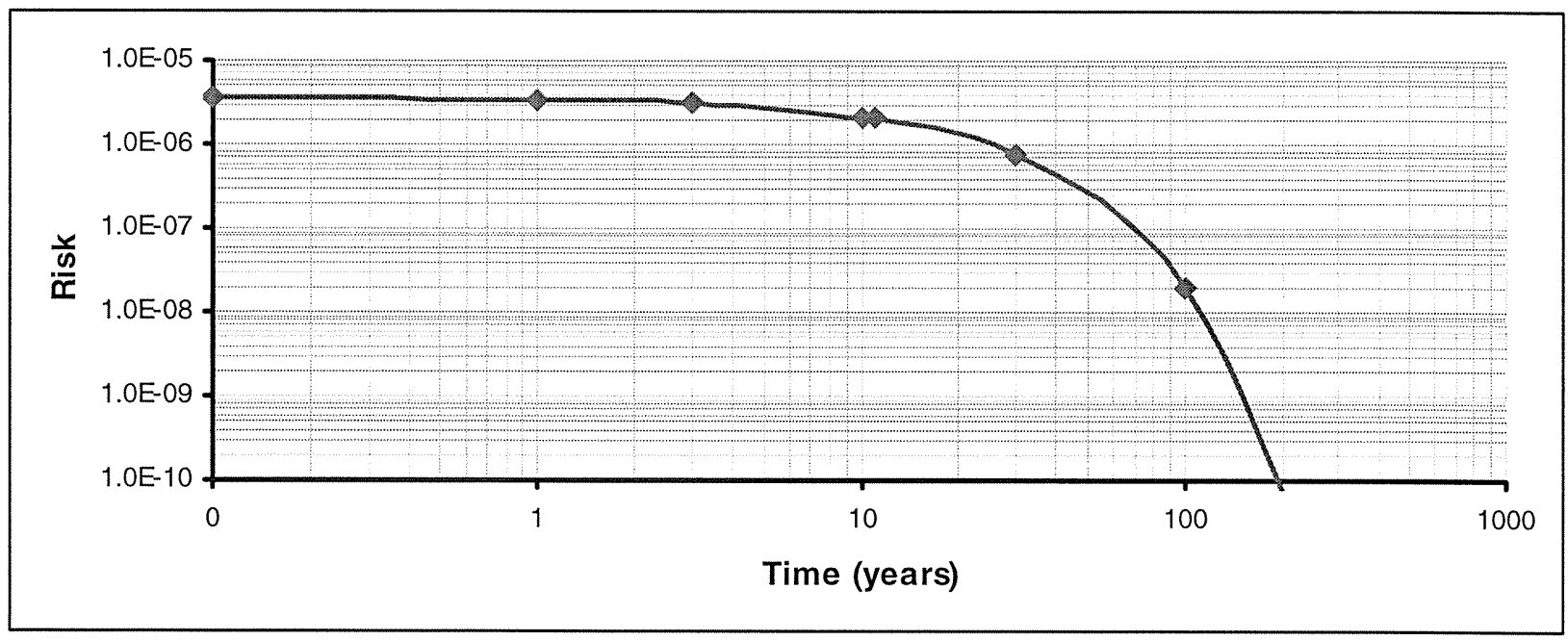

Predicted activity levels of cesium-137, cobalt-60, and strontium-90 are all significantly below the single COC/COPC RAGs. Predicted activity levels of tritium associated with soil activities in the SPA, overburden, and BCL soils are also significantly below the single COC/COPC RAGs. Tritium was not included in the overall site model for the excavation footprint, as described in Section 6.5. Tritium associated with the excavation footprint will exceed groundwater and Columbia River RAGs without the additional institutional controls documented in the 118-B-1 ESD (EPA 2007). The peak concentrations for each of these radionuclides are summarized in Table 15, but the tritium comparison is only for the combined SPA, overburden, and BCL soils. The peak concentration is the maximum value, as predicted by RESRAD modeling, from all evaluation areas, with the exception that tritium in the excavation footprint is not included because it is known that tritium will exceed RAGs without the additional institutional controls described in the 118-B-1 ESD (EPA 2007). 
Figure 14. RESRAD Analysis - Radionuclide Risk, All Pathways BCL Stockpiles.

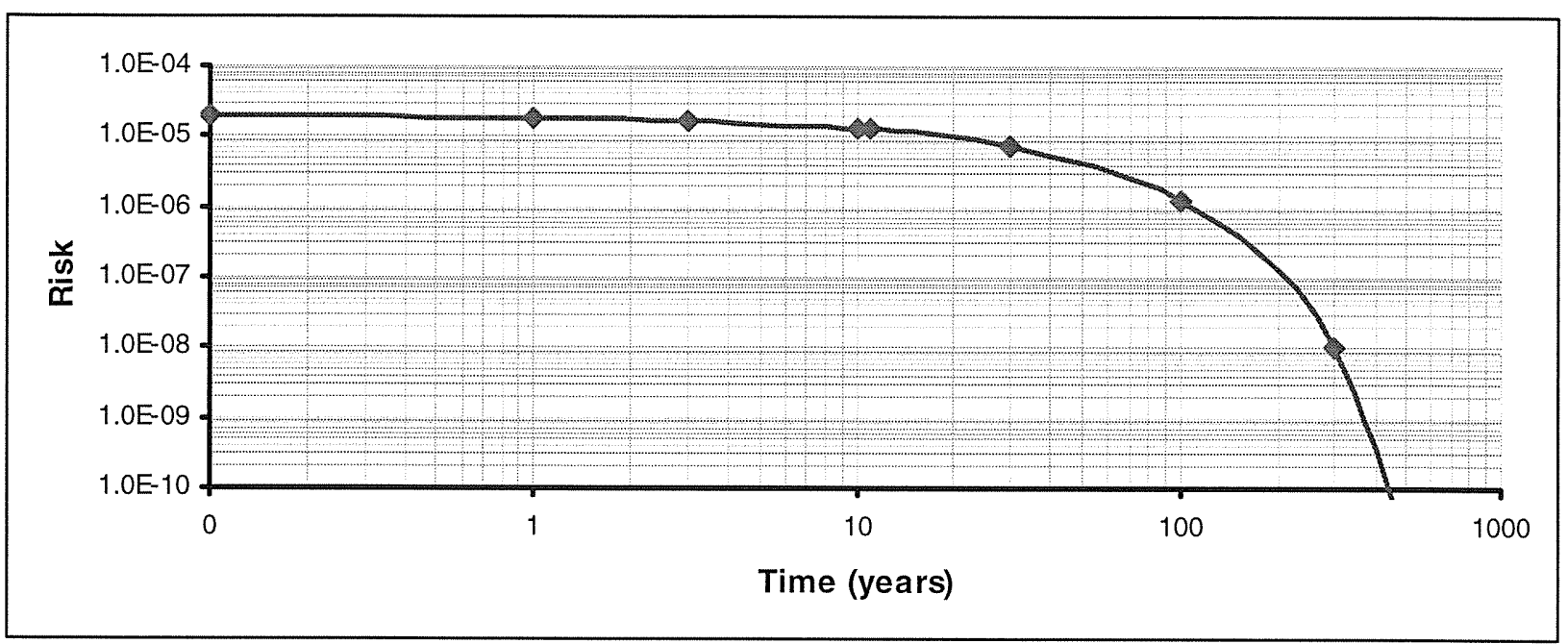

Figure 15. RESRAD Analysis - Radionuclide Risk, All Pathways - SPA Footprint.

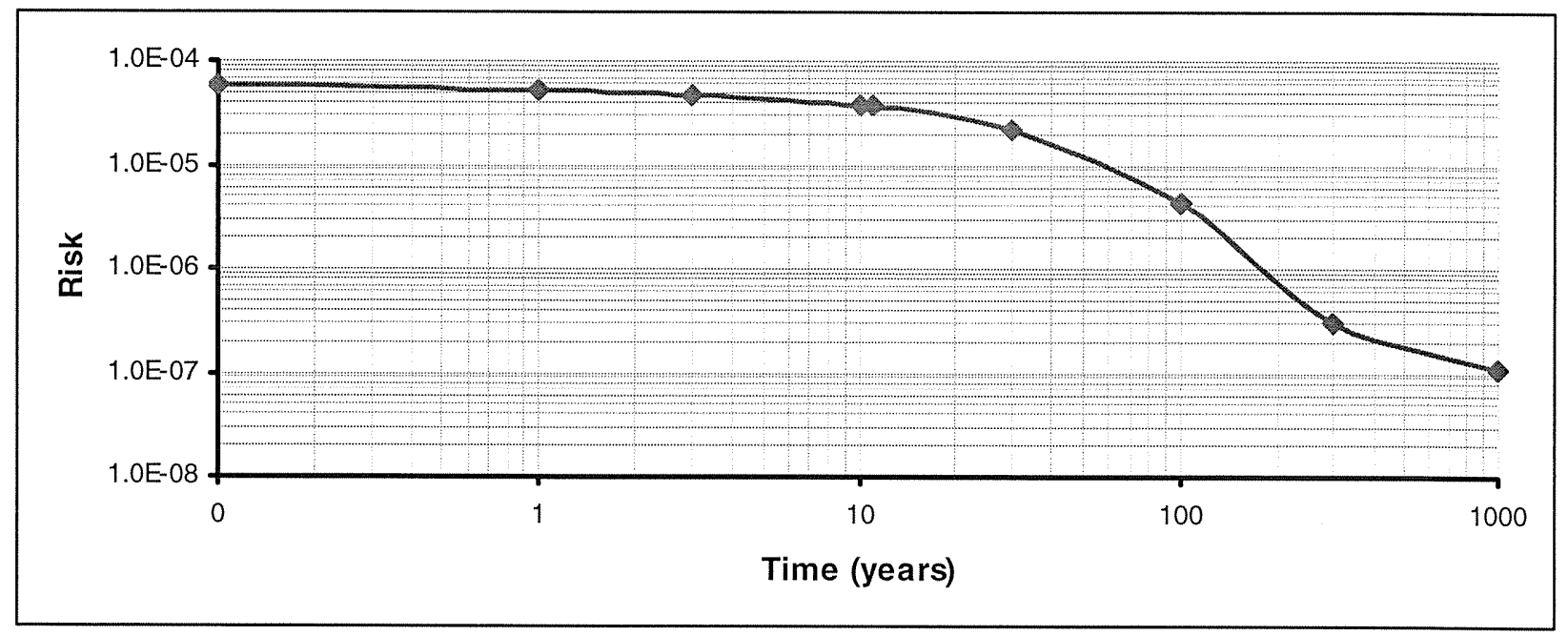

The second criterion, attainment of the National Primary Drinking Water Regulations $4 \mathrm{mrem} / \mathrm{yr}$ (beta/gamma) above background dose standard to target receptor/organ, is summarized in Figure 16, based on the results of the MCL calculation brief (Appendix C). RESRAD modeling predicts a maximum dose of $0.0786 \mathrm{mrem} / \mathrm{yr}$ to any organ (total body, bone, liver, and gastrointestinal track [lower large intestine]) within the 1,000 years of the evaluation period. Therefore, this criterion is attained.

The third criterion, meet drinking water standards for non-uranium alpha-emitters (the more stringent of $15 \mathrm{pCi} / \mathrm{L} \mathrm{MCL}$ or $1 / 25$ th of the derived concentration guide [DOE Order 5400.5]) is demonstrated in the RESRAD calculation briefs in Appendix C. The results indicate that no alpha-emitting COCs/COPCs will impact groundwater within 1,000 years. 
CVP-2007-00006

Rev. 0

Table 15. Predicted Peak Radionuclide Groundwater Concentrations for the 118-B-1 Burial Ground.

\begin{tabular}{|l|c|c|c|}
\hline \multicolumn{1}{|c|}{ Radionuclide } & $\begin{array}{c}\text { Peak Concentration } \\
(\mathrm{pCi} / \mathrm{L})^{\mathrm{a}}\end{array}$ & $\begin{array}{c}\text { RAG } \\
(\mathrm{pCi} / \mathrm{L})\end{array}$ & RAGs Attained? \\
\hline Cobalt-60 & 0.000430 & $100^{\mathrm{b}}$ & Yes \\
\hline Cesium-137 & 0.0123 & $60^{\mathrm{b}}$ & Yes \\
\hline Strontium-90 & 0.156 & $8^{\mathrm{D}}$ & Yes \\
\hline $\begin{array}{l}\text { Tritium (excluding } \\
\text { excavation footprint) }\end{array}$ & $1,440^{\mathrm{d}}$ & $20,000^{\mathrm{c}}$ & Yes \\
\hline
\end{tabular}

a The peak concentration is the maximum predicted value from the remediation footprint, overburden stockpiles, BCL material, and waste staging pile area footprint. Results are presented in the RESRAD and MCL calculation briefs (Appendix C).

b Lookup value corresponding to a single-radionuclide dose rate of $4 \mathrm{mrem} / \mathrm{yr}$.

${ }^{c}$ EPA drinking water promulgated MCL (40 CFR 141.66).

Maximum result from RESRAD modeling for the overburden stockpiles, BCL stockpiles, and waste staging pile area. Residual tritium concentrations within the remediation footprint were not considered, because institutional controls will allow for attenuation of residual vadose zone tritium contamination (EPA 2007).

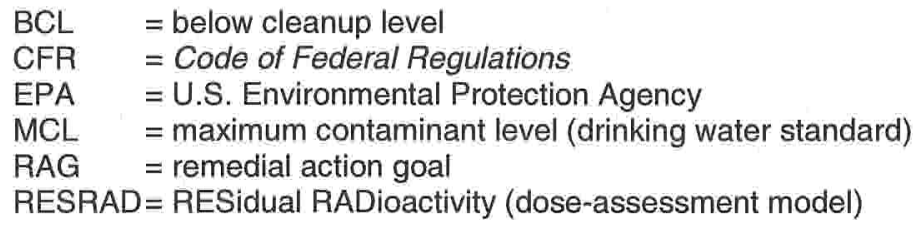

Figure 16. Dose Rate to Organs from Groundwater.

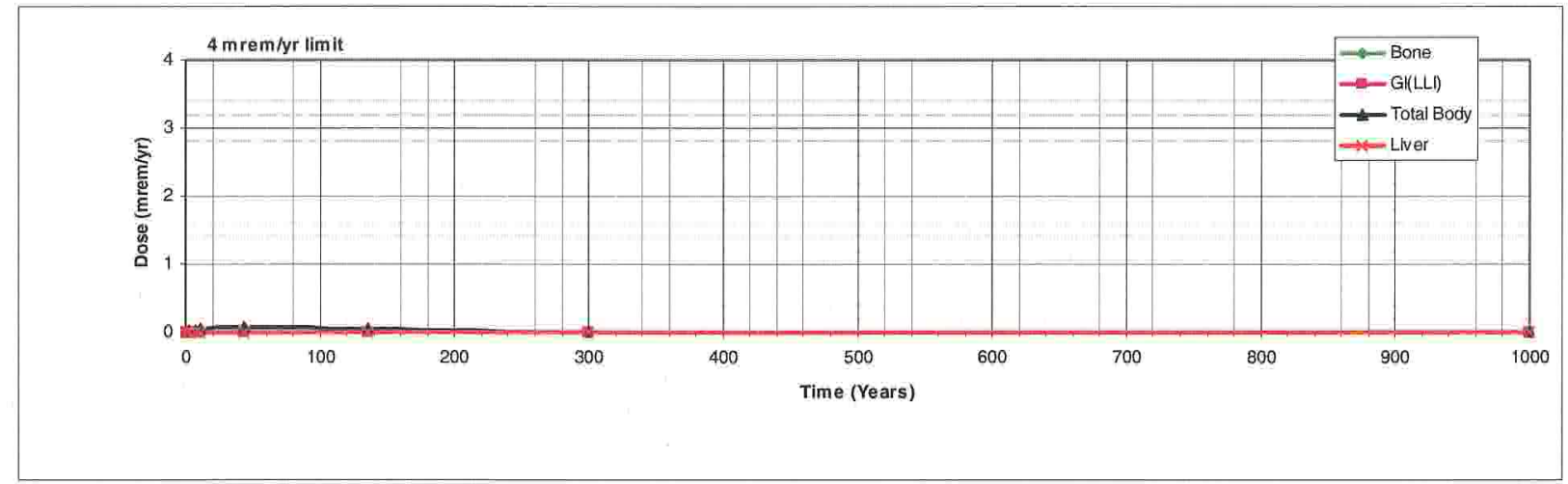

For the fourth and last criterion, meet the total uranium standard of $21.2 \mathrm{pCi} / \mathrm{L}$, no uranium isotopes were detected above background levels in the 118-B-1 Burial Ground verification samples. Therefore, this standard is met.

\subsection{DATA QUALITY ASSESSMENT}

A DQA is performed to compare the verification sampling approach and resulting analytical data with the sampling and data quality requirements specified by the project objectives and performance specifications. 
The DQA for the 118-B-1 Burial Ground established that the data are of the right type, quality, and quantity to support site verification decisions within specified error tolerances. The evaluation verified that the sample design was sufficient for the purpose of clean site verification. The detailed DQA is presented in Appendix E.

\subsection{SUMMARY FOR WASTE SITE RECLASSIFICATION}

This cleanup verification package demonstrates that remedial action at the 118-B-1 Burial Ground has achieved the remedial action objectives and corresponding RAGs established in the Burial Ground ROD (EPA 2000) and RDR/RAWP (DOE-RL 2005). The remaining soils at this site have been sampled, analyzed, and modeled. The results of verification sampling indicated that vadose zone soils beneath the burial ground contained residual tritium concentrations in excess of remedial action objectives for the protection of groundwater. The 118-B-1 ESD was approved by the Tri-Parties to leave residual tritium-contaminated soil in-place based on consideration of balancing factors, and includes institutional controls to prohibit future irrigation at the 118-B-1 waste site. The results of verification sampling show that residual concentrations of contaminants other than tritium do not preclude any other future uses (as bounded by

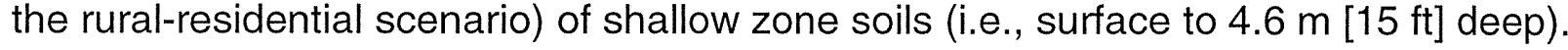
With institutional controls to prevent irrigation, the results also demonstrate that further mobilization of residual tritium-contamination to groundwater and the Columbia River will be minimized. Residual concentrations of non-tritium contaminants are also protective of groundwater and the Columbia River. The acceptability of direct exposure to residual tritium contamination in the deep vadose zone has not been demonstrated; therefore, institutional controls to prevent uncontrolled drilling or excavation into the deep zone are also required.

In accordance with this evaluation, the verification sampling and modeling results support a reclassification of the 118-B-1 Burial Ground to Interim Closed Out.

\subsection{REFERENCES}

40 CFR 141, "National Primary Drinking Water Regulations," Code of Federal Regulations, as amended.

40 CFR 300, "National Oil and Hazardous Substances Pollution Contingency Plan," Code of Federal Regulations, as amended.

ANL, 2005, RESRAD for Windows, Version 6.30, Argonne National Laboratory, Environmental Assessment Division, Argonne, Illinois.

BHI, 2001, Calculation of Total Uranium Activity Corresponding to a Maximum Contaminant Level for Total Uranium of 30 Micrograms per Liter in Groundwater, 0100X-CA-V0038, Rev. 0, Bechtel Hanford, Inc., Richland, Washington. 
BHI, 2004, 100 Area Radionuclide and Nonradionuclide Lookup Values for the 1995 Interim Remedial Action Record of Decision, 0100X-CA-V0046, Rev. 0, Bechtel Hanford Inc., Richland, Washington.

BHI, 2005, 100 Area Analogous Sites RESRAD Calculations, 0100X-CA-V0050, Rev. 0, Bechtel Hanford, Inc., Richland, Washington.

Carpenter, R. W., 1994, 100-B Area Technical Baseline Report, WHC-SD-EN-TI-220, Rev. 0, Westinghouse Hanford Company, Richland, Washington.

DOE Order 5400.5, Radiation Protection of the Public and the Environment, U.S. Department of Energy, Washington, D.C.

DOE-RL, 1996, Hanford Site Background: Part 2, Soil Background for Radionuclides, DOE/RL-96-12, Rev. 0, U.S. Department of Energy, Richland Operations Office, Richland, Washington.

DOE-RL, 2001a, 100 Area Burial Grounds Remedial Action Sampling and Analysis Plan, DOE/RL-2001-35, Rev. 0, U.S. Department of Energy, Richland Operations Office, Richland, Washington.

DOE-RL, 2001b, Hanford Site Background: Part 1, Soil Background for Nonradioactive Analytes, DOE/RL-92-24, Rev. 4, U.S. Department of Energy, Richland Operations Office, Richland, Washington.

DOE-RL, 2005, Remedial Design Report/Remedial Action Work Plan for the 100 Area, DOE/RL-96-17, Rev. 5, U.S. Department of Energy, Richland Operations Office, Richland, Washington.

DOE-RL, 2007, Tri-Party Agreement Handbook Management Procedures, RL-TPA-90-0001, Rev. 1, Guideline Number TPA-MP-14, "Maintenance of the Waste Information Data System (WIDS)," U.S. Department of Energy, Richland Operations Office, Richland, Washington.

Ecology, EPA, and DOE, 1989, Hanford Federal Facility Agreement and Consent Order, 2 vols., as amended, Washington State Department of Ecology,

U.S. Environmental Protection Agency, and U.S. Department of Energy, Olympia, Washington.

Ecology, 1994, Natural Background Soil Metals Concentrations in Washington State, Publication No. 94-115, Washington State Department of Ecology, Olympia, Washington.

Ecology, 2005, Cleanup Levels and Risk Calculations (CLARC) Database, Washington State Department of Ecology, Olympia, Washington, $<$ https://fortress.wa.gov/ecy/clarc/CLARCHome.aspx>. 
EPA, 2000, Record of Decision for the 100-BC-1, 100-BC-2, 100-DR-1, 100-DR-2, 100-FR-2, 100-HR-2, and 100-KR-2 Operable Units, Hanford Site (100 Area Burial Grounds), Benton County, Washington, U.S. Environmental Protection Agency, Region 10, Seattle, Washington.

EPA, 2007, Explanation of Significant Difference for the Interim Action Record of Decision for the 100-BC-1, 100-BC-2, 100-DR-1, 100-DR-2, 100-FR-2, 100-HR-2, and 100-KR-2 Operable Units (100 Area Burial Grounds), U. S. Environmental Protection Agency, Region 10, Seattle, Washington.

Ludowise, J. D., 2002, Report on the Test Pitting/Trenching in the 118-B-1 and 118-B-3 Burial Grounds, CCN 101408, Interoffice Memorandum to Distribution, dated August 7, 2002, Bechtel Hanford, Inc., Richland, Washington.

WAC 173-340, 1996, "Model Toxics Control Act - Cleanup," Washington Administrative Code.

WCH, 2006, Site Specific Instruction for Close-out Approach for 118-B-1, Rev. 0, SSI No. 100-BC-09, CCN 130128, Washington Closure Hanford, Richland, Washington.

WCH, 2007a, 100B/C Burial Grounds and Remaining Sites Sampling and Field Activities, Logbook EFL-1173-8, pp. 64 \& 74, Washington Closure Hanford, Richland, Washington.

WCH, 2007b, 100B/C Burial Grounds and Remaining Sites Sampling and Field Activities, Logbook EFL-1173-10, pp. 21 - 42, Washington Closure Hanford, Richland, Washington.

WCH, 2007c, 100B/C Burial Grounds and Remaining Sites Sampling and Field Activities, Logbook EFL-1173-11, pp. 61 -62, Washington Closure Hanford, Richland, Washington.

WCH, 2007d, 100B/C Burial Grounds and Remaining Sites Sampling and Field Activities, Logbook EFL-1173-12, P. 52, Washington Closure Hanford, Richland, Washington.

WCH, 2007e, 100B/C Burial Grounds and Remaining Sites Sampling and Field Activities, Logbook EFL-1173-13, pp. 12 - 13, Washington Closure Hanford, Richland, Washington. 
WDOH, 1997, State of Washington Department of Health Interim Regulatory Guidance: Hanford Guidance for Radiological Cleanup, WDOH/320-015, Rev. 1, Washington Department of Health, Richland, Washington.

WHC, 1993, Geophysical Investigation of 118-B-1 Burial Grounds, 100-B/C Area, Hanford Site, Washington, WHC-SD-EN-TI-137, Rev. 0, Westinghouse Hanford Company, Richland, Washington. 
CVP-2007-00006

Rev. 0

\section{APPENDIX A}

\section{REMEDIATION PHOTOGRAPHS}


CVP-2007-00006

Rev. 0

A-ii 
CVP-2007-00006

Rev. 0

Photograph A-1. Aerial Photograph of Remedial Activities at the 118-B-1 Burial Ground (taken July 2004).

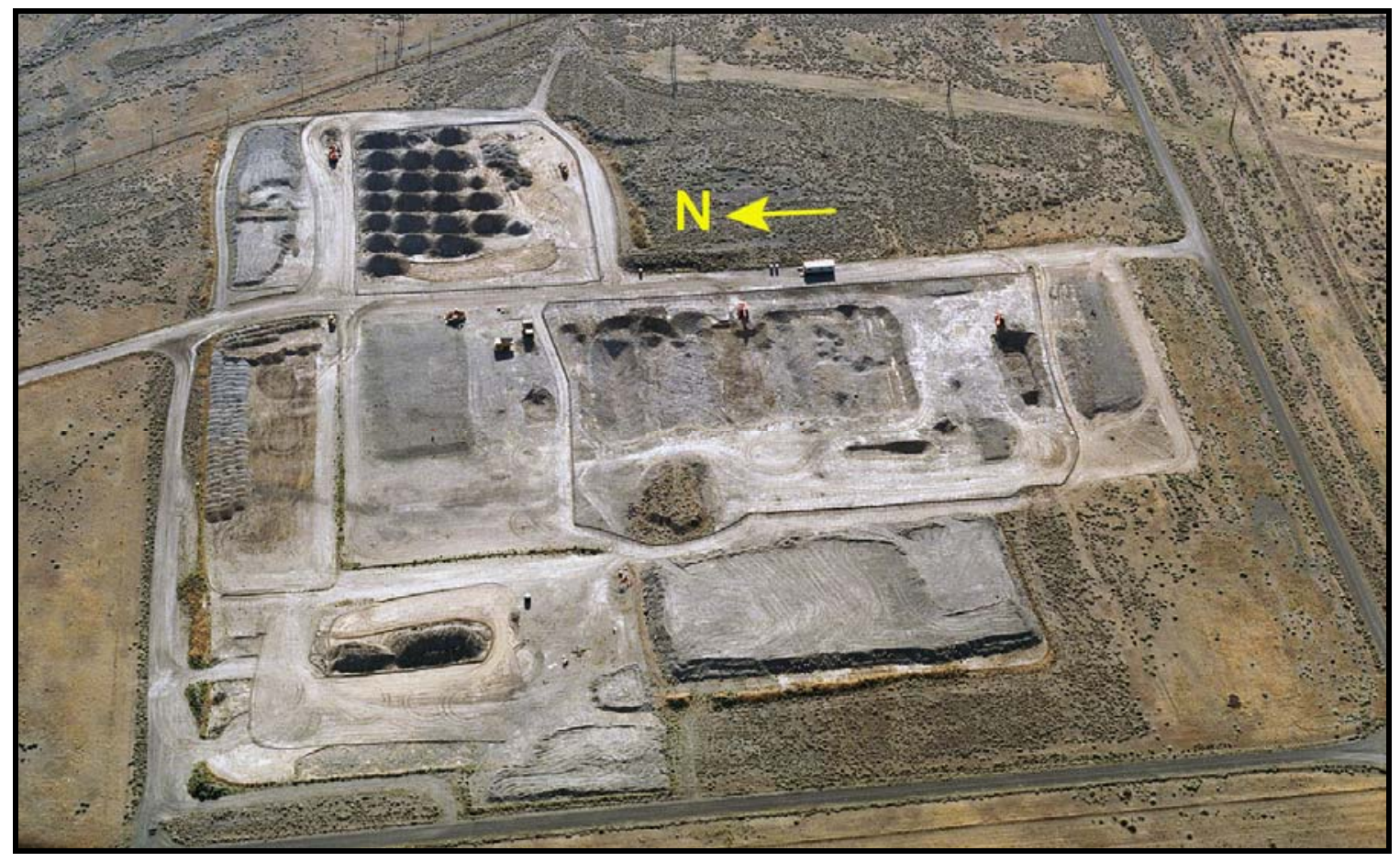

Photograph A-2. Assorted Debris in the 118-B-1 Burial Ground.

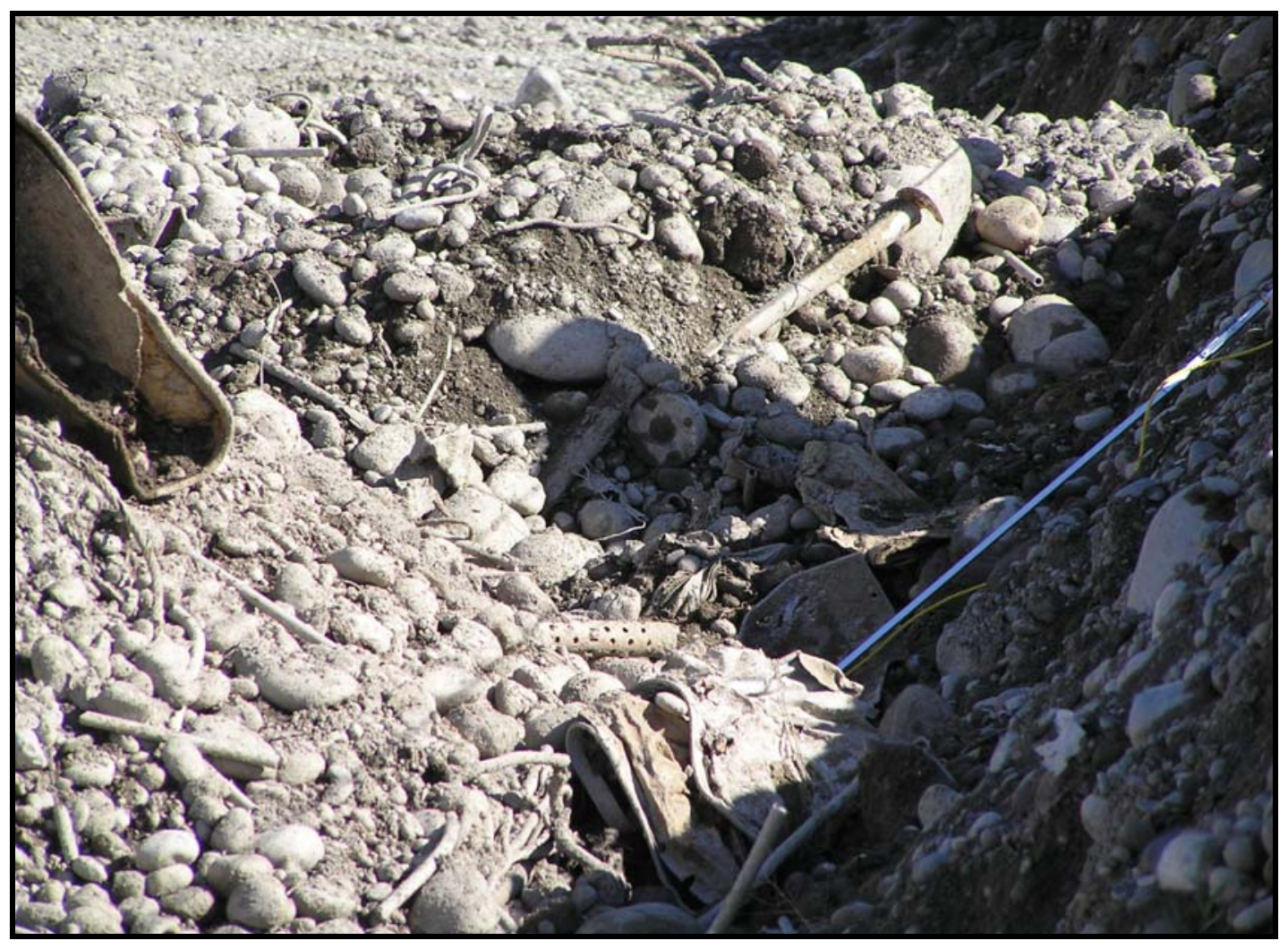


CVP-2007-00006

Rev. 0

Photograph A-3. Spacers in the 118-B-1 Burial Ground.

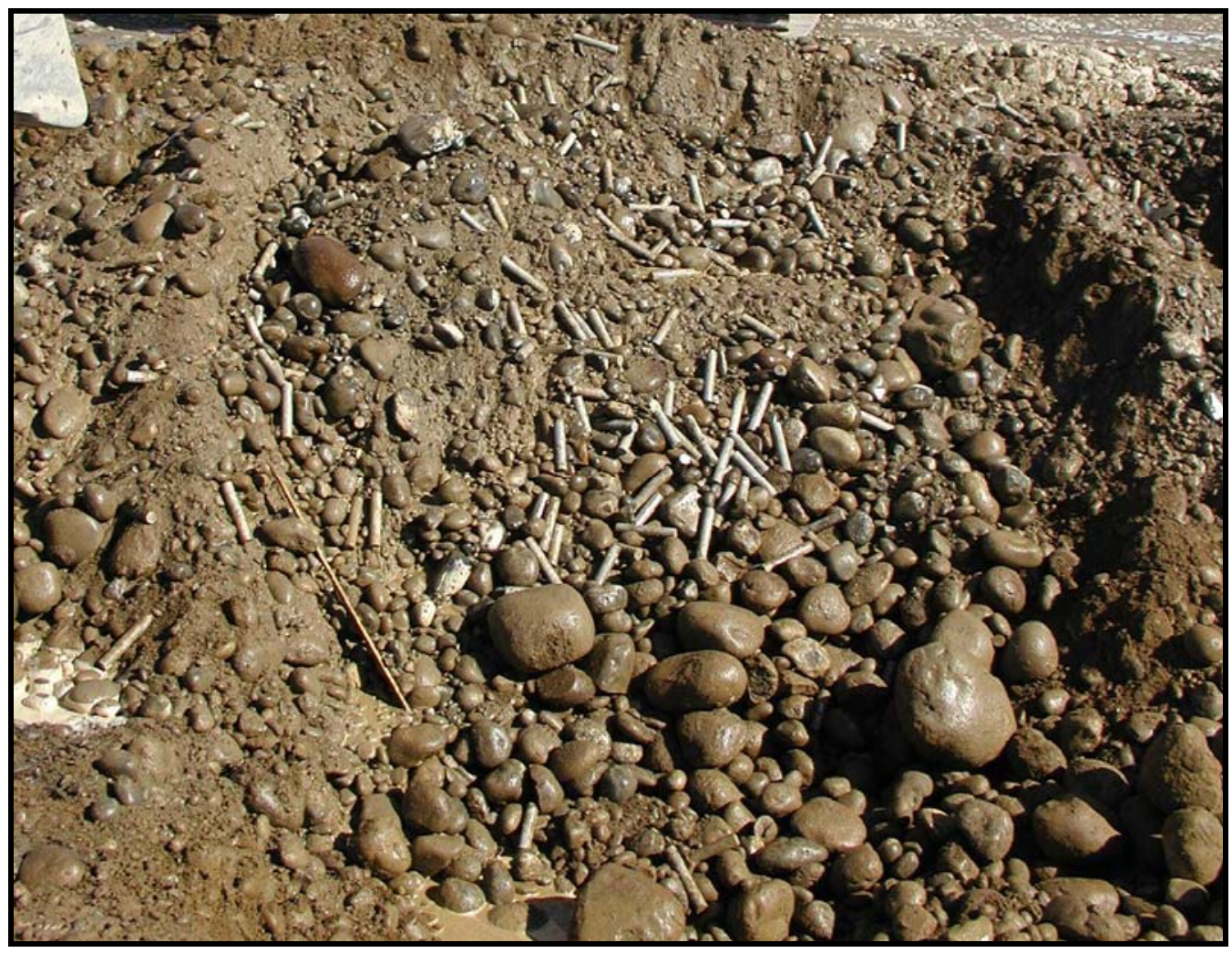

Photograph A-4. Tritium Furnaces in the 118-B-1 Burial Ground Waste Sorting Piles.

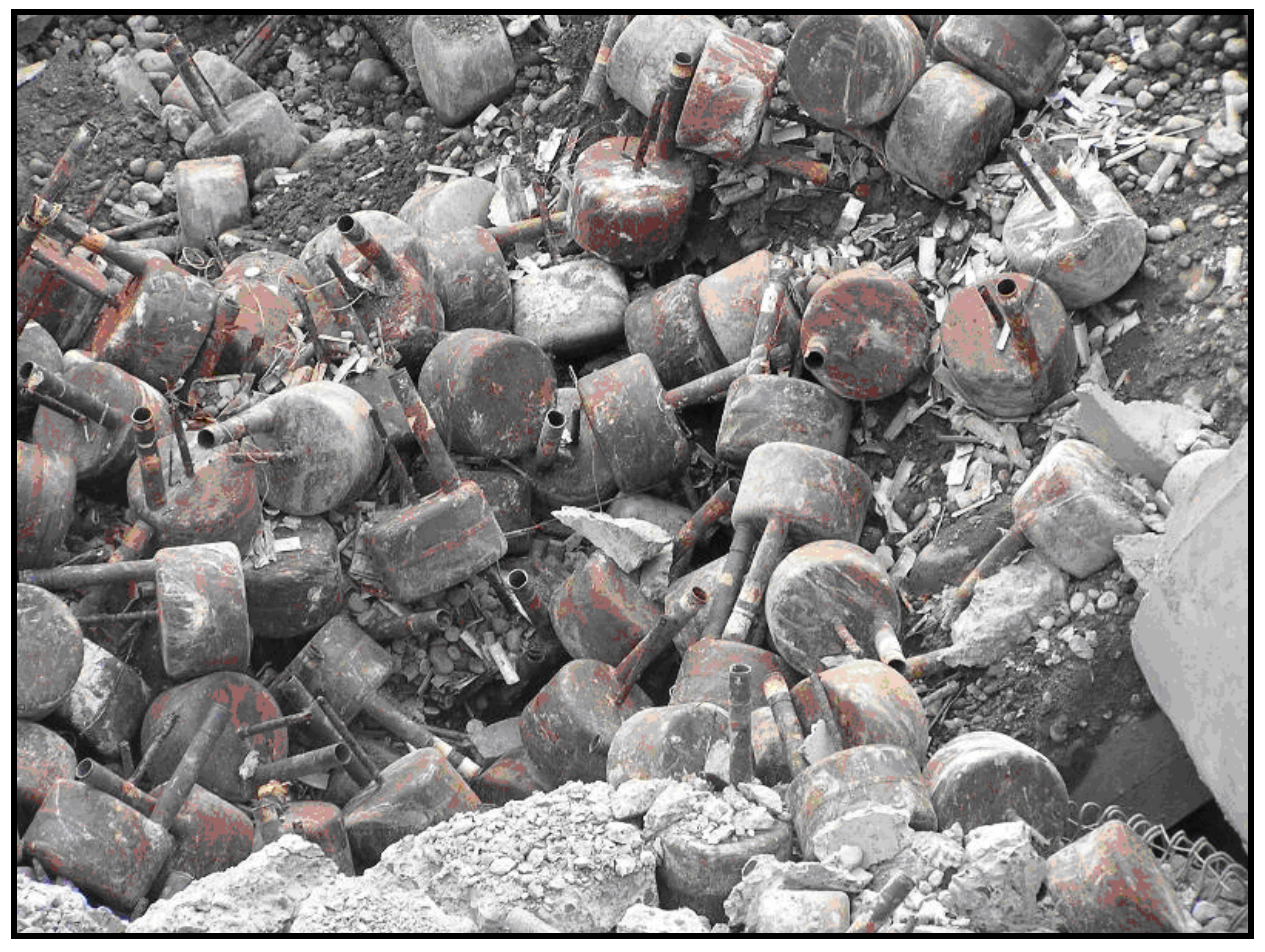


CVP-2007-00006

Rev. 0

Photograph A-5. Tritium Tubes in the 118-B-1 Waste Sorting Piles.

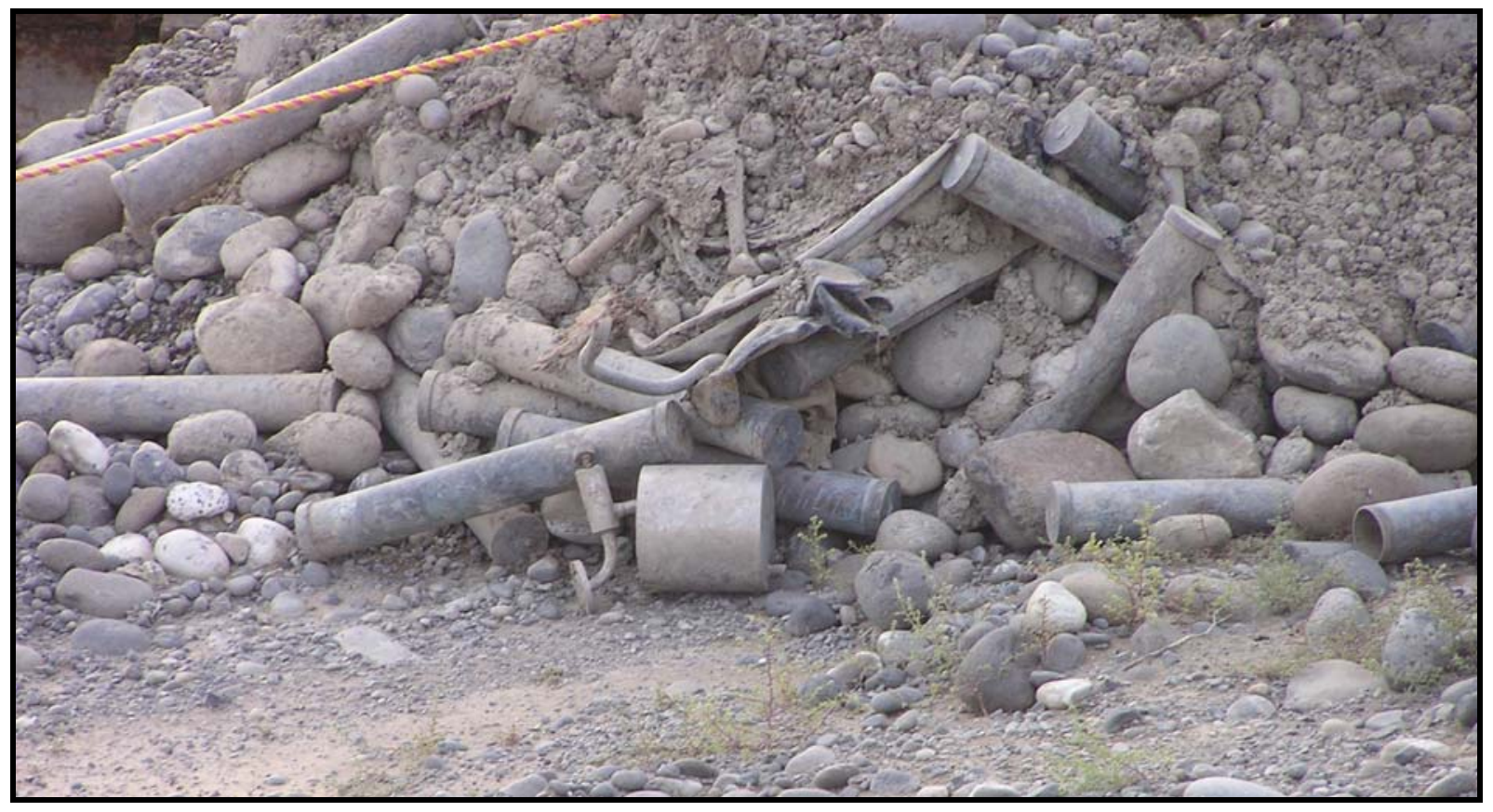

Photograph A-6. Segregation of Tritium Tubes and Other Anomalous Items.

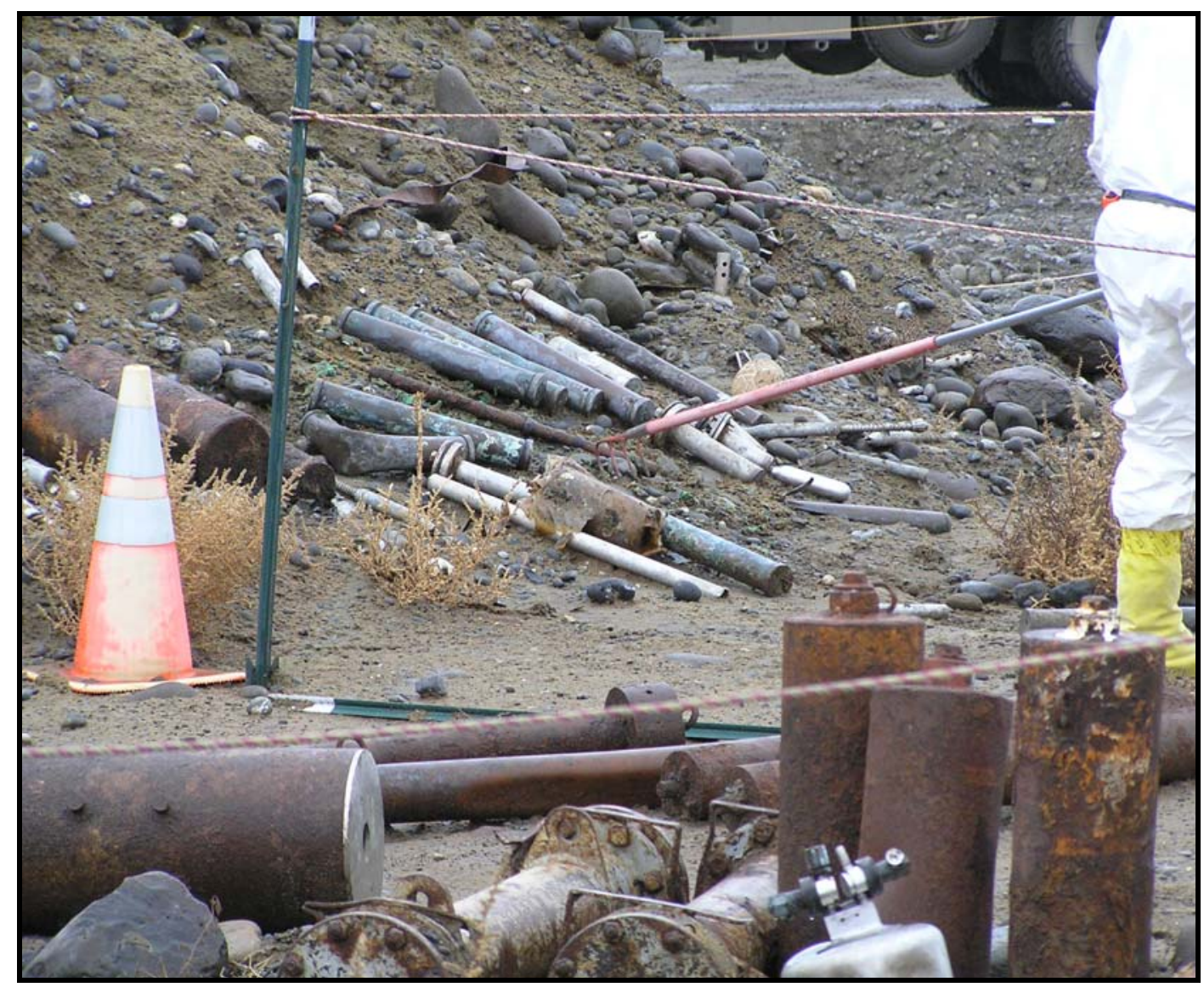


CVP-2007-00006

Photograph A-7. Segregated Tritium Tubes.

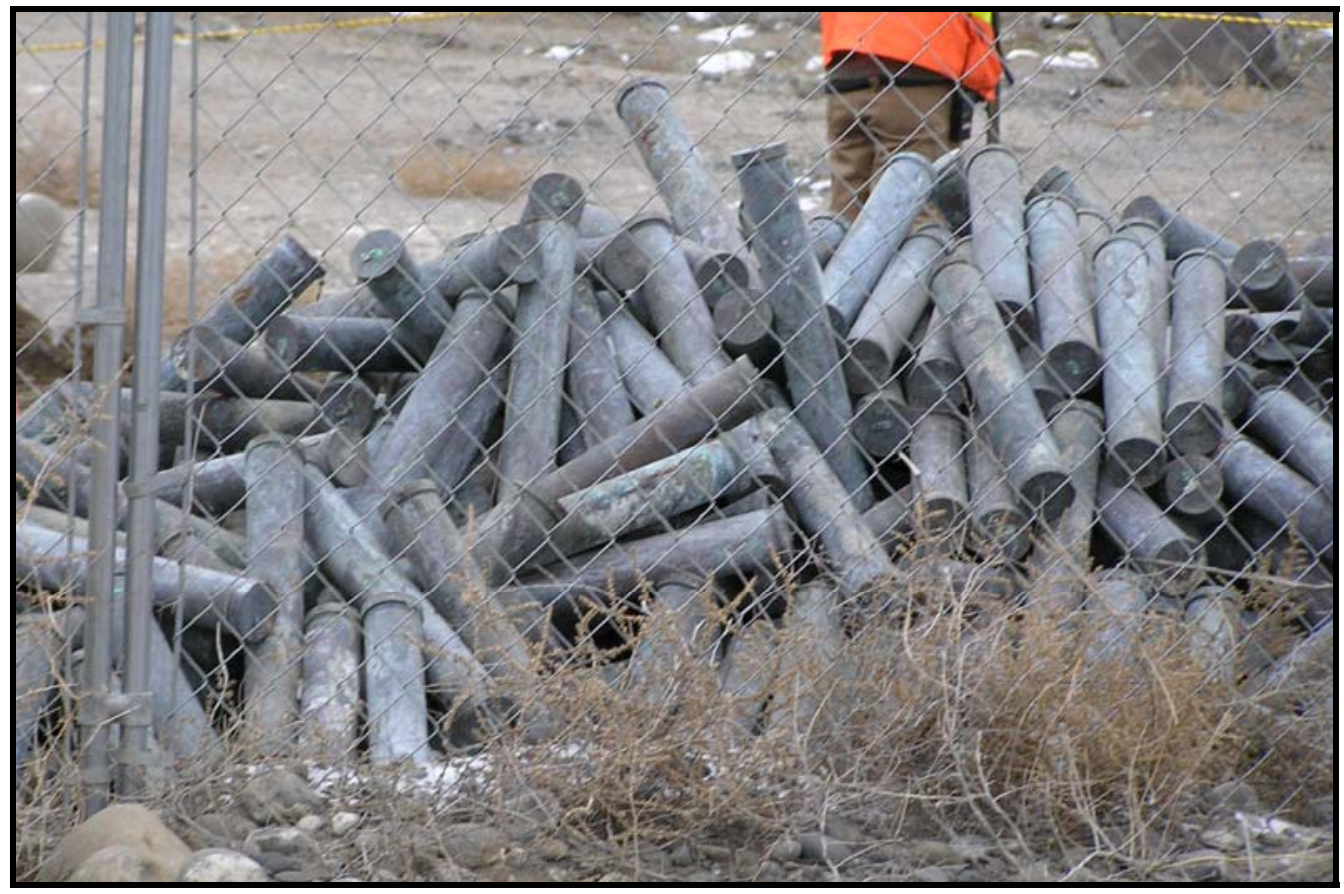

Photograph A-8. Accessing Tritium Tube Contents.

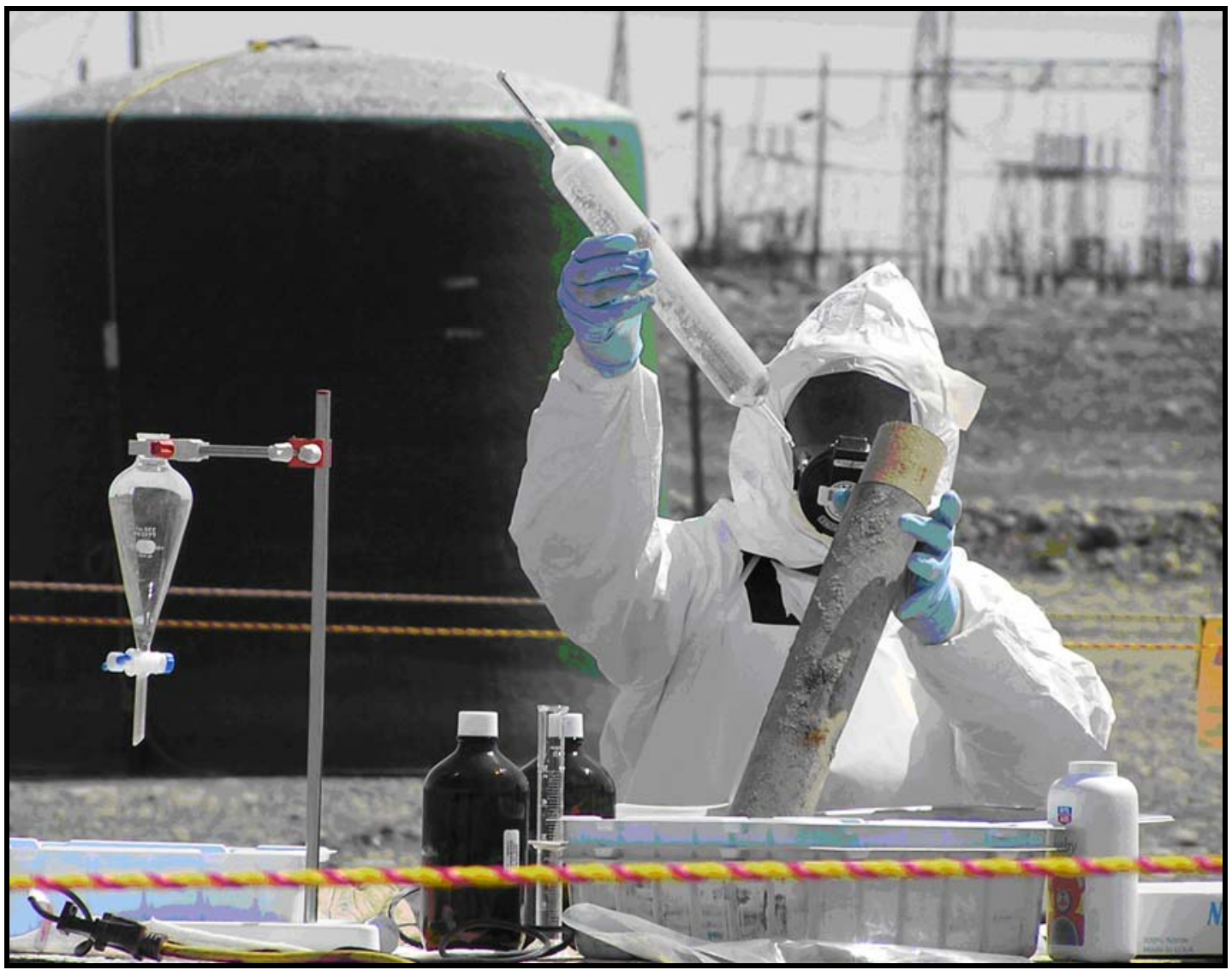


Photograph A-9. Anomalous Containers with Solid Lead Components.

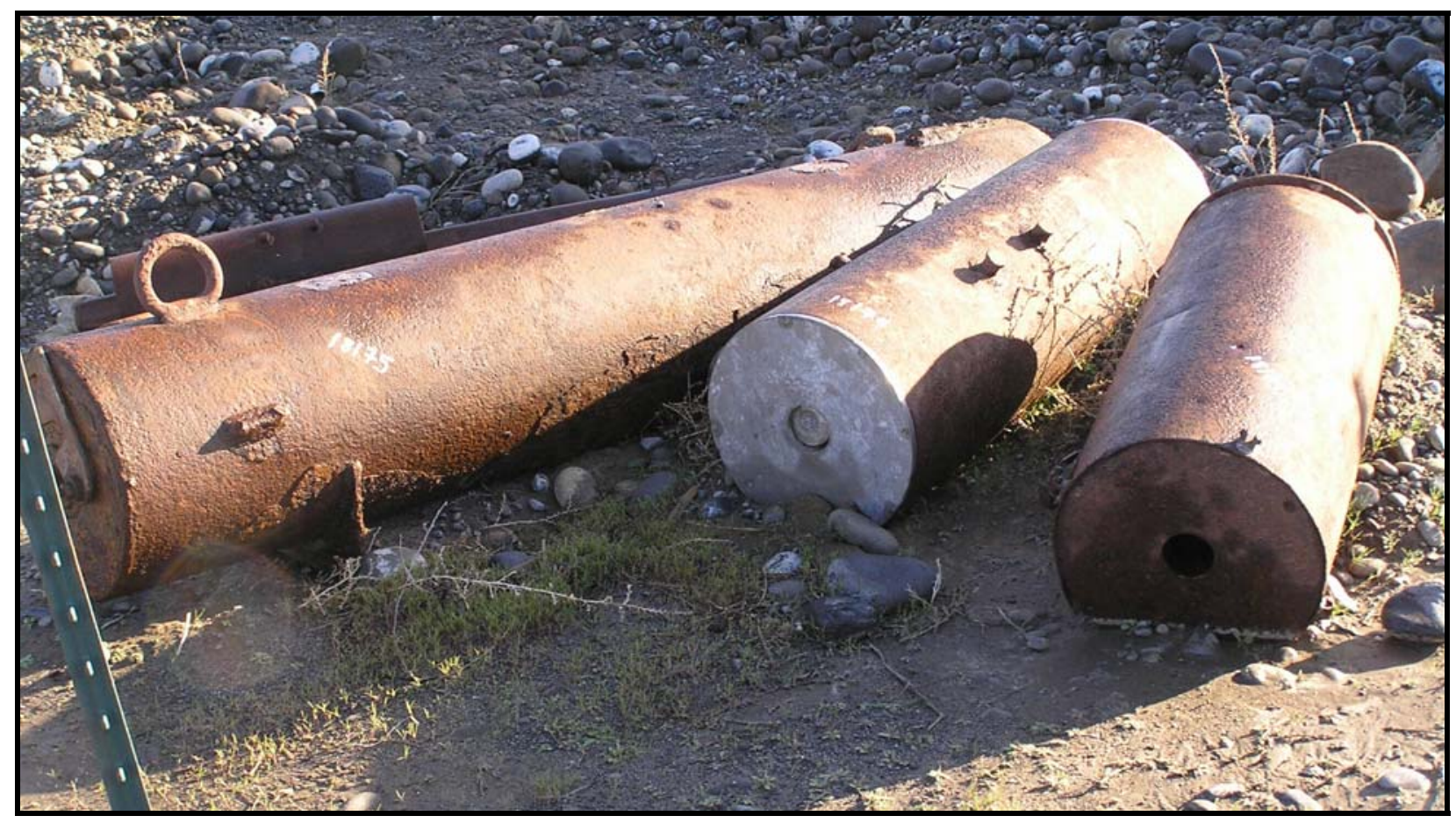

Photograph A-10. Staged Mercury Tubes and Other Suspect Mercury-Containing Items.

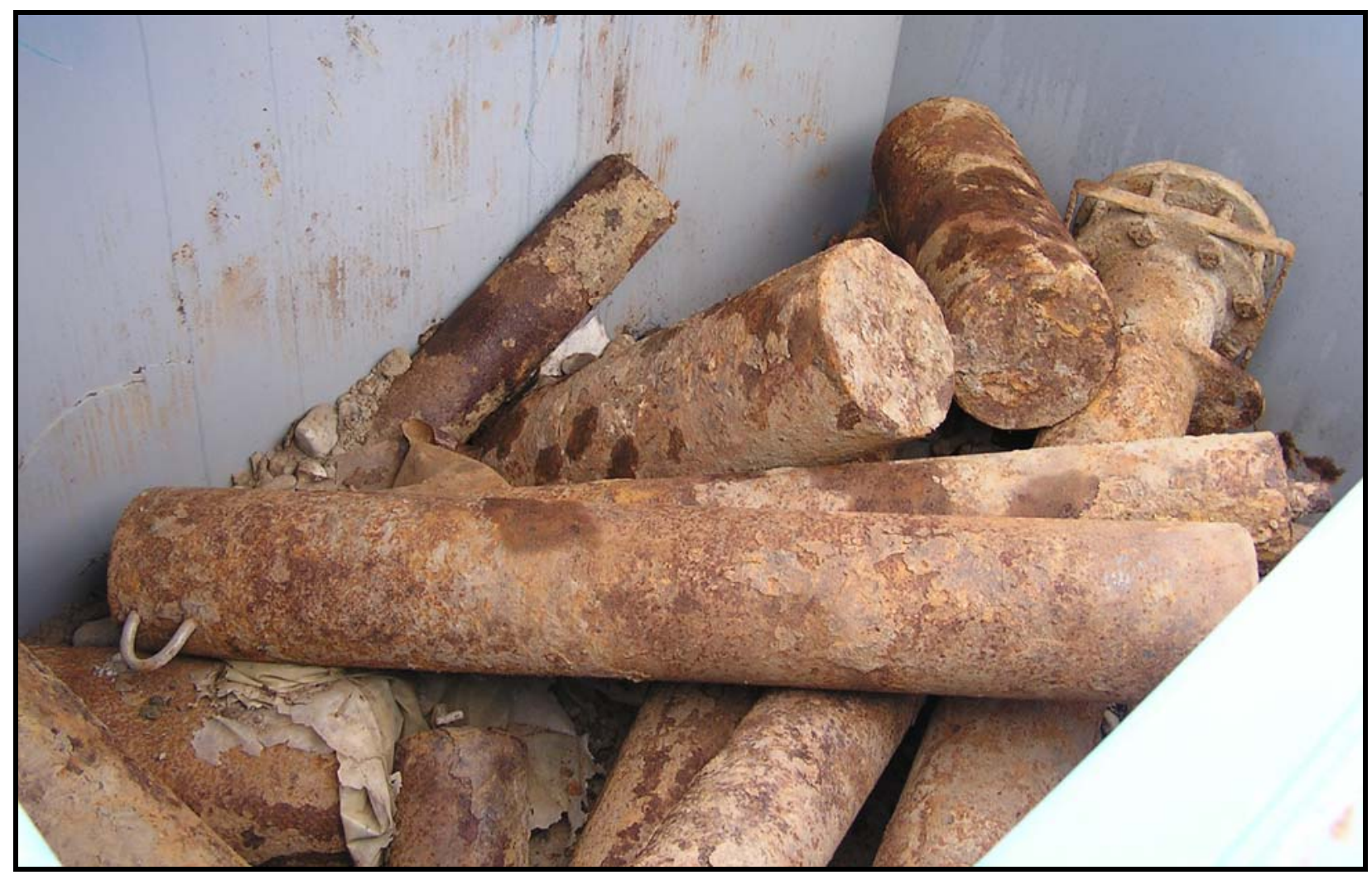


CVP-2007-00006

Photograph A-11. Moisture Traps Used in the P-10 Tritium Separation Project.

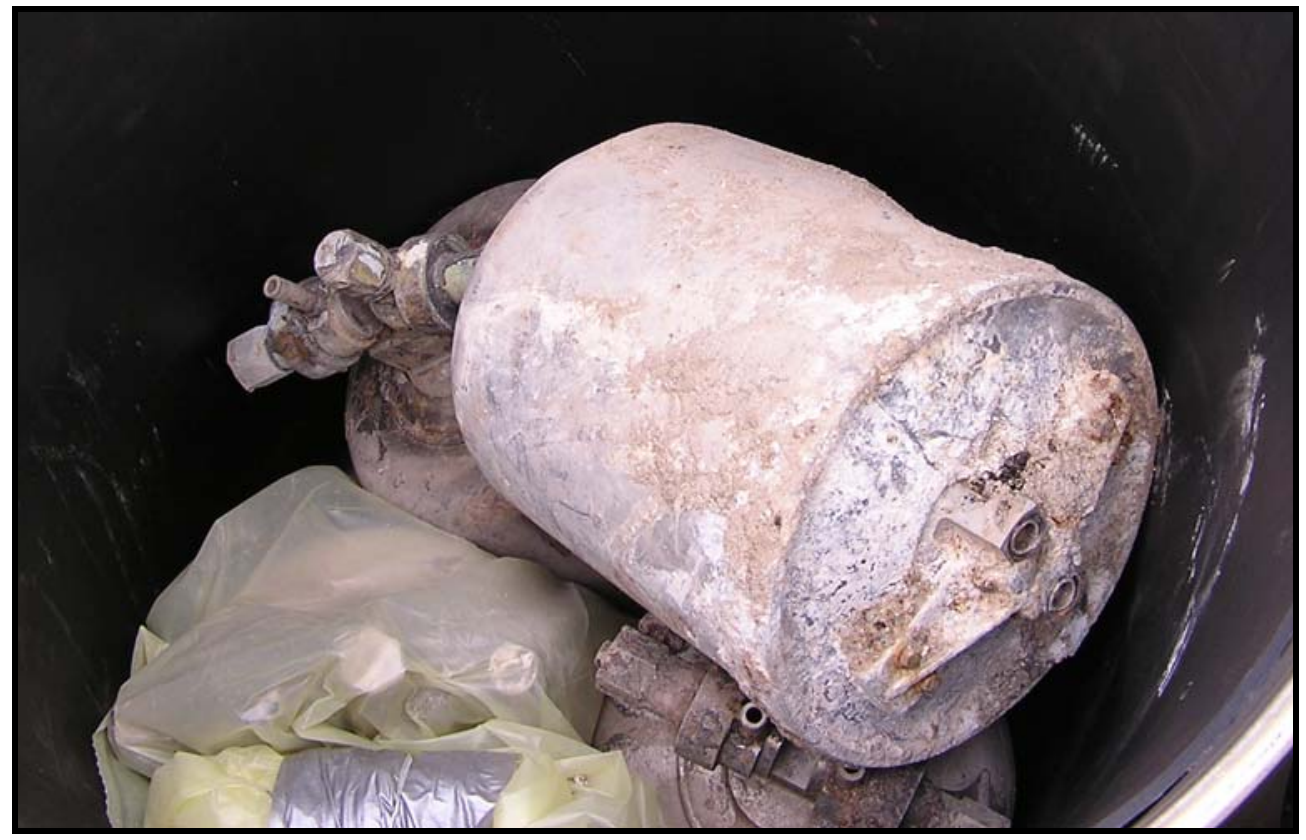

Photograph A-12. Reinforced Rubber Hoses Containing Suspect Hydraulic Oil.

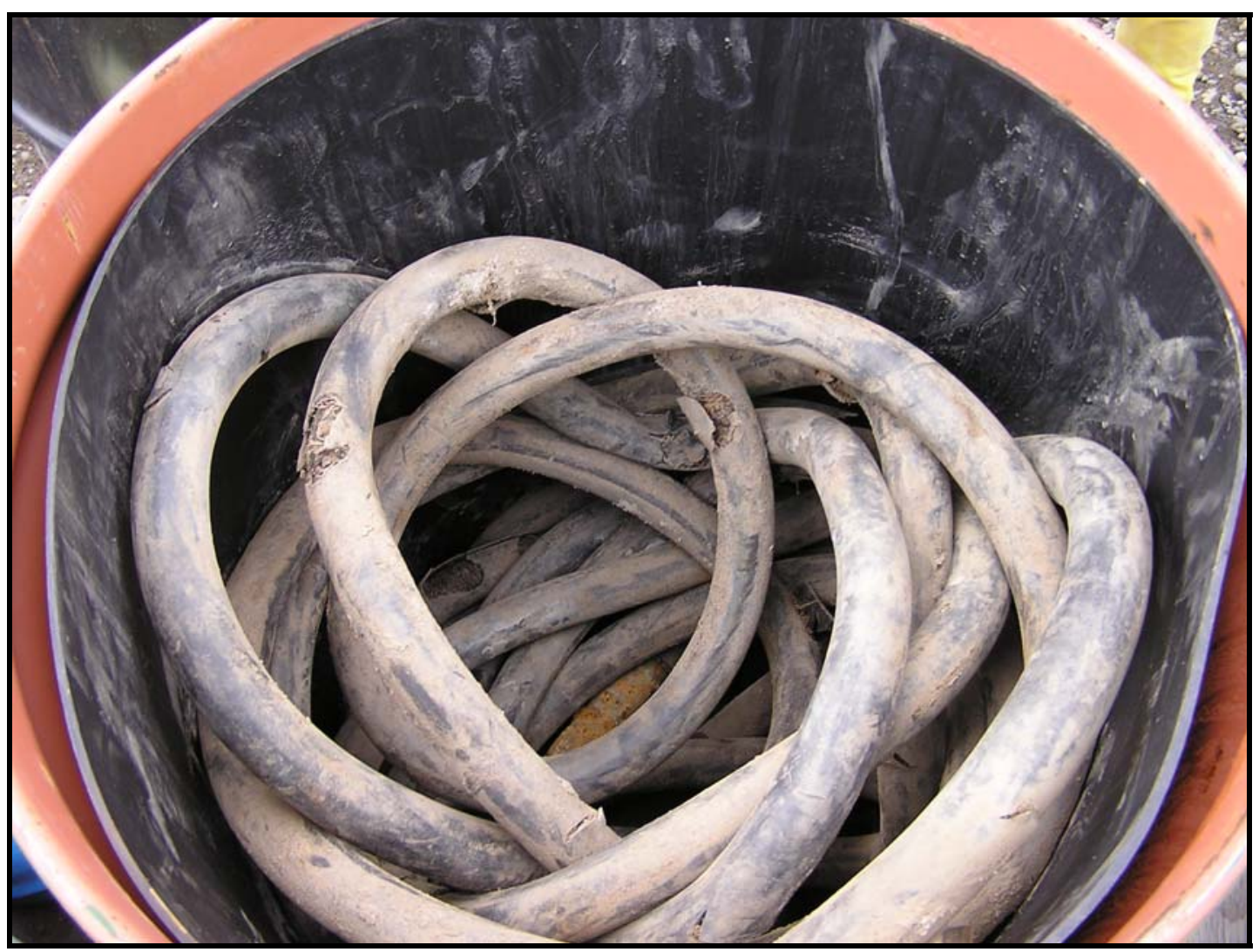


CVP-2007-00006

Rev. 0

Photograph A-13. Metal Canisters Containing Contaminated Oils and Other P-10 Tritium Separation Project Process Wastes.

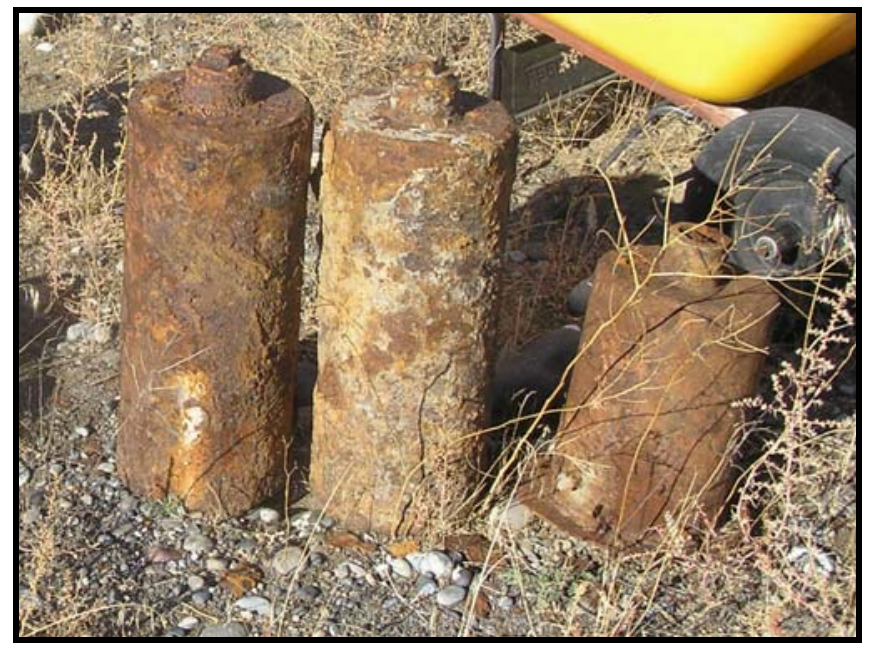

Photograph A-14. Empty Metal Container.

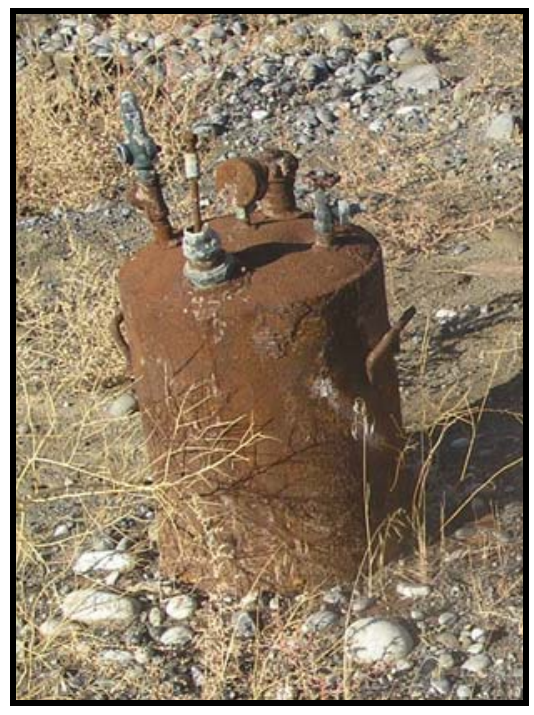


Photograph A-15. Staged Compressed Gas Cylinders and Anomalous Containers.

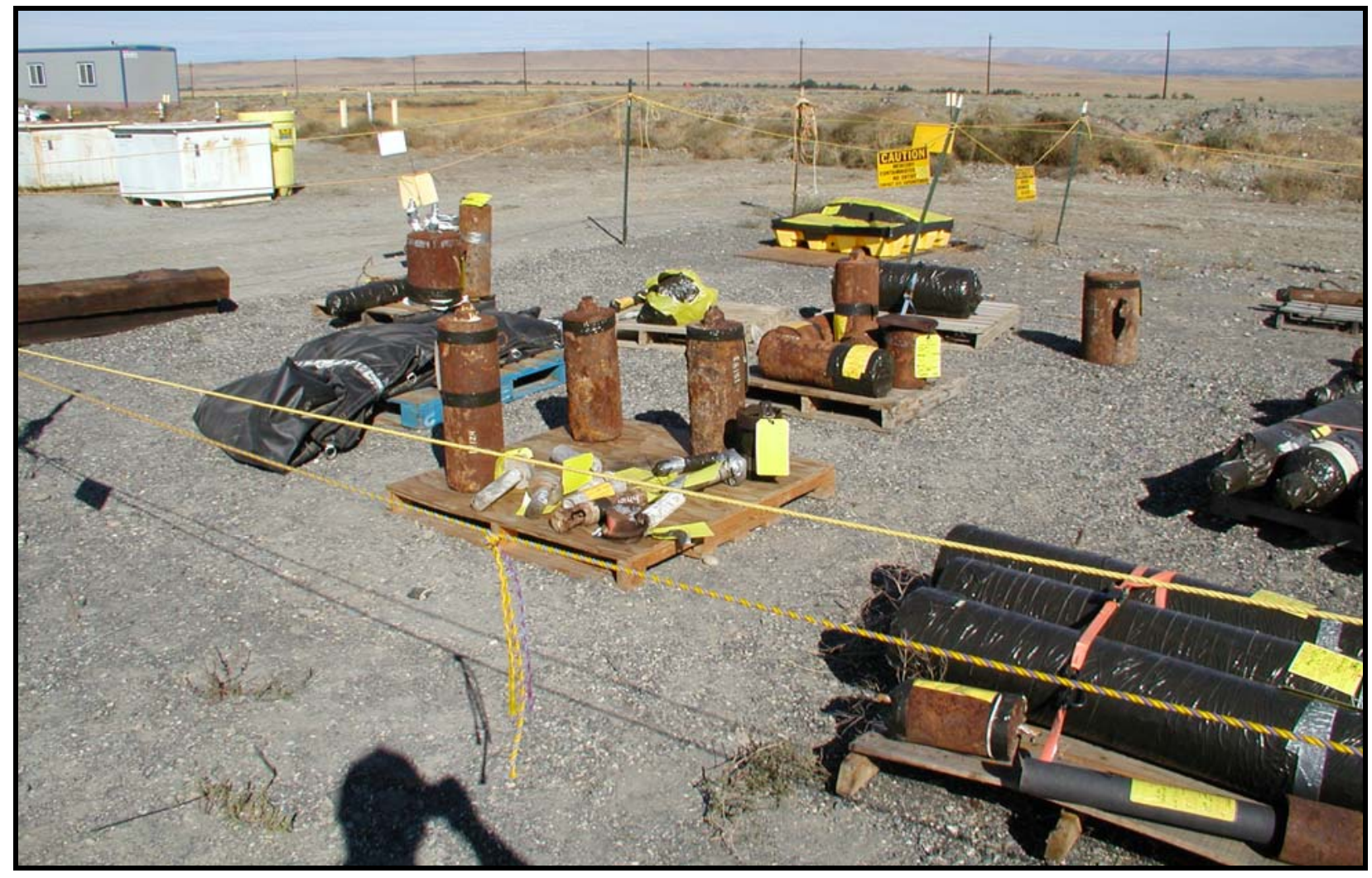


APPENDIX B

SYSTEMATIC RADIOLOGICAL SURVEYS 
CVP-2007-00006

Rev. 0

B-ii 


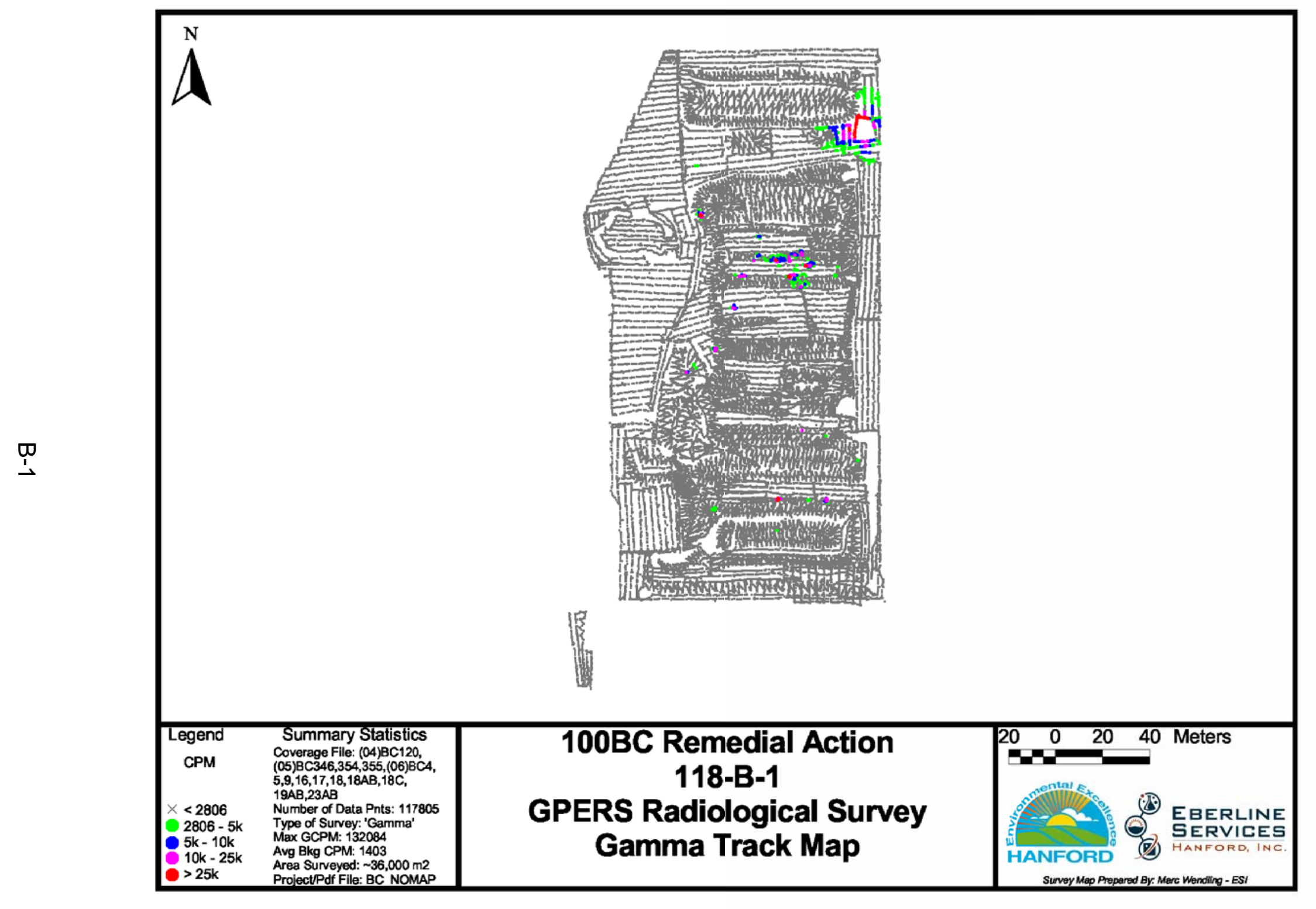

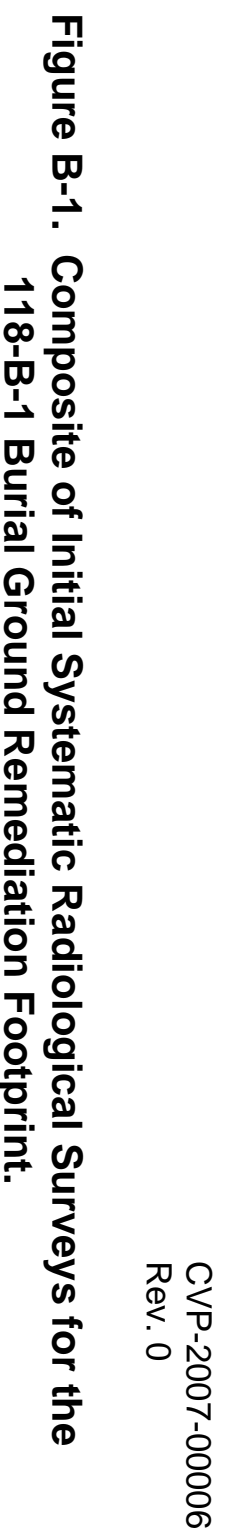




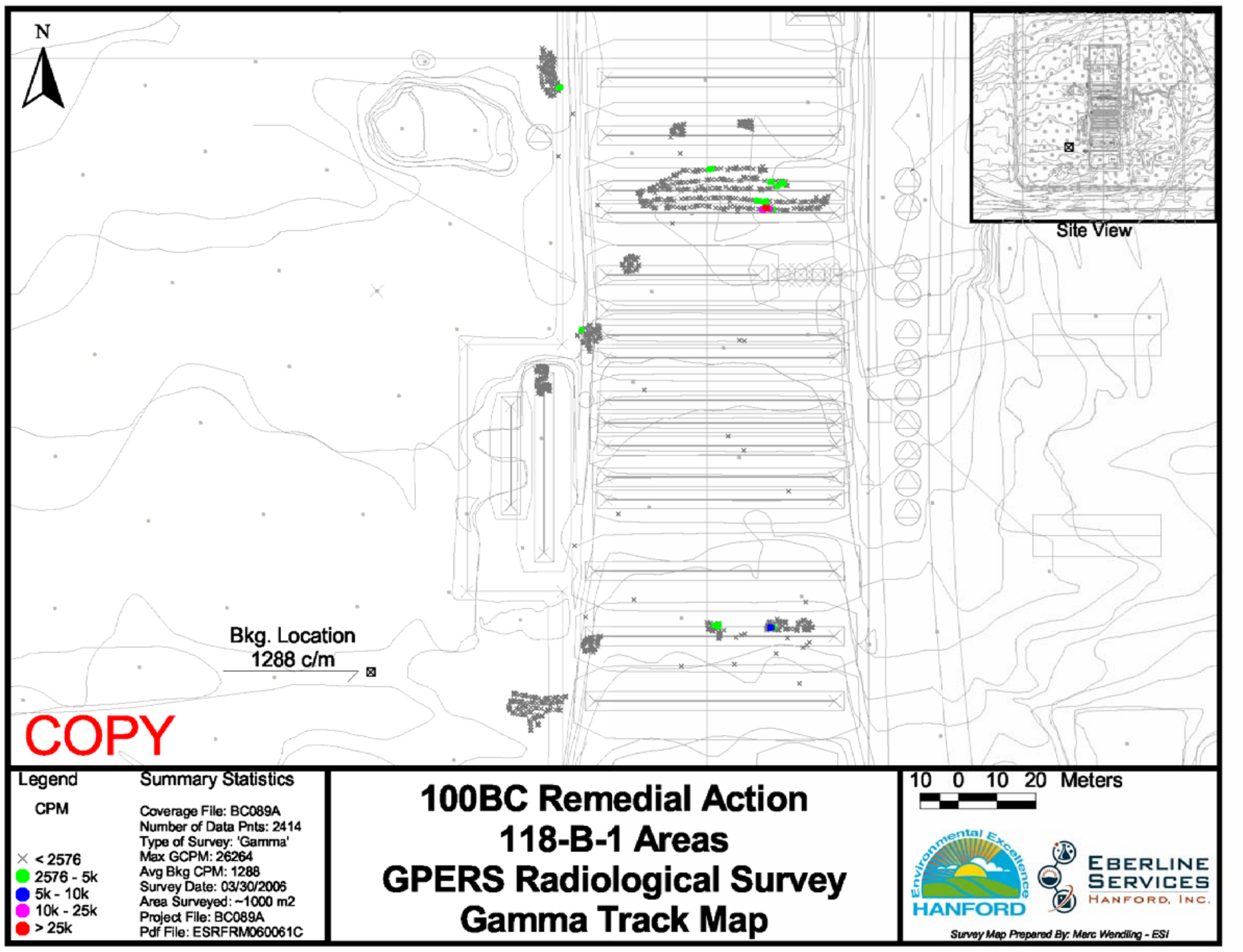

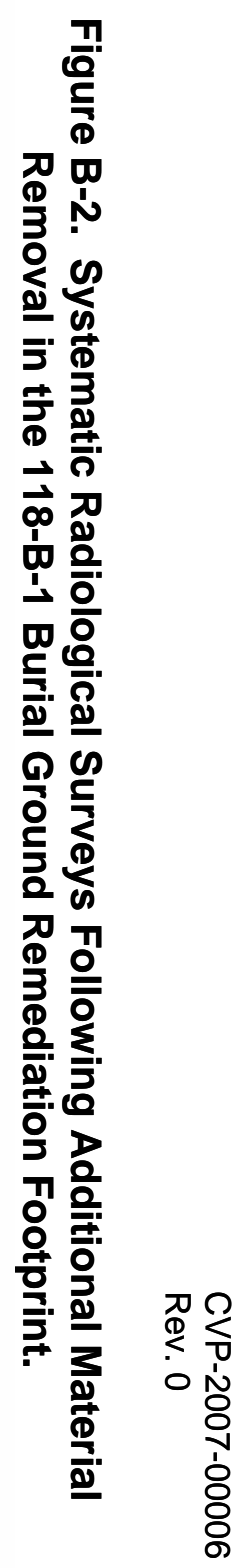




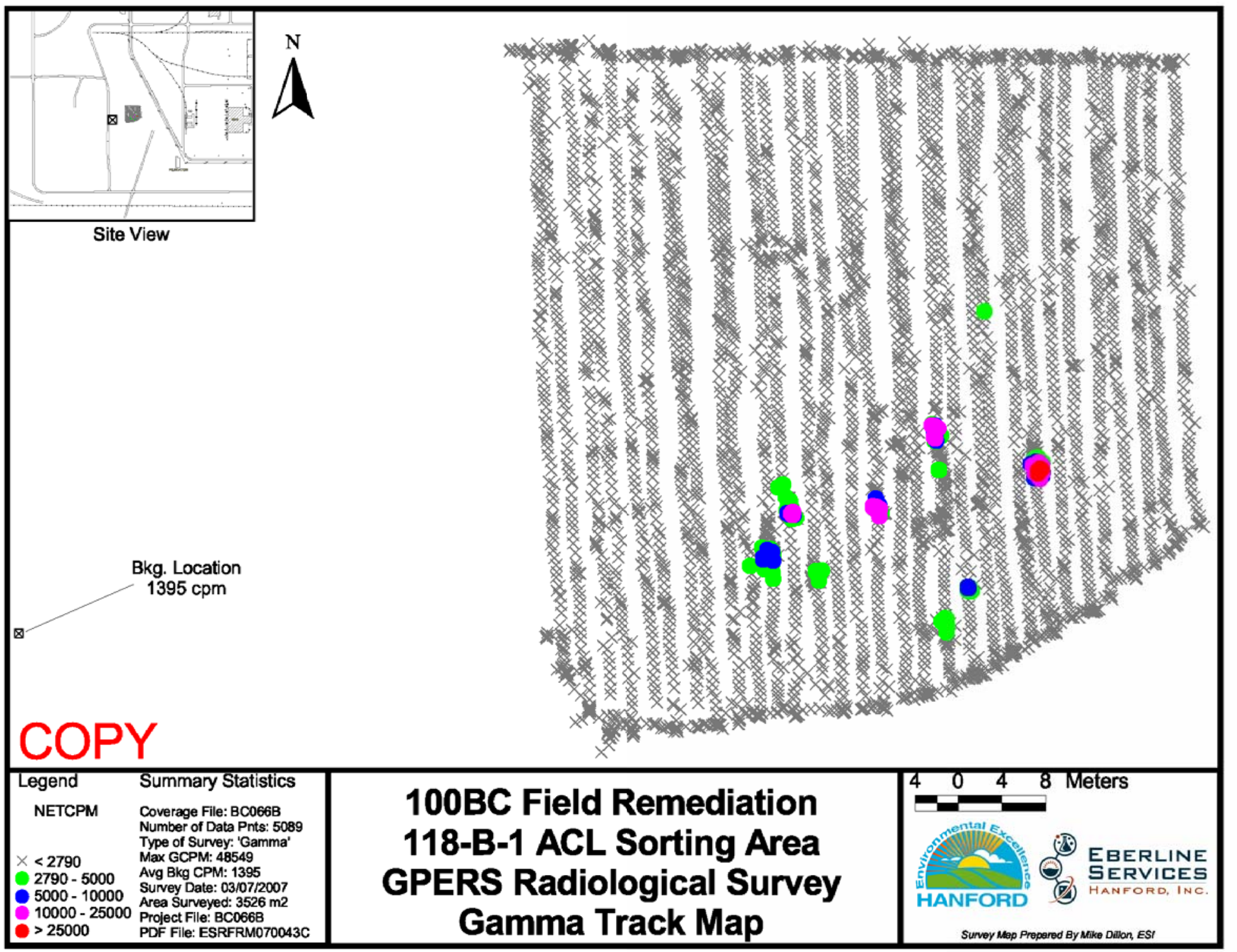

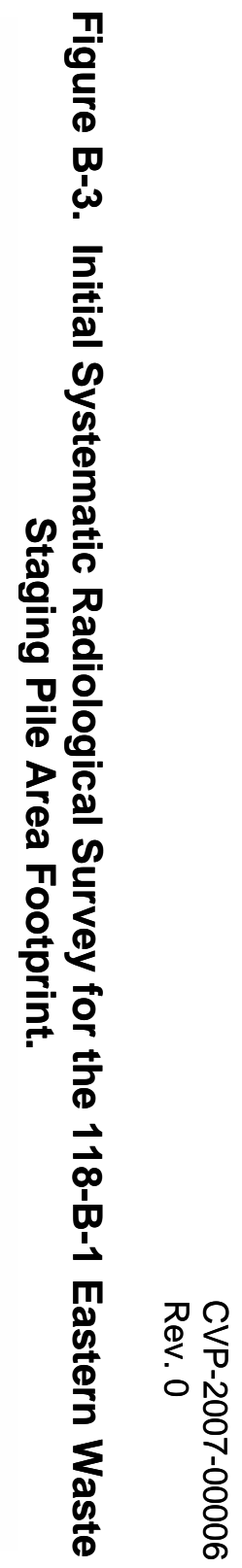




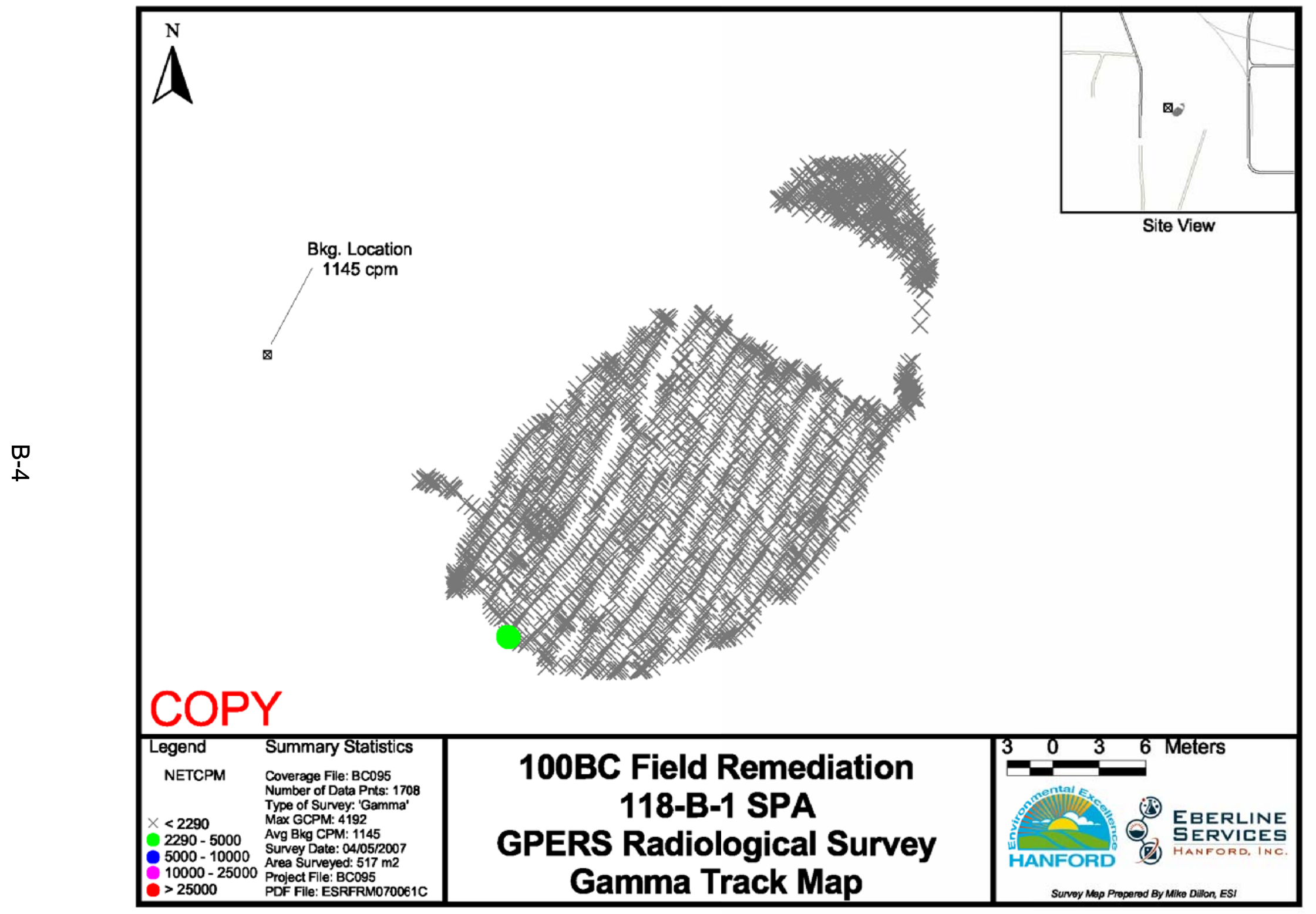

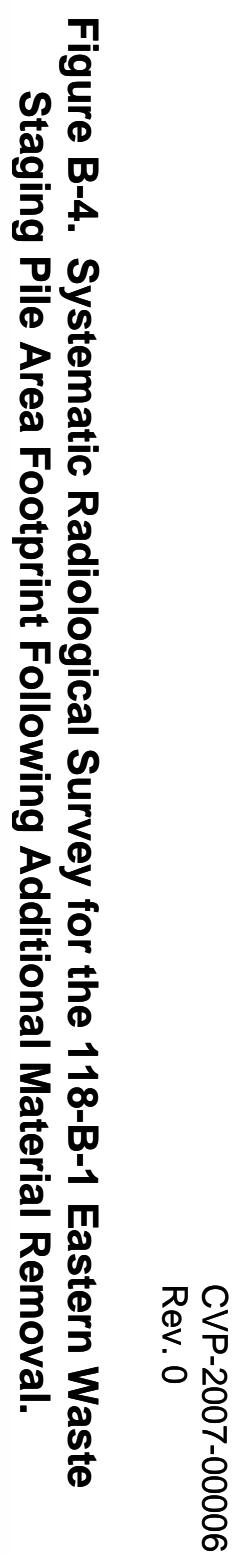




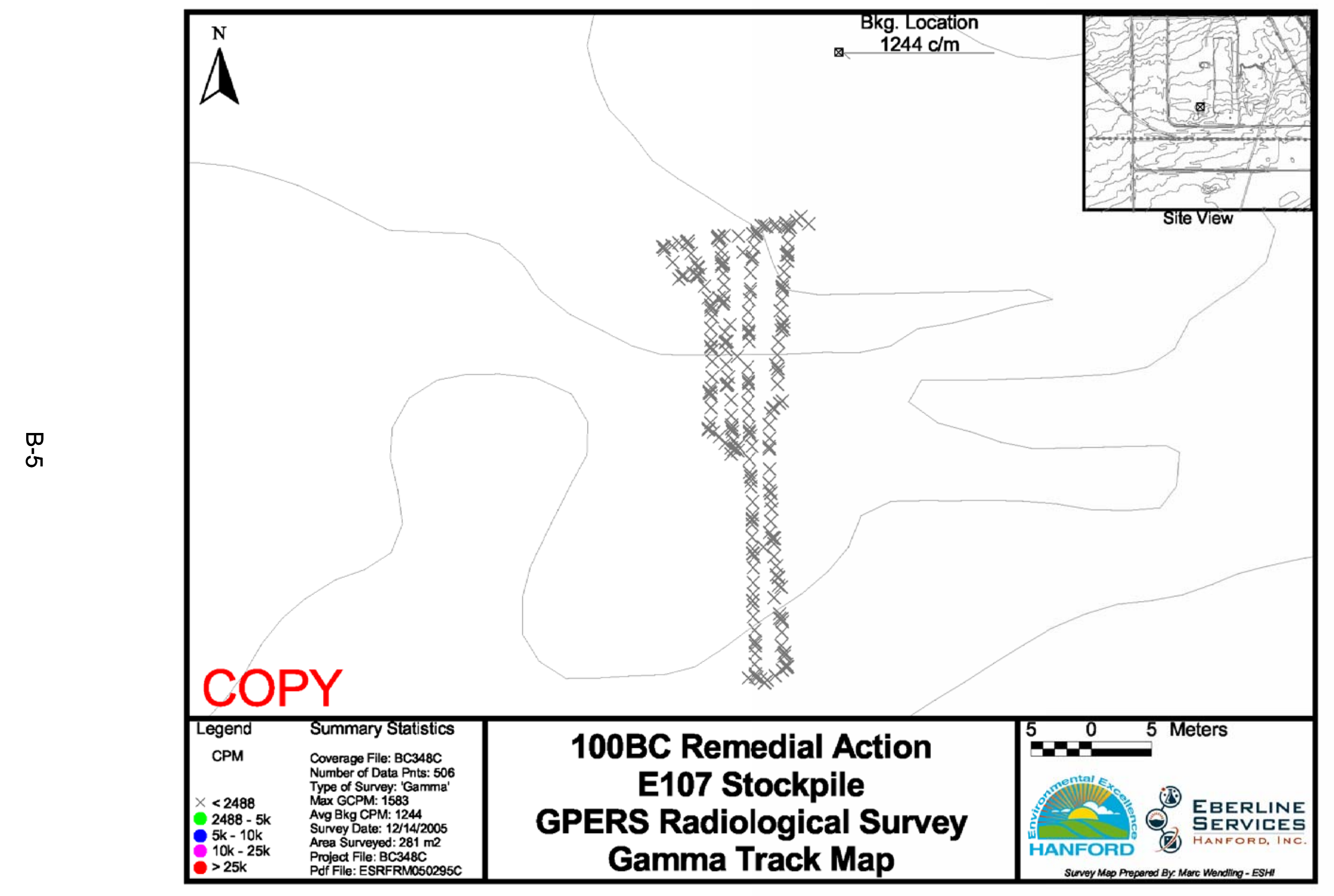

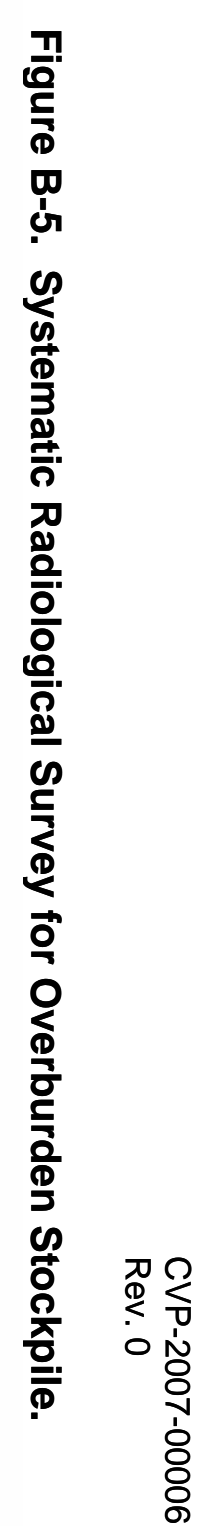




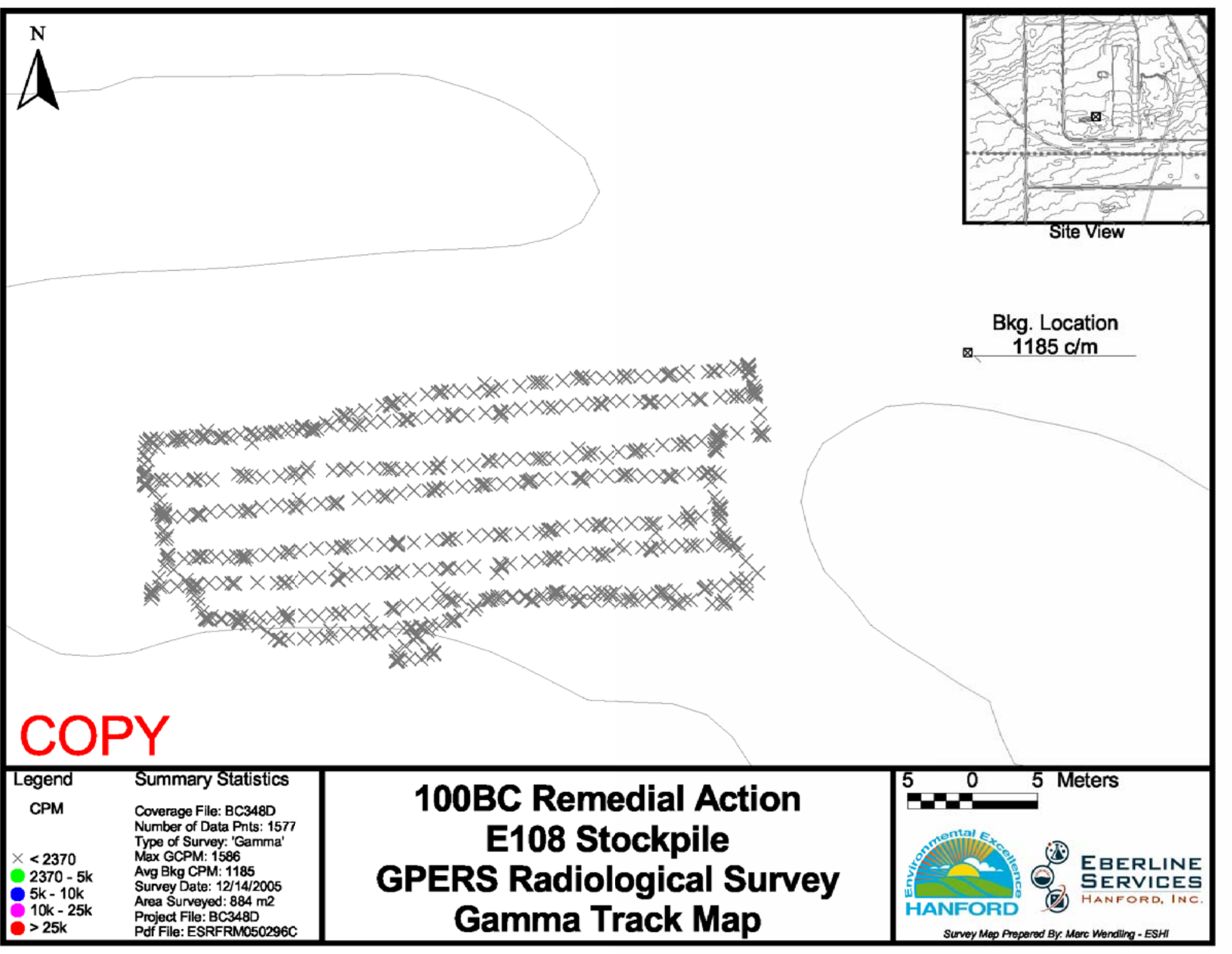

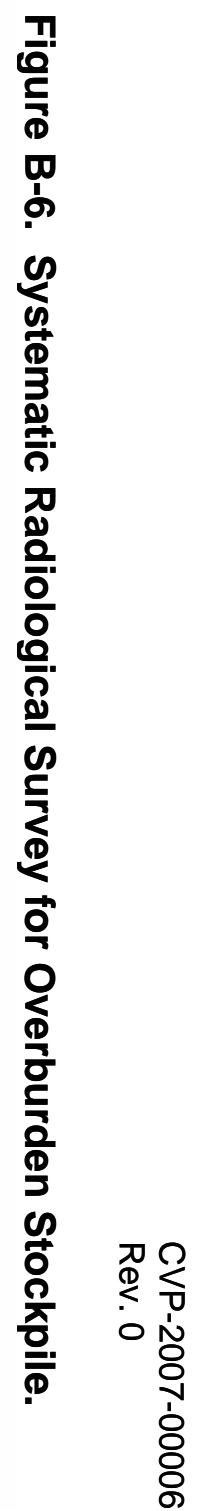




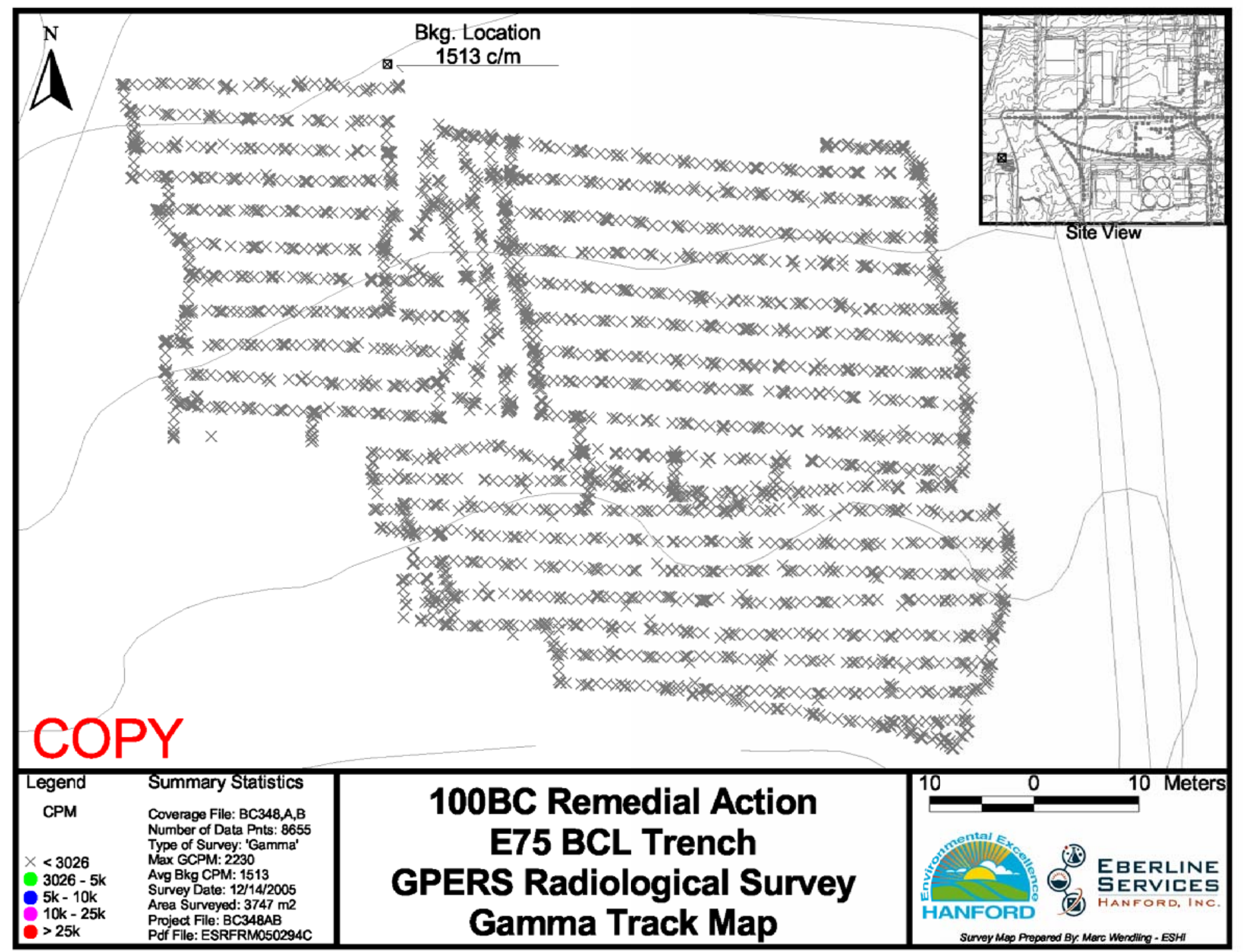

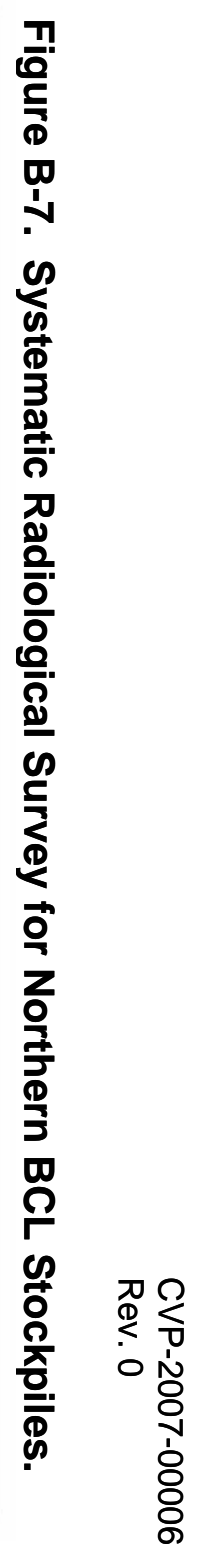




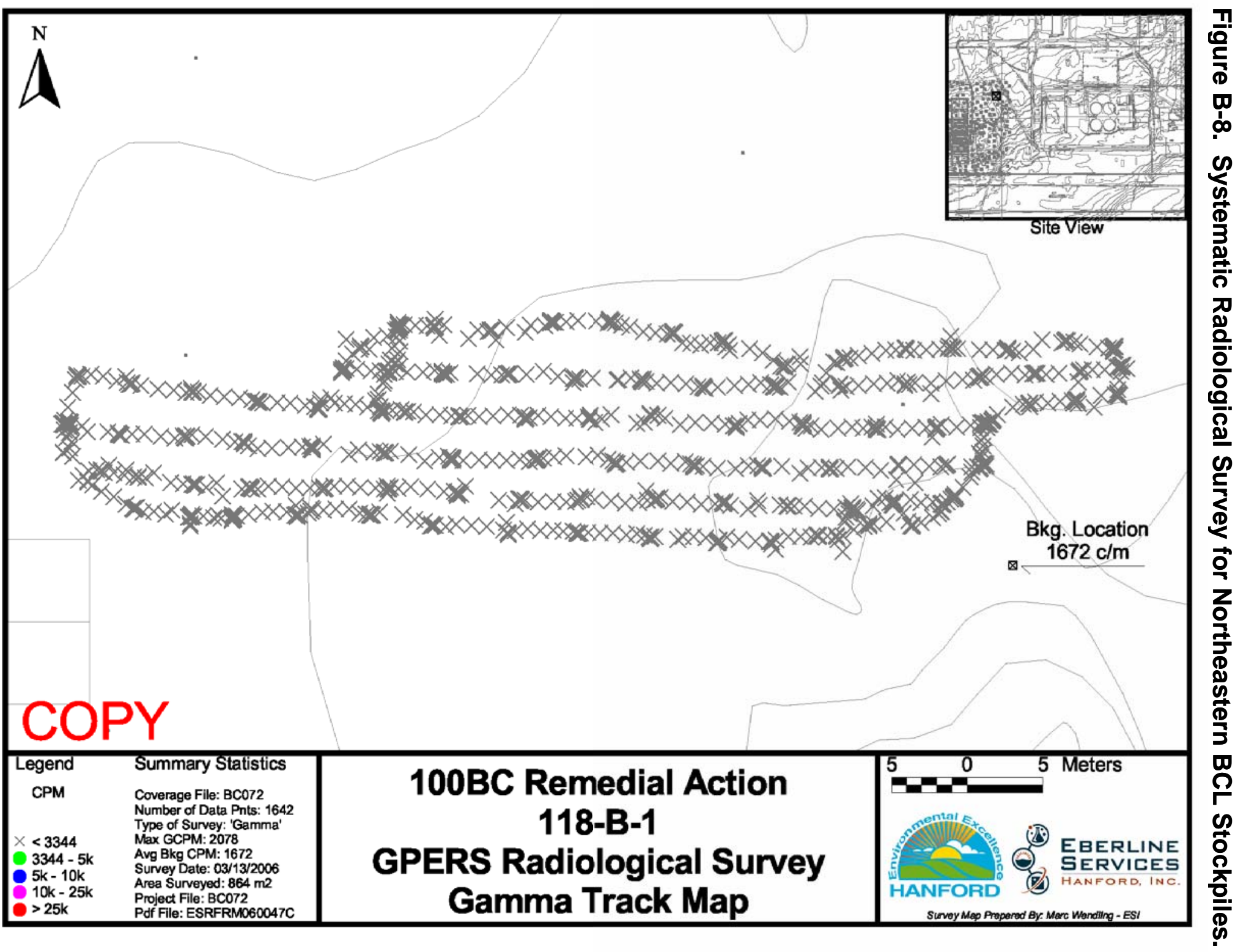

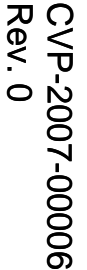




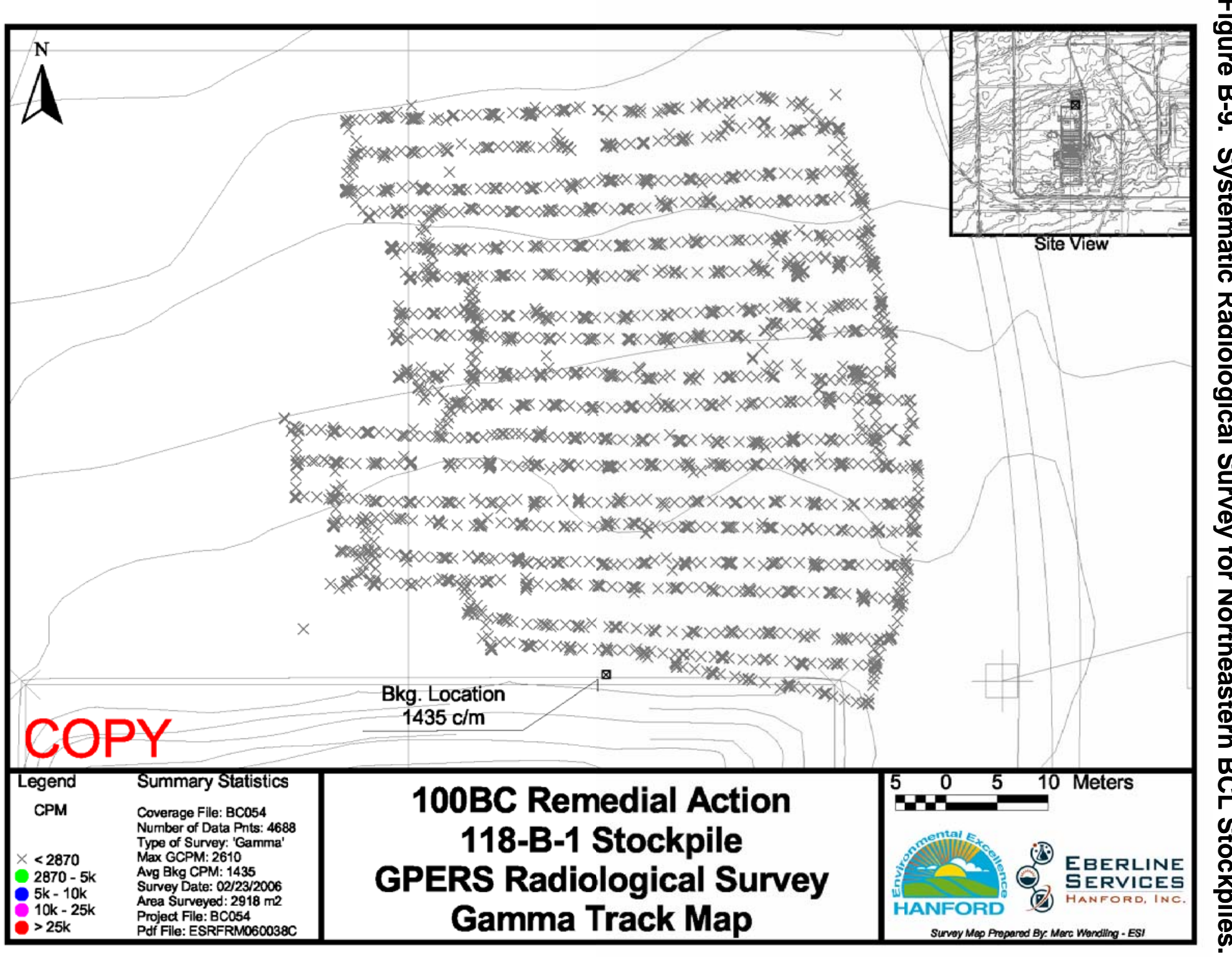

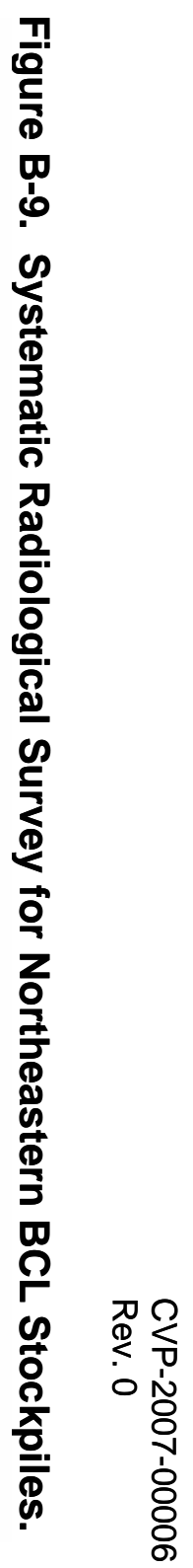




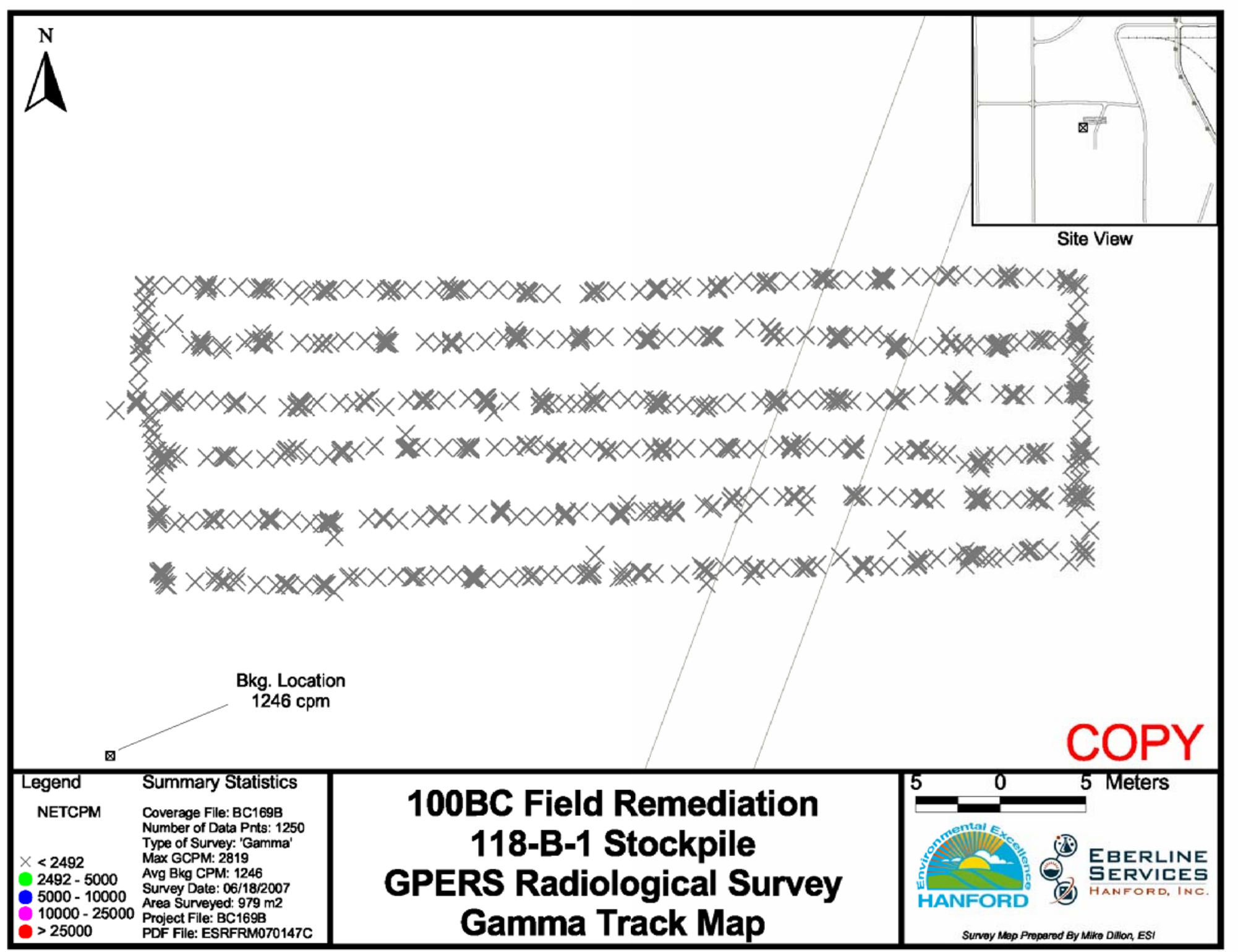

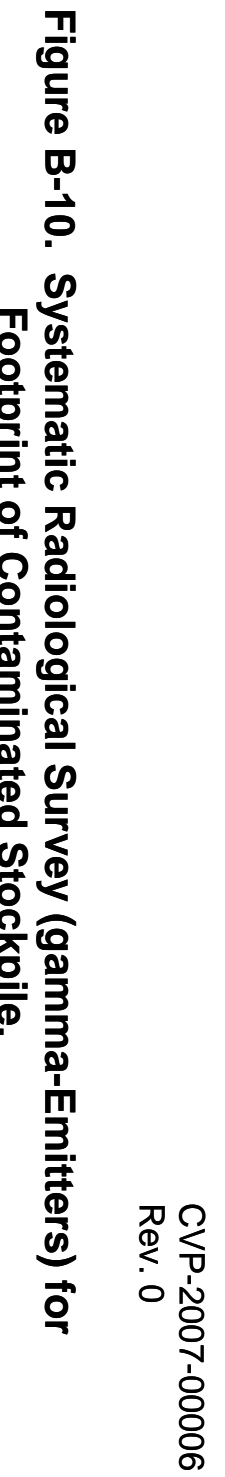




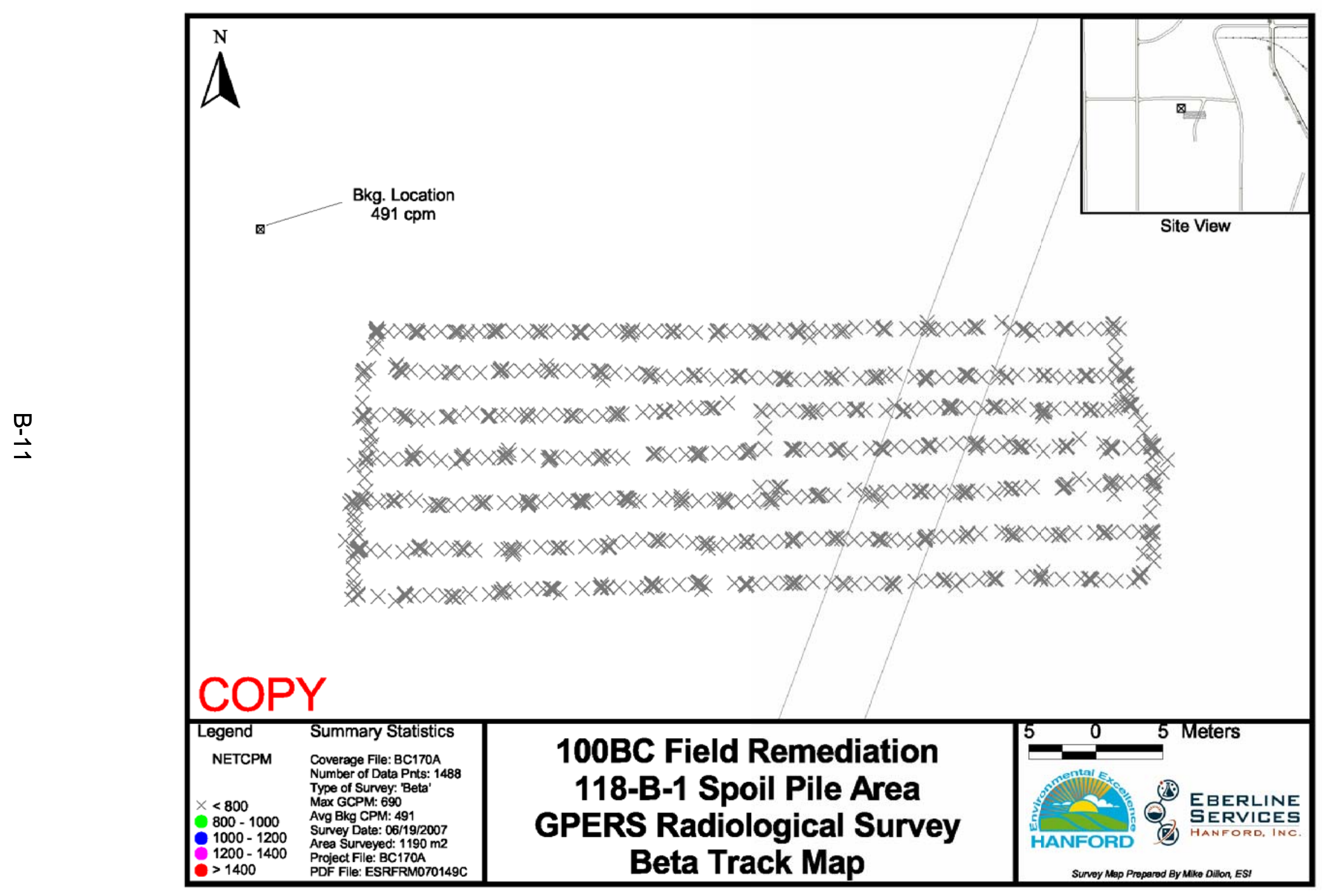

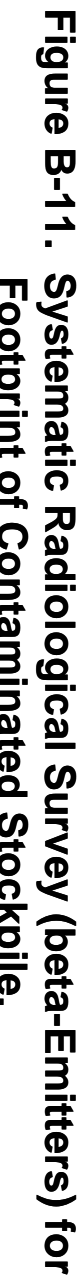






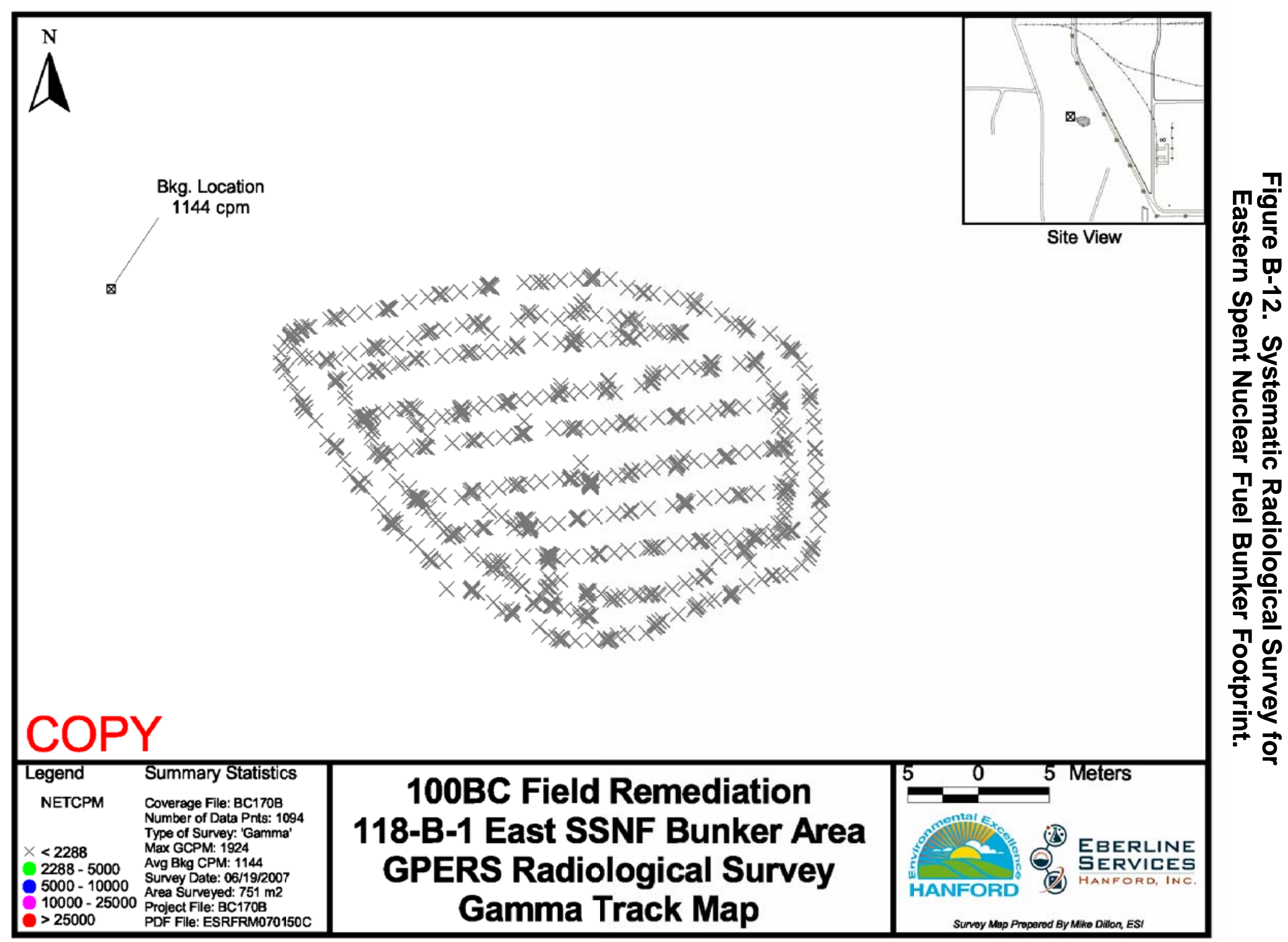

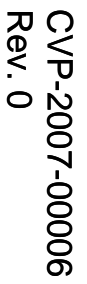




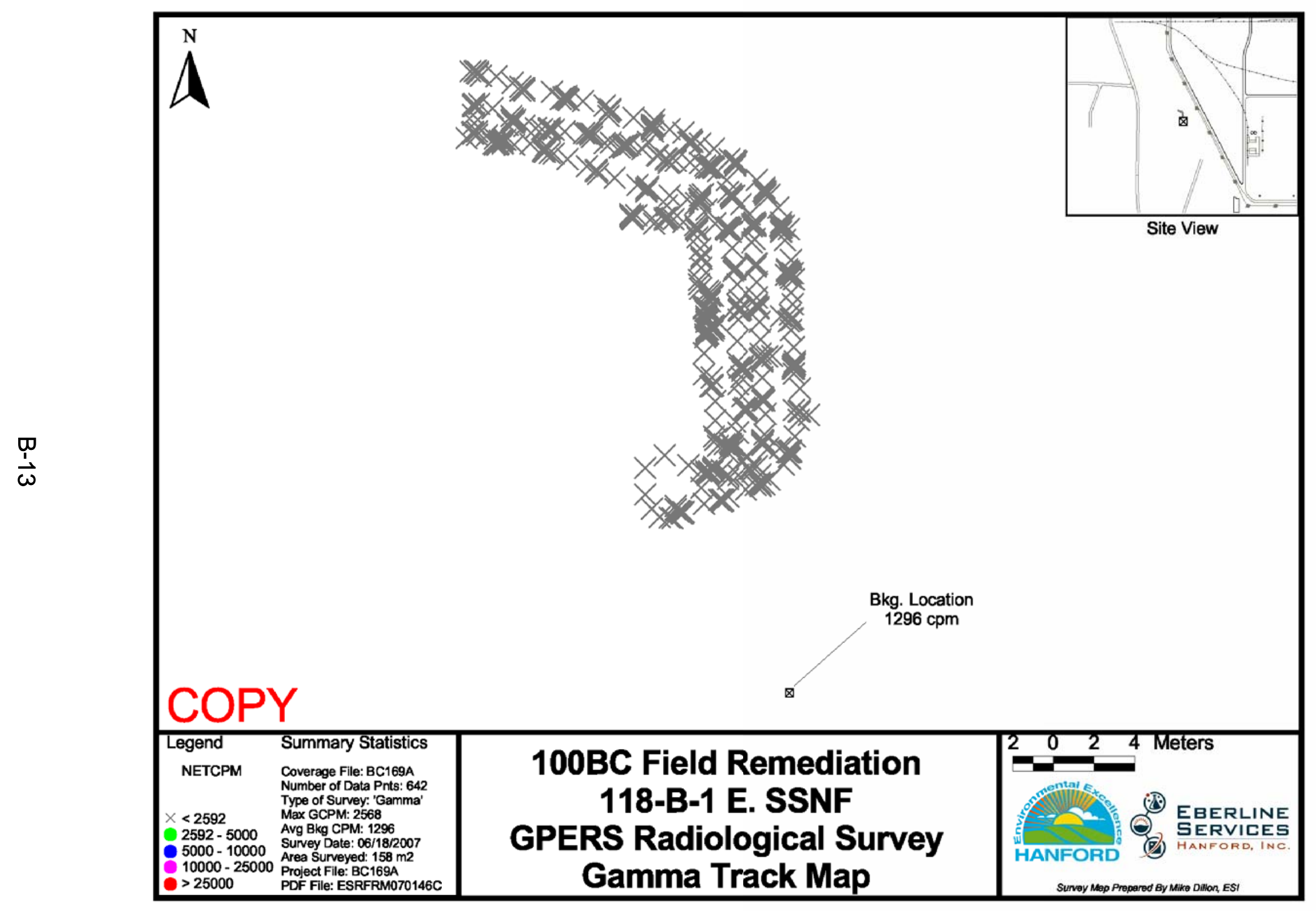

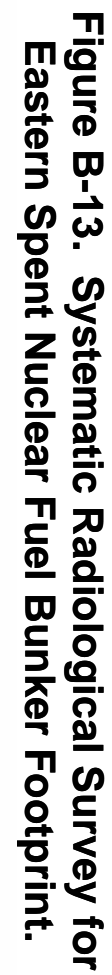

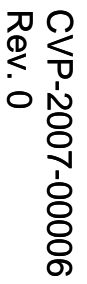




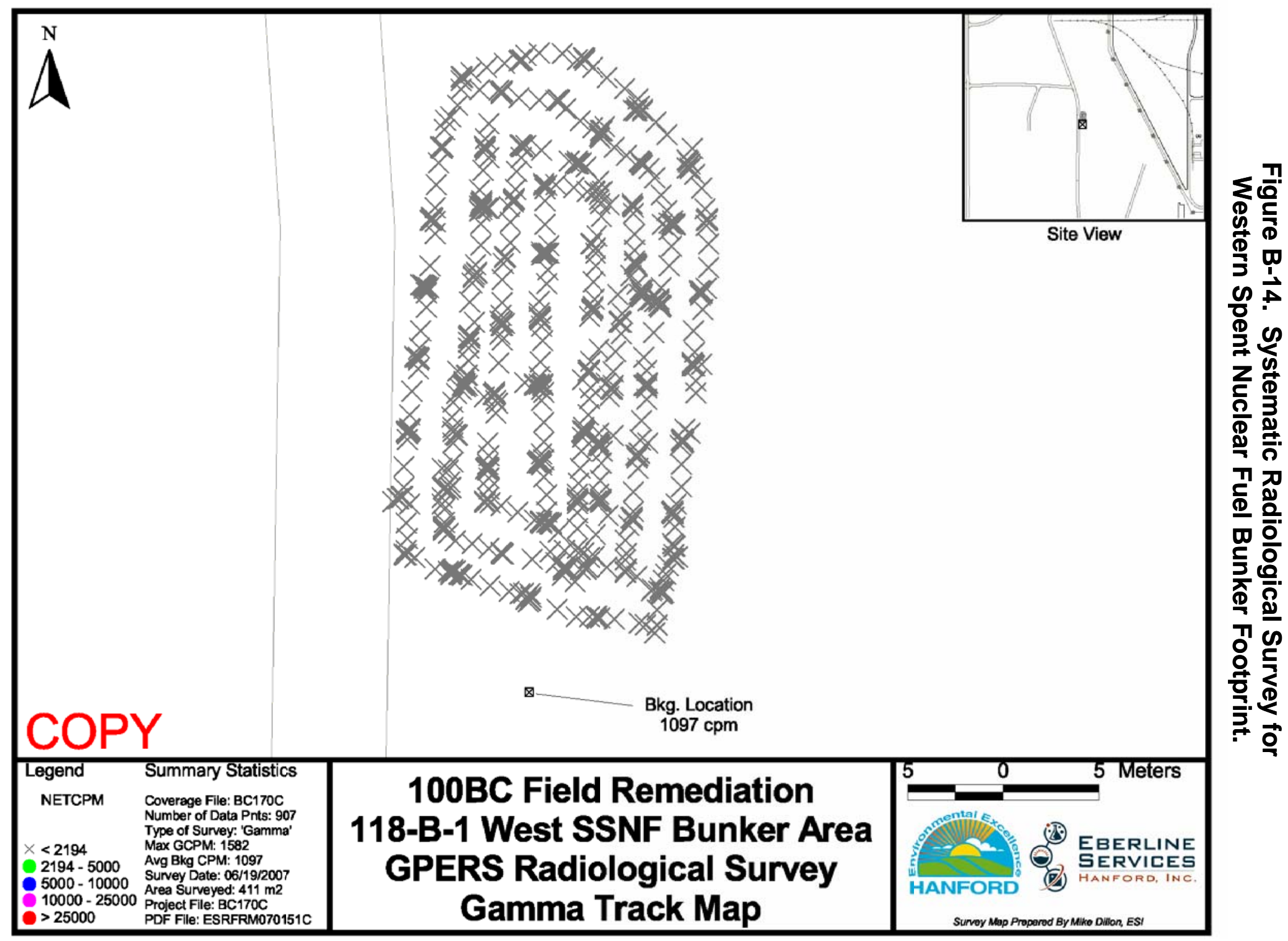

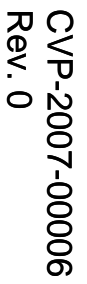




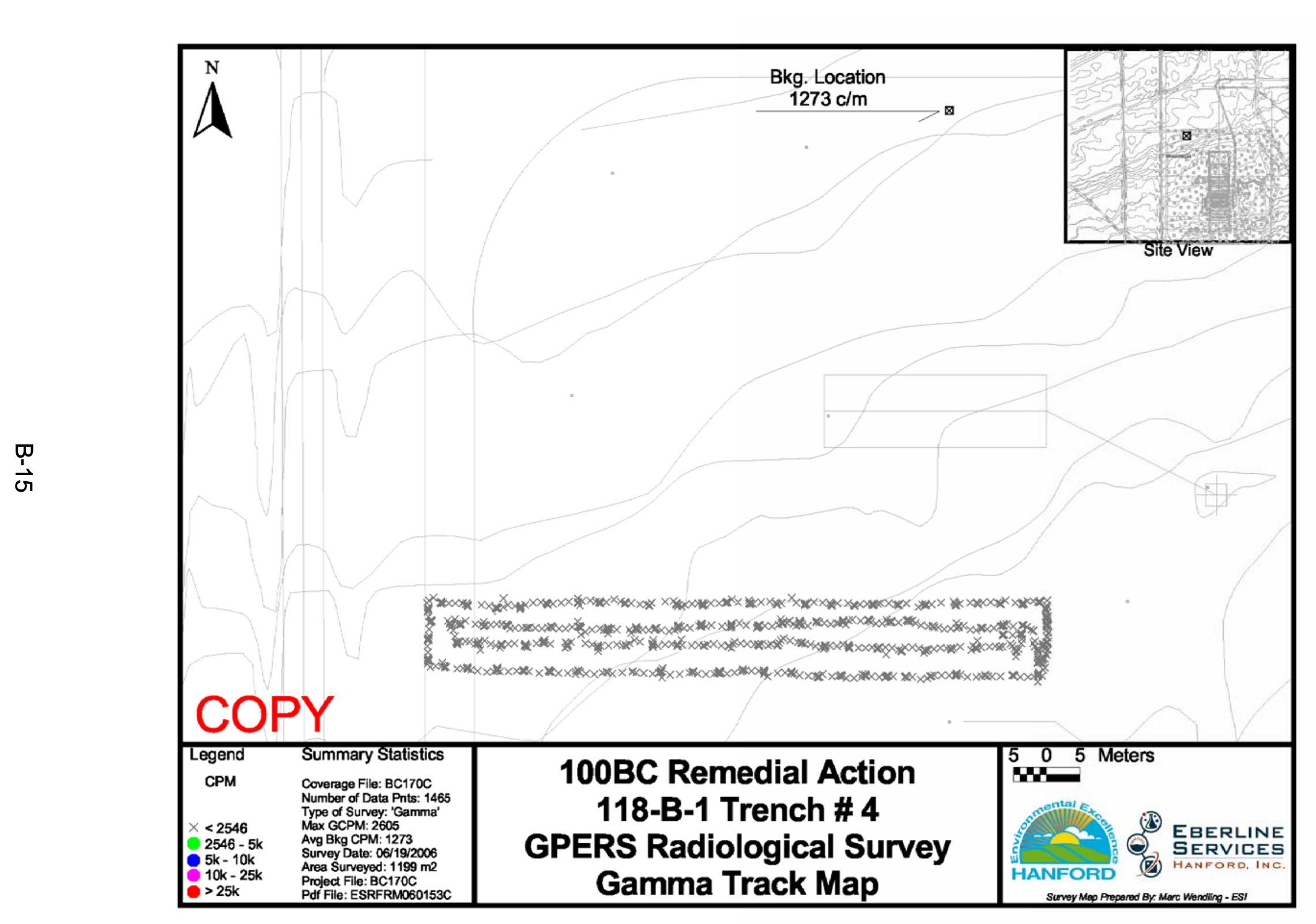




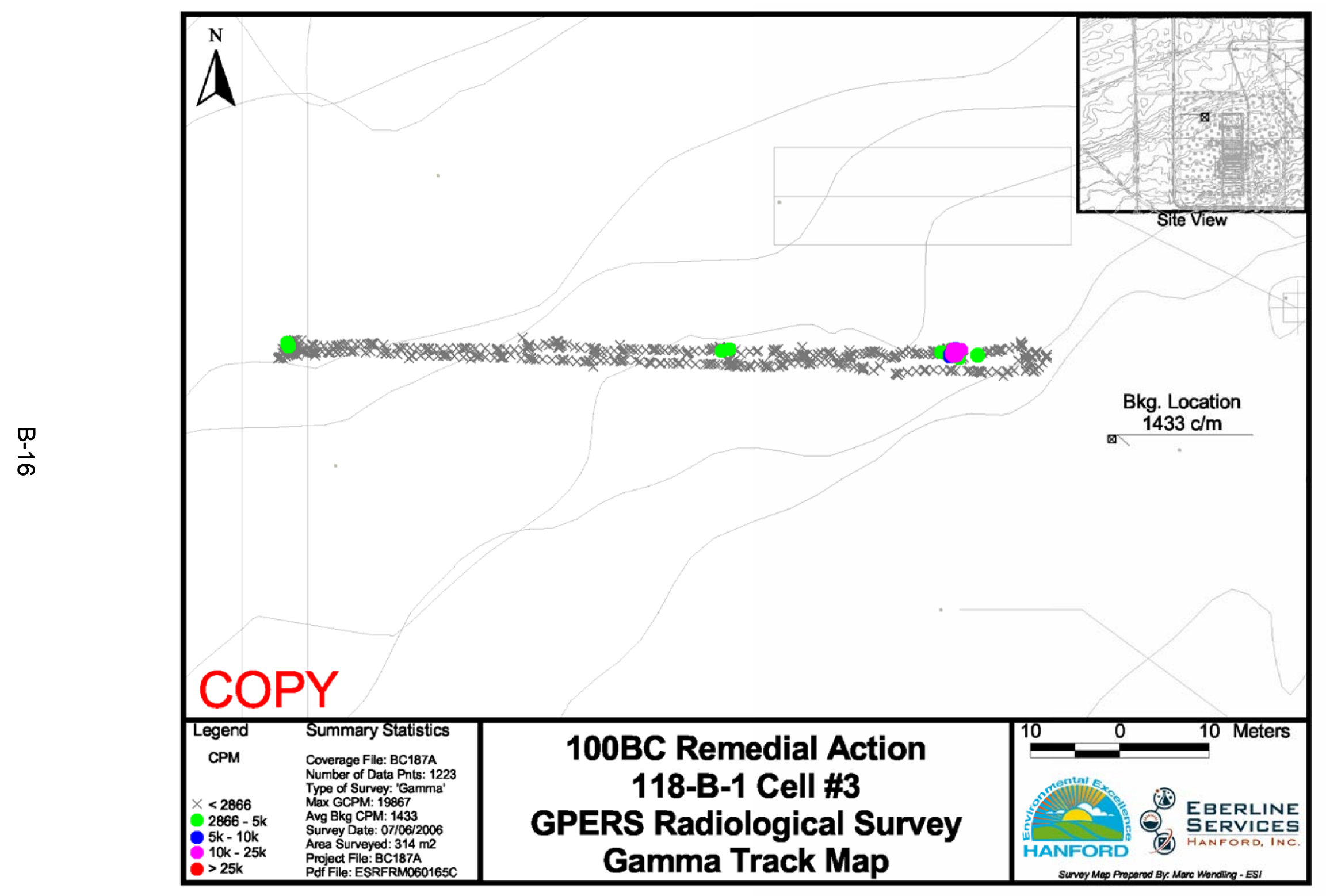

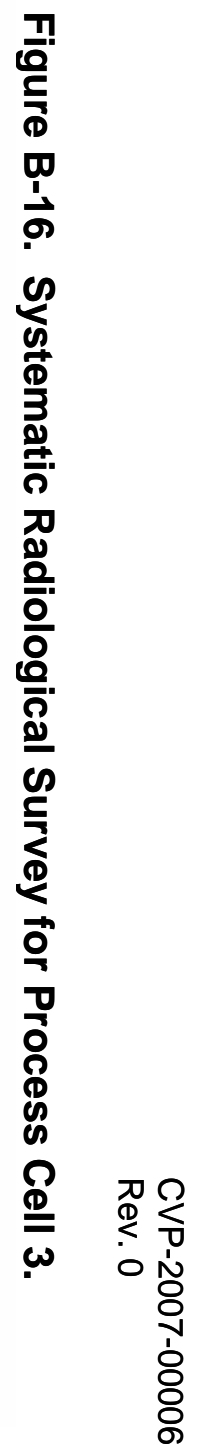




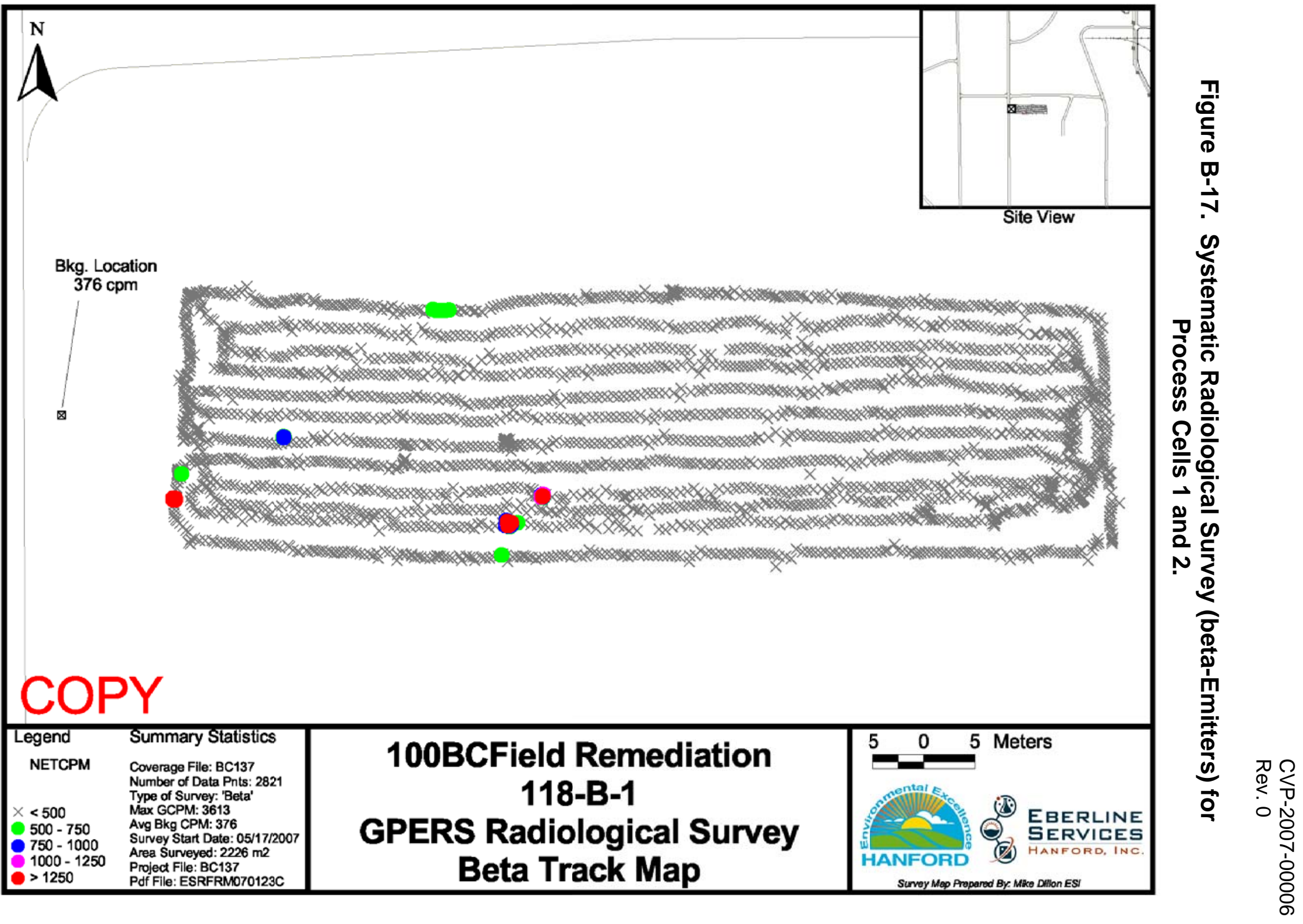




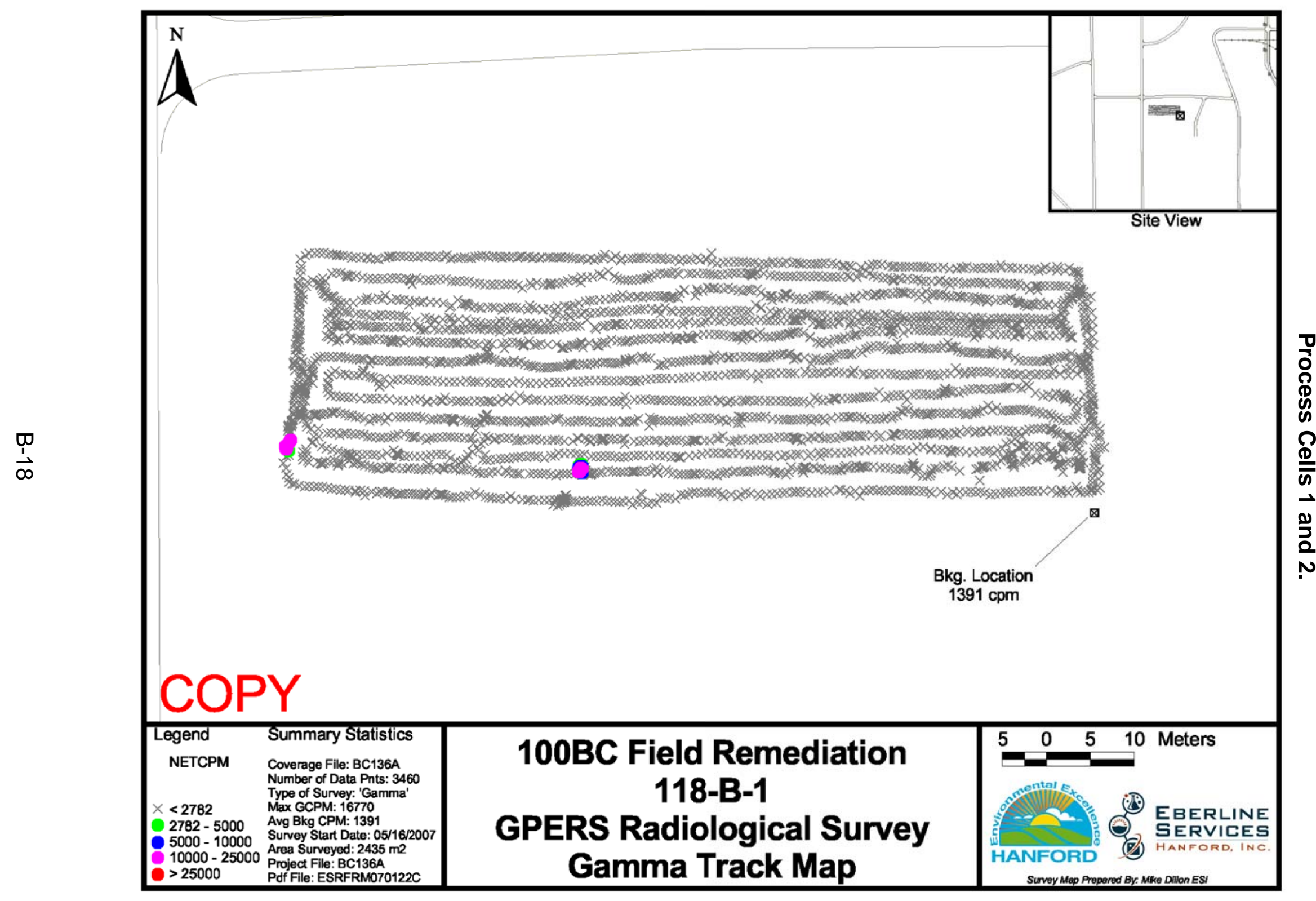

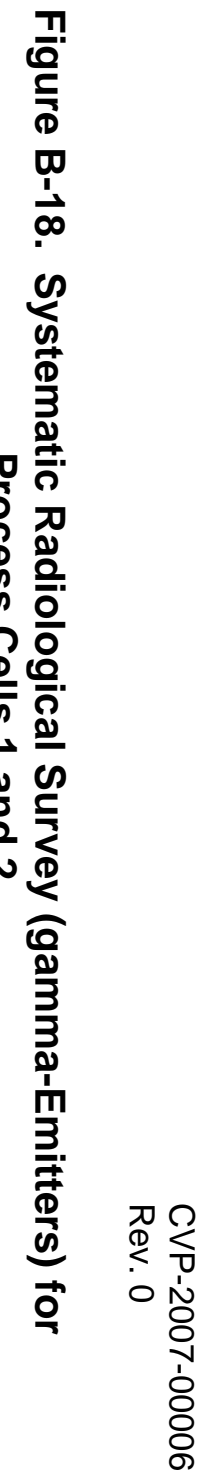




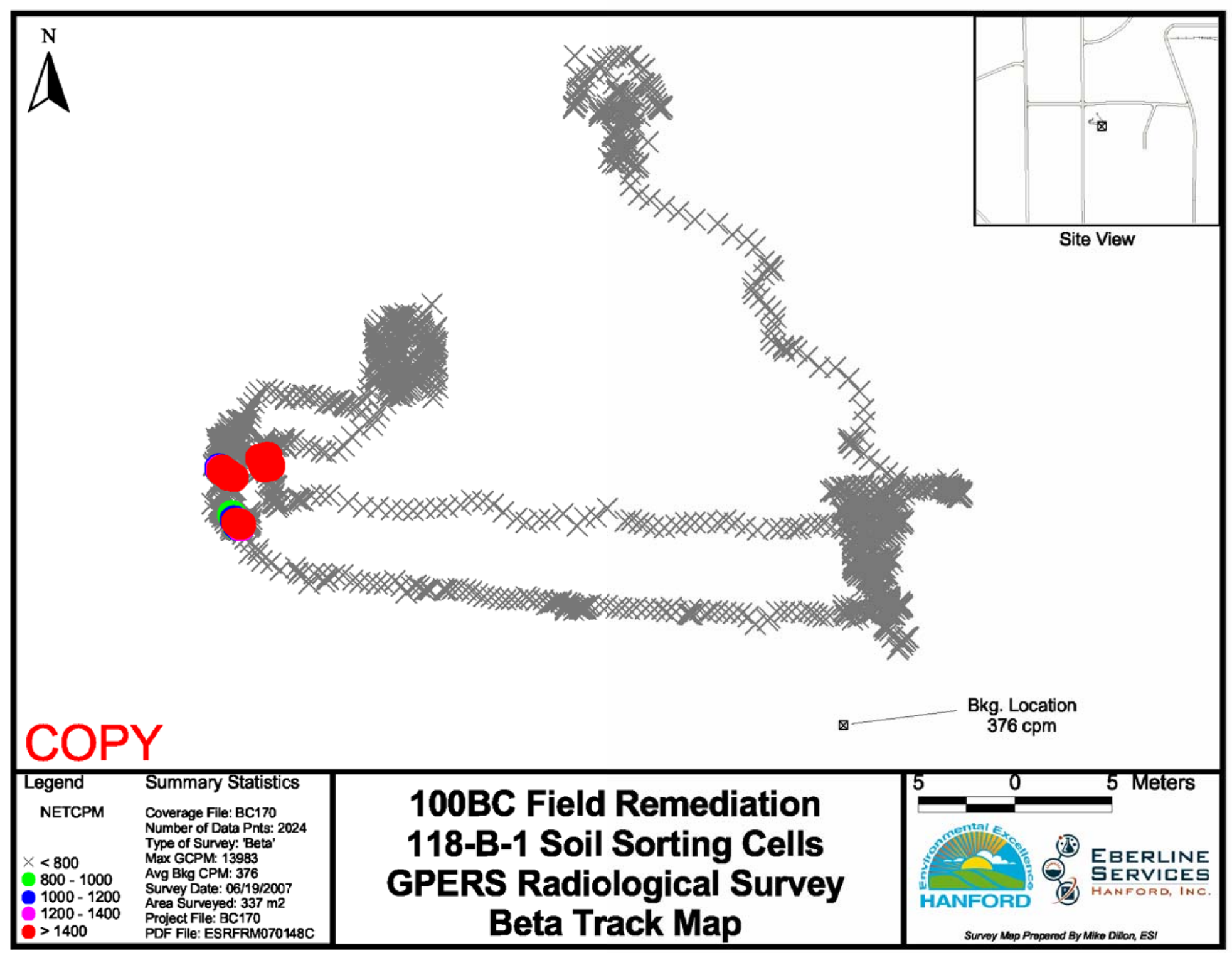

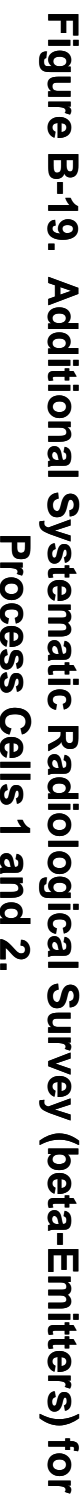

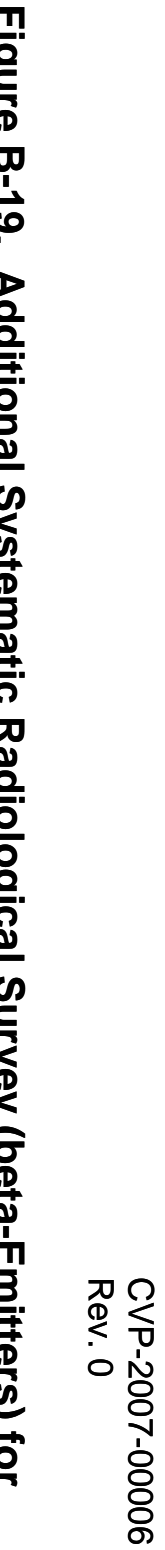




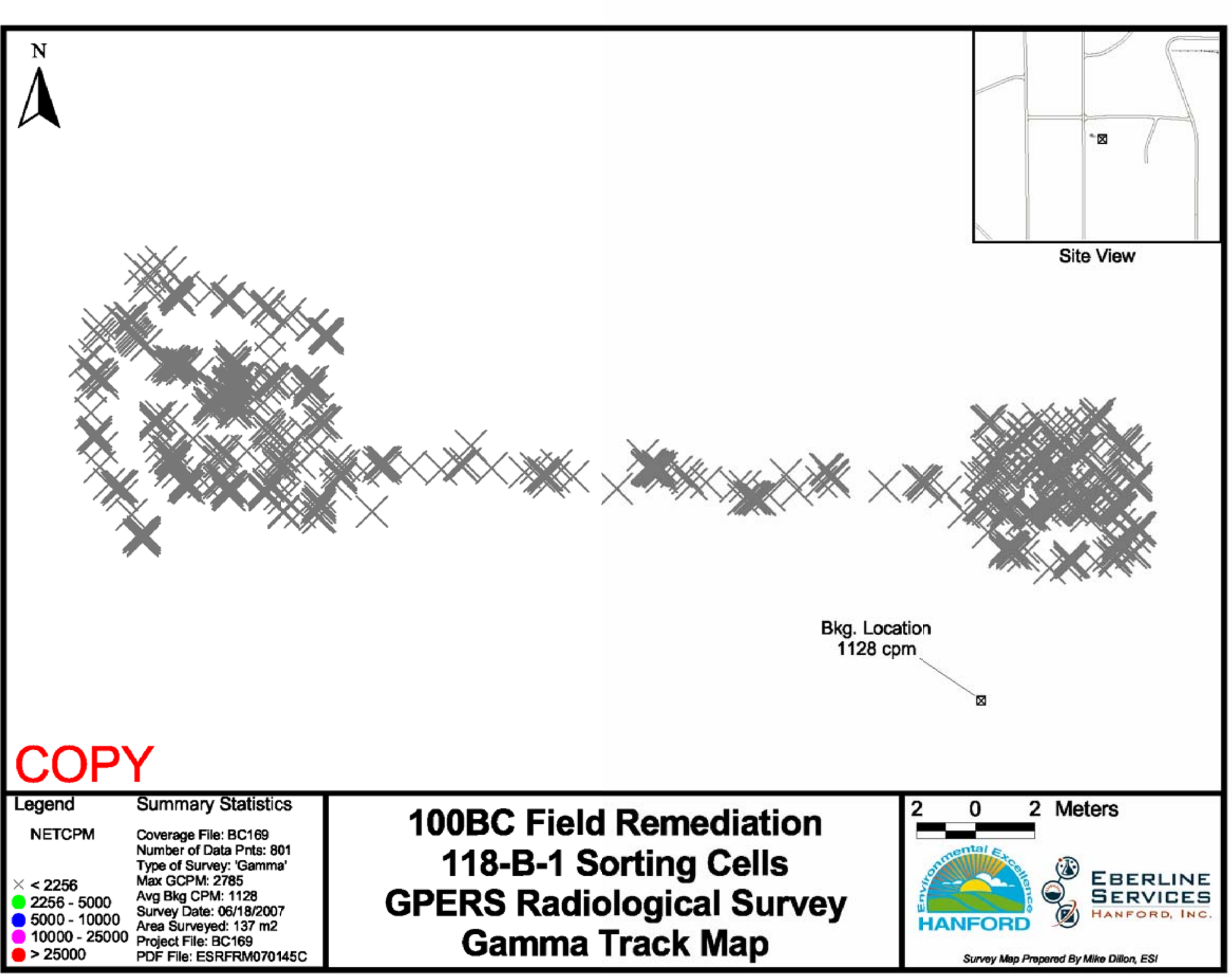

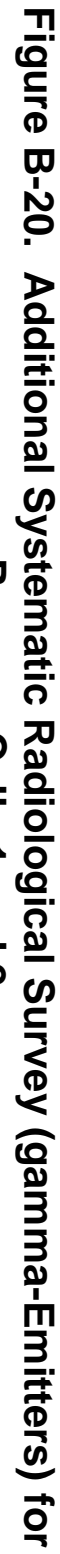




\section{APPENDIX C}

RESRAD INPUT PARAMETERS

AND CALCULATION BRIEF EXCERPTS 
CVP-2007-00006

C-ii 
CVP-2007-00006

\section{CALCULATION BRIEF EXCERPTS}




\section{DISCLAIMER FOR CALCULATIONS}

The calculations that are provided in the following appendix have been generated to document compliance with established cleanup levels. These calculations should be used in conjunction with other relevant documents in the administrative record. 


\section{CALCULATION BRIEFS}

The following calculation briefs have been prepared in accordance with ENG-1, Engineering Services, ENG-1-4.5, "Project Calculations," Washington Closure Hanford, Richland, Washington.

118-B-1 Shallow Zone, Overburden and Staging Pile Sampling Plan, 0100B-CA-V0284, Rev. 0, Washington Closure Hanford, Richland, Washington (2006).

118-B-1 Burial Ground Cleanup Verification 95\% UCL Calculations, 0100B-CA-V0299, Rev. 1, Washington Closure Hanford, Richland, Washington (2007).

118-B-1 Burial Ground Hazard Quotient and Carcinogenic Risk Calculations, 0100B-CA-V0300, Rev. 2, Washington Closure Hanford, Richland, Washington (2007).

118-B-1 Burial Ground Cleanup Verification RESRAD Calculation Brief for Overburden, Below Cleanup Level (BCL), and Staging Pile Area Soil, 0100B-CA-V0301, Rev. 0, Washington Closure Hanford, Richland, Washington (2007).

118-B-1 Combined Areas Cleanup Verification RESRAD Calculation Brief, 0100B-CA-V0308, Rev. 0, Washington Closure Hanford, Richland, Washington (2007).

118-B-1 Burial Ground Combined Areas Comparison to Drinking Water Standards (MCL) Calculation Brief, 0100B-CA-V0309, Rev. 0, Washington Closure Hanford, Richland, Washington (2007).

NOTE: The calculation briefs referenced in this appendix are kept in the active Washington Closure Hanford project files and are available upon request. When the project is completed, the files will be stored in a U.S. Department of Energy, Richland Operations Office repository. Only excerpts of the calculation briefs are included in this appendix. 
CVP-2007-00006

C-vi 


\section{CALCULATION COVER SHEET}

Project Title:

Area

Discipline

Subject

Computer Program
118-B-1 Burial Ground Sample Design $100-\mathrm{B} / \mathrm{C}$

Environmental Engineering "Calc. No. 0100B-CA-V0284

118-B-1 Shallow Zone, Overburden, BCL, and Staging Pile Sampling Plan

\begin{tabular}{lll} 
Excel Program No. Excel 2003 \\
\hline
\end{tabular}

The attached calculations have been generated to document compliance with established cleanup levels. These calculations should be used in conjuction with other relevent documents in the administrative record.

Committed Calculation $\quad \mathrm{X} \quad$ Preliminary $\square \quad$ Superseded $\square \quad$ Voided $\square$

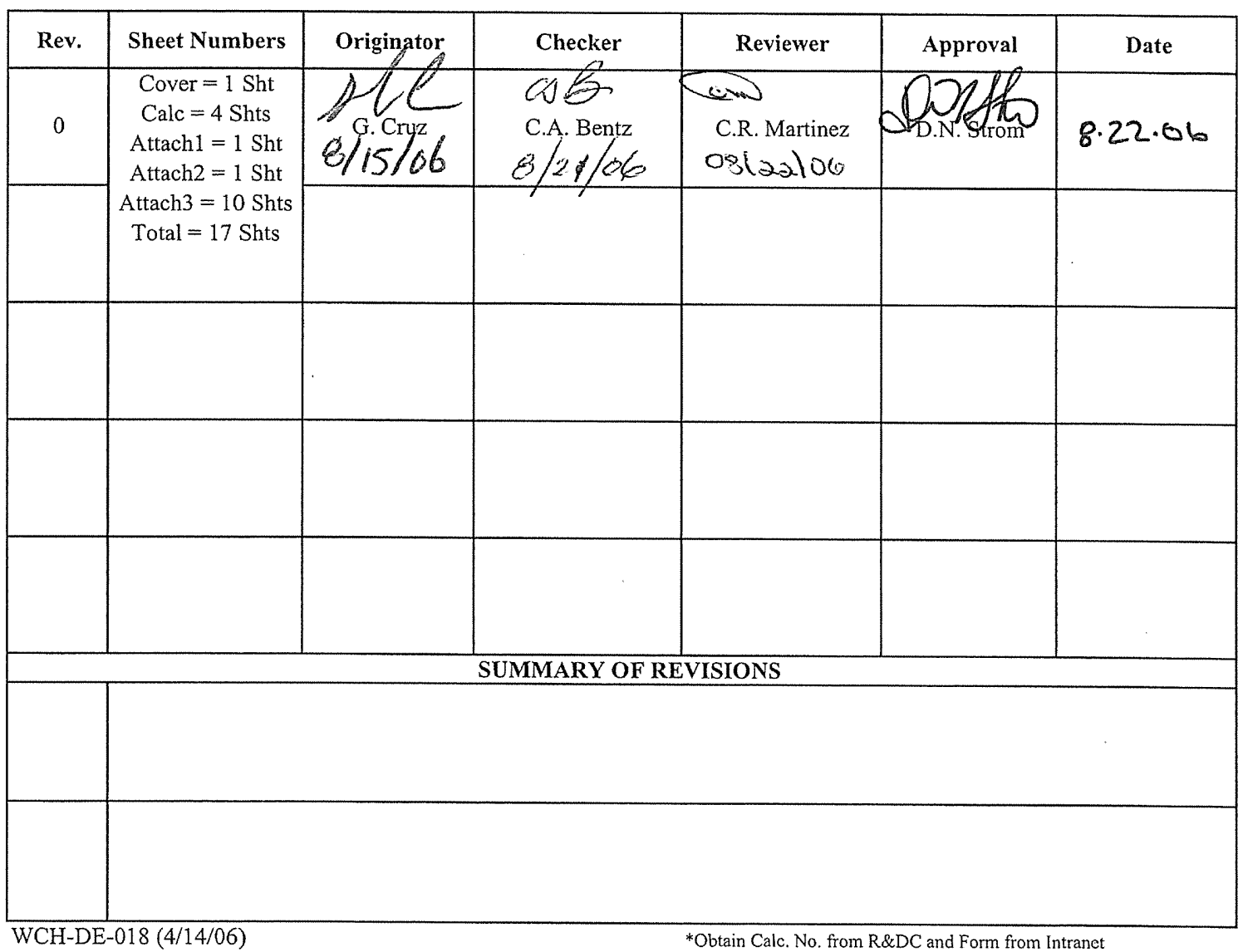


Washington Closure Hanford

Originator $\mathrm{GOHz}$

Project 118-B-1 Burial Ground Sample Design

Subject

Calc. No. $\frac{0100 \mathrm{~B}-\mathrm{CA}-\mathrm{V} 0284}{14655 \text { Checked }}$ Rev. No. $\frac{0}{\text { Date } \mathrm{E} / 2 \mathrm{2} / 06}$

and Staging Pile Sampling Plan Sheet No. 1 of 4

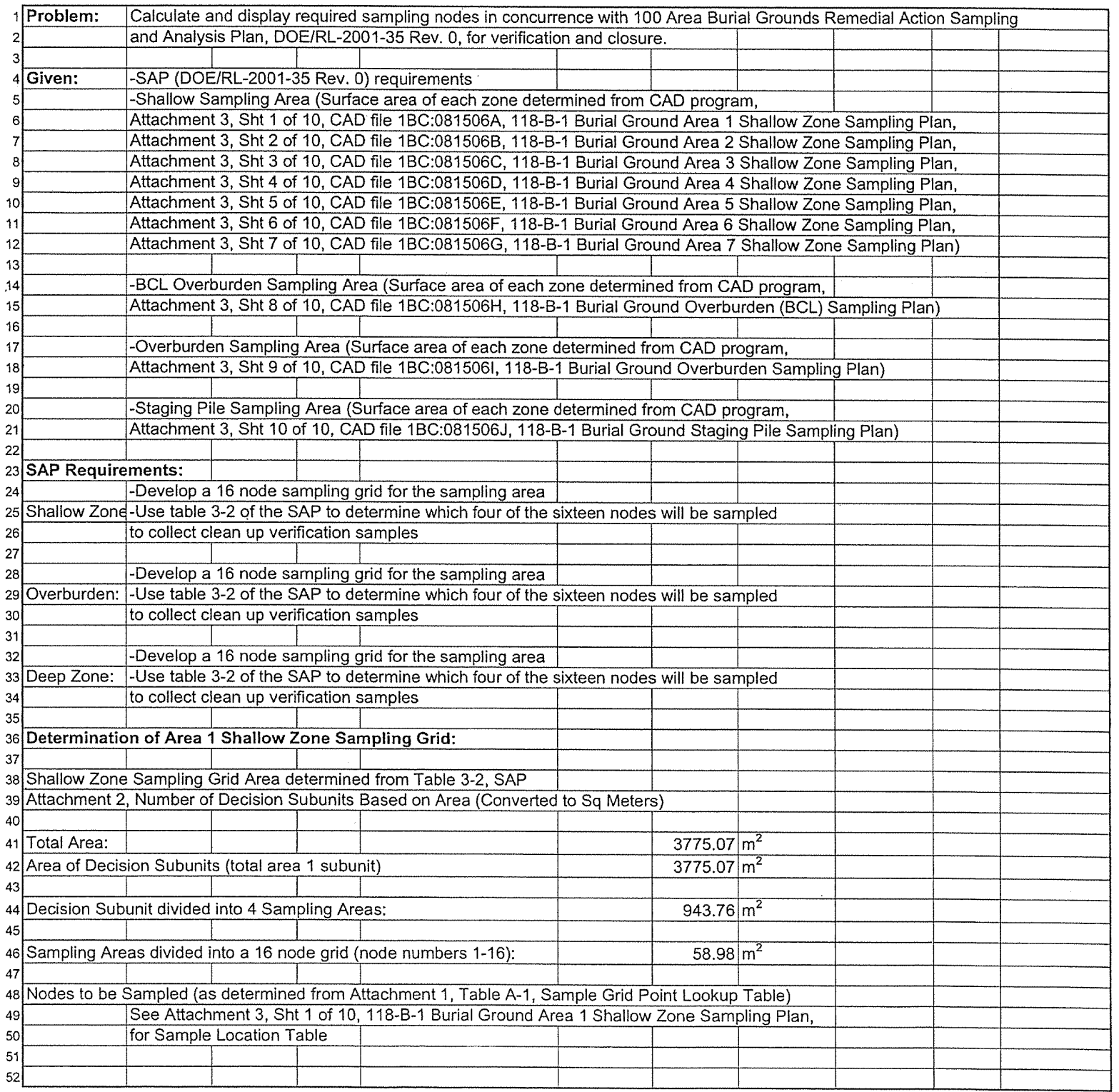


Calc. No. $\frac{0100 \mathrm{~B}-\mathrm{CA}-\mathrm{V} 0284}{14655 \text { Checked }}$ Rev. No. $\frac{0}{\text { Dob No. } Q / 2 i / \sigma 6}$

Project 118-B-1 Burial Ground Sample Design

Subject 118-B-1 Shallow Zone, Overburden, BCL, and Staging Pile Sampling Pla Sheet No. 2 of4

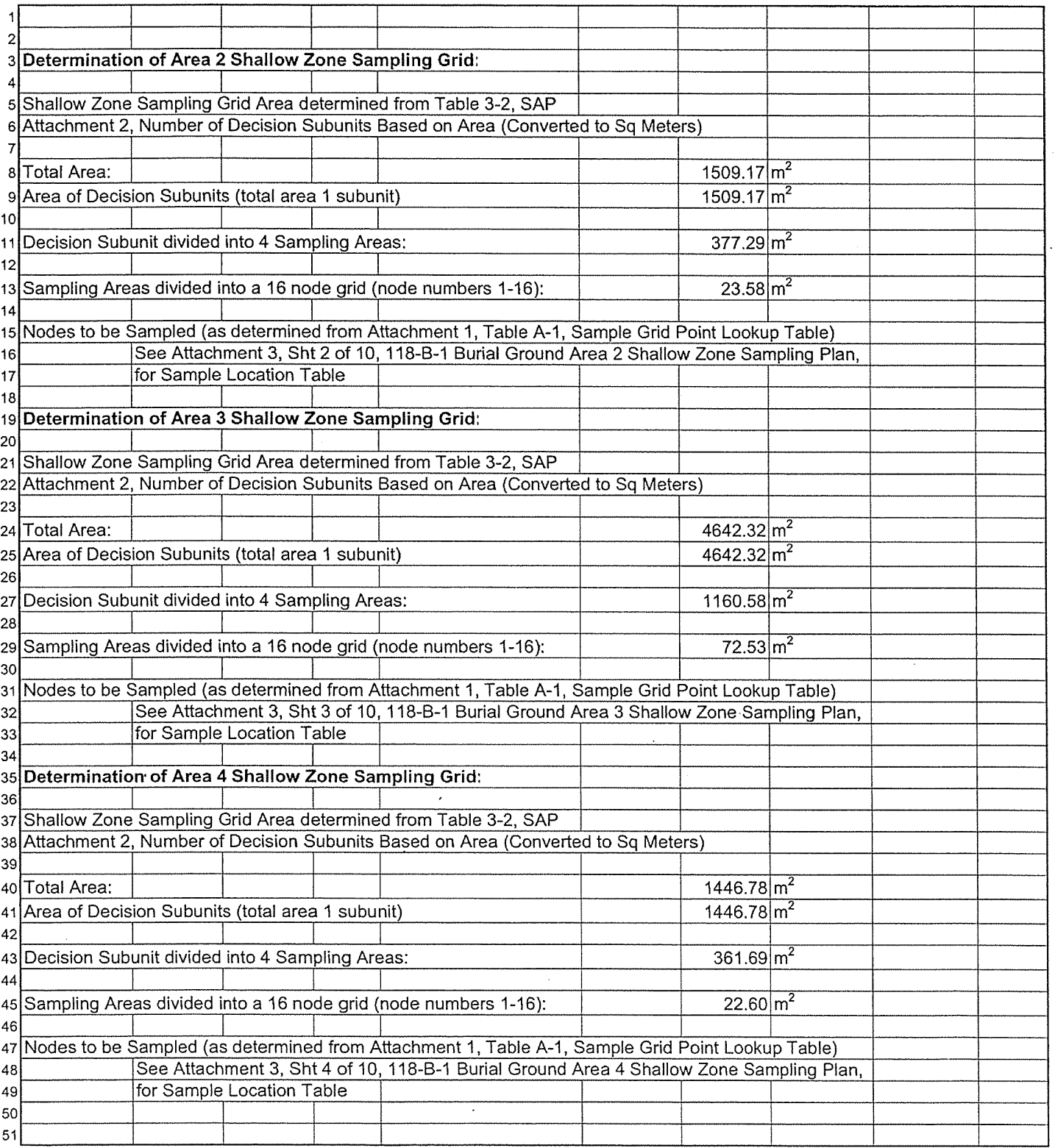


Originator G. Cruz Date 8/15/2006

Project 118-B-1 Burial Ground Sample Design Subject 118-B-1 Shallow Zone, Overburden, BCL, and Staging Pile Sampling Pla Sheet No. 3 of 4
Calc. No. 0100B-CA-V0284 Rev. No. 0 Job No: 14655 Checked C4\& Date \&/2i/ok

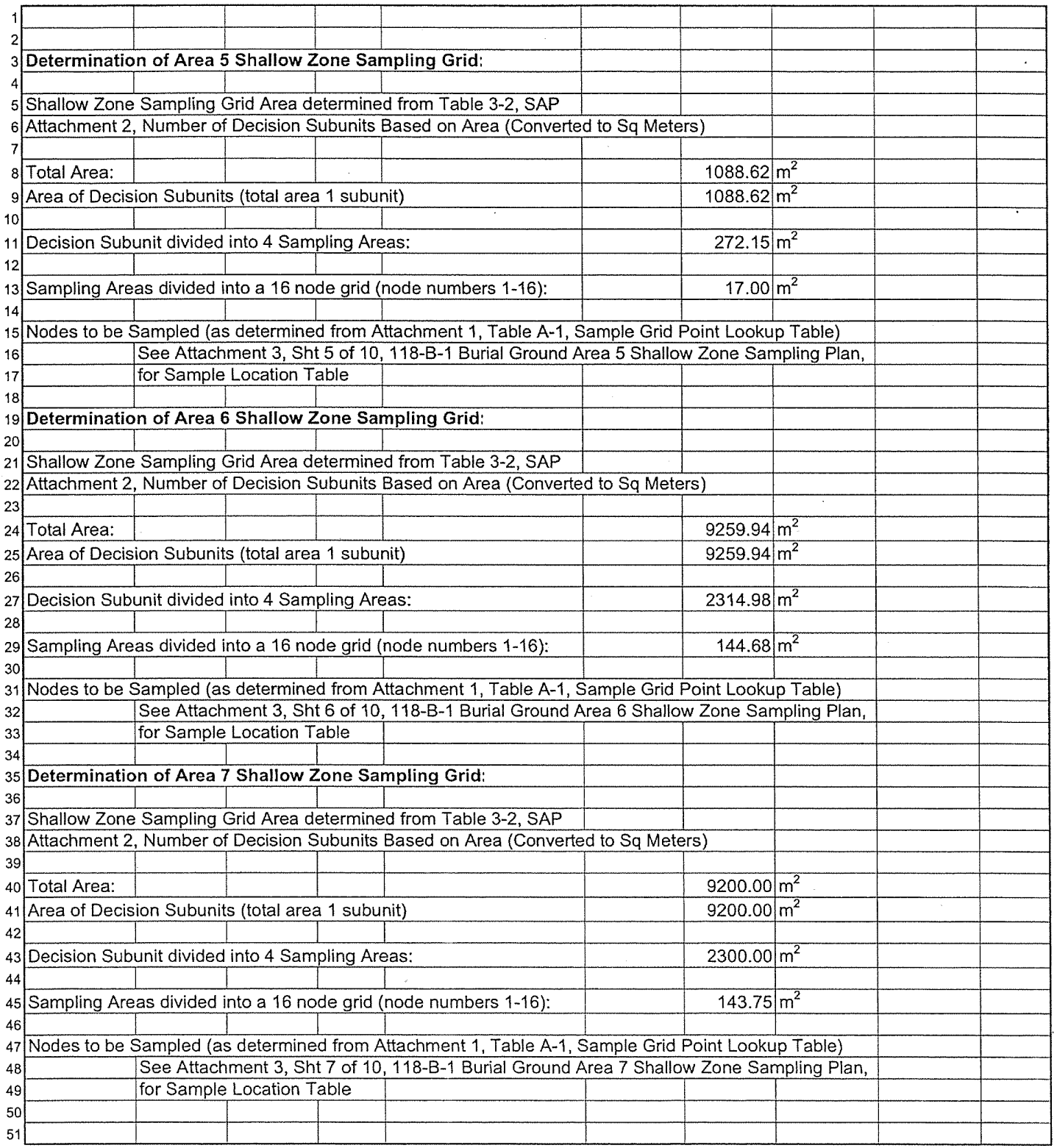




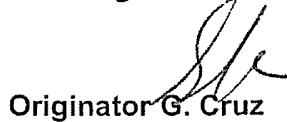

Date 8/15/2006

Calc. No. 0100B-CA-V0284 Rev. No. 0

Project 118-B-1 Burial Ground Sample Design

Job No. 14655 Checked 48 Date $8 / 2 i / 06$

Subject 118-B-1 Shallow Zone, Overburden, BCL, and Staging Pile Sampling Plai Sheet No. 4 of 4

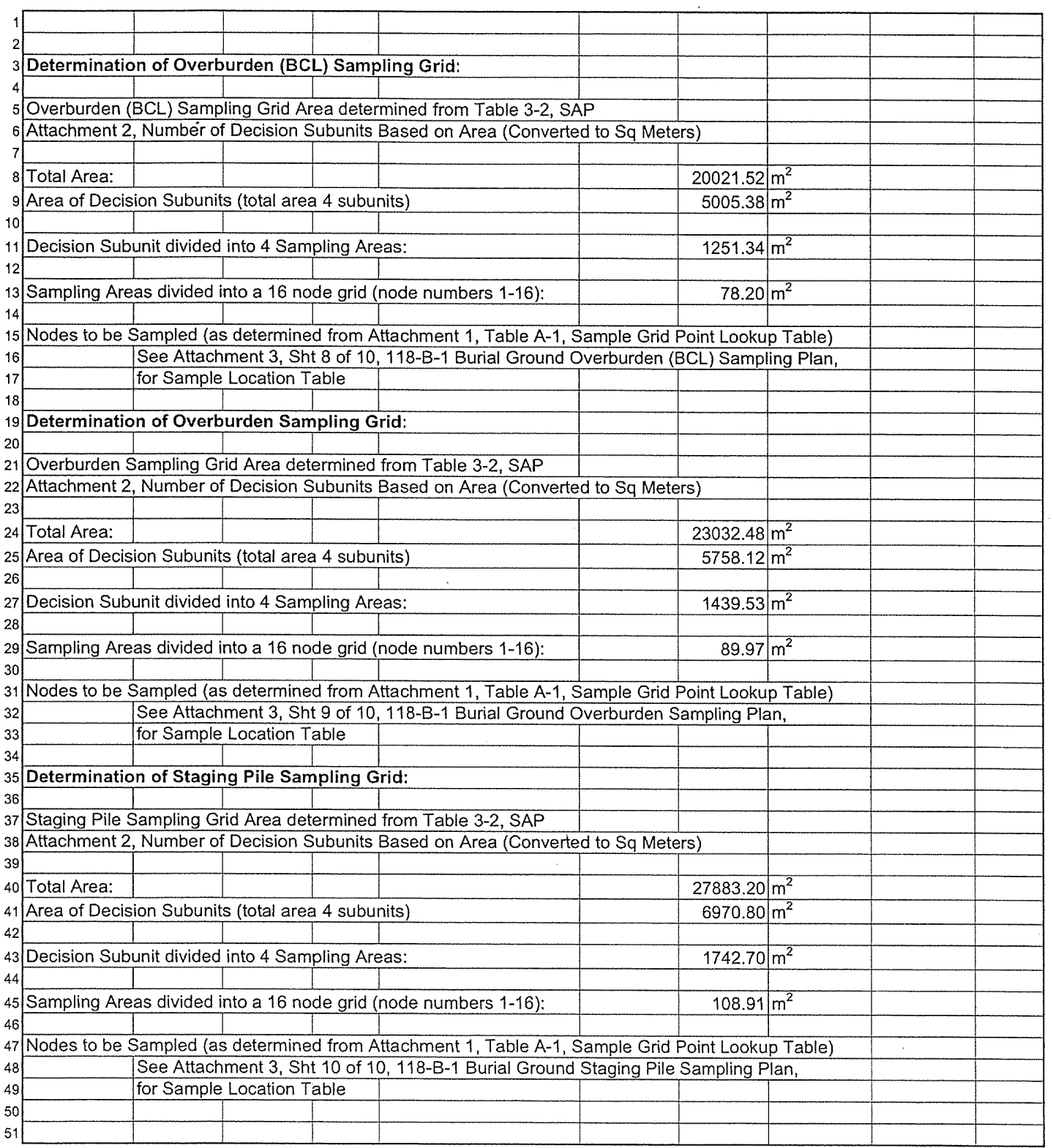




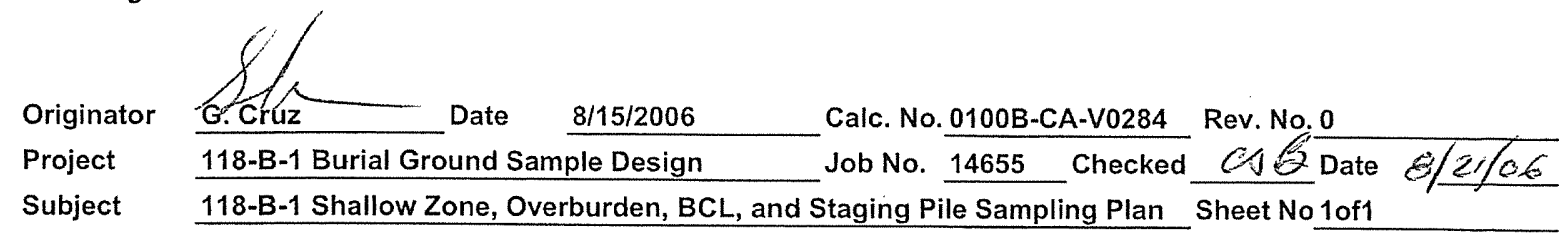

\section{ATTACHMENT 1} ${ }_{3}$ Sample Grid Point Lookup Table.

4

5

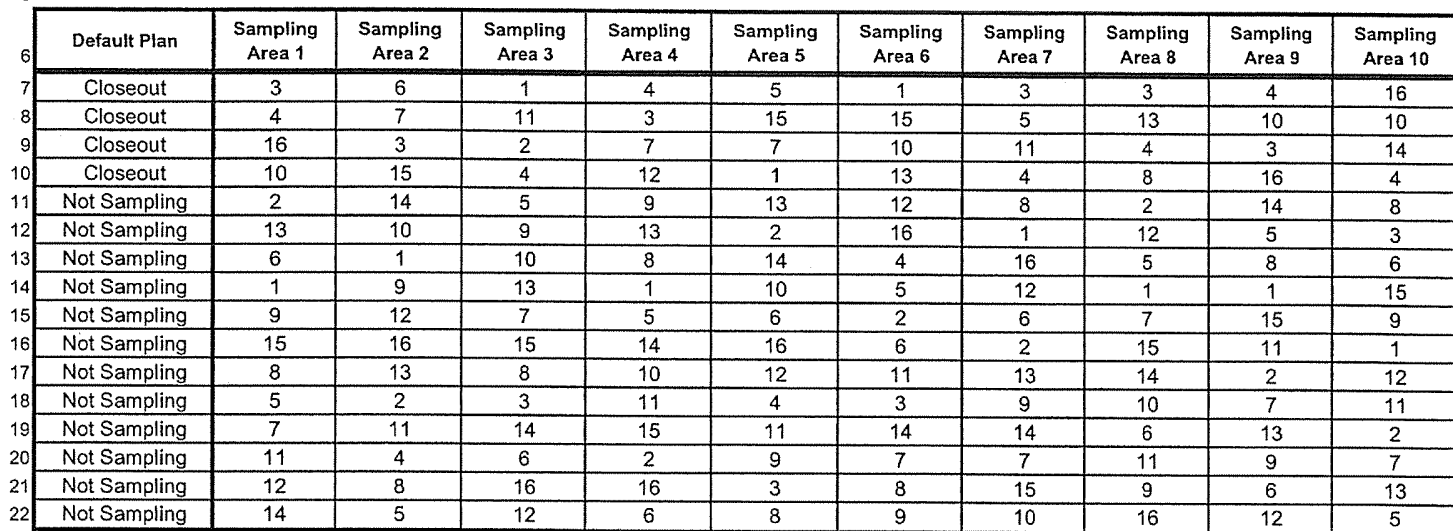

23 ** Note: Grid nodes for each sampling area in each waste site should be numbered consistently, e.g., begin numbering 24 the nodes in the northwesternmost node. Then number consecutively left to right. 


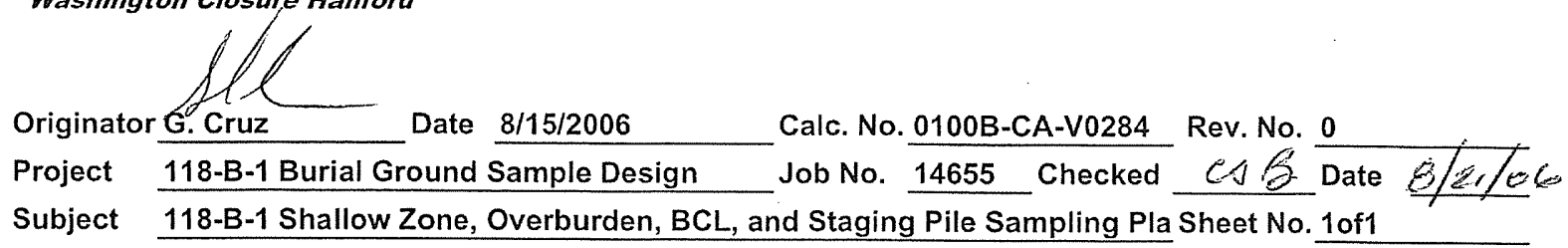

\section{ATTACHMENT 2}

${ }_{3}$ Number of Decision Subunits Based on Area.

\begin{tabular}{|c|c|c|c|c|c|}
\hline \multicolumn{6}{|c|}{ Site Verification Sampling Frequencies Based on Area. } \\
\hline Decision Unit $^{2}$ & Waste Site Size" & $\begin{array}{l}\text { Decision } \\
\text { Subunits }\end{array}$ & Blocks $^{c}$ & $\begin{array}{l}\text { Discrete } \\
\text { Samples }\end{array}$ & $\begin{array}{c}\text { Composite } \\
\text { Samples }\end{array}$ \\
\hline \multirow{3}{*}{$\begin{array}{l}\text { Shallow zone- } \\
01015 \mathrm{ft}\end{array}$} & Small: $<100,000 \mathrm{ft}^{2}$ & 1 & 4 & 16 & 4 \\
\hline & Medium: $>100,000 \mathrm{ft}^{2}<400,000 \mathrm{ft}^{2}$ & 4 & 16 & 64 & 16 \\
\hline & Large: $>400.000 \mathrm{ft}^{2}$ & 8 & 32 & 128 & 32 \\
\hline \multirow{3}{*}{$\begin{array}{l}\text { Deep Zone - } \\
>15 \mathrm{ft}\end{array}$} & Small: $<100.000 \mathrm{ft}^{2}$ & 1 & 4 & 16 & 4 \\
\hline & Medium: $>100,000 \mathrm{ft}^{2}<400,000 \mathrm{ft}^{2}$ & 4 & 16 & 64 & 16 \\
\hline & Large: $>400,000 \mathrm{ft}^{2}$ & 8 & 32 & 128 & 32 \\
\hline \multirow{3}{*}{$\begin{array}{l}\text { Overburen/layback } \\
\text { stockpiles }\end{array}$} & Small: $<100,000 \mathrm{ft}^{2}$ & 1 & 4 & 16 & 4 \\
\hline & Medium: $>100,000 \mathrm{ft}^{2}<400,000 \mathrm{ft}^{2}$ & 4 & 16 & 64 & 16 \\
\hline & Large: $>400.000 \mathrm{ft}^{2}$ & 8 & 32 & 128 & 32 \\
\hline \multirow{3}{*}{$\begin{array}{l}\text { Staging pile areas } \\
\text { (residual soil) }\end{array}$} & Small: $<100.000 \mathrm{ft}^{2}$ & 1 & 4 & 16 & 4 \\
\hline & Medium: $>100,000 \mathrm{ft}^{2}<400,000 \mathrm{ft}^{2}$ & 4 & 16 & 64 & 16 \\
\hline & Large: $>400,000 \mathrm{ft}^{2}$ & 8 & 32 & 128 & 32 \\
\hline
\end{tabular}

"The shallow zone, deep zone, overburden stockpile, and staging pile areas cach represent single decision units. The total number of decision units will vary because individual waste sites may not have a decp zone, overburden stockpile, and/or staging pile arcas.

Arca of exposed surface after excavation or area of stockpile base (as applicable)

'Decision subunits are divided into four blocks to cnsurc that random sampling locations are not bunched together in one arca 
CVP-2007-00006

C-8 


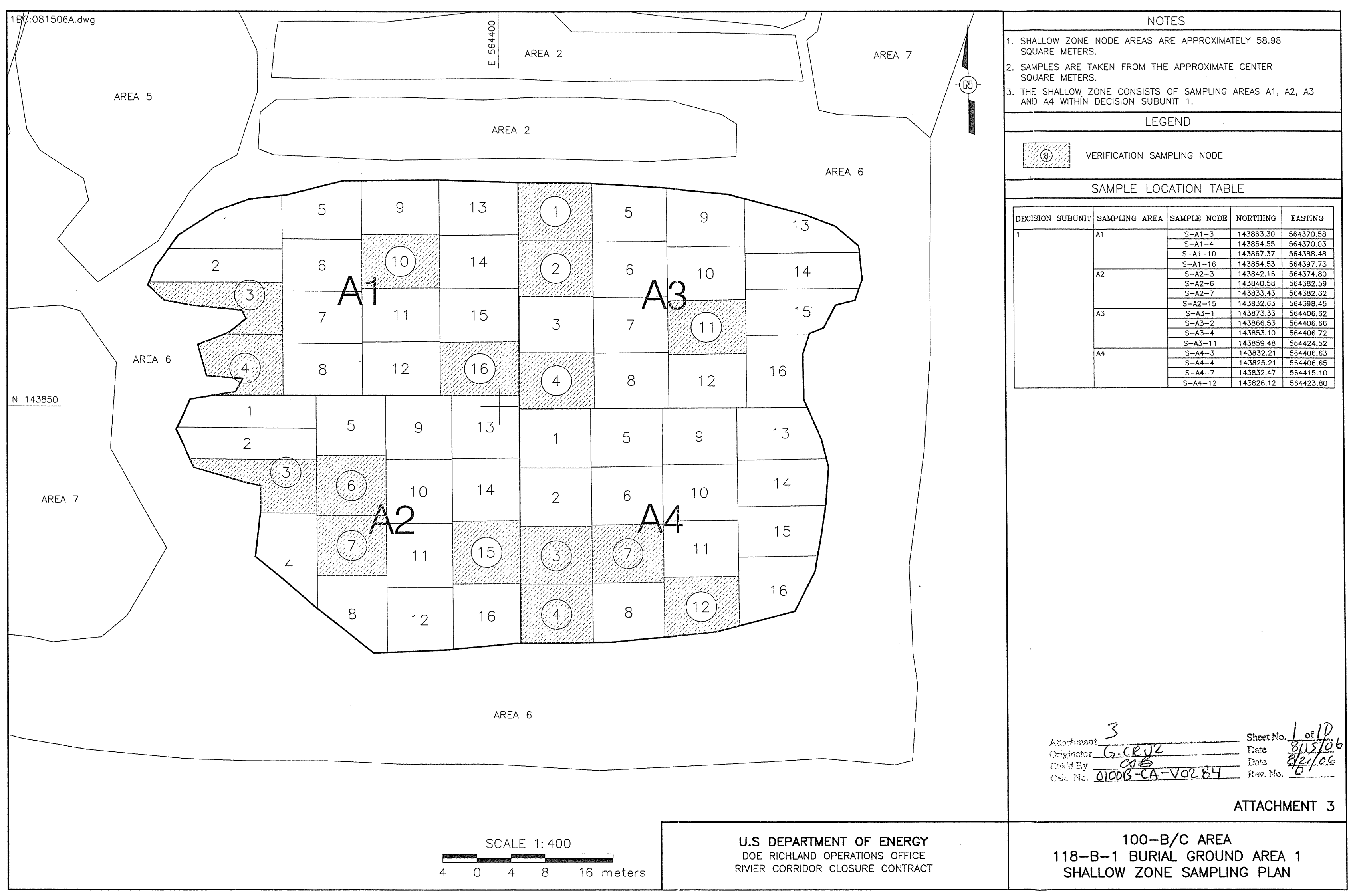




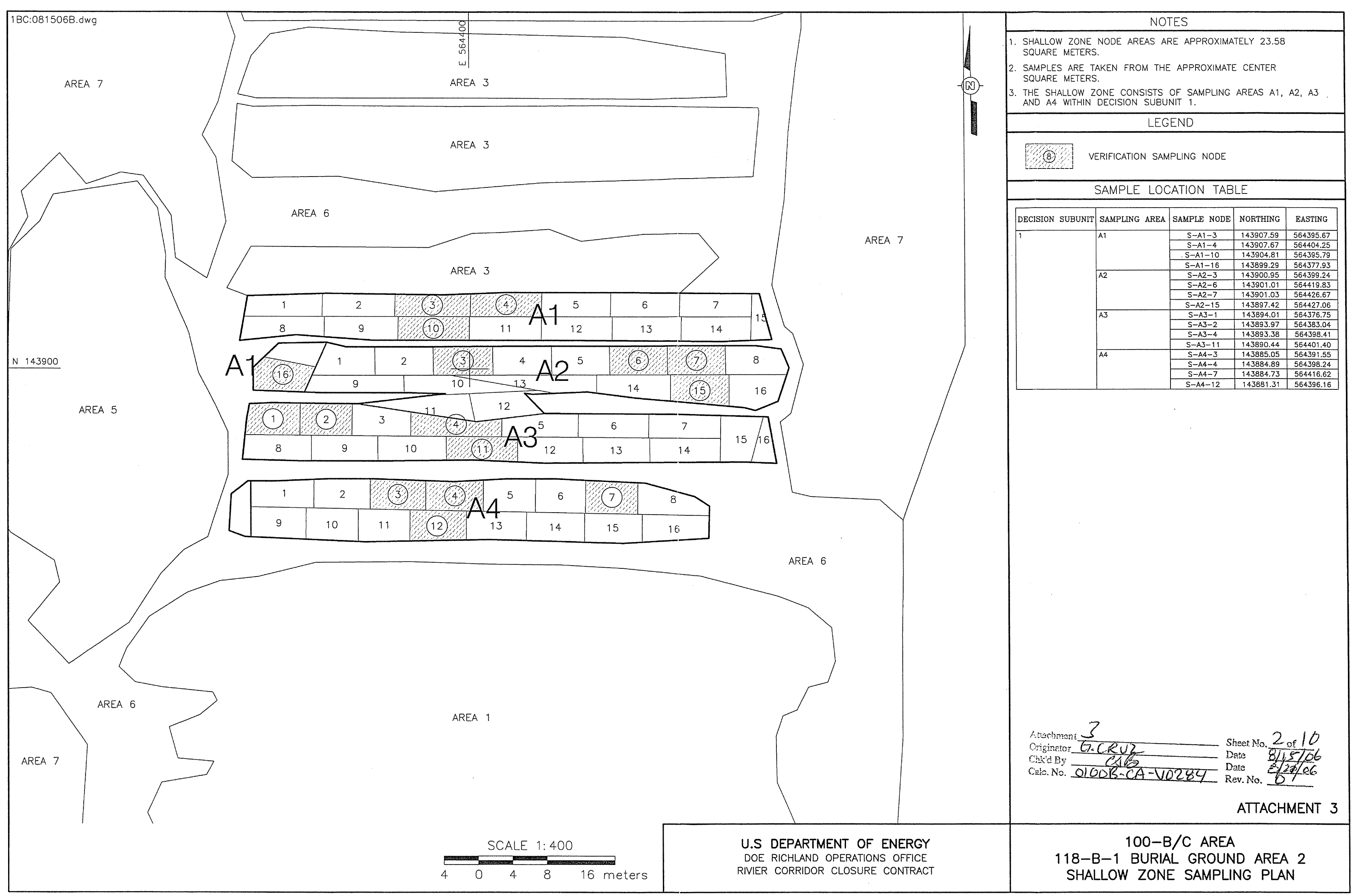




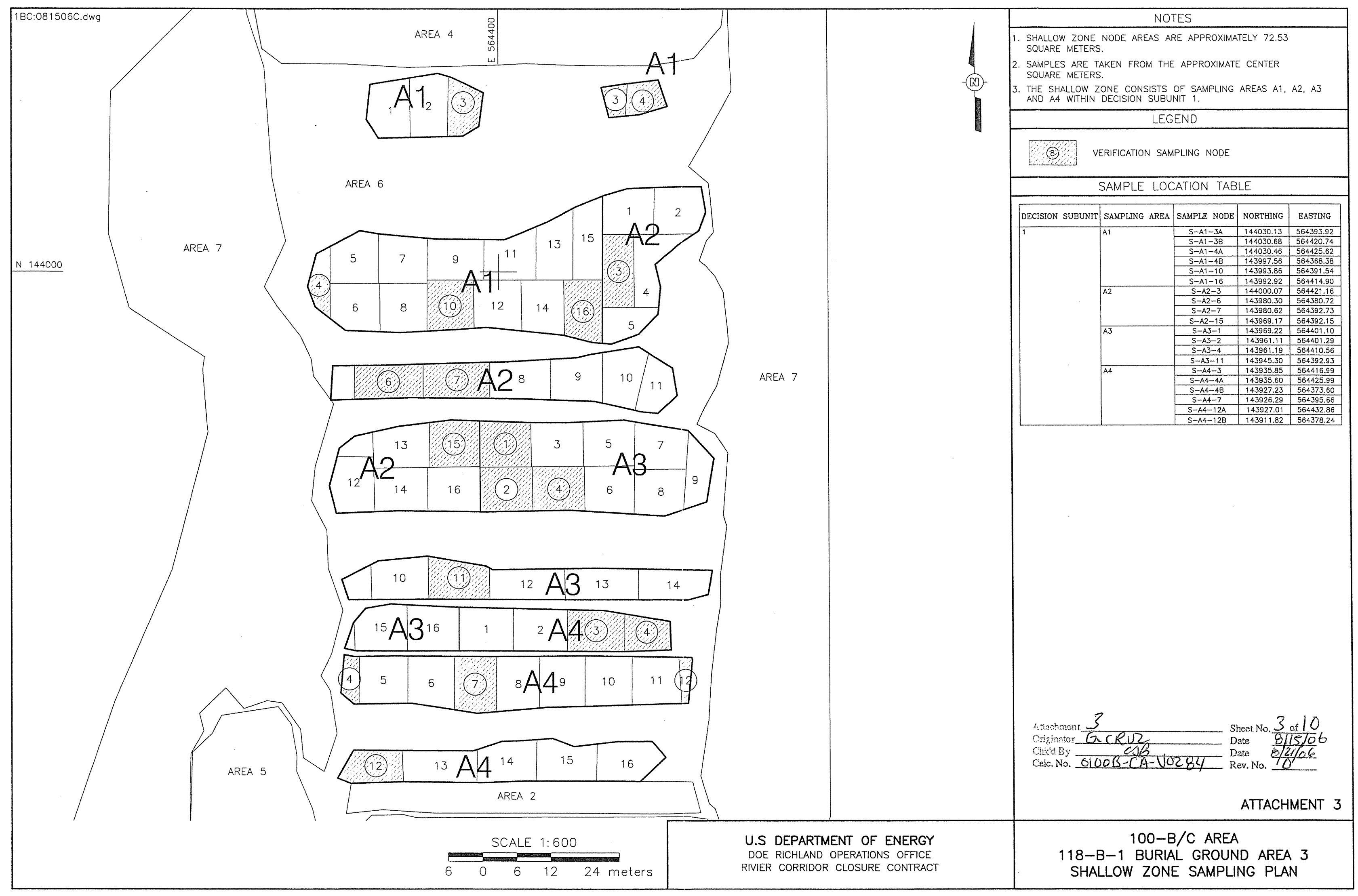




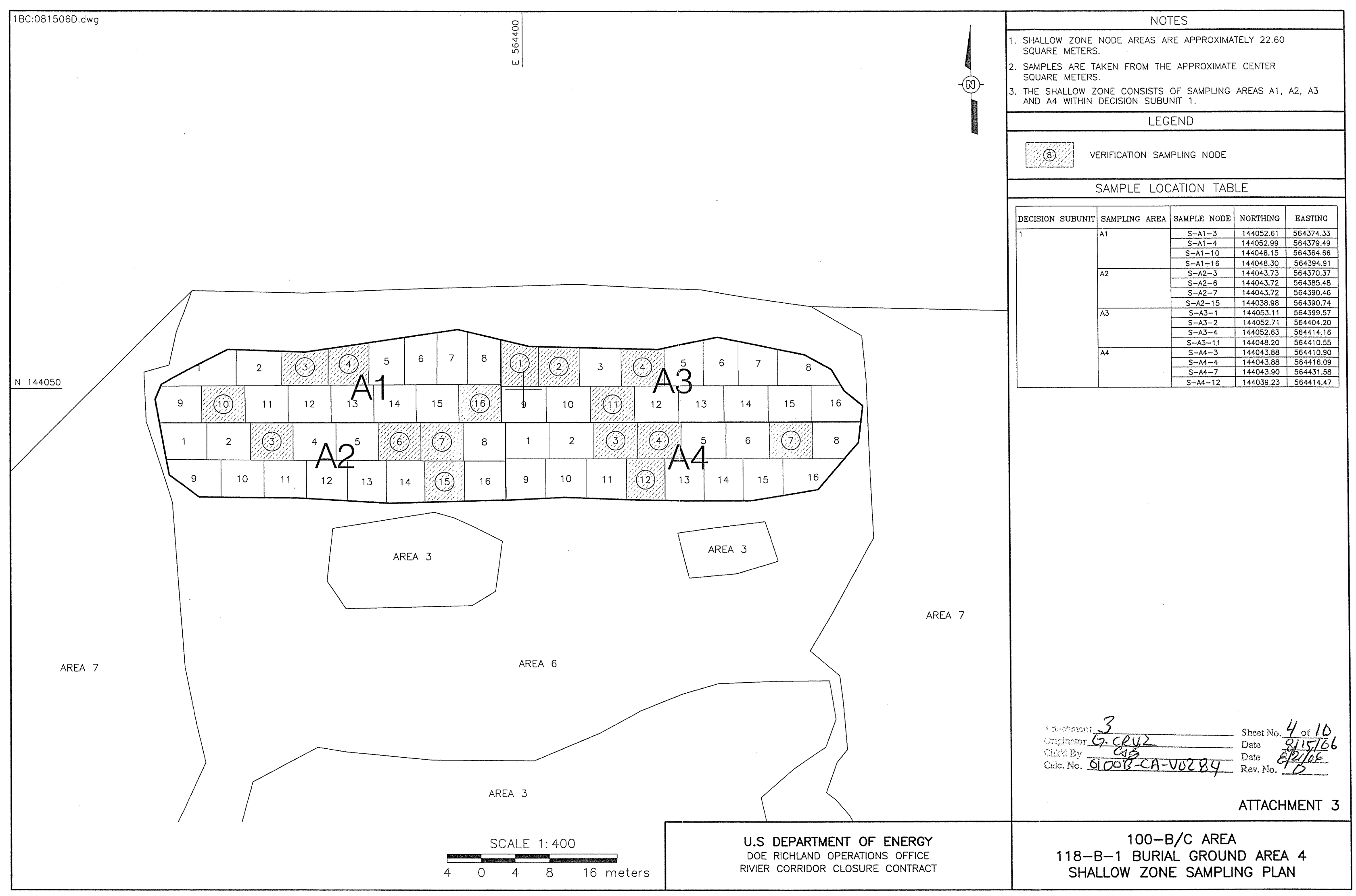




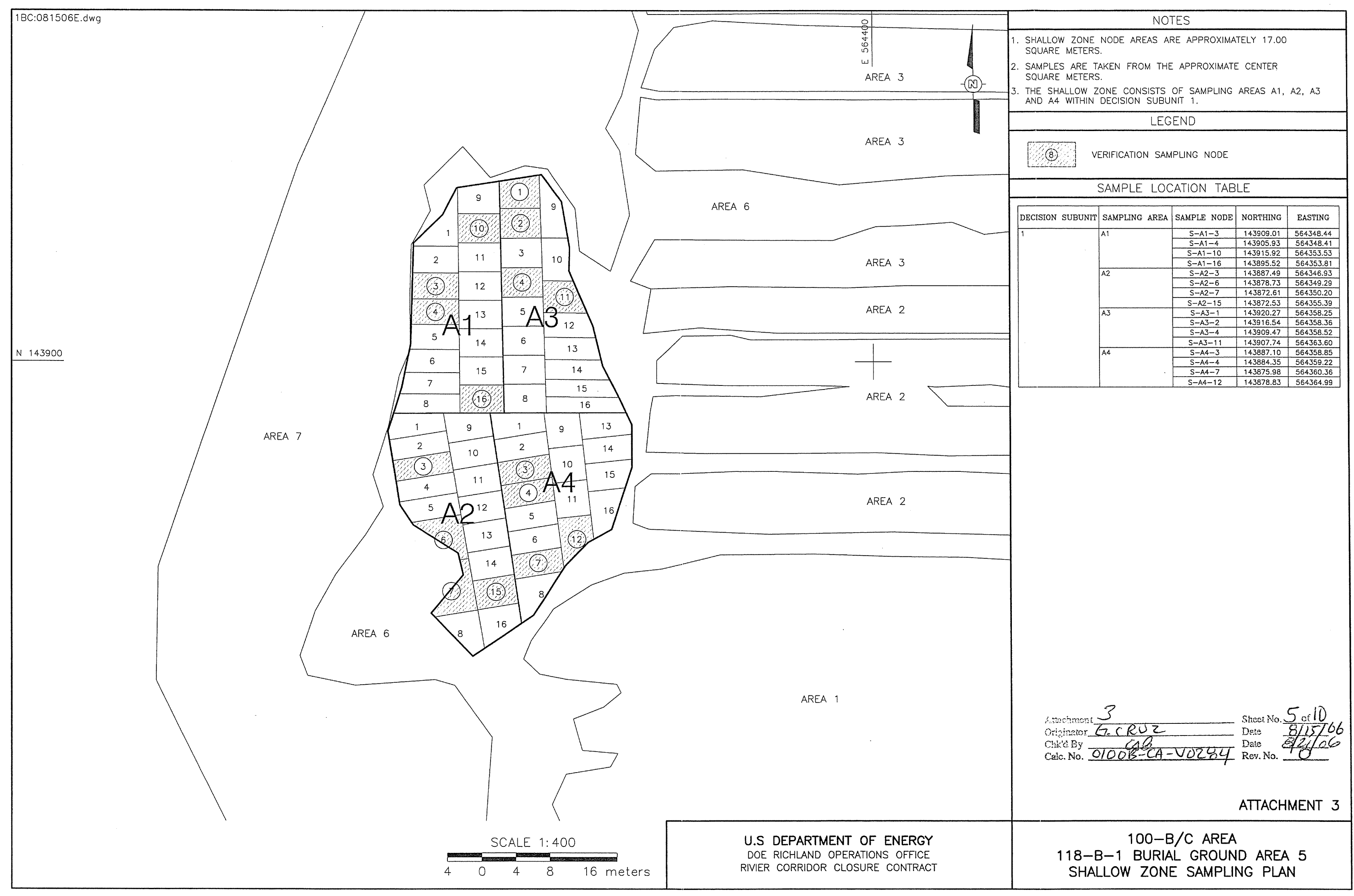




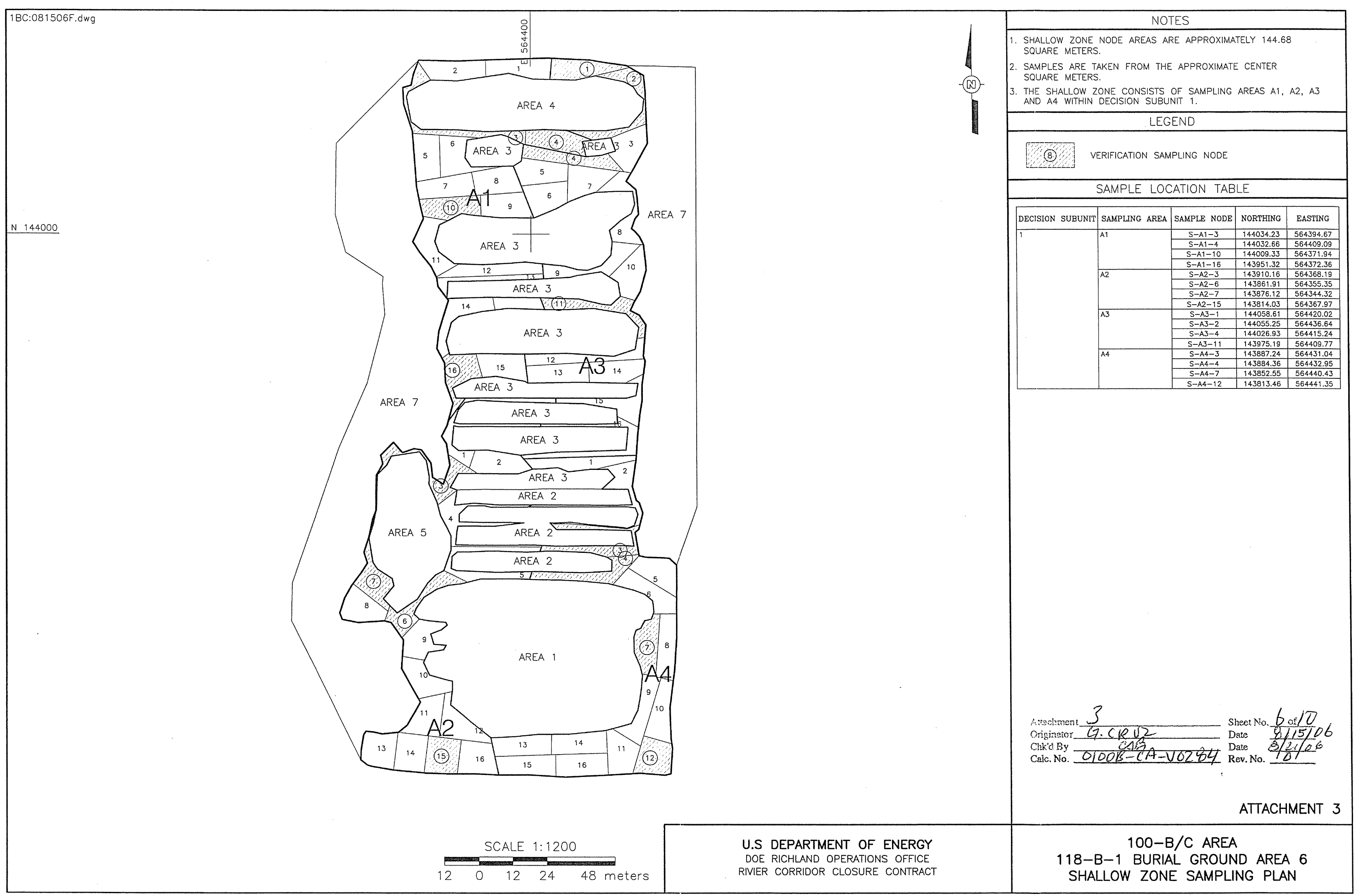




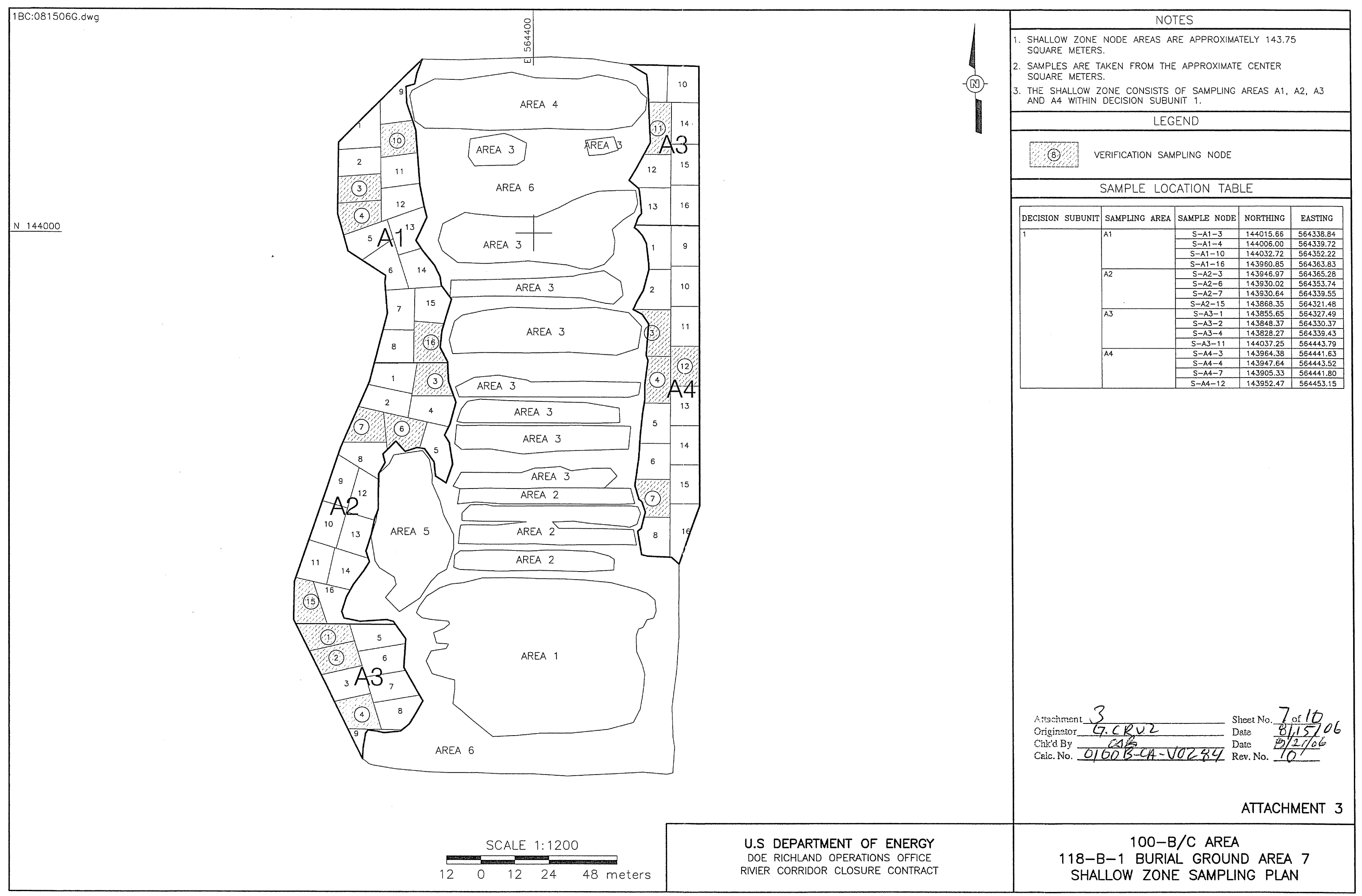




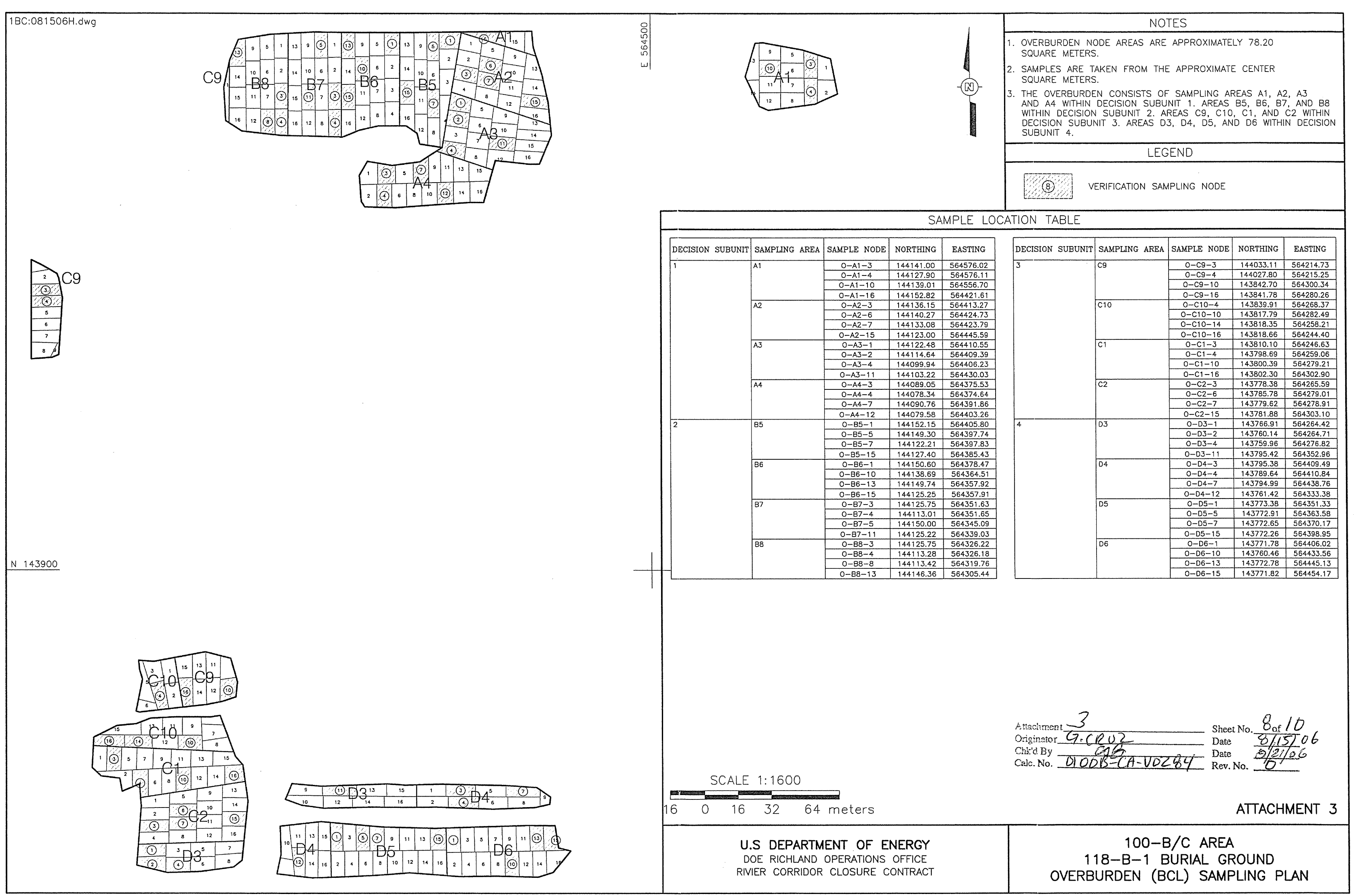




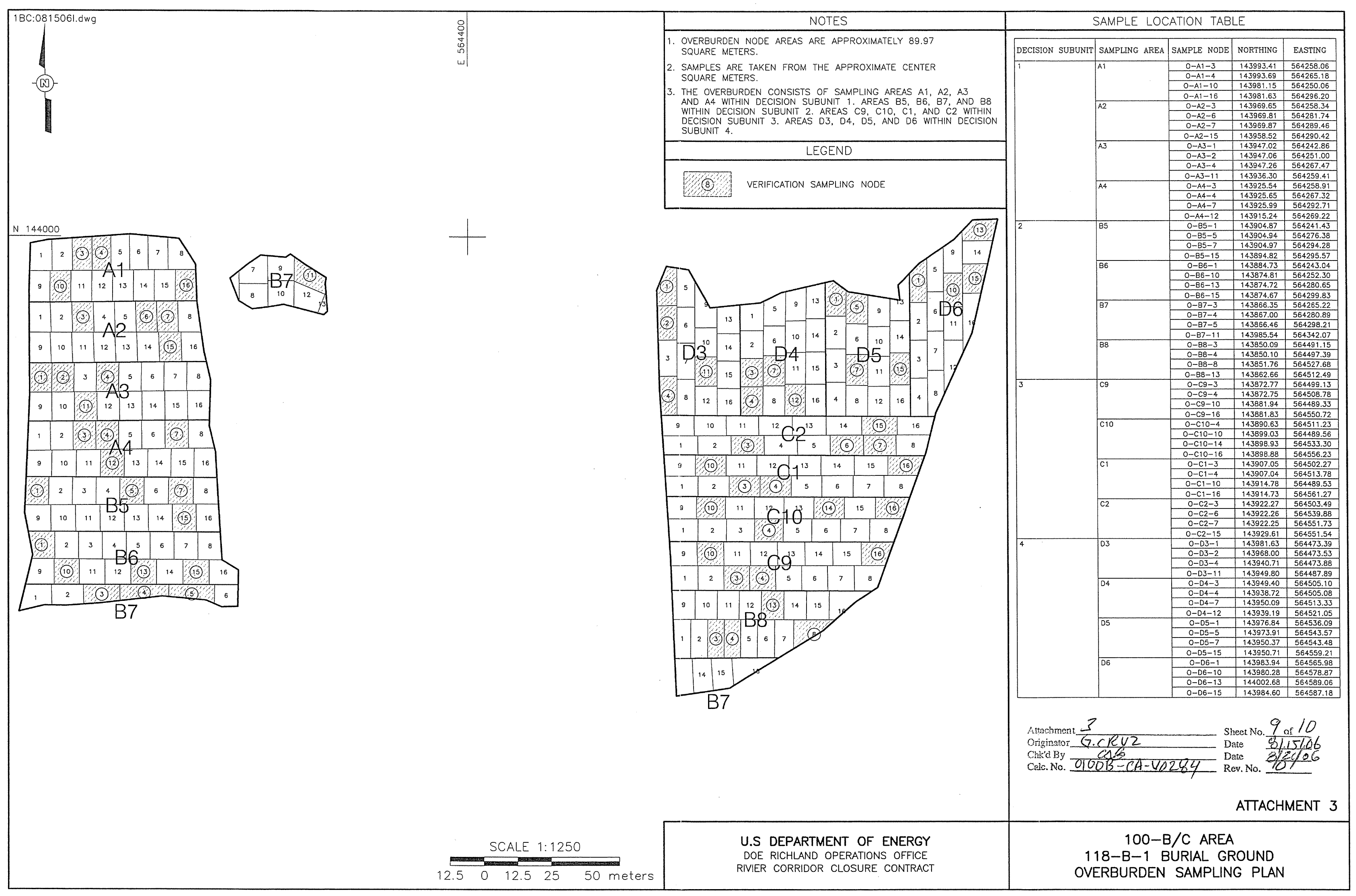




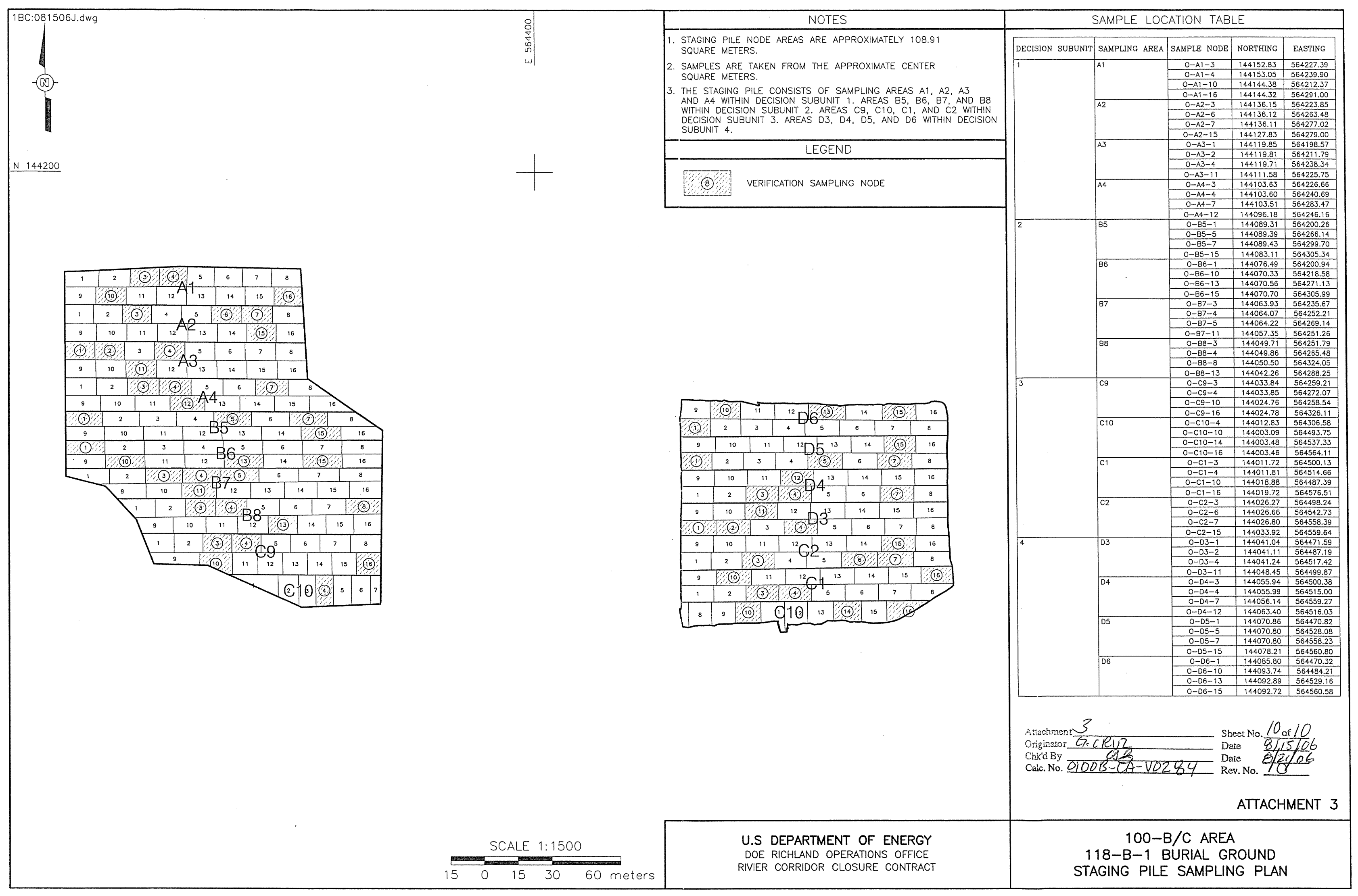




\section{CALCULATION COVER SHEET}

Project Title: 100-B/C Field Remediation

Job No. 14655

Area: $100-B / C$

Discipline: Environmental

${ }^{*}$ Calculation No: 0100B-CA-V0299

Subject: 118-B-1 Burial Ground Cleanup Verification 95\% UCL Calculations

Computer Program: Excel

Program No: Excel 2003

The attached calculations have been generated to document compliance with established cleanup levels. These calculations should be used in conjunction with other relevant documents in the administrative record.

Committed Calculation $\bar{x} \quad$ Preliminary $\Gamma \quad$ Superseded $\Gamma \quad$ Voided $\Gamma$

\begin{tabular}{|c|c|c|c|c|c|c|}
\hline Rev. & Sheet Numbers & Originator & Checker & Reviewer & Approval & Date \\
\hline 0 & $\begin{array}{c}\text { Cover }=1 \\
\text { Sheets }=61 \\
\text { Attm. } 1=113 \\
\text { Total }=175\end{array}$ & J.M. Capron & T. M. Blakley & N/A & D. N. Strom & $2 / 20 / 07$ \\
\hline 1 & $\begin{array}{c}\text { Cover }=1 \\
\text { Sheets }=61 \\
\text { Attm. } 1=113 \\
\text { Total }=175\end{array}$ & $\begin{array}{l}\text { J.M. Capron } \\
Q 215\end{array}$ & $\begin{array}{l}\text { T. M. Blakley } \\
2 . m \text {. } h \text { hang }\end{array}$ & $\mathrm{N} / \mathrm{A}$ & & 10.4 \\
\hline & & & & & & \\
\hline & & & & & & \\
\hline & & & & & & \\
\hline & & & & & & \\
\hline
\end{tabular}

SUMMARY OF REVISION

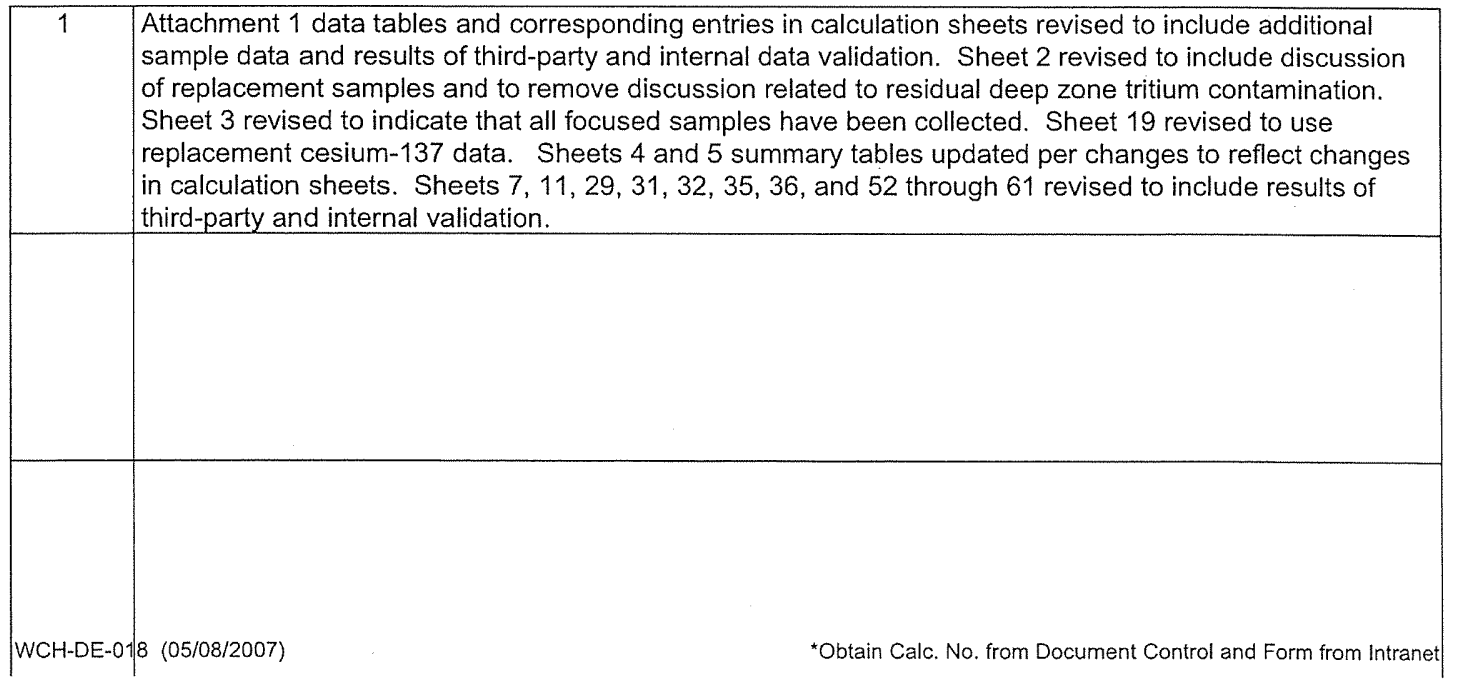




\begin{tabular}{|c|c|}
\hline Originator J.M. Capron & Date $02 / 07 / 07$ \\
\hline
\end{tabular}
Subject 118-B-1 Burial Ground Cleanup Verification 95\% UCL Calculations
Calc. No. 0100B-CA-V0299 Checked T.M. Blakley.he
Rev. No. $\frac{0}{2 / 15 \%}$

Summary

1 Purpose:

2 Calculate the 95\% upper confidence limit (UCL) values to evaluate compliance with cleanup standards for the subject site. Also,

3 perform the Washington Administrative Code (WAC) 173-340-740(7)(e) 3-part test for each nonradioactive contaminant of concern

(COC) and contaminant of potential concern (COPC) and calculate the relative percent difference (RPD) for primary-duplicate and 6 primary-split sample pairs, as necessary.

Table of Contents:

Sheets 1 to 6 - Summary

Sheets 7 to 9 - 118-B-1 Area 1 Statistical Calculations

Sheets 10 to 12 - 118-B-1 Area 2 Statistical Calculations

Sheets 13 to $15-118-\mathrm{B}-1$ Area 3 Statistical Calculations

Sheets 16 to $18-118-B-1$ Area 4 Statistical Calculations

Sheets 19 to 20 - 118-B-1 Area 5 Statistical Calculations

Sheets 21 to $23-118-B-1$ Area 6 Statistical Calculations

Sheets 24 to $26-118-B-1$ Area 7 Statistical Calculations

Sheets 27 to $29-118-B-1$ Overburden Statistical Calculations

Sheets 30 to $32-118-B-1$ BCL Material Statistical Calculations

Sheets 33 to 36 - 118-B-1 Staging Pile Area Statistical Calculations

Sheets 37 to 41 - Ecology Software (MTCAStat) Results (Overburden)

Sheets 42 to 46 - Ecology Software (MTCAStat) Results (BCL Material)

Sheets 47 to 51 - Ecology Software (MTCAStat) Results (Staging Pile Area)

Sheets 52 to 61 - Split/Duplicate Analysis

Attachment 1 - 118-B-1 Verification Sampling Results (43 sheets)

Given/References:

1) Sample Results (Attachment 1).

2) Background values and remedial action goals (RAGs) are taken from DOE-RL (1996, 2001b, 2005b) and Ecology (2005).

3) DOE-RL, 1996, Hanford Site Background: Part 2, Soil Background for Radionuclides, DOE/RL 96 12, Rev. 0, U.S. Department of Energy, Richland Operations Office, Richland, Washington.

4) DOE-RL, 2001a, 100 Area Burial Grounds Remedial Action Sampling and Analysis Plan (SAP), DOE/RL-2001-35, Rev. 0,

U.S. Department of Energy, Richland Operations Office, Richland, Washington.

5) DOE-RL, 2001b, Hanford Site Background: Part 1, Soil Background for Nonradioactive Analytes, DOE/RL-92-24, Rev. 4, U.S. Department of Energy, Richland Operations Office, Richland, Washington.

6) DOE-RL, 2005, Remedial Design Report/Remedial Action Work Plan for the 100 Area (RDR/RAWP), DOE/RL-96-17, Rev. 5, U.S. Department of Energy, Richland Operations Office, Richland, Washington.

7) Ecology, 1992, Statistical Guidance for Ecology Site Managers, Publication \#92-54, Washington Department of Ecology, Olympia, Washington.

8) Ecology, 1993, Statistical Guidance for Ecology Site Managers, Supplement S-6, Analyzing Site or Background Data with Below-detection Limit or Below-PQL Values (Censored Data Sets), Publication \#92-54, Washington Department of Ecology, Olympia, Washington.

9) Ecology, 2005, Cleanup Levels and Risk Calculations (CLARC) Database, Washington State Department of Ecology, Olympia, Washington, <https://fortress.wa.gov/ecy/clarc/CLARCHome.aspx>.

10) EPA, 1994, USEPA Contract Laboratory Program National Functional Guidelines for Inorganic Data Review, EPA 540/R-94/013. U.S. Environmental Protection Agency, Washington, D.C.

11) WAC 173-340, 1996, "Model Toxic Control Act - Cleanup," Washington Administrative Code.

Solution:

Calculation methodology is described in Ecology Pub. \#92-54 (Ecology 1992, 1993), below, and in the RDR/RAWP (DOE-RL

$2005 \mathrm{~b})$. Use data from attached worksheets to perform the $95 \%$ UCL calculation for each analyte, the

55 WAC 173-340-740(7)(e) 3-part test for nonradionuclides, and the RPD calculations, as required. The hazard quotient and

56 carcinogenic risk calculations are located in a separate calculation brief as an appendix to the Cleanup Verification Package (CVP).

58 Calculation Description:

60 The subject calculations were performed on data from soil verification samples from the subject waste site. The data were entered

61 into an EXCEL 2003 spreadsheet and calculations performed by using the built-in spreadsheet functions and/or creating formulae

62 within the cells. The statistical evaluation of data for use in accordance with the RDR/RAWP (DOE-RL 2005b) is documented by

63 this calculation. Duplicate and split RPD results are used in evaluation of data quality within the CVP for this site. 


\begin{tabular}{|c|c|c|c|}
\hline Originator J.M. Capron $A, 2$ & Date 10/03/07 & Calc. No. 0100B-CA-V0299 & Rev. No. \\
\hline $\begin{array}{l}\text { Project } \frac{100-\mathrm{B} / \mathrm{C} \text { Field Remediation }}{118-\mathrm{B}-1 \text { Burial Ground Cleanup Verification } 95 \% \text { UCL Calo }} \\
\text { Subject }\end{array}$ & Job No. 14655 & Checked T.M. Blakley dross & Date $\frac{10 / 04 / 07}{2 \text { of } 61}$ \\
\hline
\end{tabular}

Summary (continued)

\section{Methodology:}

2 For nonradioactive analytes with $\leq 50 \%$ of the data below detection limits and all detected radionuclide analytes, the statistical value 3 calculated to evaluate the effectiveness of cleanup is the $95 \%$ UCL. For nonradioactive analytes with $>50 \%$ of the data below 5 detection limits, the maximum detected value for the data set is used instead of the $95 \%$ UCL. The $95 \%$ UCL is not calculated for 6 data sets with no reported detections. The evaluation of the portion of each analyte's data set below detection limits was 7 performed by direct inspection of the attached sample results, and no further calculations were performed for those data sets 8 where $>50 \%$ of the data was below detection limits. The $95 \%$ UCL values were not calculated for radium-226, radium-228, thorium9228 , thorium-232, and potassium-40, as these isotopes are excluded from consideration as Cocs based on natural occurrence 10 and analogous site information. The $95 \%$ UCL values were also not calculated for aluminum, calcium, iron, magnesium, 11 potassium, silicon, and sodium, as no parameters to calculate cleanup values under WAC 173-340-740(3) are available in Ecology 12 (2005) or other reference databases, and these constituents are thus not considered site COCs/COPCs.

14 All calculations for uranium isotopes and americium-241 were performed using data from isotope-specific analysis rather than data 15 resulting from gamma spectroscopy. Due to data quality deficiencies, a portion of the data with reported detections for the 17 herbicide 2,4-DB in the staging pile area (SPA) were rejected; the result reported in the results summary is the maximum accepted 18 result from the SPA; additional discussion is provided in the CVP.

Initial data results from the SPA indicated the presence of tritium in sampling area A1 at a concentration that would result in a 21 predicted exceedance of groundwater protection criteria. Therefore, additional material removal was performed and a new sample 22 collected and submitted for tritium analysis. Further evaluation and calculations for tritium in the SPA were performed using the 23 replacement sample data. Initial cesium-137 results for Sampling Area A3 of Area 5 (Sample J13V13) and mercury results for 24 Focused Sample 17 (Sample J14JJ3) exceeded cleanup criteria; additional material was removed from these locations and 25 replacement samples (Samples J14JH6 and J152M1, respectively) collected for cesium-137 and mercury, respectively. All nonradionuclide data reported as being undetected are set to $1 / 2$ the detection limit value for calculation of the statistics (Ecology 1993). In cases where third-party validation requalified detected results as nondetected due to method blank contamination, but did not change the laboratory quantitation limit (i.e., bis(2-ethylhexyl)phthalate and methylene chloride in sample J13J06), 1/2 the reported value was used for calculation of statistics. For radionuclide data, calculation of the statistics is done using the reported 32 value. In cases where the laboratory does not report a value below the minimum detectable activity (MDA), half of the MDA is used 33 in the calculation. For the statistical evaluation of duplicate sample pairs, the samples are averaged before being included in the 34 data set, after adjustments for censored data as described above. Split samples are not considered within statistical evaluation.

or nonradionuclides, the WAC 173-340 statistical guidance suggests that a test for distributional form be performed on the data all radionuclide data sets, the calculations are performed assuming nonparametric distribution, so no tests for distribution are 40 performed. For nonradionuclide data sets of ten or greater, distributional testing and calculation of the $95 \%$ UCL is done using 41 Ecology's MTCAStat software (Ecology 1993). Due to differences in addressing censored data between the RDR/RAWP (DOE-RL 42 2005b) and MTCAStat coding and due to a limitation in the MTCAStat coding (no direct capability to address variable quantitation 43 limits within a data set), substitutions for censored data are performed before software input and the resulting input set treated as 44 uncensored.

WAC 173-340-740(7)(e) 3-part test is performed for nonradionuclide analytes only and determines if: the $95 \%$ UCL exceeds the most stringent cleanup limit for each $\mathrm{COPC/COC}$,

greater than $10 \%$ of the raw data exceed the most stringent cleanup limit for each COPC/COC,

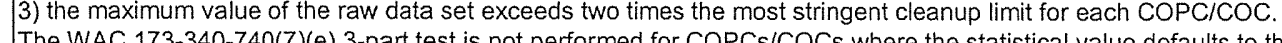

51 The WAC 173-340-740(7)(e) 3-part test is not performed for COPCs/COCs where the statistical value defaults to the maximum 52 value in the data set. Instead, direct comparison of the maximum value against site RAGs (within the CVP) is used as the 53 compliance basis.

54

55 The RPD values are evaluated for analytes detected in a primary-duplicate or primary-split sample pair for the purposes of data 56 quality assessment within the RSVP (where direct evaluation of the attached data showed that a given analyte was undetected in 57 both the primary and duplicate sample or accepted data was not available for both samples in the pair, no further calculations were 58 performed). The RPD is calculated when both the primary value and the duplicate value for a given analyte are above detection 59 limits and are greater than 5 times the target detection limit (TDL). The TDL is a laboratory detection limit pre-determined for each analytical method, listed in Table II-1 of the SAP (DOE-RL 2005a). The RPD calculations use the following formula:

$$
R P D=[|M-S| /((M+S) / 2)]^{* 100}
$$

where, $\quad M=$ main sample value $\quad S=$ split (or duplicate) sample value 
CALCULATION SHEET

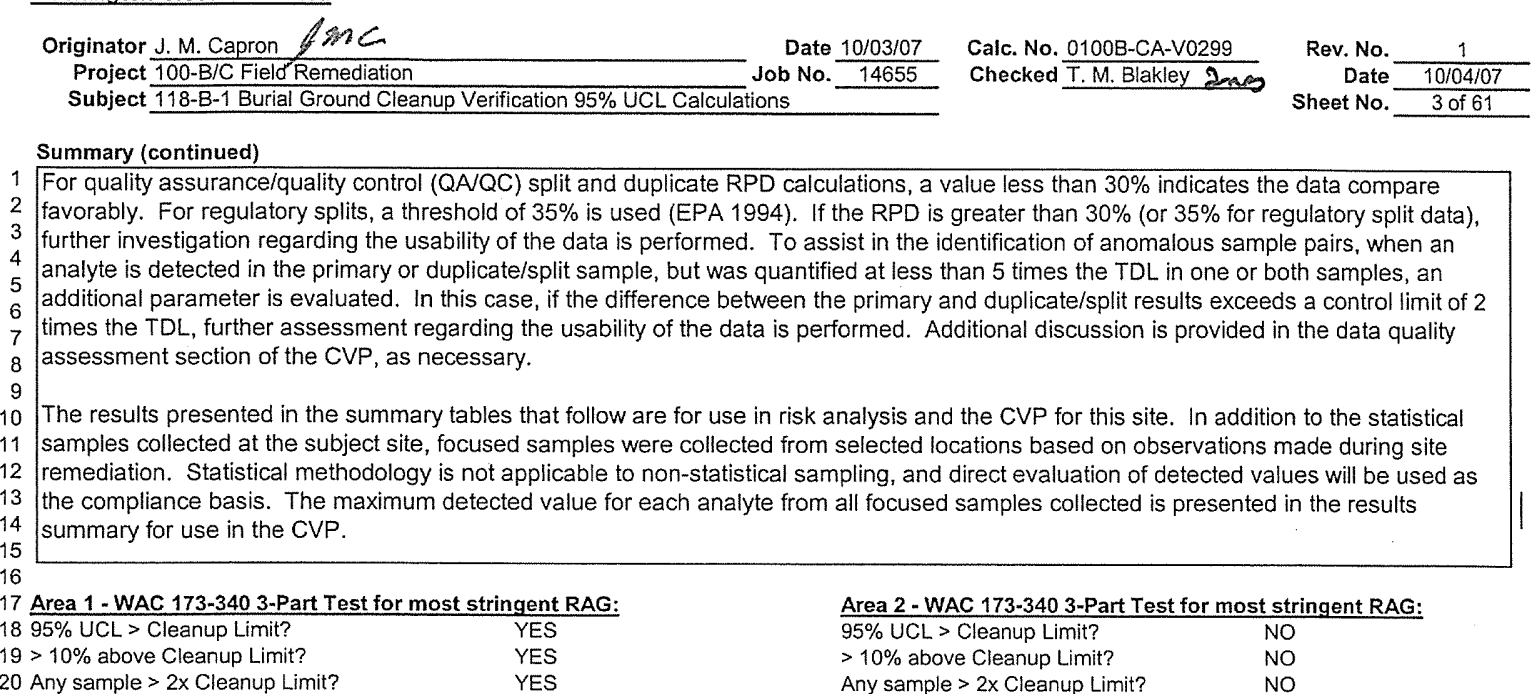

$\begin{array}{ll}95 \% \text { UCL }>\text { Cleanup Limit? } & \text { NO } \\ >10 \% \text { above Cleanup Limit? } & \text { NO } \\ \text { Any sample }>2 x \text { Cleanup Limit? } & \text { NO }\end{array}$

All data sets evaluated meet the 3-part test criteria when compared to the most stringent cleanup limit

\begin{tabular}{ll} 
Area 4 - WAC 173-340 3-Part Test for most stringent RAG: \\
\hline $95 \%$ UCL $>$ Cleanup Limit? & YES \\
$>10 \%$ above Cleanup Limit? & YES \\
Any sample $>2 \times$ Cleanup Limit? & NO
\end{tabular}

Because of the "yes" answers to the 3-part test for bis(2-ethylhexyl)phthalate, additional evaluation of the attainment of cleanup criteria will be performed.

\begin{tabular}{ll} 
Area 6 - WAC $173-340$ 3-Part Test for most stringent RAG: \\
\hline $95 \%$ UCL > Cleanup Limit? & NO \\
$>10 \%$ above Cleanup Limit? & YES \\
Any sample $>2 x$ Cleanup Limit? & NO
\end{tabular}

Because of the "yes" answer to the 3-part test for bis(2-ethythexyl)phthalate, additional evaluation of the attainment of cleanup criteria will be performed.

Overburden - WAC 173-340 3-Part Test for most stringent RAG: $95 \%$ UCL > Cleanup Limit?

$>10 \%$ above Cleanup Limit?

Any sample $>2 \times$ Cleanup Limit?

All data sets evaluated meet the 3-part test criteria when compared to the most stringent cleanup limit

\begin{tabular}{|c|c|}
\hline $95 \%$ UCL > Cleanup Limit? & NO \\
\hline$>10 \%$ above Cleanup Limit? & NO \\
\hline Any sample $>2 \times$ Cleanup Limit? & NO \\
\hline
\end{tabular}

All data sets evaluated meet the 3-part test criteria when compared to the most stringent cleanup limit 
Washington Closure Hanford

\section{CALCULATION SHEET}

Originator J. M. Capron $102 C_{\text {Date } 10 / 03 / 07}$ Checked T. M. Blakley 210 Date 10/04/07 Project 100-B/C. Field Remediation Calc. No. 0100B-CA-V0299 Subject 118-B-1 Burial Ground Cleanup Verification 95\% UCL Calculations $\quad$ Job No. $\frac{14655}{14}$

Rev. No. 1 Sheet No. $\overline{4 \text { of } 61}$

1 Summary (continued)

\begin{tabular}{|c|c|c|c|c|c|c|c|c|c|}
\hline \multirow[t]{2}{*}{2} & \multicolumn{9}{|c|}{ Results Summary - Areas 1 through $7^{2}$} \\
\hline & Analyte & Area $1^{b}$ & Area $2^{b}$ & Area $3^{b}$ & Area $4^{b}$ & Area $5^{\mathrm{b}}$ & Area $6^{b}$ & Area $7^{b}$ & Units \\
\hline 4 & Carbon-14 & 1.93 & -- & - & -- & - & - & -- & $\mathrm{pCi} / \mathrm{g}$ \\
\hline 5 & Cesium-137 & 0.201 & 0.163 & 0.776 & 0.345 & 2.40 & 0.150 & 0.517 & $\mathrm{pCi} / \mathrm{g}$ \\
\hline 6 & Cobalt-60 & 0.039 & -- & - & - & -- & - & - & $\mathrm{pCi} / \mathrm{g}$ \\
\hline 7 & Europium-152 & 0.695 & -- & - & -- & - & - & 0.098 & $\mathrm{pCi} / \mathrm{g}$ \\
\hline 8 & Strontium-90 & 0.125 & 0.172 & 2.56 & - & 1.89 & - & $\cdots$ & $\mathrm{pCi} / \mathrm{g}$ \\
\hline 9 & Tritium & 158 & 39.4 & 12.8 & - & - & 3.19 & - & $\mathrm{pCi} / \mathrm{g}$ \\
\hline 10 & Uranium-233/234 & $0(<B G)$ & $\ldots$ & -- & $\cdots$ & - & $0(<B G)$ & $0(<B G)$ & $\mathrm{pCi} / \mathrm{g}$ \\
\hline 11 & Uranium-235 & $0(<B G)$ & $\cdots$ & - & - & - & - & -- & $\mathrm{pCi} / \mathrm{g}$ \\
\hline 12 & Uranium-238 & $0(<B G)$ & -- & -- & - & - & $0(<B G)$ & $0(<B G)$ & $\mathrm{pCi} / \mathrm{g}$ \\
\hline 13 & Arsenic & 3.2 & 2.9 & 3.7 & 3.5 & 2.8 & 4.1 & 3.5 & $\mathrm{mg} / \mathrm{kg}$ \\
\hline 14 & Barium & 60.4 & 58.1 & 62.6 & 48.6 & 46.7 & 80.1 & 132 & $\mathrm{mg} / \mathrm{kg}$ \\
\hline 15 & Beryllium & 0.60 & 0.64 & 0.63 & 0.40 & 0.1 & 0.36 & 0.31 & $\mathrm{mg} / \mathrm{kg}$ \\
\hline 16 & Boron & 1.4 & 4.7 & 6.5 & 3.9 & 1.1 & 6.1 & 13.7 & $\mathrm{mg} / \mathrm{kg}$ \\
\hline 17 & Cadmium & -- & -- & -- & - & 0.20 & - & -- & $\mathrm{mg} / \mathrm{kg}$ \\
\hline 18 & Chromium (total) & 7.1 & 6.7 & 7.7 & 6.6 & 5.0 & 7.4 & 8.9 & $\mathrm{mg} / \mathrm{kg}$ \\
\hline 19 & Cobalt & 10.2 & 8.5 & 9.1 & 8.6 & 7.2 & 8.5 & 7.5 & $\mathrm{mg} / \mathrm{kg}$ \\
\hline 20 & Copper & 17.7 & 16.6 & 17.1 & 16.2 & 14.8 & 16.6 & 22.2 & $\mathrm{mg} / \mathrm{kg}$ \\
\hline 21 & Hexavalent chromium & - & 0.29 & 0.21 & 0.22 & 0.26 & 0.27 & 0.28 & $\mathrm{mg} / \mathrm{kg}$ \\
\hline 22 & Lead & 4.8 & 4.8 & 5.7 & 4.9 & 3.8 & 5.0 & 11.0 & $\mathrm{mg} / \mathrm{kg}$ \\
\hline 23 & Manganese & 381 & 321 & 367 & 334 & 290 & 323 & 329 & $\mathrm{mg} / \mathrm{kg}$ \\
\hline 24 & Mercury & 1.3 & - & 0.21 & - & 0.03 & 0.02 & 0.02 & $\mathrm{mg} / \mathrm{kg}$ \\
\hline 25 & Molybdenum & $-\cdots$ & - & -- & - & - & -- & 2.1 & $\mathrm{mg} / \mathrm{kg}$ \\
\hline 26 & Nickel & 11.2 & 10.5 & 15.9 & 10.6 & 7.4 & 11.4 & 10.6 & $\mathrm{mg} / \mathrm{kg}$ \\
\hline 27 & Vanadium & 52,0 & 54.5 & 47.5 & 49.9 & 37.4 & 49.0 & 39.8 & $\mathrm{mg} / \mathrm{kg}$ \\
\hline 28 & Zinc & 45.8 & 43.2 & 43.7 & 42.1 & 36.8 & 41.4 & 47.5 & $\mathrm{mg} / \mathrm{kg}$ \\
\hline 29 & Aroclor-1254 & -- & - & - & 0.080 & - & - & - & $\mathrm{mg} / \mathrm{kg}$ \\
\hline 30 & 2-Methylnaphthalene & - & - & -- & - & - & -- & 0.026 & $\mathrm{mg} / \mathrm{kg}$ \\
\hline 31 & bis(2-Ethylhexyl)phthalate & 0.19 & - & 0.14 & 0.41 & 0.17 & 0.32 & - & $\mathrm{mg} / \mathrm{kg}$ \\
\hline 32 & Butylbenzylphthalate & - & - & - & - & $\ldots$ & 0.024 & - & $\mathrm{mg} / \mathrm{kg}$ \\
\hline 33 & Di-n-butylphthalate & 0.12 & - & 0.16 & 0.063 & - & 0.079 & - & $\mathrm{mg} / \mathrm{kg}$ \\
\hline 34 & Diethylphthalate & -- & - & -- & 0.017 & - & $\ldots$ & 0.025 & $\mathrm{mg} / \mathrm{kg}$ \\
\hline 35 & Naphthalene & - & $\cdot-$ & -- & - & - & -- & 0.019 & $\mathrm{mg} / \mathrm{kg}$ \\
\hline 36 & Phenol & - & - & -- & 0.17 & - & 0.023 & - & $\mathrm{mg} / \mathrm{kg}$ \\
\hline 37 & 4-Methyl-2-pentanone & 0.006 & - & 0.006 & -- & -- & - & - & $\mathrm{mg} / \mathrm{kg}$ \\
\hline 38 & Acetone & 0.014 & - & 0.015 & 0.012 & 0.017 & 0.008 & 0.009 & $\mathrm{mg} / \mathrm{kg}$ \\
\hline 39 & Carbon tetrachiordie & - & - & 0.009 & 0.016 & -- & 0.007 & 0.011 & $\mathrm{mg} / \mathrm{kg}$ \\
\hline 40 & Methylene chloride & 0.019 & - & 0.019 & 0.010 & 0.013 & 0.008 & 0.008 & $\mathrm{mg} / \mathrm{kg}$ \\
\hline 41 & Aldrin & $m$ & - & -- & -- & -- & 0.00050 & $\cdots$ & $\mathrm{mg} / \mathrm{kg}$ \\
\hline 42 & alpha-Chlordane & - & -- & -- & -- & -- & -- & 0.012 & $\mathrm{mg} / \mathrm{kg}$ \\
\hline 43 & beta-BHC & - & - & -- & 0.0078 & - & - & - & $\mathrm{mg} / \mathrm{kg}$ \\
\hline 44 & 4,4'-DDE & - & - & - & 0.016 & - & - & 0.015 & $\mathrm{mg} / \mathrm{kg}$ \\
\hline 45 & Dieldrin & - & - & - & 0.0038 & - & - & 0.002 & $\mathrm{mg} / \mathrm{kg}$ \\
\hline 46 & Endosulfan sulfate & - & - & - & 0.0022 & -- & $-\cdots$ & - & $\mathrm{mg} / \mathrm{kg}$ \\
\hline 47 & Endrin & -- & $\cdots$ & $\cdots$ & 0.0015 & - & -- & - & $\mathrm{mg} / \mathrm{kg}$ \\
\hline 48 & Endrin aldehyde & $-m$ & - & - & 0.0055 & - & - & - & $\mathrm{mg} / \mathrm{kg}$ \\
\hline 49 & Endrin ketone & -- & - & $\cdots$ & -- & - & -- & 0.0078 & $\mathrm{mg} / \mathrm{kg}$ \\
\hline 50 & gamma-Chlordane & - & - & - & 0.0025 & - & $-\cdots$ & - & $\mathrm{mg} / \mathrm{kg}$ \\
\hline 51 & Methoxychlor & $\sim$ & $\cdots$ & $\ldots$ & -- & - & - & 0.0091 & $\mathrm{mg} / \mathrm{kg}$ \\
\hline 52 & 2,4-D & -- & -- & - & 0.013 & - & 0.062 & 0.023 & $\mathrm{mg} / \mathrm{kg}$ \\
\hline 53 & $2,4-D B$ & - & - & - & 0.016 & - & 0.043 & 0.027 & $\mathrm{mg} / \mathrm{kg}$ \\
\hline 54 & 2,4,5-TP & - & - & - & -- & - & 0.012 & -- & $\mathrm{mg} / \mathrm{kg}$ \\
\hline 55 & Dalapon & - & - & -- & 0.037 & - & 0.036 & 0.027 & $\mathrm{mg} / \mathrm{kg}$ \\
\hline 56 & Dicamba & - & - & - & - & - & -- & 0.013 & $\mathrm{mg} / \mathrm{kg}$ \\
\hline 57 & Dichloroprop & -- & -- & - & 0.14 & - & 0.10 & 0.050 & $\mathrm{mg} / \mathrm{kg}$ \\
\hline 58 & Pentachlorophenol & - & $\cdots$ & - & 0.014 & - & - & - & $\mathrm{mg} / \mathrm{kg}$ \\
\hline 59 & Picloram & - & $\cdots$ & - & - & -- & - & 0.014 & $\mathrm{mg} / \mathrm{kg}$ \\
\hline
\end{tabular}

$60{ }^{\mathrm{a}} \mathrm{No}$ detections were reported in any data set for COCs/COPCs not listed in this table.

$61{ }^{\circ}$ Maximum or $95 \%$ UCL result, depending on data censorship, as described in the calculation methodology.

$62 . .=$ not evaluated by laboratory analysis or not detected (for all samples in the data set)

$63 \mathrm{COC}=$ contaminant of concern

$64 \mathrm{COPC}=$ contaminant of potential concern

$65 \mathrm{UCL}=$ upper confidence limit 


\title{
Washington Closure Hanford
}

\section{CALCULATION SHEET}

Originator J.M. Capron IFC Date 10/03/07 Checked T. M. Blakley dorb Date 10/04/07 Project 100-B/C Field Remediation Calc. No. $\frac{1000 \text { B-CA-V0299 }}{14655}$

Subject 118-B-1 Burial Ground Cleanup Verification 95\% UCL Calculations Job No. $\frac{100 B-C A-V 029}{14655}$

Rev. No. 1 Sheet No. $\overline{5 \text { of } 61}$

\begin{abstract}
1 Summary (continued)
\end{abstract}

\begin{tabular}{|c|c|c|c|c|c|c|}
\hline \multicolumn{7}{|c|}{ den, BCL Material, s } \\
\hline 3 & Analyte & Overburden & $\begin{array}{c}\text { BCL } \\
\text { Material }^{\mathrm{b}}\end{array}$ & SPA $^{b}$ & $\begin{array}{l}\text { Focused } \\
\text { Samples }^{c}\end{array}$ & Units \\
\hline 4 & Americium-241 & - & - & 0.061 & - & $\mathrm{pCi} / \mathrm{g}$ \\
\hline 5 & Carbon-14 & - & -- & 1.6 & - & $\mathrm{pCi} / \mathrm{g}$ \\
\hline 6 & Cesium-137 & $0(<B G)$ & 0.159 & 0.57 & 0.353 & $\mathrm{pCi} / \mathrm{g}$ \\
\hline 7 & Cobalt-60 & - & 0.05 & 0.028 & 0.155 & $\mathrm{pCi} / \mathrm{g}$ \\
\hline 8 & Europium-152 & 0.07 & 0.068 & 0.20 & - & $\mathrm{pCi} / \mathrm{g}$ \\
\hline 9 & Nickel-63 & -- & -- & 5.05 & -- & $\mathrm{pCi} / \mathrm{g}$ \\
\hline 10 & Plutonium-239/240 & - & -- & 0.125 & -- & $\mathrm{pCi} / \mathrm{g}$ \\
\hline 11. & Strontium-90 & $0(<B G)$ & 0.120 & 0.236 & -- & $\mathrm{pCi} / \mathrm{g}$ \\
\hline 12 & Tritium & - & -- & 14.6 & 137 & $\mathrm{pCi} / \mathrm{g}$ \\
\hline 13 & Uranium-233/234 & $0(<B G)$ & $0(<B G)$ & $0(<B G)$ & $0(<B G)$ & $\mathrm{pCi} / \mathrm{g}$ \\
\hline 14 & Uranium-235 & $0(<B G)$ & $0(<B G)$ & $0(<B G)$ & $\cdots$ & $\mathrm{pCi} / \mathrm{g}$ \\
\hline 15 & Uranium-238 & $0(<B G)$ & $0(<B G)$ & $0(<B G)$ & $0(<B G)$ & $\mathrm{pCi} / \mathrm{g}$ \\
\hline 16 & Arsenic & 3.1 & 3.7 & 2.3 & 7.0 & $\mathrm{mg} / \mathrm{kg}$ \\
\hline 17 & Barium & 59.3 & 60.8 & 72.5 & 261 & $\mathrm{mg} / \mathrm{kg}$ \\
\hline 18 & Beryllium & 0.48 & 0.56 & 0.36 & 0.58 & $\mathrm{mg} / \mathrm{kg}$ \\
\hline 19 & Boron & 2.0 & 1.8 & 1.9 & 24.5 & $\mathrm{mg} / \mathrm{kg}$ \\
\hline 20 & Cadmium & 0.43 & -- & 0.53 & 0.28 & $\mathrm{mg} / \mathrm{kg}$ \\
\hline 21 & Chromium (total) & 7.2 & 8.1 & 8.3 & 16.4 & $\mathrm{mg} / \mathrm{kg}$ \\
\hline 22 & Cobalt & 8.7 & 9.2 & 7.7 & 11.3 & $\mathrm{mg} / \mathrm{kg}$ \\
\hline 23 & Copper & 16.0 & 15.9 & 14.8 & 28.0 & $\mathrm{mg} / \mathrm{kg}$ \\
\hline 24 & Hexavalent chromium & 0.23 & 0.24 & 0.27 & - & $\mathrm{mg} / \mathrm{kg}$ \\
\hline 25 & Lead & 5.1 & 5.1 & 4.7 & 34.3 & $\mathrm{mg} / \mathrm{kg}$ \\
\hline 26 & Manganese & 346 & 366 & 330 & 421 & $\mathrm{mg} / \mathrm{kg}$ \\
\hline 27 & Mercury & 0.05 & 0.03 & 0.13 & 14.5 & $\mathrm{mg} / \mathrm{kg}$ \\
\hline 28 & Molybdenum & 0.83 & 0.43 & 0.45 & 0.81 & $\mathrm{mg} / \mathrm{kg}$ \\
\hline 29 & Nickel & 10.1 & 11.1 & 10.8 & 15.2 & $\mathrm{mg} / \mathrm{kg}$ \\
\hline 30 & Selenium & 0.67 & 1.0 & -- & -- & $\mathrm{mg} / \mathrm{kg}$ \\
\hline 31 & Vanadium & 46.6 & 48.8 & 46.4 & 62.8 & $\mathrm{mg} / \mathrm{kg}$ \\
\hline 32 & Zinc & 40.0 & 41.4 & 38.8 & 65.1 & $\mathrm{mg} / \mathrm{kg}$ \\
\hline 33 & Aroclor-1242 & - & - & 0.047 & - & $\mathrm{mg} / \mathrm{kg}$ \\
\hline 34 & Aroclor-1254 & 0.038 & $\rightarrow$ & 0.17 & -- & $\mathrm{mg} / \mathrm{kg}$ \\
\hline 35 & Aroclor-1260 & -- & -- & 0.056 & -- & $\mathrm{mg} / \mathrm{kg}$ \\
\hline 36 & bis(2-Ethylhexyl)phthalate & 0.06 & 0.08 & 0.09 & 0.038 & $\mathrm{mg} / \mathrm{kg}$ \\
\hline 37 & Di-n-butylphthalate & 0.12 & 0.13 & 0.11 & - & $\mathrm{mg} / \mathrm{kg}$ \\
\hline 38 & Diethylphthalate & - & 0.019 & - & -- & $\mathrm{mg} / \mathrm{kg}$ \\
\hline 39 & Phenol & 0.022 & 0.034 & - & - & $\mathrm{mg} / \mathrm{kg}$ \\
\hline 40 & 4-Methyl-2-pentanone & 0.011 & 0.007 & - & - & $\mathrm{mg} / \mathrm{kg}$ \\
\hline 41 & Acetone & 0.039 & 0.020 & -- & 0.099 & $\mathrm{mg} / \mathrm{kg}$ \\
\hline 42 & Carbon tetrachlordie & 0.011 & 0.011 & - & $\cdots$ & $\mathrm{mg} / \mathrm{kg}$ \\
\hline 43 & Methylene chloride & 0.016 & 0.014 & 0.014 & 0.016 & $\mathrm{mg} / \mathrm{kg}$ \\
\hline 44 & Toluene & 0.002 & 0.001 & - & $\ldots$ & $\mathrm{mg} / \mathrm{kg}$ \\
\hline 45 & Trichloroethene & - & -- & 0.002 & -- & $\mathrm{mg} / \mathrm{kg}$ \\
\hline 46 & Xylenes (total) & 0.001 & 0.001 & - & $\ldots$ & $\mathrm{mg} / \mathrm{kg}$ \\
\hline 47 & Aldrin & - & - & 0.0010 & -- & $\mathrm{mg} / \mathrm{kg}$ \\
\hline 48 & alpha-Chlordane & - & - & 0.00080 & - & $\mathrm{mg} / \mathrm{kg}$ \\
\hline 49 & beta-BHC & 0.00064 & 0.0022 & 0.00082 & - & $\mathrm{mg} / \mathrm{kg}$ \\
\hline 50 & $4,4^{\prime}-\mathrm{DDD}$ & 0.0019 & - & - & -- & $\mathrm{mg} / \mathrm{kg}$ \\
\hline 51 & $4,4^{\prime}-\mathrm{DDE}$ & 0.0013 & - & 0.0065 & - & $\mathrm{mg} / \mathrm{kg}$ \\
\hline 52 & $4,4^{\prime}$-DDT & 0.0011 & 0.0016 & - & - & $\mathrm{mg} / \mathrm{kg}$ \\
\hline
\end{tabular}

$53{ }^{\mathrm{a}}$ No detections were reported in any data set for COCs/COPCs not listed in this table (continued on the following page).

54 Maximum or $95 \%$ UCL result, depending on data censorship, as described in the calculation methodology.

55 Maximum detected result from all focused samples.

$56 . .=$ not evaluated by laboratory analysis or not detected (for all samples in the data set)

$57 \mathrm{BG}=$ background

$58 \mathrm{BCL}=$ below cleanup levels

$59 \mathrm{COC}=$ contaminant of concern

$60 \mathrm{COPC}=$ contaminant of potential concern

61 SPA = staging pile area

$62 \mathrm{UCL}=$ upper confidence limit 
Washington Closure Hanford

Originator J.M. Capron 8 \& $\mathrm{C}$

Project $100-\mathrm{B} / \mathrm{C}$ Field Remediation

Subject 118-B-1 Burial Ground Cleanup Verification $95 \%$ UCL Calculations

Date $02 / 07 / 07$

\section{CALCULATION SHEET}

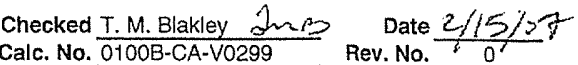
$\begin{aligned} \text { Calc. No. } & \text { Rev. No. Nob-CA-V0299 No. } \frac{0}{14655} \text { Sheet No. } 6 \text { of } 61\end{aligned}$

1 Summary (continued)

\begin{tabular}{|c|c|c|c|c|c|c|}
\hline \multicolumn{7}{|c|}{2 Results Summary - Overburden, BCL Material, SPA, and Focused Samples (continued) } \\
\hline 3 & Analyte & Overburden & $\begin{array}{c}\mathrm{BCL} \\
\text { Material }^{\mathrm{b}}\end{array}$ & $\mathrm{SPA}^{\mathrm{b}}$ & $\begin{array}{l}\text { Focused } \\
\text { Samples }^{c}\end{array}$ & Units \\
\hline 4 & Endosulfan I & 0.0028 & 0.0049 & - & - & $\mathrm{mg} / \mathrm{kg}$ \\
\hline 5 & Endosulfan II & 0.00060 & - & - & - & $\mathrm{mg} / \mathrm{kg}$ \\
\hline 6 & Endosulfan sulfate & 0.00087 & 0.00084 & 0.0019 & -- & $\mathrm{mg} / \mathrm{kg}$ \\
\hline 7 & Endrin aldehyde & 0.0019 & 0.0015 & - & -. & $\mathrm{mg} / \mathrm{kg}$ \\
\hline 8 & Endrin ketone & - & 0.0014 & -- & - & $\mathrm{mg} / \mathrm{kg}$ \\
\hline 9 & gamma-Chlordane & 0.0025 & $-\cdot$ & 0.0065 & -- & $\mathrm{mg} / \mathrm{kg}$ \\
\hline 0 & Heptachlor & -- & 0.00043 & $\cdots$ & - & $\mathrm{mg} / \mathrm{kg}$ \\
\hline 1 & Methoxychlor & 0.0067 & 0.0053 & 0.0017 & 0.0047 & $\mathrm{mg} / \mathrm{kg}$ \\
\hline 2 & 2,4-D & 0.026 & -- & 0.048 & - & $\mathrm{mg} / \mathrm{kg}$ \\
\hline 3 & $2,4-D B$ & 0.087 & 0.0098 & $0.028^{d}$ & $\cdots$ & $\mathrm{mg} / \mathrm{kg}$ \\
\hline 4 & $2,4,5-T$ & 0.0039 & $\cdots$ & 0.013 & - & $\mathrm{mg} / \mathrm{kg}$ \\
\hline 5 & $2,4,5-\mathrm{TP}$ & 0.0096 & -- & 0.043 & -- & $\mathrm{mg} / \mathrm{kg}$ \\
\hline 6 & Dalapon & 0.023 & 0.048 & 0.043 & - & $\mathrm{mg} / \mathrm{kg}$ \\
\hline$r$ & Dicamba & 0.016 & 0.013 & 0.023 & 0.0091 & $\mathrm{mg} / \mathrm{kg}$ \\
\hline 10 & Dichloroprop & 0.11 & 0.21 & 0.049 & -- & $\mathrm{mg} / \mathrm{kg}$ \\
\hline 19 & Pentachlorophenol & 0.016 & - & 0.0040 & - & $\mathrm{mg} / \mathrm{kg}$ \\
\hline
\end{tabular}

$20{ }^{\mathrm{a}} \mathrm{No}$ detections were reported in any data set for COCs/COPCs not listed in this table (continued from the previous page).

$21 \mathrm{Maximum}$ or $95 \% \mathrm{UCL}$. result, depending on data censorship, as described in the calculation methodology.

$22^{9}$ Maximum detected result from focused samples 1 through 11 and 14 through 16.

$23^{\mathrm{D}}$ Maximum accepted result.

$24--=$ not evaluated by laboratory analysis or not detected (for all samples in the data set)

$25 \mathrm{BCL}=$ below cleanup levels

$26 \mathrm{COC}=$ contaminant of concern

$27 \mathrm{COPC}=$ contaminarit of potential concern

$28 \mathrm{SPA}=$ staging pile area

$29 \mathrm{UCL}=$ upper confidence limit

30

\begin{tabular}{|c|c|c|c|c|c|c|c|c|c|c|c|}
\hline \multirow[t]{2}{*}{31} & \multicolumn{11}{|c|}{ Relative Percent Difference Results ${ }^{a}$ - } \\
\hline & Analyte & $\begin{array}{l}\text { Area } 1 \text { A1 } \\
\text { Duplicate } \\
\text { Analysis }^{\text {b }}\end{array}$ & $\begin{array}{l}\text { Area } 1 \text { A1 } \\
\text { Split } \\
\text { Analysis }^{b}\end{array}$ & $\begin{array}{l}\text { Area } 3 \text { A3 } \\
\text { Duplicate } \\
\text { Analysis }^{b}\end{array}$ & $\begin{array}{c}\text { Area } 3 \text { A3 } \\
\text { Split } \\
\text { Analysis }^{b}\end{array}$ & $\begin{array}{l}\text { Area } 4 \text { A1 } \\
\text { Duplicate } \\
\text { Analysis }^{b}\end{array}$ & $\begin{array}{c}\text { Area } 4 \text { A1 } \\
\text { Split } \\
\text { Analysis }^{b}\end{array}$ & $\begin{array}{c}\text { BCL D4 } \\
\text { Duplicate } \\
\text { Analysis }^{\mathrm{b}}\end{array}$ & $\begin{array}{c}\text { BCL B8 } \\
\text { Split } \\
\text { Analysis }^{b}\end{array}$ & $\begin{array}{c}\text { SPA B5 } \\
\text { Duplicate } \\
\text { Analysis }^{\text {b }}\end{array}$ & $\begin{array}{c}\text { SPA B5 } \\
\text { Split } \\
\text { Analysis }^{b}\end{array}$ \\
\hline 33 & Cesium-137 & $\cdots$ & $\because$ & $64 \%$ & $61 \%$ & - & - & - & -- & $\cdots$ & $\because$ \\
\hline 34 & Europium-152 & $59 \%$ & $7.5 \%$ & $\ldots$ & -- & -- & -. & $\ldots$ & - & $\ldots$ & $\ldots$ \\
\hline 35 & Potassium - 40 & $16 \%$ & -. & $12 \%$ & -. & $67 \%$ & $\ldots$ & $58 \%$ & -. & $67 \%$ & $\ldots$ \\
\hline 36 & Radium-226 & $0.14 \%$ & $\ldots$ & $\ldots$ & $-m$ & $\ldots$ & - & $56 \%$ & -- & $42 \%$ & $\ldots$ \\
\hline 37 & Radium-228 & $0.88 \%$ & - & -- & $m$ & - & $\cdots$ & - & .. & 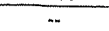 & -- \\
\hline 38 & Aluminum & $8.9 \%$ & $21 \%$ & $11 \%$ & $11 \%$ & $12 \%$ & $38 \%$ & $6.1 \%$ & $39 \%$ & $6.5 \%$ & $47 \%$ \\
\hline 39 & Barium & $12 \%$ & $20 \%$ & $8.1 \%$ & $9.4 \%$ & $27 \%$ & $41 \%$ & $12 \%$ & $25 \%$ & $6.1 \%$ & $28 \%$ \\
\hline 40 & Calcium & $10 \%$ & $0.58 \%$ & $2.2 \%$ & $22 \%$ & $19 \%$ & $36 \%$ & $2.9 \%$ & $8.7 \%$ & $1.4 \%$ & $26 \%$ \\
\hline 41 & Chromium & $16 \%$ & $2.9 \%$ & $11 \%$ & $34 \%$ & - & - & $19 \%$ & $25 \%$ & $15 \%$ & $44 \%$ \\
\hline 42 & Cobalt & $5.8 \%$ & $36 \%$ & -. & -. & -- & -- & -. & $\ldots$ & $\ldots$ & $\ldots$ \\
\hline 43 & Copper & $2.8 \%$ & $10 \%$ & $6.3 \%$ & $22 \%$ & $1.3 \%$ & $5.0 \%$ & $0 \%$ & $4.3 \%$ & $5.3 \%$ & $17 \%$ \\
\hline 44 & Iron & $7.8 \%$ & $23 \%$ & $0 \%$ & $3.0 \%$ & $2.8 \%$ & $20 \%$ & $5.8 \%$ & $12 \%$ & $4.9 \%$ & $23 \%$ \\
\hline 45 & Magnesium & $11 \%$ & $1.9 \%$ & $4.0 \%$ & $17 \%$ & $13 \%$ & $22 \%$ & $1.4 \%$ & $22 \%$ & $3.6 \%$ & $34 \%$ \\
\hline 46 & Manganese & $13 \%$ & $0.28 \%$ & $4.2 \%$ & $17 \%$ & $0.69 \%$ & $15 \%$ & $7.1 \%$ & $10 \%$ & $6.1 \%$ & $16 \%$ \\
\hline 47 & Silicon & - & - & $\ldots$ & - & $8.5 \%$ & $38 \%$ & $8.7 \%$ & $103 \%$ & $2.2 \%$ & $62 \%$ \\
\hline 48 & Sodium & $3.7 \%$ & $54 \%$ & - & 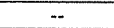 & $\ldots$ & -. & -. & -- & $\ldots$ & $\ldots$ \\
\hline 49 & Vanadium & $12 \%$ & $39 \%$ & $6.2 \%$ & $12 \%$ & $18 \%$ & $25 \%$ & $8.4 \%$ & $28 \%$ & $7.4 \%$ & $33 \%$ \\
\hline 50 & Zinc & $8.7 \%$ & $1.1 \%$ & $5.6 \%$ & $30 \%$ & $1.7 \%$ & $15 \%$ & $3.2 \%$ & $12 \%$ & $4.2 \%$ & 0010 \\
\hline
\end{tabular}

51 Quantitated results for cesium-137 (Area 4 primary-duplicate and primary-split pairs), cobalt-60 (BCL primary-split pair), radium-226 (Areas 3 and 4

52 primary-duplicate pairs), radium-228 (Area 4, BCL, and SPA primary-duplicate pairs), boron (Area 4 and SPA primary-split pairs), chromium (Area 4

53 primary-split pair), cobalt (Area 4 and SPA primary-split pairs), sodium (Area 4 and BCL primary-split pairs), aroclor-1254 (Area 4 and BCL primary-

54 split pairs), aroclor-1260 (BCL primary-split pair), 4,4'-DDE (Area 4 primary-duplicate and primary-split pairs), and 4,4'-ODT (BCL primary-split pair)

55. did not exceed the relative percent difference quality control threshold of 5XTDL, but the difference between the results exceeded the 2XTDL

56 control threshold.

57 Relative percent difference evaluation was not required for analytes not included in this table.

$58{ }^{\circ}$ The significance of relative percent difference values are discussed within the cleanup verification package for the subject site.

59 .. = analysis not required

$60 \mathrm{BCL}=$ below cleanup levels

$61 \mathrm{QA} / \mathrm{QC}=$ quality assurance/quality control

62 SPA = staging pile area 
CVP-2007-00006

Rev. 0

$+$

3

C-26 
Washington Closure Hanford

Originator J. M. Capron $9 m<$

Project 100-B/C Field Remediation
CALCULATION SHEET
Date $\frac{10 / 03 / 07}{1455}$
Job No.
Calc. No. 0100B-CA-V0299
$\begin{aligned} & \text { Rev. No. } \frac{1}{10 / 04 / 07} \\ & \text { Date } \frac{10 / 0}{7 \text { of } 61} \\ & \text { Sheet No. }\end{aligned}$

1118-B-1 Area 1 Statistical Calculations

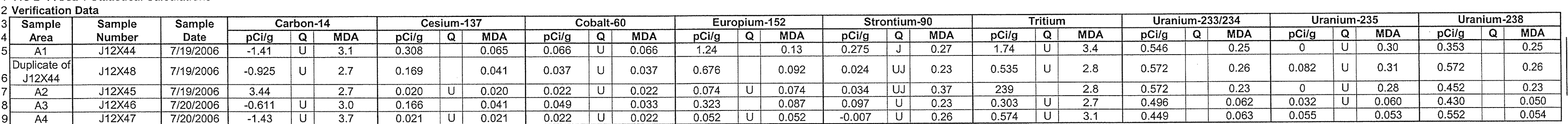

\section{Statistical Computation Input Data}

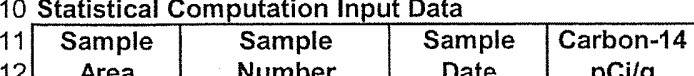

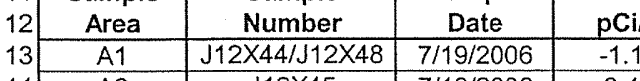

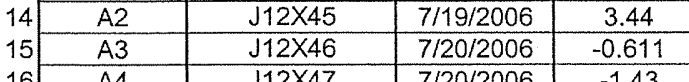

Cesium-137

Strontium-90

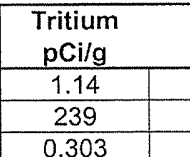

Uranium-233/234

\begin{tabular}{c|c} 
Uranium \\
pCilg \\
0.041 \\
0 \\
0 \\
0.032
\end{tabular}

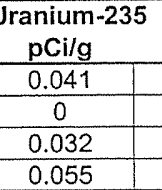

Uranium-238

17 Statistical Computations

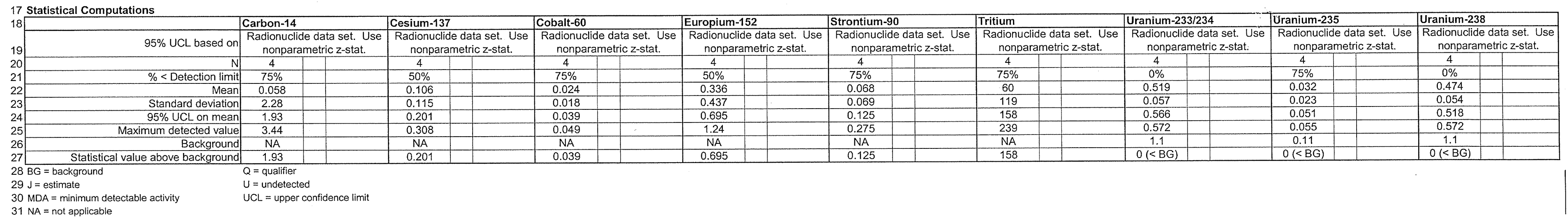


Originator J.M. Capron $\mathcal{Q}^{\text {anc }}$ Project $100-B / C$ Field Remediation
Subject 118-B-1 Burial Ground Cleanup Verification $95 \%$ UCL Calculations
CALCULATION SHEET

1 118-B-1 Area 1 Statistical Calculations (continued)

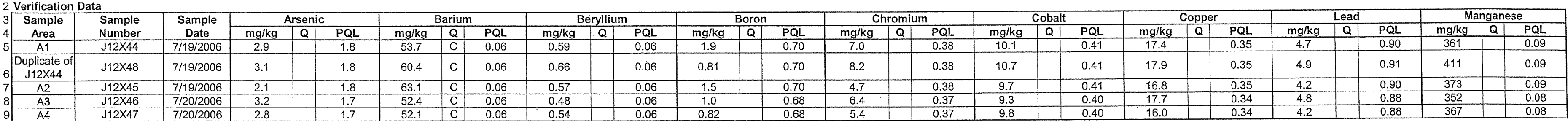

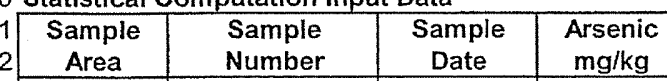

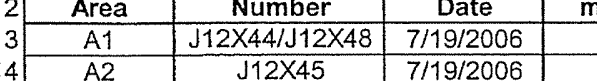

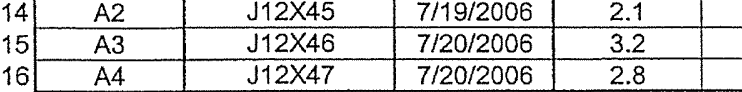

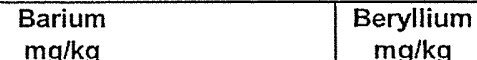
\begin{tabular}{|l|c|c|c|}
$\begin{array}{c}\text { Beryllium } \\
\mathrm{mg} / \mathrm{kg}\end{array}$ & $\begin{array}{c}\text { Boron } \\
\mathrm{mg} / \mathrm{kg}\end{array}$ & $\begin{array}{c}\text { Chromium } \\
\mathrm{mg} / \mathrm{kg}\end{array}$ \\
\hline 0.63 & & 1.5 & 7.
\end{tabular} \begin{tabular}{|c|c|}
\hline $\begin{array}{c}\text { Chromium } \\
\mathrm{mg} / \mathrm{kg}\end{array}$ & $\begin{array}{c}\text { Cobalt } \\
\mathrm{mg} / \mathrm{kg}\end{array}$ \\
\hline 76 & \\
\hline 47 & 97
\end{tabular}

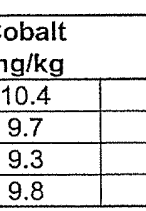
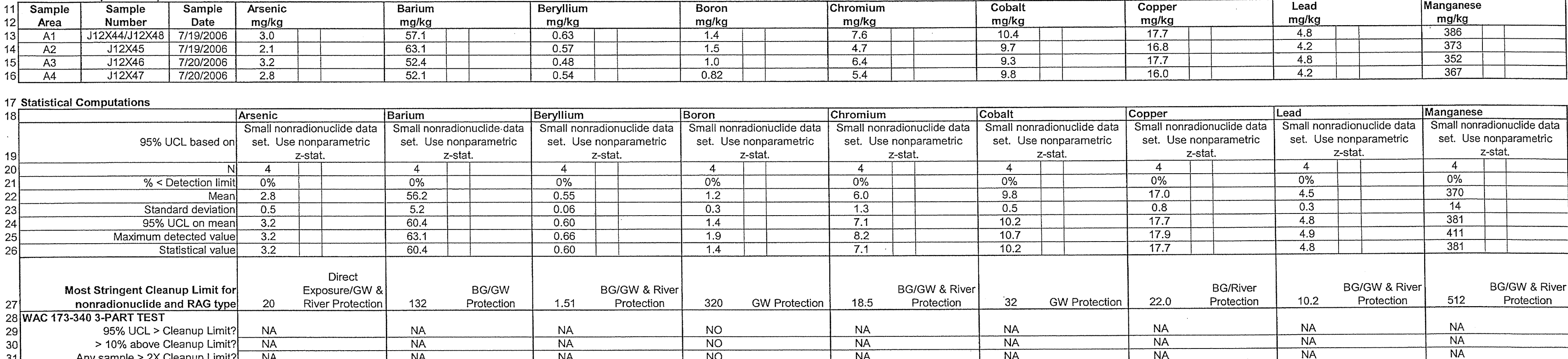

WAC 173-340 Compliance? YES background (6.5 $\mathrm{mg} / \mathrm{kg})$, the

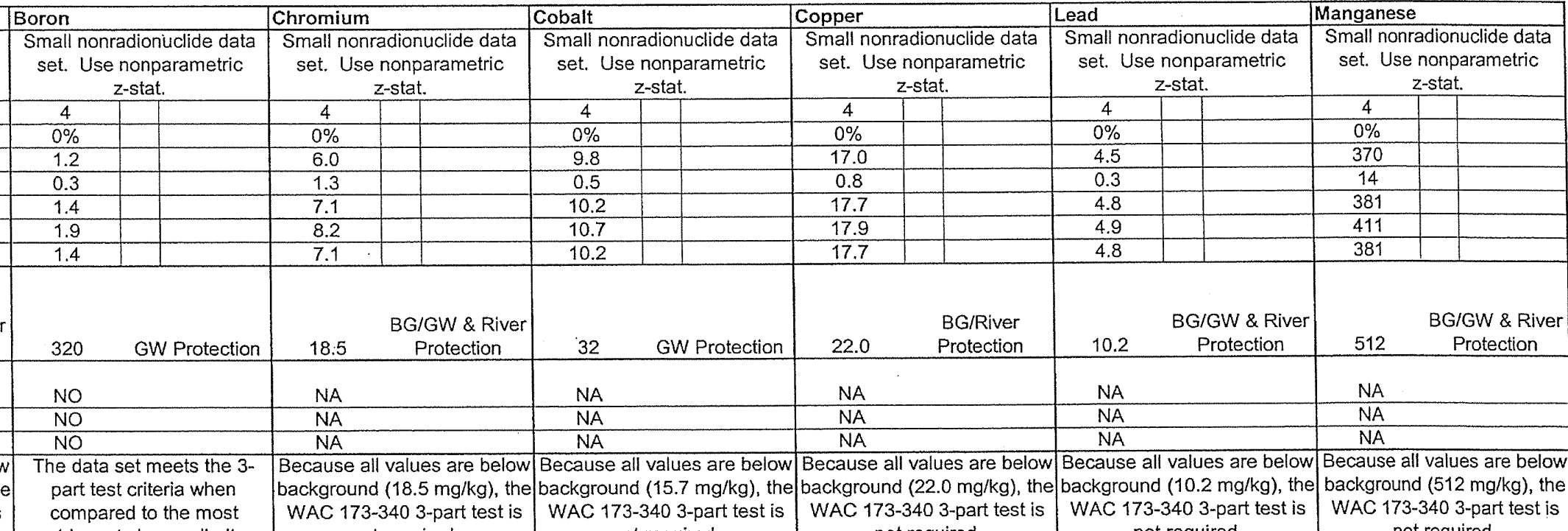
$33 \mathrm{BG}=$ background $34 \mathrm{C}=$ method blank
$35 \mathrm{GW}=$ groundwater
$36 \mathrm{WA}=$ not $37 \mathrm{PQL}=$ practical quantitation lim What required \begin{tabular}{|l|l|} 
WAC 173-340 3-part test is & background $(1.51 \mathrm{mg} / \mathrm{kg}$ ), \\
WAC 173-340 3-part test
\end{tabular}

part test criteria whe

等 $Q=$ qualifier required. not required. not required. \begin{tabular}{c}
$\begin{array}{c}73-340 \text { 3-part test is } \\
\text { not required. }\end{array}$ \\
$\begin{aligned} \text { WAC 173-340 3-par } \\
\text { not required. }\end{aligned}$ \\
\hline
\end{tabular} not required.
nosnot required. 
Originator J.M. Capron $q^{2} \mathrm{n}$ Project
Subject $100-$ B/C Field Remediation
$118-B-1$ Burial Ground Cleanup Verification $95 \%$ UCL Calculations
CALCULATION SHEET
\begin{aligned} & Date $02 / 107 / 07 \\ &$ Job No. 14655 \\ & \hline\end{aligned}
Calc. No. 0100B-CA-V0299
Checked T.M. Blakley 2 hmo
Rev. No. $\frac{0}{2 / 5 / 97}$

\section{8-B-1 Area 1 Statistical Calculations (continued)}

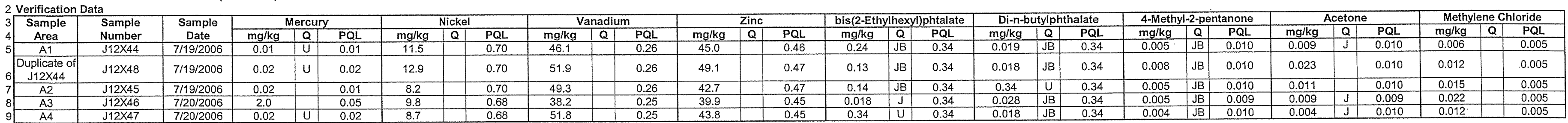

10 Statistical Computation Input Data

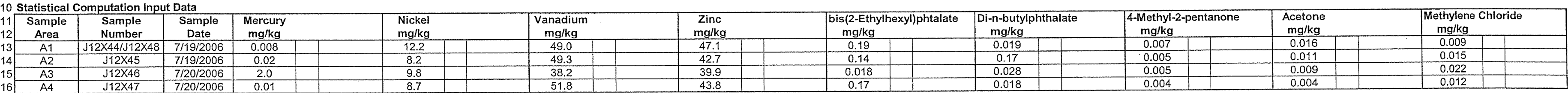

7 Statistical Computations

\begin{tabular}{|c|c|c|c|c|c|c|c|c|c|c|c|c|c|c|c|c|c|c|c|}
\hline \multirow{2}{*}{\multicolumn{2}{|c|}{$95 \%$ UCL based or }} & \multicolumn{2}{|c|}{$\begin{array}{l}\text { Mercury } \\
\text { Small nonradionuclide data } \\
\text { set. Use nonparametric } \\
\text { z-stat. }\end{array}$} & \multicolumn{2}{|c|}{$\begin{array}{l}\text { Nickel } \\
\text { Smaln nonradionuclide data } \\
\text { set. Use nonparametric } \\
\text { Z-stat. }\end{array}$} & \multicolumn{2}{|c|}{\begin{tabular}{|c|} 
Vanadium \\
Small nonradionuclide data \\
set. Use nonparametric \\
z-stat.
\end{tabular}} & \multicolumn{2}{|c|}{\begin{tabular}{|l} 
Zinc \\
$\begin{array}{c}\text { Small nonradionuclide data } \\
\text { set. Use nonparametric } \\
\text { Z-stat. }\end{array}$
\end{tabular}} & \multicolumn{2}{|c|}{\begin{tabular}{|c|} 
bis(2-Ethylhexyl)phtalate \\
Small nonradionuclide datata \\
set. Use nonparametric \\
Z-stat.
\end{tabular}} & \multicolumn{2}{|c|}{\begin{tabular}{|c|} 
Di-n-butylphthalate \\
Small Inonradionuclide data \\
set. Use nonparametric \\
Z-stat. \\
\end{tabular}} & \multicolumn{2}{|c|}{\begin{tabular}{|c|} 
4-Methyl-2-pentanone \\
Small nonradionucilide data \\
set. Use nonparametric \\
z-statt.
\end{tabular}} & \multicolumn{2}{|c|}{\begin{tabular}{|c|} 
Acetone \\
$\begin{array}{c}\text { Small nonradionuclide data } \\
\text { set. Use nonparametric } \\
\text { z-stat. }\end{array}$ \\
\end{tabular}} & \multicolumn{2}{|c|}{$\begin{array}{l}\text { Methylene Chloride } \\
\text { Small nonradionuclide data } \\
\text { set. Use nonparametric } \\
\text { z-stat. }\end{array}$} \\
\hline & & & & & & & & & & & & & & & & & & & \\
\hline & $\%<$ Detection limit & $50 \%$ & & $0 \%$ & & $0 \%$ & & $0 \%$ & & $25 \%$ & & $25 \%$ & & $0 \%$ & & $0 \%$ & & $\frac{0 \%}{0.015}$ & \\
\hline & Mean & 0.5 & $\theta$ & 9.7 & & $\frac{47.1}{60}$ & & $\frac{43.4}{30}$ & & 0.13 & & 0.06 & & $\frac{0.005}{0.001}$ & & 0.010 & & 0.015 & \\
\hline & Standard deviation & $\frac{1.0}{1.2}$ & $\square$ & $\frac{1.8}{11.2}$ & & $\frac{6.0}{550}$ & & $\frac{3.0}{45.8}$ & & 0.08 & & 0.07 & & 0.001 & & 0.005 & & $\frac{0.006}{0.019}$ & \\
\hline & $\begin{array}{l}95 \% \text { UCL on mean } \\
\text { Maximum detected value }\end{array}$ & $\frac{1.3}{2.0}$ & t & $\frac{11.2}{12.9}$ & & $\frac{52.0}{51.9}$ & & $\begin{array}{ll}45.8 \\
49.1\end{array}$ & & 0.24 & & $\frac{0.12}{0.028}$ & & 0.000 & & $\frac{.024}{0.023}$ & & 0.022 & \\
\hline & Statistical value & $\frac{.0}{1.3}$ & & 11.2 & & 52.0 & & 45.8 & & 0.19 & & 0.12 & & 0.006 & & 0.014 & & 0.019 & \\
\hline & $\begin{array}{l}\text { Most Stringent Cleanup Limit for } \\
\text { norradionuclide and RAG type }\end{array}$ & 033 & $\begin{array}{l}\text { BG/GW \& River } \\
\text { Protection }\end{array}$ & 19.1 & $\begin{array}{l}\mathrm{BG} / \mathrm{GW} \\
\text { Protection }\end{array}$ & 85.1 & $\begin{array}{l}\text { BG/GW } \\
\text { Protection }\end{array}$ & 67.8 & 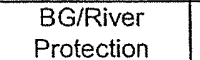 & 0.36 & River Protection & 160 & GW Protection & 64 & GW Protection & 720 & GW Protection & 0.5 & GW Protection \\
\hline & WAC 173-340 3-PART TEST & & & NA & & & & & & & & No & & & & $\mathrm{NO}$ & & & \\
\hline & & $\frac{\text { YES }}{\text { YES }}$ & & & & $\frac{N A}{N A}$ & & $\frac{N A}{N A}$ & & NO & & No & & NO & & NO & & & \\
\hline & $\begin{array}{l}>10 \% \text { above Clea } \\
\text { Any }\end{array}$ & $\frac{\text { YES }}{\text { YEC }}$ & & NA & & NA & & $\frac{N A}{N A}$ & & $\frac{N}{N 0}$ & & No & & No & & $N_{N C}$ & & NO & \\
\hline & WAC 173-340 Compliance? $\begin{array}{c}\text { Further } \\
\text { Assessment } \\
\text { Required }\end{array}$ & $\begin{array}{l}\text { Beca } \\
\text { answers } \\
\text { details } \\
\text { RESRA } \\
\text { The dat } \\
\text { part } \\
\text { comp } \\
\text { exnos }\end{array}$ & 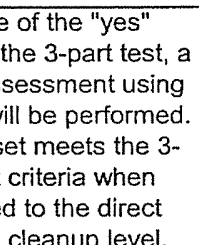 & $\begin{array}{r}\text { Because all } \\
\text { background } \\
\text { WAC 173- } \\
\text { not }\end{array}$ & $\begin{array}{l}\text { alues are below } \\
19.1 \mathrm{mg} / \mathrm{kg} \text { ), the } \\
403 \text {-part test is } \\
\text { equired. }\end{array}$ & \begin{tabular}{|} 
Because all \\
background \\
WAC 173-: \\
not
\end{tabular} & $\begin{array}{l}\text { II values are below } \\
\text { t }(85.1 \mathrm{mg} / \mathrm{kg}) \text {, the } \\
-340 \text {-part test is } \\
\text { trequired. }\end{array}$ & $\begin{array}{r}\text { Because all } \\
\text { background } \\
\text { WAC 17- } \\
\text { not }\end{array}$ & $\begin{array}{l}\text { values are below } \\
(67.8 \mathrm{mg} / \mathrm{kg}) \text {, the } \\
40 \text { 3-part test is } \\
\text { equired. }\end{array}$ & $\begin{array}{l}\text { The data } \\
\text { part tes } \\
\text { compar } \\
\text { stringen }\end{array}$ & $\begin{array}{l}\text { set meets the 3- } \\
\text { st criteria when } \\
\text { red to the most } \\
\text { nt cleanup limit. }\end{array}$ & $\begin{array}{l}\text { The datat te } \\
\text { part } \\
\text { compap } \\
\text { stringe }\end{array}$ & $\begin{array}{l}\text { set meets the 3- } \\
\text { t criteria when } \\
\text { ed to the most } \\
\text { it cleanup limit. }\end{array}$ & $\begin{array}{l}\text { The data } \\
\text { part tes } \\
\text { compar } \\
\text { stringer }\end{array}$ & $\begin{array}{l}\text { set meets the 3- } \\
t \text { criteria when } \\
\text { ed to the most } \\
t \text { cleanup limit. }\end{array}$ & $\begin{array}{l}\text { The data } \\
\text { part tes } \\
\text { compar } \\
\text { stringer }\end{array}$ & $\begin{array}{l}\text { set meets the 3- } \\
\text { it criteria when } \\
\text { ed to the most } \\
\text { it cleanup limit. }\end{array}$ & $\begin{array}{l}\text { The data } \\
\text { part tes } \\
\text { compal } \\
\text { stringer }\end{array}$ & $\begin{array}{l}\text { set meets the 3- } \\
\text { it criteria when } \\
\text { ed to the most } \\
\text { it cleanup limit. }\end{array}$ \\
\hline
\end{tabular}

33 B= method blank contamination exposured cleanup level. RAG = remedial action goal

$U=$ undetected

$35 \mathrm{GW}=$ groundwa
$36 \mathrm{~J}=$ estimate
$37 \mathrm{AA}=$ not

$38 \mathrm{PQL}=$ practical quantitation limit

$W C L=$ upper confidence limit
$W A C=$ Washington Administrative Coode 
118-B-1 Area 2 Statistical Calculations

\begin{tabular}{|c|c|c|c|c|c|c|c|c|c|c|c|c|c|c|c|c|c|c|c|c|c|c|c|c|c|c|}
\hline \multirow{3}{*}{$\begin{array}{c}\text { Sample } \\
\text { Area } \\
\text { A1 }\end{array}$} & \multirow{2}{*}{$\begin{array}{l}\text { Sample } \\
\text { Number }\end{array}$} & \multirow{2}{*}{$\begin{array}{c}\text { Sample } \\
\text { Date }\end{array}$} & \multicolumn{3}{|c|}{ Cesium-137 } & \multicolumn{3}{|c|}{ Strontium-90 } & \multicolumn{3}{|c|}{ Tritium } & \multicolumn{3}{|c|}{ Arsenic } & \multicolumn{3}{|c|}{ Barium } & \multirow{2}{*}{\multicolumn{3}{|c|}{ Beryllium }} & \multicolumn{3}{|c|}{ Boron } & \multicolumn{3}{|c|}{ Chromium } \\
\hline & & & $\mathrm{pCi} / \mathrm{g}$ & $Q$ & MDA & pcilg & Q & MDA & pCilg & Q & MDA & $\mathrm{mg} / \mathrm{kg}$ & $Q$ & PQL & $\mathrm{mg} / \mathrm{kg}$ & Q & $\mathrm{PQL}$ & & Q & $\mathrm{PQL}$ & $\mathrm{mg} / \mathrm{kg}$ & Q & $P Q L$ & $\mathrm{mg} / \mathrm{kg}$ & Q & $\mathrm{PQL}$ \\
\hline & & $7 / 20 / 2006$ & 0.223 & & 0.033 & 0.049 & $u$ & $\begin{array}{l}0.22 \\
0.23\end{array}$ & $\frac{0.150}{596}$ & U & $\frac{2.9}{28}$ & $\frac{1.8}{32}$ & & $\frac{1.7}{17}$ & $\frac{43.9}{555}$ & C & 0.06 & 0.47 & & 0.06 & $\frac{1.6}{55}$ & & $\frac{67}{68}$ & $\frac{5.5}{6.9}$ & & $\frac{0.36}{0.37}$ \\
\hline$\frac{A 2}{A 3}$ & $\frac{J 12 X}{110 x}$ & & $\frac{0.099}{0.090}$ & $\frac{U}{11}$ & $\frac{0.099}{0.040}$ & 0.248 & 11 & 0.23 & $\begin{array}{l}59.6 \\
0.236\end{array}$ & II & $\frac{2.8}{30}$ & $\frac{3.2}{26}$ & & $\frac{1.7}{17}$ & $\frac{55.5}{437}$ & $\frac{\mathrm{c}}{\mathrm{c}}$ & 0.06 & $\frac{0.62}{0.65}$ & & 0.6 & $\frac{5.5}{37}$ & & 0.68 & $\frac{6.9}{58}$ & & 0.37 \\
\hline$\frac{\mathrm{A} 3}{\mathrm{~A} 4}$ & $\frac{J 12 \times 22}{J 12 \times 53}$ & $\begin{array}{l}7 / 20 / 20000 \\
7 / 2006\end{array}$ & 0.050 & $u$ & 0.0 .040 & $\begin{array}{l}0.000 \\
0.003\end{array}$ & u & 0.24 & $\begin{array}{l}0.250 \\
-0.432\end{array}$ & $\mathrm{U}$ & $\frac{3.0}{2.7}$ & $\frac{2.0}{2.0}$ & & 1.7 & 61.0 & \begin{tabular}{|l|}
$c$ \\
$c$
\end{tabular} & 0.06 & 0.00 & & 0.06 & 2.4 & & 0.68 & $\frac{3.0}{6.5}$ & & 0.37 \\
\hline
\end{tabular}

\section{Statistical Computation Input Data}
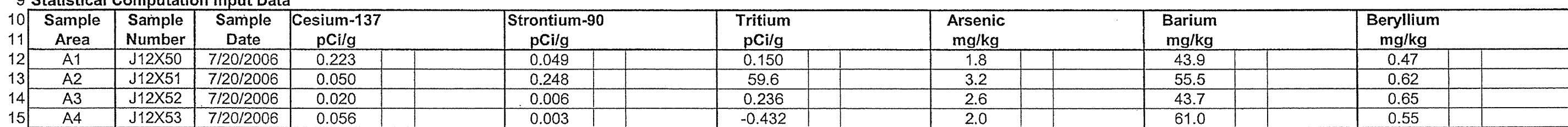

Boron $\quad$ Chromium

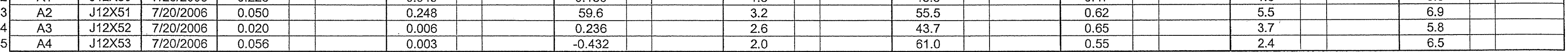

\section{Statistical Computations}

\begin{tabular}{|c|c|c|c|c|c|c|c|}
\hline 17 & & \multicolumn{2}{|c|}{ Cesium-137 } & \multicolumn{2}{|c|}{ Strontium-90 } & \multicolumn{2}{|l|}{ Tritium } \\
\hline 18 & $95 \%$ UCL based on & $\begin{array}{l}\text { Radionu } \\
\text { nonpa }\end{array}$ & $\begin{array}{l}\text { e data set. Use } \\
\text { metric z-stat. }\end{array}$ & $\begin{array}{l}\text { Radionuc } \\
\text { nonpa }\end{array}$ & $\begin{array}{l}\text { le data set. Use } \\
\text { metric z-stat. }\end{array}$ & $\begin{array}{r}\text { Radion } \\
\text { nonp }\end{array}$ & $\begin{array}{l}\text { Se data set. Use } \\
\text { metric z-stat. }\end{array}$ \\
\hline 19 & & 4 & & 4 & & 4 & \\
\hline & $\%<$ Detection limit & $50 \%$ & & $75 \%$ & & $75 \%$ & \\
\hline & & 0.087 & & 0.077 & & 14.9 & \\
\hline & Standard deviation & 0.092 & & 0.116 & & 29.8 & \\
\hline & $95 \%$ UCL on mean & & & 0.172 & & 39.4 & \\
\hline & Maximum detected value & 0.223 & & 0.248 & & 59.6 & \\
\hline $\begin{array}{l}25 \\
26\end{array}$ & Background & NA & & NA & & $\mathrm{NA}$ & \\
\hline 26 & \begin{tabular}{|l|l|} 
Statistical value above background \\
\end{tabular} & 0.163 & & 0.172 & & 39.4 & \\
\hline & $\begin{array}{r}\text { Most Stringent Cleanup Limit for } \\
\text { nonradionuclide and RAG type }\end{array}$ & & & & & & \\
\hline & WAC 173-340 3-PART TEST & & & & & & \\
\hline & $95 \%$ UCL > Cleanup Limit? & & & & & & \\
\hline 31 & $\begin{array}{c}>10 \% \text { above Cleanup Lilitit } \\
\text { Any sample }>2 X \text { Cleanup Limit? }\end{array}$ & & & & & & \\
\hline & $\begin{array}{l}\text { WAC 173-340 } \\
\text { Compliance? }\end{array}$ & & & & & & \\
\hline
\end{tabular}

\section{Arsenic}

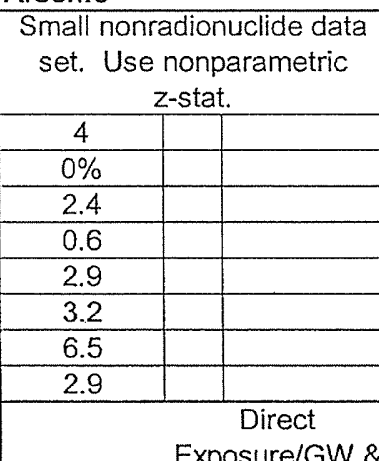

\section{River Protection}

NA

\begin{tabular}{c|c|c} 
NA & NA & NA \\
\hline Because all values are below & Because all values are below & Because all values are below
\end{tabular} \begin{tabular}{l|l|l} 
background $(6.5 \mathrm{mg} / \mathrm{kg}$ ), the & background (132 mg/kg), the \\
WAC 173-340 3-part test is & background (1.51 mg/kg), the \\
WAC 173-340 3-part test is is & WAC 173-340 3-part test is
\end{tabular}

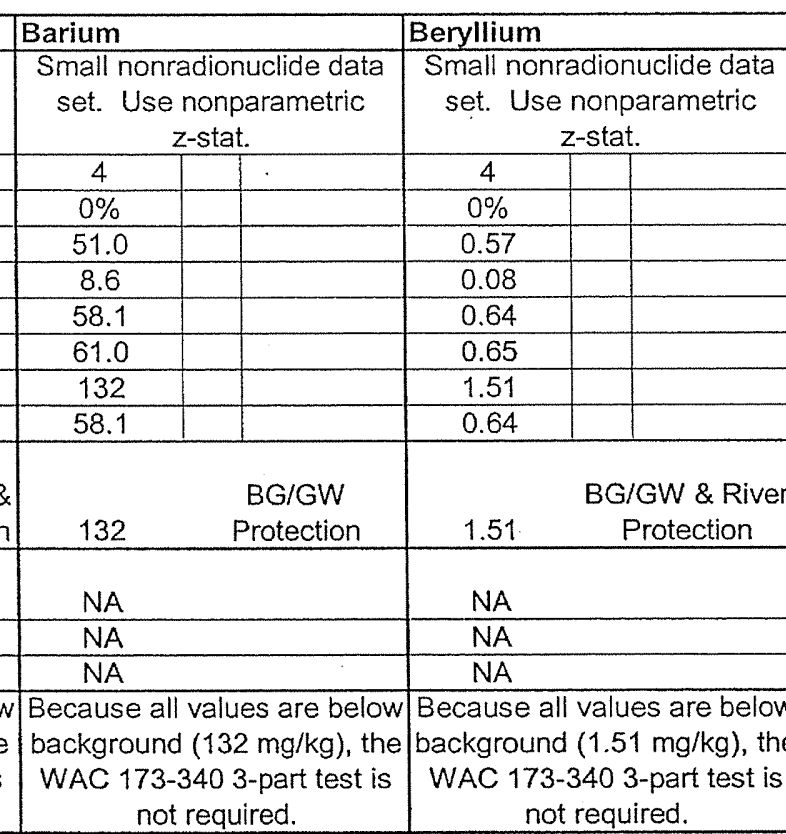

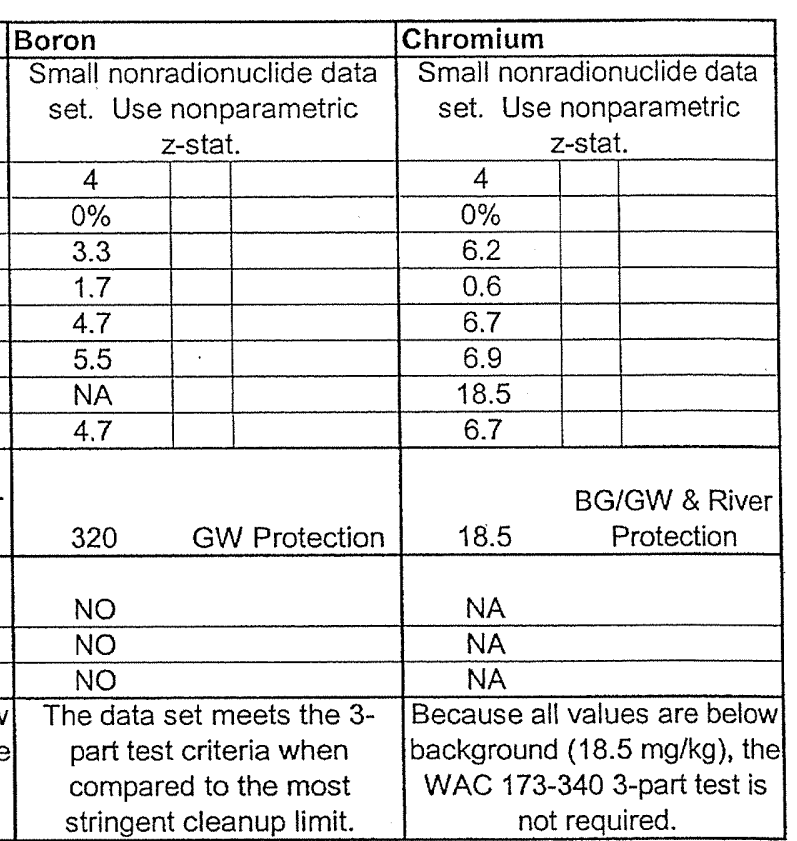

32 Complance? of background

$34 \mathrm{BG}=$ background
$35 \mathrm{C}=$ method blank

$36 \mathrm{GW}=$ groundwater

$37 \mathrm{MDA}=$ minimum de

$Q=$ qualifier
$R A G=$ remed

$\mathrm{R} A \mathrm{G}=$ remedial action goal
$U=$ undetected

UCL $=$ upper confidence limit
WAC $=$ Washington Administrafive Code 
1 118-B-1 Area 2 Statistical Calculations (continued)

\begin{tabular}{|c|c|c|c|c|c|c|c|c|c|c|c|c|c|c|c|c|c|c|c|c|c|c|c|c|c|c|}
\hline \multirow{3}{*}{\begin{tabular}{c|c} 
& Samplitio \\
& Area \\
\end{tabular}} & \multirow{3}{*}{$\begin{array}{l}\text { Dama } \\
\text { Sample } \\
\text { Number } \\
\end{array}$} & \multirow{2}{*}{$\begin{array}{c}\text { Sample } \\
\text { Date }\end{array}$} & \multicolumn{3}{|c|}{ Cobalt } & \multicolumn{3}{|c|}{ Copper } & \multicolumn{3}{|c|}{ Hexavalent Chromium } & \multicolumn{3}{|c|}{ Lead } & \multicolumn{3}{|c|}{ Manganese } & \multicolumn{3}{|c|}{ Nickel } & \multicolumn{3}{|c|}{ Vanadium } & \multicolumn{3}{|c|}{ Zinc } \\
\hline & & & $\mathrm{mg} / \mathrm{kg}$ & $Q$ & $\mathrm{PQL}$ & $\mathrm{mg} / \mathrm{kg}$ & Q & PQL & $\mathrm{mg} / \mathrm{kg}$ & $Q$ & $\mathrm{PQL}$ & $\mathrm{mg} / \mathrm{kg}$ & Q & $\mathrm{PQL}$ & $\mathrm{mg} / \mathrm{kg}$ & Q & $\mathrm{PQL}$ & $\mathrm{mg} / \mathrm{kg}$ & $Q$ & $P Q L$ & $\mathrm{mg} / \mathrm{kg}$ & $Q$ & $\mathrm{PQL}$ & $\mathrm{mg} / \mathrm{kg}$ & $Q$ & $\overline{P Q L}$ \\
\hline & & $7 / 20 / 2006$ & 8.4 & & & 15.0 & & 0.33 & 0.20 & UJ & 0.20 & 3.2 & & 0.86 & 311 & & 0.08 & 9.9 & & 0.67 & 54.0 & & 0.25 & 43.3 & & 0.45 \\
\hline$\frac{A 1}{A 2}$ & $\frac{31000}{1+2 \times 1}$ & 710100006 & $\frac{8.4}{84}$ & & 0.00 & $\frac{10.8}{168}$ & & 0.03 & 0.20 & $\mid \mathrm{UJ}$ & 0.20 & $\frac{.2}{44}$ & & 0.88 & 329 & & 0.09 & 11.0 & & 0.68 & 46.5 & & 0.26 & 41.1 & & 0.46 \\
\hline$\frac{A 2}{A 3}$ & $\sqrt{11}$ & $7 / 2$ & 8.4 & & 0.40 & $\frac{10.8}{16.2}$ & & 0.3 & 0.26 & $\frac{7}{02}$ & 0. & 4.4 & & 0.8 & $\frac{298}{298}$ & & 0.09 & 9.1. & & 0.00 & 54.7 & & 0.26 & 42.1 & & 0.46 \\
\hline$\frac{\mathrm{A3}}{\mathrm{A} 4}$ & $\begin{array}{l}\mathrm{J1} 2 \times 52 \\
112 \times 53\end{array}$ & $\frac{72012000}{71202006}$ & $\frac{8.1}{71}$ & & 0.40 & 10.2 & & 0.34 & $\begin{array}{l}0.20 \\
0.33 \\
\end{array}$ & $\mid \frac{1}{4}$ & 0.20 & $\frac{4.0}{50}$ & & 0.88 & $\frac{298}{298}$ & & 0.08 & $\frac{9.2}{8.4}$ & & 0.00 & 39.8 & & 0.25 & 36.3 & & 0.45 \\
\hline
\end{tabular}

9 Statistical Computation Input Data

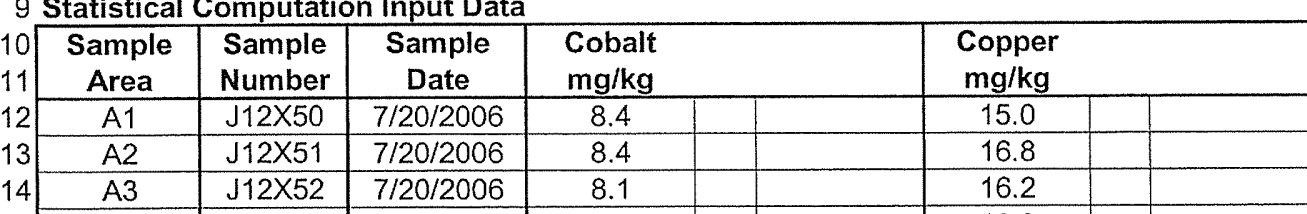

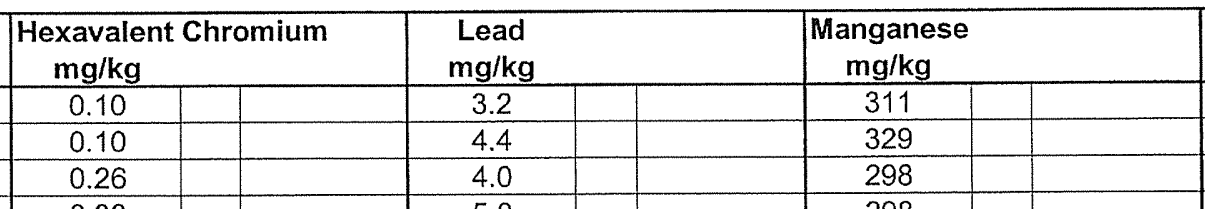

\begin{tabular}{l|l} 
Nickel & Vanadium \\
\hline
\end{tabular}

16 Statistical Computations

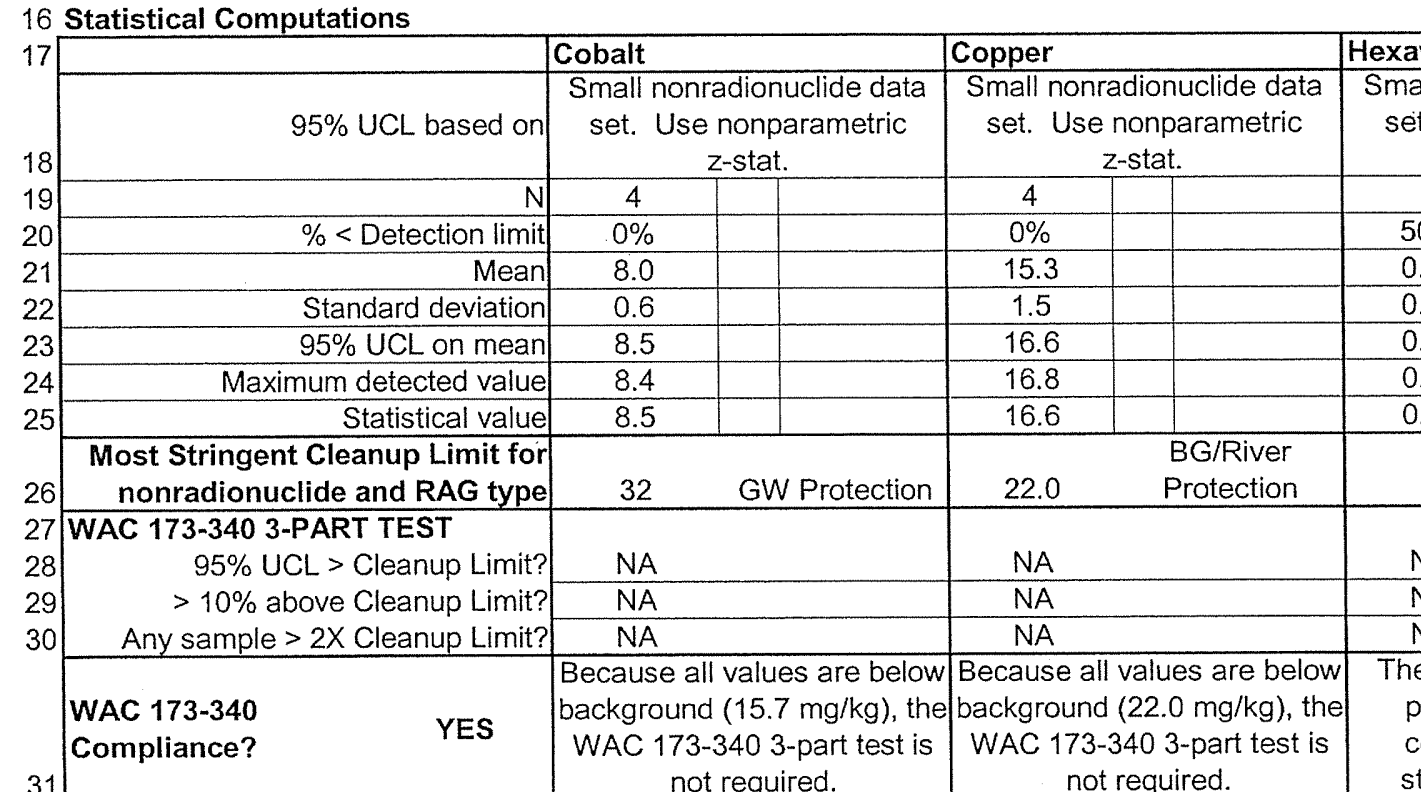
i.

\begin{tabular}{|c|l|c|c|}
\hline \multicolumn{2}{l|}{$\mathrm{mg} / \mathrm{kg}$} & $\mathrm{mg} / \mathrm{kg}$ \\
\hline 9.9 & & 54.0 \\
\hline 11.0 & & & 46.5 \\
\hline 9.2 & & 54.7 \\
\hline
\end{tabular}

YES

WAC 173-340 3-part test is is WAC 173-340 3-part test is

Xavall nonradionuclide data
set. Use nonparametric

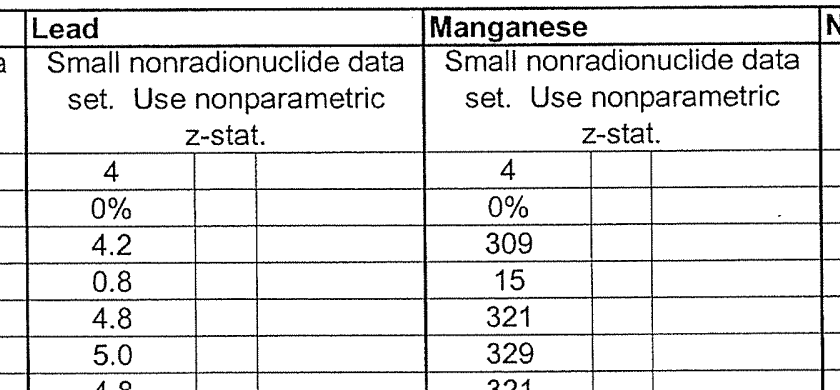

Vanadium

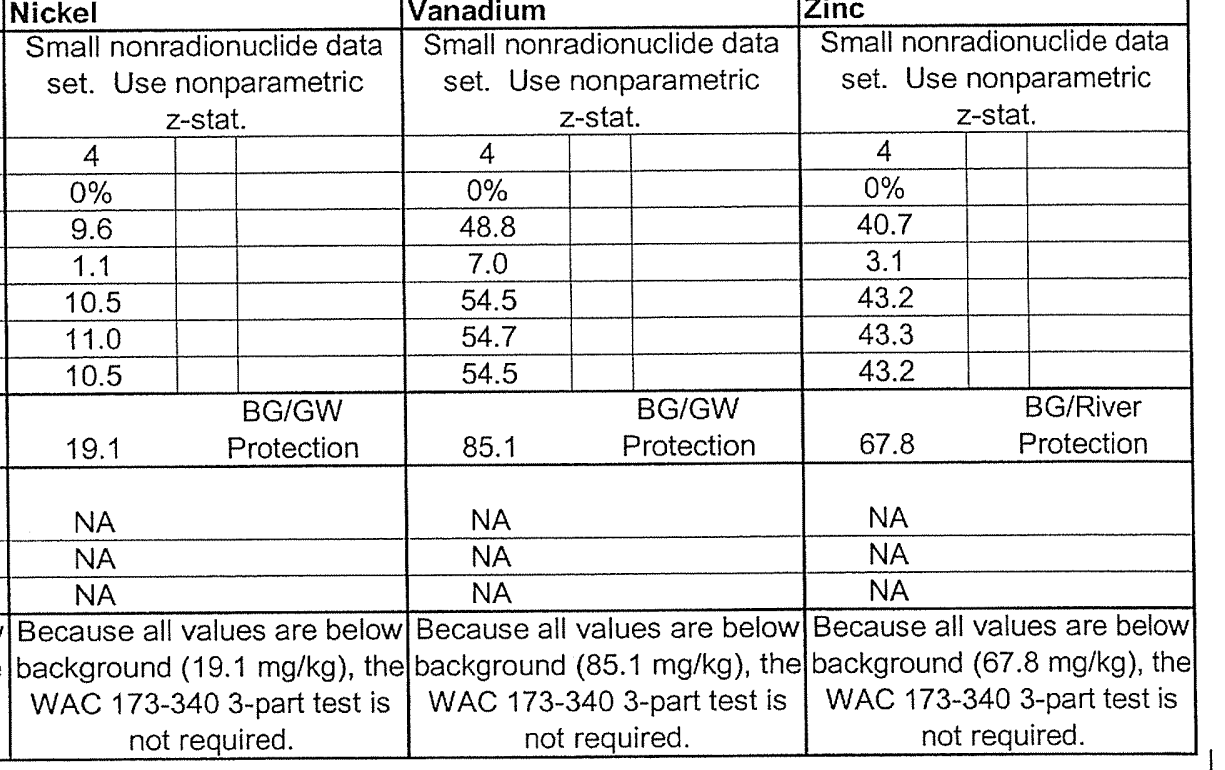

$32 B \mathrm{BG}=$ background

$33 \mathrm{GW}=$ groundwa

$34 \mathrm{~J}=$ estimate
$35 \mathrm{NA}=$ not applicable

$36 \mathrm{PQL}=$ proctical quantitation lim $Q=$ qualifier

$R A G=$ remedial action goal
$U=$ undetected

$U=$ undetected
$U C L=$ upper confidence limit

WAC $=$ Washington Administrative Code

\begin{tabular}{|c|c|c|c|c|c|c|c|c|c|c|c|}
\hline \multirow{2}{*}{\multicolumn{2}{|c|}{$\begin{array}{c}\text { set. Use nonparametric } \\
\text { z-stat. }\end{array}$}} & \multicolumn{2}{|c|}{$\begin{array}{l}\text { set. Use nonparametric } \\
\text { z-stat. }\end{array}$} & \multicolumn{2}{|c|}{$\begin{array}{c}\text { set. Use nonparametric } \\
\text { z-stat. }\end{array}$} & \multicolumn{2}{|c|}{$\begin{array}{l}z \text {-stat. } \\
\text { usmeiric }\end{array}$} & \multicolumn{2}{|c|}{ 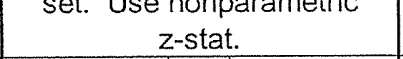 } & \multicolumn{2}{|c|}{$z$-stat. } \\
\hline & & 4 & & 4 & & 4 & & 4 & & 4 & \\
\hline $50 \%$ & & $0 \%$ & & $0 \%$ & & $0 \%$ & & $0 \%$ & & $0 \%$ & \\
\hline 0.20 & & 4.2 & & 309 & & 9.6 & & 48.8 & & 40.7 & \\
\hline 0.12 & & 0.8 & & 15 & & 1.1 & & & & 3.1 & \\
\hline 0.29 & & 4.8 & & 321 & & 10.5 & & 54.5 & & 43.2 & \\
\hline 0.33 & & 5.0 & & 329 & & 11.0 & & 54.7 & & & \\
\hline \multirow{2}{*}{\multicolumn{2}{|c|}{ er Protection }} & 4.8 & & 321 & & 10.5 & & 54.5 & & 43.2 & \\
\hline & & & $\begin{array}{c}\text { BG/GW \& River } \\
\text { Protection }\end{array}$ & & $\begin{array}{l}\text { BG/GW \& River } \\
\text { Protection }\end{array}$ & 19.1 & $\begin{array}{l}\mathrm{BG} / \mathrm{GW} \\
\text { Protection }\end{array}$ & 85.1 & $\begin{array}{l}\text { BG/GW } \\
\text { Protection }\end{array}$ & 67.8 & $\begin{array}{l}\text { BG/River } \\
\text { Protection }\end{array}$ \\
\hline \multirow{2}{*}{\multicolumn{2}{|c|}{ NO }} & \multirow{2}{*}{\multicolumn{2}{|c|}{ NA }} & \multicolumn{2}{|l|}{ NA } & \multicolumn{2}{|l|}{ NA } & \multicolumn{2}{|l|}{$\mathrm{NA}$} & \multicolumn{2}{|l|}{$\mathrm{NA}$} \\
\hline & & & & \multirow{2}{*}{\multicolumn{2}{|c|}{$\frac{N A}{N A}$}} & \multirow{2}{*}{\multicolumn{2}{|c|}{$\begin{array}{l}\text { NA } \\
\text { NA }\end{array}$}} & \multirow{2}{*}{\multicolumn{2}{|c|}{$\begin{array}{l}\text { NA } \\
\text { NA }\end{array}$}} & \\
\hline \multicolumn{2}{|l|}{$\frac{\text { NO }}{\text { NO }}$} & \multicolumn{2}{|l|}{$\frac{N A}{N A}$} & & & & & & & & \\
\hline \multicolumn{2}{|c|}{$\begin{array}{l}\text { The data set meets the } 3- \\
\text { part test criteria when } \\
\text { compared to the most }\end{array}$} & $\begin{array}{l}\text { Because } \\
\text { backgrour } \\
\text { WAC 17: }\end{array}$ & $\begin{array}{l}\text { values are below } \\
(10.2 \mathrm{mg} / \mathrm{kg}) \text {, the } \\
340 \text { 3-part test is }\end{array}$ & \multicolumn{2}{|c|}{$\begin{array}{l}\text { Because all values are } \\
\text { background }(512 \mathrm{mg} / \mathrm{kg}\end{array}$} & \multirow{2}{*}{\multicolumn{2}{|c|}{$\begin{array}{l}\text { Because all values are below } \\
\text { background }(19.1 \mathrm{mg} / \mathrm{kg} \text { ), the } \\
\text { WAC } 173-3403 \text {-part test is }\end{array}$}} & \multirow{2}{*}{\multicolumn{2}{|c|}{$\begin{array}{c}\text { Because all values are below } \\
\text { background (85.1 } \mathrm{mg} / \mathrm{kg} \text { ), the } \\
\text { WAC 173-340 3-part test is } \\
\text { not required. }\end{array}$}} & \multirow{2}{*}{\multicolumn{2}{|c|}{$\begin{array}{c}\text { Because all values are below } \\
\text { background }(67.8 \mathrm{mg} / \mathrm{kg} \text { ), the } \\
\text { WAC } 173-3403 \text {-part test is } \\
\text { not required. }\end{array}$}} \\
\hline string & cleanup limit. & & required. & & required. & & & & & & \\
\hline
\end{tabular}
compared to the most
tringent cleanup limit. not required. not required. not required. not required. 
Washington Closure Hanford

Originator J. M. Capron G 230

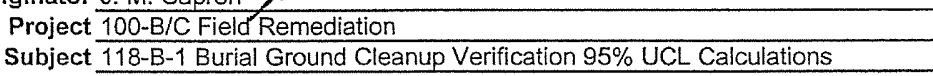

CALCULATION SHEET
Date $\frac{02107107}{14655}$
Job No.

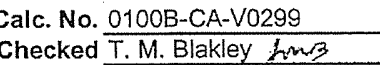

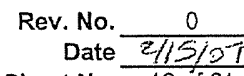
Date $\frac{2 / 15 / D T}{12 \text { of } 61}$
Sheet No.

118-B-1) Area 2 Statistical Calculations (continued)

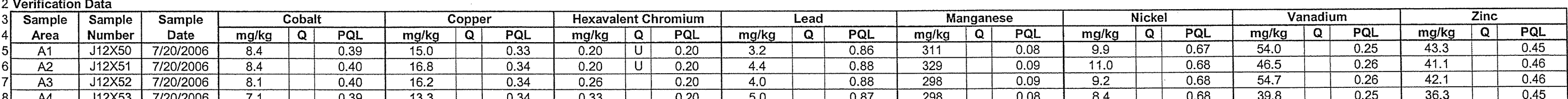

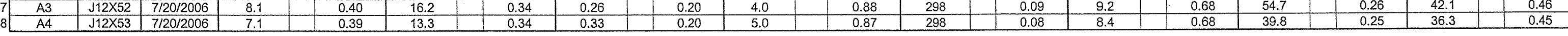

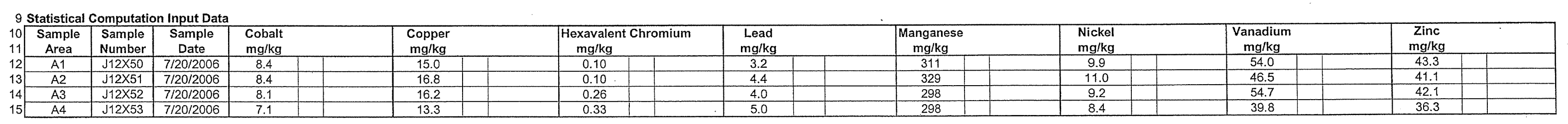

6 Statistical Computations

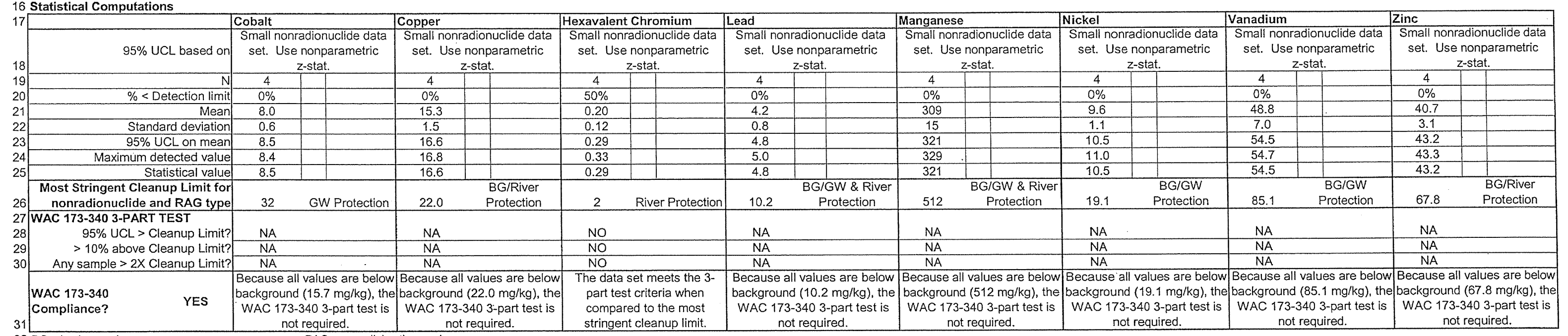

31 Complance

YES

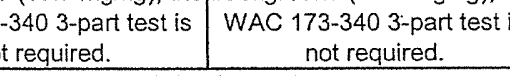

$3 \mathrm{GW}=$ groundwate
$4 \mathrm{NA}=$ not applicable

$35 \mathrm{PQL}=$ practical quantitation lim

WCL $=$ upper confidence limit
WAC = Washington Administrative Code 


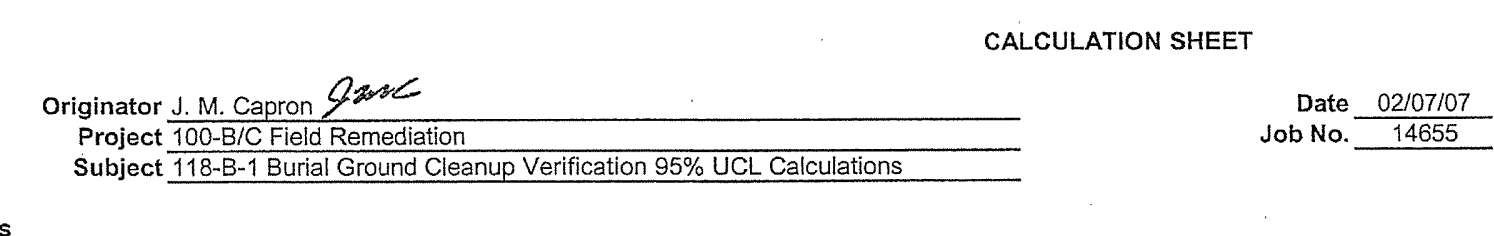

1 118-B-1 Area 3 Statistical Calculations

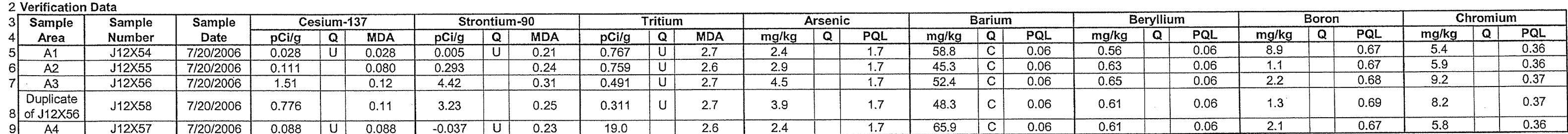

10 Statistical Computation Input Data

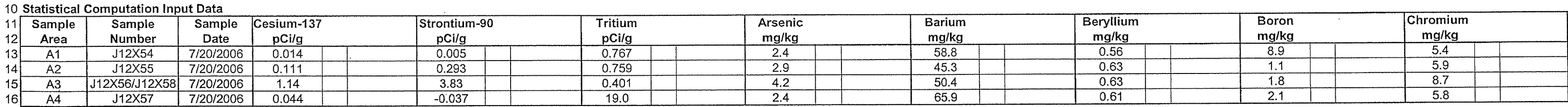

17 Statistical Computations

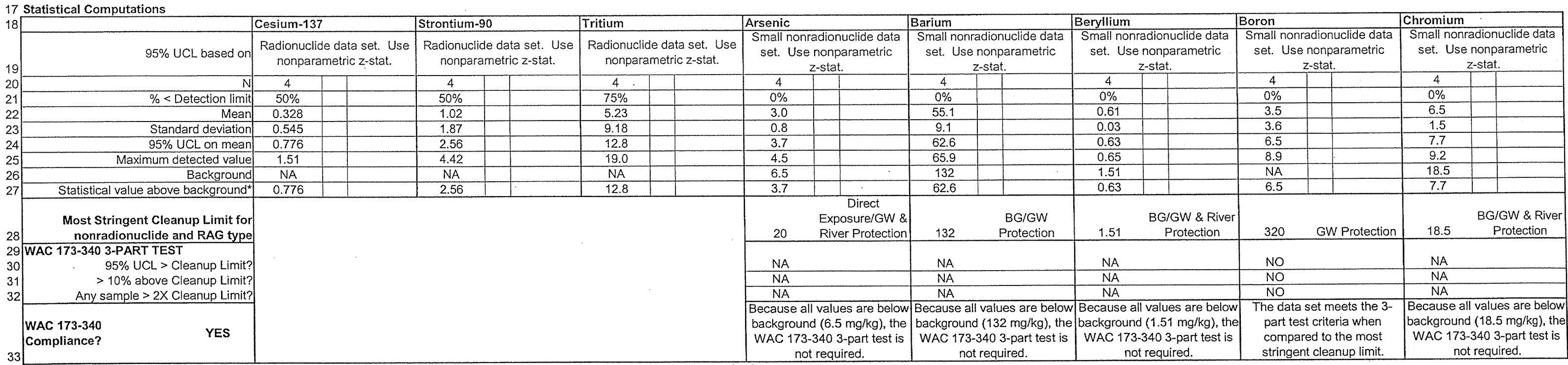

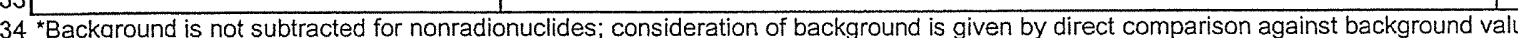

$35 \mathrm{BG}=$ background

$Q=$ qualifier
$R A G=$ remedial action goal
$U=$ undetected

$37 \mathrm{GW}=$ groundanater contamination

$39 \mathrm{NA}=$ not applicable
$40 \mathrm{PQL}=$ practical quantitation limit

$U=$ undetected
UCL = upper onfidence linit
WAC $=$ Washington Administrative Code 
CALCULATION SHEET

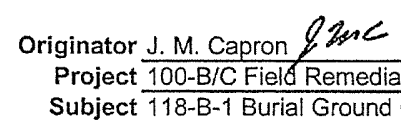

\section{118-B-1 Area 3 Statistical Calculations (continued)}

\begin{tabular}{|c|c|c|c|c|c|c|c|c|c|c|c|c|c|c|c|c|c|c|c|c|c|c|c|c|c|}
\hline \multirow{2}{*}{\begin{tabular}{l|l|l}
2 & Sartication Da \\
4 & Sarea & \\
\end{tabular}} & \multirow{2}{*}{$\begin{array}{l}\text { Sample } \\
\text { Sumber } \\
\text { Number }\end{array}$} & \multirow{2}{*}{\begin{tabular}{|c|} 
Sample \\
Date \\
$72 / 212006$ \\
\end{tabular}} & \multicolumn{3}{|c|}{ Cobalt } & \multicolumn{3}{|c|}{ Copper } & \multicolumn{3}{|c|}{ Lead } & \multicolumn{3}{|c|}{ Manganese } & \multicolumn{3}{|c|}{ Mercury } & \multicolumn{3}{|c|}{ Nickel } & & \multicolumn{3}{|c|}{ Zinc } \\
\hline & & & $\mathrm{mglkg}$ & \begin{tabular}{l|l|l|l|l|l|l} 
& \\
\end{tabular} & $\begin{array}{ll}P Q L \\
30\end{array}$ & $\mathrm{mg} / \mathrm{kg}$ & Q & $\frac{P Q L}{232}$ & $\mathrm{mg} / \mathrm{kg}$ & 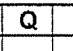 & $P Q L$ & $\begin{array}{l}\mathrm{mg} / \mathrm{kg} \\
20 \mathrm{~g}\end{array}$ & $Q$ & $P Q L$ & $\mathrm{mg} / \mathrm{kg}$ & $Q$ & $\frac{P Q L}{P Q 1}$ & $\frac{\mathrm{mg} / \mathrm{kg}}{10 \mathrm{~g}}$ & $a$ & $\begin{array}{ll}P Q L \\
067\end{array}$ & $\mathrm{mg} / \mathrm{kg}$ & \begin{tabular}{|l|l|}
$Q$ & $P Q L$ \\
\end{tabular} & $\mathrm{mg} / \mathrm{kg}$ & Q & $\frac{P Q}{0.4}$ \\
\hline$\frac{A_{1}}{A 2}$ & $\begin{array}{l}312 \times 55 \\
12 \times 55\end{array}$ & $\frac{1212012006}{712006}$ & $\begin{array}{l}7.8 \\
8.8 \\
y\end{array}$ & & $\begin{array}{l}0.39 \\
0.39 \\
\end{array}$ & $\begin{array}{r}15.6 \\
16.6 \\
\end{array}$ & & $\begin{array}{l}0.33 \\
0.34 \\
\end{array}$ & 4.6. & & $\begin{array}{l}0.86 \\
0.87 \\
\end{array}$ & $\frac{297}{320}$ & & 0.08 & $\begin{array}{l}0.04 \\
0.01 \\
\end{array}$ & u & $\begin{array}{l}0.01 \\
0.01 \\
\end{array}$ & $\frac{19.0}{9.3}$ & & 00.67 & $\frac{42.6}{49.2}$ & $\frac{0.25}{0.25}$ & $\frac{5.5}{43.2}$ & & \begin{tabular}{|l}
.45 \\
0.45 \\
0.45
\end{tabular} \\
\hline $\mathrm{A3}$ & $\sqrt{112 \times 56}$ & $7 / 20 / 2006$ & 8.9 & & 0.40 & 18.0 & & 0.34 & 6.6 & & 0.88 & 339 & & 0.08 & 0.01 & & 0.01 & 10.5 & & 0.68 & 40.0 & 0.25 & 44.3 & & 0.45 \\
\hline $8\left|\begin{array}{c}\text { ofpplicate } \\
\text { of } 122 \times 56\end{array}\right|$ & $\mathrm{J} 12 \times 58$ & $7 / 20 / 2006$ & 8.9 & & 0.40 & 16.9 & & 0.34 & 5.9 & & 0.89 & 325 & & 0.09 & 0.02 & u & 0.02 & 10.1 & & 0.69 & 37.6 & 0.26 & 41.9 & & 0.46 \\
\hline 年 & J12X57 & $7 / 2012006$ & 9.1 & & 0.39 & 16.1 & & 0.34 & 4.5 & & 0.87 & 390 & & 0.08 & 0.30 & & 0.01 & 9.6 & & 0.67 & 44.9 & 0.25 & 42.2 & & 0.4 \\
\hline
\end{tabular}

10 Statistical Computation Input Data

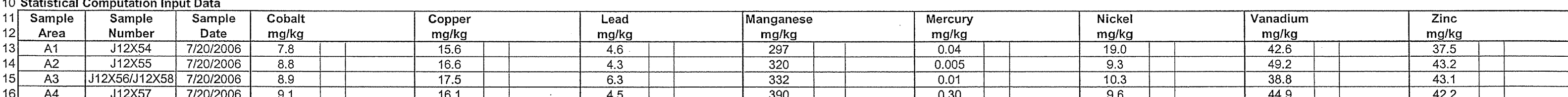

17 Statistical Computations

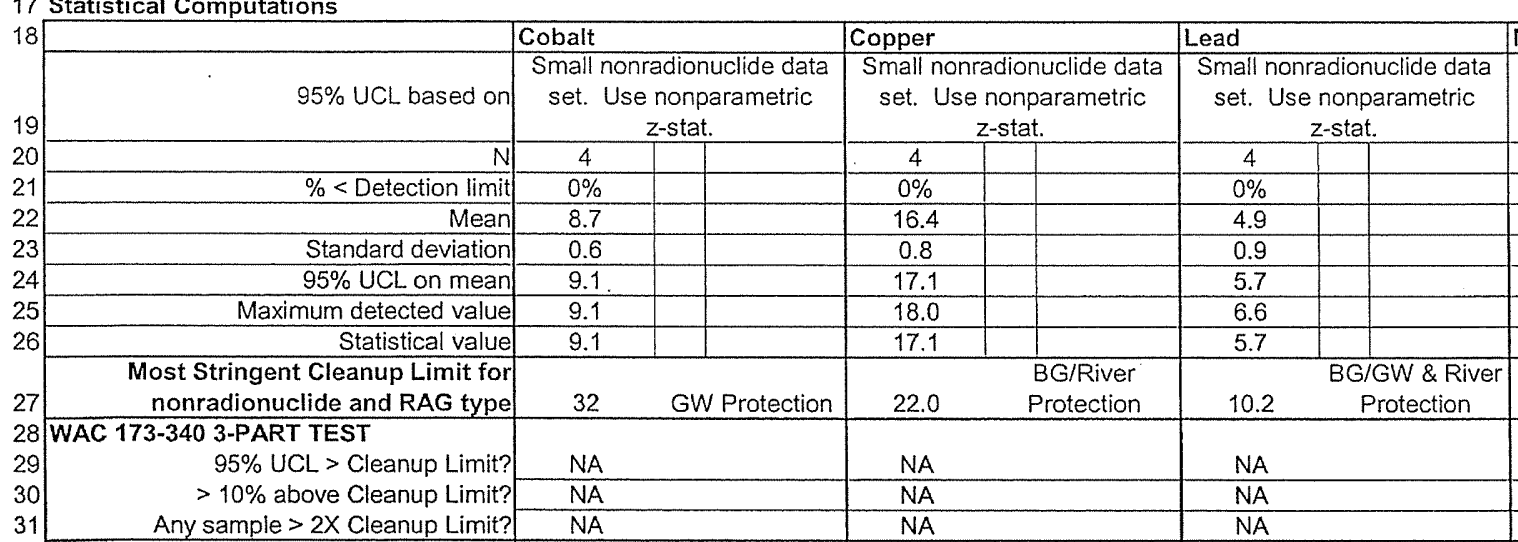
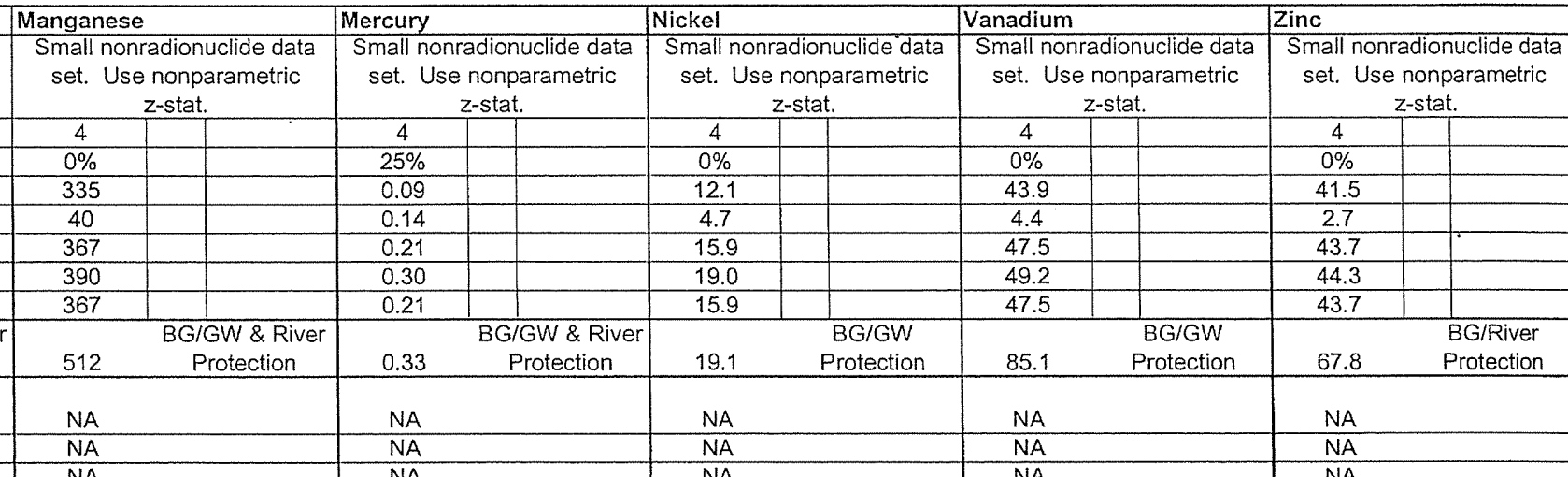

WAC $173-340$
Compliance?

YES

ecause all values are below Because all values are below Because all values are below
background $(15.7 \mathrm{mg} / \mathrm{kg}$ ), the background (22.0 $\mathrm{mg} / \mathrm{kg}$ ), the background (10.2 $\mathrm{mg} / \mathrm{kg}$ ), the
WAC $173-340$ - 3 -part test is

NA

NA

32

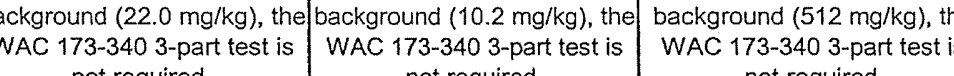

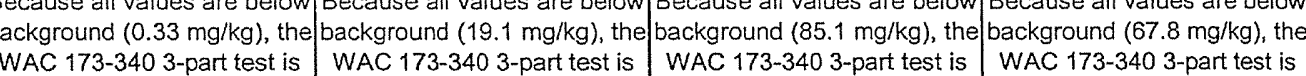

$33 \mathrm{BG}=$ background
$34 \mathrm{GW}=$ groundwater
$35 \mathrm{NA}=$ not applical

$35 \mathrm{NA}=$ not applicable
$36 \mathrm{PQL}=$ practical quantitation limit

$S=$ undelected
UCL

$36 \mathrm{QQL}=$ pracitial

WAC $=$ Washington Administrative code 
Washington Closure Hanford

CALCULATION SHEET
(a)

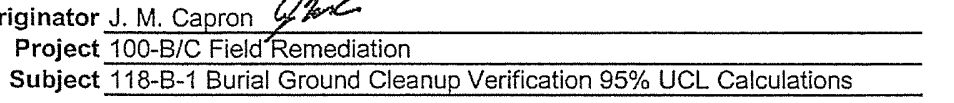
Date $\frac{02 / 07 / 07}{14655}$
Job No.

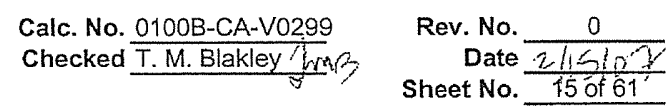

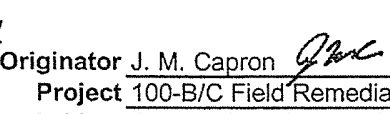

1 118-B-1 Area 3 Statistical Calculations (continued)

\begin{tabular}{|c|c|c|c|c|c|c|c|c|c|c|c|c|c|c|c|c|c|c|c|c|}
\hline Sample & Sample & & bis(2-Ethyll & hexy & phthalate & Di-n-b & utylph & thalate & 4-Meth & 1-2-pe & thanone & & ceton & & Carbor & Tetra & thoride & Methyle & $\mathrm{ne} \mathrm{Ch}$ & loride \\
\hline Area & Number & Date & $\mathrm{mg} / \mathrm{kg}$ & $Q$ & $\mathrm{PQL}$ & $\mathrm{mg} / \mathrm{kg}$ & Q & $\mathrm{PQL}$ & $\mathrm{mg} / \mathrm{kg}$ & $a$ & $\mathrm{PQL}$ & $\mathrm{mg} / \mathrm{kg}$ & $\begin{array}{l}Q \\
\end{array}$ & $\mathrm{PQL}$ & $\mathrm{mg} / \mathrm{kg}$ & 10 & $P Q L$ & $\mathrm{mg} / \mathrm{kg}$ & $Q$ & $P Q L$ \\
\hline$\frac{A 1}{A 2}$ & $\frac{\mathrm{J} 12 \times 54}{\mathrm{~J} 2 \times 54}$ & $\frac{7120 / 21}{77 / 2012}$ & \begin{tabular}{|l|}
0.017 \\
0.34
\end{tabular} & $\frac{J}{u}$ & $\begin{array}{l}0.33 \\
0.34\end{array}$ & \begin{tabular}{|l}
0.33 \\
0.34
\end{tabular} & \begin{tabular}{|l|}
$U$ \\
$u$
\end{tabular} & $\begin{array}{l}0.33 \\
0.34\end{array}$ & $\begin{array}{l}0.006 \\
0.006\end{array}$ & $|\sqrt{B}|$ & $\frac{0.010}{0.010}$ & $\frac{0.015}{0.016}$ & & $-\frac{0.0}{0.0}$ & $\frac{0.005}{0.008}$ & u & $\begin{array}{l}0.005 \\
0.005\end{array}$ & $\begin{array}{l}0.026 \\
0.005\end{array}$ & & 0.005 \\
\hline $\mathrm{A3}$ & $\mathrm{J} 12 \times 56$ & $7 / 201 / 2006$ & 0.34 & u & 0.044 & 0.027 & \begin{tabular}{|l} 
JB \\
\end{tabular} & $\frac{0.04}{0.34}$ & & $\frac{u}{u}$ & 0.010 & $\begin{array}{l}0.007 \\
0.007\end{array}$ & J & 0.010 & 0.005 & U & 0.005 & 0.005 & $\mathrm{U}$ & \\
\hline $\begin{array}{l}\text { Duplicate } \\
\text { of } 111275\end{array}$ & J12X58 & $7 / 20 / 2006$ & 0.039 & $J$ & 0.34 & 0.054 & JB & 0.34 & 0.010 & u & 0.010 & 0.008 & J & 0.010 & 0.005 & u & 0.005 & 0.005 & $u$ & 0.005 \\
\hline A4 & $\sqrt{ } 12 \times 57$ & $7 / 20 / 2006$ & 0.031 & J & 0.34 & 0.040 & $\mid \mathrm{JB}$ & 0.34 & 0.010 & u & 0.010 & 0.006 & J & 0.010 & 0.011 & & 0.005 & 0.005 & & 0.005 \\
\hline
\end{tabular}

10 Statistical Computation Input Data

\begin{tabular}{|c|c|c|c|c|c|c|c|c|}
\hline $\begin{array}{c}11 \\
12\end{array} \begin{array}{l}\text { Sample } \\
\text { Area }\end{array}$ & $\begin{array}{l}\text { Sample } \\
\text { Number }\end{array}$ & $\begin{array}{c}\text { Sample } \\
\text { Date }\end{array}$ & $\begin{array}{l}\text { bis(2-Ethylhexyl)phthalate } \\
\text { mg/kg }\end{array}$ & $\begin{array}{l}\text { Di-n-butylphthalate } \\
\text { mg/kg }\end{array}$ & $\begin{array}{l}\text { 4-Methyl-2-pentanone } \\
\mathrm{mg} / \mathrm{kg}\end{array}$ & $\begin{array}{c}\text { Acetone } \\
\mathrm{mg} / \mathrm{kg}\end{array}$ & \begin{tabular}{|c|} 
Carbon Tetrachloride \\
$\mathrm{mg} / \mathrm{kg}$
\end{tabular} & $\begin{array}{l}\text { Methylene Chlorid } \\
\mathrm{mg} / \mathrm{kg}\end{array}$ \\
\hline$\frac{A 1}{A 2}$ & $\begin{array}{l}\frac{J 12254}{J 12 \times 55} \\
\end{array}$ & $\frac{7720 / 20006}{7 / 202 / 2006}$ & \begin{tabular}{|l|}
0.017 \\
0.17
\end{tabular} & \begin{tabular}{|l|l|}
0.17 \\
0.17
\end{tabular} & \begin{tabular}{|l|l|}
0.006 \\
0.006
\end{tabular} & \begin{tabular}{|l|l}
0.015 \\
0.016
\end{tabular} & \begin{tabular}{|c|c|}
0.003 \\
0.008
\end{tabular} & \begin{tabular}{|l|l|}
0.026 \\
0.005
\end{tabular} \\
\hline$\frac{\mathrm{A} 3}{\mathrm{~A} 4}$ & $\frac{12 \times 56 / 112 \times 5}{112 \times 7}$ & $\begin{array}{ll}7 / 20 / 20006 \\
7 / 720 / 2006\end{array}$ & $\begin{array}{l}0.10 \\
0.031\end{array}$ & \begin{tabular}{|l|l|}
0.041 \\
0.040
\end{tabular} & \begin{tabular}{|l|l|}
0.005 \\
0.005
\end{tabular} & \begin{tabular}{|l|}
0.008 \\
0.006 \\
\end{tabular} & \begin{tabular}{|l|l|}
0.003 \\
0.011
\end{tabular} & \begin{tabular}{|l|l|}
0.003 \\
0.005 \\
\end{tabular} \\
\hline
\end{tabular}

17 Statistical Computations

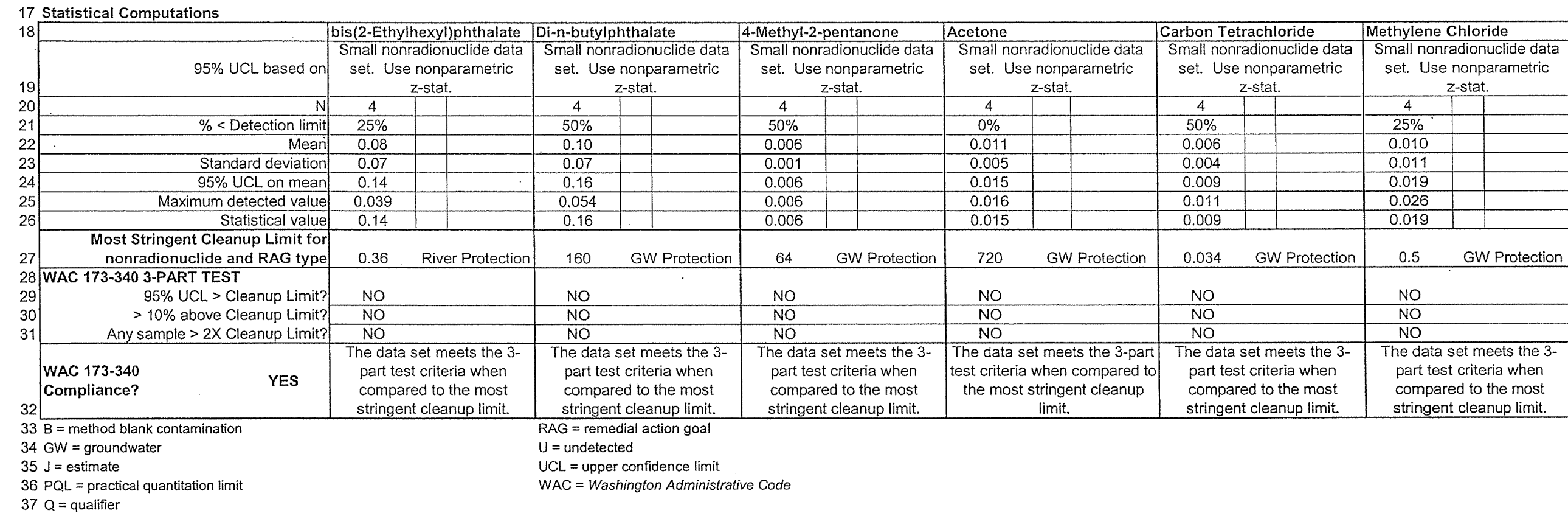


1 118-B-1 Area 4 Statistical Calculations

\begin{tabular}{|c|c|c|c|c|c|c|c|c|c|c|c|c|c|c|c|c|c|c|c|c|c|c|c|c|c|c|}
\hline \multirow{2}{*}{\begin{tabular}{|c|c|}
3 & Sample \\
4 & Area \\
5 & A1 \\
\end{tabular}} & \multirow{2}{*}{$\begin{array}{l}\text { Sample } \\
\text { Number } \\
\text { J12X61 }\end{array}$} & \multirow{2}{*}{ 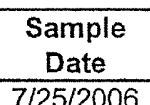 } & \multicolumn{3}{|c|}{ Cesium-137 } & \multicolumn{3}{|c|}{ Arsenic } & \multicolumn{3}{|c|}{ Barium } & \multicolumn{3}{|c|}{ Beryllium } & \multicolumn{3}{|c|}{ Chromium } & \multicolumn{3}{|c|}{ Cobalt } & \multicolumn{3}{|c|}{ Copper } & \multicolumn{3}{|c|}{ Hexavalent Chromium } \\
\hline & & & pCilg & $Q$ & MDA & $\frac{\mathrm{mg} / \mathrm{kg}}{25}$ & $Q$ & $P Q L$ & $\mathrm{mg} / \mathrm{kg}$ & $Q$ & $\mathrm{PQL}$ & $\mathrm{mg} / \mathrm{kg}$ & $Q$ & $\mathrm{PQL}$ & $\frac{\mathrm{mg} / \mathrm{kg}}{27}$ & $Q$ & $\begin{array}{l}P Q L \\
\Omega^{36}\end{array}$ & $\frac{\mathrm{mg} / \mathrm{kg}}{84}$ & $Q$ & $P Q L$ & $\frac{\mathrm{mg} / \mathrm{kg}}{155}$ & & $\frac{P Q L}{0.34}$ & & Q & $\frac{P Q L}{0.20}$ \\
\hline$\frac{\text { A1 }}{\text { Duplicate }}$ & $\frac{112 \times 61}{\sqrt{1} 2 \times 65}$ & $\begin{array}{l}7 / 25 / 2006 \\
7 / 25 / 2006\end{array}$ & $\begin{array}{l}0.738 \\
0.260\end{array}$ & & $\begin{array}{l}0.048 \\
0.033\end{array}$ & $\frac{2.5}{3.3}$ & & $\begin{array}{l}\frac{1.7}{1.7} \\
\end{array}$ & $\begin{array}{l}39.1 \\
51.4\end{array}$ & & $\begin{array}{l}0.06 \\
0.06\end{array}$ & $\begin{array}{l}0.42 \\
0.34\end{array}$ & & $\begin{array}{l}0.06 \\
0.06\end{array}$ & $\begin{array}{l}3.7 \\
5.1\end{array}$ & & $\begin{array}{l}0.36 \\
0.36\end{array}$ & 8.4 & & $\begin{array}{l}0.39 \\
0.39\end{array}$ & $\begin{array}{l}15.5 \\
15.7\end{array}$ & & $\begin{array}{l}0.34 \\
0.34\end{array}$ & $\begin{array}{l}0.30 \\
0.20\end{array}$ & $u$ & 0.20 \\
\hline 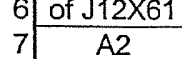 & $J 12 x$ & & 0.022 & $U$ & 0.0 & 2.8 & & 1. & 47 & & 0.0 & 0.2 & & & 5.4 & & 0. & 7. & & & 14 & & & & U & 20 \\
\hline$\frac{A 2}{A 3}$ & $\frac{12 \mathrm{AOC}}{\mathrm{J} 12 \times 63}$ & $\frac{1126}{7 / 25}$ & $\begin{array}{l}0.024 \\
0.084 \\
\end{array}$ & & $\begin{array}{ll}0.026 \\
0.047\end{array}$ & 3.9 & & 1.7 & & & & 0. & & & 6.5 & & 0.36 & 8.3 & & 0.39 & 16.5 & & 0.34 & 0.20 & U & 0.20 \\
\hline$\overline{\mathrm{A} 4}$ & $\sqrt{112 \times 64}$ & $125 / 2006$ & 0,047 & $\bar{U}$ & 0.047 & 28 & & 1.7 & 49.2 & & 0.06 & 0.40 & & 0.06 & 6.7 & & 0.36 & 8.6 & & 0.39 & 15.6 & & 0.33 & 0.24 & & 0.20 \\
\hline
\end{tabular}

10 Statistical Computation Input Data

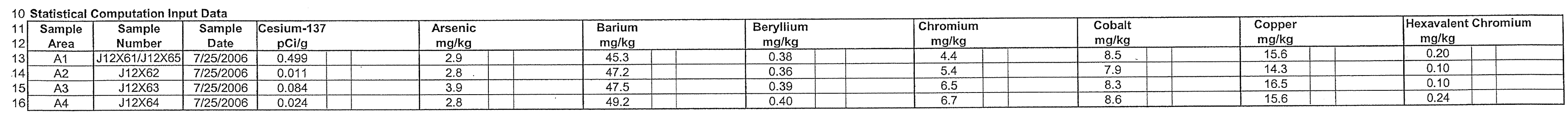

\section{Statistical Computations}

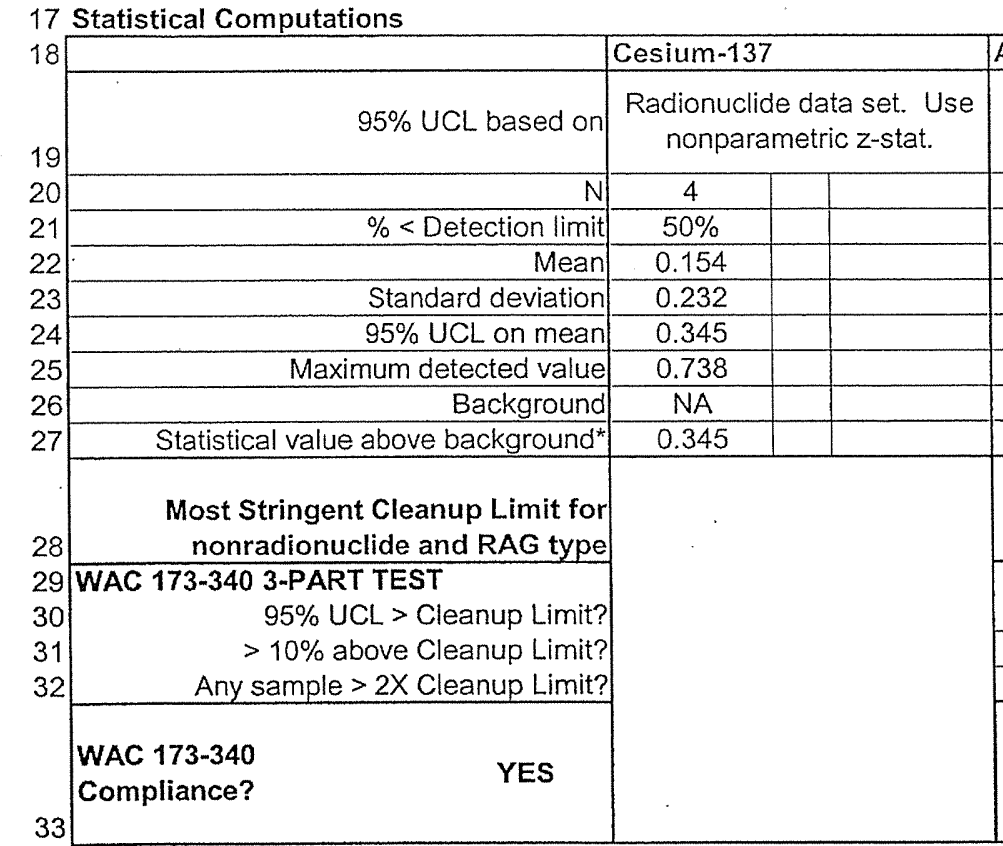

33 Compliance?
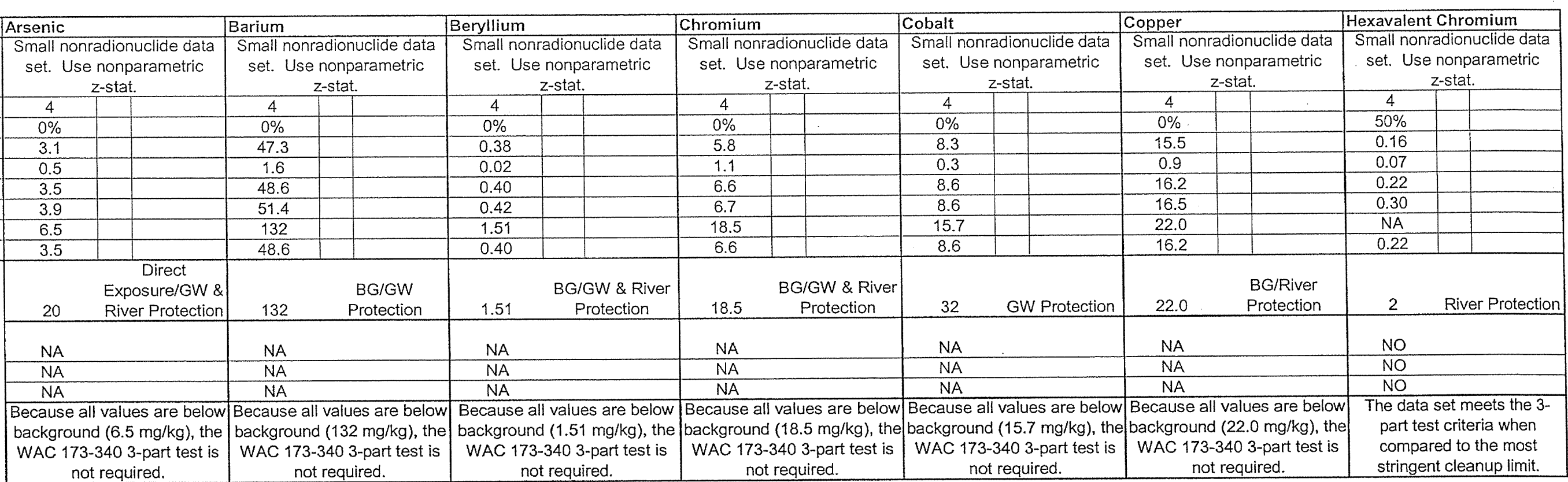

34 * Background is no
$35 \mathrm{BG}=$ background
$36 \mathrm{GW}=$ groundwater

$36 \mathrm{GW}=$ groundwater
$37 \mathrm{MDA}=$ minimum detectable activity

$38 \mathrm{NA}=$ not applicable

$39 \mathrm{PQL}=$ practical quantitation limit

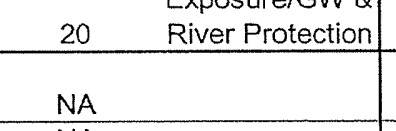

NA

\begin{tabular}{l|l|l} 
NA & NA & NA \\
\hline
\end{tabular}

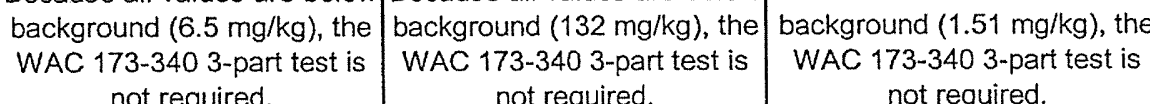
\begin{tabular}{c|c|c} 
not required. & not required. & not required. \\
&
\end{tabular}

$Q=$ qualifier
RAG $=$ remedia

$R A G=$ remedial
$U=$ undetected

UCL $=$ upper confidence limit
WAC $=$ Washington Administrative Code 
Originator J.M. Capron 9 manC Subject 118-B-B-1 Burrial Ground Cleanup Verification 95\% UCL Calculation

1118-B-1 Area 4 Statistical Calculations (continued)

\begin{tabular}{|c|c|c|c|c|c|c|c|c|c|c|c|c|c|c|c|c|c|c|c|c|c|c|c|c|c|c|}
\hline Sample & Sample & Sample & & Lea & & & garar & & & Vicke & & & nadi & & & Zinc & & bis (2-Eth) & hexyl & phthalate & $D i-n-t$ & utylph & thalate & & heno & \\
\hline$\frac{\text { Area }}{\text { A1 }}$ & $\begin{array}{l}\text { Number } \\
12 \times 61\end{array}$ & $\frac{\text { Date }}{7 / 25 / 2006}$ & $\frac{\mathrm{mg} / \mathrm{kg}}{52}$ & $Q$ & $\frac{P Q L}{0.87}$ & $\frac{\mathrm{mg} / \mathrm{kg}}{290}$ & $Q$ & $\frac{\mathrm{PQL}}{0.08}$ & $\frac{\mathrm{mg} / \mathrm{kg}}{6.8}$ & $Q$ & $\frac{P Q L}{0.67}$ & $\frac{\mathrm{mg} / \mathrm{kg}}{53.4}$ & $Q$ & $\begin{array}{l}\mathrm{PQL} \\
0.25\end{array}$ & $\frac{\mathrm{mg} / \mathrm{kg}}{41.7}$ & \begin{tabular}{|l|}
$\mathbf{Q}$ \\
$\mathrm{C}$ \\
\end{tabular} & $\frac{\mathrm{PQL}}{0.45}$ & $\frac{\mathrm{mg} / \mathrm{kg}}{0.046}$ & \begin{tabular}{|l|}
$\mathrm{Q}$ \\
$\mathrm{JB}$ \\
\end{tabular} & $\begin{array}{l}\mathrm{PQL} \\
0.34\end{array}$ & $\frac{\mathrm{mg} / \mathrm{kg}}{0.038}$ & $\begin{array}{l}Q \\
J B\end{array}$ & $\begin{array}{l}\mathrm{PQL} \\
0.34\end{array}$ & $\frac{\mathrm{mg} / \mathrm{kg}}{0.34}$ & \begin{tabular}{|l|}
$Q$ \\
$U$ \\
\end{tabular} & $\frac{P Q L}{0.34}$ \\
\hline Duplicate & J12X65 & $7 / 25 / 2006$ & 5.5 & & 0.87 & 292 & & 0.08 & 11.3 & & 0.67 & 44.8 & & 0.25 & 41.0 & c & 0.45 & 0.035 & $J B$ & 0.34 & 0.063 & $\mathrm{JB}$ & 0.34 & 0.34 & $u$ & 0.34 \\
\hline$\frac{12 \times 0}{A 2}$ & $\sqrt{112 \times 62}$ & $7 / 25 / 2006$ & 3.3 & & 0. & 299 & & 0.6 & 9.3 & & 0.66 & 43.5 & & 0.25 & 35.8 & c & 0.4 & 0.029 & JB & 0.3 & 0.0 & $\mathrm{JB}$ & 0.33 & 0.026 & $\mathrm{JB}$ & 0.33 \\
\hline$\frac{\mathrm{A} 3}{\mathrm{~A} 4}$ & $\frac{J 12 \times 63}{J 12 \times 64}$ & $\begin{array}{l}7 / 25 / 2006 \\
7 / 25 / 2006\end{array}$ & $\begin{array}{l}4.4 \\
3.6\end{array}$ & & $\begin{array}{l}0.87 \\
0.86\end{array}$ & $\begin{array}{l}334 \\
336\end{array}$ & & $\begin{array}{l}0.08 \\
0.08\end{array}$ & $\frac{10.2}{10.9}$ & & $\frac{0.67}{0.66}$ & $\frac{41.6}{50.8}$ & & 0.25 & $\frac{39.5}{42.4}$ & \begin{tabular}{|l|}
$\mathrm{C}$ \\
$\mathrm{C}$
\end{tabular} & 0.44 & $\begin{array}{l}0.60 \\
0.065\end{array}$ & \begin{tabular}{|l|}
$\mathrm{B}$ \\
$\mathrm{JB}$ \\
\end{tabular} & $\begin{array}{l}0.044 \\
0.34\end{array}$ & $\begin{array}{l}0.058 \\
0.062\end{array}$ & \begin{tabular}{|l|}
$\mathrm{JB}$ \\
$\mathrm{JB}$
\end{tabular} & $\begin{array}{l}0.34 \\
0.34\end{array}$ & $\begin{array}{l}0.34 \\
0.022\end{array}$ & JB & $\begin{array}{l}0.34 \\
0.34\end{array}$ \\
\hline
\end{tabular}

10 Statistical Computation Input Data

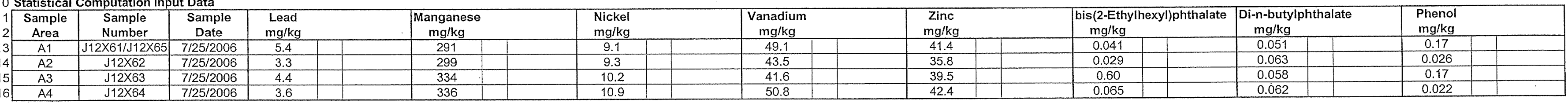

\begin{tabular}{|c|c|c|c|c|c|c|c|c|c|c|c|c|c|c|c|c|c|}
\hline \multirow[b]{3}{*}{$\begin{array}{l}19 \\
20\end{array}$} & \multirow{3}{*}{$95 \%$ UCL based on } & \multirow{2}{*}{\multicolumn{2}{|c|}{\begin{tabular}{|l|} 
Lead \\
Small nonradionuclide data \\
set. Use nonparametric
\end{tabular}}} & \multirow{2}{*}{\multicolumn{2}{|c|}{\begin{tabular}{|l|} 
Manganese \\
Small nonradionuclide data \\
set. Use nonparametric
\end{tabular}}} & \multirow{2}{*}{\multicolumn{2}{|c|}{$\begin{array}{l}\text { Nickel } \\
\text { Small nonradionuclide data } \\
\text { set. Use nonparametric }\end{array}$}} & \multirow{2}{*}{\multicolumn{2}{|c|}{\begin{tabular}{|c|} 
Vanadium \\
Small nonradionuclide data \\
set. Use nonparametric \\
$z$-stat.
\end{tabular}}} & \multirow{2}{*}{\multicolumn{2}{|c|}{\begin{tabular}{l|} 
Zinc \\
Small nonradionuclide data \\
set. Use nonparametric \\
z-stat.
\end{tabular}}} & \multirow{2}{*}{\multicolumn{2}{|c|}{\begin{tabular}{|c|} 
bis(2-Ethylhexyl)phthalate \\
Small norradionuclide data \\
set. Use nonparametric \\
z-stat.
\end{tabular}}} & \multirow{2}{*}{\multicolumn{2}{|c|}{\begin{tabular}{|l|} 
Di-n-butylphthalate \\
Small nonradionuclide data \\
set. Use nonparametric \\
z-stat.
\end{tabular}}} & \multirow{3}{*}{\multicolumn{2}{|c|}{$\begin{array}{l}\text { Phenol } \\
\begin{array}{c}\text { Small nonradionuclide data } \\
\text { set. Use nonparametric } \\
\text { z-stat. }\end{array}\end{array}$}} \\
\hline & & & & & & & & & & & & & & & & & \\
\hline & & 4 & & 4 & & 4 & & & & & & & & & & & \\
\hline & $\%<$ Detection limit & $0 \%$ & & $0 \%$ & & $0 \%$ & & $0 \%$ & & $0 \%$ & & $0 \%$ & & $0 \%$ & & $50 \%$ & \\
\hline & Mean & 4.2 & & 315 & & 9.9 & & 46.3 & & 39.8 & & 0.18 & & 0.058 & & 0.10 & \\
\hline 23 & Standard deviation & 0.9 & & 23 & & 0.8 & & 4.4 & & 2.9 & & 0.28 & & 0.006 & & 0.08 & \\
\hline & 95\% UCL on mean & 4.9 & & 334 & & 10.6 & & 49.9 & & 42.1 & & 0.41 & & 0.063 & & 0.17 & \\
\hline & Maximum detected value & 5.5 & & 336 & & 11.3 & & 53.4 & & 42.4 & & 0.60 & & 0.063 & & 0.026 & \\
\hline & $\begin{array}{r}\text { Statistical value } \\
\end{array}$ & 4.9 & & 334 & & 10.6 & & 49.9 & & 42.1 & & 0.41 & & 0.063 & & 0.17 & 11 \\
\hline & $\begin{array}{r}\text { Most Stringent Cleanup Limit for } \\
\text { nonradionuclide and RAG type }\end{array}$ & & $\begin{array}{l}\text { BG/GW \& River } \\
\text { Protection }\end{array}$ & 512 & $\begin{array}{c}\text { BG/GW \& River } \\
\text { Protection }\end{array}$ & 19.1 & $\begin{array}{l}\text { BG/GW } \\
\text { Protection }\end{array}$ & 85.1 & $\begin{array}{l}\text { BGG/GW } \\
\text { Protection }\end{array}$ & 67.8 & $\begin{array}{l}\text { BG//River } \\
\text { Protection }\end{array}$ & 0.36 & River Protection & 160 & GW Protection & 480 & GW Protection \\
\hline & WAC 173-340 3-PART TEST & & & & & & & & & & & & & & & & \\
\hline 29 & $\begin{array}{l}\text { 95\% UCL > Cleannup Limit? } \\
>10 \% \text { above Cleanup Limit? }\end{array}$ & $\frac{N A}{N A}$ & & $\frac{N A}{N A}$ & & $\frac{N A}{N A}$ & & NA & & $\frac{N A}{N A}$ & & $\begin{array}{l}\text { YES } \\
\text { YES }\end{array}$ & & $\frac{\mathrm{NO}}{\mathrm{NO}}$ & & NO & \\
\hline & Any sample $>2 X$ Cleanup Limit? & $\mathrm{NA}$ & & $\mathrm{NA}$ & & NA & & NA & & $\mathrm{NA}$ & & NO & & NO & & NO & \\
\hline & $\begin{array}{c}\text { Further } \\
\text { Assessment } \\
\text { Required }\end{array}$ & $\begin{array}{r}\text { Because a } \\
\text { backgroun } \\
\text { WAC } 173 \\
\text { no }\end{array}$ & $\begin{array}{l}\text { values are below } \\
(10.2 \mathrm{mg} / \mathrm{kg}) \text {, the } \\
340 \text { 3-part test is } \\
\text { required. }\end{array}$ & $\begin{aligned} \mid \begin{array}{l}\text { Because } \\
\text { backgrou } \\
\text { WAC } 17 \\
n\end{array}\end{aligned}$ & $\begin{array}{l}\text { II values are below } \\
\text { d ( } 512 \mathrm{mg} / \mathrm{kg}) \text {, the } \\
-340 \text {-part test is } \\
\text { t required. }\end{array}$ & $\begin{array}{r}\text { Because al } \\
\text { backgrounc } \\
\text { WAC 173- } \\
\text { no }\end{array}$ & $\begin{array}{l}\text { alues are below } \\
19.1 \mathrm{mg} / \mathrm{kg} \text {, the } \\
.03 \text {-part test is } \\
\text { equired. }\end{array}$ & $\begin{array}{r}\text { Because all } \\
\text { background } \\
\text { WAC 173- } \\
\text { not }\end{array}$ & $\begin{array}{l}\text { alues are below } \\
35.1 \mathrm{mg} / \mathrm{kg} \text { ), the } \\
03 \text {-part test is } \\
\text { quired. }\end{array}$ & \begin{tabular}{|} 
Because all \\
background \\
WAC 173-3 \\
not 1
\end{tabular} & $\begin{array}{l}\text { llues are below } \\
77.8 \mathrm{mg} / \mathrm{kg} \text { ), the } \\
0 \text { 3-part test is } \\
\text { equired. }\end{array}$ & \begin{tabular}{|c|} 
Becal \\
answers t \\
detailed \\
RESRAD \\
The date \\
part te \\
compa \\
exposu \\
exps
\end{tabular} & $\begin{array}{l}\text { se of the "yes" } \\
\text { the 3-part test, a } \\
\text { ssessment using } \\
\text { will be performed. } \\
\text { set meets the } 3- \\
\text { t criteria when } \\
\text { ed to the direct } \\
\text { e cleanup level. }\end{array}$ & $\begin{array}{l}\text { The data } \\
\text { part tes } \\
\text { compar } \\
\text { stringer }\end{array}$ & $\begin{array}{l}\text { et meets the 3- } \\
\text { criteria when } \\
\text { ed to the most } \\
\text { cleanup limit. }\end{array}$ & $\begin{array}{l}\text { The data } \\
\text { part te: } \\
\text { compa } \\
\text { stringe }\end{array}$ & $\begin{array}{l}\text { set meets the 3- } \\
\text { st criteria when } \\
\text { red to the most } \\
\text { int cleanup limit. }\end{array}$ \\
\hline
\end{tabular}

$34 \mathrm{BG}=$ backgroun

$Q=$ qualifier

$35 \mathrm{C}=$ method blank $\mathrm{c}$
$36 \mathrm{GW}=$ groundwater

$R A G=$ remedial action goal

$38 \mathrm{NA}=$ not applicable

WAC $=$ upper confidence limit
Washington Administrative Code 
Originator J.M. Mapron GarC

CALCULATION

Subject 1118-B-B-1 Field Rurial Groediation

118-B-1 Area 4 Statistical Calculations

\begin{tabular}{|c|c|c|c|c|c|c|c|c|c|c|c|c|c|c|c|c|c|}
\hline$A$ & Sample & Sample & & cetor & & Carbon & Tetrac & hloride & Methyl & ene Chloride & & 2,4-DE & & Dala & apc & Dichlo & orr \\
\hline $\mathrm{Ar}$ & & $\begin{array}{c}\text { Date } \\
7 / 25 / 2006\end{array}$ & $\frac{\mathrm{mglkg}}{0.019}$ & $a$ & $\frac{P Q L}{0.010}$ & $\frac{m g / k g}{0.013}$ & $a$ & $\begin{array}{l}\mathrm{PQL} \\
0.005\end{array}$ & $\begin{array}{l}\mathrm{mg} / \mathrm{kg} \\
0.005 \\
\end{array}$ & $\frac{P Q L}{0.005}$ & $\frac{\mathrm{mg} / \mathrm{kg}}{0.017}$ & \begin{tabular}{|l|}
$a$ \\
$u$ \\
\end{tabular} & $\begin{array}{l}\mathrm{PQL} \\
0.017\end{array}$ & $\begin{array}{l}\mathrm{mg} / \mathrm{kg} g \\
0.029\end{array}$ & $\frac{P Q L}{0.034}$ & $\frac{\frac{m g / g}{0.034}}{0.034}$ & $\frac{P Q L}{0.034}$ \\
\hline & $J 12 \times E$ & $7 / 25 / 2006$ & 0.010 & u & 0. & 0.6 & $\mathrm{~J}$ & 0. & 0.6 & 0.005 & 0.0 & J & 0.017 & 0.048 & 0.034 & 0.034 & 4 \\
\hline 5 & $\begin{array}{l}J 12 \times 62 \\
J 12 \times 63\end{array}$ & $7 / 25 / 2006$ & $\begin{array}{l}0.011 \\
0.011\end{array}$ & & $\begin{array}{l}0.010 \\
0.010\end{array}$ & $\begin{array}{l}0.014 \\
0.017 \\
\end{array}$ & & $\begin{array}{l}0.005 \\
0.005 \\
\end{array}$ & $\begin{array}{l}0.008 \\
0.012 \\
\end{array}$ & 0.005 & 0.017 & $\frac{\pi}{U}$ & $\begin{array}{l}0.011 \\
0.017\end{array}$ & $\begin{array}{l}0.032 \\
0.034\end{array}$ & .03 & $\frac{0.033}{0.047}$ & $\frac{0.033}{0.034}$ \\
\hline & & & 0.012 & & 0.010 & 0.005 & & 0.005 & 0.004 & 0000 & 0019 & & & 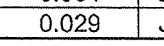 & & 1.19 & \\
\hline
\end{tabular}

10 Statistical Computation Input Data

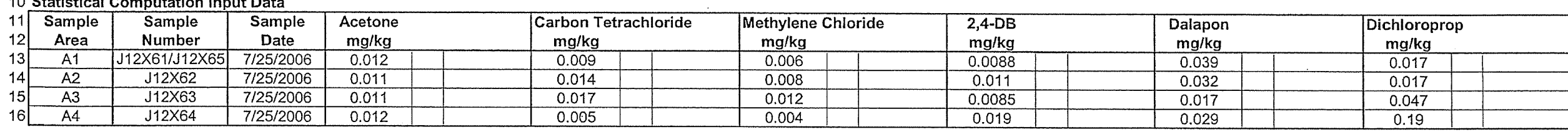

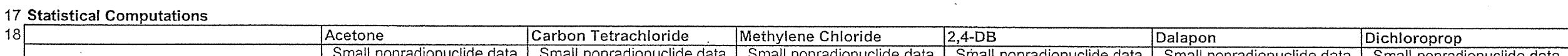

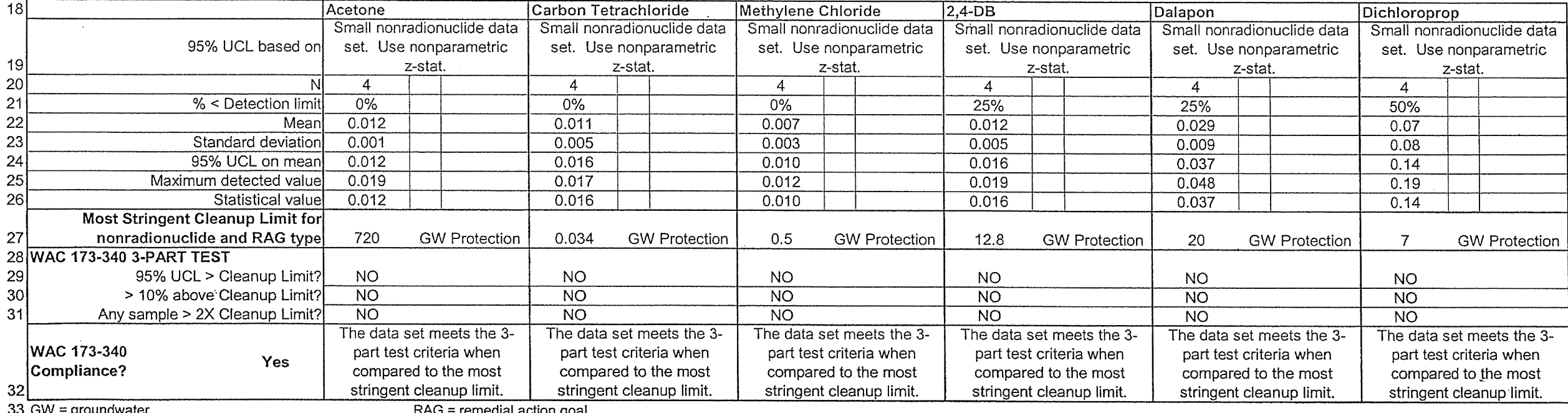

$33 \mathrm{GW}=$ groundiwater
$34 \mathrm{I}=$ interference on

$341=$ interterence on one analytical column
$35 \mathrm{~J}=$ estimation

$36 \mathrm{PQL}=$ practical quantitation limit

$\begin{aligned} & U=\text { undetected } \\ & \text { UCL }=\text { upper confidence limit } \\ & \text { WAC }=\text { Washington Administrative Code }\end{aligned}$ 
Originator J. M. Capron $9 m_{2} c$ \begin{tabular}{l} 
Project \\
Subject 1180 -B/C-B Field Remediation \\
\hline Burial Ground Cleanup Verification $95 \%$ UCL Calculations
\end{tabular}

\begin{aligned} & Date $\frac{10 / 03 / 07}{14655} \\ &$\hline\end{aligned}

Calc. No. $\frac{0100 B-C A-V 0299}{\text { Checked T. M. Blakley } 2 \text { mase }}$
.

Rev. No. $\frac{1}{\text { Date }} \frac{1}{10 / 04 / 07}$

1 118-B-1 Area 5 Statistical Calculations

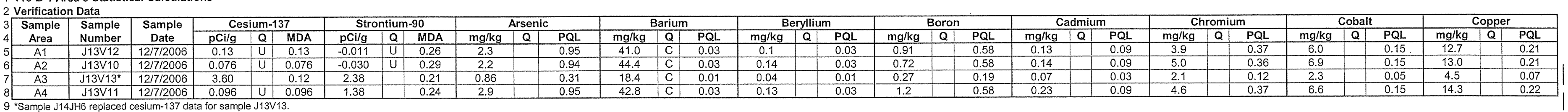

Statistical Computation input Dat

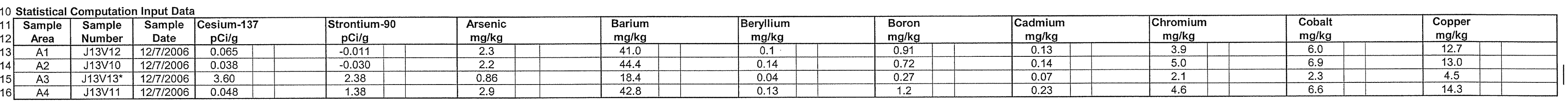

\begin{tabular}{|l|l|l|l|l|}
\hline A3 & $\mathrm{J} 13 \mathrm{~V} 13^{*}$ & $12 / 7 / 2006$ & 3.60 \\
\hline $\mathrm{A} 4$ & $\mathrm{J13V} 11$ & 127200 & \\
\hline
\end{tabular}

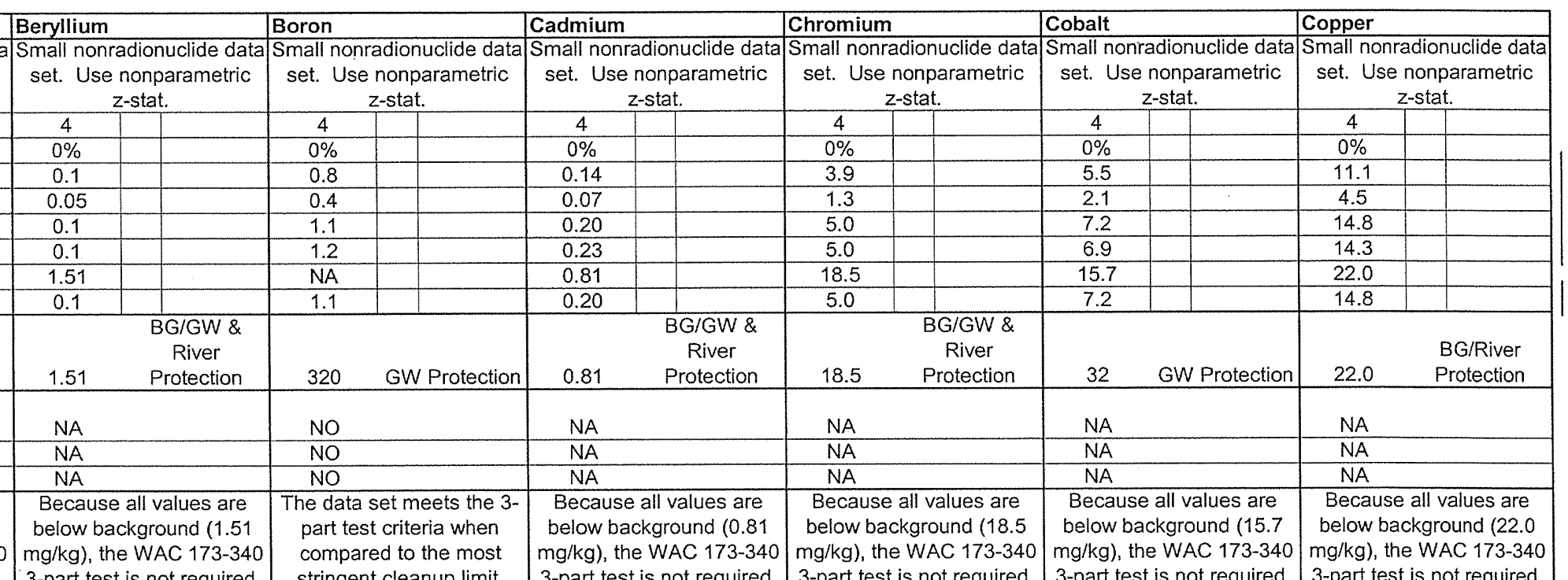

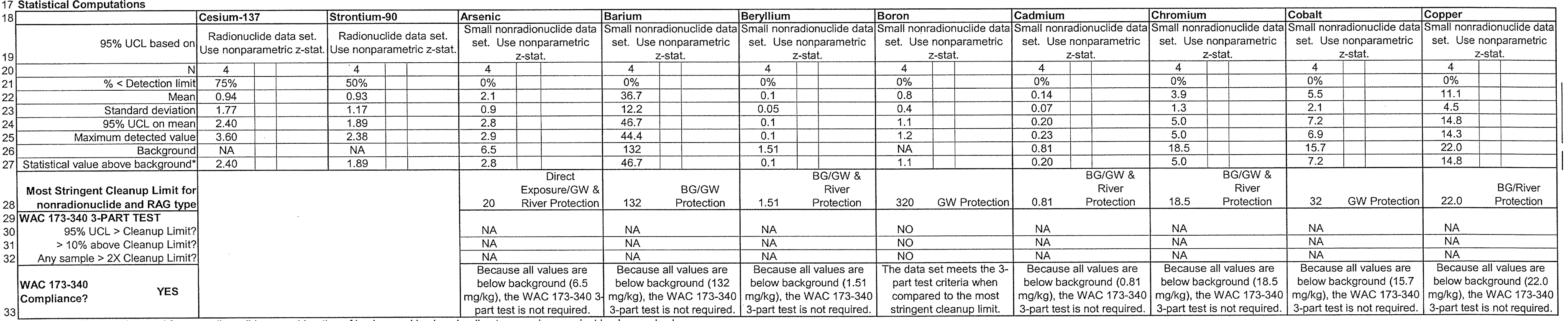

${ }_{34}^{33}$ "Background is not subtracted for nonradionuclides; consideration of background is given by direct test is not required.

$\begin{array}{ll}35 \mathrm{BG}=\text { background } & Q=\text { qualfier } \\ 36 \mathrm{C}=\text { method blank contamination } & \mathrm{RAG}=\text { remedial action goal }\end{array}$

$37 \mathrm{GW}=$ groundwater
$38 \mathrm{MDA}=$ minimum detectable activity

$39 \mathrm{NA}=$ not applicable
$40 \mathrm{PQL}=$ practical quantitition limit

UCL $=$ upper confidence limit
WAC = Washington Administrative Code 
Washington Closure Hanford

Originator J.M. Capron $9 \sin C$

Project $\frac{1 \text { 100-B/C Field Remediation }}{118-B-1 \text { Burial Ground Cleanup Verification 95\% UCL Calculations }}$
CALCULATION SHEET
Date
Job No. $\frac{02 / 07 / 07}{14655}$
Rev. No. $\frac{0}{2 / 20 / 0}$
Date
Sheet No.

1118-B-1 Area 5 Statistical Calculations (continued)
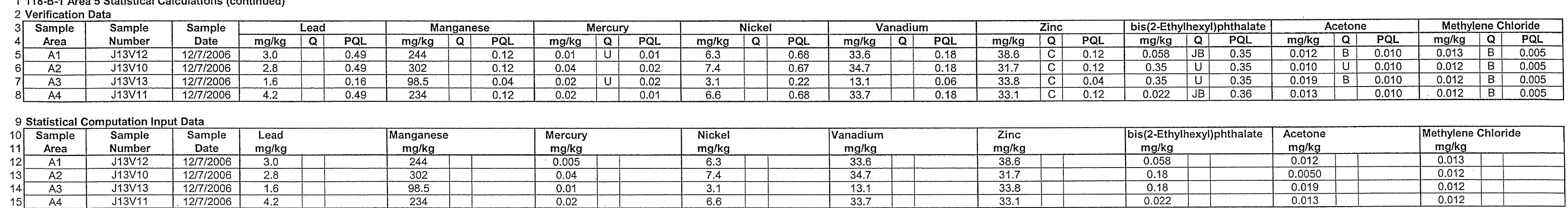

16 Statistical Computations
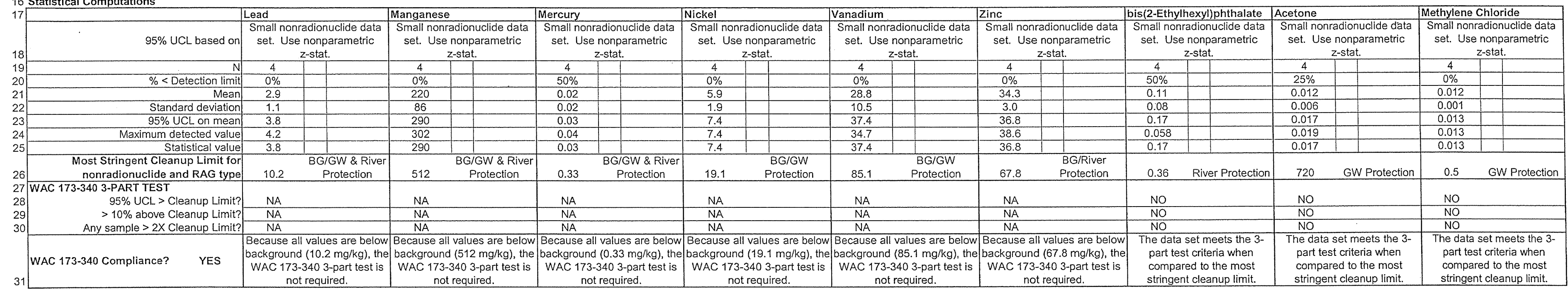

31
$B=$ method blank

$34 \mathrm{C}=$ method blank
$35 \mathrm{GW}=$ groundwater

$36 \mathrm{~J}=$ estimate
$37 \mathrm{NA}=$ not applicable

WAC 173-340 3-part test is
not require.

not required.

not required.

$173-340$ 3-part
not required.

not required.

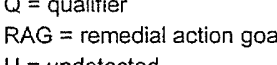

$U=$ undetected
UCL = upper confidence limit
WAC $=$ Washington Administrative Code 

$\begin{aligned} & \text { Date } 02 / 07 / 07 \\ & \text { Job No. } 14655\end{aligned}$
Calc. No. $\frac{0100 B-C A-V 0299}{\text { Checked T. M. Blakley } 2 \mathrm{mmB}}$
The
Rev. No. $\frac{0}{\text { Date }}$

118-B-1 Area 6 Statistical Calculations

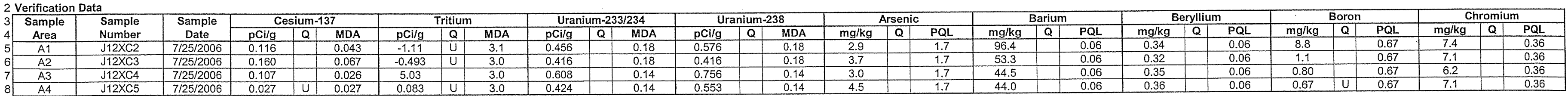

\begin{tabular}{c|c|c|c|}
\hline $\mathrm{A} 3$ & $\mathrm{~J} 12 \mathrm{XC} 4$ & $7 / 25 / 200$ \\
\hline $\mathrm{A} 4$ & $\mathrm{J12XC5}$ & $7 / 25 / 200$ \\
\hline
\end{tabular}

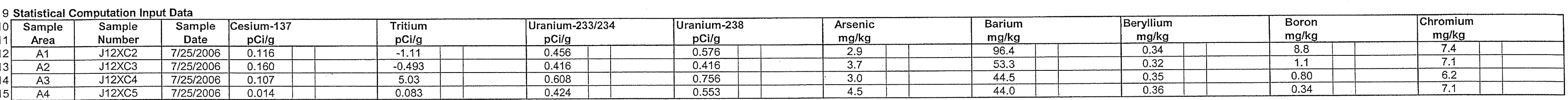

\begin{tabular}{|l|l|}
\hline A4 & J12XC5 \\
\hline
\end{tabular}

\begin{tabular}{|c|c|c|c|c|c|c|c|c|c|c|c|c|c|c|c|c|c|c|c|}
\hline \multirow[b]{3}{*}{. } & \multirow{3}{*}{$95 \%$ UCL based on } & \multicolumn{4}{|c|}{ Cesium-137 } & \multicolumn{2}{|c|}{ Uranium-233/234 } & \multicolumn{2}{|c|}{ Uranium-238 } & \multirow{2}{*}{\multicolumn{2}{|c|}{\begin{tabular}{|l|} 
Arsenic \\
Small nonredionuclide data \\
set. Use nonparametric \\
\end{tabular}}} & \multirow{2}{*}{\multicolumn{2}{|c|}{\begin{tabular}{l|} 
Barium \\
Small nonradionuclide datat \\
set. Une nonparametric \\
Zstat
\end{tabular}}} & \multirow{2}{*}{\multicolumn{2}{|c|}{\begin{tabular}{|l|} 
Beryllium \\
Small nonradionuclide data \\
set. Use nonparametric \\
$z$-stat.
\end{tabular}}} & \multirow{2}{*}{\multicolumn{2}{|c|}{\begin{tabular}{|c|} 
Boron \\
Small nonradionuclide data \\
set. Use nonparametric \\
z-stat. \\
\end{tabular}}} & \multirow{2}{*}{\multicolumn{2}{|c|}{\begin{tabular}{|c|} 
Chromium \\
$\begin{array}{c}\text { Small nonradionuclide data } \\
\text { set. Use nonnarametric } \\
\text { z-stat. }\end{array}$ \\
\end{tabular}}} \\
\hline & & \multicolumn{2}{|c|}{$\begin{array}{c}\text { Radionuclide data set. Use } \\
\text { nonparametric z-stat. }\end{array}$} & \multicolumn{2}{|c|}{$\begin{array}{c}\text { Radionuclide data set. Use } \\
\text { nonparametric z-stat. }\end{array}$} & \multicolumn{2}{|c|}{$\begin{array}{c}\text { Radionuclide data set. Use } \\
\text { nonparametric z-stat. }\end{array}$} & \multicolumn{2}{|c|}{$\begin{array}{l}\text { Radionuclide data set. Use } \\
\text { nonparametric } z \text {-stat. }\end{array}$} & & & & & & & & & & \\
\hline & & $\begin{array}{l}4 \\
\end{array}$ & & & & & & & & & & & & & & & & & \\
\hline 20 & $\%<$ Detection limit & $25 \%$ & & $75 \%$ & & $0 \%$ & & $0 \%$ & & $\frac{0 \%}{35}$ & & $0 \%$ & & $0 \%$ & & $\frac{25 \%}{28}$ & & $0 \%$ & \\
\hline & $\begin{array}{r}\text { Mean } \\
\text { Standard deviation }\end{array}$ & 0.099 & & 0.88 & 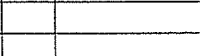 & 0.476 & & 0.575 & & $\frac{3.5}{07}$ & & $\frac{59.6}{24.9}$ & & 0.34 & & $\frac{2.8}{40}$ & & $\frac{7.0}{0.5}$ & \\
\hline & $\begin{array}{l}\text { Standard deviation } \\
95 \% \text { UCL on mean }\end{array}$ & $\frac{0.062}{0.150}$ & & $\frac{2.81}{3.19}$ & & 0.090 & & $\frac{0.140}{0.690}$ & & & & & & $\frac{0.36}{0.36}$ & & & & & \\
\hline 24 & Maximum detected value & 0.160 & & $\frac{0.19}{5.03}$ & 1 & 0.550 & & 0.056 & & $\frac{4.1}{4.5}$ & & 96.4 & & 0. & & 8.8 & & & \\
\hline & $\begin{array}{c}\text { Background } \\
\end{array}$ & $\mathrm{NA}$ & & NA & 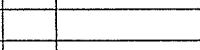 & 11 & $\theta$ & 1.1 & & 6.5 & 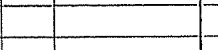 & 132 & & $\frac{1.51}{0.36}$ & +1 & $\frac{N A}{61}$ & & $\frac{18.5}{7.4}$ & \\
\hline & Most Stringent Cleanup Limit for & & & & & & & & & & $\begin{array}{c}\text { Direct } \\
\text { Exposure/GW \& }\end{array}$ & & & & & & fwe & 185 & River [ \\
\hline & $\begin{array}{l}\text { nonradionuclide and RAG type } \\
\text { WAC 173-340 3-PART TEST }\end{array}$ & & & & & & & & & & River Prot & & & & & & & & \\
\hline 9 & WAC 173-540 3 -PAR UCL >Cl & & & & & & & & & ${ }_{A} A$ & & & & & & & & NA & \\
\hline & $>1$ & & & & & & & & & NA & & NA & & NA & & NO & & $\mathrm{NA}$ & \\
\hline & $\times$ Cleanup Limit? & & & & & & & & & & & & & & & & & $N A$ & \\
\hline & WAC 173-340 Compliance? & & & & & & & & & $\begin{array}{l}\text { Because all } \\
\text { backgroun } \\
\text { WAC 173- } \\
\text { not }\end{array}$ & $\begin{array}{l}\text { values are below } \\
1(6.5 \mathrm{mg} / \mathrm{kg} \text { ), the } \\
340 \text { 3-part test is } \\
\text { required. }\end{array}$ & $\begin{array}{r}\text { Because all } \\
\text { background } \\
\text { WAC 173- } \\
\text { not }\end{array}$ & $\begin{array}{l}\text { values are below } \\
\text { (132 mg/kg), the } \\
3403 \text {-part test is } \\
\text { required. }\end{array}$ & $\begin{array}{l}\text { Because all } \\
\text { background } \\
\text { WAC 173-3 } \\
\text { not r }\end{array}$ & 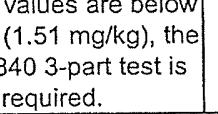 & $\begin{array}{l}\text { Ine data } \\
\text { part te: } \\
\text { compa } \\
\text { stringel }\end{array}$ & $\begin{array}{l}\text { st criteria when } \\
\text { red to the most } \\
\text { it cleanup limit. }\end{array}$ & $\begin{array}{l}\text { Secause a } \\
\text { backgroun } \\
\text { WAC r } 73 \\
\text { no }\end{array}$ & 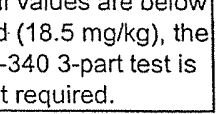 \\
\hline
\end{tabular}

32.

$34 \mathrm{BG}=$ background
$5 \mathrm{GW}=$ groundwater

$36 \mathrm{MDA}=$ minimum detectable activity

$38 \mathrm{PQL}=$ p practical quantitation limit

$Q=$ qualifier
$R A G=$ remedial action goal

$U=$ undetected
$U C L=$ upper confidence lim

UCL $=$ upper confidence limit
WAC $=$ Washington Administrative Code 
Washington Closure Hanford

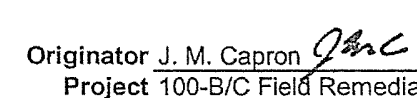

Project
Subject
100-B/C Fie-B-1 Burfal Remedialition Ground Cleanup Verification 95\% UCL Calculations
CALCULATION SHEET

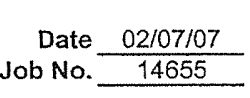
Rev. No. $\frac{0}{\text { Date } \frac{212010-3}{220613}}$

1118-B-1 Area 6 Statistical Calculations (continued)

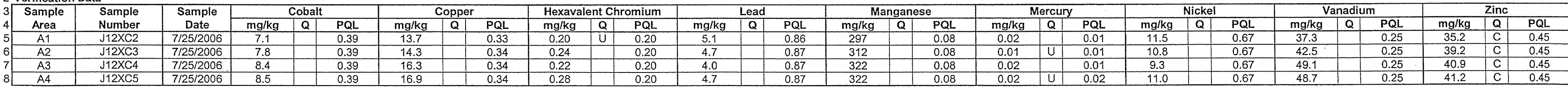

9 Statistical Computation Input Data

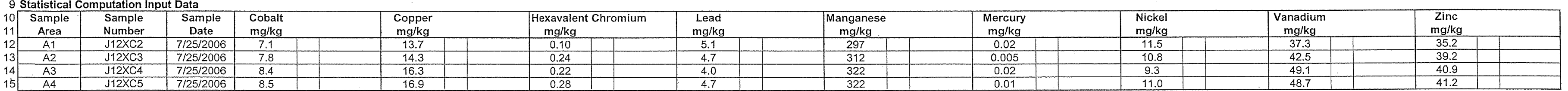

16 Statistical Computations

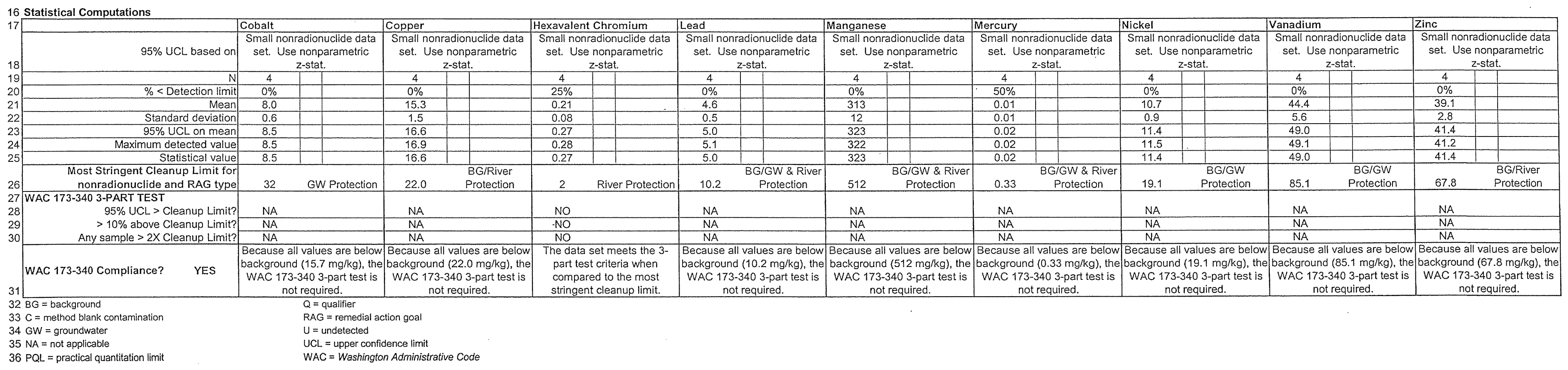


Originator J.M. Capron $9 / 8,2$ Project 100 -B/C Field Remediation $\begin{aligned} \text { Date } & 02 / 07 / 07 \\ \text { Job No. } & 14655\end{aligned}$

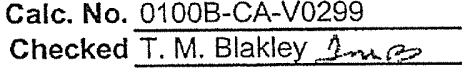

Rev. No. $\frac{0}{\text { Date } \frac{0}{2 / 20 / 07}}$

1118-B-1 Area 6 Statistical Calculations (continued)

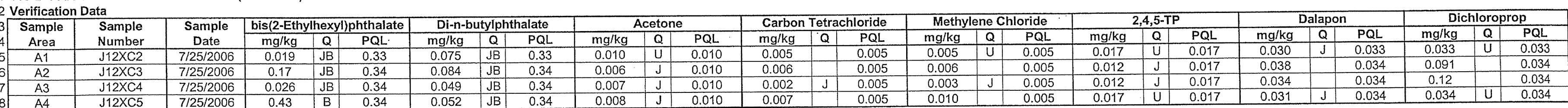

9 Statistical Computation Input Data

\section{Acetone}

\begin{tabular}{|c|c|c|c|}
\hline Area & $\begin{array}{c}\text { Number } \\
\text { Number }\end{array}$ & $\begin{array}{c}\text { Sample } \\
\text { Date }\end{array}$ & $\begin{array}{c}\text { bis(2-Ethy } \\
\mathrm{mg} / \mathrm{kg}\end{array}$ \\
\hline $\mathrm{A} 1$ & $\mathrm{j} 12 \times \mathrm{C} 2$ & $7 / 252000$ & 0.017 \\
\hline $\mathrm{A} 2$ & $112 \times C 3$ & $7 / 252000$ & 0.17 \\
\hline
\end{tabular}

\begin{tabular}{|c|c|c|}
\hline $\mathrm{A} 1$ & $\mathrm{~J} 12 \times \mathrm{X} 2$ & $7 / 25 / 2006$ \\
\hline $\mathrm{A} 2$ & $\mathrm{J12XC3}$ & $7 / 25 / 2006$ \\
\hline $\mathrm{A} 3$ & $\mathrm{J12} 2 \mathrm{CC4}$ & $7 / 2522006$ \\
\hline
\end{tabular}

\begin{tabular}{|c|c|c|c|c|c|}
\hline \multirow{2}{*}{\multicolumn{2}{|c|}{$\begin{array}{l}\mathrm{mg} / \mathrm{z} / \mathrm{kg} \\
0.019\end{array}$}} & & \\
\hline & & $\mathrm{mg} / \mathrm{kg}$ & & & \\
\hline 0.17 & & 0.084 & & 0.006 & \\
\hline 0.026 & & 0.049 & 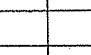 & 0.007 & \\
\hline
\end{tabular}

\begin{tabular}{|l|l|l|l|l|l|l}
\hline \multicolumn{2}{|c|}{$\mathrm{mg} / \mathrm{kg}$} & $\mathrm{mg} / \mathrm{kg}$ \\
\hline 0.005 & & 0.003 \\
\hline 0.006 & & 0.000 \\
\hline
\end{tabular}

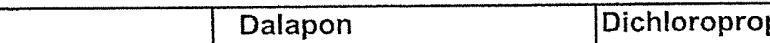

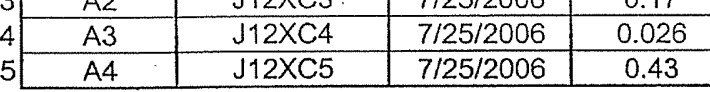

16 Statistical Computations

\begin{tabular}{|c|c|c|c|c|c|c|c|c|c|c|c|c|c|c|c|c|c|}
\hline \multirow{2}{*}{ 17] } & \multirow[b]{2}{*}{$95 \%$ UCL based on } & \multicolumn{2}{|c|}{ bis(2-Ethylhexyl)phthalate $[0$} & \multicolumn{2}{|c|}{ Di-n-butylphthalate } & & \multicolumn{2}{|c|}{ Carbon Tetrachloride } & \multicolumn{2}{|c|}{ Methylene Chloride } & \multirow{2}{*}{\multicolumn{2}{|c|}{\begin{tabular}{|l|} 
2,4,5-TP \\
Small nonradionuclide data \\
set. Use nonparametric
\end{tabular}}} & \multirow{2}{*}{\multicolumn{2}{|c|}{$\begin{array}{l}\text { Dalapon } \\
\text { Small nonradionuclide data } \\
\text { set. Use nonparametric } \\
\text { z-stat. }\end{array}$}} & \multirow{2}{*}{\multicolumn{2}{|c|}{\begin{tabular}{|l|} 
Dichloroprop \\
Small nonradionuclide data \\
set. Use nonparametric \\
z-stat. \\
\end{tabular}}} \\
\hline & & \multicolumn{2}{|c|}{$\begin{array}{c}\text { Small nonradionuclide data } \\
\text { set. Usen nonparametric } \\
\text { z-stat. }\end{array}$} & \multicolumn{2}{|c|}{$\begin{array}{l}\text { Small nonradionuclide data } \\
\text { set. Use nonparametric } \\
\text { z-stat. }\end{array}$} & \multicolumn{2}{|c|}{\begin{tabular}{|c|} 
Small nonradionuclide data \\
set. Use nonparametric \\
z-stat.
\end{tabular}} & \multicolumn{2}{|c|}{$\begin{array}{l}\text { Small nonradionuclide data } \\
\text { set. Use nonparametric } \\
\text { Z-stat. }\end{array}$} & \multicolumn{2}{|c|}{\begin{tabular}{|c|} 
Small nonradionuclide data \\
set. Use nonparametric \\
$z$-stat.
\end{tabular}} & & & & & & \\
\hline 19 & & 4 & & & & & & & & & & \multicolumn{2}{|c|}{$\begin{array}{c}\text { set. Use nonparametric } \\
\text { z-stat. }\end{array}$} & \multicolumn{2}{|c|}{$\begin{array}{c}\text { set. Use nonparametric } \\
\text { z-stat. }\end{array}$} & \multicolumn{2}{|c|}{\begin{tabular}{ll|} 
& z-stat. \\
4 & \\
\end{tabular}} \\
\hline 20 & $\%<$ Detection limit & $0 \%$ & & $0 \%$ & & $25 \%$ & & $0 \%$ & & $25 \%$ & & $50 \%$ & & $0 \%$ & & & \\
\hline 21 & Mean & 0.16 & & 0.065 & & 0.007 & & 0.005 & & 0.005 & & 0.010 & & 0.033 & & 0.06 & \\
\hline 22 & Standard deviation & 0.19 & & 0.017 & & 0.001 & & 0.002 & & 0.003 & & 0.002 & & 0.004 & & 0.05 & \\
\hline 23 & 95\% UCL on mean & 0.32 & & 0.079 & & 0.008 & & 0.007 & & 0.008 & & 0.012 & & 0.036 & & 0.10 & \\
\hline \multirow{3}{*}{26} & Maximum detecied value & 0.43 & & 0.084 & & 0.008 & & 0.007 & & & & & & 0.038 & & 0.12 & \\
\hline & Statistical value & 0.32 & & 0.079 & & 0.008 & & 0.007 & & 0.008 & 1 & 0.01 & & 0.036 & & 0.10 & \\
\hline & $\begin{array}{r}\text { Most Stringent Cleanup Limit for } \\
\text { nonradionuclide and RAG type }\end{array}$ & \multicolumn{2}{|r|}{ River Protection } & \multirow{2}{*}{\multicolumn{2}{|c|}{ GW Protection }} & \multicolumn{2}{|r|}{ GW Protection } & \multicolumn{2}{|r|}{ GW Protection } & \multicolumn{2}{|r|}{ GW Protection } & \multicolumn{2}{|r|}{ GW Protection } & \multicolumn{2}{|r|}{ GW Protection } & \multicolumn{2}{|r|}{ GW Protection } \\
\hline \multirow{4}{*}{$\begin{array}{l}27 \\
28 \\
29 \\
30\end{array}$} & WAC 173-340 3-PART TEST & \multirow{2}{*}{\multicolumn{2}{|c|}{ NO }} & \multirow{2}{*}{\multicolumn{2}{|c|}{ No }} & \multirow{2}{*}{\multicolumn{2}{|c|}{ NO }} & \multirow{2}{*}{\multicolumn{2}{|c|}{ NO }} & \multirow{2}{*}{\multicolumn{2}{|c|}{ NO }} & & & & & & \\
\hline & $95 \%$ UCL > Cleanup Limit? & & & & & & & & & & & $\frac{\text { NO }}{\text { NO }}$ & & $\frac{\text { NO }}{\text { NO }}$ & & $\frac{\text { NO }}{\text { NO }}$ & \\
\hline & $>10 \%$ above Cleanup Limit? & & & NO & & $\frac{\mathrm{NO}}{\mathrm{NO}}$ & & $\frac{\text { NO }}{\text { NO }}$ & & $\frac{\text { NO }}{\text { NO }}$ & & $\frac{\text { nO }}{\text { NO }}$ & & $\frac{\text { NO }}{\text { NO }}$ & & NO & \\
\hline & Any sample $>2 X$ Cleanup Limit?] & NO & & NO & & NO & & & & & & & & & & & \\
\hline & $\begin{array}{c}\text { Further } \\
\text { Assessment } \\
\text { Required }\end{array}$ & $\begin{array}{l}\text { Because } \\
\text { to the 3- } \\
\text { assessm } \\
\text { will be } \\
\text { set me } \\
\text { criteria } \\
\text { the direc }\end{array}$ & $\begin{array}{l}\text { frt tes "est, a detailer } \\
\text { ntt using RESRAD } \\
\text { formed. The data } \\
\text { ts the 3-part test } \\
\text { then compared to } \\
\text { exposure cleanup }\end{array}$ & $\begin{array}{l}\text { The data } \\
\text { part tes } \\
\text { compar } \\
\text { stringer }\end{array}$ & $\begin{array}{l}\text { et meets the } 3- \\
\text { criteria when } \\
\text { ed to the most } \\
\text { cleanup limit. }\end{array}$ & $\begin{array}{l}\text { The data } \\
\text { part tes } \\
\text { compal } \\
\text { stringer }\end{array}$ & $\begin{array}{l}\text { et meets the 3- } \\
\text { criteria when } \\
\text { d to the most } \\
\text { cleanup limit. }\end{array}$ & $\begin{array}{l}\text { The data } \\
\text { part tes } \\
\text { compar } \\
\text { stringer }\end{array}$ & $\begin{array}{l}\text { set meets the 3- } \\
\text { t criteria when } \\
\text { ed to the most } \\
\text { t cleanup limit. }\end{array}$ & $\begin{array}{l}\text { The data } \\
\text { part tes } \\
\text { compar } \\
\text { stringer }\end{array}$ & $\begin{array}{l}\text { set meets the 3- } \\
\text { t criteria when } \\
\text { ed to the most } \\
\text { t cleanup limit. }\end{array}$ & $\begin{array}{l}\text { The data } \\
\text { part tes } \\
\text { compar } \\
\text { stringen }\end{array}$ & $\begin{array}{l}\text { set meets the 3- } \\
t \text { criteria when } \\
\text { ed to the most } \\
t \text { cleanup limit. }\end{array}$ & $\begin{array}{l}\text { The data } \\
\text { part tes } \\
\text { compal } \\
\text { stringer }\end{array}$ & $\begin{array}{l}\text { set meets the 3- } \\
\text { st criteria when } \\
\text { red to the most } \\
\text { th cleanup limit. }\end{array}$ & $\begin{array}{l}\text { The data } \\
\text { part te. } \\
\text { compa } \\
\text { stringe }\end{array}$ & $\begin{array}{l}\text { set meets the 3- } \\
\text { st criteria when } \\
\text { red to the most } \\
\text { nt cleanup limit. }\end{array}$ \\
\hline
\end{tabular}

$32 \mathrm{~B}=$ method blank contamination

$33 \mathrm{GW}=$ groundwater
$34 \mathrm{~J}=$ estimate

$35 \mathrm{NA}=$ not applicable
$36 \mathrm{PQL}=$ practical quantitation limit level.

$U=$ undetected

$U C L=$ upper confidence limit 
CALCULATION SHEET

Washington Closure Hanford

Originator J. M. Capron QBenC

subject 118-B-1 Burial Ground Cleanup Verification 95\% UCL Calculations

Date $\frac{02 / 07 / 07}{14655}$

Calc. No. $0100 \mathrm{~B}-\mathrm{CA}-\mathrm{V} 0299$

Rev. No. $\frac{0}{2 a t e}$

Sheet No. 24 of 61

1118-B-1 Area 7 Statistical Calculations

\begin{tabular}{|c|c|c|c|c|c|c|c|c|c|c|c|c|c|c|c|c|c|c|c|c|c|c|c|c|c|c|}
\hline \multirow{3}{*}{\begin{tabular}{|l|} 
Sample \\
Area \\
5
\end{tabular}} & \multirow{3}{*}{$\begin{array}{l}\text { Sample } \\
\text { Number } \\
\end{array}$} & \multirow{2}{*}{$\begin{array}{c}\text { Sample } \\
\text { Date } \\
\end{array}$} & \multicolumn{3}{|c|}{ Cesium-137 } & \multicolumn{3}{|c|}{ Europium-152 } & \multicolumn{3}{|c|}{ Uranium-233/234 } & \multicolumn{3}{|c|}{ Uranium-238 } & \multicolumn{3}{|c|}{ Arsenic } & \multicolumn{3}{|c|}{ Barium } & \multicolumn{3}{|c|}{ Beryllium } & \multicolumn{3}{|c|}{ Boron } \\
\hline & & & pCilg & Q & MDA & $\mathrm{pCi} / \mathrm{g}$ & Q & MDA & $\mathrm{pCi} / \mathrm{g}$ & $Q$ & MDA & pCilg & Q & MDA & $\mathrm{mg} / \mathrm{kg}$ & \begin{tabular}{|l|l|l|} 
& \\
\end{tabular} & $\mathrm{PQL}$ & $\mathrm{mg} / \mathrm{kg}$ & Q & $P Q L$ & $\mathrm{mg} / \mathrm{kg}$ & $Q$ & $\mathrm{PQL}$ & $\mathrm{mg} / \mathrm{kg}$ & Q & $P Q L$ \\
\hline & & $7 / 26 / 2006$ & 0.578 & & 0.086 & 0.17 & $U$ & 0.17 & 0.473 & & 0.26 & 0.574 & & 0.26 & 3.8 & & 1.7 & 145 & & 0.06 & 0.30 & & 0.06 & $\frac{18.1}{27}$ & & 0.67 \\
\hline $\mathrm{A} 2$ & $J 12 \times C 7$ & $7 / 26 / 2006$ & 0.450 & & 0.046 & 0.12 & $U$ & 0.12 & 0.602 & & 0.27 & 0.708 & & 0.27 & 3.1 & & 1.7 & 75.1 & & 0.06 & 0.31 & & & 2.7 & & 0.66 \\
\hline A3 & $J 12 \times C 8$ & $7 / 26 / 2006$ & 0.271 & & 0.091 & 0.17 & $u$ & 0.17 & 0.718 & & 0.25 & 0.424 & & 0.25 & 3.1 & & 1.7 & 53.7 & & 0.06 & 0.19 & & 0.06 & 1.3 & & 0.67 \\
\hline $\mathrm{A} 4$ & J12XC9 & $7 / 26 / 2006$ & 0.297 & & 0.018 & 0.104 & & 0.052 & 0.292 & & 0.28 & 0.729 & & 0.28 & 2.8 & & 1.7 & 119 & & 0.06 & 0.27 & & 0.06 & 7.7 & & 0.67 \\
\hline
\end{tabular}

9 Statistical Computation Input Data

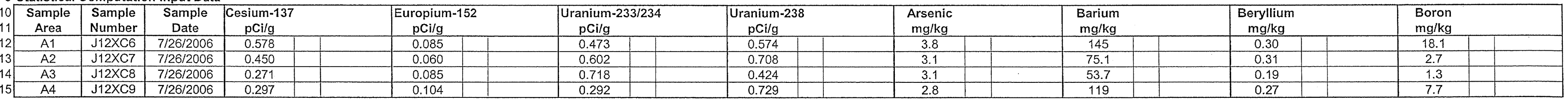

16 Statistical Computations

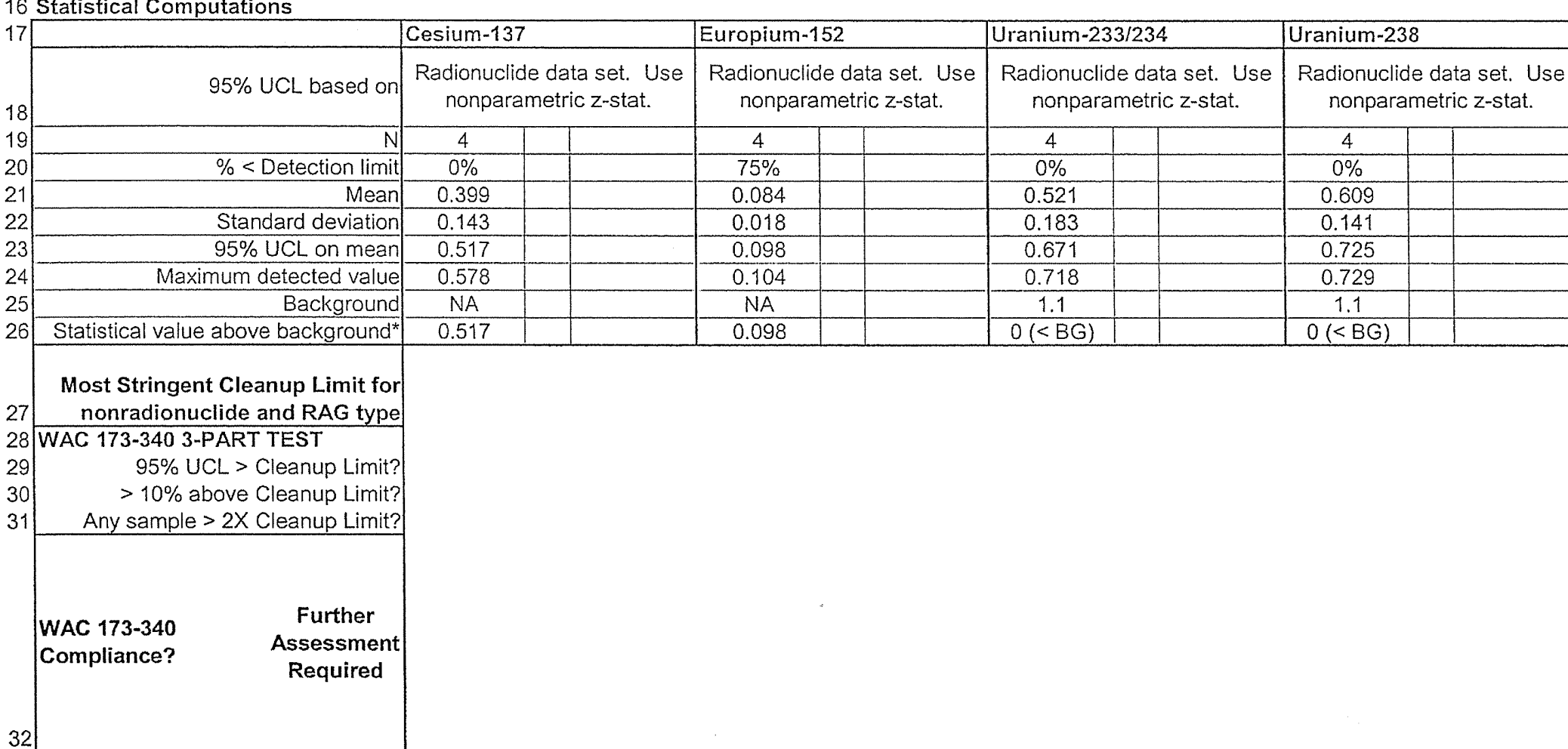

\begin{tabular}{|c|c|c|c|c|c|c|c|}
\hline \multirow{3}{*}{\multicolumn{2}{|c|}{$\begin{array}{l}\text { Arsenic } \\
\text { Small nonradionuclide data } \\
\text { set. Use nonparametric } \\
z \text {-stat. } \\
\end{array}$}} & \multirow{2}{*}{\multicolumn{2}{|c|}{$\begin{array}{l}\text { Barium } \\
\text { Small nonradionuclide data } \\
\text { set. Use nonparametric } \\
\text { z-stat. }\end{array}$}} & \multirow{2}{*}{\multicolumn{2}{|c|}{\begin{tabular}{l|} 
Beryllium \\
Small nonradionuclide data \\
set. Use nonparametric \\
z-stat.
\end{tabular}}} & \multirow{2}{*}{\multicolumn{2}{|c|}{\begin{tabular}{|c|} 
Boron \\
$\begin{array}{c}\text { Small nonradionuclide data } \\
\text { set. Use nonparametric } \\
\text { z-stat. }\end{array}$
\end{tabular}}} \\
\hline & & & & & & & \\
\hline & & 4 & & 4 & & 4 & \\
\hline $0 \%$ & & $0 \%$ & & $0 \%$ & & $0 \%$ & \\
\hline 3.2 & & 98 & & 0.27 & & 7.5 & \\
\hline 0.4 & & 41 & & 0.05 & & 7.6 & \\
\hline 3.5 & & 132 & & 0.31 & & 13.7 & \\
\hline 3.8 & & 145 & & 0.31 & & 18.1 & \\
\hline 6.5 & & 132 & & 1.51 & & NA & \\
\hline 3.5 & & 132 & & 0.31 & & 13.7 & \\
\hline \multicolumn{2}{|l|}{20} & 132 & $\begin{array}{c}\text { BG/GW } \\
\text { Protection }\end{array}$ & \multicolumn{2}{|r|}{$\begin{array}{l}\mathrm{BG} / \mathrm{GW} \text { \& River } \\
\text { Protection }\end{array}$} & \multicolumn{2}{|l|}{320} \\
\hline \multicolumn{2}{|l|}{ NA } & \multicolumn{2}{|l|}{ YES } & \multicolumn{2}{|l|}{ NA } & \multicolumn{2}{|l|}{ NO } \\
\hline \multirow{2}{*}{\multicolumn{2}{|c|}{$\frac{N A}{N A}$}} & \multirow{2}{*}{\multicolumn{2}{|c|}{$\begin{array}{l}\text { YES } \\
\text { NOO }\end{array}$}} & \multirow{2}{*}{\multicolumn{2}{|c|}{$\frac{N A}{N A}$}} & \multirow{2}{*}{\multicolumn{2}{|c|}{$\frac{N O}{N O}$}} \\
\hline & & & & & & & \\
\hline \multicolumn{2}{|c|}{$\begin{array}{c}\text { Because all values are below } \\
\text { background }(6.5 \mathrm{mg} / \mathrm{kg}) \text {, the } \\
\text { WAC } 173-3403 \text {-part test is } \\
\text { not required. }\end{array}$} & \multicolumn{2}{|c|}{$\begin{array}{l}\text { Because of the "yes" } \\
\text { answers to the 3-part test, a } \\
\text { detailed assessment using } \\
\text { RESRAD will be performed. } \\
\text { The data set meets the } 3- \\
\text { part test criteria when } \\
\text { compared to the direct } \\
\text { exposure cleanuo level. }\end{array}$} & \multicolumn{2}{|c|}{$\begin{array}{c}\text { Because all values are below } \\
\text { background (1.51 mg/kg), the } \\
\text { WAC } 173-3403-\text {-part test is } \\
\text { not required. }\end{array}$} & \multicolumn{2}{|c|}{$\begin{array}{l}\text { The data set meets the 3- } \\
\text { part test criteria when } \\
\text { compared to the most } \\
\text { stringent cleanup limit. }\end{array}$} \\
\hline
\end{tabular}

33 * Background is not subtracted for nonradionuclides; consideration of backgroun
$34 \mathrm{BG}=$ background

$34 \mathrm{BG}=$ background

$36 \mathrm{MDA}=$ minimum detectable activity

$37 \mathrm{NA}=$ not applicable

$Q=$ qualifier

$38 \mathrm{PQL}=$ practical quantitation limit

$U=$ undetected

UCL $=$ upper confidence limit
WAC $=$ Washington Administrative Code 
Originator J. M. Capron qus Project 100-B/C Field Remediation

Subject 118-B-1 Burial Ground Cleanup Verification 95\% UCL Calculation
CALCULATION SHEET

$$
\begin{aligned}
\text { Date } & 02 / 07 / 07 \\
\text { Job No. } & \frac{14655}{1465}
\end{aligned}
$$

1118-B-1 Area 7 Statistical Calculations (continued)

\begin{tabular}{|c|c|c|c|c|c|c|c|c|c|c|c|c|c|c|c|c|c|c|c|c|c|c|c|c|c|c|}
\hline \multirow{2}{*}{\begin{tabular}{l|c}
3 & Sample \\
4 & Area \\
\end{tabular}} & \multirow{2}{*}{\begin{tabular}{c|} 
Sample \\
Number
\end{tabular}} & \multirow{2}{*}{$\begin{array}{c}\text { Sample } \\
\text { Date } \\
\end{array}$} & \multicolumn{3}{|c|}{ Chromium } & \multicolumn{3}{|c|}{ Cobalt } & \multicolumn{3}{|c|}{ Copper } & \multicolumn{3}{|c|}{ Hexavalent Chromium } & \multicolumn{3}{|c|}{ Lead } & \multicolumn{3}{|c|}{ Manganese } & \multicolumn{3}{|c|}{ Nickel } & \multicolumn{3}{|c|}{ Vanadium } \\
\hline & & & $\mathrm{mg} / \mathrm{kg}$ & $Q$ & $\mathrm{PQL}$ & $\mathrm{mg} / \mathrm{kg}$ & $Q$ & $\mathrm{PQL}$ & $\mathrm{mg} / \mathrm{kg}$ & Q & $\mathrm{PQL}$ & $\mathrm{mg} / \mathrm{kg}$ & $Q$ & PQL & $\mathrm{mg} / \mathrm{kg}$ & $Q$ & $\overline{P Q L}$ & $\mathrm{mg} / \mathrm{kg}$ & Q & PQL & $\mathrm{mg} / \mathrm{kg}$ & $Q$ & $\overline{P Q L}$ & $\mathrm{mg} / \mathrm{kg}$ & $Q$ & $P Q L$ \\
\hline 5 & J12XC6 & $7 / 26 / 2006$ & 7.6 & & 0.36 & 6.3 & & 0.39 & 25.7 & & 0.33 & 0.20 & U & 0.20 & 12.9 & & 0.86 & 254 & & 0.08 & 8.6 & & 0.67 & 36.4 & & 0.25 \\
\hline $\mathrm{A} 2$ & $\mathrm{~J} 12 \times \mathrm{XC7}$ & $7 / 26 / 2006$ & 9.5 & & 0.36 & 7.6 & & 0.39 & 14.4 & & 0.33 & 0.30 & & 0.20 & 6.0 & & 0.86 & 340 & & 0.08 & 10.7 & & 0.66 & 40.9 & & 0.25 \\
\hline A3 & $J 12 \times C 8$ & $7 / 26 / 2006$ & 7.3 & & 0.37 & 6.3 & & 0.39 & 11.7 & & 0.34 & 0.27 & & 0.20 & 3.6 & & 0.87 & 270 & & 0.08 & 9.4 & & 0.67 & 32.7 & & 0.25 \\
\hline A4 & $512 \times C 9$ & $7 / 26 / 2006$ & 8.0 & & 0.36 & 7.4 & & 0.39 & 17.2 & & 0.34 & 0.20 & $u$ & 0.20 & 8.3 & & 0.87 & 318 & & 0.08 & 10.4 & & 0.67 & 38.0 & & 0.25 \\
\hline
\end{tabular}

9 Statistical Computation Input Data

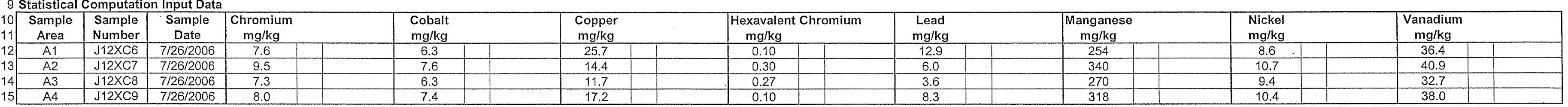

16 Statistical Computations

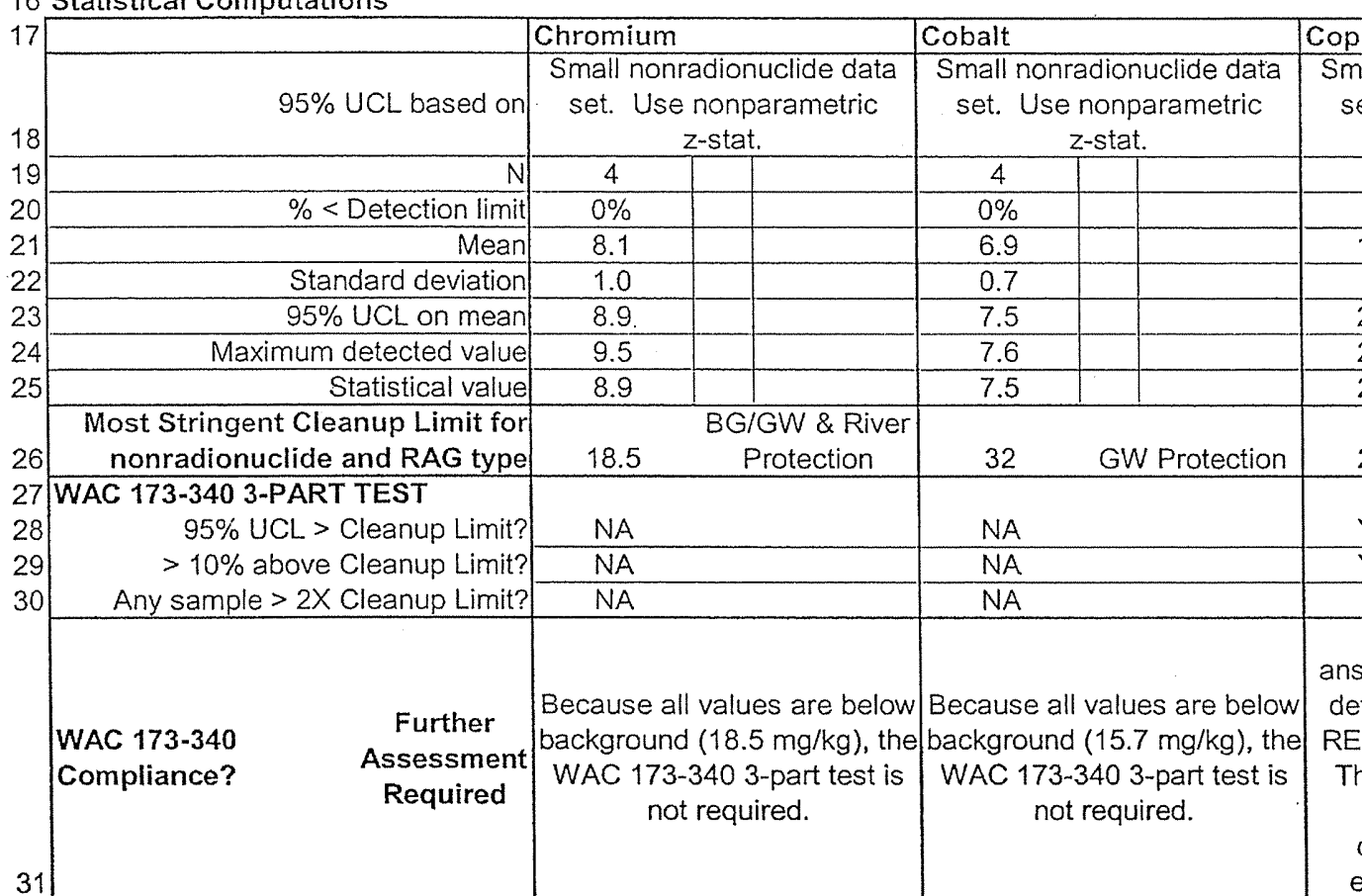
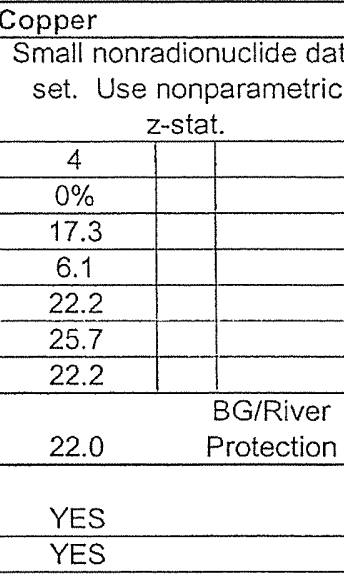

YES
YES
NO

NO etailed assessment using ESRAD will be performe The data set meets the
part test criteria when compared to the direct compared to the direct
exposure cleanup level.

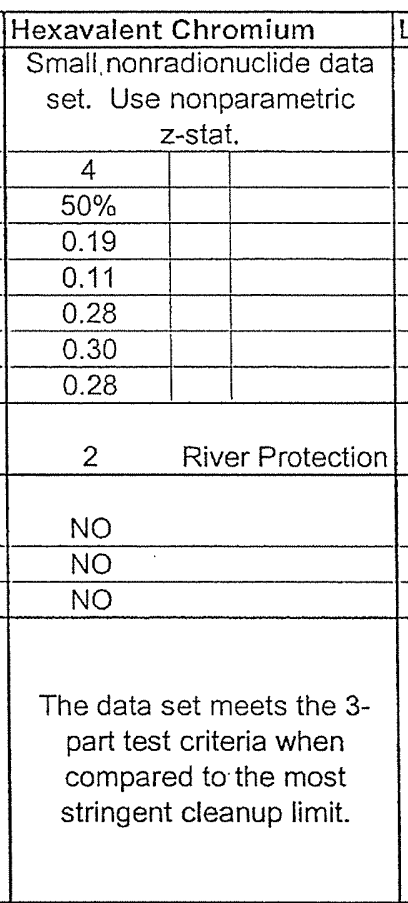

$\mathrm{RAG}=$ remedia
$U=$ undetected

WAC = Washington Admitit
$33 \mathrm{GW}=$ groundwater

$35 \mathrm{PQL}=$ practical quantitation limit

$36 \mathrm{Q}=$ qualifier

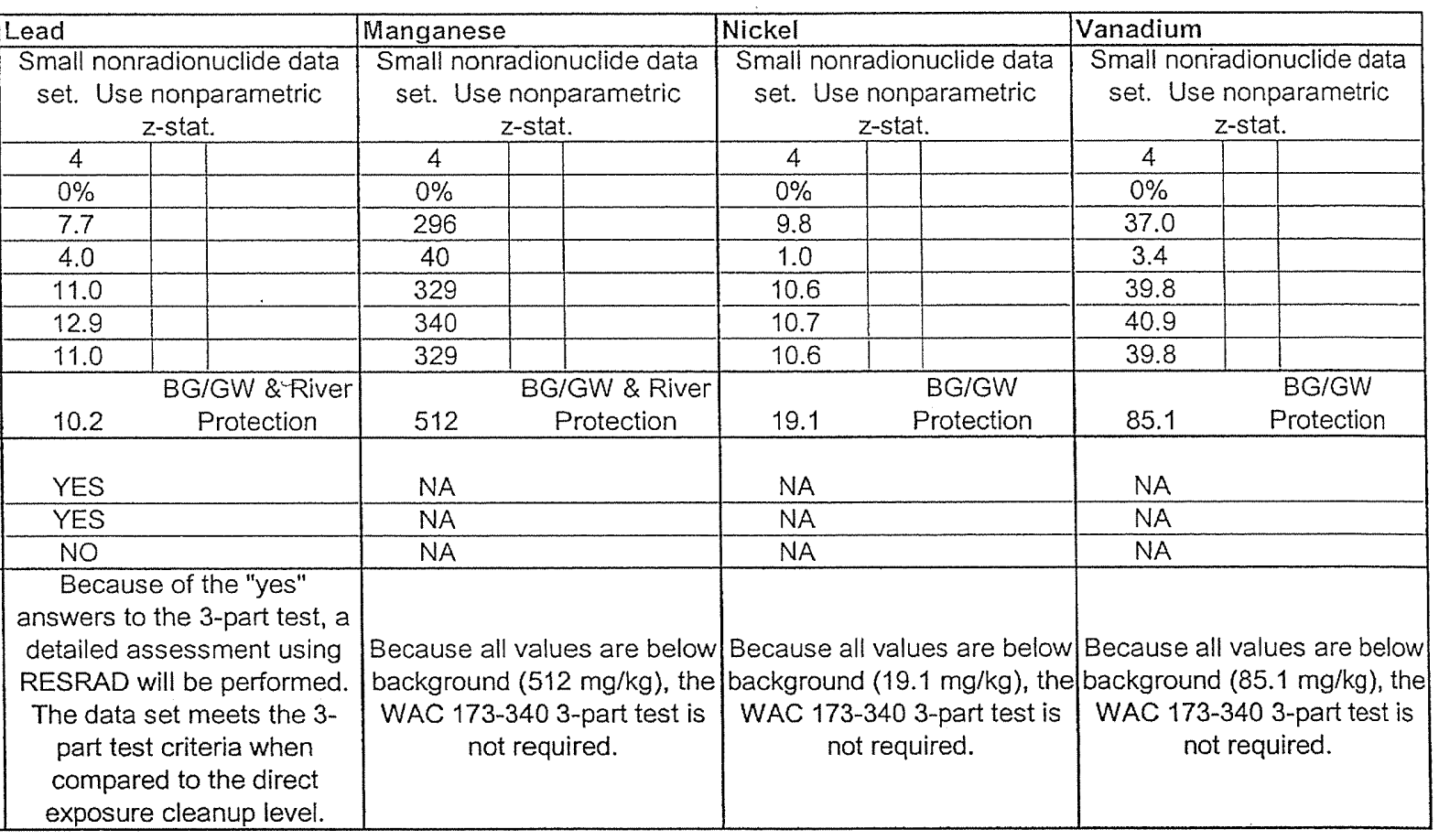

compared to the direct
Rev. No. $\frac{0}{\text { Date }}$
Calc. No. $0100 \mathrm{~B}-\mathrm{CA}-\mathrm{V} 0299$

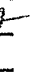


CALCULATION SHEET

Washington Closure Hanford

Originator J.M. Capron Qine

Subject 118-B-1 Burial Ground Cleanup Verification 95\% UCL Calculations
Date $02 / 07 / 07$
Job No.
Calc. No. $0100 \mathrm{~B}-\mathrm{CA}-\mathrm{V} 0299$

Checked T. M. Blakley $2 \ln B$
Rev. No. $\frac{0}{\text { Date }}$

1118-B-1 Area 7 Statistical Calculations (continued)

\begin{tabular}{|c|c|c|c|c|c|c|c|c|c|c|c|c|c|c|c|c|c|c|c|c|c|c|c|c|c|c|}
\hline \multirow{2}{*}{\begin{tabular}{|c|c|} 
& Sample \\
\end{tabular}} & \multirow{2}{*}{\begin{tabular}{l|} 
Sample \\
Number \\
\end{tabular}} & \multirow{2}{*}{$\begin{array}{c}\text { Sample } \\
\text { Date }\end{array}$} & \multicolumn{3}{|c|}{ Zinc } & \multicolumn{3}{|c|}{ Acetone } & \multicolumn{3}{|c|}{ Carbon Tetrachloride } & \multicolumn{3}{|c|}{ Methylene Chloride } & \multicolumn{3}{|c|}{ 4,4'-DDE } & \multicolumn{3}{|c|}{$2,4-D$} & \multicolumn{3}{|c|}{ Dalapon } & \multicolumn{3}{|c|}{ Dichloroprop } \\
\hline & & & $\mathrm{g} / \mathrm{kg}$ & 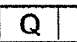 & PQL & $\mathrm{ng} / \mathrm{kg}$ & $Q$ & PQL & $\mathrm{mg} / \mathrm{kg}$ & Q & $\mathrm{PQL}$ & $\mathrm{mg} / \mathrm{kg}$ & $Q$ & PQL & $\mathrm{mg} / \mathrm{kg}$ & Q & $P Q L$ & $\mathrm{mg} / \mathrm{kg}$ & Q & $P Q L$ & $\mathrm{mg} / \mathrm{kg}$ & Q & $\mathrm{PQL}$ & $\mathrm{mg} / \mathrm{kg}$ & $Q$ & $\mathrm{PQL}$ \\
\hline A1 & $\sqrt{12 \times C 6}$ & $7 / 26 / 2006$ & 48.7 & C & 0.45 & 0.008 & J & 0.010 & 0.009 & & 0.005 & 0.010 & & 0.005 & & DJ & & & J & & & J & & & $U$ & 0.033 \\
\hline A2 & $\mathrm{J12XC7}$ & $7 / 261$ & 41.0 & \begin{tabular}{|l|l}
$\mathrm{C}$ & $\mathrm{a}$ \\
\end{tabular} & 0.44 & 0.010 & J & 0.01 & 0.0 & & 0.0 & 0.0 & u & & & UD & & & u & & & $\mathrm{J}$ & & 0.037 & & 0.033 \\
\hline A3 & $J 12 \times C 8$ & $7 / 26 /$ & 31.1 & $\begin{array}{ll}\mathrm{c} \\
\end{array}$ & 0.45 & 0.010 & u & 0.0 & 0.00 & $\mathrm{u}$ & & & u & & & UD & & & & & & J & & & $U$ & 0.034 \\
\hline A4 & $\mathrm{J12 \times C9}$ & $7 / 26 / 2006$ & 44.5 & c & 0.45 & 0.010 & u & 0.010 & 0.010 & & 0.005 & 0.006 & & 0.005 & 0.015 & DJ & 0.0013 & 0.013 & $\frac{7}{4}$ & 0. & 0.034 & u & 0.034 & 0.060 & & 0.034 \\
\hline
\end{tabular}

9 Statistical Computation Input Data

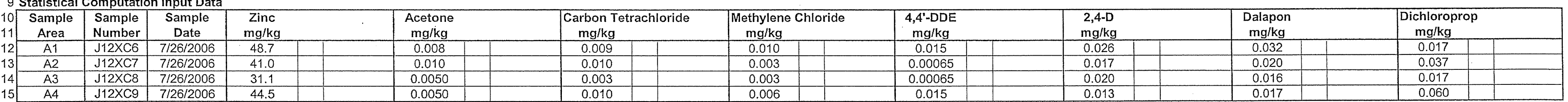

6 Statistical Computations

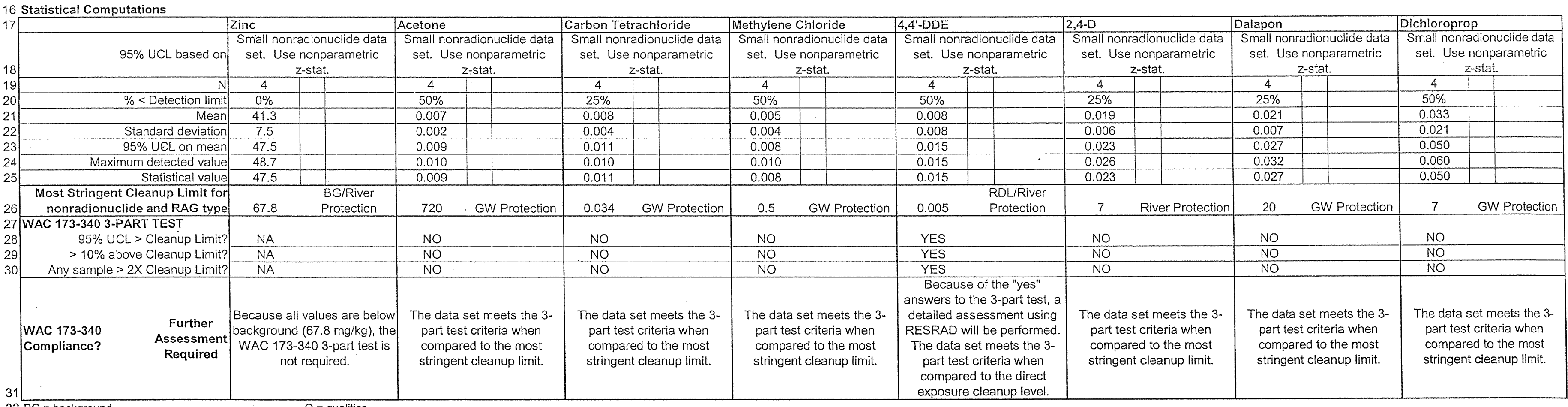

$33 \mathrm{C}=$ method blank contamination

$34 \mathrm{D}=$ diluted

$35 \mathrm{GW}=$ groundwater

$36 \mathrm{~J}=$ estimate

$38 \mathrm{PQL}=$ practical quantitation limit
$Q=$ qualifier

$\mathrm{RDL}=$ required detection lim

$U=$ undetected

$W A C=$ Washington Administrative Code 
CALCULATION SHEET

1118-B-1 Overburden Statistical Calculations

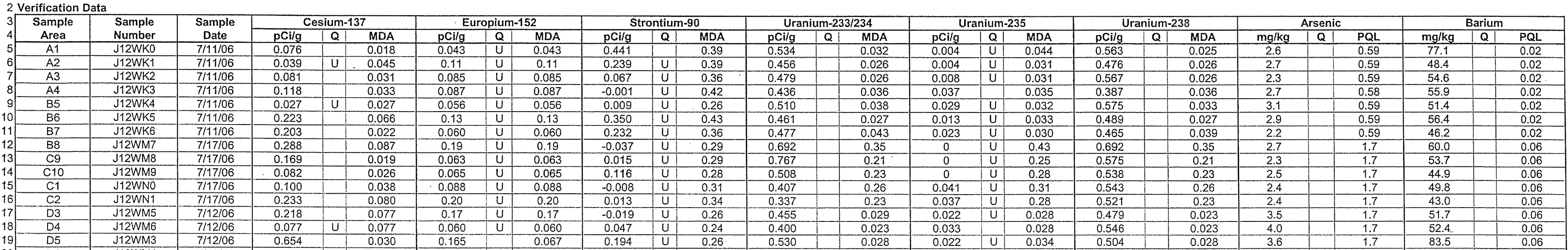

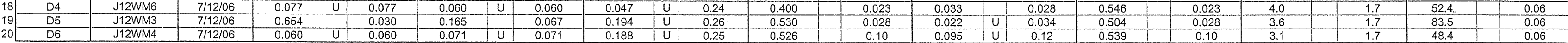

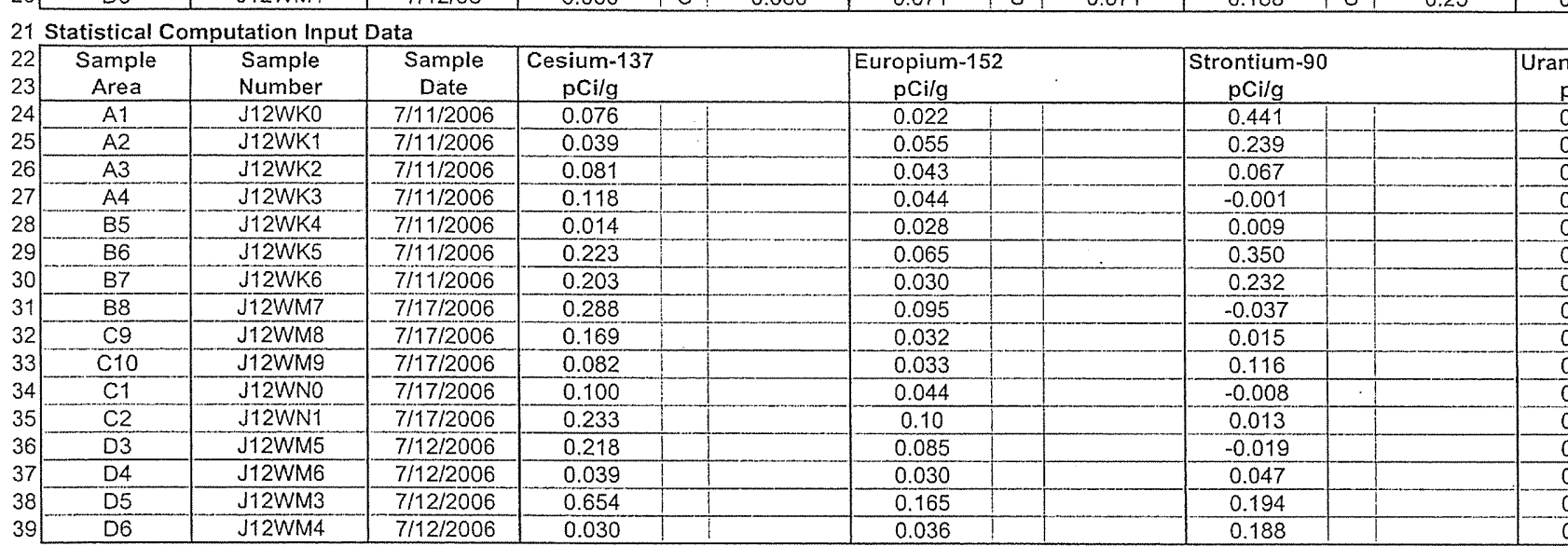

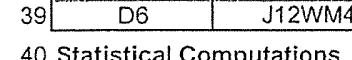

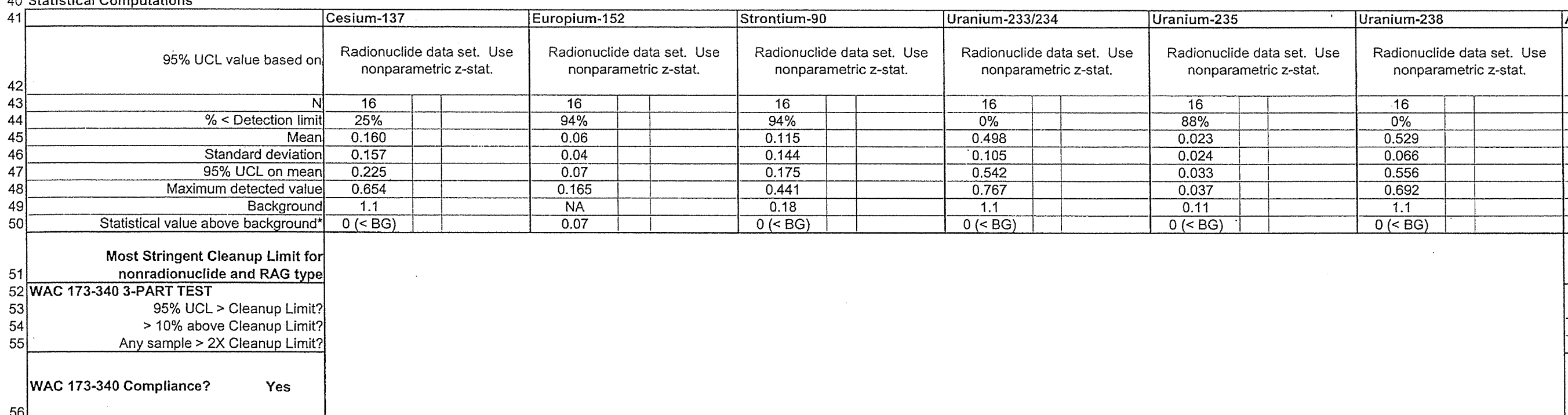

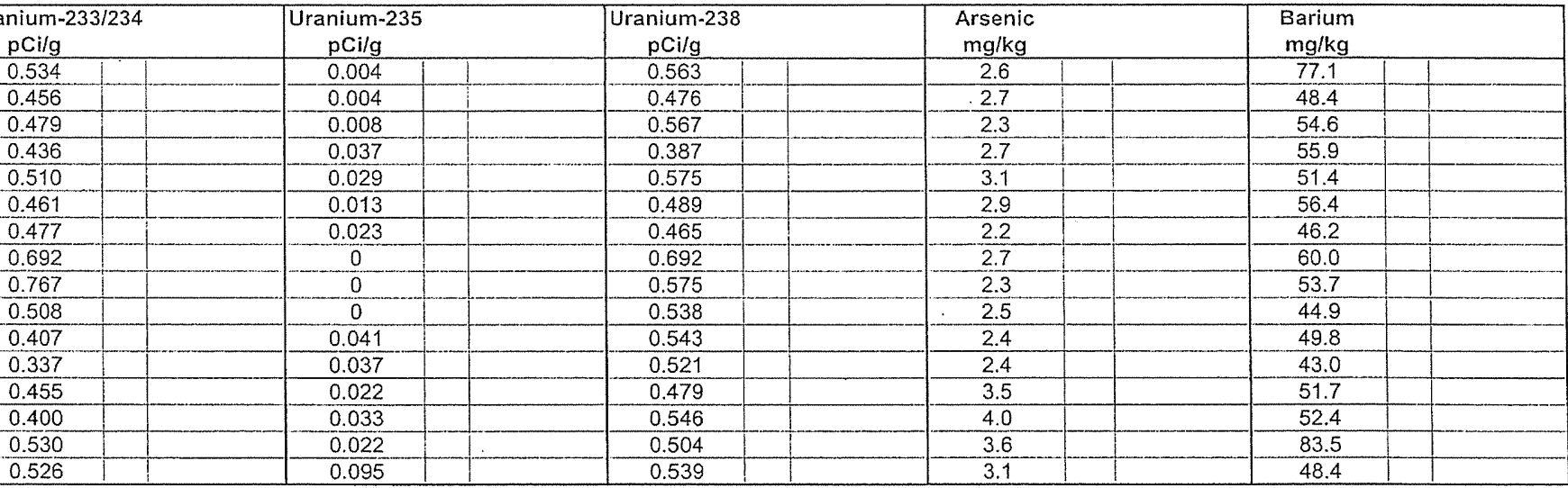

$57^{8}$ Background is not subtracted for nonradionuclides; consideration of background is given by direct comparison against background value

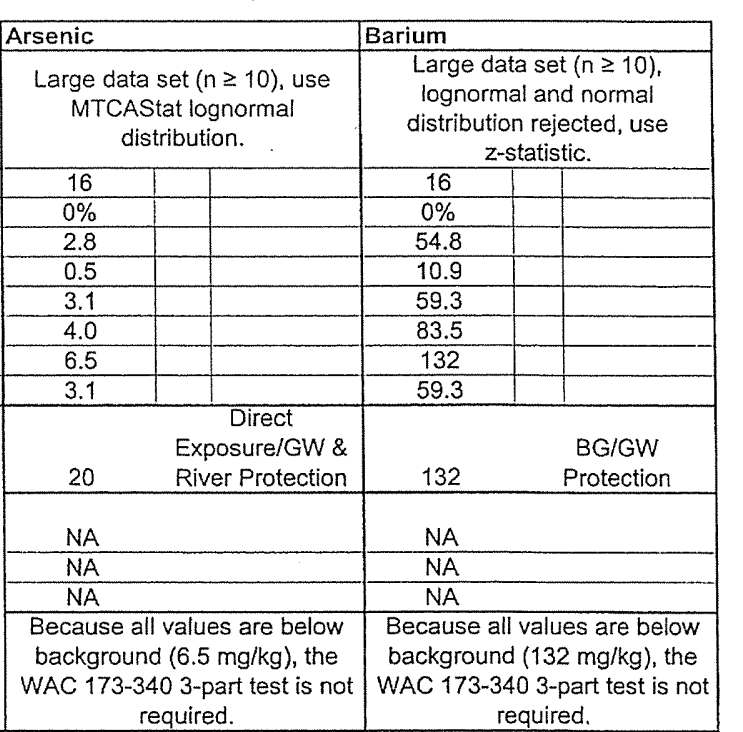

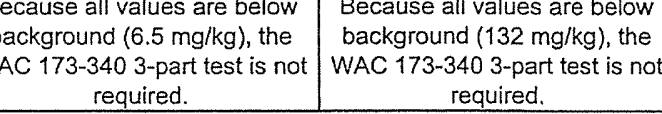


CALCULATION SHEET

118-B-1 Overburden Statistical Calculations (continued)

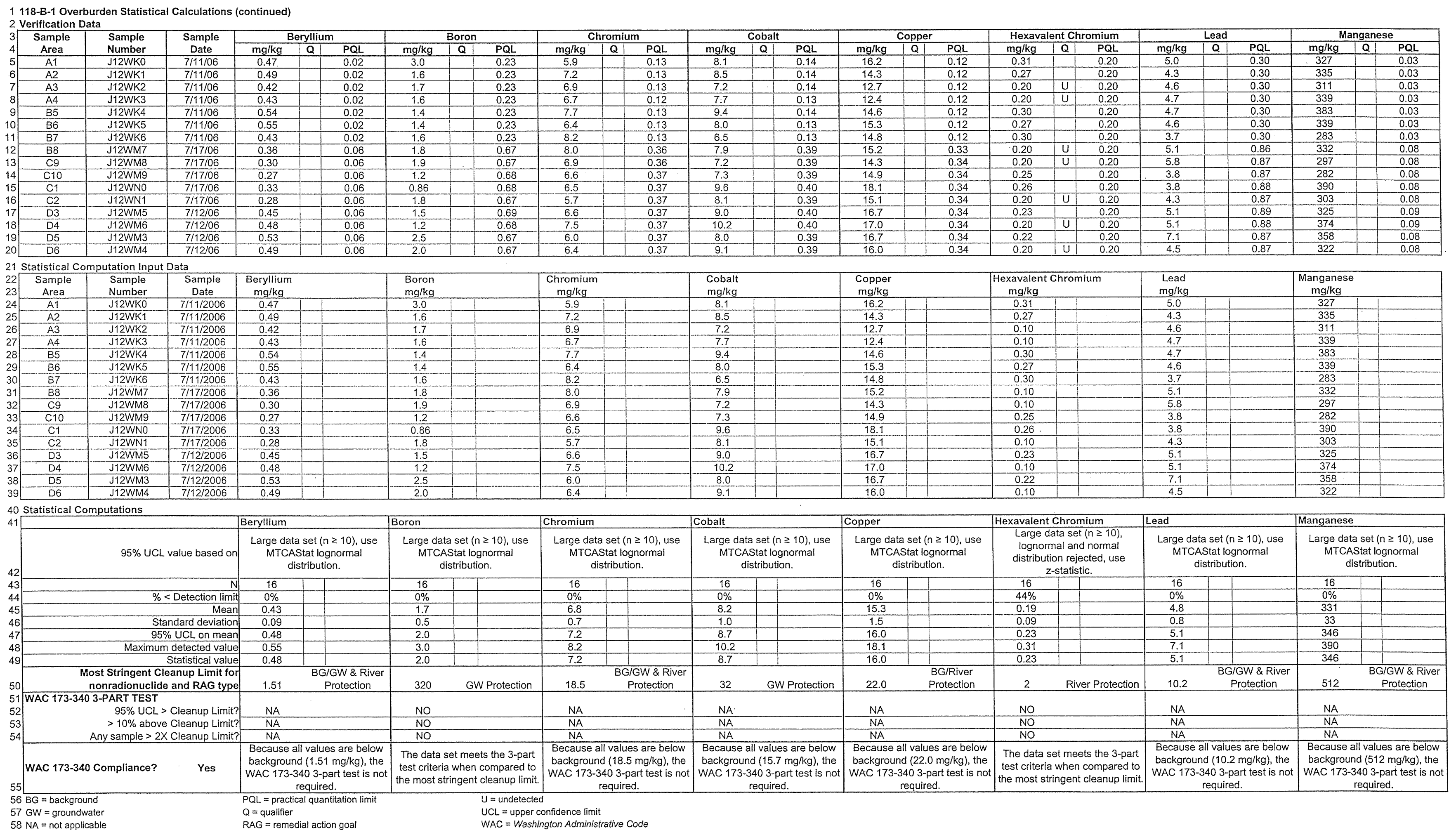


118-B-1 Overburden Statistical Calculations (continued)

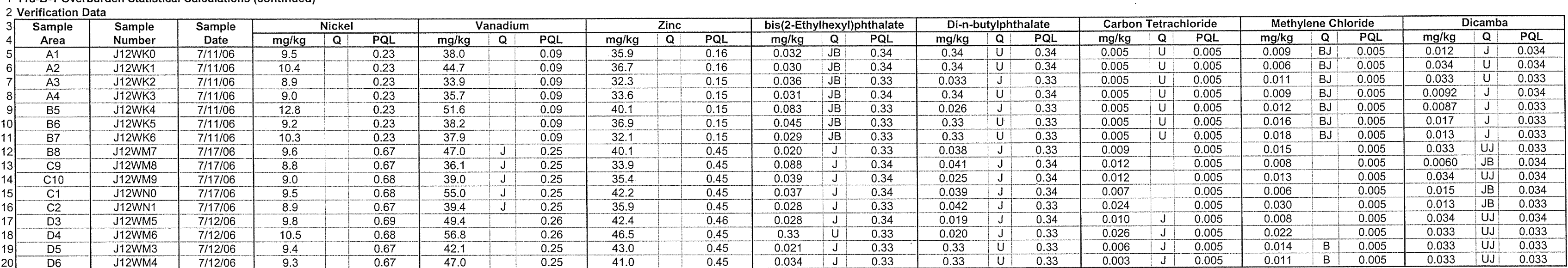

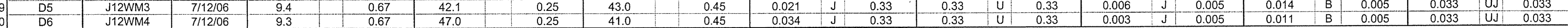

\begin{tabular}{|c|c|c|c|}
\hline \multicolumn{4}{|c|}{$\begin{array}{l}21 \text { Statistical computation nput Data } \\
2 2 \longdiv { \text { Sample } } \text { Sample sample } \\
\text { Sale }\end{array}$} \\
\hline 22 & $\begin{array}{c}\text { Sample } \\
\text { Area }\end{array}$ & $\begin{array}{l}\text { Sample } \\
\text { Number }\end{array}$ & $\begin{array}{c}\text { Sample } \\
\text { Date }\end{array}$ \\
\hline & $A 1$ & $1112 \mathrm{WK1}$ & $7 / 11 / 2006$ \\
\hline & & 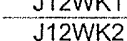 & $\frac{1712006}{7 / 11206}$ \\
\hline & A4 & J12WK3 & $7 / 11 / 2006$ \\
\hline & B5 & J12WK4 & $7111 / 2006$ \\
\hline . & $\begin{array}{l}B 6 \\
87\end{array}$ & J12WK5 & $7 / 11 / 12006$ \\
\hline & & & \\
\hline & $\mathrm{cg}$ & J12WM8 & \\
\hline 33 & C10 & J12WMg & $7 / 17 / 2006$ \\
\hline & C1 & J12WNO & $7 / 17 / 2006$ \\
\hline & $\mathrm{C} 2$ & $J 12 W N 1$ & 11722006 \\
\hline & & 12W156 & 201006 \\
\hline & 55 & 112WM33 & $2 / 2006$ \\
\hline & & & \\
\hline
\end{tabular}
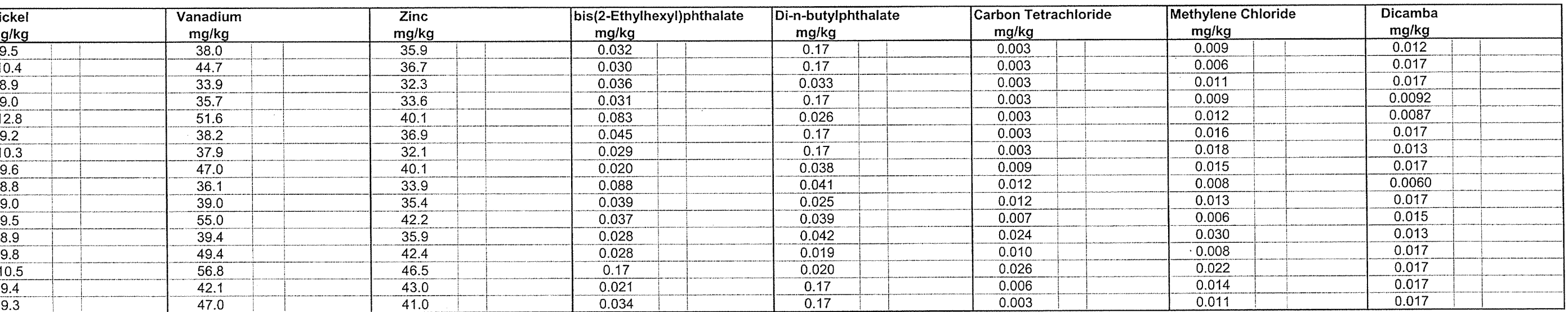

40 Statistical Computations

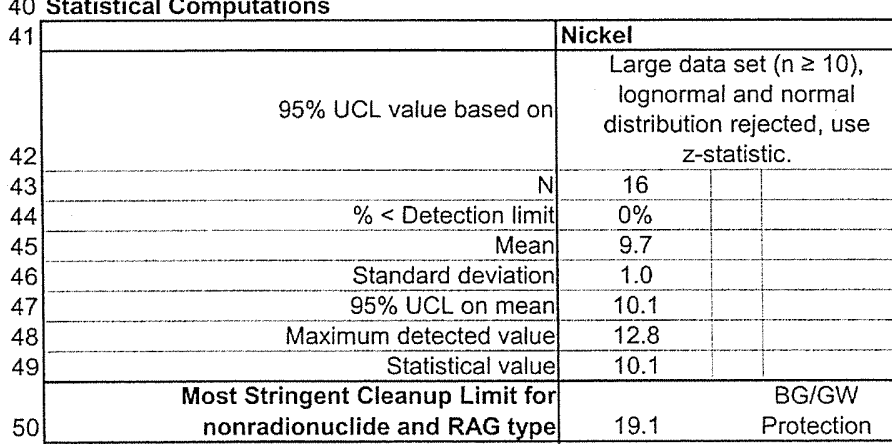

50 Most Stringent Cleanup Limitiuer for

\begin{tabular}{c|c|}
52 & $95 \%$ UCL $>$ Cleanup Limit? \\
53 & $>10 \%$ above Cleanup Limit? \\
54 & Any sample > $2 \times$ Cleanup Limit?
\end{tabular}

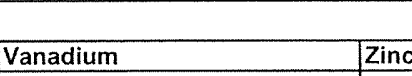

bis(2-Ethylhexyl)phthalate Di-n-butylphthalate

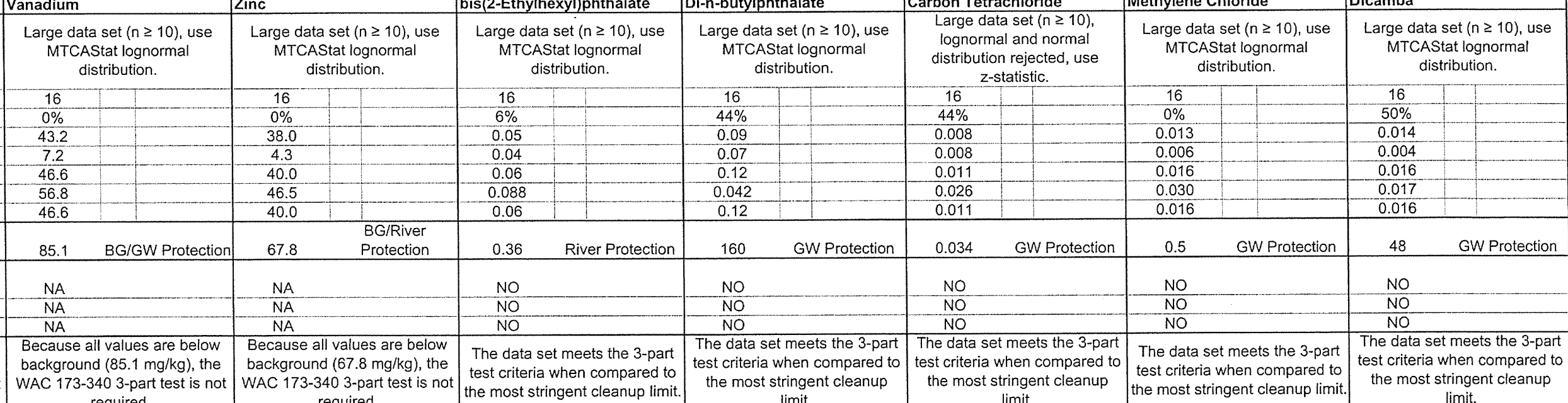

WAC 173-340 Compliance?

Yes

Because all values are below

$56 \mathrm{~B}=$ method blank contamination se

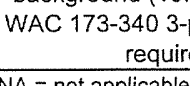

background (85.1 mg/kg), the
WAC 173-340 3-part test is not

$57 \mathrm{BG}=$ background
$58 \mathrm{GW}=$ groundwate

$\mathrm{NA}=\mathrm{not}$ tapplicable
$\mathrm{PQL}=$ practical quantitation lim

$Q=$ qualifier
$R A G=$ remedial action goal

UCL $=$ upper confdidence limit
WAC = Washington Adminisistrative Code 
1118-B-1 BCL Material Statistical Calculations

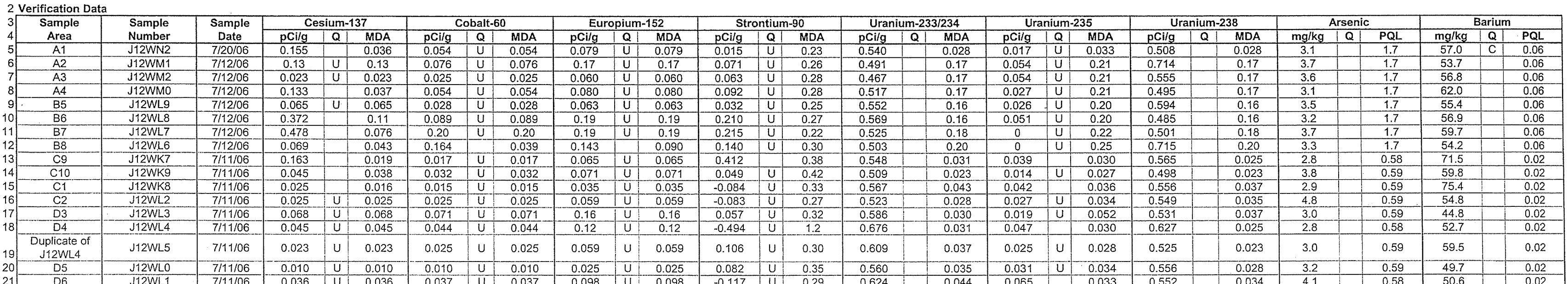

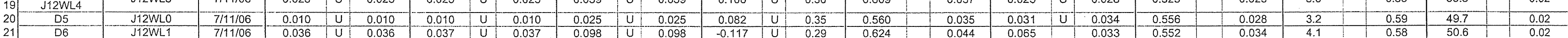
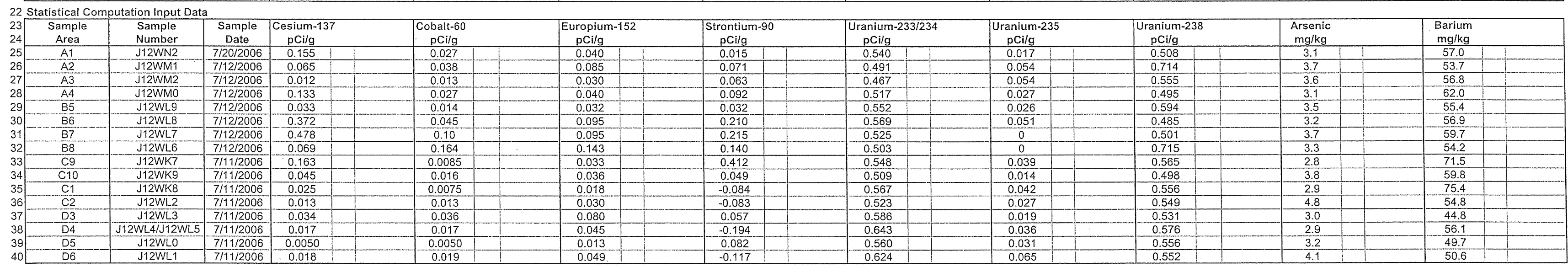

\section{Statistical Computations}

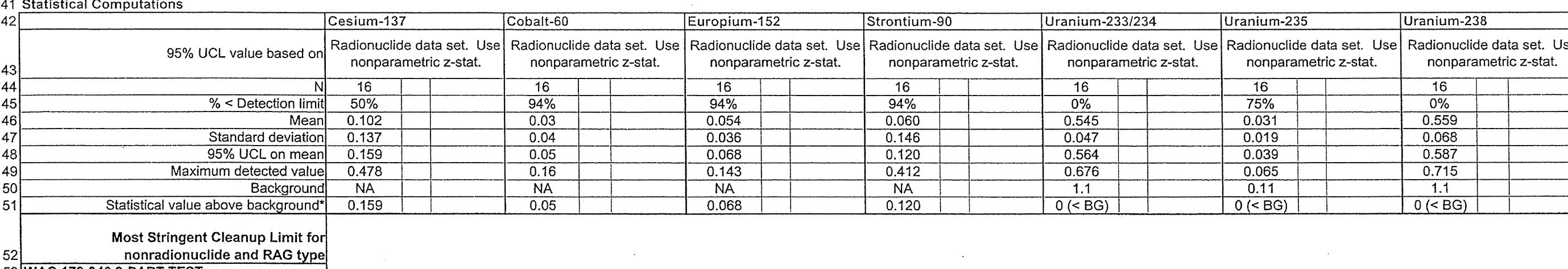

Most Stringent Cleanup Limit for
53 WAC 173-340 3-PART TRESTlide and RAG type

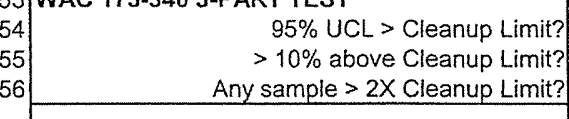

$$
\text { WAC 173-340 Compliance? Yes }
$$

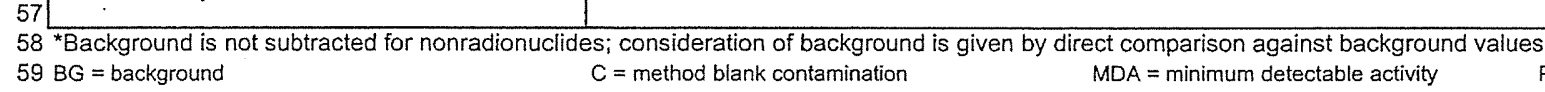

$59 \mathrm{BG}=$ background
$60 \mathrm{BCL}=$ below cleanup level

$\mathrm{C}=$ method blank con
$\mathrm{GW}=$ groundwater

MDA $=$ minimum dete
$N A=$ not applicable

$P Q L=$ practica
$Q=$ qualifier

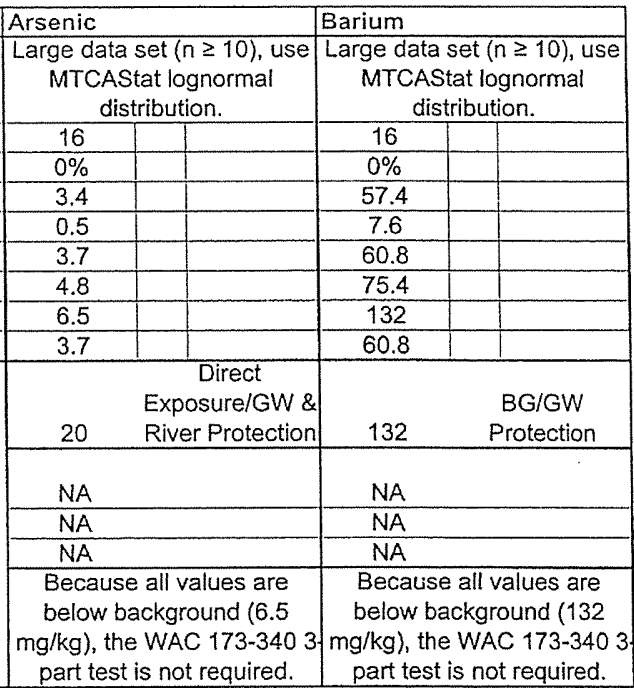

$\mathrm{CL}=$ upper confidence limit

WAC $=$ Washington Adminisistrative Code 
1 118-B-1 BCL Material Statistical Calculations (continued)

\begin{tabular}{|c|c|c|c|c|c|c|c|c|c|c|c|c|c|c|c|c|c|c|c|c|c|c|}
\hline$\sqrt[3]{\text { Sample }}$ & Sample & Sample & \multicolumn{3}{|c|}{ Beryllium } & \multicolumn{2}{|c|}{ Boron } & \multicolumn{2}{|c|}{ Chromium } & \multicolumn{2}{|c|}{ Cobalt } & \multirow{2}{*}{\multicolumn{2}{|c|}{ Copt }} & \multirow{2}{*}{\multicolumn{3}{|c|}{ Hexavalent Chromium }} & \multirow{2}{*}{\multicolumn{3}{|c|}{ Lead }} & \multirow{2}{*}{\multicolumn{3}{|c|}{ Manganese }} \\
\hline$\frac{\text { Area }}{\mathrm{A} 1}$ & $\frac{\text { Number }}{\text { J12WN2 }}$ & $\frac{\text { Date }}{7 / 2106}$ & $\frac{\mathrm{mg} / \mathrm{kg}}{0.47}$ & & 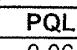 & $\begin{array}{lll}\mathrm{mg} / \mathrm{kg} & \mathrm{C} \\
\mathrm{m}\end{array}$ & & $\mathrm{mg} / \mathrm{kg}$ & $\begin{array}{ll}Q & P Q \\
\end{array}$ & $\mathrm{mg} / \mathrm{kg}$ & 000 & $\mathrm{mg} / \mathrm{kg}$ & & & & & & & & & & \\
\hline A2 & $\begin{array}{ll}\mathrm{J} 12 \mathrm{WW} \\
12 \mathrm{WM}\end{array}$ & $77 / 12100$ & $\begin{array}{l}0.47 \\
0.59\end{array}$ & & $\frac{0.06}{0.06}$ & $\frac{2.0}{16}$ & 0.68 & 6.6 & 0.3 & 9.5 & 0.40 & 15.9 & 0.34 & 0.2 & & 0.20 & 4.9 & & 0.88 & 359 & & 0.08 \\
\hline $\mathrm{A3}$ & $\mathrm{J12 \textrm {WM } 2}$ & $7 / 12 / 1$ & 0.58 & & $\frac{. .06}{0.06}$ & $\frac{1.6}{1.4}$ & 0.68 & $\frac{6.3}{72}$ & 0.3 & 8.2 & 0.40 & $\frac{16.2}{160}$ & 0.34 & 0.22 & & 0.20 & 4.5 & & 0.88 & 337 & & 0.08 \\
\hline A4 & J12WMO & & & & 0.06 & $\frac{1.4}{2.9}$ & 0.000 & $\frac{7.2}{7.2}$ & $\frac{.0 .3}{0.3}$ & $\frac{8.4}{81}$ & 0.40 & $\frac{16.0}{160}$ & $\frac{0.34}{0.34}$ & 0.28 & $\pi$ & & $\frac{4.5}{1.5}-3 x-10$ & & & $\frac{346}{322}$ & & 0.09 \\
\hline$\frac{B 5}{B 6}$ & 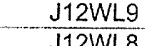 & $7 / 122$ & 0.49 & & 0.4 & 1.3 & 0.68 & 8.3 & 0.3 & 9.1 & 0.40 & $\frac{10.0}{16.2}$ & & 0.28 & & 0.2 & $\begin{array}{ll}4.0 \\
4.4\end{array}$ & & & $\frac{332}{350}$ & & 0.09 \\
\hline$\frac{B 6}{B 7}$ & & & 0.47 & & 0.0 & $\frac{1.5}{1.5}$ & 0.68 & 7.3 & 0.3 & 8.0 & 0.40 & 15.4 & 0.34 & & & 0.20 & 4.8 & & 0.000 & 3 & & -0.09 \\
\hline$B 8$ & & & $\frac{0.46}{0.43}$ & & $\frac{0.4}{0.5}$ & $\frac{1.5}{1 .}$ & 0.68 & 8.7 & 0.3 & 7.9 & 0.40 & 14.6 & 0.34 & 0.20 & u & & 4. & & 0.88 & 329 & & 0.08 \\
\hline & $\mathrm{J12W}$ & & 0.458 & & & $\frac{1.7}{2.8}$ & & $\frac{7.3}{-9.5}$ & 0.3 & 7.3 & 0.40 & $\frac{13.8}{-13.8}$ & & 0.28 & $=$ & & 4. & & 0.88 & 304 & & 0.08 \\
\hline & & & 0.60 & & 0.0 & $\frac{2.0}{1.5}$ & 0. & $\frac{9.5}{7.3}$ & 0.1. & $\frac{7.9}{9.8}$ & 0.13 & $\frac{15.4}{17.2}$ & $\frac{0.1}{0.1} \quad-1$ & 0.20 & 0 & 0.26 & $\frac{5.2}{5.5}$ & & 0.30 & $\frac{327}{382}$ & & -0.03 \\
\hline$c$ & & & $\frac{0.57}{0.58}$ & & 0.0 & $\frac{1.5}{1.2}$ & 0 & 80 & 0.1 & 0.0 & 0.14 & 14.4 & 0. & 0.20 & U & 0. & 4.5 & & 0.30 & 407 & 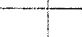 & 0.03 \\
\hline & & & 0.58 & & 0.02 & $\frac{1.2}{1.2}$ & 0 & 8.0 & & $\frac{10.8}{94}$ & $\frac{0.13}{0.14}$ & 16.9 & 0.1 & 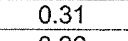 & & 0.20 & 6.5 & & 0.30 & 418 & & 0.03 \\
\hline D4 & J12WL4 & $7 / 11106$ & 0.44 & & 0.0 & $\frac{\frac{1.2}{1.3}}{1.3}$ & 0.23 & 8.8 & $\frac{0.1}{0.1}$ & $\frac{9.4}{7.9}$ & 0.14 & $\frac{14.2}{12.9}$ & 0.12 & 0.26 & & $\frac{0.20}{0.20}$ & $\frac{4.6}{4.9}$ & & $\frac{0.30}{0.30}$ & 年46 324 & & $\begin{array}{l}0.03 \\
0.03\end{array}$ \\
\hline & J1: & $7 / 11$ & 0. & & 0.8 & 1.9 & 0.23 & 7.3 & 0.1 & 7.6 & 0.14 & 12.9 & 0.12 & 0.26 & & 0.20 & 5.0 & & 0.30 & 348 & & 0.03 \\
\hline$D 6$ & $\frac{J 12 W L 0}{J 12 W L 1}$ & $7 / 11106$ & $\begin{array}{l}0.50 \\
0.58\end{array}$ & & $\begin{array}{l}0.02 \\
0.02\end{array}$ & $\frac{1.1}{1.3}$ & 0.23 & 7.1 & 0.1 & 8.8 & 0.13 & 14.3 & 0.12 & 0.27 & & 0.20 & 4.9 & & 0.30 & 339 & & \\
\hline
\end{tabular}

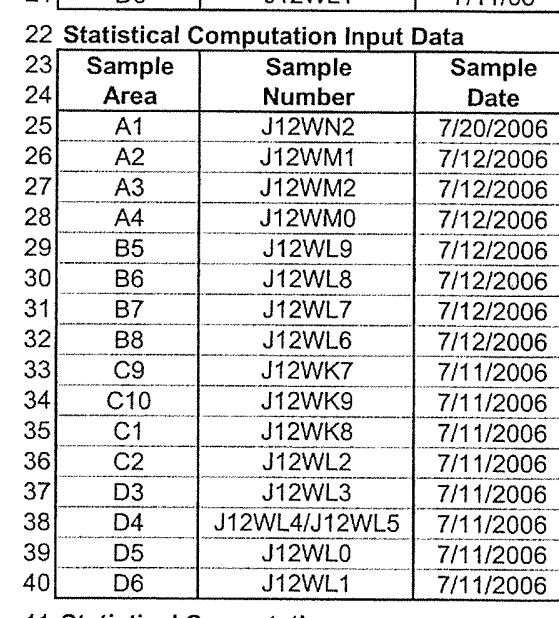

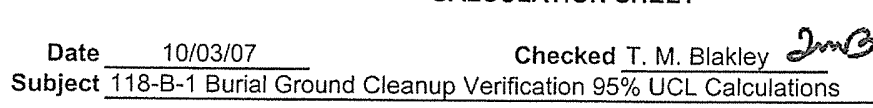

Date $10 / 04 / 07$

Calc. No. $0100 \mathrm{~B}-\mathrm{CA}-\mathrm{V} 0299$

Rev. No. $\frac{1}{31}$
Sheet No. 61
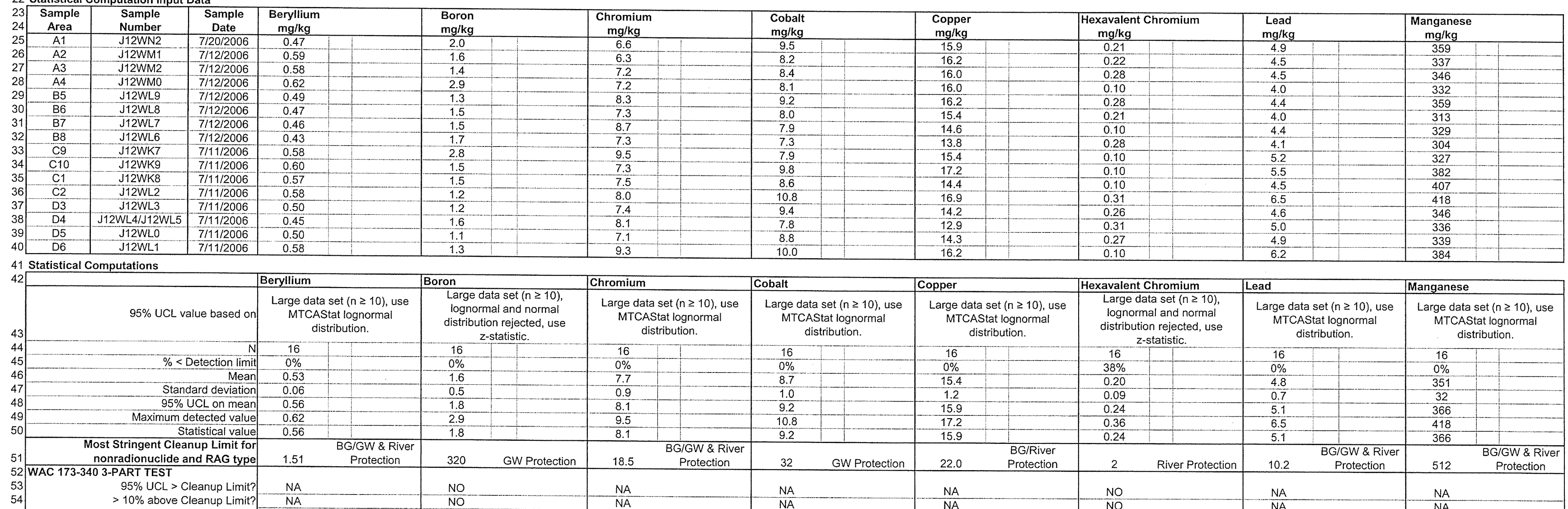

\begin{tabular}{|l|l|l|}
\hline 6.2 & 384 & \\
\hline
\end{tabular}

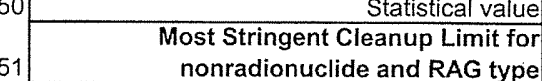

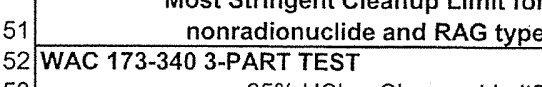

53
54 $\quad \begin{aligned} 95 \% \text { UCL }>\text { Cleanup Limit? } \\ >10 \% \text { above Cleanup Limit? }\end{aligned}$

Any sample $>2 X$ Cleanup Lim

BCL= below cleanup levels

Yes $\begin{aligned} & \text { Because all values are below } \\ & \text { backround }(1.51 \mathrm{mg} / \mathrm{kg} g) \text {, the } \\ & \text { WAC } 173.340\end{aligned}$

\begin{tabular}{l|l} 
NO & NA \\
\hline The data set meets the 3-part & Because all values are below \\
background (135 5 malk) the
\end{tabular}

$7 \mathrm{BCL}=$ below cleanup le
$8 \mathrm{BG}=$ background

\begin{tabular}{l|l} 
Yes & $\begin{array}{l}\text { background }(1.51 \mathrm{mg} \text { t } \\
\text { WAC } 173-340 \text { ) } 3 \text {-part te }\end{array}$ \\
required
\end{tabular}

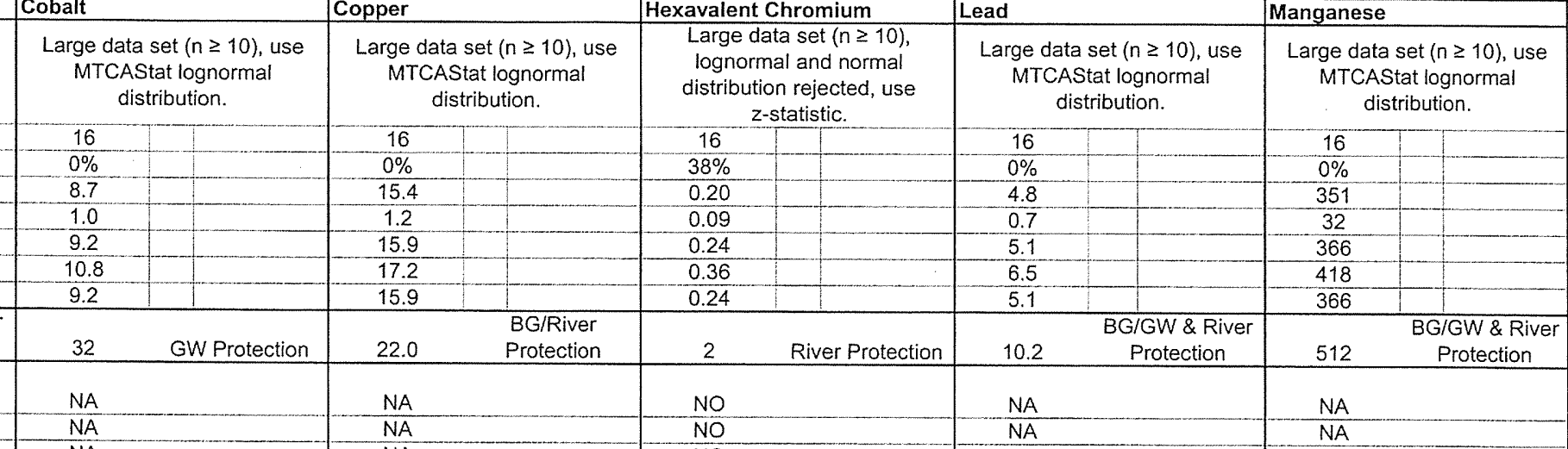

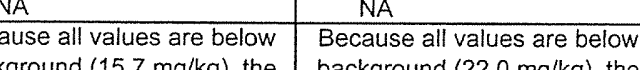

. WAC 173-340 3-part test is to

WAC 173-340 3-part test

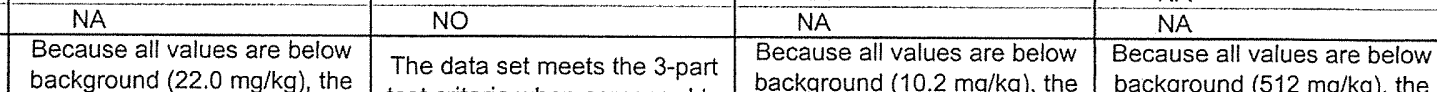

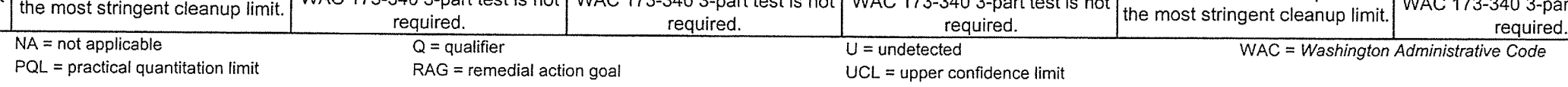

background $(512 \mathrm{mg} / \mathrm{kg})$, the
WAC $173-3403-\mathrm{part}$ test is nol 
118-B-1 BCL Material Statistical Calculations (continued)

\begin{tabular}{|c|c|c|c|c|c|c|c|c|c|c|c|c|c|c|c|c|c|c|c|c|c|c|c|c|c|}
\hline & & Sample & & bde & & & Nick: & & & $\mathrm{ad}$ & & & & & & & Di-n-bi & & & Carbon & & & Methyle & $\overline{\mathrm{neCr}}$ & \\
\hline & Number & & $\frac{n g / k g}{n}$ & & $\overrightarrow{P Q L}$ & $\mathrm{mg} / \mathrm{kg}$ & & $\overrightarrow{P Q L}$ & $\mathrm{mg} / \mathrm{kg}$ & $Q$ & $\mathrm{~g} / \mathrm{kg}$ & $Q$ & PQL & $\mathrm{mg} / \mathrm{kg}$ & $Q$ & $\mathrm{PQL}$ & $\mathrm{mg} / \mathrm{kg}$ & $Q$ & PQL & $\mathrm{mg} / \mathrm{kg}$ & $Q$ & PQL & $\mathrm{mg} / \mathrm{kg}$ & Q & $P Q L$ \\
\hline & $\frac{112 W N 2}{112 W N 1}$ & 206 & 0.82 & 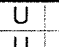 & 0.82 & 10.8 & & 0.68 & $\begin{array}{l}52.7 \\
47.8\end{array}$ & 0.25 & 43.8 & & 0.45 & 0.051 & J & 0.34 & 0.042 & $\sqrt{18}$ & 0.34 & 0.005 & U & 0.005 & 0.013 & B & 0.005 \\
\hline & & & & & & $\begin{array}{c}\frac{10.4}{10.4} \\
1\end{array}$ & & 0.68 & & 0.25 & & & 0.45 & 0.02 & $I^{-1}$ & 0.34 & $\begin{array}{l}0.34 \\
0.30\end{array}$ & $u$ & 0.34 & 0.002 & J & 0.005 & 0.006 & $B$ & 0.005 \\
\hline & J12WMO & 12106 & 0.02 & 0 & $\begin{array}{l}.0 .02 \\
0.82\end{array}$ & 9.3 & & 0.000 & $\begin{array}{l}44.0 \\
47.5\end{array}$ & 0.200 & $\frac{30.0}{39.0}$ & & $\begin{array}{l}0.46 \\
0.45\end{array}$ & 0.0 & $\frac{J}{J}$ & $\begin{array}{l}0.34 \\
0.34\end{array}$ & -0.027 & $\frac{J}{U}$ & $\begin{array}{l}0.34 \\
0.33\end{array}$ & $\begin{array}{l}0.004 \\
0.002\end{array}$ & 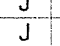 & $\begin{array}{l}0.005 \\
0.005\end{array}$ & $\begin{array}{l}0.007 \\
0.009\end{array}$ & $\frac{B}{B}$ & 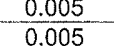 \\
\hline & J12WL9 & & 0.83 & & 0.83 & & & & 54.2 & & & & & & & 0.04 & $\frac{.34}{0.34}$ & & & $\frac{2}{1}$ & & & 0.005 & & $\frac{05}{05}$ \\
\hline & J12WL8 & $7 / 12 / 0$ & 0.82 & $u$ & 0.82 & 9.8 & & 0.68 & & 0.26 & 40.8 & & 0.45 & 0.33 & U & 0.33 & 0.33 & $\bar{U}$ & 0.33 & 0.032 & & & 0.005 & & .005 \\
\hline$B \varepsilon$ & $\frac{112 W 7}{112 W 67}$ & 711211 & $\begin{array}{l}0.82 \\
0.82\end{array}$ & $u$ & 0.82 & $\frac{11}{10}$ & & & $\begin{array}{l}42.5 \\
372\end{array}$ & 0.25 & $\frac{37.5}{32.5}$ & & 0.45 & 0.33 & $\frac{u}{4}$ & $\frac{0.33}{0.34}$ & 0.33 & 0 & 0.33 & 0.013 & & & 0.002 & $\sqrt{1}$ & $\frac{05}{25}$ \\
\hline & & & & & 0.08 & $\frac{5.4}{11.0}$ & & & 4 & & $\begin{array}{l}\frac{345}{38.5} \\
38\end{array}$ & & 0.45 & & $\sqrt{3 B}$ & & & $J$ & 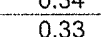 & & 11 & & & & \\
\hline $\mathrm{C}_{10}$ & & & 0.49 & & 0.28 & 11 & & 0 & 46 & 0. & 44.9 & & 0.1 & 0.036 & J & 0.34 & 0.023 & J & 0.34 & 0.005 & & & $\frac{.008}{0.008}$ & & 05 \\
\hline $\mathrm{c}$ & & & 0.39 & & 0.28 & 10.1 & & 0.23 & 43 & 0. & 38.6 & & 0. & 0. & $\mid \mathrm{JB}$ & $\frac{0.34}{0.34}$ & 0.049 & J & 0.3 & 0.005 & $\bar{U}$ & 0.005 & 0.005 & BJ & 005 \\
\hline & $\sqrt{11}$ & & 0.50 & & 0.28 & $\frac{12.5}{110}$ & & & 49 & 0.0 & 44. & & 0.1 & 23 & JB & 0.34 & 0.34 & u & 0. & 0.011 & & 0.000 & 0.018 & Du & 0.005 \\
\hline$\frac{\mathrm{DS}}{\mathrm{D} 4}$ & $\frac{N 12 W L}{J 12 W L}$ & $\frac{171106}{711106}$ & 0.422 & & -0.28 & $\frac{11.9}{9.6}$ & & $\begin{array}{l}0.23 \\
0.23\end{array}$ & $\begin{array}{l}\frac{49.6}{39.7} \\
39\end{array}$ & $\begin{array}{l}0.09 \\
0.09\end{array}$ & $\begin{array}{l}40.0 \\
35.3\end{array}$ & & $\begin{array}{l}0.15 \\
0.15\end{array}$ & $\begin{array}{l}0.029 \\
0.020\end{array}$ & $\left|\frac{B B}{J B}\right|$ & $\begin{array}{l}0.34 \\
0.33\end{array}$ & $\frac{0.018}{0.33}$ & $\frac{J}{U}$ & $\frac{0.34}{0.33}$ & $\frac{0.006}{0.006}$ & & 0.005 & $\frac{0.015}{0.012}$ & \begin{tabular}{|l|}
$B J$ \\
$B J$
\end{tabular} & $\frac{0.005}{0.005}$ \\
\hline & $\mathrm{J12V}$ & $7 / 11 / 0$ & 0.3 & & 0.2 & 10 & & 0.2 & 36 & 0. & 34 & & 0. & 0. & $|\mathrm{JB}|$ & 0.3 & 0.045 & J & 0.3 & 0.005 & $u$ & 0.005 & 0.011 & & 0.005 \\
\hline
\end{tabular}
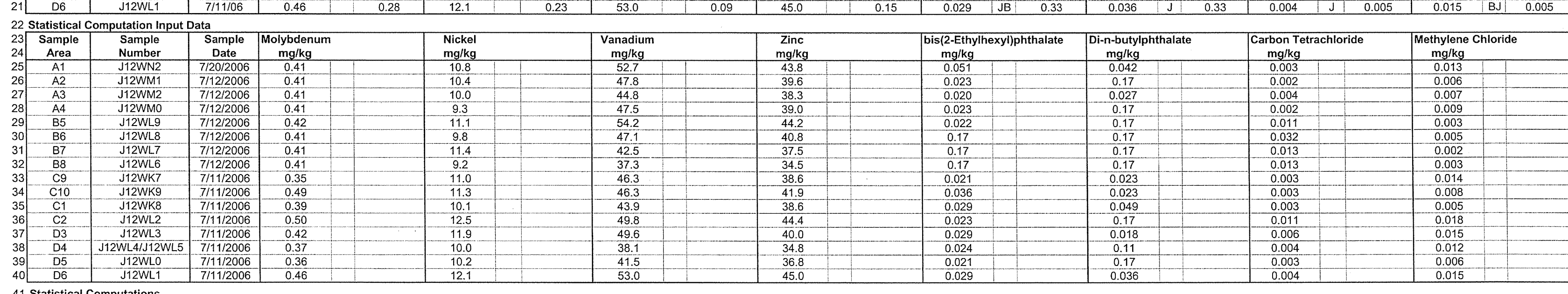

41 Statistical Computations
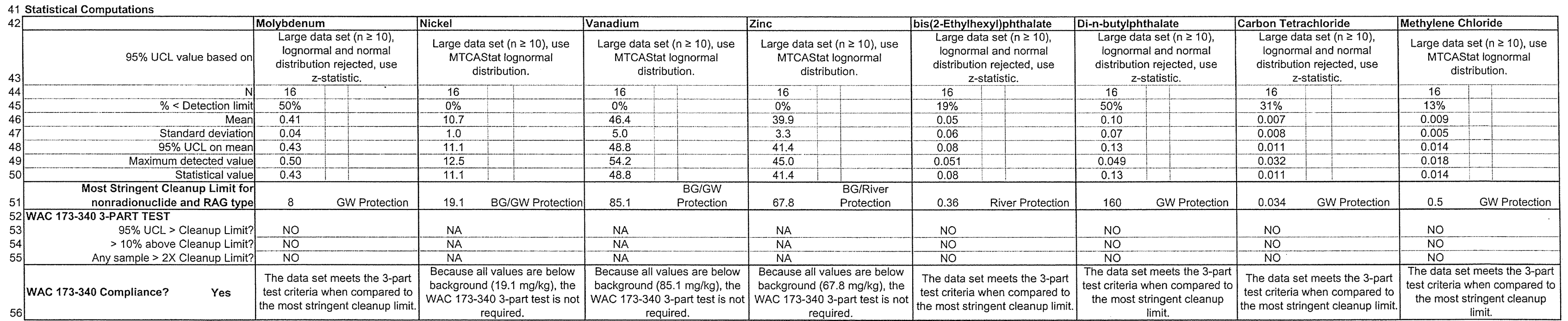
TCAStat lognorn
distribution.

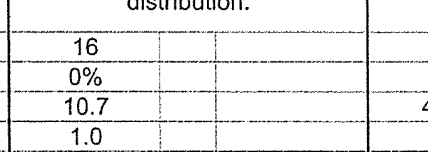

$57 \mathrm{~B}=$ method blank contamination

Yes

$58 \mathrm{BCL}=$ below cleanh
$59 \mathrm{BG}=$ background $\mathrm{GW}=$ groundwater
$J=$ estimate
$N A=$ not applicable

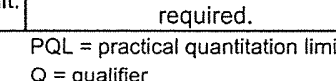
$Q=$ =
$R A$ ualacificar
$R A$ remedial action goal

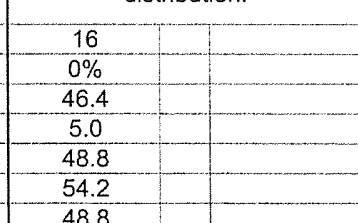
\begin{tabular}{l}
85.1 \\
\hline NA \\
\hline NA \\
\hline NA
\end{tabular} Because all values are below

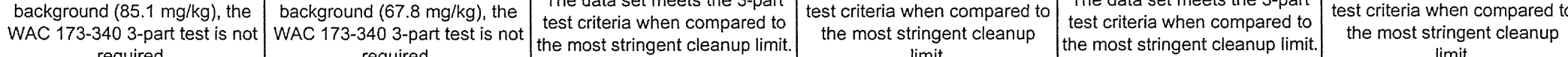

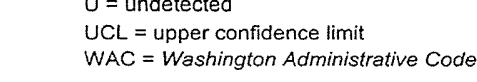


CALCULATION SHEET

1118-B-1 Staging Pile Area Statistical Calculations

\begin{tabular}{|c|c|c|c|c|c|c|c|c|c|c|c|c|c|c|c|c|c|c|c|c|c|c|c|c|c|c|}
\hline \multirow{2}{*}{\begin{tabular}{|c|c|} 
& Sample \\
& Area \\
\end{tabular}} & \multirow{2}{*}{$\begin{array}{c}\text { Sample } \\
\text { Number } \\
\end{array}$} & \multirow{2}{*}{$\begin{array}{c}\text { Sample } \\
\text { Date }\end{array}$} & \multicolumn{3}{|c|}{ Americium-241 } & \multicolumn{3}{|c|}{ Carbon-14 } & \multicolumn{3}{|c|}{ Cesium-137 } & \multicolumn{3}{|c|}{ Cobalt-60 } & \multicolumn{3}{|c|}{ Europium-152 } & \multicolumn{3}{|c|}{ Nickel-63 } & \multicolumn{3}{|c|}{ Plutonium-239/240 } & \multicolumn{3}{|c|}{ Strontium-90 } \\
\hline & & & $\mathrm{pCi/g}$ & & & pCilg & & $\overline{M D A}$ & $\mathrm{pCi} / \mathrm{g}$ & 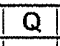 & MDA & pCilg & & MDA & pCilg & Q & MDA & pCilg & & MDA & & Q & & pcilg & & MDA \\
\hline$\frac{A 1}{A 2}$ & 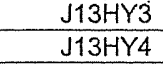 & $\frac{10 / 12106}{10 / 12106}$ & $\frac{0.039}{0}$ & $u$ & $\frac{0.30}{0.23}$ & $\frac{0.350}{0.017}$ & $\frac{U}{U}$ & $\frac{2.7}{2.5}$ & 0.217 & & $\frac{0.059}{0.034}$ & $\begin{array}{l}0.056 \\
0.030 \\
\end{array}$ & & 0.056 & $\begin{array}{l}0.16 \\
0.075\end{array}$ & $\frac{U}{u}$ & $\begin{array}{l}0.16 \\
0.055 \\
\end{array}$ & $\begin{array}{l}-0.455 \\
-0.0 .08 \\
-10\end{array}$ & \begin{tabular}{|l|l}
$u$ \\
\end{tabular} & $\frac{3.3}{3.4}$ & $\frac{0}{0.109}$ & u & $\frac{0.55}{0.42}$ & $\begin{array}{c}-0.035 \\
-0.053\end{array}$ & $\frac{U}{U}$ & $\frac{0.24}{0.26}$ \\
\hline A3 & J13HY5 & $10 / 12106$ & -0.055 & $u$ & 0.21 & -0.453 & $u$ & 2.8 & 0.158 & & 0.054 & 0.030 & U & 0.030 & $\frac{0.05}{0.17}$ & u & $\frac{0.015}{0.17}$ & $\begin{array}{l}-.0 .030 \\
0.392 \\
-10\end{array}$ & $u$ & $\frac{3.4}{3.5}$ & & U & 0.41 & 0.096 & $u$ & 0 \\
\hline $\begin{array}{l}\mathrm{A} 4 \\
\mathrm{~B} 5\end{array}$ & $\begin{array}{l}\text { J13HY6 } \\
13 H Y 7\end{array}$ & $\frac{10 / 121 / 166}{10 / 12106}$ & 0.031 & $U$ & $\frac{0.24}{0.33}$ & -0.762 & $\frac{U}{11}$ & $\frac{2.6}{2.6}$ & 0.233 & & 0.026 & $\begin{array}{l}0.025 \\
0.064\end{array}$ & $U$ & 0.025 & 0.062 & U & 0.062 & -0.597 & $\frac{U}{U 1}$ & 3.5 & 0.092 & \begin{tabular}{|l|l|}
$U$ \\
11 \\
\end{tabular} & 0.35 & 0.227 & & $\frac{0.22}{0.24}$ \\
\hline $\begin{array}{l}\text { Suplicate of } \\
13+Y Y\end{array}$ & J13309 & $10 / 12 / 06$ & 0.057 & U & 0.27 & -0.403 & $u$ & 2.7 & 0.109 & & 0.032 & 0.033 & U & 0.033 & 0.073 & $u$ & 0.073 & $\frac{0.902}{0.723}$ & $u$ & 3.5 & 0.044 & $u$ & 0.34 & 0.002 & $u$ & 0.24 \\
\hline$B 6$ & $\sqrt{13}$ & & -0.022 & $u$ & 0.17 & -0.733 & $u$ & 2.8 & 0.0 & & 0.6 & 0.037 & U & & 0.8 & u & & 2.54 & & & 0.0 & $U$ & 0.33 & 0.105 & & 0.29 \\
\hline & J13HY9 & & 0.154 & $\square$ & & & $u$ & 2.0 & & & & & & & 0.0 & 0 & & & 10 & & 0.041 & & & 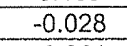 & & 8 \\
\hline$\frac{B 8}{C 9}$ & & & 0.098 & $U$ & 0.19 & $-\frac{1.02}{1.0}$ & U & 2.6 & 0.084 & $u$ & 0.084 & 0.075 & $\bar{U}$ & 0.075 & 0.21 & U & 0.21 & 1.66 & u & 2.4 & 0 & $U$ & 0.35 & -0.061 & U & 0.17 \\
\hline$\frac{c 9}{c 10}$ & $\frac{13301}{113002}$ & & 0 & $\frac{U}{11}$ & 0.20 & $\frac{-1.15}{-10^{2}}$ & $\frac{U}{4}$ & $\frac{2.6}{20}$ & 0.223 & & $\begin{array}{r}0.040 \\
0.073\end{array}$ & $\begin{array}{l}0.040 \\
0.071\end{array}$ & & 0.0 & 0.094 & $\frac{u}{11}$ & 0.094 & 0.500 & U & 2.4 & 0.314 & u & 0.40 & 0.016 & $u$ & 0.17 \\
\hline 10 & J15J02 & 101 & 0 & $u$ & $\begin{array}{l}0.110 \\
0.16\end{array}$ & $\frac{-1.02}{0.221}$ & U & $\frac{2.0}{22}$ & $\frac{0.112}{0.066}$ & & -0.073 & 0.042 & & -0.0 & 0.19 & & $\frac{0.19}{0.07}$ & -0.966 & $\frac{u}{4}$ & & $\frac{0.136}{0.088}$ & $\bar{U}$ & $\frac{0.33}{0.34}$ & $\frac{0.028}{0.166}$ & $\frac{u}{u}$ & 0.26 \\
\hline t. & J13J04 & & 0.126 & & 0.040 & -0.775 & $\frac{u}{u}$ & 2.4 & 239 & & 0.00 & 0.0 & & 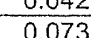 & $\frac{0.112}{0.862}$ & & 0.013 & $\frac{0.126}{246}$ & & & 2. & 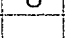 & $\frac{104}{25}$ & $\frac{0.106}{0.934}$ & & $\frac{0.17}{0.18}$ \\
\hline & J1330 & & & $\bar{J}$ & & -0.038 & Tu & 2.1 & 0.057 & U & & $\frac{0.6}{0.6}$ & & & & & 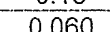 & 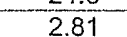 & u & & 0.26 & U & & & & $\frac{1.0}{20}$ \\
\hline$D 4$ & J13J06 & & & $U$ & 0.6 & 0.645 & U & 2 & & & & 0.6 & $\bar{U}$ & & & & & 1.66 & U & 20 & 0.02 & $\bar{U}$ & & 000 & & 18 \\
\hline$\pi$ & 1010 & & 0.036 & 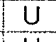 & 0.14 & -0.943 & U & 2. & 0 & & 0.0 & 0.03 & & 0. & 0.06 & U & 0.8 & 3.60 & & 2.5 & -0.012 & $\mathrm{U}$ & 0.067 & 0.109 & 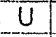 & 25 \\
\hline$D 6$ & J13J08 & $10 / 23 / 106$ & 0.046 & U & 0.18 & 11.3 & & & 0.669 & & 0 & & & & 0.17 & u & & 2.9 & iu & & 0 & Uu & & 0.131 & U & 0.31 \\
\hline
\end{tabular}

22 Statistical Computation Input Data

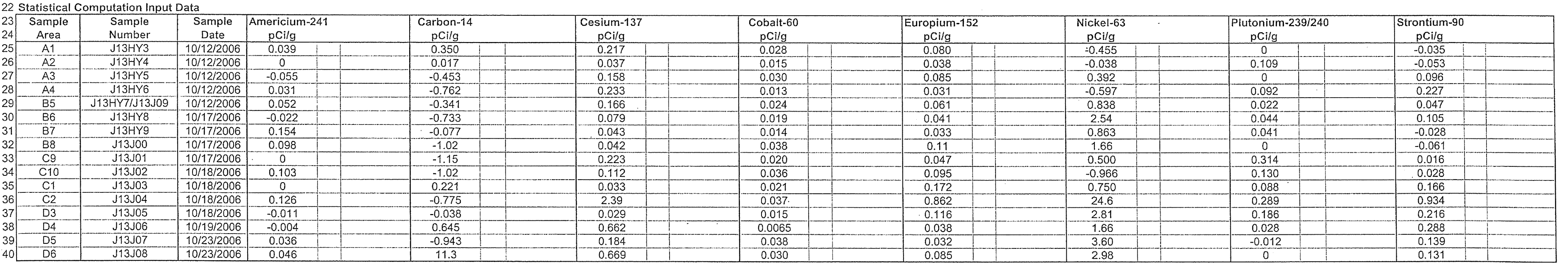

41 Statistical Computations

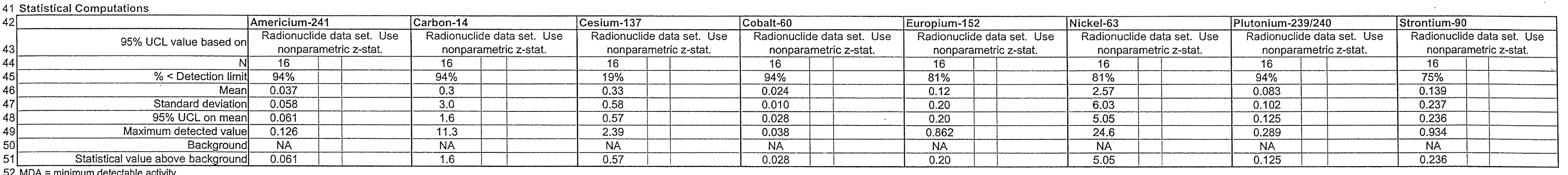

\begin{tabular}{ll|r}
50 & Background & NA \\
$51-$ Statistical value above background & 0.061
\end{tabular} $53 \mathrm{NA}=$ not applicable

$54 \mathrm{Q}=$ qualifier
$55 \mathrm{U}=$ = undetected

$55 \mathrm{U}=$ undetected
56 UCL = upper confidence limit 
CALCULATION SHEET

118-B-1 Staging Pile Area Statistical Calculations (continued)

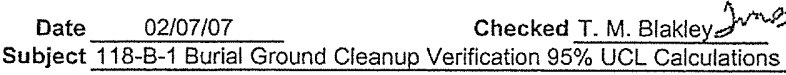

Date $212010 \%$

Calc. No.
Job No. $\frac{01008 \text {-CA.V0299 }}{14655}$

Rev. No. $\frac{0}{34}$ of 61
Sheet No.

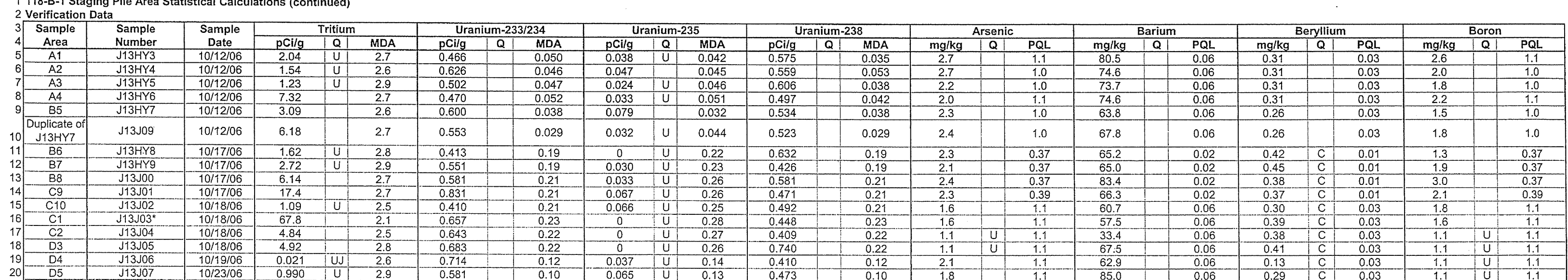

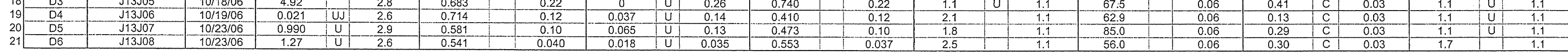

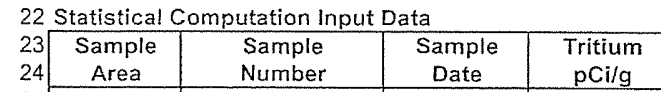
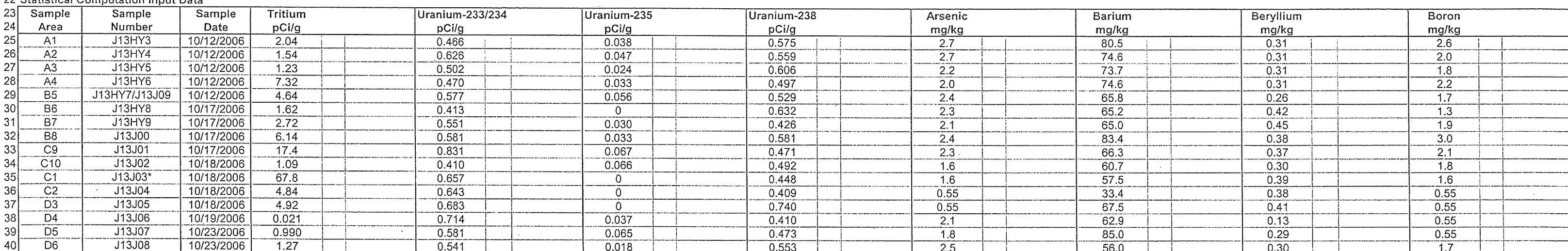

41 Statistical Computation
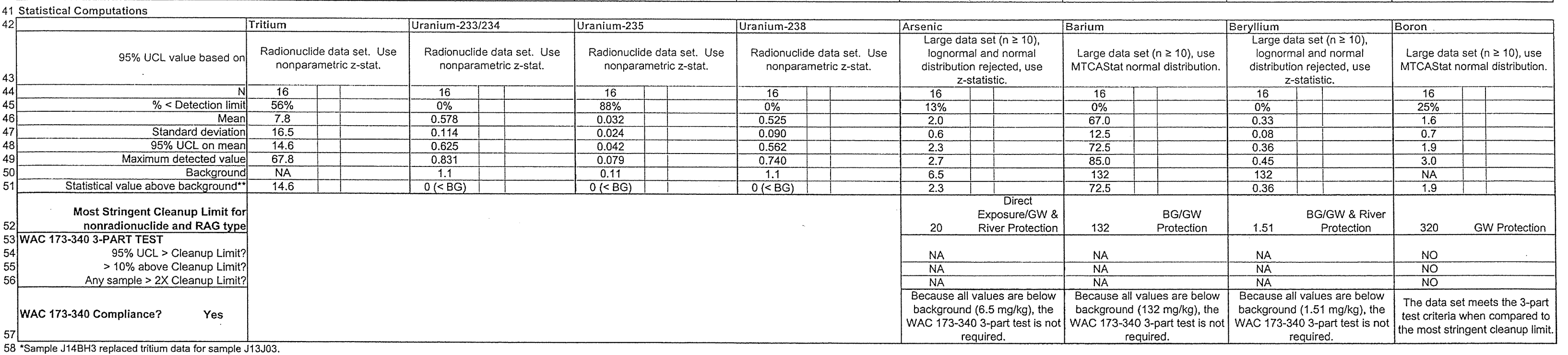

${ }_{58}^{57}$ 'Sample 14 148H3 replaced tritium datat for sample 413.303

59 "Background is not subtracte
$60 \mathrm{BG}=$ background
$61 \mathrm{C}=$ method blank contamination

$\mathrm{GW}=$ groundw
$J=$ estimate

$M O A=$ minimum dete
$N A=$ not applicable

$P Q L=$ practical
$Q=$ qualifier

Large data set $(n \geq 10)$, background ( $6.5 \mathrm{mg} / \mathrm{kg}$ ), the
WAC $173-340$ - 3 - 0 -part test is n 


\section{118-B-1 Staging Pile Area Statistical Calculations (continued)}
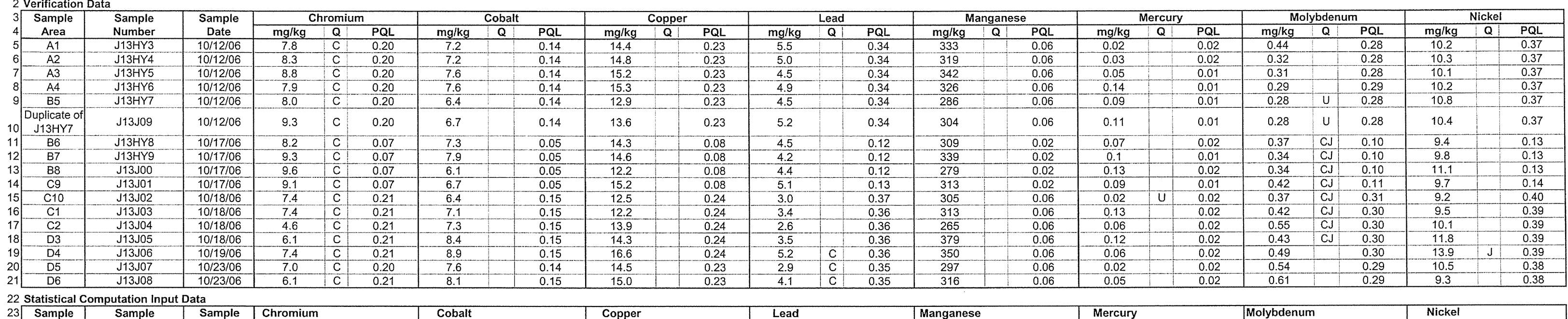

Cobalt
mglkg

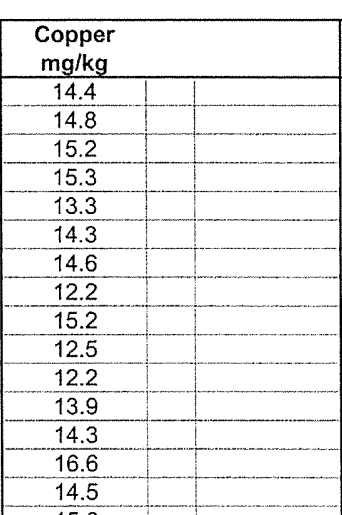

\begin{tabular}{|l|l} 
Lead & Manganese
\end{tabular}

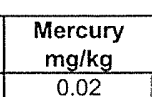

\begin{tabular}{|c}
$\substack{\text { Molybdenum } \\
\mathrm{mg} / \mathrm{kg}}$ \\
$\mathrm{O}$
\end{tabular}
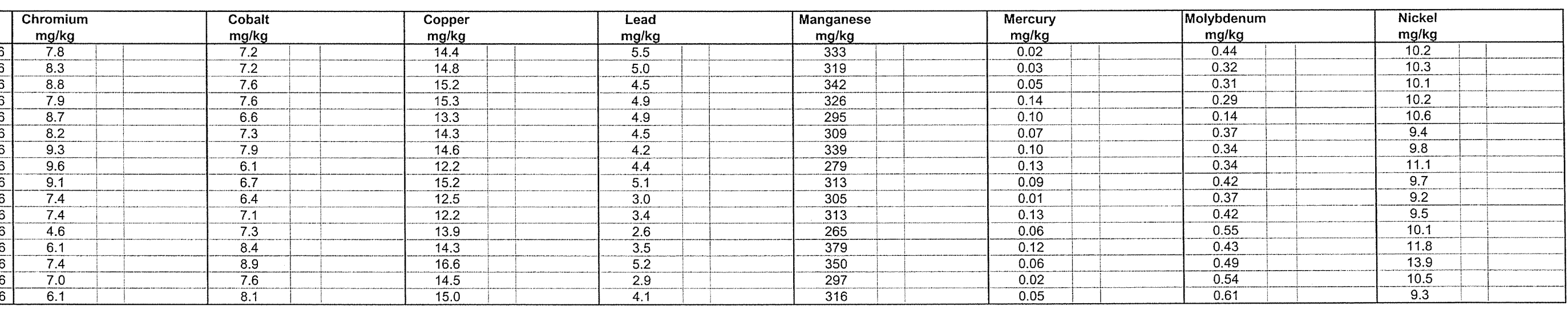

0.03
0.05
0.14
0.10

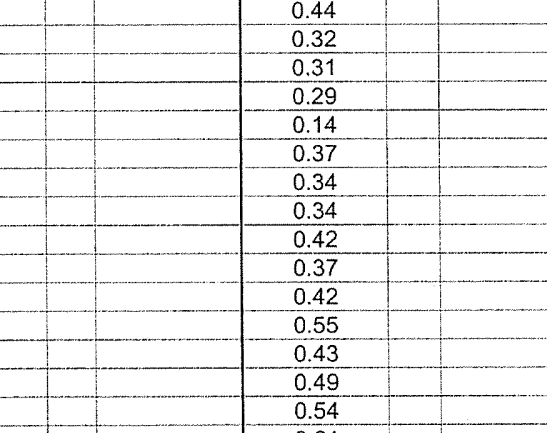

Molybdenum

Niche
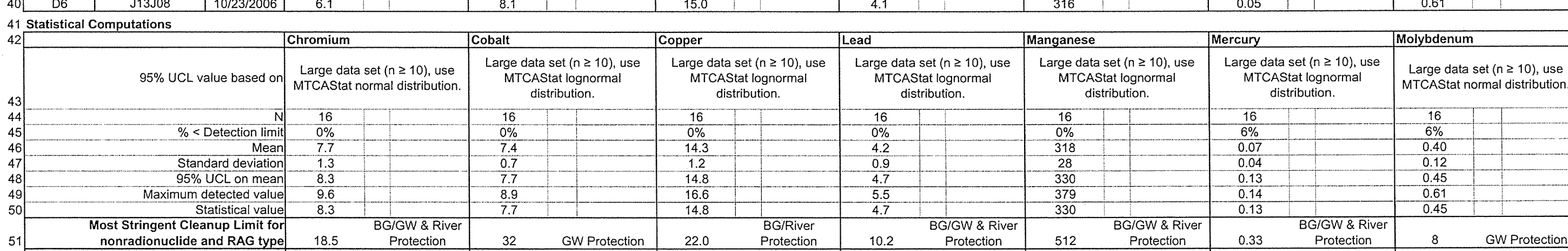

Nickel
Large data set $(n \geq 10)$,
(a)
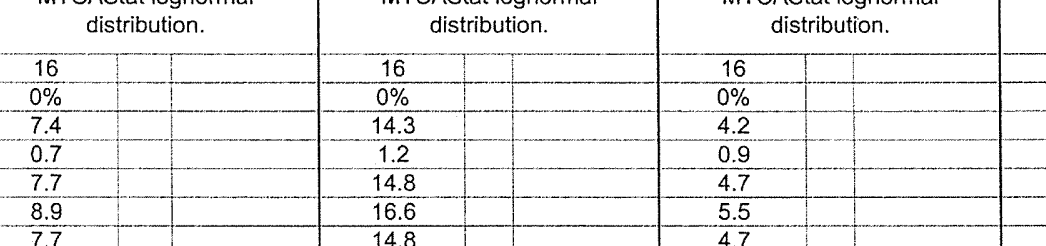

\begin{tabular}{l|l}
51 & nonradionuclide and RAG type \\
52 & WAC 173-340 3-PART TEST
\end{tabular} 53
54
55 $\quad \begin{aligned} 95 \% \text { UCL } \\ 10 \text { a Cleanup Limitit? } \\ \text { Any sampleanp L Limit }\end{aligned}$
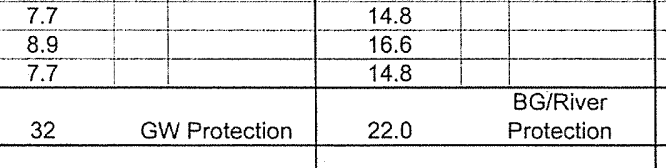

WAC 173-340 Compliance? Yes

56
57
$56=$ background
$55=$ method bank contamination wac

Because all values are below

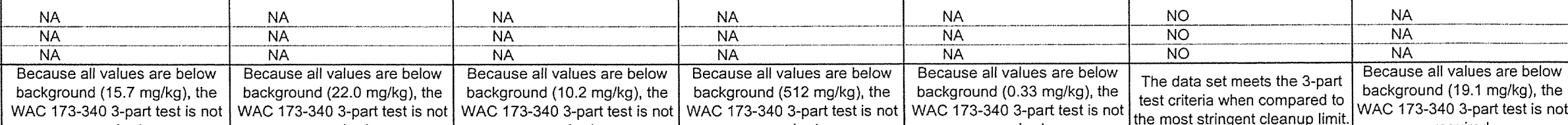
BG/River
Protection
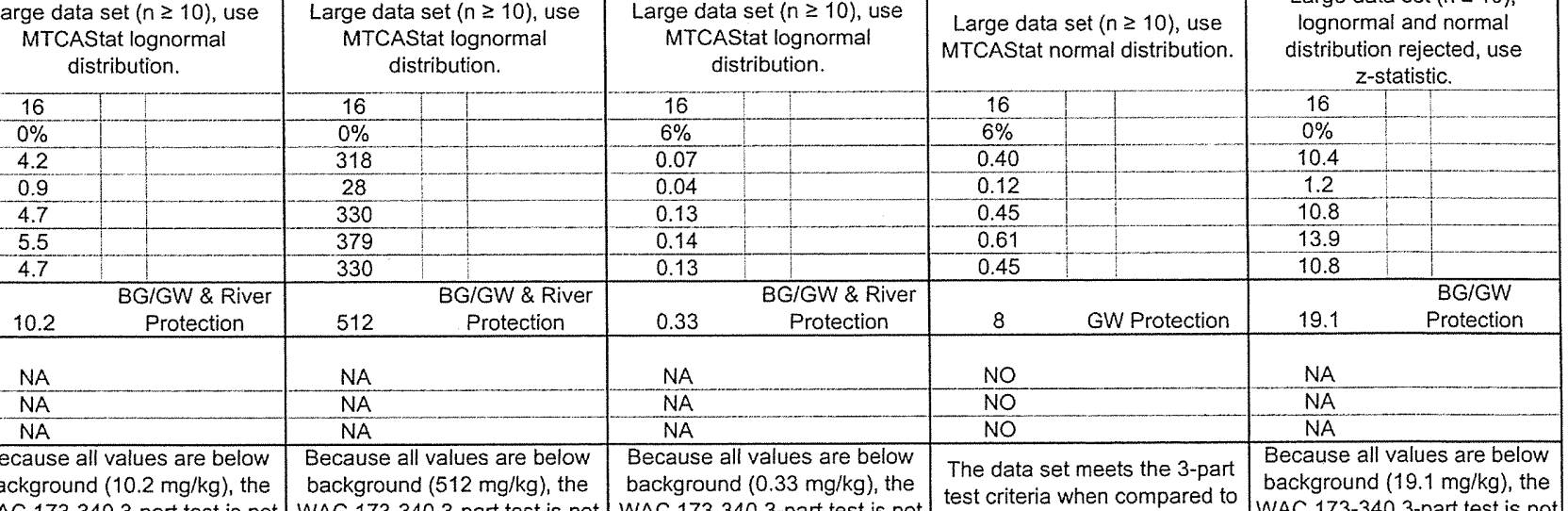

$58 \mathrm{C}=$ methoo blank co
$59 \mathrm{GW}=$ groundwater $J=$ estimate
$N A=$ not applicable
$P(2)=$ Pair $\mathrm{NA}=$ not applicable
$\mathrm{PQL}=$ pracicical quantitition limit

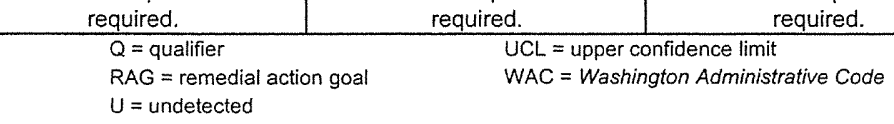
required. the most stringent cleanup lim

WAC 173-340 3-part test 


\section{8-B-1 Staging Pile Area Statistical Calculations (continued)}
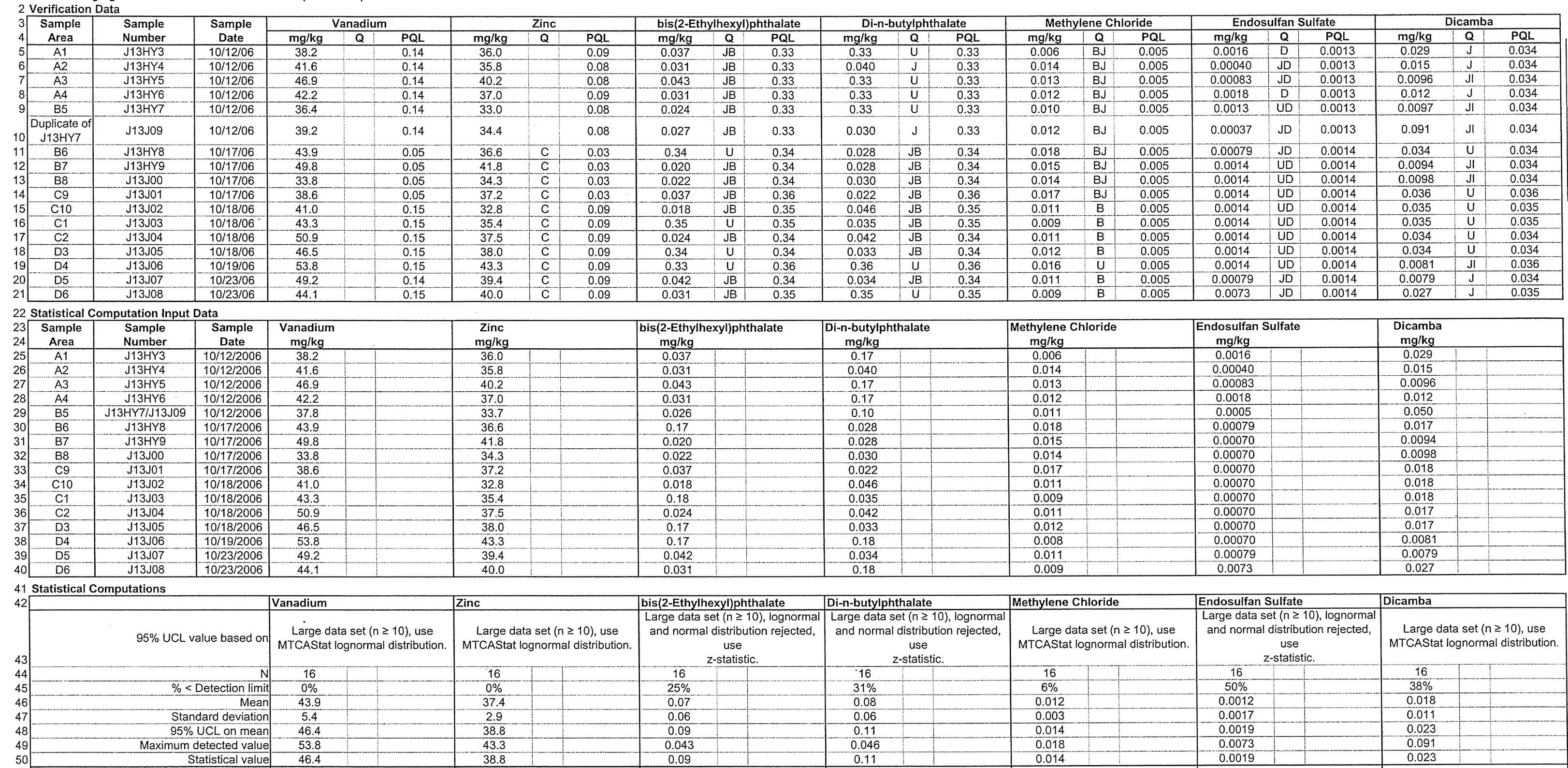

bis(2-2-Ethylhexyl)phthalate $\quad$ Di-n-butylyphthalate
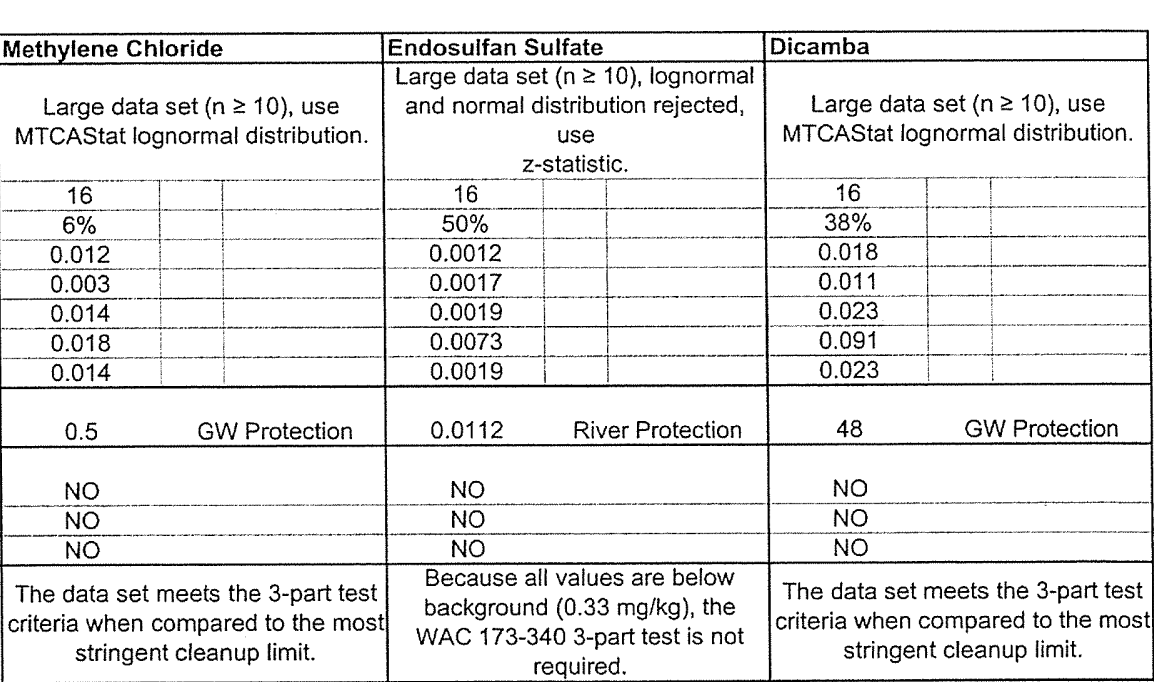

52 WAC 173-340 3 radionuclide and RAG typ

$95 \%$ UCL $>$ Cleanup Limit?
$10 \%$ above Cleanup Limit?

55 Any sample $>2 X$ Cleanup Limit?

85.1 BG/GW Protection

67.8 BG/River Protection

and normal distritio), lognor

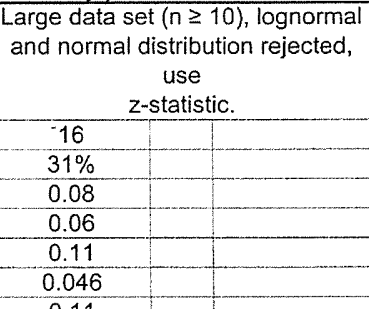
Large data set $(n \geq 10)$, use
MTCAStat lognormal distribution

$57 \mathrm{~B}=$ method blank contamination

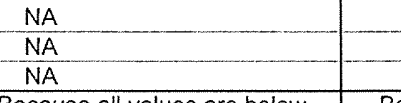

$160 \quad$ GW Protection

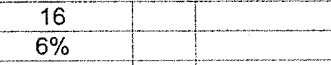

Eecause all values are below
ackkground $(85.1 \mathrm{mg} / \mathrm{kg})$, the

\begin{tabular}{l|r} 
No & No \\
NO & NO \\
\hline NO & NO
\end{tabular}

\begin{tabular}{|c|c|}
\hline & NO \\
\hline NO \\
\hline
\end{tabular}

awporion

3-part test The data set meets the 3-part test The data set meets the 3-part test

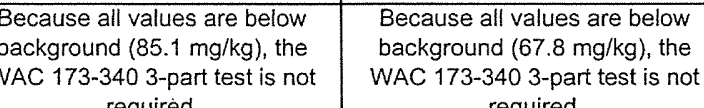

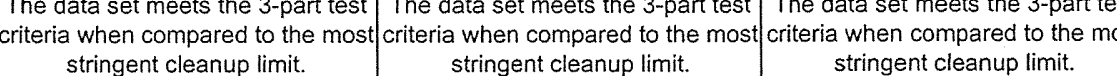

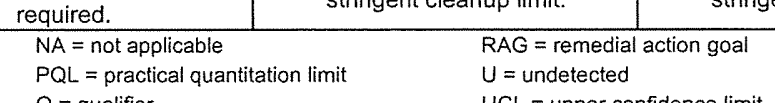

stringent cleanup limit.

UCL = upper confidence limit 
CALCULATION SHEET

Washington Closure Hanford

Originator J.M. Capron Z3n

Project $\frac{\mathrm{J} \text {. M. Capron }}{100-\mathrm{B} / \mathrm{C} \text { Field Remediation }}$

Subject $118-\mathrm{B}-1$ Burial Ground Cleanup Verification $95 \%$ UCL Calculations

Date 02/07/07 Calc. No. 0100B-CA-V0299

Job No. $\frac{14655}{14}$ Checked T.M. Blakley I $x_{3}$

Rev. No.

Date $\overline{2 / 2 \pi / 07}$

Ecology Software (MTCAStat) Results (Overburden)

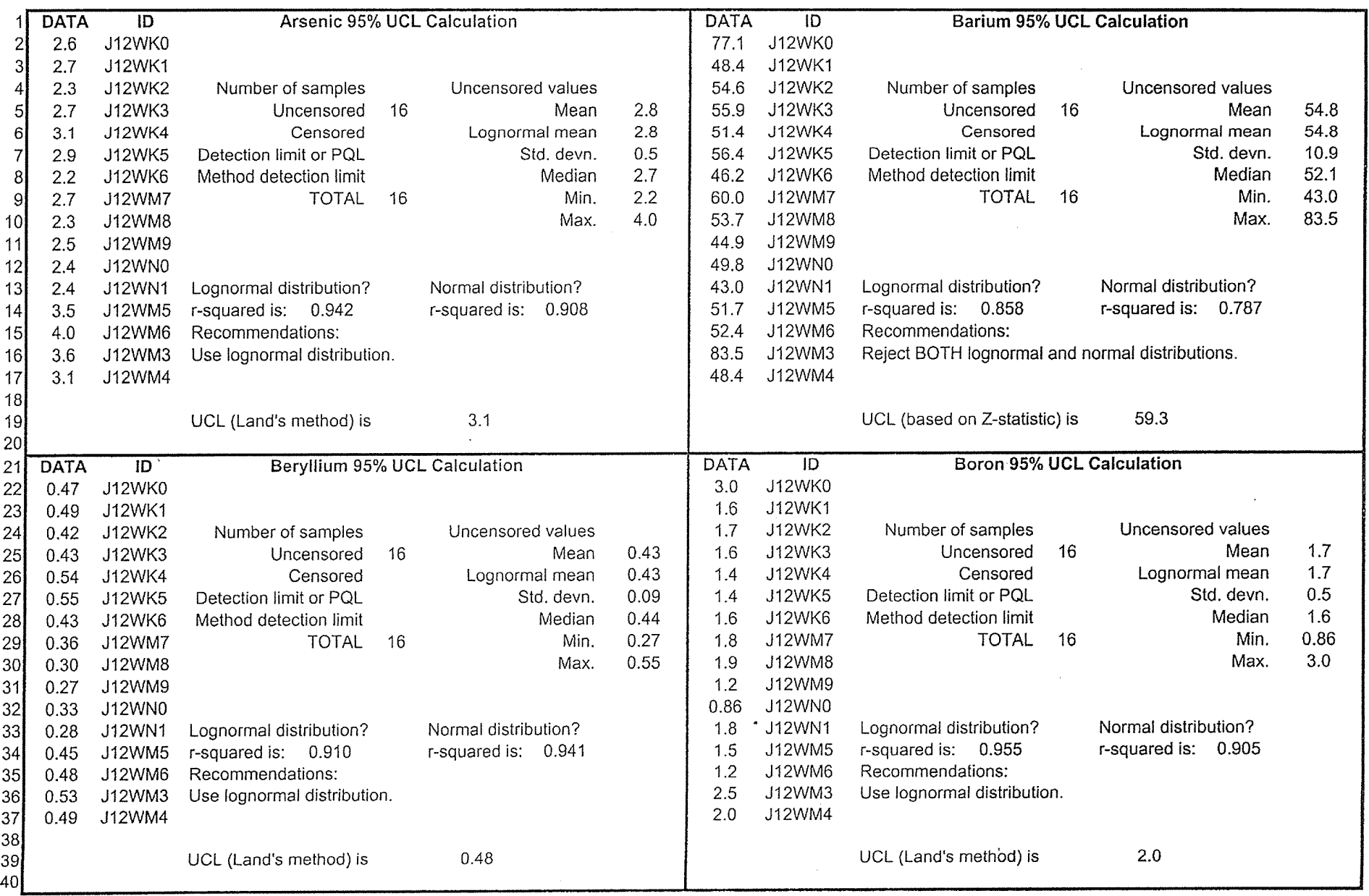

$41 \mathrm{PQL}=$ practical quantitation limi 
CALCULATION SHEET

Washington Closure Hanford

Originator J.M. Capron $/ \mathrm{mL}$

Project $\frac{100-B / C \text { Field Remediation }}{10-B-18}$ Subject 118-B-1 Burial Ground Cleanup Verification $95 \%$ UCL Calculation

02/07/07 Date $\frac{02 / 07 / 07}{14655}$ Checked T.M. Blaklex Tma
Rev. No

Date $2 / 20107$

Ecology Software (MTCAStat) Results (Overburden)

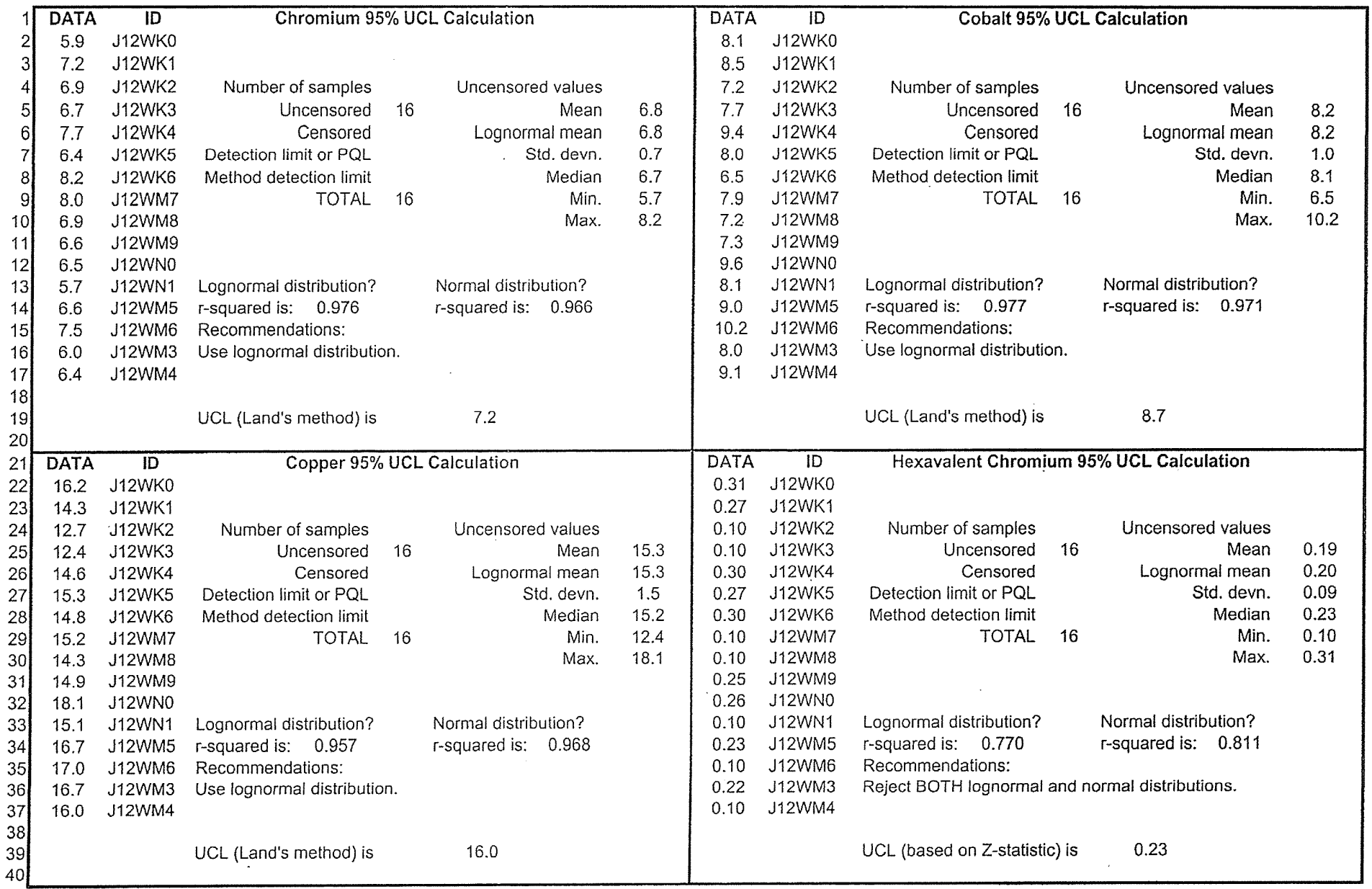


CALCULATION SHEET

Washington Closure Hanford

Qsise

Originator J.M. Capron $/ 2$ se

Subject $\frac{100-B / C \text { Field Remediation }}{118-B-1 \text { Burial Ground Cleanup Verification } 95 \% \text { UCL Calculations }}$

Date 02/07/07 Calc. No. 0100B-CA-V0299

Job No. $\frac{14655}{1}$ Checked T.M. Blakley Jim

Rev. No.

Date $2 / 20 / 07$

Ecology Software (MTCAStat) Results (Overburden)

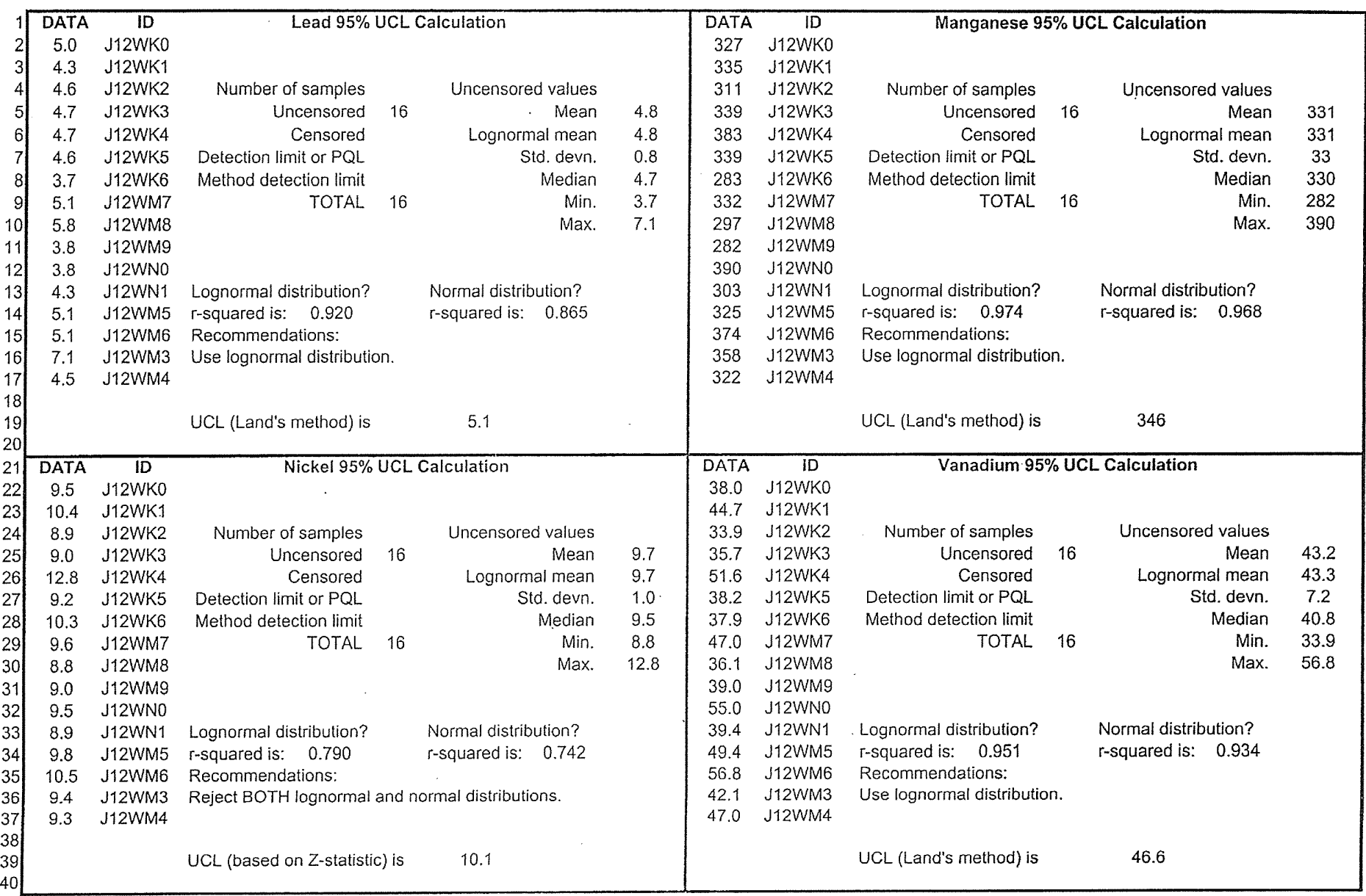

$41 \mathrm{PQL}=$ practical quantitation limi

$42 \mathrm{UCL}=$ upper confidence limit 
CALCULATION SHEET

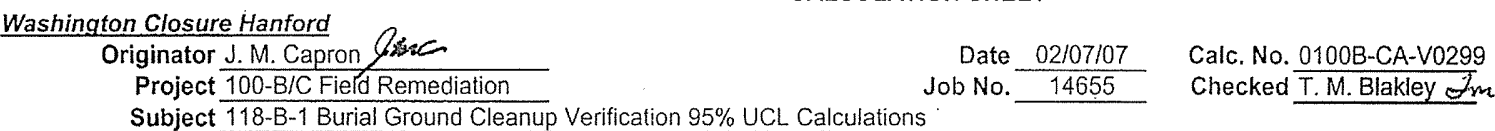

Date $02107 / 07$ Calc. No. 0100B-CA-V0299

Rev. No. $\quad 0$

Date $\overline{2 / 20107}$

Sheet No. 40 of 61

Ecology Software (MTCAStat) Results (Overburden)

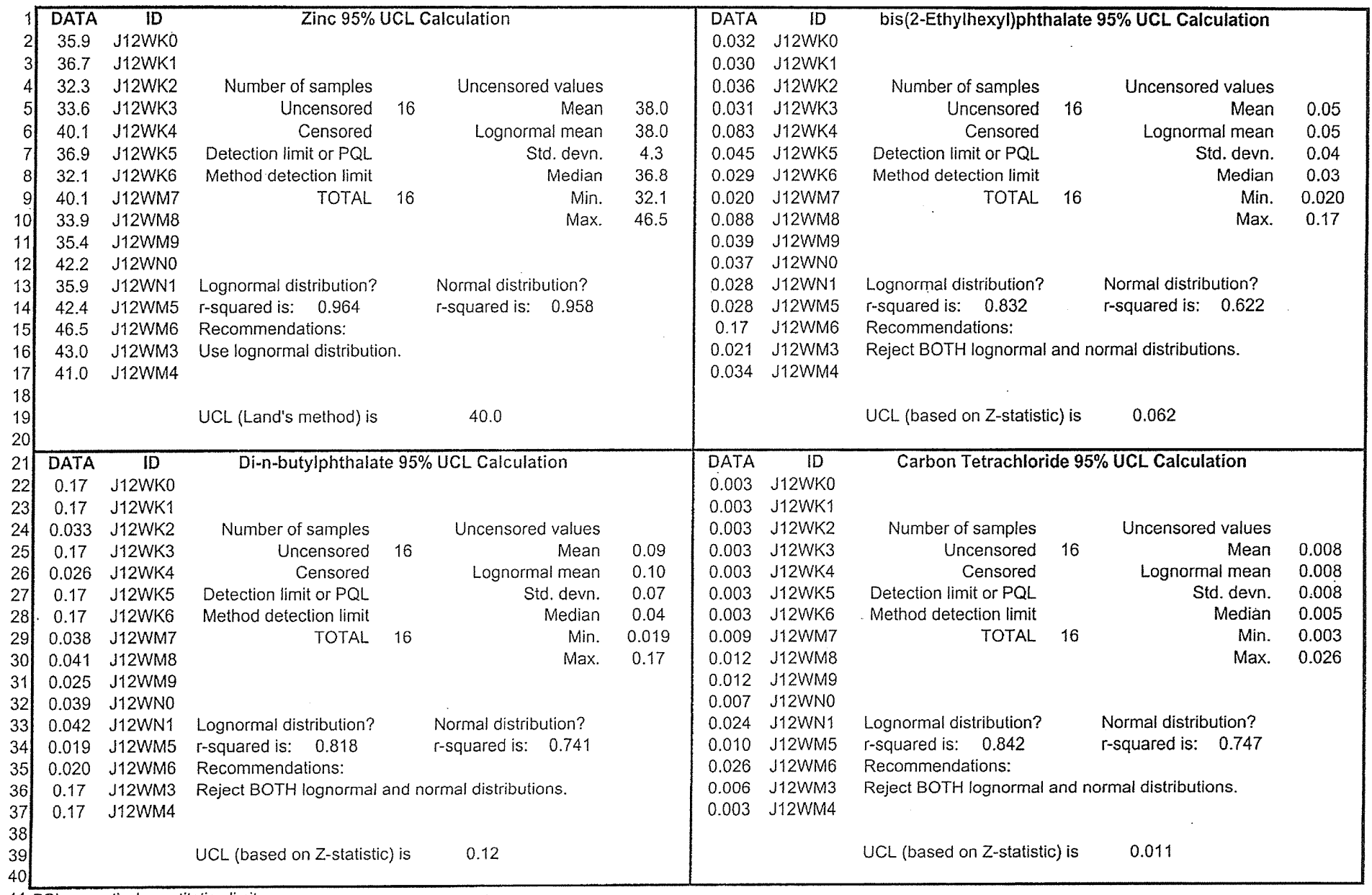

$41 \mathrm{PQL}=$ practical quantitation limit

$42 \mathrm{UCL}=$ upper confidence limit 
CALCULATION SHEET

Washington Closure Hanford

Originator J.M. Capron Qme

Project 100-B/C Field Remediation

Subject 118-B-1 Burial Ground Cleanup Verification 95\% UCL Calculations $\begin{aligned} & \text { Date } 02 / 07 / 07 \\ & 14655 \text { Calc. No. 0100B-CA-V0299 } \\ & \text { Checked T. M. Blakley } 23 m\end{aligned}$
Rev. No.

Date $2 / 2 \rightarrow / 0$

Sheet No. 41 of 61

Ecology Software (MTCAStat) Results (Overburden)

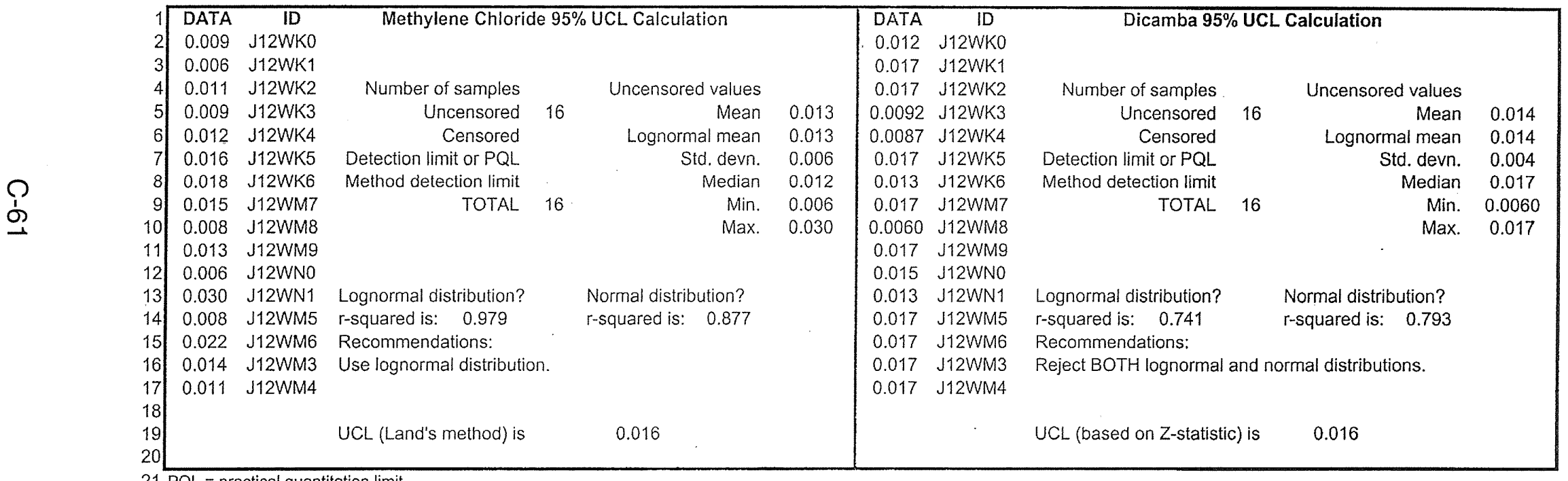

$21 \mathrm{PQL}=$ practical quantitation limit

$22 \mathrm{UCL}=$ upper confidence limit 
CALCULATION SHEET

Washington Closure Hanford

Originator J. M. Capron Gresc

Project 100-B/C Field Remediation Subject 118-B-1 Burial Ground Cleanup Verification 95\% UCL Calculations

Date $02 / 07 / 07$ Calc. No. 0100B-CA-V0299 Job No. 14655 Checked T.M. Blakley 2 mos

Rev. No.

Date $\overline{2 / 20 / \pi 7}$

Ecology Software (MTCAStat) Resuits (BCL Material)

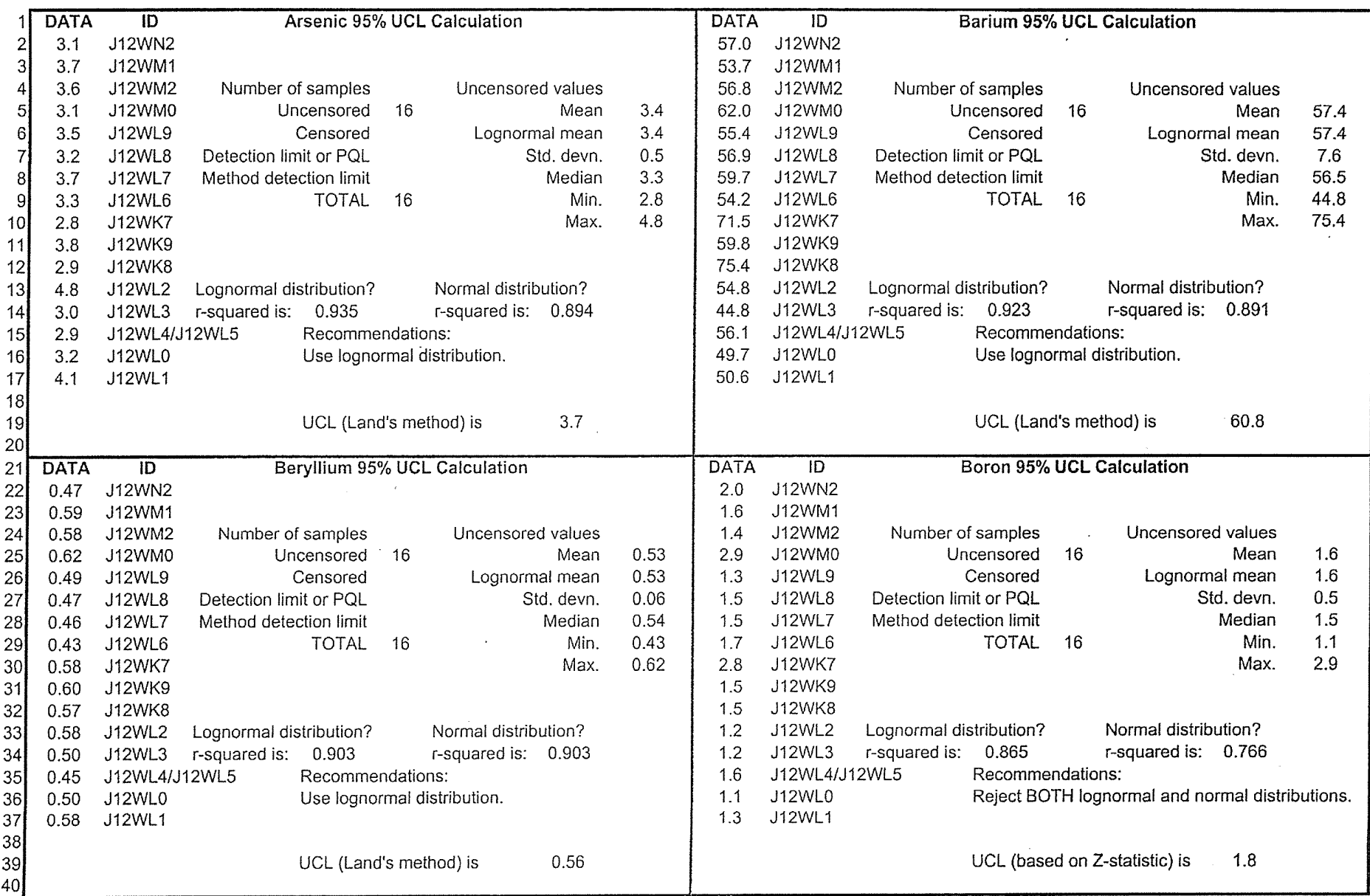

$41 \mathrm{BCL}=$ below cleanup level

$42 \mathrm{PQL}=$ practical quantitation limit

$43 \mathrm{UCL}=$ upper confidence limit 
CALCULATION SHEET

Washington Closure Hanford

Originator J. M. Capron Q Zzzc

Project 100 -B/C Field Remediation $\begin{array}{lll}\text { Date } 02 / 07 / 07 & \text { Calc. No. 0100B-CA-V0299 }\end{array}$ Job No. 14655 Checked T.M. Blakley om
Rev. No.

Date $\frac{0}{2 / 20 / 010}$

Sheet No. $\frac{2 / 20 / 132}{43 \text { of } 61}$

Ecology Software (MTCAStat) Results (BCL Material)

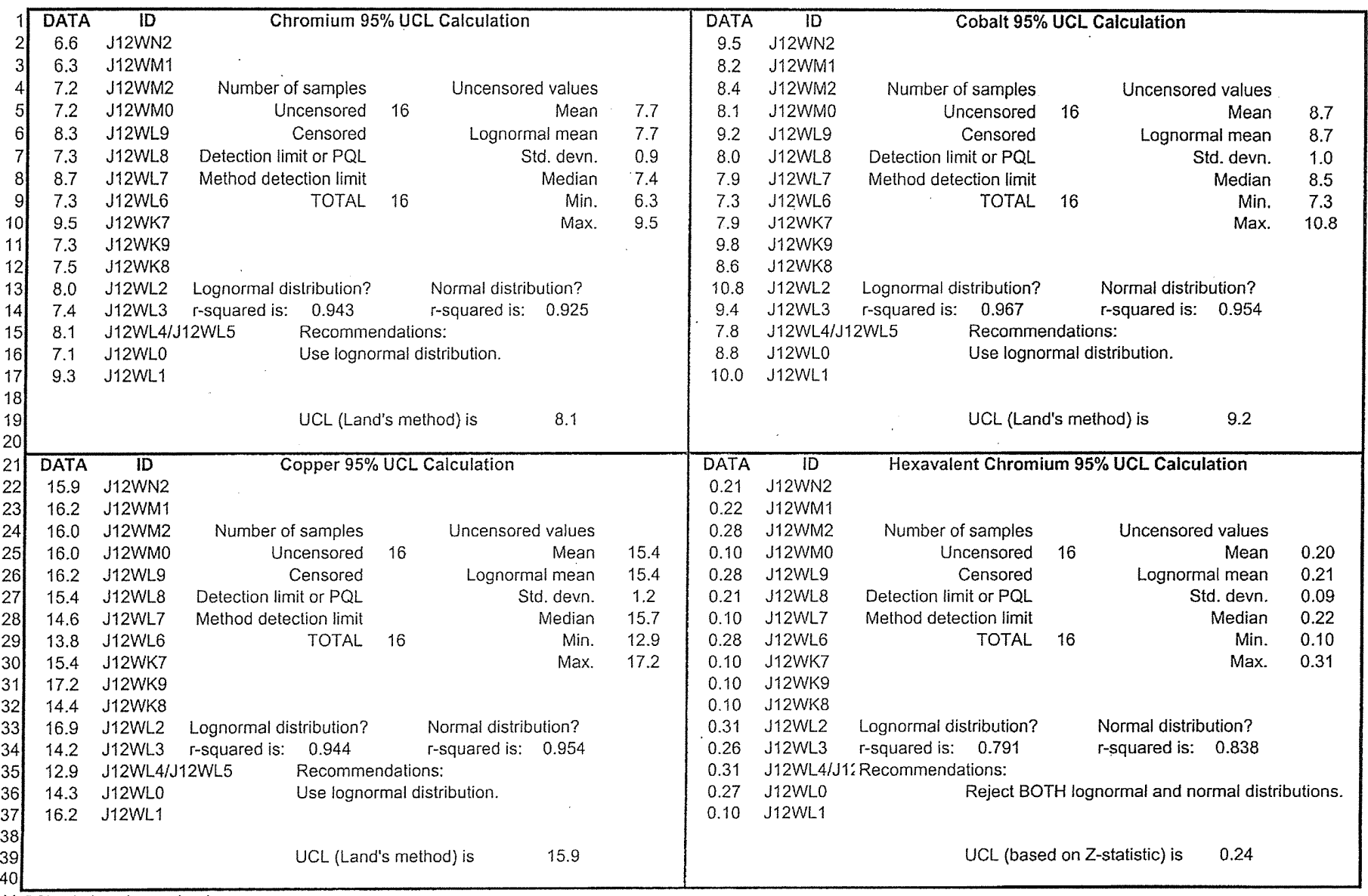

$41 \mathrm{BCL}=$ below cleanup levels

$42 \mathrm{PQL}=$ practical quantifation limit

$43 \mathrm{UCL}=$ upper confidence limit 
CALCULATION SHEET

Washington Closure Hanford

Originator J.M. Capron Gasc

Project 100-B/C Field Remediation

Subject 118-B-1 Burial Ground Cleanup Verification $95 \%$ UCL Calculations
Calc. No. 0100B-CA-V0299

Checked T.M. Blakley 7 .
Rev. No. $\quad 0$

Date 2/20/27

(20)

Ecology Software (MTCAStat) Results (BCL Material)

\begin{tabular}{|c|c|c|c|c|c|c|c|c|c|c|c|c|}
\hline$1 \longdiv { \text { DATA } }$ & ID & Lead 95\% L & UCL Calculation & & & DATA & ID & Manganese 95 & $\%$ UC & L Calculatior & & \\
\hline 4.9 & J12WN2 & & & & & 359 & J12WN2 & & & & & \\
\hline 4.5 & J12WM1 & & & & & 337 & J12WM1 & & & & & \\
\hline 4.5 & J12WM2 & Number of samples & Uncenso & red values & & 346 & J12WM2 & Number of samples & & Uncensor & ed values & \\
\hline 4.0 & J12WMO & Uncensored & 16 & Mean & 4.8 & 332 & J12WMO & Uncensored & 16 & & Mean & 351 \\
\hline 4.4 & J12WL9 & Censored & Lognor & mal mean & 4.8 & 359 & J12WL9 & Censored & & Lognom & mal mean & 351 \\
\hline 4.0 & J12WL8 & Detection limit or $\mathrm{PQL}$ & & Std. devn. & 0.7 & 313 & J12WL8 & Detection limit or $\mathrm{PQL}$ & & & Std. devn. & 32 \\
\hline 4.4 & J12WL7 & Method detection limit & & Median & 4.6 & 329 & J12WL7 & Method detection limit & & & Median & 343 \\
\hline 4.1 & J12WL6 & TOTAL & 16 & Min. & 4.0 & 304 & J12WL6 & TOTAL & 16 & & Min. & 304 \\
\hline 5.2 & J12WK7 & & & Max. & 6.5 & 327 & J12WK7 & & & & Max. & 418 \\
\hline 5.5 & J12WK9 & & & & & 382 & J12WK9 & & & & & \\
\hline 4.5 & J12WK8 & & & & & 407 & J12WK8 & & & & & \\
\hline 6.5 & J12WL2 & Lognormal distribution? & Normal dist & ribution? & & 418 & $J 12 W L 2$ & Lognormal distribution? & & Normal distri & ibution? & \\
\hline 4.6 & J12WL3 & r-squared is: $\quad 0.919$ & $r$-squared is & $: 0.882$ & & 346 & J12WL3 & r-squared is: 0.951 & & $r$-squared is: & 0.934 & \\
\hline 5.0 & J12WL4/J & Recommer & ndations: & & & 336 & J12WL4/J1 & Recommen & ndatio & & & \\
\hline 4.9 & J12WLO & Use lognor & mal distribution. & & & 339 & J12WLO & Use lognor & rmal di & istribution. & & \\
\hline 6.2 & J12WL1 & & & & & 384 & J12WL1 & & & & & \\
\hline 9 & & UCL (Land & $y^{\prime}$ s method) is & 5.1 & & & & UCL (Land" & 's met & thod) is & 366 & \\
\hline DATA & ID & Molybdenum 9 & $5 \%$ UCL Calculati & & & DATA & ID & Nickel 95\% & $\overline{\mathrm{UCLO}}$ & Galculation & & \\
\hline 0.41 & J12WN2 & & & & & 10.8 & J12WN2 & & & & & \\
\hline 0.41 & J12WM1 & & & & & 10.4 & J12WM1 & & & & & \\
\hline 0.41 & J12WM2 & Number of samples & Uncensor & ed values & & 10.0 & J12WM2 & Number of samples & & Uncensore & ed values & \\
\hline 0.41 & J12WMO & Uncensored & 16 & Mean & 0.41 & 9.3 & J12WMO & Uncensored & 16 & & Mean & 10.7 \\
\hline 0.42 & J12WL9 & Censored & Lognor & mal mean & 0.41 & 11.1 & J12WL9 & Censored & & Lognorn & mal mean & 10.7 \\
\hline 0.41 & J12WL8 & Detection limit or PQL & & Std. devn. & 0.04 & 9.8 & J12WL8 & Detection limit or $\mathrm{PQL}$ & & & Std. devn. & 1.0 \\
\hline 0.41 & J12WL7 & Method detection limit & & Median & 0.41 & 11.4 & J12WL7 & Method detection limit & & & Median & 10.6 \\
\hline 0.41 & J12WL6 & TOTAL & 16 & Min. & 0.35 & 9.2 & J12WL6 & TOTAL & 16 & & Min. & 9.2 \\
\hline 0.35 & J12WK7 & & & Max. & 0.50 & 11.0 & J12WK7 & & & & Max. & 12.5 \\
\hline 0.49 & J12WK9 & & & & & 11.3 & J12WK9 & & & & & \\
\hline 0.39 & J12WK8 & & & & & 10.1 & J12WK8 & & & & & \\
\hline 0.50 & J12WL2 & Lognormal distribution? & Normal distr & ibution? & & 12.5 & J12WL2 & Lognormal distribution? & & Normal distrit & ibution? & \\
\hline 0.42 & J12WL3 & r-squared is: 0.887 & r-squared is: & 0.871 & & 11.9 & J12WL3 & r-squared is: 0.981 & & $r$-squared is: & 0.977 & \\
\hline 0.37 & J12WL4/J & Recommer & ndations: & & & 10.0 & $J 12 W L 4 / J 1$ & Recommen & ndatior & & & \\
\hline 0.36 & J12WLO & Reject $\mathrm{BO}$ & TH lognormal and & normal distr & utions. & 10.2 & J12WLO & Use lognorr & rmal di & istribution. & & \\
\hline 0.46 & J12WL1 & & & & & 12.1 & J12WL1 & & & & & \\
\hline & & UCL (base & d on Z-statistic) is & 0.43 & & & & UCL (Land' & 's met & hod) is & 11.1 & \\
\hline
\end{tabular}


CALCULATION SHEET

Washington Closure Hanford

Originator J.M. Capron Q.2uce

Project 100-B/C Field Remediation

Subject 118-B-1 Burial Ground Cleanup Verification $95 \%$ UCL Calculation
Date 02/07/07 Calc. No. 0100B-CA-V0299 Job No. 14655 Checked T.M. Blakley 2 2 ls
Rev. No. 0

20107

Ecology Software (MTCAStat) Results (BCL Material)

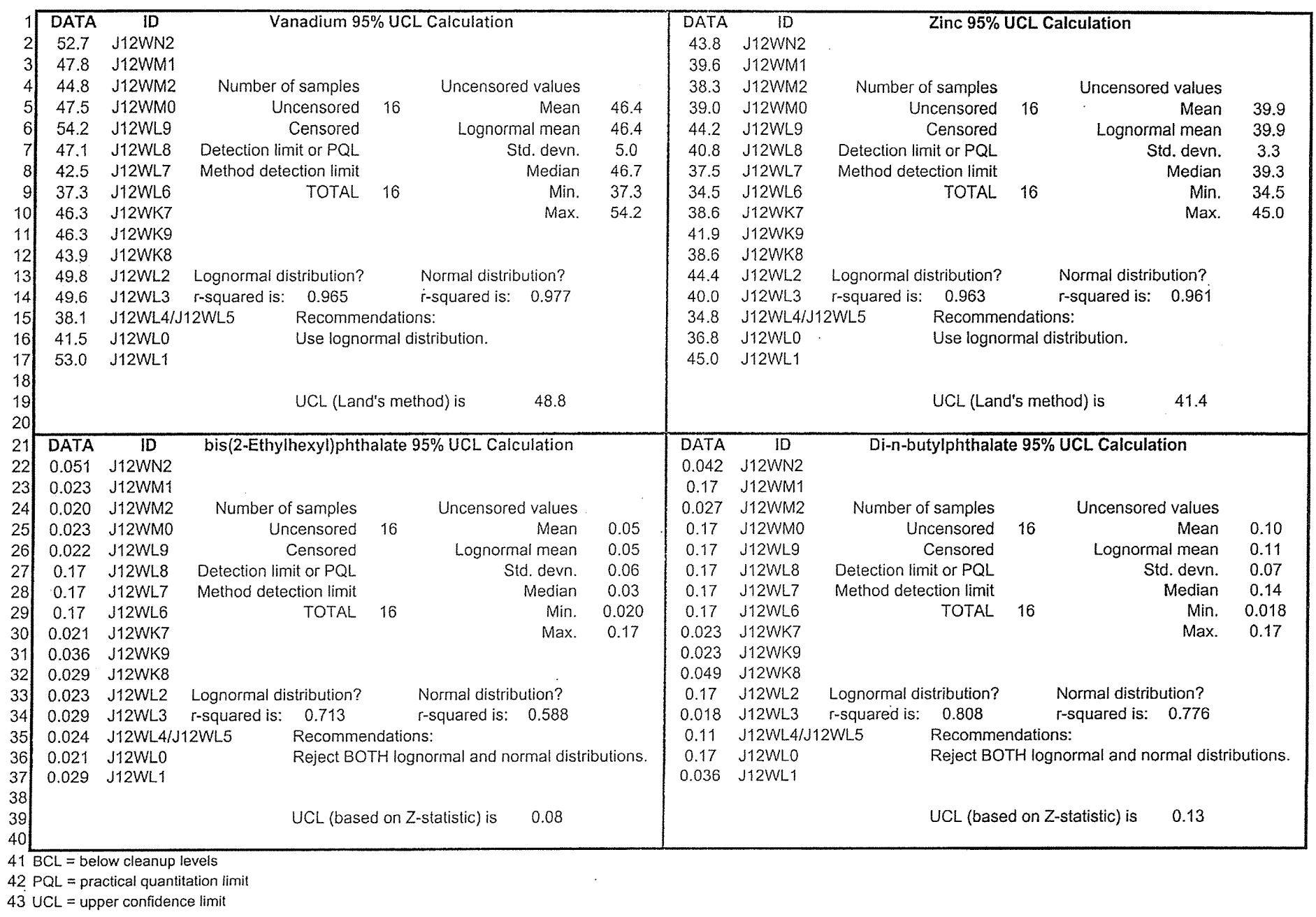


CALCULATION SHEET

Washington Closure Hanford

Originator J M. Capron Sanc

Project $100-B / C$ Field Remediation

Date $\frac{02 / 07 / 07}{14655}$ Calc. No. 0100B-CA-V0299
Job No. Checked T.M. Blakley innos

Subject $\frac{100-B / C \text { Field Remediation }}{118-B-1 \text { Burial Ground Cleanup Verification } 95 \% \text { UCL Calculations }}$

Rev. No. 0

Date $2 / 20 / 07$

Sheet No. $\frac{2}{46 \text { of } 61}$

Ecology Software (MTCAStat) Results (BCL Material)

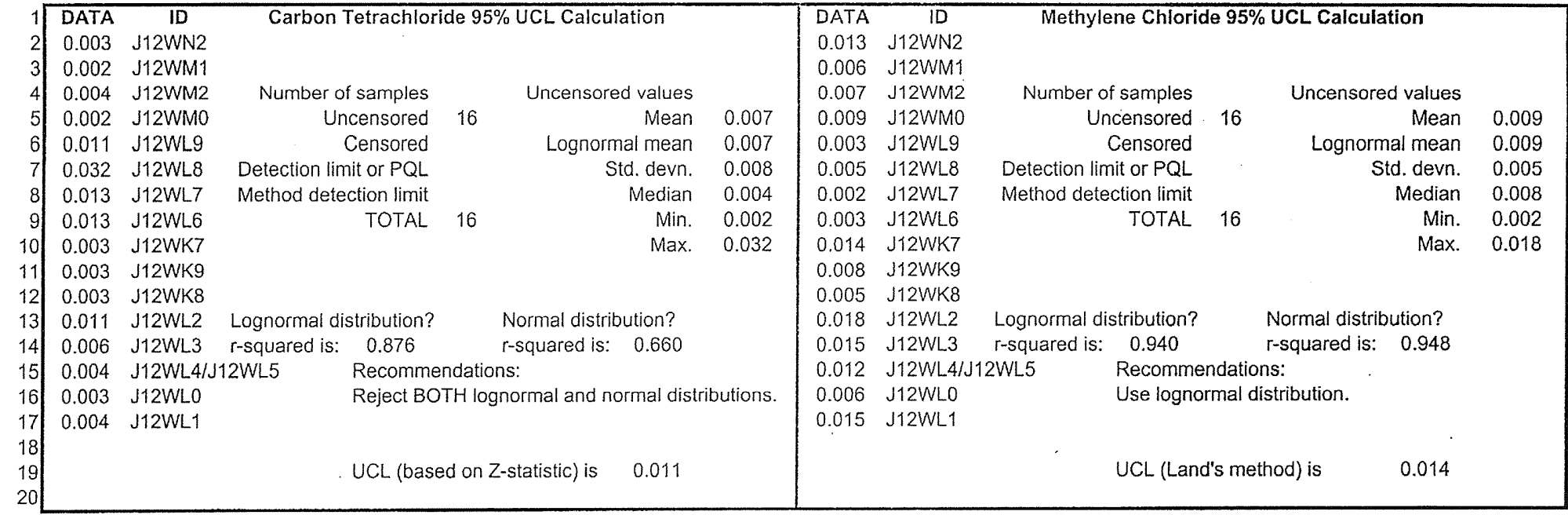

\section{$21 \mathrm{BCL}=$ below cleanup levels}

$22 \mathrm{PQL}=$ practical quantitation limi

$23 \mathrm{UCL}=$ upper confidence timit 
CALCULATION SHEET

Washington Closure Hanford

Originator J.M. Capron Q3.

Project 100-B/C Field Remediation

Subject 118-B-1 Burial Ground Cleanup Verification 95\% UCL Calculations

Date 02/07/07 Calc. No. 0100B-CA-V0299 Job No. 14655 Checked T. M. Blakley im
Rev. No. 0

Date $2 / 20 / 07$

Ecology Software (MTCAStat) Results (Staging Pile Area)

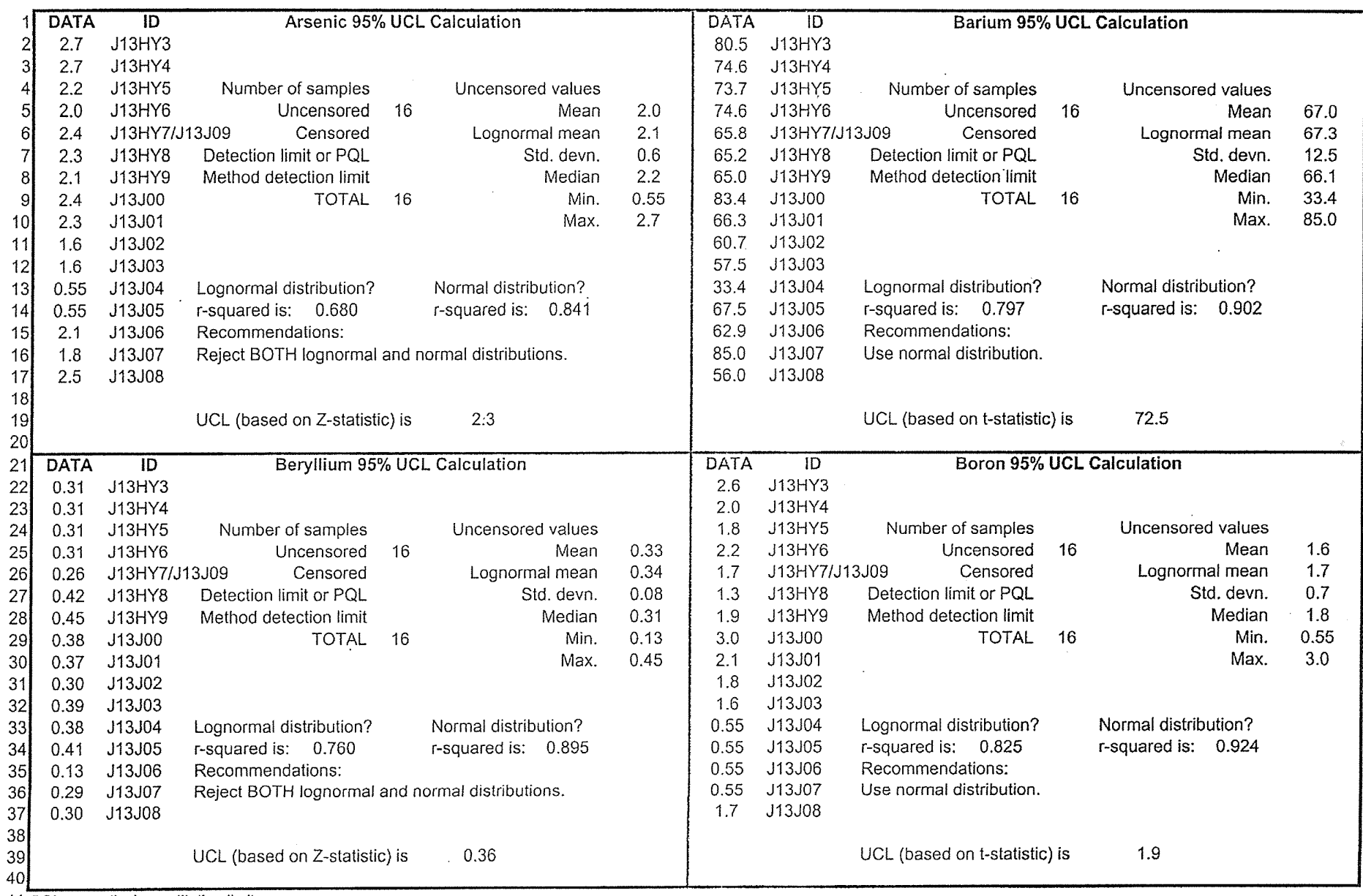




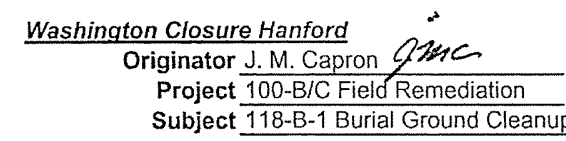

CALCULATION SHEET Subject 118-B-1 Burial Ground Cleanup Verification $95 \%$ UCL Calculations

$$
\begin{array}{cc}
\text { Date } \frac{02 / 07 / 07}{14655} \quad \text { Calc. No. } \frac{0100 B-C A-V 0299}{\text { T.M. Blakley Jng }} \\
\text { Job No. }
\end{array}
$$

Rev. No. 0

Date $2 / 20 / 07$

Ecology Software (MTCAStat) Results (Staging Pile Area)

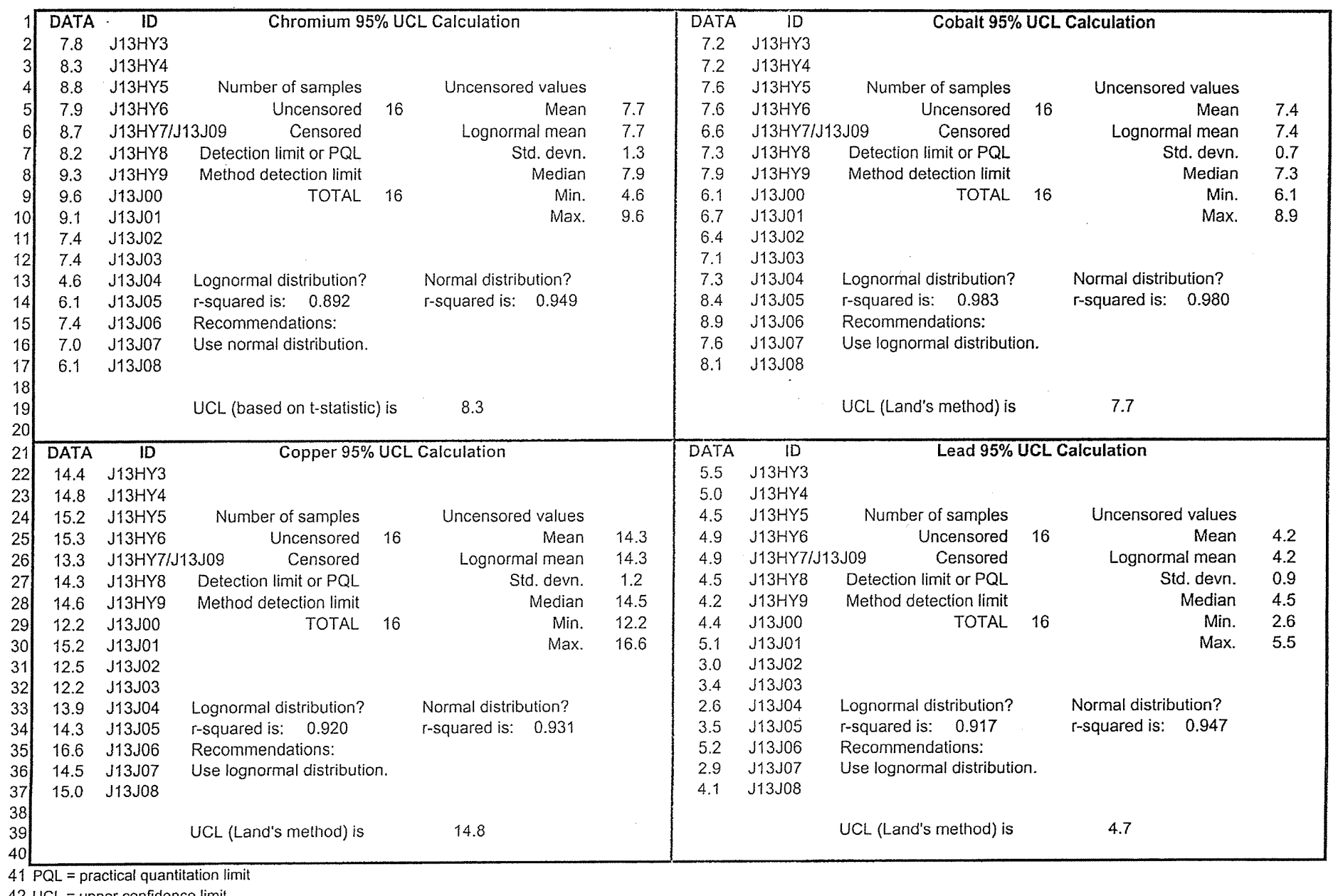


CALCULATION SHEET

\section{Washington Closure Hanford}

Originator J.M. Capron enc

Project 100-B/C Field Remediation

Subject 118-B-1 Burial Ground Cleanuo Verification 95\% UCL Calculations
Date 02/07/07 Calc. No. 0100B-CA-V0299 Job No. 14655 Checked T. M. Blakley . TraB
Rev. No. 0

Date $2 / 20107$

Ecology Software (MTCAStat) Results (Staging Pile Area)

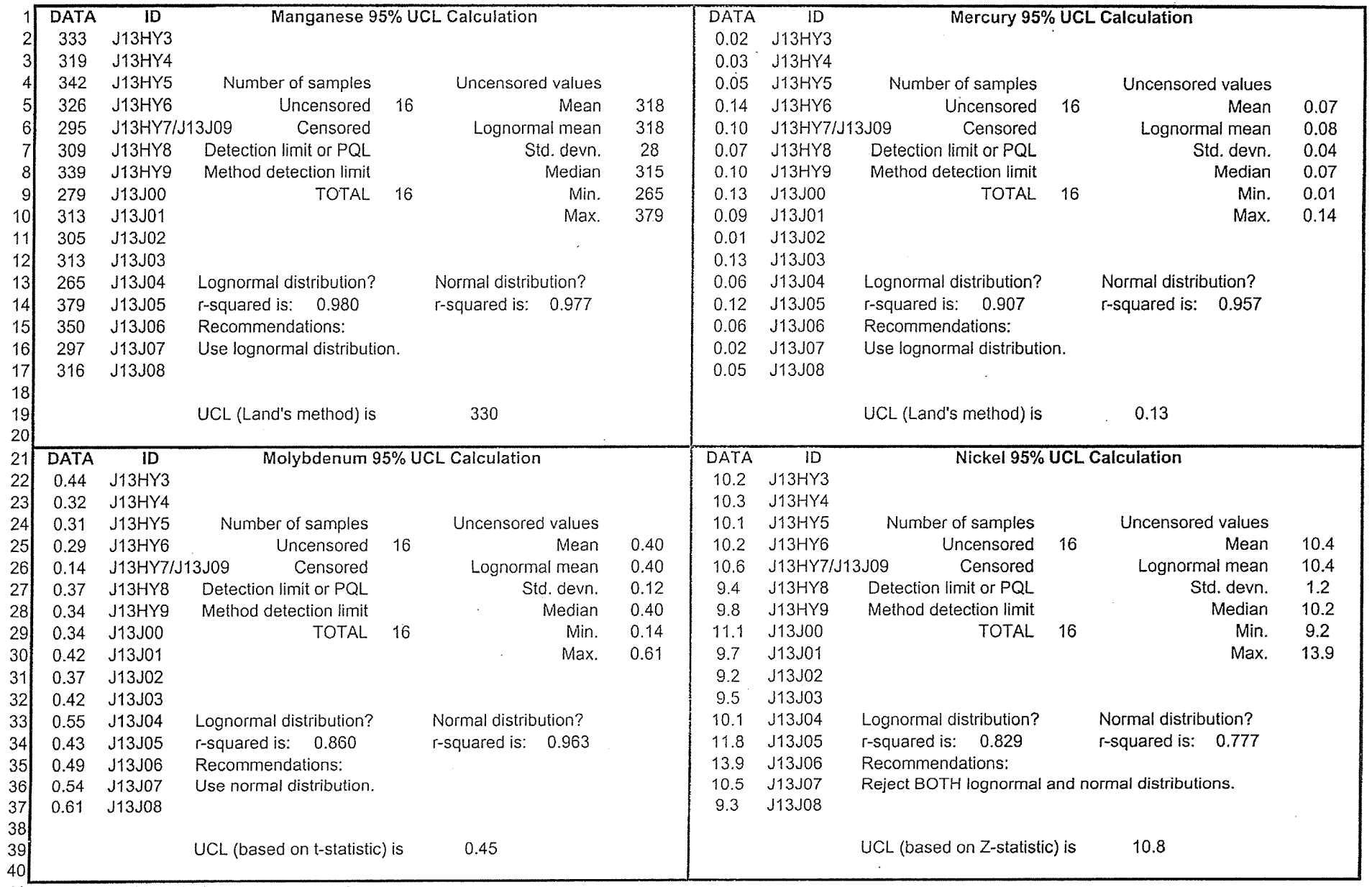


CALCULATION SHEET

Washington Closure Hanford

Originator $1 \mathrm{M} \mathrm{Capron} \mathrm{Ome}$

Date $02 / 07 / 07$ Calc. No. 0100B-CA-V0299

Project $100-B / C$ Field Remediation

Job No. 14655 Checked T.M. Blakley $2 \mathrm{~m} / \mathrm{s}$

Rev. No. 0

Date $2 / 2 n / 0$

Subject 118-B-1 Burial Ground Cleanup Verification $95 \%$ UCL Calculations

Ecology Software (MTCAStat) Results (Staging Pile Area)

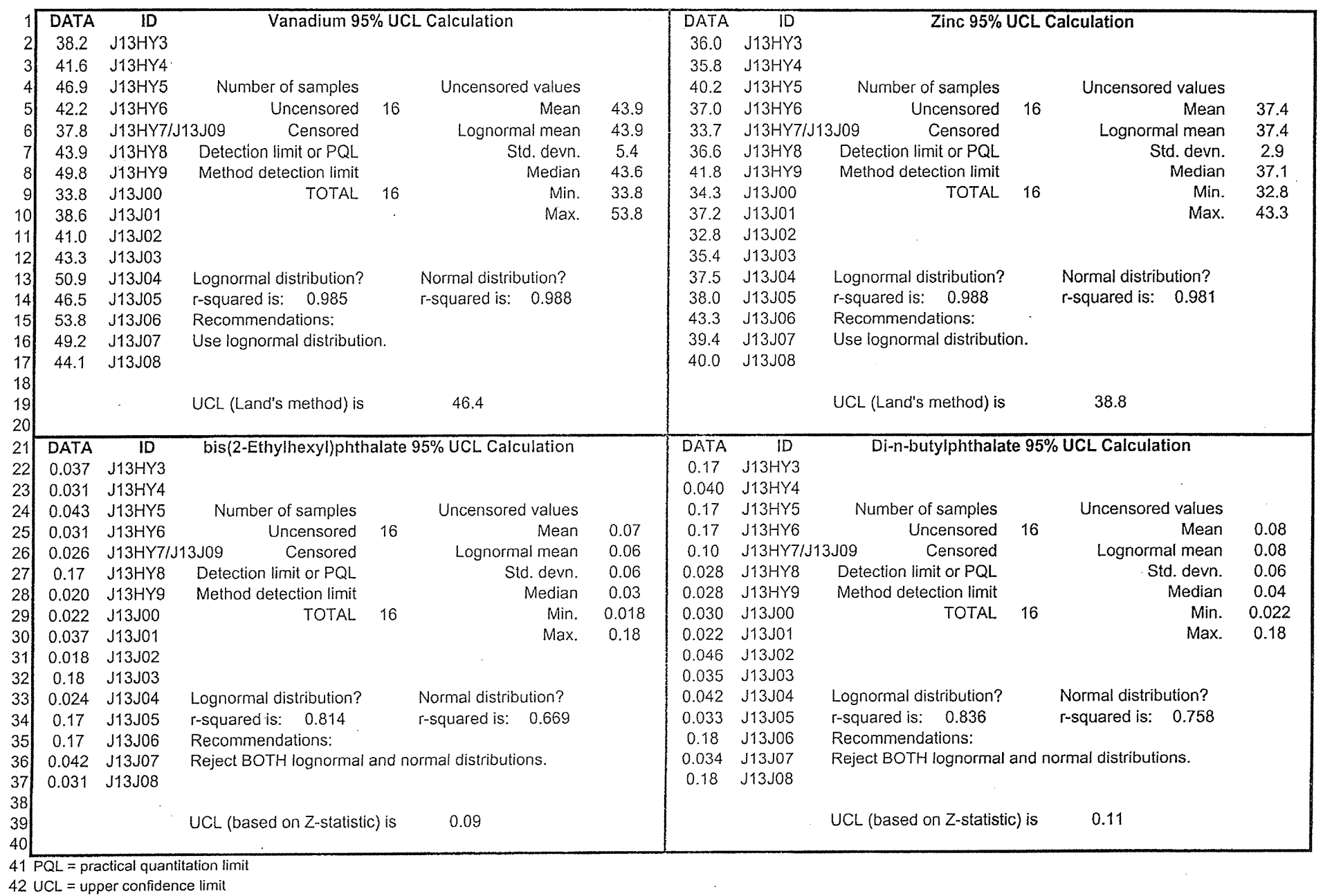


Washington Closure Hanford

CALCULATION SHEET

Originator J.M. Capron $\frac{Y}{\text { Project }}<$

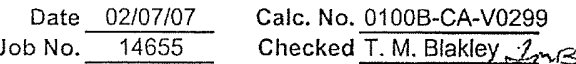

Subject 118-B-1 Burial Ground Cleanup Verification 95\% UCL Calculations

Ecology Software (MTCAStat) Results (Staging Pile Area)

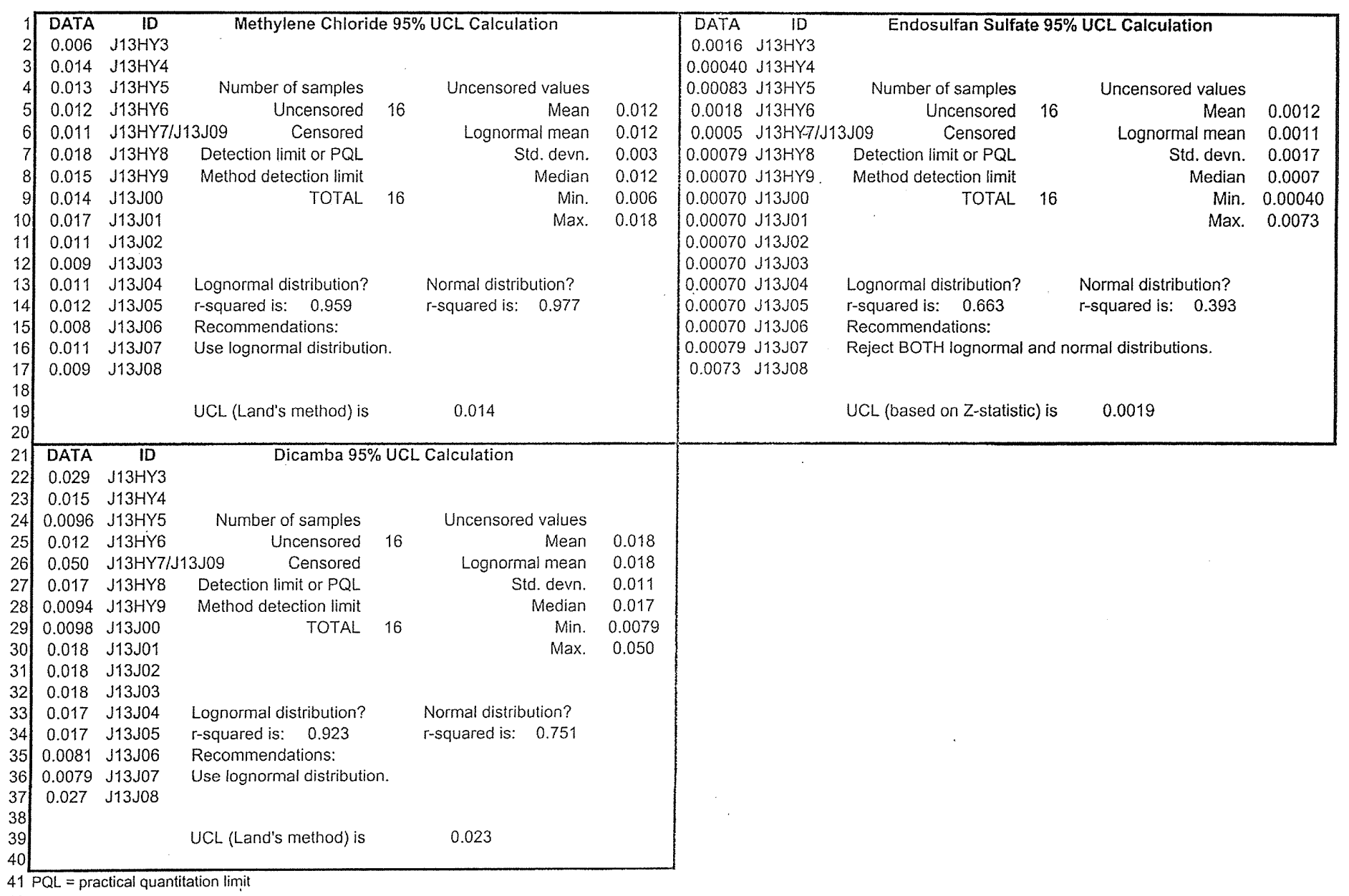

Rev. No. $\frac{0}{2(20 / 07 r}$

Dato 
CVP-2007-00006

Rev. 0 


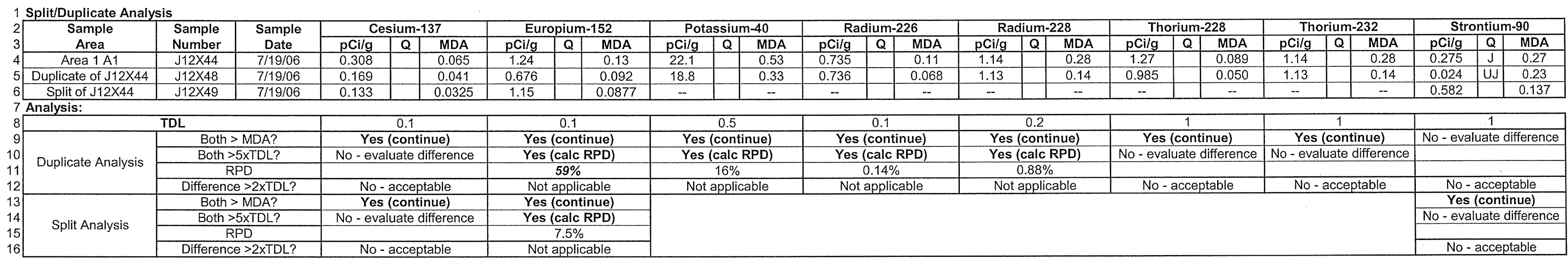

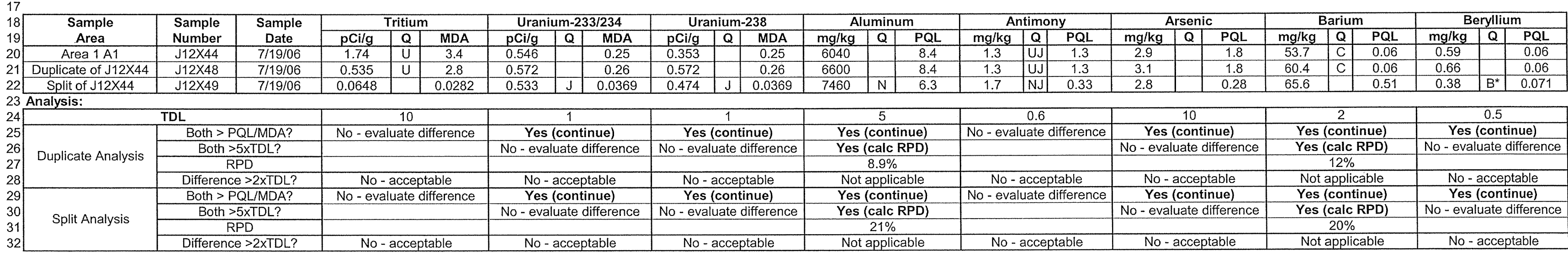

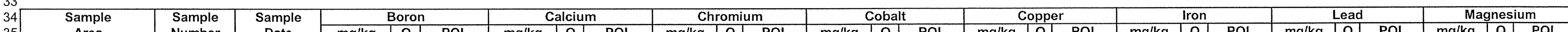

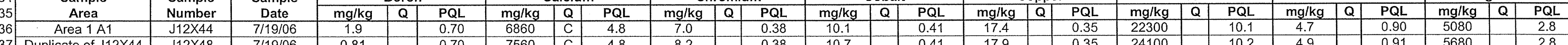

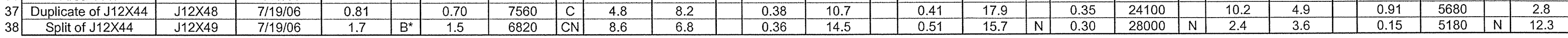
38 Split of 1

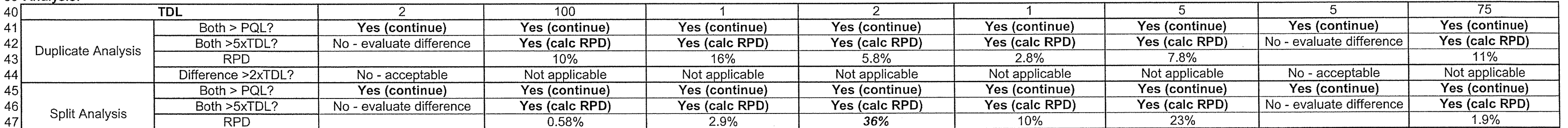

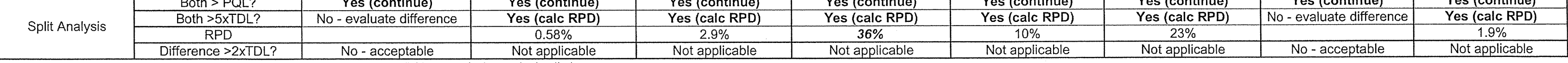
48 - 49 not reported $50 \mathrm{~B}^{*}=$ estimate

$51 \mathrm{C}=$ method blank contamination

$52 J=$ estimate

$53 \mathrm{MDA}=$ minimum detectable activity
$54 \mathrm{~N}=$ spiked analyte recavery outside control limits $Q=$ qualifier $\mathrm{RPD}=$ = relative percent difference $\mathrm{RPD}=$ relative percent difiere
$\mathrm{TDL}=$ target detection limit
$y=$ undetected 


\section{Split/Duplicate Analysis (continued)}

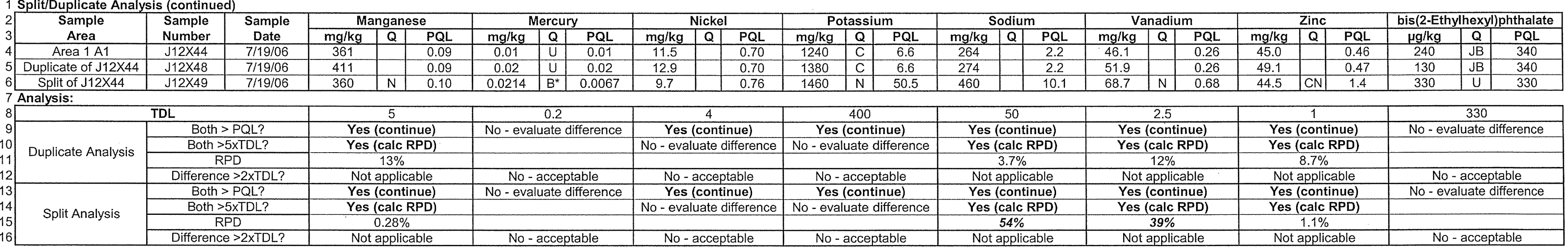

\begin{tabular}{|c|c|c|c|c|c|c|c|c|c|c|c|c|c|c|c|}
\hline & \multirow{2}{*}{$\begin{array}{c}\text { Sample } \\
\text { Area } \\
\end{array}$} & \multirow{2}{*}{$\begin{array}{l}\text { Sample } \\
\text { Number } \\
\end{array}$} & \multirow{2}{*}{$\begin{array}{c}\text { Sample } \\
\text { Date }\end{array}$} & \multicolumn{3}{|c|}{ Di-n-butylphthalate } & \multicolumn{3}{|c|}{ 4-Methyl-2-pentanone } & \multicolumn{3}{|c|}{ Acetone } & \multicolumn{3}{|c|}{ Methylene Chloride } \\
\hline & & & & $\mu \mathrm{g} / \mathrm{kg}$ & $\mathbf{Q}$ & & $\mu \mathrm{g} / \mathrm{kg}$ & $Q$ & $\mathrm{PQL}$ & $\mu \mathrm{g} / \mathrm{kg}$ & & $P Q L$ & $\mu \mathrm{g} / \mathrm{ks}$ & $Q$ & \\
\hline & & & & 19 & $\mathrm{JB}$ & 340 & 5 & JB & 10 & 9 & $\mathrm{~J}$ & 10 & & & 5 \\
\hline & Suplicate of J12X44 & $J 12 \times 48$ & & $\frac{18}{100}$ & $\mathrm{JB}$ & 340 & 8 & JB & 10 & 23 & $J$ & 10 & 12 & & \\
\hline \multirow{2}{*}{\multicolumn{16}{|c|}{$\begin{array}{ll}\text { U U } & 330 \\
& \\
330\end{array}$}} \\
\hline & & \multicolumn{2}{|l|}{ TDL } & \multirow{3}{*}{\multicolumn{3}{|c|}{$\frac{330}{\text { No- evaluate difference }}$}} & & & & & & & \multirow{2}{*}{\multicolumn{3}{|c|}{$\frac{10}{\text { Yes (continue) }}$}} \\
\hline & \multirow{3}{*}{ Duplicate Analysis } & \multirow{2}{*}{\multicolumn{2}{|c|}{$\begin{array}{l}\text { Both }>\mathrm{PLL} \text { ? } \\
\text { Both }>5 \times \mathrm{TDL} \text { ? }\end{array}$}} & & & & \multirow{2}{*}{\multicolumn{3}{|c|}{$\begin{array}{c}10 \\
\text { No - evaluate difference }\end{array}$}} & \multicolumn{3}{|c|}{ No-evaluate difference } & & & \\
\hline & & & & \multicolumn{2}{|c|}{ No - evaluate difference } & & & & & \multicolumn{3}{|c|}{ 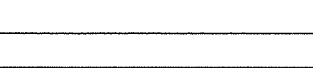 } & \multicolumn{3}{|c|}{ No - evaluate difference } \\
\hline & & \multicolumn{2}{|c|}{ Difference $>2 \times T D L ?$} & & \multirow{2}{*}{\multicolumn{3}{|c|}{$\begin{array}{l}\text { acceptable } \\
\text { iluate difference }\end{array}$}} & & \multicolumn{3}{|c|}{ No-acceptable } \\
\hline & \multirow{3}{*}{ Split Analysis } & \multirow{2}{*}{\multicolumn{2}{|c|}{$\begin{array}{l}\text { Both > PQL? } \\
\text { Both >5xTDL? }\end{array}$}} & & & & & & & & & & \multicolumn{3}{|c|}{0 - evaluate difference } \\
\hline & & & & & No - evaluate difference & & \multicolumn{3}{|c|}{ luate difference } & \multicolumn{3}{|c|}{ No - evaluate difference } & & & \\
\hline & & \multicolumn{2}{|l|}{ 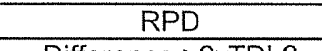 } & & & & & & & & & & & & \\
\hline
\end{tabular}

\begin{tabular}{|c|c|c|c|c|c|c|c|c|c|c|c|c|c|c|c|c|c|c|c|c|c|c|c|c|c|}
\hline Sample & Sample & Sample & & Sium- & & & ckel-t & & & ssium-40 & & lium- & & & ium- & & & ium-2 & & & rium. & & & intium & \\
\hline Area & Number & Date & pCilg & $\mathbf{Q}$ & MDA & $\mathrm{pCi} / \mathrm{g}$ & $Q$ & MDA & $\mathrm{pCi} / \mathrm{g}$ & \begin{tabular}{|l|l|} 
Q & MDA \\
\end{tabular} & $\mathrm{pCi} / \mathrm{g}$ & Q & MDA & $\mathrm{pCilg}$ & $Q$ & MDA & $\mathrm{pCil/g}$ & Q & MDA & $\mathrm{pCi} / \mathrm{g}$ & $Q$ & MDA & $\mathrm{pCi} / \mathrm{g}$ & Q & MDA \\
\hline Area 3 A3 & $\mathrm{J12 \times 56}$ & $7 / 20 / 06$ & 1.51 & & 0.12 & 1.03 & u & 4.5 & 9.50 & 1.2 & 0.200 & U & 0.21 & 0.588 & & 0.40 & 0.266 & & 0.086 & 0.588 & & 0.40 & 4.42 & & 0.31 \\
\hline Duplicate of J12X56 & $\sqrt{12 \times 58}$ & $7 / 20 / 06$ & 0.776 & & 0.11 & -0.828 & $u$ & 4.3 & 10.7 & 0.90 & 0.437 & & 0.16 & 0.87 & $u$ & 0.87 & 0.250 & & 0.079 & 0.87 & $U$ & 0.87 & 3.23 & & 0.25 \\
\hline
\end{tabular}

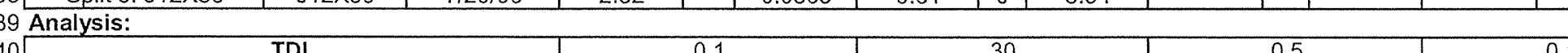

\begin{tabular}{|c|c|c|c|c|c|c|c|c|c|}
\hline \multirow{4}{*}{ Duplicate Analysis } & TDL & 0.1 & 30 & 0.5 & 0.1 & 0.2 & 1 & & 1 \\
\hline & Both > MDA? & Yes (continue) & No - evaluate difference & Yes (continue) & No - evaluate difference & No - evaluate difference & Yes (continue) & No - evaluate difference & Yes (continue) \\
\hline & $\begin{array}{c}\text { Both >5xTDL? } \\
\text { RPD }\end{array}$ & $\begin{array}{c}\text { Yes (calc RPD) } \\
64 \%\end{array}$ & & $\begin{array}{l}\text { Yes (calc RPD) } \\
11 \%\end{array}$ & & & No - evaluate difference & & No - evaluate difference \\
\hline & Difference $>2 \times \mathrm{TDL}$ ? & Not applicable & No - acceptable & \multirow{4}{*}{ Not applicable } & \multirow{4}{*}{ Yes - assess further } & \multirow{4}{*}{ No - acceptable } & \multirow{4}{*}{ No - acceptable } & \multirow{4}{*}{ No - acceptable } & No - acceptable \\
\hline \multirow{3}{*}{ Split Analysis } & Both > MDA? & Yes (continue) & No - evaluate difference & & & & & & Yes (continue) \\
\hline & $\begin{array}{l}\text { Both }>5 \times T D L ? \\
\text { RPD }\end{array}$ & $\frac{\text { Yes (calc RPD) }}{66 \%}$ & & & & & & & \\
\hline & Difference $>2 \times T D L ?$ & Not applicable & No - acceptable & & & & & & No - acceptable \\
\hline
\end{tabular}

$49-\cdots=$ not reported

$50 \mathrm{~B}=$ method blank contamination (organic cons

\begin{tabular}{|l|l|}
\hline $61 \%$ & \\
\hline ot applicable & No - acceptable \\
\hline
\end{tabular}

$\begin{array}{ll}51 \mathrm{~B}^{*}=\text { =stitiate } & \mathrm{N}=\mathrm{Q}=\text { practical quantitation limit } \\ 52 \mathrm{C}=\text { method blank contamination (inorganic consitituents) } & \mathrm{Q}=\text { qualifier } \\ & \mathrm{PD}=\text { relative percent difference }\end{array}$

$53 \mathrm{~J}=$ estimate

$\mathrm{TDL}=$ target detection limit 


\begin{tabular}{|c|c|c|c|c|c|c|c|c|c|c|c|c|c|c|c|c|c|c|c|c|c|c|c|c|c|c|}
\hline \multirow{2}{*}{\begin{tabular}{l|l}
1 S Split'Duplicate Anal \\
2 & Sample \\
3 & Area \\
\end{tabular}} & \multirow{2}{*}{$\begin{array}{l}\text { is (continue } \\
\text { Sample } \\
\text { Number }\end{array}$} & \multirow{2}{*}{$\begin{array}{c}\text { Sample } \\
\text { Date }\end{array}$} & \multicolumn{3}{|c|}{ Tritium } & \multirow{2}{*}{\multicolumn{3}{|c|}{ Aluminum }} & \multirow{2}{*}{\multicolumn{3}{|c|}{ Antimony }} & \multicolumn{3}{|c|}{ Arsenic } & \multirow{2}{*}{\multicolumn{3}{|c|}{ Barium }} & \multirow{2}{*}{\multicolumn{3}{|c|}{ Beryllium }} & \multirow{2}{*}{\multicolumn{3}{|c|}{ Boron }} & \multirow{2}{*}{\multicolumn{3}{|c|}{ Calcium }} \\
\hline & & & $\mathrm{pCi} / \mathrm{g}$ & $\begin{array}{lll}Q^{2} & & \\
\end{array}$ & MDA & $\frac{\mathrm{Al}}{\mathrm{mg} / \mathrm{kg}}$ & & $\frac{n}{P Q L}$ & $\frac{A_{1}}{\mathrm{mg} / \mathrm{kg}}$ & & $\frac{1}{P Q L}$ & $\mathrm{mg} / \mathrm{Ag}$ & $\begin{array}{l}\mathrm{rsseni} \\
\mathrm{Q} \mid\end{array}$ & PQL & $\mathrm{mg} / \mathrm{kg}$ & & $m_{P Q L}$ & & & $\mathrm{PQL}$ & $\mathrm{mg} / \mathrm{kg}$ & & PQL & $\mathrm{mg} / \mathrm{kg}$ & & \\
\hline Area 3 A3 & $J 12 \times 56$ & $7 / 20106$ & 0.491 & $\bar{U}$ & 2.7 & 6120 & & 8.1 & 1.2 & \begin{tabular}{|l|l|} 
Uuj \\
\end{tabular} & 1.2 & 4.5 & & 1.7 & 52.4 & c & 0.06 & 0.65 & & 0.06 & 2.2 & & 0.68 & 9860 & & 4.6 \\
\hline \begin{tabular}{|l|l} 
Duplicate of $\mathrm{J} 12 \times 56$ \\
\end{tabular} & $\mathrm{~J} 12 \times 58$ & $7 / 20 / 06$ & 0.311 & $U$ & 2.7 & 5500 & & 8.2 & 1.3 & us & 1.3 & 3.9 & & 1.7 & 48.3 & c) & 0.06 & 0.61 & & 0.06 & 1.3 & & 0.69 & 9650 & $\mathrm{C}$ & 4.7 \\
\hline & $\mathrm{J12 \times 60}$ & $7 / 20 / 06$ & 0.0577 & & 0.0453 & 5470 & $\mathrm{~N}$ & 6.3 & 1.1 & NJ & 0.34 & 3.0 & & 0.28 & 47.7 & & 0.51 & 0.34 & $\mathrm{~B}^{*}$ & 0.071 & 5.1 & $\mathrm{~B}^{*}$ & 1.5 & 7870 & C & 8.7 \\
\hline \multirow{2}{*}{\multicolumn{3}{|c|}{\begin{tabular}{ll}
7 & \\
7 & Analysis: \\
9 & \multicolumn{1}{c}{ TDL } \\
\cline { 2 - 2 } & Both > PQL/MDA?
\end{tabular}}} & & 10 & & \multirow{2}{*}{\multicolumn{3}{|c|}{5}} & \multirow{2}{*}{\multicolumn{3}{|c|}{0.6}} & \multirow{2}{*}{\multicolumn{3}{|c|}{10}} & & \multirow{2}{*}{\multicolumn{3}{|c|}{0.5}} & & \multirow{2}{*}{\multicolumn{3}{|c|}{100}} \\
\hline & & & \multirow{2}{*}{\multicolumn{3}{|c|}{ No - evaluate difference }} & & & & & & & & & & & & & & & & & & & & & \\
\hline \multirow{3}{*}{$\begin{array}{l}10 \text { Duplicate Analysis } \\
11\end{array}$} & Both: & TDL? & & & & Yes & (calc & & \multicolumn{3}{|c|}{ No-evaluate difference } & \multicolumn{3}{|c|}{$\begin{array}{c}\text { Yes (continue) } \\
\text { No-evaluate difference }\end{array}$} & \multicolumn{3}{|c|}{$\begin{array}{l}\text { Yes (continue) } \\
\text { Yes (calc RPD) }\end{array}$} & \multicolumn{3}{|c|}{$\begin{array}{l}\text { Yes (continue) } \\
\text { No - evaluate difference }\end{array}$} & \multicolumn{3}{|c|}{$\begin{array}{l}\text { Yes ( (continue) } \\
\text { No- evaluate difference }\end{array}$} & \multicolumn{3}{|c|}{ Yes (calc RPD) } \\
\hline & & & & & & & $11 \%$ & & & & & & & & & $8.1 \%$ & & & & & & & & & & \\
\hline & $\begin{array}{l}\text { Differenc } \\
\text { Both }>1\end{array}$ & $\begin{array}{l}\frac{2 \times I I L L ?}{2} \\
\text { UMDA? }\end{array}$ & $\frac{\text { No }}{\text { No-eve }}$ & $\begin{array}{l}\text { acce } \\
\text { luate } \\
\text { late }\end{array}$ & $\begin{array}{l}\text { able } \\
\text { fference }\end{array}$ & & applic & & $\begin{array}{l}\text { No- } \\
\text { No-eval }\end{array}$ & accep & $\begin{array}{l}\text { able } \\
\text { fference }\end{array}$ & $\frac{\text { No- }}{\text { Yes }}$ & cont & table & & appoic & $\begin{array}{l}\text { cable } \\
\text { tinue) }\end{array}$ & $\frac{\text { No- }}{\text { Yes }}$ & $\frac{\text { accer }}{\text { (cont }}$ & $\begin{array}{l}\text { ptable } \\
\text { inue) } \\
\text { inue }\end{array}$ & $\frac{\text { No- }}{\text { Yes }}$ & accep & $\begin{array}{l}\text { tiable } \\
\text { tinue) } \\
\text { inte }\end{array}$ & & appontic & \\
\hline Solit Analvsis & Both: & TDL? & & & & & (calc & & & & & No-eval & uate & ifference & & (calc & RPD) & No-eva & luate & difference & No-eval & uate c & difference & & |calc & \\
\hline & $\mathrm{F}$ & 5 & & & & & $11 \%$ & & & & & & & & & $9.4 \%$ & & & & & & & & & $22 \%$ & \\
\hline & Differens & & & acce & & & & & No- & accep & & No- & accer & & & & & & accer & & & accep & & & applic & \\
\hline
\end{tabular}

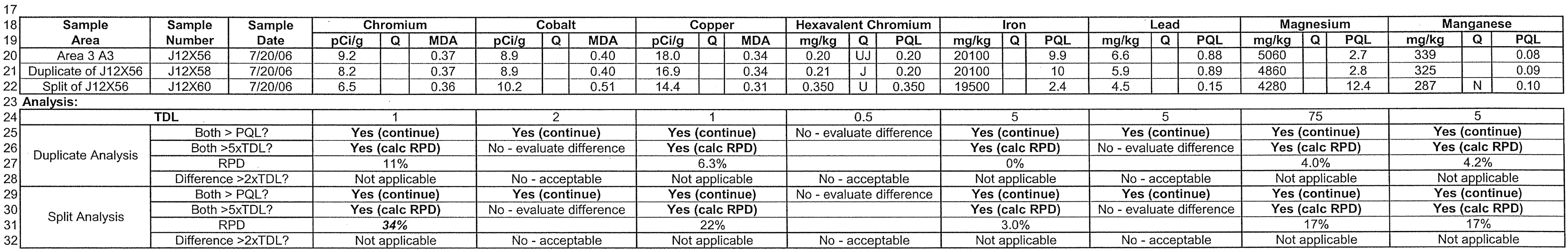

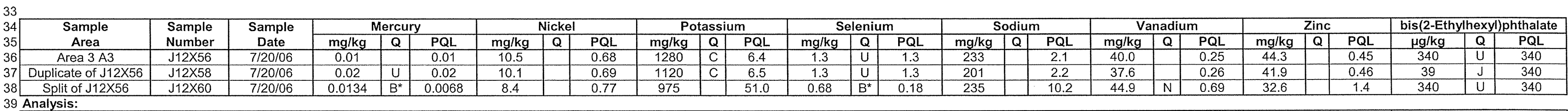

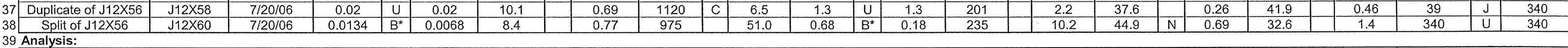

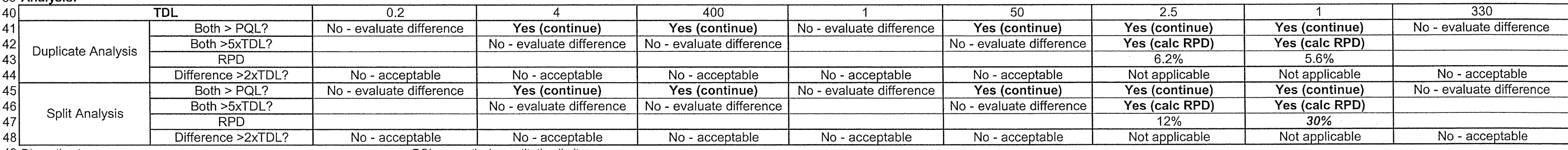
$49 \mathrm{~B}^{*}=$ estimate 
Washington Closure Hanford

Originator J. M. Capron

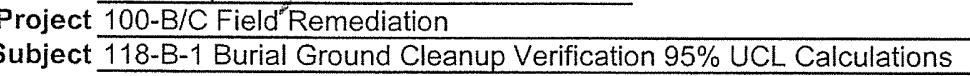

1 Split/Duplicate Analysis (continued)

\begin{tabular}{|c|c|c|c|c|c|c|c|c|}
\hline \multirow{3}{*}{\begin{tabular}{c|c}
2 & Sample \\
3 & Area \\
\end{tabular}} & \multirow{2}{*}{$\begin{array}{l}\text { Sample } \\
\text { Number }\end{array}$} & \multirow{2}{*}{$\begin{array}{c}\text { Sample } \\
\text { Date } \\
\text { Date }\end{array}$} & Di-n-1 & tyylph & halate & \multicolumn{3}{|c|}{ Acetone } \\
\hline & & & $\mu \mathrm{\mu g} / \mathrm{kg}$ & & & $\mathrm{mg} / \mathrm{kg}$ & & \\
\hline & & & 27 & $\mid J B$ & 340 & $\frac{7}{7}$ & J & 10 \\
\hline Duplicate of $\mathrm{J12 \times 56}$ & $J 12 \times 58$ & $7 / 20 / 06$ & 54 & JB & & 8 & & 10 \\
\hline Split of $\mathrm{J} 12 \times 56$ & $\mathrm{J12} 2 \times 60$ & $7 / 20 / 06$ & 340 & $u$ & 340.00 & 20 & $u$ & 20 \\
\hline \multicolumn{9}{|l|}{ Analysis: } \\
\hline \multicolumn{3}{|c|}{ TDL } & \multirow{2}{*}{\multicolumn{3}{|c|}{\begin{tabular}{|c|}
330 \\
No - evaluate difference \\
\end{tabular}}} & \multirow{2}{*}{\multicolumn{3}{|c|}{$\begin{array}{l}20 \\
\text { No-evaluate difference }\end{array}$}} \\
\hline \multirow{3}{*}{ Duplica } & \multicolumn{2}{|c|}{$\begin{array}{l}\text { Both > PQL? } \\
\text { Both >5xTDL? }\end{array}$} & & & & & & \\
\hline & & \multicolumn{3}{|c|}{ No - evaluate difference } & & & \\
\hline & \multirow{2}{*}{\multicolumn{2}{|c|}{$\begin{array}{l}\text { Both > PQL? } \\
\text { Both > 5xTDL? }\end{array}$}} & \multicolumn{3}{|c|}{ No - acceptable } & \multicolumn{3}{|c|}{ No - acceptabl } \\
\hline \multirow{2}{*}{ Split Analysis } & & & \multicolumn{3}{|c|}{ No - evaluate difference } & \multicolumn{3}{|c|}{ No- evaluate difference } \\
\hline & \multicolumn{2}{|c|}{$\frac{R P D}{\text { Difference }>2 x T D L ?}$} & & \multicolumn{3}{|c|}{ acceptable } \\
\hline
\end{tabular}

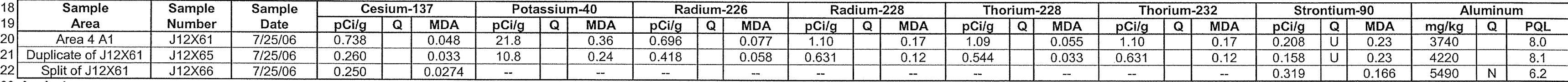

\begin{tabular}{|c|c|c|c|c|c|c|c|c|c|}
\hline \multirow{4}{*}{ Duplicate Analysis } & $\mathrm{DL}$ & 0.1 & 0.5 & 0.1 & 0.2 & 1 & 1 & 1 & 5 \\
\hline & $\begin{array}{l}\text { Both > PQL/MDA? } \\
\text { Both >5TDD/? }\end{array}$ & $\begin{array}{l}\text { Yes (continue) } \\
\end{array}$ & Yes (continue) & Yes (continue) & Yes (continue) & Yes (continue) & Yes (continue) & No - evaluate difference & Yes (continue) \\
\hline & $\begin{array}{c}\text { Both >5XXIIL? } \\
\text { RPD }\end{array}$ & No-evaluate difference & $\begin{array}{c}\text { Yes (calc RPD) } \\
67 \%\end{array}$ & No-evaluate difference & No-evaluate difference & No-evaluate difference & No - evaluate difference & & $\begin{array}{l}\text { Yes (calc RPD) } \\
12 \%\end{array}$ \\
\hline & $\begin{array}{l}\text { Difference }>2 \times T D L \text { ? } \\
\text { Both }>\text { POI/MPASA? }\end{array}$ & $\begin{array}{l}\text { Yes - assess further } \\
\text { Yes (continu) }\end{array}$ & Not applicable & Yes - assess further & Yes - assess further & No - acceptable & No - acceptable & No - acceptable & Not applicable \\
\hline \multirow[t]{2}{*}{ it Analysis } & $\begin{array}{c}\text { Both } \\
\text { Both >5xTDL? } \\
\text { RPD }\end{array}$ & $\begin{array}{c}\text { Yes (continue) } \\
\text { No - evaluate difference }\end{array}$ & & & & & & & $\begin{array}{l}\text { Yes (continue) } \\
\text { Yes (calc RPD) } \\
38^{\circ \%}\end{array}$ \\
\hline & Difference $>2 \times T D L ?$ & Yes - assess further & & & & & & No - acceptable & $\frac{38 \%}{\text { Not applicable }}$ \\
\hline
\end{tabular}

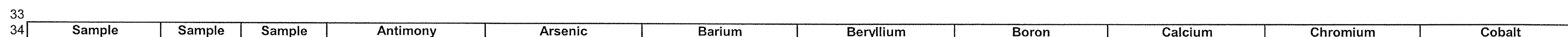

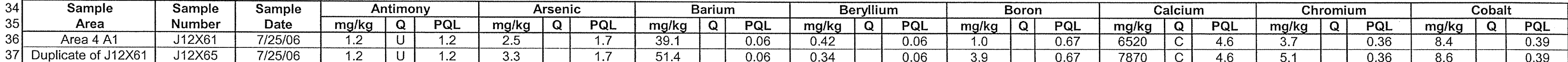

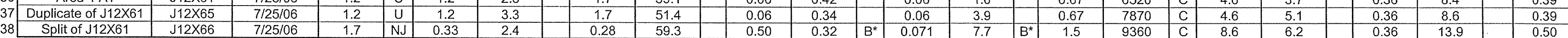
Analysis:

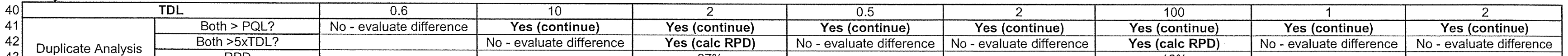
44

Split Analysis

48 .

$50 \mathrm{~B}=$ method $\mathrm{b}$

$\begin{aligned} 51 \mathrm{~B}^{*} & =\text { estimate } \\ 52 \mathrm{C} & =\text { method }\end{aligned}$

(s)

$54 \mathrm{MDA}=$ minimum detectable activity
Calc. No. $0100 B-C A-V 0299$
Checked T. M. Blakley $\partial m b$
Rev. No. $\frac{1}{\text { Date }} \frac{1}{10 / 04 / 07}$ Sheet No. 55 of 61 
Originator J.M. Capron ganc

\begin{tabular}{|c|c|c|c|c|c|c|c|c|c|c|c|c|c|c|c|c|c|c|c|c|c|c|c|c|c|}
\hline \multirow{2}{*}{\begin{tabular}{c|c}
2 & Sample \\
3 & Area \\
\end{tabular}} & \multirow{2}{*}{$\begin{array}{l}\text { Sample } \\
\text { Number }\end{array}$} & \multirow{2}{*}{$\begin{array}{c}\text { Sample } \\
\text { Date }\end{array}$} & \multicolumn{3}{|c|}{ Copper } & \multirow{2}{*}{\multicolumn{3}{|c|}{ Hexavalent Chromium }} & \multicolumn{3}{|c|}{ Iron } & \multirow{2}{*}{\multicolumn{3}{|c|}{ Lead }} & \multirow{2}{*}{\multicolumn{2}{|c|}{ Magnesium }} & \multicolumn{3}{|c|}{ Manganese } & \multicolumn{3}{|c|}{ Mercury } & \multicolumn{3}{|c|}{ Nickel } \\
\hline & & & $\mathrm{mg} / \mathrm{kg}$ & Q & $\mathrm{PQL}$ & & & & $\mathrm{mg} / \mathrm{kg}$ & $Q$ & $\mathrm{PQL}$ & & & & & & $\mathrm{mg} / \mathrm{kg}$ & & PQL & $\mathrm{mg} / \mathrm{kg}$ & Q & $\mathrm{PQL}$ & $\mathrm{mg} / \mathrm{kg}$ & Q & $\mathrm{PQL}$ \\
\hline Area 4A1 & $J 12 \times 61$ & $7 / 25 / 06$ & 15.5 & & 0.34 & 0.30 & & 0.20 & 22100 & & 9.7 & 5.2 & & 0.87 & 3970 & 2.7 & & & 0.08 & 0.01 & U & 0.01 & 6.8 & & 0.67 \\
\hline Duplicate of J12X61 & $\begin{array}{l}12 \times 65 \\
12 \times 66\end{array}$ & $\frac{7 / 25 / 06}{70506}$ & $\frac{15.7}{16.2}$ & & 0.34 & 0.20 & $U$ & 0.20 & 21500 & & 9.8 & $\frac{5.5}{6.9}$ & & 0.87 & 4510 & $\frac{2.7}{102}$ & $\frac{292}{337}$ & $N$ & 0.08 & 0.01 & $\frac{U}{B^{*}}$ & 0.01 & $\frac{11.3}{87}$ & & 0.67 \\
\hline & $J 12 \times 66$ & \multirow{2}{*}{\multicolumn{23}{|c|}{ Analysis: }} & \\
\hline \multirow{2}{*}{\multicolumn{3}{|c|}{$\begin{array}{l}\text { TDL } \\
\text { Both }>\text { PQL? }\end{array}$}} & \multirow{2}{*}{\multicolumn{3}{|c|}{$\frac{1}{\text { Yes (continue) }}$}} & & \multirow{2}{*}{\multicolumn{3}{|c|}{$\frac{5}{5}$}} & \multirow{2}{*}{\multicolumn{3}{|c|}{$\frac{5}{\text { Yes (continue) }}$}} & \multirow{2}{*}{\multicolumn{2}{|c|}{$\frac{75}{75}$}} & \multirow{2}{*}{\multicolumn{3}{|c|}{$\frac{5}{\text { Yes (continue) }}$}} & \multicolumn{3}{|c|}{0.2} & & & \\
\hline & & & & & & & & & & & & & & & & & & & & \multirow{3}{*}{\multicolumn{3}{|c|}{ No-evaluate difterence }} & \multicolumn{3}{|c|}{$\frac{4}{\text { Yes (continue) }}$} \\
\hline \multirow{3}{*}{ Duplicate Analysis } & \multicolumn{2}{|c|}{ Both >5XTDL? } & \multicolumn{3}{|c|}{ Yes (calc RPD) } & & & & \multicolumn{3}{|c|}{ Yes (calc RPD) } & \multirow{2}{*}{\multicolumn{3}{|c|}{ No - evaluate difference }} & \multirow{2}{*}{\multicolumn{2}{|c|}{$\frac{\text { Yes (calc RPD) }}{13 \%}$}} & \multicolumn{3}{|c|}{ Yes (calc RPD) } & & & & No-eve & Iluate & ifferencee \\
\hline & & & & $1.3 \%$ & & \multicolumn{3}{|c|}{ No - evaluate difference } & & $2.8 \%$ & & & & & & & & $0.69^{\circ}$ & & & & & & & \\
\hline & Differen & 2xTDL? & & applic & & No- & acce & table & Not & applit & & No- & acce & table & & applicable & & appli & & No- & accer & table & & - & \\
\hline & Both & TD! & & (cont & & No- eve & uate & lifference & & cont & & & (cont & inue) & & (continue) & & (con & nue) & No- eva & luate & difference & & (cont & nue) \\
\hline Split Analysis & Both & TTDL? & & (calc & & & & & & calc & & No-eval & uate & difference & & (calc RPD) & & (calc & & & & & No- eve & Iluate & ifference \\
\hline & Differen & S2XTDL? & & $\frac{5.0 \%}{\text { apolic }}$ & & No- & acce & table & Not & $\frac{20 \%}{20001 i}$ & & No- & acce & otable & $\mathrm{No}$ & $\frac{22 \%}{\text { applicable }}$ & & $\frac{15 \%}{\text { appli }}$ & able & No- & accer & table & & accep & table \\
\hline
\end{tabular}

\begin{tabular}{|c|c|c|c|c|c|c|c|c|c|c|c|c|c|c|c|c|c|c|c|c|c|c|c|c|c|c|}
\hline \multirow{3}{*}{\begin{tabular}{|c}
$\begin{array}{c}\text { Sample } \\
\text { Area }\end{array}$ \\
Area 4 A1
\end{tabular}} & \multirow{3}{*}{$\begin{array}{l}\text { Sample } \\
\text { Number } \\
10 \times 61\end{array}$} & \multirow{2}{*}{$\begin{array}{c}\text { Sample } \\
\text { Date }\end{array}$} & \multicolumn{3}{|c|}{ Potassium } & \multicolumn{3}{|c|}{ Selenium } & \multicolumn{3}{|c|}{ Silicon } & \multicolumn{3}{|c|}{ Sodium } & \multicolumn{3}{|c|}{ Vanadium } & \multicolumn{3}{|c|}{ Zinc } & \multicolumn{3}{|c|}{ Aroclor-1254 } & \multicolumn{3}{|c|}{ bis(2-Ethylhexyl)phthalate } \\
\hline & & & $\mathrm{mg} / \mathrm{kg}$ & $Q$ & PQL & $\mathrm{mg} / \mathrm{kg}$ & & & $\mathrm{mg} / \mathrm{kg}$ & Q & $\mathrm{PQL}$ & $\mathrm{mg} / \mathrm{kg}$ & Q & $\mathrm{PQL}$ & $\mathrm{mg} / \mathrm{kg}$ & & $\mathrm{PQL}$ & $\mathrm{mg} / \mathrm{kg}$ & $Q$ & PQL & $\mu \mathrm{g} / \mathrm{kg}$ & $Q$ & $\mathrm{PQL}$ & $\mu \mathrm{g} / \mathrm{kg}$ & 0 & PQL \\
\hline & & $7 / 25 / 06$ & 674 & & 6.3 & 1.3 & $u$ & 1.3 & 392 & J & 6.3 & 162 & & 2.1 & 53.4 & & 0.25 & 41.7 & $\mathrm{C}$ & 0.45 & & & & 46 & JB & 340 \\
\hline Duplicate of J12X61 & $\mathrm{J12 \times 65}$ & $7 / 25 / 06$ & 816 & & 6.4 & 1.3 & $u$ & 1.3 & 360 & $\mathrm{~J}$ & 6.4 & 164 & & 2.1 & 44.8 & & 0.25 & 41.0 & C & 0.45 & 57 & & 13 & 35 & JB & \\
\hline Split of J12X61 & $\mathrm{J12X66}$ & $7 / 25 / 06$ & 1160 & & 50.4 & 0.62 & $B^{*}$ & 0.17 & 577 & CNJ & 4.0 & 273 & & 10.1 & 68.4 & $|\mathrm{~N}|$ & 0.68 & 48.3 & & 1.4 & 17 & 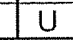 & 17 & 330 & U & \\
\hline \multicolumn{20}{|c|}{10} & & \multirow{2}{*}{\multicolumn{3}{|c|}{16.5}} & \multirow{2}{*}{\multicolumn{3}{|c|}{330}} \\
\hline \multicolumn{3}{|c|}{ TDL } & \multicolumn{3}{|c|}{400} & \multicolumn{3}{|c|}{\begin{tabular}{|c|}
1 \\
No - evaluate difference
\end{tabular}} & \multicolumn{3}{|c|}{$\frac{2}{8}$} & & & & & \multicolumn{3}{|c|}{$\frac{1}{\text { Yes (continue) }}$} & & & & & & lifference \\
\hline \multirow{2}{*}{ Duplicate Analysis } & \multicolumn{2}{|c|}{$\begin{array}{l}\text { Both }>\text { PQL? } \\
\text { Both }>5 \times \mathrm{TDL} \text { ? }\end{array}$} & \multirow{2}{*}{\multicolumn{3}{|c|}{$\begin{array}{c}\text { Yes ( (continue) } \\
\text { No - evaluate difference }\end{array}$}} & \multicolumn{3}{|c|}{ No - evaluate difference } & \multicolumn{3}{|c|}{$\begin{array}{l}\text { Yes (continue) } \\
\text { Yes (calc RPD) }\end{array}$} & \multicolumn{3}{|c|}{$\begin{array}{l}\text { Yes (continue) } \\
\text { No - evaluate difference }\end{array}$} & \multirow{2}{*}{\multicolumn{3}{|c|}{ Yes (calc RPD) }} & \multicolumn{3}{|c|}{ Yes (calc RPD) } & No- eve & luate & difference & & & \\
\hline & Dicfor & & & & & & & & & $8.5 \%$ & & & & & & & & & $1.7 \%$ & & & & & & & \\
\hline & Differen & $>2 \times T D L ?$ & & acce & table & No & accep & tible & & tapplic & & $\frac{\text { No- }}{y_{0 a}}$ & accep & table & Not & applic & cable & & tappli & able & No. & acce & ptable & & 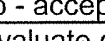 & table \\
\hline & $\frac{\text { Both }}{\text { Both }}$ & STI? & Nopev & 1000 & nie) & No - ev & uate $c$ & ifference & & (conts & & Yes & cont & nuee & & (cont & $\frac{\text { Inue) }}{\text { RPD) }}$ & & scon & $\frac{\text { nue) }}{\mathrm{RPDI}}$ & Noo-eve & the & - & No-er & 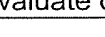 & ifference \\
\hline Split Analysis & & & TNo-ev & tude & 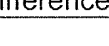 & & & & & $38 \%$ & & No - eva & the & - & & $25 \%$ & & & $15 \%$ & & & & & & & \\
\hline & Differen & $>2 \times T D L ?$ & No & acce & table & & accep & table & & t applic & & Yes-a & ssess & further & & applic & cable & & tappli & able & Yes -a & isses. & sfurther & & - accer & table \\
\hline
\end{tabular}

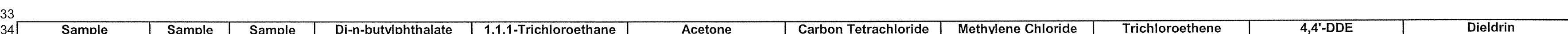

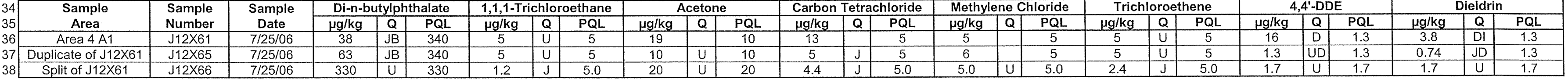
39 Analysis:

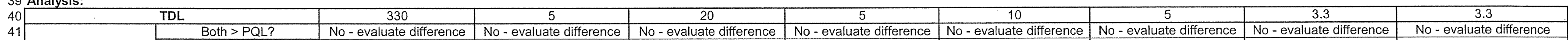
42 Duplicate Analysis Split Analysis 48 $49 \mathrm{~B}=$ method bl $50 B^{*}=e s t i m a t e$
$51 \mathrm{C}=$ method $b+10$ 
Originator J.M. Capron/mC

Subject $\frac{118-B / C \text { - Field Remediation }}{118 \text { Bural Ground Cleanup Verification } 95 \% \text { UCL Calculations }}$ \begin{aligned} & Date $\frac{10 / 03 / 07}{14655} \\ &$\hline\end{aligned}

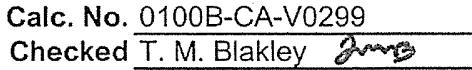

Rev. No. $\frac{1}{\text { Date }}$ Date
Sheet No. $\frac{10 / 04 / 07}{57 \text { of } 61}$

1 Split/Duplicate Analysis (continued

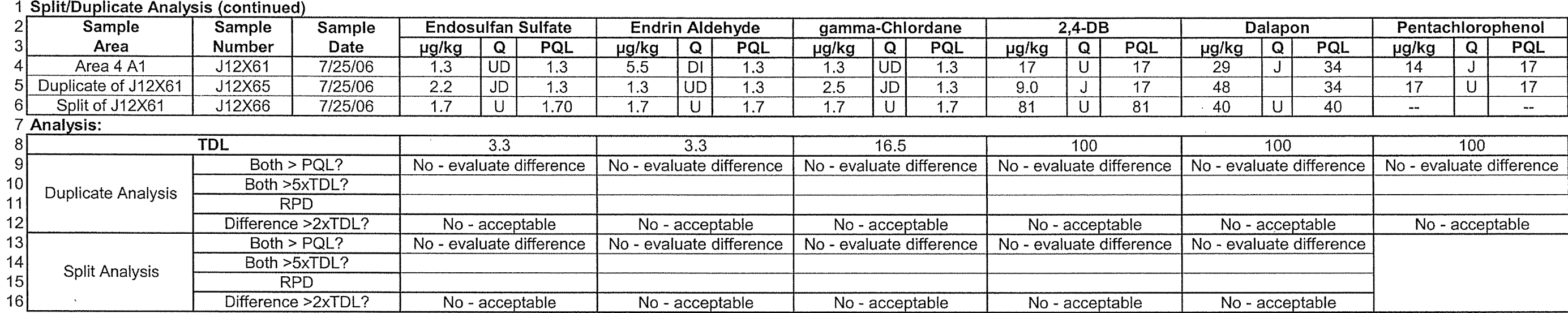

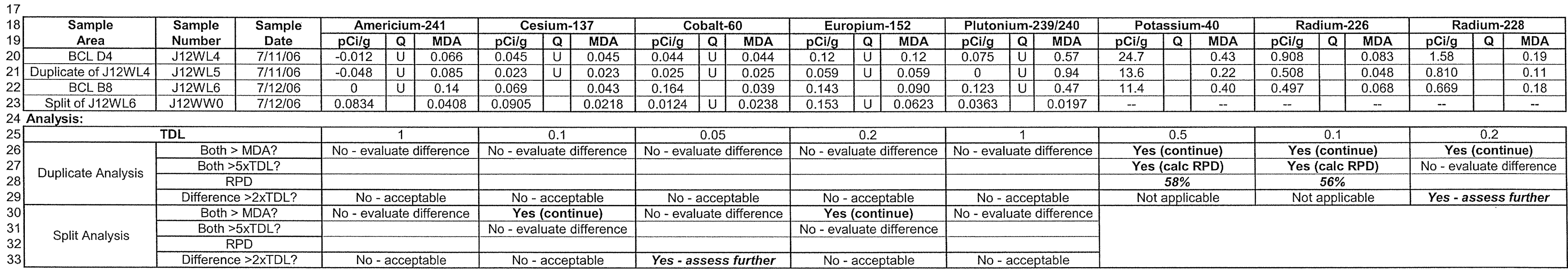

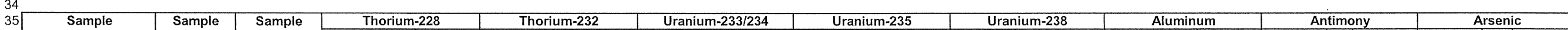

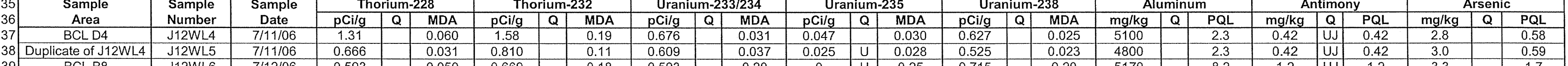

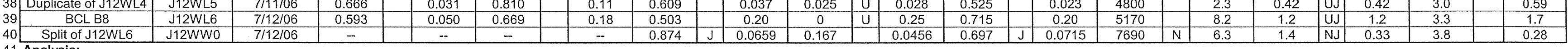

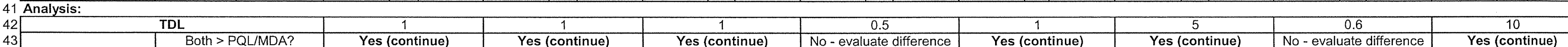

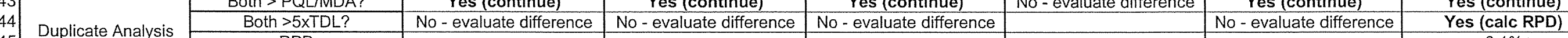
\begin{tabular}{c|c|c|c|}
446 & No - acceptable & No- acceptable \\
\cline { 2 - 4 } & Difference $>2 \times T D L ?$ & 2 &
\end{tabular}

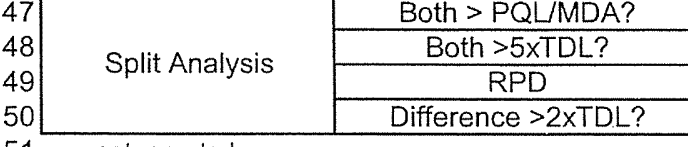
$51--=$ not reported $53 \mathrm{D}=$ diluted

$M D A=$ minimum detectable activity

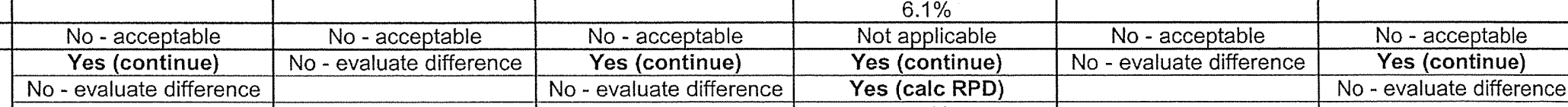
No-evaluate difference \begin{tabular}{|l|l|}
\hline No - acceptable & No - acceptable \\
\hline
\end{tabular} 


\begin{tabular}{|c|c|c|c|c|c|c|c|c|c|c|c|c|c|c|c|c|c|c|c|c|c|c|c|c|c|c|}
\hline \multirow{3}{*}{\begin{tabular}{l|c|c|}
2 & Sample \\
3 & Area \\
\end{tabular}} & \multirow{3}{*}{$\begin{array}{l}\text { Sample } \\
\text { Number } \\
\text { J12WL4 }\end{array}$} & \multirow{2}{*}{$\begin{array}{c}\text { Sample } \\
\text { Date }\end{array}$} & \multicolumn{3}{|c|}{ Barium } & \multirow{2}{*}{\multicolumn{3}{|c|}{ Beryllium }} & \multirow{2}{*}{\multicolumn{3}{|c|}{ Boron }} & \multicolumn{3}{|c|}{ Calcium } & \multirow{2}{*}{\multicolumn{3}{|c|}{ Chromium }} & \multicolumn{3}{|c|}{ Cobalt } & \multicolumn{3}{|c|}{ Copper } & \multicolumn{3}{|c|}{ Hexavalent Chromium } \\
\hline & & & $\mathrm{mg} / \mathrm{kg}$ & & $P Q L$ & $\mathrm{mg} / \mathrm{kg}$ & & PQL & & & & $\mathrm{mg} / \mathrm{kg}$ & & $\mathrm{PQL}$ & & Q & PQL & $\mathrm{mg} / \mathrm{kg}$ & & $P Q L$ & $\mathrm{mg} / \mathrm{kg}$ & $Q$ & , & $\mathrm{mg} / \mathrm{kg}$ & $Q$ & $P Q L$ \\
\hline & & $7 / 11 / 06$ & 52.7 & & & 0.44 & & 0.02 & 1.3 & & 0.23 & 5800 & \begin{tabular}{c|c} 
& \\
$c$ &
\end{tabular} & 2.1 & 8.8 & & 0.12 & 7.9 & & 0.13 & 12.9 & & 0.11 & 0.36 & & 0.20 \\
\hline $\begin{array}{l}\text { Duplicate of J12WL4 } \\
\text { BCL B8 }\end{array}$ & $\frac{J 12 W L 5}{J 12 W L 6}$ & $\frac{71 / 1106}{7 / 12106}$ & $\begin{array}{r}59.5 \\
54.2 \\
\end{array}$ & & 0.02 & $\begin{array}{l}0.45 \\
0.43\end{array}$ & & 0.02 & $\begin{array}{l}1.9 \\
1.7\end{array}$ & & 0.23 & $\frac{5970}{5750}$ & c & $\frac{2.1}{4.6}$ & $\frac{7.3}{7.3}$ & & $\frac{0.13}{0.37}$ & $\frac{7.6}{7.3}$ & & $\begin{array}{l}0.14 \\
0.40\end{array}$ & $\begin{array}{l}\frac{12.9}{13.8} \\
\end{array}$ & & $\frac{0.12}{0.34}$ & 0.26 & & $\frac{0.20}{0.20}$ \\
\hline Split of J12WL6 & J12WWO & $7 / 12106$ & 69.9 & & 0.50 & 0.34 & $\mathrm{~B}^{*}$ & 0.071 & 2.8 & $\mathrm{~B}^{*} \mathrm{C}$ & 1.5 & 6270 & $\mathrm{CN}$ & 8.6 & 9.4 & & 0.36 & 10.9 & & 0.50 & 14.4 & & 0.30 & 0.350 & UJ & 0.350 \\
\hline \multirow{2}{*}{\multicolumn{3}{|c|}{ 年 }} & & \multirow{2}{*}{\multicolumn{3}{|c|}{$\frac{0.5}{\text { Yes (continu }}$}} & & \multirow{2}{*}{\multicolumn{3}{|c|}{100}} & \multirow{2}{*}{\multicolumn{3}{|c|}{ Yes (continue) }} & & \multirow{2}{*}{\multicolumn{3}{|c|}{$\frac{1}{\text { (continue) }}$}} & \multirow{2}{*}{\multicolumn{3}{|c|}{0.5}} \\
\hline & & & & & & & & & & & & & & & & & & & & & & & & \multirow{2}{*}{\multicolumn{3}{|c|}{$\begin{array}{l}\text { Yes (continue) } \\
\text { No - evaluate differes }\end{array}$}} \\
\hline \multirow{2}{*}{ Duplicate Analysis } & \multicolumn{2}{|c|}{$\begin{array}{l}\text { Both > PQL? } \\
\text { Both }>5 \times T D L ?\end{array}$} & \multicolumn{3}{|c|}{$\begin{array}{c}\text { Yes (continue) } \\
\text { Yes (calc RPD) } \\
12 \%\end{array}$} & \multicolumn{3}{|c|}{$\begin{array}{c}\text { Yes (continue) } \\
\text { No - evaluate difference }\end{array}$} & \multicolumn{3}{|c|}{$\begin{array}{l}\text { Yes (continue) } \\
\text { No- evaluate difference }\end{array}$} & \multicolumn{3}{|c|}{$\begin{array}{l}\text { Yess continue) } \\
\text { Yes (calc RPD) }\end{array}$} & \multicolumn{3}{|c|}{ Yes (calc RPD) } & \multicolumn{3}{|c|}{ No- evaluate difference } & \multicolumn{3}{|c|}{ Yes (calc RPD) } & & & \\
\hline & Differen & T2XTDL? & & $12 \%$ & & & ac & & & a & & & $\frac{2.9 \%}{\text { apolic }}$ & & & $\frac{19 \%}{\text { applic }}$ & & & accep & & & If anolic & & No- & $e p$ & \\
\hline & & SL? & & contit & & & (cc & & & (cont) & & & (con & & & (conti & inue) & & (conti & & & $5(\operatorname{cont}$ & & $\sqrt{0-}$ & r & \\
\hline Split Analysis & Both & XTDL? & & calc $F$ & & No- eve & uate & lifference & No-ev & uate c & ifference & & (calc ) & & & (calc & & No - eval & luate di & difference & & 5 (calc & & & & \\
\hline & Differen & $\frac{D}{2 \times T D L ?}$ & Not & 25\% & & No. & acce & table & & acces & & & $\frac{8 . \%}{\text { applic }}$ & & & $\frac{20 \%}{\text { applic }}$ & cable & & accept & table & & & able & & - -accer & ptable \\
\hline
\end{tabular}

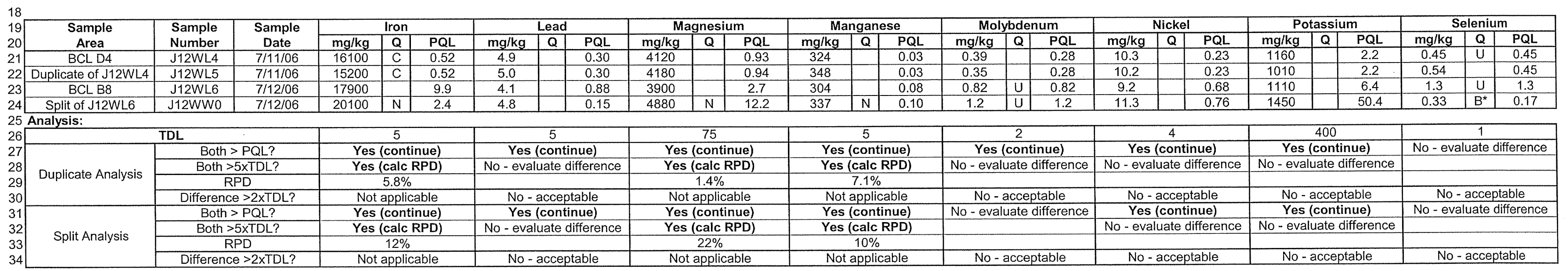

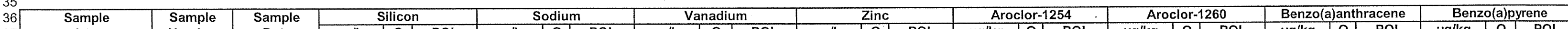

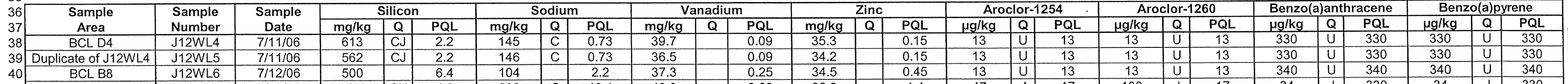

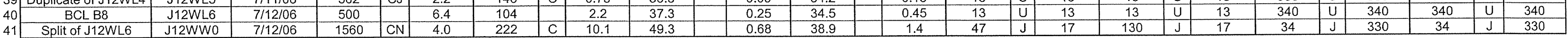

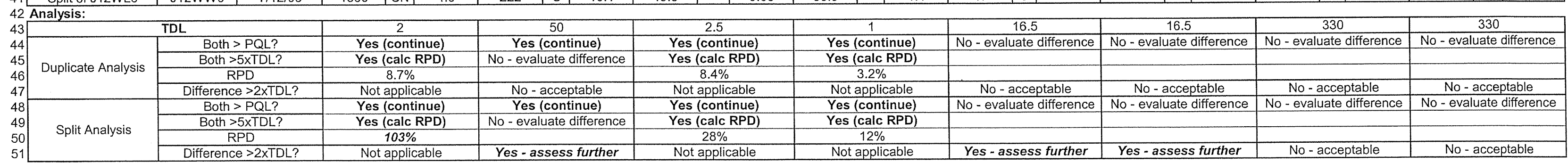
52 B* =estimate $53 \mathrm{BCL}=$ below cleanup levels

$55 \mathrm{~J}=$ estimate 
Washington Closure Hanford

Originator J. M. Capron $/ m<$

Project $\frac{100-B / C \text { Field Remediation }}{118-B-1 \text { Burial Ground Cleanup Verification } 95 \% \text { UCL Calculations }}$

\section{CALCULATION SHEET}

$\begin{aligned} \text { Date } & 10 / 03 / 07 \\ 14655 & \end{aligned}$
Calc. No. $0100 B-C A-V 0299$
Checked T. M. Blakley $2 \operatorname{mon}$

Rev. No. $\frac{1}{\text { Date }} \frac{1}{10 / 04 / 07}$
Sheet No.
59 of 61

\section{Split/Duplicate Analysis (continued)}

\begin{tabular}{|c|c|c|c|c|c|c|c|c|c|c|c|c|c|c|c|c|c|c|c|c|c|c|c|c|c|c|}
\hline \multirow{3}{*}{\begin{tabular}{|c|c|}
$\begin{array}{c}\text { Sample } \\
\text { Area }\end{array}$ \\
BCLD4 \\
\end{tabular}} & \multirow{2}{*}{$\begin{array}{l}\text { Sample } \\
\text { Number }\end{array}$} & \multirow{2}{*}{$\begin{array}{c}\text { Sample } \\
\text { Date }\end{array}$} & \multicolumn{3}{|c|}{ Benzo(b)fluoranthene } & \multicolumn{3}{|c|}{ Benzo(g,h,i)perylene } & \multicolumn{3}{|c|}{ bis(2-Ethylhexyl)phthalate } & \multicolumn{3}{|c|}{ Chrysene } & \multicolumn{3}{|c|}{ Di-n-butylphthalate } & \multicolumn{3}{|c|}{ Diethylphthalate } & \multicolumn{3}{|c|}{ Fluoranthene } & \multicolumn{3}{|c|}{ Phenanthrene } \\
\hline & & & $\mu \mathrm{g} / \mathrm{kg}$ & Q & $\mathrm{PQL}$ & $\mu \mathrm{g} / \mathrm{kg}$ & Q & $P Q L$ & $\mu \mathrm{g} / \mathrm{kg}$ & Q & $\mathrm{PQL}$ & $\mu g / \mathrm{kg}$ & Q & $P Q L$ & $\mu \mathrm{g} / \mathrm{kg}$ & Q & $\mathrm{PQL}$ & & $Q$ & & & & & $\mu \mathrm{g} / \mathrm{kg}$ & & $\mathrm{PQL}$ \\
\hline & J12WL4 & & 330 & $u$ & 330 & 330 & u & 330 & & JB & 330 & 330 & U & 330 & 330 & $u$ & 330 & 330 & $u$ & 330 & 330 & $u$ & 330 & 330 & & 230 \\
\hline \begin{tabular}{|l|} 
Duplicate of J12WL4 \\
\end{tabular} & J12WL5 & $7 / 11 / 06$ & 330 & $u$ & 330 & 330 & u & 330 & 27 & JB & 330 & 330 & $u$ & 330 & 45 & 1 & 330 & 18 & $\mathrm{~J}$ & 330 & 330 & 10 & 330 & 330 & U & 30 \\
\hline BCL B8 & J12WL6 & & 340 & 0 & 340 & 340 & $u$ & 340 & 340 & 0 & 340 & 340 & U & 340 & 340 & U & 340 & 340 & $U$ & 340 & 340 & & & 340 & $u$ & 340 \\
\hline $\begin{array}{l}\text { Split of J12WL6 } \\
\text { nalkic. }\end{array}$ & J12WW0 & $7 / 12 / 06$ & 39 & $\mid \mathrm{J}$ & 330 & 36 & $|\mathrm{~J}|$ & 330 & 280 & $\mid \mathrm{s}$ & 330 & 77 & 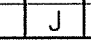 & 330 & 330 & $u$ & 330 & 330 & $u$ & 330 & 93 & J & 30 & 78 & & \\
\hline \multirow{2}{*}{\multicolumn{3}{|c|}{ Analysis: $\quad T D L$}} & \multirow{3}{*}{\multicolumn{3}{|c|}{\begin{tabular}{|c|}
330 \\
No - evaluate difference \\
\end{tabular}}} & \multicolumn{3}{|c|}{330} & \multicolumn{3}{|c|}{330} & \multicolumn{3}{|c|}{330} & \multicolumn{3}{|c|}{330} & \multicolumn{3}{|c|}{330} & \multicolumn{3}{|c|}{330} & \\
\hline & \multirow{2}{*}{\multicolumn{2}{|c|}{$\begin{array}{l}\text { Both }>5 \times T D L ? \\
\text { RPD }\end{array}$}} & & & & \multirow{2}{*}{\multicolumn{3}{|c|}{ No-evaluate difference }} & \multirow{2}{*}{\multicolumn{3}{|c|}{ No - evaluate difference }} & \multirow{2}{*}{\multicolumn{3}{|c|}{ No - evaluate difference }} & \multirow{2}{*}{\multicolumn{3}{|c|}{ No - evaluate difference }} & \multirow{2}{*}{\multicolumn{3}{|c|}{ No - evaluate difference }} & \multirow{2}{*}{\multicolumn{3}{|c|}{ No-evaluate difference }} & \multirow{2}{*}{\multicolumn{3}{|c|}{ evaluate difference }} \\
\hline \multirow{2}{*}{ Duplicate Analysis } & & & & & & & & & & & & & & & & & & & & & & & & & & \\
\hline & Differenc & $>2 \times T D L ?$ & & ac & & & $\overline{a c}$ & & & accep & ttable & & -2 & vite & & & ta & & & & & cep & & & ICC6 & \\
\hline & & & No-ev & luatec c & fference & No-eval & & ifference & No- eva & aluate d & difference & No-eva & aluatec & difference & No - eval & luated & ifference & No- eva & & ence & No - eva & luate c & erence & & & \\
\hline Split Analysis & & & & & & & & & & & & & & & & & & & & & & & & & & \\
\hline & Differenc & TLL & & & & & & & & & & & & & & & & & & & & & & & & \\
\hline
\end{tabular}

\begin{tabular}{|c|c|c|c|c|c|c|c|c|c|c|c|c|c|c|c|c|c|c|c|c|c|c|c|c|c|c|}
\hline \multirow{3}{*}{$\begin{array}{c}9_{0}^{0} \begin{array}{c}\text { Sample } \\
\text { Area }\end{array} \\
\text { BCL D4 } \\
\end{array}$} & \multirow{2}{*}{$\begin{array}{l}\text { Sample } \\
\text { Number } \\
\end{array}$} & \multirow{2}{*}{$\begin{array}{c}\begin{array}{c}\text { Sample } \\
\text { Date }\end{array} \\
\end{array}$} & \multicolumn{3}{|c|}{ Phenol } & \multicolumn{3}{|c|}{ Pyrene } & \multicolumn{3}{|c|}{ Acetone } & \multicolumn{3}{|c|}{ Carbon Tetrachloride } & \multicolumn{3}{|c|}{ Methylene Chloride } & \multicolumn{3}{|c|}{ Toluene } & \multicolumn{3}{|c|}{ 4,4'-DDT } & \multicolumn{3}{|c|}{ Methoxychlor } \\
\hline & & & $\mu \mathrm{gg} / \mathrm{kg}$ & \begin{tabular}{|l|l|l|l|}
$Q$ \\
\end{tabular} & $\frac{P Q L}{230}$ & $\mu \mathrm{g} / \mathrm{kg}$ & Q & $\mathrm{PQL}$ & $\mu g / \mathrm{kg}$ & \begin{tabular}{|l} 
Q \\
\end{tabular} & PQL & $\mu g / \mathrm{kg}$ & $Q$ & $\frac{P Q L}{5}$ & $\mu \mathrm{g} / \mathrm{kg}$ & 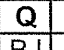 & $\frac{P Q L}{5}$ & $\mu \mathrm{gg} / \mathrm{kg}$ & Q & $\frac{P Q L}{5}$ & $\mu \mathrm{g} / \mathrm{kg}$ & \begin{tabular}{l|l} 
\\
\end{tabular} & $\mathrm{PQL}$ & $\frac{\mu \mathrm{g} / \mathrm{kg}}{17}$ & \begin{tabular}{|c|c|} 
\\
\end{tabular} & \begin{tabular}{|l}
$\mathrm{PQL}$ \\
13
\end{tabular} \\
\hline & $\begin{array}{l}\mathrm{J12WL4} \\
\mathrm{J} 12 \mathrm{WL5}\end{array}$ & $\frac{7111106}{7 / 11 / 06}$ & 330 & $\left|\frac{U}{J}\right|$ & $\frac{330}{330}$ & $\begin{array}{l}330 \\
330\end{array}$ & $\mid \frac{u}{u}$ & $\frac{330}{330}$ & $\begin{array}{l}10 \\
10\end{array}$ & $\frac{U}{U}$ & $\frac{10}{10}$ & $\frac{6}{5}$ & is & $\frac{5}{5}$ & $\frac{12}{11}$ & $\mid$\begin{tabular}{|c|}
$\mid B J$ \\
$B J$
\end{tabular} & $\begin{array}{l}5 \\
5\end{array}$ & $\begin{array}{l}5 \\
5 \\
\end{array}$ & $\frac{u}{u}$ & $\frac{5}{5}$ & $\frac{0.50}{13}$ & $\left|\begin{array}{l}\mathrm{JD} \\
\mathrm{UD}\end{array}\right|$ & $\frac{1.3}{1.3}$ & $\frac{1.7}{1.3}$ & $\frac{D}{U D}$ & \\
\hline BCL B8 & J12WL6 & $7 / 12 / 06$ & 340 & u & 340 & 340 & u & 340 & & \begin{tabular}{|l|l|l|l|}
$j$ \\
\end{tabular} & 10 & 13 & J & 5 & 2 & $\left|\begin{array}{|c|c|}\mid \mathrm{D} u \\
\end{array}\right|$ & & 5 & $u$ & & $\frac{1.3}{1.3}$ & UD & $\frac{1.3}{1.3}$ & $\frac{1.3}{1.3}$ & UD & $\frac{1.3}{1.3}$ \\
\hline \multirow{2}{*}{\multicolumn{26}{|c|}{$\begin{array}{l}\text { Split of J12WL6 } \\
\text { nalysis: }\end{array}$}} & \\
\hline & & & & & & & & & & & & & & & & & & & & & & & & & \\
\hline \multirow{3}{*}{ Duplicate Analysis } & \multirow{2}{*}{\multicolumn{2}{|c|}{$\begin{array}{l}\text { Both > PQL? } \\
\text { Both > 5xTDL? }\end{array}$}} & \multirow{2}{*}{\multicolumn{3}{|c|}{$\begin{array}{l}330 \\
\text { No - evaluate difference }\end{array}$}} & \multirow{2}{*}{\multicolumn{3}{|c|}{$\begin{array}{c}330 \\
\text { No- - evaluate difference }\end{array}$}} & \multirow{2}{*}{\multicolumn{3}{|c|}{$\begin{array}{c}20 \\
\text { No - evaluate difference }\end{array}$}} & \multicolumn{3}{|c|}{$\begin{array}{l}5 \\
\text { No - evaluate difference }\end{array}$} & \multicolumn{3}{|c|}{$\frac{10}{10}$} & \multicolumn{3}{|c|}{ No - evaluate difference } & \multicolumn{3}{|c|}{3.3} & No- eva & aluated & differenc \\
\hline & & & & & & \multicolumn{2}{|c|}{ No - evaluate difference } & & \multicolumn{2}{|c|}{ No - evaluate difterence } & & & \multicolumn{3}{|c|}{ No - evaluate difference } & \multicolumn{3}{|c|}{ No - evaluate difterence } & & & & \\
\hline & Different & P2XTDL? & & accer & & & acces & table & & $\overline{\text { accep }}$ & & No-z & accep & & No- & acceo & otable & No- & accep & table & No- - r l & accep & table & & - accep & table \\
\hline & Both & & No- eve & uate & ference & No- eve & uate & lifference & No-ev & luate & fference & No-eval & atec & fiference & No-eva & uatec & differenc & No-eva & luater & lifference & \begin{tabular}{|l} 
No-eval \\
\end{tabular} & luated & lifference & No-eva & aluated & differen \\
\hline Split Analysis & & & & & & & & & & & & & & & & & & & & & & & & & & \\
\hline & Differen & 22XTDL? & & $\overline{a c}$ & & & acc & table & & $\overline{a c}$ & & No- & ac & & No- & accep & ptable & & accep & table & & $\overline{s s}$ & further & & $\mathrm{ep}$ & ptable \\
\hline
\end{tabular}

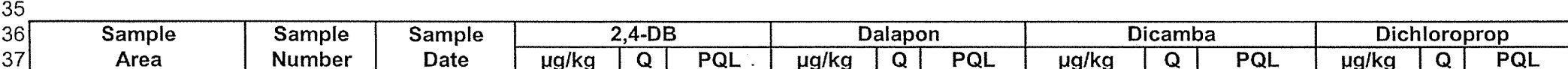

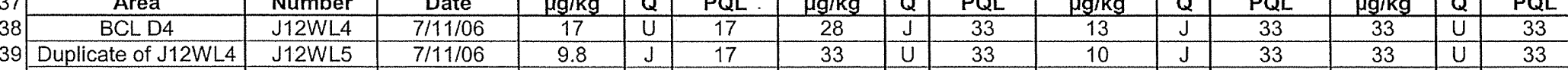

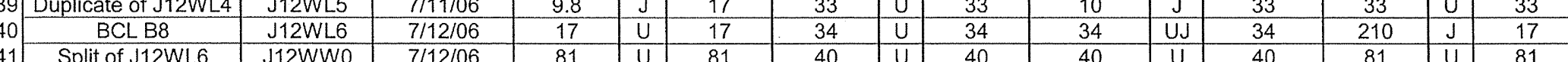

42 Analysis:

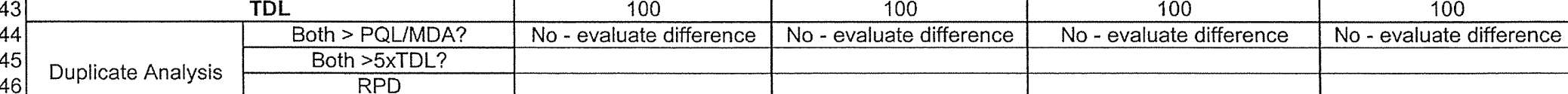

\begin{tabular}{l|l}
48 & Split Analysis \\
\hline
\end{tabular}

$52 \mathrm{~B}=$ method blank contami

$53 \mathrm{BCL}=$ below
$54 \mathrm{D}=$ diluted

\begin{tabular}{|c|c|c|c|c|}
\hline$\frac{\text { Both }>5 \times T C}{R P D}$ & & & & \\
\hline Difference $>2 \times T D L ?$ & No - acceptable & No - acceptable & No - acceptable & No - acceptable \\
\hline Both > PQL/MDA? & No - evaluate difference & No - evaluate difference & No - evaluate difference & No - evaluate difference \\
\hline $\begin{array}{l}\text { Both > 5xTDL? } \\
\text { RPD }\end{array}$ & & & & \\
\hline Difference $>2 \times T D L ?$ & No-acceptable & No - acceptable & No-acceptable & No - acceptable \\
\hline
\end{tabular}

$\mathrm{RPD}=$ relative percent differen
$\mathrm{TDL}=$ target detection

$T D L=$ target detection limit
$U=$ undetected 
Washington Closure Hanford

Originator J. M. Capron $/ 20$

Subject 118-B-1 Burial Ground Cleanup Verfication 95\% UCL Calculations

\section{CALCULATION SHEET}

Date $10 / 03 / 07$
Calc. No. 0100B-CA-V0299
Checked T. M. Blakley 2m-s

Rev. No. $\frac{1}{1010407}$

Date $\frac{1}{10 / 04 / 07}$

\begin{tabular}{|c|c|c|c|c|c|c|c|c|c|c|c|c|c|c|c|c|c|c|c|c|c|c|c|c|c|c|}
\hline \multirow{2}{*}{$\begin{array}{cc}2 & \text { Sample } \\
3 & \text { Area } \\
\end{array}$} & \multirow{2}{*}{$\begin{array}{l}\text { Sample } \\
\text { Number } \\
\end{array}$} & \multirow{2}{*}{$\begin{array}{c}\text { Sample } \\
\text { Date }\end{array}$} & \multicolumn{3}{|c|}{ Americium-241 } & \multicolumn{3}{|c|}{ Cesium-137 } & \multicolumn{3}{|c|}{ Nickel-63 } & \multicolumn{3}{|c|}{$\begin{array}{l}\text { Plutonium-239/240 } \\
\end{array}$} & \multicolumn{3}{|c|}{ Potassium-40 } & \multicolumn{3}{|c|}{ Radium-226 } & \multicolumn{3}{|c|}{ Radium-228 } & \multicolumn{3}{|c|}{ Thorium-228 } \\
\hline & & & $p \mathrm{Ci} / \mathrm{g}$ & $Q$ & MDA & $\mathrm{pCilg}$ & Q & MDA & $\mathrm{pCi} / \mathrm{g}$ & $Q$ & MDA & pCilg & \begin{tabular}{|c|}
0 \\
\end{tabular} & MDA & pCilg & \begin{tabular}{l|l} 
Q \\
\end{tabular} & MDA & pCilg & $Q$ & MDA & pCilg & Q & MDA & $\mathrm{pCi} / \mathrm{g}$ & Q & MDA \\
\hline SPAB5 & & $10 / 12 / 06$ & 0.047 & $U$ & 0.23 & 0.222 & & 0.057 & 0.952 & U & 3.5 & 0.044 & $u$ & 0.33 & 32.0 & & 0.37 & 0.931 & & 0.091 & & & 0.27 & & & 0.10 \\
\hline $\begin{array}{c}\text { Duplicate of } \mathrm{J} 13 \mathrm{HYY} \\
\text { Solit tof } \mathrm{J13HY7}\end{array}$ & J13J09 & $10 / 12 / 06$ & 0.057 & $U$ & 0.27 & 0.109 & & 0.032 & 0.723 & $u$ & 3.5 & 0 & $u$ & 0.34 & & & 0.29 & 0.607 & & 0.057 & 0.839 & & 0.11 & 0.826 & & 0.038 \\
\hline \multirow{2}{*}{\multicolumn{27}{|c|}{\begin{tabular}{|l|l} 
Split of J13HY7 & $J 13 \mathrm{~J} 10$ \\
Analysis: & TDL \\
\end{tabular}}} \\
\hline & & & \multirow{3}{*}{\multicolumn{3}{|c|}{$\begin{array}{c}1 \\
\text { No - evaluate difference }\end{array}$}} & \multirow{2}{*}{\multicolumn{3}{|c|}{$\begin{array}{c}0.1 \\
\text { Yes (continue) }\end{array}$}} & \multirow{2}{*}{\multicolumn{3}{|c|}{30}} & \multirow{2}{*}{\multicolumn{3}{|c|}{$\frac{1}{1 \text { No - evaluate difference }}$}} & \multicolumn{3}{|c|}{0.5} & \multicolumn{3}{|c|}{0.1} & \multicolumn{3}{|c|}{0.2} & \multirow{2}{*}{\multicolumn{3}{|c|}{$\frac{1}{1 n}$}} \\
\hline \multirow{4}{*}{$\begin{array}{l}\text { Duplicate Analysis } \\
0\end{array}$} & \multirow{2}{*}{\multicolumn{2}{|c|}{$\begin{array}{l}\text { Both > MDA? } \\
\text { Both > 5xTDL? }\end{array}$}} & & & & & & & & & & & & & \multirow{2}{*}{\multicolumn{3}{|c|}{$\begin{array}{l}\text { Yes (continue) } \\
\text { Yes (calc RPD) }\end{array}$}} & & & & \multirow{3}{*}{\multicolumn{3}{|c|}{$\begin{array}{c}\text { Yes (continue) } \\
\text { No-evaluate difference }\end{array}$}} & & & \\
\hline & & & & & & \multirow{2}{*}{\multicolumn{3}{|c|}{ No - evaluate difference }} & \multirow{2}{*}{\multicolumn{3}{|c|}{ No - evaluate difference }} & \multirow{2}{*}{\multicolumn{3}{|c|}{ No- evaluate difference }} & & & & \multicolumn{3}{|c|}{ Yes (calc RPD) } & & & & No- $\mathrm{ev}_{\mathrm{C}}$ & uate c & lifference \\
\hline & & & & & & & & & & & & & & & & $67 \%$ & & & $42 \%$ & & & & & & & \\
\hline & Differen & $2 \times$ TTDL? & & accer & able & No- & accep & table & & accep & able & No- & accep & table & & applic & able & & tapplic & cable & Yes-a & ssess & further & & accep & table \\
\hline & Both & DDA? & No-ev & uate & ifference & Yes & cont & & No-eve & uate $c$ & ifference & No- eva & uated & lifference & & & & & & & & & & & & \\
\hline Split Analysis & Both & TDL? & & & & No-eval & uate c & lifference & & & & & & & & & & & & & & & & & & \\
\hline & Differen & $2 \times T_{D L}$ & & accer & able & No- & cccen & table & & accer & & No & acca & table & & & & & & & & & & & & \\
\hline
\end{tabular}

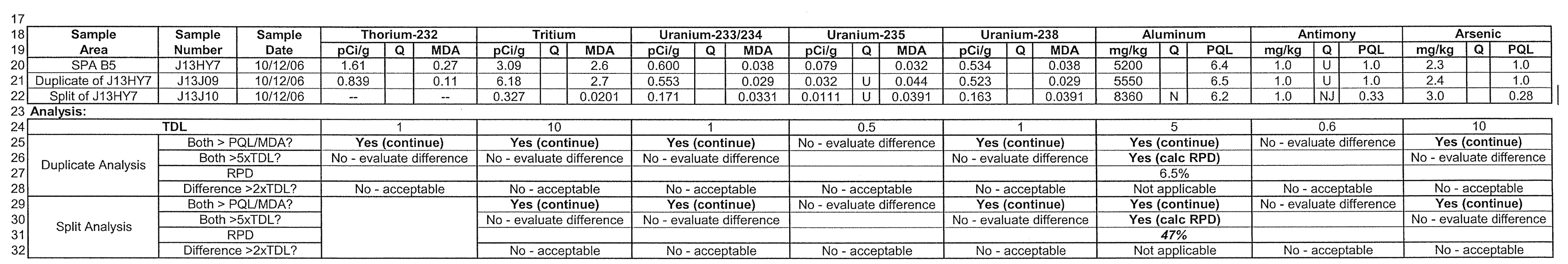

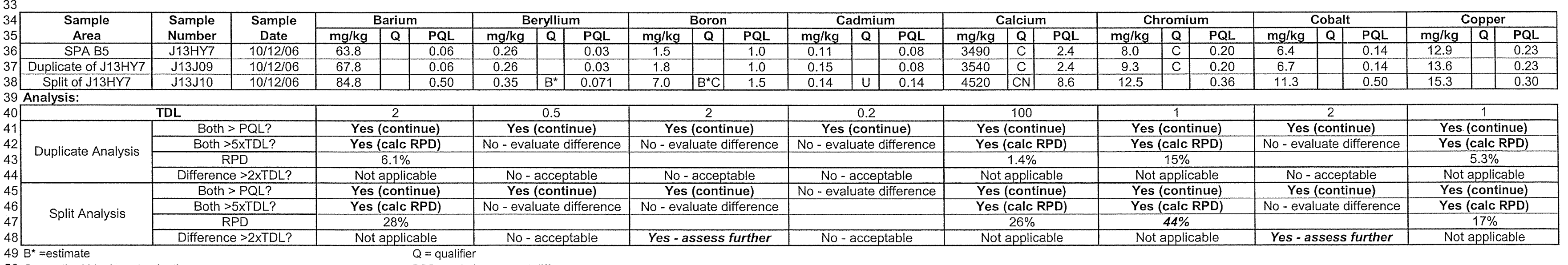

$50 \mathrm{C}=$ method blank contamination

$51 \mathrm{~J}=$ estimate
$52 \mathrm{MDA}=$ minim

M MDA = mimum detectable activity

$N=$ spiked analyte recovery outside control limits

$R P D=$ relative percent difference

$S P A=$ staging pile area
$T D L=$ target detection lim

$\mathrm{U}=$ undetected 
Washington Closure Hanford

Originator J. M. Capron 9 me

Subject $\frac{100-B / C \text { Field Remediation }}{118-B-1 \text { Burial Ground Cleanup Verification } 95 \% \text { UCL Calculations }}$
CALCULATION SHEET

Date $\frac{10 / 03 / 07}{14655}$
Calc. No. 0100B-CA-V029

Rev. No. $\frac{1}{\text { Date }} \frac{10 / 04 / 07}{610 f 61}$

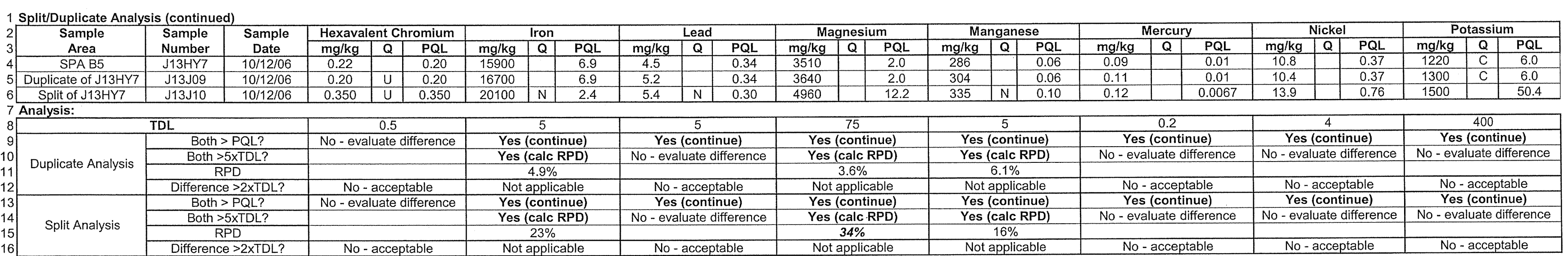

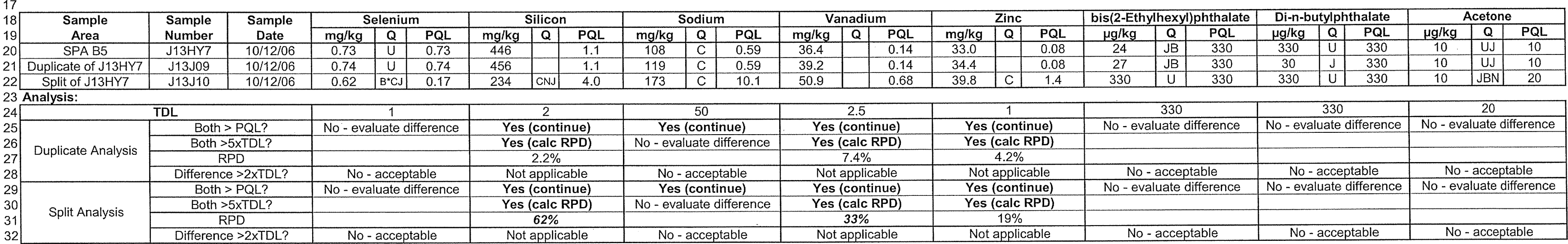

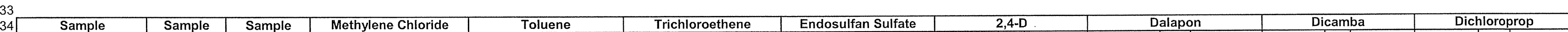

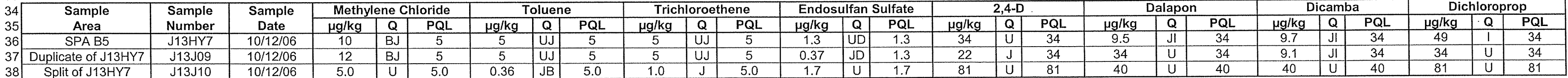

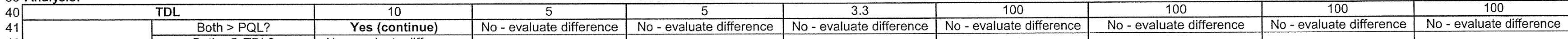

42 Duplicate Analysis

46 Split Analysis $48 \mathrm{~B}=$ method blan $50 \mathrm{~B}^{*}=$ estimate

$51 \mathrm{C}=$ method

$52 \mathrm{D}=$ diluted
$53 \mathrm{I}=$ interfere

$54 \mathrm{~J}=$ estimate

$54 \mathrm{~J}=$ estimate
$55 \mathrm{~N}=$ spiked analyte recovery outside control limits

\begin{tabular}{|c|c|c|c|c|c|c|c|c|}
\hline Both >5XTDL? & No - evaluate difference & & & & & & & \\
\hline & & & & & & & & \\
\hline Difference >2XTDL? & No - acceptable & No - acceptable & No - acceptable & No - acceptable & No - acceptable & $\begin{array}{l}\text { No - acceptable } \\
\end{array}$ & & $\frac{\text { No - - acceptable }}{\text { No - evaluate differe }}$ \\
\hline $\begin{array}{r}\text { Both > PQL? } \\
\text { Both > 5XTDL? } \\
\end{array}$ & No-evaluate difference & No - evaluate difference & No - evaluate difference & No-evaluate difference & No - evaluate difference & No - evaluate difference & No - evaluate difference & \\
\hline $\begin{aligned} R P D \\
\text { Difference }\end{aligned}$ & No - acceptable & No - acceptable & No- - acceptable & No - acceptable & No - acceptable & No - acceptable & No - acceptable & No - acceptable \\
\hline
\end{tabular}

Difference $>2 \times T D L ?$
mination (organic constituents)

Contamination (inorganic constituents)
$P Q L=$ practical quantitation limit
$Q=$ qualifier

$\mathrm{RPD}=$ relative percent difference

$\mathrm{SPA}=$ staging pile area
$\mathrm{TDL}=$ target detection limi

$U=$ undetected 
Attachment 1. 118-B-1 Verification Sampling Results.

\begin{tabular}{|c|c|c|c|c|c|c|c|c|c|c|c|c|c|c|c|c|c|}
\hline \multirow{2}{*}{ Sample Location } & \multirow{2}{*}{$\begin{array}{l}\text { Sample } \\
\text { Number }\end{array}$} & \multirow{2}{*}{$\begin{array}{c}\text { Sample } \\
\text { Date }\end{array}$} & \multicolumn{3}{|c|}{ Americium-241 } & \multicolumn{3}{|c|}{ Americium-241 GEA } & \multicolumn{3}{|c|}{ Carbon-14 } & \multicolumn{3}{|c|}{ Cesium-137 } & \multicolumn{3}{|c|}{ Cobalt-60 } \\
\hline & & & $\mathrm{pCi} / \mathrm{g}$ & $Q$ & MDA & $\mathrm{pCi} / \mathrm{g}$ & $\mathbf{Q}$ & MDA & $\mathrm{pCi} / \mathrm{g}$ & $Q$ & MDA & $\mathrm{pCi} / \mathrm{g}$ & $\mathbf{Q}$ & MDA & $\mathrm{pCi} / \mathrm{g}$ & $Q$ & MDA \\
\hline Area $1 \mathrm{~A} 1$ & $\mathrm{~J} 12 \times 44$ & $7 / 19 / 06$ & 0.080 & $\vec{U}$ & 0.61 & 0.23 & $\mathrm{U}$ & 0.23 & -1.41 & $\mathrm{U}$ & 3.1 & 0.308 & & 0.065 & 0.066 & $\mathrm{U}$ & 0.066 \\
\hline Duplicate of J12X44 & $\mathrm{J} 12 \times 48$ & $7 / 19 / 06$ & 0 & $\mathrm{U}$ & 0.65 & 0.33 & $\mathrm{U}$ & 0.33 & -0.925 & $\mathrm{U}$ & 2.7 & 0.169 & & 0.041 & 0.037 & $\mathrm{U}$ & 0.037 \\
\hline Split of J12X 44 & $\mathrm{~J} 12 \times 49$ & $7 / 19 / 06$ & 0.0285 & $\mathrm{U}$ & 0.0448 & & & & 0.230 & $\mathrm{U}$ & 0.807 & 0.133 & & 0.0325 & 0.0382 & $\mathrm{U}$ & 0.0385 \\
\hline Area $1 \mathrm{~A} 2$ & $\mathrm{~J} 12 \times 45$ & $7 / 19 / 06$ & -0.151 & $\mathrm{U}$ & 0.58 & 0.024 & $\mathrm{U}$ & 0.024 & 3.44 & & 2.7 & 0.020 & $\mathrm{U}$ & 0.020 & 0.022 & $\mathrm{U}$ & 0.022 \\
\hline Area $1 \mathrm{~A} 3$ & $\mathrm{~J} 12 \mathrm{X} 46$ & $7 / 20 / 06$ & 0.254 & $\mathrm{U}$ & 0.65 & 0.35 & $\mathrm{U}$ & 0.35 & -0.611 & $\mathrm{U}$ & 3.0 & 0.166 & & 0.041 & 0.049 & & 0.033 \\
\hline Area $1 \mathrm{~A} 4$ & $\mathrm{~J} 12 \times 47$ & $7 / 20 / 06$ & 0 & $\mathrm{U}$ & 0.60 & 0.11 & $\mathrm{U}$ & 0.11 & -1.43 & $\mathrm{U}$ & 3.7 & 0.021 & $U$ & 0.021 & 0.022 & $\mathrm{U}$ & 0.022 \\
\hline Area $2 \mathrm{Al}$ & J12X50 & $7 / 20 / 06$ & -0.046 & $\bar{U}$ & 0.35 & 0.36 & $\mathrm{U}$ & 0.36 & -0.994 & $\mathrm{U}$ & 3.2 & 0.223 & & 0.033 & 0.041 & $\mathrm{U}$ & 0.041 \\
\hline Area 2 A2 & J12X51 & $7 / 20 / 06$ & 0 & $\mathrm{U}^{\circ}$ & 0.27 & 0.086 & $U$ & 0.086 & -0.641 & $U$ & 3.2 & 0.099 & $\mathrm{U}$ & 0.099 & 0.12 & $\mathrm{U}$ & 0.12 \\
\hline Area $2 \mathrm{~A} 3$ & $\mathrm{~J} 12 \times 52$ & $7 / 20 / 06$ & 0.042 & $\mathrm{U}$ & 0.32 & 0.37 & $\mathrm{U}$ & 0.37 & -1.07 & $\mathrm{U}$ & 3.4 & 0.040 & $U$ & 0.040 & 0.042 & $\mathrm{U}$ & 0.042 \\
\hline Area $2 \mathrm{~A} 4$ & $\mathrm{J12X53}$ & $7 / 20 / 06$ & -0.040 & $\mathrm{U}$ & 0.38 & 0.14 & $\mathrm{U}$ & 0.14 & -1.01 & $U$ & 3.0 & 0.056 & & 0.028 & 0.025 & $\mathrm{U}$ & 0.025 \\
\hline Area $3 \mathrm{~A} 1$ & J12X54 & $7 / 20 / 06$ & 0 & $\mathrm{U}$ & 0.26 & 0.036 & $\mathrm{U}$ & 0.036 & -0.978 & $\mathrm{U}$ & 3.0 & 0.028 & $\mathrm{U}$ & 0.028 & 0.028 & $\mathrm{U}$ & 0.028 \\
\hline Area $3 \mathrm{~A} 2$ & $\mathrm{~J} 12 \times 55$ & $7 / 20 / 06$ & 0.046 & $U$ & 0.51 & 0.32 & $\mathrm{U}$ & 0.32 & -1.56 & $U$ & 3.0 & 0.111 & & 0.080 & 0.087 & $\mathrm{U}$ & 0.087 \\
\hline Area $3 \mathrm{~A} 3$ & J12X56 & $7 / 20 / 06$ & 0.111 & $\mathrm{U}$ & 0.35 & 0.095 & $\mathrm{U}$ & 0.095 & -1.06 & $\mathrm{U}$ & 3.0 & 1.51 & & 0.12 & 0.098 & $\mathrm{U}$ & 0.098 \\
\hline Duplicate of J12X56 & $\mathrm{J} 12 \times 58$ & $7 / 20 / 06$ & 0.170 & $\mathrm{U}$ & 0.38 & 0.094 & $\mathrm{U}$ & 0.094 & -0.855 & $\mathrm{U}$ & 3.0 & 0.776 & & 0.11 & 0.10 & $\mathrm{U}$ & 0.10 \\
\hline Split of J12X56 & $\mathrm{J} 12 \times 60$ & $7 / 20 / 06$ & & & & & & & -0.105 & $\mathrm{U}$ & 0.831 & 2.82 & & 0.0363 & -0.00405 & $U$ & 0.0401 \\
\hline Area $3 \mathrm{~A} 4$ & $\mathrm{~J} 12 \times 57$ & $7 / 20 / 06$ & 0.026 & $\mathrm{U}$ & 0.25 & 0.34 & $\mathrm{U}$ & 0.34 & -1.76 & $\mathrm{U}$ & 2.9 & 0.088 & $\mathrm{U}$ & 0.088 & 0.089 & $\mathrm{U}$ & 0.089 \\
\hline Area $4 \mathrm{Al}$ & J12X61 & $7 / 25 / 06$ & & & & 0.38 & $\mathrm{U}$ & 0.38 & -0.111 & $\mathrm{U}$ & 2.4 & 0.738 & & 0.048 & 0.084 & $\mathrm{U}$ & 0.084 \\
\hline Duplicate of J12X61 & $\mathrm{J} 12 \times 65$ & $7 / 25 / 06$ & & & & 0.20 & $\mathrm{U}$ & 0.20 & 0.241 & $\mathrm{U}$ & 2.4 & 0.260 & & 0.033 & 0.036 & $\mathrm{U}$ & 0.036 \\
\hline Split of J12X61 & $\mathrm{J} 12 \times 66$ & $7 / 25 / 06$ & & & & & & & 0.354 & $\mathrm{U}$ & 0.809 & 0.250 & & 0.0274 & 0.0220 & $\mathrm{U}$ & 0.0345 \\
\hline Area 4 A2 & $\mathrm{J} 12 \mathrm{X} 62$ & $7 / 25 / 06$ & & & & 0.030 & $\mathrm{U}$ & 0.030 & -0.860 & $\mathrm{U}$ & 2.5 & 0.022 & $\mathrm{U}$ & 0.022 & 0.028 & $\mathrm{U}$ & 0.028 \\
\hline Area $4 \mathrm{A3}$ & $\mathrm{J} 12 \times 63$ & $7 / 25 / 06$ & & & & 0.26 & $\mathrm{U}$ & 0.26 & -1.03 & $\mathrm{U}$ & 2.4 & 0.084 & & 0.047 & 0.043 & $\mathrm{U}$ & 0.043 \\
\hline Area 4 A4 & $\mathrm{J} 12 \times 64$ & $7 / 25 / 06$ & & & & 0.44 & $\mathrm{U}$ & 0.44 & 0.668 & $\mathrm{U}$ & 2.5 & 0.047 & $\mathrm{U}$ & 0.047 & 0.050 & $\mathrm{U}$ & 0.050 \\
\hline Area 5 A1 & J13V12 & $12 / 7 / 06$ & & & & 0.066 & $\mathrm{U}$ & 0.066 & 0.649 & $\mathrm{U}$ & 1.9 & 0.13 & $\mathrm{U}$ & 0.13 & 0.10 & $\mathrm{U}$ & 0.10 \\
\hline Area 5 A2 & J13V10 & $12 / 7 / 06$ & & & & 0.067 & $\mathrm{U}$ & 0.067 & 1.44 & $\mathrm{U}$ & 2.0 & 0.076 & $\mathrm{U}$ & 0.076 & 0.10 & $\mathrm{U}$ & 0.10 \\
\hline Area $5 \mathrm{~A} 3$ & $\mathrm{~J} 13 \mathrm{~V} 13$ & $12 / 7 / 06$ & & & & 0.47 & $\mathrm{U}$ & 0.47 & 1.11 & $U$ & 1.9 & 19.1 & & 0.14 & 0.082 & $U$ & 0.082 \\
\hline Area 5 A 3 resample & J14JH6 & $3 / 30 / 07$ & & & & 0.32 & $\mathrm{U}$ & 0.32 & & & & 3.60 & & 0.12 & 0.098 & $\mathrm{U}$ & 0.098 \\
\hline Area 5 A4 & J13V11 & $12 / 7 / 06$ & & & & 0.069 & $\mathrm{U}$ & 0.069 & 0.301 & $\mathrm{U}$ & 1.8 & 0.096 & $\mathrm{U}$ & 0.096 & 0.13 & $\mathrm{U}$ & 0.13 \\
\hline
\end{tabular}

Note: The following abbreviations apply to all Attachment 1 tables.

Note: Data qualified with $\mathrm{B}, \mathrm{B}^{*}, \mathrm{C}, \mathrm{D}, \mathrm{I}, \mathrm{N}$, or $\mathrm{J}$ are considered acceptable values.

$\mathrm{B}=$ method blank contamination $\quad \mathrm{OB}=$ overburden

MDA = minimum detectable activity

$\mathrm{BCL}=$ below cleanup levels

$\mathrm{C}=$ method blank contamination

$\mathrm{N}=$ spiked analyte recovery outside control limits (split samples)

$\mathrm{D}=$ diluted

$\mathrm{FS}=$ focused sample

$\mathrm{GEA}=$ gamma energy analysis

$\mathrm{PQL}=$ practical quantitation limit

$Q=$ qualifier

$\mathrm{R}=$ rejected

$I=$ interference on one analytical column $\quad U=$ undetected

Attachment Sheet No. 1 of 113

Originator J.M. Capron O/6/C Date $\frac{10 / 03 / 07}{10 / 4 / 2007}$

Checked T.M. Blakley $2 \mathrm{mB}$ Date $10 / 4 / 2007$

$J=$ estimate

Calc. No.

0100B-CA-V0299 Rev. No. 
Attachment 1. 118-B-1 Verification Sampling Results.

\begin{tabular}{|c|c|c|c|c|c|c|c|c|c|c|c|c|c|c|c|c|c|}
\hline \multirow{2}{*}{ Sample Location } & \multirow{2}{*}{$\begin{array}{c}\text { Sample } \\
\text { Number }\end{array}$} & \multirow{2}{*}{$\begin{array}{c}\text { Sample } \\
\text { Date }\end{array}$} & \multicolumn{3}{|c|}{ Americium-241 } & \multicolumn{3}{|c|}{ Americium-241 GEA } & \multicolumn{3}{|c|}{ Carbon-14 } & \multicolumn{3}{|c|}{ Cesium-137 } & \multicolumn{3}{|c|}{ Cobalt-60 } \\
\hline & & & $\mathrm{pCi} / \mathrm{g}$ & $\mathrm{Q}$ & $\mathrm{MDA}$ & $\mathrm{pCi} / \mathrm{g}$ & $Q$ & MDA & $\mathrm{pCi} / \mathrm{g}$ & $\mathrm{Q}$ & $\mathrm{MDA}$ & $\mathrm{pCi} / \mathrm{g}$ & $Q$ & MDA & $\mathrm{pCi} / \mathrm{g}$ & $Q$ & MDA \\
\hline Area $6 \mathrm{Al}$ & $\mathrm{J12XC2}$ & $7 / 25 / 06$ & -0.042 & $\mathrm{U}$ & 0.32 & 0.36 & U & 0.36 & -0.414 & U & 2.4 & 0.116 & & 0.043 & 0.063 & $\mathrm{U}$ & 0.063 \\
\hline Area $6 \mathrm{A2}$ & $.112 \times C 3$ & $7 / 25 / 06$ & 0.033 & $\mathrm{U}$ & 0.25 & 0.31 & $\mathrm{U}$ & 0.31 & -0.401 & $\mathrm{U}$ & 2.3 & 0.160 & & 0.067 & 0.077 & $\mathrm{U}$ & 0.077 \\
\hline Area $6 \mathrm{A3}$ & $.112 \times C 4$ & $7 / 25 / 06$ & 0 & $\mathrm{U}$ & 0.19 & 0.026 & U & 0.026 & 0.471 & U & 2.4 & 0.107 & & 0.026 & 0.025 & $\mathrm{U}$ & 0.025 \\
\hline Area $6 \mathrm{~A} 4$ & $112 \times C 5$ & $7 / 25 / 06$ & 0.107 & $\mathrm{U}$ & 0.20 & 0.031 & $U$ & 0.031 & 0.918 & $\mathrm{U}$ & 2.3 & 0.027 & $U$ & 0.027 & 0.028 & $\mathrm{U}$ & 0.028 \\
\hline Area $7 \mathrm{Al}$ & $.112 \times \mathrm{C} 6$ & $7 / 26 / 06$ & 0 & $\mathrm{U}$ & 0.22 & & & & 0.369 & $U$ & 2.5 & 0.578 & & 0.086 & 0.076 & U & 0.076 \\
\hline Area 7 A2 & $.112 \times C 7$ & $7 / 26 / 06$ & 0.028 & $\mathrm{U}$ & 0.21 & & & & 0.144 & U & 2.3 & 0.450 & & 0.046 & 0.037 & U & 0.037 \\
\hline Area $7 \mathrm{~A} 3$ & $.112 \times \mathrm{C} 8$ & $7 / 26 / 06$ & 0 & $\mathrm{U}$ & 0.23 & & & & -0.422 & $\mathrm{U}$ & 2.2 & 0.271 & & 0.091 & 0.082 & U & 0.082 \\
\hline Area $7 \mathrm{~A} 4$ & $.112 \times \mathrm{C} 9$ & $7 / 26 / 06$ & 0.058 & $\mathrm{U}$ & 0.22 & & & & -0.574 & $U$ & 2.2 & 0.297 & & 0.018 & 0.022 & $\mathrm{U}$ & 0.022 \\
\hline OB A1 & $.112 \mathrm{WKO}$ & $7 / 11 / 06$ & 0 & $\mathrm{U}$ & 0.24 & 0.097 & $\mathrm{U}$ & 0.097 & 2.77 & $\mathrm{U}$ & 4.4 & 0.076 & & 0.018 & 0.016 & $\mathrm{U}$ & 0.016 \\
\hline $\mathrm{OB} \mathrm{A2}$ & $112 \mathrm{WK} 1$ & $7 / 11 / 06$ & -0.035 & $\mathrm{U}$ & 0.27 & 0.41 & $\mathrm{U}$ & 0.41 & 1.86 & $\mathrm{U}$ & 4.0 & 0.039 & $\mathrm{U}$ & 0.045 & 0.045 & $\mathrm{U}$ & 0.045 \\
\hline $\mathrm{OB} \mathrm{A3}$ & $\mathrm{J12} \mathrm{WK} 2$ & $7 / 11 / 06$ & 0.111 & $U$ & 0.27 & 0.24 & $\mathrm{U}$ & 0.24 & 1.35 & $\mathrm{U}$ & 3.9 & 0.081 & & 0.031 & 0.038 & $U$ & 0.038 \\
\hline OB A4 & J12WK3 & $7 / 11 / 06$ & 0 & $\mathrm{U}$ & 0.29 & 0.30 & $\mathrm{U}$ & 0.30 & 2.69 & $\mathrm{U}$ & 3.9 & 0.118 & & 0.033 & 0.040 & $\mathrm{U}$ & 0.040 \\
\hline OB B5 & $\mathrm{J} 12 \mathrm{~W} / \mathrm{K} 4$ & $7 / 11 / 06$ & 0.103 & $\mathrm{U}$ & 0.49 & 0.17 & $\mathrm{U}$ & 0.17 & 0.951 & U & 3.6 & 0.027 & $U$ & 0.027 & 0.028 & $\mathrm{U}$ & 0.028 \\
\hline OB B6 & J12WK5 & $7 / 11 / 06$ & 0 & $\mathrm{U}$ & 0.29 & 0.26 & $\mathrm{U}$ & 0.26 & 2.38 & $\mathrm{U}$ & 3.9 & 0.223 & & 0.066 & 0.060 & $\mathrm{U}$ & 0.060 \\
\hline OB B7 & $J 12 \mathrm{~W} / \mathrm{K} 6$ & $7 / 11 / 06$ & -0.062 & $\mathrm{U}$ & 0.24 & 0.029 & $\mathrm{U}$ & 0.029 & 1.37 & $\mathrm{U}$ & 5.0 & 0.203 & & 0.022 & 0.028 & $\mathrm{U}$ & 0.028 \\
\hline OB BS & $112 \mathrm{WM} 7$ & $7 / 17 / 06$ & 0.022 & $U$ & 0.17 & 0.34 & $\mathrm{U}$ & 0.34 & 0.568 & $U$ & 3.0 & 0.288 & & 0.087 & 0.079 & $\mathrm{U}$ & 0.079 \\
\hline OB C9 & J12WM8 & $7 / 17 / 06$ & 0.088 & $\mathrm{U}$ & 0.17 & 0.097 & $\mathrm{U}$ & 0.097 & -0.308 & $\mathrm{U}$ & 3.1 & 0.169 & & 0.019 & 0.018 & $\mathrm{U}$ & 0.018 \\
\hline $\mathrm{OBC} 10$ & I12WM9 & $7 / 17 / 06$ & -0.050 & $\mathrm{U}$ & 0.19 & 0.035 & $\mathrm{U}$ & 0.035 & 1.30 & $\mathrm{U}$ & 2.6 & 0.082 & & 0.026 & 0.026 & $\mathrm{U}$ & 0.026 \\
\hline$O B C]$ & J12WNO & $7 / 17 / 06$ & 0.060 & $\mathrm{U}$ & 0.29 & 0.34 & $\mathrm{U}$ & 0.34 & 0.893 & $\mathrm{U}$ & 2.6 & 0.100 & & 0.038 & 0.036 & $\mathrm{U}$ & 0.036 \\
\hline$O B C 2$ & $112 \mathrm{WN1}$ & $7 / 17 / 06$ & 0.097 & $\mathrm{U}$ & 0.25 & 0.35 & $\mathrm{U}$ & 0.35 & 1.01 & $\mathrm{U}$ & 2.5 & 0.233 & & 0.080 & 0.094 & $\mathrm{U}$ & 0.094 \\
\hline OB D3 & J12WM5 & $7 / 12 / 06$ & -0.037 & $U$ & 0.18 & 0.32 & $\mathrm{U}$ & 0.32 & 0.763 & $\mathrm{U}$ & 4.2 & 0.218 & & 0.077 & 0.080 & $\mathrm{U}$ & 0.080 \\
\hline OB D4 & J12WM6 & $7 / 12 / 06$ & 0.041 & $U$ & 0.10 & 0.029 & $\mathrm{U}$ & 0.029 & 1.73 & $\mathrm{U}$ & 3.8 & 0.077 & $\mathrm{U}$ & 0.077 & 0.028 & U & 0.028 \\
\hline OB D5 & $.112 W M 3$ & $7 / 12 / 06$ & 0 & $\mathrm{U}$ & 0.18 & 0.15 & $\mathrm{U}$ & 0.15 & 2.68 & $\mathrm{U}$ & 4.4 & 0.654 & & 0.030 & 0.047 & $\mathrm{U}$ & 0.047 \\
\hline OB D6 & $112 \mathrm{WM} 4$ & $7 / 12 / 06$ & 0.019 & $\mathrm{U}$ & 0.14 & 0.24 & $\mathrm{U}$ & 0.24 & 1.21 & $\mathrm{U}$ & 3.7 & 0.060 & $\mathrm{U}$ & 0.060 & 0.035 & $\mathrm{U}$ & 0.035 \\
\hline$B C L A I$ & $\mathrm{J12} \mathrm{WN} 2$ & $7 / 20 / 06$ & -0.087 & $\mathrm{U}$ & 0.33 & 0.21 & $\mathrm{U}$ & 0.21 & -1.74 & U & 3.1 & 0.155 & & 0.036 & 0.054 & $\mathrm{U}$ & 0.054 \\
\hline $\mathrm{BCL} \mathrm{A2}$ & $.112 \mathrm{WM} 1$ & $7 / 12 / 06$ & 0.013 & $\mathrm{U}$ & 0.15 & 0.33 & $\mathrm{U}$ & 0.33 & 1.05 & $\mathrm{U}$ & 3.5 & 0.13 & $\mathrm{U}$ & 0.13 & 0.076 & $\mathrm{U}$ & 0.076 \\
\hline $\mathrm{BCLA} 3$ & $112 \mathrm{WM} 2$ & $7 / 12 / 06$ & -0.049 & $\mathrm{U}$ & 0.15 & 0.029 & $\mathrm{U}$ & 0.029 & 1.74 & $\mathrm{U}$ & 3.8 & 0.023 & $\mathrm{U}$ & 0.023 & 0.025 & $\mathrm{U}$ & 0.025 \\
\hline $\mathrm{BCL} \mathrm{A4}$ & $.112 \mathrm{~W} / \mathrm{M} 0$ & $7 / 12 / 06$ & 0.017 & U & 0.13 & 0.16 & $U$ & 0.16 & 1.14 & $\mathrm{U}$ & 3.7 & 0.133 & & 0.037 & 0.054 & $U$ & 0.054 \\
\hline$B C L B 5$ & I12WL9 & $7 / 12 / 06$ & -0.065 & $\mathrm{U}$ & 0.16 & 0.029 & $\mathrm{U}$ & 0.029 & 0.888 & $U$ & 3.8 & 0.065 & $\mathrm{U}$ & 0.065 & 0.028 & U & 0.028 \\
\hline
\end{tabular}

Attachment 1 Sheet No. 2 of 113

Checked

J. M. Capron Date $\quad 02 / 07 / 07$

Calc. No.

T. M. Blakley

Date

0100B-CA-V0299 
Attachment 1. 118-B-1 Verification Sampling Results.

\begin{tabular}{|c|c|c|c|c|c|c|c|c|c|c|c|c|c|c|c|c|c|}
\hline \multirow{2}{*}{ Sample Location } & \multirow{2}{*}{$\begin{array}{c}\text { Sample } \\
\text { Number } \\
\end{array}$} & \multirow{2}{*}{$\begin{array}{c}\text { Sample } \\
\text { Date }\end{array}$} & \multicolumn{3}{|c|}{ Americium-241 } & \multicolumn{3}{|c|}{ Americium-241 GEA } & \multicolumn{3}{|c|}{ Carbon-14 } & \multicolumn{3}{|c|}{ Cesium-137 } & \multicolumn{3}{|c|}{ Cobalt-60 } \\
\hline & & & $\mathrm{pCi} / \mathrm{g}$ & $Q$ & MDA & $\mathrm{pCi} / \mathrm{g}$ & $Q$ & MDA & $\mathrm{pCi} / \mathrm{g}$ & $Q$ & MDA & $\mathrm{pCi} / \mathrm{g}$ & Q & MDA & $\mathrm{pCi} / \mathrm{g}$ & $\mathbf{Q}$ & MDA \\
\hline $\mathrm{BCL} B 6$ & J12WL8 & $7 / 12 / 06$ & -0.016 & $\mathrm{U}$ & 0.16 & 0.34 & $\mathrm{U}$ & 0.34 & 1.15 & $\mathrm{U}$ & 3.7 & 0.372 & & 0.11 & 0.089 & $\mathrm{U}$ & 0.089 \\
\hline BCL B7 & J12WL7 & $7 / 12 / 06$ & -0.031 & $\mathrm{U}$ & 0.15 & 0.35 & $\mathrm{U}$ & 0.35 & 2.20 & $\mathrm{U}$ & 3.4 & 0.478 & & 0.076 & 0.20 & $\mathrm{U}$ & 0.20 \\
\hline BCL B8 & J12WL6 & $7 / 12 / 06$ & 0 & $\mathrm{U}$ & 0.14 & 0.27 & $\mathrm{U}$ & 0.27 & 1.02 & $\mathrm{U}$ & 3.7 & 0.069 & & 0.043 & 0.164 & & 0.039 \\
\hline Split of J12WL6 & J12WW0 & $7 / 12 / 06$ & 0.0834 & & 0.0408 & & & & 0.547 & $\mathrm{U}$ & 0.754 & 0.0905 & & 0.0218 & 0.0124 & $\mathrm{U}$ & 0.0238 \\
\hline BCL C9 & J12WK7 & $7 / 11 / 06$ & -0.008 & $\mathrm{U}$ & 0.068 & 0.16 & $\mathrm{U}$ & 0.16 & 0.267 & $\mathrm{U}$ & 4.4 & 0.163 & & 0.019 & 0.017 & $\mathrm{U}$ & 0.017 \\
\hline BCL C10 & $\mathrm{J12WK9}$ & $7 / 11 / 06$ & -0.013 & $\mathrm{U}$ & 0.066 & 0.14 & $\mathrm{U}$ & 0.14 & 0.948 & $\mathrm{U}$ & 4.0 & 0.045 & & 0.038 & 0.032 & $\mathrm{U}$ & 0.032 \\
\hline $\mathrm{BCL}$ C1 & J12WK8 & $7 / 11 / 06$ & 0.043 & $\mathrm{U}$ & 0.052 & 0.10 & $\mathrm{U}$ & 0.10 & 1.70 & $\mathrm{U}$ & 3.5 & 0.025 & & 0.016 & 0.015 & $\mathrm{U}$ & 0.015 \\
\hline $\mathrm{BCL}$ C2 & J12WL2 & $7 / 11 / 06$ & -0.009 & $\mathrm{U}$ & 0.048 & 0.14 & $\mathrm{U}$ & 0.14 & 0.247 & $\mathrm{U}$ & 4.0 & 0.025 & $\mathrm{U}$ & 0.025 & 0.025 & $\mathrm{U}$ & 0.025 \\
\hline BCL D3 & $\mathrm{J} 12 \mathrm{WL} 3$ & $7 / 11 / 06$ & 0.037 & $\mathrm{U}$ & 0.092 & 0.31 & $\mathrm{U}$ & 0.31 & 1.07 & $\mathrm{U}$ & 3.6 & 0.068 & $\mathrm{U}$ & 0.068 & 0.071 & $\mathrm{U}$ & 0.071 \\
\hline BCL D4 & $\mathrm{J} 12 \mathrm{WL} 4$ & $7 / 11 / 06$ & -0.012 & $\mathrm{U}$ & 0.066 & 0.43 & $\mathrm{U}$ & 0.43 & 1.78 & $\mathrm{U}$ & 4.0 & 0.045 & U & 0.045 & 0.044 & $\mathrm{U}$ & 0.044 \\
\hline Duplicate of J12WL4 & J12WL5 & $7 / 11 / 06$ & -0.048 & $\mathrm{U}$ & 0.085 & 0.14 & $\mathrm{U}$ & 0.14 & 0.942 & $\mathrm{U}$ & 4.2 & 0.023 & $\mathrm{U}$ & 0.023 & 0.025 & $\mathrm{U}$ & 0.025 \\
\hline BCL D5 & J12WL0 & $7 / 11 / 06$ & -0.042 & $\mathrm{U}$ & 0.086 & 0.058 & $\mathrm{U}$ & 0.058 & 2.46 & $\mathrm{U}$ & 3.9 & 0.010 & $\mathrm{U}$ & 0.010 & 0.010 & $\mathrm{u}$ & 0.010 \\
\hline BCL D6 & $\mathrm{J} 12 \mathrm{WL} 1$ & $7 / 11 / 06$ & 0.004 & $\mathrm{U}$ & 0.070 & 0.37 & $\mathrm{U}$ & 0.37 & 0.905 & $\mathrm{U}$ & 4.0 & 0.036 & $\mathrm{U}$ & 0.036 & 0.037 & $\mathrm{U}$ & 0.037 \\
\hline SPAA1 & J13HY3 & $10 / 12 / 06$ & 0.039 & $\mathrm{U}$ & 0.30 & 0.63 & U & 0.63 & 0.350 & $\mathrm{U}$ & 2.7 & 0.217 & & 0.059 & 0.056 & $\mathrm{U}$ & 0.056 \\
\hline SPA A2 & $\mathrm{J13HY4}$ & $10 / 12 / 06$ & 0 & $\mathrm{U}$ & 0.23 & 0.17 & $\mathrm{U}$ & 0.17 & 0.017 & $\mathrm{U}$ & 2.5 & 0.037 & & 0.034 & 0.030 & $\mathrm{U}$ & 0.030 \\
\hline SPA A3 & J13HY5 & $10 / 12 / 06$ & -0.055 & $\mathrm{U}$ & 0.21 & 0.62 & $\mathrm{U}$ & 0.62 & -0.453 & $\mathrm{U}$ & 2.8 & 0.158 & & 0.058 & 0.060 & $\mathrm{U}$ & 0.060 \\
\hline SPA A4 & J13HY6 & $10 / 12 / 06$ & 0.031 & $\mathrm{U}$ & 0.24 & 0.14 & $\mathrm{U}$ & 0.14 & -0.762 & $\mathrm{U}$ & 2.6 & 0.233 & & 0.026 & 0.025 & $\mathrm{U}$ & 0.025 \\
\hline SPA B5 & $\mathrm{J} 13 \mathrm{HY} 7$ & $10 / 12 / 06$ & 0.047 & $\mathrm{U}$ & 0.23 & 0.63 & $\mathrm{U}$ & 0.63 & -0.278 & $\mathrm{U}$ & 2.6 & 0.222 & & 0.057 & 0.064 & $\mathrm{U}$ & 0.064 \\
\hline Duplicate of J13HY7 & J13J09 & $10 / 12 / 06$ & 0.057 & U & 0.27 & 0.17 & $\mathrm{U}$ & 0.17 & -0.403 & $\mathrm{U}$ & 2.7 & 0.109 & & 0.032 & 0.033 & $\mathrm{U}$ & 0.033 \\
\hline Split of J13HY7 & $\mathrm{J} 13 \mathrm{~J} 10$ & $10 / 12 / 06$ & 0.311 & $\mathrm{~J}$ & 0.0465 & & & & -0.197 & $\mathrm{U}$ & 0.862 & 0.121 & & 0.0213 & 0.0117 & $\mathrm{U}$ & 0.0233 \\
\hline SPA B6 & J13HY8 & $10 / 17 / 06$ & -0.022 & $\mathrm{U}$ & 0.17 & 0.050 & $\mathrm{U}$ & 0.050 & -0.733 & $\mathrm{U}$ & 2.8 & 0.079 & & 0.026 & 0.037 & $\mathrm{U}$ & 0.037 \\
\hline SPA B7 & J13HY9 & $10 / 17 / 06$ & 0.154 & $\mathrm{U}$ & 0.20 & 0.039 & $\mathrm{U}$ & 0.039 & -0.077 & $\mathrm{U}$ & 2.8 & 0.043 & & 0.031 & 0.027 & $\mathrm{U}$ & 0.027 \\
\hline SPA B8 & $\mathrm{J} 13 \mathrm{~J} 00$ & $10 / 17 / 06$ & 0.098 & $\mathrm{U}$ & 0.19 & 0.76 & $U$ & 0.76 & -1.02 & $\mathrm{U}$ & 2.6 & 0.084 & $\mathrm{U}$ & 0.084 & 0.075 & $\mathrm{U}$ & 0.075 \\
\hline SPAC9 & $\mathrm{J} 13 . \mathrm{J} 01$ & $10 / 17 / 06$ & 0 & $\mathrm{U}$ & 0.20 & 0.20 & U & 0.20 & -1.15 & $\mathrm{U}$ & 2.6 & 0.223 & & 0.040 & 0.040 & $\mathrm{U}$ & 0.040 \\
\hline SPA C10 & $\mathrm{J} 13 \mathrm{~J} 02$ & $10 / 18 / 06$ & 0.103 & $\mathrm{U}$ & 0.16 & 0.70 & $\mathrm{U}$ & 0.70 & -1.02 & $\mathrm{U}$ & 2.0 & 0.112 & & 0.073 & 0.071 & $\mathrm{U}$ & 0.071 \\
\hline $\mathrm{SPACl}$ & $\mathrm{J} 13 \mathrm{~J} 03$ & $10 / 18 / 06$ & 0 & $\mathrm{U}$ & 0.16 & 0.047 & $\mathrm{U}$ & 0.047 & 0.221 & $\mathrm{U}$ & 2.2 & 0.066 & $\mathrm{U}$ & 0.066 & 0.042 & $\mathrm{U}$ & 0.042 \\
\hline SPAC2 & $\mathrm{J} 13 \mathrm{~J} 04$ & 10/18/06 & 0.126 & & 0.040 & 0.53 & $\mathrm{U}$ & 0.53 & -0.775 & $\mathrm{U}$ & 1.9 & 2.39 & & 0.060 & 0.073 & $\mathrm{U}$ & 0.073 \\
\hline SPA D3 & $\mathrm{J} 13 \mathrm{~J} 05$ & 10/18/06 & -0.011 & $\mathrm{U}$ & 0.045 & 0.038 & $\mathrm{U}$ & 0.038 & -0.038 & $\mathrm{U}$ & 2.1 & 0.057 & $\mathrm{U}$ & 0.057 & 0.030 & $\mathrm{U}$ & 0.030 \\
\hline SPA D4 & $\mathrm{J} 13 \mathrm{J06}$ & 10/19/06 & -0.004 & $\mathrm{U}$ & 0.057 & 0.016 & $\mathrm{U}$ & 0.016 & 0.645 & $\mathrm{U}$ & 2.1 & 0.662 & & 0.013 & 0.013 & U & 0.013 \\
\hline SPA D5 & 313307 & $10 / 23 / 06$ & 0.036 & $\mathrm{U}$ & 0.14 & 0.036 & $U$ & 0.036 & -0.943 & $\mathrm{U}$ & 2.9 & 0.184 & & 0.020 & 0.038 & & 0.029 \\
\hline SPA D6 & $\mathrm{J} 13308$ & $10 / 23 / 06$ & 0.046 & U & 0.18 & 0.57 & $\mathrm{U}$ & 0.57 & 11.3 & & 2.6 & 0.669 & & 0.056 & 0.060 & U & 0.060 \\
\hline FS-12 & $\mathrm{J14Y75}$ & $6 / 27 / 07$ & -0.060 & $\mathrm{U}$ & 0.228 & 0.169 & $\mathrm{U}$ & 0.169 & & & & 0.353 & & 0.075 & 0.155 & & 0.081 \\
\hline FS-13 & J14Y76 & $6 / 27 / 07$ & -0.033 & U & 0.250 & 0.074 & $\mathrm{U}$ & 0.074 & & & & 0.117 & $\mathrm{U}$ & 0.117 & 0.090 & $\mathrm{U}$ & 0.090 \\
\hline FS-14 & $\mathrm{J12XB3}$ & $7 / 19 / 06$ & & & & 0.077 & $\mathrm{U}$ & 0.077 & & & & 0.086 & $\mathrm{U}$ & 0.086 & 0.12 & $\mathrm{U}$ & 0.12 \\
\hline FS-17 & $\mathrm{J} 14 \mathrm{JJ} 3$ & $3 / 5 / 07$ & & & & 0.16 & $\mathrm{U}$ & 0.16 & & & & 0.080 & $\mathrm{U}$ & 0.080 & 0.051 & $\mathrm{U}$ & 0.051 \\
\hline FS-18 & J14L38 & $3 / 5 / 07$ & & & & 0.046 & U & 0.046 & & & & 0.057 & $\mathrm{U}$ & 0.057 & 0.072 & $\mathrm{U}$ & 0.072 \\
\hline FS-19 & J14L39 & $3 / 5 / 07$ & & & & 0.057 & $\mathrm{U}$ & 0.057 & & & & 0.069 & U & 0.069 & 0.070 & $\mathrm{U}$ & 0.070 \\
\hline
\end{tabular}

\begin{tabular}{|c|c|c|c|}
\hline Attachment & 1 & Sheet No. & 3 of 113 \\
\hline Originator & J.M. Capron & Date & $10 / 03 / 07$ \\
\hline Checked & T.M. Blakley & Date & \\
\hline Calc. No. & $0100 \mathrm{~B}-\mathrm{CA}-\mathrm{V} 0299$ & Rev. No. & 1 \\
\hline
\end{tabular}


Attachment 1. 118-B-1 Verification Sampling Results.

\begin{tabular}{|c|c|c|c|c|c|c|c|c|c|c|c|c|c|c|c|c|c|}
\hline \multirow{2}{*}{ Sample Location } & \multirow{2}{*}{$\begin{array}{l}\text { Sample } \\
\text { Number }\end{array}$} & \multirow{2}{*}{$\begin{array}{c}\text { Sample } \\
\text { Date }\end{array}$} & \multicolumn{3}{|c|}{ Europium-152 } & \multicolumn{3}{|c|}{ Europium-154 } & \multicolumn{3}{|c|}{ Europium-155 } & \multicolumn{3}{|c|}{ Nickel-63 } & \multicolumn{3}{|c|}{ Plutonium-238 } \\
\hline & & & $\mathrm{pCi} / \mathrm{g}$ & $\mathrm{Q}$ & MDA & $\mathrm{pCi} / \mathrm{g}$ & $Q$ & MDA & $\mathrm{pCi} / \mathrm{g}$ & $Q$ & MDA & $\mathrm{pCi} / \mathrm{g}$ & $\mathbf{Q}$ & MDA & $\mathrm{pCi} / \mathrm{g}$ & $Q$ & MDA \\
\hline Area $1 \mathrm{~A} 1$ & $\mathrm{~J} 12 \times 44$ & $7 / 19 / 06$ & 1.24 & & 0.13 & 0.19 & $\mathrm{U}$ & 0.19 & 0.14 & $\mathrm{U}$ & 0.14 & 2.28 & $\mathrm{U}$ & 2.6 & 0 & $\mathrm{U}$ & 1.5 \\
\hline Duplicate of J12X44 & $\mathrm{J} 12 \mathrm{X} 48$ & $7 / 19 / 06$ & 0.676 & & 0.092 & 0.12 & $\mathrm{U}$ & 0.12 & 0.21 & $\mathrm{U}$ & 0.21 & 1.15 & $\mathrm{U}$ & 2.7 & 0 & $\mathrm{U}$ & 0.96 \\
\hline Split of J12X44 & $\mathrm{J} 12 \mathrm{X} 49$ & $7 / 19 / 06$ & 1.15 & & 0.0877 & 0.0700 & $\mathrm{U}$ & 0.112 & 0.0352 & $\mathrm{U}$ & 0.110 & 4.41 & $\mathrm{U}$ & 5.46 & 0.00 & $\mathrm{U}$ & 0.0304 \\
\hline Area $1 \mathrm{~A} 2$ & $\mathrm{~J} 12 \times 45$ & $7 / 19 / 06$ & 0.074 & $\mathrm{U}$ & 0.074 & 0.14 & $\mathrm{U}$ & 0.14 & 0.045 & $\mathrm{U}$ & 0.045 & 0 & $\mathrm{U}$ & 2.6 & -0.085 & $\mathrm{U}$ & 0.65 \\
\hline Area $1 \mathrm{A3}$ & $\mathrm{J} 12 \mathrm{X} 46$ & $7 / 20 / 06$ & 0.323 & & 0.087 & 0.12 & $\mathrm{U}$ & 0.12 & 0.14 & $U$ & 0.14 & 0.177 & $\mathrm{U}$ & 3.9 & 0.019 & $\mathrm{U}$ & 0.060 \\
\hline Area $1 \mathrm{~A} 4$ & $\mathrm{~J} 12 \mathrm{X} 47$ & $7 / 20 / 06$ & 0.052 & $\mathrm{U}$ & 0.052 & 0.065 & $\mathrm{U}$ & 0.065 & 0.070 & $\mathrm{U}$ & 0.070 & -1.38 & $\mathrm{U}$ & 4.1 & 0.009 & $\mathrm{U}$ & 0.11 \\
\hline Area $2 \mathrm{A1}$ & $\mathrm{J} 12 \times 50$ & $7 / 20 / 06$ & 0.097 & $\mathrm{U}$ & 0.097 & 0.13 & $\mathrm{U}$ & 0.13 & 0.15 & $U$ & 0.15 & -1.55 & $\mathrm{U}$ & 3.7 & 0.201 & $\mathrm{U}$ & 0.77 \\
\hline Area $2 \mathrm{~A} 2$ & J12X51 & $7 / 20 / 06$ & 0.13 & $\mathrm{U}$ & 0.13 & 0.41 & $\mathrm{U}$ & 0.41 & 0.092 & $\mathrm{U}$ & 0.092 & -0.044 & $\mathrm{U}$ & 3.9 & 0 & $\mathrm{U}$ & 1.1 \\
\hline Area $2 \mathrm{~A} 3$ & $\mathrm{~J} 12 \times 52$ & $7 / 20 / 06$ & 0.097 & $\mathrm{U}$ & 0.097 & 0.13 & $\mathrm{U}$ & 0.13 & 0.14 & $\mathrm{U}$ & 0.14 & -2.48 & $\mathrm{U}$ & 4.4 & 0.117 & $\mathrm{U}$ & 0.90 \\
\hline Area $2 \mathrm{~A} 4$ & $\mathrm{~J} 12 \times 53$ & $7 / 20 / 06$ & 0.063 & $\mathrm{U}$ & 0.063 & 0.075 & $U$ & 0.075 & 0.085 & $\mathrm{U}$ & 0.085 & -1.47 & $\mathrm{U}$ & 3.9 & -0.071 & $\mathrm{U}$ & 0.54 \\
\hline Area $3 \mathrm{Al}$ & $\mathrm{J12X54}$ & $7 / 20 / 06$ & 0.060 & $\mathrm{U}$ & 0.060 & 0.096 & $\mathrm{U}$ & 0.096 & 0.055 & $\mathrm{U}$ & 0.055 & -1.33 & $\mathrm{U}$ & 4.3 & -0.119 & $\mathrm{U}$ & 0.91 \\
\hline Area $3 \mathrm{~A} 2$ & $\mathrm{~J} 12 \times 55$ & $7 / 20 / 06$ & 0.17 & $\mathrm{U}$ & 0.17 & 0.29 & $\mathrm{U}$ & 0.29 & 0.19 & $\mathrm{U}$ & 0.19 & 0.472 & $\mathrm{U}$ & 4.6 & 0.076 & $\mathrm{U}$ & 0.58 \\
\hline Area $3 \mathrm{~A} 3$ & $\mathrm{~J} 12 \times 56$ & $7 / 20 / 06$ & 0.18 & $\mathrm{U}$ & 0.18 & 0.37 & $\mathrm{U}$ & 0.37 & 0.10 & $U$ & 0.10 & 1.03 & $\mathrm{U}$ & 4.5 & -0.089 & $\mathrm{U}$ & 0.68 \\
\hline Duplicate of J12X56 & $\mathrm{J} 12 \times 58$ & $7 / 20 / 06$ & 0.16 & $\mathrm{U}$ & 0.16 & 0.32 & $\mathrm{U}$ & 0.32 & 0.098 & $\mathrm{U}$ & 0.098 & -0.828 & $\mathrm{U}$ & 4.3 & -0.054 & $U$ & 0.41 \\
\hline Split of J12X56 & $\mathrm{J} 12 \times 60$ & $7 / 20 / 06$ & 0.274 & $\mathrm{U}$ & 0.114 & -0.00265 & $\mathrm{U}$ & 0.118 & 0.0518 & $\mathrm{U}$ & 0.0879 & 6.61 & $\mathrm{~J}$ & 5.94 & & & \\
\hline Area $3 \mathrm{~A} 4$ & $\mathrm{~J} 12 \times 57$ & $7 / 20 / 06$ & 0.19 & $\mathrm{U}$ & 0.19 & 0.30 & $\mathrm{U}$ & 0.30 & 0.21 & $\mathrm{U}$ & 0.21 & 0.192 & $\mathrm{U}$ & 4.2 & 0.098 & $\mathrm{U}$ & 0.75 \\
\hline Area 4A1 & $\mathrm{J} 12 \times 61$ & $7 / 25 / 06$ & 0.11 & $\mathrm{U}$ & 0.11 & 0.13 & $\mathrm{U}$ & 0.13 & 0.17 & $\mathrm{U}$ & 0.17 & -0.037 & $\mathrm{U}$ & 3.3 & & & \\
\hline Duplicate of J12X61 & $\mathrm{J} 12 \times 65$ & $7 / 25 / 06$ & 0.068 & $\mathrm{U}$ & 0.068 & 0.10 & $\mathrm{U}$ & 0.10 & 0.092 & $\mathrm{U}$ & 0.092 & -0.356 & $\mathrm{U}$ & 3.5 & & & \\
\hline Split of J12X61 & $\mathrm{J} 12 \times 66$ & $7 / 25 / 06$ & -0.00874 & $\mathrm{U}$ & 0.0738 & -0.00678 & $\mathrm{U}$ & 0.0850 & 0.0317 & $U$ & 0.0846 & 1.36 & $\mathrm{U}$ & 5.97 & & & \\
\hline Area 4 A2 & $\mathrm{J} 12 \times 62$ & $7 / 25 / 06$ & 0.058 & $\mathrm{U}$ & 0.058 & 0.086 & $\mathrm{U}$ & 0.086 & 0.063 & $\mathrm{U}$ & 0.063 & -0.735 & $\mathrm{U}$ & 3.6 & & & \\
\hline Area 4 A3 & $\mathrm{J} 12 \times 63$ & $7 / 25 / 06$ & 0.083 & $\mathrm{U}$ & 0.083 & 0.14 & $\mathrm{U}$ & 0.14 & 0.12 & $\mathrm{U}$ & 0.12 & 0.724 & $\mathrm{U}$ & 3.3 & & & \\
\hline Area 4 A4 & $\mathrm{J} 12 \times 64$ & $7 / 25 / 06$ & 0.12 & $U$ & 0.12 & 0.16 & $\mathrm{U}$ & 0.16 & 0.17 & $\mathrm{U}$ & 0.17 & 0.463 & $\mathrm{U}$ & 3.4 & & & \\
\hline Area $5 \mathrm{Al}$ & J13V12 & $12 / 7 / 06$ & 0.12 & $\mathrm{U}$ & 0.12 & 0.35 & $\mathrm{U}$ & 0.35 & 0.082 & $\mathrm{U}$ & 0.082 & 2.89 & $\mathrm{U}$ & 3.9 & & & \\
\hline Area $5 \mathrm{~A} 2$ & J13V10 & $12 / 7 / 06$ & 0.13 & $\mathrm{U}$ & 0.13 & 0.33 & $\mathrm{U}$ & 0.33 & 0.080 & $\mathrm{U}$ & 0.080 & 1.50 & $\mathrm{U}$ & 2.5 & & & \\
\hline Area $5 \mathrm{~A} 3$ & $\mathrm{~J} 13 \mathrm{~V} 13$ & $12 / 7 / 06$ & 0.36 & $\mathrm{U}$ & 0.36 & 0.25 & $\mathrm{U}$ & 0.25 & 0.34 & $\mathrm{U}$ & 0.34 & 2.41 & $\mathrm{U}$ & 4.0 & & & \\
\hline Area 5 A3 resample & J14JH6 & $3 / 30 / 07$ & 0.27 & $\mathrm{U}$ & 0.27 & 0.31 & $\mathrm{U}$ & 0.31 & 0.25 & $\mathrm{U}$ & 0.25 & & & & & & \\
\hline Area $5 \mathrm{~A} 4$ & J13V11 & $12 / 7 / 06$ & 0.14 & $U$ & 0.14 & 0.39 & $\mathrm{U}$ & 0.39 & 0.087 & $\mathrm{U}$ & 0.087 & 0.740 & $U$ & 3.9 & & & \\
\hline Area $6 \mathrm{Al}$ & $\mathrm{J} 12 \mathrm{XC} 2$ & $7 / 25 / 06$ & 0.10 & $\mathrm{U}$ & 0.10 & 0.12 & $\mathrm{U}$ & 0.12 & 0.14 & $\mathrm{U}$ & 0.14 & -0.897 & $\mathrm{U}$ & 3.4 & -0.192 & $\mathrm{U}$ & 0.92 \\
\hline Area $6 \mathrm{~A} 2$ & $\mathrm{~J} 12 \mathrm{XC} 3$ & $7 / 25 / 06$ & 0.16 & $\mathrm{U}$ & 0.16 & 0.25 & $\mathrm{U}$ & 0.25 & 0.18 & $\mathrm{U}$ & 0.18 & -0.430 & $\mathrm{U}$ & 3.4 & -0.068 & $U$ & 0.66 \\
\hline Area $6 \mathrm{~A} 3$ & $\mathrm{~J} 12 \mathrm{XC4}$ & $7 / 25 / 06$ & 0.055 & $\mathrm{U}$ & 0.055 & 0.083 & $\mathrm{U}$ & 0.083 & 0.049 & $\mathrm{U}$ & 0.049 & 1.13 & $\mathrm{U}$ & 3.5 & 0 & $U$ & 0.62 \\
\hline Area $6 \mathrm{~A} 4$ & J12XC5 & $7 / 25 / 06$ & 0.064 & $\mathrm{U}$ & 0.064 & 0.10 & $U$ & 0.10 & 0.072 & $\mathrm{U}$ & 0.072 & 0.122 & $\mathrm{U}$ & 3.6 & -0.110 & $\mathrm{U}$ & 0.84 \\
\hline
\end{tabular}

\begin{tabular}{|c|c|c|c|}
\hline Attachment & 1 & Sheet No. & 4 of 113 \\
\hline Originator & J. M. Capron & Date & $10 / 03 / 07$ \\
\hline Checked & T.M. Blakley & Date & \\
\hline Calc. No. & $0100 \mathrm{~B}-\mathrm{CA}-\mathrm{V} 0299$ & Rev. No. & 1 \\
\hline
\end{tabular}


Attachment 1. 118-B-1 Verification Sampling Results.

\begin{tabular}{|c|c|c|c|c|c|c|c|c|c|c|c|c|c|c|c|c|c|}
\hline \multirow{2}{*}{ Sample Location } & \multirow{2}{*}{$\begin{array}{l}\text { Sample } \\
\text { Number }\end{array}$} & \multirow{2}{*}{$\begin{array}{c}\text { Sample } \\
\text { Date }\end{array}$} & \multicolumn{3}{|c|}{ Europium-152 } & \multicolumn{3}{|c|}{ Europium-154 } & \multicolumn{3}{|c|}{ Europium-155 } & \multicolumn{3}{|c|}{ Nickel-63 } & \multicolumn{3}{|c|}{ Plutonium-238 } \\
\hline & & & $\mathrm{pCi} / \mathrm{g}$ & $\mathrm{Q}$ & MDA & $\mathrm{pCi} / \mathrm{g}$ & $Q$ & $\mathrm{MDA}$ & $\mathrm{pCi} / \mathrm{g}$ & $Q$ & MDA & $\mathrm{pCi} / \mathrm{g}$ & $\mathrm{Q}$ & MDA & $\mathrm{pCi} / \mathrm{g}$ & $\mathrm{Q}$ & $\mathrm{MDA}$ \\
\hline Area $7 \mathrm{Al}$ & $.112 \times \mathrm{C} 6$ & $7 / 26 / 06$ & 0.17 & $\mathrm{U}$ & 0.17 & 0.23 & $\mathrm{U}$ & 0.23 & 0.17 & $\mathrm{U}$ & 0.17 & -1.37 & U & 3.7 & -0.104 & $\mathrm{U}$ & 1.0 \\
\hline Area $7 \mathrm{A2}$ & $.112 \times C 7$ & $7 / 26 / 06$ & 0.12 & $\mathrm{U}$ & 0.12 & 0.12 & $\mathrm{U}$ & 0.12 & 0.15 & $\mathrm{U}$ & 0.15 & -0.140 & $\mathrm{U}$ & 3.8 & 0.267 & $\mathrm{U}$ & 0.85 \\
\hline Area $7 \mathrm{A3}$ & $112 \times C 8$ & $7 / 26 / 06$ & 0.17 & $\mathrm{U}$ & 0.17 & 0.24 & U & 0.24 & 0.19 & $\mathrm{U}$ & 0.19 & 0.516 & $\mathrm{U}$ & 3.5 & -0.071 & $\mathrm{U}$ & 0.54 \\
\hline Area 7 A4 & $.112 \times C 9$ & $7 / 26 / 06$ & 0.104 & & 0.052 & 0.067 & $\mathrm{U}$ & 0.067 & 0.072 & $\mathrm{U}$ & 0.072 & 2.41 & $\mathrm{U}$ & 3.7 & -0.129 & $\mathrm{U}$ & 0.98 \\
\hline OBAl & $112 \mathrm{WK} 0$ & $7 / 11 / 06$ & 0.043 & $\mathrm{U}$ & 0.043 & 0.052 & $\mathrm{U}$ & 0.052 & 0.084 & $\mathrm{U}$ & 0.084 & -2.07 & $\mathrm{U}$ & 3.8 & 0 & U & 0.91 \\
\hline OB A2 & J12WKI & $7 / 11 / 06$ & 0.11 & $\mathrm{U}$ & 0.11 & 0.15 & $\mathrm{U}$ & 0.15 & 0.20 & $U$ & 0.20 & -1.18 & U & 3.8 & 0.101 & $\mathrm{U}$ & 0.77 \\
\hline $\mathrm{OBA3}$ & J12WK2 & $7 / 11 / 06$ & 0.085 & $\mathrm{U}$ & 0.085 & 0.14 & $\mathrm{U}$ & 0.14 & 0.12 & $\mathrm{U}$ & 0.12 & 1.39 & $U$ & 4.2 & 0.138 & $U$ & 1.1 \\
\hline $\mathrm{OB} \mathrm{A4}$ & $112 \mathrm{WK} 3$ & $7 / 11 / 06$ & 0.087 & $U$ & 0.087 & 0.11 & $\mathrm{U}$ & 0.11 & 0.12 & $\mathrm{U}$ & 0.12 & -0.205 & $\mathrm{U}$ & 3.7 & 0 & $\mathrm{U}$ & 0.66 \\
\hline OB B5 & $\mathrm{J12WK} 4$ & $7 / 11 / 06$ & 0.056 & $U$ & 0.056 & 0.087 & $\mathrm{U}$ & 0.087 & 0.084 & $U$ & 0.084 & -0.160 & $U$ & 3.6 & 0 & $\mathrm{U}$ & 0.65 \\
\hline $\mathrm{OB} \mathrm{B6}$ & J12WKS & $7 / 11 / 06$ & 0.13 & $\mathrm{U}$ & 0.13 & 0.20 & $\mathrm{U}$ & 0.20 & 0.14 & $\mathrm{U}$ & 0.14 & -0.628 & $U$ & 3.8 & -0.103 & $\mathrm{U}$ & 0.79 \\
\hline OB B7 & $112 \mathrm{WK} 6$ & $7 / 11 / 06$ & 0.060 & $U$ & 0.060 & 0.085 & $\mathrm{U}$ & 0.085 & 0.059 & $\mathrm{U}$ & 0.059 & 1.10 & $U$ & 3.8 & 0.062 & $\mathrm{U}$ & 0.68 \\
\hline OB.B8 & 112 WM7 & $7 / 17 / 06$ & 0.19 & $\mathrm{U}$ & 0.19 & 0.29 & $U$ & 0.29 & 0.22 & $\mathrm{U}$ & 0.22 & 1.07 & U & 2.6 & 0.012 & $\mathrm{U}$ & 0.16 \\
\hline OBC9 & $112 \mathrm{WMS}$ & $7 / 17 / 06$ & 0.063 & $\mathrm{U}$ & 0.063 & 0.055 & U & 0.055 & 0.059 & $U$ & 0.059 & 0.384 & U & 2.7 & 0 & $\mathrm{U}$ & 0.24 \\
\hline OB C 10 & 112WM9 & $7 / 17 / 06$ & 0.065 & U & 0.065 & 0.089 & $\mathrm{U}$ & 0.089 & 0.053 & $\mathrm{U}$ & 0.053 & 0.127 & $\mathrm{U}$ & 2.6 & -0.067 & $\mathrm{U}$ & 0.52 \\
\hline $\mathrm{OBCl}$ & J12WNO & $7 / 17 / 06$ & 0.088 & $\mathrm{U}$ & 0.088 & 0.13 & $\mathrm{U}$ & 0.13 & 0.14 & $U$ & 0.14 & 0.297 & $\mathrm{U}$ & 2.6 & -0.028 & $U$ & 0.20 \\
\hline $\mathrm{OBC}$ & $.12 \mathrm{WN}$ & $7 / 17 / 06$ & 0.20 & $\mathrm{U}$ & 0.20 & 0.31 & $\mathrm{U}$ & 0.31 & 0.23 & $\mathrm{U}$ & 0.23 & 0.373 & $U$ & 2.6 & 0.006 & $\mathrm{U}$ & 0.087 \\
\hline OB D3 & J12WM5 & $7 / 12 / 06$ & 0.17 & $\mathrm{U}$ & 0.17 & 0.25 & $\mathrm{U}$ & 0.25 & 0.18 & $\mathrm{U}$ & 0.18 & -0.491 & $\mathrm{U}$ & 3.7 & 0 & $U$ & 0.61 \\
\hline $\mathrm{OB} \mathrm{D4}$ & $112 \mathrm{~W} M 6$ & $7 / 12 / 06$ & 0.060 & $\mathrm{U}$ & 0.060 & 0.089 & $\mathrm{U}$ & 0.089 & 0.080 & $\mathrm{U}$ & 0.080 & -1.67 & $\mathrm{U}$ & 3.9 & 0.047 & $\mathrm{U}$ & 0.17 \\
\hline OB D5 & I12WM3 & $7 / 12 / 06$ & 0.165 & & 0.067 & 0.078 & $U$ & 0.078 & 0.088 & $\mathrm{U}$ & 0.088 & -0.394 & $\mathrm{U}$ & 3.9 & 0 & $U$ & 1.2 \\
\hline OB D6 & $.112 \mathrm{WM} 4$ & $7 / 12 / 06$ & 0.071 & U & 0.071 & 0.11 & $\mathrm{U}$ & 0.11 & 0.10 & $\mathrm{U}$ & 0.10 & -0.703 & $\mathrm{U}$ & 3.7 & 0 & $U$ & 0.64 \\
\hline $\mathrm{BCL} A \mathrm{l}$ & $.112 W N 2$ & $7 / 20 / 06$ & 0.079 & $\mathrm{U}$ & 0.079 & 0.10 & $\mathrm{U}$ & 0.10 & 0.098 & $\mathrm{U}$ & 0.098 & 0.285 & $\mathrm{U}$ & 4.2 & 0.006 & $\mathrm{U}$ & 0.11 \\
\hline $\mathrm{BCL} \mathrm{A2}$ & $.112 \mathrm{~W} M \mathrm{Ml}$ & $7 / 12 / 06$ & 0.17 & $U$ & 0.17 & 0.25 & $U$ & 0.25 & 0.19 & $\mathrm{U}$ & 0.19 & -1.60 & $\mathrm{U}$ & 3.8 & -0.067 & $\mathrm{U}$ & 0.65 \\
\hline $\mathrm{BCL} A 3$ & $J 12 W M 2$ & $7 / 12 / 06$ & 0.060 & $\mathrm{U}$ & 0.060 & 0.084 & $\mathrm{U}$ & 0.084 & 0.058 & $\mathrm{U}$ & 0.058 & 0.258 & $U$ & 3.9 & -0.120 & $\mathrm{U}$ & 1.3 \\
\hline $\mathrm{BCL} \mathrm{A4}$ & $112 \mathrm{WMO}$ & $7 / 12 / 06$ & 0.080 & $\mathrm{U}$ & 0.080 & 0.12 & $U$ & 0.12 & 0.080 & $U$ & 0.080 & -1.74 & $U$ & 3.8 & -0.086 & $U$ & 0.41 \\
\hline BCL B5 & J12WL9 & $7 / 12 / 06$ & 0.063 & $\mathrm{U}$ & 0.063 & 0.095 & $U$ & 0.095 & 0.060 & $\mathrm{U}$ & 0.060 & -0.677 & $U$ & 3.6 & 0.112 & $\mathrm{U}$ & 0.43 \\
\hline $\mathrm{BCL} \mathrm{B6}$ & 112 WL.8 & $7 / 12 / 06$ & 0.19 & $\mathrm{U}$ & 0.19 & 0.26 & $\mathrm{U}$ & 0.26 & 0.20 & $\mathrm{U}$ & 0.20 & -1.19 & $U$ & 3.7 & -0.160 & $\mathrm{U}$ & 0.77 \\
\hline BCL B7 & $112 W L 7$ & $7 / 12 / 06$ & 0.19 & $\mathrm{U}$ & 0.19 & 0.27 & $\mathrm{U}$ & 0.27 & 0.20 & U & 0.20 & -0.342 & $\mathrm{U}$ & 3.8 & 0 & $\mathrm{U}$ & 0.43 \\
\hline$B C L B S$ & $112 \mathrm{WLG}$ & $7 / 12 / 06$ & 0.143 & & 0.090 & 0.13 & U & 0.13 & 0.12 & $\mathrm{U}$ & 0.12 & 0.840 & U & 4.0 & 0.061 & $\mathrm{U}$ & 0.47 \\
\hline & 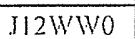 & $7 / 12106$ & 0.153 & $U$ & 0.0623 & -000325 & $\mathrm{U}$ & 0.0675 & 0.0281 & $U$ & T & (2) & $U$ & +2 & .00727 & - & - \\
\hline
\end{tabular}

$B C L B S$

Attachment

Originator

Checked

Calc. No.
$\frac{1}{J}$

T.M. Blakley

$0100 \mathrm{~B}-\mathrm{CA}-\mathrm{V} 0299$
Sheet No 5 of 113

Date $02 / 07 / 07$ Date Rev. No. 
Attachment 1. 118-B-1 Verification Sampling Results.

\begin{tabular}{|c|c|c|c|c|c|c|c|c|c|c|c|c|c|c|c|c|c|}
\hline \multirow{2}{*}{ Sample Location } & \multirow{2}{*}{$\begin{array}{l}\text { Sample } \\
\text { Number }\end{array}$} & \multirow{2}{*}{$\begin{array}{c}\text { Sample } \\
\text { Date }\end{array}$} & \multicolumn{3}{|c|}{ Europium-152 } & \multicolumn{3}{|c|}{ Europium-154 } & \multicolumn{3}{|c|}{ Europium-155 } & \multicolumn{3}{|c|}{ Nickel-63 } & \multicolumn{3}{|c|}{ Plutonium-238 } \\
\hline & & & $\mathrm{pCi} / \mathrm{g}$ & $Q$ & MDA & $\mathrm{pCi} / \mathrm{g}$ & $\mathbf{Q}$ & MDA & $\mathrm{pCi} / \mathrm{g}$ & $Q$ & MDA & $\mathrm{pCi} / \mathrm{g}$ & $\mathbf{Q}$ & MDA & $\mathrm{pCi} / \mathrm{g}$ & $Q$ & MDA \\
\hline BCL C9 & J12WK7 & $7 / 11 / 06$ & 0.065 & $U$ & 0.065 & 0.057 & $\mathrm{U}$ & 0.057 & 0.10 & $U$ & 0.10 & -0.288 & $U$ & 3.7 & -0.040 & $\mathrm{U}$ & 0.23 \\
\hline $\mathrm{BCL}$ C10 & J12WK9 & $7 / 11 / 06$ & 0.071 & U & 0.071 & 0.11 & $\mathrm{U}$ & 0.11 & 0.079 & $\mathrm{U}$ & 0.079 & 0.158 & $\mathrm{U}$ & 3.6 & -0.104 & $\mathrm{U}$ & 0.80 \\
\hline $\mathrm{BCL} \mathrm{Cl}$ & J12WK8 & $7 / 11 / 06$ & 0.035 & $\mathrm{U}$ & 0.035 & 0.051 & $\mathrm{U}$ & 0.051 & 0.050 & $U$ & 0.050 & 1.28 & $\mathrm{U}$ & 3.9 & 0.161 & $\mathrm{U}$ & 1.2 \\
\hline $\mathrm{BCL} C 2$ & J12WL2 & $7 / 11 / 06$ & 0.059 & $\mathrm{U}$ & 0.059 & 0.078 & $\mathrm{U}$ & 0.078 & 0.085 & $U$ & 0.085 & -1.12 & $\mathrm{U}$ & 4.8 & 0 & $\mathrm{U}$ & 0.54 \\
\hline BCL D3 & J12WL3 & $7 / 11 / 06$ & 0.16 & $\mathrm{U}$ & 0.16 & 0.24 & $\mathrm{U}$ & 0.24 & 0.17 & $U$ & 0.17 & -0.529 & $\mathrm{U}$ & 3.7 & 0 & $\mathrm{U}$ & 0.89 \\
\hline BCL D4 & J12WL4 & $7 / 11 / 06$ & 0.12 & $\mathrm{U}$ & 0.12 & 0.14 & $\mathrm{U}$ & 0.14 & 0.17 & $\mathrm{U}$ & 0.17 & -1.57 & $\mathrm{U}$ & 3.5 & -0.075 & $\mathrm{U}$ & 0.57 \\
\hline Duplicate of J12WL4 & J12WL5 & $7 / 11 / 06$ & 0.059 & $\mathrm{U}$ & 0.059 & 0.078 & $\mathrm{U}$ & 0.078 & 0.084 & $\mathrm{U}$ & 0.084 & -1.87 & $\mathrm{U}$ & 3.6 & 0 & $\mathrm{U}$ & 0.94 \\
\hline BCL D5 & J12WLO & $7 / 11 / 06$ & 0.025 & $\mathrm{U}$ & 0.025 & 0.032 & $\mathrm{U}$ & 0.032 & 0.042 & $U$ & 0.042 & -0.466 & $\mathrm{U}$ & 3.8 & 0 & $\mathrm{U}$ & 0.76 \\
\hline BCL D6 & J12WL1 & $7 / 11 / 06$ & 0.098 & $U$ & 0.098 & 0.13 & $\mathrm{U}$ & 0.13 & 0.14 & $\mathrm{U}$ & 0.14 & -2.02 & $\mathrm{U}$ & 3.6 & -0.068 & $\mathrm{U}$ & 0.52 \\
\hline SPA Al & J13HY3 & $10 / 12 / 06$ & 0.16 & $\mathrm{U}$ & 0.16 & 0.20 & $\mathrm{U}$ & 0.20 & 0.21 & $\mathrm{U}$ & 0.21 & -0.455 & $\mathrm{U}$ & 3.3 & 0 & $\mathrm{U}$ & 0.55 \\
\hline SPA A2 & J13HY4 & $10 / 12 / 06$ & 0.075 & $\mathrm{U}$ & 0.075 & 0.097 & $\mathrm{U}$ & 0.097 & 0.10 & $\mathrm{U}$ & 0.10 & -0.038 & $\mathrm{U}$ & 3.4 & 0.055 & $\mathrm{U}$ & 0.42 \\
\hline SPA A3 & J13HY5 & $10 / 12 / 06$ & 0.17 & $\mathrm{U}$ & 0.17 & 0.20 & $\mathrm{U}$ & 0.20 & 0.21 & $\mathrm{U}$ & 0.21 & 0.392 & $\mathrm{U}$ & 3.5 & 0 & $\mathrm{U}$ & 0.42 \\
\hline SPA A4 & J13HY6 & $10 / 12 / 06$ & 0.062 & $\mathrm{U}$ & 0.062 & 0.074 & $\mathrm{U}$ & 0.074 & 0.084 & $\mathrm{U}$ & 0.084 & -0.597 & $\mathrm{U}$ & 3.5 & -0.046 & $U$ & 0.44 \\
\hline SPA B5 & J13HY7 & $10 / 12 / 06$ & 0.17 & $\mathrm{U}$ & 0.17 & 0.21 & $\mathrm{U}$ & 0.21 & 0.21 & $\mathrm{U}$ & 0.21 & 0.952 & $\mathrm{U}$ & 3.5 & 0.087 & $\mathrm{U}$ & 0.42 \\
\hline Duplicate of J13HY7 & J13509 & $10 / 12 / 06$ & 0.073 & $\mathrm{U}$ & 0.073 & 0.097 & $\mathrm{U}$ & 0.097 & 0.10 & $U$ & 0.10 & 0.723 & $\mathrm{U}$ & 3.5 & 0 & $\mathrm{U}$ & 0.34 \\
\hline Split of J13HY7 & $\mathrm{J} 13 \mathrm{~J} 10$ & $10 / 12 / 06$ & 0.000190 & $\mathrm{U}$ & 0.0461 & 0.0112 & $\mathrm{U}$ & 0.0700 & 0.0331 & $\mathrm{U}$ & 0.0579 & 5.13 & & 5.07 & -0.00376 & $\mathrm{U}$ & 0.0531 \\
\hline SPA B6 & J13HY8 & $10 / 17 / 06$ & 0.081 & $\mathrm{U}$ & 0.081 & 0.11 & $\mathrm{U}$ & 0.11 & 0.075 & $\mathrm{U}$ & 0.075 & 2.54 & & 2.4 & 0.044 & $\bar{U}$ & 0.33 \\
\hline SPA B7 & J13HY9 & $10 / 17 / 06$ & 0.065 & $\mathrm{U}$ & 0.065 & 0.11 & $\mathrm{U}$ & 0.11 & 0.061 & $\mathrm{U}$ & 0.061 & 0.863 & $\mathrm{U}$ & 2.5 & 0.041 & $\mathrm{U}$ & 0.31 \\
\hline SPA B8 & J13J00 & $10 / 17 / 06$ & 0.21 & $\mathrm{U}$ & 0.21 & 0.26 & $\mathrm{U}$ & 0.26 & 0.26 & $U$ & 0.26 & 1.66 & $\mathrm{U}$ & 2.4 & -0.046 & $\mathrm{U}$ & 0.36 \\
\hline SPA C9 & J13J01 & $10 / 17 / 06$ & 0.094 & $\mathrm{U}$ & 0.094 & 0.13 & $\mathrm{U}$ & 0.13 & 0.12 & $\mathrm{U}$ & 0.12 & 0.500 & $\mathrm{U}$ & 2.4 & -0.052 & $\mathrm{U}$ & 0.40 \\
\hline SPA C10 & $\mathrm{J} 13 \mathrm{~J} 02$ & $10 / 18 / 06$ & 0.19 & $\mathrm{U}$ & 0.19 & 0.24 & $\mathrm{U}$ & 0.24 & 0.24 & $\mathrm{U}$ & 0.24 & -0.966 & $\mathrm{U}$ & 3.6 & 0.043 & $\mathrm{U}$ & 0.33 \\
\hline SPA C 1 & $\mathrm{~J} 13 \mathrm{~J} 03$ & $10 / 18 / 06$ & 0.172 & & 0.070 & 0.13 & $\mathrm{U}$ & 0.13 & 0.073 & $\mathrm{U}$ & 0.073 & 0.750 & $\mathrm{U}$ & 3.5 & -0.132 & $\mathrm{U}$ & 0.49 \\
\hline SPA C2 & J13J04 & $10 / 18 / 06$ & 0.862 & & 0.13 & 0.18 & $\mathrm{U}$ & 0.18 & 0.18 & $\mathrm{U}$ & 0.18 & 24.6 & & 3.8 & 0.128 & $\mathrm{U}$ & 0.36 \\
\hline SPAD3 & $\mathrm{J13J05}$ & $10 / 18 / 06$ & 0.116 & & 0.060 & 0.097 & $\mathrm{U}$ & 0.097 & 0.059 & $\mathrm{U}$ & 0.059 & 2.81 & $\mathrm{U}$ & 3.9 & 0.149 & $\mathrm{U}$ & 0.36 \\
\hline SPA D4 & J13.J06 & $10 / 19 / 06$ & 0.076 & $\mathrm{U}$ & 0.076 & 0.038 & $\mathrm{U}$ & 0.038 & 0.033 & $\mathrm{U}$ & 0.033 & 1.66 & $\mathrm{U}$ & 3.8 & -0.028 & $U$ & 0.27 \\
\hline SPA D5 & $513 J 07$ & $10 / 23 / 06$ & 0.063 & $\mathrm{U}$ & 0.063 & 0.094 & $\mathrm{U}$ & 0.094 & 0.053 & $\mathrm{U}$ & 0.053 & 3.60 & & 2.5 & -0.031 & $\mathrm{U}$ & 0.11 \\
\hline SPA D6 & J13J08 & $10 / 23 / 06$ & 0.17 & $\mathrm{U}$ & 0.17 & 0.20 & $\mathrm{U}$ & 0.20 & 0.20 & $U$ & 0.20 & 2.98 & $\mathrm{U}$ & 3.2 & -0.043 & $\mathrm{U}$ & 0.33 \\
\hline FS-12 & J14Y75 & $6 / 27 / 07$ & 0.239 & $\mathrm{U}$ & 0.239 & 0.256 & $\mathrm{U}$ & 0.256 & 0.174 & $\mathrm{U}$ & 0.174 & & & & -0.037 & $\mathrm{U}$ & 0.282 \\
\hline FS-13 & $\mathrm{J14Y76}$ & $6 / 27 / 07$ & 0.227 & $\mathrm{U}$ & 0.227 & 0.249 & $\mathrm{U}$ & 0.249 & 0.156 & U & 0.156 & & & & 0 & $\mathrm{U}$ & 0.374 \\
\hline FS-14 & $\mathrm{J} 12 \mathrm{XB} 3$ & $7 / 19 / 06$ & 0.13 & $\mathrm{U}$ & 0.13 & 0.32 & $\mathrm{U}$ & 0.32 & 0.084 & $U$ & 0.084 & & & & & & \\
\hline FS-17 & $\mathrm{J} 14 \mathrm{JJ3}$ & $3 / 5 / 07$ & 0.11 & $\mathrm{U}$ & 0.11 & 0.15 & $\mathrm{U}$ & 0.15 & 0.12 & U & 0.12 & & & & & & \\
\hline FS-18 & J14L38 & $3 / 5 / 07$ & 0.083 & $\mathrm{U}$ & 0.083 & 0.12 & $\mathrm{U}$ & 0.12 & 0.071 & $\mathrm{U}$ & 0.071 & & & & & & \\
\hline FS-19 & J14L39 & $3 / 5 / 07$ & 0.16 & $\mathrm{U}$ & 0.16 & 0.21 & $\mathrm{U}$ & 0.21 & 0.11 & $\mathrm{U}$ & 0.11 & & & & & & \\
\hline
\end{tabular}


Attachment 1. 118-B-1 Verification Sampling Results.

\begin{tabular}{|c|c|c|c|c|c|c|c|c|c|c|c|c|c|c|c|c|c|}
\hline \multirow{2}{*}{ Sample Location } & \multirow{2}{*}{$\begin{array}{c}\text { Sample } \\
\text { Number }\end{array}$} & \multirow{2}{*}{$\begin{array}{c}\text { Sample } \\
\text { Date }\end{array}$} & \multicolumn{3}{|c|}{ Plutonium-239/240 } & \multicolumn{3}{|c|}{ Plutonium-241 } & \multicolumn{3}{|c|}{ Potassium-40 } & \multicolumn{3}{|c|}{ Radium-226 } & \multicolumn{3}{|c|}{ Radium-228 } \\
\hline & & & $\mathrm{pCi} / \mathrm{g}$ & $Q$ & MDA & $\mathrm{pCi} / \mathrm{g}$ & $Q$ & MDA & $\mathrm{pCi} / \mathrm{g}$ & $Q$ & MDA & $\mathrm{pCi} / \mathrm{g}$ & $\mathbf{Q}$ & MDA & $\mathrm{pCi} / \mathrm{g}$ & $\mathbf{Q}$ & MDA \\
\hline Area $1 \mathrm{Al}$ & $\mathrm{J12X} 44$ & $7 / 19 / 06$ & 0 & $\mathrm{U}$ & 1.5 & 0.477 & $\mathrm{U}$ & 15 & 22.1 & & 0.53 & 0.735 & & 0.11 & 1.14 & & 0.28 \\
\hline Duplicate of J12X44 & $\mathrm{J} 12 \mathrm{X} 48$ & $7 / 19 / 06$ & 0 & $\mathrm{U}$ & 0.96 & -3.76 & $\mathrm{U}$ & 15 & 18.8 & & 0.33 & 0.736 & & 0.068 & 1.13 & & 0.14 \\
\hline Split of J12X44 & $\mathrm{J} 12 \times 49$ & $7 / 19 / 06$ & 0.00 & $\mathrm{U}$ & 0.0304 & 0.409 & $\mathrm{U}$ & 1.99 & & & & & & & & & \\
\hline Area $1 \mathrm{~A} 2$ & $\mathrm{~J} 12 \mathrm{X} 45$ & $7 / 19 / 06$ & 0 & $\mathrm{U}$ & 0.65 & 5.29 & $\mathrm{U}$ & 15 & 11.0 & & 0.17 & 0.416 & & 0.039 & 0.624 & & 0.089 \\
\hline Area $1 \mathrm{A3}$ & $\mathrm{J} 12 \mathrm{X} 46$ & $7 / 20 / 06$ & 0.044 & $\mathrm{U}$ & 0.048 & 6.64 & $\mathrm{U}$ & 22 & 19.6 & & 0.33 & 0.722 & & 0.072 & 1.34 & & 0.14 \\
\hline Area $1 \mathrm{~A} 4$ & $\mathrm{~J} 12 \mathrm{X} 47$ & $7 / 20 / 06$ & 0 & $\mathrm{U}$ & 0.070 & 7.47 & $\mathrm{U}$ & 20 & 10.1 & & 0.19 & 0.344 & & 0.040 & 0.606 & & 0.092 \\
\hline Area $2 \mathrm{Al}$ & $\mathrm{J} 12 \times 50$ & $7 / 20 / 06$ & 0 & U & 0.77 & 8.88 & $\mathrm{U}$ & 23 & 20.0 & & 0.40 & 0.655 & & 0.065 & 0.973 & & 0.16 \\
\hline Area 2 A2 & $\mathrm{J12X51}$ & $7 / 20 / 06$ & 0 & $\mathrm{U}$ & 1.1 & 2.24 & $\mathrm{U}$ & 25 & 9.05 & & 1.5 & 0.192 & $U$ & 0.20 & 0.742 & & 0.40 \\
\hline Area $2 \mathrm{~A} 3$ & $J 12 \times 52$ & $7 / 20 / 06$ & 0 & $\mathrm{U}$ & 0.89 & 15.8 & $\mathrm{U}$ & 25 & 20.1 & & 0.41 & 0.651 & & 0.073 & 1.12 & & 0.14 \\
\hline Area $2 \mathrm{~A} 4$ & $\mathrm{~J} 12 \times 53$ & $7 / 20 / 06$ & 0 & $\mathrm{U}$ & 0.54 & 13.0 & $\mathrm{U}$ & 24 & 12.9 & & 0.28 & 0.456 & & 0.049 & 0.764 & & 0.11 \\
\hline Area $3 \mathrm{Al}$ & $\mathrm{J} 12 \mathrm{X} 54$ & $7 / 20 / 06$ & 0.119 & $\bar{U}$ & 0.91 & 14.3 & $U$ & 23 & 13.1 & & 0.26 & 0.487 & & 0.047 & 0.715 & & 0.12 \\
\hline Area $3 \mathrm{~A} 2$ & $\mathrm{~J} 12 \times 55$ & $7 / 20 / 06$ & 0.076 & $\mathrm{U}$ & 0.58 & 9.52 & $\mathrm{U}$ & 25 & 26.4 & & 0.84 & 0.965 & & 0.16 & 1.46 & & 0.34 \\
\hline Area $3 \mathrm{~A} 3$ & $\mathrm{~J} 12 \times 56$ & $7 / 20 / 06$ & 0.622 & $\mathrm{U}$ & 0.68 & 5.57 & $\mathrm{U}$ & 23 & 9.50 & & 1.2 & 0.200 & $\mathrm{U}$ & 0.21 & 0.588 & & 0.40 \\
\hline Duplicate of $J 12 \times 56$ & $\mathrm{~J} 12 \mathrm{X} 58$ & $7 / 20 / 06$ & 0.216 & $\mathrm{U}$ & 0.41 & 0.998 & $\mathrm{U}$ & 24 & 10.7 & & 0.90 & 0.437 & & 0.16 & 0.87 & $U$ & 0.87 \\
\hline Split of J12X56 & $J 12 \times 60$ & $7 / 20 / 06$ & & & & & & & & & & & & & & & \\
\hline Area $3 \mathrm{~A} 4$ & $\mathrm{~J} 12 \times 57$ & $7 / 20 / 06$ & 0 & $\mathrm{U}$ & 0.75 & 9.85 & $U$ & 23 & 26.5 & & 0.96 & 0.972 & & 0.16 & 1.61 & & 0.38 \\
\hline Area 4 A1 & $\mathrm{J} 12 \times 61$ & $7 / 25 / 06$ & & & & & & & 21.8 & & 0.36 & 0.696 & & 0.077 & 1.10 & & 0.17 \\
\hline Duplicate of J12X61 & $\mathrm{J} 12 \times 65$ & $7 / 25 / 06$ & & & & & & & 10.8 & & 0.24 & 0.418 & & 0.058 & 0.631 & & 0.12 \\
\hline Split of J12X61 & $\mathrm{J} 12 \times 66$ & $7 / 25 / 06$ & & & & & & & & & & & & & & & \\
\hline Area 4 A2 & $\mathrm{J} 12 \times 62$ & $7 / 25 / 06$ & & & & & & & 12.0 & & 0.20 & 0.377 & & 0.043 & 0.719 & & 0.092 \\
\hline Area 4 A3 & $\mathrm{J} 12 \times 63$ & $7 / 25 / 06$ & & & & & & & 11.4 & & 0.31 & 0.407 & & 0.083 & 0.743 & & 0.19 \\
\hline Area 4 A4 & $\mathrm{J} 12 \times 64$ & $7 / 25 / 06$ & & & & & & & 23.4 & & 0.45 & 0.825 & & 0.091 & 1.32 & & 0.21 \\
\hline Area $5 \mathrm{Al}$ & $\mathrm{J} 13 \mathrm{~V} 12$ & $12 / 7 / 06$ & & & & & & & 9.58 & & 1.4 & 0.381 & & 0.17 & 0.50 & $\mathrm{U}$ & 0.50 \\
\hline Area $5 \mathrm{A2}$ & J13V10 & $12 / 7 / 06$ & & & & & & & 10.3 & & 1.2 & 0.394 & & 0.13 & 0.872 & & 0.33 \\
\hline Area $5 \mathrm{~A} 3$ & J13V13 & $12 / 7 / 06$ & & & & & & & 9.85 & & 0.87 & 0.273 & & 0.19 & 0.455 & & 0.32 \\
\hline Area $5 \mathrm{~A} 3$ resample & $\mathrm{J} 14 \mathrm{JH} 6$ & $3 / 30 / 07$ & & & & & & & 8.84 & & 1.1 & 0.293 & & 0.14 & 0.925 & & 0.37 \\
\hline Area $5 \mathrm{~A} 4$ & J13V11 & $12 / 7 / 06$ & & & & & & & 9.80 & & 1.4 & 0.274 & & 0.17 & 0.716 & & 0.48 \\
\hline Area $6 \mathrm{Al}$ & $\mathrm{J12XC2}$ & $7 / 25 / 06$ & 0.096 & $\bar{U}$ & 0.73 & -13.0 & $\mathrm{U}$ & 23 & 24.1 & & 0.32 & 0.870 & & 0.075 & 1.31 & & 0.15 \\
\hline Area $6 \mathrm{~A} 2$ & $\mathrm{~J} 12 \mathrm{XC} 3$ & $7 / 25 / 06$ & 0.068 & $\mathrm{U}$ & 0.52 & -15.6 & $\mathrm{U}$ & 23 & 27.8 & & 0.76 & 1.05 & & 0.13 & 1.38 & & 0.35 \\
\hline Area $6 \mathrm{~A} 3$ & $\mathrm{~J} 12 \mathrm{XC} 4$ & $7 / 25 / 06$ & 0 & $\mathrm{U}$ & 0.62 & -5.92 & $\mathrm{U}$ & 23 & 12.0 & & 0.23 & 0.433 & & 0.042 & 0.690 & & 0.092 \\
\hline Area $6 \mathrm{A4}$ & $\mathrm{J} 12 \mathrm{XC5}$ & $7 / 25 / 06$ & 0 & $\mathrm{U}$ & 0.84 & -2.03 & $\mathrm{U}$ & 24 & 13.3 & & 0.25 & 0.458 & & 0.046 & 0.705 & & 0.11 \\
\hline
\end{tabular}

\begin{tabular}{|c|c|c|c|}
\hline Attachment & 1 & Sheet No & 7 of 113 \\
\hline Originator & J.M. Capron & Date & $10 / 03 / 07$ \\
\hline Checked & T. M. Blakley & Date & \\
\hline Calc. No. & 0100B-CA-V0299 & Rev. No. & 1 \\
\hline
\end{tabular}


Attachment 1. 118-B-1 Verification Sampling Results.

\begin{tabular}{|c|c|c|c|c|c|c|c|c|c|c|c|c|c|c|c|c|c|}
\hline \multirow{2}{*}{ Sample Location } & \multirow{2}{*}{$\begin{array}{c}\text { Sample } \\
\text { Number }\end{array}$} & \multirow{2}{*}{$\begin{array}{c}\text { Sample } \\
\text { Date }\end{array}$} & \multicolumn{3}{|c|}{ Plutonium-239/240 } & \multicolumn{3}{|c|}{ Plutonium-241 } & \multicolumn{3}{|c|}{ Potassium -40} & \multicolumn{3}{|c|}{ Radium-226 } & \multicolumn{3}{|c|}{ Radium-228 } \\
\hline & & & $\mathrm{pCi} / \mathrm{g}$ & $Q$ & MDA & $\mathrm{pCi} / \mathrm{g}$ & $Q$ & MDA & $\mathrm{pCi} / \mathrm{g}$ & $\mathbf{Q}$ & MDA & $\mathrm{pCi} / \mathrm{g}$ & $\mathrm{Q}$ & MDA & $\mathrm{pCi} / \mathrm{g}$ & $Q$ & MDA \\
\hline Area $7 \mathrm{Al}$ & $\mathrm{J12XC6}$ & $7 / 26 / 06$ & 0 & $\mathrm{U}$ & 0.80 & -3.98 & $\mathrm{U}$ & 24 & 26.8 & & 0.61 & 1.12 & & 0.13 & 1.48 & & 0.34 \\
\hline Area $7 \mathrm{~A} 2$ & $\mathrm{~J} 12 \mathrm{XC7}$ & $7 / 26 / 06$ & 0 & $\mathrm{U}$ & 0.68 & -1.1 .4 & $\mathrm{U}$ & 23 & 26.5 & & 0.38 & 1.04 & & 0.075 & 1.44 & & 0.18 \\
\hline Area $7 \mathrm{~A} 3$ & $\mathrm{~J} 12 \mathrm{XC} 8$ & $7 / 26 / 06$ & 0.071 & $\mathrm{U}$ & 0.54 & -7.39 & $\mathrm{U}$ & 28 & 33.6 & & 0.59 & 1.17 & & 0.15 & 2.14 & & 0.31 \\
\hline Area $7 \mathrm{~A} 4$ & $\mathrm{~J} 12 \mathrm{XC} 9$ & $7 / 26 / 06$ & 0.129 & $\mathrm{U}$ & 0.98 & -12.3 & $\mathrm{U}$ & 26 & 12.8 & & 0.21 & 0.536 & & 0.039 & 0.728 & & 0.088 \\
\hline $\mathrm{OBA1}$ & J12WK0 & $7 / 11 / 06$ & 0.119 & $\mathrm{U}$ & 0.91 & 11.8 & $\mathrm{U}$ & 26 & 11.5 & & 0.18 & 0.453 & & 0.032 & 0.698 & & 0.080 \\
\hline $\mathrm{OB} \mathrm{A2}$ & J12WK1 & $7 / 11 / 06$ & 0 & $\mathrm{U}$ & 0.77 & -6.81 & $\mathrm{U}$ & 24 & 22.7 & & 0.37 & 0.805 & & 0.085 & 1.23 & & 0.17 \\
\hline $\mathrm{OB} \mathrm{A3}$ & $\mathrm{J} 12 \mathrm{WK} 2$ & $7 / 11 / 06$ & 0 & $\mathrm{U}$ & 1.1 & -1.46 & $\mathrm{U}$ & 26 & 12.0 & & 0.37 & 0.530 & & 0.062 & 0.686 & & 0.19 \\
\hline OB A4 & J12WK3 & $7 / 11 / 06$ & 0.086 & $\mathrm{U}$ & 0.66 & -1.91 & $U$ & 27 & 23.1 & & 0.26 & 0.827 & & 0.057 & 1.22 & & 0.14 \\
\hline OB B5 & J12WK4 & $7 / 11 / 06$ & 0.428 & $\mathrm{U}$ & 0.65 & 2.93 & $\mathrm{U}$ & 26 & 11.8 & & 0.24 & 0.435 & & 0.048 & 0.694 & & 0.099 \\
\hline OB B6 & J12WK5 & $7 / 11 / 06$ & 0 & $\mathrm{U}$ & 0.79 & 3.37 & $U$ & 26 & 26.4 & & 0.64 & 0.808 & & 0.10 & 1.58 & & 0.27 \\
\hline OB B7 & J12WK6 & $7 / 11 / 06$ & 0.062 & $\mathrm{U}$ & 0.47 & -0.418 & $\mathrm{U}$ & 26 & 13.1 & & 0.24 & 0.442 & & 0.046 & 0.640 & & 0.11 \\
\hline OB B8 & J12WM7 & $7 / 17 / 06$ & 0 & $\mathrm{U}$ & 0.11 & -3.51 & $\mathrm{U}$ & 13 & 28.8 & & 0.70 & 0.910 & & 0.16 & 1.53 & & 0.35 \\
\hline $\mathrm{OBC} C$ & J12WM8 & $7 / 17 / 06$ & 0.053 & $\mathrm{U}$ & 0.10 & 1.58 & $\mathrm{U}$ & 13 & 12.9 & & 0.18 & 0.464 & & 0.034 & 0.751 & & 0.070 \\
\hline $\mathrm{OB} \mathrm{C10}$ & J12WM9 & $7 / 17 / 06$ & 0 & $\mathrm{U}$ & 0.52 & 2.71 & $\mathrm{U}$ & 12 & 13.1 & & 0.21 & 0.488 & & 0.047 & 0.883 & & 0.12 \\
\hline $\mathrm{OBC} 1$ & J12WN0 & $7 / 17 / 06$ & 0 & $\mathrm{U}$ & 0.11 & 11.4 & $\mathrm{U}$ & 24 & 20.0 & & 0.34 & 0.778 & & 0.072 & 1.13 & & 0.15 \\
\hline $\mathrm{OBC} 2$ & J12WN1 & $7 / 17 / 06$ & 0.013 & $\mathrm{U}$ & 0.049 & 0.461 & $\mathrm{U}$ & 28 & 28.8 & & 0.82 & 0.869 & & 0.19 & 1.70 & & 0.35 \\
\hline OB D3 & J12WM5 & $7 / 12 / 06$ & -0.080 & $\mathrm{U}$ & 0.61 & 6.21 & $\mathrm{U}$ & 15 & 22.9 & & 0.87 & 0.851 & & 0.14 & 1.21 & & 0.31 \\
\hline OB D4 & J12WM6 & $7 / 12 / 06$ & 0.035 & $\mathrm{U}$ & 0.089 & 5.46 & $\mathrm{U}$ & 12 & 12.6 & & 0.25 & 0.494 & & 0.050 & 0.795 & & 0.092 \\
\hline OB D5 & J12WM3 & $7 / 12 / 06$ & 0.128 & $\mathrm{U}$ & 0.98 & 6.06 & $\mathrm{U}$ & 14 & 11.6 & & 0.26 & 0.442 & & 0.051 & 0.643 & & 0.12 \\
\hline OB D6 & J12WM4 & $7 / 12 / 06$ & 0 & $\mathrm{U}$ & 0.64 & 10.1 & $\mathrm{U}$ & 14 & 9.87 & & 0.29 & 0.382 & & 0.051 & 0.608 & & 0.12 \\
\hline $\mathrm{BCL} A 1$ & $\mathrm{~J} 12 \mathrm{WN} 2$ & $7 / 20 / 06$ & 0.012 & $\mathrm{U}$ & 0.064 & 15.1 & $\mathrm{U}$ & 19 & 10.8 & & 0.32 & 0.402 & & 0.049 & 0.644 & & 0.15 \\
\hline $\mathrm{BCL} \mathrm{A2}$ & J12WM1 & $7 / 12 / 06$ & 0.135 & U & 0.52 & 4.34 & $\mathrm{U}^{\prime}$ & 14 & 25.3 & & 0.77 & 0.857 & & 0.14 & 1.47 & & 0.31 \\
\hline $\mathrm{BCL} \mathrm{A} 3$ & J12WM2 & $7 / 12 / 06$ & 0 & $\mathrm{U}$ & 0.92 & 8.67 & $\mathrm{U}$ & 12 & 12.5 & & 0.25 & 0.460 & & 0.047 & 0.783 & & 0.12 \\
\hline $\mathrm{BCL} \mathrm{A4}$ & J12WM0 & $7 / 12 / 06$ & 0.086 & $\mathrm{U}$ & 0.33 & 4.87 & $\mathrm{U}$ & 16 & 24.7 & & 0.36 & 0.838 & & 0.073 & 1.38 & & 0.19 \\
\hline $\mathrm{BCL} \mathrm{B5}$ & J12WL9 & $7 / 12 / 06$ & 0 & $\mathrm{U}$ & 0.43 & 7.48 & $\mathrm{U}$ & 14 & 12.7 & & 0.26 & 0.469 & & 0.047 & 0.872 & & 0.13 \\
\hline BCL B6 & J12WL8 & $7 / 12 / 06$ & 0.080 & $\mathrm{U}$ & 0.61 & 4.87 & $\mathrm{U}$ & 12 & 25.6 & & 0.89 & 0.900 & & 0.12 & 1.62 & & 0.31 \\
\hline BCL B7 & J12WL7 & $7 / 12 / 06$ & 0 & $\mathrm{U}$ & 0.35 & 10.3 & $U$ & 13 & 30.1 & & 0.87 & 0.960 & & 0.15 & 1.38 & & 0.38 \\
\hline BCL B8 & J12WL6 & $7 / 12 / 06$ & 0.123 & $\mathrm{U}$ & 0.47 & 7.93 & $\mathrm{U}$ & 14 & 11.4 & & 0.40 & 0.497 & & 0.068 & 0.669 & & 0.18 \\
\hline Split of J12WL6 & J12WW0 & $7 / 12 / 06$ & 0.0363 & & 0.0197 & -0.354 & $\mathrm{U}$ & 2.30 & & & & & & & & & \\
\hline
\end{tabular}

Attachment

Originator
Checked

Calc. No. $\frac{1}{\text { J. M. Capron }}$

T. M. Blakley

0100B-CA-V0299
Sheet No. 8 of 113

Date $\quad 10 / 03 / 07$ Date

Rev. No. 
Attachment 1. 118-B-1 Verification Sampling Results.

\begin{tabular}{|c|c|c|c|c|c|c|c|c|c|c|c|c|c|c|c|c|c|}
\hline \multirow{2}{*}{ Sample Location } & \multirow{2}{*}{$\begin{array}{l}\text { Sample } \\
\text { Number }\end{array}$} & \multirow{2}{*}{$\begin{array}{c}\text { Sample } \\
\text { Date }\end{array}$} & \multicolumn{3}{|c|}{ Plutonium-239/240 } & \multicolumn{3}{|c|}{ Plutonium-241 } & \multicolumn{3}{|c|}{ Potassium-40 } & \multicolumn{3}{|c|}{ Radium-226 } & \multicolumn{3}{|c|}{ Radium-228 } \\
\hline & & & $\mathrm{pCi} / \mathrm{g}$ & $Q$ & MDA & $\overline{p C i} / g$ & $Q$ & MDA & $\mathrm{pCi} / \mathrm{g}$ & $\mathbf{Q}$ & MDA & $\mathrm{pCi} / \mathrm{g}$ & $Q$ & MDA & $\mathrm{pCi} / \mathrm{g}$ & $Q$ & $\overline{M D A}$ \\
\hline BCL C9 & J12WK7 & $7 / 11 / 06$ & 0.040 & $\mathrm{U}$ & 0.077 & 7.84 & $\mathrm{U}$ & 29 & 21.4 & & 0.16 & 0.794 & & 0.033 & 1.28 & & 0.065 \\
\hline $\mathrm{BCL}$ C10 & J12WK9 & $7 / 11 / 06$ & 0 & $\mathrm{U}$ & 0.80 & -2.70 & $\mathrm{U}$ & 31 & 25.3 & & 0.35 & 0.778 & & 0.058 & 1.34 & & 0.15 \\
\hline $\mathrm{BCL} C 1$ & J12WK8 & $7 / 11 / 06$ & 0 & $\mathrm{U}$ & 1.2 & -0.406 & $\mathrm{U}$ & 25 & 11.6 & & 0.14 & 0.466 & & 0.027 & 0.741 & & 0.058 \\
\hline $\mathrm{BCL} \mathrm{C2}$ & J12WL2 & $7 / 11 / 06$ & 0 & $\mathrm{U}$ & 0.54 & 6.52 & $\mathrm{U}$ & 27 & 13.6 & & 0.25 & 0.487 & & 0.050 & 0.836 & & 0.11 \\
\hline $\mathrm{BCL}$ D3 & J12WL3 & $7 / 11 / 06$ & 0 & $\mathrm{U}$ & 0.89 & 8.21 & $\mathrm{U}$ & 28 & 26.0 & & 0.80 & 0.957 & & 0.11 & 1.34 & & 0.31 \\
\hline BCL D4 & J12WL4 & $7 / 11 / 06$ & 0.075 & $\bar{U}$ & 0.57 & 6.26 & $\bar{U}$ & 33 & 24.7 & & 0.43 & 0.908 & & 0.083 & 1.58 & & 0.19 \\
\hline Duplicate of J12WL4 & J12WL5 & $7 / 11 / 06$ & 0 & $\mathrm{U}$ & 0.94 & 11.4 & $\mathrm{U}$ & 30 & 13.6 & & 0.22 & 0.508 & & 0.048 & 0.810 & & 0.11 \\
\hline BCL D5 & J12WLO & $7 / 11 / 06$ & 0.099 & $\mathrm{U}$ & 0.76 & 3.18 & $\mathrm{U}$ & 27 & 12.6 & & 0.10 & 0.458 & & 0.020 & 0.800 & & 0.050 \\
\hline BCL D6 & J12WL1 & $7 / 11 / 06$ & 0 & $\mathrm{U}$ & 0.52 & 13.4 & $\mathrm{U}$ & 31 & 22.2 & & 0.40 & 0.803 & & 0.071 & 1.29 & & 0.15 \\
\hline$\overline{\text { SPA A1 }}$ & J13HY3 & $10 / 12 / 06$ & 0 & $\bar{U}$ & 0.55 & 6.43 & $\mathrm{U}$ & 22 & 24.3 & & 0.62 & 0.991 & & 0.094 & 1.46 & & 0.25 \\
\hline SPA A2 & $\mathrm{J} 13 \mathrm{HY} 4$ & $10 / 12 / 06$ & 0.109 & $\mathrm{U}$ & 0.42 & 13.1 & $\mathrm{U}$ & 23 & 14.4 & & 0.32 & 0.574 & & 0.055 & 0.896 & & 0.13 \\
\hline SPA A3 & J13HY5 & $10 / 12 / 06$ & 0 & U & 0.41 & 7.02 & $\mathrm{U}$ & 22 & 28.8 & & 0.38 & 1.04 & & 0.10 & 1.54 & & 0.25 \\
\hline SPA A4 & J13HY6 & $10 / 12 / 06$ & 0.092 & $\mathrm{U}$ & 0.35 & 4.26 & $\mathrm{U}$ & 21 & 12.6 & & 0.26 & 0.583 & & 0.048 & 0.860 & & 0.099 \\
\hline SPA B5 & J13HY7 & $10 / 12 / 06$ & 0.044 & $\mathrm{U}$ & 0.33 & 12.6 & $\mathrm{U}$ & 23 & 32.0 & & 0.37 & 0.931 & & 0.091 & 1.61 & & 0.27 \\
\hline Duplicate of J13HY7 & J13J09 & $10 / 12 / 06$ & 0 & $\mathrm{U}$ & 0.34 & 9.76 & $\mathrm{U}$ & 23 & 15.9 & & 0.29 & 0.607 & & 0.057 & 0.839 & & 0.11 \\
\hline Split of J13HY7 & J13J10 & $10 / 12 / 06$ & 0.195 & & 0.0450 & 0.348 & $U$ & 2.17 & & & & & & & & & \\
\hline SPA B6 & J13HY8 & $10 / 17 / 06$ & 0.044 & $\mathrm{U}$ & 0.33 & 0.898 & $\mathrm{U}$ & 24 & 15.0 & & 0.37 & 0.502 & & 0.070 & 0.903 & & 0.16 \\
\hline SPA B7 & J13HY9 & $10 / 17 / 06$ & 0.041 & $\mathrm{U}$ & 0.31 & -16.8 & $\mathrm{U}$ & 22 & 12.9 & & 0.41 & 0.608 & & 0.052 & 0.827 & & 0.15 \\
\hline SPA B8 & $\mathrm{J} 13 \mathrm{~J} 00$ & $10 / 17 / 06$ & 0 & $\mathrm{U}$ & 0.35 & -15.1 & $\mathrm{U}$ & 25 & 29.3 & & 2.7 & 1.10 & & 0.12 & 1.76 & & 0.32 \\
\hline SPA C9 & J13J01 & $10 / 17 / 06$ & 0.314 & $\mathrm{U}$ & 0.40 & -25.4 & $\mathrm{U}$ & 27 & 17.1 & & 0.30 & 0.620 & & 0.068 & 0.958 & & 0.16 \\
\hline SPA C 10 & $\mathrm{~J} 13 \mathrm{~J} 02$ & $10 / 18 / 06$ & 0.130 & $\mathrm{U}$ & 0.33 & -11.2 & $\mathrm{U}$ & 26 & 29.5 & & 2.6 & 0.955 & & 0.11 & 1.65 & & 0.28 \\
\hline SPA Cl & $\mathrm{J} 13 \mathrm{~J} 03$ & $10 / 18 / 06$ & 0.088 & $\mathrm{U}$ & 0.34 & -19.2 & $\mathrm{U}$ & 26 & 13.8 & & 0.30 & 0.523 & & 0.066 & 0.816 & & 0.13 \\
\hline SPA C2 & $\mathrm{J} 13 \mathrm{~J} 04$ & $10 / 18 / 06$ & 0.289 & & 0.25 & -6.01 & $\mathrm{U}$ & 26 & 21.0 & & 0.48 & 0.610 & & 0.093 & 1.04 & & 0.24 \\
\hline SPA D3 & J13.05 & $10 / 18 / 06$ & 0.186 & $\mathrm{U}$ & 0.28 & -8.46 & $\mathrm{U}$ & 26 & 13.9 & & 0.25 & 0.507 & & 0.053 & 0.856 & & 0.098 \\
\hline SPA D4 & J13J06 & $10 / 19 / 06$ & 0.028 & $\mathrm{U}$ & 0.22 & -11.2 & $\mathrm{U}$ & 30 & 13.3 & & 0.12 & 0.498 & & 0.021 & 0.791 & & 0.047 \\
\hline SPA D5 & $\mathrm{J} 13 \mathrm{~J} 07$ & $10 / 23 / 06$ & -0.012 & $\mathrm{U}$ & 0.067 & -1.35 & $\mathrm{U}$ & 28 & 12.2 & & 0.25 & 0.453 & & 0.049 & 0.686 & & 0.12 \\
\hline SPA D6 & J13.J08 & $10 / 23 / 06$ & 0 & $\mathrm{U}$ & 0.33 & 8.17 & $\mathrm{U}$ & 27 & 22.6 & & 0.56 & 0.933 & & 0.094 & 1.27 & & 0.24 \\
\hline FS-12 & J14Y75 & $6 / 27 / 07$ & 0.111 & $\mathrm{U}$ & 0.282 & 3.50 & UJ & 16.2 & 6.04 & & 0.754 & 0.293 & & 0.144 & 0.406 & & 0.261 \\
\hline FS-13 & $\mathrm{J} 14 \mathrm{Y} 76$ & $6 / 27 / 07$ & 0 & $\mathrm{U}$ & 0.374 & 1.73 & $\mathrm{UJ}$ & 12.7 & 5.18 & & 0.706 & 0.188 & & 0.144 & 0.450 & & 0.329 \\
\hline FS-14 & $\mathrm{J} 12 \mathrm{XB3}$ & $7 / 19 / 06$ & & & & & & & 7.94 & & 1.2 & 0.356 & & 0.16 & 0.81 & $\mathrm{U}$ & 0.81 \\
\hline FS-17 & $\mathrm{J} 14 \mathrm{JJ} 3$ & $3 / 5 / 07$ & & & & & & & 8.74 & & 0.59 & 0.292 & & 0.087 & 0.560 & & 0.19 \\
\hline FS-18 & J14L38 & $3 / 5 / 07$ & & & & & & & 10.7 & & 0.38 & 0.476 & & 0.074 & 0.720 & & 0.11 \\
\hline FS-19 & J14L39 & $3 / 5 / 07$ & & & & & & & 10.8 & & 0.71 & 0.454 & & 0.12 & 0.707 & & 0.29 \\
\hline
\end{tabular}

\begin{tabular}{|c|c|c|c|}
\hline Attachment & 1 & Sheet No. & 9 of 113 \\
\hline Origi & J.M. Capron & Date & $10 / 03 / 07$ \\
\hline hecked & T. M. Blakley & Date & \\
\hline alc. No. & 0100B-CA-V0299 & Rev. No. & 1 \\
\hline
\end{tabular}


Attachment 1. 118-B-1 Verification Sampling Results.

\begin{tabular}{|c|c|c|c|c|c|c|c|c|c|c|c|c|c|c|c|c|c|}
\hline \multirow[t]{2}{*}{ Sample Location } & \multirow{2}{*}{$\begin{array}{l}\text { Sample } \\
\text { Number }\end{array}$} & \multirow{2}{*}{$\begin{array}{c}\text { Sample } \\
\text { Date }\end{array}$} & \multicolumn{3}{|c|}{ Silver-108m } & \multicolumn{3}{|c|}{ Thorium-228 } & \multicolumn{3}{|c|}{ Thorium-232 } & \multicolumn{3}{|c|}{$\begin{array}{c}\text { Total beta } \\
\text { Radiostrontium }\end{array}$} & \multicolumn{3}{|c|}{ Tritium } \\
\hline & & & $\mathrm{pCi} / \mathrm{g}$ & $Q$ & MDA & $\mathrm{pCi} / \mathrm{g}$ & $Q$ & MDA & $\mathrm{pCi} / \mathrm{g}$ & $Q$ & MDA & $\mathrm{pCi} / \mathrm{g}$ & Q & MDA & $\mathrm{pCi} / \mathrm{g}$ & $\mathbf{Q}$ & MDA \\
\hline Area $1 \mathrm{Al}$ & $\mathrm{J} 12 \times 44$ & $7 / 19 / 06$ & 0.040 & $\mathrm{U}$ & 0.040 & 1.27 & & 0.089 & 1.14 & & 0.28 & 0.275 & $\mathrm{~J}$ & 0.27 & 1.74 & $\mathrm{U}$ & 3.4 \\
\hline Duplicate of J12X44 & $\mathrm{J} 12 \times 48$ & $7 / 19 / 06$ & 0.026 & $\mathrm{U}$ & 0.026 & 0.985 & & 0.050 & 1.13 & & 0.14 & 0.024 & UJ & 0.23 & 0.535 & $\mathrm{U}$ & 2.8 \\
\hline Split of J12X44 & $\mathrm{J} 12 \times 49$ & $7 / 19 / 06$ & -0.00444 & $\mathrm{U}$ & 0.0260 & & & & & & & 0.582 & & 0.137 & 0.0648 & & 0.0282 \\
\hline Area $1 \mathrm{~A} 2$ & $\mathrm{~J} 12 \times 45$ & $7 / 19 / 06$ & 0.013 & $\mathrm{U}$ & 0.013 & 0.673 & & 0.039 & 0.624 & & 0.089 & 0.034 & UJ & 0.37 & 239 & & 2.8 \\
\hline Area $1 \mathrm{~A} 3$ & $\mathrm{~J} 12 \times 46$ & $7 / 20 / 06$ & 0.025 & $\mathrm{U}$ & 0.025 & 1.01 & & 0.047 & 1.34 & & 0.14 & 0.097 & $\mathrm{U}$ & 0.23 & 0.303 & $\mathrm{U}$ & 2.7 \\
\hline Area $1 \mathrm{~A} 4$ & $\mathrm{~J} 12 \times 47$ & $7 / 20 / 06$ & 0.015 & $U$ & 0.015 & 0.468 & & 0.026 & 0.606 & & 0.092 & -0.007 & $\mathrm{U}$ & 0.26 & 0.574 & $\mathrm{U}$ & 3.1 \\
\hline Area $2 \mathrm{Al}$ & $\mathrm{J} 12 \times 50$ & $7 / 20 / 06$ & 0.028 & $\mathrm{U}$ & 0.028 & 0.903 & & 0.053 & 0.973 & & 0.16 & 0.049 & $\mathrm{U}$ & 0.22 & 0.150 & $\mathrm{U}$ & 2.9 \\
\hline Area 2 A2 & $512 \times 51$ & $7 / 20 / 06$ & 0.050 & $\mathrm{U}$ & 0.050 & 0.271 & & 0.077 & 0.742 & & 0.40 & 0.248 & & 0.23 & 59.6 & & 2.8 \\
\hline Area $2 \mathrm{~A} 3$ & $\mathrm{~J} 12 \times 52$ & $7 / 20 / 06$ & 0.027 & $U$ & 0.027 & 1.00 & & 0.053 & 1.12 & & 0.14 & 0.006 & $\mathrm{U}$ & 0.23 & 0.236 & $\mathrm{U}$ & 3.0 \\
\hline Area $2 \mathrm{~A} 4$ & $\mathrm{~J} 12 \times 53$ & $7 / 20 / 06$ & 0.018 & $U$ & 0.018 & 0.620 & & 0.031 & 0.764 & & 0.11 & 0.003 & $\mathrm{U}$ & 0.24 & -0.432 & $\mathrm{U}$ & 2.7 \\
\hline Area $3 \mathrm{Al}$ & $\mathrm{J} 12 \times 54$ & $7 / 20 / 06$ & 0.018 & $\mathrm{U}$ & 0.018 & 0.637 & & 0.031 & 0.715 & & 0.12 & 0.005 & $\mathrm{U}$ & 0.21 & 0.767 & $\mathrm{U}$ & 2.7 \\
\hline Area $3 \mathrm{~A} 2$ & $\mathrm{~J} 12 \times 55$ & $7 / 20 / 06$ & 0.051 & $\mathrm{U}$ & 0.051 & 1.41 & & 0.12 & 1.46 & & 0.34 & 0.293 & & 0.24 & 0.759 & $U$ & 2.6 \\
\hline Area $3 \mathrm{~A} 3$ & $\mathrm{~J} 12 \times 56$ & $7 / 20 / 06$ & 0.070 & $U$ & 0.070 & 0.266 & & 0.086 & 0.588 & & 0.40 & 4.42 & & 0.31 & 0.491 & $\mathrm{U}$ & 2.7 \\
\hline Duplicate of $112 \times 56$ & $\mathrm{~J} 12 \times 58$ & $7 / 20 / 06$ & 0.058 & $\mathrm{U}$ & 0.058 & 0.250 & & 0.079 & 0.87 & $\mathrm{U}$ & 0.87 & 3.23 & & 0.25 & 0.311 & $\mathrm{U}$ & 2.7 \\
\hline Split of J12X56 & $\mathrm{J} 12 \times 60$ & $7 / 20 / 06$ & 0.00198 & $\mathrm{U}$ & 0.0323 & & & & & & & 3.43 & & 0.175 & 0.0577 & & 0.0453 \\
\hline Area $3 \mathrm{~A} 4$ & $\mathrm{~J} 12 \times 57$ & $7 / 20 / 06$ & 0.055 & $\mathrm{U}$ & 0.055 & 1.46 & & 0.14 & 1.61 & & 0.38 & -0.037 & $\mathrm{U}$ & 0.23 & 19.0 & & 2.6 \\
\hline Area 4 Al & $J 12 \times 61$ & $7 / 25 / 06$ & 0.030 & $\mathrm{U}$ & 0.030 & 1.09 & & 0.055 & 1.10 & & 0.17 & 0.208 & U & 0.23 & -0.056 & $\mathrm{U}$ & 3.0 \\
\hline Duplicate of J12X61 & $\mathrm{J} 12 \times 65$ & $7 / 25 / 06$ & 0.020 & $\mathrm{U}$ & 0.020 & 0.544 & & 0.033 & 0.631 & & 0.12 & 0.158 & $\mathrm{U}$ & 0.23 & -0.936 & $\mathrm{U}$ & 3.1 \\
\hline Split of J12X61 & $\mathrm{J12X66}$ & $7 / 25 / 06$ & 0.00648 & $\mathrm{U}$ & 0.0234 & & & & & & & 0.319 & & 0.166 & 0.0303 & $\mathrm{U}$ & 0.0361 \\
\hline Area 4A2 & $\mathrm{J} 12 \times 62$ & $7 / 25 / 06$ & 0.016 & $\mathrm{U}$ & 0.016 & 0.588 & & 0.029 & 0.719 & & 0.092 & -0.077 & $\mathrm{U}$ & 0.24 & 1.14 & $U$ & 3.1 \\
\hline Area 4 A3 & $\mathrm{J} 12 \times 63$ & $7 / 25 / 06$ & 0.025 & $\mathrm{U}$ & 0.025 & 0.579 & & 0.039 & 0.743 & & 0.19 & -0.035 & $\mathrm{U}$ & 0.23 & -0.228 & $\mathrm{U}$ & 3.1 \\
\hline Area $4 \mathrm{~A} 4$ & $\mathrm{~J} 12 \mathrm{X} 64$ & $7 / 25 / 06$ & 0.033 & $\mathrm{U}$ & 0.033 & 1.26 & & 0.064 & 1.32 & & 0.21 & -0.021 & $\mathrm{U}$ & 0.22 & -1.07 & $\mathrm{U}$ & 3.2 \\
\hline Area 5 A1 & J13V12 & $12 / 7 / 06$ & 0.044 & $\mathrm{U}$ & 0.044 & 0.293 & & 0.068 & 0.50 & $\mathrm{U}$ & 0.50 & -0.011 & $\mathrm{U}$ & 0.26 & 0.741 & $\mathrm{U}$ & 3.3 \\
\hline Area $5 \mathrm{~A} 2$ & J13V10 & $12 / 7 / 06$ & 0.044 & $\mathrm{U}$ & 0.044 & 0.283 & & 0.058 & 0.872 & & 0.33 & -0.030 & $\mathrm{U}$ & 0.29 & 2.18 & $\mathrm{U}$ & 3.5 \\
\hline Area $5 \mathrm{~A} 3$ & J13V13 & $12 / 7 / 06$ & 0.12 & $\mathrm{U}$ & 0.12 & 0.538 & & 0.18 & 0.455 & & 0.32 & 2.38 & & 0.21 & 0.369 & $U$ & 3.3 \\
\hline Area $5 \mathrm{~A} 3$ resample & J14JH6 & $3 / 30 / 07$ & 0.085 & $\mathrm{U}$ & 0.085 & 0.430 & & 0.14 & 0.925 & & 0.37 & & & & & & \\
\hline Area $5 \mathrm{~A} 4$ & J13V11 & $12 / 7 / 06$ & 0.048 & $\mathrm{U}$ & 0.048 & 0.301 & & 0.068 & 0.716 & & 0.48 & 1.38 & & 0.24 & 1.70 & $\mathrm{U}$ & 3.3 \\
\hline Area $6 \mathrm{Al}$ & $\mathrm{J} 12 \mathrm{XC} 2$ & $7 / 25 / 06$ & 0.027 & $\mathrm{U}$ & 0.027 & 1.23 & & 0.050 & 1.31 & & 0.15 & -0.052 & $\mathrm{U}$ & 0.23 & -1.11 & $\mathrm{U}$ & 3.1 \\
\hline Area 6 A2 & $\mathrm{J} 12 \mathrm{XC} 3$ & $7 / 25 / 06$ & 0.051 & $\mathrm{U}$ & 0.051 & 1.50 & & 0.10 & 1.38 & & 0.35 & 0.043 & $\mathrm{U}$ & 0.22 & -0.493 & $\mathrm{U}$ & 3.0 \\
\hline Area $6 \mathrm{~A} 3$ & $\mathrm{~J} 12 \mathrm{XC4}$ & $7 / 25 / 06$ & 0.014 & $\mathrm{U}$ & 0.014 & 0.590 & & 0.027 & 0.690 & & 0.092 & 0.010 & $\mathrm{U}$ & 0.24 & 5.03 & & 3.0 \\
\hline Area $6 \mathrm{A4}$ & $\mathrm{J12XC5}$ & $7 / 25 / 06$ & 0.015 & $\mathrm{U}$ & 0.015 & 0.639 & & 0.030 & 0.705 & & 0.11 & -0.048 & $\mathrm{U}$ & 0.30 & 0.083 & $\mathrm{U}$ & 3.0 \\
\hline
\end{tabular}

Attachment 1 Sheet No. 10 of 113 $\begin{array}{llll}\text { Originator } & \text { J.M. Capron Date } & 10 / 03 / 07 \\ \text { Checked } & \text { T.M. Blakley } & \end{array}$ Calc. No. N100B-CA-V0299 Rev. No. 
Attachment 1. 118-B-1 Verification Sampling Results.

\begin{tabular}{|c|c|c|c|c|c|c|c|c|c|c|c|c|c|c|c|c|c|}
\hline \multirow[t]{2}{*}{ Sample Location } & \multirow{2}{*}{$\begin{array}{l}\text { Sample } \\
\text { Number }\end{array}$} & \multirow{2}{*}{$\begin{array}{l}\text { Sample } \\
\text { Date }\end{array}$} & \multicolumn{3}{|c|}{ Silver-108m } & \multicolumn{3}{|c|}{ Thorium-228 } & \multicolumn{3}{|c|}{ Thorium-232 } & \multicolumn{3}{|c|}{$\begin{array}{c}\text { Total beta } \\
\text { Radiostrontium }\end{array}$} & \multicolumn{3}{|c|}{ Tritium } \\
\hline & & & $\mathrm{pCi} / \mathrm{g}$ & $Q$ & MDA & $\mathrm{pCi} / \mathrm{g}$ & $\mathrm{Q}$ & MDA & $\mathrm{pCi} / \mathrm{g}$ & Q & MDA & $\mathrm{pCi} / \mathrm{g}$ & Q & MDA & $\mathrm{pCi} / \mathrm{g}$ & $Q$ & MDA \\
\hline Area $7 \mathrm{Al}$ & $\mathrm{J} 12 \times \mathrm{C} 6$ & $7 / 26 / 06$ & 0.049 & $U$ & 0.049 & 1.42 & & 0.11 & 1.48 & & 0.34 & 0.219 & $\mathrm{U}$ & 0.27 & -1.72 & UJ & 3.4 \\
\hline Area $7 \mathrm{~A} 2$ & $\mathrm{~J} 12 \mathrm{XC7}$ & $7 / 26 / 06$ & 0.029 & $\mathrm{U}$ & 0.029 & 1.43 & & 0.054 & 1.44 & & 0.18 & -0.045 & $\mathrm{U}$ & 0.33 & -0.580 & UJ & 3.1 \\
\hline Area 7 A3 & $\mathrm{J} 12 \mathrm{XC} 8$ & $7 / 26 / 06$ & 0.054 & $\mathrm{U}$ & 0.054 & 1.80 & & 0.11 & 2.14 & & 0.31 & 0.031 & $\mathrm{U}$ & 0.30 & -1.30 & UJ & 3.1 \\
\hline Area 7 A4 & $\mathrm{J} 12 \times \mathrm{C} 9$ & $7 / 26 / 06$ & 0.015 & $\mathrm{U}$ & 0.015 & 0.595 & & 0.026 & 0.728 & & 0.088 & 0.051 & $\mathrm{U}$ & 0.33 & -0.879 & UJ & 3.1 \\
\hline $\mathrm{OBA1}$ & J12WK0 & $7 / 11 / 06$ & 0.013 & $\mathrm{U}$ & 0.013 & 0.563 & & 0.021 & 0.698 & & 0.080 & 0.441 & & 0.39 & -2.08 & $\mathrm{U}$ & 5.4 \\
\hline $\mathrm{OB} \mathrm{A2}$ & J12WK1 & $7 / 11 / 06$ & 0.030 & $\mathrm{U}$ & 0.030 & 1.17 & & 0.056 & 1.23 & & 0.17 & 0.239 & $\mathrm{U}$ & 0.39 & 0.823 & $\mathrm{U}$ & 4.8 \\
\hline $\mathrm{OBA} 3$ & J12WK2 & $7 / 11 / 06$ & 0.023 & $\mathrm{U}$ & 0.023 & 0.805 & & 0.065 & 0.686 & & 0.19 & 0.067 & U & 0.36 & -0.736 & U & 4.7 \\
\hline $\mathrm{OB} \mathrm{A4}$ & J12WK3 & $7 / 11 / 06$ & 0.023 & $\mathrm{U}$ & 0.023 & 1.21 & & 0.043 & 1.22 & & 0.14 & -0.001 & $\mathrm{U}$ & 0.42 & 0.269 & $\mathrm{U}$ & 4.7 \\
\hline $\mathrm{OB} \mathrm{B5}$ & J12WK4 & $7 / 11 / 06$ & 0.018 & $\mathrm{U}$ & 0.018 & 0.568 & & 0.028 & 0.694 & & 0.099 & 0.009 & $\mathrm{U}$ & 0.26 & -2.31 & $\mathrm{U}$ & 4.5 \\
\hline OB B6 & J12WK5 & $7 / 11 / 06$ & 0.040 & $U$ & 0.040 & 1.42 & & 0.088 & 1.58 & & 0.27 & 0.350 & $\mathrm{U}$ & 0.43 & -0.731 & $\mathrm{U}$ & 4.6 \\
\hline OB B7 & J12WK6 & $7 / 11 / 06$ & 0.017 & $\mathrm{U}$ & 0.017 & 0.590 & & 0.029 & 0.640 & & 0.11 & 0.232 & $\mathrm{U}$ & 0.36 & -1.11 & $\mathrm{U}$ & 5.5 \\
\hline $\mathrm{OB} \mathrm{B8}$ & $\mathrm{J} 12 \mathrm{WM} 7$ & $7 / 17 / 06$ & 0.057 & $\mathrm{U}$ & 0.057 & 1.49 & & 0.14 & 1.53 & & 0.35 & -0.037 & $\mathrm{U}$ & 0.29 & 1.84 & $U$ & 2.7 \\
\hline OB C9 & J12WM8 & $7 / 17 / 06$ & 0.013 & $\mathrm{U}$ & 0.013 & 0.635 & & 0.021 & 0.751 & & 0.070 & 0.015 & $\mathrm{U}$ & 0.29 & 0.850 & $\mathrm{U}$ & 2.8 \\
\hline $\mathrm{OBC} 10$ & J12WM9 & $7 / 17 / 06$ & 0.017 & $\mathrm{U}$ & 0.017 & 0.640 & & 0.031 & 0.883 & & 0.12 & 0.116 & $\mathrm{U}$ & 0.28 & 1.30 & $\mathrm{U}$ & 2.4 \\
\hline $\mathrm{OB} \mathrm{Cl}$ & J12WNO & $7 / 17 / 06$ & 0.027 & $\mathrm{U}$ & 0.027 & 1.05 & & 0.048 & 1.13 & & 0.15 & -0.008 & $U$ & 0.31 & 1.96 & $\mathrm{U}$ & 2.3 \\
\hline $\mathrm{OBC} 2$ & $\mathrm{~J} 12 \mathrm{WN} 1$ & $7 / 17 / 06$ & 0.059 & $U$ & 0.059 & 1.42 & & 0.13 & 1.70 & & 0.35 & 0.013 & $\mathrm{U}$ & 0.34 & 0.468 & $\mathrm{U}$ & 2.3 \\
\hline OB D3 & J12WM5 & $7 / 12 / 06$ & 0.052 & $\mathrm{U}$ & 0.052 & 1.33 & & 0.12 & 1.21 & & 0.31 & -0.019 & $\mathrm{U}$ & 0.26 & 1.72 & $\mathrm{U}$ & 4.4 \\
\hline OB D4 & J12WM6 & $7 / 12 / 06$ & 0.025 & $\mathrm{U}$ & 0.025 & 0.612 & & 0.027 & 0.795 & & 0.092 & 0.047 & $\mathrm{U}$ & 0.24 & -0.621 & $\mathrm{U}$ & 3.9 \\
\hline OB D5 & J12WM3 & $7 / 12 / 06$ & 0.021 & $\mathrm{U}$ & 0.021 & 0.538 & & 0.034 & 0.643 & & 0.12 & 0.194 & $\mathrm{U}$ & 0.26 & 1.45 & $\mathrm{U}$ & 4.6 \\
\hline OB D6 & J12WM4 & $7 / 12 / 06$ & 0.023 & $\mathrm{U}$ & 0.023 & 0.467 & & 0.038 & 0.608 & & 0.12 & 0.188 & $\mathrm{U}$ & 0.25 & 1.39 & $\mathrm{U}$ & 3.8 \\
\hline $\mathrm{BCL} \mathrm{Al}$ & $\mathrm{J} 12 \mathrm{WN} 2$ & $7 / 20 / 06$ & 0.021 & $\mathrm{U}$ & 0.021 & 0.534 & & 0.039 & 0.644 & & 0.15 & 0.015 & $\mathrm{U}$ & 0.23 & 2.03 & $\mathrm{U}$ & 2.7 \\
\hline $\mathrm{BCL} A 2$ & J12WM1 & $7 / 12 / 06$ & 0.053 & $\mathrm{U}$ & 0.053 & 1.36 & & 0.11 & 1.47 & & 0.31 & 0.071 & $\mathrm{U}$ & 0.26 & -0.621 & $\mathrm{U}$ & 3.6 \\
\hline $\mathrm{BCL} A 3$ & J12WM2 & $7 / 12 / 06$ & 0.016 & $\mathrm{U}$ & 0.016 & 0.604 & & 0.030 & 0.783 & & 0.12 & 0.063 & U & 0.28 & -1.03 & $\mathrm{U}$ & 3.9 \\
\hline BCL A4 & J12WM0 & $7 / 12 / 06$ & 0.025 & $\mathrm{U}$ & 0.025 & 1.34 & & 0.058 & 1.38 & & 0.19 & 0.092 & $\mathrm{U}$ & 0.28 & -0.884 & $\mathrm{U}$ & 3.7 \\
\hline BCL B5 & J12WL9 & $7 / 12 / 06$ & 0.017 & $\mathrm{U}$ & 0.017 & 0.649 & & 0.030 & 0.872 & & 0.13 & 0.032 & U & 0.25 & 0 & $\mathrm{U}$ & 4.0 \\
\hline BCL B6 & J12WL8 & $7 / 12 / 06$ & 0.055 & $\mathrm{U}$ & 0.055 & 1.50 & & 0.13 & 1.62 & & 0.31 & 0.210 & $\mathrm{U}$ & 0.27 & -0.762 & $\mathrm{U}$ & 3.9 \\
\hline BCL B7 & J12WL7 & $7 / 12 / 06$ & 0.057 & $\mathrm{U}$ & 0.057 & 1.48 & & 0.12 & 1.38 & & 0.38 & 0.215 & U & 0.22 & 0.819 & $\mathrm{U}$ & 3.7 \\
\hline BCL B8 & J12WL6 & $7 / 12 / 06$ & 0.025 & $\mathrm{U}$ & 0.025 & 0.593 & & 0.050 & 0.669 & & 0.18 & 0.140 & $\mathrm{U}$ & 0.30 & -0.050 & $\mathrm{U}$ & 3.8 \\
\hline Split of J12WL6 & J12WW0 & $7 / 12 / 06$ & 0.000659 & $\mathrm{U}$ & 0.0159 & & & & & & & 0.126 & U & 0.151 & 0.0476 & $\mathrm{U}$ & 0.0625 \\
\hline & & & & & & & & & & \multirow{4}{*}{\multicolumn{2}{|c|}{$\begin{array}{l}\text { Attachment } \\
\text { Originator } \\
\text { Checked } \\
\text { Calc. No. }\end{array}$}} & \multicolumn{3}{|c|}{1} & \multirow{4}{*}{$\begin{array}{l}\text { Sheet No. } \\
\text { Date } \\
\text { Date } \\
\text { Rev. No. }\end{array}$} & \multicolumn{2}{|c|}{11 of 113} \\
\hline & & & & & & & & & & & & \multirow{3}{*}{\multicolumn{3}{|c|}{$\begin{array}{l}\text { J. M. Capron } \\
\text { T. M. Blakley } \\
\text { 0100B-CA-V0299 }\end{array}$}} & & \multicolumn{2}{|c|}{$10 / 03 / 07$} \\
\hline & & & & & & & & & & & & & & & & & \\
\hline & & & & & & & & & & & & & & & & & 0 \\
\hline
\end{tabular}


Attachment 1. 118-B-1 Verification Sampling Results.

\begin{tabular}{|c|c|c|c|c|c|c|c|c|c|c|c|c|c|c|c|c|c|}
\hline \multirow[t]{2}{*}{ Sample Location } & \multirow{2}{*}{$\begin{array}{l}\text { Sample } \\
\text { Number }\end{array}$} & \multirow{2}{*}{$\begin{array}{c}\text { Sample } \\
\text { Date }\end{array}$} & \multicolumn{3}{|c|}{ Silver-108m } & \multicolumn{3}{|c|}{ Thorium-228 } & \multicolumn{3}{|c|}{ Thorium-232 } & \multicolumn{3}{|c|}{$\begin{array}{c}\text { Total beta } \\
\text { Radiostrontium }\end{array}$} & \multicolumn{3}{|c|}{ Tritium } \\
\hline & & & $\mathrm{pCi} / \mathrm{g}$ & $Q$ & MDA & $\mathrm{pCi} / \mathrm{g}$ & $Q$ & MDA & $\mathrm{pCi} / \mathrm{g}$ & $Q$ & MDA & $\mathrm{pCi} / \mathrm{g}$ & Q & MDA & $\mathrm{pCi} / \mathrm{g}$ & $\mathbf{Q}$ & MDA \\
\hline BCL C9 & $\mathrm{J} 12 \mathrm{WK} 7$ & $7 / 11 / 06$ & 0.012 & $\mathrm{u}$ & 0.012 & 1.13 & & 0.023 & 1.28 & & 0.065 & 0.412 & & 0.38 & 1.41 & $\mathrm{U}$ & 5.2 \\
\hline $\mathrm{BCL}$ C10 & $\mathrm{J} 12 \mathrm{WK} 9$ & $7 / 11 / 06$ & 0.022 & $\mathrm{U}$ & 0.022 & 1.41 & & 0.050 & 1.34 & & 0.15 & 0.049 & U & 0.42 & -0.567 & $\mathrm{U}$ & 5.0 \\
\hline $\mathrm{BCLCl}$ & J12WK8 & $7 / 11 / 06$ & 0.010 & $\mathrm{U}$ & 0.010 & 0.760 & & 0.025 & 0.741 & & 0.058 & -0.084 & U & 0.33 & 0.955 & $\mathrm{U}$ & 4.4 \\
\hline $\mathrm{BCL} C 2$ & J12WL2 & $7 / 11 / 06$ & 0.019 & $\mathrm{U}$ & 0.019 & 0.633 & & 0.031 & 0.836 & & 0.11 & -0.083 & u & 0.27 & -0.142 & $\mathrm{U}$ & 5.0 \\
\hline BCL D3 & J12WL3 & $7 / 11 / 06$ & 0.049 & $\mathrm{U}$ & 0.049 & 1.42 & & 0.11 & 1.34 & & 0.31 & 0.057 & U & 0.32 & -1.58 & $\mathrm{U}$ & 4.4 \\
\hline BCL D4 & J12WL4 & $7 / 11 / 06$ & 0.033 & $\mathrm{U}$ & 0.033 & 1.31 & & 0.060 & 1.58 & & 0.19 & -0.494 & u & 1.2 & 0.209 & $\mathrm{U}$ & 4.9 \\
\hline Duplicate of J12WL4 & J12WLS & $7 / 11 / 06$ & 0.019 & $\mathrm{U}$ & 0.019 & 0.666 & & 0.031 & 0.810 & & 0.11 & 0.106 & U & 0.30 & 1.37 & $U$ & 5.0 \\
\hline BCL D5 & J12WL0 & $7 / 11 / 06$ & 0.007 & $\mathrm{U}$ & 0.007 & 0.600 & & 0.012 & 0.800 & & 0.050 & 0.082 & $\mathrm{U}$ & 0.35 & -1.91 & $\mathrm{U}$ & 4.6 \\
\hline BCL D6 & $\mathrm{J} 12 \mathrm{WL} 1$ & $7 / 11 / 06$ & 0.027 & $U$ & 0.027 & 1.18 & & 0.052 & 1.29 & & 0.15 & -0.117 & $\mathrm{u}$ & 0.29 & -1.77 & $\mathrm{U}$ & 5.0 \\
\hline SPA A1 & $\mathrm{J} 13 \mathrm{HY} 3$ & $10 / 12 / 06$ & 0.044 & $\mathrm{U}$ & 0.044 & 1.60 & & 0.095 & 1.46 & & 0.25 & -0.035 & U & 0.24 & 2.04 & $\mathrm{U}$ & 2.7 \\
\hline SPA A2 & J13HY4 & $10 / 12 / 06$ & 0.020 & $\mathrm{U}$ & 0.020 & 0.754 & & 0.038 & 0.896 & & 0.13 & -0.053 & U & 0.26 & 1.54 & $\mathrm{U}$ & 2.6 \\
\hline SPA A3 & J13HY5 & $10 / 12 / 06$ & 0.044 & $\mathrm{U}$ & 0.044 & 1.76 & & 0.10 & 1.54 & & 0.25 & 0.096 & $\mathrm{u}$ & 0.26 & 1.23 & $\mathrm{U}$ & 2.9 \\
\hline SPA A4 & J13HY6 & $10 / 12 / 06$ & 0.017 & $\mathrm{U}$ & 0.017 & 0.726 & & 0.032 & 0.860 & & 0.099 & 0.227 & & 0.22 & 7.32 & & 2.7 \\
\hline SPA B5 & $\mathrm{J} 13 \mathrm{HY} 7$ & $10 / 12 / 06$ & 0.045 & $\mathrm{U}$ & 0.045 & 1.74 & & 0.10 & 1.61 & & 0.27 & 0.092 & $\mathrm{u}$ & 0.24 & 3.09 & & 2.6 \\
\hline Duplicate of J13HY7 & J13509 & $10 / 12 / 06$ & 0.019 & $\mathrm{U}$ & 0.019 & 0.826 & & 0.038 & 0.839 & & 0.11 & 0.002 & $\mathrm{U}$ & 0.24 & 6.18 & & 2.7 \\
\hline Split of J13HY7 & $\mathrm{J} 13 \mathrm{~J} 10$ & $10 / 12 / 06$ & -0.00461 & $\mathrm{U}$ & 0.0138 & & & & & & & 0.102 & $u$ & 0.176 & 0.327 & & 0.0201 \\
\hline SPA B6 & $\mathrm{J} 13 \mathrm{HY} 8$ & $10 / 17 / 06$ & 0.022 & $\mathrm{U}$ & 0.022 & 0.810 & & 0.038 & 0.903 & & 0.16 & 0.105 & U & 0.29 & 1.62 & $\mathrm{U}$ & 2.8 \\
\hline SPAB7 & $\mathrm{J} 13 \mathrm{HY} 9$ & $10 / 17 / 06$ & 0.018 & $\mathrm{U}$ & 0.018 & 0.776 & & 0.034 & 0.827 & & 0.15 & -0.028 & $\mathrm{u}$ & 0.18 & 2.72 & $\mathrm{U}$ & 2.9 \\
\hline SPA B8 & $\mathrm{J} 13 \mathrm{~J} 00$ & $10 / 17 / 06$ & 0.056 & $\mathrm{U}$ & 0.056 & 1.86 & & 0.12 & 1.76 & & 0.32 & -0.061 & $\mathrm{U}$ & 0.17 & 6.14 & & 2.7 \\
\hline SPA C9 & $\mathrm{J13 \textrm {J } 0 1}$ & $10 / 17 / 06$ & 0.027 & $\mathrm{U}$ & 0.027 & 0.884 & & 0.049 & 0.958 & & 0.16 & 0.016 & $U$ & 0.17 & 17.4 & & 2.7 \\
\hline SPA Cl0 & $\mathrm{J13J02}$ & $10 / 18 / 06$ & 0.050 & $\mathrm{U}$ & 0.050 & 1.69 & & 0.12 & 1.65 & & 0.28 & 0.028 & $\mathrm{U}$ & 0.26 & 1.09 & $\mathrm{U}$ & 2.5 \\
\hline SPACl & $\mathrm{J} 13 \mathrm{~J} 03$ & $10 / 18 / 06$ & 0.022 & $\mathrm{U}$ & 0.022 & 0.705 & & 0.036 & 0.816 & & 0.13 & 0.166 & $\mathrm{U}$ & 0.17 & 476 & & 4.2 \\
\hline SPA Cl resample & $\mathrm{J} / 4 \mathrm{BH} 3$ & $1 / 12 / 07$ & & & & & & & & & & & & & 67.8 & & 2.1 \\
\hline SPA C2 & $\mathrm{J} 13 \mathrm{~J} 04$ & $10 / 18 / 06$ & 0.045 & $\mathrm{U}$ & 0.045 & 1.11 & & 0.10 & 1.04 & & 0.24 & 0.934 & & 0.18 & 4.84 & & 2.5 \\
\hline SPA D3 & $\mathrm{J} 13 \mathrm{~J} 05$ & $10 / 18 / 06$ & 0.018 & $\mathrm{U}$ & 0.018 & 0.764 & & 0.030 & 0.856 & & 0.098 & 0.216 & & 0.20 & 4.92 & & 2.8 \\
\hline SPA D4 & $\mathrm{J13506}$ & $10 / 19 / 06$ & 0.008 & $\mathrm{U}$ & 0.008 & 0.715 & & 0.013 & 0.791 & & 0.047 & 0.288 & & 0.18 & 0.021 & UJ & 2.6 \\
\hline SPA D5 & $\mathrm{J} 13 \mathrm{~J} 07$ & $10 / 23 / 06$ & 0.018 & $\mathrm{U}$ & 0.018 & 0.606 & & 0.032 & 0.686 & & 0.12 & 0.139 & $U$ & 0.25 & 0.990 & $\mathrm{U}$ & 2.9 \\
\hline SPA D6 & $\mathrm{J} 13 \mathrm{~J} 08$ & $10 / 23 / 06$ & 0.042 & $U$ & 0.042 & 1.40 & & 0.096 & 1.27 & & 0.24 & 0.131 & U & 0.31 & 1.27 & $\mathrm{U}$ & 2.6 \\
\hline FS-3 & $\mathrm{J} 12 \mathrm{R} 26$ & $7 / 10 / 06$ & & & & & & & & & & & & & 0.029 & $\mathrm{U}$ & 3.1 \\
\hline FS-4 & $\mathrm{J} 12 \mathrm{R} 27$ & $7 / 10 / 06$ & & & & & & & & & & & & & 0.456 & $\mathrm{U}$ & 3.0 \\
\hline FS-11 & $\mathrm{J} 12 \mathrm{R} 34$ & $7 / 10 / 06$ & & & & & & & & & & & & & 107 & & 3.1 \\
\hline FS-12 & $\mathrm{J} 14 \mathrm{Y} 75$ & $6 / 27 / 07$ & 0.066 & $\mathrm{U}$ & 0.066 & 0.424 & & 0.135 & 0.406 & & 0.261 & & & & & & \\
\hline FS-13 & $\mathrm{J} 14 \mathrm{Y} 76$ & $6 / 27 / 07$ & 0.059 & $\mathrm{U}$ & 0.059 & 0.543 & & 0.158 & 0.450 & & 0.329 & & & & & & \\
\hline FS-14 & $\mathrm{J} 12 \mathrm{XB} 3$ & $7 / 19 / 06$ & 0.048 & $\mathrm{U}$ & 0.048 & 0.220 & & 0.066 & 0.81 & $\mathrm{U}$ & 0.81 & & & & & & \\
\hline FS-17 & $\mathrm{J} 14 \mathrm{JJ} 3$ & $3 / 5 / 07$ & 0.034 & $\bar{U}$ & 0.034 & 0.490 & & 0.074 & 0.560 & & 0.19 & & & & 7.40 & & 2.3 \\
\hline FS-17 resample & J152M! & $5 / 10 / 07$ & & & & & & & & & & & & & 3.24 & & 2.7 \\
\hline FS-18 & $\mathrm{J14L38}$ & $3 / 5 / 07$ & 0.023 & $\mathrm{U}$ & 0.023 & 0.594 & & 0.043 & 0.720 & & 0.11 & & & & 9.20 & & 2.4 \\
\hline FS-19 & J14L39 & $3 / 5 / 07$ & 0.048 & $\mathrm{U}$ & 0.048 & 0.821 & & 0.11 & 0.707 & & 0.29 & & & & 137 & & 2.5 \\
\hline & & & & & & & & & & Atta & hment & & 1 & & Sheet N & 12 & $\frac{\text { of } 113}{103 / 07}$ \\
\hline & & & & & & & & & & $\begin{array}{l}\text { Orig } \\
\text { Chec }\end{array}$ & $\begin{array}{l}\text { ator } \\
\text { ed }\end{array}$ & $\frac{\text { J.M. Cap }}{\text { T.M. Bla }}$ & & & $\begin{array}{l}\text { Date } \\
\text { Date }\end{array}$ & & \\
\hline & & & & & & & & & & Calc & No. & $0100 \mathrm{~B}-\mathrm{C}$ & $\frac{\mathrm{A}-\mathrm{V} 0}{\mathrm{~V}}$ & & Rev. No. & & 1 \\
\hline
\end{tabular}


Attachment 1. 118-B-1 Verification Sampling Results.

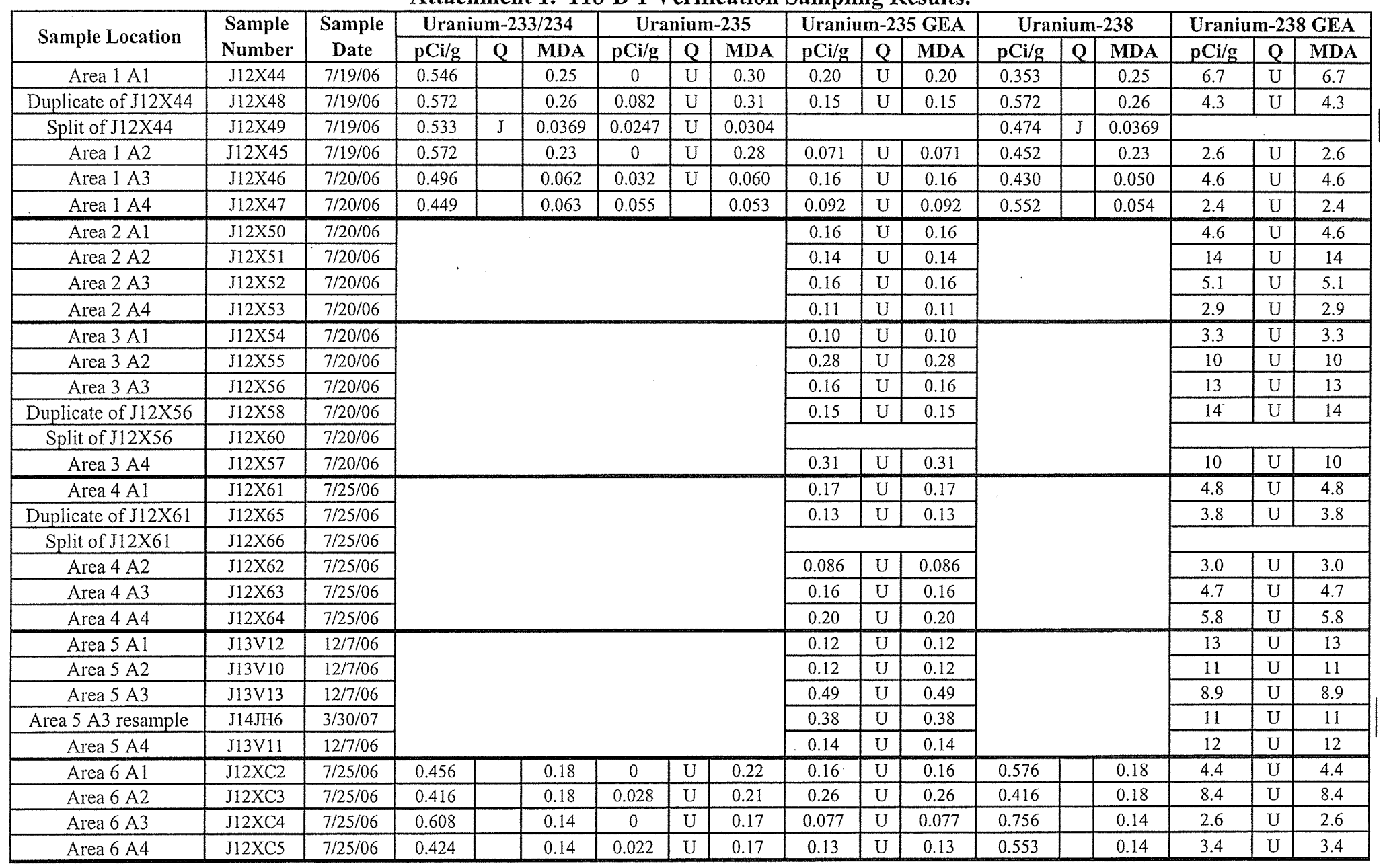

\begin{tabular}{|c|c|c|c|}
\hline Attachment & 1 & Sheet No. & 13 of 113 \\
\hline Originator & J. M. Capron & Date & $10 / 03 / 07$ \\
\hline Checked & T. M. Blakley & Date & \\
\hline Calc. No. & $0100 \mathrm{~B}-\mathrm{CA}-\mathrm{V} 0299$ & Rev. No & 1 \\
\hline
\end{tabular}


Attachment 1. 118-B-1 Verification Sampling Results.

\begin{tabular}{|c|c|c|c|c|c|c|c|c|c|c|c|c|c|c|c|c|c|}
\hline \multirow{2}{*}{ Sample Location } & \multirow{2}{*}{$\begin{array}{c}\text { Sample } \\
\text { Number }\end{array}$} & \multirow{2}{*}{$\begin{array}{c}\text { Sample } \\
\text { Date }\end{array}$} & \multicolumn{3}{|c|}{ Uranium-233/234 } & \multicolumn{3}{|c|}{ Uranium-235 } & \multicolumn{3}{|c|}{ Uranium-235 GEA } & \multicolumn{3}{|c|}{ Uranium-238 } & \multicolumn{3}{|c|}{ Uranium-238 GEA } \\
\hline & & & $\mathrm{pCi} / \mathrm{g}$ & $\mathbf{Q}$ & MDA & $\mathrm{pCi} / \mathrm{g}$ & $\mathbf{Q}$ & MDA & $\mathrm{pCi} / \mathrm{g}$ & $Q$ & MDA & $\mathrm{pCi} / \mathrm{g}$ & $\mathbf{Q}$ & MDA & $\mathrm{pCi} / \mathrm{g}$ & $Q$ & MDA \\
\hline Area $7 \mathrm{Al}$ & $\mathrm{J} 12 \times \mathrm{XC} 6$ & $7 / 26 / 06$ & 0.473 & & 0.26 & 0 & $\mathrm{U}$ & 0.31 & 0.25 & $\mathrm{U}$ & 0.25 & 0.574 & & 0.26 & 8.8 & $\vec{U}$ & 8.8 \\
\hline Area $7 \mathrm{A2}$ & $\mathrm{J} 12 \times \mathrm{C} 7$ & $7 / 26 / 06$ & 0.602 & & 0.27 & 0.086 & $\mathrm{U}$ & 0.33 & 0.17 & $\mathrm{U}$ & 0.17 & 0.708 & & 0.27 & 4.5 & $\mathrm{U}$ & 4.5 \\
\hline Area $7 \mathrm{~A} 3$ & $\mathrm{~J} 12 \mathrm{XC} 8$ & $7 / 26 / 06$ & 0.718 & & 0.25 & 0.040 & $\mathrm{U}$ & 0.30 & 0.28 & $\mathrm{U}$ & 0.28 & 0.424 & & 0.25 & 8.9 & $\bar{U}$ & 8.9 \\
\hline Area $7 \mathrm{~A} 4$ & $\mathrm{~J} 12 \mathrm{XC} 9$ & $7 / 26 / 06$ & 0.292 & & 0.28 & 0.044 & $U$ & 0.34 & 0.085 & $\mathrm{U}$ & 0.085 & 0.729 & & 0.28 & 2.3 & $\mathrm{U}$ & 2.3 \\
\hline $\mathrm{OBAl}$ & J12WK0 & $7 / 11 / 06$ & 0.534 & & 0.032 & 0.004 & $\mathrm{U}$ & 0.044 & 0.067 & $\mathrm{U}$ & 0.067 & 0.563 & & 0.025 & 2.0 & U & 2.0 \\
\hline $\mathrm{OB} \mathrm{A2}$ & $\mathrm{J} 12 \mathrm{WK} 1$ & $7 / 11 / 06$ & 0.456 & & 0.026 & 0.004 & $\mathrm{U}$ & 0.031 & 0.18 & $\mathrm{U}$ & 0.18 & 0.476 & & 0.026 & 4.9 & $\mathrm{U}$ & 4.9 \\
\hline $\mathrm{OB} \mathrm{A3}$ & $\mathrm{J} 12 \mathrm{WK} 2$ & $7 / 11 / 06$ & 0.479 & & 0.026 & 0.008 & U & 0.031 & 0.15 & $\mathrm{U}$ & 0.15 & 0.567 & & 0.026 & 4.1 & $\mathrm{U}$ & 4.1 \\
\hline $\mathrm{OB} \mathrm{A4}$ & J12WK3 & $7 / 11 / 06$ & 0.436 & & 0.036 & 0.037 & & 0.035 & 0.13 & $\mathrm{U}$ & 0.13 & 0.387 & & 0.036 & 3.9 & $\mathrm{U}$ & 3.9 \\
\hline OB B5 & $\mathrm{J} 12 \mathrm{WK} 4$ & $7 / 11 / 06$ & 0.510 & & 0.038 & 0.029 & $\mathrm{U}$ & 0.032 & 0.10 & $\mathrm{U}$ & 0.10 & 0.575 & & 0.033 & 3.2 & $\mathrm{U}$ & 3.2 \\
\hline OB B6 & J12WK5 & $7 / 11 / 06$ & 0.461 & & 0.027 & 0.013 & $\mathrm{U}$ & 0.033 & 0.20 & $\mathrm{U}$ & 0.20 & 0.489 & & 0.027 & 7.0 & $\mathrm{U}$ & 7.0 \\
\hline OB B7 & J12WK6 & $7 / 11 / 06$ & 0.477 & & 0.043 & 0.023 & $U$ & 0.030 & 0.085 & $\mathrm{U}$ & 0.085 & 0.465 & & 0.039 & 3.0 & $\mathrm{U}$ & 3.0 \\
\hline $\mathrm{OB} \mathrm{B} 8$ & J12WM7 & $7 / 17 / 06$ & 0.692 & & 0.35 & 0 & $\mathrm{U}$ & 0.43 & 0.32 & $\mathrm{U}$ & 0.32 & 0.692 & & 0.35 & 11 & $\mathrm{U}$ & 11 \\
\hline OB C9 & J12WM8 & $7 / 17 / 06$ & 0.767 & & 0.21 & 0 & $U$ & 0.25 & 0.076 & $\mathrm{U}$ & 0.076 & 0.575 & & 0.21 & 2.0 & $\mathrm{U}$ & 2.0 \\
\hline $\mathrm{OB} \mathrm{C10}$ & J12WM9 & $7 / 17 / 06$ & 0.508 & & 0.23 & 0 & $\mathrm{U}$ & 0.28 & 0.097 & $\mathrm{U}$ & 0.097 & 0.538 & & 0.23 & 3.1 & $\mathrm{U}$ & 3.1 \\
\hline $\mathrm{OB} \mathrm{Cl}$ & J12WN0 & $7 / 17 / 06$ & 0.407 & & 0.26 & 0.041 & $\mathrm{U}$ & 0.31 & 0.15 & $\mathrm{U}$ & 0.15 & 0.543 & & 0.26 & 4.2 & $\mathrm{U}$ & 4.2 \\
\hline $\mathrm{OBC} 2$ & $\mathrm{~J} 12 \mathrm{WN} 1$ & $7 / 17 / 06$ & 0.337 & & 0.23 & 0.037 & $\mathrm{U}$ & 0.28 & 0.32 & $\mathrm{U}$ & 0.32 & 0.521 & & 0.23 & 10 & $\mathrm{U}$ & 10 \\
\hline OB D3 & J12WM5 & $7 / 12 / 06$ & 0.455 & & 0.029 & 0.022 & $\mathrm{U}$ & 0.028 & 0.26 & $\mathrm{U}$ & 0.26 & 0.479 & & 0.023 & 8.9 & U & 8.9 \\
\hline OB D4 & J12WM6 & $7 / 12 / 06$ & 0.400 & & 0.023 & 0.033 & & 0.028 & 0.088 & $U$ & 0.088 & 0.546 & & 0.023 & 3.0 & $\mathrm{U}$ & 3.0 \\
\hline OB D5 & J12WM3 & $7 / 12 / 06$ & 0.530 & & 0.028 & 0.022 & $\mathrm{U}$ & 0.034 & 0.10 & $\mathrm{U}$ & 0.10 & 0.504 & & 0.028 & 3.0 & $\mathrm{U}$ & 3.0 \\
\hline OB D6 & J12WM4 & $7 / 12 / 06$ & 0.526 & & 0.10 & 0.095 & $\mathrm{U}$ & 0.12 & 0.13 & $\mathrm{U}$ & 0.13 & 0.539 & & 0.10 & 4.0 & $\mathrm{U}$ & 4.0 \\
\hline $\mathrm{BCLA1}$ & J12WN2 & $7 / 20 / 06$ & 0.540 & & 0.028 & 0.017 & $\mathrm{U}$ & 0.033 & 0.14 & $\mathrm{U}$ & 0.14 & 0.508 & & 0.028 & 3.8 & $\mathrm{U}$ & 3.8 \\
\hline BCL A2 & J12WMI & $7 / 12 / 06$ & 0.491 & & 0.17 & 0.054 & $\mathrm{U}$ & 0.21 & 0.27 & $\mathrm{U}$ & 0.27 & 0.714 & & 0.17 & 9.5 & $\mathrm{U}$ & 9.5 \\
\hline $\mathrm{BCL} A 3$ & J12WM2 & $7 / 12 / 06$ & 0.467 & & 0.17 & 0.054 & $\mathrm{U}$ & 0.21 & 0.087 & $\mathrm{U}$ & 0.087 & 0.555 & & 0.17 & 3.0 & $\mathrm{U}$ & 3.0 \\
\hline BCL A4 & J12WM0 & $7 / 12 / 06$ & 0.517 & & 0.17 & 0.027 & $\mathrm{U}$ & 0.21 & 0.13 & $\mathrm{U}$ & 0.13 & 0.495 & & 0.17 & 4.4 & $\mathrm{U}$ & 4.4 \\
\hline BCL B5 & J12WL9 & $7 / 12 / 06$ & 0.552 & & 0.16 & 0.026 & $\mathrm{U}$ & 0.20 & 0.083 & $\mathrm{U}$ & 0.083 & 0.594 & & 0.16 & 3.1 & $\mathrm{U}$ & 3.1 \\
\hline BCL B6 & J12WL8 & $7 / 12 / 06$ & 0.569 & & 0.16 & 0.051 & $\mathrm{U}$ & 0.20 & 0.29 & $\mathrm{U}$ & 0.29 & 0.485 & & 0.16 & 9.3 & $\mathrm{U}$ & 9.3 \\
\hline BCL B7 & J12WL7 & $7 / 12 / 06$ & 0.525 & & 0.18 & 0 & $\mathrm{U}$ & 0.22 & 0.29 & $\mathrm{U}$ & 0.29 & 0.501 & & 0.18 & 9.7 & $\mathrm{U}$ & 9.7 \\
\hline $\mathrm{BCL} B 8$ & J12WL6 & $7 / 12 / 06$ & 0.503 & & 0.20 & 0 & $\mathrm{U}$ & 0.25 & 0.15 & $U$ & 0.15 & 0.715 & & 0.20 & 4.6 & $\mathrm{U}$ & 4.6 \\
\hline Split of J12WL6 & J12WW0 & $7 / 12 / 06$ & 0.874 & $\mathrm{~J}$ & 0.0659 & 0.167 & & 0.0456 & & & & 0.697 & $\mathrm{~J}$ & 0.0715 & & & \\
\hline
\end{tabular}

\begin{tabular}{|c|c|c|c|}
\hline Attachment & 1 & Sheet No. & 14 of 113 \\
\hline Originator & J.M. Capron & Date & $10 / 03 / 07$ \\
\hline Checked & T. M. Blakley & Date & \\
\hline Calc. No. & 0100B-CA-V0299 & Rev. No. & 1 \\
\hline
\end{tabular}


Attachment 1. 118-B-1 Verification Sampling Results.

\begin{tabular}{|c|c|c|c|c|c|c|c|c|c|c|c|c|c|c|c|c|c|}
\hline \multirow{2}{*}{ Sample Location } & \multirow{2}{*}{$\begin{array}{c}\text { Sample } \\
\text { Number }\end{array}$} & \multirow{2}{*}{$\begin{array}{l}\text { Sample } \\
\text { Date }\end{array}$} & \multicolumn{3}{|c|}{ Uranium-233/234 } & \multicolumn{3}{|c|}{ Uranium-235 } & \multicolumn{3}{|c|}{ Uranium-235 GEA } & \multicolumn{3}{|c|}{ Uranium-238 } & \multicolumn{3}{|c|}{ Uranium-238 GEA } \\
\hline & & & $\mathrm{pCi} / \mathrm{g}$ & $\mathrm{Q}$ & MDA & $\mathrm{pCi} / \mathrm{g}$ & $Q$ & MDA & $\mathrm{pCi} / \mathrm{g}$ & $Q$ & MDA & $\mathrm{pCi} / \mathrm{g}$ & Q & MDA & $\mathrm{pCi} / \mathrm{g}$ & Q & MDA \\
\hline BCL C9 & J12WK7 & $7 / 11 / 06$ & 0.548 & & 0.031 & 0.039 & & 0.030 & 0.071 & $U$ & 0.071 & 0.565 & & 0.025 & 2.0 & $U$ & 2.0 \\
\hline $\mathrm{BCL}$ C10 & J12WK9 & $7 / 11 / 06$ & 0.509 & & 0.023 & 0.014 & $\mathrm{U}$ & 0.027 & 0.13 & $\mathrm{U}$ & 0.13 & 0.498 & & 0.023 & 3.9 & $\mathrm{U}$ & 3.9 \\
\hline $\mathrm{BCL} \mathrm{Cl}$ & J12WK8 & $7 / 11 / 06$ & 0.567 & & 0.043 & 0.042 & & 0.036 & 0.057 & $\mathrm{U}$ & 0.057 & 0.556 & & 0.037 & 1.8 & $\mathrm{U}$ & 1.8 \\
\hline $\mathrm{BCL} \mathrm{C2}$ & J12WL2 & $7 / 11 / 06$ & 0.523 & & 0.028 & 0.027 & $\mathrm{U}$ & 0.034 & 0.097 & $\mathrm{U}$ & 0.097 & 0.549 & & 0.035 & 2.9 & $\mathrm{U}$ & 2.9 \\
\hline BCL D3 & J12WL3 & $7 / 11 / 06$ & 0.586 & & 0.030 & 0.019 & $\mathrm{U}$ & 0.052 & 0.26 & $\mathrm{U}$ & 0.26 & 0.531 & & 0.037 & 8.3 & $\mathrm{U}$ & 8.3 \\
\hline BCL D4 & J12WL4 & $7 / 11 / 06$ & 0.676 & & 0.031 & 0.047 & & 0.030 & 0.19 & $U$ & 0.19 & 0.627 & & 0.025 & 5.2 & $\mathrm{U}$ & 5.2 \\
\hline Duplicate of J12WL4 & J12WL5 & $7 / 11 / 06$ & 0.609 & & 0.037 & 0.025 & $U$ & 0.028 & 0.099 & U & 0.099 & 0.525 & & 0.023 & 2.8 & $\mathrm{U}$ & 2.8 \\
\hline BCL D5 & J12WL0 & $7 / 11 / 06$ & 0.560 & & 0.035 & 0.031 & $U$ & 0.034 & 0.040 & $\mathrm{U}$ & 0.040 & 0.556 & & 0.028 & 1.4 & $\mathrm{U}$ & 1.4 \\
\hline BCL D6 & J12WL1 & $7 / 11 / 06$ & 0.624 & & 0.044 & 0.065 & & 0.033 & 0.16 & $\mathrm{U}$ & 0.16 & 0.552 & & 0.034 & 4.7 & $\mathrm{U}$ & 4.7 \\
\hline SPA A1 & J13HY3 & $10 / 12 / 06$ & 0.466 & & 0.050 & 0.038 & $\mathrm{U}$ & 0.042 & 0.28 & $\mathrm{U}$ & 0.28 & 0.575 & & 0.035 & 6.8 & $\mathrm{U}$ & 6.8 \\
\hline SPA A2 & $\mathrm{J} 13 \mathrm{HY} 4$ & $10 / 12 / 06$ & 0.626 & & 0.046 & 0.047 & & 0.045 & 0.14 & $\mathrm{U}$ & 0.14 & 0.559 & & 0.053 & 3.4 & $\mathrm{U}$ & 3.4 \\
\hline SPA A3 & J13HY5 & $10 / 12 / 06$ & 0.502 & & 0.047 & 0.024 & $\mathrm{U}$ & 0.046 & 0.28 & $\mathrm{U}$ & 0.28 & 0.606 & & 0.038 & 6.9 & $U$ & 6.9 \\
\hline SPA A4 & J13HY6 & $10 / 12 / 06$ & 0.470 & & 0.052 & 0.033 & $U$ & 0.051 & 0.11 & $\mathrm{U}$ & 0.11 & 0.497 & & 0.042 & 2.8 & U & 2.8 \\
\hline SPA B5 & J13HY7 & $10 / 12 / 06$ & 0.600 & & 0.038 & 0.079 & & 0.032 & 0.29 & $\mathrm{U}$ & 0.29 & 0.534 & & 0.038 & 7.5 & $\mathrm{U}$ & 7.5 \\
\hline Duplicate of J13HY7 & $\mathrm{J} 13 \mathrm{~J} 09$ & $10 / 12 / 06$ & 0.553 & & 0.029 & 0.032 & $\mathrm{U}$ & 0.044 & 0.13 & U & 0.13 & 0.523 & & 0.029 & 3.4 & $\mathrm{U}$ & 3.4 \\
\hline Split of J13HY7 & $\mathrm{J} 13 \mathrm{~J} 10$ & $10 / 12 / 06$ & 0.171 & & 0.0331 & 0.0111 & $\mathrm{U}$ & 0.0391 & & & & 0.163 & & 0.0391 & & & \\
\hline SPA B6 & J13HY8 & $10 / 17 / 06$ & 0.413 & & 0.19 & 0 & $U$ & 0.22 & 0.12 & $\mathrm{U}$ & 0.12 & 0.632 & & 0.19 & 4.2 & $\mathrm{U}$ & 4.2 \\
\hline SPA B7 & J13HY9 & $10 / 17 / 06$ & 0.551 & & 0.19 & 0.030 & $\mathrm{U}$ & 0.23 & 0.097 & $\mathrm{U}$ & 0.097 & 0.426 & & 0.19 & 3.6 & U & 3.6 \\
\hline SPA B8 & $\mathrm{J} 13 \mathrm{~J} 00$ & $10 / 17 / 06$ & 0.581 & & 0.21 & 0.033 & $U$ & 0.26 & 0.35 & $\mathrm{U}$ & 0.35 & 0.581 & & 0.21 & 9.2 & $\mathrm{U}$ & 9.2 \\
\hline SPA C9 & $\mathrm{J} 13 \mathrm{~J} 01$ & $10 / 17 / 06$ & 0.831 & & 0.21 & 0.067 & $\mathrm{U}$ & 0.26 & 0.17 & $\mathrm{U}$ & 0.17 & 0.471 & & 0.21 & 4.3 & U & 4.3 \\
\hline SPACI0 & $\mathrm{J} 13 \mathrm{~J} 02$ & $10 / 18 / 06$ & 0.410 & & 0.21 & 0.066 & $\mathrm{U}$ & 0.25 & 0.32 & $\mathrm{U}$ & 0.32 & 0.492 & & 0.21 & 8.4 & $\mathrm{U}$ & 8.4 \\
\hline SPA Cl & $\mathrm{J} 13 \mathrm{~J} 03$ & $10 / 18 / 06$ & 0.657 & & 0.23 & 0 & $\mathrm{U}$ & 0.28 & 0.12 & U & 0.12 & 0.448 & & 0.23 & 4.2 & $\mathrm{U}$ & 4.2 \\
\hline SPA C2 & $\mathrm{J} 13 \mathrm{~J} 04$ & $10 / 18 / 06$ & 0.643 & & 0.22 & 0 & U & 0.27 & 0.25 & U & 0.25 & 0.409 & & 0.22 & 6.6 & U & 6.6 \\
\hline SPAD3 & J13J05 & $10 / 18 / 06$ & 0.683 & & 0.22 & 0 & $\mathrm{U}$ & 0.26 & 0.092 & U & 0.092 & 0.740 & & 0.22 & 3.3 & $\mathrm{U}$ & 3.3 \\
\hline SPA D4 & J13J06 & $10 / 19 / 06$ & 0.714 & & 0.12 & 0.037 & U & 0.14 & 0.055 & $\mathrm{U}$ & 0.055 & 0.410 & & 0.12 & 1.3 & U & 1.3 \\
\hline SPA D5 & $\mathrm{J} 13 \mathrm{~J} 07$ & $10 / 23 / 06$ & 0.581 & & 0.10 & 0.065 & $\mathrm{U}$ & 0.13 & 0.095 & U & 0.095 & 0.473 & & 0.10 & 3.5 & U & 3.5 \\
\hline SPA D6 & $\mathrm{J} 13 \mathrm{~J} 08$ & $10 / 23 / 06$ & 0.541 & & 0.040 & 0.018 & $\mathrm{U}$ & 0.035 & 0.27 & U & 0.27 & 0.553 & & 0.037 & 6.5 & $\mathrm{U}$ & 6.5 \\
\hline FS-12 & J14Y75 & $6 / 27 / 07$ & 0.587 & & 0.173 & 0.027 & $\mathrm{U}$ & 0.209 & 0.318 & $\mathrm{U}$ & 0.318 & 0.564 & & 0.173 & 10.5 & $\mathrm{U}$ & 10.5 \\
\hline FS-13 & J14Y76 & $6 / 27 / 07$ & 0.461 & & 0.186 & 0.029 & $U$ & 0.225 & 0.283 & U & 0.283 & 0.510 & & 0.186 & 9.31 & $\mathrm{U}$ & 9.31 \\
\hline FS-14 & $\mathrm{J} 12 \mathrm{XB} 3$ & $7 / 19 / 06$ & & & & & & & 0.13 & $\mathrm{U}$ & 0.13 & & & & 13 & $\mathrm{U}$ & 13 \\
\hline FS-17 & J14JJ3 & $3 / 5 / 07$ & & & & & & & 0.18 & $\mathrm{U}$ & 0.18 & & & & 5.4 & $\mathrm{U}$ & 5.4 \\
\hline FS-18 & J14L38 & $3 / 5 / 07$ & & & & & & & 0.13 & $\mathrm{U}$ & 0.13 & & & & 3.7 & $\mathrm{U}$ & 3.7 \\
\hline FS-19 & J14L39 & $3 / 5 / 07$ & & & & & & & 0.18 & U & 0.18 & & & & 7.4 & $\mathrm{U}$ & 7.4 \\
\hline
\end{tabular}

Attachment

Sheet No. 15 of 113

\begin{tabular}{|c|c|c|c|}
\hline Attachment & I M Capron & $\begin{array}{l}\text { Sheet No. } \\
\text { Date. }\end{array}$ & $\frac{15 \text { of } 113}{10 / 03 / 07}$ \\
\hline $\begin{array}{l}\text { Uriginator } \\
\text { Checked }\end{array}$ & $\frac{\text { T. M. Blakley }}{\text { T. }}$ & Date & $10 / 03707$ \\
\hline Calc. No. & $0100 \mathrm{~B}-\mathrm{CA}-\mathrm{V} 0299$ & Rev. No. & 1 \\
\hline
\end{tabular}


Attachment 1. 118-B-1 Verification Sampling Results.

\begin{tabular}{|c|c|c|c|c|c|c|c|c|c|c|c|c|c|c|c|c|c|c|c|c|}
\hline \multirow{2}{*}{ Sample Location } & \multirow{2}{*}{$\begin{array}{c}\text { Sample } \\
\text { Number }\end{array}$} & \multirow{2}{*}{$\begin{array}{c}\text { Sample } \\
\text { Date }\end{array}$} & \multicolumn{3}{|c|}{ Aluminum } & \multicolumn{3}{|c|}{ Antimony } & \multicolumn{3}{|c|}{ Arsenic } & \multicolumn{3}{|c|}{ Barium } & \multicolumn{3}{|c|}{ Beryllium } & \multicolumn{3}{|c|}{ Boron } \\
\hline & & & $\mathrm{mg} / \mathrm{kg}$ & $\mathbf{Q}$ & PQL & $\mathrm{mg} / \mathrm{kg}$ & $Q$ & PQL & $\mathrm{mg} / \mathrm{kg}$ & $\mathbf{Q}$ & PQL & $\mathrm{mg} / \mathrm{kg}$ & Q & PQL & $\mathrm{mg} / \mathrm{kg}$ & Q & PQL & $\mathrm{mg} / \mathrm{kg}$ & $Q$ & PQL \\
\hline Area $1 \mathrm{Al}$ & $\mathrm{J} 12 \times 44$ & $7 / 19 / 06$ & 6040 & & 8.4 & 1.3 & UJ & 1.3 & 2.9 & & 1.8 & 53.7 & C & 0.06 & 0.59 & & 0.06 & 1.9 & & 0.70 \\
\hline Duplicate of J12X 44 & $\mathrm{~J} 12 \times 48$ & $7 / 19 / 06$ & 6600 & & 8.4 & 1.3 & UJ & 1.3 & 3.1 & & 1.8 & 60.4 & $\mathrm{C}$ & 0.06 & 0.66 & & 0.06 & 0.81 & & 0.70 \\
\hline Split of J12X44 & $\mathrm{J} 12 \times 49$ & $7 / 19 / 06$ & 7460 & $\mathrm{~N}$ & 6.3 & 1.7 & $\mathrm{NJ}$ & 0.33 & 2.8 & & 0.28 & 65.6 & & 0.51 & 0.38 & $B^{*}$ & 0.071 & 1.7 & $\mathrm{~B}^{*}$ & 1.5 \\
\hline Area 1 A2 & $\mathrm{J} 12 \times 45$ & $7 / 19 / 06$ & 4460 & & 8.4 & 1.3 & $\mathrm{UJ}$ & 1.3 & 2.1 & & 1.8 & 63.1 & $\mathrm{C}$ & 0.06 & 0.57 & & 0.06 & 1.5 & & 0.70 \\
\hline Area $1 \mathrm{~A} 3$ & $\mathrm{J12X46}$ & $7 / 20 / 06$ & 5420 & & 8.1 & 1.2 & UJ & 1.2 & 3.2 & & 1.7 & 52.4 & $\mathrm{C}$ & 0.06 & 0.48 & & 0.06 & 1.0 & & 0.68 \\
\hline Area $1 \mathrm{~A} 4$ & $\mathrm{~J} 12 \times 47$ & $7 / 20 / 06$ & 4910 & & 8.1 & 1.2 & $\mathrm{UJ}$ & 1.2 & 2.8 & & 1.7 & 52.1 & $\mathrm{C}$ & 0.06 & 0.54 & & 0.06 & 0.82 & & 0.68 \\
\hline Area $2 \mathrm{Al}$ & $\mathrm{J} 12 \times 50$ & $7 / 20 / 06$ & 3900 & & 8.0 & 1.2 & UI & 1.2 & 1.8 & & 1.7 & 43.9 & C & 0.06 & 0.47 & & 0.06 & 1.6 & & 0.67 \\
\hline Area $2 \mathrm{~A} 2$ & $\mathrm{~J} 12 \times 51$ & $7 / 20 / 06$ & 5170 & & 8.2 & 1.3 & \begin{tabular}{|l|}
$\mathrm{UJ}$ \\
\end{tabular} & 1.3 & 3.2 & & 1.7 & 55.5 & C & 0.06 & 0.62 & & 0.06 & 5.5 & & 0.68 \\
\hline Area $2 \mathrm{~A} 3$ & $\mathrm{~J} 12 \mathrm{X} 52$ & $7 / 20 / 06$ & 5050 & & 8.2 & 1.3 & UJ & 1.3 & 2.6 & & 1.7 & 43.7 & C & 0.06 & 0.65 & & 0.06 & 3.7 & & 0.68 \\
\hline Area $2 \mathrm{~A} 4$ & $\mathrm{~J} 12 \times 53$ & $7 / 20 / 06$ & 4930 & & 8.1 & 1.2 & \begin{tabular}{|l|}
$\mathrm{UJ}$ \\
\end{tabular} & 1.2 & 2.0 & & 1.7 & 61.0 & C & 0.06 & 0.55 & & 0.06 & 2.4 & & 0.68 \\
\hline Area $3 \mathrm{Al}$ & $\mathrm{J} 12 \times 54$ & $7 / 20 / 06$ & 4240 & & 8.0 & 1.2 & UJ & 1.2 & 2.4 & & 1.7 & 58.8 & C & 0.06 & 0.56 & & 0.06 & 8.9 & & 0.67 \\
\hline Area $3 \mathrm{~A} 2$ & $312 \times 55$ & $7 / 20 / 06$ & 4820 & & 8.1 & 1.2 & UJ & 1.2 & 2.9 & & 1.7 & 45.3 & C & 0.06 & 0.63 & & 0.06 & 1.1 & & 0.67 \\
\hline Area $3 \mathrm{~A} 3$ & $\mathrm{~J} 12 \times 56$ & $7 / 20 / 06$ & 6120 & & 8.1 & 1.2 & UJ & 1.2 & 4.5 & & 1.7 & 52.4 & $\mathrm{C}$ & 0.06 & 0.65 & & 0.06 & 2.2 & & 0.68 \\
\hline Duplicate of J12X56 & $512 \times 58$ & $7 / 20106$ & 5500 & & 8.2 & 1.3 & UJ & 1.3 & 3.9 & & 1.7 & 48.3 & $\mathrm{C}$ & 0.06 & 0.61 & & 0.06 & 1.3 & & 0.69 \\
\hline Split of J12X56 & $\mathrm{J} 12 \times 60$ & $7 / 20 / 06$ & 5470 & $\mathrm{~N}$ & 6.3 & 1.1 & $\mathrm{NJ}$ & 0.34 & 3.0 & & 0.28 & 47.7 & & 0.51 & 0.34 & $\mathrm{~B}^{*}$ & 0.071 & 5.1 & $\mathrm{~B}^{*}$ & 1.5 \\
\hline Area $3 \mathrm{~A} 4$ & $\mathrm{~J} 12 \times 57$ & $7 / 20 / 06$ & 4640 & & 8.1 & 1.2 & UJ & 1.2 & 2.4 & & 1.7 & 65.9 & $\mathrm{C}$ & 0.06 & 0.61 & & 0.06 & 2.1 & & 0.67 \\
\hline Area $4 \mathrm{Al}$ & $312 \times 61$ & $7 / 25 / 06$ & 3740 & & 8.0 & 1.2 & $\mathrm{U}$ & 1.2 & 2.5 & & 1.7 & 39.1 & & 0.06 & 0.42 & & 0.06 & 1.0 & & 0.67 \\
\hline Duplicate of J12X61 & $312 \times 65$ & $7 / 25 / 06$ & 4220 & & 8.1 & 1.2 & $\mathrm{U}$ & 1.2 & 3.3 & & 1.7 & 51.4 & & 0.06 & 0.34 & & 0.06 & 3.9 & & 0.67 \\
\hline Split of J12X61 & $512 \times 66$ & $7 / 25 / 06$ & 5490 & $\mathrm{~N}$ & 6.2 & 1.7 & $\mathrm{NJ}$ & 0.33 & 2.4 & & 0.28 & 59.3 & & 0.50 & 0.32 & $\mathrm{~B}^{*}$ & 0.071 & 7.7 & $\mathrm{~B}^{*}$ & 1.5 \\
\hline Area 4 A2 & $J 12 \times 62$ & $7 / 25 / 06$ & 4140 & & 7.9 & 1.2 & $\mathrm{U}$ & 1.2 & 2.8 & & 1.7 & 47.2 & & 0.06 & 0.36 & & 0.06 & 0.66 & $\mathrm{U}$ & 0.66 \\
\hline Area $4 \mathrm{~A} 3$ & $\mathrm{~J} 12 \times 63$ & $7 / 25 / 06$ & 4840 & & 8.1 & 1.2 & $\mathrm{U}$ & 1.2 & 3.9 & & 1.7 & 47.5 & & 0.06 & 0.39 & & 0.06 & 0.67 & $\mathrm{U}$ & 0.67 \\
\hline Area 4 A4 & $\mathrm{J} 12 \times 64$ & $7 / 25 / 06$ & 5270 & & 8.0 & 1.2 & $\mathrm{U}$ & 1.2 & 2.8 & & 1.7 & 49.2 & & 0.06 & 0.40 & & 0.06 & 0.66 & $\mathrm{U}$ & 0.66 \\
\hline Area $5 \mathrm{Al}$ & J13V12 & $12 / 7 / 06$ & 3450 & & 6.5 & 0.77 & UJ & 0.77 & 2.3 & & 0.95 & 41.0 & C & 0.03 & 0.1 & & 0.03 & 0.91 & & 0.58 \\
\hline Area $5 \mathrm{~A} 2$ & $313 \vee 10$ & $12 / 7 / 06$ & 3970 & & 6.4 & 0.76 & $\mathrm{UJ}$ & 0.76 & 2.2 & & 0.94 & 44.4 & $\mathrm{C}$ & 0.03 & 0.14 & & 0.03 & 0.72 & & 0.58 \\
\hline Area $5 \mathrm{~A} 3$ & J13V13 & $12 / 7 / 06$ & 1550 & & 2.1 & 0.25 & $\mathrm{UJ}$ & 0.25 & 0.86 & & 0.31 & 18.4 & C & 0.01 & 0.04 & & 0.01 & 0.27 & & 0.19 \\
\hline Area $5 \mathrm{~A} 4$ & J13V11 & $12 / 7 / 06$ & 3990 & & 6.5 & 0.77 & $\mathrm{UJ}$ & 0.77 & 2.9 & & 0.95 & 42.8 & $\mathrm{C}$ & 0.03 & 0.13 & & 0.03 & 1.2 & & 0.58 \\
\hline Area $6 \mathrm{Al}$ & $\mathrm{J} 12 \mathrm{XC} 2$ & $7 / 25 / 06$ & 5070 & & 8.0 & 1.2 & $U$ & 1.2 & 2.9 & & 1.7 & 96.4 & & 0.06 & 0.34 & & 0.06 & 8.8 & & 0.67 \\
\hline Area $6 \mathrm{~A} 2$ & $\mathrm{~J} 12 \mathrm{XC} 3$ & $7 / 25 / 06$ & 5190 & & 8.1 & 1.2 & $\mathrm{U}$ & 1.2 & 3.7 & & 1.7 & 53.3 & & 0.06 & 0.32 & & 0.06 & 1.1 & & 0.67 \\
\hline Area $6 \mathrm{~A} 3$ & $\mathrm{~J} 12 \mathrm{XC} 4$ & $7 / 25 / 06$ & 4710 & & 8.1 & 1.2 & $\mathrm{U}$ & 1.2 & 3.0 & & 1.7 & 44.5 & & 0.06 & 0.35 & & 0.06 & 0.80 & & 0.67 \\
\hline Area $6 \mathrm{~A} 4$ & $\mathrm{~J} 12 \times \mathrm{C} 5$ & $7 / 25 / 06$ & 5270 & & 8.1 & 1.2 & $\mathrm{U}$ & 1.2 & 4.5 & & 1.7 & 44.0 & & 0.06 & 0.36 & & 0.06 & 0.67 & $\mathrm{U}$ & 0.67 \\
\hline Area $7 \mathrm{Al}$ & $\mathrm{J} 12 \mathrm{XC} 6$ & $7 / 26 / 06$ & 4980.00 & & 8.00 & 1.2 & UJ & 1.2 & 3.8 & & 1.7 & 145 & & 0.06 & 0.30 & & 0.06 & 18.1 & & 0.67 \\
\hline Area $7 \mathrm{~A} 2$ & $312 \times C 7$ & $7 / 26 / 06$ & 6090 & & 7.9 & 1.2 & UJ & 1.2 & 3.1 & & 1.7 & 75.1 & & 0.06 & 0.31 & & 0.06 & 2.7 & & 0.66 \\
\hline Area $7 \mathrm{A3}$ & $\mathrm{J} 12 \mathrm{XC} 8$ & $7 / 26 / 06$ & 4610 & & 8.1 & 1.2 & UJ & 1.2 & 3.1 & & 1.7 & 53.7 & & 0.06 & 0.19 & & 0.06 & 1.3 & & 0.67 \\
\hline Area $7 \mathrm{~A} 4$ & J12XC9 & $7 / 26 / 06$ & 5610 & & 8.1 & 1.2 & $\mathrm{UJ}$ & 1.2 & 2.8 & & 1.7 & 119 & & 0.06 & 0.27 & & 0.06 & 7.7 & & 0.67 \\
\hline
\end{tabular}

\begin{tabular}{llll} 
Attachment & \multicolumn{1}{c}{1} & Sheet No. & 16 of 113 \\
Originator & J.M. Mapron & $10 / 03 / 07$ \\
Checked & T.M. Blakley & Date & 1 \\
Calc. No. & &
\end{tabular}


Attachment 1. 118-B-1 Verification Sampling Results.

\begin{tabular}{|c|c|c|c|c|c|c|c|c|c|c|c|c|c|c|c|c|c|c|c|c|}
\hline \multirow{2}{*}{ Sample Location } & \multirow{2}{*}{$\begin{array}{c}\text { Sample } \\
\text { Number }\end{array}$} & \multirow{2}{*}{$\begin{array}{c}\text { Sample } \\
\text { Date }\end{array}$} & \multicolumn{3}{|c|}{ Aluminum } & \multicolumn{3}{|c|}{ Antimony } & \multicolumn{3}{|c|}{ Arsenic } & \multicolumn{3}{|c|}{ Barium } & \multicolumn{3}{|c|}{ Beryllium } & \multicolumn{3}{|c|}{ Boron } \\
\hline & & & $\mathrm{mg} / \mathrm{kg}$ & $Q$ & PQL & $\mathrm{mg} / \mathrm{kg}$ & $Q$ & $\mathrm{PQL}$ & $\mathrm{mg} / \mathrm{kg}$ & $Q$ & PQL & $\mathrm{mg} / \mathrm{kg}$ & $Q$ & $\mathrm{PQL}$ & $\mathrm{mg} / \mathrm{kg}$ & $Q$ & PQL & $\mathrm{mg} / \mathrm{kg}$ & $Q$ & PQL \\
\hline OB A.1 & $\mathrm{J} 12 \mathrm{WK} 0$ & $7 / 11 / 06$ & 4440 & & 2.3 & 0.43 & UJ & 0.43 & 2.6 & & 0.59 & 77.1 & & 0.02 & 0.47 & & 0.02 & 3.0 & & 0.23 \\
\hline $\mathrm{OB} A 2$ & $\mathrm{~J} 12 \mathrm{WK} 1$ & $7 / 11 / 06$ & 4930 & & 2.3 & 0.43 & UJ & 0.43 & 2.7 & & 0.59 & 48.4 & & 0.02 & 0.49 & & 0.02 & 1.6 & & 0.23 \\
\hline OB A3 & $\mathrm{J} 12 \mathrm{WK} 2$ & $7 / 11 / 06$ & 4650 & & 2.3 & 0.42 & UJ & 0.42 & 2.3 & & 0.59 & 54.6 & & 0.02 & 0.42 & & 0.02 & 1.7 & & 0.23 \\
\hline $\mathrm{OB} \mathrm{A4}$ & $\mathrm{J} 12 \mathrm{WK} 3$ & $7 / 11 / 06$ & 4350 & & 2.3 & 0.42 & UJ & 0.42 & 2.7 & & 0.58 & 55.9 & & 0.02 & 0.43 & & 0.02 & 1.6 & & 0.23 \\
\hline OB B5 & $\mathrm{J12WK4}$ & $7 / 11 / 06$ & 5330 & & 2.3 & 0.42 & UJ & 0.42 & 3.1 & & 0.59 & 51.4 & & 0.02 & 0.54 & & 0.02 & 1.4 & & 0.23 \\
\hline $\mathrm{OB} \mathrm{B6}$ & J12WK 5 & $7 / 11 / 06$ & 4810 & & 2.3 & 0.42 & UJ & 0.42 & 2.9 & & 0.59 & 56.4 & & 0.02 & 0.55 & & 0.02 & 1.4 & & 0.23 \\
\hline OB B7 & J12WK6 & $7 / 11 / 06$ & 4720 & & 2.3 & 0.42 & $\mathrm{UJ}$ & 0.42 & 2.2 & & 0.59 & 46.2 & & 0.02 & 0.43 & & 0.02 & 1.6 & & 0.23 \\
\hline OB B8 & J12WM7 & $7 / 17 / 06$ & 5540 & & 8.0 & 1.2 & UJ & 1.2 & 2.7 & & 1.7 & 60.0 & & 0.06 & 0.36 & & 0.06 & 1.8 & & 0.67 \\
\hline OB C9 & J12WM8 & $7 / 17 / 06$ & 4610 & & 8.1 & 1.2 & UJ & 1.2 & 2.3 & & 1.7 & 53.7 & & 0.06 & 0.30 & & 0.06 & 1.9 & & 0.67 \\
\hline $\mathrm{OBC} 10$ & J12WM9 & $7 / 17 / 06$ & 4440 & & 8.1 & 1.2 & UJ & 1.2 & 2.5 & & 1.7 & 44.9 & & 0.06 & 0.27 & & 0.06 & 1.2 & & 0.68 \\
\hline $\mathrm{OBCl}$ & J12WNO & 7/17/06, & 5080 & & 8.1 & 1.2 & UJ & 1.2 & 2.4 & & 1.7 & .49 .8 & & 0.06 & 0.33 & & 0.06 & 0.86 & & 0.68 \\
\hline $\mathrm{OB} C 2$ & $\mathrm{~J} 12 \mathrm{WN} 1$ & $7 / 17 / 06$ & 4510 & & 8.1 & 1.2 & UJ & 1.2 & 2.4 & & 1.7 & 43.0 & & 0.06 & 0.28 & & 0.06 & 1.8 & & 0.67 \\
\hline OB D3 & .112 WM5 & $7 / 12 / 06$ & 5160 & & 8.2 & 1.3 & UJ & 1.3 & 3.5 & & 1.7 & 51.7 & & 0.06 & 0.45 & & 0.06 & 1.5 & & 0.69 \\
\hline OB D4 & J12WM6 & $7 / 12 / 06$ & 5810 & & 8.2 & 1.2 & $\mathrm{UJ}$ & 1.2 & 4.0 & & 1.7 & 52.4 & & 0.06 & 0.48 & & 0.06 & 1.2 & & 0.68 \\
\hline OB DS & J12WM3 & $7 / 12 / 06$ & 4990 & & 8.1 & 1.2 & UJ & 1.2 & 3.6 & & 1.7 & 83.5 & & 0.06 & 0.53 & & 0.06 & 2.5 & & 0.67 \\
\hline OB D6 & J12WM4 & $7 / 12 / 06$ & 5170 & & 8.1 & 1.2 & UJ & 1.2 & 3.1 & & 1.7 & 48.4 & & 0.06 & 0.49 & & 0.06 & 2.0 & & 0.67 \\
\hline BCL Al & $J 12 W N 2$ & $7 / 20 / 06$ & 5200 & & 8.1 & 1.2 & UJ & 1.2 & 3.1 & & 1.7 & 57.0 & C & 0.06 & 0.47 & & 0.06 & 2.0 & & 0.68 \\
\hline $\mathrm{BCL} A 2$ & J12WM1 & $7 / 12 / 06$ & 4960 & & 8.1 & 1.2 & U. & 1.2 & 3.7 & & 1.7 & 53.7 & & 0.06 & 0.59 & & 0.06 & 1.6 & & 0.68 \\
\hline $\mathrm{BCL} A 3$ & J12WM2 & $7 / 12 / 06$ & 5420 & & 8.2 & 1.3 & UJ & 1.3 & 3.6 & & 1.7 & 56.8 & & 0.06 & 0.58 & & 0.06 & 1.4 & & 0.68 \\
\hline $\mathrm{BCLA4}$ & J12WMO & $7 / 12 / 06$ & 4890 & & 8.2 & 1.2 & UJ & 1.2 & 3.1 & & 1.7 & 62.0 & & 0.06 & 0.62 & & 0.06 & 2.9 & & 0.68 \\
\hline BCL B5 & J12WL9 & $7 / 12 / 06$ & 5850 & & 8.2 & 1.3 & UJ & 1.3 & 3.5 & & 1.7 & 55.4 & & 0.06 & 0.49 & & 0.06 & 1.3 & & 0.68 \\
\hline BCL B6 & J12WL8 & $7 / 12 / 06$ & 5450 & & 8.2 & 1.3 & UJ & 1.3 & 3.2 & & 1.7 & 56.9 & & 0.06 & 0.47 & & 0.06 & 1.5 & & 0.68 \\
\hline $\mathrm{BCL} B 7$ & J12WL7 & $7 / 12 / 06$ & 5800 & & 8.1 & 1.2 & UJ & 1.2 & 3.7 & & 1.7 & 59.7 & & 0.06 & 0.46 & & 0.06 & 1.5 & & 0.68 \\
\hline BCL B 8 & J12WL6 & $7 / 12 / 06$ & 5170 & & 8.2 & 1.2 & UJ & 1.2 & 3.3 & & 1.7 & 54.2 & & 0.06 & 0.43 & & 0.06 & 1.7 & & 0.68 \\
\hline Split of J12WL6 & J12WW0 & $7 / 12 / 06$ & 7690 & $\mathrm{~N}$ & 6.3 & 1.4 & NJ & 0.33 & 3.8 & & 0.28 & 69.9 & & 0.50 & 0.34 & B* & 0.071 & 2.8 & $\mathrm{~B}^{*} \mathrm{C}$ & 1.5 \\
\hline BCL C9 & J12WK7 & $7 / 11 / 06$ & 5880 & & 2.3 & 0.42 & UJ & 0.42 & 2.8 & & 0.58 & 71.5 & & 0.02 & 0.58 & & 0.02 & 2.8 & & 0.23 \\
\hline $\mathrm{BCLC} 10$ & $312 \mathrm{WK} 9$ & $7 / 11 / 06$ & 5840 & & 2.3 & 0.43 & UJ & 0.43 & 3.8 & & 0.59 & 59.8 & & 0.02 & 0.60 & & 0.02 & 1.5 & & 0.23 \\
\hline $\mathrm{BCLC1}$ & J12WK8 & $7 / 11 / 06$ & 5540 & & 2.3 & 0.43 & UJ & 0.43 & 2.9 & & 0.59 & 75.4 & & 0.02 & 0.57 & & 0.02 & 1.5 & & 0.23 \\
\hline $\mathrm{BCLC2}$ & J12WL2 & $7 / 11 / 06$ & 5750 & & 2.3 & 0.42 & UJ & 0.42 & 4.8 & & 0.59 & 54.8 & & 0.02 & 0.58 & & 0.02 & 1.2 & & 0.23 \\
\hline BCL D3 & J12WL3 & $7 / 11 / 06$ & 5550 & & 2.3 & 0.43 & UJ & 0.43 & 3.0 & & 0.59 & 44.8 & & 0.02 & 0.50 & & 0.02 & 1.2 & & 0.23 \\
\hline BCL D4 & J12WL4 & $7 / 11 / 06$ & 5100 & & 2.3 & 0.42 & UJ & 0.42 & 2.8 & & 0.58 & 52.7 & & 0.02 & 0.44 & & 0.02 & 1.3 & & 0.23 \\
\hline Duplicate of J12WL4 & J12WL5 & $7 / 11 / 06$ & 4800 & & 2.3 & 0.42 & UJ & 0.42 & 3.0 & & 0.59 & 59.5 & & 0.02 & 0.45 & & 0.02 & 1.9 & & 0.23 \\
\hline BCL D5 & J12WLO & $7 / 11 / 06$ & 5020 & & 2.3 & 0.42 & UJ & 0.42 & 3.2 & & 0.59 & 49.7 & & 0.02 & 0.50 & & 0.02 & 1.1 & & 0.23 \\
\hline BCL D6 & J12WL1 & 7/11/06 & 6610 & & 2.3 & 0.42 & UJ & 0.42 & 4.1 & & 0.58 & 50.6 & & 0.02 & 0.58 & & 0.02 & 1.3 & & 0.23 \\
\hline
\end{tabular}

Attachment 1 Sheet No. 17 of 113

Originator J.M. Capron Date 10/03/07

Checked T.M. Blakley

Calc. No. $\quad$ 0100B-CA-V0299 Rev. No. 
Attachment 1. 118-B-1 Verification Sampling Results.

\begin{tabular}{|c|c|c|c|c|c|c|c|c|c|c|c|c|c|c|c|c|c|c|c|c|}
\hline \multirow{2}{*}{ Sample Location } & \multirow{2}{*}{$\begin{array}{c}\text { Sample } \\
\text { Number } \\
\end{array}$} & \multirow{2}{*}{$\begin{array}{c}\text { Sample } \\
\text { Date }\end{array}$} & \multicolumn{3}{|c|}{ Aluminum } & \multicolumn{3}{|c|}{ Antimony } & \multicolumn{3}{|c|}{ Arsenic } & \multicolumn{3}{|c|}{ Barium } & \multicolumn{3}{|c|}{ Beryllium } & \multicolumn{3}{|c|}{ Boron } \\
\hline & & & $\mathrm{mg} / \mathrm{kg}$ & $Q$ & $\mathrm{PQL}$ & $\mathrm{mg} / \mathrm{kg}$ & $Q$ & PQL & $\mathrm{mg} / \mathrm{kg}$ & $Q$ & PQL & $\mathrm{mg} / \mathrm{kg}$ & $\mathbf{Q}$ & PQL & $\mathrm{mg} / \mathrm{kg}$ & $\mathbf{Q}$ & PQL & $\mathrm{mg} / \mathrm{kg}$ & $Q$ & PQL \\
\hline SPA Al & J13HY3 & $10 / 12 / 06$ & 5520 & & 6.5 & 1.0 & $\mathrm{U}$ & 1.0 & 2.7 & & 1.1 & 80.5 & & 0.06 & 0.31 & & 0.03 & 2.6 & & 1.1 \\
\hline SPA A2 & J13HY4 & $10 / 12 / 06$ & 5660 & & 6.4 & 1.0 & $\mathrm{U}$ & 1.0 & 2.7 & & 1.0 & 74.6 & & 0.06 & 0.31 & & 0.03 & 2.0 & & 1.0 \\
\hline SPA A3 & J13HY5 & $10 / 12 / 06$ & 6080 & & 6.4 & 1.0 & $\mathrm{U}$ & 1.0 & 2.2 & & 1.0 & 73.7 & & 0.06 & 0.31 & & 0.03 & 1.8 & & 1.0 \\
\hline SPA A4 & J13HY6 & $10 / 12 / 06$ & 5570 & & 6.5 & 1.0 & U & 1.0 & 2.0 & & 1.1 & 74.6 & & 0.06 & 0.31 & & 0.03 & 2.2 & & 1.1 \\
\hline SPA B5 & J13HY7 & $10 / 12 / 06$ & 5200 & & 6.4 & 1.0 & $\mathrm{U}$ & 1.0 & 2.3 & & 1.0 & 63.8 & & 0.06 & 0.26 & & 0.03 & 1.5 & & 1.0 \\
\hline Duplicate of J13HY7 & $\mathrm{J} 13 \mathrm{~J} 09$ & $10 / 12 / 06$ & 5550 & & 6.5 & 1.0 & $\mathrm{U}$ & 1.0 & 2.4 & & 1.0 & 67.8 & & 0.06 & 0.26 & & 0.03 & 1.8 & & 1.0 \\
\hline Split of J13HY7 & $\mathrm{J} 13 \mathrm{J10}$ & $10 / 12 / 06$ & 8360 & $\mathrm{~N}$ & 6.2 & 1.0 & $\mathrm{NJ}$ & 0.33 & 3.0 & & 0.28 & 84.8 & & 0.50 & 0.35 & $\mathrm{~B}^{*}$ & 0.071 & 7.0 & $\mathrm{~B}^{*} \mathrm{C}$ & 1.5 \\
\hline SPA B6 & J13HY8 & $10 / 17 / 06$ & 5430 & & 2.3 & 0.36 & U & 0.36 & 2.3 & & 0.37 & 65.2 & & 0.02 & 0.42 & C & 0.01 & 1.3 & & 0.37 \\
\hline SPA B7 & J13HY9 & $10 / 17 / 06$ & 5850 & & 2.3 & 0.36 & $\mathrm{U}$ & 0.36 & 2.1 & & 0.37 & 65.0 & & 0.02 & 0.45 & $\mathrm{C}$ & 0.01 & 1.9 & & 0.37 \\
\hline SPA B8 & $\mathrm{J} 13 \mathrm{~J} 00$ & $10 / 17 / 06$ & 5800 & & 2.3 & 0.36 & $\mathrm{U}$ & 0.36 & 2.4 & & 0.37 & 83.4 & & 0.02 & 0.38 & C & 0.01 & 3.0 & & 0.37 \\
\hline SPA C9 & J13J01 & $10 / 17 / 06$ & 5350 & & 2.4 & 0.38 & $U$ & 0.38 & 2.3 & & 0.39 & 66.3 & & 0.02 & 0.37 & $\mathrm{C}$ & 0.01 & 2.1 & & 0.39 \\
\hline SPA C10 & $\mathrm{J} 13 \mathrm{~J} 02$ & $10 / 18 / 06$ & 4900 & & 7.0 & 1.1 & UJ & 1.1 & 1.6 & & 1.1 & 60.7 & & 0.06 & 0.30 & C & 0.03 & 1.8 & & 1.1 \\
\hline SPACl & $\mathrm{J} 13 \mathrm{~J} 03$ & $10 / 18 / 06$ & 4890 & & 6.9 & 1.1 & UJ & 1.1 & 1.6 & & 1.1 & 57.5 & & 0.06 & 0.39 & C & 0.03 & 1.6 & & 1.1 \\
\hline SPA C2 & .113 .04 & $10 / 18 / 06$ & 3590 & & 6.9 & 1.1 & UJ & 1.1 & 1.1 & $\mathrm{U}$ & 1.1 & 33.4 & & 0.06 & 0.38 & $\mathrm{C}$ & 0.03 & 1.1 & $\mathrm{U}$ & 1.1 \\
\hline SPA D3 & J13J05 & $10 / 18 / 06$ & 4730 & & 6.8 & 1.1 & UJ & 1.1 & 1.1 & $\mathrm{U}$ & 1.1 & 67.5 & & 0.06 & 0.41 & $\mathrm{C}$ & 0.03 & 1.1 & $\mathrm{U}$ & 1.1 \\
\hline SPA D4 & $\mathrm{J} 13.06$ & $10 / 19 / 06$ & 5300 & $\mathrm{C}$ & 6.9 & 1.1 & UJ & 1.1 & 2.1 & & 1.1 & 62.9 & & 0.06 & 0.13 & $\mathrm{C}$ & 0.03 & 1.1 & $\mathrm{U}$ & 1.1 \\
\hline SPA D5 & $\mathrm{J} 13 \mathrm{~J} 07$ & $10 / 23 / 06$ & 5140 & $\mathrm{C}$ & 6.6 & 1.0 & UJ & 1.0 & 1.8 & & 1.1 & 85.0 & & 0.06 & 0.29 & $\mathrm{C}$ & 0.03 & 1.1 & $\mathrm{U}$ & 1.1 \\
\hline SPA D6 & 313108 & $10 / 23 / 06$ & 4730 & $\mathrm{C}$ & 6.7 & 1.1 & \begin{tabular}{|l|}
$\mathrm{UJ}$ \\
\end{tabular} & 1.1 & 2.5 & & 1.1 & 56.0 & & 0.06 & 0.30 & $\mathrm{C}$ & 0.03 & 1.7 & & 1.1 \\
\hline FS-2 & $\mathrm{J} 12 \mathrm{R} 25$ & $7 / 10 / 06$ & 4360 & & 8.1 & 1.2 & \begin{tabular}{|l|}
$\mathrm{UJ}$ \\
\end{tabular} & 1.2 & 1.7 & $\mathrm{U}$ & 1.7 & 52.3 & & 0.06 & 0.30 & & 0.06 & 1.1 & & 0.67 \\
\hline FS-3 & $J 12 R 26$ & $7 / 10 / 06$ & 7200 & & 8.2 & 1.2 & UJ & 1.2 & 4.0 & & 1.7 & 55.5 & & 0.06 & 0.42 & & 0.06 & 1.8 & & 0.68 \\
\hline FS-5 & $J 12 R 28$ & $7 / 10 / 06$ & 6170 & & 6.5 & 1.0 & UJ & 1.0 & 4.2 & & 1.1 & 53.0 & & 0.06 & 0.50 & & 0.03 & 2.3 & & 1.1 \\
\hline FS-6 & J12R29 & $7 / 10 / 06$ & 10100 & & 8.1 & 1.2 & UJ & 1.2 & 4.4 & & 1.7 & 209 & & 0.06 & 0.58 & & 0.06 & 16.1 & & 0.67 \\
\hline FS-7 & $J 13 P 85$ & $11 / 15 / 06$ & 4600 & & 6.5 & 0.77 & U.J & 0.77 & 1.7 & & 0.96 & 50.0 & C & 0.03 & 0.24 & & 0.03 & 0.61 & & 0.59 \\
\hline FS-8 & $\mathrm{J} 12 \mathrm{R} 31$ & $7 / 10 / 06$ & 6770 & & 8.2 & 1.3 & UJ & 1.3 & 2.1 & & 1.7 & 261 & & 0.06 & 0.47 & & 0.06 & 24.5 & & 0.68 \\
\hline FS-9 & $.12 R 32$ & $7 / 10 / 06$ & 6200 & & 8.2 & 1.3 & UJ & 1.3 & 2.9 & & 1.7 & 61.6 & & 0.06 & 0.37 & & 0.06 & 13.1 & & 0.69 \\
\hline FS-11 & $\mathrm{J12R34}$ & $7 / 10 / 06$ & 6980 & & 6.5 & 1.0 & UJ & 1.0 & 7.0 & & 1.1 & 61.0 & & 0.06 & 0.46 & & 0.03 & 1.6 & & 1.1 \\
\hline FS-14 & $.112 \times B 3$ & $7 / 19 / 06$ & 4700 & & 8.8 & 1.3 & \begin{tabular}{|l|} 
U. \\
\end{tabular} & 1.3 & 1.9 & & 1.9 & 45.5 & $\mathrm{C}$ & 0.06 & 0.51 & & 0.06 & 4.1 & & 0.73 \\
\hline FS-15 & $113 \mathrm{NX} 4$ & $11 / 14 / 06$ & 3630 & & 6.5 & 0.77 & UJ & 0.77 & 1.4 & & 0.95 & 38.6 & $\mathrm{C}$ & 0.03 & 0.18 & & 0.03 & 0.58 & $\mathrm{U}$ & 0.58 \\
\hline FS-16 & J13P84 & $11 / 15 / 06$ & 3730 & & 6.3 & 0.75 & UJ & 0.75 & 1.0 & & 0.93 & 36.8 & $\mathrm{C}$ & 0.03 & 0.23 & & 0.03 & 0.57 & $U$ & 0.57 \\
\hline FS-17 & J14.J3 & $3 / 5 / 07$ & 6480 & & 1.4 & 0.31 & UJ & 0.31 & 2.8 & & 0.40 & 64.3 & & 0.02 & 0.40 & & 0.01 & 1.7 & & 0.37 \\
\hline FS-17 resample & $\mathrm{J} 152 \mathrm{M} 1$ & $5 / 10 / 07$ & 6200 & & 1.4 & 0.31 & $U$ & 0.31 & 2.7 & & 0.39 & 63.4 & & 0.02 & 0.46 & & 0.01 & 1.8 & & 0.37 \\
\hline FS-18 & J14L38 & $3 / 5 / 07$ & 5780 & & 1.4 & 0.31 & $\mathrm{UJ}$ & 0.31 & 2.6 & & 0.40 & 61.3 & & 0.02 & 0.35 & & 0.01 & 1.8 & & 0.37 \\
\hline FS-19 & J14L39 & $3 / 5 / 07$ & 5380 & & 1.4 & 0.31 & UJ & 0.31 & 2.3 & & 0.40 & 57.6 & & 0.02 & 0.35 & & 0.01 & 1.3 & & 0.37 \\
\hline Equipment blank & $\mathrm{J} 12 \times 59$ & $7 / 20 / 06$ & 151 & & 8.1 & 1.2 & $\mathrm{UJ}$ & 1.2 & 1.7 & $\mathrm{U}$ & 1.7 & 3.9 & C & 0.06 & 0.18 & & 0.06 & 0.67 & $\bar{U}$ & 0.67 \\
\hline
\end{tabular}

\begin{tabular}{|c|c|c|c|}
\hline Attachment & 1 & Sheet No. & 18 of 113 \\
\hline Originator & J. M. Capron & Date & $10 / 03 / 07$ \\
\hline Checked & T. M. Blakley & Date & \\
\hline Calc. No. & 0100B-CA-V0299 & Rev. No. & 1 \\
\hline
\end{tabular}


Attachment 1. 118-B-1 Verification Sampling Results.

\begin{tabular}{|c|c|c|c|c|c|c|c|c|c|c|c|c|c|c|c|c|c|c|c|c|}
\hline \multirow[t]{2}{*}{ Sample Location } & \multirow{2}{*}{$\begin{array}{l}\text { Sample } \\
\text { Number }\end{array}$} & \multirow{2}{*}{$\begin{array}{l}\text { Sample } \\
\text { Date }\end{array}$} & \multicolumn{3}{|c|}{ Cadmium } & \multicolumn{3}{|c|}{ Calcium } & \multicolumn{3}{|c|}{ Chromium } & \multicolumn{3}{|c|}{ Cobalt } & \multicolumn{3}{|c|}{ Copper } & \multicolumn{3}{|c|}{$\begin{array}{l}\text { Hexavalent } \\
\text { Chromium }\end{array}$} \\
\hline & & & $\mathrm{mg} / \mathrm{kg}$ & $Q$ & PQL & $\mathrm{mg} / \mathrm{kg}$ & $Q$ & PQL & $\mathrm{mg} / \mathrm{kg}$ & $Q$ & PQL & $\mathrm{mg} / \mathrm{kg}$ & $Q$ & PQL & $\mathrm{mg} / \mathrm{kg}$ & $Q$ & PQL & $\mathrm{mg} / \mathrm{kg}$ & $Q$ & PQL \\
\hline Area $1 \mathrm{Al}$ & $\mathrm{J} 12 \times 44$ & $7 / 19 / 06$ & 0.20 & $\mathrm{U}$ & 0.20 & 6860 & $\mathrm{C}$ & 4.8 & 7.0 & & 0.38 & 10.1 & & 0.41 & 17.4 & & 0.35 & 0.20 & $\mathrm{U}$ & 0.20 \\
\hline Duplicate of J12X44 & $\mathrm{J} 12 \times 48$ & $7 / 19 / 06$ & 0.20 & $\mathrm{U}$ & 0.20 & 7560 & $\mathrm{C}$ & 4.8 & 8.2 & & 0.38 & 10.7 & & 0.41 & 17.9 & & 0.35 & 0.20 & $\mathrm{U}$ & 0.20 \\
\hline Split of J12X44 & $\mathrm{J} 12 \times 49$ & $7 / 19 / 06$ & 0.14 & UNJ & 0.14 & 6820 & $\mathrm{CN}$ & 8.6 & 6.8 & & 0.36 & 14.5 & & 0.51 & 15.7 & $\mathrm{~N}$ & 0.30 & 0.350 & UJ & 0.350 \\
\hline Area $1 \mathrm{~A} 2$ & $\mathrm{~J} 12 \times 45$ & $7 / 19 / 06$ & 0.20 & $\mathrm{U}$ & 0.20 & 7280 & $\mathrm{C}$ & 4.8 & 4.7 & & 0.38 & 9.7 & & 0.41 & 16.8 & & 0.35 & 0.20 & $U$ & 0.20 \\
\hline Area $1 \mathrm{~A} 3$ & J12X46 & $7 / 20 / 06$ & 0.20 & $U$ & 0.20 & 7150 & $\mathrm{C}$ & 4.6 & 6.4 & & 0.37 & 9.3 & & 0.40 & 17.7 & & 0.34 & 0.20 & $\mathrm{UJ}$ & 0.20 \\
\hline Area $1 \mathrm{~A} 4$ & $\mathrm{~J} 12 \times 47$ & $7 / 20 / 06$ & 0.20 & $\mathrm{U}$ & 0.20 & 7870 & $\mathrm{C}$ & 4.6 & 5.4 & & 0.37 & 9.8 & & 0.40 & 16.0 & & 0.34 & 0.20 & UJ & 0.20 \\
\hline Area $2 \mathrm{Al}$ & $\mathrm{J} 12 \times 50$ & $7 / 20 / 06$ & 0.20 & $\mathrm{U}$ & 0.20 & 5360 & $\mathrm{C}$ & 4.6 & 5.5 & & 0.36 & 8.4 & & 0.39 & 15.0 & & 0.33 & 0.20 & UJ & 0.20 \\
\hline Area $2 \mathrm{~A} 2$ & $J 12 \times 51$ & $7 / 20 / 06$ & 0.20 & $\mathrm{U}$ & 0.20 & 8200 & C & 4.7 & 6.9 & & 0.37 & 8.4 & & 0.40 & 16.8 & & 0.34 & 0.20 & UJ & 0.20 \\
\hline Area $2 \mathrm{~A} 3$ & $\mathrm{~J} 12 \times 52$ & $7 / 20 / 06$ & 0.20 & $U$ & 0.20 & 9080 & $\mathrm{C}$ & 4.7 & 5.8 & & 0.37 & 8.1 & & 0.40 & 16.2 & & 0.34 & 0.26 & $\mathrm{~J}$ & 0.20 \\
\hline Area $2 \mathrm{~A} 4$ & $\mathrm{~J} 12 \times 53$ & $7 / 20 / 06$ & 0.20 & $\mathrm{U}$ & 0.20 & 5120 & $\mathrm{C}$ & 4.6 & 6.5 & & 0.37 & 7.1 & & 0.39 & 13.3 & & 0.34 & 0.33 & $J$ & 0.20 \\
\hline Area $3 \mathrm{Al}$ & $\mathrm{J} 12 \times 54$ & $7 / 20 / 06$ & 0.20 & $\mathrm{U}$ & 0.20 & 6080 & $\mathrm{C}$ & 4.6 & 5.4 & & 0.36 & 7.8 & & 0.39 & 15.6 & & 0.33 & 0.20 & UJ & 0.20 \\
\hline Area $3 \mathrm{~A} 2$ & $\mathrm{~J} 12 \times 55$ & $7 / 20 / 06$ & 0.20 & $\mathrm{U}$ & 0.20 & 7310 & C & 4.6 & 5.9 & & 0.36 & 8.8 & & 0.39 & 16.6 & & 0.34 & 0.20 & UJ & 0.20 \\
\hline Area $3 \mathrm{A3}$ & $.112 \times 56$ & $7 / 20 / 06$ & 0.20 & $\mathrm{U}$ & 0.20 & 9860 & C & 4.6 & 9.2 & & 0.37 & 8.9 & & 0.40 & 18.0 & & 0.34 & 0.20 & UJ & 0.20 \\
\hline Duplicate of J12X56 & $\mathrm{J} 12 \times 58$ & $7 / 20 / 06$ & 0.20 & $\mathrm{U}$ & 0.20 & 9650 & $\mathrm{C}$ & 4.7 & 8.2 & & 0.37 & 8.9 & & 0.40 & 16.9 & & 0.34 & 0.21 & $\mathrm{~J}$ & 0.20 \\
\hline Split of J12X56 & $.112 \times 60$ & $7 / 20 / 06$ & 0.14 & UNJ & 0.14 & 7870 & $\mathrm{C}$ & 8.7 & 6.5 & & 0.36 & 10.2 & & 0.51 & 14.4 & & 0.31 & 0.350 & UJ & 0.350 \\
\hline Area 3 A4 & $\mathrm{J} 12 \times 57$ & $7 / 20 / 06$ & 0.20 & $\mathrm{U}$ & 0.20 & 6680 & $\mathrm{C}$ & 4.6 & 5.8 & & 0.36 & 9.1 & & 0.39 & 16.1 & & 0.34 & 0.20 & $\mathrm{UJ}$ & 0.20 \\
\hline Area $4 \mathrm{Al}$ & $\mathrm{J} 12 \times 61$ & $7 / 25 / 06$ & 0.20 & $\mathrm{U}$ & 0.20 & 6520 & C & 4.6 & 3.7 & & 0.36 & 8.4 & & 0.39 & 15.5 & & 0.34 & 0.30 & & 0.20 \\
\hline Duplicate of J12X61 & $\mathrm{J} 12 \times 65$ & $7 / 25 / 06$ & 0.20 & $\mathrm{U}$ & 0.20 & 7870 & C & 4.6 & 5.1 & & 0.36 & 8.6 & & 0.39 & 15.7 & & 0.34 & 0.20 & $\mathrm{U}$ & 0.20 \\
\hline Split of J12X61 & $112 \times 66$ & $7 / 25 / 06$ & 0.14 & UNJ & 0.14 & 9360 & C & 8.6 & 6.2 & & 0.36 & 13.9 & & 0.50 & 16.3 & & 0.30 & 0.350 & $\mathrm{U}$ & 0.350 \\
\hline Area 4 A2 & $\mathrm{J} 12 \times 62$ & $7 / 25 / 06$ & 0.19 & $\mathrm{U}$ & 0.19 & 5490 & C & 4.5 & 5.4 & & 0.36 & 7.9 & & 0.39 & 14.3 & & 0.33 & 0.20 & $\mathrm{U}$ & 0.20 \\
\hline Area 4 A3 & $112 \times 63$ & $7 / 25 / 06$ & 0.20 & $\mathrm{U}$ & 0.20 & 8920 & $\mathrm{C}$ & 4.6 & 6.5 & & 0.36 & 8.3 & & 0.39 & 16.5 & & 0.34 & 0.20 & $\mathrm{U}$ & 0.20 \\
\hline Area 4 A4 & $\mathrm{J} 12 \times 64$ & $7 / 25 / 06$ & 0.19 & $\mathrm{U}$ & 0.19 & 5770 & $\mathrm{C}$ & 4.5 & 6.7 & & 0.36 & 8.6 & & 0.39 & 15.6 & & 0.33 & 0.24 & & 0.20 \\
\hline Area $5 \mathrm{Al}$ & $\mathrm{J} 13 \mathrm{~V} 12$ & $12 / 7 / 06$ & 0.13 & & 0.09 & 4550 & $\mathrm{CJ}$ & 3.8 & 3.9 & & 0.37 & 6.0 & & 0.15 & 12.7 & & 0.21 & 0.21 & $\mathrm{U}$ & 0.21 \\
\hline Area $5 \mathrm{A2}$ & I13V10 & $12 / 7 / 06$ & 0.14 & & 0.09 & 5050 & $\mathrm{CJ}$ & 3.8 & 5.0 & & 0.36 & 6.9 & & 0.15 & 13.0 & & 0.21 & 0.21 & $\mathrm{U}$ & 0.21 \\
\hline Area $5 \mathrm{~A} 3$ & $113 \mathrm{~V} 13$ & $12 / 7 / 06$ & 0.07 & & 0.03 & 1770 & $\mathrm{CJ}$ & 1.3 & 2.1 & & 0.12 & 2.3 & & 0.05 & 4.5 & & 0.07 & 0.26 & & 0.21 \\
\hline Area $5 \mathrm{~A} 4$ & J13V11 & $12 / 7 / 06$ & 0.23 & & 0.09 & 11900 & $\mathrm{CJ}$ & 3.8 & 4.6 & & 0.37 & 6.6 & & 0.15 & 14.3 & & 0.22 & 0.21 & $\mathrm{U}$ & 0.21 \\
\hline Area $6 \mathrm{Al}$ & $\mathrm{J} 12 \times \mathrm{C} 2$ & $7 / 25 / 06$ & 0.20 & $\mathrm{U}$ & 0.20 & 5890 & C & 4.6 & 7.4 & & 0.36 & 7.1 & & 0.39 & 13.7 & & 0.33 & 0.20 & $\mathrm{U}$ & 0.20 \\
\hline Area $6 \mathrm{~A} 2$ & $\mathrm{J12 \times C3}$ & $7 / 25 / 06$ & 0.20 & $\mathrm{U}$ & 0.20 & 6030 & C & 4.6 & 7.1 & & 0.36 & 7.8 & & 0.39 & 14.3 & & 0.34 & 0.24 & & 0.20 \\
\hline Area $6 \mathrm{~A} 3$ & $J 12 \times C 4$ & $7 / 25 / 06$ & 0.20 & $\mathrm{U}$ & 0.20 & 10100 & $\mathrm{C}$ & 4.6 & 6.2 & & 0.36 & 8.4 & & 0.39 & 16.3 & & 0.34 & 0.22 & & 0.20 \\
\hline Area $6 \mathrm{~A} 4$ & $312 \times \mathrm{C} 5$ & $7 / 25 / 06$ & 0.20 & $\mathrm{U}$ & 0.20 & 14500 & C & 4.6 & 7.1 & & 0.36 & 8.5 & & 0.39 & 16.9 & & 0.34 & 0.28 & & 0.20 \\
\hline Area $7 \mathrm{Al}$ & $512 \times \mathrm{C} 6$ & $7 / 26 / 06$ & 0.19 & $\mathrm{U}$ & 0.19 & 7420 & C & 4.6 & 7.6 & & 0.36 & 6.3 & & 0.39 & 25.7 & & 0.33 & 0.20 & $\mathrm{U}$ & 0.20 \\
\hline Area $7 \mathrm{~A} 2$ & $\mathrm{J12 \times C7}$ & $7 / 26 / 06$ & 0.19 & $\mathrm{U}$ & 0.19 & 4550 & $\mathrm{C}$ & 4.5 & 9.5 & & 0.36 & 7.6 & & 0.39 & 14.4 & & 0.33 & 0.30 & & 0.20 \\
\hline Area $7 \mathrm{A3}$ & $\mathrm{J1} 2 \mathrm{XC} 8$ & $7 / 26 / 06$ & 0.20 & $\mathrm{U}$ & 0.20 & 4270 & C & 4.6 & 7.3 & & 0.37 & 6.3 & & 0.39 & 11.7 & & 0.34 & 0.27 & & 0.20 \\
\hline \multirow[t]{5}{*}{ Area 7 A4 } & $312 \times C 9$ & $7 / 26 / 06$ & 0.20 & $\mathrm{U}$ & 0.20 & 6150 & C & 4.6 & 8.0 & & 0.36 & 7.4 & & 0.39 & 17.2 & & 0.34 & 0.20 & $\mathrm{U}$ & 0.20 \\
\hline & & & & & & & & & & & & & \multirow{4}{*}{\multicolumn{2}{|c|}{$\begin{array}{l}\text { Attachment } \\
\text { Originator } \\
\text { Checked } \\
\text { Calc. No. }\end{array}$}} & \multicolumn{3}{|c|}{1} & \multirow{4}{*}{$\begin{array}{l}\text { Sheet No. } \\
\text { Date } \\
\text { Date } \\
\text { Rev. No. }\end{array}$} & \multicolumn{2}{|c|}{19 of 113} \\
\hline & & & & & & & & & & & & & & & \multirow{2}{*}{\multicolumn{3}{|c|}{ J. M. Capron }} & & \multicolumn{2}{|c|}{$10 / 03 / 07$} \\
\hline & & & & & & & & & & & & & & & & & & & & \\
\hline & & & & & & & & & & & & & & & \multicolumn{3}{|c|}{ 0100B-CA-V0299 } & & & 1 \\
\hline
\end{tabular}


Attachment 1. 118-B-1 Verification Sampling Results.

\begin{tabular}{|c|c|c|c|c|c|c|c|c|c|c|c|c|c|c|c|c|c|c|c|c|}
\hline \multirow[t]{2}{*}{ Sample Location } & \multirow{2}{*}{$\begin{array}{l}\text { Sample } \\
\text { Number }\end{array}$} & \multirow{2}{*}{$\begin{array}{l}\text { Sample } \\
\text { Date }\end{array}$} & \multicolumn{3}{|c|}{ Cadmium } & \multicolumn{3}{|c|}{ Calcium } & \multicolumn{3}{|c|}{ Chromium } & \multicolumn{3}{|c|}{ Cobalt } & \multicolumn{3}{|c|}{ Copper } & \multicolumn{3}{|c|}{$\begin{array}{l}\text { Hexavalent } \\
\text { Chromium }\end{array}$} \\
\hline & & & $\mathrm{mg} / \mathrm{kg}$ & $Q$ & PQL & $\mathrm{mg} / \mathrm{kg}$ & $Q$ & PQL & $\mathrm{mg} / \mathrm{kg}$ & $Q$ & PQL & $\mathrm{mg} / \mathrm{kg}$ & Q & PQL & $\mathrm{mg} / \mathrm{kg}$ & $Q$ & $\mathrm{PQL}$ & $\mathrm{mg} / \mathrm{kg}$ & $Q$ & $\mathrm{PQL}$ \\
\hline $\mathrm{OB} \mathrm{Al}$ & $\mathrm{J} 12 \mathrm{WK} 0$ & $7 / 11 / 06$ & 0.41 & $\mathrm{U}$ & 0.41 & 5710 & C & 2.1 & 5.9 & & 0.13 & 8.1 & & 0.14 & 16.2 & & 0.12 & 0.31 & & 0.20 \\
\hline $\mathrm{OB} \mathrm{A2}$ & J12WK1 & $7 / 11 / 06$ & 0.41 & $\mathrm{U}$ & 0.41 & 5300 & C & 2.1 & 7.2 & & 0.13 & 8.5 & & 0.14 & 14.3 & & 0.12 & 0.27 & & 0.20 \\
\hline $\mathrm{OBA3}$ & J12WK2 & $7 / 11 / 06$ & 0.43 & & 0.41 & 4350 & $\mathrm{C}$ & 2.1 & 6.9 & & 0.13 & 7.2 & & 0.14 & 12.7 & & 0.12 & 0.20 & $\mathrm{U}$ & 0.20 \\
\hline $\mathrm{OB} \mathrm{A4}$ & $\mathrm{J} 12 \mathrm{WK} 3$ & $7 / 11 / 06$ & 0.42 & & 0.40 & 4610 & C & 2.1 & 6.7 & & 0.12 & 7.7 & & 0.13 & 12.4 & & 0.12 & 0.20 & $\mathrm{U}$ & 0.20 \\
\hline OB B5 & J12WK4 & $7 / 11 / 06$ & 0.41 & $\mathrm{U}$ & 0.41 & 5650 & C & 2.1 & 7.7 & & 0.13 & 9.4 & & 0.14 & 14.6 & & 0.12 & 0.30 & & 0.20 \\
\hline $\mathrm{OB} B 6$ & J12WK5 & $7 / 11 / 06$ & 0.40 & $\mathrm{U}$ & 0.40 & 5680 & C & 2.1 & 6.4 & & 0.13 & 8.0 & & 0.13 & 15.3 & & 0.12 & 0.27 & & 0.20 \\
\hline OB B7 & J12WK6 & $7 / 11 / 06$ & 0.40 . & $\mathrm{U}$ & 0.40 & 5590 & $\mathrm{C}$ & 2.1 & 8.2 & & 0.13 & 6.5 & & 0.13 & 14.8 & & 0.12 & 0.30 & & 0.20 \\
\hline OB B8 & J12WM7 & $7 / 17 / 06$ & 0.28 & & 0.20 & 4360 & C & 4.6 & 8.0 & & 0.36 & 7.9 & & 0.39 & 15.2 & & 0.33 & 0.20 & $\mathrm{U}$ & 0.20 \\
\hline OB C9 & J12WM8 & $7 / 17 / 06$ & 0.31 & & 0.20 & 3660 & $\mathrm{C}$ & 4.6 & 6.9 & & 0.36 & 7.2 & & 0.39 & 14.3 & & 0.34 & 0.20 & $\mathrm{U}$ & 0.20 \\
\hline $\mathrm{OBC}$ & J12WM9 & $7 / 17 / 06$ & 0.32 & & 0.20 & 3570 & $\mathrm{C}$ & 4.6 & 6.6 & & 0.37 & 7.3 & & 0.39 & 14.9 & & 0.34 & 0.25 & & 0.20 \\
\hline $\mathrm{OBCl}$ & J12WN0 & $7 / 17 / 06$ & 0.32 & & 0.20 & 4930 & C & 4.6 & 6.5 & & 0.37 & 9.6 & & 0.40 & 18.1 & & 0.34 & 0.26 & & 0.20 \\
\hline $\mathrm{OBC} 2$ & $J 12 \mathrm{WN} 1$ & $7 / 17 / 06$ & 0.26 & & 0.20 & 4760 & C & 4.6 & 5.7 & & 0.37 & 8.1 & & 0.39 & 15.1 & & 0.34 & 0.20 & $\mathrm{U}$ & 0.20 \\
\hline OB D3 & J12WM5 & $7 / 12 / 06$ & 0.20 & U & 0.20 & 5900 & & 4.7 & 6.6 & & 0.37 & 9.0 & & 0.40 & 16.7 & & 0.34 & 0.23 & & 0.20 \\
\hline OB D4 & J12WM6 & $7 / 12 / 06$ & 0.20 & $\mathrm{U}$ & 0.20 & 6750 & & 4.6 & 7.5 & & 0.37 & 10.2 & & 0.40 & 17.0 & & 0.34 & 0.20 & $\mathrm{U}$ & 0.20 \\
\hline OB D5 & J12WM3 & $7 / 12 / 06$ & 0.20 & $\mathrm{U}$ & 0.20 & 4690 & & 4.6 & 6.0 & & 0.37 & 8.0 & & 0.39 & 16.7 & & 0.34 & 0.22 & & 0.20 \\
\hline OB D6 & J12WM4 & $7 / 12 / 06$ & 0.20 & $\mathrm{U}$ & 0.20 & 6400 & & 4.6 & 6.4 & & 0.37 & 9.1 & & 0.39 & 16.0 & & 0.34 & 0.20 & $\mathrm{U}$ & 0.20 \\
\hline BCL A1 & J12WN2 & $7 / 20 / 06$ & 0.20 & $\mathrm{U}$ & 0.20 & 7000 & $\mathrm{C}$ & 4.6 & 6.6 & & 0.37 & 9.5 & & 0.40 & 15.9 & & 0.34 & 0.21 & $\mathrm{~J}$ & 0.20 \\
\hline BCL A2 & J12WM1 & $7 / 12 / 06$ & 0.20 & $\mathrm{U}$ & 0.20 & 6110 & & 4.6 & 6.3 & & 0.37 & 8.2 & & 0.40 & 16.2 & & 0.34 & 0.22 & & 0.20 \\
\hline $\mathrm{BCL} A 3$ & J12WM2 & $7 / 12 / 06$ & 0.20 & $\mathrm{U}$ & 0.20 & 6800 & & 4.7 & 7.2 & & 0.37 & 8.4 & & 0.40 & 16.0 & & 0.34 & 0.28 & & 0.20 \\
\hline $\mathrm{BCL} \mathrm{A4}$ & J12WM0 & $7 / 12 / 06$ & 0.20 & $\mathrm{U}$ & 0.20 & 4880 & & 4.6 & 7.2 & & 0.37 & 8.1 & & 0.40 & 16.0 & & 0.34 & 0.20 & $\mathrm{U}$ & 0.20 \\
\hline BCL B5 & J12WL9 & $7 / 12 / 06$ & 0.20 & $\mathrm{U}$ & 0.20 & 7180 & & 4.7 & 8.3 & & 0.37 & 9.2 & & 0.40 & 16.2 & & 0.34 & 0.28 & & 0.20 \\
\hline $\mathrm{BCL} B 6$ & J12WL8 & $7 / 12 / 06$ & 0.20 & $\mathrm{U}$ & 0.20 & 6180 & & 4.7 & 7.3 & & 0.37 & 8.0 & & 0.40 & 15.4 & & 0.34 & 0.21 & & 0.20 \\
\hline BCL B7 & J12WL7 & $7 / 12 / 06$ & 0.20 & $\mathrm{U}$ & 0.20 & 6120 & & 4.6 & 8.7 & & 0.37 & 7.9 & & 0.40 & 14.6 & & 0.34 & 0.20 & $\mathrm{U}$ & 0.20 \\
\hline BCL B8 & J12WL6 & $7 / 12 / 06$ & 0.20 & $U$ & 0.20 & 5750 & & 4.6 & 7.3 & & 0.37 & 7.3 & & 0.40 & 13.8 & & 0.34 & 0.28 & & 0.20 \\
\hline Split of J12WL6 & J12WW0 & $7 / 12 / 06$ & 0.14 & UNJ & 0.14 & 6270 & $\mathrm{CN}$ & 8.6 & 9.4 & & 0.36 & 10.9 & & 0.50 & 14.4 & $\mathrm{~N}$ & 0.30 & 0.350 & UJ & 0.350 \\
\hline BCL C9 & $112 \mathrm{WK} 7$ & $7 / 11 / 06$ & 0.40 & $\mathrm{U}$ & 0.40 & 5360 & $\mathrm{C}$ & 2.1 & 9.5 & & 0.12 & 7.9 & & 0.13 & 15.4 & & 0.11 & 0.20 & $\mathrm{U}$ & 0.20 \\
\hline $\mathrm{BCL} \mathrm{ClO}$ & J12WK9 & $7 / 11 / 06$ & 0.41 & $\mathrm{U}$ & 0.41 & 9220 & $\mathrm{C}$ & 2.1 & 7.3 & & 0.13 & 9.8 & & 0.14 & 17.2 & & 0.12 & 0.20 & $\mathrm{U}$ & 0.20 \\
\hline $\mathrm{BCL} \mathrm{Cl}$ & J12WK8 & $7 / 11 / 06$ & 0.41 & $\mathrm{U}$ & 0.41 & 6140 & $\mathrm{C}$ & 2.1 & 7.5 & & 0.13 & 8.6 & & 0.14 & 14.4 & & 0.12 & 0.20 & $\mathrm{U}$ & 0.20 \\
\hline $\mathrm{BCL}$ C2 & J12WL2 & $7 / 11 / 06$ & 0.40 & $\mathrm{U}$ & 0.40 & 11600 & $\mathrm{C}$ & 2.1 & 8.0 & & 0.12 & 10.8 & & 0.13 & 16.9 & & 0.12 & 0.31 & & 0.20 \\
\hline BCL D3 & J12WL3 & $7 / 11 / 06$ & 0.41 & $\mathrm{U}$ & 0.41 & 7130 & $\mathrm{C}$ & 2.1 & 7.4 & & 0.13 & 9.4 & & 0.14 & 14.2 & & 0.12 & 0.26 & & 0.20 \\
\hline BCL D4 & J12WL4 & $7 / 111 / 06$ & 0.40 & $\mathrm{U}$ & 0.40 & 5800 & $\mathrm{C}$ & 2.1 & 8.8 & & 0.12 & 7.9 & & 0.13 & 12.9 & & 0.11 & 0.36 & & 0.20 \\
\hline Duplicate of J12WL4 & J12WL5 & $7 / 11 / 06$ & 0.41 & $\mathrm{U}$ & 0.41 & 5970 & $\mathrm{C}$ & 2.1 & 7.3 & & 0.13 & 7.6 & & 0.14 & 12.9 & & 0.12 & 0.26 & & 0.20 \\
\hline BCL D5 & J12WLO & $7 / 11 / 06$ & 0.40 & $\mathrm{U}$ & 0.40 & 6820 & $\mathrm{C}$ & 2.1 & 7.1 & & 0.13 & 8.8 & & 0.13 & 14.3 & & 0.12 & 0.27 & & 0.20 \\
\hline BCL D6 & J12WL1 & $7 / 11 / 06$ & 0.40 & $\mathrm{U}$ & 0.40 & 10400 & $\mathrm{C}$ & 2.1 & 9.3 & & 0.12 & 10.0 & & 0.13 & 16.2 & & 0.11 & 0.20 & $\mathrm{U}$ & 0.20 \\
\hline
\end{tabular}

\begin{tabular}{llll} 
Attachment & \multicolumn{1}{c}{1} & Sheet No. 20 of 113 \\
Originator & D.M. Capron & Date & $10 / 03 / 07$ \\
Checked & T. M. Blakley & Date & \\
Calc. No. & 0100B-CA-V0299 Rev. No. &
\end{tabular} 
Attachment 1. 118-B-1 Verification Sampling Results.

\begin{tabular}{|c|c|c|c|c|c|c|c|c|c|c|c|c|c|c|c|c|c|c|c|c|}
\hline \multirow[t]{2}{*}{ Sample Location } & \multirow{2}{*}{$\begin{array}{l}\text { Sample } \\
\text { Number }\end{array}$} & \multirow{2}{*}{$\begin{array}{c}\text { Sample } \\
\text { Date }\end{array}$} & \multicolumn{3}{|c|}{ Cadmium } & \multicolumn{3}{|c|}{ Calcium } & \multicolumn{3}{|c|}{ Chromium } & \multicolumn{3}{|c|}{ Cobalt } & \multicolumn{3}{|c|}{ Copper } & \multicolumn{3}{|c|}{$\begin{array}{l}\text { Hexavalent } \\
\text { Chromium }\end{array}$} \\
\hline & & & $\mathrm{mg} / \mathrm{kg}$ & Q & $\mathrm{PQL}$ & $\mathrm{mg} / \mathrm{kg}$ & $Q$ & $\mathrm{PQL}$ & $\mathrm{mg} / \mathrm{kg}$ & $Q$ & $\mathrm{PQL}$ & $\mathrm{mg} / \mathrm{kg}$ & Q & $\mathrm{PQL}$ & $\mathrm{mg} / \mathrm{kg}$ & $Q$ & PQL & $\mathrm{mg} / \mathrm{kg}$ & $\mathbf{Q}$ & $\mathrm{PQL}$ \\
\hline SPAAl & J13HY3 & $10 / 12 / 06$ & 0.16 & & 0.09 & 4020 & $\mathrm{C}$ & 2.4 & 7.8 & $\mathrm{C}$ & 0.20 & 7.2 & & 0.14 & 14.4 & & 0.23 & 0.20 & $\mathrm{U}$ & 0.20 \\
\hline SPA A2 & J13HY4 & $10 / 12 / 06$ & 0.12 & & 0.08 & 3460 & $\mathrm{C}$ & 2.4 & 8.3 & $\mathrm{C}$ & 0.20 & 7.2 & & 0.14 & 14.8 & & 0.23 & 0.20 & $\mathrm{U}$ & 0.20 \\
\hline SPA A3 & J13HY5 & $10 / 12 / 06$ & 0.15 & & 0.08 & 3630 & $\mathrm{C}$ & 2.4 & 8.8 & $\mathrm{C}$ & 0.20 & 7.6 & & 0.14 & 15.2 & & 0.23 & 0.20 & $\bar{U}$ & 0.20 \\
\hline SPA A4 & J13HY6 & $10 / 12 / 06$ & 0.13 & & 0.09 & 4260 & $\mathrm{C}$ & 2.5 & 7.9 & $\mathrm{C}$ & 0.20 & 7.6 & & 0.14 & 15.3 & & 0.23 & 0.20 & $\bar{U}$ & 0.20 \\
\hline SPA B5 & J13HY7 & $10 / 12 / 06$ & 0.11 & & 0.08 & 3490 & $\mathrm{C}$ & 2.4 & 8.0 & $\mathrm{C}$ & 0.20 & 6.4 & & 0.14 & 12.9 & & 0.23 & 0.22 & & 0.20 \\
\hline Duplicate of J13HY7 & J13J09 & $10 / 12 / 06$ & 0.15 & & 0.08 & 3540 & $\mathrm{C}$ & 2.4 & 9.3 & $\mathrm{C}$ & 0.20 & 6.7 & & 0.14 & 13.6 & & 0.23 & 0.20 & $\mathrm{U}$ & 0.20 \\
\hline Split of J13HY7 & $\mathrm{J13J10}$ & $10 / 12 / 06$ & 0.14 & $\mathrm{U}$ & 0.14 & 4520 & $\mathrm{CN}$ & 8.6 & 12.5 & & 0.36 & 11.3 & & 0.50 & 15.3 & & 0.30 & 0.350 & $\mathrm{U}$ & 0.350 \\
\hline SPA B6 & J13HY8 & $10 / 17 / 06$ & 0.03 & 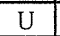 & 0.03 & 3960 & $\mathrm{C}$ & 0.86 & 8.2 & $\mathrm{C}$ & 0.07 & 7.3 & & 0.05 & 14.3 & & 0.08 & 0.20 & $\mathrm{U}$ & 0.20 \\
\hline SPA B7 & J13HY9 & $10 / 17 / 06$ & 0.03 & $\begin{array}{ll}\mathrm{U} \\
\end{array}$ & 0.03 & 4490 & $\mathrm{C}$ & 0.87 & 9.3 & $C$ & 0.07 & 7.9 & & 0.05 & 14.6 & & 0.08 & 0.21 & $\mathrm{U}$ & 0.21 \\
\hline SPA B8 & $\mathrm{J} 13 \mathrm{~J} 00$ & $10 / 17 / 06$ & 0.03 & $\mathrm{U}$ & 0.03 & 3470 & $\mathrm{C}$ & 0.86 & 9.6 & $\mathrm{C}$ & 0.07 & 6.1 & & 0.05 & 12.2 & & 0.08 & 0.20 & $\mathrm{U}$ & 0.20 \\
\hline SPAC9 & J13J01 & $10 / 17 / 06$ & 0.03 & $\mathrm{U}$ & 0.03 & 3630 & $\mathrm{C}$ & 0.90 & 9.1 & $\mathrm{C}$ & 0.07 & 6.7 & & 0.05 & 15.2 & & 0.08 & 0.22 & $\mathrm{U}$ & 0.22 \\
\hline SPA C10 & $\mathrm{J} 13 \mathrm{~J} 02$ & $10 / 18 / 06$ & 0.09 & $\mathrm{U}$ & 0.09 & 3220 & $\mathrm{C}$ & 2.6 & 7.4 & $\mathrm{C}$ & 0.21 & 6.4 & & 0.15 & 12.5 & & 0.24 & 0.27 & & 0.21 \\
\hline SPA C1 & $\mathrm{J} 13.103$ & $10 / 18 / 06$ & 0.09 & $\mathrm{U}$ & 0.09 & 3560 & $\mathrm{C}$ & 2.6 & 7.4 & $\mathrm{C}$ & 0.21 & 7.1 & & 0.15 & 12.2 & & 0.24 & 0.21 & $\mathrm{U}$ & 0.21 \\
\hline SPA C2 & $\mathrm{J13J04}$ & $10 / 18 / 06$ & 0.09 & $\mathrm{U}$ & 0.09 & 5250 & $\mathrm{C}$ & 2.6 & 4.6 & $\mathrm{C}$ & 0.21 & 7.3 & & 0.15 & 13.9 & & 0.24 & 0.21 & $\mathrm{U}$ & 0.21 \\
\hline SPA D3 & $\mathrm{J} 13 \mathrm{~J} 05$ & $10 / 18 / 06$ & 0.09 & $\mathrm{U}$ & 0.09 & 4300 & $\mathrm{C}$ & 2.6 & 6.1 & $\mathrm{C}$ & 0.21 & 8.4 & & 0.15 & 14.3 & & 0.24 & 0.21 & $\mathrm{U}$ & 0.21 \\
\hline SPA D4 & $\mathrm{J} 13 \mathrm{~J} 06$ & $10 / 19 / 06$ & 0.53 & & 0.09 & 5800 & $\mathrm{CJ}$ & 2.6 & 7.4 & $\mathrm{C}$ & 0.21 & 8.9 & & 0.15 & 16.6 & & 0.24 & 0.22 & $\mathrm{U}$ & 0.22 \\
\hline SPA D5 & J13.107 & $10 / 23 / 06$ & 0.09 & $\mathrm{U}$ & 0.09 & 4780 & $\mathrm{CJ}$ & 2.5 & 7.0 & $\mathrm{C}$ & 0.20 & 7.6 & & 0.14 & 14.5 & & 0.23 & 0.23 & & 0.21 \\
\hline SPA D6 & $\mathrm{J} 13 \mathrm{~J} 08$ & $10 / 23 / 06$ & 0.09 & $\mathrm{U}$ & 0.09 & 5140 & $\mathrm{CJ}$ & 2.5 & 6.1 & $\mathrm{C}$ & 0.21 & 8.1 & & 0.15 & 15.0 & & 0.23 & 0.21 & $\mathrm{U}$ & 0.21 \\
\hline FS-2 & $\mathrm{J12R} 25$ & $7 / 10 / 06$ & 0.20 & $\mathrm{U}$ & 0.20 & 3770 & & 4.6 & 5.2 & & 0.37 & 7.5 & & 0.39 & 14.4 & & 0.34 & & & \\
\hline FS-3 & $\mathrm{J} 12 \mathrm{R} 26$ & $7 / 10 / 06$ & 0.28 & & 0.20 & 7570 & & 4.7 & 9.6 & & 0.37 & 11.3 & & 0.40 & 21.0 & & 0.34 & & & \\
\hline FS-5 & $\mathrm{J} 12 \mathrm{R} 28$ & $7 / 10 / 06$ & 0.09 & UC & 0.09 & 9270 & $\mathrm{C}$ & 2.5 & 7.1 & $\mathrm{C}$ & 0.20 & 8.8 & & 0.14 & 18.3 & & 0.23 & & & \\
\hline FS-6 & $312 R 29$ & $7 / 10 / 06$ & 0.20 & $\mathrm{U}$ & 0.20 & 11700 & & 4.6 & 15.9 & & 0.36 & 9.6 & & 0.39 & 28.0 & & 0.34 & & & \\
\hline FS-7 & $\mathrm{J} 13 \mathrm{P} 85$ & $11 / 15 / 06$ & 0.09 & $\mathrm{U}$ & 0.09 & 5590 & C & 3.9 & 5.2 & & 0.37 & 6.9 & & 0.15 & 14.2 & & 0.22 & & & \\
\hline FS-8 & $\mathrm{J} 12 \mathrm{R} 31$ & $7 / 10 / 06$ & 0.20 & $\mathrm{U}$ & 0.20 & 8560 & & 4.7 & 7.6 & & 0.37 & 6.8 & & 0.40 & 13.7 & & 0.34 & & & \\
\hline FS-9 & $\mathrm{J} 12 \mathrm{R} 32$ & $7 / 10 / 06$ & 0.20 & $\mathrm{U}$ & 0.20 & 9520 & & 4.7 & 8.0 & & 0.37 & 8.7 & & 0.40 & 18.2 & & 0.34 & & & \\
\hline FS-11 & $\mathrm{J} 12 \mathrm{R} 34$ & $7 / 10 / 06$ & 0.28 & $\mathrm{C}$ & 0.09 & 9980 & $\mathrm{C}$ & 2.5 & 16.4 & $\mathrm{C}$ & 0.20 & 8.7 & & 0.14 & 18.5 & & 0.23 & & & \\
\hline FS-14 & $\mathrm{J} 12 \times \mathrm{XB} 3$ & $7 / 19 / 06$ & 0.21 & $\mathrm{U}$ & 0.21 & 5300 & $\mathrm{C}$ & 5.0 & 5.4 & & 0.40 & 8.8 & & 0.43 & 16.7 & & 0.37 & & & \\
\hline FS-15 & $.113 \mathrm{NX} 4$ & $11 / 14 / 06$ & 0.09 & $\mathrm{U}$ & 0.09 & 4140 & $\mathrm{C}$ & 3.8 & 4.8 & & 0.37 & 7.4 & & 0.15 & 13.5 & & 0.22 & & & \\
\hline FS-16 & J13P84 & $11 / 15 / 06$ & 0.09 & $\mathrm{U}$ & 0.09 & 4260 & $\mathrm{C}$ & 3.7 & 3.9 & & 0.36 & 7.2 & & 0.15 & 13.8 & & 0.21 & & & \\
\hline FS-17 & $\mathrm{J14 \textrm {JJ } 3}$ & $3 / 5 / 07$ & 0.13 & & 0.03 & 5920 & & 0.74 & 10.0 & & 0.08 & 9.0 & & 0.09 & 18.1 & & 0.12 & & & \\
\hline FS-17 resample & $\mathrm{J} 152 \mathrm{M} 1$ & $5 / 10 / 07$ & 0.03 & $\mathrm{U}$ & 0.03 & 5090 & & 0.72 & 7.9 & & 0.08 & 8.6 & & 0.09 & 15.4 & & 0.12 & & & \\
\hline FS-18 & J14L38 & $3 / 5 / 07$ & 0.17 & & 0.03 & 4420 & & 0.74 & 7.6 & & 0.08 & 7.9 & & 0.09 & 15.2 & & 0.12 & & & \\
\hline FS-19 & J14L39 & $3 / 5 / 07$ & 0.17 & & 0.03 & 4390 & & 0.73 & 7.7 & & 0.08 & 7.5 & & 0.09 & 14.6 & & 0.12 & & & \\
\hline Equipment blank & $\mathrm{J12 \times 59}$ & $7 / 20 / 06$ & 0.20 & $\mathrm{U}$ & 0.20 & 89.5 & $\mathrm{C}$ & 4.6 & 0.71 & & 0.36 & 0.39 & U & 0.39 & 0.34 & $U$ & 0.34 & & & \\
\hline
\end{tabular}

\begin{tabular}{llll} 
Attachment & \multicolumn{1}{c}{1} & Sheet No. 21 of 113 \\
\cline { 2 - 3 } Originator & D.M. Capron & $10 / 03 / 07$ \\
Checked & T.M. Blakley & Date & \\
Calc. No. & O100B-CA-V0299 Rev. No. & &
\end{tabular}


Attachment 1. 118-B-1 Verification Sampling Results.

\begin{tabular}{|c|c|c|c|c|c|c|c|c|c|c|c|c|c|c|c|c|c|c|c|c|}
\hline \multirow{2}{*}{ Sample Location } & \multirow{2}{*}{$\begin{array}{c}\text { Sample } \\
\text { Number }\end{array}$} & \multirow{2}{*}{$\begin{array}{c}\text { Sample } \\
\text { Date }\end{array}$} & \multicolumn{3}{|c|}{ Iron } & \multicolumn{3}{|c|}{ Lead } & \multicolumn{3}{|c|}{ Magnesium } & \multicolumn{3}{|c|}{ Manganese } & \multicolumn{3}{|c|}{ Mercury } & \multicolumn{3}{|c|}{ Molybdenum } \\
\hline & & & $\mathrm{mg} / \mathrm{kg}$ & $\mathbf{Q}$ & PQL & $\mathrm{mg} / \mathrm{kg}$ & Q & PQL & $\mathrm{mg} / \mathrm{kg}$ & $\mathrm{Q}$ & PQL & $\begin{array}{ll}\mathrm{mg} / \mathrm{kg} \\
\end{array}$ & $Q$ & PQL & $\mathrm{mg} / \mathrm{kg}$ & Q & PQL & $\mathrm{mg} / \mathrm{kg}$ & 0 & PQL \\
\hline Area $1 \mathrm{~A} 1$ & $\mathrm{~J} 12 \mathrm{X} 44$ & $7 / 19 / 06$ & 22300 & & 10.1 & 4.7 & & 0.90 & 5080 & & 2.8 & 361 & & 0.09 & 0.01 & $\vec{U}$ & 0.01 & 0.84 & $\mathrm{U}$ & 0.84 \\
\hline Duplicate of J12X44 & $\mathrm{J} 12 \mathrm{X} 48$ & $7 / 19 / 06$ & 24100 & & 10.2 & 4.9 & & 0.91 & 5680 & & 2.8 & 411 & & 0.09 & 0.02 & $\mathrm{U}$ & 0.02 & 0.85 & $\mathrm{U}$ & 0.85 \\
\hline Split of J12X44 & $\mathrm{J} 12 \times 49$ & $7 / 19 / 06$ & 28000 & $\mathrm{~N}$ & 2.4 & 3.6 & & 0.15 & 5180 & $\mathrm{~N}$ & 12.3 & 360 & $\mathrm{~N}$ & 0.10 & 0.0214 & $\mathrm{~B}^{*}$ & 0.0067 & 1.2 & $\mathrm{U}$ & 1.2 \\
\hline Area $1 \mathrm{~A} 2$ & $\mathrm{~J} 12 \times 45$ & $7 / 19 / 06$ & 23200 & & 10.2 & 4.2 & & 0.90 & 4320 & & 2.8 & 373 & & 0.09 & 0.02 & & 0.01 & 0.84 & $\mathrm{U}$ & 0.84 \\
\hline Area $1 \mathrm{~A} 3$ & $\mathrm{~J} 12 \times 46$ & $7 / 20 / 06$ & 21700 & & 9.9 & 4.8 & & 0.88 & 4820 & & 2.7 & 352 & & 0.08 & 2.0 & & 0.05 & 0.82 & $\mathrm{U}$ & 0.82 \\
\hline Area $1 \mathrm{~A} 4$ & $J 12 \times 47$ & $7 / 20 / 06$ & 24100 & & 9.9 & 4.2 & & 0.88 & 4630 & & 2.7 & 367 & & 0.08 & 0.02 & $\mathrm{U}$ & 0.02 & 0.82 & $\mathrm{U}$ & 0.82 \\
\hline Area $2 \mathrm{Al}$ & $\mathrm{J} 12 \times 50$ & $7 / 20 / 06$ & 21300 & & 9.7 & $\overline{3.2}$ & & 0.86 & 4030 & & 2.7 & 311 & & 0.08 & 0.01 & $\mathrm{U}$ & $\overline{0.01}$ & $\overline{0.81}$ & $\overline{\mathrm{U}}$ & 0.81 \\
\hline Arca 2 A2 & $J 12 \times 51$ & $7 / 20 / 06$ & 20500 & & 9.9 & 4.4 & & 0.88 & 4760 & & 2.8 & 329 & & 0.09 & 0.01 & $\mathrm{U}$ & 0.01 & 0.83 & $\bar{U}$ & 0.83 \\
\hline Area $2 \mathrm{~A} 3$ & $\mathrm{~J} 12 \times 52$ & $7 / 20 / 06$ & 21800 & & 9.9 & 4.0 & & 0.88 & 4530 & & 2.8 & 298 & & 0.09 & 0.02 & $\mathrm{U}$ & 0.02 & 0.83 & $\bar{U}$ & 0.83 \\
\hline Area $2 \mathrm{~A} 4$ & $\mathrm{~J} 12 \times 53$ & $7 / 20 / 06$ & 17500 & & 9.8 & 5.0 & & 0.87 & 3710 & & 2.7 & 298 & & 0.08 & 0.02 & $\mathrm{U}$ & 0.02 & 0.82 & $\bar{U}$ & 0.82 \\
\hline Area $3 \mathrm{Al}$ & $J 12 \times 54$ & $7 / 20 / 06$ & 18800 & & 9.7 & 4.6 & & 0.86 & 3880 & & 2.7 & 297 & & 0.08 & 0.04 & & 0.01 & 0.81 & $\mathrm{U}$ & 0.81 \\
\hline Area $3 \mathrm{~A} 2$ & $\mathrm{~J} 12 \times 55$ & $7 / 20 / 06$ & 22100 & & 9.8 & 4.3 & & 0.87 & 4670 & & 2.7 & 320 & & 0.08 & 0.01 & $\mathrm{U}$ & 0.01 & 0.81 & $\mathrm{U}$ & 0.81 \\
\hline Area $3 \mathrm{~A} 3$ & $.112 \times 56$ & $7 / 20 / 06$ & 20100 & & 9.9 & 6.6 & & 0.88 & 5060 & & 2.7 & 339 & & 0.08 & 0.01 & & 0.01 & 0.82 & $\mathrm{U}$ & 0.82 \\
\hline Duplicate of $\mathrm{J12 \times 56}$ & $\mathrm{J12 \times 58}$ & $7 / 20 / 06$ & 20100 & & 10 & 5.9 & & 0.89 & 4860 & & 2.8 & 325 & & 0.09 & 0.02 & $\mathrm{U}$ & 0.02 & 0.83 & $\mathrm{U}$ & 0.83 \\
\hline Split of J12X56 & $\mathrm{J} 12 \times 60$ & $7 / 20 / 06$ & 19500 & & 2.4 & 4.5 & & 0.15 & 4280 & & 12.4 & 287 & $\mathrm{~N}$ & 0.10 & 0.0134 & $\mathrm{~B}^{*}$ & 0.0068 & 1.2 & $\mathrm{UNJ}$ & 1.2 \\
\hline Area $3 \mathrm{~A} 4$ & $J 12 \times 57$ & $7 / 20 / 06$ & 20500 & & 9.8 & 4.5 & & 0.87 & 4240 & & 2.7 & 390 & & 0.08 & 0.30 & & 0.01 & 0.81 & $\mathrm{U}$ & 0.81 \\
\hline Area $4 \mathrm{Al}$ & $\mathrm{J} 12 \times 61$ & $7 / 25 / 06$ & 22100 & & 9.7 & 5.2 & & 0.87 & 3970 & & 2.7 & 290 & & 0.08 & 0.01 & $U$ & 0.01 & 0.81 & $\mathrm{U}$ & 0.81 \\
\hline Duplicate of J12X61 & $\mathrm{J} 12 \times 65$ & $7 / 25 / 06$ & 21500 & & 9.8 & 5.5 & & 0.87 & 4510 & & 2.7 & 292 & & 0.08 & 0.01 & $\mathrm{U}$ & 0.01 & 0.81 & $\mathrm{U}$ & 0.81 \\
\hline Split of J12X61 & $\mathrm{J} 12 \times 66$ & $7 / 25 / 06$ & 27000 & & 2.4 & 6.8 & & 0.15 & 4950 & & 12.2 & 337 & $\mathrm{~N}$ & 0.10 & 0.0306 & $\mathrm{~B}^{*}$ & 0.0067 & 1.2 & UNJ & 1.2 \\
\hline Area $4 \mathrm{A2}$ & $\mathrm{J} 12 \times 62$ & $7 / 25 / 06$ & 19200 & & 9.6 & 3.3 & & 0.85 & 3820 & & 2.7 & 299 & & 0.08 & 0.01 & $\mathrm{U}$ & 0.01 & 0.80 & $\mathrm{U}$ & 0.80 \\
\hline Area $4 \mathrm{A3}$ & $\mathrm{J} 12 \times 63$ & $7 / 25 / 06$ & 20200 & & 9.8 & 4.4 & & 0.87 & 4770 & & 2.7 & 334 & & 0.08 & 0.01 & $\mathrm{U}$ & 0.01 & 0.81 & $\mathrm{U}$ & 0.81 \\
\hline Area 4 A4 & $\mathrm{J} 12 \times 64$ & $7 / 25 / 06$ & 22300 & & 9.7 & 3.6 & & 0.86 & 4800 & & 2.7 & 336 & & 0.08 & 0.01 & $\mathrm{U}$ & 0.01 & 0.80 & $\mathrm{U}$ & 0.80 \\
\hline Area $5 \mathrm{Al}$ & $113 \mathrm{~V} 12$ & $12 / 7 / 06$ & 16000 & $\mathrm{C}$ & 7.5 & 3.0 & & 0.49 & 3070 & $\mathrm{C}$ & 1.4 & 244 & & 0.12 & 0.01 & $\mathrm{U}$ & 0.01 & 0.49 & $\mathrm{U}$ & 0.49 \\
\hline Area $5 \mathrm{~A} 2$ & J13V10 & $12 / 7 / 06$ & 16700 & $\mathrm{C}$ & 7.4 & 2.8 & & 0.49 & 3440 & $\mathrm{C}$ & 1.4 & 302 & & 0.12 & 0.04 & & 0.02 & 0.49 & $U$ & 0.49 \\
\hline Area $5 \mathrm{~A} 3$ & $\mathrm{~J} 13 \mathrm{~V} 13$ & $12 / 7 / 06$ & 5920 & $\mathrm{C}$ & 2.5 & 1.6 & & 0.16 & 1280 & $\mathrm{C}$ & 0.47 & 98.5 & & 0.04 & 0.02 & $\mathrm{U}$ & 0.02 & 0.16 & $\mathrm{U}$ & 0.16 \\
\hline Area $5 \mathrm{~A} 4$ & J13V11 & $12 / 7 / 06$ & 16300 & $\mathrm{C}$ & 7.5 & 4.2 & & 0.49 & 4100 & $\mathrm{C}$ & 1.4 & 234 & & 0.12 & 0.02 & & 0.01 & 0.49 & $\mathrm{U}$ & 0.49 \\
\hline Area $6 \mathrm{Al}$ & $\mathrm{J} 12 \mathrm{XC2}$ & $7 / 25 / 06$ & 17200 & & 9.7 & 5.1 & & 0.86 & 4250 & & 2.7 & 297 & & 0.08 & 0.02 & & 0.01 & 0.81 & $\mathrm{U}$ & 0.81 \\
\hline Area $6 \mathrm{~A} 2$ & $\mathrm{~J} 12 \mathrm{XC} 3$ & $7 / 25 / 06$ & 19300 & & 9.8 & 4.7 & & 0.87 & 4070 & & 2.7 & 312 & & 0.08 & 0.01 & U & 0.01 & 0.81 & U & 0.81 \\
\hline Area $6 \mathrm{~A} 3$ & $\mathrm{~J} 12 \times \mathrm{C} 4$ & $7 / 25 / 06$ & 21500 & & 9.8 & 4.0 & & 0.87 & 4510 & & 2.7 & 322 & & 0.08 & 0.02 & & 0.01 & 0.81 & $\mathrm{U}$ & 0.81 \\
\hline Area $6 \mathrm{~A} 4$ & $\mathrm{~J} 12 \mathrm{XC} 5$ & $7 / 25 / 06$ & 22100 & & 9.8 & 4.7 & & 0.87 & 5040 & & 2.7 & 322 & & 0.08 & 0.02 & $\mathrm{U}$ & 0.02 & 0.81 & $U$ & 0.81 \\
\hline Area $7 \mathrm{Al}$ & $\mathrm{J} 12 \mathrm{XC} 6$ & $7 / 26 / 06$ & 17000 & & 9.7 & 12.9 & & 0.86 & 4440 & & 2.7 & 254 & & 0.08 & 0.02 & $\mathrm{U}$ & 0.02 & 2.1 & & 0.81 \\
\hline Area $7 \mathrm{~A} 2$ & $112 \times C 7$ & $7 / 26 / 06$ & 18700 & & 9.6 & 6.0 & & 0.86 & 4140 & & 2.7 & 340 & & 0.08 & 0.01 & $\mathrm{U}$ & 0.01 & 0.80 & $\mathrm{U}$ & 0.80 \\
\hline Area $7 \mathrm{~A} 3$ & $\mathrm{~J} 12 \mathrm{XC} 8$ & $7 / 26 / 06$ & 15900 & & 9.8 & 3.6 & & 0.87 & 3410 & & 2.7 & 270 & & 0.08 & 0.02 & $\mathrm{U}$ & 0.02 & 0.81 & $\mathrm{U}$ & 0.81 \\
\hline Area $7 \mathrm{~A} 4$ & $312 \times \mathrm{C} 9$ & $7 / 26 / 06$ & 18000 & & 9.8 & 8.3 & & 0.87 & 4220 & & 2.7 & 318 & & 0.08 & 0.02 & & 0.02 & 0.81 & $\mathrm{U}$ & 0.81 \\
\hline
\end{tabular}

Attachment

Originator

Checked T.M. Blakley

Calc. No. $\quad$ Rev. No.

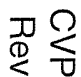

○ ̃ 
Attachment 1. 118-B-1 Verification Sampling Results.

\begin{tabular}{|c|c|c|c|c|c|c|c|c|c|c|c|c|c|c|c|c|c|c|c|c|}
\hline \multirow{2}{*}{ Sample Location } & \multirow{2}{*}{$\begin{array}{c}\text { Sample } \\
\text { Number }\end{array}$} & \multirow{2}{*}{$\begin{array}{c}\text { Sample } \\
\text { Date }\end{array}$} & \multicolumn{3}{|c|}{ Iron } & \multicolumn{3}{|c|}{ Lead } & \multicolumn{3}{|c|}{ Maguesium } & \multicolumn{3}{|c|}{ Manganese } & \multicolumn{3}{|c|}{ Mercury } & \multicolumn{3}{|c|}{ Molybdenum } \\
\hline & & & $\mathrm{mg} / \mathrm{kg}$ & $Q$ & PQL & $\mathrm{mg} / \mathrm{kg}$ & $Q$ & $\mathrm{PQL}$ & $\mathrm{mg} / \mathrm{kg}$ & $Q$ & $\mathrm{PQL}$ & $\mathrm{mg} / \mathrm{kg}$ & Q & $\mathrm{PQL}$ & $\mathrm{mg} / \mathrm{kg}$ & $Q$ & $\mathrm{PQL}$ & $\mathrm{mg} / \mathrm{kg}$ & $\mathbf{Q}$ & PQL \\
\hline $\mathrm{OBAl}$ & J12WKO & 7/11/06 & 16800 & C & 0.52 & 5.0 & & 0.30 & 4110 & & 0.94 & 327 & & 0.03 & 0.01 & $\mathrm{U}$ & 0.01 & 0.43 & & 0.28 \\
\hline $\mathrm{OB} \mathrm{A2}$ & JI2WKI & $7 / 11 / 06$ & 18200 & C & 0.52 & 4.3 & & 0.30 & 4280 & & 0.94 & 335 & & 0.03 & 0.02 & $\mathrm{U}$ & 0.02 & 0.33 & & 0.28 \\
\hline $\mathrm{OBA} 3$ & J12WK2 & $7 / 11 / 06$ & 14800 & $\mathrm{C}$ & 0.52 & 4.6 & & 0.30 & 3660 & & 0.94 & 311 & & 0.03 & 0.02 & $\mathrm{U}$ & 0.02 & 0.38 & & 0.28 \\
\hline $\mathrm{OB} \mathrm{A4}$ & J12WK3 & $7 / 11 / 06$ & 14800 & $\mathrm{C}$ & 0.52 & 4.7 & & 0.30 & 3610 & & 0.93 & 339 & & 0.03 & 0.01 & $\mathrm{U}$ & 0.01 & 0.41 & & 0.28 \\
\hline OB B5 & J12WK4 & $7 / 11 / 06$ & 20000 & $\mathrm{C}$ & 0.52 & 4.7 & & 0.30 & 4540 & & 0.94 & 383 & & 0.03 & 0.01 & $\mathrm{U}$ & 0.01 & 0.42 & & 0.28 \\
\hline OB B6 & J12WK5 & $7 / 11 / 06$ & 17100 & C & 0.52 & 4.6 & & 0.30 & 4050 & & 0.94 & 339 & & 0.03 & 0.01 & $\mathrm{U}$ & 0.01 & 0.28 & U & 0.28 \\
\hline OB B7 & J12WK6 & $7 / 11 / 06$ & 15600 & $C$ & 0.52 & 3.7 & & 0.30 & 3950 & & 0.94 & 283 & & 0.03 & 0.01 & $\mathrm{U}$ & 0.01 & 0.34 & & 0.28 \\
\hline OB B8 & J12WM7 & $7 / 17 / 06$ & 20100 & & 9.7 & 5.1 & & 0.86 & 4020 & & 2.7 & 332 & & 0.08 & 0.01 & $\mathrm{U}$ & 0.01 & 0.81 & $\mathrm{U}$ & 0.81 \\
\hline OB C9 & J12WM8 & $7 / 17 / 06$ & 17200 & & 9.8 & 5.8 & & 0.87 & 3560 & & 2.7 & 297 & & 0.08 & 0.01 & $\mathrm{U}$ & 0.01 & 0.81 & $\mathrm{U}$ & $0: 81$ \\
\hline $\mathrm{OBC} 10$ & J12WM9 & $7 / 17 / 06$ & 17800 & & 9.8 & 3.8 & & 0.87 & 3700 & & 2.7 & 282 & & 0.08 & 0.02 & $\mathrm{U}$ & 0.02 & 0.82 & $\mathrm{U}$ & 0.82 \\
\hline $\mathrm{OBCl}$ & J12WNO & $7 / 17 / 06$ & 24700 & & 9.9 & 3.8 & & 0.88 & 4450 & & 2.7 & 390 & & 0.08 & 0.02 & $U$ & 0.02 & 0.82 & $\mathrm{U}$ & 0.82 \\
\hline $\mathrm{OBC} 2$ & $\mathrm{~J} 12 \mathrm{WN} 1$ & $7 / 17 / 06$ & 19700 & & 9.8 & 4.3 & & 0.87 & 4210 & & 2.7 & 303 & & 0.08 & 0.02 & & 0.01 & 0.82 & $U$ & 0.82 \\
\hline $\mathrm{OBD} 3$ & J12WM5 & $7 / 12 / 06$ & 22800 & & 10 & 5.1 & & 0.89 & 4580 & & 2.8 & 325 & & 0.09 & 0.02 & & 0.01 & 0.83 & $\mathrm{U}$ & 0.83 \\
\hline $\mathrm{OBD}$ 4 & J12WM6 & $7 / 12 / 06$ & 25100 & & 9.9 & 5.1 & & 0.88 & 5270 & & 2.7 & 374 & & 0.09 & 0.02 & $U$ & 0.02 & 0.82 & $\mathrm{U}$ & 0.82 \\
\hline OB D5 & J12WM3 & $7 / 12 / 06$ & 19600 & & 9.8 & 7.1 & & 0.87 & 4080 & & 2.7 & 358 & & 0.08 & 0.05 & & 0.02 & 0.82 & $\mathrm{U}$ & 0.82 \\
\hline $\mathrm{OBD6}$ & J12WM4 & $7 / 12 / 06$ & 22000 & & 9.8 & 4.5 & & 0.87 & 4530 & & 2.7 & 322 & & 0.08 & 0.02 & & 0.01 & 0.81 & $\mathrm{U}$ & 0.81 \\
\hline $\mathrm{BCLAI}$ & $\mathrm{J} 12 \mathrm{WN} 2$ & $7 / 20 / 06$ & 22300 & & 9.9 & 4.9 & & 0.88 & 4620 & & 2.7 & 359 & & 0.08 & 0.03 & & 0.02 & 0.82 & $\mathrm{U}$ & 0.82 \\
\hline $\mathrm{BCL} \mathrm{A2}$ & J12WM1 & $7 / 12 / 06$ & 20700 & & 9.9 & 4.5 & & 0.88 & 4280 & & 2.7 & 337 & & 0.08 & 0.01 & $\mathrm{U}$ & 0.01 & 0.82 & $\mathrm{U}$ & 0.82 \\
\hline $\mathrm{BCL} A 3$ & J12WM2 & $7 / 12 / 06$ & 19700 & & 9.9 & 4.5 & & 0.88 & 4250 & & 2.8 & 346 & & 0.09 & 0.02 & $\mathrm{U}$ & 0.02 & 0.82 & $\mathrm{U}$ & 0.82 \\
\hline $\mathrm{BCLA4}$ & J12WMO & $7 / 12 / 06$ & $20300^{\circ}$ & & 9.9 & 4.0 & & 0.88 & 4110 & & 2.7 & 332 & & 0.09 & 0.01 & $\mathrm{U}$ & 0.01 & 0.82 & $U$ & 0.82 \\
\hline BCL B5 & J12WL9 & $7 / 12 / 06$ & 23400 & & 9.9 & 4.4 & & 0.88 & 4880 & & 2.8 & 359 & & 0.09 & 0.02 & $\mathrm{U}$ & 0.02 & 0.83 & $\mathrm{U}$ & 0.83 \\
\hline BCL B6 & J12WL8 & $7 / 12 / 06$ & 21000 & & 9.9 & 4.0 & & 0.88 & 4240 & & 2.8 & 313 & & 0.09 & 0.01 & $\mathrm{U}$ & 0.01 & 0.82 & $\mathrm{U}$ & 0.82 \\
\hline $\mathrm{BCL} B 7$ & J12WL7 & $7 / 12 / 06$ & 19500 & & 9.9 & 4.4 & & 0.88 & 4400 & & 2.7 & 329 & & 0.08 & 0.01 & $\mathrm{U}$ & 0.01 & 0.82 & $\mathrm{U}$ & 0.82 \\
\hline BCL B 8 & J12WL6 & $7 / 12 / 06$ & 17900 & & 9.9 & 4.1 & & 0.88 & 3900 & & 2.7 & 304 & & 0.08 & 0.02 & $\mathrm{U}$ & 0.02 & 0.82 & $\mathrm{U}$ & 0.82 \\
\hline Split of J12WL6 & J12WW0 & $7 / 12 / 06$ & 20100 & $\mathrm{~N}$ & 2.4 & 4.8 & & 0.15 & 4880 & $N$ & 12.2 & 337 & $\mathrm{~N}$ & 0.10 & 0.0067 & UN & 0.0067 & 1.2 & $\mathrm{U}$ & 1.2 \\
\hline $\mathrm{BCLC} 9$ & J12WK7 & $7 / 11 / 06$ & 18400 & $C$ & 0.52 & 5.2 & & 0.30 & 4360 & & 0.93 & 327 & & 0.03 & 0.02 & & 0.01 & 0.35 & & 0.28 \\
\hline $\mathrm{BCLC10}$ & J12WK9 & $7 / 11 / 06$ & 21200 & $C$ & 0.52 & 5.5 & & 0.30 & 5370 & & 0.94 & 382 & & 0.03 & 0.01 & $\mathrm{U}$ & 0.01 & 0.49 & & 0.28 \\
\hline $\mathrm{BCLC1}$ & J12WK8 & $7 / 11 / 06$ & 18900 & $\mathrm{C}$ & 0.52 & 4.5 & & 0.30 & 4380 & & 0.94 & 407 & & 0.03 & 0.01 & $\mathrm{U}$ & 0.01 & 0.39 & & 0.28 \\
\hline $\mathrm{BCLC2}$ & $\mathrm{J} 12 \mathrm{WL} 2$ & $7 / 11 / 06$ & 21300 & $\mathrm{C}$ & 0.52 & 6.5 & & 0.30 & 5970 & & 0.93 & 418 & & 0.03 & 0.01 & $\mathrm{U}$ & 0.01 & 0.50 & & 0.28 \\
\hline $\mathrm{BCLD3}$ & J12WL3 & $7 / 11 / 06$ & 20500 & $\mathrm{C}$ & 0.52 & 4.6 & & 0.30 & 4940 & & 0.94 & 346 & & 0.03 & 0.01 & $\mathrm{U}$ & 0.01 & 0.42 & & 0.28 \\
\hline BCL D4 & J12WLA & $7 / 11 / 06$ & 16100 & $C$ & 0.52 & 4.9 & & 0.30 & 4120 & & 0.93 & 324 & & 0.03 & 0.01 & $\mathrm{U}$ & 0.01 & 0.39 & & 0.28 \\
\hline Duplicate of J12WL4 & J12WL5 & $7 / 11 / 06$ & 15200 & C & 0.52 & 5.0 & & 0.30 & 4180 & & 0.94 & 348 & & 0.03 & 0.01 & $\mathrm{U}$ & 0.01 & 0.35 & & 0.28 \\
\hline BCL D5 & J12WLO & $7 / 11 / 06$ & 17600 & $\mathrm{C}$ & 0.52 & 4.9 & & 0.30 & 4640 & & 0.94 & 339 & & 0.03 & 0.01 & $\mathrm{U}$ & 0.01 & 0.36 & & 0.28 \\
\hline BCLD6 & J12WL1 & $7 / 11 / 06$ & 21600 & C & 0.52 & 6.2 & & 0.30 & 5960 & & 0.93 & 384 & & 0.03 & 0.01 & $\mathrm{U}$ & 0.01 & 0.46 & & 0.28 \\
\hline
\end{tabular}

Attachment 
Attachment 1. 118-B-1 Verification Sampling Results.

\begin{tabular}{|c|c|c|c|c|c|c|c|c|c|c|c|c|c|c|c|c|c|c|c|c|}
\hline \multirow{2}{*}{ Sample Location } & \multirow{2}{*}{$\begin{array}{c}\text { Sample } \\
\text { Number }\end{array}$} & \multirow{2}{*}{$\begin{array}{c}\text { Sample } \\
\text { Date }\end{array}$} & \multicolumn{3}{|c|}{ Iron } & \multicolumn{3}{|c|}{ Lead } & \multicolumn{3}{|c|}{ Magnesium } & \multicolumn{3}{|c|}{ Manganese } & \multicolumn{3}{|c|}{ Mercury } & \multicolumn{3}{|c|}{ Molybdenum } \\
\hline & & & $\mathrm{mg} / \mathrm{kg}$ & Q & PQL & $\mathrm{mg} / \mathrm{kg}$ & $Q$ & PQL & $\mathrm{mg} / \mathrm{kg}$ & $Q$ & PQL & $\mathrm{mg} / \mathrm{kg}$ & $Q$ & PQL & $\mathrm{mg} / \mathrm{kg}$ & $Q$ & $\mathrm{PQL}$ & $\mathrm{mg} / \mathrm{kg}$ & $Q$ & PQL \\
\hline SPAAI & J13HY3 & $10 / 12 / 06$ & 16700 & & 6.9 & 5.5 & & 0.34 & 3680 & & 2.0 & 333 & & 0.06 & 0.02 & & 0.02 & 0.44 & & 0.28 \\
\hline SPA A2 & J13HY4 & $10 / 12 / 06$ & 18000 & & 6.9 & 5.0 & & 0.34 & 3790 & & 2.0 & 319 & & 0.06 & 0.03 & & 0.02 & 0.32 & & 0.28 \\
\hline SPA A3 & J13HY5 & $10 / 12 / 06$ & 19200 & & 6.9 & 4.5 & & 0.34 & 3770 & & 2.0 & 342 & & 0.06 & 0.05 & & 0.01 & 0.31 & & 0.28 \\
\hline SPA A4 & J13HY6 & $10 / 12 / 06$ & 18200 & & 6.9 & 4.9 & & 0.34 & 3850 & & 2.1 & 326 & & 0.06 & 0.14 & & 0.01 & 0.29 & & 0.29 \\
\hline SPA B5 & J13HY7 & $10 / 12 / 06$ & 15900 & & 6.9 & 4.5 & & 0.34 & 3510 & & 2.0 & 286 & & 0.06 & 0.09 & & 0.01 & 0.28 & $\mathrm{U}$ & 0.28 \\
\hline Duplicate of $J 13 \mathrm{HY} 7$ & 513.09 & $10 / 12 / 06$ & 16700 & & 6.9 & 5.2 & & 0.34 & 3640 & & 2.0 & 304 & & 0.06 & 0.11 & & 0.01 & 0.28 & $\mathrm{U}$ & 0.28 \\
\hline Split of J13HY7 & $\$ 13 \mathrm{~J} 10$ & $10 / 12 / 06$ & 20100 & $\mathrm{~N}$ & 2.4 & 5.4 & $\mathrm{~N}$ & 0.30 & 4960 & & 12.2 & 335 & $\mathrm{~N}$ & 0.10 & 0.12 & & 0.0067 & 1.2 & UN & 1.2 \\
\hline SPA B6 & J13HY8 & $10 / 17 / 06$ & 18200 & C & 2.4 & 4.5 & & 0.12 & 3590 & $\mathrm{C}$ & 0.72 & 309 & & 0.02 & 0.07 & & 0.02 & 0.37 & $\mathrm{CJ}$ & 0.10 \\
\hline SPA B7 & J13HY9 & $10 / 17 / 06$ & 20100 & C & 2.5 & 4.2 & & 0.12 & 3910 & $\mathrm{C}$ & 0.73 & 339 & & 0.02 & 0.1 & & 0.01 & 0.34 & $\mathrm{CJ}$ & 0.10 \\
\hline SPA B8 & $\mathrm{J} 13.100$ & $10 / 17 / 06$ & 15400 & C & 2.4 & 4.4 & & 0.12 & 3630 & $\mathrm{C}$ & 0.72 & 279 & & 0.02 & 0.13 & & 0.02 & 0.34 & $\mathrm{CJ}$ & 0.10 \\
\hline SPA C9 & J13.01 & $10 / 17 / 06$ & 17900 & C & 2.6 & 5.1 & & 0.13 & 3750 & $\mathrm{C}$ & 0.76 & 313 & & 0.02 & 0.09 & & 0.01 & 0.42 & $\mathrm{CJ}$ & 0.11 \\
\hline SPA C10 & $\mathrm{J} 13 \mathrm{~J} 02$ & $10 / 18 / 06$ & 17000 & C & 7.4 & 3.0 & & 0.37 & 3430 & $\mathrm{C}$ & 2.2 & 305 & & 0.06 & 0.02 & $\mathrm{U}$ & 0.02 & 0.37 & $\mathrm{CJ}$ & 0.31 \\
\hline SPACl & $\mathrm{J} 13 \mathrm{~J} 03$ & $10 / 18 / 06$ & 17900 & C & 7.4 & 3.4 & & 0.36 & 3540 & $\mathrm{C}$ & 2.2 & 313 & & 0.06 & 0.13 & & 0.02 & 0.42 & $\mathrm{CJ}$ & 0.30 \\
\hline SPA C2 & $\mathrm{J} 13.104$ & $10 / 18 / 06$ & 20500 & C & 7.3 & 2.6 & & 0.36 & 3920 & $\mathrm{C}$ & 2.2 & 265 & & 0.06 & 0.06 & & 0.02 & 0.55 & $\mathrm{CJ}$ & 0.30 \\
\hline SPA D3 & $\mathrm{J} 13 \mathrm{~J} 05$ & $10 / 18 / 06$ & 19400 & C & 7.3 & 3.5 & & 0.36 & 3730 & $\mathrm{C}$ & 2.2 & 379 & & 0.06 & 0.12 & & 0.02 & 0.43 & $\mathrm{CJ}$ & 0.30 \\
\hline SPA D4 & $\mathrm{J} 13.306$ & $10 / 19 / 06$ & 23300 & & 7.4 & 5.2 & $\mathrm{C}$ & 0.36 & 4260 & & 2.2 & 350 & & 0.06 & 0.06 & & 0.02 & 0.49 & & 0.30 \\
\hline SPA D5 & J13.07 & $10 / 23 / 06$ & 20700 & & 7.0 & 2.9 & $\mathrm{C}$ & 0.35 & 4020 & & 2.1 & 297 & & 0.06 & 0.02 & & 0.02 & 0.54 & & 0.29 \\
\hline SPA D6 & $\mathrm{J} 13 \mathrm{~J} 08$ & $10 / 23 / 06$ & 20200 & & 7.1 & 4.1 & $\mathrm{C}$ & 0.35 & 3960 & & 2.1 & 316 & & 0.06 & 0.05 & & 0.02 & 0.61 & & 0.29 \\
\hline FS-2 & $\mathrm{J12R25}$ & $7 / 10 / 06$ & 19200 & & 9.8 & 2.4 & & 0.87 & 3690 & & 2.7 & 296 & & 0.08 & & & & 0.82 & $\mathrm{U}$ & 0.82 \\
\hline FS-3 & $\mathrm{J12R26}$ & $7 / 10 / 06$ & 24300 & & 9.9 & 5.4 & & 0.88 & 5950 & & 2.8 & 421 & & 0.09 & & & & 0.82 & $\mathrm{U}$ & 0.82 \\
\hline FS-5 & $\mathrm{J} 12 \mathrm{R} 28$ & $7 / 10 / 06$ & 21500 & & 6.9 & 6.5 & $\mathrm{~J}$ & 0.34 & 5030 & $\mathrm{C}$ & 2.1 & 352 & & 0.06 & 0.24 & & 0.01 & 0.54 & $\mathrm{CJ}$ & 0.29 \\
\hline FS-6 & J12R29 & $7 / 10 / 06$ & 21600 & & 9.8 & 12.0 & & 0.87 & 5920 & & 2.7 & 405 & & 0.08 & & & & 0.81 & $U$ & 0.81 \\
\hline FS-7 & J13P85 & $11 / 15 / 06$ & 17400 & & 7.5 & 3.5 & & 0.49 & 3510 & & 1.4 & 261 & & 0.12 & 0.97 & & 0.02 & 0.49 & $\mathrm{U}$ & 0.49 \\
\hline FS-8 & $\mathrm{J12R} 31$ & $7 / 10 / 06$ & 17100 & & 9.9 & 11.4 & & 0.88 & 4030 & & 2.8 & 314 & & 0.09 & & & & 0.82 & $\mathrm{U}$ & 0.82 \\
\hline FS-9 & $\mathrm{J} 12 \mathrm{R} 32$ & $7 / 10 / 06$ & 21000 & & 10 & 5.4 & & 0.89 & 5470 & & 2.8 & 361 & & 0.09 & & & & 0.83 & $\mathrm{U}$ & 0.83 \\
\hline FS-11 & $\mathrm{J} 12 \mathrm{R} 34$ & $7 / 10 / 06$ & 22200 & & 6.9 & 34.3 & $\mathrm{~J}$ & 0.34 & 5790 & $\mathrm{C}$ & 2.1 & 404 & & 0.06 & 0.24 & & 0.01 & 0.81 & $\mathrm{C}$ & 0.29 \\
\hline FS-14 & $\mathrm{J} 12 \mathrm{XB3}$ & $7 / 19 / 06$ & 23000 & & 10.6 & 3.5 & & 0.94 & 4110 & & 3.0 & 331 & & 0.09 & 0.02 & $\mathrm{U}$ & 0.02 & 0.88 & $\mathrm{U}$ & 0.88 \\
\hline FS-15 & $\mathrm{J} 13 \mathrm{NX} 4$ & $11 / 14 / 06$ & 19400 & & 7.5 & 7.0 & & 0.49 & 3680 & & 1.4 & 253 & & 0.12 & 10.3 & & 0.23 & 0.49 & $U$ & 0.49 \\
\hline FS-16 & $\mathrm{J} 13 \mathrm{PP} 84$ & $11 / 15 / 06$ & 19300 & & 7.3 & 2.7 & & 0.48 & 3430 & & 1.4 & 262 & & 0.12 & 0.06 & & 0.02 & 0.48 & $\mathrm{U}$ & 0.48 \\
\hline FS-17 & J14.J.J3 & $3 / 5 / 07$ & 24500 & & 2.9 & 4.8 & & 0.28 & 4650 & & 0.72 & 370 & & 0.02 & 81.0 & & 1.6 & 0.43 & & 0.13 \\
\hline FS-17 resample & $\mathrm{J} 152 \mathrm{Ml}$ & $5 / 10 / 07$ & 23400 & & 2.9 & 4.5 & & 0.27 & 4510 & & 0.70 & 375 & & 0.02 & 6.9 & & 0.09 & 0.41 & & 0.13 \\
\hline FS-18 & J14L38 & $3 / 5 / 07$ & 21600 & & 2.9 & 4.1 & & 0.28 & 3800 & & 0.72 & 334 & & 0.02 & 1.8 & & 0.05 & 0.40 & & 0.13 \\
\hline FS-19 & J14L,39 & $3 / 5 / 07$ & 20200 & & 2.9 & 3.9 & & 0.27 & 3790 & & 0.71 & 315 & & 0.02 & 14.5 & & 0.78 & 0.35 & & 0.13 \\
\hline Equipment blank & $512 \times 59$ & $7 / 20 / 06$ & 1560 & & 9.8 & 1.2 & & 0.87 & 28.6 & & 2.7 & 24.6 & & 0.08 & 0.02 & $U$ & 0.02 & 0.81 & $\mathrm{U}$ & 0.81 \\
\hline
\end{tabular}

\begin{tabular}{|c|c|c|c|}
\hline Attachment & 1 & Sheet No. & 24 of 113 \\
\hline Originator & J.M. Capron & Date & $10 / 03 / 07$ \\
\hline Checked & T. M. Blakley & Date & \\
\hline Calc. No. & $0100 \mathrm{~B}-\mathrm{CA}-\mathrm{V} 0299$ & Rev. No. & 1 \\
\hline
\end{tabular}


Attachment 1. 118-B-1 Verification Sampling Results.

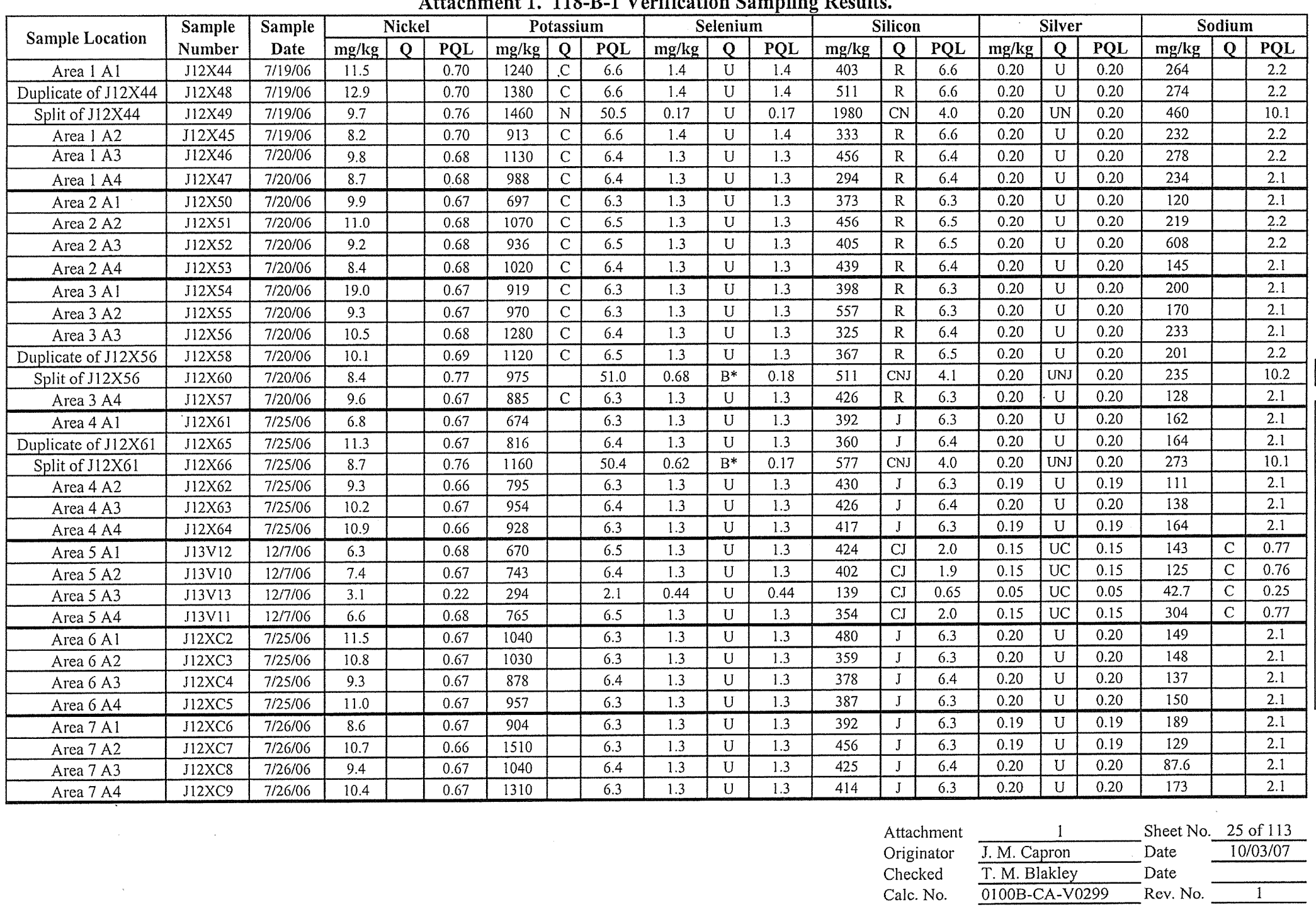

$\begin{array}{ll}\text { Checked } & \text { T.M. Blakley } \\ \text { O100B-CA-V02 } & \end{array}$

Date No. 
Attachment 1. 118-B-1 Verification Sampling Results.

\begin{tabular}{|c|c|c|c|c|c|c|c|c|c|c|c|c|c|c|c|c|c|c|c|c|}
\hline \multirow{2}{*}{ Sample Location } & \multirow{2}{*}{$\begin{array}{c}\text { Sample } \\
\text { Number }\end{array}$} & \multirow{2}{*}{$\begin{array}{c}\text { Sample } \\
\text { Date }\end{array}$} & \multicolumn{3}{|c|}{ Nickel } & \multicolumn{3}{|c|}{ Potassium } & \multicolumn{3}{|c|}{ Selenium } & \multicolumn{3}{|c|}{ Silicon } & \multicolumn{3}{|c|}{ Silver } & \multicolumn{3}{|c|}{ Sodium } \\
\hline & & & $\mathrm{mg} / \mathrm{kg}$ & $Q$ & $\mathrm{PQL}$ & $\mathrm{mg} / \mathrm{kg}$ & $\mathbf{Q}$ & PQL & $\mathrm{mg} / \mathrm{kg}$ & $\mathbf{Q}$ & PQL & $\mathrm{mg} / \mathrm{kg}$ & $Q$ & PQL & $\mathrm{mg} / \mathrm{kg}$ & $\mathbf{Q}$ & PQL & $\mathrm{mg} / \mathrm{kg}$ & $Q$ & PQL \\
\hline $\mathrm{OB} \mathrm{A1}$ & J12WKO & $7 / 11 / 06$ & 9.5 & & 0.23 & 905 & & 2.2 & 0.52 & & 0.46 & 547 & $\mathrm{CJ}$ & 2.2 & 0.07 & U & 0.07 & 122 & $\mathrm{C}$ & 0.74 \\
\hline OB A2 & J12WK1 & $7 / 11 / 06$ & 10.4 & & 0.23 & 975 & & 2.2 & 0.65 & & 0.46 & 513 & $\mathrm{CJ}$ & 2.2 & 0.07 & $\mathrm{U}$ & 0.07 & 127 & $\mathrm{C}$ & 0.74 \\
\hline$O B A 3$ & J12WK2 & $7 / 11 / 06$ & 8.9 & & 0.23 & 1100 & & 2.2 & 0.45 & $\mathrm{U}$ & 0.45 & 592 & $\mathrm{CJ}$ & 2.2 & 0.07 & $\mathrm{U}$ & 0.07 & 107 & $\mathrm{C}$ & 0.73 \\
\hline $\mathrm{OB} \mathrm{A4}$ & J12WK3 & $7 / 11 / 06$ & 9.0 & & 0.23 & 1060 & & 2.2 & 0.45 & $\mathrm{U}$ & 0.45 & 696 & $\mathrm{CJ}$ & 2.2 & 0.07 & $\mathrm{U}$ & 0.07 & 102 & $\mathrm{C}$ & 0.73 \\
\hline OB B5 & J12WK4 & $7 / 11 / 06$ & 12.8 & & 0.23 & 1040 & & 2.2 & 0.67 & & 0.45 & 462 & $\mathrm{CJ}$ & 2.2 & 0.07 & $\mathrm{U}$ & 0.07 & 119 & $\mathrm{C}$ & 0.73 \\
\hline OB B6 & J12WK5 & $7 / 11 / 06$ & 9.2 & & 0.23 & 1050 & & 2.2 & 0.45 & $\mathrm{U}$ & 0.45 & 437 & $\mathrm{CJ}$ & 2.2 & 0.07 & $\mathrm{U}$ & 0.07 & 124 & $\mathrm{C}$ & 0.73 \\
\hline OB B7 & J12WK 6 & $7 / 11 / 06$ & 10.3 & & 0.23 & 968 & & 2.2 & 0.45 & $\mathrm{U}$ & 0.45 & 496 & $\mathrm{CJ}$ & 2.2 & 0.07 & $\mathrm{U}$ & 0.07 & 124 & $\mathrm{C}$ & 0.73 \\
\hline OB B8 & J12WM7 & $7 / 17 / 06$ & 9.6 & & 0.67 & 1170 & & 6.3 & 1.3 & $\mathrm{U}$ & 1.3 & 395 & $\mathrm{~J}$ & 6.3 & 0.20 & $\mathrm{U}$ & 0.20 & 137 & & 7.0 \\
\hline $\mathrm{OBC}$ C & J12WM8 & $7 / 17 / 06$ & 8.8 & & 0.67 & 1160 & & 6.3 & 1.3 & $\mathrm{U}$ & 1.3 & 550 & $J$ & 6.3 & 0.20 & $\mathrm{U}$ & 0.20 & 103 & & 7.0 \\
\hline $\mathrm{OBC} 10$ & J12WM9 & $7 / 17 / 06$ & 9.0 & & 0.68 & 1080 & & 6.4 & 1.3 & $\mathrm{U}$ & 1.3 & 429 & $\mathrm{~J}$ & 6.4 & 0.20 & $\mathrm{U}$ & 0.20 & 120 & & 7.1 \\
\hline $\mathrm{OBCl}$ & J12WNO & $7 / 17 / 06$ & 9.5 & & 0.68 & 1010 & & 6.4 & 1.3 & $\mathrm{U}$ & 1.3 & 414 & $\mathrm{~J}$ & 6.4 & 0.20 & $\mathrm{U}$ & 0.20 & 160 & & 7.1 \\
\hline $\mathrm{OBC} 2$ & J12WN1 & $7 / 17 / 06$ & 8.9 & & 0.67 & 969 & & 6.4 & 1.3 & $\mathrm{U}$ & 1.3 & 493 & $\mathrm{~J}$ & 6.4 & 0.20 & $\mathrm{U}$ & 0.20 & 103 & & 7.1 \\
\hline OB D3 & J12WM5 & $7 / 12 / 06$ & 9.8 & & 0.69 & 1100 & & 6.5 & 1.3 & $\mathrm{U}$ & 1.3 & 370 & & 6.5 & 0.20 & U & 0.20 & 130 & & 2.2 \\
\hline OB D4 & J12WM6 & $7 / 12 / 06$ & 10.5 & & 0.68 & 1190 & & 6.4 & 1.3 & $\mathrm{U}$ & 1.3 & 417 & & 6.4 & 0.20 & $\mathrm{U}$ & 0.20 & 154 & & 2.2 \\
\hline $\mathrm{OB} \mathrm{D5}$ & I12WM3 & $7 / 12 / 06$ & 9.4 & & 0.67 & 1090 & & 6.4 & 1.3 & $\mathrm{u}$ & 1.3 & 435 & & 6.4 & 0.20 & $\mathrm{U}$ & 0.20 & 170 & & 2.1 \\
\hline OB D6 & J12WM4 & $7 / 12 / 06$ & 9.3 & & 0.67 & 1150 & & 6.4 & 1.3 & $\mathrm{U}$ & 1.3 & 391 & & 6.4 & 0.20 & $\mathrm{U}$ & 0.20 & 129 & & 2.1 \\
\hline $\mathrm{BCLAl}$ & $\mathrm{J12WN2}$ & $7 / 20 / 06$ & 10.8 & & 0.68 & 1050 & $\mathrm{C}$ & 6.4 & 1.3 & $\mathrm{U}$ & 1.3 & 438 & $\mathrm{R}$ & 6.4 & 0.20 & $\mathrm{U}$ & 0.20 & 180 & & 2.1 \\
\hline $\mathrm{BCLA} 2$ & J12WM1 & $7 / 12 / 06$ & 10.4 & & 0.68 & 1020 & & 6.4 & 1.3 & $\mathrm{U}$ & 1.3 & 451 & & 6.4 & 0.20 & $\mathrm{U}$ & 0.20 & 142 & & 2.2 \\
\hline $\mathrm{BCL} \mathrm{A} 3$ & J12WM2 & $7 / 12 / 06$ & 10.0 & & 0.68 & 1110 & & 6.5 & 1.3 & $\mathrm{U}$ & 1.3 & 448 & & 6.5 & 0.20 & U & 0.20 & 121 & & 2.2 \\
\hline $\mathrm{BCL} \mathrm{A4}$ & J12WM0 & $7 / 12 / 06$ & 9.3 & & 0.68 & 1000 & & 6.4 & 1.3 & $\mathrm{U}$ & 1.3 & 465 & & 6.4 & 0.20 & $\mathrm{U}$ & 0.20 & 126 & & 2.2 \\
\hline BCL B5 & J12WL9 & $7 / 12 / 06$ & 11.1 & & 0.68 & 1160 & & 6.5 & 1.3 & $\mathrm{U}$ & 1.3 & 438 & & 6.5 & 0.20 & $\mathrm{U}$ & 0.20 & 170 & & 2.2 \\
\hline BCL B6 & J12WL8 & $7 / 12 / 06$ & 9.8 & & 0.68 & 1080 & & 6.4 & 1.3 & $\mathrm{U}$ & 1.3 & 479 & & 6.4 & 0.20 & $\mathrm{U}$ & 0.20 & 136 & & 2.2 \\
\hline BCL B7 & J12WL7 & $7 / 12 / 06$ & 11.4 & & 0.68 & 1200 & & 6.4 & 1.3 & $\mathrm{U}$ & 1.3 & 414 & & 6.4 & 0.20 & $\mathrm{U}$ & 0.20 & 123 & & 2.2 \\
\hline BCL B8 & J12WL6 & $7 / 12 / 06$ & 9.2 & & 0.68 & 1110 & & 6.4 & 1.3 & $\mathrm{U}$ & 1.3 & 500 & & 6.4 & 0.20 & $\mathrm{U}$ & 0.20 & 104 & & 2.2 \\
\hline Split of J12WL6 & J12WW0 & $7 / 12 / 06$ & 11.3 & & 0.76 & 1450 & & 50.4 & 0.33 & $\mathrm{~B}^{*}$ & 0.17 & 1560 & $\mathrm{CN}$ & 4.0 & 0.20 & UNJ & 0.20 & 222 & $\mathrm{C}$ & 10.1 \\
\hline BCL C9 & I12WK7 & $7 / 11 / 06$ & 11.0 & & 0.23 & 1180 & & 2.2 & 0.45 & $\mathrm{U}$ & 0.45 & 380 & $\mathrm{CJ}$ & 2.2 & 0.07 & $\mathrm{U}$ & 0.07 & 172 & $\mathrm{C}$ & 0.73 \\
\hline $\mathrm{BCL} \mathrm{ClO}$ & J12WK9 & $7 / 11 / 06$ & 11.3 & & 0.23 & 1200 & & 2.2 & 0.49 & & 0.46 & 451 & $\mathrm{CJ}$ & 2.2 & 0.07 & $\mathrm{U}$ & 0.07 & 220 & $\mathrm{C}$ & 0.74 \\
\hline $\mathrm{BCLCl}$ & J12WK8 & $7 / 11 / 06$ & 10.1 & & 0.23 & 1270 & & 2.2 & 0.45 & $\mathrm{U}$ & 0.45 & 470 & $\mathrm{CJ}$ & 2.2 & 0.07 & $\mathrm{U}$ & 0.07 & 189 & C & 0.74 \\
\hline $\mathrm{BCLC2}$ & J12WL2 & $7 / 11 / 06$ & 12.5 & & 0.23 & 1140 & & 2.2 & 0.80 & & 0.45 & 532 & $\mathrm{CJ}$ & 2.2 & 0.07 & $\mathrm{U}$ & 0.07 & 239 & C & 0.73 \\
\hline BCL D3 & J12WL3 & $7 / 11 / 06$ & 11.9 & & 0.23 & 973 & & 2.2 & 1.0 & & 0.45 & 462 & $\mathrm{CI}$ & 2.2 & 0.07 & $\mathrm{U}$ & 0.07 & 162 & C & 0.73 \\
\hline BCL D4 & J12WL4 & $7 / 11 / 06$ & 9.6 & & 0.23 & 1130 & & 2.2 & 0.76 & & 0.45 & 561 & $\mathrm{CJ}$ & 2.2 & 0.07 & $\mathrm{U}$ & 0.07 & 145 & C & 0.73 \\
\hline Duplicate of J12WL4 & J12WLS & $7 / 11 / 06$ & 10.3 & & 0.23 & 1160 & & 2.2 & 0.45 & $\mathrm{U}$ & 0.45 & 613 & $\mathrm{CJ}$ & 2.2 & 0.07 & $\mathrm{U}$ & 0.07 & 146 & $\mathrm{C}$ & 0.73 \\
\hline $\mathrm{BCL}$ D5 & J12WLO & $7 / 11 / 06$ & 10.2 & & 0.23 & 1010 & & 2.2 & 0.54 & & 0.45 & 562 & $\mathrm{Cr}$ & 2.2 & 0.07 & $\mathrm{U}$ & 0.07 & 134 & $\mathrm{C}$ & 0.73 \\
\hline BCL D6 & J12WL1 & $7 / 11 / 06$ & 12.1 & & 0.23 & 1180 & & 2.2 & 0.63 & & 0.45 & 438 & $\mathrm{CJ}$ & 2.2 & 0.07 & $U$ & 0.07 & 205 & $\mathrm{C}$ & 0.73 \\
\hline
\end{tabular}

\begin{tabular}{|c|c|c|c|}
\hline Attachment & 1 & Sheet No. & 26 of 113 \\
\hline Originator & J.M. Capron & Date & $10 / 03 / 07$ \\
\hline Checked & T.M. Blakley & Date & \\
\hline Calc. No. & $0100 \mathrm{~B}-\mathrm{CA}-\mathrm{V} 0299$ & Rev. No. & 1 \\
\hline
\end{tabular}


Attachment 1. 118-B-1 Verification Sampling Results.

\begin{tabular}{|c|c|c|c|c|c|c|c|c|c|c|c|c|c|c|c|c|c|c|c|c|}
\hline \multirow{2}{*}{ Sample Location } & \multirow{2}{*}{$\begin{array}{l}\text { Sample } \\
\text { Number }\end{array}$} & \multirow{2}{*}{$\begin{array}{c}\text { Sample } \\
\text { Date }\end{array}$} & \multicolumn{3}{|c|}{ Nickel } & \multicolumn{3}{|c|}{ Potassium } & \multicolumn{3}{|c|}{ Selenium } & \multicolumn{3}{|c|}{ Silicon } & \multicolumn{3}{|c|}{ Silver } & \multicolumn{3}{|c|}{ Sodium } \\
\hline & & & $\mathrm{mg} / \mathrm{kg}$ & $Q$ & $\mathrm{PQL}$ & $\mathrm{mg} / \mathrm{kg}$ & $Q$ & PQL & $\mathrm{mg} / \mathrm{kg}$ & $Q$ & PQL & $\mathrm{mg} / \mathrm{kg}$ & $Q$ & PQL & $\mathrm{mg} / \mathrm{kg}$ & $Q$ & PQL & $\mathrm{mg} / \mathrm{kg}$ & $\mathrm{Q}$ & PQL \\
\hline SPAA1 & $313 \mathrm{HY} 3$ & $10 / 12 / 06$ & 10.2 & & 0.37 & 1210 & $\mathrm{C}$ & 6.0 & 0.74 & U & 0.74 & 423 & & 1.1 & 0.31 & U & 0.31 & 161 & $\mathrm{C}$ & 0.60 \\
\hline SPA A2 & J13HY4 & $10 / 12 / 06$ & 10.3 & & 0.37 & 1140 & $\mathrm{C}$ & 6.0 & 0.73 & $\mathrm{U}$ & 0.73 & 465 & & 1.1 & 0.31 & $\mathrm{U}$ & 0.31 & 104 & $\mathrm{C}$ & 0.59 \\
\hline SPA A3 & J13HY5 & $10 / 12 / 06$ & 10.1 & & 0.37 & 1250 & $\mathrm{C}$ & 6.0 & 0.73 & $\mathrm{U}$ & 0.73 & 430 & & 1.1 & 0.31 & $\mathrm{U}$ & 0.31 & 137 & $\mathrm{C}$ & 0.59 \\
\hline SPA A4 & ग13HY6 & $10 / 12 / 06$ & 10.2 & & 0.37 & 1160 & $\mathrm{C}$ & 6.1 & 0.74 & $\mathrm{U}$ & 0.74 & 459 & & 1.1 & 0.31 & $\mathrm{U}$ & 0.31 & 176 & $\mathrm{C}$ & 0.60 \\
\hline SPA B5 & J13HY7 & $10 / 12 / 06$ & 10.8 & & 0.37 & 1220 & $\mathrm{C}$ & 6.0 & 0.73 & $\mathrm{U}$ & 0.73 & 446 & & 1.1 & 0.31 & $\mathrm{U}$ & 0.31 & 108 & $\mathrm{C}$ & 0.59 \\
\hline Duplicate of J13HY7 & $\mathrm{J} 13 \mathrm{~J} 09$ & $10 / 12 / 06$ & 10.4 & & 0.37 & 1300 & $\mathrm{C}$ & 6.0 & 0.74 & $\mathrm{U}$ & 0.74 & 456 & & 1.1 & 0.31 & $\mathrm{U}$ & 0.361 & 119 & $\mathrm{C}$ & 0.59 \\
\hline Split of J13HY7 & $\mathrm{J} 13 \mathrm{~J} 10$ & $10 / 12 / 06$ & 13.9 & & 0.76 & 1500 & & 50.4 & 0.62 & $\mathrm{~B}^{*} \mathrm{CJ}$ & 0.17 & 234 & $\mathrm{CNJ}$ & 4.0 & 0.20 & UNJ & 0.20 & 173 & $\mathrm{C}$ & 10.1 \\
\hline SPAB6 & J13HY8 & $10 / 17 / 06$ & 9.4 & & 0.13 & 1170 & & 2.1 & 0.26 & $\mathrm{U}$ & 0.26 & 334 & $\mathrm{~J}$ & 0.40 & 0.11 & $U$ & 0.11 & 112 & $\mathrm{C}$ & 0.21 \\
\hline SPA B7 & J13HY9 & $10 / 17 / 06$ & 9.8 & & 0.13 & 1040 & & 2.1 & 0.26 & $\mathrm{U}$ & 0.26 & 317 & $\mathrm{~J}$ & 0.40 & 0.11 & $\mathrm{U}$ & 0.11 & 164 & $\mathrm{C}$ & 0.21 \\
\hline SPA B8 & $\mathrm{J} 13 \mathrm{~J} 00$ & $10 / 17 / 06$ & 11.1 & & 0.13 & 1250 & & 2.1 & 0.26 & U & 0.26 & 556 & J & 0.40 & 0.11 & $\mathrm{U}$ & 0.11 & 134 & C & 0.21 \\
\hline SPA C9 & $\mathrm{J} 13 \mathrm{~J} 01$ & $10 / 17 / 06$ & 9.7 & & 0.14 & 1270 & & 2.2 & 0.27 & $U$ & 0.27 & 385 & $\mathrm{~J}$ & 0.42 & 0.12 & $\mathrm{U}$ & 0.12 & 114 & $\mathrm{C}$ & 0.22 \\
\hline SPA C10 & $\mathrm{J} 13 \mathrm{~J} 02$ & $10 / 18 / 06$ & 9.2 & & 0.40 & 981 & & 6.5 & 0.79 & $\mathrm{U}$ & 0.79 & 305 & & 1.2 & 0.34 & $\mathrm{U}$ & 0.34 & 105 & $\mathrm{C}$ & 0.64 \\
\hline SPA Cl & $\mathrm{J} 13 \mathrm{~J} 03$ & $10 / 18 / 06$ & 9.5 & & 0.39 & 979 & & 6.4 & 0.79 & $\mathrm{U}$ & 0.79 & 401 & & 1.2 & 0.33 & $\mathrm{U}$ & 0.33 & 104 & $\mathrm{C}$ & 0.64 \\
\hline SPA C2 & $\mathrm{J} 13 \mathrm{~J} 04$ & $10 / 18 / 06$ & 10.1 & & 0.39 & 570 & & 6.4 & 0.79 & U & 0.79 & 305 & & 1.2 & 0.33 & $\mathrm{U}$ & 0.33 & 121 & C & 0.64 \\
\hline SPA D3 & $J 13.05$ & $10 / 18 / 06$ & 11.8 & & 0.39 & 810 & & 6.3 & 0.78 & $\mathrm{U}$ & 0.78 & 347 & & 1.2 & 0.33 & $\mathrm{U}$ & 0.33 & 125 & $\mathrm{C}$ & 0.63 \\
\hline SPA D4 & J13.06 & $10 / 19 / 06$ & 13.9 & $\mathrm{~J}$ & 0.39 & 924 & & 6.4 & 0.79 & $\mathrm{U}$ & 0.79 & 450 & \begin{tabular}{|l|}
$\mathrm{CJ}$ \\
\end{tabular} & 1.2 & 0.33 & U & 0.33 & 133 & $\mathrm{C}$ & 0.64 \\
\hline SPA D5 & $113 \mathrm{~J} 07$ & $10 / 23 / 06$ & 10.5 & & 0.38 & 806 & & 6.1 & 0.75 & $\mathrm{U}$ & 0.75 & 548 & C & 1.2 & 0.32 & $\mathrm{U}$ & 0.32 & 133 & C & 0.61 \\
\hline SPA D6 & $\mathrm{J} 13 \mathrm{~J} 08$ & $10 / 23 / 06$ & 9.3 & & 0.38 & 934 & & 6.2 & 0.76 & $\mathrm{U}$ & 0.76 & 528 & C & 1.2 & 0.32 & $\mathrm{U}$ & 0.32 & 143 & C & 0.62 \\
\hline FS-2 & $J 12 R 25$ & $7 / 10 / 06$ & 8.8 & & 0.67 & 715 & & 6.4 & 1.3 & $\mathrm{U}$ & 1.3 & 438 & & 6.4 & 0.20 & $\mathrm{U}$ & 0.20 & 95.4 & & 2.1 \\
\hline FS-3 & $J 12 R 26$ & $7 / 10 / 06$ & 13.2 & & 0.68 & 1670 & & 6.4 & 1.3 & $\mathrm{U}$ & 0.13 & 433 & & 6.4 & 0.20 & $\mathrm{U}$ & 0.20 & 254 & & 2.2 \\
\hline FS-5 & $\mathrm{J} 12 \mathrm{R} 28$ & $7 / 10 / 06$ & 10.4 & & 0.37 & 1360 & $\mathrm{C}$ & 6.1 & 0.74 & $\mathrm{U}$ & 0.74 & 611 & $\mathrm{C}$ & 1.1 & 0.31 & $\mathrm{U}$ & 0.31 & 283 & C & 0.60 \\
\hline FS- 6 & $112 R 29$ & $7 / 10 / 06$ & 15.2 & & 0.67 & 1920 & & 6.4 & 1.3 & $\mathrm{U}$ & 1.3 & 629 & & 6.4 & 0.20 & $\mathrm{U}$ & 0.20 & 324 & & 2.1 \\
\hline FS-7 & J13P85 & $11 / 15 / 06$ & 8.2 & & 0.68 & 874 & $\mathrm{C}$ & 6.5 & 1.3 & $\mathrm{U}$ & 1.3 & 447 & $\mathrm{C}$ & 2.0 & 0.15 & U & 0.15 & 223 & $\mathrm{C}$ & 0.77 \\
\hline FS-8 & $\mathrm{J} 12 \mathrm{R} 31$ & $7 / 10 / 06$ & 9.0 & & 0.68 & 1380 & & 6.4 & 1.3 & $\mathrm{U}$ & 1.3 & 718 & & 6.4 & 0.20 & $\mathrm{U}$ & 0.20 & 326 & & 2.2 \\
\hline FS-9 & $\mathrm{J12R} 32$ & $7 / 10 / 06$ & 11.9 & & 0.69 & 1580 & & 6.5 & 1.3 & $\mathrm{U}$ & 1.3 & 417 & & 6.5 & 0.20 & U & 0.20 & 1000 & & 2.2 \\
\hline FS-11 & $\mathrm{J} 12 \mathrm{R} 34$ & $7 / 10 / 06$ & 14.3 & & 0.37 & 1490 & $\mathrm{C}$ & 6.1 & 0.74 & $\mathrm{U}$ & 0.74 & 680 & $\mathrm{C}$ & 1.1 & 0.31 & $\mathrm{U}$ & 0.31 & 408 & C & 0.60 \\
\hline FS-14 & $512 \times B 3$ & $7 / 19 / 06$ & 9.0 & & 0.73 & 785 & $\mathrm{C}$ & 6.9 & 1.4 & $\mathrm{U}$ & 1.4 & 392 & $\mathrm{R}$ & 6.9 & 0.21 & $\mathrm{U}$ & 0.21 & 130 & & 2.3 \\
\hline FS-15 & $\mathrm{J} 13 \mathrm{NX} 4$ & $11 / 14 / 06$ & 9.4 & & 0.68 & 524 & $\mathrm{C}$ & 6.5 & 1.3 & $\mathrm{U}$ & 1.3 & 626 & $\mathrm{C}$ & 2.0 & 0.15 & $\mathrm{U}$ & 0.15 & 110 & $\mathrm{C}$ & 0.77 \\
\hline FS- 16 & $\mathrm{~J} 13 \mathrm{P} 84$ & $11 / 15 / 06$ & 7.8 & & 0.66 & 513 & $\mathrm{C}$ & 6.4 & 1.3 & U & 1.3 & 426 & $\mathrm{C}$ & 1.9 & 0.15 & $U$ & 0.15 & 130 & $\mathrm{C}$ & 0.75 \\
\hline FS-17 & $\mathrm{J} 14 \mathrm{JJ} 3$ & $3 / 5 / 07$ & 11.9 & & 0.19 & 1300 & & 4.8 & 0.36 & $\mathrm{U}$ & 0.36 & 486 & $\mathrm{R}$ & 0.41 & 0.09 & $\mathrm{U}$ & 0.09 & 159 & & 2.9 \\
\hline FS-17 resample & $\mathrm{J152 \textrm {M } 1}$ & $5 / 10 / 07$ & 10.1 & & 0.18 & 1260 & & 4.7 & 0.36 & $\mathrm{U}$ & 0.36 & 746 & & 0.40 & 0.09 & $\mathrm{U}$ & 0.09 & 193 & & 0.42 \\
\hline FS-18 & $\mathrm{J} 14 \mathrm{~L} 38$ & $3 / 5 / 07$ & 9.3 & & 0.19 & 1210 & & 4.8 & 0.36 & $\mathrm{U}$ & 0.36 & 447 & $\mathrm{R}$ & 0.41 & 0.09 & $\mathrm{U}$ & 0.09 & 144 & & 2.9 \\
\hline FS-19 & $\mathrm{J} 14 \mathrm{~L} 39$ & $3 / 5 / 07$ & 10.0 & & 0.18 & 1130 & & 4.7 & 0.36 & $\mathrm{U}$ & 0.36 & 455 & $\mathrm{R}$ & 0.41 & 0.09 & $\mathrm{U}$ & 0.09 & 146 & & 2.9 \\
\hline Equipment blank & $\mathrm{J} 12 \times 59$ & $7 / 20 / 06$ & 0.67 & $\bar{U}$ & 0.67 & 66.4 & $\mathrm{C}$ & 6.4 & 1.3 & $\mathrm{U}$ & 1.3 & 117 & $\mathrm{R}$ & 6.4 & 0.20 & $\mathrm{U}$ & 0.20 & 28.8 & & 2.1 \\
\hline
\end{tabular}

\begin{tabular}{|c|c|c|c|}
\hline Attachment & 1 & Sheet No. & 27 of 113 \\
\hline Originator & J. M. Capron & Date & $10 / 03 / 07$ \\
\hline Checked & T. M. Blakley & Date & \\
\hline Calc. No. & $0100 \mathrm{~B}-\mathrm{CA}-\mathrm{V} 0299$ & Rev. No. & 1 \\
\hline
\end{tabular}


Attachment 1. 118-B-1 Verification Sampling Results.

\begin{tabular}{|c|c|c|c|c|c|c|c|c|}
\hline \multirow{2}{*}{ Sample Location } & \multirow{2}{*}{$\begin{array}{l}\text { Sample } \\
\text { Number }\end{array}$} & \multirow{2}{*}{$\begin{array}{c}\text { Sample } \\
\text { Date }\end{array}$} & \multicolumn{3}{|c|}{ Vanadium } & \multicolumn{3}{|c|}{ Zine } \\
\hline & & & $\mathrm{mg} / \mathrm{kg}$ & $Q$ & PQL & $\mathrm{mg} / \mathrm{kg}$ & $Q$ & PQL \\
\hline Area $1 \mathrm{Al}$ & $\mathrm{J} 12 \times 44$ & $7 / 19 / 06$ & 46.1 & & $0.26^{\circ}$ & 45.0 & & 0.46 \\
\hline Duplicate of J12X44 & $2 \times 48$ & $7 / 19 / 06$ & 51.9 & & 0.26 & 49.1 & & 0.47 \\
\hline Split of J12X44 & $J 12 \times 49$ & $7 / 19 / 06$ & 68.7 & $\mathrm{~N}$ & 0.68 & 44.5 & $\mathrm{CN}$ & 1.4 \\
\hline Area $1 \mathrm{~A} 2$ & $2 \times 45$ & $19 / 06$ & 49.3 & & 0.26 & 42.7 & & 0.47 \\
\hline Area $1 \mathrm{~A} 3$ & $\mathrm{~J} 12 \times 46$ & $7 / 20 / 06$ & 38.2 & & 0.25 & 39.9 & & 0.45 \\
\hline Area $1 \mathrm{~A} 4$ & $\mathrm{~J} 12 \times 47$ & $7 / 20 / 06$ & 51.8 & & 0.25 & 43.8 & & 0.45 \\
\hline Area $2 \mathrm{Al}$ & $\mathrm{J} 12 \times 50$ & $7 / 20 / 06$ & 54.0 & & 0.25 & 43.3 & & 0.45 \\
\hline Area $2 \mathrm{~A} 2$ & $\mathrm{~J} 12 \times 51$ & $7 / 20 / 06$ & 46.5 & & 0.26 & 41.1 & & 0.46 \\
\hline Area $2 \mathrm{~A} 3$ & $\mathrm{~J} 12 \times 52$ & $7 / 20 / 06$ & 54.7 & & 0.26 & 42.1 & & 0.46 \\
\hline Area 2 A4 & $\mathrm{J} 12 \times 53$ & $7 / 20 / 06$ & 39.8 & & 0.25 & 36.3 & & 0.45 \\
\hline Area $3 \mathrm{Al}$ & $\mathrm{J} 12 \times 54$ & $7 / 20 / 06$ & 42.6 & & 0.25 & 37.5 & & 0.45 \\
\hline Area $3 \mathrm{~A} 2$ & $\mathrm{~J} 12 \times 55$ & 7/20/06 & 49.2 & & 0.25 & 43.2 & & 0.45 \\
\hline Area $3 \mathrm{~A} 3$ & $\mathrm{J12 \times 56}$ & $7 / 20 / 06$ & 40.0 & & 0.25 & 44.3 & & 0.45 \\
\hline Duplicate of J12X56 & $\mathrm{J} 12 \times 58$ & 7/20/06 & 37.6 & & 0.26 & 41.9 & & 0.46 \\
\hline Split of $112 \times 56$ & $J 12 \times 60$ & $7 / 20 / 06$ & 44.9 & $\mathrm{~N}$ & 0.69 & 32.6 & & 1.4 \\
\hline Area 3 A4 & $112 \times 57$ & 7/20/06 & 44.9 & & 0.25 & 42.2 & & 0.45 \\
\hline Area $4 \mathrm{Al}$ & $512 \times 61$ & $7 / 25 / 06$ & 53.4 & & 0.25 & 41.7 & $\mathrm{C}$ & 0.45 \\
\hline Duplicate of $J 12 \times 61$ & 65 & $7 / 25 / 06$ & 44.8 & & 0.25 & 41.0 & $\mathrm{C}$ & 0.45 \\
\hline Split of J12X61 & 66 & $7 / 25 / 06$ & 68.4 & $\mathrm{~N}$ & 0.68 & 48.3 & & 1.4 \\
\hline Area $4 \mathrm{~A} 2$ & 62 & $7 / 25 / 06$ & 43.5 & & 0.25 & 35.8 & $\mathrm{C}$ & 0.45 \\
\hline Area $4 \mathrm{~A} 3$ & $\mathrm{~J} 12 \times 63$ & $7 / 25 / 06$ & 41.6 & & 0.25 & 39.5 & C & 0.45 \\
\hline Area 4 A4 & $.112 \times 64$ & $7 / 25 / 06$ & 50.8 & & 0.25 & 42.4 & $\mathrm{C}$ & 0.44 \\
\hline Area $5 \mathrm{~A} 1$ & $\mathrm{~V} 12$ & $12 / 7 / 06$ & 33.6 & & 0.18 & 38.6 & C & 0.12 \\
\hline Area 5 A2 & 10 & $12 / 7 / 06$ & 34.7 & & 0.18 & 31.7 & C & 0.12 \\
\hline Area $5 \mathrm{~A} 3$ & 13 & $12 / 7 / 06$ & 13.1 & & 0.06 & 33.8 & C & 0.04 \\
\hline Area $5 \mathrm{~A} 4$ & 11 & $12 / 7 / 06$ & 33.7 & & 0.18 & 33.1 & C & 0.12 \\
\hline Area $6 \mathrm{Al}$ & $\mathrm{exC2}$ & $7 / 25 / 06$ & 37.3 & & 0.25 & 35.2 & C & 0.45 \\
\hline Area $6 \mathrm{~A} 2$ & $\mathrm{XC3}$ & $7 / 25 / 06$ & 42.5 & & 0.25 & 39.2 & C & 0.45 \\
\hline Area $6 \mathrm{~A} 3$ & $312 \times C 4$ & $7 / 25 / 06$ & 49.1 & & 0.25 & 40.9 & C & 0.45 \\
\hline Area $6 \mathrm{A4}$ & $.12 \times C S$ & $7 / 25 / 06$ & 48.7 & & 0.25 & 41.2 & $\mathrm{C}$ & 0.45 \\
\hline Area $7 \mathrm{~A} 1$ & $\mathrm{J12XC6}$ & $7 / 26 / 06$ & 36.4 & & 0.25 & 48.7 & C & 0.45 \\
\hline Area 7 A2 & $512 \times C 7$ & $7 / 26 / 06$ & 40.9 & & 0.25 & 41.0 & $\mathrm{C}$ & 0.44 \\
\hline Area 7 A3 & $\mathrm{J} 12 \mathrm{XC8}$ & $7 / 26 / 06$ & 32.7 & & 0.25 & 31.1 & C & 0.45 \\
\hline Area $7 \mathrm{~A} 4$ & $\mathrm{J12XC9}$ & $7 / 26 / 06$ & 38.0 & & 0.25 & 44.5 & C & 0.45 \\
\hline
\end{tabular}

\begin{tabular}{|c|c|c|c|c|c|}
\hline \multirow[t]{2}{*}{ Sample Location } & \multirow{2}{*}{$\begin{array}{l}\text { Sample } \\
\text { Number }\end{array}$} & \multirow{2}{*}{$\begin{array}{l}\text { Sample } \\
\text { Date }\end{array}$} & \multicolumn{3}{|c|}{$\begin{array}{l}\text { Total Petroleum } \\
\text { Hydrocarbons }\end{array}$} \\
\hline & & & $\mathrm{mg} / \mathrm{kg}$ & $\mathbf{Q}$ & PQL \\
\hline Area $1 \mathrm{~A} 1$ & $\mathrm{~J} 12 \times 44$ & $7 / 19 / 06$ & 135 & $\mathrm{U}$ & 135 \\
\hline Duplicate of J12X44 & $\mathrm{J} 12 \times 48$ & $7 / 19 / 06$ & 135 & $\bar{U}$ & 135 \\
\hline Split of J12X44 & $\mathrm{J} 12 \times 49$ & $7 / 19 / 06$ & 173 & UN & 173 \\
\hline Area $1 \mathrm{~A} 2$ & $\mathrm{~J} 12 \mathrm{X} 45$ & $7 / 19 / 06$ & 134 & $\mathrm{U}$ & 134 \\
\hline Area $1 \mathrm{~A} 3$ & $\mathrm{J12X} 46$ & $7 / 20 / 06$ & 134 & $\mathrm{U}$ & 134 \\
\hline Area $1 \mathrm{~A} 4$ & $\mathrm{~J} 12 \times 47$ & $7 / 20 / 06$ & 133 & $\mathrm{U}$ & 133 \\
\hline Area $2 \mathrm{Al}$ & $\mathrm{J} 12 \times 50$ & $7 / 20 / 06$ & 133 & $\mathrm{U}$ & 133 \\
\hline Area $2 \mathrm{~A} 2$ & $J 12 \times 51$ & $7 / 20 / 06$ & 135 & $\mathrm{U}$ & 135 \\
\hline Area $2 \mathrm{A3}$ & $\mathrm{J12} 2 \times 52$ & $7 / 20 / 06$ & 134 & $\mathrm{U}$ & 134 \\
\hline Area 2 A4 & $\mathrm{J} 12 \times 53$ & $7 / 20 / 06$ & 134 & $\mathrm{U}$ & 134 \\
\hline Area 3A1 & $\mathrm{J} 12 \times 54$ & $7 / 20 / 06$ & 135 & $\mathrm{U}$ & 135 \\
\hline Area $3 \mathrm{~A} 2$ & $\mathrm{~J} 12 \times 55$ & $7 / 20 / 06$ & 134 & $\mathrm{U}$ & 134 \\
\hline Area $3 \mathrm{A3}$ & $\mathrm{J12 \times 56}$ & $7 / 20 / 06$ & 135 & U & 135 \\
\hline Duplicate of J12X56 & $\mathrm{J} 12 \times 58$ & $7 / 20 / 06$ & 136 & $\mathrm{U}$ & 136 \\
\hline Split of J12X56 & $\mathrm{J} 12 \times 60$ & $7 / 20 / 06$ & 173 & UNJ & 173 \\
\hline Area $3 \mathrm{~A} 4$ & $\mathrm{~J} 12 \times 57$ & $7 / 20 / 06$ & 134 & $\mathrm{U}$ & 134 \\
\hline Area 4Al & $312 \times 61$ & $7 / 25 / 06$ & 134 & $\mathrm{U}$ & 134 \\
\hline Duplicate of J12X61 & $J 12 \times 65$ & $7 / 25 / 06$ & 134 & $\mathrm{U}$ & 134 \\
\hline Split of J12X61 & $\mathrm{J} 12 \times 66$ & $7 / 25 / 06$ & 173 & UNJ & 173 \\
\hline Area 4A2 & $\mathrm{J} 12 \mathrm{2} 62$ & $7 / 25 / 06$ & 133 & $\mathrm{U}$ & 133 \\
\hline Area $4 \mathrm{~A} 3$ & $\mathrm{~J} 12 \times 63$ & $7 / 25 / 06$ & 133 & $\mathrm{U}$ & 133 \\
\hline Area 4 A4 & $\mathrm{J} 12 \times 64$ & $7 / 25 / 06$ & 134 & U & 134 \\
\hline Area $5 \mathrm{Al}$ & $\mathrm{J} 13 \mathrm{~V} 12$ & $12 / 7 / 06$ & 139 & $\mathrm{U}$ & 139 \\
\hline Area $5 \mathrm{~A} 2$ & $\mathrm{J13V} 10$ & $12 / 7 / 06$ & 139 & $\mathrm{U}$ & 139 \\
\hline Area $5 \mathrm{~A} 3$ & $\mathrm{~J} 13 \mathrm{~V} 13$ & $12 / 7 / 06$ & 139 & U & 139 \\
\hline Area $5 \mathrm{A4}$ & J13V11 & $12 / 7 / 06$ & 142 & U & 142 \\
\hline Area $6 \mathrm{~A} 1$ & J13D51 & $9 / 5 / 06$ & 133 & U & 133 \\
\hline Area $6 \mathrm{~A} 2$ & J13D52 & $9 / 5 / 06$ & 134 & $\mathrm{U}$ & 134 \\
\hline Area $6 \mathrm{~A} 3$ & J13D53 & $9 / 5 / 06$ & 133 & U & 133 \\
\hline Area $6 \mathrm{~A} 4$ & J13D54 & $9 / 5 / 06$ & 133 & U & 133 \\
\hline Area $7 \mathrm{Al}$ & J13D55 & $9 / 5 / 06$ & 133 & U & 133 \\
\hline Area 7 A2 & J13D56 & $9 / 5 / 06$ & 133 & $\mathrm{U}$ & 133 \\
\hline Area $7 \mathrm{A3}$ & J13D57 & $9 / 5 / 06$ & 134 & $\mathrm{U}$ & 134 \\
\hline Area $7 \mathrm{~A} 4$ & J13D58 & $9 / 5 / 06$ & 133 & $\mathrm{U}$ & 133 \\
\hline
\end{tabular}

Attachment Originator

Checked J.M Capron 1 Sheet No. 28 of 113

Calc. No. T. M. Blakley Sheet No.
Date $\frac{\frac{28 \text { of } 113}{10 / 03 / 07}}{-1}$ 
Attachment 1. 118-B-1 Verification Sampling Results.

\begin{tabular}{|c|c|c|c|c|c|c|c|c|}
\hline \multirow{2}{*}{ Sample Location } & \multirow{2}{*}{$\begin{array}{l}\text { Sample } \\
\text { Number }\end{array}$} & \multirow{2}{*}{$\begin{array}{c}\text { Sample } \\
\text { Date }\end{array}$} & \multicolumn{3}{|c|}{ Vanadium } & \multicolumn{3}{|c|}{ Zinc } \\
\hline & & & $\mathrm{mg} / \mathrm{kg}$ & $Q$ & PQL & $\mathrm{mg} / \mathrm{kg}$ & $Q$ & PQL \\
\hline OBAI & $\mathrm{J} 12 \mathrm{WK} 0$ & $7 / 11 / 06$ & 38.0 & & 0.09 & 35.9 & & 0.16 \\
\hline $\mathrm{OB} \mathrm{A2}$ & $\mathrm{J12 \textrm {WK } 1}$ & $7 / 11 / 06$ & 44.7 & & 0.09 & 36.7 & & 0.16 \\
\hline $\mathrm{OBA} 3$ & $312 \mathrm{WK} 2$ & $7 / 11 / 06$ & 33.9 & & 0.09 & 32.3 & & 0.15 \\
\hline OB A4 & $\mathrm{J12WK3}$ & $7 / 11 / 06$ & 35.7 & & 0.09 & 33.6 & & 0.15 \\
\hline $\mathrm{OB} \mathrm{B5}$ & $\mathrm{J} 12 \mathrm{WK} 4$ & $7 / 11 / 06$ & 51.6 & & 0.09 & 40.1 & & 0.15 \\
\hline $\mathrm{OB} B 6$ & J12WK5 & $7 / 11 / 06$ & 38.2 & & 0.09 & 36.9 & & 0.15 \\
\hline OB B7 & J12WK6 & $7 / 11 / 06$ & 37.9 & & 0.09 & 32.1 & & 0.15 \\
\hline OB B8 & J12WM7 & $7 / 17 / 06$ & 47.0 & $J$ & 0.25 & 40.1 & & 0.45 \\
\hline $\mathrm{OB} C 9$ & J12WM8 & $7 / 17 / 06$ & 36.1 & $\mathrm{~J}$ & 0.25 & 33.9 & & 0.45 \\
\hline $\mathrm{OBC} 10$ & J12WM9 & $7 / 17 / 06$ & 39.0 & $y$ & 0.25 & 35.4 & & 0.45 \\
\hline $\mathrm{OBCl}$ & $\mathrm{J} 12 \mathrm{WN} 0$ & $7 / 17 / 06$ & 55.0 & $J$ & 0.25 & 42.2 & & 0.45 \\
\hline $\mathrm{OBC} 2$ & $\mathrm{~J} 12 \mathrm{WN} 1$ & $7 / 17 / 06$ & 39.4 & $\mathrm{~J}$ & 0.25 & 35.9 & & 0.45 \\
\hline OB D3 & J12WM5 & $7 / 12 / 06$ & 49.4 & & 0.26 & 42.4 & & 0.46 \\
\hline OB D4 & J12WM6 & $7 / 12 / 06$ & 56.8 & & 0.26 & 46.5 & & 0.45 \\
\hline OB D5 & J12WM3 & $7 / 12 / 06$ & 42.1 & & 0.25 & 43.0 & & 0.45 \\
\hline OB D6 & Ji2WM4 & $7 / 12 / 06$ & 47.0 & & 0.25 & 41.0 & & 0.45 \\
\hline $\mathrm{BCL} A \mathrm{l}$ & $\mathrm{J} 12 \mathrm{WN} 2$ & $7 / 20 / 06$ & 52.7 & & 0.25 & 43.8 & & 0.45 \\
\hline $\mathrm{BCL} A 2$ & $\mathrm{~J} 12 \mathrm{WM} 1$ & $7 / 12 / 06$ & 47.8 & & 0.25 & 39.6 & & 0.45 \\
\hline $\mathrm{BCL} \mathrm{A} 3$ & J12WM2 & $7 / 12 / 06$ & 44.8 & & 0.26 & 38.3 & & 0.46 \\
\hline BCL A4 & J12WM0 & $7 / 12 / 06$ & 47.5 & & 0.26 & 39.0 & & 0.45 \\
\hline BCL B5 & J12WL9 & $7 / 12 / 06$ & 54.2 & & 0.26 & 44.2 & & 0.46 \\
\hline BCL B6 & J12WL8 & $7 / 12 / 06$ & 47.1 & & 0.26 & 40.8 & & 0.45 \\
\hline BCL B7 & J12WL7 & $7 / 12 / 06$ & 42.5 & & 0.25 & 37.5 & & 0.45 \\
\hline BCL B8 & J12WL6 & $7 / 12 / 06$ & 37.3 & & 0.25 & 34.5 & & 0.45 \\
\hline Split of J12WL6 & J12WW0 & $7 / 12 / 06$ & 49.3 & & 0.68 & 38.9 & & 1.4 \\
\hline BCL C9 & J12WK7 & $7 / 11 / 06$ & 46.3 & & 0.09 & 38.6 & & 0.15 \\
\hline $\mathrm{BCLC10}$ & J12WK9 & $7 / 11 / 06$ & 46.3 & & 0.09 & 41.9 & & 0.16 \\
\hline $\mathrm{BCL} \mathrm{Cl}$ & J12WK8 & $7 / 11 / 06$ & 43.9 & & 0.09 & 38.6 & & 0.15 \\
\hline $\mathrm{BCL}$ C2 & J12WL2 & $7 / 11 / 06$ & 49.8 & & 0.09 & 44.4 & & 0.15 \\
\hline BCL D3. & J12WL3 & $7 / 11 / 06$ & 49.6 & & 0.09 & 40.0 & & 0.15 \\
\hline $\mathrm{BCL}$ D4 & J12WL4 & $7 / 11 / 06$ & 39.7 & & 0.09 & 35.3 & & 0.15 \\
\hline Duplicate of J12WL4 & J12WL5 & $7 / 11 / 06$ & 36.5 & & 0.09 & 34.2 & & 0.15 \\
\hline BCL D5 & J12WLO & $7 / 11 / 06$ & 41.5 & & 0.09 & 36.8 & & 0.15 \\
\hline BCL D6 & $\mathrm{J} 12 \mathrm{WLI}$ & $7 / 11 / 06$ & 53.0 & & 0.09 & 45.0 & & 0.15 \\
\hline
\end{tabular}

\begin{tabular}{|c|c|c|c|c|c|}
\hline \multirow{2}{*}{ Sample Location } & \multirow{2}{*}{$\begin{array}{c}\text { Sample } \\
\text { Number }\end{array}$} & \multirow{2}{*}{$\begin{array}{c}\text { Sample } \\
\text { Date }\end{array}$} & \multicolumn{3}{|c|}{$\begin{array}{c}\text { Total Petroleum } \\
\text { Hydrocarbons }\end{array}$} \\
\cline { 4 - 6 } & & & mg/kg & Q & PQL \\
\hline OB A1 & J12WK0 & $7 / 11 / 06$ & 134 & $\mathrm{U}$ & 134 \\
\hline OB A2 & J12WK1 & $7 / 11 / 06$ & 133 & $\mathrm{U}$ & 133 \\
\hline OB A3 & J12WK2 & $7 / 11 / 06$ & 133 & $\mathrm{U}$ & 133 \\
\hline OB A4 & J12WK3 & $7 / 11 / 06$ & 134 & $\mathrm{U}$ & 134 \\
\hline OB B5 & J12WK4 & $7 / 11 / 06$ & 134 & $\mathrm{U}$ & 134 \\
\hline OB B6 & J12WK5 & $7 / 11 / 06$ & 134 & $\mathrm{U}$ & 134 \\
\hline OB B7 & J12WK6 & $7 / 11 / 06$ & 133 & $\mathrm{U}$ & 133 \\
\hline OB B8 & J12WM7 & $7 / 17 / 06$ & 133 & $\mathrm{U}$ & 133 \\
\hline OB C9 & J12WM8 & $7 / 17 / 06$ & 132 & $\mathrm{U}$ & 132 \\
\hline OB C10 & J12WM9 & $7 / 17 / 06$ & 132 & $\mathrm{U}$ & 132 \\
\hline OB C1 & J12WN0 & $7 / 17 / 06$ & 132 & $\mathrm{U}$ & 132 \\
\hline OB C2 & J12WN1 & $7 / 17 / 06$ & 133 & $\mathrm{U}$ & 133 \\
\hline OB D3 & J12WM5 & $7 / 12 / 06$ & 134 & $\mathrm{U}$ & 134 \\
\hline OB D4 & J12WM6 & $7 / 12 / 06$ & 133 & $\mathrm{U}$ & 133 \\
\hline OB D5 & J12WM3 & $7 / 12 / 06$ & 134 & $\mathrm{U}$ & 134 \\
\hline OB D6 & J12WM4 & $7 / 12 / 06$ & 134 & $\mathrm{U}$ & 134 \\
\hline BCL A1 & J12WN2 & $7 / 20 / 06$ & 134 & $\mathrm{U}$ & 134 \\
\hline BCL A2 & J12WM1 & $7 / 12 / 06$ & 133 & $\mathrm{U}$ & 133 \\
\hline BCL A3 & J12WM2 & $7 / 12 / 06$ & 133 & $\mathrm{U}$ & 133 \\
\hline BCL A4 & J12WM0 & $7 / 12 / 06$ & 133 & $\mathrm{U}$ & 133 \\
\hline BCL B5 & J12WL9 & $7 / 12 / 06$ & 133 & $\mathrm{U}$ & 133 \\
\hline BCL B6 & J12WL8 & $7 / 12 / 06$ & 134 & $\mathrm{U}$ & 134 \\
\hline BCL B7 & J12WL7 & $7 / 12 / 06$ & 132 & $\mathrm{U}$ & 132 \\
\hline BCL B8 & J12WL6 & $7 / 12 / 06$ & 134 & $\mathrm{U}$ & 134 \\
\hline Split of J12WL6 & J12WW0 & $7 / 12 / 06$ & 173 & $\mathrm{UNJ}$ & 173 \\
\hline BCL C9 & J12WK7 & $7 / 11 / 06$ & 133 & $\mathrm{U}$ & 133 \\
\hline BCL C10 & J12WK9 & $7 / 11 / 06$ & 134 & $\mathrm{U}$ & 134 \\
\hline BCL C1 & J12WK8 & $7 / 11 / 06$ & 133 & $\mathrm{U}$ & 133 \\
\hline BCL C2 & J12WL2 & $7 / 11 / 06$ & 134 & $\mathrm{U}$ & 134 \\
\hline BCL D3 & J12WL3 & $7 / 11 / 06$ & 134 & $\mathrm{U}$ & 134 \\
\hline BCL D4 & J12WL4 & $7 / 11 / 06$ & 133 & $\mathrm{U}$ & 133 \\
\hline Duplicate of J12WL4 & J12WL5 & $7 / 11 / 06$ & 134 & $\mathrm{U}$ & 134 \\
\hline BCL D5 & J12WL0 & $7 / 11 / 06$ & 133 & $\mathrm{U}$ & 133 \\
\hline BCL D6 & J12WL1 & $7 / 11 / 06$ & 133 & $\mathrm{U}$ & 133 \\
\hline & & & & & \\
\hline
\end{tabular}

Attachment

Originator

?

\begin{tabular}{|c|c|c|}
\hline 1 & \multirow{4}{*}{$\begin{array}{l}\text { Sheet No. } \\
\text { Date } \\
\text { Date } \\
\text { Rev. No. }\end{array}$} & 29 of 113 \\
\hline J.M. Capron & & $10 / 03 / 07$ \\
\hline T. M. Blakley & & \\
\hline $0100 \mathrm{~B}-\mathrm{CA}-\mathrm{V} 0299$ & & 1 \\
\hline
\end{tabular}


Attachment 1. 118-B-1 Verification Sampling Results.

\begin{tabular}{|c|c|c|c|c|c|c|c|c|c|c|c|c|c|c|}
\hline \multirow{3}{*}{$\frac{\text { Sample Location }}{\text { SPA A1 }}$} & \multirow{3}{*}{$\begin{array}{c}\begin{array}{c}\text { Sample } \\
\text { Number }\end{array} \\
\text { J13HY3 }\end{array}$} & \multirow{3}{*}{$\begin{array}{c}\begin{array}{c}\text { Sample } \\
\text { Date }\end{array} \\
10 / 12 / 06\end{array}$} & \multicolumn{3}{|c|}{ Vanadium } & \multicolumn{3}{|c|}{ Zinc } & \multirow{3}{*}{ Sample Location } & \multirow{3}{*}{$\begin{array}{l}\text { Sample } \\
\text { Number }\end{array}$} & \multirow{3}{*}{$\begin{array}{c}\text { Sample } \\
\text { Date }\end{array}$} & \multirow{2}{*}{\multicolumn{3}{|c|}{$\begin{array}{l}\text { Total Petroleum } \\
\text { Hydrocarbons }\end{array}$}} \\
\hline & & & \multirow{2}{*}{\begin{tabular}{|c|}
$\mathrm{mg} / \mathrm{kg}$ \\
38.2 \\
\end{tabular}} & \multirow[t]{2}{*}{$Q$} & \multirow{2}{*}{$\frac{\mathrm{PQL}}{0.14}$} & \multirow{2}{*}{\begin{tabular}{|c|}
$\mathrm{mg} / \mathrm{kg}$ \\
36.0
\end{tabular}} & \multirow[t]{2}{*}{$Q$} & \multirow{2}{*}{$\begin{array}{c}\text { PQL } \\
0.09\end{array}$} & & & & & & \\
\hline & & & & & & & & & & & & $\mathrm{mg} / \mathrm{kg}$ & $Q$ & PQL \\
\hline SPA A2 & $\mathrm{J} 13 \mathrm{HY} 4$ & $10 / 12 / 06$ & 41.6 & & 0.14 & 35.8 & & 0.08 & SPAA1 & $\mathrm{J} 13 \mathrm{HYY} 3$ & $10 / 12 / 06$ & 135 & $\mathrm{U}$ & 135 \\
\hline SPA A3 & J13HYS & $10 / 12 / 06$ & 46.9 & & 0.14 & 40.2 & & 0.08 & SPA A2 & J13HY4 & $10 / 12 / 06$ & 133 & $\mathrm{U}$ & 133 \\
\hline SPA A4 & J13HY6 & $10 / 12 / 06$ & 42.2 & & 0.14 & 37.0 & & 0.09 & SPA A3 & J13HY5 & $10 / 12 / 06$ & 134 & $\mathrm{U}$ & 134 \\
\hline SPA B5 & J13HY7 & $10 / 12 / 06$ & 36.4 & & 0.14 & 33.0 & & 0.08 & SPA A4 & J13HY6 & $10 / 12 / 06$ & 134 & $\mathrm{U}$ & 134 \\
\hline Duplicate of J13HY7 & $\mathrm{J} 13 \mathrm{~J} 09$ & $10 / 12 / 06$ & 39.2 & & 0.14 & 34.4 & & 0.08 & SPA B5 & $\mathrm{J} 13 \mathrm{HY} 7$ & $10 / 12 / 06$ & 133 & $\mathrm{U}$ & 133 \\
\hline Split of J13HY7 & $\mathrm{J} 13 \mathrm{~J} 10$ & $10 / 12 / 06$ & 50.9 & & 0.68 & 39.8 & $\mathrm{C}$ & 1.4 & Duplicate of J13HY7 & $\mathrm{J} 13 \mathrm{~J} 09$ & $10 / 12 / 06$ & 133 & $\mathrm{U}$ & 133 \\
\hline SPA B6 & J13HY8 & $10 / 17 / 06$ & 43.9 & & 0.05 & 36.6 & $\mathrm{C}$ & 0.03 & Split of J13HY7 & $\mathrm{J} 13 \mathrm{~J} 10$ & $10 / 12 / 06$ & 173 & U & 173 \\
\hline SPA B7 & J13HY9 & $10 / 17 / 06$ & 49.8 & & 0.05 & 41.8 & C & 0.03 & SPA B6 & J13HY8 & $10 / 17 / 06$ & 136 & $\mathrm{U}$ & 136 \\
\hline SPA B8 & J13J00 & $10 / 17 / 06$ & 33.8 & & 0.05 & 34.3 & $\mathrm{C}$ & 0.03 & SPA B7 & J13HY9 & $10 / 17 / 06$ & 137 & $\mathrm{U}$ & 137 \\
\hline SPAC9 & J13J01 & $10 / 17 / 06$ & 38.6 & & 0.05 & 37.2 & $\mathrm{C}$ & 0.03 & SPA B8 & $\mathrm{J} 13 \mathrm{~J} 00$ & $10 / 17 / 06$ & 136 & $\mathrm{U}$ & 136 \\
\hline SPA C10 & $\mathrm{J} 13 \mathrm{~J} 02$ & $10 / 18 / 06$ & 41.0 & & 0.15 & 32.8 & $\mathrm{C}$ & 0.09 & SPA C9 & $J 13301$ & $10 / 17 / 06$ & 142 & $\mathrm{U}$ & 142 \\
\hline SPA Cl & $\mathrm{J} 13 \mathrm{~J} 03$ & $10 / 18 / 06$ & 43.3 & & 0.15 & 35.4 & $\mathrm{C}$ & 0.09 & SPA CIO & $\mathrm{J} 13 \mathrm{y} 02$ & $10 / 18 / 06$ & 137 & $\mathrm{U}$ & 137 \\
\hline SPA C2 & $\mathrm{J} 13 \mathrm{~J} 04$ & $10 / 18 / 06$ & 50.9 & & 0.15 & 37.5 & $\mathrm{C}$ & 0.09 & SPA C1 & $\mathrm{J} 13 \mathrm{~J} 03$ & $10 / 18 / 06$ & 137 & $\mathrm{U}$ & 137 \\
\hline SPA D3 & J13J05 & $10 / 18 / 06$ & 46.5 & & 0.15 & 38.0 & $\mathrm{C}$ & 0.09 & SPA C2 & $\mathrm{J} 13 \mathrm{~J} 04$ & $10 / 18 / 06$ & 136 & $\mathrm{U}$ & 136 \\
\hline SPA D4 & J13J06 & $10 / 19 / 06$ & 53.8 & & 0.15 & 43.3 & $\mathrm{C}$ & 0.09 & SPA D3 & J13.05 & $10 / 18 / 06$ & 137 & $\mathrm{U}$ & 137 \\
\hline SPAD5 & J13J07 & $10 / 23 / 06$ & 49.2 & & 0.14 & 39.4 & C & 0.09 & SPA D4 & J13J06 & $10 / 19 / 06$ & 144 & $\mathrm{U}$ & 144 \\
\hline SPA D6 & J13J08 & $10 / 23 / 06$ & 44.1 & & 0.15 & 40.0 & $\mathrm{C}$ & 0.09 & SPAD5 & J13J07 & $10 / 23 / 06$ & 137 & $\mathrm{U}$ & 137 \\
\hline FS-2 & J12R25 & $7 / 10 / 06$ & 45.4 & & 0.25 & 35.8 & & 0.45 & SPAD6 & $\mathrm{J} 13 \mathrm{~J} 08$ & $10 / 23 / 06$ & 138 & $\mathrm{U}$ & 138 \\
\hline FS-3 & J12R26 & $7 / 10 / 06$ & 46.0 & & 0.26 & 52.5 & & 0.45 & $\mathrm{FS}-1$ & J12R24 & $7 / 10 / 06$ & 132 & $\mathrm{U}$ & 132 \\
\hline FS-5 & $J 12 R 28$ & $7 / 10 / 06$ & 37.4 & $\mathrm{C}$ & 0.14 & 47.7 & & 0.09 & FS-10 & $J 12 R 33$ & $12 / 7 / 06$ & 144 & $\mathrm{U}$ & 144 \\
\hline
\end{tabular}

\begin{tabular}{|c|c|c|c|c|c|c|c|c|}
\hline & & & & & & & & \\
\hline FS-7 & J13P85 & $11 / 15 / 06$ & 30.0 & & 0.18 & 31.4 & $\mathrm{C}$ & 0.12 \\
\hline FS-8 & J12R31 & $7 / 10 / 06$ & 43.7 & & 0.26 & 43.1 & & 0.45 \\
\hline FS-9 & J12R32 & $7 / 10 / 06$ & 46.7 & & 0.26 & 45.8 & & 0.46 \\
\hline FS-11 & $\mathrm{J} 12 \mathrm{R} 34$ & $7 / 10 / 06$ & 42.1 & $\mathrm{C}$ & 0.14 & 65.1 & & 0.09 \\
\hline FS-14 & $\mathrm{J} 12 \times \mathrm{B} 3$ & $7 / 19 / 06$ & 59.9 & & 0.27 & 44.7 & & 0.49 \\
\hline FS-15 & J13NX4 & $11 / 14 / 06$ & 41.7 & & 0.18 & 34.9 & $\mathrm{C}$ & 0.12 \\
\hline FS-16 & J13P84 & $11 / 15 / 06$ & 46.6 & & 0.18 & 35.5 & $\mathrm{C}$ & 0.12 \\
\hline FS-17 & J14JJ33 & $3 / 5 / 07$ & 62.8 & & 0.1 & 47.6 & & 0.03 \\
\hline FS-17 resample & $\mathrm{J} 152 \mathrm{M} 1$ & $5 / 10 / 07$ & 53.8 & & 0.1 & 45.1 & & 0.03 \\
\hline FS-18 & $\mathrm{J} 14 \mathrm{~L} 38$ & $3 / 5 / 07$ & 53.2 & & 0.1 & 41.7 & & 0.03 \\
\hline FS-19 & J14L39 & $3 / 5 / 07$ & 49.4 & & 0.1 & 40.0 & & 0.03 \\
\hline Equipment blank & $\mathrm{J} 12 \times 59$ & $7 / 20 / 06$ & 0.49 & & 0.25 & 4.8 & & 0.45 \\
\hline
\end{tabular}

Attachment Originator Checked Calc. No.

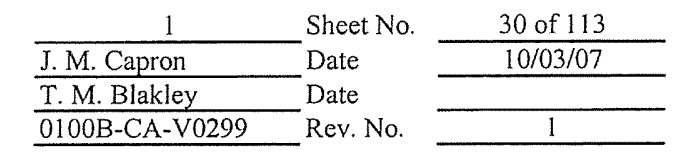

ग

$\circ$ ì 


\begin{tabular}{|c|c|c|c|}
\hline Sample Location & $\begin{array}{l}\text { Sample } \\
\text { Number }\end{array}$ & $\begin{array}{c}\text { Sample } \\
\text { Date }\end{array}$ & Asbestos Result \\
\hline Area $1 \mathrm{Al}$ & $J 12 \times 39$ & $7 / 19 / 06$ & None detected \\
\hline Duplicate of J12X44 & $\mathrm{J} 12 \times 43$ & $7 / 19 / 06$ & None detected \\
\hline Area $1 \mathrm{~A} 2$ & $\mathrm{~J} 12 \times 40$ & $7 / 19 / 06$ & None detected \\
\hline Area $1 \mathrm{~A} 3$ & $J 12 \times 41$ & $7 / 20 / 06$ & None detected \\
\hline Area $1 \mathrm{A4}$ & $\mathrm{J} 12 \times 42$ & $7 / 20 / 06$ & None detected \\
\hline Area $6 \mathrm{Al}$ & J12XB4 & $7 / 25 / 06$ & None detected \\
\hline Area $6 \mathrm{~A} 2$ & $\mathrm{~J} 12 \mathrm{XB} 5$ & $7 / 25 / 06$ & None detected \\
\hline Area $6 \mathrm{A3}$ & $\mathrm{J12XB6}$ & $7 / 25 / 06$ & None detected \\
\hline Area $6 \mathrm{A4}$ & $\mathrm{J} 12 \mathrm{XB7}$ & $7 / 25 / 06$ & None detected \\
\hline Area $7 \mathrm{Al}$ & J12XB8 & $7 / 26 / 06$ & None detected \\
\hline Area 7 A2 & J12XB9 & $7 / 26 / 06$ & None detected \\
\hline Area $7 \mathrm{A3}$ & $J 12 \times C 0$ & $7 / 26 / 06$ & None detected \\
\hline Area $7 \mathrm{~A} 4$ & $\mathrm{~J} 12 \mathrm{XC1}$ & $.7 / 26 / 06$ & None detected \\
\hline $\mathrm{OBAl}$ & J12WN3 & $7 / 11 / 06$ & None detected \\
\hline $\mathrm{OB} \mathrm{A} 2$ & J12WN4 & $7 / 11 / 06$ & None detected \\
\hline$O B A 3$ & J12WN5 & $7 / 11 / 06$ & None detected \\
\hline $\mathrm{OB} \mathrm{A} 4$ & $\mathrm{~J} 12 \mathrm{WN} 6$ & $7 / 11 / 06$ & None detected \\
\hline OB B5 & J12WN7 & $7 / 11 / 06$ & None detected \\
\hline OB B6 & J12WN8 & $7 / 11 / 06$ & None detected \\
\hline OB B7 & J12WN9 & $7 / 11 / 06$ & None detected \\
\hline OB B8 & J12WT0 & $7 / 17 / 06$ & None detected \\
\hline OB C9 & $\mathrm{J} 12 \mathrm{WTI}$ & $7 / 17 / 06$ & None detected \\
\hline $\mathrm{OBC} 10$ & J12WT2 & $7 / 17 / 06$ & None detected \\
\hline $\mathrm{OBCl}$ & $\mathrm{J12WT3}$ & $7 / 17 / 06$ & None detected \\
\hline $\mathrm{OBC} 2$ & J12WT4 & $7 / 17 / 06$ & None detected \\
\hline OB D3 & J12WR8 & $7 / 12 / 06$ & None detected \\
\hline OB D4 & J12WR9 & $7 / 12 / 06$ & None detected \\
\hline OBD5 & J12WR6 & $7 / 12 / 06$ & None detected \\
\hline OB D6 & J12WR7 & $7 / 12 / 06$ & None detected \\
\hline
\end{tabular}

\begin{tabular}{|c|c|c|c|}
\hline Sample Location & $\begin{array}{c}\text { Sample } \\
\text { Number }\end{array}$ & $\begin{array}{c}\text { Sample } \\
\text { Date }\end{array}$ & Asbestos Result \\
\hline BCLA1 & J12WT5 & $7 / 20 / 06$ & None detected \\
\hline $\mathrm{BCL} A 2$ & J12WR4 & $7 / 12 / 06$ & None detected \\
\hline $\mathrm{BCL} \mathrm{A3}$ & J12WR5 & $7 / 12 / 06$ & None detected \\
\hline BCL A4 & J12WR3 & $7 / 12 / 06$ & None detected \\
\hline $\mathrm{BCL} B 5$ & J12WR2 & $7 / 12 / 06$ & None detected \\
\hline BCL B6 & $\mathrm{J} 12 \mathrm{WR} 1$ & $7 / 12 / 06$ & None detected \\
\hline BCL B7 & J12WR0 & $7 / 12 / 06$ & None detected \\
\hline BCL B8 & J12WP9 & $7 / 12 / 06$ & None detected \\
\hline $\mathrm{BCL}$ C9 & J12WP0 & $7 / 11 / 06$ & None detected \\
\hline BCL C10 & J12WP2 & $7 / 11 / 06$ & None detected \\
\hline $\mathrm{BCL} \mathrm{Cl}$ & J12WP1 & $7 / 11 / 06$ & None detected \\
\hline $\mathrm{BCLC2}$ & J12WP5 & $7 / 11 / 06$ & None detected \\
\hline BCL D3 & J12WP6 & $7 / 11 / 06$ & None detected \\
\hline BCL D4 & J12WP7 & $7 / 11 / 06$ & None detected \\
\hline Duplicate of J12WL4 & J12WP8 & $7 / 11 / 06$ & None detected \\
\hline BCL D5 & J12WP3 & $7 / 11 / 06$ & None detected \\
\hline BCL D6 & J12WP4 & 7/11/06 & None detected \\
\hline SPAAl & $\mathrm{J13.111}$ & $10 / 12 / 06$ & $<1 \%$ chrysotile asbestos \\
\hline SPA A2 & $\mathrm{J13J12}$ & $10 / 12 / 06$ & None detected \\
\hline SPA A3 & $\mathrm{J} 13 \mathrm{~J} 13$ & $10 / 12 / 06$ & None detected \\
\hline SPA A4 & $\mathrm{J} 13 \mathrm{~J} 14$ & $10 / 12 / 06$ & None detected \\
\hline SPA B5 & $\mathrm{J} 13 \mathrm{~J} 15$ & $10 / 12 / 06$ & None detected \\
\hline Duplicate of J13HY7 & $\mathrm{J} 13 \mathrm{~J} 27$ & $10 / 12 / 06$ & None detected \\
\hline SPA B6 & $\mathrm{J} 13 \mathrm{~J} 16$ & $10 / 17 / 06$ & None detected \\
\hline SPA B7 & $\mathrm{J} 13 \mathrm{J17}$ & $10 / 17 / 06$ & None detected \\
\hline SPA B8 & $\mathrm{J} 13 \mathrm{~J} 18$ & $10 / 17 / 06$ & None detected \\
\hline SPA C9 & $\mathrm{J} 13 \mathrm{J19}$ & $10 / 17 / 06$ & None detected \\
\hline SPA ClO & $\mathrm{J} 13 \mathrm{~J} 20$ & $10 / 18 / 06$ & None detected \\
\hline SPA C1 & $\mathrm{J} 13 \mathrm{~J} 21$ & $10 / 18 / 06$ & None detected \\
\hline SPA C2 & $\mathrm{J} 13 \mathrm{~J} 22$ & $10 / 18 / 06$ & None detected \\
\hline SPA D3 & $\mathrm{J} 13 \mathrm{~J} 23$ & $10 / 18 / 06$ & None detected \\
\hline SPA D4 & $\mathrm{J} 13 \sqrt{24}$ & $10 / 19 / 06$ & None detected \\
\hline SPA D5 & $\mathrm{J} 13 \mathrm{~J} 25$ & $10 / 23 / 06$ & None detected \\
\hline SPAD6 & J13.26 & $10 / 23 / 06$ & None detected \\
\hline
\end{tabular}

Attachment

Originator J.M. Capron

Checked T.M. Blakley

Calc. No. 0100B-CA-V0299
Sheet No. 31 of 113 Date $10 / 03 / 07$ Date Rev. No. 0 
Attachment 1. 118-B-1 Verification Sampling Results.

\begin{tabular}{|c|c|c|c|c|c|c|c|c|c|c|c|c|}
\hline \multirow[t]{2}{*}{ Constituents } & \multicolumn{3}{|c|}{$\begin{array}{c}\text { J12X44 } \\
\text { Area } 1 \text { A1 } \\
\text { Sample Date } 7 / 19 / 06\end{array}$} & \multicolumn{3}{|c|}{$\begin{array}{c}\mathrm{J} 12 \mathrm{X} 48 \\
\text { Duplicate of J12X44 } \\
\text { Sample Date } 7 / 19 / 06 \\
\end{array}$} & \multicolumn{3}{|c|}{$\begin{array}{c}12 X 49 \\
\text { Split of J12X44 } \\
\text { Sample Date } 7 / 19 / 06 \\
\end{array}$} & \multicolumn{3}{|c|}{$\begin{array}{c}\mathrm{J} 12 \mathrm{X} 45 \\
\text { Area } 1 \mathrm{A2} \\
\text { Sample Date } 7 / 19 / 06\end{array}$} \\
\hline & $\mu \mathrm{g} / \mathrm{kg}$ & $Q$ & $\mathbf{P Q L}$ & $\mu \mathrm{g} / \mathrm{kg}$ & $\mathbf{Q}$ & $\mathbf{P Q L}$ & $\mu \mathrm{g} / \mathrm{kg}$ & $\mathbf{Q}$ & PQL & $\mu \mathrm{g} / \mathrm{kg}$ & $\mathbf{Q}$ & PQL \\
\hline \multicolumn{13}{|c|}{ Polychlorinated Biphenyls } \\
\hline Aroclor-1016 & 13 & $\mathrm{U}$ & 13 & 13 & $\mathrm{U}$ & 13 & 17 & $\mathrm{U}$ & 17 & 13 & $\mathrm{U}$ & 13 \\
\hline Aroclor-1221 & 13 & U & 13 & 13 & $\mathrm{U}$ & 13 & 17 & $\mathrm{U}$ & 17 & 13 & $\mathrm{U}$ & 13 \\
\hline Aroclor-1232 & 13 & $\mathrm{U}$ & 13 & 13 & $\mathrm{U}$ & 13 & 17 & $\mathrm{U}$ & 17 & 13 & $\mathrm{U}$ & 13 \\
\hline Aroclor-1242 & 13 & $\mathrm{U}$ & 13 & 13 & $\mathrm{U}$ & 13 & 17 & $\mathrm{U}$ & 17 & 13 & $\mathrm{U}$ & 13 \\
\hline Aroclor-1248 & 13 & U & 13 & 13 & $\mathrm{U}$ & 13 & 17 & $\mathrm{U}$ & 17 & 13 & $\mathrm{U}$ & 13 \\
\hline Aroclor 1254 & 13 & $\mathrm{U}$ & 13 & 13 & $\mathrm{U}$ & 13 & 17 & $\mathrm{U}$ & 17 & 13 & $\mathrm{U}$ & 13 \\
\hline Aroclor- 1260 & 13 & $\mathrm{U}$ & 13 & 13 & $\mathrm{U}$ & 13 & 17 & $\mathrm{U}$ & 17 & 13 & $\mathrm{U}$ & 13 \\
\hline \multicolumn{13}{|c|}{ Semivolatile Organic Compounds } \\
\hline 1,2,4-Trichlorobenzene & 340 & UJ & 340 & 340 & $\mathrm{UJ}$ & 340 & 330 & $\mathrm{U}$ & 330 & 340 & UJ & 340 \\
\hline 1,2-Dichlorobenzene & 340 & $\mathrm{U}$ & 340 & 340 & U & 340 & 330 & U & 330 & 340 & $\mathrm{U}$ & 340 \\
\hline 1,3-Dichlorobenzene & 340 & $\mathrm{U}$ & 340 & 340 & $\mathrm{U}$ & 340 & 330 & U & 330 & 340 & $\mathrm{U}$ & 340 \\
\hline 1,4-Dichlorobenzene & 340 & $\mathrm{U}$ & 340 & 340 & $U$ & 340 & 330 & U & 330 & 340 & $\mathrm{U}$ & 340 \\
\hline 2,4,5-Trichlorophenol & 850 & U & 850 & 850 & $U$ & 850 & 330 & $U$ & 330 & 840 & $\mathrm{U}$ & 840 \\
\hline 2,4,6-Trichlorophenol & 340 & $\mathrm{U}$ & 340 & 340 & $\mathrm{U}$ & 340 & 330 & $U$ & 330 & 340 & $U$ & 340 \\
\hline 2,4-Dichlorophenol & 340 & $\mathrm{U}$ & 340 & 340 & $U$ & 340 & 330 & $U$ & 330 & 340 & $\mathrm{U}$ & 340 \\
\hline 2,4-Dimethylphenol & 340 & UJ & 340 & 340 & $\mathrm{UJ}$ & 340 & 330 & $\mathrm{U}$ & 330 & 340 & UJ & 340 \\
\hline 2,4-Dinitrophenol & 850 & $U$ & 850 & 850 & $\mathrm{U}$ & 850 & 1600 & UR & 1600 & 840 & $\mathrm{U}$ & 840 \\
\hline 2,4-Dinitrotoluene & 340 & $\mathrm{U}$ & 340 & 340 & $\bar{U}$ & 340 & 330 & U & 330 & 340 & $\mathrm{U}$ & 340 \\
\hline 2,6-Dinitrotoluene & 340 & $\mathrm{U}$ & 340 & 340 & $\mathrm{U}$ & 340 & 330 & $\mathrm{U}$ & 330 & 340 & $\mathrm{U}$ & 340 \\
\hline 2-Chloronaphthalene & 340 & $\mathrm{U}$ & 340 & 340 & $\mathrm{U}$ & 340 & 330 & $\mathrm{U}$ & 330 & 340 & $\mathrm{U}$ & 340 \\
\hline 2-Chlorophenol & 340 & $\mathrm{U}$ & 340 & 340 & $\mathrm{U}$ & 340 & 330 & $\mathrm{U}$ & 330 & 340 & $\mathrm{U}$ & 340 \\
\hline 2-Methylnaphthalene & 340 & UJ & 340 & 340 & UJ & 340 & 330 & U & 330 & 340 & UJ & 340 \\
\hline 2-Methylphenol (cresol, o-) & 340 & $\mathrm{U}$ & 340 & 340 & $\mathrm{U}$ & 340 & 330 & $\mathrm{U}$ & 330 & 340 & $\mathrm{U}$ & 340 \\
\hline 2-Nitroaniline & 850 & $\mathrm{U}$ & 850 & 850 & $\mathrm{U}$ & 850 & 1600 & $\mathrm{U}$ & 1600 & 840 & U & 840 \\
\hline 2-Nitrophenol & 340 & $\mathrm{U}$ & 340 & 340 & $\mathrm{U}$ & 340 & 330 & $U$ & 330 & 340 & $\mathrm{U}$ & 340 \\
\hline 3,3'-Dichlorobenzidine & 340 & $U$ & 340 & 340 & $\mathrm{U}$ & 340 & 1600 & $\mathrm{U}$ & 1600 & 340 & $\mathrm{U}$ & 340 \\
\hline 3-Nitroaniline & 850 & U & 850 & 850 & $\mathrm{U}$ & 850 & 1600 & U & 1600 & 840 & $\mathrm{U}$ & 840 \\
\hline 4,6-Dinitro-2-methylphenol & 850 & $\mathrm{U}$ & 850 & 850 & $U$ & 850 & 1600 & $\mathrm{U}$ & 1600 & 840 & $\mathrm{U}$ & 840 \\
\hline 4-Bromophenyl-phenylether & 340 & $U$ & 340 & 340 & $U$ & 340 & 330 & $\mathrm{U}$ & 330 & 340 & $\mathrm{U}$ & 340 \\
\hline 4-Chloro-3-methylphenol & 340 & UJ & 340 & 340 & UJ & 340 & 330 & $U$ & 330 & 340 & UJ & 340 \\
\hline 4-Chloroaniline & 340 & $\mathrm{U}$ & 340 & 340 & $\mathrm{U}$ & 340 & 330 & $\mathrm{U}$ & 330 & 340 & $\mathrm{U}$ & 340 \\
\hline 4-Chlorophenyl-phenylether & 340 & $\mathrm{U}$ & 340 & 340 & U & 340 & 330 & $U$ & 330 & 340 & $\mathrm{U}$ & 340 \\
\hline 4-Methylphenol (p-cresol) & 340 & $U$ & 340 & 340 & $\mathrm{U}$ & 340 & 670 & $\mathrm{U}$ & 670 & 340 & $\mathrm{U}$ & 340 \\
\hline 4-Nitroaniline & 850 & $U$ & 850 & 850 & $\mathrm{U}$ & 850 & 1600 & $\mathrm{U}$ & 1600 & 840 & $\mathrm{U}$ & 840 \\
\hline 4-Nitrophenol & 850 & $U$ & 850 & 850 & $\mathrm{U}$ & 850 & 1600 & $\mathrm{U}$ & 1600 & 840 & $\mathrm{U}$ & 840 \\
\hline Acenaphthene & 340 & $\mathrm{U}$ & 340 & 340 & $\mathrm{U}$ & 340 & 330 & $\mathrm{U}$ & 330 & 340 & $U$ & 340 \\
\hline Acenaphthylene & 340 & $\mathrm{U}$ & 340 & 340 & $U$ & 340 & 330 & $\mathrm{U}$ & 330 & 340 & $\mathrm{U}$ & 340 \\
\hline Anthracene & 340 & $\mathrm{U}$ & 340 & 340 & $\mathrm{U}$ & 340 & 330 & $\mathrm{U}$ & 330 & 340 & $\mathrm{U}$ & 340 \\
\hline Benzo(a)anthracene & 340 & $\mathrm{U}$ & 340 & 340 & $\mathrm{U}$ & 340 & 330 & $\mathrm{U}$ & 330 & 340 & $\mathrm{U}$ & 340 \\
\hline Benzo(a)pyrene & 340 & $\mathrm{U}$ & 340 & 340 & $\mathrm{U}$ & 340 & 330 & $\mathrm{U}$ & 330 & 340 & $\mathrm{U}$ & 340 \\
\hline Benzo(b)fluoranthene & 340 & $\bar{U}$ & 340 & 340 & $\mathrm{U}$ & 340 & 330 & $\mathrm{U}$ & 330 & 340 . & $\mathrm{U}$ & 340 \\
\hline Benzo(g,h,i)perylene & 340 & U & 340 & 340 & $\mathrm{U}$ & 340 & 330 & $\mathrm{U}$ & 330 & 340 & $\mathrm{U}$ & 340 \\
\hline Benzo(k)fluoranthene & 340 & U & 340 & 340 & $\mathrm{U}$ & 340 & 330 & $\mathrm{U}$ & 330 & 340 & $\mathrm{U}$ & 340 \\
\hline bis(2-Chloro-1-methylethyl)ether & 340 & $\mathrm{U}$ & 340 & 340 & $\mathrm{U}$ & 340 & 330 & $\mathrm{U}$ & 330 & 340 & $\mathrm{U}$ & 340 \\
\hline bis(2-Chloroethoxy)methane & 340 & $\mathrm{U}$ & 340 & 340 & $\mathrm{U}$ & 340 & 330 & $\mathrm{U}$ & 330 & 340 & $\mathrm{U}$ & 340 \\
\hline bis(2-Chloroethyl)ether & 340 & $\mathrm{U}$ & 340 & 340 & $\mathrm{U}$ & 340 & 330 & $\mathrm{U}$ & 330 & 340 & $\mathrm{U}$ & 340 \\
\hline bis(2-Ethylhexyl)phthalate & 240 & JB & 340 & 130 & $\mathrm{JB}$ & 340 & 330 & $\mathrm{U}$ & 330 & 140 & $\mathrm{JB}$ & 340 \\
\hline
\end{tabular}

Attachment

Originator

Checked

Calc. No.
J. M. Capron

T. M. Blakley

0100B-CA-V0299
Sheet No. 32 of 113

Date $10 / 04 / 07$

Date

Rev. No. 1 
Attachment 1. 118-B-1 Verification Sampling Results.

\begin{tabular}{|c|c|c|c|c|c|c|c|c|c|c|c|c|}
\hline \multirow[t]{2}{*}{ Constituents } & \multicolumn{3}{|c|}{$\begin{array}{c}\text { J12X44 } \\
\text { Area 1 A1 } \\
\text { Sample Date } 7 / 19 / 06\end{array}$} & \multicolumn{3}{|c|}{$\begin{array}{c}\mathrm{J} 12 \mathrm{X} 48 \\
\text { Duplicate of } \mathrm{J} 12 \mathrm{X} 44 \\
\text { Sample Date } 7 / 19 / 06\end{array}$} & \multicolumn{3}{|c|}{$\begin{array}{c}\text { J12X49 } \\
\text { Split of J12X44 } \\
\text { Sample Date } 7 / 19 / 06\end{array}$} & \multicolumn{3}{|c|}{$\begin{array}{c}\text { J12X45 } \\
\text { Area } 1 \text { A2 } \\
\text { Sample Date } 7 / 19 / 06\end{array}$} \\
\hline & $\mu \mathrm{g} / \mathrm{kg}$ & $\mathbf{Q}$ & PQL & $\mu \mathrm{g} / \mathrm{kg}$ & $Q$ & PQL & $\mu \mathrm{g} / \mathrm{kg}$ & $Q$ & MDL & $\mu \mathrm{g} / \mathrm{kg}$ & $Q$ & PQL \\
\hline \multicolumn{13}{|c|}{ Semivolatile Organic Compounds (continued) } \\
\hline Butylbenzylphthalate & 340 & $\mathrm{U}$ & 340 & 340 & $\mathrm{U}$ & 340 & 330 & $\mathrm{U}$ & 330 & 340 & $\mathrm{U}$ & 340 \\
\hline Carbazole & 340 & $\mathrm{U}$ & 340 & 340 & $\mathrm{U}$ & 340 & 330 & $\mathrm{U}$ & 330 & 340 & $U$ & 340 \\
\hline Chrysene & 340 & $\mathrm{U}$ & 340 & 340 & $\mathrm{U}$ & 340 & 330 & $\mathrm{U}$ & 330 & 340 & $\mathrm{U}$ & 340 \\
\hline Di-n-butylphthalate & 19 & $\mathrm{JB}$ & 340 & 18 & $\mathrm{JB}$ & 340 & 330 & $\mathrm{U}$ & 330 & 340 & $\mathrm{U}$ & 340 \\
\hline Di-n-octylphthalate & 340 & $\mathrm{U}$ & 340 & 340 & $\mathrm{U}$ & 340 & 330 & $\mathrm{U}$ & 330 & 340 & $\mathrm{U}$ & 340 \\
\hline Dibenz $(\mathrm{a}, \mathrm{h})$ anthracene & 340 & $\mathrm{U}$ & 340 & 340 & $\mathrm{U}$ & 340 & 330 & $\mathrm{U}$ & 330 & 340 & $\mathrm{U}$ & 340 \\
\hline Dibenzofuran & 340 & $\bar{U}$ & 340 & 340 & $\mathrm{U}$ & 340 & 330 & $\mathrm{U}$ & 330 & 340 & $\mathrm{U}$ & 340 \\
\hline Diethylphthalate & 340 & $\mathrm{U}$ & 340 & 340 & $\mathrm{U}$ & 340 & 330 & $\bar{U}$ & 330 & 340 & $\mathrm{U}$ & 340 \\
\hline Dimethylphthalate & 340 & $\mathrm{U}$ & 340 & 340 & $\bar{U}$ & 340 & 330 & $\mathrm{U}$ & 330 & 340 & $\mathrm{U}$ & 340 \\
\hline Fluoranthene & 340 & $\mathrm{U}$ & 340 & 340 & $\mathrm{U}$ & 340 & 330 & $\mathrm{U}$ & 330 & 340 & $\mathrm{U}$ & 340 \\
\hline Fluorene & 340 & U & 340 & 340 & $\mathrm{U}$ & 340 & 330 & $\mathrm{U}$ & 330 & 340 & $\mathrm{U}$ & 340 \\
\hline Hexachlorobenzene & 340 & $\mathrm{U}$ & 340 & 340 & $\mathrm{U}$ & 340 & 330 & $\mathrm{U}$ & 330 & 340 & $U$ & 340 \\
\hline Hexachlorobutadiene & 340 & $\mathrm{U}$ & 340 & 340 & $\mathrm{U}$ & 340 & 330 & $\mathrm{U}$ & 330 & 340 & $\mathrm{U}$ & 340 \\
\hline Hexachlorocyclopentadiene & 340 & $\mathrm{U}$ & 340 & 340 & $\mathrm{U}$ & 340 & 1600 & $\mathrm{U}$ & 1600 & 340 & $\mathrm{U}$ & 340 \\
\hline Hexachloroethane & 340 & $\mathrm{U}$ & 340 & 340 & $U$ & 340 & 330 & $\mathrm{U}$ & 330 & 340 & $\mathrm{U}$ & 340 \\
\hline Indeno(1,2,3-cd)pyrene & 340 & $\mathrm{U}$ & 340 & 340 & $\mathrm{U}$ & 340 & 330 & $\mathrm{U}$ & 330 & 340 & $\mathrm{U}$ & 340 \\
\hline Isophorone & 340 & UJ & 340 & 340 & UJ & 340 & 330 & $\mathrm{U}$ & 330 & 340 & UJ & 340 \\
\hline N-Nitroso-di-n-dipropylamine & 340 & $\mathrm{U}$ & 340 & 340 & $\mathrm{U}$ & 340 & 330 & $\mathrm{U}$ & 330 & 340 & $\mathrm{U}$ & 340 \\
\hline N-Nitrosodiphenylamine & 340 & $\mathrm{U}$ & 340 & 340 & $\mathrm{U}$ & 340 & 330 & $\mathrm{U}$ & 330 & 340 & $\mathrm{U}$ & 340 \\
\hline Naphthalene & 340 & $\mathrm{U}$ & 340 & 340 & $\mathrm{U}$ & 340 & 330 & $\mathrm{U}$ & 330 & 340 & $\mathrm{U}$ & 340 \\
\hline Nitrobenzene & 340 & UJ & 340 & 340 & UJ & 340 & 330 & $\bar{U}$ & 330 & 340 & UJ & 340 \\
\hline Pentachlorophenol & 850 & $\mathrm{U}$ & 850 & 850 & $\mathrm{U}$ & 850 & 1600 & $\mathrm{U}$ & 1600 & 840 & $\mathrm{U}$ & 840 \\
\hline Phenanthrene & 340 & $\mathrm{U}$ & 340 & 340 & $\mathrm{U}$ & 340 & 330 & $\mathrm{U}$ & 330 & 340 & $\mathrm{U}$ & 340 \\
\hline Phenol & 340 & $\mathrm{U}$ & 340 & 340 & $\mathrm{U}$ & 340 & 330 & $\mathrm{U}$ & 330 & 340 & $\mathrm{U}$ & 340 \\
\hline Pyrene & 340 & $\mathrm{U}$ & 340 & 340 & $\mathrm{U}$ & 340 & 330 & $\mathrm{U}$ & 330 & 340 & $\mathrm{U}$ & 340 \\
\hline
\end{tabular}

Attachment

Originator

Checked

Calc. No.

\begin{tabular}{|c|}
\hline 1 \\
\hline J. M. Capron \\
\hline T. M. Blakley \\
\hline 0100B-CA-V0299 \\
\hline
\end{tabular}

Sheet No. $\frac{33 \text { of } 113}{10 / 04 / 07}$
Date 11
Date
Rev. No. 1 
Attachment 1. 118-B-1 Verification Sampling Results.

\begin{tabular}{|c|c|c|c|c|c|c|c|c|c|c|c|c|}
\hline \multirow[t]{2}{*}{ Constituents } & \multicolumn{3}{|c|}{$\begin{array}{c}\mathrm{J} 12 \mathrm{X} 44 \\
\text { Area } 1 \mathrm{A1} \\
\text { Sample Date } 7 / 19 / 06\end{array}$} & \multicolumn{3}{|c|}{\begin{tabular}{|c|}
$\mathrm{J12X} 48$ \\
Duplicate of J12X44 \\
Sample Date $7 / 19 / 06$ \\
\end{tabular}} & \multicolumn{3}{|c|}{$\begin{array}{c}\mathrm{J} 12 \times 49 \\
\text { Split of J12X44 } \\
\text { Sample Date } 7 / 19 / 06\end{array}$} & \multicolumn{3}{|c|}{$\begin{array}{c}\text { J12X45 } \\
\text { Area } 1 \text { A2 } \\
\text { Sample Date } 7 / 19 / 06\end{array}$} \\
\hline & $\mu \mathrm{g} / \mathrm{kg}$ & $Q$ & $\mathrm{PQL}$ & $\mu \mathrm{g} / \mathrm{kg}$ & $\mathbf{Q}$ & PQL & $\mu \mathrm{g} / \mathrm{kg}$ & $Q$ & MDL & $\mu \mathrm{g} / \mathrm{kg}$ & $Q$ & PQL \\
\hline \multicolumn{13}{|c|}{ Volatile Organic Compounds } \\
\hline 1,1,1-Trichloroethane & 5 & $\mathrm{U}$ & 5 & 5 & $\mathrm{U}$ & 5 & 5.1 & $\mathrm{U}$ & 5.1 & 5 & $\mathrm{U}$ & 5 \\
\hline 1,1,2,2-Tetrachloroethane & 5 & $\mathrm{U}$ & 5 & 5 & $\mathrm{U}$ & 5 & 5.1 & $\mathrm{U}$ & 5.1 & 5 & $\mathrm{U}$ & 5 \\
\hline 1,1,2-Trichloroethane & 5 & $U$ & 5 & 5 & $\mathrm{U}$ & 5 & 5.1 & $\mathrm{U}$ & 5.1 & 5 & $\mathrm{U}$ & 5 \\
\hline 1,1-Dichloroethane & 5 & $\mathrm{U}$ & 5 & 5 & $\mathrm{U}$ & 5 & 5.1 & $\mathrm{U}$ & 5.1 & 5 & $\mathrm{U}$ & 5 \\
\hline 1,1-Dichloroethene & 5 & $\mathrm{U}$ & 5 & 5 & $\mathrm{U}$ & 5 & 5.1 & $\mathrm{U}$ & 5.1 & 5 & $\mathrm{U}$ & 5 \\
\hline 1,2-Dichloroethane & 5 & $\mathrm{U}$ & 5 & 5 & $\mathrm{U}$ & 5 & 5.1 & $\mathrm{U}$ & 5.1 & 5 & $\mathrm{U}$ & 5 \\
\hline 1,2-Dichloroethene (total) & 5 & U & 5 & 5 & $\mathrm{U}$ & 5 & 10 & $\mathrm{U}$ & 10 & 5 & $\mathrm{U}$ & 5 \\
\hline 1,2-Dichloropropane & 5 & $\mathrm{U}$ & 5 & 5 & $\mathrm{U}$ & 5 & 5.1 & $\mathrm{U}$ & 5.1 & 5 & $\mathrm{U}$ & 5 \\
\hline 2-Butanone & 10 & $\mathrm{U}$ & 10 & 10 & $\mathrm{U}$ & 10 & 10 & $\mathrm{U}$ & 10 & 10 & $\mathrm{U}$ & 10 \\
\hline 2-Hexanone & 10 & $\mathrm{U}$ & 10 & 10 & $\mathrm{U}$ & 10 & 20 & $\mathrm{U}$ & 20 & 10 & $\mathrm{U}$ & 10 \\
\hline 4-Methyl-2-pentanone & 5 & $\mathrm{JB}$ & 10 & 8 & $\mathrm{JB}$ & 10 & 10 & $\mathrm{U}$ & 10 & 5 & $\mathrm{JB}$ & 10 \\
\hline Acetone & 9 & $\mathrm{~J}$ & 10 & 23 & $\mathrm{~J}$ & 10 & 20 & U & 20 & 11 & $\mathrm{~J}$ & 10 \\
\hline Benzene & 5 & $\mathrm{U}$ & 5 & 5 & $\mathrm{U}$ & 5 & 5.1 & $\mathrm{U}$ & 5.1 & 5 & $\mathrm{U}$ & 5 \\
\hline Bromodichloromethane & 5 & $\mathrm{U}$ & 5 & 5 & $\mathrm{U}$ & 5 & 5.1 & $\mathrm{U}$ & 5.1 & 5 & $\mathrm{U}$ & 5 \\
\hline Bromoform & 5 & $\mathrm{U}$ & 5 & 5 & $\mathrm{U}$ & 5 & 5.1 & $\mathrm{U}$ & 5.1 & 5 & $\mathrm{U}$ & 5 \\
\hline Bromomethane & 10 & $\mathrm{U}$ & 10 & 10 & $\mathrm{U}$ & 10 & 10 & UN & 10 & 10 & $\mathrm{U}$ & 10 \\
\hline Carbon disulfide & 5 & $\mathrm{U}$ & 5 & 5 & $\mathrm{U}$ & 5 & 5.1 & $\mathrm{U}$ & 5.1 & 5 & $\mathrm{U}$ & 5 \\
\hline Carbon tetrachloride & 5 & $\mathrm{U}$ & 5 & 5 & $\mathrm{U}$ & 5 & 5.1 & $\mathrm{U}$ & 5.1 & 5 & $\mathrm{U}$ & 5 \\
\hline Chlorobenzene & 5 & $\mathrm{U}$ & 5 & 5 & $\mathrm{U}$ & 5 & 5.1 & $\mathrm{U}$ & 5.1 & 5 & $\mathrm{U}$ & 5 \\
\hline Chloroethane & 10 & $\mathrm{U}$ & 10 & 10 & U & 10 & 10 & $\mathrm{U}$ & 10 & 10 & $\mathrm{U}$ & 10 \\
\hline Chloroform & 5 & 0 & 5 & 5 & $\mathrm{U}$ & 5 & 5.1 & $\mathrm{U}$ & 5.1 & 5 & $\mathrm{U}$ & 5 \\
\hline Chloromethane & 10 & $\mathrm{U}$ & 10 & 10 & $\mathrm{U}$ & 10 & 10 & $\mathrm{U}$ & 10 & 10 & $\mathrm{U}$ & 10 \\
\hline cis-1,2-Dichloroethylene & 5 & U & 5 & 5 & $\mathrm{U}$ & 5 & & & & 5 & $\mathrm{U}$ & 5 \\
\hline cis-1,3-Dichloropropene & 5 & $\mathrm{U}$ & 5 & 5 & $\mathrm{U}$ & 5 & 5.1 & $\mathrm{U}$ & 5.1 & 5 & $\mathrm{U}$ & 5 \\
\hline Dibromochloromethane & 5 & $\mathrm{U}$ & 5 & 5 & $\mathrm{U}$ & 5 & 5.1 & $\mathrm{U}$ & 5.1 & 5 & $\mathrm{U}$ & 5 \\
\hline Ethylbenzene & 5 & $\mathrm{U}$ & 5 & 5 & $\mathrm{U}$ & 5 & 5.1 & $\mathrm{U}$ & 5.1 & 5 & $\mathrm{U}$ & 5 \\
\hline Methylene chloride & 6 & & 5 & 12 & & 5 & 5.1 & U & 5.1 & 15 & & 5 \\
\hline Styrene & 5 & U & 5 & 5 & $\mathrm{U}$ & 5 & 5.1 & $\mathrm{U}$ & 5.1 & 5 & $\mathrm{U}$ & 5 \\
\hline Tetrachloroethene & 5 & $\mathrm{U}$ & 5 & 5 & $\mathrm{U}$ & 5 & 5.1 & UN & 5.1 & 5 & $\mathrm{U}$ & 5 \\
\hline Toluene & 5 & $\mathrm{U}$ & 5 & 5 & $\mathrm{U}$ & 5 & 5.1 & $\mathrm{U}$ & 5.1 & 5 & $\mathrm{U}$ & 5 \\
\hline trans-1,2-Dichloroethylene & 5 & $\mathrm{U}$ & 5 & 5 & $\mathrm{U}$ & 5 & & & & 5 & $\mathrm{U}$ & 5 \\
\hline trans-1,3-Dichloropropene & 5 & $\mathrm{U}$ & 5 & 5 & $\mathrm{U}$ & 5 & 5.1 & U & 5.1 & 5 & $\mathrm{U}$ & 5 \\
\hline Trichloroethene & 5 & $\mathrm{U}$ & 5 & 5 & $\mathrm{U}$ & 5 & 5.1 & U & 5.1 & 5 & $\mathrm{U}$ & 5 \\
\hline Vinyl chloride & 10 & $\mathrm{U}$ & 10 & 10 & $\mathrm{U}$ & 10 & 5.1 & $\mathrm{U}$ & 5.1 & 10 & $\mathrm{U}$ & 10 \\
\hline Xylenes (total) & 5 & $\mathrm{U}$ & 5 & 5 & $\mathrm{U}$ & 5 & 10 & $\mathrm{U}$ & 10 & 5 & $\mathrm{U}$ & 5 \\
\hline & & & & Attachn & & & 1 & & & heet No. & & of 113 \\
\hline & & & & Origina & & J.M. C & oron & & & Datc & & $04 / 07$ \\
\hline & & & & Checke & & T.M. B & akley & & & Date & & \\
\hline & & & & Calc. $\mathrm{N}$ & & $0100 \mathrm{~B}-$ & $\mathrm{A}-\mathrm{V} 029$ & & & v. No & & 1 \\
\hline
\end{tabular}


Attachment 1. 118-B-1 Verification Sampling Results.

\begin{tabular}{|c|c|c|c|c|c|c|c|c|c|c|c|c|}
\hline \multirow[t]{2}{*}{ Constituents } & \multicolumn{3}{|c|}{$\begin{array}{c}\text { J12X46 } \\
\text { Area } 1 \mathrm{A3} \\
\text { Sample Date } 7 / 20 / 06\end{array}$} & \multicolumn{3}{|c|}{$\begin{array}{c}\text { J12X47 } \\
\text { Area 1 A4 } \\
\text { Sample Date } 7 / 20 / 06\end{array}$} & \multicolumn{3}{|c|}{$\begin{array}{c}\mathrm{J12X54} \\
\text { Area } 3 \mathrm{A1} \\
\text { Sample Date } 7 / 20 / 06 \\
\end{array}$} & \multicolumn{3}{|c|}{$\begin{array}{c}\text { J12X55 } \\
\text { Area } 3 \text { A2 } \\
\text { Sample Date 7/20/06 }\end{array}$} \\
\hline & $\mu \mathrm{g} / \mathrm{kg}$ & $\mathbf{Q}$ & PQL & $\mu \mathrm{g} / \mathrm{kg}$ & $Q$ & PQL & $\mu \mathrm{g} / \mathrm{kg}$ & $Q$ & PQL & $\mu \mathrm{g} / \mathrm{kg}$ & $Q$ & PQL \\
\hline \multicolumn{13}{|c|}{ Polychlorinated Biphenyls } \\
\hline Aroclor-1016 & 13 & $\mathrm{U}$ & 13 & 14 & $\mathrm{U}$ & 14 & 13 & $\mathrm{U}$ & 13 & 13 & $\mathrm{U}$ & 13 \\
\hline Aroclor-1221 & 13 & $\mathrm{U}$ & 13 & 14 & $\mathrm{U}$ & 14 & 13 & $\mathrm{U}$ & 13 & 13 & $\bar{U}$ & 13 \\
\hline Aroclor -1232 & 13 & $\mathrm{U}$ & 13 & 14 & $\mathrm{U}$ & 14 & 13 & $\mathrm{U}$ & 13 & 13 & $\mathrm{U}$ & 13 \\
\hline Aroclor- 1242 & 13 & $\mathrm{U}$ & 13 & 14 & $\mathrm{U}$ & 14 & 13 & U & 13 & 13 & $\mathrm{U}$ & 13 \\
\hline Aroclor- 1248 & 13 & $\mathrm{U}$ & 13 & 14 & $\mathrm{U}$ & 14 & 13 & U & 13 & 13 & $\mathrm{U}$ & 13 \\
\hline Aroclor- 1254 & 13 & $\mathrm{U}$ & 13 & 14 & $\mathrm{U}$ & 14 & 13 & $\mathrm{U}$ & 13 & 13 & $\mathrm{U}$ & 13 \\
\hline Aroclor- 1260 & 13 & $\mathrm{U}$ & 13 & 14 & $\mathrm{U}$ & 14 & 13 & $\mathrm{U}$ & 13 & 13 & $\mathrm{U}$ & 13 \\
\hline \multicolumn{13}{|c|}{ Semivolatile Organic Compounds } \\
\hline 1,2,4-Trichlorobenzene & 340 & $\mathrm{U}$ & 340 & 340 & $\mathrm{U}$ & 340 & 330 & $\mathrm{U}$ & 330 & 340 & $\mathrm{U}$ & 340 \\
\hline 1,2-Dichlorobenzene & 340 & $\mathrm{U}$ & 340 & 340 & $\mathrm{U}$ & 340 & 330 & $\mathrm{U}$ & 330 & 340 & $\mathrm{U}$ & 340 \\
\hline 1,3-Dichlorobenzene & 340 & $U$ & 340 & 340 & $\mathrm{U}$ & 340 & 330 & $\mathrm{U}$ & 330 & 340 & $\mathrm{U}$ & 340 \\
\hline 1,4-Dichlorobenzene & 340 & $\mathrm{U}$ & 340 & 340 & $\mathrm{U}$ & 340 & 330 & $\mathrm{U}$ & 330 & 340 & $\mathrm{U}$ & 340 \\
\hline 2,4,5-Trichlorophenol & 840 & $\mathrm{U}$ & 840 & 850 & $\mathrm{U}$ & 850 & 840 & $\mathrm{U}$ & 840 & 840 & $\mathrm{U}$ & 840 \\
\hline 2,4,6-Trichlorophenol & 340 & $\mathrm{U}$ & 340 & 340 & $\mathrm{U}$ & 340 & 330 & $\mathrm{U}$ & 330 & 340 & $\mathrm{U}$ & 340 \\
\hline 2,4-Dichlorophenol & 340 & $\mathrm{U}$ & 340 & 340 & $\mathrm{U}$ & 340 & 330 & $\mathrm{U}$ & 330 & 340 & $\mathrm{U}$ & 340 \\
\hline 2,4-Dimethylphenol & 340 & UJ & 340 & 340 & UJ & 340 & 330 & $\mathrm{UJ}$ & 330 & 340 & UJ & 340 \\
\hline 2,4-Dinitrophenol & 840 & $\mathrm{U}$ & 840 & 850 & $\mathrm{U}$ & 850 & 840 & $\mathrm{U}$ & 840 & 840 & $\mathrm{U}$ & 840 \\
\hline 2,4-Dinitrotoluene & 340 & $\mathrm{U}$ & 340 & 340 & $\mathrm{U}$ & 340 & 330 & $\mathrm{U}$ & 330 & 340 & $\mathrm{U}$ & 340 \\
\hline 2,6-Dinitrotoluene & 340 & $\mathrm{U}$ & 340 & 340 & $\mathrm{U}$ & 340 & 330 & $\mathrm{U}$ & 330 & 340 & $\mathrm{U}$ & 340 \\
\hline 2-Chloronaphthalene & 340 & $\mathrm{U}$ & 340 & 340 & U & 340 & 330 & $\mathrm{U}$ & 330 & 340 & $\mathrm{U}$ & 340 \\
\hline 2-Chlorophenol & 340 & $\mathrm{U}$ & 340 & 340 & $\mathrm{U}$ & 340 & 330 & U & 330 & 340 & $\mathrm{U}$ & 340 \\
\hline 2-Methylnaphthalene & 340 & $\mathrm{U}$ & 340 & 340 & $\mathrm{U}$ & 340 & 330 & $\mathrm{U}$ & 330 & 340 & $\mathrm{U}$ & 340 \\
\hline 2-Methylphenol (cresol, o-) & 340 & U & 340 & 340 & $\mathrm{U}$ & 340 & 330 & $U$ & 330 & 340 & $\mathrm{U}$ & 340 \\
\hline 2-Nitroaniline & 840 & $\mathrm{U}$ & 840 & 850 & $\mathrm{U}$ & 850 & 840 & $\mathrm{U}$ & 840 & 840 & $\mathrm{U}$ & 840 \\
\hline 2-Nitrophenol & 340 & $\mathrm{U}$ & 340 & 340 & $\mathrm{U}$ & 340 & 330 & $\mathrm{U}$ & 330 & 340 & $\mathrm{U}$ & 340 \\
\hline 3,3'-Dichlorobenzidine & 340 & $\mathrm{U}$ & 340 & 340 & $\mathrm{U}$ & 340 & 330 & $\mathrm{U}$ & 330 & 340 & $\mathrm{U}$ & 340 \\
\hline 3-Nitroaniline & 840 & $\mathrm{U}$ & 840 & 850 & $\mathrm{U}$ & 850 & 840 & $\mathrm{U}$ & 840 & 840 & $\mathrm{U}$ & 840 \\
\hline 4,6-Dinitro-2-methylphenol & 840 & $\mathrm{U}$ & 840 & 850 & $\mathrm{U}$ & 850 & 840 & U & 840 & 840 & $\mathrm{U}$ & 840 \\
\hline 4-Bromophenyl-phenylether & 340 & U & 340 & 340 & $\mathrm{U}$ & 340 & 330 & $\mathrm{U}$ & 330 & 340 & $\mathrm{U}$ & 340 \\
\hline 4-Chloro-3-methylphenol & 340 & UJ & 340 & 340 & UJ & 340 & 330 & UJ & 330 & 340 & UJ & 340 \\
\hline 4-Chloroaniline & 340 & $\mathrm{U}$ & 340 & 340 & $\mathrm{U}$ & 340 & 330 & $\mathrm{U}$ & 330 & 340 & $\mathrm{U}$ & 340 \\
\hline 4-Chlorophenyl-phenylether & 340 & $\mathrm{U}$ & 340 & 340 & $\mathrm{U}$ & 340 & 330 & $\mathrm{U}$ & 330 & 340 & $\mathrm{U}$ & 340 \\
\hline 4-Methylphenol (p-cresol) & 340 & $\mathrm{U}$ & 340 & 340 & $\mathrm{U}$ & 340 & 330 & $\mathrm{U}$ & 330 & 340 & $\mathrm{U}$ & 340 \\
\hline 4-Nitroaniline & 840 & $\mathrm{U}$ & 840 & 850 & $\mathrm{U}$ & 850 & 840 & $\mathrm{U}$ & 840 & 840 & $\mathrm{U}$ & 840 \\
\hline 4-Nitrophenol & 840 & $\mathrm{U}$ & 840 & 850 & $\mathrm{U}$ & 850 & 840 & $\mathrm{U}$ & 840 & 840 & $\mathrm{U}$ & 840 \\
\hline Acenaphthene & 340 & $\mathrm{U}$ & 340 & 340 & $\mathrm{U}$ & 340 & 330 & $\mathrm{U}$ & 330 & 340 & $\mathrm{U}$ & 340 \\
\hline Acenaphthylene & 340 & $\mathrm{U}$ & 340 & 340 & $\mathrm{U}$ & 340 & 330 & $\mathrm{U}$ & 330 & 340 & $\mathrm{U}$ & 340 \\
\hline Anthracene & 340 & U & 340 & 340 & $\mathrm{U}$ & 340 & 330 & $\mathrm{U}$ & 330 & 340 & $\mathrm{U}$ & 340 \\
\hline Benzo(a)anthracene & 340 & U & 340 & 340 & $\mathrm{U}$ & 340 & 330 & $\mathrm{U}$ & 330 & 340 & $\mathrm{U}$ & 340 \\
\hline Benzo(a)pyrene & 340 & $\mathrm{U}$ & 340 & 340 & $\mathrm{U}$ & 340 & 330 & $\mathrm{U}$ & 330 & 340 & $\mathrm{U}$ & 340 \\
\hline Benzo(b)fluoranthene & 340 & $\mathrm{U}$ & 340 & 340 & $\mathrm{U}$ & 340 & 330 & $\mathrm{U}$ & 330 & 340 & $\mathrm{U}$ & 340 \\
\hline Benzo $(g, h, i)$ perylene & 340 & $\mathrm{U}$ & 340 & 340 & $\mathrm{U}$ & 340 & 330 & U & 330 & 340 & $\mathrm{U}$ & 340 \\
\hline Benzo(k)fluoranthene & 340 & $\mathrm{U}$ & 340 & 340 & U & 340 & 330 & $\mathrm{U}$ & 330 & 340 & $\mathrm{U}$ & 340 \\
\hline bis(2-Chloro-1-methylethyl)ether & 340 & $\mathrm{U}$ & 340 & 340 & U & 340 & 330 & $\mathrm{U}$ & 330 & 340 & $\mathrm{U}$ & 340 \\
\hline bis(2-Chloroethoxy)methane & 340 & $\mathrm{U}$ & 340 & 340 & $\mathrm{U}$ & 340 & 330 & $\mathrm{U}$ & 330 & 340 & $\mathrm{U}$ & 340 \\
\hline bis(2-Chloroethyl)ether & 340 & U & 340 & 340 & $\mathrm{U}$ & 340 & 330 & $\mathrm{U}$ & 330 & 340 & $\mathrm{U}$ & 340 \\
\hline bis(2-Ethylhexyl)phthalate & 18 & $\mathrm{~J}$ & 340 & 340 & $\mathrm{U}$ & 340 & 17 & $\mathrm{~J}$ & 330 & 340 & U & 340 \\
\hline
\end{tabular}

Attachment

Originator

Checked

Calc. No.

$\frac{\frac{1}{\text { J. M. Capron }}}{\text { T. M. Blakley }}$

Sheet No. 35 of 113 Date 10/04/07 Date Rev. No. 1 
Attachment 1. 118-B-1 Verification Sampling Results.

\begin{tabular}{|c|c|c|c|c|c|c|c|c|c|c|c|c|}
\hline \multirow[t]{2}{*}{ Constituents } & \multicolumn{3}{|c|}{$\begin{array}{c}\text { J12X46 } \\
\text { Area } 1 \mathrm{A3} \\
\text { Sample Date } 7 / 20 / 06\end{array}$} & \multicolumn{3}{|c|}{$\begin{array}{c}\text { J12X47 } \\
\text { Area } 1 \text { A4 } \\
\text { Sample Date 7/20/06 }\end{array}$} & \multicolumn{3}{|c|}{$\begin{array}{c}\text { J12X54 } \\
\text { Area } 3 \text { A1 } \\
\text { Sample Date } 7 / 20 / 06\end{array}$} & \multicolumn{3}{|c|}{$\begin{array}{c}\text { J12X55 } \\
\text { Area } 3 \text { A2 } \\
\text { Sample Date } 7 / 20 / 06\end{array}$} \\
\hline & $\mu \mathrm{g} / \mathrm{kg}$ & $\mathbf{Q}$ & PQL & $\mu \mathrm{g} / \mathrm{kg}$ & $\mathbf{Q}$ & PQL & $\mu \mathrm{g} / \mathrm{kg}$ & $Q$ & MDL & $\mu \mathrm{g} / \mathrm{kg}$ & $\mathbf{Q}$ & PQL \\
\hline \multicolumn{13}{|c|}{ Semivolatile Organic Compounds (continued) } \\
\hline Butylbenzylphthalate & 340 & $\mathrm{U}$ & 340 & 340 & $\mathrm{U}$ & 340 & 330 & $\mathrm{U}$ & 330 & 340 & $\mathrm{U}$ & 340 \\
\hline Carbazole & 340 & $\mathrm{U}$ & 340 & 340 & $\mathrm{U}$ & 340 & 330 & $\mathrm{U}$ & 330 & 340 & $\mathrm{U}$ & 340 \\
\hline Chrysene & 340 & $\mathrm{U}$ & 340 & 340 & $\mathrm{U}$ & 340 & 330 & $\mathrm{U}$ & 330 & 340 & $\mathrm{U}$ & 340 \\
\hline Di-n-butylphthalate & 28 & $\mathrm{JB}$ & 340 & 18 & $\mathrm{JB}$ & 340 & 330 & $\mathrm{U}$ & 330 & 340 & $\mathrm{U}$ & 340 \\
\hline Di-n-octylphthalate & 340 & $\mathrm{U}$ & 340 & 340 & $\mathrm{U}$ & 340 & 330 & $\mathrm{U}$ & 330 & 340 & $\mathrm{U}$ & 340 \\
\hline Dibenz $(\mathrm{a}, \mathrm{h})$ anthracene & 340 & $\mathrm{U}$ & 340 & 340 & $\mathrm{U}$ & 340 & 330 & $\mathrm{U}$ & 330 & 340 & $\mathrm{U}$ & 340 \\
\hline Dibenzofuran & 340 & $\mathrm{U}$ & 340 & 340 & $\mathrm{U}$ & 340 & 330 & $\mathrm{U}$ & 330 & 340 & $\mathrm{U}$ & 340 \\
\hline Diethylphthalate & 340 & $\mathrm{U}$ & 340 & 340 & $\mathrm{U}$ & 340 & 330 & $\mathrm{U}$ & 330 & 340 & $\mathrm{U}$ & 340 \\
\hline Dimethylphthalate & 340 & $\mathrm{U}$ & 340 & 340 & $\mathrm{U}$ & 340 & 330 & $\mathrm{U}$ & 330 & 340 & $\mathrm{U}$ & 340 \\
\hline Fluoranthene & 340 & $\mathrm{U}$ & 340 & 340 & $\mathrm{U}$ & 340 & 330 & $\mathrm{U}$ & 330 & 340 & $\mathrm{U}$ & 340 \\
\hline Fluorene & 340 & $\mathrm{U}$ & 340 & 340 & $\bar{U}$ & 340 & 330 & $\mathrm{U}$ & 330 & 340 & $\mathrm{U}$ & 340 \\
\hline Hexachlorobenzene & 340 & $\mathrm{U}$ & 340 & 340 & $\mathrm{U}$ & 340 & 330 & $\mathrm{U}$ & 330 & 340 & $\mathrm{U}$ & 340 \\
\hline Hexachlorobutadiene & 340 & $\mathrm{U}$ & 340 & 340 & $\mathrm{U}$ & 340 & 330 & $\mathrm{U}$ & 330 & 340 & $\mathrm{U}$ & 340 \\
\hline Hexachlorocyclopentadiene & 340 & $\mathrm{U}$ & 340 & 340 & $\mathrm{U}$ & 340 & 330 & $\mathrm{U}$ & 330 & 340 & $\mathrm{U}$ & 340 \\
\hline Hexachloroethane & 340 & $\mathrm{U}$ & 340 & 340 & $\mathrm{U}$ & 340 & 330 & $\mathrm{U}$ & 330 & 340 & $\mathrm{U}$ & 340 \\
\hline Indeno(1,2,3-cd)pyrene & 340 & $\mathrm{U}$ & 340 & 340 & $\mathrm{U}$ & 340 & 330 & $\mathrm{U}$ & 330 & 340 & $\mathrm{U}$ & 340 \\
\hline Isophorone & 340 & UJ & 340 & 340 & UT & 340 & 330 & UJ & 330 & 340 & UJ & 340 \\
\hline N-Nitroso-di-n-dipropylamine & 340 & $\mathrm{U}$ & 340 & 340 & $\mathrm{U}$ & 340 & 330 & $\mathrm{U}$ & 330 & 340 & $\mathrm{U}$ & 340 \\
\hline N-Nitrosodiphenylamine & 340 & $\mathrm{U}$ & 340 & 340 & $\mathrm{U}$ & 340 & 330 & $\mathrm{U}$ & 330 & 340 & $\mathrm{U}$ & 340 \\
\hline Naphthalene & 340 & $\mathrm{U}$ & 340 & 340 & $\mathrm{U}$ & 340 & 330 & $\mathrm{U}$ & 330 & 340 & $\mathrm{U}$ & 340 \\
\hline Nitrobenzene & 340 & $\mathrm{U}$ & 340 & 340 & $\mathrm{U}$ & 340 & 330 & $\mathrm{U}$ & 330 & 340 & $\mathrm{U}$ & 340 \\
\hline Pentachlorophenol & 840 & $\mathrm{U}$ & 840 & 850 & $\mathrm{U}$ & 850 & 840 & $\mathrm{U}$ & 840 & 840 & $\mathrm{U}$ & 840 \\
\hline Phenanthrene & 340 & $\mathrm{U}$ & 340 & 340 & $\mathrm{U}$ & 340 & 330 & $U$ & 330 & 340 & $\mathrm{U}$ & 340 \\
\hline Phenol & 340 & $\mathrm{U}$ & 340 & 340 & $\mathrm{U}$ & 340 & 330 & $\mathrm{U}$ & 330 & 340 & U & 340 \\
\hline Pyrene & 340 & $\mathrm{U}$ & 340 & 340 & $\mathrm{U}$ & 340 & 330 & $\mathrm{U}$ & 330 & 340 & U & 340 \\
\hline
\end{tabular}

Attachment Originator

Checked

Calc. No.

$\frac{1}{\frac{1}{\text { J. M. Capron }}}$

Sheet No. 36 of 113

Date $10 / 04 / 07$

Date

Rev. No. 1 
Attachment 1. 118-B-1 Verification Sampling Results.

\begin{tabular}{|c|c|c|c|c|c|c|c|c|c|c|c|c|}
\hline \multirow[t]{2}{*}{ Constituents } & \multicolumn{3}{|c|}{$\begin{array}{c}\text { J12X46 } \\
\text { Area } 1 \text { A3 } \\
\text { Sample Date } 7 / 20 / 06\end{array}$} & \multicolumn{3}{|c|}{$\begin{array}{c}\text { J12X47 } \\
\text { Area } 1 \text { A4 } \\
\text { Sample Date } 7 / 20 / 06 \\
\end{array}$} & \multicolumn{3}{|c|}{$\begin{array}{c}\text { J12X54 } \\
\text { Area } 3 \text { A1 } \\
\text { Sample Date } 7 / 20 / 06\end{array}$} & \multicolumn{3}{|c|}{$\begin{array}{c}\text { J12X55 } \\
\text { Area } 3 \text { A2 } \\
\text { Sample Date } 7 / 20 / 06 \\
\end{array}$} \\
\hline & $\mu \mathrm{g} / \mathrm{kg}$ & $\mathbf{Q}$ & PQL & $\mu \mathrm{g} / \mathrm{kg}$ & $Q$ & $\mathrm{PQL}$ & $\mu \mathrm{g} / \mathrm{kg}$ & $\mathbf{Q}$ & $\mathrm{MOL}$ & $\mu \mathrm{g} / \mathrm{kg}$ & $Q$ & PQL \\
\hline \multicolumn{13}{|c|}{ Volatile Organic Compounds } \\
\hline 1,1,1-Trichloroethane & 5 & $\mathrm{U}$ & 5 & 5 & $\mathrm{U}$ & 5 & 5 & $\mathrm{U}$ & 5 & 5 & $\mathrm{U}$ & 5 \\
\hline $1,1,2,2$-Tetrachloroethane & 5 & $U$ & 5 & 5 & $\mathrm{U}$ & 5 & 5 & $\mathrm{U}$ & 5 & 5 & $\mathrm{U}$ & 5 \\
\hline 1,1,2-Trichloroethane & 5 & $\mathrm{U}$ & 5 & 5 & $\mathrm{U}$ & 5 & 5 & $\mathrm{U}$ & 5 & 5 & U & 5 \\
\hline 1,1-Dichloroethane & 5 & $\mathrm{U}$ & 5 & 5 & $\mathrm{U}$ & 5 & 5 & $\mathrm{U}$ & 5 & 5 & $\mathrm{U}$ & 5 \\
\hline 1,1-Dichloroethene & 5 & $\mathrm{U}$ & 5 & 5 & $\mathrm{U}$ & 5 & 5 & $\mathrm{U}$ & 5 & 5 & $\mathrm{U}$ & 5 \\
\hline 1,2-Dichloroethane & 5 & $U$ & 5 & 5 & $\mathrm{U}$ & 5 & 5 & $\mathrm{U}$ & 5 & 5 & $\mathrm{U}$ & 5 \\
\hline 1,2-Dichloroethene (total) & 5 & $\mathrm{U}$ & 5 & 5 & $\mathrm{U}$ & 5 & 5 & $\mathrm{U}$ & 5 & 5 & $\mathrm{U}$ & 5 \\
\hline 1,2-Dichloropropane & 5 & $\mathrm{U}$ & 5 & 5 & $\mathrm{U}$ & 5 & 5 & $\mathrm{U}$ & 5 & 5 & $\mathrm{U}$ & 5 \\
\hline 2-Butanone & 9 & $\mathrm{U}$ & 9 & 10 & $\mathrm{U}$ & 10 & 10 & U & 10 & 10 & $\mathrm{U}$ & 10 \\
\hline 2-Hexanone & 9 & $\mathrm{U}$ & 9 & 10 & $\mathrm{U}$ & 10 & 10 & $\mathrm{U}$ & 10 & 10 & $\mathrm{U}$ & 10 \\
\hline 4-Methyl-2-pentanone & 5 & JB & 9 & 4 & $\mathrm{JB}$ & 10 & 6 & $\mathrm{JB}$ & 10 & 6 & $\mathrm{JB}$ & 10 \\
\hline Acetone & 9 & $\mathrm{~J}$ & 9 & 4 & $\mathrm{~J}$ & 10 & 15 & & 10 & 16 & & 10 \\
\hline Benzene & 5 & $\mathrm{U}$ & 5 & 5 & $\mathrm{U}$ & 5 & 5 & $\mathrm{U}$ & 5 & 5 & $\mathrm{U}$ & 5 \\
\hline Bromodichloromethane & 5 & $\mathrm{U}$ & 5 & 5 & $\mathrm{U}$ & 5 & 5 & $\mathrm{U}$ & 5 & 5 & $\mathrm{U}$ & 5 \\
\hline Bromoform & 5 & $\mathrm{U}$ & 5 & 5 & $U$ & 5 & 5 & $\mathrm{U}$ & 5 & 5 & $\mathrm{U}$ & 5 \\
\hline Bromomethane & 9 & $\mathrm{U}$ & 9 & 10 & $\mathrm{U}$ & 10 & 10 & $\mathrm{U}$ & 10 & 10 & $\mathrm{U}$ & 10 \\
\hline Carbon disulfide & 5 & $\mathrm{U}$ & 5 & 5 & $\mathrm{U}$ & 5 & 5 & $\mathrm{U}$ & 5 & 5 & $\mathrm{U}$ & 5 \\
\hline Carbon tetrachloride & 5 & $\mathrm{U}$ & 5 & 5 & $\mathrm{U}$ & 5 & 5 & $\mathrm{U}$ & 5 & 8 & & 5 \\
\hline Chlorobenzene & 5 & U & 5 & 5 & U & 5 & 5 & $\mathrm{U}$ & 5 & 5 & U & 5 \\
\hline Chloroethane & 9 & $\mathrm{U}$ & 9 & 10 & $\mathrm{U}$ & 10 & 10 & $\mathrm{U}$ & 10 & 10 & $\mathrm{U}$ & 10 \\
\hline Chloroform & 5 & U & 5 & 5 & $\mathrm{U}$ & 5 & 5 & $\mathrm{U}$ & 5 & 5 & $\mathrm{U}$ & 5 \\
\hline Chloromethane & 9 & $\mathrm{U}$ & 9 & 10 & $\mathrm{U}$ & 10 & 10 & $\mathrm{U}$ & 10 & 10 & $\mathrm{U}$ & 10 \\
\hline cis-1,2-Dichloroethylene & 5 & $\mathrm{U}$ & 5 & 5 & $\mathrm{U}$ & 5 & 5 & $\mathrm{U}$ & 5 & 5 & $\mathrm{U}$ & 5 \\
\hline cis-1,3-Dichloropropene & 5 & $\mathrm{U}$ & 5 & 5 & $\mathrm{U}$ & 5 & 5 & $\mathrm{U}$ & 5 & 5 & $\mathrm{U}$ & 5 \\
\hline Dibromochloromethane & 5 & $U$ & 5 & 5 & $\mathrm{U}$ & 5 & 5 & $\mathrm{U}$ & 5 & 5 & $\mathrm{U}$ & 5 \\
\hline Ethylbenzene & 5 & $\mathrm{U}$ & 5 & 5 & $\mathrm{U}$ & 5 & 5 & $\mathrm{U}$ & 5 & 5 & $\mathrm{U}$ & 5 \\
\hline Methylene chloride & 22 & & 5 & 12 & & 5 & 26 & & 5 & 5 & & 5 \\
\hline Styrene & 5 & $\mathrm{U}$ & 5 & 5 & $\mathrm{U}$ & 5 & 5 & $\mathrm{U}$ & 5 & 5 & $\mathrm{U}$ & 5 \\
\hline Tetrachloroethene & 5 & $\mathrm{U}$ & 5 & 5 & $\mathrm{U}$ & 5 & 5 & $\mathrm{U}$ & 5 & 5 & $\mathrm{U}$ & 5 \\
\hline \begin{tabular}{|l} 
Toluene \\
\end{tabular} & 5 & $\mathrm{U}$ & 5 & 5 & $\mathrm{U}$ & 5 & 5 & $\mathrm{U}$ & 5 & 5 & $\mathrm{U}$ & 5 \\
\hline trans-1,2-Dichloroethylene & 5 & $\mathrm{U}$ & 5 & 5 & $\mathrm{U}$ & 5 & 5 & $\mathrm{U}$ & 5 & 5 & $\mathrm{U}$ & 5 \\
\hline trans-1,3-Dichloropropene & 5 & $\mathrm{U}$ & 5 & 5 & $\mathrm{U}$ & 5 & 5 & $\mathrm{U}$ & 5 & 5 & $\mathrm{U}$ & 5 \\
\hline \begin{tabular}{|l|} 
Trichloroethene \\
\end{tabular} & 5 & U & 5 & 5 & $\mathrm{U}$ & 5 & 5 & $\mathrm{U}$ & 5 & 5 & $\mathrm{U}$ & 5 \\
\hline Vinyl chloride & 9 & $\mathrm{U}$ & 9 & 10 & $\mathrm{U}$ & 10 & 10 & $\mathrm{U}$ & 10 & 10 & $\mathrm{U}$ & 10 \\
\hline Xylenes (total) & 5 & $\mathrm{U}$ & 5 & 5 & $\mathrm{U}$ & 5 & 5 & $\mathrm{U}$ & 5 & 5 & $\mathrm{U}$ & 5 \\
\hline & & & & Attachm & & & 1 & & & Sheet No. & 37 & of 113 \\
\hline & & & & Origina & & J.M. C & rron & & & Date & & $07 / 07$ \\
\hline & & & & Checker & & T.M. B & akley & & & Date & & \\
\hline & & & & Calc. N & & 0100B- & A-V029 & & & Rev. No. & & 0 \\
\hline
\end{tabular}


Attachment 1. 118-B-1 Verification Sampling Results.

\begin{tabular}{|c|c|c|c|c|c|c|c|c|c|c|c|c|}
\hline \multirow[t]{2}{*}{ Constituents } & \multicolumn{3}{|c|}{$\begin{array}{c}\text { J12X56 } \\
\text { Area } 3 \text { A3 } \\
\text { Sample Date } 7 / 20 / 06 \\
\end{array}$} & \multicolumn{3}{|c|}{$\begin{array}{c}\text { J12X58 } \\
\text { Duplicate of J12X56 } \\
\text { Sample Date } 7 / 20 / 06 \\
\end{array}$} & \multicolumn{3}{|c|}{$\begin{array}{c}\mathrm{J} 12 \times 60 \\
\text { Split of } \mathrm{J} 12 \times 56 \\
\text { Sample Date } 7 / 20 / 06 \\
\end{array}$} & \multicolumn{3}{|c|}{$\begin{array}{c}\text { J12X57 } \\
\text { Area } 3 \text { A4 } \\
\text { Sample Date } 7 / 20 / 06\end{array}$} \\
\hline & $\mu \mathrm{g} / \mathrm{kg}$ & $\mathrm{Q}$ & PQL & $\mu \mathrm{g} / \mathrm{kg}$ & $Q$ & PQL & $\mu \mathrm{g} / \mathrm{kg}$ & $\mathbf{Q}$ & $\mathrm{PQL}$ & $\mu \mathrm{g} / \mathrm{kg}$ & $\mathbf{Q}$ & PQL \\
\hline \multicolumn{13}{|c|}{ Polychlorinated Biphenyls } \\
\hline Aroclor-1016 & 14 & $\mathrm{U}$ & 14 & 14 & $\mathrm{U}$ & 14 & 17 & U & 17 & 13 & U & 13 \\
\hline Aroclor- 1221 & 14 & U & 14 & 14 & $\mathrm{U}$ & 14 & 17 & $\mathrm{U}$ & 17 & 13 & $\mathrm{U}$ & 13 \\
\hline Aroclor- 1232 & 14 & U & 14 & 14 & $U$ & 14 & 17 & $\mathrm{U}$ & 17 & 13 & $\mathrm{U}$ & 13 \\
\hline Aroclor- 1242 & 14 & $\mathrm{U}$ & 14 & 14 & $\mathrm{U}$ & 14 & 17 & $\mathrm{U}$ & 17 & 13 & $\mathrm{U}$ & 13 \\
\hline Aroclor- 1248 & 14 & $\mathrm{U}$ & 14 & 14 & $\mathrm{U}$ & 14 & 17 & $\mathrm{U}$ & 17 & 13 & $\mathrm{U}$ & 13 \\
\hline Aroclor- 1254 & 14 & $\mathrm{U}$ & 14 & 14 & $\mathrm{U}$ & 14 & 17 & $\mathrm{U}$ & 17 & 13 & $\mathrm{U}$ & 13 \\
\hline Aroclor- 1260 & 14 & $\mathrm{U}$ & 14 & 14 & $\mathrm{U}$ & 14 & 17 & $\mathrm{U}$ & 17 & 13 & $\mathrm{U}$ & 13 \\
\hline \multicolumn{13}{|c|}{ Semivolatile Organic Compounds } \\
\hline 1,2,4-Trichlorobenzene & 340 & $\mathrm{U}$ & 340 & 340 & $\mathrm{U}$ & 340 & 340 & $\mathrm{U}$ & 340 & 340 & $\mathrm{U}$ & 340 \\
\hline 1,2-Dichlorobenzene & 340 & $\mathrm{U}$ & 340 & 340 & $\mathrm{U}$ & 340 & 340 & $\mathrm{U}$ & 340 & 340 & $\mathrm{U}$ & 340 \\
\hline 1,3-Dichlorobenzene & 340 & $\mathrm{U}$ & 340 & 340 & $\mathrm{U}$ & 340 & 340 & $\mathrm{U}$ & 340 & 340 & $\mathrm{U}$ & 340 \\
\hline 1,4-Dichlorobenzene & 340 & $\mathrm{U}$ & 340 & 340 & $\mathrm{U}$ & 340 & 340 & $\mathrm{U}$ & 340 & 340 & $\mathrm{U}$ & 340 \\
\hline 2,4,5-Trichlorophenol & 850 & $\mathrm{U}$ & 850 & 850 & $\mathrm{U}$ & 850 & 340 & $\mathrm{U}$ & 340 & 840 & $\mathrm{U}$ & 840 \\
\hline 2,4,6-Trichlorophenol & 340 & $\mathrm{U}$ & 340 & 340 & $\mathrm{U}$ & 340 & 340 & $\mathrm{U}$ & 340 & 340 & $U$ & 340 \\
\hline 2,4-Dichlorophenol & 340 & $\mathrm{U}$ & 340 & 340 & $\mathrm{U}$ & 340 & 340 & $\mathrm{U}$ & 340 & 340 & $\mathrm{U}$ & 340 \\
\hline 2,4-Dimethylphenol & 340 & UJ & 340 & 340 & UJ & 340 & 340 & $\mathrm{U}$ & 340 & 340 & $\mathrm{UJ}$ & 340 \\
\hline 2,4-Dinitrophenol & 850 & $\mathrm{U}$ & 850 & 850 & $\mathrm{U}$ & 850 & 1600 & UJ & 1600 & 840 & $\mathrm{U}$ & 840 \\
\hline 2,4-Dinitrotoluene & 340 & $\mathrm{U}$ & 340 & 340 & $\mathrm{U}$ & 340 & 340 & $\mathrm{U}$ & 340 & 340 & $\mathrm{U}$ & 340 \\
\hline 2,6-Dinitrotoluene & 340 & $\mathrm{U}$ & 340 & 340 & $\mathrm{U}$ & 340 & 340 & $\mathrm{U}$ & 340 & 340 & U & 340 \\
\hline 2-Chloronaphthalene & 340 & $\mathrm{U}$ & 340 & 340 & $\mathrm{U}$ & 340 & 340 & $\mathrm{U}$ & 340 & 340 & $\mathrm{U}$ & 340 \\
\hline 2-Chlorophenol & 340 & $\mathrm{U}$ & 340 & 340 & $\mathrm{U}$ & 340 & 340 & $U$ & 340 & 340 & $\mathrm{U}$ & 340 \\
\hline 2-Methylnaphthalene & 340 & $\mathrm{U}$ & 340 & 340 & $\mathrm{U}$ & 340 & 340 & U & 340 & 340 & $\mathrm{U}$ & 340 \\
\hline 2-Methylphenol (cresol, o-) & 340 & $\mathrm{U}$ & 340 & 340 & $\mathrm{U}$ & 340 & 340 & $\mathrm{U}$ & 340 & 340 & $\mathrm{U}$ & 340 \\
\hline 2-Nitroaniline & 850 & $\mathrm{U}$ & 850 & 850 & $\mathrm{U}$ & 850 & 1600 & U & 1600 & 840 & $\mathrm{U}$ & 840 \\
\hline 2-Nitrophenol & 340 & $\mathrm{U}$ & 340 & 340 & $\mathrm{U}$ & 340 & 340 & $\mathrm{U}$ & .340 & 340 & $\mathrm{U}$ & 340 \\
\hline 3,3'-Dichlorobenzidine & 340 & $\mathrm{U}$ & 340 & 340 & $\mathrm{U}$ & 340 & 1600 & $\mathrm{U}$ & 1600 & 340 & $\mathrm{U}$ & 340 \\
\hline 3-Nitroaniline & 850 & $\mathrm{U}$ & 850 & 850 & $\mathrm{U}$ & 850 & 1600 & $\mathrm{U}$ & 1600 & 840 & $\mathrm{U}$ & 840 \\
\hline 4,6-Dinitro-2-methylphenol & 850 & $\mathrm{U}$ & 850 & 850 & $\mathrm{U}$ & 850 & 1600 & $U$ & 1600 & 840 & $\mathrm{U}$ & 840 \\
\hline 4-Bromophenyl-phenylether & 340 & $\mathrm{U}$ & 340 & 340 & $\mathrm{U}$ & 340 & 340 & $\mathrm{U}$ & 340 & 340 & $\mathrm{U}$ & 340 \\
\hline 4-Chloro-3-methylphenol & 340 & UJ & 340 & 340 & $\mathrm{UJ}$ & 340 & 340 & $\mathrm{UJ}$ & 340 & 340 & UJ & 340 \\
\hline 4-Chloroaniline & 340 & $\mathrm{U}$ & 340 & 340 & $\mathrm{U}$ & 340 & 340 & $\mathrm{U}$ & 340 & 340 & $\mathrm{U}$ & 340 \\
\hline 4-Chlorophenyl-phenylether & 340 & $\mathrm{U}$ & 340 & 340 & $\mathrm{U}$ & 340 & 340 & $\mathrm{U}$ & 340 & 340 & $\mathrm{U}$ & 340 \\
\hline 4-Methylphenol (p-cresol) & 340 & $\mathrm{U}$ & 340 & 340 & $\mathrm{U}$ & 340 & 670 & $\mathrm{U}$ & 670 & 340 & $\mathrm{U}$ & 340 \\
\hline 4-Nitroaniline & 850 & $\mathrm{U}$ & 850 & 850 & $\mathrm{U}$ & 850 & 1600 & $\mathrm{U}$ & 1600 & 840 & $U$ & 840 \\
\hline 4-Nitrophenol & 850 & $\mathrm{U}$ & 850 & 850 & $\mathrm{U}$ & 850 & 1600 & $\mathrm{U}$ & 1600 & 840 & $\mathrm{U}$ & 840 \\
\hline Acenaphthene & 340 & $\mathrm{U}$ & 340 & 340 & $\mathrm{U}$ & 340 & 340 & $\mathrm{U}$ & 340 & 340 & $U$ & 340 \\
\hline Acenaphthylene & 340 & $\mathrm{U}$ & 340 & 340 & $\mathrm{U}$ & 340 & 340 & $\mathrm{U}$ & 340 & 340 & $\mathrm{U}$ & 340 \\
\hline Anthracene & 340 & $U$ & 340 & 340 & $\mathrm{U}$ & 340 & 340 & $\mathrm{U}$ & 340 & 340 & $\mathrm{U}$ & 340 \\
\hline Benzo(a)anthracene & 340 & $\mathrm{U}$ & 340 & 340 & $\mathrm{U}$ & 340 & 340 & $\mathrm{U}$ & 340 & 340 & $\mathrm{U}$ & 340 \\
\hline Benzo(a)pyrene & 340 & $\mathrm{U}$ & 340 & 340 & $\mathrm{U}$ & 340 & 340 & $\mathrm{U}$ & 340 & 340 & $\mathrm{U}$ & 340 \\
\hline Benzo(b)fluoranthene & 340 & $\mathrm{U}$ & 340 & 340 & $\mathrm{U}$ & 340 & 340 & $\mathrm{U}$ & 340 & 340 & $\mathrm{U}$ & 340 \\
\hline Benzo(g, $h, \mathrm{i})$ perylene & 340 & $\mathrm{U}$ & 340 & 340 & $\mathrm{U}$ & 340 & 340 & $\mathrm{U}$ & 340 & 340 & $\mathrm{U}$ & 340 \\
\hline Benzo(k)fluoranthene & 340 & U & 340 & 340 & $\mathrm{U}$ & 340 & 340 & $\mathrm{U}$ & 340 & 340 & $\mathrm{U}$ & 340 \\
\hline bis(2-Chloro-1-methylethyl)ether & 340 & $\mathrm{U}$ & 340 & 340 & $\mathrm{U}$ & 340 & 340 & $\mathrm{U}$ & 340 & 340 & $\mathrm{U}$ & 340 \\
\hline bis(2-Chloroethoxy)methane & 340 & U & 340 & 340 & $\mathrm{U}$ & 340 & 340 & $\mathrm{U}$ & 340 & 340 & $\mathrm{U}$ & 340 \\
\hline bis(2-Chloroethyl)ether & 340 & $\mathrm{U}$ & 340 & 340 & U & 340 & 340 & $\mathrm{U}$ & 340 & 340 & $\mathrm{U}$ & 340 \\
\hline bis(2-Ethylhexyl)phthalate & 340 & $\mathrm{U}$ & 340 & 39 & $\mathrm{~J}$ & 340 & 340 & U & 340 & 31 & $\mathrm{~J}$ & 340 \\
\hline
\end{tabular}

Attachment

Originator

Checked

Calc. No.

$\frac{1}{\frac{1}{\text { J.M. Capron }}} \frac{}{\text { T. M. Blakley }}$

Sheet No. 38 of 113

Date $\frac{380 f 113}{10 / 04 / 07}$

Date

Rev. No. 
Rev. 0

Attachment 1. 118-B-1 Verification Sampling Results.

\begin{tabular}{|c|c|c|c|c|c|c|c|c|c|c|c|c|}
\hline \multirow[t]{2}{*}{ Constituents } & \multicolumn{3}{|c|}{$\begin{array}{c}\mathrm{J} 12 \times 56 \\
\text { Area } 3 \mathrm{A3} \\
\text { Sample Date } 7 / 20 / 06\end{array}$} & \multicolumn{3}{|c|}{$\begin{array}{c}\mathrm{J12X58} \\
\text { Duplicate of } \mathrm{J12X56} \\
\text { Sample Date } 7 / 20 / 06\end{array}$} & \multicolumn{3}{|c|}{$\begin{array}{c}\mathrm{J} 12 \mathrm{X} 60 \\
\text { Split of J12X56 } \\
\text { Sample Date } 7 / 20 / 06\end{array}$} & \multicolumn{3}{|c|}{$\begin{array}{c}\text { J12X57 } \\
\text { Area } 3 \text { A4 } \\
\text { Sample Date } 7 / 20 / 06\end{array}$} \\
\hline & $\mu \mathrm{g} / \mathrm{kg}$ & $\mathbf{Q}$ & PQL & $\mu \mathrm{g} / \mathrm{kg}$ & $\mathbf{Q}$ & PQL & $\mu \mathrm{g} / \mathrm{kg}$ & $\mathbf{Q}$ & MDL & $\mu \mathrm{g} / \mathrm{kg}$ & $Q$ & PQL \\
\hline \multicolumn{13}{|c|}{ Semivolatile Organic Compounds (continued) } \\
\hline Butylbenzylphthalate & 340 & $\mathrm{U}$ & 340 & 340 & $\mathrm{U}$ & 340 & 340 & $\mathrm{U}$ & 340 & 340 & $\mathrm{U}$ & 340 \\
\hline Carbazole & 340 & $\mathrm{U}$ & 340 & 340 & $\mathrm{U}$ & 340 & 340 & $U$ & 340 & 340 & $\bar{U}$ & 340 \\
\hline Chrysene & 340 & $\mathrm{U}$ & 340 & 340 & $\mathrm{U}$ & 340 & 340 & $\mathrm{U}$ & 340 & 340 & U & 340 \\
\hline Di-n-butylphthalate & 27 & $\mathrm{JB}$ & 340 & 54 & $\mathrm{JB}$ & 340 & 340 & $\mathrm{U}$ & 340 & 40 & $\mathrm{JB}$ & 340 \\
\hline Di-n-octylphthalate & 340 & $\mathrm{U}$ & 340 & 340 & $\mathrm{U}$ & 340 & 340 & $\mathrm{U}$ & 340 & 340 & $\mathrm{U}$ & 340 \\
\hline Dibenz $(\mathrm{a}, \mathrm{h})$ anthracene & 340 & $\mathrm{U}$ & 340 & 340 & $\mathrm{U}$ & 340 & 340 & $\mathrm{U}$ & 340 & 340 & $\mathrm{U}$ & 340 \\
\hline Dibenzofuran & 340 & $U$ & 340 & 340 & $\mathrm{U}$ & 340 & 340 & $\mathrm{U}$ & 340 & 340 & $\mathrm{U}$ & 340 \\
\hline Diethylphthalate & 340 & $\mathrm{U}$ & 340 & 340 & $\mathrm{U}$ & 340 & 340 & $\mathrm{U}$ & 340 & 340 & $\mathrm{U}$ & 340 \\
\hline Dimethylphthalate & 340 & $\mathrm{U}$ & 340 & 340 & U & 340 & 340 & $\mathrm{U}$ & 340 & 340 & $\mathrm{U}$ & 340 \\
\hline Fluoranthene & 340 & $\mathrm{U}$ & 340 & 340 & $\mathrm{U}$ & 340 & 340 & $\mathrm{U}$ & 340 & 340 & $\mathrm{U}$ & 340 \\
\hline Fluorene & 340 & $\mathrm{U}$ & 340 & 340 & $\mathrm{U}$ & 340 & 340 & U & 340 & 340 & U & 340 \\
\hline Hexachlorobenzene & 340 & $\mathrm{U}$ & 340 & 340 & $\mathrm{U}$ & 340 & 340 & $\mathrm{U}$ & 340 & 340 & $\mathrm{U}$ & 340 \\
\hline Hexachlorobutadiene & 340 & $\bar{U}$ & 340 & 340 & $\mathrm{U}$ & 340 & 340 & $\mathrm{U}$ & 340 & 340 & $\mathrm{U}$ & 340 \\
\hline Hexachlorocyclopentadiene & 340 & $\mathrm{U}$ & 340 & 340 & $\mathrm{U}$ & 340 & 1600 & $\mathrm{U}$ & 1600 & 340 & $\mathrm{U}$ & 340 \\
\hline Hexachloroethane & 340 & $\mathrm{U}$ & 340 & 340 & $\mathrm{U}$ & 340 & 340 & $\mathrm{U}$ & 340 & 340 & $\mathrm{U}$ & 340 \\
\hline Indeno(1,2,3-cd)pyrene & 340 & $\mathrm{U}$ & 340 & 340 & $\mathrm{U}$ & 340 & 340 & $\mathrm{U}$ & 340 & 340 & U & 340 \\
\hline Isophorone & 340 & UJ & 340 & 340 & UJ & 340 & 340 & $\mathrm{U}$ & 340 & 340 & UJ & 340 \\
\hline N-Nitroso-di-n-dipropylamine & 340 & $\mathrm{U}$ & 340 & 340 & U & 340 & 340 & $\mathrm{U}$ & 340 & 340 & $\mathrm{U}$ & 340 \\
\hline N-Nitrosodiphenylamine & 340 & $\mathrm{U}$ & 340 & 340 & $\mathrm{U}$ & 340 & 340 & $\mathrm{U}$ & 340 & 340 & $\mathrm{U}$ & 340 \\
\hline Naphthalene & 340 & $\mathrm{U}$ & 340 & 340 & $\mathrm{U}$ & 340 & 340 & U & 340 & 340 & $\bar{U}$ & 340 \\
\hline Nitrobenzene & 340 & $\mathrm{U}$ & 340 & 340 & $\mathrm{U}$ & 340 & 340 & $\mathrm{U}$ & 340 & 340 & $\mathrm{U}$ & 340 \\
\hline Pentachlorophenol & 850 & $\mathrm{U}$ & 850 & 850 & $\mathrm{U}$ & 850 & 1600 & $\mathrm{U}$ & 1600 & 840 & $\mathrm{U}$ & 840 \\
\hline Phenanthrene & 340 & $\mathrm{U}$ & 340 & 340 & $\mathrm{U}$ & 340 & 340 & $\mathrm{U}$ & 340 & 340 & $\mathrm{U}$ & 340 \\
\hline Phenol & 340 & $\mathrm{U}$ & 340 & 340 & $\mathrm{U}$ & 340 & 340 & $\mathrm{U}$ & 340 & 340 & $\mathrm{U}$ & 340 \\
\hline Pyrene & 340 & $\overline{\mathrm{U}}$ & 340 & 340 & $\mathrm{U}$ & 340 & 340 & $\mathrm{U}$ & 340 & 340 & $\overline{\mathrm{U}}$ & 340 \\
\hline
\end{tabular}

Attachment

Originator

Checked

Calc. No.

$\frac{\text { J. M. Capron }}{\text { T.M. Blakley }}$

Sheet No. 39 of 113

Date $\frac{39 / 04 / 07}{107}$

Date

Rev. No. 
Attachment 1. 118-B-1 Verification Sampling Results.

\begin{tabular}{|c|c|c|c|c|c|c|c|c|c|c|c|c|}
\hline \multirow[t]{2}{*}{ Constituents } & \multicolumn{3}{|c|}{$\begin{array}{c}\text { J12X56 } \\
\text { Area } 3 \text { A3 } \\
\text { Sample Date } 7 / 20 / 06\end{array}$} & \multicolumn{3}{|c|}{$\begin{array}{c}\mathrm{J} 12 \times 58 \\
\text { Duplicate of } \mathrm{J12 \times 56} \\
\text { Sample Date } 7 / 20 / 06\end{array}$} & \multicolumn{3}{|c|}{$\begin{array}{c}\mathrm{J12 \times 60} \\
\text { Split of J12X56 } \\
\text { Sample Date } 7 / 20 / 06 \\
\end{array}$} & \multicolumn{3}{|c|}{$\begin{array}{c}\text { J12X57 } \\
\text { Area } 3 \text { A4 } \\
\text { Sample Date } 7 / 20 / 06\end{array}$} \\
\hline & $\mu \mathrm{g} / \mathrm{kg}$ & $Q$ & PQL & $\mu \mathrm{g} / \mathrm{kg}$ & $Q$ & PQL & $\mu \mathrm{g} / \mathrm{kg}$ & $Q$ & $\mathrm{MDL}$ & $\mu g / \mathrm{kg}$ & $Q$ & PQL \\
\hline \multicolumn{13}{|c|}{ Volatile Organic Compounds } \\
\hline 1,1,1-Trichloroethane & 5 & $\mathrm{U}$ & 5 & 5 & $\mathrm{U}$ & 5 & 5.1 & $\mathrm{U}$ & 5.1 & 5 & $\mathrm{U}$ & 5 \\
\hline 1,1,2,2-Tetrachloroethane & 5 & $\mathrm{U}$ & 5 & 5 & U & 5 & 5.1 & $\mathrm{U}$ & 5.1 & 5 & $\mathrm{U}$ & 5 \\
\hline 1,1,2-Trichloroethane & 5 & $\mathrm{U}$ & 5 & 5 & $\mathrm{U}$ & 5 & 5.1 & $\mathrm{U}$ & 5.1 & 5 & $\mathrm{U}$ & 5 \\
\hline 1,1-Dichloroethane & 5 & $\mathrm{U}$ & 5 & 5 & $\mathrm{U}$ & 5 & 5.1 & $\mathrm{U}$ & 5.1 & 5 & $\mathrm{U}$ & 5 \\
\hline 1,1-Dichloroethene & 5. & $\mathrm{U}$ & 5 & 5 & $\mathrm{U}$ & 5 & 5.1 & $\mathrm{U}$ & 5.1 & 5 & $\mathrm{U}$ & 5 \\
\hline 1,2-Dichloroethane & 5 & $\mathrm{U}$ & 5 & 5 & $\mathrm{U}$ & 5 & 5.1 & $\mathrm{U}$ & 5.1 & 5 & $\mathrm{U}$ & 5 \\
\hline 1,2-Dichloroethene (total) & 5 & $\mathrm{U}$ & 5 & 5 & U & 5 & 10 & $\mathrm{U}$ & 10 & 5 & $\mathrm{U}$ & 5 \\
\hline 1,2-Dichloropropane & 5 & U & 5 & 5 & $\mathrm{U}$ & 5 & 5.1 & $\mathrm{U}$ & 5.1 & 5 & $\mathrm{U}$ & 5 \\
\hline 2-Butanone & 10 & $\mathrm{U}$ & 10 & 10 & $\mathrm{U}$ & 10 & 10 & $\mathrm{U}$ & 10 & 10 & $U$ & 10 \\
\hline 2-Hexanone & 10 & $\mathrm{U}$ & 10 & 10 & $\mathrm{U}$ & 10 & 20 & $\mathrm{U}$ & 20 & 10 & $\mathrm{U}$ & 10 \\
\hline 4-Methyl-2-pentanone & 10 & $\mathrm{U}$ & 10 & 10 & $\mathrm{U}$ & 10 & 10 & $\mathrm{U}$ & 10 & 10 & $U$ & 10 \\
\hline Acetone & 7 & $\mathrm{~J}$ & 10 & 8 & $\mathrm{~J}$ & 10 & 20 & $\mathrm{U}$ & 20 & 6 & $\mathrm{~J}$ & 10 \\
\hline Benzene & 5 & U & 5 & 5 & $\mathrm{U}$ & 5 & 5.1 & $\mathrm{U}$ & 5.1 & 5 & $\mathrm{U}$ & 5 \\
\hline Bromodichloromethane & 5 & $\mathrm{U}$ & 5 & 5 & $\mathrm{U}$ & 5 & 5.1 & $\mathrm{U}$ & 5.1 & 5 & $\mathrm{U}$ & 5 \\
\hline Bromoform & 5 & $U$ & 5 & 5 & $\mathrm{U}$ & 5 & 5.1 & $\mathrm{U}$ & 5.1 & 5 & $\mathrm{U}$ & 5 \\
\hline Bromomethane & 10 & U & 10 & 10 & $\mathrm{U}$ & 10 & 10 & $\mathrm{U}$ & 10 & 10 & $\mathrm{U}$ & 10 \\
\hline Carbon disulfide & 5 & $\mathrm{U}$ & 5 & 5 & $\mathrm{U}$ & 5 & 5.1 & $\mathrm{U}$ & 5.1 & 5 & $\mathrm{U}$ & 5 \\
\hline Carbon tetrachloride & 5 & $\mathrm{U}$ & 5 & 5 & $\mathrm{U}$ & 5 & 5.1 & $\mathrm{U}$ & 5.1 & 11 & & 5 \\
\hline Chlorobenzene & 5 & $\mathrm{U}$ & 5 & 5 & $\mathrm{U}$ & 5 & 5.1 & $\mathrm{U}$ & 5.1 & 5 & $\mathrm{U}$ & 5 \\
\hline Chloroethane & 10 & $\mathrm{U}$ & 10 & 10 & $\mathrm{U}$ & 10 & 10 & $\mathrm{U}$ & 10 & 10 & $\mathrm{U}$ & 10 \\
\hline Chloroform & 5 & $\mathrm{U}$ & 5 & 5 & $\mathrm{U}$ & 5 & 5.1 & $\mathrm{U}$ & 5.1 & 5 & $\mathrm{U}$ & 5 \\
\hline Chloromethane & 10 & $\mathrm{U}$ & 10 & 10 & U & 10 & 10 & $\mathrm{U}$ & 10 & 10 & $\mathrm{U}$ & 10 \\
\hline cis-1,2-Dichloroethylene & 5 & $\mathrm{U}$ & 5 & 5 & $\mathrm{U}$ & 5 & & & & 5 & $\mathrm{U}$ & 5 \\
\hline cis-1,3-Dichloropropene & 5 & $\mathrm{U}$ & 5 & 5 & $\mathrm{U}$ & 5 & 5.1 & $\mathrm{U}$ & 5.1 & 5 & $\mathrm{U}$ & 5 \\
\hline Dibromochloromethane & 5 & $\mathrm{U}$ & 5 & 5 & $\mathrm{U}$ & 5 & 5.1 & U & 5.1 & 5 & $\mathrm{U}$ & 5 \\
\hline Ethylbenzene & 5 & $\mathrm{U}$ & 5 & 5 & $\mathrm{U}$ & 5 & 5.1 & $\mathrm{U}$ & 5.1 & 5 & $\mathrm{U}$ & 5 \\
\hline Methylene chloride & 5 & $\mathrm{U}$ & 5 & 5 & $\mathrm{U}$ & 5 & 5.1 & $U$ & 5.1 & 5 & $\frac{\mathrm{J}}{\mathrm{J}}$ & 5 \\
\hline Styrene & 5 & U & 5 & 5 & U & 5 & 5.1 & U & 5.1 & 5 & $\mathrm{U}$ & 5 \\
\hline Tetrachloroethene & 5 & $\mathrm{U}$ & 5 & 5 & $\mathrm{U}$ & 5 & 5.1 & UN & 5.1 & 5 & $\mathrm{U}$ & 5 \\
\hline Toluene & 5 & $\mathrm{U}$ & 5 & 5 & $\mathrm{U}$ & 5 & 5.1 & UN & 5.1 & 5 & $\mathrm{U}$ & 5 \\
\hline trans - 1,2-Dichloroethylene & 5 & $\mathrm{U}$ & 5 & 5 & $\mathrm{U}$ & 5 & & & & 5 & $\mathrm{U}$ & 5 \\
\hline trans-1,3-Dichloropropene & 5 & $\mathrm{U}$ & 5 & 5 & $U$ & 5 & 5.1 & $\mathrm{U}$ & 5.1 & 5 & $\mathrm{U}$ & 5 \\
\hline Trichloroethene & 5 & $\mathrm{U}$ & 5 & 5 & U & 5 & 5.1 & $\mathrm{U}$ & 5.1 & 5 & $\mathrm{U}$ & 5 \\
\hline \begin{tabular}{|l} 
Vinyl chloride \\
\end{tabular} & 10 & $\mathrm{U}$ & 10 & 10 & U & 10 & 5.1 & $\mathrm{U}$ & 5.1 & 10 & $\mathrm{U}$ & 10 \\
\hline Xylenes (total) & 5 & $\mathrm{U}$ & 5 & 5 & $\mathrm{U}$ & 5 & 10 & $\mathrm{U}$ & 10 & 5 & U & 5 \\
\hline & & & & Attachm & & & 1 & & & heet No. & & f 113 \\
\hline & & & & Origina & & J.M. C & pron & & & Date & & $07 / 07$ \\
\hline & & & & Checke & & T.M. B & akley & & & Date & & \\
\hline & & & & Calc. N & & 0100B- & $A-V 029$ & & & Rev. No. & & 0 \\
\hline
\end{tabular}


Attachment 1. 118-B-1 Verification Sampling Results.

\begin{tabular}{|c|c|c|c|c|c|c|c|c|c|c|c|c|}
\hline \multirow[t]{2}{*}{ Constituents } & \multicolumn{3}{|c|}{$\begin{array}{c}\text { J12X61 } \\
\text { Area } 4 \text { A1 } \\
\text { Sample Date } 7 / 25 / 06\end{array}$} & \multicolumn{3}{|c|}{$\begin{array}{c}\mathrm{J} 12 \mathrm{X} 65 \\
\text { Duplicate of } \mathrm{J12X61} \\
\text { Sample Date } 7 / 25 / 06 \\
\end{array}$} & \multicolumn{3}{|c|}{$\begin{array}{c}\mathrm{J12X66} \\
\text { Split of } \mathrm{J} 12 \times 61 \\
\text { Sample Date } 7 / 25 / 06 \\
\end{array}$} & \multicolumn{3}{|c|}{$\begin{array}{c}\text { J12X62 } \\
\text { Area } 4 \text { A2 } \\
\text { Sample Date 7/25/06 }\end{array}$} \\
\hline & $\mu \mathrm{g} / \mathrm{kg}$ & Q & PQL & $\mu \mathrm{g} / \mathrm{kg}$ & $\mathbf{Q}$ & PQL & $\mu \mathrm{g} / \mathrm{kg}$ & Q & $\mathbf{P Q L}$ & $\mu \mathrm{g} / \mathrm{kg}$ & $\mathrm{Q}$ & PQL \\
\hline \multicolumn{13}{|c|}{ Polychlorinated Biphenyls } \\
\hline Aroclor-1016 & 13 & $\mathrm{U}$ & 13 & 13 & $\mathrm{U}$ & 13 & 17 & $\mathrm{U}$ & 17 & 13 & $\overline{\mathrm{U}}$ & 13 \\
\hline Aroclor-1221 & 13 & U & 13 & 13 & $\mathrm{U}$ & 13 & 17 & $\mathrm{U}$ & 17 & 13 & $\bar{U}$ & 13 \\
\hline Aroclor-1232 & 13 & $\mathrm{U}$ & 13 & 13 & $\mathrm{U}$ & 13 & 17 & $\mathrm{U}$ & 17 & 13 & $\mathrm{U}$ & 13 \\
\hline Aroclor- 1242 & 13 & U & 13 & 13 & $\mathrm{U}$ & 13 & 17 & $\mathrm{U}$ & 17 & 13 & $\mathrm{U}$ & 13 \\
\hline Aroclor- 1248 & 13 & $\mathrm{U}$ & 13 & 13 & $\mathrm{U}$ & 13 & 17 & $\mathrm{U}$ & 17 & 13 & $\mathrm{U}$ & 13 \\
\hline Aroclor -1254 & 80 & & 13 & 57 & & 13 & 17 & $\mathrm{U}$ & 17 & 13 & $\mathrm{U}$ & 13 \\
\hline Aroclor- 1260 & 13 & $\mathrm{U}$ & 13 & 13 & $\mathrm{U}$ & 13 & 17 & $\mathrm{U}$ & 17 & 13 & $\mathrm{U}$ & 13 \\
\hline \multicolumn{13}{|c|}{ Semivolatile Organic Compounds } \\
\hline 1,2,4-Trichlorobenzene & 340 & UJ & 340 & 340 & UJ & 340 & 330 & $\mathrm{U}$ & 330 & 330 & UJ & 330 \\
\hline 1,2-Dichlorobenzene & 340 & $\mathrm{U}$ & 340 & 340 & $\mathrm{U}$ & 340 & 330 & $\mathrm{U}$ & 330 & 330 & $\mathrm{U}$ & 330 \\
\hline 1,3-Dichlorobenzene & 340 & $\mathrm{U}$ & 340 & 340 & $\mathrm{U}$ & 340 & 330 & $\mathrm{U}$ & 330 & 330 & $\mathrm{U}$ & 330 \\
\hline 1,4-Dichlorobenzene & 340 & $\mathrm{U}$ & 340 & 340 & $\mathrm{U}$ & 340 & 330 & $\mathrm{U}$ & 330 & 330 & $\mathrm{U}$ & 330 \\
\hline 2,4,5-Trichlorophenol & 840 & $\mathrm{U}$ & 840 & 840 & $\mathrm{U}$ & 840 & 330 & $\mathrm{U}$ & 330 & 840 & $\mathrm{U}$ & 840 \\
\hline 2,4,6-Trichlorophenol & 340 & $\mathrm{U}$ & 340 & 340 & $\mathrm{U}$ & 340 & 330 & $\mathrm{U}$ & 330 & 330 & $\mathrm{U}$ & 330 \\
\hline 2,4-Dichlorophenol & 340 & $\mathrm{U}$ & 340 & 340 & $\mathrm{U}$ & 340 & 330 & $\mathrm{U}$ & 330 & 330 & $\mathrm{U}$ & 330 \\
\hline 2,4-Dimethylphenol & 340 & $\mathrm{U}$ & 340 & 340 & $\mathrm{U}$ & 340 & 330 & $\mathrm{U}$ & 330 & 330 & U & 330 \\
\hline 2,4-Dinitrophenol & 840 & $\mathrm{U}$ & 840 & 840 & $\mathrm{U}$ & 840 & 1600 & UJ & 1600 & 840 & $\mathrm{U}$ & 840 \\
\hline 2,4-Dinitrotoluene & 340 & $\mathrm{U}$ & 340 & 340 & $\mathrm{U}$ & 340 & 330 & $\mathrm{U}$ & 330 & 330 & $\mathrm{U}$ & 330 \\
\hline 2,6-Dinitrotoluene & 340 & $\mathrm{U}$ & 340 & 340 & $\mathrm{U}$ & 340 & 330 & $\mathrm{U}$ & 330 & 330 & $\mathrm{U}$ & 330 \\
\hline 2-Chloronaphthalene & 340 & $\mathrm{U}$ & 340 & 340 & $\mathrm{U}$ & 340 & 330 & $\mathrm{U}$ & 330 & 330 & $\mathrm{U}$ & 330 \\
\hline 2-Chlorophenol & 340 & $\mathrm{U}$ & 340 & 340 & $\mathrm{U}$ & 340 & 330 & $\mathrm{U}$ & 330 & 330 & $\mathrm{U}$ & 330 \\
\hline 2-Methylnaphthalene & 340 & $\mathrm{UJ}$ & 340 & 340 & $\mathrm{UJ}$ & 340 & 330 & $\mathrm{U}$ & 330 & 330 & UJ & 330 \\
\hline 2-Methylphenol (cresol, o-) & 340 & $\mathrm{U}$ & 340 & 340 & $\mathrm{U}$ & 340 & 330 & $\mathrm{U}$ & 330 & 330 & $\mathrm{U}$ & 330 \\
\hline 2-Nitroaniline & 840 & $\mathrm{U}$ & 840 & 840 & $\mathrm{U}$ & 840 & 1600 & $\mathrm{U}$ & 1600 & 840 & $\mathrm{U}$ & 840 \\
\hline 2-Nitrophenol & 340 & $\mathrm{U}$ & 340 & 340 & $\mathrm{U}$ & 340 & 330 & $\mathrm{U}$ & 330 & 330 & $\mathrm{U}$ & 330 \\
\hline 3,3'-Dichlorobenzidine & 340 & $\mathrm{U}$ & 340 & 340 & $\mathrm{U}$ & 340 & 1600 & $U$ & 1600 & 330 & $\mathrm{U}$ & 330 \\
\hline 3-Nitroaniline & 840 & $\mathrm{U}$ & 840 & 840 & $\mathrm{U}$ & 840 & 1600 & $\mathrm{U}$ & 1600 & 840 & $\mathrm{U}$ & 840 \\
\hline 4,6-Dinitro-2-methylphenol & 840 & $\mathrm{U}$ & 840 & 840 & $\mathrm{U}$ & 840 & 1600 & $\mathrm{U}$ & 1600 & 840 & $\mathrm{U}$ & 840 \\
\hline 4-Bromophenyl-phenylether & 340 & $\mathrm{U}$ & 340 & 340 & $\mathrm{U}$ & 340 & 330 & $\mathrm{U}$ & 330 & 330 & $\mathrm{U}$ & 330 \\
\hline 4-Chloro-3-methylphenol & 340 & $\mathrm{U}$ & 340 & 340 & $\mathrm{U}$ & 340 & 330 & $\mathrm{U}$ & 330 & 330 & $\mathrm{U}$ & 330 \\
\hline 4-Chloroaniline & 340 & $\mathrm{U}$ & 340 & 340 & $\mathrm{U}$ & 340 & 330 & $\mathrm{U}$ & 330 & 330 & $\mathrm{U}$ & 330 \\
\hline 4-Chlorophenyl-phenylether & 340 & $\mathrm{U}$ & 340 & 340 & $\mathrm{U}$ & 340 & 330 & $\mathrm{U}$ & 330 & 330 & $\mathrm{U}$ & 330 \\
\hline 4-Methylphenol (p-cresol) & 340 & $\mathrm{U}$ & 340 & 340 & $\mathrm{U}$ & 340 & 670 & $\mathrm{U}$ & 670 & 330 & $\mathrm{U}$ & 330 \\
\hline 4-Nitroaniline & 840 & $\mathrm{U}$ & 840 & 840 & $\mathrm{U}$ & 840 & 1600 & $\mathrm{U}$ & 1600 & 840 & $\mathrm{U}$ & 840 \\
\hline 4-Nitrophenol & 840 & $\mathrm{U}$ & 840 & 840 & $\mathrm{U}$ & 840 & 1600 & $\mathrm{U}$ & 1600 & 840 & $\mathrm{U}$ & 840 \\
\hline Acenaphthene & 340 & $\mathrm{U}$ & 340 & 340 & $\mathrm{U}$ & 340 & 330 & $\mathrm{U}$ & 330 & 330 & U & 330 \\
\hline Acenaphthylene & 340 & $\mathrm{U}$ & 340 & 340 & $\mathrm{U}$ & 340 & 330 & U & 330 & 330 & $\mathrm{U}$ & 330 \\
\hline Anthracene & 340 & U & 340 & 340 & U & 340 & 330 & U & 330 & 330 & $\mathrm{U}$ & 330 \\
\hline Benzo(a)anthracene & 340 & $\mathrm{U}$ & 340 & 340 & $\mathrm{U}$ & 340 & 330 & $\mathrm{U}$ & 330 & 330 & $\mathrm{U}$ & 330 \\
\hline Benzo(a)pyrene & 340 & $\mathrm{U}$ & 340 & 340 & $\mathrm{U}$ & 340 & 330 & $\mathrm{U}$ & 330 & 330 & $\mathrm{U}$ & 330 \\
\hline Benzo(b)fluoranthene & 340 & $\mathrm{U}$ & 340 & 340 & $\mathrm{U}$ & 340 & 330 & U & 330 & 330 & U & 330 \\
\hline Benzo(g,h,i)perylene & 340 & $\mathrm{U}$ & 340 & 340 & $\mathrm{U}$ & 340 & 330 & $\mathrm{U}$ & 330 & 330 & $\mathrm{U}$ & 330 \\
\hline Benzo(k)fluoranthene & 340 & $\mathrm{U}$ & 340 & 340 & U & 340 & 330 & U & 330 & 330 & $\mathrm{U}$ & 330 \\
\hline bis(2-Chloro-1-methylethyl)ether & 340 & $\mathrm{U}$ & 340 & 340 & $\mathrm{U}$ & 340 & 330 & $\mathrm{U}$ & 330 & 330 & $\mathrm{U}$ & 330 \\
\hline bis(2-Chloroethoxy)methane & 340 & $\mathrm{U}$ & 340 & 340 & $\mathrm{U}$ & 340 & 330 & $\mathrm{U}$ & 330 & 330 & $\mathrm{U}$ & 330 \\
\hline bis(2-Chloroethyl)ether & 340 & $\mathrm{U}$ & 340 & 340 & $\mathrm{U}$ & 340 & 330 & $\mathrm{U}$ & 330 & 330 & $\mathrm{U}$ & 330 \\
\hline bis(2-Ethylhexyl)phthalate & 46 & JB & 340 & 35 & $\mathrm{JB}$ & 340 & 330 & $\mathrm{U}$ & 330 & 29 & $\mathrm{JB}$ & 330 \\
\hline & & & & Attachn & & & 1 & & & et No. & & of 113 \\
\hline & & & & Origina & & J.M.C & ron & & & Date & & $04 / 07$ \\
\hline & & & & Checke & & T.M. B & ikley & & & Date & & \\
\hline & & & & Calc. $N$ & & $0100 \mathrm{~B}$ & $\mathrm{~A}-\mathrm{V} 02$ & & & Rev. No. & & 1 \\
\hline
\end{tabular}


Rev. 0

Attachment 1. 118-B-1 Verification Sampling Results.

\begin{tabular}{|c|c|c|c|c|c|c|c|c|c|c|c|c|}
\hline \multirow[t]{2}{*}{ Constituents } & \multicolumn{3}{|c|}{$\begin{array}{c}\text { J12X61 } \\
\text { Area } 4 \mathrm{A1} \\
\text { Sample Date } 7 / 25 / 06\end{array}$} & \multicolumn{3}{|c|}{$\begin{array}{c}\text { J12X65 } \\
\text { Duplicate of J12X61 } \\
\text { Sample Date } 7 / 25 / 06\end{array}$} & \multicolumn{3}{|c|}{$\begin{array}{c}\text { J12X66 } \\
\text { Split of J12X61 } \\
\text { Sample Date } 7 / 25 / 06\end{array}$} & \multicolumn{3}{|c|}{$\begin{array}{c}\text { J12X62 } \\
\text { Area } 4 \mathrm{A2} \\
\text { Sample Date 7/25/06 }\end{array}$} \\
\hline & $\mu \mathrm{g} / \mathrm{kg}$ & $Q$ & $\mathrm{PQL}$ & $\mu \mathrm{g} / \mathrm{kg}$ & $Q$ & PQL & $\mu \mathrm{g} / \mathrm{kg}$ & $Q$ & MDL & $\mu \mathrm{g} / \mathrm{kg}$ & $Q$ & $\mathbf{P Q L}$ \\
\hline \multicolumn{13}{|c|}{ Semivolatile Organic Compounds (continued) } \\
\hline Butylbenzylphthalate & 340 & $\mathrm{U}$ & 340 & 340 & $\mathrm{U}$ & 340 & 330 & $U$ & 330 & 330 & $\bar{U}$ & 330 \\
\hline Carbazole & 340 & $\mathrm{U}$ & 340 & 340 & $U$ & 340 & 330 & $\mathrm{U}$ & 330 & 330 & $\mathrm{U}$ & 330 \\
\hline Chrysene & 340 & $\mathrm{U}$ & 340 & 340 & $\mathrm{U}$ & 340 & 330 & $\mathrm{U}$ & 330 & 330 & $\mathrm{U}$ & 330 \\
\hline Di-n-butylphthalate & 38 & $\mathrm{JB}$ & 340 & 63 & $\mathrm{JB}$ & 340 & 330 & $\mathrm{U}$ & 330 & 63 & $\mathrm{JB}$ & 330 \\
\hline Di-n-octylphthalate & 340 & $\mathrm{U}$ & 340 & 340 & $\mathrm{U}$ & 340 & 330 & $\mathrm{U}$ & 330 & 330 & $\mathrm{U}$ & 330 \\
\hline Dibenz $(\mathrm{a}, \mathrm{h})$ anthracene & 340 & $\mathrm{U}$ & 340 & 340 & $\mathrm{U}$ & 340 & 330 & $\mathrm{U}$ & 330 & 330 & $\mathrm{U}$ & 330 \\
\hline Dibenzofuran & 340 & $\mathrm{U}$ & 340 & 340 & $\mathrm{U}$ & 340 & 330 & $\mathrm{U}$ & 330 & 330 & $\mathrm{U}$ & 330 \\
\hline Diethylphthalate & 340 & $\mathrm{U}$ & 340 & 340 & $\mathrm{U}$ & 340 & 330 & $\mathrm{U}$ & 330 & 17 & $\mathrm{~J}$ & 330 \\
\hline Dimethylphthalate & 340 & $\mathrm{U}$ & 340 & 340 & $\mathrm{U}$ & 340 & 330 & $\mathrm{U}$ & 330 & 330 & $\mathrm{U}$ & 330 \\
\hline Fluoranthene & 340 & $\mathrm{U}$ & 340 & 340 & $\mathrm{U}$ & 340 & 330 & $\mathrm{U}$ & 330 & 330 & $\mathrm{U}$ & 330 \\
\hline Fluorene & 340 & $\mathrm{U}$ & 340 & 340 & $\mathrm{U}$ & 340 & 330 & $\mathrm{U}$ & 330 & 330 & $\mathrm{U}$ & 330 \\
\hline Hexachlorobenzene & 340 & $\mathrm{U}$ & 340 & 340 & $\mathrm{U}$ & 340 & 330 & $\mathrm{U}$ & 330 & 330 & $\mathrm{U}$ & 330 \\
\hline Hexachlorobutadiene & 340 & U & 340 & 340 & $\mathrm{U}$ & 340 & 330 & $\mathrm{U}$ & 330 & 330 & $\mathrm{U}$ & 330 \\
\hline Hexachlorocyclopentadiene & 340 & $\mathrm{U}$ & 340 & 340 & $\mathrm{U}$ & 340 & 1600 & $U$ & 1600 & 330 & $\mathrm{U}$ & 330 \\
\hline Hexachloroethane & 340 & $\mathrm{U}$ & 340 & 340 & $\mathrm{U}$ & 340 & 330 & $\mathrm{U}$ & 330 & 330 & $\mathrm{U}$ & 330 \\
\hline Indeno(1,2,3-cd)pyrene & 340 & $U$ & 340 & 340 & $\mathrm{U}$ & 340 & 330 & $\mathrm{U}$ & 330 & 330 & $\mathrm{U}$ & 330 \\
\hline Isophorone & 340 & $\mathrm{U}$ & 340 & 340 & $\mathrm{U}$ & 340 & 330 & $\mathrm{U}$ & 330 & 330 & $\mathrm{U}$ & 330 \\
\hline N-Nitroso-di-n-dipropylamine & 340 & $\mathrm{U}$ & 340 & 340 & $\mathrm{U}$ & 340 & 330 & $\mathrm{U}$ & 330 & 330 & $\mathrm{U}$ & 330 \\
\hline $\mathrm{N}$-Nitrosodiphenylamine & 340 & $\mathrm{U}$ & 340 & 340 & $\mathrm{U}$ & 340 & 330 & $\mathrm{U}$ & 330 & 330 & $\mathrm{U}$ & 330 \\
\hline Naphthalene & 340 & $\mathrm{U}$ & 340 & 340 & $\mathrm{U}$ & 340 & 330 & $\mathrm{U}$ & 330 & 330 & $\mathrm{U}$ & 330 \\
\hline Nitrobenzene & 340 & $\mathrm{U}$ & 340 & 340 & $\mathrm{U}$ & 340 & 330 & $\mathrm{U}$ & 330 & 330 & $\mathrm{U}$ & 330 \\
\hline Pentachlorophenol & 840 & $\mathrm{U}$ & 840 & 840 & $\mathrm{U}$ & 840 & 1600 & $\mathrm{U}$ & 1600 & 840 & $\mathrm{U}$ & 840 \\
\hline Phenanthrene & 340 & $\mathrm{U}$ & 340 & 340 & $\mathrm{U}$ & 340 & 330 & $\mathrm{U}$ & 330 & 330 & $\mathrm{U}$ & 330 \\
\hline Phenol & 340 & $\mathrm{U}$ & 340 & 340 & $U$ & 340 & 330 & $\mathrm{U}$ & 330 & 26 & $\mathrm{JB}$ & 330 \\
\hline Pyrene & 340 & $\mathrm{U}$ & 340 & 340 & U & 340 & 330 & $\mathrm{U}$ & 330 & 330 & $\mathrm{U}$ & 330 \\
\hline & & & & Attachn & & & 1 & & & eet No. & 42 & of 113 \\
\hline & & & & Origina & & J.M.C & ron & & & Date & & $07 / 07$ \\
\hline & & & & $\begin{array}{l}\text { Checke } \\
\text { Calc. N }\end{array}$ & & T. M. B & kley & & & Date & & \\
\hline & & & & Calc. $N$ & & $0100 \mathrm{~B}-$ & $A-V 0299$ & & & Rev. No & & 0 \\
\hline
\end{tabular}


Attachment 1. 118-B-1 Verification Sampling Results.

\begin{tabular}{|c|c|c|c|c|c|c|c|c|c|c|c|c|}
\hline \multirow[t]{2}{*}{ Constituents } & \multicolumn{3}{|c|}{$\begin{array}{c}\text { J12X61 } \\
\text { Area 4A1 } \\
\text { Sample Date } 7 / 25 / 06\end{array}$} & \multicolumn{3}{|c|}{$\begin{array}{c}J 12 X 65 \\
\text { Duplicate of J12X61 } \\
\text { Sample Date } 7 / 25 / 06\end{array}$} & \multicolumn{3}{|c|}{$\begin{array}{c}\text { J12X66 } \\
\text { Split of J12X61 } \\
\text { Sample Date } 7 / 25 / 06 \\
\end{array}$} & \multicolumn{3}{|c|}{$\begin{array}{c}\text { J12X62 } \\
\text { Area 4 A2 } \\
\text { Sample Date } 7 / 25 / 06\end{array}$} \\
\hline & $\mu \mathrm{g} / \mathrm{kg}$ & $\mathbf{Q}$ & $\mathrm{PQL}$ & $\mu \mathrm{g} / \mathrm{kg}$ & $Q$ & $\mathrm{PQL}$ & $\mu \mathrm{g} / \mathrm{kg}$ & $Q$ & MDL & $\mu \mathrm{g} / \mathrm{kg}$ & $\mathbf{Q}$ & $\mathrm{PQL}$ \\
\hline \multicolumn{13}{|c|}{ Volatile Organic Compounds } \\
\hline 1,1,1-Trichloroethane & 5 & $\mathrm{U}$ & 5 & 5 & $\mathrm{U}$ & 5 & 1.2 & $\mathrm{~J}$ & 5.0 & 5 & $\mathrm{U}$ & 5 \\
\hline 1,1,2,2-Tetrachloroethane & 5 & $\mathrm{U}$ & 5 & 5 & $\mathrm{U}$ & 5 & 5.0 & $\mathrm{U}$ & 5.0 & 5 & $\mathrm{U}$ & 5 \\
\hline 1,1,2-Trichloroethane & 5 & $\mathrm{U}$ & 5 & 5 & $\mathrm{U}$ & 5 & 5.0 & $\mathrm{U}$ & 5.0 & 5 & $\mathrm{U}$ & 5 \\
\hline 1,1-Dichloroethane & 5 & $\mathrm{U}$ & 5 & 5 & $\mathrm{U}$ & 5 & 5.0 & $\mathrm{U}$ & 5.0 & 5 & $\mathrm{U}$ & 5 \\
\hline 1,1-Dichloroethene & 5 & $\mathrm{U}$ & 5 & 5 & $\mathrm{U}$ & 5 & 5.0 & $\mathrm{U}$ & 5.0 & 5 & $\mathrm{U}$ & 5 \\
\hline 1,2-Dichloroethane & 5 & $\bar{U}$ & 5 & 5 & $\mathrm{U}$ & 5 & 5.0 & $\mathrm{U}$ & 5.0 & 5 & $\mathrm{U}$ & 5 \\
\hline 1,2-Dichloroethene (total) & 5 & $\mathrm{U}$ & 5 & 5 & $\mathrm{U}$ & 5 & 10 & $\mathrm{U}$ & 10 & 5 & $\mathrm{U}$ & 5 \\
\hline 1,2-Dichloropropane & 5 & $\mathrm{U}$ & 5 & 5 & $\mathrm{U}$ & 5 & 5.0 & $\mathrm{U}$ & 5.0 & 5 & $\mathrm{U}$ & 5 \\
\hline 2-Butanone & 10 & $\mathrm{U}$ & 10 & 10 & $\mathrm{U}$ & 10 & 10 & $\mathrm{U}$ & 10 & 10 & $\mathrm{U}$ & 10 \\
\hline 2-Hexanone & 10 & $\mathrm{U}$ & 10 & 10 & $\mathrm{U}$ & 10 & 20 & $\mathrm{U}$ & 20 & 10 & U & 10 \\
\hline 4-Methyl-2-pentanone & 10 & $\mathrm{U}$ & 10 & 10 & $\mathrm{U}$ & 10 & 10 & $\mathrm{U}$ & 10 & 10 & $\mathrm{U}$ & 10 \\
\hline Acetone & 19 & & 10 & 10 & $\mathrm{U}$ & 10 & 20 & $\mathrm{U}$ & 20 & 11 & & 10 \\
\hline Benzene & 5 & $\mathrm{U}$ & 5 & 5 & $\mathrm{U}$ & 5 & 5.0 & $\mathrm{U}$ & 5.0 & 5 & $U$ & 5 \\
\hline Bromodichloromethane & 5 & $\mathrm{U}$ & 5 & 5 & $\mathrm{U}$ & 5 & 5.0 & $\mathrm{U}$ & 5.0 & 5 & U & 5 \\
\hline Bromoform & 5 & $\mathrm{U}$ & 5 & 5 & $\mathrm{U}$ & 5 & 5.0 & $\mathrm{U}$ & 5.0 & 5 & $U$ & 5 \\
\hline Bromomethane & 10 & $\mathrm{U}$ & 10 & 10 & $U$ & 10 & 10 & $\mathrm{U}$ & 10 & 10 & $\mathrm{U}$ & 10 \\
\hline Carbon disulfide & 5 & $\mathrm{U}$ & 5 & 5 & $\mathrm{U}$ & 5 & 5.0 & $\mathrm{U}$ & 5.0 & 5 & $\mathrm{U}$ & 5 \\
\hline Carbon tetrachloride & 13 & & 5 & 5 & $\mathrm{~J}$ & 5 & 4.4 & $\mathrm{~J}$ & 5.0 & 14 & & 5 \\
\hline Chlorobenzene & 5 & U & 5 & 5 & $\mathrm{U}$ & 5 & 5.0 & $\mathrm{U}$ & 5.0 & 5 & $\mathrm{U}$ & 5 \\
\hline Chloroethane & 10 & $\mathrm{U}$ & 10 & 10 & $\mathrm{U}$ & 10 & 10 & $\mathrm{U}$ & 10 & 10 & $\mathrm{U}$ & 10 \\
\hline Chloroform & 5 & $\mathrm{U}$ & 5 & 5 & $\mathrm{U}$ & 5 & 5.0 & $\mathrm{U}$ & 5.0 & 5 & U & 5 \\
\hline Chloromethane & 10 & $U$ & 10 & 10 & $\mathrm{U}$ & 10 & 10 & $\mathrm{U}$ & 10 & 10 & $\mathrm{U}$ & 10 \\
\hline cis-1,2-Dichloroethylene & 5 & $\mathrm{U}$ & 5 & 5 & $\mathrm{U}$ & 5 & & & & 5 & $\mathrm{U}$ & 5 \\
\hline cis-1,3-Dichloropropene & 5 & $\mathrm{U}$ & 5 & 5 & $\mathrm{U}$ & 5 & 5.0 & $\mathrm{U}$ & 5.0 & 5 & $\mathrm{U}$ & 5 \\
\hline Dibromochloromethane & 5 & $\mathrm{U}$ & 5 & 5 & $\mathrm{U}$ & 5 & 5.0 & $\mathrm{U}$ & 5.0 & 5 & $\mathrm{U}$ & 5 \\
\hline Ethylbenzene & 5 & $\mathrm{U}$ & 5 & 5 & $\mathrm{U}$ & 5 & 5.0 & $\mathrm{U}$ & 5.0 & 5 & $\mathrm{U}$ & 5 \\
\hline Methylene chloride & 5 & & 5 & 6 & & 5 & 5.0 & $\mathrm{U}$ & 5.0 & 8 & & 5 \\
\hline Styrene & 5 & $\mathrm{U}$ & 5 & 5 & $\mathrm{U}$ & 5 & 5.0 & $\mathrm{U}$ & 5.0 & 5 & $\mathrm{U}$ & 5 \\
\hline Tetrachloroethene & 5 & $\mathrm{U}$ & 5 & 5 & $\mathrm{U}$ & 5 & 5.0 & UN & 5.0 & 5 & $\mathrm{U}$ & 5 \\
\hline \begin{tabular}{|l|} 
Toluene \\
\end{tabular} & 5 & $\mathrm{U}$ & 5 & 5 & $\mathrm{U}$ & 5 & 5.0 & UN & 5.0 & 5 & $\mathrm{U}$ & 5 \\
\hline trans-1,2-Dichloroethylene & 5 & $\mathrm{U}$ & 5 & 5 & $\mathrm{U}$ & 5 & & & & 5 & $U$ & 5 \\
\hline trans-1,3-Dichloropropene & 5 & $\mathrm{U}$ & 5 & 5 & $\mathrm{U}$ & 5 & 5.0 & $\mathrm{U}$ & 5.0 & 5 & $\mathrm{U}$ & 5 \\
\hline Trichloroethene & 5 & $U$ & 5 & 5 & $\mathrm{U}$ & 5 & 2.4 & $\mathrm{~J}$ & 5.0 & 5 & $\mathrm{U}$ & 5 \\
\hline Vinyl chloride & 10 & $\mathrm{U}$ & 10 & 10 & $U$ & 10 & 5.0 & $\mathrm{U}$ & 5.0 & 10 & $\mathrm{U}$ & 10 \\
\hline Xylenes (total) & 5 & $\mathrm{U}$ & 5 & 5 & $\mathrm{U}$ & 5 & 10 & $\mathrm{U}$ & 10 & 5 & $\mathrm{U}$ & 5 \\
\hline & & & & Attachm & & & 1 & & & Sheet No. & & of 113 \\
\hline & & & & Originat & & J.M.C & pron & & & Date & & $07 / 07$ \\
\hline & & & & Checkec & & T.M.B & akley & & & Date & & \\
\hline & & & & Calc. $\mathrm{N}$ & & $0100 \mathrm{~B}$ & $\mathrm{~A}-\mathrm{V} 029$ & & & Rev, No. & & 0 \\
\hline
\end{tabular}


Attachment 1. 118-B-1 Verification Sampling Results.

\begin{tabular}{|c|c|c|c|c|c|c|c|c|c|c|c|c|}
\hline \multirow[t]{2}{*}{ Constituents } & \multicolumn{3}{|c|}{$\begin{array}{c}\text { J12X61 } \\
\text { Area 4 A1 } \\
\text { Sample Date } 7 / 25 / 06\end{array}$} & \multicolumn{3}{|c|}{$\begin{array}{c}\mathrm{J12X65} \\
\text { Duplicate of } \mathrm{J12X61} \\
\text { Sample Date } 7 / 25 / 06 \\
\end{array}$} & \multicolumn{3}{|c|}{$\begin{array}{c}\text { J12X66 } \\
\text { Split of } \mathrm{J} 12 \mathrm{X} 61 \\
\text { Sample Date } 7 / 25 / 06 \\
\end{array}$} & \multicolumn{3}{|c|}{$\begin{array}{c}\text { J12X62 } \\
\text { Area } 4 \text { A2 } \\
\text { Sample Date } 7 / 25 / 06\end{array}$} \\
\hline & $\mu \mathrm{g} / \mathrm{kg}$ & $\bar{Q}$ & PQL & $\mu \mathrm{g} / \mathrm{kg}$ & $\mathbf{Q}$ & $\mathbf{P Q L}$ & $\mu \mathrm{g} / \mathrm{kg}$ & $\mathbf{Q}$ & MDL & $\mu \mathrm{g} / \mathrm{kg}$ & $\bar{Q}$ & PQL \\
\hline \multicolumn{13}{|c|}{ Pesticides } \\
\hline Aldrin & 1.3 & UD & 1.3 & 1.3 & UD & 1.3 & 1.7 & $\mathrm{U}$ & 1.7 & 1.3 & UD & 1.3 \\
\hline alpha-BHC & 1.3 & UD & 1.3 & 1.3 & UD & 1.3 & 1.7 & U & 1.7 & 1.3 & UD & 1.3 \\
\hline alpha-Chlordane & 1.3 & UD & 1.3 & 1.3 & UD & 1.3 & 1.7 & $\mathrm{U}$ & 1.7 & 1.3 & UD & 1.3 \\
\hline beta-BHC & 1.3 & UD & 1.3 & 1.3 & UD & 1.3 & 1.7 & $\mathrm{U}$ & 1.7 & 7.8 & $\mathrm{JD}$ & 1.3 \\
\hline delta-BHC & 1.3 & UD & 1.3 & 1.3 & UD & 1.3 & 1.7 & $\bar{U}$ & 1.7 & 1.3 & UD & 1.3 \\
\hline Dichlorodiphenyldichloroethane & 1.3 & UD & 1.3 & 1.3 & UD & 1.3 & 1.7 & $\mathrm{U}$ & 1.7 & 1.3 & UD & 1.3 \\
\hline Dichlorodiphenyldichloroethylene & 16 & $\mathrm{D}$ & 1.3 & 1.3 & UD & 1.3 & 1.7 & $\mathrm{U}$ & 1.7 & 1.3 & UD & 1.3 \\
\hline Dichlorodiphenyltrichloroethane & 1.3 & UD & 1.3 & 1.3 & UD & 1.3 & 1.7 & $\mathrm{U}$ & 1.7 & 1.3 & UD & 1.3 \\
\hline Dieldrin & 3.8 & DI & 1.3 & 0.74 & $\mathrm{JD}$ & 1.3 & 1.7 & $\bar{U}$ & 1.7 & 1.3 & UD & 1.3 \\
\hline Endosulfan I & 1.3 & UD & 1.3 & 1.3 & UD & 1.3 & 1.7 & $\mathrm{U}$ & 1.7 & 1.3 & UD & 1.3 \\
\hline Endosulfan II & 1.3 & UD & 1.3 & 1.3 & UD & 1.3 & 1.7 & $\mathrm{U}$ & 1.7 & 1.3 & UD & 1.3 \\
\hline Endosulfan sulfate & 1.3 & UD & 1.3 & 2.2 & $\mathrm{JD}$ & 1.3 & 1.7 & $\mathrm{U}$ & 1.7 & 1.3 & UD & 1.3 \\
\hline Endrin & 1.3 & UD & 1.3 & 1.3 & UD & 1.3 & 1.7 & $\bar{U}$ & 1.7 & 1.5 & JDI & 1.3 \\
\hline Endrin aldehyde & 5.5 & DI & 1.3 & 1.3 & UD & 1.3 & 1.7 & $\mathrm{U}$ & 1.7 & 1.3 & UD & 1.3 \\
\hline Endrin ketone & 1.3 & UD & 1.3 & 1.3 & UD & 1.3 & 1.7 & $\mathrm{U}$ & 1.7 & 1.3 & UD & 1.3 \\
\hline gamma-BHC (Lindane) & 1.3 & UD & 1.3 & 1.3 & UD & 1.3 & 1.7 & $\mathrm{U}$ & 1.7 & 1.3 & UD & 1.3 \\
\hline gamma-Chlordane & 1.3 & UD & 1.3 & 2.5 & JD & 1.3 & 1.7 & $\mathrm{U}$ & 1.7 & 1.3 & UD & 1.3 \\
\hline Heptachlor & 1.3 & UD & 1.3 & 1.3 & UD & 1.3 & 1.7 & $\mathrm{U}$ & 1.7 & 1.3 & UD & 1.3 \\
\hline Heptachlor epoxide & 1.3 & UD & 1.3 & 1.3 & UD & 1.3 & 1.7 & $\mathrm{U}$ & 1.7 & 1.3 & UD & 1.3 \\
\hline Methoxychlor & 1.3 & UD & 1.3 & 1.3 & UD & 1.3 & 3.3 & $\bar{U}$ & 3.3 & 1.3 & UD & 1.3 \\
\hline Toxaphene & 13 & UDJ & 13 & 13 & UDJ & 13 & 68 & $\mathrm{U}$ & 68 & 13 & UDJ & 13 \\
\hline \multicolumn{13}{|c|}{ Herbicides } \\
\hline $2,4,5-\mathrm{T}$ & 17 & $\bar{U}$ & 17 & 17 & $\mathrm{U}$ & 17 & 20 & $\mathrm{U}$ & 20 & 17 & $\mathrm{U}$ & 17 \\
\hline $2,4-\mathrm{D}$ & 34 & $\mathrm{U}$ & 34 & 34 & $\mathrm{U}$ & 34 & 81 & $\mathrm{U}$ & 81 & 13 & $\mathrm{~J}$ & 34 \\
\hline $2,4,5-\mathrm{TP}$ & 17 & $\mathrm{U}$ & 17 & 17 & $\mathrm{U}$ & 17 & 20 & $\mathrm{U}$ & 20 & 17 & $\mathrm{U}$ & 17 \\
\hline MCPP & & & & & & & 8100 & $\mathrm{U}$ & 8100 & & & \\
\hline $\mathrm{MCPA}$ & & & & & & & 8100 & $\mathrm{U}$ & 8100 & & & \\
\hline Dinoseb & 17 & $\mathrm{U}$ & 17 & 17 & $\mathrm{U}$ & 17 & 25 & $\mathrm{UN}$ & 25 & 17 & $\mathrm{U}$ & 17 \\
\hline $2,4-\mathrm{DB}$ & 17 & $\bar{U}$ & 17 & 9.0 & $\mathrm{~J}$ & 17 & 81 & $\mathrm{U}$ & 81 & 11 & $\mathrm{JI}$ & 17 \\
\hline Dalapon & 29 & $\mathrm{~J}$ & 34 & 48 & & 34 & 40 & $\mathrm{U}$ & 40 & 32 & $\mathrm{~J}$ & 33 \\
\hline Dicamba & 34 & $\mathrm{U}$ & 34 & 34 & $\mathrm{U}$ & 34 & 40 & $\mathrm{U}$ & 40 & 33 & $\mathrm{U}$ & 33 \\
\hline Dichloroprop & 34 & $\mathrm{U}$ & 34 & 34 & $\mathrm{U}$ & 34 & 81 & $\mathrm{U}$ & 81 & 33 & $\mathrm{U}$ & 33 \\
\hline Pentachlorophenol & 14 & $\mathrm{~J}$ & 17 & 17 & $\mathrm{U}$ & 17 & & & & 17 & $\mathrm{U}$ & 17 \\
\hline Picloram & 17 & UJ & 17 & 17 & UJ & 17 & & & & 17 & UJ & 17 \\
\hline
\end{tabular}

Attachment

Originator

Checked

Calc. No.

\begin{tabular}{l}
\hline J.M. Capron \\
\hline T. M. Blakley \\
\hline $0100 \mathrm{~B}-\mathrm{CA}-\mathrm{V} 0299$ \\
\hline
\end{tabular}

Sheet No. $\frac{44 \text { of } 113}{10 / 04 / 07}$
Date
Date
Rev. No. 
Attachment 1. 118-B-1 Verification Sampling Results.

\begin{tabular}{|c|c|c|c|c|c|c|c|c|c|c|c|c|}
\hline \multirow[t]{2}{*}{ Constituents } & \multicolumn{3}{|c|}{$\begin{array}{c}\text { J12X63 } \\
\text { Area } 4 \text { A3 } \\
\text { Sample Date 7/25/06 } \\
\end{array}$} & \multicolumn{3}{|c|}{$\begin{array}{c}\text { J12X64 } \\
\text { Area } 4 \text { A4 } \\
\text { Sample Date } 7 / 25 / 06 \\
\end{array}$} & \multicolumn{3}{|c|}{$\begin{array}{c}\text { J13V12 } \\
\text { Area } 5 \text { A1 } \\
\text { Sample Date 12/7/06 } \\
\end{array}$} & \multicolumn{3}{|c|}{$\begin{array}{c}\text { J13V10 } \\
\text { Area } 5 \text { A2 } \\
\text { Sample Date 12/7/06 } \\
\end{array}$} \\
\hline & $\mu \mathrm{g} / \mathrm{kg}$ & $\mathbf{Q}$ & PQL & $\mu \mathrm{g} / \mathrm{kg}$ & $\mathbf{Q}$ & $\overline{P Q L}$ & $\mu \mathrm{g} / \mathrm{kg}$ & $\mathrm{Q}$ & PQL & $\mu \mathrm{g} / \mathrm{kg}$ & $Q$ & $\mathrm{PQL}$ \\
\hline \multicolumn{13}{|c|}{ Polychlorinated Biphenyls } \\
\hline Aroclor-1016 & 13 & $\mathrm{U}$ & 13 & 13 & $\mathrm{U}$ & 13 & 14 & UJ & 14 & 14 & $\mathrm{UJ}$ & 14 \\
\hline Aroclor-1221 & 13 & $\mathrm{U}$ & 13 & 13 & $\mathrm{U}$ & 13 & 14 & $\mathrm{U}$ & 14 & 14 & $\mathrm{U}$ & 14 \\
\hline Aroclor-1232 & 13 & $\mathrm{U}$ & 13 & 13 & U & 13 & 14 & $\mathrm{U}$ & 14 & 14 & \begin{tabular}{|l|l}
$\mathrm{U}$ \\
\end{tabular} & 14 \\
\hline Aroclor-1242 & 13 & $\mathrm{U}$ & 13 & 13 & $\mathrm{U}$ & 13 & 14 & $\mathrm{U}$ & 14 & 14 & $\mathrm{U}$ & 14 \\
\hline Aroclor -1248 & 13 & $\mathrm{U}$ & 13 & 13 & $\mathrm{U}$ & 13 & 14 & $\mathrm{U}$ & 14 & 14 & $\mathrm{U}$ & 14 \\
\hline Aroclor-1254 & 13 & $\mathrm{U}$ & 13 & 13 & $\mathrm{U}$ & 13 & 14 & $\mathrm{U}$ & 14 & 14 & $\mathrm{U}$ & 14 \\
\hline Aroclor- 1260 & 13 & $\mathrm{U}$ & 13 & 13 & $\mathrm{U}$ & 13 & 14 & $\mathrm{UJ}$ & 14 & 14 & UJ & 14 \\
\hline \multicolumn{13}{|c|}{ Semivolatile Organic Compounds } \\
\hline 1,2,4-Trichlorobenzene & 340 & UJ & 340 & 340 & UJ & 340 & 350 & $\mathrm{UJ}$ & 350 & 350 & \begin{tabular}{|l|l}
$\mathrm{UJ}$ \\
\end{tabular} & 350 \\
\hline 1,2-Dichlorobenzene & 340 & $\mathrm{U}$ & 340 & 340 & $\mathrm{U}$ & 340 & 350 & $\mathrm{U}$ & 350 & 350 & $\mathrm{U}$ & 350 \\
\hline 1,3-Dichlorobenzene & 340 & $\mathrm{U}$ & 340 & 340 & $\mathrm{U}$ & 340 & 350 & $\mathrm{U}$ & 350 & 350 & $\mathrm{U}$ & 350 \\
\hline 1,4-Dichlorobenzene & 340 & $\mathrm{U}$ & 340 & 340 & $\mathrm{U}$ & 340 & 350 & $\mathrm{U}$ & 350 & 350 & $\mathrm{U}$ & 350 \\
\hline 2,4,5-Trichlorophenol & 840 & $\mathrm{U}$ & 840 & 840 & $\mathrm{U}$ & 840 & 880 & $\mathrm{U}$ & 880 & 880 & U & 880 \\
\hline 2,4,6-Trichlorophenol & 340 & $\mathrm{U}$ & 340 & 340 & $\mathrm{U}$ & 340 & 350 & $\mathrm{U}$ & 350 & 350 & U & 350 \\
\hline 2,4-Dichlorophenol & 340 & $\mathrm{U}$ & 340 & 340 & $\mathrm{U}$ & 340 & 350 & $\mathrm{UJ}$ & 350 & 350 & $\mathrm{UJ}$ & 350 \\
\hline 2,4-Dimethylphenol & 340 & $\mathrm{U}$ & 340 & 340 & $\mathrm{U}$ & 340 & 350 & UJ & 350 & 350 & UJ & 350 \\
\hline 2,4-Dinitrophenol & 840 & $\mathrm{U}$ & 840 & 840 & $\mathrm{U}$ & 840 & 880 & $\mathrm{U}$ & 880 & 880 & $\mathrm{U}$ & 880 \\
\hline 2,4-Dinitrotoluene & 340 & $\mathrm{U}$ & 340 & 340 & $\mathrm{U}$ & 340 & 350 & $\mathrm{U}$ & 350 & 350 & $\mathrm{U}$ & 350 \\
\hline 2,6-Dinitrotoluene & 340 & $\mathrm{U}$ & 340 & 340 & $\mathrm{U}$ & 340 & 350 & $\mathrm{U}$ & 350 & 350 & $\mathrm{U}$ & 350 \\
\hline 2-Chloronaphthalene & 340 & $\mathrm{U}$ & 340 & 340 & $\mathrm{U}$ & 340 & 350 & $\mathrm{U}$ & 350 & 350 & $\mathrm{U}$ & 350 \\
\hline 2-Chlorophenol & 340 & $\mathrm{U}$ & 340 & 340 & $\mathrm{U}$ & 340 & 350 & $\mathrm{U}$ & 350 & 350 & $\mathrm{U}$ & 350 \\
\hline 2-Methylnaphthalene & 340 & $\mathrm{UJ}$ & 340 & 340 & $\mathrm{UJ}$ & 340 & 350 & $\mathrm{UJ}$ & 350 & 350 & UJ & 350 \\
\hline 2-Methylphenol (cresol, o-) & 340 & $\mathrm{U}$ & 340 & 340 & $\mathrm{U}$ & 340 & 350 & $\mathrm{U}$ & 350 & 350 & $\mathrm{U}$ & 350 \\
\hline 2-Nitroaniline & 840 & $\mathrm{U}$ & 840 & 840 & $\mathrm{U}$ & 840 & 880 & $\mathrm{U}$ & 880 & 880 & $\mathrm{U}$ & 880 \\
\hline 2-Nitrophenol & 340 & $\mathrm{U}$ & 340 & 340 & $\mathrm{U}$ & 340 & 350 & UJ & 350 & 350 & $\mathrm{UJ}$ & 350 \\
\hline 3,3'-Dichlorobenzidine & 340 & $\mathrm{U}$ & 340 & 340 & $\mathrm{U}$ & 340 & 350 & $\mathrm{U}$ & 350 & 350 & $\mathrm{U}$ & 350 \\
\hline 3-Nitroaniline & 840 & $\mathrm{U}$ & 840 & 840 & $\mathrm{U}$ & 840 & 880 & $\mathrm{U}$ & 880 & 880 & $\mathrm{U}$ & 880 \\
\hline 4,6-Dinitro-2-methylphenol & 840 & $\mathrm{U}$ & 840 & 840 & $\mathrm{U}$ & 840 & 880 & $\mathrm{U}$ & 880 & 880 & $\mathrm{U}$ & 880 \\
\hline 4-Bromophenyl-phenylether & 340 & $\mathrm{U}$ & 340 & 340 & $\mathrm{U}$ & 340 & 350 & $\mathrm{U}$ & 350 & 350 & $\mathrm{U}$ & 350 \\
\hline 4-Chloro-3-methylphenol & 340 & $\mathrm{U}$ & 340 & 340 & $\mathrm{U}$ & 340 & 350 & UJ & 350 & 350 & $\mathrm{UJ}$ & 350 \\
\hline 4-Chloroaniline & 340 & $\mathrm{U}$ & 340 & 340 & $\mathrm{U}$ & 340 & 350 & $\mathrm{U}$ & 350 & 350 & $\mathrm{U}$ & 350 \\
\hline 4-Chlorophenyl-phenylether & 340 & $\mathrm{U}$ & 340 & 340 & $\mathrm{U}$ & 340 & 350 & $\mathrm{U}$ & 350 & 350 & $\mathrm{U}$ & 350 \\
\hline 4-Methylphenol (p-cresol) & 340 & $\mathrm{U}$ & 340 & 340 & $\mathrm{U}$ & 340 & 350 & $\mathrm{U}$ & 350 & 350 & $\mathrm{U}$ & 350 \\
\hline 4-Nitroaniline & 840 & $\mathrm{U}$ & 840 & 840 & $\mathrm{U}$ & 840 & 880 & UR & 880 & 880 & UR & 880 \\
\hline 4-Nitrophenol & 840 & $\mathrm{U}$ & 840 & 840 & $\mathrm{U}$ & 840 & 880 & $\mathrm{U}$ & 880 & 880 & $\mathrm{U}$ & 880 \\
\hline Acenaphthene & 340 & $\mathrm{U}$ & 340 & 340 & $\mathrm{U}$ & 340 & 350 & $\mathrm{U}$ & 350 & 350 & $\mathrm{U}$ & 350 \\
\hline Acenaphthylene & 340 & $\mathrm{U}$ & 340 & 340 & $\mathrm{U}$ & 340 & 350 & $\mathrm{U}$ & 350 & 350 & $\mathrm{U}$ & 350 \\
\hline Anthracene & 340 & $\mathrm{U}$ & 340 & 340 & $\mathrm{U}$ & 340 & 350 & $\mathrm{U}$ & 350 & 350 & $\mathrm{U}$ & 350 \\
\hline Benzo(a)anthracene & 340 & $\mathrm{U}$ & 340 & 340 & $\mathrm{U}$ & 340 & 350 & $\mathrm{U}$ & 350 & 350 & $\mathrm{U}$ & 350 \\
\hline Benzo(a)pyrene & 340 & $\mathrm{U}$ & 340 & 340 & $\mathrm{U}$ & 340 & 350 & $\mathrm{U}$ & 350 & 350 & $\mathrm{U}$ & 350 \\
\hline Benzo(b)fluoranthene & 340 & $\mathrm{U}$ & 340 & 340 & $\mathrm{U}$ & 340 & 350 & $\mathrm{U}$ & 350 & 350 & $\mathrm{U}$ & 350 \\
\hline Benzo $(\mathrm{g}, \mathrm{h}, \mathrm{i})$ perylene & 340 & $\mathrm{U}$ & 340 & 340 & $\mathrm{U}$ & 340 & 350 & $\mathrm{U}$ & 350 & 350 & U & 350 \\
\hline Benzo(k)fluoranthene & 340 & $\mathrm{U}$ & 340 & 340 & $\mathrm{U}$ & 340 & 350 & $\mathrm{U}$ & 350 & 350 & $\mathrm{U}$ & 350 \\
\hline bis(2-Chloro-1-methylethyl)ether & 340 & $\mathrm{U}$ & 340 & 340 & $\mathrm{U}$ & 340 & 350 & $\mathrm{U}$ & 350 & 350 & $\mathrm{U}$ & 350 \\
\hline bis(2-Chloroethoxy)methane & 340 & $\mathrm{U}$ & 340 & 340 & $\mathrm{U}$ & 340 & 350 & $\mathrm{U}$ & 350 & 350 & $\mathrm{U}$ & 350 \\
\hline bis(2-Chloroethyl)ether & 340 & $\mathrm{U}$ & 340 & 340 & $\mathrm{U}$ & 340 & 350 & $\mathrm{U}$ & 350 & 350 & $\mathrm{U}$ & 350 \\
\hline bis(2-Ethylhexyl)phthalate & 600 & $\mathrm{~B}$ & 340 & 65 & $\mathrm{JB}$ & 340 & 58 & JB & 350 & 350 & $\mathrm{U}$ & 350 \\
\hline
\end{tabular}

Attachment

Originator

Checked

Calc. No.

$\frac{1}{\frac{1}{\text { J. M. Capron }}} \frac{1}{\text { T. M. Blakley }}$

Sheet No. 45 of 113

Date $10 / 04 / 07$.

Date

Rev. No. 
Rev. 0

Attachment 1. 118-B-1 Verification Sampling Results.

\begin{tabular}{|c|c|c|c|c|c|c|c|c|c|c|c|c|}
\hline \multirow[t]{2}{*}{ Constituents } & \multicolumn{3}{|c|}{$\begin{array}{c}\text { J12X63 } \\
\text { Area } 4 \text { A3 } \\
\text { Sample Date } 7 / 25 / 06\end{array}$} & \multicolumn{3}{|c|}{$\begin{array}{c}\text { J12X64 } \\
\text { Area } 4 \text { A4 } \\
\text { Sample Date } 7 / 25 / 06\end{array}$} & \multicolumn{3}{|c|}{$\begin{array}{c}\text { J13V12 } \\
\text { Area } 5 \text { A1 } \\
\text { Sample Date 12/7/06 }\end{array}$} & \multicolumn{3}{|c|}{$\begin{array}{c}\text { J13V10 } \\
\text { Area } 5 \text { A2 } \\
\text { Sample Date 12/7/06 }\end{array}$} \\
\hline & $\mu \mathrm{g} / \mathrm{kg}$ & $\mathbf{Q}$ & PQL & $\mu \mathrm{g} / \mathrm{kg}$ & Q & PQL & $\mu \mathrm{g} / \mathrm{kg}$ & $\mathbf{Q}$ & MDL & $\mu \mathrm{g} / \mathrm{kg}$ & $Q$ & PQL \\
\hline \multicolumn{13}{|c|}{ Semivolatile Organic Compounds (continued) } \\
\hline Butylbenzylphthalate & 340 & $\mathrm{U}$ & 340 & 340 & $\mathrm{U}$ & 340 & 350 & $\bar{U}$ & 350 & 350 & $\mathrm{U}$ & 350 \\
\hline Carbazole & 340 & $\mathrm{U}$ & 340 & 340 & $\mathrm{U}$ & 340 & 350 & $\mathrm{U}$ & 350 & 350 & $\mathrm{U}$ & 350 \\
\hline Chrysene & 340 & $\mathrm{U}$ & 340 & 340 & $\mathrm{U}$ & 340 & 350 & $\mathrm{U}$ & 350 & 350 & $\mathrm{U}$ & 350 \\
\hline Di-n-butylphthalate & 58 & $\mathrm{JB}$ & 340 & 62 & $\mathrm{JB}$ & 340 & 350 & $\mathrm{U}$ & 350 & 350 & $\mathrm{U}$ & 350 \\
\hline Di-n-octylphthalate & 340 & $\mathrm{U}$ & 340 & 340 & $\mathrm{U}$ & 340 & 350 & $\mathrm{U}$ & 350 & 350 & $\mathrm{U}$ & 350 \\
\hline Dibenz $(\mathrm{a}, \mathrm{h})$ anthracene & 340 & $\mathrm{U}$ & 340 & 340 & $\mathrm{U}$ & 340 & 350 & $\mathrm{U}$ & 350 & 350 & $\mathrm{U}$ & 350 \\
\hline Dibenzofuran & 340 & $\mathrm{U}$ & 340 & 340 & $U$ & 340 & 350 & $\mathrm{U}$ & 350 & 350 & $\mathrm{U}$ & 350 \\
\hline Diethylphthalate & 340 & $\mathrm{U}$ & 340 & 340 & $U$ & 340 & 350 & $\bar{U}$ & 350 & 350 & $\mathrm{U}$ & 350 \\
\hline Dimethylphthalate & 340 & $\mathrm{U}$ & 340 & 340 & $\mathrm{U}$ & 340 & 350 & $\mathrm{U}$ & 350 & 350 & $\mathrm{U}$ & 350 \\
\hline Fluoranthene & 340 & $\mathrm{U}$ & 340 & 340 & $\mathrm{U}$ & 340 & 350 & $\mathrm{U}$ & 350 & 350 & $\mathrm{U}$ & 350 \\
\hline Fluorene & 340 & $\mathrm{U}$ & 340 & 340 & $\mathrm{U}$ & 340 & 350 & $\mathrm{U}$ & 350 & 350 & $\mathrm{U}$ & 350 \\
\hline Hexachlorobenzene & 340 & $\mathrm{U}$ & 340 & 340 & $\mathrm{U}$ & 340 & 350 & $\mathrm{U}$ & 350 & 350 & $\mathrm{U}$ & 350 \\
\hline Hexachlorobutadiene & 340 & $\mathrm{U}$ & 340 & 340 & $\mathrm{U}$ & 340 & 350 & $\mathrm{U}$ & 350 & 350 & $\mathrm{U}$ & 350 \\
\hline Hexachlorocyclopentadiene & 340 & $\mathrm{U}$ & 340 & 340 & $\mathrm{U}$ & 340 & 350 & $\mathrm{U}$ & 350 & 880 & $\mathrm{U}$ & 880 \\
\hline Hexachloroethane & 340 & $\mathrm{U}$ & 340 & 340 & $\mathrm{U}$ & 340 & 350 & $\mathrm{U}$ & 350 & 350 & $\mathrm{U}$ & 350 \\
\hline Indeno(1,2,3-cd)pyrene & 340 & $\mathrm{U}$ & 340 & 340 & $\mathrm{U}$ & 340 & 350 & $\mathrm{U}$ & 350 & 350 & $\mathrm{U}$ & 350 \\
\hline Isophorone & 340 & $\mathrm{U}$ & 340 & 340 & $\mathrm{U}$ & 340 & 350 & UJ & 350 & 350 & UJ & 350 \\
\hline N-Nitroso-di-n-dipropylamine & 340 & $\mathrm{U}$ & 340 & 340 & $\mathrm{U}$ & 340 & 350 & $\mathrm{U}$ & 350 & 350 & $\mathrm{U}$ & 350 \\
\hline N-Nitrosodiphenylamine & 340 & $\mathrm{U}$ & 340 & 340 & U & 340 & 350 & UJ & 350 & 350 & UJ & 350 \\
\hline Naphthalene & 340 & U & 340 & 340 & U & 340 & 350 & $\mathrm{U}$ & 350 & 350 & $\mathrm{U}$ & 350 \\
\hline Nitrobenzene & 340 & $\mathrm{U}$ & 340 & 340 & $\mathrm{U}$ & 340 & 350 & UJ & 350 & 350 & UJ & 350 \\
\hline Pentachlorophenol & 840 & $\mathrm{U}$ & 840 & 840 & $\mathrm{U}$ & 840 & 880 & $\mathrm{U}$ & 880 & 880 & $\mathrm{U}$ & 880 \\
\hline Phenanthrene & 340 & $\mathrm{U}$ & 340 & 340 & $\mathrm{U}$ & 340 & 350 & $\mathrm{U}$ & 350 & 350 & $\bar{U}$ & 350 \\
\hline Phenol & 340 & $\mathrm{U}$ & 340 & 22 & $\mathrm{JB}$ & 340 & 350 & $\mathrm{U}$ & 350 & 350 & $\mathrm{U}$ & 350 \\
\hline Pyrene & 340 & $\mathrm{U}$ & 340 & 340 & $\mathrm{U}$ & 340 & 350 & $\mathrm{U}$ & 350 & 350 & $\mathrm{U}$ & 350 \\
\hline & & & & Attachn & & & 1 & & & heet No. & & of 113 \\
\hline & & & & Originat & & J.M.C & oron & & & Dat & & $04 / 07$ \\
\hline & & & & Checker & & T. M. B & akley & & & Date & & \\
\hline & & & & Calc. N & & $0100 \mathrm{~B}-$ & $\mathrm{A}-\mathrm{V} 029$ & & & Rev. No & & 1 \\
\hline
\end{tabular}


Rev. 0

Attachment 1. 118-B-1 Verification Sampling Results.

\begin{tabular}{|c|c|c|c|c|c|c|c|c|c|c|c|c|}
\hline \multirow[t]{2}{*}{ Constituents } & \multicolumn{3}{|c|}{$\begin{array}{c}\text { J12X63 } \\
\text { Area } 4 \text { A3 } \\
\text { Sample Date } 7 / 25 / 06\end{array}$} & \multicolumn{3}{|c|}{$\begin{array}{c}\text { J12X64 } \\
\text { Area } 4 \text { A4 } \\
\text { Sample Date } 7 / 25 / 06\end{array}$} & \multicolumn{3}{|c|}{$\begin{array}{c}\text { J13V12 } \\
\text { Area } 5 \text { A1 } \\
\text { Sample Date 12/7/06 }\end{array}$} & \multicolumn{3}{|c|}{$\begin{array}{c}\text { J13V10 } \\
\text { Area } 5 \text { A2 } \\
\text { Sample Date 12/7/06 }\end{array}$} \\
\hline & $\mu \mathrm{g} / \mathrm{kg}$ & $Q$ & PQL & $\mu \mathrm{g} / \mathrm{kg}$ & $Q$ & PQL & $\mu \mathrm{g} / \mathrm{kg}$ & $\mathbf{Q}$ & MDL & $\mu \mathrm{g} / \mathrm{kg}$ & $Q$ & PQL \\
\hline \multicolumn{13}{|c|}{ Volatile Organic Compounds } \\
\hline 1,1,1-Trichloroethane & 5 & $\mathrm{U}$ & 5 & 5 & U & 5 & 5 & $\mathrm{U}$ & 5 & 5 & $\mathrm{U}$ & 5 \\
\hline 1,1,2,2-Tetrachloroethane & 5 & $\mathrm{U}$ & 5 & 5 & $\mathrm{U}$ & 5 & 5 & UJ & 5 & 5 & UJ & 5 \\
\hline 1,1,2-Trichloroethane & 5 & $U$ & 5 & 5 & U & 5 & 5 & UJ & 5 & 5 & UJ & 5 \\
\hline 1,1-Dichloroethane & 5 & $\mathrm{U}$ & 5 & 5 & $\mathrm{U}$ & 5 & 5 & $\mathrm{U}$ & 5 & 5 & $\mathrm{U}$ & 5 \\
\hline 1,1-Dichloroethene & 5 & $\mathrm{U}$ & 5 & 5 & $\mathrm{U}$ & 5 & 5 & $\mathrm{U}$ & 5 & 5 & $\mathrm{U}$ & 5 \\
\hline 1,2-Dichloroethane & 5 & $\mathrm{U}$ & 5 & 5 & $\mathrm{U}$ & 5 & 5 & $\mathrm{U}$ & 5 & 5 & $\mathrm{U}$ & 5 \\
\hline 1,2-Dichloroethene (total) & 5 & $\mathrm{U}$ & 5 & 5 & $\mathrm{U}$ & 5 & 5 & $\mathrm{U}$ & 5 & 5 & $\mathrm{U}$ & 5 \\
\hline 1,2-Dichloropropane & 5 & $\mathrm{U}$ & 5 & 5 & $\mathrm{U}$ & 5 & 5 & $\mathrm{U}$ & 5 & 5 & $\mathrm{U}$ & 5 \\
\hline 2-Butanone & 10 & $\mathrm{U}$ & 10 & 10 & $\mathrm{U}$ & 10 & 10 & $\mathrm{U}$ & 10 & 10 & $\mathrm{U}$ & 10 \\
\hline 2-Hexanone & 10 & $\mathrm{U}$ & 10 & 10 & $\mathrm{U}$ & 10 & 10 & $\mathrm{U}$ & 10 & 10 & $\mathrm{U}$ & 10 \\
\hline 4-Methyl-2-pentanone & 10 & $\mathrm{U}$ & 10 & 10 & $\mathrm{U}$ & 10 & 10 & UJ & 10 & 10 & $\mathrm{UJ}$ & 10 \\
\hline Acetone & 11 & & 10 & 12 & & 10 & 12 & $\mathrm{~B}$ & 10 & 10 & $\mathrm{U}$ & 10 \\
\hline Benzene & 5 & $\mathrm{U}$ & 5 & 5 & $\mathrm{U}$ & 5 & 5 & $\mathrm{U}$ & 5 & 5 & $\mathrm{U}$ & 5 \\
\hline Bromodichloromethane & 5 & $\mathrm{U}$ & 5 & 5 & $\mathrm{U}$ & 5 & 5 & $\mathrm{U}$ & 5 & 5 & $U$ & 5 \\
\hline Bromoform & 5 & $\mathrm{U}$ & 5 & 5 & $\mathrm{U}$ & 5 & 5 & $\mathrm{UJ}$ & 5 & 5 & $\mathrm{UJ}$ & 5 \\
\hline Bromomethane & 10 & $\mathrm{U}$ & 10 & 10 & $\mathrm{U}$ & 10 & 10 & $\mathrm{U}$ & 10 & 10 & $\mathrm{U}$ & 10 \\
\hline Carbon disulfide & 5 & $\mathrm{U}$ & 5 & 5 & $\mathrm{U}$ & 5 & 5 & U & 5 & 5 & $\mathrm{U}$ & 5 \\
\hline Carbon tetrachloride & 17 & & 5 & 5 & & 5 & 5 & U & 5 & 5 & $\mathrm{U}$ & 5 \\
\hline Chlorobenzene & 5 & $\mathrm{U}$ & 5 & 5 & $\mathrm{U}$ & 5 & 5 & $\mathrm{U}$ & 5 & 5 & U & 5 \\
\hline Chloroethane & 10 & $\mathrm{U}$ & 10 & 10 & $\mathrm{U}$ & 10 & 10 & $\mathrm{U}$ & 10 & 10 & $\mathrm{U}$ & 10 \\
\hline Chloroform & 5 & U & 5 & 5 & $\mathrm{U}$ & 5 & 5 & $\mathrm{U}$ & 5 & 5 & $\mathrm{U}$ & 5 \\
\hline Chloromethane & 10 & $\mathrm{U}$ & 10 & 10 & $\mathrm{U}$ & 10 & 10 & U & 10 & 10 & $\mathrm{U}$ & 10 \\
\hline cis-1,2-Dichloroethylene & 5 & $\mathrm{U}$ & 5 & 5 & $\mathrm{U}$ & 5 & 5 & $\mathrm{U}$ & 5 & 5 & $\mathrm{U}$ & 5 \\
\hline cis-1,3-Dichloropropene & 5 & $\mathrm{U}$ & 5 & 5 & $\mathrm{U}$ & 5 & 5 & $\mathrm{U}$ & 5 & 5 & $\mathrm{U}$ & 5 \\
\hline Dibromochloromethane & 5 & $\bar{U}$ & 5 & 5 & $\mathrm{U}$ & 5 & 5 & UJ & 5 & 5 & $\mathrm{UJ}$ & 5 \\
\hline Ethylbenzene & 5 & $\mathrm{U}$ & 5 & 5 & $\mathrm{U}$ & 5 & 5 & $\mathrm{U}$ & 5 & 5 & $\mathrm{U}$ & 5 \\
\hline Methylene chloride & 12 & & 5 & 4 & $\mathrm{~J}$ & 5 & 13 & $\mathrm{~B}$ & 5 & 12 & $\mathrm{~B}$ & 5 \\
\hline Styrene & 5 & U & 5 & 5 & $\mathrm{U}$ & 5 & 5 & $\mathrm{U}$ & 5 & 5 & $\mathrm{U}$ & 5 \\
\hline Tetrachloroethene & 5 & $U$ & 5 & 5 & $\mathrm{U}$ & 5 & 5 & $\mathrm{U}$ & 5 & 5 & $\mathrm{U}$ & 5 \\
\hline Toluene & 5 & $U$ & 5 & 5 & $\mathrm{U}$ & 5 & 5 & $\mathrm{U}$ & 5 & 5 & $\mathrm{U}$ & 5 \\
\hline trans-1,2-Dichloroethylene & 5 & $U$ & 5 & 5 & $\mathrm{U}$ & 5 & 5 & $\mathrm{U}$ & 5 & 5 & $\mathrm{U}$ & 5 \\
\hline trans-1,3-Dichloropropene & 5 & $\mathrm{U}$ & 5 & 5 & $\mathrm{U}$ & 5 & 5 & UJ & 5 & 5 & UJ & 5 \\
\hline Trichloroethene & 5 & $\mathrm{U}$ & 5 & 5 & $\mathrm{U}$ & 5 & 5 & $\mathrm{U}$ & 5 & 5 & $\mathrm{U}$ & 5 \\
\hline Vinyl chloride & 10 & $\mathrm{U}$ & 10 & 10 & $\mathrm{U}$ & 10 & 10 & $\mathrm{U}$ & 10 & 10 & $\mathrm{U}$ & 10 \\
\hline Xylenes (total) & 5 & $\mathrm{U}$ & 5 & 5 & $\mathrm{U}$ & 5 & 5 & $\mathrm{U}$ & 5 & 5 & $\mathrm{U}$ & 5 \\
\hline & & & & Attachm & & & 1 & & & heet No. & & of 113 \\
\hline & & & & Originat & & J.M. Ca & pron & & & Date & & $04 / 07$ \\
\hline & & & & Checked & & T.M.B & akley & & & Date & & \\
\hline & & & & Calc. Nc & & $0100 \mathrm{~B}-$ & $\mathrm{A}-\mathrm{V} 029$ & & & Rev. No. & & 1 \\
\hline
\end{tabular}


Attachment 1. 118-B-1 Verification Sampling Results.

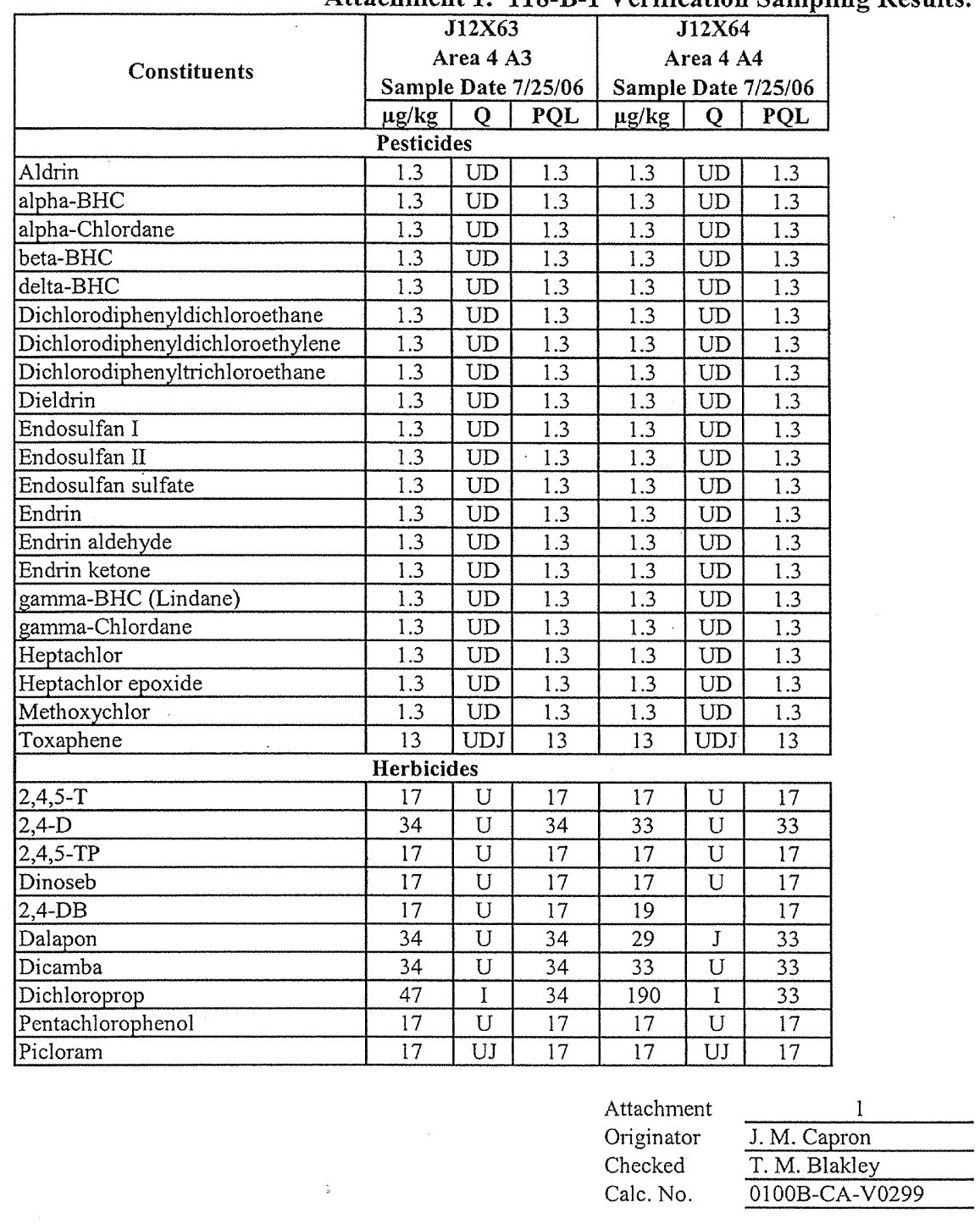


Attachment 1. 118-B-1 Verification Sampling Results.

\begin{tabular}{|c|c|c|c|c|c|c|c|c|c|c|c|c|}
\hline \multirow[t]{2}{*}{ Constituents } & \multicolumn{3}{|c|}{$\begin{array}{c}\text { J13V13 } \\
\text { Area } 5 \mathrm{A3} \\
\text { Sample Date } 12 / 7 / 06\end{array}$} & \multicolumn{3}{|c|}{$\begin{array}{c}\text { J13V11 } \\
\text { Area } 5 \text { A4 } \\
\text { Sample Date 12/7/06 } \\
\end{array}$} & \multicolumn{3}{|c|}{\begin{tabular}{|c|}
$\mathrm{J} 12 \mathrm{XC2}$ \\
Area $6 \mathrm{A1}$ \\
Sample Date $7 / 25 / 06$ \\
\end{tabular}} & \multicolumn{3}{|c|}{$\begin{array}{c}\text { J12XC3 } \\
\text { Area } 6 \text { A2 } \\
\text { Sample Date } 7 / 25 / 06 \\
\end{array}$} \\
\hline & $\mu \mathrm{g} / \mathrm{kg}$ & $Q$ & PQL & $\mu \mathrm{g} / \mathrm{kg}$ & Q & PQL & $\mu \mathrm{g} / \mathrm{kg}$ & $\mathrm{Q}$ & PQL & $\mu \mathrm{g} / \mathrm{kg}$ & $\mathrm{Q}$ & PQL \\
\hline \multicolumn{13}{|c|}{ Polychlorinated Biphenyls } \\
\hline Aroclor-1016 & 14 & $\mathrm{UJ}$ & 14 & 14 & $\mathrm{UJ}$ & 14 & 13 & $\mathrm{U}$ & 13 & 13 & $\mathrm{U}$ & 13 \\
\hline Aroclor-1221 & 14 & $\mathrm{U}$ & 14 & 14 & $\mathrm{U}$ & 14 & 13 & $\mathrm{U}$ & 13 & 13 & $\mathrm{U}$ & 13 \\
\hline Aroclor-1232 & 14 & $\mathrm{U}$ & 14 & 14 & $\mathrm{U}$ & 14 & 13 & $\mathrm{U}$ & 13 & 13 & $\mathrm{U}$ & 13 \\
\hline Aroclor- 1242 & 14 & $\mathrm{U}$ & 14 & 14 & $U$ & 14 & 13 & $\mathrm{U}$ & 13 & 13 & $\mathrm{U}$ & 13 \\
\hline Aroclor-1248 & 14 & $\mathrm{U}$ & 14 & 14 & $\mathrm{U}$ & 14 & 13 & $\mathrm{U}$ & 13 & 13 & $U$ & 13 \\
\hline Aroclor-1254 & 14 & $\mathrm{U}$ & 14 & 14 & $\mathrm{U}$ & 14 & 13 & U & 13 & 13 & $U$ & 13 \\
\hline Aroclor-1260 & 14 & UJ & 14 & 14 & UJ & 14 & 13 & $\mathrm{U}$ & 13 & 13 & $\mathrm{U}$ & 13 \\
\hline \multicolumn{13}{|c|}{ Semivolatile Organic Compounds } \\
\hline 1,2,4-Trichlorobenzene & 350 & UJ & 350 & 360 & UJ & 360 & 330 & UJ & 330 & 340 & UJ & 340 \\
\hline 1,2-Dichlorobenzene & 350 & $U$ & 350 & 360 & $\mathrm{U}$ & 360 & 330 & $\mathrm{U}$ & 330 & 340 & $U$ & 340 \\
\hline 1,3-Dichlorobenzene & 350 & $\mathrm{U}$ & 350 & 360 & $\mathrm{U}$ & 360 & 330 & $\mathrm{U}$ & 330 & 340 & $\mathrm{U}$ & 340 \\
\hline 1,4-Dichlorobenzene & 350 & $\mathrm{U}$ & 350 & 360 & $\mathrm{U}$ & 360 & 330 & $\mathrm{U}$ & 330 & 340 & $\bar{U}$ & 340 \\
\hline 2,4,5-Trichlorophenol & 870 & $\mathrm{U}$ & 870 & 890 & $\mathrm{U}$ & 890 & 840 & $\mathrm{U}$ & 840 & 840 & $\mathrm{U}$ & 840 \\
\hline 2,4,6-Trichlorophenol & 350 & $\mathrm{U}$ & 350 & 360 & $\mathrm{U}$ & 360 & 330 & $\mathrm{U}$ & 330 & 340 & $\mathrm{U}$ & 340 \\
\hline 2,4-Dichlorophenol & 350 & UJ & 350 & 360 & UJ & 360 & 330 & $\mathrm{U}$ & 330 & 340 & $\mathrm{U}$ & 340 \\
\hline 2,4-Dimethylphenol & 350 & UJ & 350 & 360 & UJ & 360 & 330 & $\mathrm{U}$ & 330 & 340 & $\mathrm{U}$ & 340 \\
\hline 2,4-Dinitrophenol & 870 & $\mathrm{U}$ & 870 & 890 & $\mathrm{U}$ & 890 & 840 & $\mathrm{U}$ & 840 & 840 & $\mathrm{U}$ & 840 \\
\hline 2,4-Dinitrotoluene & 350 & $\mathrm{U}$ & 350 & 360 & $\mathrm{U}$ & 360 & 330 & $\mathrm{U}$ & 330 & 340 & $\mathrm{U}$ & 340 \\
\hline 2,6-Dinitrotoluene & 350 & $\mathrm{U}$ & 350 & 360 & $\mathrm{U}$ & 360 & 330 & $\mathrm{U}$ & 330 & 340 & $\mathrm{U}$ & 340 \\
\hline 2-Chloronaphthalene & 350 & $\mathrm{U}$ & 350 & 360 & $\mathrm{U}$ & 360 & 330 & $\mathrm{U}$ & 330 & 340 & $\mathrm{U}$ & 340 \\
\hline 2-Chlorophenol & 350 & $\mathrm{U}$ & 350 & 360 & $\mathrm{U}$ & 360 & 330 & $\mathrm{U}$ & 330 & 340 & $\mathrm{U}$ & 340 \\
\hline 2-Methylnaphthalene & 350 & UJ & 350 & 360 & $\mathrm{UJ}$ & 360 & 330 & UJ & 330 & 340 & UJ & 340 \\
\hline 2-Methylphenol (cresol, o-) & 350 & $\mathrm{U}$ & 350 & 360 & $\mathrm{U}$ & 360 & 330 & $\mathrm{U}$ & 330 & 340 & $\mathrm{U}$ & 340 \\
\hline 2-Nitroaniline & 870 & $\mathrm{U}$ & 870 & 890 & $\mathrm{U}$ & 890 & 840 & $\mathrm{U}$ & 840 & 840 & $\mathrm{U}$ & 840 \\
\hline 2-Nitrophenol & 350 & UJ & 350 & 360 & $\mathrm{UJ}$ & 360 & 330 & $\mathrm{U}$ & 330 & 340 & $\mathrm{U}$ & 340 \\
\hline 3,3'-Dichlorobenzidine & 350 & $\mathrm{U}$ & 350 & 360 & $\mathrm{U}$ & 360 & 330 & $\mathrm{U}$ & 330 & 340 & $\mathrm{U}$ & 340 \\
\hline 3-Nitroaniline & 870 & $\mathrm{U}$ & 870 & 890 & $\mathrm{U}$ & 890 & 840 & $\mathrm{U}$ & 840 & 840 & $\mathrm{U}$ & 840 \\
\hline 4,6-Dinitro-2-methylphenol & 870 & $\bar{U}$ & 870 & 890 & $\mathrm{U}$ & 890 & 840 & $\bar{U}$ & 840 & 840 & $\mathrm{U}$ & 840 \\
\hline 4-Bromophenyl-phenylether & 350 & $\mathrm{U}$ & 350 & 360 & $\mathrm{U}$ & 360 & 330 & $\mathrm{U}$ & 330 & 340 & $\mathrm{U}$ & 340 \\
\hline 4-Chloro-3-methylphenol & 350 & UJ & 350 & 360 & UJ & 360 & 330 & $\mathrm{U}$ & 330 & 340 & $\mathrm{U}$ & 340 \\
\hline 4-Chloroaniline & 350 & $\mathrm{U}$ & 350 & 360 & $U$ & 360 & 330 & $\mathrm{U}$ & 330 & 340 & $\mathrm{U}$ & 340 \\
\hline 4-Chlorophenyl-phenylether & 350 & $\mathrm{U}$ & 350 & 360 & $\mathrm{U}$ & 360 & 330 & $\mathrm{U}$ & 330 & 340 & $\mathrm{U}$ & 340 \\
\hline 4-Methylphenol (p-cresol) & 350 & $\mathrm{U}$ & 350 & 360 & $\mathrm{U}$ & 360 & 330 & $\mathrm{U}$ & 330 & 340 & $\mathrm{U}$ & 340 \\
\hline 4-Nitroaniline & 870 & UR & 870 & 890 & UR & 890 & 840 & $\mathrm{U}$ & 840 & 840 & $\mathrm{U}$ & 840 \\
\hline 4-Nitrophenol & 870 & U & 870 & 890 & $\mathrm{U}$ & 890 & 840 & $\mathrm{U}$ & 840 & 840 & $\mathrm{U}$ & 840 \\
\hline Acenaphthene & 350 & $\mathrm{U}$ & 350 & 360 & $\mathrm{U}$ & 360 & 330 & $\mathrm{U}$ & 330 & 340 & $\mathrm{U}$ & 340 \\
\hline Acenaphthylene & 350 & $\mathrm{U}$ & 350 & 360 & $\mathrm{U}$ & 360 & 330 & $\mathrm{U}$ & 330 & 340 & $\mathrm{U}$ & 340 \\
\hline Anthracene & 350 & $\mathrm{U}$ & 350 & 360 & $\mathrm{U}$ & 360 & 330 & $\mathrm{U}$ & 330 & 340 & $\mathrm{U}$ & 340 \\
\hline Benzo(a)anthracene & 350 & $\mathrm{U}$ & 350 & 360 & $\mathrm{U}$ & 360 & 330 & $\mathrm{U}$ & 330 & 340 & $\mathrm{U}$ & 340 \\
\hline Benzo(a)pyrene & 350 & U & 350 & 360 & $\mathrm{U}$ & 360 & 330 & $\mathrm{U}$ & 330 & 340 & $\mathrm{U}$ & 340 \\
\hline Benzo(b)fluoranthene & 350 & $\mathrm{U}$ & 350 & 360 & $\mathrm{U}$ & 360 & 330 & $\mathrm{U}$ & 330 & 340 & $\mathrm{U}$ & 340 \\
\hline Benzo(g,h,i)perylene & 350 & $\mathrm{U}$ & 350 & 360 & U & 360 & 330 & $\mathrm{U}$ & 330 & 340 & $\mathrm{U}$ & 340 \\
\hline Benzo(k)fluoranthene & 350 & $\mathrm{U}$ & 350 & 360 & $\mathrm{U}$ & 360 & 330 & $\mathrm{U}$ & 330 & 340 & $\mathrm{U}$ & 340 \\
\hline bis(2-Chloro-1-methylethyl)ether & 350 & U & 350 & 360 & $\mathrm{U}$ & 360 & 330 & $\mathrm{U}$ & 330 & 340 & $\mathrm{U}$ & 340 \\
\hline bis(2-Chloroethoxy)methane & 350 & U & 350 & 360 & $\mathrm{U}$ & 360 & 330 & $\mathrm{U}$ & 330 & 340 & $\mathrm{U}$ & 340 \\
\hline bis(2-Chloroethyl)ether & 350 & $\mathrm{U}$ & 350 & 360 & $\mathrm{U}$ & 360 & 330 & $\mathrm{U}$ & 330 & 340 & $\mathrm{U}$ & 340 \\
\hline bis(2-Ethylhexyl)phthalate & 350 & $\mathrm{U}$ & 350 & 22 & $\mathrm{JB}$ & 360 & 19 & $\mathrm{JB}$ & 330 & 170 & $\mathrm{JB}$ & 340 \\
\hline
\end{tabular}

Attachment Originator

Checked

Calc. No.

$\frac{1}{\frac{1}{\text { J.M. Capron }}} \frac{}{\text { T.M. Blakley }}$

Sheet No. 49 of 113

Date $\frac{40 / 04 / 07}{10}$

Date Rev. No. 
Attachment 1. 118-B-1 Verification Sampling Results.

\begin{tabular}{|c|c|c|c|c|c|c|c|c|c|c|c|c|}
\hline \multirow[t]{2}{*}{ Constituents } & \multicolumn{3}{|c|}{$\begin{array}{c}\text { J13V13 } \\
\text { Area } 5 \text { A3 } \\
\text { Sample Date } 12 / 7 / 06\end{array}$} & \multicolumn{3}{|c|}{$\begin{array}{c}\text { J13V11 } \\
\text { Area } 5 \text { A4 } \\
\text { Sample Date 12/7/06 }\end{array}$} & \multicolumn{3}{|c|}{$\begin{array}{c}\text { J12XC2 } \\
\text { Area } 6 \text { A1 } \\
\text { Sample Date } 7 / 25 / 06\end{array}$} & \multicolumn{3}{|c|}{$\begin{array}{c}\text { J12XC3 } \\
\text { Area } 6 \text { A2 } \\
\text { Sample Date } 7 / 25 / 06\end{array}$} \\
\hline & $\mu \mathrm{g} / \mathrm{kg}$ & $\mathbf{Q}$ & PQL & $\mu \mathrm{g} / \mathrm{kg}$ & $\mathbf{Q}$ & PQL & $\mu \mathrm{g} / \mathrm{kg}$ & $\mathbf{Q}$ & MDL & $\mu \mathrm{g} / \mathrm{kg}$ & Q & PQL \\
\hline \multicolumn{13}{|c|}{ Semivolatile Organic Compounds (continued) } \\
\hline Butylbenzylphthalate & 350 & $\mathrm{U}$ & 350 & 360 & $\mathrm{U}$ & 360 & 330 & $\mathrm{U}$ & 330 & 24 & $\mathrm{~J}$ & 340 \\
\hline Carbazole & 350 & $\mathrm{U}$ & 350 & 360 & $\mathrm{U}$ & 360 & 330 & $\mathrm{U}$ & 330 & 340 & $\mathrm{U}$ & 340 \\
\hline Chrysene & 350 & $\mathrm{U}$ & 350 & 360 & $\mathrm{U}$ & 360 & 330 & $\mathrm{U}$ & 330 & 340 & U & 340 \\
\hline Di-n-butylphthalate & 350 & $\mathrm{U}$ & 350 & 360 & $\mathrm{U}$ & 360 & 75 & $\mathrm{JB}$ & 330 & 84 & $\mathrm{JB}$ & 340 \\
\hline Di-n-octylphthalate & 350 & $\mathrm{U}$ & 350 & 360 & $\mathrm{U}$ & 360 & 330 & $\mathrm{U}$ & 330 & 340 & U & 340 \\
\hline Dibenz(a,h)anthracene & 350 & $\mathrm{U}$ & 350 & 360 & $\mathrm{U}$ & 360 & 330 & $\mathrm{U}$ & 330 & 340 & $\mathrm{U}$ & 340 \\
\hline Dibenzofuran & 350 & $\mathrm{U}$ & 350 & 360 & U & 360 & 330 & $\mathrm{U}$ & 330 & 340 & $\mathrm{U}$ & 340 \\
\hline Diethylphthalate & 350 & $\mathrm{U}$ & 350 & 360 & $\mathrm{U}$ & 360 & 330 & $\mathrm{U}$ & 330 & 340 & $\mathrm{U}$ & 340 \\
\hline Dimethylphthalate & 350 & $\mathrm{U}$ & 350 & 360 & $\mathrm{U}$ & 360 & 330 & $\mathrm{U}$ & 330 & 340 & $\mathrm{U}$ & 340 \\
\hline Fluoranthene & 350 & $\mathrm{U}$ & 350 & 360 & $\mathrm{U}$ & 360 & 330 & $\mathrm{U}$ & 330 & 340 & $\mathrm{U}$ & 340 \\
\hline Fluorene & 350 & $\mathrm{U}$ & 350 & 360 & $\mathrm{U}$ & 360 & 330 & $\mathrm{U}$ & 330 & 340 & U & 340 \\
\hline Hexachlorobenzene & 350 & $\mathrm{U}$ & 350 & 360 & $U$ & 360 & 330 & $\mathrm{U}$ & 330 & 340 & $\mathrm{U}$ & 340 \\
\hline Hexachlorobutadiene & 350 & $\mathrm{U}$ & 350 & 360 & $\mathrm{U}$ & 360 & 330 & $U$ & 330 & 340 & U & 340 \\
\hline Hexachlorocyclopentadiene & 350 & $\mathrm{U}$ & 350 & 360 & $\mathrm{U}$ & 360 & 330 & $\mathrm{U}$ & 330 & 340 & $\mathrm{U}$ & 340 \\
\hline Hexachloroethane & 350 & $\mathrm{U}$ & 350 & 360 & $\bar{U}$ & 360 & 330 & $\mathrm{U}$ & 330 & 340 & $\mathrm{U}$ & 340 \\
\hline Indeno(1,2,3-cd)pyrene & 350 & U & 350 & 360 & $\mathrm{U}$ & 360 & 330 & U & 330 & 340 & $\mathrm{U}$ & 340 \\
\hline Isophorone & 350 & UJ & 350 & 360 & UJ & 360 & 330 & $U$ & 330 & 340 & $\mathrm{U}$ & 340 \\
\hline N-Nitroso-di-n-dipropylamine & 350 & $\mathrm{U}$ & 350 & 360 & $\mathrm{U}$ & 360 & 330 & U & 330 & 340 & $U$ & 340 \\
\hline N-Nitrosodiphenylamine & 350 & UJ & 350 & 360 & UJ & 360 & 330 & $\mathrm{U}$ & 330 & 340 & $\mathrm{U}$ & 340 \\
\hline Naphthalene & 350 & $\mathrm{U}$ & 350 & 360 & $\mathrm{U}$ & 360 & 330 & $\mathrm{U}$ & 330 & 340 & $\mathrm{U}$ & 340 \\
\hline Nitrobenzene & 350 & UJ & 350 & 360 & UJ & 360 & 330 & $\bar{U}$ & 330 & 340 & $\mathrm{U}$ & 340 \\
\hline Pentachlorophenol & 870 & $\mathrm{U}$ & 870 & 890 & $\mathrm{U}$ & 890 & 840 & $\mathrm{U}$ & 840 & 840 & $\mathrm{U}$ & 840 \\
\hline Phenanthrene & 350 & $\mathrm{U}$ & 350 & 360 & $\mathrm{U}$ & 360 & 330 & $\mathrm{U}$ & 330 & 340 & $\mathrm{U}$ & 340 \\
\hline Phenol & 350 & $\mathrm{U}$ & 350 & 360 & $\mathrm{U}$ & 360 & 330 & $\bar{U}$ & 330 & 23 & $\mathrm{JB}$ & 340 \\
\hline Pyrene & 350 & $\mathrm{U}$ & 350 & 360 & $\mathrm{U}$ & 360 & 330 & $\mathrm{U}$ & 330 & 340 & $U$ & 340 \\
\hline
\end{tabular}

Attachment

Originator

Checked

Calc. No.

\begin{tabular}{l}
\hline J.M. Capron \\
\hline T. M. Blakley \\
\hline 0100B-CA-V0299 \\
\hline
\end{tabular}

Sheet No. 50 of 113

Date $10 / 04 / 07$

Date

Rev. No. 1 
Attachment 1. 118-B-1 Verification Sampling Results.

\begin{tabular}{|c|c|c|c|c|c|c|c|c|c|c|c|c|}
\hline \multirow[t]{2}{*}{ Constituents } & \multicolumn{3}{|c|}{\begin{tabular}{|c|} 
J13V13 \\
Area 5 A3 \\
Sample Date 12/7/06 \\
\end{tabular}} & \multicolumn{3}{|c|}{$\begin{array}{c}\text { J13V11 } \\
\text { Area } 5 \text { A4 } \\
\text { Sample Date 12/7/06 }\end{array}$} & \multicolumn{3}{|c|}{$\begin{array}{c}\text { J12XC2 } \\
\text { Area } 6 \mathrm{A1} \\
\text { Sample Date } 7 / 25 / 06\end{array}$} & \multicolumn{3}{|c|}{$\begin{array}{c}\text { J12XC3 } \\
\text { Area } 6 \text { A2 } \\
\text { Sample Date } 7 / 25 / 06 \\
\end{array}$} \\
\hline & $\mu \mathrm{g} / \mathrm{kg}$ & $\mathbf{Q}$ & PQL & $\mu \mathrm{g} / \mathrm{kg}$ & $\mathbf{Q}$ & PQL & $\mu \mathrm{g} / \mathrm{kg}$ & $\mathbf{Q}$ & MDL & $\mu \mathrm{g} / \mathrm{kg}$ & $\bar{Q}$ & PQL \\
\hline \multicolumn{13}{|c|}{ Volatile Organic Compounds } \\
\hline $1,1,1$-Trichloroethane & 5 & $\mathrm{U}$ & 5 & 5 & $\mathrm{U}$ & 5 & 5 & $\mathrm{U}$ & 5 & 5 & $\mathrm{U}$ & 5 \\
\hline 1,1,2,2-Tetrachloroethane & 5 & $\mathrm{UJ}$ & 5 & 5 & UJ & 5 & 5 & $\mathrm{U}$ & 5 & 5 & $\mathrm{U}$ & 5 \\
\hline 1,1,2-Trichloroethane & 5 & UJ & 5 & 5 & $\mathrm{UJ}$ & 5 & 5 & $\mathrm{U}$ & 5 & 5 & $\mathrm{U}$ & 5 \\
\hline 1,1-Dichloroethane & 5 & $\mathrm{U}$ & 5 & 5 & $\mathrm{U}$ & 5 & 5 & $\mathrm{U}$ & 5 & 5 & $\mathrm{U}$ & 5 \\
\hline 1,1-Dichloroethene & 5 & $\mathrm{U}$ & 5 & 5 & $\mathrm{U}$ & 5 & 5 & $\mathrm{U}$ & 5 & 5 & $\mathrm{U}$ & 5 \\
\hline 1,2-Dichloroethane & 5 & $U$ & 5 & 5 & $\mathrm{U}$ & 5 & 5 & $\mathrm{U}$ & 5 & 5 & $\mathrm{U}$ & 5 \\
\hline 1,2-Dichloroethene (total) & 5 & $\mathrm{U}$ & 5 & 5 & $\mathrm{U}$ & 5 & 5 & $\mathrm{U}$ & 5 & 5 & $\mathrm{U}$ & 5 \\
\hline 1,2-Dichloropropane & 5 & $\mathrm{U}$ & 5 & 5 & $\mathrm{U}$ & 5 & 5 & $\mathrm{U}$ & 5 & 5 & $\mathrm{U}$ & 5 \\
\hline 2-Butanone & 10 & $\mathrm{U}$ & 10 & 10 & $\mathrm{U}$ & 10 & 10 & $\mathrm{U}$ & 10 & 10 & $\mathrm{U}$ & 10 \\
\hline 2-Hexanone & 10 & $\mathrm{U}$ & 10 & 10 & $\mathrm{U}$ & 10 & 10 & $\mathrm{U}$ & 10 & 10 & $\mathrm{U}$ & 10 \\
\hline 4-Methyl-2-pentanone & 10 & $\mathrm{UJ}$ & 10 & 10 & UJ & 10 & 10 & $\mathrm{U}$ & 10 & 10 & $\mathrm{U}$ & 10 \\
\hline Acetone & 19 & $\mathrm{~B}$ & 10 & 13 & & 10 & 10 & $\mathrm{U}$ & 10 & 6 & $\mathrm{~J}$ & 10 \\
\hline Benzene & 5 & $\mathrm{U}$ & 5 & 5 & $\mathrm{U}$ & 5 & 5 & $\mathrm{U}$ & 5 & 5 & $\mathrm{U}$ & 5 \\
\hline Bromodichloromethane & 5 & $\mathrm{U}$ & 5 & 5 & $\mathrm{U}$ & 5 & 5 & $U$ & 5 & 5 & $\mathrm{U}$ & 5 \\
\hline Bromoform & 5 & $\mathrm{UJ}$ & 5 & 5 & UJ & 5 & 5 & $\mathrm{U}$ & 5 & 5 & $\mathrm{U}$ & 5 \\
\hline Bromomethane & 10 & $U$ & 10 & 10 & $\mathrm{U}$ & 10 & 10 & $\mathrm{U}$ & 10 & 10 & $\mathrm{U}$ & 10 \\
\hline Carbon disulfide & 5 & $\mathrm{U}$ & 5 & 5 & $\mathrm{U}$ & 5 & 5 & $\mathrm{U}$ & 5 & 5 & $\mathrm{U}$ & 5 \\
\hline Carbon tetrachloride & 5 & $\mathrm{U}$ & 5 & 5 & $\mathrm{U}$ & 5 & 5 & & 5 & 6 & & 5 \\
\hline Chlorobenzene & 5 & $\mathrm{U}$ & 5 & 5 & $\mathrm{U}$ & 5 & 5 & $\mathrm{U}$ & 5 & 5 & $\mathrm{U}$ & 5 \\
\hline Chloroethane & 10 & $\mathrm{U}$ & 10 & 10 & $\mathrm{U}$ & 10 & 10 & $\mathrm{U}$ & 10 & 10 & $\mathrm{U}$ & 10 \\
\hline Chloroform & 5 & $\mathrm{U}$ & 5 & 5 & $\mathrm{U}$ & 5 & 5 & $\mathrm{U}$ & 5 & 5 & $\mathrm{U}$ & 5 \\
\hline Chloromethane & 10 & $\mathrm{U}$ & 10 & 10 & $\mathrm{U}$ & 10 & 10 & $\mathrm{U}$ & 10 & 10 & $\mathrm{U}$ & 10 \\
\hline cis-1,2-Dichloroethylene & 5 & $\mathrm{U}$ & 5 & 5 & $\mathrm{U}$ & 5 & 5 & $\mathrm{U}$ & 5 & 5 & $\mathrm{U}$ & 5 \\
\hline cis-1,3-Dichloropropene & 5 & $\mathrm{U}$ & 5 & 5 & $\mathrm{U}$ & 5 & 5 & $\mathrm{U}$ & 5 & 5 & $\mathrm{U}$ & 5 \\
\hline Dibromochloromethane & 5 & $\mathrm{UJ}$ & 5 & 5 & $\mathrm{UJ}$ & 5 & 5 & $\mathrm{U}$ & 5 & 5 & $\mathrm{U}$ & 5 \\
\hline Ethylbenzene & 5 & $\mathrm{U}$ & 5 & 5 & $\mathrm{U}$ & 5 & 5 & $\mathrm{U}$ & 5 & 5 & $\mathrm{U}$ & 5 \\
\hline Methylene chloride & 12 & B & 5 & 12 & $\mathrm{~B}$ & 5 & 5 & $\mathrm{U}$ & 5 & 6 & & 5 \\
\hline Styrene & 5 & $\mathrm{U}$ & 5 & 5 & $\mathrm{U}$ & 5 & 5 & $\mathrm{U}$ & 5 & 5 & $\mathrm{U}$ & 5 \\
\hline Tetrachloroethene & 5 & $\mathrm{U}$ & 5 & 5 & $\mathrm{U}$ & 5 & 5 & $\mathrm{U}$ & 5 & 5 & $\mathrm{U}$ & 5 \\
\hline Toluene & 5 & $\mathrm{U}$ & 5 & 5 & $\mathrm{U}$ & 5 & 5 & $\mathrm{U}$ & 5 & 5 & $\mathrm{U}$ & 5 \\
\hline trans-1,2-Dichloroethylene & 5 & $\mathrm{U}$ & 5 & 5 & $\mathrm{U}$ & 5 & 5 & $\mathrm{U}$ & 5 & 5 & $\mathrm{U}$ & 5 \\
\hline trans-1,3-Dichloropropene & 5 & $\mathrm{UJ}$ & 5 & 5 & UJ & 5 & 5 & $\mathrm{U}$ & 5 & 5 & $\mathrm{U}$ & 5 \\
\hline Trichloroethene & 5 & $\mathrm{U}$ & 5 & 5 & $\mathrm{U}$ & 5 & 5 & $\mathrm{U}$ & 5 & 5 & $\mathrm{U}$ & 5 \\
\hline Vinyl chloride & 10 & $\mathrm{U}$ & 10 & 10 & $\mathrm{U}$ & 10 & 10 & $\mathrm{U}$ & 10 & 10 & $\mathrm{U}$ & 10 \\
\hline Xylenes (total) & 5 & $\mathrm{U}$ & 5 & 5 & $\mathrm{U}$ & 5 & 5 & $\mathrm{U}$ & 5 & 5 & $\mathrm{U}$ & 5 \\
\hline & & & & Attachn & & & 1 & & & Sheet No. & & of 113 \\
\hline & & & & Originat & & J.M. Ca & oron & & & Date & & $04 / 07$ \\
\hline & & & & Checkes & & T.M. B & kley & & & Date & & \\
\hline & & & & Calc. N & & $0100 \mathrm{~B}-\mathrm{C}$ & $\mathrm{A}-\mathrm{V} 0299$ & & & Rev. No & & 1 \\
\hline
\end{tabular}


CVP-2007-00006

Rev. 0

Attachment 1. 118-B-1 Verification Sampling Results.

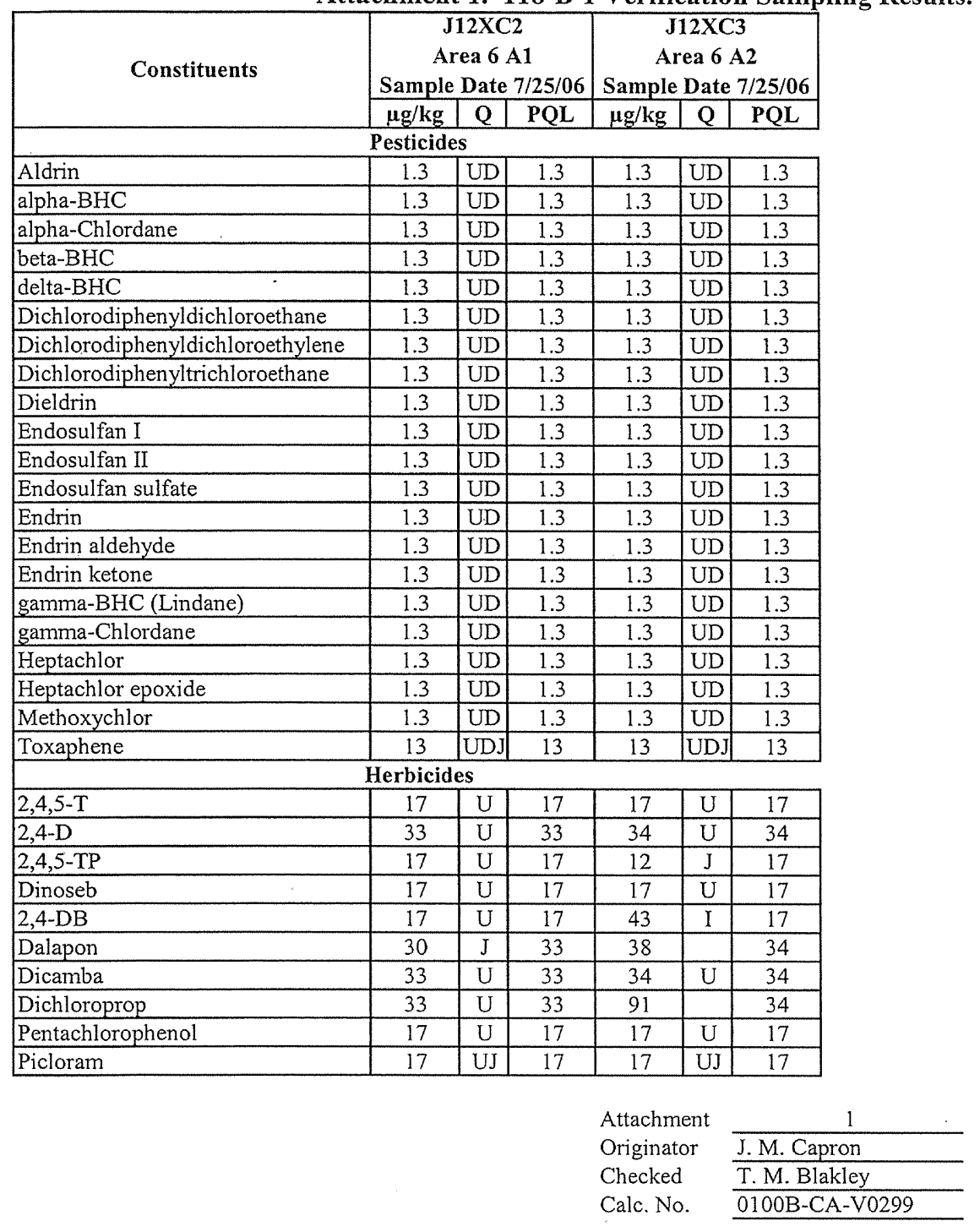


Attachment 1. 118-B-1 Verification Sampling Results.

\begin{tabular}{|c|c|c|c|c|c|c|c|c|c|c|c|c|}
\hline \multirow[t]{2}{*}{ Constituents } & \multicolumn{3}{|c|}{$\begin{array}{c}\text { J12XC4 } \\
\text { Area } 6 \mathrm{A3} \\
\text { Sample Date } 7 / 25 / 06\end{array}$} & \multicolumn{3}{|c|}{$\begin{array}{c}\text { J12XC5 } \\
\text { Area } 6 \text { A4 } \\
\text { Sample Date } 7 / 25 / 06 \\
\end{array}$} & \multicolumn{3}{|c|}{$\begin{array}{c}\text { J12XC6 } \\
\text { Area } 7 \text { A1 } \\
\text { Sample Date } 7 / 26 / 06 \\
\end{array}$} & \multicolumn{3}{|c|}{$\begin{array}{c}\text { J12XC7 } \\
\text { Area } 7 \text { A2 } \\
\text { Sample Date } 7 / 26 / 06\end{array}$} \\
\hline & $\mu \mathrm{g} / \mathrm{kg}$ & $\mathbf{Q}$ & PQL & $\mu \mathrm{g} / \mathrm{kg}$ & $Q$ & PQL & $\mu \mathrm{g} / \mathrm{kg}$ & $Q$ & PQL & $\mu \mathrm{g} / \mathrm{kg}$ & $\mathbf{Q}$ & PQL \\
\hline \multicolumn{13}{|c|}{ Polychlorinated Biphenyls } \\
\hline Aroclor-1016 & 13 & $\mathrm{U}$ & 13 & 13 & $U$ & 13 & 13 & $\mathrm{U}$ & 13 & 13 & $\mathrm{U}$ & 13 \\
\hline Aroclor- 1221 & 13 & $\mathrm{U}$ & 13 & 13 & $\mathrm{U}$ & 13 & 13 & $\mathrm{U}$ & 13 & 13 & $\mathrm{U}$ & 13 \\
\hline Aroclor- 1232 & 13 & $\mathrm{U}$ & 13 & 13 & $\mathrm{U}$ & 13 & 13 & $\mathrm{U}$ & 13 & 13 & $\mathrm{U}$ & 13 \\
\hline Aroclor- 1242 & 13 & $\mathrm{U}$ & 13 & 13 & $\mathrm{U}$ & 13 & 13 & $\mathrm{U}$ & 13 & 13 & U & 13 \\
\hline Aroclor-1248 & 13 & $\mathrm{U}$ & 13 & 13 & $U$ & 13 & 13 & $\mathrm{U}$ & 13 & 13 & $\mathrm{U}$ & 13 \\
\hline Aroclor- 1254 & 13 & $\mathrm{U}$ & 13 & 13 & $\mathrm{U}$ & 13 & 13 & $\mathrm{U}$ & 13 & 13 & $\mathrm{U}$ & 13 \\
\hline Aroclor -1260 & 13 & $\mathrm{U}$ & 13 & 13 & $\mathrm{U}$ & 13 & 13 & $\mathrm{U}$ & 13 & 13 & $\mathrm{U}$ & 13 \\
\hline \multicolumn{13}{|c|}{ Semivolatile Organic Compounds } \\
\hline 1,2,4-Trichlorobenzene & 340 & $\mathrm{UJ}$ & 340 & 340 & $\mathrm{UJ}$ & 340 & 330 & UJ & 330 & 330 & $\mathrm{UJ}$ & 330 \\
\hline 1,2-Dichlorobenzene & 340 & $\mathrm{U}$ & 340 & 340 & $U$ & 340 & 330 & $\mathrm{U}$ & 330 & 330 & $\mathrm{U}$ & 330 \\
\hline 1,3-Dichlorobenzene & 340 & $\mathrm{U}$ & 340 & 340 & $\mathrm{U}$ & 340 & 330 & $\mathrm{U}$ & 330 & 330 & $\mathrm{U}$ & 330 \\
\hline 1,4-Dichlorobenzene & 340 & $\mathrm{U}$ & 340 & 340 & $\mathrm{U}$ & 340 & 330 & $\mathrm{U}$ & 330 & 330 & $\mathrm{U}$ & 330 \\
\hline 2,4,5-Trichlorophenol & 840 & $\mathrm{U}$ & 840 & 840 & $\mathrm{U}$ & 840 & 840 & $\mathrm{U}$ & 840 & 840 & $\mathrm{U}$ & 840 \\
\hline 2,4,6-Trichlorophenol & 340 & $\mathrm{U}$ & 340 & 340 & $\mathrm{U}$ & 340 & 330 & $\mathrm{U}$ & 330 & 330 & $\mathrm{U}$ & 330 \\
\hline 2,4-Dichlorophenol & 340 & U & 340 & 340 & $\mathrm{U}$ & 340 & 330 & $\mathrm{U}$ & 330 & 330 & $\mathrm{U}$ & 330 \\
\hline 2,4-Dimethylphenol & 340 & $\mathrm{U}$ & 340 & 340 & $\mathrm{U}$ & 340 & 330 & $\mathrm{U}$ & 330 & 330 & $\mathrm{U}$ & 330 \\
\hline 2,4-Dinitrophenol & 840 & $\mathrm{U}$ & 840 & 840 & $\mathrm{U}$ & 840 & 840 & UJ & 840 & 840 & UJ & 840 \\
\hline 2,4-Dinitrotoluene & 340 & $\mathrm{U}$ & 340 & 340 & $\mathrm{U}$ & 340 & 330 & $\mathrm{U}$ & 330 & 330 & $\mathrm{U}$ & 330 \\
\hline 2,6-Dinitrotoluene & 340 & $\mathrm{U}$ & 340 & 340 & $\mathrm{U}$ & 340 & 330 & $\mathrm{U}$ & 330 & 330 & $\mathrm{U}$ & 330 \\
\hline 2-Chloronaphthalene & 340 & $\mathrm{U}$ & 340 & 340 & $\mathrm{U}$ & 340 & 330 & $\mathrm{U}$ & 330 & 330 & $\mathrm{U}$ & 330 \\
\hline 2-Chlorophenol & 340 & $\mathrm{U}$ & 340 & 340 & $\mathrm{U}$ & 340 & 330 & $\mathrm{U}$ & 330 & 330 & $\mathrm{U}$ & 330 \\
\hline 2-Methylnaphthalene & 340 & UJ & 340 & 340 & $\mathrm{UJ}$ & 340 & 26 & $\mathrm{~J}$ & 330 & 330 & $\mathrm{U}$ & 330 \\
\hline 2-Methylphenol (cresol, o-) & 340 & $\mathrm{U}$ & 340 & 340 & $\mathrm{U}$ & 340. & 330 & $\mathrm{U}$ & 330 & 330 & $\mathrm{U}$ & 330 \\
\hline 2-Nitroaniline & 840 & $\mathrm{U}$ & 840 & 840 & $\mathrm{U}$ & 840 & 840 & $\mathrm{U}$ & 840 & 840 & $\mathrm{U}$ & 840 \\
\hline 2-Nitrophenol & 340 & $\mathrm{U}$ & 340 & 340 & $\mathrm{U}$ & 340 & 330 & $\mathrm{U}$ & 330 & 330 & $\mathrm{U}$ & 330 \\
\hline 3,3'-Dichlorobenzidine & 340 & $\mathrm{U}$ & 340 & 340 & $\mathrm{U}$ & 340 & 330 & $\mathrm{U}$ & 330 & 330 & $\mathrm{U}$ & 330 \\
\hline 3-Nitroaniline & 840 & $\mathrm{U}$ & 840 & 840 & $\mathrm{U}$ & 840 & 840 & $\mathrm{U}$ & 840 & 840 & $\mathrm{U}$ & 840 \\
\hline 4,6-Dinitro-2-methylphenol & 840 & $\mathrm{U}$ & 840 & 840 & $\mathrm{U}$ & 840 & 840 & $U$ & 840 & 840 & U & 840 \\
\hline 4-Bromophenyl-phenylether & 340 & $\mathrm{U}$ & 340 & 340 & $\mathrm{U}$ & 340 & 330 & $\mathrm{U}$ & 330 & 330 & $\mathrm{U}$ & 330 \\
\hline 4-Chloro-3-methylphenol & 340 & $\mathrm{U}$ & 340 & 340 & $\mathrm{U}$ & 340 & 330 & $\mathrm{U}$ & 330 & 330 & $\mathrm{U}$ & 330 \\
\hline 4-Chloroaniline & 340 & $\mathrm{U}$ & 340 & 340 & $\mathrm{U}$ & 340 & 330 & $\mathrm{U}$ & 330 & 330 & $\mathrm{U}$ & 330 \\
\hline 4-Chlorophenyl-phenylether & 340 & $\mathrm{U}$ & 340 & 340 & $\mathrm{U}$ & 340 & 330 & $\mathrm{U}$ & 330 & 330 & $\mathrm{U}$ & 330 \\
\hline 4-Methylphenol (p-cresol) & 340 & $\mathrm{U}$ & 340 & 340 & $\mathrm{U}$ & 340 & 330 & $\mathrm{U}$ & 330 & 330 & $\mathrm{U}$ & 330 \\
\hline 4-Nitroaniline & 840 & $\mathrm{U}$ & 840 & 840 & $\mathrm{U}$ & 840 & 840 & $\mathrm{U}$ & 840 & 840 & $\mathrm{U}$ & 840 \\
\hline 4-Nitrophenol & 840 & $\mathrm{U}$ & 840 & 840 & $\mathrm{U}$ & 840 & 840 & $\mathrm{U}$ & 840 & 840 & $\mathrm{U}$ & 840 \\
\hline Acenaphthene & 340 & $\mathrm{U}$ & 340 & 340 & $U$ & 340 & 330 & $\mathrm{U}$ & 330 & 330 & $\mathrm{U}$ & 330 \\
\hline Acenaphthylene & 340 & $\mathrm{U}$ & 340 & 340 & $\mathrm{U}$ & 340 & 330 & $\mathrm{U}$ & 330 & 330 & $\mathrm{U}$ & 330 \\
\hline Anthracene & 340 & $\mathrm{U}$ & 340 & 340 & $\mathrm{U}$ & 340 & 330 & $\mathrm{U}$ & 330 & 330 & $\mathrm{U}$ & 330 \\
\hline Benzo(a)anthracene & 340 & $\mathrm{U}$ & 340 & 340 & $\mathrm{U}$ & 340 & 330 & $\mathrm{U}$ & 330 & 330 & $\mathrm{U}$ & 330 \\
\hline Benzo(a)pyrene & 340 & U & 340 & 340 & $\mathrm{U}$ & 340 & 330 & $\mathrm{U}$ & 330 & 330 & $\mathrm{U}$ & 330 \\
\hline Benzo(b)fluoranthene & 340 & $\mathrm{U}$ & 340 & 340 & $\mathrm{U}$ & 340 & 330 & $\mathrm{U}$ & 330 & 330 & $\mathrm{U}$ & 330 \\
\hline Benzo(g,h,i)perylene & 340 & $\mathrm{U}$ & 340 & 340 & $\mathrm{U}$ & 340 & 330 & $\mathrm{U}$ & 330 & 330 & $\mathrm{U}$ & 330 \\
\hline Benzo(k)fluoranthene & 340 & $\mathrm{U}$ & 340 & 340 & $\mathrm{U}$ & 340 & 330 & $\mathrm{U}$ & 330 & 330 & $\mathrm{U}$ & 330 \\
\hline bis(2-Chloro-1-methylethyl)ether & 340 & $\mathrm{U}$ & 340 & 340 & $\mathrm{U}$ & 340 & 330 & $\mathrm{U}$ & 330 & 330 & $\mathrm{U}$ & 330 \\
\hline bis(2-Chloroethoxy)methane & 340 & $\mathrm{U}$ & 340 & 340 & U & 340 & 330 & $\mathrm{U}$ & 330 & 330 & U & 330 \\
\hline bis(2-Chloroethyl)ether & 340 & $\mathrm{U}$ & 340 & 340 & $\mathrm{U}$ & 340 & 330 & $\mathrm{U}$ & 330 & 330 & $\mathrm{U}$ & 330 \\
\hline bis(2-Ethylhexyl)phthalate & 26 & $\mathrm{JB}$ & 340 & 430 & $\mathrm{~B}$ & 340 & 330 & $\mathrm{U}$ & 330 & 330 & $U$ & 330 \\
\hline & & & & Attachn & & & 1 & & & Sheet No. & & of 113 \\
\hline & & & & Origina & & J.M.Ca & pron & & & Date & & 104/07 \\
\hline & & & & Checke & & T.M. B1 & akley & & & Date & & \\
\hline & & & & Calc. N & & $0100 \mathrm{~B}$ & A-V02 & & & Rev. No. & & 1 \\
\hline
\end{tabular}


Rev. 0

Attachment 1. 118-B-1 Verification Sampling Results.

\begin{tabular}{|c|c|c|c|c|c|c|c|c|c|c|c|c|}
\hline \multirow[t]{2}{*}{ Constituents } & \multicolumn{3}{|c|}{$\begin{array}{c}\text { J12XC4 } \\
\text { Area } 6 \mathrm{A3} \\
\text { Sample Date } 7 / 25 / 06 \\
\end{array}$} & \multicolumn{3}{|c|}{$\begin{array}{c}\text { J12XC5 } \\
\text { Area } 6 \text { A4 } \\
\text { Sample Date } 7 / 25 / 06 \\
\end{array}$} & \multicolumn{3}{|c|}{$\begin{array}{c}\text { J12XC6 } \\
\text { Area } 7 \text { A1 } \\
\text { Sample Date } 7 / 26 / 06\end{array}$} & \multicolumn{3}{|c|}{$\begin{array}{c}\text { J12XC7 } \\
\text { Area } 7 \mathrm{~A} 2 \\
\text { Sample Date } 7 / 26 / 06 \\
\end{array}$} \\
\hline & $\mu \mathrm{g} / \mathrm{kg}$ & $Q$ & PQL & $\mu \mathrm{g} / \mathrm{kg}$ & $\mathbf{Q}$ & PQL & $\mu \mathrm{g} / \mathrm{kg}$ & $Q$ & MDL & $\mu \mathrm{g} / \mathrm{kg}$ & Q & $\mathrm{PQL}$ \\
\hline \multicolumn{13}{|c|}{ Semivolatile Organic Compounds (continued) } \\
\hline Butylbenzylphthalate & 340 & $\mathrm{U}$ & 340 & 340 & $\mathrm{U}$ & 340 & 330 & $\mathrm{U}$ & 330 & 330 & $\mathrm{U}$ & 330 \\
\hline Carbazole & 340 & $\mathrm{U}$ & 340 & 340 & $\mathrm{U}$ & 340 & 330 & $\mathrm{U}$ & 330 & 330 & $\mathrm{U}$ & 330 \\
\hline Chrysene & 340 & $\mathrm{U}$ & 340 & 340 & $\mathrm{U}$ & 340 & 330 & $\mathrm{U}$ & 330 & 330 & $\mathrm{U}$ & 330 \\
\hline Di-n-butylphthalate & 49 & $\mathrm{JB}$ & 340 & 52 & $\mathrm{JB}$ & 340 & 330 & $\mathrm{U}$ & 330 & 330 & $\mathrm{U}$ & 330 \\
\hline Di-n-octylphthalate & 340 & $\mathrm{U}$ & 340 & 340 & $U$ & 340 & 330 & $\mathrm{U}$ & 330 & 330 & $\mathrm{U}$ & 330 \\
\hline Dibenz $(a, h)$ anthracene & 340 & $\mathrm{U}$ & 340 & 340 & $\mathrm{U}$ & 340 & 330 & $\mathrm{U}$ & 330 & 330 & $\mathrm{U}$ & 330 \\
\hline Dibenzofuran & 340 & $\mathrm{U}$ & 340 & 340 & $\mathrm{U}$ & 340 & 330 & $\mathrm{U}$ & 330 & 330 & $\mathrm{U}$ & 330 \\
\hline Diethylphthalate & 340 & $\mathrm{U}$ & 340 & 340 & $\mathrm{U}$ & 340 & 330 & $\mathrm{U}$ & 330 & 25 & $\mathrm{~J}$ & 330 \\
\hline Dimethylphthalate & 340 & $\mathrm{U}$ & 340 & 340 & $\mathrm{U}$ & 340 & 330 & $\mathrm{U}$ & 330 & 330 & $\mathrm{U}$ & 330 \\
\hline Fluoranthene & 340 & $\mathrm{U}$ & 340 & 340 & $\mathrm{U}$ & 340 & 330 & $\mathrm{U}$ & 330 & 330 & $\mathrm{U}$ & 330 \\
\hline Fluorene & 340 & $\mathrm{U}$ & 340 & 340 & $\mathrm{U}$ & 340 & 330 & $\mathrm{U}$ & 330 & 330 & $\mathrm{U}$ & 330 \\
\hline Hexachlorobenzene & 340 & $\mathrm{U}$ & 340 & 340 & $\mathrm{U}$ & 340 & 330 & $\mathrm{U}$ & 330 & 330 & $\mathrm{U}$ & 330 \\
\hline Hexachlorobutadiene & 340 & $\mathrm{U}$ & 340 & 340 & $\mathrm{U}$ & 340 & 330 & $\mathrm{U}$ & 330 & 330 & $\mathrm{U}$ & 330 \\
\hline Hexachlorocyclopentadiene & 340 & $\mathrm{U}$ & 340 & 340 & $\mathrm{U}$ & 340 & 330 & $\mathrm{U}$ & 330 & 330 & U & 330 \\
\hline Hexachloroethane & 340 & $\mathrm{U}$ & 340 & 340 & $\mathrm{U}$ & 340 & 330 & $\mathrm{U}$ & 330 & 330 & $\mathrm{U}$ & 330 \\
\hline Indeno( $1,2,3$-cd)pyrene & 340 & $\mathrm{U}$ & 340 & 340 & $\mathrm{U}$ & 340 & 330 & $\mathrm{U}$ & 330 & 330 & $\mathrm{U}$ & 330 \\
\hline Isophorone & 340 & U & 340 & 340 & $\mathrm{U}$ & 340 & 330 & $\mathrm{U}$ & 330 & 330 & $\mathrm{U}$ & 330 \\
\hline N-Nitroso-di-n-dipropylamine & 340 & $\mathrm{U}$ & 340 & 340 & $\mathrm{U}$ & 340 & 330 & $\mathrm{U}$ & 330 & 330 & $\mathrm{U}$ & 330 \\
\hline N-Nitrosodiphenylamine & 340 & $\mathrm{U}$ & 340 & 340 & $\mathrm{U}$ & 340 & 330 & $\mathrm{U}$ & 330 & 330 & $\mathrm{U}$ & 330 \\
\hline Naphthalene & 340 & $\mathrm{U}$ & 340 & 340. & $\mathrm{U}$ & 340 & 19 & $\mathrm{~J}$ & 330 & 330 & $\mathrm{U}$ & 330 \\
\hline Nitrobenzene & 340 & $\mathrm{U}$ & 340 & 340 & $\mathrm{U}$ & 340 & 330 & $\mathrm{U}$ & 330 & 330 & $\mathrm{U}$ & 330 \\
\hline Pentachlorophenol & 840 & $\mathrm{U}$ & 840 & 840 & $\mathrm{U}$ & 840 & 840 & $\mathrm{U}$ & 840 & 840 & $\mathrm{U}$ & 840 \\
\hline Phenanthrene & 340 & $\mathrm{U}$ & 340 & 340 & $\mathrm{U}$ & 340 & 330 & $\mathrm{U}$ & 330 & 330 & $\mathrm{U}$ & 330 \\
\hline Phenol & 340 & $\mathrm{U}$ & 340 & 340 & $\mathrm{U}$ & 340 & 330 & $\mathrm{U}$ & 330 & 330 & $\mathrm{U}$ & 330 \\
\hline Pyrene & 340 & $\mathrm{U}$ & 340 & 340 & $\mathrm{U}$ & 340 & 330 & $\mathrm{U}$ & 330 & 330 & $\mathrm{U}$ & 330 \\
\hline
\end{tabular}

Attachment

Originator

Checked

J.M. Capron

Calc. No.
T. M. Blakley

0100B-CA-V0299
Sheet No. 54 of 113

Date $\underline{02 / 07 / 07}$

Date

Rev. No.
0 
Attachment 1. 118-B-1 Verification Sampling Results.

\begin{tabular}{|c|c|c|c|c|c|c|c|c|c|c|c|c|}
\hline \multirow[t]{2}{*}{ Constituents } & \multicolumn{3}{|c|}{$\begin{array}{c}\text { J12XC4 } \\
\text { Area } 6 \mathrm{A3} \\
\text { Sample Date } 7 / 25 / 06 \\
\end{array}$} & \multicolumn{3}{|c|}{$\begin{array}{c}\text { J12XC5 } \\
\text { Area } 6 \mathrm{A4} \\
\text { Sample Date } 7 / 25 / 06 \\
\end{array}$} & \multicolumn{3}{|c|}{$\begin{array}{c}\text { J12XC6 } \\
\text { Area } 7 \text { A1 } \\
\text { Sample Date 7/26/06 }\end{array}$} & \multicolumn{3}{|c|}{$\begin{array}{c}\text { J12XC7 } \\
\text { Area } 7 \text { A2 } \\
\text { Sample Date 7/26/06 } \\
\end{array}$} \\
\hline & $\mu \mathrm{g} / \mathrm{kg}$ & $Q$ & PQL & $\mu \mathrm{g} / \mathrm{kg}$ & $Q$ & PQL & $\mu \mathrm{g} / \mathrm{kg}$ & $\mathbf{Q}$ & MDL & $\mu \mathrm{g} / \mathrm{kg}$ & $Q$ & $\mathrm{PQL}$ \\
\hline \multicolumn{13}{|c|}{ Volatile Organic Compounds } \\
\hline 1,1,1-Trichloroethane & 5 & $\mathrm{U}$ & 5 & 5 & $\mathrm{U}$ & 5 & 5 & $\mathrm{U}$ & 5 & 5 & $\mathrm{U}$ & 5 \\
\hline 1,1,2,2-Tetrachloroethane & 5 & $\mathrm{U}$ & 5 & 5 & $\mathrm{U}$ & 5 & 5 & $\mathrm{U}$ & 5 & 5 & $\mathrm{U}$ & 5 \\
\hline 1,1,2-Trichloroethane & 5 & $\mathrm{U}$ & 5 & 5 & $\mathrm{U}$ & 5 & 5 & $\mathrm{U}$ & 5 & 5 & $\mathrm{U}$ & 5 \\
\hline 1,1-Dichloroethane & 5 & $U$ & 5 & 5 & $\mathrm{U}$ & 5 & 5 & $U$ & 5 & 5 & $\mathrm{U}$ & 5 \\
\hline 1,1-Dichloroethene & 5 & $\mathrm{U}$ & 5 & 5 & $\mathrm{U}$ & 5 & 5 & $\mathrm{U}$ & 5 & 5 & $\mathrm{U}$ & 5 \\
\hline 1,2-Dichloroethane & 5 & $\mathrm{U}$ & 5 & 5 & $\mathrm{U}$ & 5 & 5 & $\mathrm{U}$ & 5 & 5 & $\mathrm{U}$ & 5 \\
\hline 1,2-Dichloroethene (total) & 5 & $\mathrm{U}$ & 5 & 5 & $\mathrm{U}$ & 5 & 5 & $\mathrm{U}$ & 5 & 5 & $\mathrm{U}$ & 5 \\
\hline 1,2-Dichloropropane & 5 & $\mathrm{U}$ & 5 & 5 & $\mathrm{U}$ & 5 & 5 & U & 5 & 5 & $\mathrm{U}$ & 5 \\
\hline 2-Butanone & 10 & $\mathrm{U}$ & 10 & 10 & $\mathrm{U}$ & 10 & 10 & $\mathrm{U}$ & 10 & 10 & $\mathrm{U}$ & 10 \\
\hline 2-Hexanone & 10 & $\mathrm{U}$ & 10 & 10 & $\mathrm{U}$ & 10 & 10 & $\mathrm{U}$ & 10 & 10 & $\mathrm{U}$ & 10 \\
\hline 4-Methyl-2-pentanone & 10 & $\mathrm{U}$ & 10 & 10 & $\mathrm{U}$ & 10 & 10 & $\mathrm{U}$ & 10 & 10 & $\mathrm{U}$ & 10 \\
\hline Acetone & 7 & $\mathrm{~J}$ & 10 & 8 & $\mathrm{~J}$ & 10 & 8 & $\mathrm{~J}$ & 10 & 10 & $\mathrm{~J}$ & 10 \\
\hline Benzene & 5 & $\mathrm{U}$ & 5 & 5 & $\mathrm{U}$ & 5 & 5 & $\mathrm{U}$ & 5 & 5 & $\mathrm{U}$ & 5 \\
\hline Bromodichloromethane & 5 & $\mathrm{U}$ & 5 & 5 & $\mathrm{U}$ & 5 & 5 & $\mathrm{U}$ & 5 & 5 & $\mathrm{U}$ & 5 \\
\hline Bromoform & 5 & $\mathrm{U}$ & 5 & 5 & $\mathrm{U}$ & 5 & 5 & $\mathrm{U}$ & 5 & 5 & $\mathrm{U}$ & 5 \\
\hline Bromomethane & 10 & $\mathrm{U}$ & 10 & 10 & $\mathrm{U}$ & 10 & 10 & $\mathrm{U}$ & 10 & 10 & $U$ & 10 \\
\hline Carbon disulfide & 5 & $\mathrm{U}$ & 5 & 5 & $\mathrm{U}$ & 5 & 5 & $\mathrm{U}$ & 5 & 5 & $\mathrm{U}$ & 5 \\
\hline Carbon tetrachloride & 2 & $\mathrm{~J}$ & 5 & 7 & & 5 & 9 & & 5 & 10 & & 5 \\
\hline Chlorobenzene & 5 & $\mathrm{U}$ & 5 & 5 & $\mathrm{U}$ & 5 & 5 & $\mathrm{U}$ & 5 & 5 & $\mathrm{U}$ & 5 \\
\hline Chloroethane & 10 & $\mathrm{U}$ & 10 & 10 & $\mathrm{U}$ & 10 & 10 & $\mathrm{U}$ & 10 & 10 & $\mathrm{U}$ & 10 \\
\hline Chloroform & 5 & $\mathrm{U}$ & 5 & 5 & $\mathrm{U}$ & 5 & 5 & $\mathrm{U}$ & 5 & 5 & $\mathrm{U}$ & 5 \\
\hline Chloromethane & 10 & $\mathrm{U}$ & 10 & 10 & $\mathrm{U}$ & 10 & 10 & $\mathrm{U}$ & 10 & 10 & U & 10 \\
\hline cis-1,2-Dichloroethylene & 5 & $\mathrm{U}$ & 5 & 5 & $\mathrm{U}$ & 5 & 5 & $\mathrm{U}$ & 5 & 5 & $\mathrm{U}$ & 5 \\
\hline cis-1,3-Dichloropropene & 5 & $\mathrm{U}$ & 5 & 5 & $\mathrm{U}$ & 5 & 5 & $\mathrm{U}$ & 5 & 5 & $\mathrm{U}$ & 5 \\
\hline Dibromochloromethane & 5 & $\mathrm{U}$ & 5 & 5 & $\mathrm{U}$ & 5 & 5 & $\mathrm{U}$ & 5 & 5 & $U$ & 5 \\
\hline Ethylbenzene & 5 & $\mathrm{U}$ & 5 & 5 & $\mathrm{U}$ & 5 & 5 & $\mathrm{U}$ & 5 & 5 & $\mathrm{U}$ & 5 \\
\hline Methylene chloride & 3 & $\mathrm{~J}$ & 5 & 10 & & 5 & 10 & & 5 & 5 & $\mathrm{U}$ & 5 \\
\hline Styrene & 5 & $\mathrm{U}$ & 5 & 5 & $\mathrm{U}$ & 5 & 5 & $\mathrm{U}$ & 5 & 5 & $\mathrm{U}$ & 5 \\
\hline Tetrachloroethene & 5 & $\mathrm{U}$ & 5 & 5 & $\mathrm{U}$ & 5 & 5 & $\mathrm{U}$ & 5 & 5 & $\mathrm{U}$ & 5 \\
\hline Toluene & 5 & $\mathrm{U}$ & 5 & 5 & $\mathrm{U}$ & 5 & 5 & $\mathrm{U}$ & 5 & 5 & $\mathrm{U}$ & 5 \\
\hline trans-1,2-Dichloroethylene & 5 & $\mathrm{U}$ & 5 & 5 & $\mathrm{U}$ & 5 & 5 & $\mathrm{U}$ & 5 & 5 & $\mathrm{U}$ & 5 \\
\hline trans-1,3-Dichloropropene & 5 & $\mathrm{U}$ & 5 & 5 & $\mathrm{U}$ & 5 & 5 & U & 5 & 5 & $\mathrm{U}$ & 5 \\
\hline Trichloroethene & 5 & $\mathrm{U}$ & 5 & 5 & $\mathrm{U}$ & 5 & 5 & $\mathrm{U}$ & 5 & 5 & $\mathrm{U}$ & 5 \\
\hline Vinyl chloride & 10 & $\mathrm{U}$ & 10 & 10 & U & 10 & 10 & $\mathrm{U}$ & 10 & 10 & $\mathrm{U}$ & 10 \\
\hline Xylenes (total) & 5 & $\mathrm{U}$ & 5 & 5 & $\mathrm{U}$ & 5 & 5 & $\mathrm{U}$ & 5 & 5 & $\mathrm{U}$ & 5 \\
\hline & & & & Attachn & & & 1 & & & Sheet No. & 55 & of 113 \\
\hline & & & & Origina & & J.M.Ca & pron & & & Date & & $07 / 07$ \\
\hline & & & & Checke & & T.M. Bl & akley & & & Dat & & \\
\hline & & & & Calc. $\mathrm{N}$ & & 0100B-C & $A-V 029$ & & & Rev. No & & 0 \\
\hline
\end{tabular}


Attachment 1. 118-B-1 Verification Sampling Results.

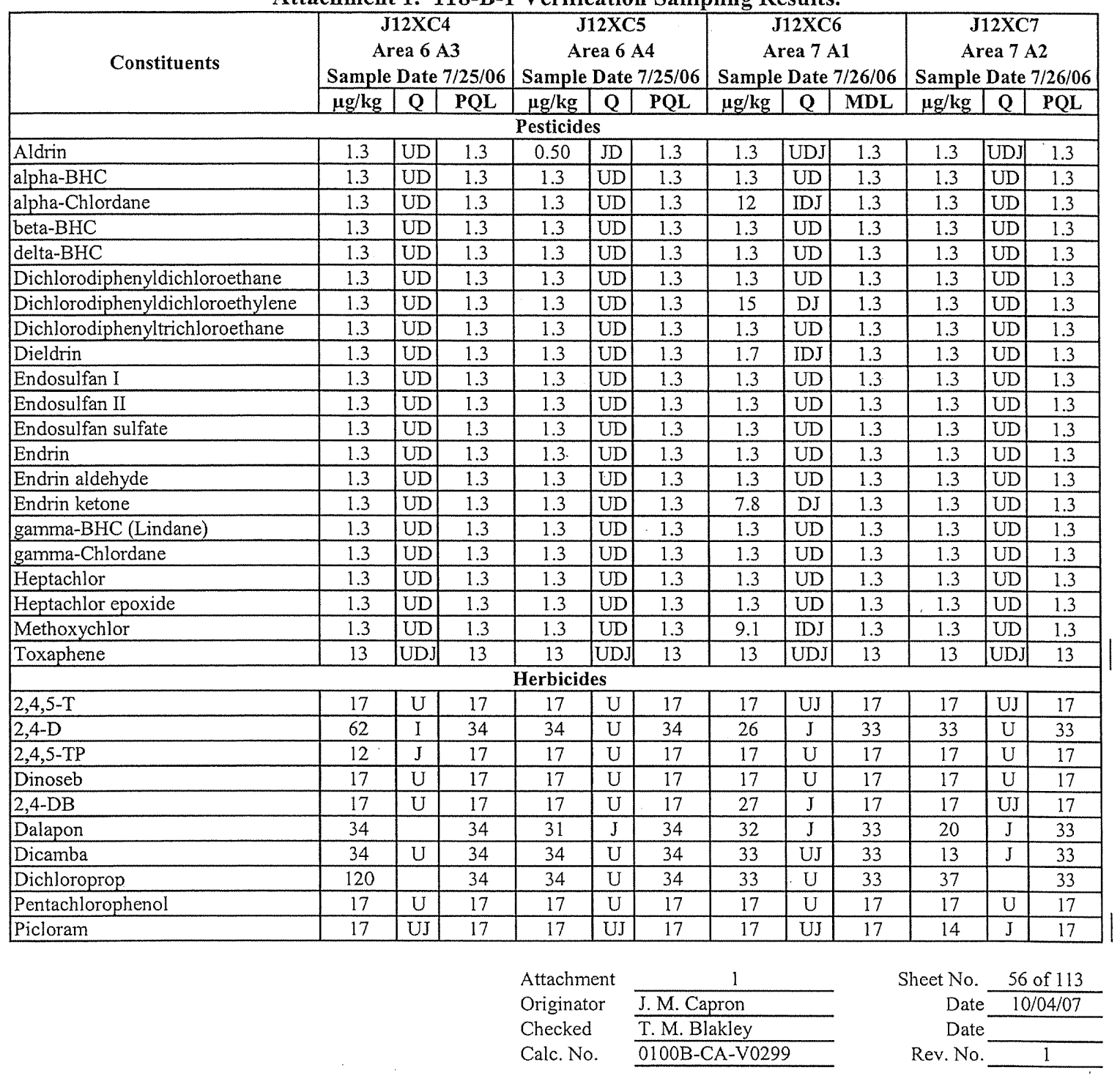


Attachment 1. 118-B-1 Verification Sampling Results.

\begin{tabular}{|c|c|c|c|c|c|c|c|c|c|c|c|c|}
\hline \multirow[t]{2}{*}{ Constituents } & \multicolumn{3}{|c|}{$\begin{array}{c}\text { J12XC8 } \\
\text { Area } 7 \text { A3 } \\
\text { Sample Date } 7 / 26 / 06 \\
\end{array}$} & \multicolumn{3}{|c|}{$\begin{array}{c}\text { J12XC9 } \\
\text { Area } 7 \text { A4 } \\
\text { Sample Date } 7 / 26 / 06\end{array}$} & \multicolumn{3}{|c|}{$\begin{array}{c}\text { J12WK0 } \\
\text { OB A1 } \\
\text { Sample Date } 7 / 11 / 06\end{array}$} & \multicolumn{3}{|c|}{\begin{tabular}{|c|} 
J12WK1 \\
OB A2 \\
Sample Date $7 / 11 / 06$ \\
\end{tabular}} \\
\hline & $\mu \mathrm{g} / \mathrm{kg}$ & $Q$ & PQL & $\mu \mathrm{g} / \mathrm{kg}$ & $\mathbf{Q}$ & PQL & $\mu \mathrm{g} / \mathrm{kg}$ & $\mathrm{Q}$ & PQL & $\mu \mathrm{g} / \mathrm{kg}$ & $\mathbf{Q}$ & $\mathrm{PQL}$ \\
\hline \multicolumn{13}{|c|}{ Polychlorinated Biphenyls } \\
\hline Aroclor-1016 & 13 & $\mathrm{U}$ & 13 & 13 & $\mathrm{U}$ & 13 & 13 & U & 13 & 13 & $\mathrm{U}$ & 13 \\
\hline Aroclor-1221 & 13 & $\mathrm{U}$ & 13 & 13 & $\mathrm{U}$ & 13 & 13 & $\mathrm{U}$ & 13 & 13 & $\mathrm{U}$ & 13 \\
\hline Aroclor- 1232 & 13 & $\mathrm{U}$ & 13 & 13 & $\mathrm{U}$ & 13 & 13 & $\mathrm{U}$ & 13 & 13 & $\mathrm{U}$ & 13 \\
\hline Aroclor- 1242 & 13 & $\mathrm{U}$ & 13 & 13 & $\dot{\mathrm{U}}$ & 13 & 13 & $\mathrm{U}$ & 13 & 13 & $\mathrm{U}$ & 13 \\
\hline Aroclor-1248 & 13 & $\mathrm{U}$ & 13 & 13 & $\mathrm{U}$ & 13 & 13 & $\mathrm{U}$ & 13 & 13 & $\mathrm{U}$ & 13 \\
\hline Aroclor-1254 & 13 & $\mathrm{U}$ & 13 & 13 & $\mathrm{U}$ & 13 & 13 & $\mathrm{U}$ & 13 & 13 & $\mathrm{U}$ & 13 \\
\hline Aroclor- 1260 & 13 & $\mathrm{U}$ & 13 & 13 & $U$ & 13 & 13 & $\mathrm{U}$ & 13 & 13 & $\mathrm{U}$ & 13 \\
\hline \multicolumn{13}{|c|}{ Semivolatile Organic Compounds } \\
\hline 1,2,4-Trichlorobenzene & 340 & UJ & 340 & 340 & UJ & 340 & 340 & $\mathrm{UJ}$ & 340 & 340 & UJ & 340 \\
\hline 1,2-Dichlorobenzene & 340 & $\mathrm{U}$ & 340 & 340 & $\mathrm{U}$ & 340 & 340 & $\mathrm{U}$ & 340 & 340 & $\mathrm{U}$ & 340 \\
\hline 1,3-Dichlorobenzene & 340 & $\mathrm{U}$ & 340 & 340 & $\mathrm{U}$ & 340 & 340 & $\mathrm{U}$ & 340 & 340 & U & 340 \\
\hline 1,4-Dichlorobenzene & 340 & $\mathrm{U}$ & 340 & 340 & $\mathrm{U}$ & 340 & 340 & $\mathrm{U}$ & 340 & 340 & $\mathrm{U}$ & 340 \\
\hline 2,4,5-Trichlorophenol & 840 & $\mathrm{U}$ & 840 & 840 & $\mathrm{U}$ & 840 & 840 & $\mathrm{U}$ & 840 & 840 & U & 840 \\
\hline 2,4,6-Trichlorophenol & 340 & $\mathrm{U}$ & 340 & 340 & $\mathrm{U}$ & 340 & 340 & $\mathrm{U}$ & 340 & 340 & $\mathrm{U}$ & 340 \\
\hline 2,4-Dichlorophenol & 340 & $\mathrm{U}$ & 340 & 340 & $\mathrm{U}$ & 340 & 340 & $\mathrm{U}$ & 340 & 340 & $\mathrm{U}$ & 340 \\
\hline 2,4-Dimethylphenol & 340 & $\mathrm{U}$ & 340 & 340 & $\mathrm{U}$ & 340 & 340 & UJ & 340 & 340 & UJ & 340 \\
\hline 2,4-Dinitrophenol & 840 & $\mathrm{UJ}$ & 840 & 840 & UJ & 840 & 840 & UR & 840 & 840 & UR & 840 \\
\hline 2,4-Dinitrotoluene & 340 & $\mathrm{U}$ & 340 & 340 & $\mathrm{U}$ & 340 & 340 & $\mathrm{U}$ & 340 & 340 & $U$ & 340 \\
\hline 2,6-Dinitrotoluene & 340 & $\mathrm{U}$ & 340 & 340 & $U$ & 340 & 340 & $\mathrm{U}$ & 340 & 340 & U & 340 \\
\hline 2-Chloronaphthalene & 340 & $\mathrm{U}$ & 340 & 340 & $\mathrm{U}$ & 340 & 340 & $\mathrm{U}$ & 340 & 340 & $\mathrm{U}$ & 340 \\
\hline 2-Chlorophenol & 340 & $\mathrm{U}$ & 340 & 340 & $\mathrm{U}$ & 340 & 340 & $\mathrm{U}$ & 340 & 340 & $\mathrm{U}$ & 340 \\
\hline 2-Methylnaphthalene & 340 & $\mathrm{U}$ & 340 & 340 & U & 340 & 340 & $\mathrm{UJ}$ & 340 & 340 & UJ & 340 \\
\hline 2-Methylphenol (cresol, o-) & 340 & $\mathrm{U}$ & 340 & 340 & $\mathrm{U}$ & 340 & 340 & $\mathrm{U}$ & 340 & 340 & U & 340 \\
\hline 2-Nitroaniline & 840 & $\mathrm{U}$ & 840 & 840 & $\mathrm{U}$ & 840 & 840 & $\mathrm{U}$ & 840 & 840 & $\mathrm{U}$ & 840 \\
\hline 2-Nitrophenol & 340 & $\mathrm{U}$ & 340 & 340 & $\mathrm{U}$ & 340 & 340 & $\mathrm{U}$ & 340 & 340 & $\mathrm{U}$ & 340 \\
\hline 3,3'-Dichlorobenzidine & 340 & $\mathrm{U}$ & 340 & 340 & $\mathrm{U}$ & 340 & 340 & $\mathrm{U}$ & 340 & 340 & $\mathrm{U}$ & 340 \\
\hline 3-Nitroaniline & 840 & $\mathrm{U}$ & 840 & 840 & $\mathrm{U}$ & 840 & 840 & $\mathrm{U}$ & 840 & 840 & $\mathrm{U}$ & 840 \\
\hline 4,6-Dinitro-2-methylphenol & 840 & $\mathrm{U}$ & 840 & 840 & $\mathrm{U}$ & 840 & 840 & $\mathrm{U}$ & 840 & 840 & $\mathrm{U}$ & 840 \\
\hline 4-Bromophenyl-phenylether & 340 & $\mathrm{U}$ & 340 & 340 & $\mathrm{U}$ & 340 & 340 & $\mathrm{U}$ & 340 & 340 & $\mathrm{U}$ & 340 \\
\hline 4-Chloro-3-methylphenol & 340 & $\mathrm{U}$ & 340 & 340 & $\mathrm{U}$ & 340 & 340 & $\mathrm{U}$ & 340 & 340 & $\mathrm{U}$ & 340 \\
\hline 4-Chloroaniline & 340 & $\mathrm{U}$ & 340 & 340 & $\mathrm{U}$ & 340 & 340 & $\mathrm{U}$ & 340 & 340 & $\mathrm{U}$ & 340 \\
\hline 4-Chlorophenyl-phenylether & 340 & $\mathrm{U}$ & 340 & 340 & $\mathrm{U}$ & 340 & 340 & $\mathrm{U}$ & 340 & 340 & $\mathrm{U}$ & 340 \\
\hline 4-Methylphenol (p-cresol) & 340 & $\mathrm{U}$ & 340 & 340 & $\mathrm{U}$ & 340 & 340 & $\mathrm{U}$ & 340 & 340 & $\mathrm{U}$ & 340 \\
\hline 4-Nitroaniline & 840 & $\mathrm{U}$ & 840 & 840 & $\mathrm{U}$ & 840 & 840 & $\mathrm{U}$ & 840 & 840 & $\mathrm{U}$ & 840 \\
\hline 4-Nitrophenol & 840 & $U$ & 840 & 840 & $\mathrm{U}$ & 840 & 840 & $\mathrm{U}$ & 840 & 840 & $\mathrm{U}$ & 840 \\
\hline Acenaphthene & 340 & $\mathrm{U}$ & 340 & 340 & $\mathrm{U}$ & 340 & 340 & $\mathrm{U}$ & 340 & 340 & $\bar{U}$ & 340 \\
\hline Acenaphthylene & 340 & $\mathrm{U}$ & 340 & 340 & $\mathrm{U}$ & 340 & 340 & $\mathrm{U}$ & 340 & 340 & $\mathrm{U}$ & 340 \\
\hline Anthracene & 340 & $\mathrm{U}$ & 340 & 340 & $\mathrm{U}$ & 340 & 340 & $\mathrm{U}$ & 340 & 340 & $\mathrm{U}$ & 340 \\
\hline Benzo(a)anthracene & 340 & $\mathrm{U}$ & 340 & 340 & $\mathrm{U}$ & 340 & 340 & $U$ & 340 & 340 & $\mathrm{U}$ & 340 \\
\hline Benzo(a)pyrene & 340 & $\bar{U}$ & 340 & 340 & $\mathrm{U}$ & 340 & 340 & $\mathrm{U}$ & 340 & 340 & $\mathrm{U}$ & 340 \\
\hline Benzo(b)fluoranthene & 340 & $\mathrm{U}$ & 340 & 340 & $\mathrm{U}$ & 340 & 340 & $\mathrm{U}$ & 340 & 340 & $U$ & 340 \\
\hline Benzo(g,h,i)perylene & 340 & $\mathrm{U}$ & 340 & 340 & $\mathrm{U}$ & 340 & 340 & $\mathrm{U}$ & 340 & 340 & $\mathrm{U}$ & 340 \\
\hline Benzo(k)fluoranthene & 340 & $\mathrm{U}$ & 340 & 340 & $\mathrm{U}$ & 340 & 340 & $\mathrm{U}$ & 340 & 340 & $\mathrm{U}$ & 340 \\
\hline bis(2-Chloro-1-methylethyl)ether & 340 & $\mathrm{U}$ & 340 & 340 & $\mathrm{U}$ & 340 & 340 & $\mathrm{U}$ & 340 & 340 & $\mathrm{U}$ & 340 \\
\hline bis(2-Chloroethoxy)methane & 340 & $\mathrm{U}$ & 340 & 340 & $\mathrm{U}$ & 340 & 340 & $\mathrm{U}$ & 340 & 340 & $\mathrm{U}$ & 340 \\
\hline bis(2-Chloroethyl)ether & 340 & U & 340 & 340 & $\mathrm{U}$ & 340 & 340 & $\mathrm{U}$ & 340 & 340 & $\mathrm{U}$ & 340 \\
\hline bis(2-Ethylhexyl)phthalate & 330 & $\mathrm{U}$ & 340 & 330 & $\mathrm{U}$ & 340 & 32 & $\mathrm{JB}$ & 340 & 30 & $\mathrm{JB}$ & 340 \\
\hline & & & & Attachn & & & 1 & & & neet No. & & of 113 \\
\hline & & & & Origina & & J.M. Ca & ron & & & Date & & $104 / 07$ \\
\hline & & & & Checke & & T.M. Bl & kley & & & Date & & \\
\hline & & & & Calc. $N$ & & $0100 \mathrm{~B}-\mathrm{C}$ & $\mathrm{A}-\mathrm{V} 02$ & & & Rev. No. & & 1 \\
\hline
\end{tabular}


Attachment 1. 118-B-1 Verification Sampling Results.

\begin{tabular}{|c|c|c|c|c|c|c|c|c|c|c|c|c|}
\hline \multirow[t]{2}{*}{ Constituents } & \multicolumn{3}{|c|}{$\begin{array}{c}\text { J12XC8 } \\
\text { Area } 7 \text { A3 } \\
\text { Sample Date } 7 / 26 / 06\end{array}$} & \multicolumn{3}{|c|}{$\begin{array}{c}\text { J12XC9 } \\
\text { Area } 7 \text { A4 } \\
\text { Sample Date } 7 / 26 / 06\end{array}$} & \multicolumn{3}{|c|}{$\begin{array}{c}\text { J12WK0 } \\
\text { OB A1 } \\
\text { Sample Date } 7 / 11 / 06\end{array}$} & \multicolumn{3}{|c|}{$\begin{array}{c}\text { J12WK1 } \\
\text { OB A2 } \\
\text { Sample Date } 7 / 11 / 06\end{array}$} \\
\hline & $\mu \mathrm{g} / \mathrm{kg}$ & $Q$ & PQL & $\mu \mathrm{g} / \mathrm{kg}$ & $\mathbf{Q}$ & PQL & $\mu \mathrm{g} / \mathrm{kg}$ & $Q$ & MDL & $\mu \mathrm{g} / \mathrm{kg}$ & $Q$ & PQL \\
\hline \multicolumn{13}{|c|}{ Semivolatile Organic Compounds (continued) } \\
\hline Butylbenzylphthalate & 340 & $\mathrm{U}$ & 340 & 340 & $\mathrm{U}$ & 340 & 340 & $\mathrm{U}$ & 340 & 340 & $\mathrm{U}$ & 340 \\
\hline Carbazole & 340 & $\mathrm{U}$ & 340 & 340 & $\mathrm{U}$ & 340 & 340 & $\mathrm{U}$ & 340 & 340 & $\mathrm{U}$ & 340 \\
\hline Chrysene & 340 & $\mathrm{U}$ & 340 & 340 & $\mathrm{U}$ & 340 & 340 & $\mathrm{U}$ & 340 & 340 & $\mathrm{U}$ & 340 \\
\hline Di-n-butylphthalate & 330 & $U$ & 340 & 330 & $\mathrm{U}$ & 340 & 340 & $\mathrm{U}$ & 340 & 340 & $\mathrm{U}$ & 340 \\
\hline Di-n-octylphthalate & 340 & $\mathrm{U}$ & 340 & 340 & $U$ & 340 & 340 & $\mathrm{U}$ & 340 & 340 & $\mathrm{U}$ & 340 \\
\hline Dibenz(a,h)anthracene & 340 & $\mathrm{U}$ & 340 & 340 & U & 340 & 340 & $\mathrm{U}$ & 340 & 340 & $\mathrm{U}$ & 340 \\
\hline Dibenzofuran & 340 & $\mathrm{U}$ & 340 & 340 & $\mathrm{U}$ & 340 & 340 & $\mathrm{U}$ & 340 & 340 & $\mathrm{U}$ & 340 \\
\hline Diethylphthalate & 340 & $\mathrm{U}$ & 340 & 340 & U & 340 & 340 & $\mathrm{U}$ & 340 & 340 & $\bar{U}$ & 340 \\
\hline Dimethylphthalate & 340 & $\mathrm{U}$ & 340 & 340 & $\mathrm{U}$ & 340 & 340 & $\mathrm{U}$ & 340 & 340 & $\mathrm{U}$ & 340 \\
\hline Fluoranthene & 340 & $\mathrm{U}$ & 340 & 340 & U & 340 & 340 & $\mathrm{U}$ & 340 & 340 & $\mathrm{U}$ & 340 \\
\hline Fluorene & 340 & $U$ & 340 & 340 & $\mathrm{U}$ & 340 & 340 & $\mathrm{U}$ & 340 & 340 & $\mathrm{U}$ & 340 \\
\hline Hexachlorobenzene & 340 & $\mathrm{U}$ & 340 & 340 & U & 340 & 340 & $\mathrm{U}$ & 340 & 340 & $\mathrm{U}$ & 340 \\
\hline Hexachlorobutadiene & 340 & $\mathrm{U}$ & 340 & 340 & $\mathrm{U}$ & 340 & 340 & $\mathrm{U}$ & 340 & 340 & $\mathrm{U}$ & 340 \\
\hline Hexachlorocyclopentadiene & 340 & $\mathrm{U}$ & 340 & 340 & $\mathrm{U}$ & 340 & 340 & $\mathrm{U}$ & 340 & 340 & $\mathrm{U}$ & 340 \\
\hline Hexachloroethane & 340 & $\mathrm{U}$ & 340 & 340 & U & 340 & 340 & $\mathrm{U}$ & 340 & 340 & $\mathrm{U}$ & 340 \\
\hline Indeno(1,2,3-cd)pyrene & 340 & $\mathrm{U}$ & 340 & 340 & $\mathrm{U}$ & 340 & 340 & $\mathrm{U}$ & 340 & 340 & $\mathrm{U}$ & 340 \\
\hline Isophorone & 340 & $\mathrm{U}$ & 340 & 340 & $\mathrm{U}$ & 340 & 340 & UJ & 340 & 340 & UJ & 340 \\
\hline $\mathrm{N}$-Nitroso-di-n-dipropylamine & 340 & $\mathrm{U}$ & 340 & 340 & $\mathrm{U}$ & 340 & 340 & $\mathrm{U}$ & 340 & 340 & $\mathrm{U}$ & 340 \\
\hline N-Nitrosodiphenylamine & 340 & $\mathrm{U}$ & 340 & 340 & $\mathrm{U}$ & 340 & 340 & $\mathrm{U}$ & 340 & 340 & $\mathrm{U}$ & 340 \\
\hline Naphthalene & 340 & $\mathrm{U}$ & 340 & 340 & $\mathrm{U}$ & 340 & 340 & $\mathrm{U}$ & 340 & 340 & $\mathrm{U}$ & 340 \\
\hline Nitrobenzene & 340 & $\mathrm{U}$ & 340 & 340 & U & 340 & 340 & $\mathrm{U}$ & 340 & 340 & $\mathrm{U}$ & 340 \\
\hline Pentachlorophenol & 840 & $\mathrm{U}$ & 840 & 840 & $\mathrm{U}$ & 840 & 840 & $\mathrm{U}$ & 840 & 840 & $\mathrm{U}$ & 840 \\
\hline Phenanthrene & 340 & $\mathrm{U}$ & 340 & 340 & $\mathrm{U}$ & 340 & 340 & $\mathrm{U}$ & 340 & 340 & $\mathrm{U}$ & 340 \\
\hline Phenol & 330 & $\mathrm{U}$ & 340 & 340 & $\mathrm{U}$ & 340 & 340 & $\mathrm{U}$ & 340 & 340 & $\mathrm{U}$ & 340 \\
\hline Pyrene & 340 & $\mathrm{U}$ & 340 & 340 & $\mathrm{U}$ & 340 & 340 & $\mathrm{U}$ & 340 & 340 & $\mathrm{U}$ & 340 \\
\hline
\end{tabular}

Attachment

Originato

Checked

Calc. No.
J. M. Capron

T. M. Blakley

0100B-CA-V0299
Sheet No. 58 of 113

Date $\overline{10 / 04 / 07}$

Date

Rev. No. 1 
Attachment 1. 118-B-1 Verification Sampling Results.

\begin{tabular}{|c|c|c|c|c|c|c|c|c|c|c|c|c|}
\hline \multirow[t]{2}{*}{ Constituents } & \multicolumn{3}{|c|}{$\begin{array}{c}\text { J12XC8 } \\
\text { Area } 7 \text { A3 } \\
\text { Sample Date } 7 / 26 / 06\end{array}$} & \multicolumn{3}{|c|}{$\begin{array}{c}\text { J12XC9 } \\
\text { Area } 7 \text { A4 } \\
\text { Sample Date } 7 / 26 / 06\end{array}$} & \multicolumn{3}{|c|}{$\begin{array}{c}\text { J12WK0 } \\
\text { OB A1 } \\
\text { Sample Date } 7 / 11 / 06\end{array}$} & \multicolumn{3}{|c|}{$\begin{array}{c}\text { J12WK1 } \\
\text { OB A2 } \\
\text { Sample Date } 7 / 11 / 06 \\
\end{array}$} \\
\hline & $\mu \mathrm{g} / \mathrm{kg}$ & $Q$ & PQL & $\mu \mathrm{g} / \mathrm{kg}$ & $\mathbf{Q}$ & PQL & $\mu \mathrm{g} / \mathrm{kg}$ & $\mathbf{Q}$ & MDL & $\mu \mathrm{g} / \mathrm{kg}$ & $\mathbf{Q}$ & $\mathrm{PQL}$ \\
\hline \multicolumn{13}{|c|}{ Volatile Organic Compounds } \\
\hline 1,1,1-Trichloroethane & 5 & $\mathrm{U}$ & 5 & 5 & $\mathrm{U}$ & 5 & 5 & $\mathrm{U}$ & 5 & 5 & $\mathrm{U}$ & 5 \\
\hline 1,1,2,2-Tetrachloroethane & 5 & $\mathrm{U}$ & 5 & 5 & $\mathrm{U}$ & 5 & 5 & $\mathrm{U}$ & 5 & 5 & $\mathrm{U}$ & 5 \\
\hline 1,1,2-Trichloroethane & 5 & $\mathrm{U}$ & 5 & 5 & $\mathrm{U}$ & 5 & 5 & $\mathrm{U}$ & 5 & 5 & $\mathrm{U}$ & 5 \\
\hline 1,1-Dichloroethane & 5 & $\mathrm{U}$ & 5 & 5 & $\mathrm{U}$ & 5 & 5 & $\mathrm{U}$ & 5 & 5 & $\bar{U}$ & 5 \\
\hline 1,1-Dichloroethene & 5 & $\mathrm{U}$ & 5 & 5 & $\mathrm{U}$ & 5 & 5 & $\mathrm{U}$ & 5 & 5 & $\mathrm{U}$ & 5 \\
\hline 1,2-Dichloroethane & 5 & $\mathrm{U}$ & 5 & 5 & $\mathrm{U}$ & 5 & 5 & $\mathrm{U}$ & 5 & 5 & $\mathrm{U}$ & 5 \\
\hline 1,2-Dichloroethene (total) & 5 & $\mathrm{U}$ & 5 & 5 & $\mathrm{U}$ & 5 & 5 & U & 5 & 5 & $\mathrm{U}$ & 5 \\
\hline 1,2-Dichloropropane & 5 & $\mathrm{U}$ & 5 & 5 & $\mathrm{U}$ & 5 & 5 & $\mathrm{U}$ & 5 & 5 & U & 5 \\
\hline 2-Butanone & 10 & $\mathrm{U}$ & 10 & 10 & $\mathrm{U}$ & 10 & 10 & $\mathrm{U}$ & 10 & 10 & $\mathrm{U}$ & 10 \\
\hline 2-Hexanone & 10 & $\mathrm{U}$ & 10 & 10 & $\mathrm{U}$ & 10 & 10 & $\mathrm{U}$ & 10 & 10 & $\mathrm{U}$ & 10 \\
\hline 4-Methyl-2-pentanone & 10 & $\mathrm{U}$ & 10 & 10 & $\mathrm{U}$ & 10 & 10 & $U$ & 10 & 10 & $\bar{U}$ & 10 \\
\hline Acetone & 10 & $\mathrm{U}$ & 10 & 10 & $\mathrm{U}$ & 10 & 10 & $\mathrm{U}$ & 10 & 10 & $\mathrm{U}$ & 10 \\
\hline Benzene & 5 & $\mathrm{U}$ & 5 & 5 & U & 5 & 5 & $\mathrm{U}$ & 5 & 5 & $\mathrm{U}$ & 5 \\
\hline Bromodichloromethane & 5 & $\mathrm{U}$ & 5 & 5 & $\mathrm{U}$ & 5 & 5 & $\mathrm{U}$ & 5 & 5 & $\mathrm{U}$ & 5 \\
\hline Bromoform & 5 & $\mathrm{U}$ & 5 & 5 & $\mathrm{U}$ & 5 & 5 & $\mathrm{U}$ & 5 & 5 & $\mathrm{U}$ & 5 \\
\hline Bromomethane & 10 & $\mathrm{U}$ & 10 & 10 & $\mathrm{U}$ & 10 & 10 & $\mathrm{U}$ & 10 & 10 & $\mathrm{U}$ & 10 \\
\hline Carbon disulfide & 5 & U & 5 & 5 & $\mathrm{U}$ & 5 & 5 & $\mathrm{U}$ & 5 & 5 & $\mathrm{U}$ & 5 \\
\hline Carbon tetrachloride & 5 & $\mathrm{U}$ & 5 & 10 & & 5 & 5 & $\mathrm{U}$ & 5 & 5 & $\mathrm{U}$ & 5 \\
\hline Chlorobenzene & 5 & $\mathrm{U}$ & 5 & 5 & $\mathrm{U}$ & 5 & 5 & $\mathrm{U}$ & 5 & 5 & $\mathrm{U}$ & 5 \\
\hline Chloroethane & 10 & $\mathrm{U}$ & 10 & 10 & U & 10 & 10 & $\mathrm{U}$ & 10 & 10 & $\mathrm{U}$ & 10 \\
\hline Chloroform & 5 & $\mathrm{U}$ & 5 & 5 & $\mathrm{U}$ & 5 & 5 & $\mathrm{U}$ & 5 & 5 & $\mathrm{U}$ & 5 \\
\hline Chloromethane & 10 & $\mathrm{U}$ & 10 & 10 & $\mathrm{U}$ & 10 & 10 & $\mathrm{U}$ & 10 & 10 & $\mathrm{U}$ & 10 \\
\hline cis-1,2-Dichloroethylene & 5 & $\mathrm{U}$ & 5 & 5 & $\mathrm{U}$ & 5 & 5 & $\mathrm{U}$ & 5 & 5 & $\mathrm{U}$ & 5 \\
\hline cis-1,3-Dichloropropene & 5 & $\mathrm{U}$ & 5 & 5 & $\mathrm{U}$ & 5 & 5 & $\mathrm{U}$ & 5 & 5 & $\mathrm{U}$ & 5 \\
\hline Dibromochloromethane & 5 & $\mathrm{U}$ & 5 & 5 & $\mathrm{U}$ & 5 & 5 & $\mathrm{U}$ & 5 & 5 & $\mathrm{U}$ & 5 \\
\hline Ethylbenzene & 5 & $\mathrm{U}$ & 5 & 5 & $\mathrm{U}$ & 5 & 5 & $\mathrm{U}$ & 5 & 5 & $\mathrm{U}$ & 5 \\
\hline Methylene chloride & 5 & $\mathrm{U}$ & 5 & 6 & & 5 & 9 & $\mathrm{BJ}$ & 5 & 6 & $\mathrm{BJ}$ & 5 \\
\hline Styrene & 5 & $\mathrm{U}$ & 5 & 5 & $U$ & 5 & 5 & $\mathrm{U}$ & 5 & 5 & $\mathrm{U}$ & 5 \\
\hline Tetrachloroethene & 5 & $\mathrm{U}$ & 5 & 5 & $\mathrm{U}$ & 5 & 5 & $\mathrm{U}$ & 5 & 5 & $\mathrm{U}$ & 5 \\
\hline Toluene & 5 & $\mathrm{U}$ & 5 & 5 & $\mathrm{U}$ & 5 & 5 & $\mathrm{U}$ & 5 & 5 & $\mathrm{U}$ & 5 \\
\hline trans-1,2-Dichloroethylene & 5 & $\mathrm{U}$ & 5 & 5 & $\mathrm{U}$ & 5 & 5 & $\mathrm{U}$ & 5 & 5 & $\mathrm{U}$ & 5 \\
\hline trans-1,3-Dichloropropene & 5 & $\mathrm{U}$ & 5 & 5 & $\mathrm{U}$ & 5 & 5 & $\mathrm{U}$ & 5 & 5 & U & 5 \\
\hline Trichloroethene & 5 & $\mathrm{U}$ & 5 & 5 & $\mathrm{U}$ & 5 & 5 & $\mathrm{U}$ & 5 & 5 & $\mathrm{U}$ & 5 \\
\hline Vinyl chloride & 10 & $\mathrm{U}$ & 10 & 10 & $\mathrm{U}$ & 10 & 10 & $\mathrm{U}$ & 10 & 10 & $\mathrm{U}$ & 10 \\
\hline Xylenes (total) & 5 & $\mathrm{U}$ & 5 & 5 & $\mathrm{U}$ & 5 & 5 & $\mathrm{U}$ & 5 & 5 & $\mathrm{U}$ & 5 \\
\hline & & & & Attachn & & & 1 & & & Sheet No. & & of 113 \\
\hline & & & & Originat & & J.M. Ca & oron & & & Date & & $04 / 07$ \\
\hline & & & & Checke & & T.M. B & akley & & & Date & & \\
\hline & & & & Calc. N & & $0100 \mathrm{~B}-\mathrm{C}$ & $\mathrm{A}-\mathrm{V} 029$ & & & Rev. No & & 1 \\
\hline
\end{tabular}


Attachment 1. 118-B-1 Verification Sampling Results.

\begin{tabular}{|c|c|c|c|c|c|c|c|c|c|c|c|c|}
\hline \multirow[t]{2}{*}{ Constituents } & \multicolumn{3}{|c|}{\begin{tabular}{|c|} 
J12XC8 \\
Area 7 A3 \\
Sample Date $7 / 26 / 06$
\end{tabular}} & \multicolumn{3}{|c|}{$\begin{array}{c}\text { J12XC9 } \\
\text { Area } 7 \text { A4 } \\
\text { Sample Date } 7 / 26 / 06\end{array}$} & \multicolumn{3}{|c|}{$\begin{array}{c}\text { J12WK0 } \\
\text { OB A1 } \\
\text { Sample Date } 7 / 11 / 06\end{array}$} & \multicolumn{3}{|c|}{$\begin{array}{c}\text { J12WK1 } \\
\text { OB A2 } \\
\text { Sample Date } 7 / 11 / 06\end{array}$} \\
\hline & $\mu \mathrm{g} / \mathrm{kg}$ & $\mathbf{Q}$ & PQL & $\mu \mathrm{g} / \mathrm{kg}$ & $\mathbf{Q}$ & PQL & $\mu \mathrm{g} / \mathrm{kg}$ & Q & MDL & $\mu \mathrm{g} / \mathrm{kg}$ & $\mathbf{Q}$ & PQL \\
\hline \multicolumn{13}{|c|}{ Pesticides } \\
\hline Aldrin & 1.3 & UDJ & 1.3 & 1.3 & UDJ & 1.3 & 1.3 & UD & 1.3 & 1.3 & UD & 1.3 \\
\hline alpha-BHC & 1.3 & UD & 1.3 & 1.3 & UD & 1.3 & 1.3 & UDJ & 1.3 & 1.3 & UDJ & 1.3 \\
\hline alpha-Chlordane & 1.3 & UD & 1.3 & 1.3 & UD & 1.3 & 1.3 & UD & 1.3 & 1.3 & UD & 1.3 \\
\hline beta-BHC & 1.3 & UD & 1.3 & 1.3 & UD & 1.3 & 0.64 & $\mathrm{JD}$ & 1.3 & 1.3 & UD & 1.3 \\
\hline delta-BHC & 1.3 & UD & 1.3 & 1.3 & UD & 1.3 & 1.3 & UDJ & 1.3 & 1.3 & UDJ & 1.3 \\
\hline Dichlorodiphenyldichloroethane & 1.3 & UD & 1.3 & 1.3 & UD & 1.3 & 1.3 & UD & 1.3 & 1.3 & UD & 1.3 \\
\hline Dichlorodiphenyldichloroethylene & 1.3 & UD & 1.3 & 15 & DJ & 1.3 & 1.3 & UDJ & 1.3 & 1.3 & UDJ & 1.3 \\
\hline Dichlorodiphenyltrichloroethane & 1.3 & UD & 1.3 & 1.3 & UD & 1.3 & 1.3 & UD & 1.3 & 1.3 & UD & 1.3 \\
\hline Dieldrin & 1.3 & UD & 1.3 & 1.3 & UD & 1.3 & 1.3 & UD & 1.3 & 1.3 & UD & 1.3 \\
\hline Endosulfan I & 1.3 & UD & 1.3 & 1.3 & UD & 1.3 & 1.3 & UD & 1.3 & 1.3 & UD & 1.3 \\
\hline Endosulfan II & 1.3 & UD & 1.3 & 1.3 & UD & 1.3 & 1.3 & UD & 1.3 & 1.3 & UD & 1.3 \\
\hline Endosulfan sulfate & 1.3 & UD & 1.3 & 1.3 & UD & 1.3 & 1.3 & UD & 1.3 & 1.3 & UD & 1.3 \\
\hline Endrin & 1.3 & UD & 1.3 & 1.3 & UD & 1.3 & 1.3 & UD & 1.3 & 1.3 & UD & 1.3 \\
\hline Endrin aldehyde & 1.3 & UD & 1.3 & 1.3 & UD & 1.3 & 1.3 & UD & 1.3 & 1.3 & UD & 1.3 \\
\hline Endrin ketone & 1.3 & UD & 1.3 & 1.3 & UD & 1.3 & 1.3 & UD & 1.3 & 1.3 & UD & 1.3 \\
\hline gamma-BHC (Lindane) & 1.3 & UD & 1.3 & 1.3 & UD & 1.3 & 1.3 & UDJ & 1.3 & 1.3 & UDJ & 1.3 \\
\hline gamma-Chlordane & 1.3 & UD & 1.3 & 1.3 & UD & 1.3 & 1.3 & UD & 1.3 & 1.3 & UD & 1.3 \\
\hline Heptachlor & 1.3 & UD & 1.3 & 1.3 & UD & 1.3 & 1.3 & UD & 1.3 & 1.3 & UD & 1.3 \\
\hline Heptachlor epoxide & 1.3 & UD & 1.3 & 1.3 & UD & 1.3 & 1.3 & UD & 1.3 & 1.3 & UD & 1.3 \\
\hline Methoxychlor & 1.3 & UD & 1.3 & 1.3 & UD & 1.3 & 2.7 & $\mathrm{D}$ & 1.3 & 1.3 & UD & 1.3 \\
\hline Toxaphene & 13 & UDJ & 13 & 13 & UDJ & 13 & 13 & UD & 13 & 13 & UD & 13 \\
\hline \multicolumn{13}{|c|}{ Herbicides } \\
\hline $2,4,5-T$ & 17 & $\mathrm{UJ}$ & 17 & 17 & UJ & 17 & 17 & $\mathrm{UJ}$ & 17 & 17 & UJ & 17 \\
\hline 2,4-D & 20 & $\mathrm{~J}$ & 34 & 13 & $\mathrm{~J}$ & 34 & 34 & $\mathrm{UJ}$ & 34 & 34 & UJ & 34 \\
\hline $2,4,5-\mathrm{TP}$ & 17 & $\mathrm{U}$ & 17 & 17 & $\mathrm{U}$ & 17 & 17 & UJ & 17 & 17 & UJ & 17 \\
\hline Dinoseb & 17 & $\mathrm{U}$ & 17 & 17 & $\mathrm{U}$ & 17 & 17 & $\mathrm{UJ}$ & 17 & 17 & UJ & 17 \\
\hline 2,4-DB & 17 & $\mathrm{UJ}$ & 17 & 17 & $\mathrm{UJ}$ & 17 & 17 & UJ & 17 & 17 & UJ & 17 \\
\hline Dalapon & 16 & $\mathrm{~J}$ & 34 & 34 & $\mathrm{U}$ & 34 & 34 & UJ & 34 & 34 & UJ & 34 \\
\hline Dicamba & 34 & UJ & 34 & 34 & UJ & 34 & 12 & $\mathrm{~J}$ & 34 & 34 & UJ & 34 \\
\hline Dichloroprop & 34 & $\mathrm{U}$ & 34 & 60 & & 34 & 34 & UJ & 34 & 34 & UJ & 34 \\
\hline Pentachlorophenol & 17 & $\mathrm{U}$ & 17 & 17 & $\mathrm{U}$ & 17 & 17 & $\mathrm{UJ}$ & 17 & 17 & UJ & 17 \\
\hline Picloram & 17 & UJ & 17 & 17 & $\mathrm{UJ}$ & 17 & 17 & UR & 17 & 17 & UR & 17 \\
\hline
\end{tabular}

Attachment

Originator

Checked

Calc. No.

\begin{tabular}{l}
$\frac{1}{\text { J.M. Capron }}$ \\
$\frac{\text { T. M. Blakley }}{\text { 0100B-CA-V0299 }}$ \\
\hline
\end{tabular}

Sheet No. 60 of 113

Date $10 / 04 / 07$

Date

Rev. No. $\frac{1}{1}$ 
Attachment 1. 118-B-1 Verification Sampling Results.

\begin{tabular}{|c|c|c|c|c|c|c|c|c|c|c|c|c|}
\hline \multirow[t]{2}{*}{ Constituents } & \multicolumn{3}{|c|}{$\begin{array}{c}\text { J12WK2 } \\
\text { OB A3 } \\
\text { Sample Date } 7 / 11 / 06 \\
\end{array}$} & \multicolumn{3}{|c|}{\begin{tabular}{|c|} 
J12WK3 \\
OB A4 \\
Sample Date $7 / 11 / 06$ \\
\end{tabular}} & \multicolumn{3}{|c|}{$\begin{array}{c}\text { J12WK4 } \\
\text { OB B5 } \\
\text { Sample Date } 7 / 11 / 06 \\
\end{array}$} & \multicolumn{3}{|c|}{$\begin{array}{c}\text { J12WK5 } \\
\text { OB B6 } \\
\text { Sample Date } 7 / 11 / 06 \\
\end{array}$} \\
\hline & $\mu \mathrm{g} / \mathrm{kg}$ & Q & PQL & $\mu \mathrm{g} / \mathrm{kg}$ & $Q$ & PQL & $\mu \mathrm{g} / \mathrm{kg}$ & $\mathrm{Q}$ & PQL & $\mu \mathrm{g} / \mathrm{kg}$ & $\mathrm{Q}$ & PQL \\
\hline \multicolumn{13}{|c|}{ Polychlorinated Biphenyls } \\
\hline Aroclor- 1016 & 13 & $\mathrm{U}$ & 13 & 13 & $\mathrm{U}$ & 13 & 13 & $\mathrm{U}$ & 13 & 13 & $\mathrm{U}$ & 13 \\
\hline Aroclor-1221 & 13 & $\mathrm{U}$ & 13 & 13 & $\mathrm{U}$ & 13 & 13 & $\mathrm{U}$ & 13 & 13 & $\mathrm{U}$ & 13 \\
\hline Aroclor-1232 & 13 & $\mathrm{U}$ & 13 & 13 & $U$ & 13 & 13 & $U$ & 13 & 13 & $\mathrm{U}$ & 13 \\
\hline Aroclor-1242 & 13 & $\mathrm{U}$ & 13 & 13 & $U$ & 13 & 13 & $U$ & 13 & 13 & $\mathrm{U}$ & 13 \\
\hline Aroclor-1248 & 13 & $\mathrm{U}$ & 13 & 13 & $U$ & 13 & 13 & $\mathrm{U}$ & 13 & 13 & $U$ & 13 \\
\hline Aroclor-1254 & 13 & $\mathrm{U}$ & 13 & 13 & $\mathrm{U}$ & 13 & 13 & $\mathrm{U}$ & 13 & 13 & $\mathrm{U}$ & 13 \\
\hline Aroclor- 1260 & 13 & $\mathrm{U}$ & 13 & 13 & $U$ & 13 & 13 & $\mathrm{U}$ & 13 & 13 & $\mathrm{U}$ & 13 \\
\hline \multicolumn{13}{|c|}{ Semivolatile Organic Compounds } \\
\hline 1,2,4-Trichlorobenzene & 330 & UJ & 330 & 340 & $\mathrm{UJ}$ & 340 & 330 & UJ & 330 & 330 & UJ & 330 \\
\hline 1,2-Dichlorobenzene & 330 & $\mathrm{U}$ & 330 & 340 & $\mathrm{U}$ & 340 & 330 & $\mathrm{U}$ & 330 & 330 & $\mathrm{U}$ & 330 \\
\hline 1,3-Dichlorobenzene & 330 & $\mathrm{U}$ & 330 & 340 & $\mathrm{U}$ & 340 & 330 & $\mathrm{U}$ & 330 & 330 & $\mathrm{U}$ & 330 \\
\hline 1,4-Dichlorobenzene & 330 & $\mathrm{U}$ & 330 & 340 & $\mathrm{U}$ & 340 & 330 & $\mathrm{U}$ & 330 & 330 & $\mathrm{U}$ & 330 \\
\hline 2,4,5-Trichlorophenol & 840 & $U$ & 840 & 840 & $\mathrm{U}$ & 840 & 840 & $\mathrm{U}$ & 840 & 840 & $\mathrm{U}$ & 840 \\
\hline 2,4,6-Trichlorophenol & 330 & $\mathrm{U}$ & 330 & 340 & $\mathrm{U}$ & 340 & 330 & $U$ & 330 & 330 & $\mathrm{U}$ & 330 \\
\hline 2,4-Dichlorophenol & 330 & $\mathrm{U}$ & 330 & 340 & $\mathrm{U}$ & 340 & 330 & $\mathrm{U}$ & 330 & 330 & U & 330 \\
\hline 2,4-Dimethylphenol & 330 & $\mathrm{UJ}$ & 330 & 340 & $\mathrm{UJ}$ & 340 & 330 & UJ & 330 & 330 & UJ & 330 \\
\hline 2,4-Dinitrophenol & 840 & UR & 840 & 840 & UR & 840 & 840 & UR & 840 & 840 & UR & 840 \\
\hline 2,4-Dinitrotoluene & 330 & $\mathrm{U}$ & 330 & 340 & $\mathrm{U}$ & 340 & 330 & $\mathrm{U}$ & 330 & 330 & $\mathrm{U}$ & 330 \\
\hline 2,6-Dinitrotoluene & 330 & $\mathrm{U}$ & 330 & 340 & $\mathrm{U}$ & 340 & 330 & $\mathrm{U}$ & 330 & 330 & $\mathrm{U}$ & 330 \\
\hline 2-Chloronaphthalene & 330 & $\mathrm{U}$ & 330 & 340 & $\mathrm{U}$ & 340 & 330 & $\mathrm{U}$ & 330 & 330 & $\mathrm{U}$ & 330 \\
\hline 2-Chlorophenol & 330 & $\mathrm{U}$ & 330 & 340 & $\mathrm{U}$ & 340 & 330 & $\mathrm{U}$ & 330 & 330 & $\mathrm{U}$ & 330 \\
\hline 2-Methylnaphthalene & 330 & UJ & 330 & 340 & UJ & 340 & 330 & UJ & 330 & 330 & UJ & 330 \\
\hline 2-Methylphenol (cresol, o-) & 330 & $U$ & 330 & 340 & $\mathrm{U}$ & 340 & 330 & $\mathrm{U}$ & 330 & 330 & $\mathrm{U}$ & 330 \\
\hline 2-Nitroaniline & 840 & $\mathrm{U}$ & 840 & 840 & $\mathrm{U}$ & 840 & 840 & $\mathrm{U}$ & 840 & 840 & U & 840 \\
\hline 2-Nitrophenol & 330 & $\mathrm{U}$ & 330 & 340 & $\mathrm{U}$ & 340 & 330 & $\mathrm{U}$ & 330 & 330 & $\mathrm{U}$ & 330 \\
\hline 3,3'-Dichlorobenzidine & 330 & $\mathrm{U}$ & 330 & 340 & $\mathrm{U}$ & 340 & 330 & $\mathrm{U}$ & 330 & 330 & $\mathrm{U}$ & 330 \\
\hline 3-Nitroaniline & 840 & $\mathrm{U}$ & 840 & 840 & $\mathrm{U}$ & 840 & 840 & $\mathrm{U}$ & 840 & 840 & $\mathrm{U}$ & 840 \\
\hline 4,6-Dinitro-2-methylphenol & 840 & $\mathrm{U}$ & 840 & 840 & $\mathrm{U}$ & 840 & 840 & $\mathrm{U}$ & 840 & 840 & $\mathrm{U}$ & 840 \\
\hline 4-Bromophenyl-phenylether & 330 & $\mathrm{U}$ & 330 & 340 & $\mathrm{U}$ & 340 & 330 & $U$ & 330 & 330 & $\mathrm{U}$ & 330 \\
\hline 4-Chloro-3-methylphenol & 330 & $\mathrm{U}$ & 330 & 340 & $\mathrm{U}$ & 340 & 330 & $U$ & 330 & 330 & $\mathrm{U}$ & 330 \\
\hline 4-Chloroaniline & 330 & $\mathrm{U}$ & 330 & 340 & $\mathrm{U}$ & 340 & 330 & $\mathrm{U}$ & 330 & 330 & $\mathrm{U}$ & 330 \\
\hline 4-Chlorophenyl-phenylether & 330 & $\mathrm{U}$ & 330 & 340 & $\mathrm{U}$ & 340 & 330 & $\mathrm{U}$ & 330 & 330 & $\mathrm{U}$ & 330 \\
\hline 4-Methylphenol (p-cresol) & 330 & $\mathrm{U}$ & 330 & 340 & $\mathrm{U}$ & 340 & 330 & $\mathrm{U}$ & 330 & 330 & $\mathrm{U}$ & 330 \\
\hline 4-Nitroaniline & 840 & $\mathrm{U}$ & 840 & 840 & $\mathrm{U}$ & 840 & 840 & $\mathrm{U}$ & 840 & 840 & $\mathrm{U}$ & 840 \\
\hline 4-Nitrophenol & 840 & $\mathrm{U}$ & 840 & 840 & $\mathrm{U}$ & 840 & 840 & $\mathrm{U}$ & 840 & 840 & U & 840 \\
\hline Acenaphthene & 330 & $\mathrm{U}$ & 330 & 340 & $\mathrm{U}$ & 340 & 330 & $\mathrm{U}$ & 330 & 330 & U & 330 \\
\hline Acenaphthylene & 330 & $\mathrm{U}$ & 330 & 340 & $\mathrm{U}$ & 340 & 330 & $\mathrm{U}$ & 330 & 330 & $\mathrm{U}$ & 330 \\
\hline Anthracene & 330 & $\mathrm{U}$ & 330 & 340 & $\mathrm{U}$ & 340 & 330 & $\mathrm{U}$ & 330 & 330 & $\mathrm{U}$ & 330 \\
\hline Benzo(a)anthracene & 330 & U & 330 & 340 & $\mathrm{U}$ & 340 & 330 & $\mathrm{U}$ & 330 & 330 & $\mathrm{U}$ & 330 \\
\hline Benzo(a)pyrene & 330 & $\mathrm{U}$ & 330 & 340 & $\mathrm{U}$ & 340 & 330 & $\mathrm{U}$ & 330 & 330 & $\mathrm{U}$ & 330 \\
\hline Benzo(b)fluoranthene & 330 & $\mathrm{U}$ & 330 & 340 & $\mathrm{U}$ & 340 & 330 & $\mathrm{U}$ & 330 & 330 & $U$ & 330 \\
\hline Benzo $(\mathrm{g}, \mathrm{h}, \mathrm{i})$ perylene & 330 & $\mathrm{U}$ & 330 & 340 & $\mathrm{U}$ & 340 & 330 & $\mathrm{U}$ & 330 & 330 & $\mathrm{U}$ & 330 \\
\hline Benzo(k)fluoranthene & 330 & $\mathrm{U}$ & 330 & 340 & $\mathrm{U}$ & 340 & 330 & $\mathrm{U}$ & 330 & 330 & $\mathrm{U}$ & 330 \\
\hline bis(2-Chloro-1-methylethyl)ether & 330 & $\mathrm{U}$ & 330 & 340 & $\mathrm{U}$ & 340 & 330 & $\mathrm{U}$ & 330 & 330 & $U$ & 330 \\
\hline \begin{tabular}{|l|} 
bis(2-Chloroethoxy)methane \\
\end{tabular} & 330 & U & 330 & 340 & $\mathrm{U}$ & 340 & 330 & $\mathrm{U}$ & 330 & 330 & $\mathrm{U}$ & 330 \\
\hline bis(2-Chloroethyl)ether & 330 & $\mathrm{U}$ & 330 & 340 & $\mathrm{U}$ & 340 & 330 & $\mathrm{U}$ & 330 & 330 & $\mathrm{U}$ & 330 \\
\hline bis(2-Ethylhexyl)phthalate & 36 & $\mathrm{JB}$ & 330 & 31 & $\mathrm{JB}$ & 340 & 83 & $\mathrm{JB}$ & 330 & 45 & JB & 330 \\
\hline
\end{tabular}

Attachment

Originator

Checked

Calc. No.

$\frac{1}{\frac{1}{\text { J. M. Capron }}} \frac{\text { T. M. Blakley }}{\text { 0100B-CA-V0299 }}$

Sheet No. 61 of 113 Date $10 / 04 / 07$

Date

Rev. No. $+1$ 
Attachment 1. 118-B-1 Verification Sampling Results.

\begin{tabular}{|c|c|c|c|c|c|c|c|c|c|c|c|c|}
\hline \multirow[t]{2}{*}{ Constituents } & \multicolumn{3}{|c|}{\begin{tabular}{|c|} 
J12WK2 \\
OB A3 \\
Sample Date $7 / 11 / 06$
\end{tabular}} & \multicolumn{3}{|c|}{$\begin{array}{c}\text { J12WK3 } \\
\text { OB A4 } \\
\text { Sample Date } 7 / 11 / 06\end{array}$} & \multicolumn{3}{|c|}{$\begin{array}{c}\text { J12WK4 } \\
\text { OB B5 } \\
\text { Sample Date } 7 / 11 / 06\end{array}$} & \multicolumn{3}{|c|}{$\begin{array}{c}\text { J12WK5 } \\
\text { OB B6 } \\
\text { Sample Date } 7 / 11 / 06 \\
\end{array}$} \\
\hline & $\mu \mathrm{g} / \mathrm{kg}$ & $Q$ & PQL & $\mu \mathrm{g} / \mathrm{kg}$ & $Q$ & PQL & $\mu \mathrm{g} / \mathrm{kg}$ & $Q$ & MDL & $\mu \mathrm{g} / \mathrm{kg}$ & $\mathrm{Q}$ & PQL \\
\hline \multicolumn{13}{|c|}{ Semivolatile Organic Compounds (continued) } \\
\hline Butylbenzylphthalate & 330 & $\mathrm{U}$ & 330 & 340 & $\mathrm{U}$ & 340 & 330 & $\mathrm{U}$ & 330 & 330 & $\mathrm{U}$ & 330 \\
\hline Carbazole & 330 & $\mathrm{U}$ & 330 & 340 & $\mathrm{U}$ & 340 & 330 & $\mathrm{U}$ & 330 & 330 & $\mathrm{U}$ & 330 \\
\hline Chrysene & 330 & $\mathrm{U}$ & 330 & 340 & $\mathrm{U}$ & 340 & 330 & $\bar{U}$ & 330 & 330 & $\mathrm{U}$ & 330 \\
\hline Di-n-butylphthalate & 33 & $\mathrm{~J}$ & 330 & 340 & $\mathrm{U}$ & 340 & 26 & $\mathrm{~J}$ & 330 & 330 & $\mathrm{U}$ & 330 \\
\hline Di-n-octylphthalate & 330 & $\mathrm{U}$ & 330 & 340 & $\mathrm{U}$ & 340 & 330 & $\mathrm{U}$ & 330 & 330 & $\mathrm{U}$ & 330 \\
\hline Dibenz(a,h)anthracene & 330 & $\mathrm{U}$ & 330 & 340 & $\mathrm{U}$ & 340 & 330 & $\mathrm{U}$ & 330 & 330 & $\mathrm{U}$ & 330 \\
\hline Dibenzofuran & 330 & $\mathrm{U}$ & 330 & 340 & $\mathrm{U}$ & 340 & 330 & $\mathrm{U}$ & 330 & 330 & $\mathrm{U}$ & 330 \\
\hline Diethylphthalate & 330 & $\bar{U}$ & 330 & 340 & $\mathrm{U}$ & 340 & 330 & $\mathrm{U}$ & 330 & 330 & $\mathrm{U}$ & 330 \\
\hline Dimethylphthalate & 330 & $\mathrm{U}$ & 330 & 340 & $\mathrm{U}$ & $340^{\circ}$ & 330 & $\mathrm{U}$ & 330 & 330 & $\mathrm{U}$ & 330 \\
\hline Fluoranthene & 330 & $\mathrm{U}$ & 330 & 340 & $\mathrm{U}$ & 340 & 330 & $\mathrm{U}$ & 330 & 330 & $\mathrm{U}$ & 330 \\
\hline Fluorene & 330 & $\mathrm{U}$ & 330 & 340 & $\mathrm{U}$ & 340 & 330 & $\mathrm{U}$ & 330 & 330 & $\mathrm{U}$ & 330 \\
\hline Hexachlorobenzene & 330 & $\mathrm{U}$ & 330 & 340 & $\mathrm{U}$ & 340 & 330 & $\bar{U}$ & 330 & 330 & $U$ & 330 \\
\hline Hexachlorobutadiene & 330 & $\mathrm{U}$ & 330 & 340 & $\mathrm{U}$ & 340 & 330 & $\mathrm{U}$ & 330 & 330 & $\mathrm{U}$ & 330 \\
\hline Hexachlorocyclopentadiene & 330 & $\mathrm{U}$ & 330 & 340 & $\mathrm{U}$ & 340 & 330 & $\mathrm{U}$ & 330 & 330 & $\mathrm{U}$ & 330 \\
\hline Hexachloroethane & 330 & $\mathrm{U}$ & 330 & 340 & $\mathrm{U}$ & 340 & 330 & $\mathrm{U}$ & 330 & 330 & $\mathrm{U}$ & 330 \\
\hline Indeno(1,2,3-cd)pyrene & 330 & $\mathrm{U}$ & 330 & 340 & $\mathrm{U}$ & 340 & 330 & $\mathrm{U}$ & 330 & 330 & $\mathrm{U}$ & 330 \\
\hline Isophorone & 330 & $\mathrm{UJ}$ & 330 & 340 & UJ & 340 & 330 & UJ & 330 & 330 & $\mathrm{UJ}$ & 330 \\
\hline N-Nitroso-di-n-dipropylamine & 330 & $\mathrm{U}$ & 330 & 340 & $\mathrm{U}$ & 340 & 330 & $\mathrm{U}$ & 330 & 330 & $\mathrm{U}$ & 330 \\
\hline N-Nitrosodiphenylamine & 330 & $\mathrm{U}$ & 330 & 340 & $\mathrm{U}$ & 340 & 330 & $\mathrm{U}$ & 330 & 330 & $\mathrm{U}$ & 330 \\
\hline Naphthalene & 330 & $\mathrm{U}$ & 330 & 340 & $\mathrm{U}$ & 340 & 330 & $\mathrm{U}$ & 330 & 330 & $\mathrm{U}$ & 330 \\
\hline Nitrobenzene & 330 & $\mathrm{U}$ & 330 & 340 & $\mathrm{U}$ & 340 & 330 & $\mathrm{U}$ & 330 & 330 & $\mathrm{U}$ & 330 \\
\hline Pentachlorophenol & 840 & U & 840 & 840 & $\mathrm{U}$ & 840 & 840 & $\mathrm{U}$ & 840 & 840 & U & 840 \\
\hline \begin{tabular}{|l} 
Phenanthrene \\
\end{tabular} & 330 & $\mathrm{U}$ & 330 & 340 & $\mathrm{U}$ & 340 & 330 & $\mathrm{U}$ & 330 & 330 & $\mathrm{U}$ & 330 \\
\hline Phenol & 22 & $\mathrm{~J}$ & 330 & 340 & $\mathrm{U}$ & 340 & 330 & $\mathrm{U}$ & 330 & 330 & $\mathrm{U}$ & 330 \\
\hline Pyrene & 330 & $\mathrm{U}$ & 330 & 340 & $U$ & 340 & 330 & $\mathrm{U}$ & 330 & 330 & $\mathrm{U}$ & 330 \\
\hline
\end{tabular}

Attachment

Originator
Checked

Calc. No.
J. M. Capron

T. M. Blakley

0100B-CA-V0299
Sheet No. 62 of 113

Date $10 / 04 / 07$

Date

Rev. No. 
Attachment 1. 118-B-1 Verification Sampling Results.

\begin{tabular}{|c|c|c|c|c|c|c|c|c|c|c|c|c|}
\hline \multirow[t]{2}{*}{ Constituents } & \multicolumn{3}{|c|}{$\begin{array}{c}\text { J12WK2 } \\
\text { OB A3 } \\
\text { Sample Date 7/11/06 }\end{array}$} & \multicolumn{3}{|c|}{$\begin{array}{c}\text { J12WK3 } \\
\text { OB A4 } \\
\text { Sample Date } 7 / 11 / 06 \\
\end{array}$} & \multicolumn{3}{|c|}{$\begin{array}{c}\text { J12WK4 } \\
\text { OB B5 } \\
\text { Sample Date 7/11/06 }\end{array}$} & \multicolumn{3}{|c|}{\begin{tabular}{|c|} 
J12WK5 \\
OB B6 \\
Sample Date $7 / 11 / 06$ \\
\end{tabular}} \\
\hline & $\mu \mathrm{g} / \mathrm{kg}$ & $\mathbf{Q}$ & PQL & $\mu \mathrm{g} / \mathrm{kg}$ & Q & PQL & $\mu \mathrm{g} / \mathrm{kg}$ & Q & MDL & $\mu \mathrm{g} / \mathrm{kg}$ & $\mathrm{Q}$ & PQL \\
\hline \multicolumn{13}{|c|}{ Volatile Organic Compounds } \\
\hline 1,1,1-Trichloroethane & 5 & $\mathrm{U}$ & 5 & 5 & $\mathrm{U}$ & 5 & 5 & $\mathrm{U}$ & 5 & 5 & $\mathrm{U}$ & 5 \\
\hline 1,1,2,2-Tetrachloroethane & 5 & $\mathrm{U}$ & 5 & 5 & U & 5 & 5 & $\mathrm{U}$ & 5 & 5 & $\mathrm{U}$ & 5 \\
\hline 1,1,2-Trichloroethane & 5 & $\mathrm{U}$ & 5 & 5 & $\mathrm{U}$ & 5 & 5 & $\mathrm{U}$ & 5 & 5 & $\mathrm{U}$ & 5 \\
\hline 1,1-Dichloroethane & 5 & $\mathrm{U}$ & 5 & 5 & $\mathrm{U}$ & 5 & 5 & $\mathrm{U}$ & 5 & 5 & $\mathrm{U}$ & 5 \\
\hline 1,1-Dichloroethene & 5 & U & 5 & 5 & $\mathrm{U}$ & 5 & 5 & $\mathrm{U}$ & 5 & 5 & $\mathrm{U}$ & 5 \\
\hline 1,2-Dichloroethane & 5 & $\mathrm{U}$ & 5 & 5 & $\mathrm{U}$ & 5 & 5 & $\mathrm{U}$ & 5 & 5 & $\mathrm{U}$ & 5 \\
\hline 1,2-Dichloroethene (total) & 5 & $\mathrm{U}$ & 5 & 5 & $\mathrm{U}$ & 5 & 5 & $\mathrm{U}$ & 5 & 5 & $\mathrm{U}$ & 5 \\
\hline 1,2-Dichloropropane & 5 & $\mathrm{U}$ & 5 & 5 & $\mathrm{U}$ & 5 & 5 & $\mathrm{U}$ & 5 & 5 & $\mathrm{U}$ & 5 \\
\hline 2-Butanone & 10 & $\mathrm{U}$ & 10 & 10 & $\mathrm{U}$ & 10 & 10 & $\mathrm{U}$ & 10 & 10 & $\mathrm{U}$ & 10 \\
\hline 2-Hexanone & 10 & $\mathrm{U}$ & 10 & 10 & $\mathrm{U}$ & 10 & 10 & $\mathrm{U}$ & 10 & 10 & $\mathrm{U}$ & 10 \\
\hline 4-Methyl-2-pentanone & 10 & $\mathrm{U}$ & 10 & 10 & $\mathrm{U}$ & 10 & 10 & $\mathrm{U}$ & 10 & 10 & $\mathrm{U}$ & 10 \\
\hline Acetone & 10 & $\mathrm{U}$ & 10 & 10 & $\mathrm{U}$ & 10 & 10 & $\mathrm{U}$ & 10 & 38 & & 38 \\
\hline Benzene & 5 & $\mathrm{U}$ & 5 & 5 & $\mathrm{U}$ & 5 & 5 & $\mathrm{U}$ & 5 & 5 & $\mathrm{U}$ & 5 \\
\hline Bromodichloromethane & 5 & $\mathrm{U}$ & 5 & 5 & $\mathrm{U}$ & 5 & 5 & $\mathrm{U}$ & 5 & 5 & $\mathrm{U}$ & 5 \\
\hline Bromoform & 5 & $\mathrm{U}$ & 5 & 5 & $\mathrm{U}$ & 5 & 5 & $\mathrm{U}$ & 5 & 5 & $\mathrm{U}$ & 5 \\
\hline Bromomethane & 10 & $\mathrm{U}$ & 10 & 10 & $\mathrm{U}$ & 10 & 10 & $\mathrm{U}$ & 10 & 10 & $\mathrm{U}$ & 10 \\
\hline Carbon disulfide & 5 & $\mathrm{U}$ & 5 & 5 & $\mathrm{U}$ & 5 & 5 & $\mathrm{U}$ & 5 & 5 & U & 5 \\
\hline Carbon tetrachloride & 5 & $\mathrm{U}$ & 5 & 5 & $\mathrm{U}$ & 5 & 5 & $\mathrm{U}$ & 5 & 5 & $\mathrm{U}$ & 5 \\
\hline Chlorobenzene & 5 & $\mathrm{U}$ & 5 & 5 & $\mathrm{U}$ & 5 & 5 & $\mathrm{U}$ & 5 & 5 & $\mathrm{U}$ & 5 \\
\hline Chloroethane & 10 & $\mathrm{U}$ & 10 & 10 & $\mathrm{U}$ & 10 & 10 & $\mathrm{U}$ & 10 & 10 & $\mathrm{U}$ & 10 \\
\hline Chloroform & 5 & $\mathrm{U}$ & 5 & 5 & $\mathrm{U}$ & 5 & 5 & $\mathrm{U}$ & 5 & 5 & $\mathrm{U}$ & 5 \\
\hline Chloromethane & 10 & $\mathrm{U}$ & 10 & 10 & $\mathrm{U}$ & 10 & 10 & $\mathrm{U}$ & 10 & 10 & $\mathrm{U}$ & 10 \\
\hline cis-1,2-Dichloroethylene & 5 & $\mathrm{U}$ & 5 & 5 & $\mathrm{U}$ & 5 & 5 & U & 5 & 5 & $\mathrm{U}$ & 5 \\
\hline cis-1,3-Dichloropropene & 5 & $\mathrm{U}$ & 5 & 5 & $\mathrm{U}$ & 5 & 5 & $\mathrm{U}$ & 5 & 5 & $\mathrm{U}$ & 5 \\
\hline Dibromochloromethane & 5 & $\mathrm{U}$ & 5 & 5 & $\mathrm{U}$ & 5 & 5 & $\mathrm{U}$ & 5 & 5 & $\mathrm{U}$ & 5 \\
\hline Ethylbenzene & 5 & $\mathrm{U}$ & 5 & 5 & $\mathrm{U}$ & 5 & 5 & $\mathrm{U}$ & 5 & 5 & $\mathrm{U}$ & 5 \\
\hline Methylene chloride & 11 & $\mathrm{BJ}$ & 5 & 9 & $\mathrm{BJ}$ & 5 & 12 & $\mathrm{BJ}$ & 5 & 16 & $\mathrm{BJ}$ & 5 \\
\hline Styrene & 5 & $\mathrm{U}$ & 5 & 5 & $\mathrm{U}$ & 5 & 5 & $\mathrm{U}$ & 5 & 5 & U & 5 \\
\hline Tetrachloroethene & 5 & $\mathrm{U}$ & 5 & 5 & $\mathrm{U}$ & 5 & 5 & $\mathrm{U}$ & 5 & 5 & $\mathrm{U}$ & 5 \\
\hline Toluene & 5 & $\mathrm{U}$ & 5 & 5 & $\mathrm{U}$ & 5 & 5 & $\mathrm{U}$ & 5 & 5 & $U$ & 5 \\
\hline trans-1,2-Dichloroethylene & 5 & $\mathrm{U}$ & 5 & 5 & $\mathrm{U}$ & 5 & 5 & $\mathrm{U}$ & 5 & 5 & $\mathrm{U}$ & 5 \\
\hline trans-1,3-Dichloropropene & 5 & $\mathrm{U}$ & 5 & 5 & $\mathrm{U}$ & 5 & 5 & $\mathrm{U}$ & 5 & 5 & $\mathrm{U}$ & 5 \\
\hline Trichloroethene & 5 & $\mathrm{U}$ & 5 & 5 & $\mathrm{U}$ & 5 & 5 & $\mathrm{U}$ & 5 & 5 & $\mathrm{U}$ & 5 \\
\hline Vinyl chloride & 10 & $\mathrm{U}$ & 10 & 10 & $\mathrm{U}$ & 10 & 10 & $\mathrm{U}$ & 10 & 10 & $\mathrm{U}$ & 10 \\
\hline Xylenes (total) & 5 & $\mathrm{U}$ & 5 & 5 & $\mathrm{U}$ & 5 & 5 & $\mathrm{U}$ & 5 & 5 & $\mathrm{U}$ & 5 \\
\hline & & & & Attachn & & & 1 & & & Sheet No. & & of 113 \\
\hline & & & & Origina & & J.M. Ca & pron & & & Date & & $104 / 07$ \\
\hline & & & & Checke & & T.M.Bl & akley & & & Date & & \\
\hline & & & & Calc. $\mathrm{N}$ & & $0100 \mathrm{~B}-\mathrm{C}$ & $\mathrm{A}-\mathrm{V} 0295$ & & & Rev. No. & & 1 \\
\hline
\end{tabular}


Attachment 1. 118-B-1 Verification Sampling Results.

\begin{tabular}{|c|c|c|c|c|c|c|c|c|c|c|c|c|}
\hline \multirow[t]{2}{*}{ Constituents } & \multicolumn{3}{|c|}{\begin{tabular}{|c|} 
J12WK2 \\
OB A3 \\
Sample Date $7 / 11 / 06$ \\
\end{tabular}} & \multicolumn{3}{|c|}{$\begin{array}{c}\text { J12WK3 } \\
\text { OB A4 } \\
\text { Sample Date } 7 / 11 / 06\end{array}$} & \multicolumn{3}{|c|}{$\begin{array}{c}\text { J12WK4 } \\
\text { OB B5 } \\
\text { Sample Date } 7 / 11 / 06\end{array}$} & \multicolumn{3}{|c|}{\begin{tabular}{|c|} 
J12WK5 \\
OB B6 \\
Sample Date $7 / 11 / 06$ \\
\end{tabular}} \\
\hline & $\mu \mathrm{g} / \mathrm{kg}$ & $\mathbf{Q}$ & PQL & $\mu \mathrm{g} / \mathrm{kg}$ & $\mathbf{Q}$ & PQL & $\mu \mathrm{g} / \mathrm{kg}$ & $\mathbf{Q}$ & MDL & $\mu \mathrm{g} / \mathrm{kg}$ & $\mathbf{Q}$ & PQL \\
\hline \multicolumn{13}{|c|}{ Pesticides } \\
\hline Aldrin & 1.3 & UD & 1.3 & 1.3 & UD & 1.3 & 1.3 & UD & 1.3 & 1.3 & UD & 1.3 \\
\hline alpha-BHC & 1.3 & UDJ & 1.3 & 1.3 & UDJ & 1.3 & 1.3 & UDI & 1.3 & 1.3 & UDJ & 1.3 \\
\hline alpha-Chlordane & 1.3 & UD & 1.3 & 1.3 & UD & 1.3 & 1.3 & UD & 1.3 & 1.3 & UD & 1.3 \\
\hline beta-BHC & 1.3 & UD & 1.3 & 1.3 & UD & 1.3 & 1.3 & UD & 1.3 & 1.3 & UD & 1.3 \\
\hline delta-BHC & 1.3 & UDJ & 1.3 & 1.3 & UDJ & 1.3 & 1.3 & UDJ & 1.3 & 1.3 & UDJ & 1.3 \\
\hline Dichlorodiphenyldichloroethane & 1.3 & UD & 1.3 & 1.3 & UD & 1.3 & 1.3 & UD & 1.3 & 1.3 & UD & 1.3 \\
\hline Dichlorodiphenyldichloroethylene & 1.3 & UDJ & 1.3 & 1.3 & UDJ & 1.3 & 1.3 & UDJ & 1.3 & 1.3 & UDJ & 1.3 \\
\hline Dichlorodiphenyltrichloroethane & 1.1 & $\mathrm{JD}$ & 1.3 & 0.74 & $\mathrm{JD}$ & 1.3 & 1.3 & UD & 1.3 & 0.94 & JD & 1.3 \\
\hline Dieldrin & 1.3 & UD & 1.3 & 1.3 & UD & 1.3 & 1.3 & UD & 1.3 & 1.3 & UD & 1.3 \\
\hline Endosulfan I & 1.3 & UD & 1.3 & 1.3 & UD & 1.3 & 1.3 & UD & 1.3 & 1.3 & UD & 1.3 \\
\hline Endosulfan II & 1.3 & UD & 1.3 & 1.3 & UD & 1.3 & 1.3 & UD & 1.3 & 1.3 & UD & 1.3 \\
\hline Endosulfan sulfate & 1.3 & UD & 1.3 & 1.3 & UD & 1.3 & 1.3 & UD & 1.3 & 1.3 & UD & 1.3 \\
\hline Endrin & 1.3 & UD & 1.3 & 1.3 & UD & 1.3 & 1.3 & UD & 1.3 & 1.3 & UD & 1.3 \\
\hline Endrin aldehyde & 1.3 & UD & 1.3 & 1.3 & UD & 1.3 & 1.3 & UD & 1.3 & 1.3 & UD & 1.3 \\
\hline Endrin ketone & 1.3 & UD & 1.3 & 1.3 & UD & 1.3 & 1.3 & UD & 1.3 & 1.3 & UD & 1.3 \\
\hline gamma-BHC (Lindane) & 1.3 & UDJ & 1.3 & 1.3 & UDJ & 1.3 & 1.3 & UDJ & 1.3 & 1.3 & UDJ & 1.3 \\
\hline gamma-Chlordane & 1.3 & UD & 1.3 & 1.3 & UD & 1.3 & 1.3 & UD & 1.3 & 1.3 & UD & 1.3 \\
\hline Heptachlor & 1.3 & UD & 1.3 & 1.3 & UD & 1.3 & 1.3 & UD & 1.3 & 1.3 & UD & 1.3 \\
\hline Heptachlor epoxide & 1.3 & UD & 1.3 & 1.3 & UD & 1.3 & 1.3 & UD & 1.3 & 1.3 & UD & 1.3 \\
\hline Methoxychlor & 1.4 & JDI & 1.3 & 1.3 & UD & 1.3 & 5.5 & $\mathrm{D}$ & 1.3 & 1.3 & UD & 1.3 \\
\hline Toxaphene & 13 & UD & 13 & 13 & UD & 13 & 13 & UD & 13 & 13 & UD & 13 \\
\hline \multicolumn{13}{|c|}{ Herbicides } \\
\hline $2,4,5-\mathrm{T}$ & 17 & $\mathrm{U}$ & 17 & 17 & $\mathrm{U}$ & 17 & 17 & $\mathrm{U}$ & 17 & 17 & $\mathrm{U}$ & 17 \\
\hline $2,4-\mathrm{D}$ & 33 & $U$ & 33 & 34 & $\mathrm{U}$ & 34 & 33 & $\mathrm{U}$ & 33 & 33 & $\mathrm{U}$ & 33 \\
\hline $2,4,5-\mathrm{TP}$ & 17 & $\mathrm{U}$ & 17 & 17 & $\mathrm{U}$ & 17 & 17 & $\mathrm{U}$ & 17 & 17 & $\mathrm{U}$ & 17 \\
\hline Dinoseb & 17 & $\mathrm{U}$ & 17 & 17 & $\mathrm{U}$ & 17 & 17 & $\mathrm{U}$ & 17 & 17 & $\mathrm{U}$ & 17 \\
\hline 2,4-DB & 17 & $\mathrm{U}$ & 17 & 17 & $\mathrm{U}$ & 17 & 17 & $\mathrm{U}$ & 17 & 17 & $\mathrm{U}$ & 17 \\
\hline Dalapon & 33 & $\mathrm{U}$ & 33 & 34 & $U$ & 34 & 33 & $\mathrm{U}$ & 33 & 20 & $\mathrm{~J}$ & 33 \\
\hline Dicamba & 33 & $\mathrm{U}$ & 33 & 9.2 & $\mathrm{~J}$ & 34 & 8.7 & $\mathrm{~J}$ & 33 & 17 & $\mathrm{~J}$ & 33 \\
\hline Dichloroprop & 33 & $\mathrm{U}$ & 33 & 34 & $\mathrm{U}$ & 34 & 33 & $\mathrm{U}$ & 33 & 33 & $\mathrm{U}$ & 33 \\
\hline Pentachlorophenol & 17 & U & 17 & 16 & $\mathrm{~J}$ & 17 & 17 & $\mathrm{U}$ & 17 & 17 & $\mathrm{U}$ & 17 \\
\hline Picloram & 17 & UR & 17 & 17 & UR & 17 & 17 & UR & 17 & 17 & UR & 17 \\
\hline & & & & \multirow{4}{*}{\multicolumn{2}{|c|}{$\begin{array}{l}\text { Attachment } \\
\text { Originator } \\
\text { Checked } \\
\text { Calc. No. }\end{array}$}} & \multicolumn{3}{|c|}{1} & \multicolumn{2}{|c|}{ Sheet No. } & \multicolumn{2}{|c|}{64 of 113} \\
\hline & & & & & & \multicolumn{4}{|c|}{ J. M. Capron } & Dat & \multicolumn{2}{|c|}{$10 / 04 / 07$} \\
\hline & & & & & & \multicolumn{3}{|c|}{ T. M. Blakley } & \multicolumn{4}{|c|}{ Date } \\
\hline & & & & & & $0100 \mathrm{~B}-\mathrm{C}$ & $A-V 0299$ & & & Rev. No & & 1 \\
\hline
\end{tabular}


Attachment 1. 118-B-1 Verification Sampling Results.

\begin{tabular}{|c|c|c|c|c|c|c|c|c|c|c|c|c|}
\hline \multirow[t]{2}{*}{ Constituents } & \multicolumn{3}{|c|}{$\begin{array}{c}\text { J12WK6 } \\
\text { OB B7 } \\
\text { Sample Date } 7 / 11 / 06 \\
\end{array}$} & \multicolumn{3}{|c|}{\begin{tabular}{|c|} 
J12WM7 \\
OB B8 \\
Sample Date $7 / 17 / 06$ \\
\end{tabular}} & \multicolumn{3}{|c|}{$\begin{array}{c}\text { J12WM8 } \\
\text { OB C9 } \\
\text { Sample Date } 7 / 17 / 06 \\
\end{array}$} & \multicolumn{3}{|c|}{\begin{tabular}{|c|} 
J12WM9 \\
OB C10 \\
Sample Date $7 / 17 / 06$ \\
\end{tabular}} \\
\hline & $\mu \mathrm{g} / \mathrm{kg}$ & $Q$ & PQL & $\mu \mathrm{g} / \mathrm{kg}$ & $\mathbf{Q}$ & PQL & $\mu \mathrm{g} / \mathrm{kg}$ & $\mathbf{Q}$ & PQL & $\mu \mathrm{g} / \mathrm{kg}$ & $\mathbf{Q}$ & PQL \\
\hline \multicolumn{13}{|c|}{ Polychlorinated Biphenyls } \\
\hline Aroclor-1016 & 13 & $\mathrm{U}$ & 13 & 13 & $\mathrm{U}$ & 13 & 13 & $\mathrm{U}$ & 13 & 13 & $\mathrm{U}$ & 13 \\
\hline Aroclor-1221 & 13 & $\mathrm{U}$ & 13 & 13 & $\mathrm{U}$ & 13 & 13 & $\mathrm{U}$ & 13 & 13 & $\mathrm{U}$ & 13 \\
\hline Aroclor-1232 & 13 & $\mathrm{U}$ & 13 & 13 & $\mathrm{U}$ & 13 & 13 & $\mathrm{U}$ & 13 & 13 & $\mathrm{U}$ & 13 \\
\hline Aroclor-1242 & 13 & $\mathrm{U}$ & 13 & 13 & $\mathrm{U}$ & 13 & 13 & $\mathrm{U}$ & 13 & 13 & $\mathrm{U}$ & 13 \\
\hline Aroclor-1248 & 13 & $\mathrm{U}$ & 13 & 13 & $\mathrm{U}$ & 13 & 13 & $\mathrm{U}$ & 13 & 13 & $U$ & 13 \\
\hline Aroclor-1254 & 38 & & 13 & 13 & $\mathrm{U}$ & 13 & 13 & U & 13 & 13 & $\mathrm{U}$ & 13 \\
\hline Aroclor-1260 & 13 & $\mathrm{U}$ & 13 & 13 & $\mathrm{U}$ & 13 & 13 & $\mathrm{U}$ & 13 & 13 & $\mathrm{U}$ & 13 \\
\hline \multicolumn{13}{|c|}{ Semivolatile Organic Compounds } \\
\hline 1,2,4-Trichlorobenzene & 330 & UJ & 330 & 330 & UJ & 330 & 340 & UJ & 340 & 340 & UJ & 340 \\
\hline 1,2-Dichlorobenzene & 330 & $\mathrm{U}$ & 330 & 330 & $\mathrm{U}$ & 330 & 340 & $\mathrm{U}$ & 340 & 340 & $\mathrm{U}$ & 340 \\
\hline 1,3-Dichlorobenzene & 330 & $\mathrm{U}$ & 330 & 330 & $\mathrm{U}$ & 330 & 340 & $\mathrm{U}$ & 340 & 340 & $\mathrm{U}$ & 340 \\
\hline 1,4-Dichlorobenzene & 330 & $\mathrm{U}$ & 330 & 330 & $\mathrm{U}$ & 330 & 340 & $\mathrm{U}$ & 340 & 340 & $\mathrm{U}$ & 340 \\
\hline 2,4,5-Trichlorophenol & 840 & $\mathrm{U}$ & 840 & 840 & $\mathrm{U}$ & 840 & 840 & $\mathrm{U}$ & 840 & 840 & $\mathrm{U}$ & 840 \\
\hline 2,4,6-Trichlorophenol & 330 & $\mathrm{U}$ & 330 & 330 & $\mathrm{U}$ & 330 & 340 & $\mathrm{U}$ & 340 & 340 & $\mathrm{U}$ & 340 \\
\hline 2,4-Dichlorophenol & 330 & $\mathrm{U}$ & 330 & 330 & $\mathrm{U}$ & 330 & 340 & $\mathrm{U}$ & 340 & 340 & $\mathrm{U}$ & 340 \\
\hline 2,4-Dimethylphenol & 330 & UJ & 330 & 330 & $\mathrm{U}$ & 330 & 340 & $\mathrm{U}$ & 340 & 340 & $\mathrm{U}$ & 340 \\
\hline 2,4-Dinitrophenol & 840 & UR & 840 & 840 & $\mathrm{U}$ & 840 & 840 & $\mathrm{U}$ & 840 & 840 & $\mathrm{U}$ & 840 \\
\hline 2,4-Dinitrotoluene & 330 & $\mathrm{U}$ & 330 & 330 & $\mathrm{U}$ & 330 & 340 & $\mathrm{U}$ & 340 & 340 & $\mathrm{U}$ & 340 \\
\hline 2,6-Dinitrotoluene & 330 & $\mathrm{U}$ & 330 & 330 & $\mathrm{U}$ & 330 & 340 & $\mathrm{U}$ & 340 & 340 & $\mathrm{U}$ & 340 \\
\hline 2-Chloronaphthalene & 330 & $\mathrm{U}$ & 330 & 330 & $\mathrm{U}$ & 330 & 340 & $\mathrm{U}$ & 340 & 340 & $\mathrm{U}$ & 340 \\
\hline 2-Chlorophenol & 330 & $\mathrm{U}$ & 330 & 330 & $\mathrm{U}$ & 330 & 340 & $\mathrm{U}$ & 340 & 340 & $\mathrm{U}$ & 340 \\
\hline 2-Methylnaphthalene & 330 & $\mathrm{UJ}$ & 330 & 330 & $\mathrm{U}$ & 330 & 340 & $\mathrm{U}$ & 340 & 340 & $\mathrm{U}$ & 340 \\
\hline 2-Methylphenol (cresol, o-) & 330 & U & 330 & 330 & $\mathrm{U}$ & 330 & 340 & $\mathrm{U}$ & 340 & 340 & $\mathrm{U}$ & 340 \\
\hline 2-Nitroaniline & 840 & $\mathrm{U}$ & 840 & 840 & $\mathrm{U}$ & 840 & 840 & $\mathrm{U}$ & 840 & 840 & $\mathrm{U}$ & 840 \\
\hline 2-Nitrophenol & 330 & $\mathrm{U}$ & 330 & 330 & $\mathrm{U}$ & 330 & 340 & U & 340 & 340 & $\mathrm{U}$ & 340 \\
\hline 3,3'-Dichlorobenzidine & 330 & $\mathrm{U}$ & 330 & 330 & $\mathrm{U}$ & 330 & 340 & $\mathrm{U}$ & 340 & 340 & $\mathrm{U}$ & 340 \\
\hline 3-Nitroaniline & 840 & $\mathrm{U}$ & 840 & 840 & $\mathrm{U}$ & 840 & 840 & $\mathrm{U}$ & 840 & 840 & $\mathrm{U}$ & 840 \\
\hline 4,6-Dinitro-2-methylphenol & 840 & $\mathrm{U}$ & 840 & 840 & $\mathrm{U}$ & 840 & 840 & $\mathrm{U}$ & 840 & 840 & $\mathrm{U}$ & 840 \\
\hline 4-Bromophenyl-phenylether & 330 & $\mathrm{U}$ & 330 & 330 & $\mathrm{U}$ & 330 & 340 & $\mathrm{U}$ & 340 & 340 & $\mathrm{U}$ & 340 \\
\hline 4-Chloro-3-methylphenol & 330 & $\mathrm{U}$ & 330 & 330 & $\mathrm{U}$ & 330 & 340 & $\mathrm{U}$ & 340 & 340 & $\mathrm{U}$ & 340 \\
\hline 4-Chloroaniline & 330 & $\mathrm{U}$ & 330 & 330 & $\mathrm{U}$ & 330 & 340 & $\mathrm{U}$ & 340 & 340 & $\mathrm{U}$ & 340 \\
\hline 4-Chlorophenyl-phenylether & 330 & $\mathrm{U}$ & 330 & 330 & $\mathrm{U}$ & 330 & 340 & $\mathrm{U}$ & 340 & 340 & $\mathrm{U}$ & 340 \\
\hline 4-Methylphenol (p-cresol) & 330 & U & 330 & 330 & $\mathrm{U}$ & 330 & 340 & $\mathrm{U}$ & 340 & 340 & $\mathrm{U}$ & 340 \\
\hline 4-Nitroaniline & 840 & $\mathrm{U}$ & 840 & 840 & $\mathrm{U}$ & 840 & 840 & $\mathrm{U}$ & 840 & 840 & $\mathrm{U}$ & 840 \\
\hline 4-Nitrophenol & 840 & $\mathrm{U}$ & 840 & 840 & $\mathrm{U}$ & 840 & 840 & $\mathrm{U}$ & 840 & 840 & $\mathrm{U}$ & 840 \\
\hline Acenaphthene & 330 & $\mathrm{U}$ & 330 & 330 & $\mathrm{U}$ & 330 & 340 & $\mathrm{U}$ & 340 & 340 & $\mathrm{U}$ & 340 \\
\hline Acenaphthylene & 330 & U & 330 & 330 & $\mathrm{U}$ & 330 & 340 & $\mathrm{U}$ & 340 & 340 & $\mathrm{U}$ & 340 \\
\hline Anthracene & 330 & $\mathrm{U}$ & 330 & 330 & $\mathrm{U}$ & 330 & 340 & $\mathrm{U}$ & 340 & 340 & $\mathrm{U}$ & 340 \\
\hline Benzo(a)anthracene & 330 & $\mathrm{U}$ & 330 & 330 & $\mathrm{U}$ & 330 & 340 & $\mathrm{U}$ & 340 & 340 & $\mathrm{U}$ & 340 \\
\hline Benzo(a)pyrene & 330 & U & 330 & 330 & $\mathrm{U}$ & 330 & 340 & $\mathrm{U}$ & 340 & 340 & $\mathrm{U}$ & 340 \\
\hline Benzo(b)fluoranthene & 330 & $\mathrm{U}$ & 330 & 330 & $\mathrm{U}$ & 330 & 340 & $\mathrm{U}$ & 340 & 340 & $\mathrm{U}$ & 340 \\
\hline Benzo $(\mathrm{g}, \mathrm{h}, \mathrm{i})$ perylene & 330 & $\mathrm{U}$ & 330 & 330 & $\mathrm{U}$ & 330 & 340 & $\mathrm{U}$ & 340 & 340 & $\mathrm{U}$ & 340 \\
\hline Benzo(k)fluoranthene & 330 & U & 330 & 330 & $\mathrm{U}$ & 330 & 340 & $\mathrm{U}$ & 340 & 340 & U & 340 \\
\hline bis(2-Chloro-1-methylethyl)ether & 330 & $\mathrm{U}$ & 330. & 330 & $\mathrm{U}$ & 330 & 340 & $\mathrm{U}$ & 340 & 340 & $\mathrm{U}$ & 340 \\
\hline bis(2-Chloroethoxy)methane & 330 & $\mathrm{U}$ & 330 & 330 & $\mathrm{U}$ & 330 & 340 & $\mathrm{U}$ & 340 & 340 & $\mathrm{U}$ & 340 \\
\hline bis(2-Chloroethyl)ether & 330 & $\mathrm{U}$ & 330 & 330 & $\mathrm{U}$ & 330 & 340 & $\mathrm{U}$ & 340 & 340 & $\mathrm{U}$ & 340 \\
\hline bis(2-Ethylhexyl)phthalate & 29 & $\mathrm{JB}$ & 330 & 20 & $\mathrm{~J}$ & 330 & 88 & $\mathrm{~J}$ & 340 & 39 & $\mathrm{~J}$ & 340 \\
\hline
\end{tabular}

Attachmen

Originator

Checked

Calc. No.

\begin{tabular}{l}
$\frac{1}{\text { J. M. Capron }}$ \\
\hline T. M. Blakley \\
\hline 0100B-CA-V0299
\end{tabular}

Sheet No. 65 of 113

Date $\frac{65 / 04 / 07}{10 / 13}$

Date

Rev. No. $\frac{1}{1}$ 
Attachment 1. 118-B-1 Verification Sampling Results.

\begin{tabular}{|c|c|c|c|c|c|c|c|c|c|c|c|c|}
\hline \multirow[t]{2}{*}{ Constituents } & \multicolumn{3}{|c|}{\begin{tabular}{|c|} 
J12WK6 \\
OB B7 \\
Sample Date $7 / 11 / 06$
\end{tabular}} & \multicolumn{3}{|c|}{\begin{tabular}{|c|} 
J12WM7 \\
OB B8 \\
Sample Date $7 / 17 / 06$ \\
\end{tabular}} & \multicolumn{3}{|c|}{$\begin{array}{c}\text { J12WM8 } \\
\text { OB C9 } \\
\text { Sample Date } 7 / 17 / 06 \\
\end{array}$} & \multicolumn{3}{|c|}{$\begin{array}{c}\text { J12WM9 } \\
\text { OB C10 } \\
\text { Sample Date 7/17/06 } \\
\end{array}$} \\
\hline & $\mu \mathrm{g} / \mathrm{kg}$ & $Q$ & PQL & $\mu \mathrm{g} / \mathrm{kg}$ & $\mathbf{Q}$ & PQL & $\mu \mathrm{g} / \mathrm{kg}$ & $\mathbf{Q}$ & MDL & $\mu \mathrm{g} / \mathrm{kg}$ & Q & PQL \\
\hline \multicolumn{13}{|c|}{ Semivolatile Organic Compounds (continued) } \\
\hline Butylbenzylphthalate & 330 & $U$ & 330 & 330 & $\mathrm{U}$ & 330 & 340 & $\mathrm{U}$ & 340 & 340 & $\mathrm{U}$ & 340 \\
\hline Carbazole & 330 & $\mathrm{U}$ & 330 & 330 & $\mathrm{U}$ & 330 & 340 & $\mathrm{U}$ & 340 & 340 & $\mathrm{U}$ & 340 \\
\hline Chrysene & 330 & $\mathrm{U}$ & 330 & 330 & $\mathrm{U}$ & 330 & 340 & $\mathrm{U}$ & 340 & 340 & $\mathrm{U}$ & 340 \\
\hline Di-n-butylphthalate & 330 & $\mathrm{U}$ & 330 & 38 & $\mathrm{~J}$ & 330 & 41 & $\mathrm{~J}$ & 340 & 25 & $\mathrm{~J}$ & 340 \\
\hline Di-n-octylphthalate & 330 & $\mathrm{U}$ & 330. & 330 & $\mathrm{U}$ & 330 & 340 & $\mathrm{U}$ & 340 & 340 & $\mathrm{U}$ & 340 \\
\hline Dibenz(a,h)anthracene & 330 & $\mathrm{U}$ & 330 & 330 & $\mathrm{U}$ & 330 & 340 & $\mathrm{U}$ & 340 & 340 & U & 340 \\
\hline Dibenzofuran & 330 & $\mathrm{U}$ & 330 & 330 & $\mathrm{U}$ & 330 & 340 & $\mathrm{U}$ & 340 & 340 & $\mathrm{U}$ & 340 \\
\hline Diethylphthalate & 330 & $\mathrm{U}$ & 330 & 330 & $\mathrm{U}$ & 330 & 340 & U & 340 & 340 & $\mathrm{U}$ & 340 \\
\hline Dimethylphthalate & 330 & $\mathrm{U}$ & 330 & 330 & $\mathrm{U}$ & 330 & 340 & $\mathrm{U}$ & 340 & 340 & U & 340 \\
\hline \begin{tabular}{|l|} 
Fluoranthene \\
\end{tabular} & 330 & $\mathrm{U}$ & 330 & 330 & $\mathrm{U}$ & 330 & 340 & $\mathrm{U}$ & 340 & 340 & $\mathrm{U}$ & 340 \\
\hline Fluorene & 330 & $\mathrm{U}$ & 330 & 330 & $\mathrm{U}$ & 330 & 340 & U & 340 & 340 & $\mathrm{U}$ & 340 \\
\hline Hexachlorobenzene & 330 & $\mathrm{U}$ & 330 & 330 & $\mathrm{U}$ & 330 & 340 & $\mathrm{U}$ & 340 & 340 & $\mathrm{U}$ & 340 \\
\hline Hexachlorobutadiene & 330 & $\mathrm{U}$ & 330 & 330 & $\mathrm{U}$ & 330 & 340 & $\mathrm{U}$ & 340 & 340 & $\mathrm{U}$ & 340 \\
\hline Hexachlorocyclopentadiene & 330 & $\mathrm{U}$ & 330 & 330 & $\mathrm{U}$ & 330 & 340 & $\mathrm{U}$ & 340 & 340 & $\mathrm{U}$ & 340 \\
\hline Hexachloroethane & 330 & $\mathrm{U}$ & 330 & 330 & $\mathrm{U}$ & 330 & 340 & $\mathrm{U}$ & 340 & 340 & $\mathrm{U}$ & 340 \\
\hline Indeno(1,2,3-cd)pyrene & 330 & $\mathrm{U}$ & 330 & 330 & $\mathrm{U}$ & 330 & 340 & $\mathrm{U}$ & 340 & 340 & $\mathrm{U}$ & 340 \\
\hline Isophorone & 330 & $\mathrm{UJ}$ & 330 & 330 & $\mathrm{U}$ & 330 & 340 & $\mathrm{U}$ & 340 & 340 & $\mathrm{U}$ & 340 \\
\hline N-Nitroso-di-n-dipropylamine & 330 & $\mathrm{U}$ & 330 & 330 & $\mathrm{U}$ & 330 & 340 & $\mathrm{U}$ & 340 & 340 & $\mathrm{U}$ & 340 \\
\hline N-Nitrosodiphenylamine & 330 & $\mathrm{U}$ & 330 & 330 & $\mathrm{U}$ & 330 & 340 & $\mathrm{U}$ & 340 & 340 & $\mathrm{U}$ & 340 \\
\hline Naphthalene & 330 & $\mathrm{U}$ & 330 & 330 & $\mathrm{U}$ & 330 & 340 & $\mathrm{U}$ & 340 & 340 & $\mathrm{U}$ & 340 \\
\hline Nitrobenzene & 330 & $\mathrm{U}$ & 330 & 330 & $\mathrm{U}$ & 330 & 340 & $\mathrm{U}$ & 340 & 340 & $\mathrm{U}$ & 340 \\
\hline Pentachlorophenol & 840 & $\mathrm{U}$ & 840 & 840 & $\mathrm{U}$ & 840 & 840 & $\mathrm{U}$ & 840 & 840 & $\mathrm{U}$ & 840 \\
\hline Phenanthrene & 330 & $\mathrm{U}$ & 330 & 330 & $\mathrm{U}$ & 330 & 340 & $U$ & 340 & 340 & $\mathrm{U}$ & 340 \\
\hline Phenol & 330 & $\mathrm{U}$ & 330 & 330 & $\mathrm{U}$ & 330 & 340 & $\mathrm{U}$ & 340 & 340 & $\mathrm{U}$ & 340 \\
\hline Pyrene & 330 & $\mathrm{U}$ & 330 & 330 & $\mathrm{U}$ & 330 & 340 & $\mathrm{U}$ & 340 & 340 & $\mathrm{U}$ & 340 \\
\hline
\end{tabular}

Attachment

Originator

Checked

Calc. No.

$\frac{1}{\frac{1}{\text { J. M. Capron }}} \frac{}{\text { T. M. Blakley }}$

Sheet No. 66 of 113 Date $10 / 04 / 07$ Date Rev. No. 
Attachment 1. 118-B-1 Verification Sampling Results.

\begin{tabular}{|c|c|c|c|c|c|c|c|c|c|c|c|c|}
\hline \multirow[t]{2}{*}{ Constituents } & \multicolumn{3}{|c|}{\begin{tabular}{|c|} 
J12WK6 \\
OB B7 \\
Sample Date 7/11/06 \\
\end{tabular}} & \multicolumn{3}{|c|}{\begin{tabular}{|c|} 
J12WM7 \\
OB B8 \\
Sample Date 7/17/06 \\
\end{tabular}} & \multicolumn{3}{|c|}{\begin{tabular}{|c|} 
J12WM8 \\
OB C9 \\
Sample Date $7 / 17 / 06$ \\
\end{tabular}} & \multicolumn{3}{|c|}{\begin{tabular}{|c|} 
J12WM9 \\
OB C10 \\
Sample Date $7 / 17 / 0$ \\
\end{tabular}} \\
\hline & $\mu \mathrm{g} / \mathrm{kg}$ & $\mathbf{Q}$ & PQL & $\mu \mathrm{g} / \mathrm{kg}$ & $\mathbf{Q}$ & PQL & $\mu \mathrm{g} / \mathrm{kg}$ & $\mathbf{Q}$ & MDL & $\mu \mathrm{g} / \mathrm{kg}$ & $\mathbf{Q}$ & PQL \\
\hline \multicolumn{13}{|c|}{ Volatile Organic Compounds } \\
\hline 1,1,1-Trichloroethane & 5 & $\mathrm{U}$ & 5 & 5 & $\mathrm{U}$ & 5 & 5 & $\mathrm{U}$ & 5 & 5 & $\mathrm{U}$ & 5 \\
\hline 1,1,2,2-Tetrachloroethane & 5 & $\mathrm{U}$ & 5 & 5 & $\mathrm{U}$ & 5 & 5 & $\mathrm{U}$ & 5 & 5 & $\mathrm{U}$ & 5 \\
\hline 1,1,2-Trichloroethane & 5 & $\mathrm{U}$ & 5 & 5 & $\mathrm{U}$ & 5 & 5 & $\mathrm{U}$ & 5 & 5 & $\mathrm{U}$ & 5 \\
\hline 1,1-Dichloroethane & 5 & $\mathrm{U}$ & 5 & 5 & $\mathrm{U}$ & 5 & 5 & $\mathrm{U}$ & 5 & 5 & $\mathrm{U}$ & 5 \\
\hline 1,1-Dichloroethene & 5 & $\mathrm{U}$ & 5 & 5 & $\mathrm{U}$ & 5 & 5 & $\mathrm{U}$ & 5 & 5 & $\mathrm{U}$ & 5 \\
\hline 1,2-Dichloroethane & 5 & $\mathrm{U}$ & 5 & 5 & $\mathrm{U}$ & 5 & 5 & $\mathrm{U}$ & 5 & 5 & $\mathrm{U}$ & 5 \\
\hline 1,2-Dichloroethene (total) & 5 & $\mathrm{U}$ & 5 & 5 & $\bar{U}$ & 5 & 5 & $\mathrm{U}$ & 5 & 5 & $\mathrm{U}$ & 5 \\
\hline 1,2-Dichloropropane & 5 & $\mathrm{U}$ & 5 & 5 & $\mathrm{U}$ & 5 & 5 & $\mathrm{U}$ & 5 & 5 & U & 5 \\
\hline 2-Butanone & 10 & $\mathrm{U}$ & 10 & 10 & $\mathrm{U}$ & 10 & 10 & $\mathrm{U}$ & 10 & 10 & $\mathrm{U}$ & 10 \\
\hline 2-Hexanone & 10 & $\mathrm{U}$ & 10 & 10 & U & 10 & 10 & $\mathrm{U}$ & 10 & 10 & U & 10 \\
\hline 4-Methyl-2-pentanone & 10 & $\mathrm{U}$ & 10 & 10 & $\mathrm{~B}$ & 10 & 10 & $\mathrm{JB}$ & 10 & 11 & $\mathrm{~B}$ & 10 \\
\hline Acetone & 10 & $\mathrm{U}$ & 10 & 34 & & 10 & 28 & & 10 & 24 & & 10 \\
\hline Benzene & 5 & $\mathrm{U}$ & 5 & 5 & $\mathrm{U}$ & 5 & 5 & $\mathrm{U}$ & 5 & 5 & $\mathrm{U}$ & 5 \\
\hline Bromodichloromethane & 5 & $\mathrm{U}$ & 5 & 5 & $\mathrm{U}$ & 5 & 5 & $\mathrm{U}$ & 5 & 5 & $\mathrm{U}$ & 5 \\
\hline Bromoform & 5 & $\mathrm{U}$ & 5 & 5 & $\mathrm{U}$ & 5 & 5 & $\mathrm{U}$ & 5 & 5 & $\mathrm{U}$ & 5 \\
\hline Bromomethane & 10 & $\mathrm{U}$ & 10 & 10 & $\mathrm{U}$ & 10 & 10 & $\mathrm{U}$ & 10 & 10 & $\mathrm{U}$ & 10 \\
\hline Carbon disulfide & 5 & $\mathrm{U}$ & 5 & 5 & $\mathrm{U}$ & 5 & 5 & $\mathrm{U}$ & 5 & 5 & $\mathrm{U}$ & 5 \\
\hline Carbon tetrachloride & 5 & $\mathrm{U}$ & 5 & 9 & & 5 & 12 & & 5 & 12 & & 5 \\
\hline Chlorobenzene & 5 & $\mathrm{U}$ & 5 & 5 & $\mathrm{U}$ & 5 & 5 & $\mathrm{U}$ & 5 & 5 & $\mathrm{U}$ & 5 \\
\hline Chloroethane & 10 & $\mathrm{U}$ & 10 & 10 & $\mathrm{U}$ & 10 & 10 & $\mathrm{U}$ & 10 & 10 & $\mathrm{U}$ & 10 \\
\hline Chloroform & 5 & $\mathrm{U}$ & 5 & 5 & $\mathrm{U}$ & 5 & 5 & $\mathrm{U}$ & 5 & 5 & $\mathrm{U}$ & 5 \\
\hline Chloromethane & 10 & $\mathrm{U}$ & 10 & 10 & $\mathrm{U}$ & 10 & 10 & $\mathrm{U}$ & 10 & 10 & $\mathrm{U}$ & 10 \\
\hline cis-1,2-Dichloroethylene & 5 & $\mathrm{U}$ & 5 & 5 & $\mathrm{U}$ & 5 & 5 & $\mathrm{U}$ & 5 & 5 & $\mathrm{U}$ & 5 \\
\hline cis-1,3-Dichloropropene & 5 & $\mathrm{U}$ & 5 & 5 & $\mathrm{U}$ & 5 & 5 & $\mathrm{U}$ & 5 & 5 & $\mathrm{U}$ & 5 \\
\hline Dibromochloromethane & 5 & $\mathrm{U}$ & 5 & 5 & $\mathrm{U}$ & 5 & 5 & $\mathrm{U}$ & 5 & 5 & $\mathrm{U}$ & 5 \\
\hline Ethylbenzene & 5 & $\mathrm{U}$ & 5 & 5 & $\mathrm{U}$ & 5 & 5 & $\mathrm{U}$ & 5 & 5 & $\mathrm{U}$ & 5 \\
\hline Methylene chloride & 18 & $\mathrm{BJ}$ & 5 & 15 & & 5 & 8 & & 5 & 13 & & 5 \\
\hline Styrene & 5 & $\mathrm{U}$ & 5 & 5 & $\mathrm{U}$ & 5 & 5 & $\mathrm{U}$ & 5 & 5 & $\mathrm{U}$ & 5 \\
\hline Tetrachloroethene & 5 & $\mathrm{U}$ & 5 & 5 & $\mathrm{U}$ & 5 & 5 & $\mathrm{U}$ & 5 & 5 & $\mathrm{U}$ & 5 \\
\hline Toluene & 5 & $\mathrm{U}$ & 5 & 1 & $\mathrm{JB}$ & 5 & 1 & $\mathrm{JB}$ & 5 & 1 & $\mathrm{JB}$ & 5 \\
\hline trans-1,2-Dichloroethylene & 5 & $\mathrm{U}$ & 5 & 5 & $\mathrm{U}$ & 5 & 5 & $\mathrm{U}$ & 5 & 5 & $\mathrm{U}$ & 5 \\
\hline trans-1,3-Dichloropropene & 5 & $\mathrm{U}$ & 5 & 5 & $\mathrm{U}$ & 5 & 5 & $\mathrm{U}$ & 5 & 5 & $\mathrm{U}$ & 5 \\
\hline Trichloroethene & 5 & U & 5 & 5 & U & 5 & 5 & $\mathrm{U}$ & 5 & 5 & $\mathrm{U}$ & 5 \\
\hline Vinyl chloride & 10 & $\mathrm{U}$ & 10 & 10 & $\mathrm{U}$ & 10 & 10 & $\mathrm{U}$ & 10 & 10 & $\mathrm{U}$ & 10 \\
\hline Xylenes (total) & 5 & $\mathrm{U}$ & 5 & 1 & $\mathrm{~J}$ & 5 & 5 & $\mathrm{U}$ & 5 & 5 & $\mathrm{U}$ & 5 \\
\hline & & & & Attach & & & 1 & & & Sheet No. & & of 113 \\
\hline & & & & Origin & & J.M.Ca & pron & & & Date & & $104 / 07$ \\
\hline & & & & Checke & & T.M. B & akley & & & Date & & \\
\hline & & & & Calc. $N$ & & $0100 \mathrm{~B}-1$ & $\mathrm{AA}-\mathrm{V} 029$ & & & Rev. No & & 1 \\
\hline
\end{tabular}


Rev. 0

Attachment 1. 118-B-1 Verification Sampling Results.

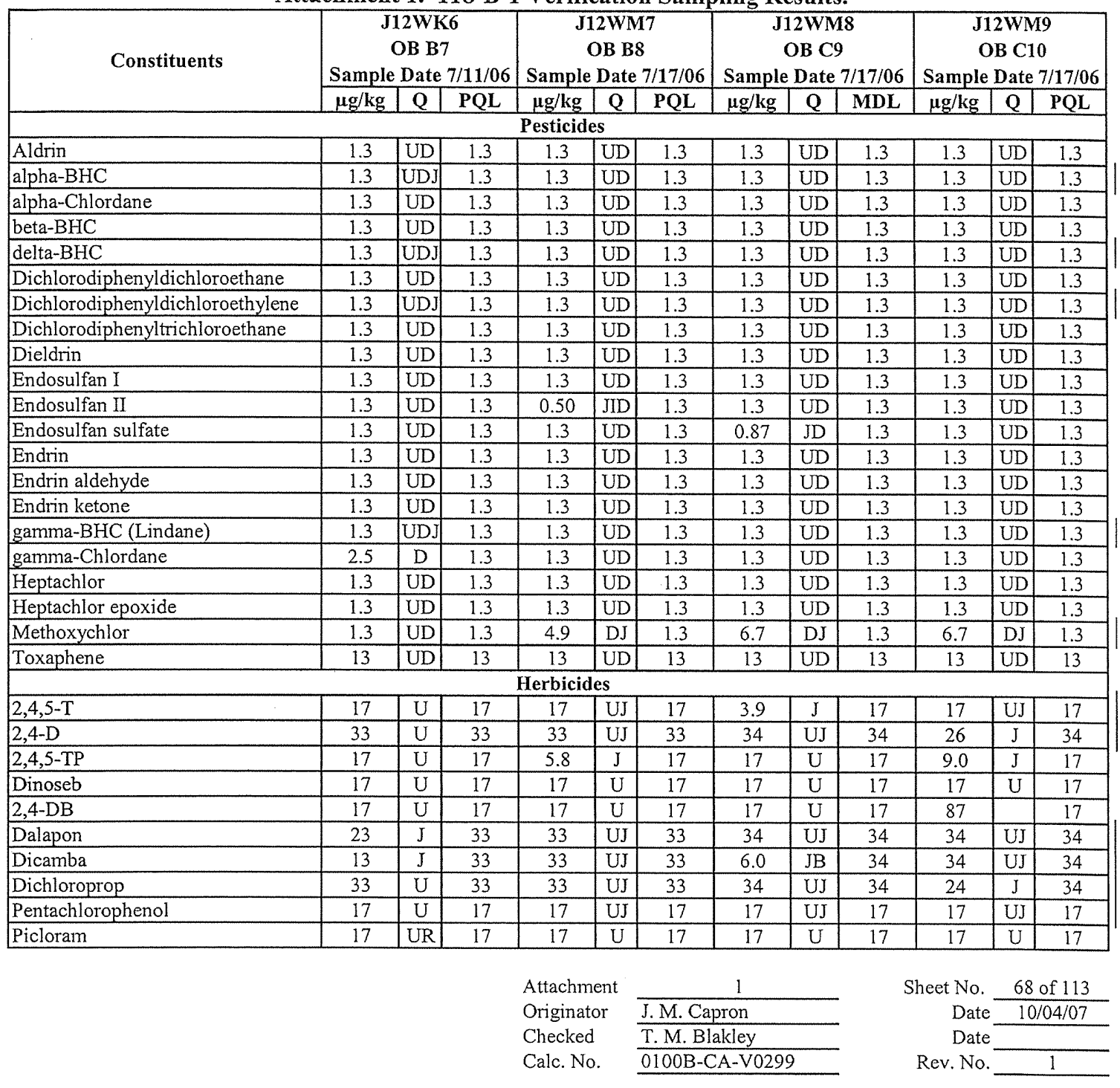


Attachment 1. 118-B-1 Verification Sampling Results.

\begin{tabular}{|c|c|c|c|c|c|c|c|c|c|c|c|c|}
\hline \multirow[t]{2}{*}{ Constituents } & \multicolumn{3}{|c|}{$\begin{array}{c}\text { J12WN0 } \\
\text { OB C1 } \\
\text { Sample Date } 7 / 17 / 06\end{array}$} & \multicolumn{3}{|c|}{$\begin{array}{c}\text { J12WN1 } \\
\text { OB C2 } \\
\text { Sample Date } 7 / 17 / 06\end{array}$} & \multicolumn{3}{|c|}{$\begin{array}{c}\text { J12WM5 } \\
\text { OB D3 } \\
\text { Sample Date } 7 / 12 / 06\end{array}$} & \multicolumn{3}{|c|}{\begin{tabular}{|c|} 
J12WM6 \\
OB D4 \\
Sample Date 7/12/06
\end{tabular}} \\
\hline & $\mu \mathrm{g} / \mathrm{kg}$ & $\mathbf{Q}$ & PQL & $\mu \mathrm{g} / \mathrm{kg}$ & $\mathbf{Q}$ & PQL & $\mu \mathrm{g} / \mathrm{kg}$ & $\mathbf{Q}$ & PQL & $\mu \mathrm{g} / \mathrm{kg}$ & $\mathbf{Q}$ & PQL \\
\hline \multicolumn{13}{|c|}{ Polychlorinated Biphenyls } \\
\hline Aroclor-1016 & 13 & $\mathrm{U}$ & 13 & 13 & U & 13 & 13 & $\mathrm{U}$ & 13 & 13 & UJ & 13 \\
\hline Aroclor-1221 & 13 & $\mathrm{U}$ & 13 & 13 & $\mathrm{U}$ & 13 & 13 & $\mathrm{U}$ & 13 & 13 & UJ & 13 \\
\hline Aroclor-1232 & 13 & $\bar{U}$ & 13 & 13 & $\mathrm{U}$ & 13 & 13 & $\mathrm{U}$ & 13 & 13 & UJ & 13 \\
\hline Aroclor- 1242 & 13 & $\mathrm{U}$ & 13 & 13 & $\mathrm{U}$ & 13 & 13 & $\mathrm{U}$ & 13 & 13 & UJ & 13 \\
\hline Aroclor-1248 & 13 & $\mathrm{U}$ & 13 & 13 & $\mathrm{U}$ & 13 & 13 & $\mathrm{U}$ & 13 & 13 & UJ & 13 \\
\hline Aroclor-1254 & 13 & $\mathrm{U}$ & 13 & 13 & $\mathrm{U}$ & 13 & 13 & $\mathrm{U}$ & 13 & 13 & UJ & 13 \\
\hline Aroclor-1260 & 13 & $\mathrm{U}$ & 13 & 13 & $\mathrm{U}$ & 13 & 13 & $\mathrm{U}$ & 13 & 13 & UJ & 13 \\
\hline \multicolumn{13}{|c|}{ Semivolatile Organic Compounds } \\
\hline 1,2,4-Trichlorobenzene & 340 & UJ & 340 & 330 & $\overline{U J}$ & 330 & 340 & $\mathrm{U}$ & 340 & 330 & $\mathrm{U}$ & 330 \\
\hline 1,2-Dichlorobenzene & 340 & $\mathrm{U}$ & 340 & 330 & $\mathrm{U}$ & 330 & 340 & $\mathrm{U}$ & 340 & 330 & $\mathrm{U}$ & 330 \\
\hline 1,3-Dichlorobenzene & 340 & $\mathrm{U}$ & 340 & 330 & $\mathrm{U}$ & 330 & 340 & $\mathrm{U}$ & 340 & 330 & $\mathrm{U}$ & 330 \\
\hline 1,4-Dichlorobenzene & 340 & $\mathrm{U}$ & 340 & 330 & $\bar{U}$ & 330 & 340 & $\mathrm{U}$ & 340 & 330 & $\mathrm{U}$ & 330 \\
\hline 2,4,5-Trichlorophenol & 840 & $\mathrm{U}$ & 840 & 840 & $\mathrm{U}$ & 840 & 840 & $\mathrm{U}$ & 840 & 840 & $\mathrm{U}$ & 840 \\
\hline 2,4,6-Trichlorophenol & 340 & $U$ & 340 & 330 & $\mathrm{U}$ & 330 & 340 & $\mathrm{U}$ & 340 & 330 & $\mathrm{U}$ & 330 \\
\hline 2,4-Dichlorophenol & 340 & U & 340 & 330 & $\mathrm{U}$ & 330 & 340 & $\mathrm{U}$ & 340 & 330 & $\mathrm{U}$ & 330 \\
\hline 2,4-Dimethylphenol & 340 & $\mathrm{U}$ & 340 & 330 & $\mathrm{U}$ & 330 & 340 & $\mathrm{U}$ & 340 & 330 & $\bar{U}$ & 330 \\
\hline 2,4-Dinitrophenol & 840 & $\mathrm{U}$ & 840 & 840 & $\bar{U}$ & 840 & 840 & $\bar{U}$ & 840 & 840 & $\bar{U}$ & 840 \\
\hline 2,4-Dinitrotoluene & 340 & $\bar{U}$ & 340 & 330 & $\bar{U}$ & 330 & 340 & $\bar{U}$ & 340 & 330 & $\mathrm{U}$ & 330 \\
\hline 2,6-Dinitrotoluene & 340 & $\bar{U}$ & 340 & 330 & $\mathrm{U}$ & 330 & 340 & $\mathrm{U}$ & 340 & 330 & $\mathrm{U}$ & 330 \\
\hline 2-Chloronaphthalene & 340 & $\mathrm{U}$ & 340 & 330 & $\mathrm{U}$ & 330 & 340 & $\bar{U}$ & 340 & 330 & $\mathrm{U}$ & 330 \\
\hline 2-Chlorophenol & 340 & U & 340 & 330 & $\mathrm{U}$ & 330 & 340 & $\mathrm{U}$ & 340 & 330 & $\mathrm{U}$ & 330 \\
\hline 2-Methylnaphthalene & 340 & $U$ & 340 & 330 & $\mathrm{U}$ & 330 & 340 & $\mathrm{U}$ & 340 & 330 & $\mathrm{U}$ & 330 \\
\hline 2-Methylphenol (cresol, o-) & 340 & $\mathrm{U}$ & 340 & 330 & $\mathrm{U}$ & 330 & 340 & $U$ & 340 & 330 & $\mathrm{U}$ & 330 \\
\hline 2-Nitroaniline & 840 & $\mathrm{U}$ & 840 & 840 & $\mathrm{U}$ & 840 & 840 & $\mathrm{U}$ & 840 & 840 & $\mathrm{U}$ & 840 \\
\hline 2-Nitrophenol & 340 & $\mathrm{U}$ & 340 & 330 & $\mathrm{U}$ & 330 & 340 & $\mathrm{U}$ & 340 & 330 & $\mathrm{U}$ & 330 \\
\hline 3,3'-Dichlorobenzidine & 340 & $\mathrm{U}$ & 340 & 330 & $\mathrm{U}$ & 330 & 340 & $\mathrm{U}$ & 340 & 330 & $\mathrm{U}$ & 330 \\
\hline 3-Nitroaniline & 840 & $\mathrm{U}$ & 840 & 840 & $\mathrm{U}$ & 840 & 840 & $\mathrm{U}$ & 840 & 840 & U & 840 \\
\hline 4,6-Dinitro-2-methylphenol & 840 & $\mathrm{U}$ & 840 & 840 & $\mathrm{U}$ & 840 & 840 & $\mathrm{U}$ & 840 & 840 & $\mathrm{U}$ & 840 \\
\hline 4-Bromophenyl-phenylether & 340 & $\mathrm{U}$ & 340 & 330 & $\mathrm{U}$ & 330 & 340 & $\mathrm{U}$ & 340 & 330 & $\mathrm{U}$ & 330 \\
\hline 4-Chloro-3-methylphenol & 340 & $\mathrm{U}$ & 340 & 330 & $\mathrm{U}$ & 330 & 340 & $\mathrm{U}$ & 340 & 330 & $\mathrm{U}$ & 330 \\
\hline 4-Chloroaniline & 340 & $\mathrm{U}$ & 340 & 330 & $\mathrm{U}$ & 330 & 340 & $\mathrm{U}$ & 340 & 330 & $\mathrm{U}$ & 330 \\
\hline 4-Chlorophenyl-phenylether & 340 & $\mathrm{U}$ & 340 & 330 & $\mathrm{U}$ & 330 & 340 & $\mathrm{U}$ & 340 & 330 & $\mathrm{U}$ & 330 \\
\hline 4-Methylphenol (p-cresol) & 340 & $\mathrm{U}$ & 340 & 330 & $\mathrm{U}$ & 330 & 340 & $\mathrm{U}$ & 340 & 330 & $\mathrm{U}$ & 330 \\
\hline 4-Nitroaniline & 840 & $\mathrm{U}$ & 840 & 840 & $\mathrm{U}$ & 840 & 840 & $\mathrm{U}$ & 840 & 840 & $\mathrm{U}$ & 840 \\
\hline 4-Nitrophenol & 840 & $\mathrm{U}$ & 840 & 840 & $\mathrm{U}$ & 840 & 840 & $\mathrm{U}$ & 840 & 840 & U & 840 \\
\hline Acenaphthene & 340 & $\mathrm{U}$ & 340 & 330 & $U$ & 330 & 340 & $\mathrm{U}$ & 340 & 330 & $\bar{U}$ & 330 \\
\hline Acenaphthylene & 340 & U & 340 & 330 & $\bar{U}$ & 330 & 340 & $\mathrm{U}$ & 340 & 330 & U & 330 \\
\hline Anthracene & 340 & $\mathrm{U}$ & 340 & 330 & $\bar{U}$ & 330 & 340 & $\mathrm{U}$ & 340 & 330 & U & 330 \\
\hline Benzo(a)anthracene & 340 & $\mathrm{U}$ & 340 & 330 & $\mathrm{U}$ & 330 & 340 & $\mathrm{U}$ & 340 & 330 & $\mathrm{U}$ & 330 \\
\hline Benzo(a)pyrene & 340 & $\mathrm{U}$ & 340 & 330 & $\mathrm{U}$ & 330 & 340 & $\mathrm{U}$ & 340 & 330 & $\mathrm{U}$ & 330 \\
\hline Benzo(b)fluoranthene & 340 & $\bar{U}$ & 340 & 330 & $\mathrm{U}$ & 330 & 340 & $\mathrm{U}$ & 340 & 330 & $\mathrm{U}$ & 330 \\
\hline Benzo(g,h,i)perylene & 340 & $\bar{U}$ & 340 & 330 & $\mathrm{U}$ & 330 & 340 & $\mathrm{U}$ & 340 & 330 & $\mathrm{U}$ & 330 \\
\hline Benzo(k)fluoranthene & 340 & $\mathrm{U}$ & 340 & 330 & $\mathrm{U}$ & 330 & 340 & $\mathrm{U}$ & 340 & 330 & $\mathrm{U}$ & 330 \\
\hline bis(2-Chloro-1-methylethyl)ether & 340 & $\mathrm{U}$ & 340 & 330 & $\mathrm{U}$ & 330 & 340 & $\mathrm{U}$ & 340 & 330 & $\mathrm{U}$ & 330 \\
\hline bis(2-Chloroethoxy)methane & 340 & $\mathrm{U}$ & 340 & 330 & $\mathrm{U}$ & 330 & 340 & $\mathrm{U}$ & 340 & 330 & $\mathrm{U}$ & 330 \\
\hline bis(2-Chloroethyl)ether & 340 & $\mathrm{U}$ & 340 & 330 & $\mathrm{U}$ & 330 & 340 & $\mathrm{U}$ & 340 & 330 & $\mathrm{U}$ & 330 \\
\hline bis(2-Ethylhexyl)phthalate & 37 & $\mathrm{~J}$ & 340 & 28 & $\mathrm{~J}$ & 330 & 28 & $\mathrm{~J}$ & 340 & 330 & $\mathrm{U}$ & 330 \\
\hline & & & & Attachr & & & 1 & & & et No. & & $f 113$ \\
\hline & & & & Origina & & $\overline{J . M . C a}$ & ron & & & Dat & & $104 / 07$ \\
\hline & & & & Checke & & T.M. Bl & kley & & & Dat & & \\
\hline & & & & Calc. $\mathrm{N}$ & & $0100 \mathrm{~B}-\mathrm{C}$ & A-V029 & & & Rev. No & & 1 \\
\hline
\end{tabular}


Attachment 1. 118-B-1 Verification Sampling Results.

\begin{tabular}{|c|c|c|c|c|c|c|c|c|c|c|c|c|}
\hline \multirow[t]{2}{*}{ Constituents } & \multicolumn{3}{|c|}{\begin{tabular}{|c|} 
J12WN0 \\
OB C1 \\
Sample Date 7/17/06
\end{tabular}} & \multicolumn{3}{|c|}{\begin{tabular}{|c|} 
J12WN1 \\
OB C2 \\
Sample Date 7/17/06 \\
\end{tabular}} & \multicolumn{3}{|c|}{$\begin{array}{c}\text { J12WM5 } \\
\text { OB D3 } \\
\text { Sample Date } 7 / 12 / 06 \\
\end{array}$} & \multicolumn{3}{|c|}{\begin{tabular}{|c|} 
J12WM6 \\
OB D4 \\
Sample Date $7 / 12 / 06$ \\
\end{tabular}} \\
\hline & $\mu \mathrm{g} / \mathrm{kg}$ & $\mathbf{Q}$ & PQL & $\mu \mathrm{g} / \mathrm{kg}$ & $\mathbf{Q}$ & PQL & $\mu \mathrm{g} / \mathrm{kg}$ & $Q$ & MDL & $\mu \mathrm{g} / \mathrm{kg}$ & $Q$ & PQL \\
\hline \multicolumn{13}{|c|}{ Semivolatile Organic Compounds (continued) } \\
\hline Butylbenzylphthalate & 340 & $\mathrm{U}$ & 340 & 330 & $\mathrm{U}$ & 330 & 340 & $\mathrm{U}$ & 340 & 330 & $\mathrm{U}$ & 330 \\
\hline Carbazole & 340 & $\mathrm{U}$ & 340 & 330 & $\mathrm{U}$ & 330 & 340 & $\mathrm{U}$ & 340 & 330 & $\mathrm{U}$ & 330 \\
\hline Chrysene & 340 & $U$ & 340 & 330 & $\mathrm{U}$ & 330 & 340 & $\mathrm{U}$ & 340 & 330 & $\mathrm{U}$ & 330 \\
\hline Di-n-butylphthalate & 39 & $\mathrm{~J}$ & 340 & 42 & $\mathrm{~J}$ & 330 & 19 & $\mathrm{~J}$ & 340 & 20 & $\mathrm{~J}$ & 330 \\
\hline Di-n-octylphthalate & 340 & $\mathrm{U}$ & 340 & 330 & $\mathrm{U}$ & 330 & 340 & $\mathrm{U}$ & 340 & 330 & $\mathrm{U}$ & 330 \\
\hline Dibenz(a,h)anthracene & 340 & $\mathrm{U}$ & 340 & 330 & $\mathrm{U}$ & 330 & 340 & $\mathrm{U}$ & 340 & 330 & $\mathrm{U}$ & 330 \\
\hline Dibenzofuran & 340 & $\bar{U}$ & 340 & 330 & $\mathrm{U}$ & 330 & 340 & $\mathrm{U}$ & 340 & 330 & $\mathrm{U}$ & 330 \\
\hline Diethylphthalate & 340 & $\mathrm{U}$ & 340 & 330 & $\mathrm{U}$ & 330 & 340 & $\mathrm{U}$ & 340 & 330 & $\mathrm{U}$ & 330 \\
\hline Dimethylphthalate & 340 & $\mathrm{U}$ & 340 & 330 & $\mathrm{U}$ & 330 & 340 & $\mathrm{U}$ & 340 & 330 & $\mathrm{U}$ & 330 \\
\hline Fluoranthene & 440 & $\mathrm{U}$ & 340 & 330 & $\bar{U}$ & 330 & 340 & $\bar{U}$ & 340 & 330 & $\mathrm{U}$ & 330 \\
\hline Fluorene & 40 & $\mathrm{U}$ & 340 & 330 & $U$ & 330 & 340 & $\overline{\mathrm{U}}$ & 340 & 330 & $\mathrm{U}$ & 330 \\
\hline Hexachlorobenzene & 40 & $\mathrm{U}$ & 340 & 330 & $U$ & 330 & 340 & $\mathrm{U}$ & 340 & 330 & $\mathrm{U}$ & 330 \\
\hline Hexachlorobutadiene & 340 & $\mathrm{U}$ & 340 & 330 & $\mathrm{U}$ & 330 & 340 & $\mathrm{U}$ & 340 & 330 & $\mathrm{U}$ & 330 \\
\hline Hexachlorocyclopentadiene & 340 & $\mathrm{U}$ & 340 & 330 & $\mathrm{U}$ & 330 & 340 & $\mathrm{U}$ & 340 & 330 & $\mathrm{U}$ & 330 \\
\hline Hexachloroethane & 340 & $\mathrm{U}$ & 340 & 330 & $\mathrm{U}$ & 330 & 340 & $\mathrm{U}$ & 340 & 330 & $\mathrm{U}$ & 330 \\
\hline Indeno( $1,2,3$-cd)pyrene & 340 & $\mathrm{U}$ & 340 & 330 & $\mathrm{U}$ & 330 & 340 & $\mathrm{U}$ & 340 & 330 & $\mathrm{U}$ & 330 \\
\hline Isophorone & 340 & $\mathrm{U}$ & 340 & 330 & $\mathrm{U}$ & 330 & 340 & $\mathrm{U}$ & 340 & 330 & $\mathrm{U}$ & 330 \\
\hline N-Nitroso-di-n-diprop & 340 & U & 340 & 330 & $\mathrm{U}$ & 330 & 340 & $\mathrm{U}$ & 340 & 330 & $\mathrm{U}$ & 330 \\
\hline N-Nitrosodiphenylamine & 340 & $\mathrm{U}$ & 340 & 330 & $\mathrm{U}$ & 330 & 340 & $\mathrm{U}$ & 340 & 330 & $\mathrm{U}$ & 330 \\
\hline Naphthalene & 340 & $\mathrm{U}$ & 340 & 330 & $\mathrm{U}$ & 330 & 340 & $\mathrm{U}$ & 340 & 330 & $\mathrm{U}$ & 330 \\
\hline Nitrobenzene & 340 & $\mathrm{U}$ & 340 & 330 & $\mathrm{U}$ & 330 & 340 & $\mathrm{U}$ & 340 & 330 & $\mathrm{U}$ & 330 \\
\hline Pentachlorophenol & 840 & $\mathrm{U}$ & 840 & 840 & $\mathrm{U}$ & 840 & 840 & $\bar{U}$ & 840 & 840 & $\bar{U}$ & 840 \\
\hline Phenanthrene & 340 & $\mathrm{U}$ & 340 & 330 & $\mathrm{U}$ & 330 & 340 & $\mathrm{U}$ & 340 & 330 & $U$ & 330 \\
\hline Phenol & 340 & $\mathrm{U}$ & 340 & 330 & $\mathrm{U}$ & 330 & 340 & $U$ & 340 & 330 & $U$ & 330 \\
\hline Pyrene & 340 & $\mathrm{U}$ & 340 & 330 & $\mathrm{U}$ & 330 & 340 & $U$ & 340 & 330 & $\mathrm{U}$ & 330 \\
\hline
\end{tabular}

Attachment

Originator

Checked

Calc. No.

$\frac{1}{\frac{1}{\text { J. M. Capron }}} \frac{}{\text { T. M. Blakley }}$

Sheet No. $\frac{70 \text { of } 113}{0}$
Date $\frac{02 / 07 / 07}{0}$
Date
Rev. No. 
Attachment 1. 118-B-1 Verification Sampling Results.

\begin{tabular}{|c|c|c|c|c|c|c|c|c|c|c|c|c|}
\hline \multirow[t]{2}{*}{ Constituents } & \multicolumn{3}{|c|}{$\begin{array}{c}\text { J12WN0 } \\
\text { OB C1 } \\
\text { Sample Date } 7 / 17 / 06\end{array}$} & \multicolumn{3}{|c|}{$\begin{array}{c}\text { J12WN1 } \\
\text { OB C2 } \\
\text { Sample Date } 7 / 17 / 06\end{array}$} & \multicolumn{3}{|c|}{$\begin{array}{c}\text { J12WM5 } \\
\text { OB D3 } \\
\text { Sample Date 7/12/06 }\end{array}$} & \multicolumn{3}{|c|}{$\begin{array}{c}\text { J12WM6 } \\
\text { OB D4 } \\
\text { Sample Date 7/12/06 }\end{array}$} \\
\hline & $\mu \mathrm{g} / \mathrm{kg}$ & $Q$ & PQL & $\mu \mathrm{g} / \mathrm{kg}$ & $\mathbf{Q}$ & PQL & $\mu \mathrm{g} / \mathrm{kg}$ & $\mathbf{Q}$ & MDL & $\mu \mathrm{g} / \mathrm{kg}$ & Q & PQL \\
\hline \multicolumn{13}{|c|}{ Volatile Organic Compounds } \\
\hline 1,1,1-Trichloroethane & 5 & $\mathrm{U}$ & 5 & 5 & $\bar{U}$ & 5 & 5 & UJ & 5 & 5 & UJ & 5 \\
\hline 1,1,2,2-Tetrachloroethane & 5 & $\mathrm{U}$ & 5 & 5 & $U$ & 5 & 5 & UJ & 5 & 5 & UJ & 5 \\
\hline 1,1,2-Trichloroethane & 5 & $\mathrm{U}$ & 5 & 5 & $\mathrm{U}$ & 5 & 5 & UJ & 5 & 5 & UJ & 5 \\
\hline 1,1-Dichloroethane & 5 & $\mathrm{U}$ & 5 & 5 & $\mathrm{U}$ & 5 & 5 & UJ & 5 & 5 & UJ & 5 \\
\hline 1,1-Dichloroethene & 5 & $\mathrm{U}$ & 5 & 5 & $\mathrm{U}$ & 5 & 5 & UJ & 5 & 5 & UJ & 5 \\
\hline 1,2-Dichloroethane & 5 & $\mathrm{U}$ & 5 & 5 & $\mathrm{U}$ & 5 & 5 & UJ & 5 & 5 & UJ & 5 \\
\hline 1,2-Dichloroethene (total) & 5 & $\mathrm{U}$ & 5 & 5 & $\mathrm{U}$ & 5 & 5 & UJ & 5 & 5 & UJ & 5 \\
\hline 1,2-Dichloropropane & 5 & $\mathrm{U}$ & 5 & 5 & $\mathrm{U}$ & 5 & 5 & UJ & 5 & 5 & \begin{tabular}{|l|l} 
UJ \\
\end{tabular} & 5 \\
\hline 2-Butanone & 10 & $\mathrm{U}$ & 10 & 9 & $\mathrm{U}$ & 9 & 10 & UJ & 10 & 10 & UJ & 10 \\
\hline 2-Hexanone & 10 & $\mathrm{U}$ & 10 & 9 & $\mathrm{U}$ & 9 & 10 & UJ & 10 & 10 & \begin{tabular}{|l|l}
$\mathrm{UJ}$ \\
\end{tabular} & 10 \\
\hline 4-Methyl-2-pentanone & 8 & $\mathrm{JB}$ & 10 & 9 & U & 9 & 6 & $\mathrm{~J}$ & 10 & 10 & UJ & 10 \\
\hline Acetone & 26 & & 10 & 39 & & 9 & 7 & $\mathrm{~J}$ & 10 & 10 & $\mathrm{UJ}$ & 10 \\
\hline Benzene & 5 & $\mathrm{U}$ & 5 & 5 & $\mathrm{U}$ & 5 & 5 & UJ & 5 & 5 & UJ & 5 \\
\hline Bromodichloromethane & 5 & $\mathrm{U}$ & 5 & 5 & $\mathrm{U}$ & 5 & 5 & UJ & 5 & 5 & UJ & 5 \\
\hline Bromoform & 5 & $\mathrm{U}$ & 5 & 5 & $\mathrm{U}$ & 5 & 5 & UJ & 5 & 5 & UJ & 5 \\
\hline Bromomethane & 10 & $\mathrm{U}$ & 10 & 9 & $\mathrm{U}$ & 9 & 10 & UJ & 10 & 10 & $\mathrm{UJ}$ & 10 \\
\hline Carbon disulfide & 5 & $\mathrm{U}$ & 5 & 5 & U & 5 & 5 & UJ & 5 & 5 & UJ & 5 \\
\hline Carbon tetrachloride & 7 & & 5 & 24 & & 5 & 10 & $\mathrm{~J}$ & 5 & 26 & $\mathrm{~J}$ & 5 \\
\hline Chlorobenzene & 5 & $\mathrm{U}$ & 5 & 5 & $\mathrm{U}$ & 5 & 5 & $\mathrm{UJ}$ & 5 & 5 & UJ & 5 \\
\hline Chloroethane & 10 & $\mathrm{U}$ & 10 & 9 & $\mathrm{U}$ & 9 & 10 & UJ & 10 & 10 & UJ & 10 \\
\hline Chloroform & 5 & $\mathrm{U}$ & 5 & 5 & $\mathrm{U}$ & 5 & 5 & $\mathrm{UJ}$ & 5 & 5 & UJ & 5 \\
\hline Chloromethane & 10 & $\mathrm{U}$ & 10 & 9 & $\mathrm{U}$ & 9 & 10 & $\mathrm{UJ}$ & 10 & 10 & UJ & 10 \\
\hline cis-1,2-Dichloroethylene & 5 & $\mathrm{U}$ & 5 & 5 & $\mathrm{U}$ & 5 & 5 & $\mathrm{UJ}$ & 5 & 5 & \begin{tabular}{|l|}
$\mathrm{UJ}$ \\
\end{tabular} & 5 \\
\hline cis-1,3-Dichloropropene & 5 & $\mathrm{U}$ & 5 & 5 & $\mathrm{U}$ & 5 & 5 & $\mathrm{UJ}$ & 5 & 5 & UJ & 5 \\
\hline Dibromochloromethane & 5 & $\mathrm{U}$ & 5 & 5 & $\mathrm{U}$ & 5 & 5 & UJ & 5 & 5 & UJ & 5 \\
\hline Ethylbenzene & 5 & $\mathrm{U}$ & 5 & 5 & $\bar{U}$ & 5 & 5 & UJ & 5 & 5 & UJ & 5 \\
\hline Methylene chloride & 6 & & 5 & 30 & & 5 & 8 & $\mathrm{~J}$ & 5 & 22 & $\mathrm{~J}$ & 5 \\
\hline Styrene & 5 & $\mathrm{U}$ & 5 & 5 & $\mathrm{U}$ & 5 & 5 & UJ & 5 & 5 & UJ & 5 \\
\hline Tetrachloroethene & 5 & $\mathrm{U}$ & 5 & 5 & $\mathrm{U}$ & 5 & 5 & UJ & 5 & 5 & UJ & 5 \\
\hline Toluene & 1 & JB & 5 & 2 & $\mathrm{JB}$ & 5 & 2 & $\mathrm{~J}$ & 5 & 1 & $\mathrm{~J}$ & 5 \\
\hline trans-1,2-Dichloroethylene & 5 & $\mathrm{U}$ & 5 & 5 & $\mathrm{U}$ & 5 & 5 & $\mathrm{UJ}$ & 5 & 5 & UJ & 5 \\
\hline trans-1,3-Dichloropropene & 5 & $\mathrm{U}$ & 5 & 5 & U & 5 & 5 & UJ & 5 & 5 & UJ & 5 \\
\hline Trichloroethene & 5 & $\bar{U}$ & 5 & 5 & $\bar{U}$ & 5 & 5 & $\overline{U J}$ & 5 & 5 & UJ & 5 \\
\hline Vinyl chloride & 10 & $\mathrm{U}$ & 10 & 9 & $\mathrm{U}$ & 9 & 10 & UJ & 10 & 10 & \begin{tabular}{|l|}
$U J$ \\
\end{tabular} & 10 \\
\hline Xylenes (total) & 5 & $\mathrm{U}$ & 5 & 5 & $\mathrm{U}$ & 5 & 5 & UJ & 5 & 5 & UI & 5 \\
\hline & & & & Attachm & & & 1 & & & heet No. & & of 113 \\
\hline & & & & Originat & & J.M. Ca & pron & & & Date & & $104 / 07$ \\
\hline & & & & Checked & & T.M.Bl & akley & & & Date & & \\
\hline & & & & Calc. N & & $0100 \mathrm{~B}-\mathrm{C}$ & $A-V 029$ & & & Rev. No. & & 1 \\
\hline
\end{tabular}


Rev. 0

Attachment 1. 118-B-1 Verification Sampling Results.

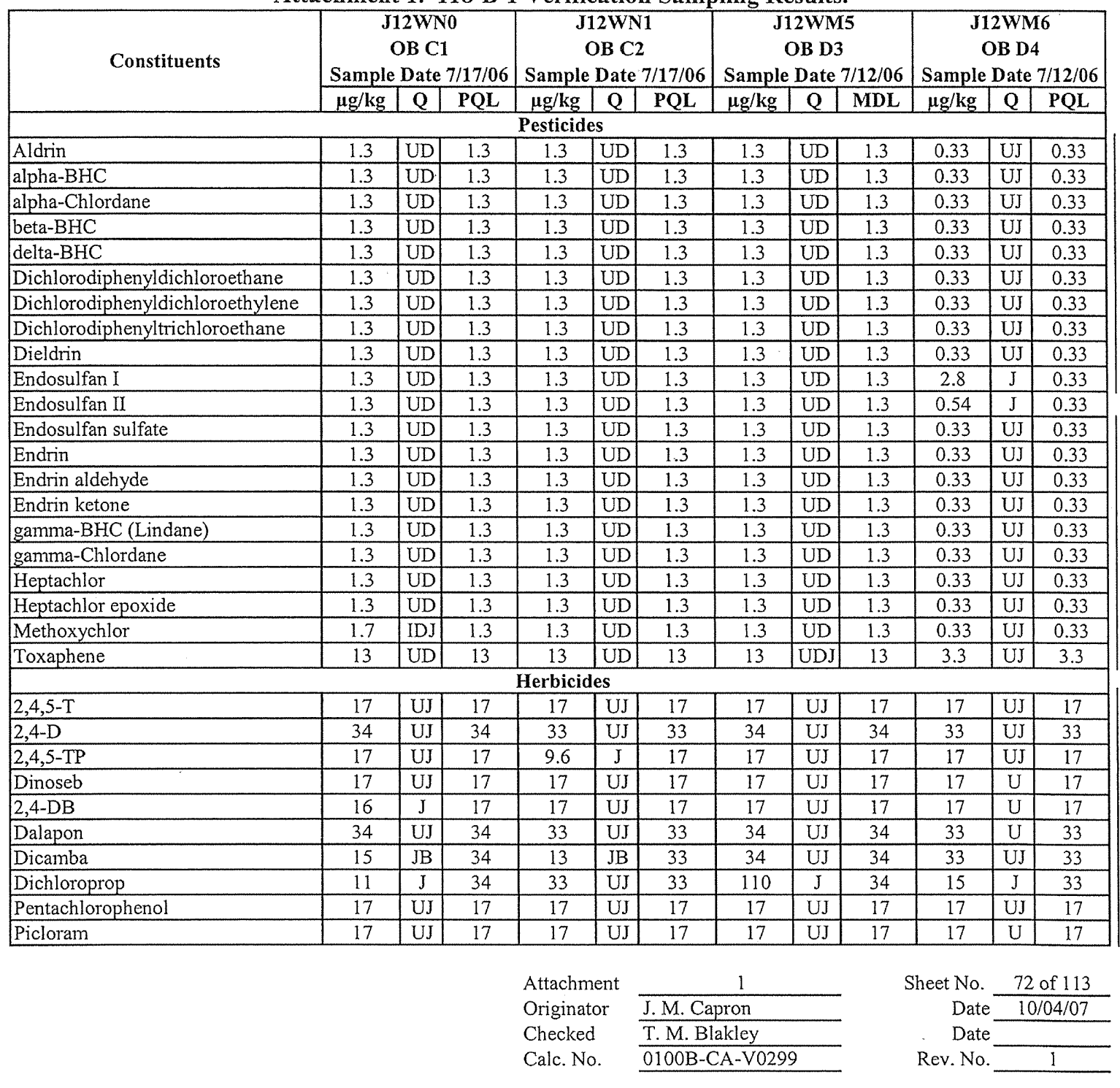


Attachment 1. 118-B-1 Verification Sampling Results.

\begin{tabular}{|c|c|c|c|c|c|c|c|c|c|c|c|c|}
\hline \multirow[t]{2}{*}{ Constituents } & \multicolumn{3}{|c|}{$\begin{array}{c}\text { J12WM3 } \\
\text { OB D5 } \\
\text { Sample Date } 7 / 12 / 06\end{array}$} & \multicolumn{3}{|c|}{$\begin{array}{c}\text { J12WM4 } \\
\text { OB D6 } \\
\text { Sample Date } 7 / 12 / 06\end{array}$} & \multicolumn{3}{|c|}{$\begin{array}{c}\text { J12WN2 } \\
\text { BCL A1 } \\
\text { Sample Date 7/20/06 }\end{array}$} & \multicolumn{3}{|c|}{\begin{tabular}{|c|} 
J12WM1 \\
BCL A2 \\
Sample Date $7 / 12 / 06$
\end{tabular}} \\
\hline & $\mu \mathrm{g} / \mathrm{kg}$ & $\mathbf{Q}$ & PQL & $\mu \mathrm{g} / \mathrm{kg}$ & $\mathbf{Q}$ & PQL & $\mu \mathrm{g} / \mathrm{kg}$ & $\mathrm{Q}$ & PQL & $\mu \mathrm{g} / \mathrm{kg}$ & $\mathbf{Q}$ & PQL \\
\hline \multicolumn{13}{|c|}{ Polychlorinated Biphenyls } \\
\hline Aroclor-1016 & 13 & $\mathrm{U}$ & 13 & 13 & $\mathrm{U}$ & 13 & 13 & $\mathrm{U}$ & 13 & 13 & $\mathrm{U}$ & 13 \\
\hline Aroclor-1221 & 13 & $\mathrm{U}$ & 13 & 13 & $\mathrm{U}$ & 13 & 13 & $\mathrm{U}$ & 13 & 13 & $\mathrm{U}$ & 13 \\
\hline Aroclor-1232 & 13 & $\mathrm{U}$ & 13 & 13 & $\mathrm{U}$ & 13 & 13 & $\mathrm{U}$ & 13 & 13 & $\mathrm{U}$ & 13 \\
\hline Aroclor-1242 & 13 & $\mathrm{U}$ & 13 & 13 & $\mathrm{U}$ & 13 & 13 & $\mathrm{U}$ & 13 & 13 & $\mathrm{U}$ & 13 \\
\hline Aroclor- 1248 & 13 & $\mathrm{U}$ & 13 & 13 & $\mathrm{U}$ & 13 & 13 & $\mathrm{U}$ & 13 & 13 & $\mathrm{U}$ & 13 \\
\hline Aroclor-1254 & 13 & $\mathrm{U}$ & 13 & 13 & $\mathrm{U}$ & 13 & 13 & $\bar{U}$ & 13 & 13 & $\mathrm{U}$ & 13 \\
\hline Aroclor- 1260 & 13 & $\mathrm{U}$ & 13 & 13 & $\mathrm{U}$ & 13 & 13 & $\mathrm{U}$ & 13 & 13 & $\mathrm{U}$ & 13 \\
\hline \multicolumn{13}{|c|}{ Semivolatile Organic Compounds } \\
\hline 1,2,4-Trichlorobenzene & 330 & $\mathrm{U}$ & 330 & 330 & U & 330 & 340 & $\overline{\mathrm{UJ}}$ & 340 & 340 & $\mathrm{U}$ & 340 \\
\hline 1,2-Dichlorobenzene & 330 & $\mathrm{U}$ & 330 & 330 & $\mathrm{U}$ & 330 & 340 & $\mathrm{U}$ & 340 & 340 & $\mathrm{U}$ & 340 \\
\hline 1,3-Dichlorobenzene & 330 & $\mathrm{U}$ & 330 & 330 & $\mathrm{U}$ & 330 & 340 & $\mathrm{U}$ & 340 & 340 & $\mathrm{U}$ & 340 \\
\hline 1,4-Dichlorobenzene & 330 & $\mathrm{U}$ & 330 & 330 & $\mathrm{U}$ & 330 & 340 & $\mathrm{U}$ & 340 & 340 & $\mathrm{U}$ & 340 \\
\hline 2,4,5-Trichlorophenol & 840 & $\mathrm{U}$ & 840 & 830 & $\mathrm{U}$ & 830 & 840 & $\mathrm{U}$ & 840 & 840 & $\mathrm{U}$ & 840 \\
\hline 2,4,6-Trichlorophenol & 330 & $\mathrm{U}$ & 330 & 330 & $\mathrm{U}$ & 330 & 340 & $\mathrm{U}$ & 340 & 340 & $\mathrm{U}$ & 340 \\
\hline 2,4-Dichlorophenol & 330 & $\mathrm{U}$ & 330 & 330 & $\mathrm{U}$ & 330 & 340 & $\mathrm{U}$ & 340 & 340 & $\mathrm{U}$ & 340 \\
\hline 2,4-Dimethylphenol & 330 & $\mathrm{U}$ & 330 & 330 & $\mathrm{U}$ & 330 & 340 & $\mathrm{UJ}$ & 340 & 340 & $\mathrm{U}$ & 340 \\
\hline 2,4-Dinitrophenol & 840 & $\mathrm{U}$ & 840 & 830 & $\mathrm{U}$ & 830 & 840 & $\mathrm{U}$ & 840 & 840 & U & 840 \\
\hline 2,4-Dinitrotoluene & 330 & $\mathrm{U}$ & 330 & 330 & $\mathrm{U}$ & 330 & 340 & $\mathrm{U}$ & 340 & 340 & $\mathrm{U}$ & 340 \\
\hline 2,6-Dinitrotoluene & 330 & $\mathrm{U}$ & 330 & 330 & $\mathrm{U}$ & 330 & 340 & $\mathrm{U}$ & 340 & 340 & $\mathrm{U}$ & 340 \\
\hline 2-Chloronaphthalene & 330 & $\mathrm{U}$ & 330 & 330 & $\mathrm{U}$ & 330 & 340 & $\mathrm{U}$ & 340 & 340 & $\mathrm{U}$ & 340 \\
\hline 2-Chlorophenol & 330 & $\mathrm{U}$ & 330 & 330 & $\mathrm{U}$ & 330 & 340 & $\mathrm{U}$ & 340 & 340 & $\mathrm{U}$ & 340 \\
\hline 2-Methylnaphthalene & 330 & $\mathrm{U}$ & 330 & 330 & $\mathrm{U}$ & 330 & 340 & UJ & 340 & 340 & $\mathrm{U}$ & 340 \\
\hline 2-Methylphenol (cresol, o-) & 330 & $\mathrm{U}$ & 330 & 330 & U & 330 & 340 & $\mathrm{UJ}$ & 340 & 340 & $\mathrm{U}$ & 340 \\
\hline 2-Nitroaniline & 840 & $\mathrm{U}$ & 840 & 830 & $\mathrm{U}$ & 830 & 840 & $\mathrm{U}$ & 840 & 840 & $\mathrm{U}$ & 840 \\
\hline 2-Nitrophenol & 330 & $\mathrm{U}$ & 330 & 330 & $\mathrm{U}$ & 330 & 340 & $\mathrm{U}$ & 340 & 340 & $\mathrm{U}$ & 340 \\
\hline 3,3'-Dichlorobenzidine & 330 & U & 330 & 330 & $\mathrm{U}$ & 330 & 340 & $\mathrm{U}$ & 340 & 340 & $\mathrm{U}$ & 340 \\
\hline 3-Nitroaniline & 840 & U & 840 & 830 & $\mathrm{U}$ & 830 & 840 & $\mathrm{U}$ & 840 & 840 & $\mathrm{U}$ & 840 \\
\hline 4,6-Dinitro-2-methylphenol & 840 & $\mathrm{U}$ & 840 & 830 & $\mathrm{U}$ & 830 & 840 & $\mathrm{U}$ & 840 & 840 & $\mathrm{U}$ & 840 \\
\hline 4-Bromophenyl-phenylether & 330 & $\mathrm{U}$ & 330 & 330 & $\mathrm{U}$ & 330 & 340 & $\mathrm{U}$ & 340 & 340 & $\mathrm{U}$ & 340 \\
\hline 4-Chloro-3-methylphenol & 330 & $\mathrm{U}$ & 330 & 330 & $\mathrm{U}$ & 330 & 340 & $\mathrm{UJ}$ & 340 & 340 & $\mathrm{U}$ & 340 \\
\hline 4-Chloroaniline & 330 & $\mathrm{U}$ & 330 & 330 & $\mathrm{U}$ & 330 & 340 & $\mathrm{U}$ & 340 & 340 & $\mathrm{U}$ & 340 \\
\hline 4-Chlorophenyl-phenylether & 330 & $\mathrm{U}$ & 330 & 330 & $\mathrm{U}$ & 330 & 340 & $\mathrm{U}$ & 340 & 340 & $\mathrm{U}$ & 340 \\
\hline 4-Methylphenol (p-cresol) & 330 & $\mathrm{U}$ & 330 & 330 & $\mathrm{U}$ & 330 & 340 & $\mathrm{UJ}$ & 340 & 340 & U & 340 \\
\hline 4-Nitroaniline & 840 & $\mathrm{U}$ & 840 & 830 & $\mathrm{U}$ & 830 & 840 & $\mathrm{U}$ & 840 & 840 & $\mathrm{U}$ & 840 \\
\hline 4-Nitrophenol & 840 & $\mathrm{U}$ & 840 & 830 & $\mathrm{U}$ & 830 & 840 & $\bar{U}$ & 840 & 840 & $\bar{U}$ & 840 \\
\hline Acenaphthene & 330 & $\mathrm{U}$ & 330 & 330 & $\mathrm{U}$ & 330 & 340 & $\mathrm{U}$ & 340 & 340 & $\mathrm{U}$ & 340 \\
\hline Acenaphthylene & 330 & U & 330 & 330 & $\mathrm{U}$ & 330 & 340 & $\mathrm{U}$ & 340 & 340 & $\mathrm{U}$ & 340 \\
\hline Anthracene & 330 & $\mathrm{U}$ & 330 & 330 & $\mathrm{U}$ & 330 & 340 & $\mathrm{U}$ & 340 & 340 & $\mathrm{U}$ & 340 \\
\hline Benzo(a)anthracene & 330 & $\mathrm{U}$ & 330 & 330 & $\mathrm{U}$ & 330 & 340 & $\mathrm{U}$ & 340 & 340 & $\mathrm{U}$ & 340 \\
\hline Benzo(a)pyrene & 330 & $\mathrm{U}$ & 330 & 330 & $\mathrm{U}$ & 330 & 340 & $\mathrm{U}$ & 340 & 340 & $\mathrm{U}$ & 340 \\
\hline Benzo(b)fluoranthene & 330 & $\mathrm{U}$ & 330 & 330 & $\mathrm{U}$ & 330 & 340 & $\mathrm{U}$ & 340 & 340 & $\mathrm{U}$ & 340 \\
\hline Benzo $(\mathrm{g}, \mathrm{h}, \mathrm{i})$ perylene & 330 & $\mathrm{U}$ & 330 & 330 & $\mathrm{U}$ & 330 & 340 & $\mathrm{U}$ & 340 & 340 & $\mathrm{U}$ & 340 \\
\hline Benzo(k)fluoranthene & 330 & $\mathrm{U}$ & 330 & 330 & $\mathrm{U}$ & 330 & 340 & $\mathrm{U}$ & 340 & 340 & $\mathrm{U}$ & 340 \\
\hline bis(2-Chloro-1-methylethyl)ether & 330 & $\bar{U}$ & 330 & 330 & $\mathrm{U}$ & 330 & 340 & $\mathrm{U}$ & 340 & 340 & $\mathrm{U}$ & 340 \\
\hline bis(2-Chloroethoxy)methane & 330 & $\mathrm{U}$ & 330 & 330 & $\mathrm{U}$ & 330 & 340 & $\mathrm{U}$ & 340 & 340 & $\mathrm{U}$ & 340 \\
\hline bis(2-Chloroethyl)ether & 330 & $\mathrm{U}$ & 330 & 330 & $\mathrm{U}$ & 330 & 340 & $\mathrm{U}$ & 340 & 340 & $\mathrm{U}$ & 340 \\
\hline bis(2-Ethylhexyl)phthalate & 21 & $\mathrm{~J}$ & 330 & 34 & $\mathrm{~J}$ & 330 & 51 & $\mathrm{~J}$ & 340 & 23 & $\mathrm{~J}$ & 340 \\
\hline
\end{tabular}

Attachment

Originator

Checked

Calc. No.

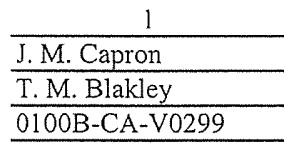

Sheet No. $\frac{73 \text { of } 113}{10 / 04 / 07}$
Date
Date
Rev. No. 1 
Rev. 0

Attachment 1. 118-B-1 Verification Sampling Results.

\begin{tabular}{|c|c|c|c|c|c|c|c|c|c|c|c|c|}
\hline \multirow[t]{2}{*}{ Constituents } & \multicolumn{3}{|c|}{$\begin{array}{c}\text { J12WM3 } \\
\text { OB D5 } \\
\text { Sample Date } 7 / 12 / 06\end{array}$} & \multicolumn{3}{|c|}{$\begin{array}{c}\text { J12WM4 } \\
\text { OB D6 } \\
\text { Sample Date } 7 / 12 / 06 \\
\end{array}$} & \multicolumn{3}{|c|}{$\begin{array}{c}\text { J12WN2 } \\
\text { BCL A1 } \\
\text { Sample Date 7/20/06 } \\
\end{array}$} & \multicolumn{3}{|c|}{\begin{tabular}{|c|} 
J12WM1 \\
BCL A2 \\
Sample Date 7/12/06 \\
\end{tabular}} \\
\hline & $\mu \mathrm{g} / \mathrm{kg}$ & $\mathbf{Q}$ & PQL & $\mu \mathrm{g} / \mathrm{kg}$ & $\mathbf{Q}$ & PQL & $\mu \mathrm{g} / \mathrm{kg}$ & $\mathbf{Q}$ & MDL & $\mu \mathrm{g} / \mathrm{kg}$ & Q & PQL \\
\hline \multicolumn{13}{|c|}{ Semivolatile Organic Compounds (continued) } \\
\hline Butylbenzylphthalate & 330 & $\mathrm{U}$ & 330 & 330 & $\mathrm{U}$ & 330 & 340 & $\mathrm{U}$ & 340 & 340 & $\mathrm{U}$ & 340 \\
\hline Carbazole & 330 & $\mathrm{U}$ & 330 & 330 & $\mathrm{U}$ & 330 & 340 & $\mathrm{U}$ & 340 & 340 & $U$ & 340 \\
\hline Chrysene & 330 & $\mathrm{U}$ & 330 & 330 & $\mathrm{U}$ & 330 & 340 & $\mathrm{U}$ & 340 & 340 & $\mathrm{U}$ & 340 \\
\hline Di-n-butylphthalate & 330 & $\mathrm{U}$ & 330 & 330 & $\mathrm{U}$ & 330 & 42 & JB & 340 & 340 & $\mathrm{U}$ & 340 \\
\hline Di-n-octylphthalate & 330 & U & 330 & 330 & $\mathrm{U}$ & 330 & 340 & $\mathrm{U}$ & 340 & 340 & $\mathrm{U}$ & 340 \\
\hline Dibenz $(\mathrm{a}, \mathrm{h})$ anthracene & 330 & $U$ & 330 & 330 & $\mathrm{U}$ & 330 & 340 & $\mathrm{U}$ & 340 & 340 & $\mathrm{U}$ & 340 \\
\hline Dibenzofuran & 330 & $\mathrm{U}$ & 330 & 330 & $\mathrm{U}$ & 330 & 340 & $\mathrm{U}$ & 340 & 340 & $\mathrm{U}$ & 340 \\
\hline Diethylphthalate & 330 & $\mathrm{U}$ & 330 & 330 & $\mathrm{U}$ & 330 & 340 & $\mathrm{U}$ & 340 & 340 & $\mathrm{U}$ & 340 \\
\hline Dimethylphthalate & 330 & $\mathrm{U}$ & 330 & 330 & $\mathrm{U}$ & 330 & 340 & $\mathrm{U}$ & 340 & 340 & $\mathrm{U}$ & 340 \\
\hline Fluoranthene & 330 & $\mathrm{U}$ & 330 & 330 & $\mathrm{U}$ & 330 & 340 & $\mathrm{U}$ & 340 & 340 & $\mathrm{U}$ & 340 \\
\hline Fluorene & 330 & $\mathrm{U}$ & 330 & 330 & $\mathrm{U}$ & 330 & 340 & $\mathrm{U}$ & 340 & 340 & $\mathrm{U}$ & 340 \\
\hline Hexachlorobenzene & 330 & $\mathrm{U}$ & 330 & 330 & $\mathrm{U}$ & 330 & 340 & $\mathrm{U}$ & 340 & 340 & $\mathrm{U}$ & 340 \\
\hline Hexachlorobutadiene & 330 & $\mathrm{U}$ & 330 & 330 & $\mathrm{U}$ & 330 & 340 & $\mathrm{U}$ & 340 & 340 & $\mathrm{U}$ & 340 \\
\hline Hexachlorocyclopentadiene & 330 & $\mathrm{U}$ & 330 & 330 & $\mathrm{U}$ & 330 & 340 & $\mathrm{U}$ & 340 & 340 & $\mathrm{U}$ & 340 \\
\hline Hexachloroethane & 330 & $\mathrm{U}$ & 330 & 330 & $\mathrm{U}$ & 330 & 340 & $\mathrm{U}$ & 340 & 340 & $\mathrm{U}$ & 340 \\
\hline Indeno(1,2,3-cd)pyrene & 330 & $\mathrm{U}$ & 330 & 330 & $\mathrm{U}$ & 330 & 340 & $\mathrm{U}$ & 340 & 340 & $\mathrm{U}$ & 340 \\
\hline Isophorone & 330 & $\mathrm{U}$ & 330 & 330 & $\mathrm{U}$ & 330 & 340 & UJ & 340 & 340 & $\mathrm{U}$ & 340 \\
\hline N-Nitroso-di-n-dipropylamine & 330 & $\mathrm{U}$ & 330 & 330 & $\mathrm{U}$ & 330 & 340 & $\mathrm{U}$ & 340 & 340 & U & 340 \\
\hline $\mathrm{N}$-Nitrosodiphenylamine & 330 & $\mathrm{U}$ & 330 & 330 & $\mathrm{U}$ & 330 & 340 & $\mathrm{U}$ & 340 & 340 & $\mathrm{U}$ & 340 \\
\hline Naphthalene & 330 & $\mathrm{U}$ & 330 & 330 & $\mathrm{U}$ & 330 & 340 & $\mathrm{U}$ & 340 & 340 & $\mathrm{U}$ & 340 \\
\hline Nitrobenzene & 330 & $\mathrm{U}$ & 330 & 330 & $\mathrm{U}$ & 330 & 340 & $\mathrm{U}$ & 340 & 340 & $\bar{U}$ & 340 \\
\hline Pentachlorophenol & 840 & $\mathrm{U}$ & 840 & 830 & $\mathrm{U}$ & 830 & 840 & $\mathrm{U}$ & 840 & 840 & $\mathrm{U}$ & 840 \\
\hline Phenanthrene & 330 & $\mathrm{U}$ & 330 & 330 & $\mathrm{U}$ & 330 & 340 & $\mathrm{U}$ & 340 & 340 & $\mathrm{U}$ & 340 \\
\hline Phenol & 330 & $\mathrm{U}$ & 330 & 330 & $\mathrm{U}$ & 330 & 340 & $\mathrm{U}$ & 340 & 340 & $\mathrm{U}$ & 340 \\
\hline Pyrene & 330 & $\mathrm{U}$ & 330 & 330 & $\mathrm{U}$ & 330 & 340 & $\mathrm{U}$ & 340 & 340 & $\mathrm{U}$ & 340 \\
\hline & & & & Attachn & & & 1 & & & & & of 113 \\
\hline & & & & Origina & & J.M. Ca & rron & & & Date & & $04 / 07$ \\
\hline & & & & Checked & & T.M.Bl & kley & & & Date & & \\
\hline & & & & Calc. N & & $0100 \mathrm{~B}-\mathrm{C}$ & $A-V 0299$ & & & Rev. No. & & 1 \\
\hline
\end{tabular}


Attachment 1. 118-B-1 Verification Sampling Results.

\begin{tabular}{|c|c|c|c|c|c|c|c|c|c|c|c|c|}
\hline \multirow[t]{2}{*}{ Constituents } & \multicolumn{3}{|c|}{$\begin{array}{c}\text { J12WM3 } \\
\text { OB D5 } \\
\text { Sample Date } 7 / 12 / 06\end{array}$} & \multicolumn{3}{|c|}{$\begin{array}{c}\text { J12WM4 } \\
\text { OB D6 } \\
\text { Sample Date 7/12/06 }\end{array}$} & \multicolumn{3}{|c|}{$\begin{array}{c}\text { J12WN2 } \\
\text { BCL A1 } \\
\text { Sample Date 7/20/06 }\end{array}$} & \multicolumn{3}{|c|}{\begin{tabular}{|c|} 
J12WM1 \\
BCL A2 \\
Sample Date 7/12/06
\end{tabular}} \\
\hline & $\mu \mathrm{g} / \mathrm{kg}$ & $Q$ & PQL & $\mu \mathrm{g} / \mathrm{kg}$ & $Q$ & PQL & $\mu \mathrm{g} / \mathrm{kg}$ & $Q$ & MDL & $\mu \mathrm{g} / \mathrm{kg}$ & $\mathbf{Q}$ & PQL \\
\hline \multicolumn{13}{|c|}{ Volatile Organic Compounds } \\
\hline 1,1,1-Trichloroethane & 5 & $\mathrm{U}$ & 5 & 5 & $\mathrm{U}$ & 5 & 5 & $\mathrm{U}$ & 5 & 5 & $\mathrm{U}$ & 5 \\
\hline 1,1,2,2-Tetrachloroethane & 5 & $\mathrm{U}$ & 5 & 5 & $\mathrm{U}$ & 5 & 5 & $\mathrm{U}$ & 5 & 5 & $\mathrm{U}$ & 5 \\
\hline 1,1,2-Trichloroethane & 5 & $\mathrm{U}$ & 5 & 5 & $\mathrm{U}$ & 5 & 5 & $\mathrm{U}$ & 5 & 5 & $\mathrm{U}$ & 5 \\
\hline 1,1-Dichloroethane & 5 & $\mathrm{U}$ & 5 & 5 & $\mathrm{U}$ & 5 & 5 & $\mathrm{U}$ & 5 & 5 & $\mathrm{U}$ & 5 \\
\hline 1,1-Dichloroethene & 5 & $\mathrm{U}$ & 5 & 5 & $\mathrm{U}$ & 5 & 5 & $\mathrm{U}$ & 5 & 5 & $U$ & 5 \\
\hline 1,2-Dichloroethane & 5 & $\mathrm{U}$ & 5 & 5 & $\mathrm{U}$ & 5 & 5 & $\mathrm{U}$ & 5 & 5 & $\mathrm{U}$ & 5 \\
\hline 1,2-Dichloroethene (total) & 5 & $\mathrm{U}$ & 5 & 5 & $\mathrm{U}$ & 5 & 5 & $\mathrm{U}$ & 5 & 5 & $\mathrm{U}$ & 5 \\
\hline 1,2-Dichloropropane & 5 & U & 5 & 5 & U & 5 & 5 & $\mathrm{U}$ & 5 & 5 & $\mathrm{U}$ & 5 \\
\hline 2-Butanone & 10 & U & 10 & 9 & U & 9 & 10 & $U$ & 10 & 10 & $U$ & 10 \\
\hline 2-Hexanone & 10 & $\mathrm{U}$ & 10 & 9 & $\mathrm{U}$ & 9 & 10 & $\mathrm{U}$ & 10 & 10 & $\mathrm{U}$ & 10 \\
\hline 4-Methyl-2-pentanone & 10 & $\mathrm{U}$ & 10 & 9 & $\mathrm{U}$ & 9 & 7 & $\mathrm{JB}$ & 10 & 10 & $U$ & 10 \\
\hline Acetone & 10 & $\mathrm{U}$ & 10 & 9 & $\mathrm{U}$ & 9 & 12 & & 10 & 10 & $\mathrm{U}$ & 10 \\
\hline Benzene & 5 & $\mathrm{U}$ & 5 & 5 & $\mathrm{U}$ & 5 & 5 & $\mathrm{U}$ & 5 & 5 & $\mathrm{U}$ & 5 \\
\hline Bromodichloromethane & 5 & $\mathrm{U}$ & 5 & 5 & $\mathrm{U}$ & 5 & 5 & $\mathrm{U}$ & 5 & 5 & $\mathrm{U}$ & 5 \\
\hline \begin{tabular}{|l|} 
Bromoform \\
\end{tabular} & 5 & $\mathrm{U}$ & 5 & 5 & $\mathrm{U}$ & 5 & 5 & U & 5 & 5 & $\mathrm{U}$ & 5 \\
\hline Bromomethane & 10 & $\mathrm{U}$ & 10 & 9 & $\mathrm{U}$ & 9 & 10 & $\mathrm{U}$ & 10 & 10 & $\mathrm{U}$ & 10 \\
\hline Carbon disulfide & 5 & $\mathrm{U}$ & 5 & 5 & $\mathrm{U}$ & 5 & 5 & $\mathrm{U}$ & 5 & 5 & $\mathrm{U}$ & 5 \\
\hline Carbon tetrachloride & 6 & $\mathrm{~J}$ & 5 & 3 & $\mathrm{~J}$ & 5 & 5 & $\mathrm{U}$ & 5 & 2 & $\mathrm{~J}$ & 5 \\
\hline Chlorobenzene & 5 & $\mathrm{U}$ & 5 & 5 & $\mathrm{U}$ & 5 & 5 & $\bar{U}$ & 5 & 5 & $\mathrm{U}$ & 5 \\
\hline Chloroethane & 10 & $\mathrm{U}$ & 10 & 9 & U & 9 & 10 & $\mathrm{U}$ & 10 & 10 & $\mathrm{U}$ & 10 \\
\hline Chloroform & 5 & $\mathrm{U}$ & 5 & 5 & $\mathrm{U}$ & 5 & 5 & $\bar{U}$ & 5 & 5 & $\mathrm{U}$ & 5 \\
\hline Chloromethane & 10 & $\mathrm{U}$ & 10 & 9 & $\mathrm{U}$ & 9 & 10 & $\mathrm{U}$ & 10 & 10 & $\mathrm{U}$ & 10 \\
\hline cis-1,2-Dichloroethylene & 5 & $\mathrm{U}$ & 5 & 5 & U & 5 & 5 & $\mathrm{U}$ & 5 & 5 & $\mathrm{U}$ & 5 \\
\hline cis-1,3-Dichloropropene & 5 & $\mathrm{U}$ & 5 & 5 & $\mathrm{U}$ & 5 & 5 & $\mathrm{U}$ & 5 & 5 & $\mathrm{U}$ & 5 \\
\hline Dibromochloromethane & 5 & $\mathrm{U}$ & 5 & 5 & $\mathrm{U}$ & 5 & 5 & $\mathrm{U}$ & 5 & 5 & $\mathrm{U}$ & 5 \\
\hline Ethylbenzene & 5 & $\mathrm{U}$ & 5 & 5 & $\mathrm{U}$ & 5 & 5 & $\mathrm{U}$ & 5 & 5 & $\mathrm{U}$ & 5 \\
\hline \begin{tabular}{|l} 
Methylene chloride \\
\end{tabular} & 14 & $\mathrm{~B}$ & 5 & 11 & $\mathrm{~B}$ & 5 & 13 & & 5 & 6 & $\mathrm{~B}$ & 5 \\
\hline Styrene & 5 & U & 5 & 5 & $\mathrm{U}$ & 5 & 5 & $\mathrm{U}$ & 5 & 5 & $\mathrm{U}$ & 5 \\
\hline \begin{tabular}{|l} 
Tetrachloroethene \\
\end{tabular} & 5 & $\mathrm{U}$ & 5 & 5 & $\mathrm{U}$ & 5 & 5 & $\mathrm{U}$ & 5 & 5 & $\bar{U}$ & 5 \\
\hline Toluene & 5 & $U$ & 5 & 5 & $\mathrm{U}$ & 5 & 5 & $\mathrm{U}$ & 5 & 5 & $\mathrm{U}$ & 5 \\
\hline \begin{tabular}{|l} 
trans-1,2-Dichloroethylene \\
\end{tabular} & 5 & $\mathrm{U}$ & 5 & 5 & U & 5 & 5 & $\mathrm{U}$ & 5 & 5 & $\mathrm{U}$ & 5 \\
\hline trans-1,3-Dichloropropene & 5 & $\mathrm{U}$ & 5 & 5 & $\mathrm{U}$ & 5 & 5 & $\mathrm{U}$ & 5 & 5 & $U$ & 5 \\
\hline Trichloroethene & 5 & $\mathrm{U}$ & 5 & 5 & $\mathrm{U}$ & 5 & 5 & $\mathrm{U}$ & 5 & 5 & $\mathrm{U}$ & 5 \\
\hline \begin{tabular}{|l} 
Vinyl chloride \\
\end{tabular} & 10 & $\mathrm{U}$ & 10 & 9 & $\mathrm{U}$ & 9 & 10 & $U$ & 10 & 10 & $\mathrm{U}$ & 10 \\
\hline Xylenes (total) & 5 & $\mathrm{U}$ & 5 & 5 & $\mathrm{U}$ & 5 & 5 & $\mathrm{U}$ & 5 & 5 & $\mathrm{U}$ & 5 \\
\hline & & & & Attachr & & & 1 & & & heet No. & & of 113 \\
\hline & & & & Origina & & J.M. Ce & pron & & & Date & & $/ 04 / 07$ \\
\hline & & & & Checke & & T.M.B & akley & & & Date & & \\
\hline & & & & Calc. $N$ & & $0100 \mathrm{~B}-$ & $\mathrm{A}-\mathrm{V} 02$ & & & Rev. No. & & 1 \\
\hline
\end{tabular}


Attachment 1. 118-B-1 Verification Sampling Results.

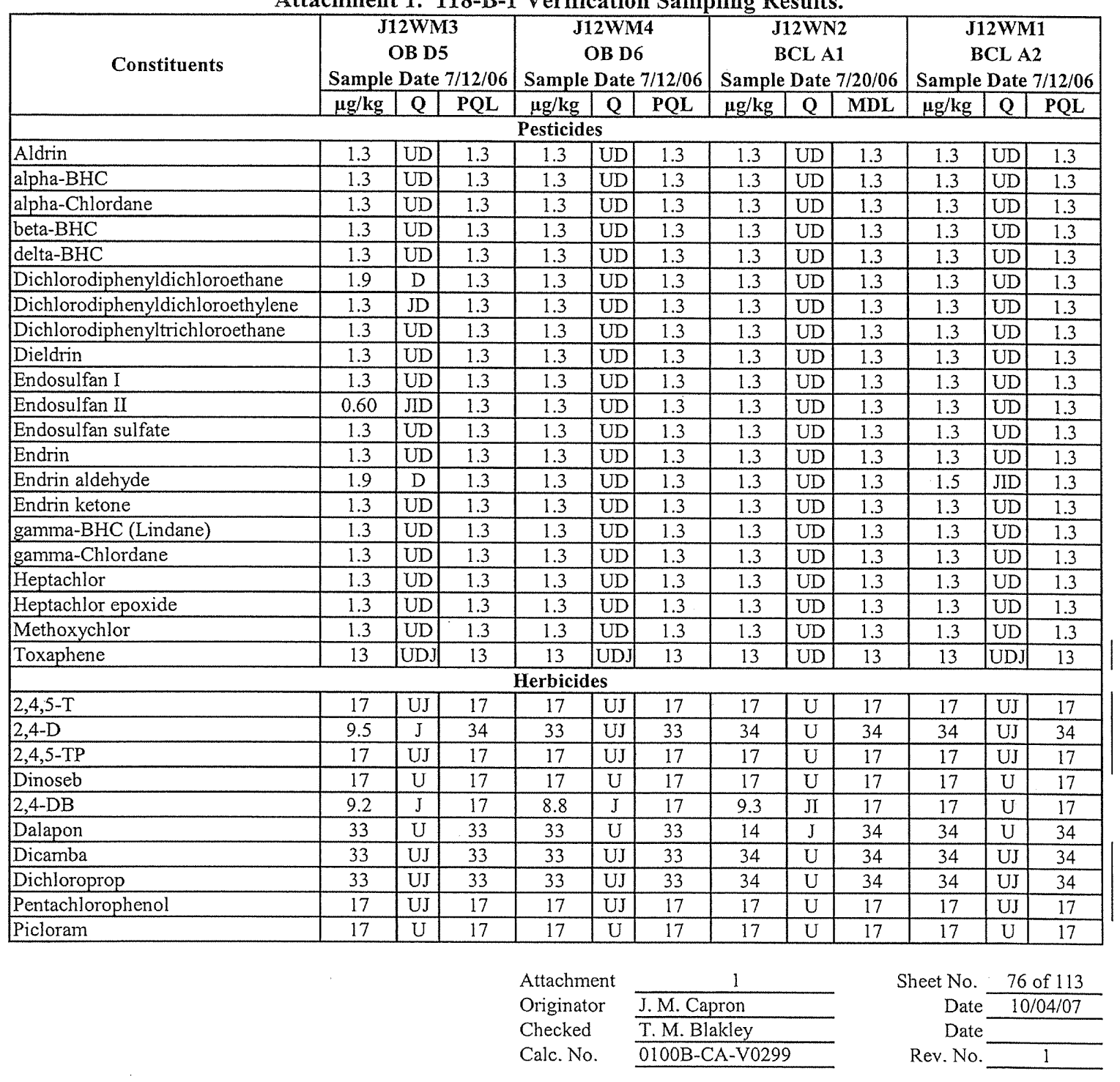


Attachment 1. 118-B-1 Verification Sampling Results.

\begin{tabular}{|c|c|c|c|c|c|c|c|c|c|c|c|c|}
\hline \multirow[t]{2}{*}{ Constituents } & \multicolumn{3}{|c|}{$\begin{array}{c}\text { J12WM2 } \\
\text { BCL A3 } \\
\text { Sample Date } 7 / 12 / 06\end{array}$} & \multicolumn{3}{|c|}{$\begin{array}{c}\text { J12WM0 } \\
\text { BCL A4 } \\
\text { Sample Date } 7 / 12 / 06\end{array}$} & \multicolumn{3}{|c|}{$\begin{array}{c}\text { J12WL9 } \\
\text { BCL B5 } \\
\text { Sample Date } 7 / 12 / 06\end{array}$} & \multicolumn{3}{|c|}{\begin{tabular}{|c|} 
J12WL8 \\
BCL B6 \\
Sample Date 7/12/06
\end{tabular}} \\
\hline & $\mu \mathrm{g} / \mathrm{kg}$ & $Q$ & PQL & $\mu \mathrm{g} / \mathrm{kg}$ & $\mathbf{Q}$ & PQL & $\mu \mathrm{g} / \mathrm{kg}$ & $Q$ & PQL & $\mu \mathrm{g} / \mathrm{kg}$ & $\mathrm{Q}$ & PQL \\
\hline \multicolumn{13}{|c|}{ Polychlorinated Biphenyls } \\
\hline Aroclor-1016 & 13 & $\mathrm{U}$ & 13 & 13 & $\mathrm{U}$ & 13 & 13 & UJ & 13 & 13 & $\mathrm{U}$ & 13 \\
\hline Aroclor-1221 & 13 & $U$ & 13 & 13 & $\mathrm{U}$ & 13 & 13 & UJ & 13 & 13 & $\mathrm{U}$ & 13 \\
\hline Aroclor-1232 & 13 & $\mathrm{U}$ & 13 & 13 & $\mathrm{U}$ & 13 & 13 & UJ & 13 & 13 & 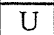 & 13 \\
\hline Aroclor-1242 & 13 & $\mathrm{U}$ & 13 & 13 & $U$ & 13 & 13 & UJ & 13 & 13 & $U$ & 13 \\
\hline Aroclor-1248 & 13 & U & 13 & 13 & $\mathrm{U}$ & 13 & 13 & UJ & 13 & 13 & $\mathrm{U}$ & 13 \\
\hline Aroclor-1254 & 13 & $\mathrm{U}$ & 13 & 13 & $\mathrm{U}$ & 13 & 13 & UJ & 13 & 13 & $\mathrm{U}$ & 13 \\
\hline Aroclor-1260 & 13 & $\mathrm{U}$ & 13 & 13 & $\mathrm{U}$ & 13 & 13 & UJ & 13 & 13 & $\mathrm{U}$ & 13 \\
\hline \multicolumn{13}{|c|}{ Semivolatile Organic Compounds } \\
\hline 1,2,4-Trichlorobenzene & 340 & $\mathrm{U}$ & 340 & 330 & $\mathrm{U}$ & 330 & 340 & U & 340 & 330 & 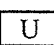 & 330 \\
\hline 1,2-Dichlorobenzene & 340 & $\mathrm{U}$ & 340 & 330 & $\mathrm{U}$ & 330 & 340 & U & 340 & 330 & $\mathrm{U}$ & 330 \\
\hline 1,3-Dichlorobenzene & 340 & $\mathrm{U}$ & 340 & 330 & $\mathrm{U}$ & 330 & 340 & $\mathrm{U}$ & 340 & 330 & $\mathrm{U}$ & 330 \\
\hline 1,4-Dichlorobenzene & 340 & $\mathrm{U}$ & 340 & 330 & $\mathrm{U}$ & 330 & 340 & $\mathrm{U}$ & 340 & 330 & $\mathrm{U}$ & 330 \\
\hline 2,4,5-Trichlorophenol & 840 & $\mathrm{U}$ & 840 & 840 & $\mathrm{U}$ & 840 & 840 & $\mathrm{U}$ & 840 & 840 & $\mathrm{U}$ & 840 \\
\hline 2,4,6-Trichlorophenol & 340 & $\mathrm{U}$ & 340 & 330 & $\bar{U}$ & 330 & 340 & $\mathrm{U}$ & 340 & 330 & $U$ & 330 \\
\hline 2,4-Dichlorophenol & 340 & $\mathrm{U}$ & 340 & 330 & $\mathrm{U}$ & 330 & 340 & $\mathrm{U}$ & 340 & 330 & $\mathrm{U}$ & 330 \\
\hline 2,4-Dimethylphenol & 340 & $\mathrm{U}$ & 340 & 330 & $\mathrm{U}$ & 330 & 340 & $\mathrm{U}$ & 340 & 330 & U & 330 \\
\hline 2,4-Dinitrophenol & 840 & $\mathrm{U}$ & 840 & 840 & $\mathrm{U}$ & 840 & 840 & $\mathrm{U}$ & 840 & 840 & $\mathrm{U}$ & 840 \\
\hline 2,4-Dinitrotoluene & 340 & $\mathrm{U}$ & 340 & 330 & $\mathrm{U}$ & 330 & 340 & $\mathrm{U}$ & 340 & 330 & U & 330 \\
\hline 2,6-Dinitrotoluene & 340 & $\mathrm{U}$ & 340 & 330 & $\mathrm{U}$ & 330 & 340 & U & 340 & 330 & U & 330 \\
\hline 2-Chloronaphthalene & 340 & $\mathrm{U}$ & 340 & 330 & $\mathrm{U}$ & 330 & 340 & $\mathrm{U}$ & 340 & 330 & $U$ & 330 \\
\hline 2-Chlorophenol & 340 & $\mathrm{U}$ & 340 & 330 & $\mathrm{U}$ & 330 & 340 & $\mathrm{U}$ & 340 & 330 & $U$ & 330 \\
\hline 2-Methylnaphthalene & 340 & $\mathrm{U}$ & 340 & 330 & $\mathrm{U}$ & 330 & 340 & $\mathrm{U}$ & 340 & 330 & U & 330 \\
\hline 2-Methylphenol (cresol, o-) & 340 & $\mathrm{U}$ & 340 & 330 & $\mathrm{U}$ & 330 & 340 & $\mathrm{U}$ & 340 & 330 & U & 330 \\
\hline 2-Nitroaniline & 840 & $\mathrm{U}$ & 840 & 840 & $\mathrm{U}$ & 840 & 840 & $\mathrm{U}$ & 840 & 840 & $\mathrm{U}$ & 840 \\
\hline 2-Nitrophenol & 340 & $\mathrm{U}$ & 340 & 330 & $\mathrm{U}$ & 330 & 340 & $\mathrm{U}$ & 340 & 330 & U & 330 \\
\hline 3,3'-Dichlorobenzidine & 340 & $\mathrm{U}$ & 340 & 330 & $\bar{U}$ & 330 & 340 & $\mathrm{U}$ & 340 & 330 & $\mathrm{U}$ & 330 \\
\hline 3-Nitroaniline & 840 & $\mathrm{U}$ & 840 & 840 & $\mathrm{U}$ & 840 & 840 & $\mathrm{U}$ & 840 & 840 & $\mathrm{U}$ & 840 \\
\hline 4,6-Dinitro-2-methylphenol & 840 & $\mathrm{U}$ & 840 & 840 & $\mathrm{U}$ & 840 & 840 & $U$ & 840 & 840 & $\mathrm{U}$ & 840 \\
\hline 4-Bromophenyl-phenylether & 340 & $\mathrm{U}$ & 340 & 330 & $\mathrm{U}$ & 330 & 340 & $\mathrm{U}$ & 340 & 330 & $\mathrm{U}$ & 330 \\
\hline 4-Chloro-3-methylphenol & 340 & $\mathrm{U}$ & 340 & 330 & $\mathrm{U}$ & 330 & 340 & $\mathrm{U}$ & 340 & 330 & $\mathrm{U}$ & 330 \\
\hline 4-Chloroaniline & 340 & $\mathrm{U}$ & 340 & 330 & $\mathrm{U}$ & 330 & 340 & $\mathrm{U}$ & 340 & 330 & $\mathrm{U}$ & 330 \\
\hline 4-Chlorophenyl-phenylether & 340 & $\mathrm{U}$ & 340 & 330 & $\mathrm{U}$ & 330 & 340 & $\mathrm{U}$ & 340 & 330 & $\mathrm{U}$ & 330 \\
\hline 4-Methylphenol (p-cresol) & 340 & $\mathrm{U}$ & 340 & 330 & $\mathrm{U}$ & 330 & 340 & $\mathrm{U}$ & 340 & 330 & $\mathrm{U}$ & 330 \\
\hline 4-Nitroaniline & 840 & U & 840 & 840 & $\mathrm{U}$ & 840 & 840 & $\mathrm{U}$ & 840 & 840 & U & 840 \\
\hline 4-Nitrophenol & 840 & $\mathrm{U}$ & 840 & 840 & $\mathrm{U}$ & 840 & 840 & $\mathrm{U}$ & 840 & 840 & $\mathrm{U}$ & 840 \\
\hline Acenaphthene & 340 & $\mathrm{U}$ & 340 & 330 & $\mathrm{U}$ & 330 & 340 & U & 340 & 330 & U & 330 \\
\hline Acenaphthylene & 340 & $\mathrm{U}$ & 340 & 330 & $\mathrm{U}$ & 330 & 340 & $\mathrm{U}$ & 340 & 330 & $\mathrm{U}$ & 330 \\
\hline $\begin{array}{l}\text { Anthracene } \\
\end{array}$ & 340 & $\mathrm{U}$ & 340 & 330 & $\mathrm{U}$ & 330 & 340 & $\mathrm{U}$ & 340 & 330 & $\mathrm{U}$ & 330 \\
\hline Benzo(a)anthracene & 340 & $\mathrm{U}$ & 340 & 330 & $\mathrm{U}$ & 330 & 340 & $\mathrm{U}$ & 340 & 330 & $\mathrm{U}$ & 330 \\
\hline Benzo(a)pyrene & 340 & $\mathrm{U}$ & 340 & 330 & $\mathrm{U}$ & 330 & 340 & $\mathrm{U}$ & 340 & 330 & U & 330 \\
\hline Benzo(b)fluoranthene & 340 & $\mathrm{U}$ & 340 & 330 & $\mathrm{U}$ & 330 & 340 & $\mathrm{U}$ & 340 & 330 & $\mathrm{U}$ & 330 \\
\hline Benzo(g,h,i)perylene & 340 & $\mathrm{U}$ & 340 & 330 & $\mathrm{U}$ & 330 & 340 & $\mathrm{U}$ & 340 & 330 & U & 330 \\
\hline Benzo(k)fluoranthene & 340 & $\mathrm{U}$ & 340 & 330 & $\mathrm{U}$ & 330 & 340 & $\mathrm{U}$ & 340 & 330 & $\mathrm{U}$ & 330 \\
\hline bis(2-Chloro-1-methylethyl)ether & 340 & $\mathrm{U}$ & 340 & 330 & $\mathrm{U}$ & 330 & 340 & $\mathrm{U}$ & 340 & 330 & U & 330 \\
\hline bis(2-Chloroethoxy)methane & 340 & U & 340 & 330 & $\mathrm{U}$ & 330 & 340 & $\mathrm{U}$ & 340 & 330 & $\mathrm{U}$ & 330 \\
\hline bis(2-Chloroethyl)ether & 340 & $\mathrm{U}$ & 340 & 330 & $\mathrm{U}$ & 330 & 340 & $\mathrm{U}$ & 340 & 330 & $\mathrm{U}$ & 330 \\
\hline bis(2-Ethylhexyl)phthalate & 20 & $\mathrm{~J}$ & 340 & 23 & $\mathrm{~J}$ & 330 & 22 & $\mathrm{~J}$ & 340 & 330 & U & 330 \\
\hline
\end{tabular}

Attachment

Originator

Checked

Calc. No.
J.M. Capron

T. M. Blakley

0100B-CA-V0299
Sheet No. 77 of 113

Date $10 / 04 / 07$

Date

Rev. No. 1 
Attachment 1. 118-B-1 Verification Sampling Results.

\begin{tabular}{|c|c|c|c|c|c|c|c|c|c|c|c|c|}
\hline & \multicolumn{3}{|c|}{$\begin{array}{c}\text { J12WM2 } \\
\text { BCL A3 } \\
\text { Sample Date 7/12/06 } \\
\end{array}$} & \multicolumn{3}{|c|}{$\begin{array}{c}\text { J12WM0 } \\
\text { BCL A4 } \\
\text { Sample Date 7/12/06 }\end{array}$} & \multicolumn{3}{|c|}{$\begin{array}{c}\text { J12WL9 } \\
\text { BCL B5 } \\
\text { Sample Date } 7 / 12 / 06\end{array}$} & \multicolumn{3}{|c|}{\begin{tabular}{|c|} 
J12WL8 \\
BCL B6 \\
Sample Date 7/12/06 \\
\end{tabular}} \\
\hline & $\mu \mathrm{g} / \mathrm{kg}$ & $Q$ & PQL & $\mu \mathrm{g} / \mathrm{kg}$ & $\mathbf{Q}$ & PQL & $\mu \mathrm{g} / \mathrm{kg}$ & Q & MDL & $\mu \mathrm{g} / \mathrm{kg}$ & Q & PQL \\
\hline \multicolumn{13}{|c|}{ Semivolatile Organic Compounds (continued) } \\
\hline Butylbenzylphthalate & 340 & $\mathrm{U}$ & 340 & 330 & $\mathrm{U}$ & 330 & 340 & $\mathrm{U}$ & 340 & 330 & $\bar{U}$ & 330 \\
\hline Carbazole & 340 & $\mathrm{U}$ & 340 & 330 & $\mathrm{U}$ & 330 & 340 & $\mathrm{U}$ & 340 & 330 & $\mathrm{U}$ & 330 \\
\hline Chrysene & 340 & $\mathrm{U}$ & 340 & 330 & $\mathrm{U}$ & 330 & 340 & $\mathrm{U}$ & 340 & 330 & $\mathrm{U}$ & 330 \\
\hline Di-n-butylphthalate & 27 & $\mathrm{~J}$ & 340 & 330 & $\mathrm{U}$ & 330 & 340 & $\mathrm{U}$ & 340 & 330 & U & 330 \\
\hline Di-n-octylphthalate & 340 & $\mathrm{U}$ & 340 & 330 & $\mathrm{U}$ & 330 & 340 & $\mathrm{U}$ & 340 & 330 & $\mathrm{U}$ & 330 \\
\hline Dibenz $(a, h)$ anthracene & 340 & $\mathrm{U}$ & 340 & 330 & $\mathrm{U}$ & 330 & 340 & $\mathrm{U}$ & 340 & 330 & $\mathrm{U}$ & 330 \\
\hline Dibenzofuran & 340 & $\mathrm{U}$ & 340 & 330 & $\mathrm{U}$ & 330 & 340 & $\mathrm{U}$ & 340 & 330 & $\mathrm{U}$ & 330 \\
\hline Diethylphthalate & 340 & $\mathrm{U}$ & 340 & 330 & $\mathrm{U}$ & 330 & 340 & $\mathrm{U}$ & 340 & 330 & $\mathrm{U}$ & 330 \\
\hline Dimethylphthalate & 340 & $\mathrm{U}$ & 340 & 330 & $\mathrm{U}$ & 330 & 340 & $\mathrm{U}$ & 340 & 330 & $\mathrm{U}$ & 330 \\
\hline Fluoranthene & 340 & U & 340 & 330 & $\mathrm{U}$ & 330 & 340 & $\mathrm{U}$ & 340 & 330 & $\mathrm{U}$ & 330 \\
\hline Fluorene & 340 & U & 340 & 330 & $\mathrm{U}$ & 330 & 340 & $\mathrm{U}$ & 340 & 330 & $\mathrm{U}$ & 330 \\
\hline Hexachlorobenzene & 340 & $\mathrm{U}$ & 340 & 330 & $\mathrm{U}$ & 330 & 340 & $\mathrm{U}$ & 340 & 330 & $\mathrm{U}$ & 330 \\
\hline Hexachlorobutadiene & 340 & $\mathrm{U}$ & 340 & 330 & $\mathrm{U}$ & 330 & 340 & $\mathrm{U}$ & 340 & 330 & $\mathrm{U}$ & 330 \\
\hline Hexachlorocyclopentadiene & 340 & $\mathrm{U}$ & 340 & 330 & $\mathrm{U}$ & 330 & 340 & $\mathrm{U}$ & 340 & 330 & $\bar{U}$ & 330 \\
\hline Hexachloroethane & 340 & $\mathrm{U}$ & 340 & 330 & $\mathrm{U}$ & 330 & 340 & $\mathrm{U}$ & 340 & 330 & $\mathrm{U}$ & 330 \\
\hline Indeno(1,2,3-cd)pyrene & 340 & $\mathrm{U}$ & 340 & 330 & $\mathrm{U}$ & 330 & 340 & $\mathrm{U}$ & 340 & 330 & $\mathrm{U}$ & 330 \\
\hline Isophorone & 340 & $\mathrm{U}$ & 340 & 330 & $U$ & 330 & 340 & $\mathrm{U}$ & 340 & 330 & $\mathrm{U}$ & 330 \\
\hline N-Nitroso-di-n-dipropylamine & 340 & $\mathrm{U}$ & 340 & 330 & $\mathrm{U}$ & 330 & 340 & $\mathrm{U}$ & 340 & 330 & $\mathrm{U}$ & 330. \\
\hline N-Nitrosodiphenylamine & 340 & $\mathrm{U}$ & 340 & 330 & $\mathrm{U}$ & 330 & 340 & $\mathrm{U}$ & 340 & 330 & $\mathrm{U}$ & 330 \\
\hline Naphthalene & 340 & $\mathrm{U}$ & 340 & 330 & $\mathrm{U}$ & 330 & 340 & $\mathrm{U}$ & 340 & 330 & $\mathrm{U}$ & 330 \\
\hline Nitrobenzene & 340 & $\mathrm{U}$ & 340 & 330 & $\mathrm{U}$ & 330 & 340 & $\mathrm{U}$ & 340 & 330 & $\mathrm{U}$ & 330 \\
\hline Pentachlorophenol & 840 & $\mathrm{U}$ & 840 & 840 & $\mathrm{U}$ & 840 & 840 & $\mathrm{U}$ & 840 & 840 & $\mathrm{U}$ & 840 \\
\hline Phenanthrene & 340 & $\mathrm{U}$ & 340 & 330 & $\mathrm{U}$ & 330 & 340 & $\mathrm{U}$ & 340 & 330 & $\mathrm{U}$ & 330 \\
\hline Phenol & 340 & $\mathrm{U}$ & 340 & 330 & $\mathrm{U}$ & 330 & 340 & $\mathrm{U}$ & 340 & 330 & $\mathrm{U}$ & 330 \\
\hline Pyrene & 340 & U & 340 & 330 & $\mathrm{U}$ & 330 & 340 & $\mathrm{U}$ & 340 & 330 & U & 330 \\
\hline & & & & Attachr & & & 1 & & & Sheet No. & & of 113 \\
\hline & & & & Origina & & J.M. Ca & ron & & & Date & & $107 / 07$ \\
\hline & & & & Checke & & T.M.B & kley & & & Dat & & \\
\hline & & & & Calc. $N$ & & $0100 \mathrm{~B}-\mathrm{C}$ & $\mathrm{A}-\mathrm{V} 0299$ & & & Rev. No & & 0 \\
\hline
\end{tabular}


Attachment 1. 118-B-1 Verification Sampling Results.

\begin{tabular}{|c|c|c|c|c|c|c|c|c|c|c|c|c|}
\hline \multirow[t]{2}{*}{ Constituents } & \multicolumn{3}{|c|}{$\begin{array}{c}\text { J12WM2 } \\
\text { BCL A3 } \\
\text { Sample Date } 7 / 12 / 06 \\
\end{array}$} & \multicolumn{3}{|c|}{\begin{tabular}{|c|} 
J12WM0 \\
BCL A4 \\
Sample Date 7/12/06 \\
\end{tabular}} & \multicolumn{3}{|c|}{$\begin{array}{c}\text { J12WL9 } \\
\text { BCL B5 } \\
\text { Sample Date 7/12/06 }\end{array}$} & \multicolumn{3}{|c|}{$\begin{array}{c}\text { J12WL8 } \\
\text { BCL B6 } \\
\text { Sample Date 7/12/06 }\end{array}$} \\
\hline & $\mu \mathrm{g} / \mathrm{kg}$ & $\mathbf{Q}$ & PQL & $\mu \mathrm{g} / \mathrm{kg}$ & $Q$ & PQL & $\mu \mathrm{g} / \mathrm{kg}$ & $Q$ & MDL & $\mu \mathrm{g} / \mathrm{kg}$ & $Q$ & PQL \\
\hline \multicolumn{13}{|c|}{ Volatile Organic Compounds } \\
\hline 1,1,1-Trichloroethane & 5 & $\mathrm{U}$ & 5 & 5 & $\mathrm{U}$ & 5 & 5 & $\mathrm{U}$ & 5 & 5 & $\mathrm{U}$ & 5 \\
\hline 1,1,2,2-Tetrachloroethane & 5 & $\mathrm{U}$ & 5 & 5 & $\mathrm{U}$ & 5 & 5 & $\mathrm{U}$ & 5 & 5 & $\mathrm{U}$ & 5 \\
\hline 1,1,2-Trichloroethane & 5 & $\mathrm{U}$ & 5 & 5 & $\mathrm{U}$ & 5 & 5 & $\mathrm{U}$ & 5 & 5 & $\mathrm{U}$ & 5 \\
\hline 1,1-Dichloroethane & 5 & $\mathrm{U}$ & 5 & 5 & $\mathrm{U}$ & 5 & 5 & $\mathrm{U}$ & 5 & 5 & $\mathrm{U}$ & 5 \\
\hline 1,1-Dichloroethene & 5 & $\mathrm{U}$ & 5 & 5 & $\mathrm{U}$ & 5 & 5 & $\mathrm{U}$ & 5 & 5 & $\mathrm{U}$ & 5 \\
\hline 1,2-Dichloroethane & 5 & $\mathrm{U}$ & 5 & 5 & $\mathrm{U}$ & 5 & 5 & $\mathrm{U}$ & 5 & 5 & $\mathrm{U}$ & 5 \\
\hline 1,2-Dichloroethene (total) & 5 & $\mathrm{U}$ & 5 & 5 & $\mathrm{U}$ & 5 & 5 & $\mathrm{U}$ & 5 & 5 & $U$ & 5 \\
\hline 1,2-Dichloropropane & 5 & $\mathrm{U}$ & 5 & 5 & $\mathrm{U}$ & 5 & 5 & U & 5 & 5 & $\mathrm{U}$ & 5 \\
\hline 2-Butanone & 10 & $\mathrm{U}$ & 10 & 10 & $\mathrm{U}$ & 10 & 10 & $\mathrm{U}$ & 10 & 10 & $\mathrm{U}$ & 10 \\
\hline 2-Hexanone & 10 & $\mathrm{U}$ & 10 & 10 & $\mathrm{U}$ & 10 & 10 & $\mathrm{U}$ & 10 & 10 & $\mathrm{U}$ & 10 \\
\hline 4-Methyl-2-pentanone & 10 & $\mathrm{U}$ & 10 & 10 & $\mathrm{U}$ & 10 & 10 & $\mathrm{U}$ & 10 & 10 & $\mathrm{U}$ & 10 \\
\hline Acetone & 10 & U & 10 & 10 & $\mathrm{U}$ & 10 & 10 & $\mathrm{U}$ & 10 & 10 & $\mathrm{U}$ & 10 \\
\hline Benzene & 5 & U & 5 & 5 & $\mathrm{U}$ & 5 & 5 & $\mathrm{U}$ & 5 & 5 & $\mathrm{U}$ & 5 \\
\hline Bromodichloromethane & 5 & U & 5 & 5 & $\mathrm{U}$ & 5 & 5 & $\mathrm{U}$ & 5 & 5 & $\mathrm{U}$ & 5 \\
\hline Bromoform & 5 & U & 5 & 5 & $\mathrm{U}$ & 5 & 5 & $\mathrm{U}$ & 5 & 5 & $\mathrm{U}$ & 5 \\
\hline Bromomethane & 10 & $\mathrm{U}$ & 10 & 10 & $\mathrm{U}$ & 10 & 10 & $\mathrm{U}$ & 10 & 10 & $\mathrm{U}$ & 10 \\
\hline Carbon disulfide & 5 & $\mathrm{U}$ & 5 & 5 & $\mathrm{U}$ & 5 & 5 & $\mathrm{U}$ & 5 & 5 & $U$ & 5 \\
\hline Carbon tetrachloride & 4 & $\mathrm{~J}$ & 5 & 2 & $\mathrm{~J}$ & 5 & 11 & $\mathrm{~J}$ & 5 & 32 & $\mathrm{~J}$ & 5 \\
\hline Chlorobenzene & 5 & $\mathrm{U}$ & 5 & 5 & $\mathrm{U}$ & 5 & 5 & $\mathrm{U}$ & 5 & 5 & $\mathrm{U}$ & 5 \\
\hline Chloroethane & 10 & $\mathrm{U}$ & 10 & 10 & $\mathrm{U}$ & 10 & 10 & $\mathrm{U}$ & 10 & 10 & $\mathrm{U}$ & 10 \\
\hline Chloroform & 5 & $\mathrm{U}$ & 5 & 5 & $\mathrm{U}$ & 5 & 5 & $\mathrm{U}$ & 5 & 5 & $\mathrm{U}$ & 5 \\
\hline Chloromethane & 10 & $\mathrm{U}$ & 10 & 10 & $\mathrm{U}$ & 10 & 10 & $\mathrm{U}$ & 10 & 10 & $\mathrm{U}$ & 10 \\
\hline cis-1,2-Dichloroethylene & 5 & $\mathrm{U}$ & 5 & 5 & $\mathrm{U}$ & 5 & 5 & $\mathrm{U}$ & 5 & 5 & $\mathrm{U}$ & 5 \\
\hline cis-1,3-Dichloropropene & 5 & $\mathrm{U}$ & 5 & 5 & $\mathrm{U}$ & 5 & 5 & $\mathrm{U}$ & 5 & 5 & $\mathrm{U}$ & 5 \\
\hline Dibromochloromethane & 5 & $\mathrm{U}$ & 5 & 5 & $\mathrm{U}$ & 5 & 5 & $\mathrm{U}$ & 5 & 5 & $\mathrm{U}$ & 5 \\
\hline Ethylbenzene & 5 & $\mathrm{U}$ & 5 & 5 & $\mathrm{U}$ & 5 & 5 & $\mathrm{U}$ & 5 & 5 & $\mathrm{U}$ & 5 \\
\hline Methylene chloride & 7 & $\mathrm{~B}$ & 5 & 9 & $\mathrm{~B}$ & 5 & 5 & $\mathrm{U}$ & 5 & 5 & & 5 \\
\hline Styrene & 5 & $\mathrm{U}$ & 5 & 5 & $\mathrm{U}$ & 5 & 5 & $\mathrm{U}$ & 5 & 5 & $\mathrm{U}$ & 5 \\
\hline Tetrachloroethene & 5 & $\mathrm{U}$ & 5 & 5 & $\mathrm{U}$ & 5 & 5 & $\mathrm{U}$ & 5 & 5 & $\mathrm{U}$ & 5 \\
\hline Toluene & 5 & $\mathrm{U}$ & 5 & 5 & $\mathrm{U}$ & 5 & 5 & $\mathrm{U}$ & 5 & 1 & $\mathrm{~J}$ & 5 \\
\hline trans-1,2-Dichloroethylene & 5 & $\mathrm{U}$ & 5 & 5 & $\mathrm{U}$ & 5 & 5 & $\mathrm{U}$ & 5 & 5 & $\mathrm{U}$ & 5 \\
\hline trans-1,3-Dichloropropene & 5 & $\mathrm{U}$ & 5 & 5 & $\mathrm{U}$ & 5 & 5 & $\mathrm{U}$ & 5 & 5 & $\mathrm{U}$ & 5 \\
\hline Trichloroethene & 5 & $\mathrm{U}$ & 5 & 5 & $\mathrm{U}$ & 5 & 5 & $\mathrm{U}$ & 5 & 5 & $\mathrm{U}$ & 5 \\
\hline Vinyl chloride & 10 & U & 10 & 10 & $\mathrm{U}$ & 10 & 10 & $\mathrm{U}$ & 10 & 10 & $\mathrm{U}$ & 10 \\
\hline Xylenes (total) & 5 & $\mathrm{U}$ & 5 & 1 & $\mathrm{~J}$ & 5 & 5 & $\mathrm{U}$ & 5 & 5 & $\mathrm{U}$ & 5 \\
\hline & & & & Attachm & & & 1 & & & eet No. & & of 113 \\
\hline & & & & Originat & & J.M. Ca & ron & & & Date & & $04 / 07$ \\
\hline & & & & Checked & & T.M. B1 & kley & & & Date & & \\
\hline & & & & Calc. No & & $0100 \mathrm{~B}-\mathrm{C}$ & $\mathrm{A}-\mathrm{V} 0299$ & & & Rev. No. & & 1 \\
\hline
\end{tabular}


Attachment 1. 118-B-1 Verification Sampling Results.

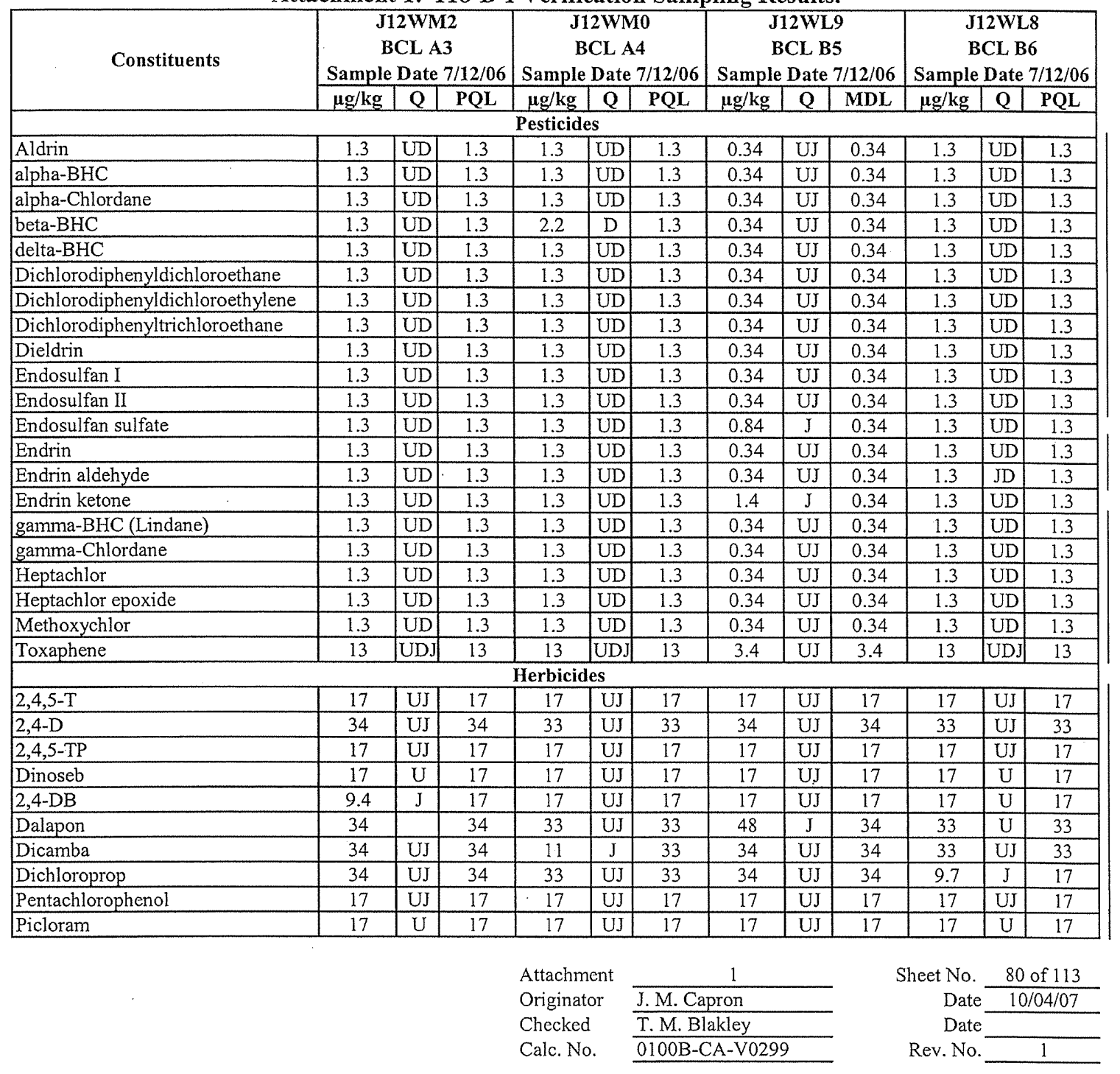


Attachment 1. 118-B-1 Verification Sampling Results.

\begin{tabular}{|c|c|c|c|c|c|c|c|c|c|c|c|c|}
\hline \multirow[t]{2}{*}{ Constituents } & \multicolumn{3}{|c|}{\begin{tabular}{|c|} 
J12WL7 \\
BCL B7 \\
Sample Date 7/12/06 \\
\end{tabular}} & \multicolumn{3}{|c|}{$\begin{array}{c}\text { J12WL6 } \\
\text { BCL B8 } \\
\text { Sample Date } 7 / 12 / 06\end{array}$} & \multicolumn{3}{|c|}{$\begin{array}{c}\text { J12WW0 } \\
\text { Split of J12WL6 } \\
\text { Sample Date } 7 / 12 / 06\end{array}$} & \multicolumn{3}{|c|}{$\begin{array}{c}\text { J12WK7 } \\
\text { BCL C9 } \\
\text { Sample Date } 7 / 11 / 06\end{array}$} \\
\hline & $\mu \mathrm{g} / \mathrm{kg}$ & $Q$ & PQL & $\mu \mathrm{g} / \mathrm{kg}$ & $Q$ & PQL & $\mu \mathrm{g} / \mathrm{kg}$ & $\mathrm{Q}$ & PQL & $\mu \mathrm{g} / \mathrm{kg}$ & $Q$ & $\mathrm{PQL}$ \\
\hline \multicolumn{13}{|c|}{ Polychlorinated Biphenyls } \\
\hline Aroclor-1016 & 13 & $\mathrm{U}$ & 13 & 13 & $\mathrm{U}$ & 13 & 17 & $\mathrm{U}$ & 17 & 13 & $\mathrm{U}$ & 13 \\
\hline Aroclor-1221 & 13 & $\mathrm{U}$ & 13 & 13 & $\mathrm{U}$ & 13 & 17 & $\mathrm{U}$ & 17 & 13 & $\mathrm{U}$ & 13 \\
\hline Aroclor-1232 & 13 & $\mathrm{U}$ & 13 & 13 & $\mathrm{U}$ & 13 & 17 & $\mathrm{U}$ & 17 & 13 & $\mathrm{U}$ & 13 \\
\hline Aroclor -1242 & 13 & $\mathrm{U}$ & 13 & 13 & $\mathrm{U}$ & 13 & 17 & $\mathrm{U}$ & 17 & 13 & $\mathrm{U}$ & 13 \\
\hline Aroclor- 1248 & 13 & $\mathrm{U}$ & 13 & 13 & $\mathrm{U}$ & 13 & 17 & $\mathrm{U}$ & 17 & 13 & $\mathrm{U}$ & 13 \\
\hline Aroclor-1254 & 13 & $\mathrm{U}$ & 13 & 13 & $U$ & 13 & 47 & $\mathrm{~J}$ & 17 & 13 & $\mathrm{U}$ & 13 \\
\hline Aroclor- 1260 & 13 & $\mathrm{U}$ & 13 & 13 & U & 13 & 130 & $\mathrm{~J}$ & 17 & 13 & $\mathrm{U}$ & 13 \\
\hline \multicolumn{13}{|c|}{ Semivolatile Organic Compounds } \\
\hline 1,2,4-Trichlorobenzene & 330 & U & 330 & 340 & $\mathrm{U}$ & 340 & 330 & $\mathrm{U}$ & 330 & 330 & UJ & 330 \\
\hline 1,2-Dichlorobenzene & 330 & U & 330 & 340 & $\mathrm{U}$ & 340 & 330 & $\mathrm{U}$ & 330 & 330 & $\mathrm{U}$ & 330 \\
\hline 1,3-Dichlorobenzene & 330 & U & 330 & 340 & $\mathrm{U}$ & 340 & 330 & $\mathrm{U}$ & 330 & 330 & $\mathrm{U}$ & 330 \\
\hline 1,4-Dichlorobenzene & 330 & $\mathrm{U}$ & 330 & 340 & $\mathrm{U}$ & 340 & 330 & $U$ & 330 & 330 & $\mathrm{U}$ & 330 \\
\hline 2,4,5-Trichlorophenol & 830 & $\mathrm{U}$ & 830 & 840 & $\mathrm{U}$ & 840 & 330 & $\mathrm{U}$ & 330 & 840 & $\mathrm{U}$ & 840 \\
\hline 2,4,6-Trichlorophenol & 330 & $\mathrm{U}$ & 330 & 340 & U & 340 & 330 & $U$ & 330 & 330 & U & 330 \\
\hline 2,4-Dichlorophenol & 330 & $\mathrm{U}$ & 330 & 340 & $\mathrm{U}$ & 340 & 330 & $\mathrm{U}$ & 330 & 330 & $\mathrm{U}$ & 330 \\
\hline 2,4-Dimethylphenol & 330 & $\mathrm{U}$ & 330 & 340 & $\mathrm{U}$ & 340 & 330 & $\mathrm{U}$ & 330 & 330 & UJ & 330 \\
\hline 2,4-Dinitrophenol & 830 & $\mathrm{U}$ & 830 & 840 & $\mathrm{U}$ & 840 & 1600 & $\mathrm{U}$ & 1600 & 840 & UR & 840 \\
\hline 2,4-Dinitrotoluene & 330 & $\mathrm{U}$ & 330 & 340 & $\mathrm{U}$ & 340 & 330 & UN & 330 & 330 & $\mathrm{U}$ & 330 \\
\hline 2,6-Dinitrotoluene & 330 & $\mathrm{U}$ & 330 & 340 & $\mathrm{U}$ & 340 & 330 & UN & 330 & 330 & $\mathrm{U}$ & 330 \\
\hline 2-Chloronaphthalene & 330 & $\mathrm{U}$ & 330 & 340 & $\mathrm{U}$ & 340 & 330 & $\mathrm{U}$ & 330 & 330 & $\mathrm{U}$ & 330 \\
\hline 2-Chlorophenol & 330 & $\mathrm{U}$ & 330 & 340 & $\mathrm{U}$ & 340 & 330 & $\mathrm{U}$ & 330 & 330 & $\mathrm{U}$ & 330 \\
\hline 2-Methylnaphthalene & 330 & $\mathrm{U}$ & 330 & 340 & $\mathrm{U}$ & 340 & 330 & $\mathrm{U}$ & 330 & 330 & UJ & 330 \\
\hline 2-Methylphenol (cresol, o-) & 330 & $\mathrm{U}$ & 330 & 340 & $\mathrm{U}$ & 340 & 330 & $\mathrm{U}$ & 330 & 330 & $\mathrm{U}$ & 330 \\
\hline 2-Nitroaniline & 830 & U & 830 & 840 & $\mathrm{U}$ & 840 & 1600 & $\mathrm{U}$ & 1600 & 840 & $\mathrm{U}$ & 840 \\
\hline 2-Nitrophenol & 330 & $\mathrm{U}$ & 330 & 340 & $\mathrm{U}$ & 340 & 330 & $\mathrm{U}$ & 330 & 330 & $\mathrm{U}$ & 330 \\
\hline 3,3'-Dichlorobenzidine & 330 & $\mathrm{U}$ & 330 & 340 & $\mathrm{U}$ & 340 & 1600 & $\mathrm{U}$ & 1600 & 330 & $\mathrm{U}$ & 330 \\
\hline 3-Nitroaniline & 830 & $\mathrm{U}$ & 830 & 840 & $\mathrm{U}$ & 840 & 1600 & $\mathrm{U}$ & 1600 & 840 & $\mathrm{U}$ & 840 \\
\hline 4,6-Dinitro-2-methylphenol & 830 & $\mathrm{U}$ & 830 & 840 & $\mathrm{U}$ & 840 & 1600 & $\mathrm{U}$ & 1600 & 840 & $\mathrm{U}$ & 840 \\
\hline 4-Bromophenyl-phenylether & 330 & $\mathrm{U}$ & 330 & 340 & $\mathrm{U}$ & 340 & 330 & $\mathrm{U}$ & 330 & 330 & $\mathrm{U}$ & 330 \\
\hline 4-Chloro-3-methylphenol & 330 & $\mathrm{U}$ & 330 & 340 & $\mathrm{U}$ & 340 & 330 & $\mathrm{U}$ & 330 & 330 & $\mathrm{U}$ & 330 \\
\hline 4-Chloroaniline & 330 & $\mathrm{U}$ & 330 & 340 & $\mathrm{U}$ & 340 & 330 & $\mathrm{U}$ & 330 & 330 & $\mathrm{U}$ & 330 \\
\hline 4-Chlorophenyl-phenylether & 330 & $\mathrm{U}$ & 330 & 340 & $\mathrm{U}$ & 340 & 330 & $\mathrm{U}$ & 330 & 330 & $\mathrm{U}$ & 330 \\
\hline 4-Methylphenol (p-cresol) & 330 & $\mathrm{U}$ & 330 & 340 & $\mathrm{U}$ & 340 & 670 & $\mathrm{U}$ & 670 & 330 & $\mathrm{U}$ & 330 \\
\hline 4-Nitroaniline & 830 & $\mathrm{U}$ & 830 & 840 & $\mathrm{U}$ & 840 & 1600 & $\mathrm{U}$ & 1600 & 840 & $\mathrm{U}$ & 840 \\
\hline 4-Nitrophenol & 830 & $\mathrm{U}$ & 830 & 840 & $\mathrm{U}$ & 840 & 1600 & $\mathrm{U}$ & 1600 & 840 & U & 840 \\
\hline Acenaphthene & 330 & $\mathrm{U}$ & 330 & 340 & $\mathrm{U}$ & 340 & 330 & $\mathrm{U}$ & 330 & 330 & $\mathrm{U}$ & 330 \\
\hline Acenaphthylene & 330 & $\mathrm{U}$ & 330 & 340 & $U$ & 340 & 330 & $\bar{U}$ & 330 & 330 & $\mathrm{U}$ & 330 \\
\hline Anthracene & 330 & $\mathrm{U}$ & 330 & 340 & $\mathrm{U}$ & 340 & 330 & $\mathrm{U}$ & 330 & 330 & $\mathrm{U}$ & 330 \\
\hline Benzo(a)anthracene & 330 & $\mathrm{U}$ & 330 & 340 & $\mathrm{U}$ & 340 & 34 & $\mathrm{~J}$ & 330 & 330 & $\mathrm{U}$ & 330 \\
\hline Benzo(a)pyrene & 330 & $\mathrm{U}$ & 330 & 340 & $\mathrm{U}$ & 340 & 34 & $\mathrm{~J}$ & 330 & 330 & $\mathrm{U}$ & 330 \\
\hline Benzo(b)fluoranthene & 330 & $\mathrm{U}$ & 330 & 340 & $\mathrm{U}$ & 340 & 39 & $\mathrm{~J}$ & 330 & 330 & $\mathrm{U}$ & 330 \\
\hline Benzo(g,h,i)perylene & 330 & $\mathrm{U}$ & 330 & 340 & $\mathrm{U}$ & 340 & 36 & $\mathrm{~J}$ & 330 & 330 & $\mathrm{U}$ & 330 \\
\hline Benzo(k)fluoranthene & 330 & $\mathrm{U}$ & 330 & 340 & $\mathrm{U}$ & 340 & 330 & $\mathrm{U}$ & 330 & 330 & $\mathrm{U}$ & 330 \\
\hline bis(2-Chloro-1-methylethyl)ether & 330 & $\mathrm{U}$ & 330 & 340 & $\mathrm{U}$ & 340 & 330 & $\mathrm{U}$ & 330 & 330 & $\mathrm{U}$ & 330 \\
\hline bis(2-Chloroethoxy)methane & 330 & $\mathrm{U}$ & 330 & 340 & $\mathrm{U}$ & 340 & 330 & $\mathrm{U}$ & 330 & 330 & $\mathrm{U}$ & 330 \\
\hline bis(2-Chloroethyl)ether & 330 & $\mathrm{U}$ & 330 & 340 & $\mathrm{U}$ & 340 & 330 & $\mathrm{U}$ & 330 & 330 & $\mathrm{U}$ & 330 \\
\hline bis(2-Ethylhexyl)phthalate & 330 & $\mathrm{U}$ & 330 & 340 & $\mathrm{U}$ & 340 & 280 & $\mathrm{~J}$ & 330 & 21 & $\mathrm{JB}$ & 330 \\
\hline
\end{tabular}

Attachment

Originator

Checked

Calc. No.

\begin{tabular}{|c|}
\hline J.M. Capron \\
\hline T. M. Blakley \\
\hline $0100 B-C A-V 0299$ \\
\hline
\end{tabular}

Sheet No. 81 of 113 Date $10 / 04 / 07$ Date

Rev. No. 1 
Attachment 1. 118-B-1 Verification Sampling Results.

\begin{tabular}{|c|c|c|c|c|c|c|c|c|c|c|c|c|}
\hline \multirow[t]{2}{*}{ Constituents } & \multicolumn{3}{|c|}{$\begin{array}{c}\text { J12WL7 } \\
\text { BCL B7 } \\
\text { Sample Date 7/12/06 } \\
\end{array}$} & \multicolumn{3}{|c|}{\begin{tabular}{|c|} 
J12WL6 \\
BCL B8 \\
Sample Date 7/12/06 \\
\end{tabular}} & \multicolumn{3}{|c|}{$\begin{array}{c}\text { J12WW0 } \\
\text { Split of J12WL6 } \\
\text { Sample Date 7/12/06 } \\
\end{array}$} & \multicolumn{3}{|c|}{\begin{tabular}{|c|} 
J12WK7 \\
BCL C9 \\
Sample Date 7/11/06 \\
\end{tabular}} \\
\hline & $\mu \mathrm{g} / \mathrm{kg}$ & $\mathbf{Q}$ & PQL & $\mu \mathrm{g} / \mathrm{kg}$ & $\mathbf{Q}$ & $\overline{\mathbf{P Q L}}$ & $\mu \mathrm{g} / \mathrm{kg}$ & $\mathbf{Q}$ & MDL & $\mu \mathrm{g} / \mathrm{kg}$ & $\mathbf{Q}$ & PQL \\
\hline \multicolumn{13}{|c|}{ Semivolatile Organic Compounds (continued) } \\
\hline Butylbenzylphthalate & 330 & $\mathrm{U}$ & 330 & 340 & $\mathrm{U}$ & 340 & 330 & $\mathrm{U}$ & 330 & 330 & $\mathrm{U}$ & 330 \\
\hline Carbazole & 330 & $\mathrm{U}$ & 330 & 340 & $\mathrm{U}$ & 340 & 330 & $\mathrm{U}$ & 330 & 330 & $\mathrm{U}$ & 330 \\
\hline Chrysene & 330 & $\mathrm{U}$ & 330 & 340 & $\mathrm{U}$ & 340 & 77 & $\mathrm{~J}$ & 330 & 330 & $\mathrm{U}$ & 330 \\
\hline Di-n-butylphthalate & 330 & $\mathrm{U}$ & 330 & 340 & $\mathrm{U}$ & 340 & 330 & $\mathrm{U}$ & 330 & 23 & $\mathrm{~J}$ & 330 \\
\hline Di-n-octylphthalate & 330 & $U$ & 330 & 340 & $\mathrm{U}$ & 340 & 330 & $\mathrm{U}$ & 330 & 330 & $\mathrm{U}$ & 330 \\
\hline Dibenz $(a, h)$ anthracene & 330 & $\mathrm{U}$ & 330 & 340 & $\mathrm{U}$ & 340 & 330 & $\mathrm{U}$ & 330 & 330 & $\mathrm{U}$ & 330 \\
\hline Dibenzofuran & 330 & $\mathrm{U}$ & 330 & 340 & $\mathrm{U}$ & 340 & 330 & $\mathrm{U}$ & 330 & 330 & $\mathrm{U}$ & 330 \\
\hline Diethylphthalate & 330 & $\mathrm{U}$ & 330 & 340 & U & 340 & 330 & $\mathrm{U}$ & 330 & 330 & $\mathrm{U}$ & 330 \\
\hline Dimethylphthalate & 330 & $\mathrm{U}$ & 330 & 340 & $\mathrm{U}$ & 340 & 330 & $\mathrm{U}$ & 330 & 330 & $\mathrm{U}$ & 330 \\
\hline Fluoranthene & 330 & $\mathrm{U}$ & 330 & 340 & $\mathrm{U}$ & 340 & 93 & $\mathrm{~J}$ & 330 & 330 & $\mathrm{U}$ & 330 \\
\hline Fluorene & 330 & $\mathrm{U}$ & 330 & 340 & U & 340 & 330 & $\mathrm{U}$ & 330 & 330 & $\mathrm{U}$ & 330 \\
\hline Hexachlorobenzene & 330 & $\mathrm{U}$ & 330 & 340 & $\mathrm{U}$ & 340 & 330 & $\mathrm{U}$ & 330 & 330 & $\mathrm{U}$ & 330 \\
\hline Hexachlorobutadiene & 330 & $\mathrm{U}$ & 330 & 340 & $\mathrm{U}$ & 340 & 330 & $\mathrm{U}$ & 330 & 330 & $\mathrm{U}$ & 330 \\
\hline Hexachlorocyclopentadiene & 330 & $\mathrm{U}$ & 330 & 340 & $\mathrm{U}$ & 340 & 1600 & $\mathrm{U}$ & 1600 & 330 & $\mathrm{U}$ & 330 \\
\hline Hexachloroethane & 330 & $\mathrm{U}$ & 330 & 340 & $\mathrm{U}$ & 340 & 330 & $\mathrm{U}$ & 330 & 330 & $\mathrm{U}$ & 330 \\
\hline Indeno(1,2,3-cd)pyrene & 330 & $\mathrm{U}$ & 330 & 340 & $\mathrm{U}$ & 340 & 330 & $\mathrm{U}$ & 330 & 330 & $\mathrm{U}$ & 330 \\
\hline Isophorone & 330 & $\mathrm{U}$ & 330 & 340 & $\mathrm{U}$ & 340 & 330 & $\mathrm{U}$ & 330 & 330 & UJ & 330 \\
\hline N-Nitroso-di-n-dipropylamine & 330 & $\mathrm{U}$ & 330 & 340 & $\mathrm{U}$ & 340 & 330 & $\mathrm{U}$ & 330 & 330 & $\mathrm{U}$ & 330 \\
\hline N-Nitrosodiphenylamine & 330 & $\mathrm{U}$ & 330 & 340 & $\mathrm{U}$ & 340 & 330 & $\mathrm{U}$ & 330 & 330 & $\mathrm{U}$ & 330 \\
\hline Naphthalene & 330 & $\mathrm{U}$ & 330 & 340 & $\mathrm{U}$ & 340 & 330 & $\mathrm{U}$ & 330 & 330 & $\mathrm{U}$ & 330 \\
\hline Nitrobenzene & 330 & $\mathrm{U}$ & 330 & 340 & $\mathrm{U}$ & 340 & 330 & $\mathrm{U}$ & 330 & 330 & $\mathrm{U}$ & 330 \\
\hline Pentachlorophenol & 830 & $\mathrm{U}$ & 830 & 840 & $\mathrm{U}$ & 840 & 1600 & $\mathrm{U}$ & 1600 & 840 & $\mathrm{U}$ & 840 \\
\hline Phenanthrene & 330 & $\mathrm{U}$ & 330 & 340 & $\mathrm{U}$ & 340 & 78 & $\mathrm{~J}$ & 330 & 330 & $\mathrm{U}$ & 330 \\
\hline Phenol & 330 & $\mathrm{U}$ & 330 & 340 & $\mathrm{U}$ & 340 & 330 & $\mathrm{U}$ & 330 & 330 & U & 330 \\
\hline Pyrene & 330 & $\mathrm{U}$ & 330 & 340 & $\mathrm{U}$ & 340 & 87 & $\mathrm{~J}$ & 330 & 330 & $\mathrm{U}$ & 330 \\
\hline \multirow{4}{*}{ · } & & & & \multicolumn{2}{|c|}{ Attachment } & \multicolumn{3}{|c|}{1} & \multicolumn{2}{|c|}{ Sheet No. } & \multicolumn{2}{|c|}{82 of 113} \\
\hline & & & & \multirow{2}{*}{\multicolumn{2}{|c|}{$\begin{array}{l}\text { Originator } \\
\text { Checked }\end{array}$}} & \multicolumn{3}{|c|}{ J.M. Capron } & & Date & \multicolumn{2}{|c|}{$10 / 04 / 07$} \\
\hline & & & & & & T.M. Bl & akley & & & Date & & \\
\hline & & & & \multicolumn{2}{|c|}{ Calc. No. } & \multicolumn{3}{|c|}{ 0100B-CA-V0299 } & & Rev. No & & 1 \\
\hline
\end{tabular}


Attachment 1. 118-B-1 Verification Sampling Results.

\begin{tabular}{|c|c|c|c|c|c|c|c|c|c|c|c|c|}
\hline \multirow[t]{2}{*}{ Constituents } & \multicolumn{3}{|c|}{$\begin{array}{c}\text { J12WL7 } \\
\text { BCL B7 } \\
\text { Sample Date 7/12/06 } \\
\end{array}$} & \multicolumn{3}{|c|}{$\begin{array}{c}\text { J12WL6 } \\
\text { BCL B8 } \\
\text { Sample Date } 7 / 12 / 06\end{array}$} & \multicolumn{3}{|c|}{$\begin{array}{c}\text { J12WW0 } \\
\text { Split of J12WL6 } \\
\text { Sample Date } 7 / 12 / 06 \\
\end{array}$} & \multicolumn{3}{|c|}{$\begin{array}{c}\text { J12WK7 } \\
\text { BCL C9 } \\
\text { Sample Date 7/11/06 }\end{array}$} \\
\hline & $\mu \mathrm{g} / \mathrm{kg}$ & $\mathrm{Q}$ & PQL & $\mu \mathrm{g} / \mathrm{kg}$ & Q & PQL & $\mu \mathrm{g} / \mathrm{kg}$ & $Q$ & MDL & $\mu \mathrm{g} / \mathrm{kg}$ & $\mathbf{Q}$ & PQL \\
\hline \multicolumn{13}{|c|}{ Volatile Organic Compounds } \\
\hline 1,1,1-Trichloroethane & 5 & $\mathrm{U}$ & 5 & 5 & $\mathrm{U}$ & 5 & 5.0 & $\mathrm{U}$ & 5.0 & 5 & $\mathrm{U}$ & 5 \\
\hline 1,1,2,2-Tetrachloroethane & 5 & $U$ & 5 & 5 & $\mathrm{U}$ & 5 & 5.0 & $\mathrm{U}$ & 5.0 & 5 & $\mathrm{U}$ & 5 \\
\hline 1,1,2-Trichloroethane & 5 & $\mathrm{U}$ & 5 & 5 & $\mathrm{U}$ & 5 & 5.0 & $\mathrm{U}$ & 5.0 & 5 & $\mathrm{U}$ & 5 \\
\hline 1,1-Dichloroethane & 5 & $\mathrm{U}$ & 5 & 5 & $\mathrm{U}$ & 5 & 5.0 & $\mathrm{U}$ & 5.0 & 5 & $\mathrm{U}$ & 5 \\
\hline 1,1-Dichloroethene & 5 & $\mathrm{U}$ & 5 & 5 & $\mathrm{U}$ & 5 & 5.0 & $\mathrm{U}$ & 5.0 & 5 & $\mathrm{U}$ & 5 \\
\hline 1,2-Dichloroethane & 5 & $\mathrm{U}$ & 5 & 5 & $\bar{U}$ & 5 & 5.0 & $\mathrm{U}$ & 5.0 & 5 & $\mathrm{U}$ & 5 \\
\hline 1,2-Dichloroethene (total) & 5 & $\mathrm{U}$ & 5 & 5 & $\mathrm{U}$ & 5 & 10 & $\mathrm{U}$ & 10 & 5 & $\mathrm{U}$ & 5 \\
\hline 1,2-Dichloropropane & 5 & $\mathrm{U}$ & 5 & 5 & $\mathrm{U}$ & 5 & 5.0 & $\mathrm{U}$ & 5.0 & 5 & $\mathrm{U}$ & 5 \\
\hline 2-Butanone & 9 & $\mathrm{U}$ & 9 & 10 & $\mathrm{U}$ & 10 & 10 & $\mathrm{U}$ & 10 & 10 & $\mathrm{U}$ & 10 \\
\hline 2-Hexanone & 9 & $\mathrm{U}$ & 9 & 10 & $\mathrm{U}$ & 10 & 20 & $\mathrm{U}$ & 20 & 10 & $\mathrm{U}$ & 10 \\
\hline 4-Methyl-2-pentanone & 9 & $\mathrm{U}$ & 9 & 10 & $\mathrm{U}$ & 10 & 10 & $\mathrm{U}$ & 10 & 10 & $\mathrm{U}$ & 10 \\
\hline Acetone & 9 & $U$ & 9 & 2 & $\mathrm{~J}$ & 10 & 20 & $\mathrm{U}$ & 20 & 18 & & 10 \\
\hline Benzene & 5 & $\mathrm{U}$ & 5 & 5 & $\mathrm{U}$ & 5 & 5.0 & $\mathrm{U}$ & 5.0 & 5 & $\mathrm{U}$ & 5 \\
\hline Bromodichloromethane & 5 & $\mathrm{U}$ & 5 & 5 & $\mathrm{U}$ & 5 & 5.0 & $U$ & 5.0 & 5 & $\mathrm{U}$ & 5 \\
\hline Bromoform & 5 & $\mathrm{U}$ & 5 & 5 & $\mathrm{U}$ & 5 & 5.0 & $\mathrm{U}$ & 5.0 & 5 & $\mathrm{U}$ & 5 \\
\hline Bromomethane & 9 & $\mathrm{U}$ & 9 & 10 & $\mathrm{U}$ & 10 & 10 & $\mathrm{U}$ & 10 & 10 & $\mathrm{U}$ & 10 \\
\hline Carbon disulfide & 5 & $\mathrm{U}$ & 5 & 5 & $\mathrm{U}$ & 5 & 5.0 & $\mathrm{U}$ & 5.0 & 5 & $\mathrm{U}$ & 5 \\
\hline Carbon tetrachloride & 13 & $\mathrm{~J}$ & 5 & 13 & $\mathrm{~J}$ & 5 & 5.0 & $\mathrm{U}$ & 5.0 & 5 & $\mathrm{U}$ & 5 \\
\hline Chlorobenzene & 5 & $\mathrm{U}$ & 5 & 5 & $\mathrm{U}$ & 5 & 5.0 & $U$ & 5.0 & 5 & $\mathrm{U}$ & 5 \\
\hline Chloroethane & 9 & $\mathrm{U}$ & 9 & 10 & $\mathrm{U}$ & 10 & 10 & $\mathrm{U}$ & 10 & 10 & $\mathrm{U}$ & 10 \\
\hline Chloroform & 5 & $\mathrm{U}$ & 5 & 5 & $\mathrm{U}$ & 5 & 5.0 & $\mathrm{U}$ & 5.0 & 5 & $\mathrm{U}$ & 5 \\
\hline Chloromethane & 9 & $\mathrm{U}$ & 9 & 10 & $\mathrm{U}$ & 10 & 10 & $\mathrm{U}$ & 10 & 10 & $\mathrm{U}$ & 10 \\
\hline cis-1,2-Dichloroethylene & 5 & $\mathrm{U}$ & 5 & 5 & $U$ & 5 & & & & 5 & $\mathrm{U}$ & 5 \\
\hline cis-1,3-Dichloropropene & 5 & $\mathrm{U}$ & 5 & 5 & $\mathrm{U}$ & 5 & 5.0 & $\mathrm{U}$ & 5.0 & 5 & $\mathrm{U}$ & 5 \\
\hline Dibromochloromethane & 5 & $\mathrm{U}$ & 5 & 5 & $\mathrm{U}$ & 5 & 5.0 & $\mathrm{U}$ & 5.0 & 5 & $\mathrm{U}$ & 5 \\
\hline Ethylbenzene & 5 & $\mathrm{U}$ & 5 & 5 & $\mathrm{U}$ & 5 & 5.0 & $\mathrm{U}$ & 5.0 & 5 & $\mathrm{U}$ & 5 \\
\hline Methylene chloride & 2 & $\mathrm{~J}$ & 5 & 5 & $\mathrm{U}$ & 5 & 5.0 & $\mathrm{U}$ & 5.0 & 14 & $\mathrm{BJ}$ & 5 \\
\hline Styrene & 5 & $\mathrm{U}$ & 5 & 5 & $\mathrm{U}$ & 5 & 5.0 & $\mathrm{U}$ & 5.0 & 5 & $\mathrm{U}$ & 5 \\
\hline Tetrachloroethene & 5 & $\mathrm{U}$ & 5 & 5 & $\mathrm{U}$ & 5 & 5.0 & U & 5.0 & 5 & $\mathrm{U}$ & 5 \\
\hline Toluene & 5 & $\mathrm{U}$ & 5 & 5 & $\mathrm{U}$ & 5 & 0.68 & $\mathrm{~J}$ & 5.0 & 5 & $\mathrm{U}$ & 5 \\
\hline trans-1,2-Dichloroethylene & 5 & $\mathrm{U}$ & 5 & 5 & $\mathrm{U}$ & 5 & & & & 5 & $\mathrm{U}$ & 5 \\
\hline trans - 1,3-Dichloropropene & 5 & $\mathrm{U}$ & 5 & 5 & $\mathrm{U}$ & 5 & 5.0 & $\mathrm{U}$ & 5.0 & 5 & $\mathrm{U}$ & 5 \\
\hline Trichloroethene & 5 & $\mathrm{U}$ & 5 & 5 & $\mathrm{U}$ & 5 & 5.0 & $\mathrm{U}$ & 5.0 & 5 & $\mathrm{U}$ & 5 \\
\hline Vinyl chloride & 9 & $\mathrm{U}$ & 9 & 10 & $\mathrm{U}$ & 10 & 5.0 & $\mathrm{U}$ & 5.0 & 10 & $\mathrm{U}$ & 10 \\
\hline Xylenes (total) & 5 & $\mathrm{U}$ & 5 & 5 & $\mathrm{U}$ & 5 & 10 & $\mathrm{U}$ & 10 & 5 & $\mathrm{U}$ & 5 \\
\hline
\end{tabular}

Attachment

Originator

Checked

J. M. Capron

Calc. No.
T. M. Blakley

0100B-CA-V0299
Sheet No. 83 of 113

Date $\frac{83 / 0 / 1 / 07}{10 / 04}$

Date

Rev. No. 1 
Attachment 1. 118-B-1 Verification Sampling Results.

\begin{tabular}{|c|c|c|c|c|c|c|c|c|c|c|c|c|}
\hline \multirow[t]{2}{*}{ Constituents } & \multicolumn{3}{|c|}{$\begin{array}{c}\text { J12WL7 } \\
\text { BCL B7 } \\
\text { Sample Date 7/12/06 } \\
\end{array}$} & \multicolumn{3}{|c|}{$\begin{array}{c}\text { J12WL6 } \\
\text { BCL B8 } \\
\text { Sample Date 7/12/06 } \\
\end{array}$} & \multicolumn{3}{|c|}{$\begin{array}{c}\text { J12WW0 } \\
\text { Split of J12WL6 } \\
\text { Sample Date 7/12/06 } \\
\end{array}$} & \multicolumn{3}{|c|}{$\begin{array}{c}\text { J12WK7 } \\
\text { BCL C9 } \\
\text { Sample Date } 7 / 11 / 06 \\
\end{array}$} \\
\hline & $\mu \mathrm{g} / \mathrm{kg}$ & $\mathbf{Q}$ & PQL & $\mu \mathrm{g} / \mathrm{kg}$ & $\mathbf{Q}$ & PQL & $\mu \mathrm{g} / \mathrm{kg}$ & $\mathbf{Q}$ & MDL & $\mu \mathrm{g} / \mathrm{kg}$ & $\mathrm{Q}$ & PQL \\
\hline \multicolumn{13}{|c|}{ Pesticides } \\
\hline Aldrin & 1.3 & UD & 1.3 & 1.3 & UD & 1.3 & 17 & UD & 17 & 1.3 & UD & 1.3 \\
\hline alpha-BHC & 1.3 & UD & 1.3 & 1.3 & UD & 1.3 & 17 & UD & 17 & 1.3 & UDJ & 1.3 \\
\hline alpha-Chlordane & 1.3 & UD & 1.3 & 1.3 & UD & 1.3 & 17 & UD & 17 & 1.3 & UD & 1.3 \\
\hline beta-BHC & 1.3 & UD & 1.3 & 1.3 & UD & 1.3 & 17 & UD & 17 & 1.3 & UD & 1.3 \\
\hline delta-BHC & 1.3 & UD & 1.3 & 1.3 & UD & 1.3 & 17 & UD & 17 & 1.3 & UDJ & 1.3 \\
\hline Dichlorodiphenyldichloroethane & 1.3 & UD & 1.3 & 1.3 & UD & 1.3 & 17 & UD & 17 & 1.3 & UD & 1.3 \\
\hline Dichlorodiphenyldichloroethylene & 1.3 & UD & 1.3 & 1.3 & UD & 1.3 & 17 & UD & 17 & 1.3 & UDJ & 1.3 \\
\hline Dichlorodiphenyltrichloroethane & 1.6 & JD & 1.3 & 1.3 & UD & 1.3 & 17 & UD & 17 & 1.3 & UD & 1.3 \\
\hline Dieldrin & 1.3 & UD & 1.3 & 1.3 & UD & 1.3 & 17 & UD & 17 & 1.3 & UD & 1.3 \\
\hline Endosulfan I & 1.3 & UD & 1.3 & 1.3 & UD & 1.3 & 17 & UD & 17 & 1.3 & UD & 1.3 \\
\hline Endosulfan II & 1.3 & UD & 1.3 & 1.3 & UD & 1.3 & 17 & UD & 17 & 1.3 & UD & 1.3 \\
\hline Endosulfan sulfate & 1.3 & UD & 1.3 & 1.3 & UD & 1.3 & 17 & UD & 17 & 1.3 & UD & 1.3 \\
\hline Endrin & 1.3 & UD & 1.3 & 1.3 & UD & 1.3 & 17 & UD & 17 & 1.3 & UD & 1.3 \\
\hline \begin{tabular}{|l|} 
Endrin aldehyde \\
\end{tabular} & 1.3 & $\mathrm{JD}$ & 1.3 & 1.3 & UD & 1.3 & 17 & UD & 17 & 1.3 & UD & 1.3 \\
\hline Endrin ketone & 1.3 & UD & 1.3 & 1.3 & UD & 1.3 & 17 & UD & 17 & 1.3 & UD & 1.3 \\
\hline gamma-BHC (Lindane) & 1.3 & UD & 1.3 & 1.3 & UD & 1.3 & 17 & UD & 17 & 1.3 & UDJ & 1.3 \\
\hline gamma-Chlordane & 1.3 & UD & 1.3 & 1.3 & UD & 1.3 & 17 & UD & 17 & 1.3 & UD & 1.3 \\
\hline Heptachlor & 1.3 & UD & 1.3 & 1.3 & UD & 1.3 & 17 & UD & 17 & 1.3 & UD & 1.3 \\
\hline Heptachlor epoxide & 1.3 & UD & 1.3 & 1.3 & UD & 1.3 & 17 & UD & 17 & 1.3 & UD & 1.3 \\
\hline Methoxychlor & 1.3 & UD & 1.3 & 1.3 & UD & 1.3 & 33 & UD & 33 & 3.7 & D & 1.3 \\
\hline Toxaphene & 13 & UDJ & 13 & 13 & UDJ & 13 & 680 & UD & 680 & 13 & UD & 13 \\
\hline \multicolumn{13}{|c|}{ Herbicides } \\
\hline $2,4,5-\mathrm{T}$ & 17 & UJ & 17 & 17 & UJ & 17 & 20 & $\mathrm{U}$ & 20 & 17 & $\mathrm{U}$ & 17 \\
\hline 2,4-D & 33 & UJ & 33 & 34 & UJ & 34 & 81 & U & 81 & 33 & $\mathrm{U}$ & 33 \\
\hline $2,4,5-\mathrm{TP}$ & 17 & UJ & 17 & 17 & UJ & 17 & 20 & $\mathrm{U}$ & 20 & 17 & $\mathrm{U}$ & 17 \\
\hline MCPP & & & & & & & 8100 & $\mathrm{U}$ & 8100 & & & \\
\hline MCPA & & & & & & & 8100 & $\mathrm{U}$ & 8100 & & & \\
\hline Dinoseb & 17 & U & 17 & 17 & $\mathrm{U}$ & 17 & 25 & $\mathrm{U}$ & 25 & 17 & $\mathrm{U}$ & 17 \\
\hline $2,4-\mathrm{DB}$ & 17 & $\mathrm{U}$ & 17 & 17 & $\mathrm{U}$ & 17 & 81 & $\mathrm{U}$ & 81 & 17 & $\mathrm{U}$ & 17 \\
\hline Dalapon & 33 & $\mathrm{U}$ & 33 & 34 & $\mathrm{U}$ & 34 & 40 & $\mathrm{U}$ & 40 & 33 & $\mathrm{U}$ & 33 \\
\hline Dicamba & 33 & UJ & 33 & 34 & $\mathrm{UJ}$ & 34 & 40 & $\mathrm{U}$ & 40 & 12 & $\mathrm{~J}$ & 33 \\
\hline Dichloroprop & 10 & $\mathrm{~J}$ & 33 & 210 & $\mathrm{~J}$ & 17 & 81 & $\mathrm{U}$ & 81 & 11 & $\mathrm{~J}$ & 33 \\
\hline Pentachlorophenol & 17 & $\mathrm{UJ}$ & 17 & 17 & UJ & 17 & & & & 17 & $\mathrm{U}$ & 17 \\
\hline \begin{tabular}{|l} 
Picloram \\
\end{tabular} & 17 & $\mathrm{U}$ & 17 & 17 & $\mathrm{U}$ & 17 & & & & 17 & UR & 17 \\
\hline
\end{tabular}

Attachment

Originator

Checked

Calc. No.

$\frac{1}{\frac{1}{\text { J. M. Capron }}} \frac{1 \text { T. M. Blakley }}{\text { 0100B-CA-V0299 }}$

Sheet No. 84 of 113 Date $10 / 04 / 07$ Date

Rev. No. $\overline{1}$ 
Attachment 1. 118-B-1 Verification Sampling Results.

\begin{tabular}{|c|c|c|c|c|c|c|c|c|c|c|c|c|}
\hline \multirow[t]{2}{*}{ Constituents } & \multicolumn{3}{|c|}{$\begin{array}{c}\text { J12WK9 } \\
\text { BCL C10 } \\
\text { Sample Date } 7 / 11 / 06\end{array}$} & \multicolumn{3}{|c|}{$\begin{array}{c}\text { J12WK8 } \\
\text { BCL C1 } \\
\text { Sample Date 7/11/06 } \\
\end{array}$} & \multicolumn{3}{|c|}{$\begin{array}{c}\text { J12WL2 } \\
\text { BCL C2 } \\
\text { Sample Date 7/11/06 } \\
\end{array}$} & \multicolumn{3}{|c|}{$\begin{array}{c}\text { J12WL3 } \\
\text { BCL D3 } \\
\text { Sample Date 7/11/06 } \\
\end{array}$} \\
\hline & $\mu \mathrm{g} / \mathrm{kg}$ & Q & PQL & $\mu \mathrm{g} / \mathrm{kg}$ & $\mathbf{Q}$ & PQL & $\mu \mathrm{g} / \mathrm{kg}$ & $\mathbf{Q}$ & PQL & $\mu \mathrm{g} / \mathrm{kg}$ & Q & PQL \\
\hline \multicolumn{13}{|c|}{ Polychlorinated Biphenyls } \\
\hline Aroclor-1016 & 13 & $\mathrm{U}$ & 13 & 13 & $\mathrm{U}$ & 13 & 13 & $\mathrm{U}$ & 13 & 13 & $\mathrm{U}$ & 13 \\
\hline Aroclor-1221 & 13 & $\mathrm{U}$ & 13 & 13 & $\mathrm{U}$ & 13 & 13 & $\mathrm{U}$ & 13 & 13 & $\mathrm{U}$ & 13 \\
\hline Aroclor-1232 & 13 & $\mathrm{U}$ & 13 & 13 & $\mathrm{U}$ & 13 & 13 & $\mathrm{U}$ & 13 & 13 & $\mathrm{U}$ & 13 \\
\hline Aroclor-1242 & 13 & $\mathrm{U}$ & 13 & 13 & $\mathrm{U}$ & 13 & 13 & $\mathrm{U}$ & 13 & 13 & $\mathrm{U}$ & 13 \\
\hline Aroclor-1248 & 13 & $\mathrm{U}$ & 13 & 13 & $\mathrm{U}$ & 13 & 13 & U & 13 & 13 & $\mathrm{U}$ & 13 \\
\hline Aroclor -1254 & 13 & $\mathrm{U}$ & 13 & 13 & $\mathrm{U}$ & 13 & 13 & $\mathrm{U}$ & 13 & 13 & $\mathrm{U}$ & 13 \\
\hline Aroclor- 1260 & 13 & $\mathrm{U}$ & 13 & 13 & $\mathrm{U}$ & 13 & 13 & $\mathrm{U}$ & 13 & 13 & $\mathrm{U}$ & 13 \\
\hline \multicolumn{13}{|c|}{ Semivolatile Organic Compounds } \\
\hline 1,2,4-Trichlorobenzene & 340 & UJ & 340 & 340 & UJ & 340 & 340 & UJ & 340 & 340 & UJ & 340 \\
\hline 1,2-Dichlorobenzene & 340 & $U$ & 340 & 340 & $U$ & 340 & 340 & $\mathrm{U}$ & 340 & 340 & $\mathrm{U}$ & 340 \\
\hline 1,3-Dichlorobenzene & 340 & $\mathrm{U}$ & 340 & 340 & $\bar{U}$ & 340 & 340 & $\mathrm{U}$ & 340 & 340 & $\mathrm{U}$ & 340 \\
\hline 1,4-Dichlorobenzene & 340 & $\mathrm{U}$ & 340 & 340 & U & 340 & 340 & $\mathrm{U}$ & 340 & 340 & $\mathrm{U}$ & 340 \\
\hline 2,4,5-Trichlorophenol & 840 & $U$ & 840 & 840 & $\mathrm{U}$ & 840 & 840 & $\mathrm{U}$ & 840 & 840 & $\mathrm{U}$ & 840 \\
\hline 2,4,6-Trichlorophenol & 340 & $\mathrm{U}$ & 340 & 340 & $\mathrm{U}$ & 340 & 340 & $\mathrm{U}$ & 340 & 340 & $\mathrm{U}$ & 340 \\
\hline 2,4-Dichlorophenol & 340 & $\mathrm{U}$ & 340 & 340 & $\mathrm{U}$ & 340 & 340 & $\mathrm{U}$ & 340 & 340 & \begin{tabular}{|l|}
$U$ \\
\end{tabular} & 340 \\
\hline 2,4-Dimethylphenol & 340 & UJ & 340 & 340 & $\mathrm{UJ}$ & 340 & 340 & UJ & 340 & 340 & UJ & 340 \\
\hline 2,4-Dinitrophenol & 840 & UR & 840 & 840 & UR & 840 & 840 & UR & 840 & 840 & UR & 840 \\
\hline 2,4-Dinitrotoluene & 340 & $\mathrm{U}$ & 340 & 340 & $\mathrm{U}$ & 340 & 340 & $\mathrm{U}$ & 340 & 340 & U & 340 \\
\hline 2,6-Dinitrotoluene & 340 & $\mathrm{U}$ & 340 & 340 & $\mathrm{U}$ & 340 & 340 & $\mathrm{U}$ & 340 & 340 & $\mathrm{U}$ & 340 \\
\hline 2-Chloronaphthalene & 340 & $\mathrm{U}$ & 340 & 340 & $\mathrm{U}$ & 340 & 340 & $\mathrm{U}$ & 340 & 340 & $\mathrm{U}$ & 340 \\
\hline 2-Chlorophenol & 340 & $\mathrm{U}$ & 340 & 340 & $\mathrm{U}$ & 340 & 340 & $\mathrm{U}$ & 340 & 340 & $\mathrm{U}$ & 340 \\
\hline 2-Methylnaphthalene & 340 & UJ & 340 & 340 & UJ & 340 & 340 & UJ & 340 & 340 & $\mathrm{UJ}$ & 340 \\
\hline 2-Methylphenol (cresol, o-) & 340 & $\mathrm{U}$ & 340 & 340 & $\mathrm{U}$ & 340 & 340 & $\mathrm{U}$ & 340 & 340 & $\mathrm{U}$ & 340 \\
\hline 2-Nitroaniline & 840 & $\mathrm{U}$ & 840 & 840 & $\mathrm{U}$ & 840 & 840 & $\mathrm{U}$ & 840 & 840 & $\mathrm{U}$ & 840 \\
\hline 2-Nitrophenol & 340 & $\mathrm{U}$ & 340 & 340 & $\mathrm{U}$ & 340 & 340 & $\mathrm{U}$ & 340 & 340 & $\mathrm{U}$ & 340 \\
\hline 3,3'-Dichlorobenzidine & 340 & $\mathrm{U}$ & 340 & 340 & $\mathrm{U}$ & 340 & 340 & $\mathrm{U}$ & 340 & 340 & $\mathrm{U}$ & 340 \\
\hline 3-Nitroaniline & 840 & $\mathrm{U}$ & 840 & 840 & $\mathrm{U}$ & 840 & 840 & $\mathrm{U}$ & 840 & 840 & $\mathrm{U}$ & 840 \\
\hline 4,6-Dinitro-2-methylphenol & 840 & $\mathrm{U}$ & 840 & 840 & $\mathrm{U}$ & 840 & 840 & $\mathrm{U}$ & 840 & 840 & $\mathrm{U}$ & 840 \\
\hline 4-Bromophenyl-phenylether & 340 & $\mathrm{U}$ & 340 & 340 & $\mathrm{U}$ & 340 & 340 & $\mathrm{U}$ & 340 & 340 & $\mathrm{U}$ & 340 \\
\hline 4-Chloro-3-methylphenol & 340 & $\mathrm{U}$ & 340 & 340 & $\mathrm{U}$ & 340 & 340 & $\mathrm{U}$ & 340 & 340 & $\mathrm{U}$ & 340 \\
\hline 4-Chloroaniline & 340 & $\mathrm{U}$ & 340 & 340 & $\mathrm{U}$ & 340 & 340 & $\mathrm{U}$ & 340 & 340 & $\mathrm{U}$ & 340 \\
\hline 4-Chlorophenyl-phenylether & 340 & $\mathrm{U}$ & 340 & 340 & $\mathrm{U}$ & 340 & 340 & $\mathrm{U}$ & 340 & 340 & $\mathrm{U}$ & 340 \\
\hline 4-Methylphenol (p-cresol) & 340 & $\mathrm{U}$ & 340 & 340 & $\mathrm{U}$ & 340 & 340 & $\mathrm{U}$ & 340 & 340 & $\mathrm{U}$ & 340 \\
\hline 4-Nitroaniline & 840 & $\mathrm{U}$ & 840 & 840 & $\mathrm{U}$ & 840 & 840 & $\mathrm{U}$ & 840 & 840 & $\mathrm{U}$ & 840 \\
\hline 4-Nitrophenol & 840 & $\mathrm{U}$ & 840 & 840 & $\mathrm{U}$ & 840 & 840 & $\mathrm{U}$ & 840 & 840 & $\mathrm{U}$ & 840 \\
\hline Acenaphthene & 340 & $\mathrm{U}$ & 340 & 340 & $\mathrm{U}$ & 340 & 340 & $\mathrm{U}$ & 340 & 340 & $\mathrm{U}$ & 340 \\
\hline Acenaphthylene & 340 & $\mathrm{U}$ & 340 & 340 & $\mathrm{U}$ & 340 & 340 & $\mathrm{U}$ & 340 & 340 & $\mathrm{U}$ & 340 \\
\hline Anthracene & 340 & $\mathrm{U}$ & 340 & 340 & $\mathrm{U}$ & 340 & 340 & $\mathrm{U}$ & 340 & 340 & $\mathrm{U}$ & 340 \\
\hline Benzo(a)anthracene & 340 & $\mathrm{U}$ & 340 & 340 & $\mathrm{U}$ & 340 & 340 & $\mathrm{U}$ & 340 & 340 & $\mathrm{U}$ & 340 \\
\hline Benzo(a)pyrene & 340 & $\mathrm{U}$ & 340 & 340 & $\mathrm{U}$ & 340 & 340 & $\mathrm{U}$ & 340 & 340 & $\mathrm{U}$ & 340 \\
\hline Benzo(b)fluoranthene & 340 & $\mathrm{U}$ & 340 & 340 & $\mathrm{U}$ & 340 & 340 & U & 340 & 340 & $\mathrm{U}$ & 340 \\
\hline Benzo(g,h,i)perylene & 340 & $\mathrm{U}$ & 340 & 340 & $\mathrm{U}$ & 340 & 340 & $\mathrm{U}$ & 340 & 340 & $\mathrm{U}$ & 340 \\
\hline Benzo(k)fluoranthene & 340 & $\mathrm{U}$ & 340 & 340 & $U$ & 340 & 340 & $\mathrm{U}$ & 340 & 340 & $U$ & 340 \\
\hline bis(2-Chloro-1-methylethyl)ether & 340 & U & 340 & 340 & $U$ & 340 & 340 & $\mathrm{U}$ & 340 & 340 & $\mathrm{U}$ & 340 \\
\hline bis(2-Chloroethoxy)methane & 340 & $\mathrm{U}$ & 340 & 340 & $\mathrm{U}$ & 340 & 340 & $\mathrm{U}$ & 340 & 340 & $\mathrm{U}$ & 340 \\
\hline bis(2-Chloroethyl)ether & 340 & $\mathrm{U}$ & 340 & 340 & $\mathrm{U}$ & 340 & 340 & $\mathrm{U}$ & 340 & 340 & $\mathrm{U}$ & 340 \\
\hline bis(2-Ethylhexyl)phthalate & 36 & JB & 340 & 29 & $\mathrm{JB}$ & 340 & 23 & $\mathrm{JB}$ & 340 & 29 & $\mathrm{JB}$ & 340 \\
\hline
\end{tabular}

Attachment

Originator

Checked

Calc. No.

\begin{tabular}{l}
$\frac{1}{\text { J.M. Capron }}$ \\
\hline T. M. Blakley \\
\hline 0100B-CA-V0299
\end{tabular}

Sheet No. 85 of 113 Date $10 / 04 / 07$ Date

Rev. No. 1 
Attachment 1. 118-B-1 Verification Sampling Results.

\begin{tabular}{|c|c|c|c|c|c|c|c|c|c|c|c|c|}
\hline \multirow[t]{2}{*}{ Constituents } & \multicolumn{3}{|c|}{$\begin{array}{c}\text { J12WK9 } \\
\text { BCL C10 } \\
\text { Sample Date } 7 / 11 / 06\end{array}$} & \multicolumn{3}{|c|}{\begin{tabular}{|c|} 
J12WK8 \\
BCL C1 \\
Sample Date $7 / 11 / 06$ \\
\end{tabular}} & \multicolumn{3}{|c|}{$\begin{array}{c}\text { J12WL2 } \\
\text { BCL C2 } \\
\text { Sample Date } 7 / 11 / 06\end{array}$} & \multicolumn{3}{|c|}{\begin{tabular}{|c|} 
J12WL3 \\
BCL D3 \\
Sample Date 7/11/06
\end{tabular}} \\
\hline & $\mu \mathrm{g} / \mathrm{kg}$ & $\mathbf{Q}$ & PQL & $\mu \mathrm{g} / \mathrm{kg}$ & $\mathbf{Q}$ & PQL & $\mu \mathrm{g} / \mathrm{kg}$ & $\mathbf{Q}$ & MDL & $\mu \mathrm{g} / \mathrm{kg}$ & $\mathbf{Q}$ & PQL \\
\hline \multicolumn{13}{|c|}{ Semivolatile Organic Compounds (continued) } \\
\hline Butylbenzylphthalate & 340 & $\mathrm{U}$ & 340 & 340 & $\mathrm{U}$ & 340 & 340 & $\mathrm{U}$ & 340 & 340 & $\mathrm{U}$ & 340 \\
\hline Carbazole & 340 & $\mathrm{U}$ & 340 & 340 & $\mathrm{U}$ & 340 & 340 & $\mathrm{U}$ & 340 & 340 & $\mathrm{U}$ & 340 \\
\hline Chrysene & 340 & $\bar{U}$ & 340 & 340 & $\mathrm{U}$ & 340 & 340 & $\mathrm{U}$ & 340 & 340 & $\mathrm{U}$ & 340 \\
\hline Di-n-butylphthalate & 23 & $\mathrm{~J}$ & 340 & 49 & $\mathrm{~J}$ & 340 & 340 & $\mathrm{U}$ & 340 & 18 & $\mathrm{~J}$ & 340 \\
\hline Di-n-octylphthalate & 340 & $\mathrm{U}$ & 340 & 340 & $\mathrm{U}$ & 340 & 340 & $\mathrm{U}$ & 340 & 340 & $\mathrm{U}$ & 340 \\
\hline Dibenz(a,h)anthracene & 340 & $\mathrm{U}$ & 340 & 340 & $\mathrm{U}$ & 340 & 340 & $\mathrm{U}$ & 340 & 340 & $\mathrm{U}$ & 340 \\
\hline Dibenzofuran & 340 & $\mathrm{U}$ & 340 & 340 & $\mathrm{U}$ & 340 & 340 & $\mathrm{U}$ & 340 & 340 & $\mathrm{U}$ & 340 \\
\hline Diethylphthalate & 340 & $\mathrm{U}$ & 340 & 19 & $\mathrm{~J}$ & 340 & 340 & $\mathrm{U}$ & 340 & 340 & $\mathrm{U}$ & 340 \\
\hline Dimethylphthalate & 340 & $\mathrm{U}$ & 340 & 340 & $\mathrm{U}$ & 340 & 340 & $\mathrm{U}$ & 340 & 340 & $\mathrm{U}$ & 340 \\
\hline Fluoranthene & 340 & $\mathrm{U}$ & 340 & 340 & $\mathrm{U}$ & 340 & 340 & $\mathrm{U}$ & 340 & 340 & $\mathrm{U}$ & 340 \\
\hline Fluorene & 340 & $\mathrm{U}$ & 340 & 340 & $\mathrm{U}$ & 340 & 340 & $\mathrm{U}$ & 340 & 340 & $\mathrm{U}$ & 340 \\
\hline Hexachlorobenzene & 340 & $\mathrm{U}$ & 340 & 340 & $\mathrm{U}$ & 340 & 340 & $\mathrm{U}$ & 340 & 340 & $\mathrm{U}$ & 340 \\
\hline Hexachlorobutadiene & 340 & $\mathrm{U}$ & 340 & 340 & $\mathrm{U}$ & 340 & 340 & $\mathrm{U}$ & 340 & 340 & $\mathrm{U}$ & 340 \\
\hline Hexachlorocyclopentadiene & 340 & $\mathrm{U}$ & 340 & 340 & $\mathrm{U}$ & 340 & 340 & $\mathrm{U}$ & 340 & 340 & $\mathrm{U}$ & 340 \\
\hline Hexachloroethane & 340 & $\mathrm{U}$ & 340 & 340 & $\mathrm{U}$ & 340 & 340 & $\mathrm{U}$ & 340 & 340 & $\mathrm{U}$ & 340 \\
\hline Indeno(1,2,3-cd)pyrene & 340 & $\mathrm{U}$ & 340 & 340 & $\mathrm{U}$ & 340 & 340 & $\mathrm{U}$ & 340 & 340 & $\mathrm{U}$ & 340 \\
\hline Isophorone & 340 & UJ & 340 & 340 & UJ & 340 & 340 & UJ & 340 & 340 & $\mathrm{UJ}$ & 340 \\
\hline N-Nitroso-di-n-dipropylamine & 340 & U & 340 & 340 & $\mathrm{U}$ & 340 & 340 & $\mathrm{U}$ & 340 & 340 & $\mathrm{U}$ & 340 \\
\hline N-Nitrosodiphenylamine & 340 & $\mathrm{U}$ & 340 & 340 & $\mathrm{U}$ & 340 & 340 & $\mathrm{U}$ & 340 & 340 & $\mathrm{U}$ & 340 \\
\hline Naphthalene & 340 & $\mathrm{U}$ & 340 & 340 & $\mathrm{U}$ & 340 & 340 & $\mathrm{U}$ & 340 & 340 & $\mathrm{U}$ & 340 \\
\hline Nitrobenzene & 340 & U & 340 & 340 & $\mathrm{U}$ & 340 & 340 & $\mathrm{U}$ & 340 & 340 & $\mathrm{U}$ & 340 \\
\hline Pentachlorophenol & 840 & $\mathrm{U}$ & 840 & 840 & $\mathrm{U}$ & 840 & 840 & $\mathrm{U}$ & 840 & 840 & $\mathrm{U}$ & 840 \\
\hline Phenanthrene & 340 & $U$ & 340 & 340 & $\mathrm{U}$ & 340 & 340 & $\mathrm{U}$ & 340 & 340 & $\mathrm{U}$ & 340 \\
\hline Phenol & 340 & $\mathrm{U}$ & 340 & 27 & $\mathrm{~J}$ & 340 & 340 & $\mathrm{U}$ & 340 & 27 & $\mathrm{~J}$ & 340 \\
\hline Pyrene & 340 & $\mathrm{U}$ & 340 & 340 & $\mathrm{U}$ & 340 & 340 & $\mathrm{U}$ & 340 & 340 & $\mathrm{U}$ & 340 \\
\hline & & & & Attachm & & & 1 & & & et No. & & $f 113$ \\
\hline & & & & Originat & & J.M. Ca & ron & & & Date & & $104 / 07$ \\
\hline & & & & Checked & & T.M. Bl & kley & & & Date & & \\
\hline & & & & Calc. Nc & & $0100 \mathrm{~B}-\mathrm{C}$ & A-V0299 & & & Rev. No. & & 1 \\
\hline
\end{tabular}


Attachment 1. 118-B-1 Verification Sampling Results.

\begin{tabular}{|c|c|c|c|c|c|c|c|c|c|c|c|c|}
\hline \multirow[t]{2}{*}{ Constituents } & \multicolumn{3}{|c|}{$\begin{array}{c}\text { J12WK9 } \\
\text { BCL C10 } \\
\text { Sample Date 7/11/06 }\end{array}$} & \multicolumn{3}{|c|}{$\begin{array}{c}\text { J12WK8 } \\
\text { BCL C1 } \\
\text { Sample Date 7/11/06 } \\
\end{array}$} & \multicolumn{3}{|c|}{$\begin{array}{c}\text { J12WL2 } \\
\text { BCL C2 } \\
\text { Sample Date 7/11/06 }\end{array}$} & \multicolumn{3}{|c|}{$\begin{array}{c}\text { J12WL3 } \\
\text { BCL D3 } \\
\text { Sample Date 7/11/06 } \\
\end{array}$} \\
\hline & $\mu \mathrm{g} / \mathrm{kg}$ & $Q$ & PQL & $\mu \mathrm{g} / \mathrm{kg}$ & Q & PQL & $\mu \mathrm{g} / \mathrm{kg}$ & $\mathbf{Q}$ & MDL & $\mu \mathrm{g} / \mathrm{kg}$ & $\mathrm{Q}$ & PQL \\
\hline \multicolumn{13}{|c|}{ Volatile Organic Compounds } \\
\hline 1,1,1-Trichloroethane & 5 & $\mathrm{U}$ & 5 & 5 & $\mathrm{U}$ & 5 & 5 & $\mathrm{U}$ & 5 & 5 & $U$ & 5 \\
\hline 1,1,2,2-Tetrachloroethane & 5 & $\mathrm{U}$ & 5 & 5 & $\mathrm{U}$ & 5 & 5 & $\mathrm{U}$ & 5 & 5 & $\mathrm{U}$ & 5 \\
\hline 1,1,2-Trichloroethane & 5 & $\mathrm{U}$ & 5 & 5 & $\mathrm{U}$ & 5 & 5 & $\mathrm{U}$ & 5 & 5 & $\mathrm{U}$ & 5 \\
\hline 1,1-Dichloroethane & 5 & $\mathrm{U}$ & 5 & 5 & $\mathrm{U}$ & 5 & 5 & $\mathrm{U}$ & 5 & 5 & $\mathrm{U}$ & 5 \\
\hline 1,1-Dichloroethene & 5 & $\mathrm{U}$ & 5 & 5 & $\mathrm{U}$ & 5 & 5 & $\mathrm{U}$ & 5 & 5 & $\mathrm{U}$ & 5 \\
\hline 1,2-Dichloroethane & 5 & $\mathrm{U}$ & 5 & 5 & $\mathrm{U}$ & 5 & 5 & $\mathrm{U}$ & 5 & 5 & $U$ & 5 \\
\hline 1,2-Dichloroethene (total) & 5 & $\mathrm{U}$ & 5 & 5 & $\mathrm{U}$ & 5 & 5 & $\mathrm{U}$ & 5 & 5 & $\mathrm{U}$ & 5 \\
\hline 1,2-Dichloropropane & 5 & $\mathrm{U}$ & 5 & 5 & $\mathrm{U}$ & 5 & 5 & $\mathrm{U}$ & 5 & 5 & $\mathrm{U}$ & 5 \\
\hline 2-Butanone & 10 & $\mathrm{U}$ & 10 & 10 & $\mathrm{U}$ & 10 & 10 & $\bar{U}$ & 10 & 10 & $\bar{U}$ & 10 \\
\hline 2-Hexanone & 10 & $\mathrm{U}$ & 10 & 10 & $U$ & 10 & 10 & $\mathrm{U}$ & 10 & 10 & $\bar{U}$ & 10 \\
\hline 4-Methyl-2-pentanone & 10 & $\mathrm{U}$ & 10 & 10 & $\mathrm{U}$ & 10 & 10 & $\mathrm{U}$ & 10 & 10 & $U$ & 10 \\
\hline Acetone & 10 & $\mathrm{U}$ & 10 & 10 & $\mathrm{U}$ & 10 & 20 & & 10 & 10 & $\mathrm{U}$ & 10 \\
\hline Benzene & 5 & $\mathrm{U}$ & 5 & 5 & $\mathrm{U}$ & 5 & 5 & $\mathrm{U}$ & 5 & 5 & $\mathrm{U}$ & 5 \\
\hline Bromodichloromethane & 5 & $\mathrm{U}$ & 5 & 5 & $\mathrm{U}$ & 5 & 5 & $\mathrm{U}$ & 5 & 5 & $\mathrm{U}$ & 5 \\
\hline Bromoform & 5 & $\mathrm{U}$ & 5 & 5 & $\mathrm{U}$ & 5 & 5 & $\mathrm{U}$ & 5 & 5 & $\mathrm{U}$ & 5 \\
\hline Bromomethane & 10 & $\mathrm{U}$ & 10 & 10 & $\mathrm{U}$ & 10 & 10 & U & 10 & 10 & $\mathrm{U}$ & 10 \\
\hline Carbon disulfide & 5 & $\mathrm{U}$ & 5 & 5 & $U$ & 5 & 5 & $\mathrm{U}$ & 5 & 5 & $\mathrm{U}$ & 5 \\
\hline Carbon tetrachloride & 5 & $\mathrm{U}$ & 5 & 5 & $U$ & 5 & 11 & & 5 & 6 & & 5 \\
\hline Chlorobenzene & 5 & $\mathrm{U}$ & 5 & 5 & $\mathrm{U}$ & 5 & 5 & $\mathrm{U}$ & 5 & 5 & $\mathrm{U}$ & 5 \\
\hline Chloroethane & 10 & $\mathrm{U}$ & 10 & 10 & $\mathrm{U}$ & 10 & 10 & $\mathrm{U}$ & 10 & 10 & $\mathrm{U}$ & 10 \\
\hline Chloroform & 5 & $\mathrm{U}$ & 5 & 5 & $\mathrm{U}$ & 5 & 5 & $\mathrm{U}$ & 5 & 5 & $\mathrm{U}$ & 5 \\
\hline Chloromethane & 10 & $\mathrm{U}$ & 10 & 10 & $\mathrm{U}$ & 10 & 10 & $\mathrm{U}$ & 10 & 10 & $\mathrm{U}$ & 10 \\
\hline cis-1,2-Dichloroethylene & 5 & $\mathrm{U}$ & 5 & 5 & $U$ & 5 & 5 & $\mathrm{U}$ & 5 & 5 & $\mathrm{U}$ & 5 \\
\hline cis-1,3-Dichloropropene & 5 & $\mathrm{U}$ & 5 & 5 & $\mathrm{U}$ & 5 & 5 & $\mathrm{U}$ & 5 & 5 & $\mathrm{U}$ & 5 \\
\hline Dibromochloromethane & 5 & $\mathrm{U}$ & 5 & 5 & $\mathrm{U}$ & 5 & 5 & $\mathrm{U}$ & 5 & 5 & $\mathrm{U}$ & 5 \\
\hline Ethylbenzene & 5 & $\mathrm{U}$ & 5 & 5 & $\mathrm{U}$ & 5 & 5 & $\mathrm{U}$ & 5 & 5 & $\mathrm{U}$ & 5 \\
\hline Methylene chloride & 8 & $\mathrm{BJ}$ & 5 & 5 & BJ & 5 & 18 & $\mathrm{BJ}$ & 5 & 15 & $\mathrm{BJ}$ & 5 \\
\hline Styrene & 5 & $\mathrm{U}$ & 5 & 5 & $\mathrm{U}$ & 5 & 5 & $\mathrm{U}$ & 5 & 5 & U & 5 \\
\hline Tetrachloroethene & 5 & $\mathrm{U}$ & 5 & 5 & $\mathrm{U}$ & 5 & 5 & $\mathrm{U}$ & 5 & 5 & $\mathrm{U}$ & 5 \\
\hline Toluene & 5 & $\mathrm{U}$ & 5 & 5 & $\mathrm{U}$ & 5 & 5 & $\mathrm{U}$ & 5 & 5 & $\mathrm{U}$ & 5 \\
\hline trans-1,2-Dichloroethylene & 5 & $\mathrm{U}$ & 5 & 5 & $U$ & 5 & 5 & $\mathrm{U}$ & 5 & 5 & $\mathrm{U}$ & 5 \\
\hline trans-1,3-Dichloropropene & 5 & $\mathrm{U}$ & 5 & 5 & $\mathrm{U}$ & 5 & 5 & $\mathrm{U}$ & 5 & 5 & $\mathrm{U}$ & 5 \\
\hline Trichloroethene & 5 & $U$ & 5 & 5 & $\mathrm{U}$ & 5 & 5 & $\mathrm{U}$ & 5 & 5 & $\mathrm{U}$ & 5 \\
\hline Vinyl chloride & 10 & $\mathrm{U}$ & 10 & 10 & $\mathrm{U}$ & 10 & 10 & $\mathrm{U}$ & 10 & 10 & $\mathrm{U}$ & 10 \\
\hline Xylenes (total) & 5 & $\mathrm{U}$ & 5 & 5 & $\mathrm{U}$ & 5 & 5 & $\mathrm{U}$ & 5 & 5 & $\mathrm{U}$ & 5 \\
\hline & & & & Attachm & & & 1 & & & Sheet No. & & f 113 \\
\hline & & & & Originat & & J.M. Ca & rron & & & Date & & $04 / 07$ \\
\hline & & & & Checkec & & T.M. Bl & kley & & & Date & & \\
\hline & & & & Calc. No & & $0100 \mathrm{~B}-\mathrm{C}$ & $\mathrm{A}-\mathrm{V} 0299$ & & & Rev. No & & 1 \\
\hline
\end{tabular}


Attachment 1. 118-B-1 Verification Sampling Results.

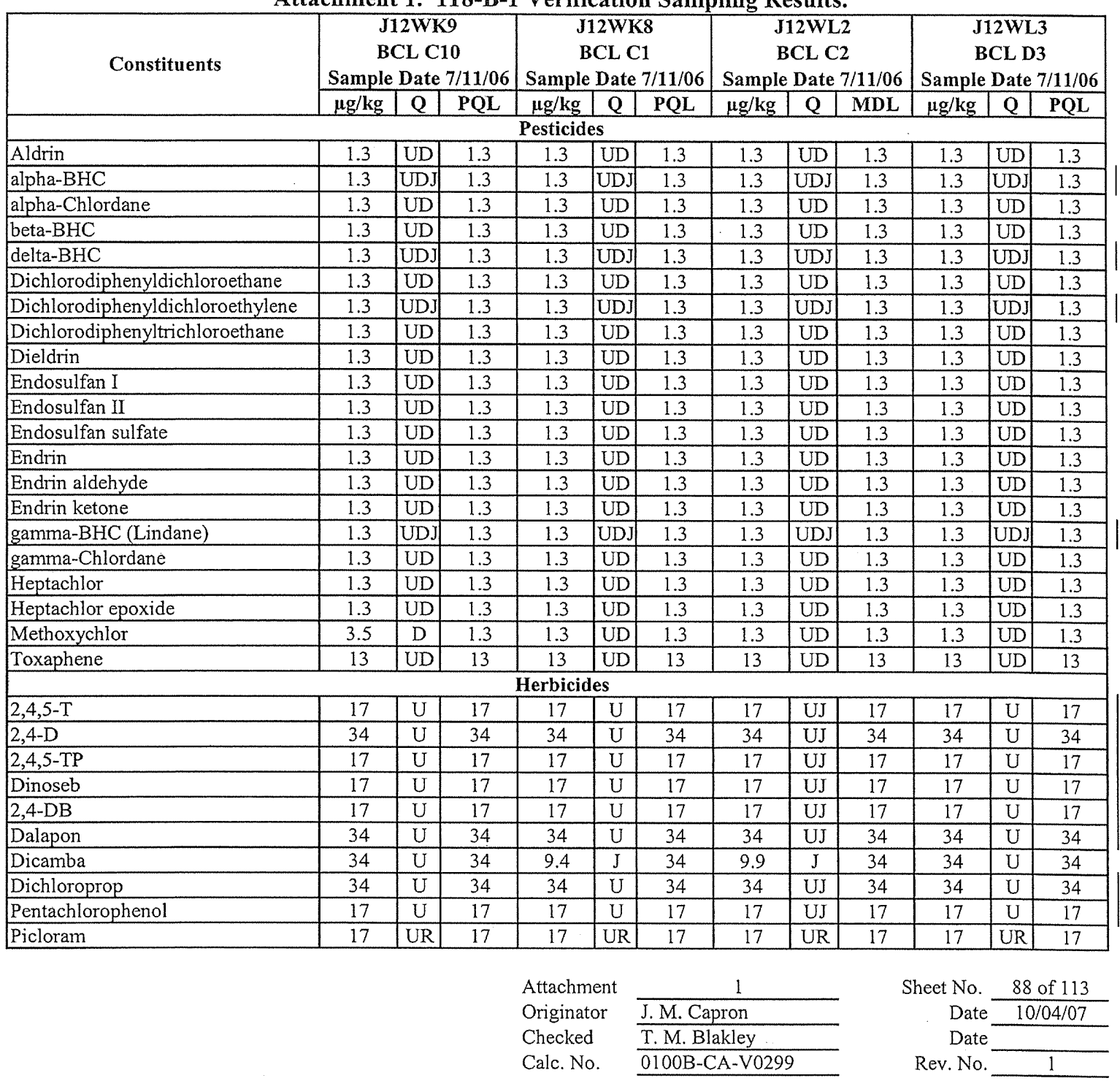


Attachment 1. 118-B-1 Verification Sampling Results.

\begin{tabular}{|c|c|c|c|c|c|c|c|c|c|c|c|c|}
\hline \multirow[t]{2}{*}{ Constituents } & \multicolumn{3}{|c|}{$\begin{array}{c}\text { J12WL4 } \\
\text { BCL D4 } \\
\text { Sample Date 7/11/06 } \\
\end{array}$} & \multicolumn{3}{|c|}{$\begin{array}{c}\text { J12WL5 } \\
\text { Duplicate of J12WL4 } \\
\text { Sample Date } 7 / 11 / 06 \\
\end{array}$} & \multicolumn{3}{|c|}{$\begin{array}{c}\text { J12WL0 } \\
\text { BCL D5 } \\
\text { Sample Date 7/11/06 } \\
\end{array}$} & \multicolumn{3}{|c|}{\begin{tabular}{|c|} 
J12WL1 \\
BCL D6 \\
Sample Date 7/11/06 \\
\end{tabular}} \\
\hline & $\mu \mathrm{g} / \mathrm{kg}$ & $\mathbf{Q}$ & PQL & $\mu \mathrm{g} / \mathrm{kg}$ & Q & PQL & $\mu \mathrm{g} / \mathrm{kg}$ & $\mathbf{Q}$ & PQL & $\mu \mathrm{g} / \mathrm{kg}$ & $\mathrm{Q}$ & PQL \\
\hline \multicolumn{13}{|c|}{ Polychlorinated Biphenyls } \\
\hline Aroclor-1016 & 13 & $\mathrm{U}$ & 13 & 13 & $\mathrm{U}$ & 13 & 13 & $\mathrm{U}$ & 13 & 13 & $\mathrm{U}$ & 13 \\
\hline Aroclor-1221 & 13 & $\mathrm{U}$ & 13 & 13 & $\mathrm{U}$ & 13 & 13 & $\mathrm{U}$ & 13 & 13 & $\mathrm{U}$ & 13 \\
\hline Aroclor-1232 & 13 & $U$ & 13 & 13 & $\mathrm{U}$ & 13 & 13 & $\mathrm{U}$ & 13 & 13 & $\mathrm{U}$ & 13 \\
\hline Aroclor-1242 & 13 & $\mathrm{U}$ & 13 & 13 & $\mathrm{U}$ & 13 & 13 & $\mathrm{U}$ & 13 & 13 & $\mathrm{U}$ & 13 \\
\hline Aroclor-1248 & 13 & $\mathrm{U}$ & 13 & 13 & $\mathrm{U}$ & 13 & 13 & $\mathrm{U}$ & 13 & 13 & $\mathrm{U}$ & 13 \\
\hline Aroclor-1254 & 13 & $\mathrm{U}$ & 13 & 13 & $\mathrm{U}$ & 13 & 13 & $\mathrm{U}$ & 13 & 13 & $\mathrm{U}$ & 13 \\
\hline Aroclor -1260 & 13 & $\mathrm{U}$ & 13 & 13 & $\mathrm{U}$ & 13 & 13 & $\mathrm{U}$ & 13 & 13 & $\mathrm{U}$ & 13 \\
\hline \multicolumn{13}{|c|}{ Semivolatile Organic Compounds } \\
\hline 1,2,4-Trichlorobenzene & 330 & $\mathrm{UJ}$ & 330 & 330 & UJ & 330 & 330 & UJ & 330 & 330 & UJ & 330 \\
\hline 1,2-Dichlorobenzene & 330 & $\mathrm{U}$ & 330 & 330 & $\bar{U}$ & 330 & 330 & $\mathrm{U}$ & 330 & 330 & $\mathrm{U}$ & 330 \\
\hline 1,3-Dichlorobenzene & 330 & $\mathrm{U}$ & 330 & 330 & $\mathrm{U}$ & 330 & 330 & $\mathrm{U}$ & 330 & 330 & $\mathrm{U}$ & 330 \\
\hline 1,4-Dichlorobenzene & 330 & $\mathrm{U}$ & 330 & 330 & $\mathrm{U}$ & 330 & 330 & $\mathrm{U}$ & 330 & 330 & $\mathrm{U}$ & 330 \\
\hline 2,4,5-Trichlorophenol & 840 & $\mathrm{U}$ & 840 & 840 & $\mathrm{U}$ & 840 & 840 & $\mathrm{U}$ & 840 & 840 & $\mathrm{U}$ & 840 \\
\hline 2,4,6-Trichlorophenol & 330 & $\mathrm{U}$ & 330 & 330 & $\mathrm{U}$ & 330 & 330 & $\mathrm{U}$ & 330 & 330 & $\mathrm{U}$ & 330 \\
\hline 2,4-Dichlorophenol & 330 & $\mathrm{U}$ & 330 & 330 & $\mathrm{U}$ & 330 & 330 & $\mathrm{U}$ & 330 & 330 & $\mathrm{U}$ & 330 \\
\hline 2,4-Dimethylphenol & 330 & $\mathrm{UJ}$ & 330 & 330 & $\mathrm{UJ}$ & 330 & 330 & $\mathrm{UJ}$ & 330 & 330 & $\mathrm{UJ}$ & 330 \\
\hline 2,4-Dinitrophenol & 840 & UR & 840 & 840 & UR & 840 & 840 & UR & 840 & 840 & UR & 840 \\
\hline 2,4-Dinitrotoluene & 330 & $\mathrm{U}$ & 330 & 330 & U & 330 & 330 & $\mathrm{U}$ & 330 & 330 & $\mathrm{U}$ & 330 \\
\hline 2,6-Dinitrotoluene & 330 & $\mathrm{U}$ & 330 & 330 & $\mathrm{U}$ & 330 & 330 & $\mathrm{U}$ & 330 & 330 & $\mathrm{U}$ & 330 \\
\hline 2-Chloronaphthalene & 330 & $\mathrm{U}$ & 330 & 330 & $\mathrm{U}$ & 330 & 330 & $\mathrm{U}$ & 330 & 330 & $\mathrm{U}$ & 330 \\
\hline 2-Chlorophenol & 330 & $\mathrm{U}$ & 330 & 330 & $U$ & 330 & 330 & $\mathrm{U}$ & 330 & 330 & $\mathrm{U}$ & 330 \\
\hline 2-Methylnaphthalene & 330 & $\mathrm{UJ}$ & 330 & 330 & UJ & 330 & 330 & UJ & 330 & 330 & $\mathrm{UJ}$ & 330 \\
\hline 2-Methylphenol (cresol, o-) & 330 & $\mathrm{U}$ & 330 & 330 & $\mathrm{U}$ & 330 & 330 & $\mathrm{U}$ & 330 & 330 & $\mathrm{U}$ & 330 \\
\hline 2-Nitroaniline & 840 & $\mathrm{U}$ & 840 & 840 & $\mathrm{U}$ & 840 & 840 & $\mathrm{U}$ & 840 & 840 & $\mathrm{U}$ & 840 \\
\hline 2-Nitrophenol & 330 & $\mathrm{U}$ & 330 & 330 & $\mathrm{U}$ & 330 & 330 & $\mathrm{U}$ & 330 & 330 & $U$ & 330 \\
\hline 3,3'-Dichlorobenzidine & 330 & $\mathrm{U}$ & 330 & 330 & $\mathrm{U}$ & 330 & 330 & $U$ & 330 & 330 & $\mathrm{U}$ & 330 \\
\hline 3-Nitroaniline & 840 & $\mathrm{U}$ & 840 & 840 & $\mathrm{U}$ & 840 & 840 & $U$ & 840 & 840 & $\mathrm{U}$ & 840 \\
\hline 4,6-Dinitro-2-methylphenol & 840 & $\mathrm{U}$ & 840 & 840 & $\mathrm{U}$ & 840 & 840 & $\mathrm{U}$ & 840 & 840 & $\mathrm{U}$ & 840 \\
\hline 4-Bromophenyl-phenylether & 330 & $\mathrm{U}$ & 330 & 330 & $\mathrm{U}$ & 330 & 330 & $\mathrm{U}$ & 330 & 330 & $\mathrm{U}$ & 330 \\
\hline 4-Chloro-3-methylphenol & 330 & $\mathrm{U}$ & 330 & 330 & $\mathrm{U}$ & 330 & 330 & $\mathrm{U}$ & 330 & 330 & U & 330 \\
\hline 4-Chloroaniline & 330 & $\mathrm{U}$ & 330 & 330 & $\mathrm{U}$ & 330 & 330 & $\mathrm{U}$ & 330 & 330 & $\mathrm{U}$ & 330 \\
\hline 4-Chlorophenyl-phenylether & 330 & $\mathrm{U}$ & 330 & 330 & $\mathrm{U}$ & 330 & 330 & $\mathrm{U}$ & 330 & 330 & $\mathrm{U}$ & 330 \\
\hline 4-Methylphenol (p-cresol) & 330 & $\mathrm{U}$ & 330 & 330 & $\mathrm{U}$ & 330 & 330 & $\mathrm{U}$ & 330 & 330 & $\mathrm{U}$ & 330 \\
\hline 4-Nitroaniline & 840 & $\mathrm{U}$ & 840 & 840 & $\mathrm{U}$ & 840 & 840 & $\mathrm{U}$ & 840 & 840 & U & 840 \\
\hline 4-Nitrophenol & 840 & $U$ & 840 & 840 & $\mathrm{U}$ & 840 & 840 & $\mathrm{U}$ & 840 & 840 & $\mathrm{U}$ & 840 \\
\hline Acenaphthene & 330 & $U$ & 330 & 330 & $\mathrm{U}$ & 330 & 330 & $\mathrm{U}$ & 330 & 330 & $\mathrm{U}$ & 330 \\
\hline Acenaphthylene & 330 & $\mathrm{U}$ & 330 & 330 & $\mathrm{U}$ & 330 & 330 & $\mathrm{U}$ & 330 & 330 & $\mathrm{U}$ & 330 \\
\hline Anthracene & 330 & $\mathrm{U}$ & 330 & 330 & $\mathrm{U}$ & 330 & 330 & $\mathrm{U}$ & 330 & 330 & $\mathrm{U}$ & 330 \\
\hline Benzo(a)anthracene & 330 & $\mathrm{U}$ & 330 & 330 & $\mathrm{U}$ & 330 & 330 & $\mathrm{U}$ & 330 & 330 & $\mathrm{U}$ & 330 \\
\hline Benzo(a)pyrene & 330 & $\mathrm{U}$ & 330 & 330 & $\mathrm{U}$ & 330 & 330 & $\mathrm{U}$ & 330 & 330 & $\mathrm{U}$ & 330 \\
\hline Benzo(b)fluoranthene & 330 & $\mathrm{U}$ & 330 & 330 & $\mathrm{U}$ & 330 & 330 & $\mathrm{U}$ & 330 & 330 & $\mathrm{U}$ & 330 \\
\hline Benzo(g,h,i)perylene & 330 & $U$ & 330 & 330 & $\mathrm{U}$ & 330 & 330 & $U$ & 330 & 330 & $\mathrm{U}$ & 330 \\
\hline Benzo(k)fluoranthene & 330 & $\mathrm{U}$ & 330 & 330 & $\mathrm{U}$ & 330 & 330 & $\mathrm{U}$ & 330 & 330 & $\mathrm{U}$ & 330 \\
\hline bis(2-Chloro-1-methylethyl)ether & 330 & $\mathrm{U}$ & 330 & 330 & $\mathrm{U}$ & 330 & 330 & $\mathrm{U}$ & 330 & 330 & $\mathrm{U}$ & 330 \\
\hline bis(2-Chloroethoxy)methane & 330 & $\mathrm{U}$ & 330 & 330 & $\mathrm{U}$ & 330 & 330 & $\mathrm{U}$ & 330 & 330 & $\mathrm{U}$ & 330 \\
\hline bis(2-Chloroethyl)ether & 330 & $\mathrm{U}$ & 330 & 330 & $\mathrm{U}$ & 330 & 330 & $\mathrm{U}$ & 330 & 330 & $\mathrm{U}$ & 330 \\
\hline bis(2-Ethylhexyl)phthalate & 20 & $\mathrm{JB}$ & 330 & 27 & $\mathrm{JB}$ & 330 & 21 & $\mathrm{JB}$ & 330 & 29 & JB & 330 \\
\hline & & & & Attachn & & & 1 & & & Sheet No. & & of 113 \\
\hline & & & & Originat & & J.M. Ca & ron & & & Dat & & 104/07 \\
\hline & & & & Checkes & & T.M. B1 & kley & & & Date & & \\
\hline & & & & Calc. N & & $0100 \mathrm{~B}-\mathrm{C}$ & $\mathrm{A}-\mathrm{V} 0299$ & & & Rev. No. & & 1 \\
\hline
\end{tabular}


Attachment 1. 118-B-1 Verification Sampling Results.

\begin{tabular}{|c|c|c|c|c|c|c|c|c|c|c|c|c|}
\hline \multirow[t]{2}{*}{ Constituents } & \multicolumn{3}{|c|}{\begin{tabular}{|c|} 
J12WL4 \\
BCL D4 \\
Sample Date $7 / 11 / 06$
\end{tabular}} & \multicolumn{3}{|c|}{\begin{tabular}{|c|} 
J12WL5 \\
Duplicate of J12WL4 \\
Sample Date 7/11/06
\end{tabular}} & \multicolumn{3}{|c|}{$\begin{array}{c}\text { J12WL0 } \\
\text { BCL D5 } \\
\text { Sample Date 7/11/06 }\end{array}$} & \multicolumn{3}{|c|}{$\begin{array}{c}\text { J12WL1 } \\
\text { BCL D6 } \\
\text { Sample Date } 7 / 11 / 06\end{array}$} \\
\hline & $\mu \mathrm{g} / \mathrm{kg}$ & $\mathbf{Q}$ & PQL & $\mu \mathrm{g} / \mathrm{kg}$ & $\mathbf{Q}$ & PQL & $\mu \mathrm{g} / \mathrm{kg}$ & $\mathbf{Q}$ & MDL & $\mu \mathrm{g} / \mathrm{kg}$ & $\mathbf{0}$ & PQL \\
\hline \multicolumn{13}{|c|}{ Semivolatile Organic Compounds (continued) } \\
\hline Butylbenzylphthalate & 330 & $\mathrm{U}$ & 330 & 330 & $\mathrm{U}$ & 330 & 330 & $\mathrm{U}$ & 330 & 330 & $\overline{\mathrm{U}}$ & 330 \\
\hline Carbazole & 330 & $\mathrm{U}$ & 330 & 330 & $\mathrm{U}$ & 330 & 330 & $\mathrm{U}$ & 330 & 330 & $\bar{U}$ & 330 \\
\hline Chrysene & 330 & $\mathrm{U}$ & 330 & 330 & $\bar{U}$ & 330 & 330 & $\bar{U}$ & 330 & 330 & $\bar{U}$ & 330 \\
\hline Di-n-butylphthalate & 330 & U & 330 & 45 & $\mathrm{~J}$ & 330 & 330 & $\mathrm{U}$ & 330 & 36 & $\mathrm{~J}$ & 330 \\
\hline Di-n-octylphthalate & 330 & $\mathrm{U}$ & 330 & 330 & U & 330 & 330 & $U$ & 330 & 330 & $\mathrm{U}$ & 330 \\
\hline Dibenz $(\mathrm{a}, \mathrm{h})$ anthracene & 330 & U & 330 & 330 & U & 330 & 330 & $\mathrm{U}$ & 330 & 330 & $\mathrm{U}$ & 330 \\
\hline Dibenzofuran & 330 & $\mathrm{U}$ & 330 & 330 & $\mathrm{U}$ & 330 & 330 & $\mathrm{U}$ & 330 & 330 & $\mathrm{U}$ & 330 \\
\hline Diethylphthalate & 330 & U & 330 & 18 & $\mathrm{~J}$ & 330 & 330 & $\mathrm{U}$ & 330 & 330 & $\mathrm{U}$ & 330 \\
\hline Dimethylphthalate & 330 & U & 330 & 330 & $\mathrm{U}$ & 330 & 330 & U & 330 & 330 & $\mathrm{U}$ & 330 \\
\hline Fluoranthene & 330 & U & 330 & 330 & $\mathrm{U}$ & 330 & 330 & $\mathrm{U}$ & 330 & 330 & $\mathrm{U}$ & 330 \\
\hline Fluorene & 330 & $\mathrm{U}$ & 330 & 330 & $U$ & 330 & 330 & $\mathrm{U}$ & 330 & 330 & $\mathrm{U}$ & 330 \\
\hline Hexachlorobenzene & 330 & $\mathrm{U}$ & 330 & 330 & $\mathrm{U}$ & 330 & 330 & $\bar{U}$ & 330 & 330 & $\mathrm{U}$ & 330 \\
\hline Hexachlorobutadiene & 330 & U & 330 & 330 & $\mathrm{U}$ & 330 & 330 & $\mathrm{U}$ & 330 & 330 & $\mathrm{U}$ & 330 \\
\hline Hexachlorocyclopentadiene & 330 & $\mathrm{U}$ & 330 & 330 & $\mathrm{U}$ & 330 & 330 & $\mathrm{U}$ & 330 & 330 & $\mathrm{U}$ & 330 \\
\hline Hexachloroethane & 330 & U & 330 & 330 & $\mathrm{U}$ & 330 & 330 & $\mathrm{U}$ & 330 & 330 & $\mathrm{U}$ & 330 \\
\hline Indeno( $1,2,3-\mathrm{cd})$ pyrene & 330 & $\mathrm{U}$ & 330 & 330 & $U$ & 330 & 330 & $\mathrm{U}$ & 330 & 330 & $\mathrm{U}$ & 330 \\
\hline Isophorone & 330 & UJ & 330 & 330 & UJ & 330 & 330 & UJ & 330 & 330 & UJ & 330 \\
\hline N-Nitroso-di-n-dipropylamine & 330 & $\mathrm{U}$ & 330 & 330 & $U$ & 330 & 330 & $\mathrm{U}$ & 330 & 330 & $\mathrm{U}$ & 330 \\
\hline N-Nitrosodiphenylamine & 330 & $\mathrm{U}$ & 330 & 330 & U & 330 & 330 & $\mathrm{U}$ & 330 & 330 & $U$ & 330 \\
\hline Naphthalene & 330 & U & 330 & 330 & $\mathrm{U}$ & 330 & 330 & $\mathrm{U}$ & 330 & 330 & $\mathrm{U}$ & 330 \\
\hline Nitrobenzene & 330 & $\mathrm{U}$ & 330 & 330 & $\mathrm{U}$ & 330 & 330 & $\mathrm{U}$ & 330 & 330 & $U$ & 330 \\
\hline Pentachlorophenol & 840 & $\mathrm{U}$ & 840 & 840 & $\mathrm{U}$ & 840 & 840 & $\mathrm{U}$ & 840 & 840 & $\mathrm{U}$ & 840 \\
\hline Phenanthrene & 330 & U & 330 & 330 & $\mathrm{U}$ & 330 & 330 & $\mathrm{U}$ & 330 & 330 & $\mathrm{U}$ & 330 \\
\hline Phenol & 330 & $\mathrm{U}$ & 330 & 34 & $\mathrm{~J}$ & 330 & 330 & $\mathrm{U}$ & 330 & 20 & $\mathrm{~J}$ & 330 \\
\hline Pyrene & 330 & $\bar{U}$ & 330 & 330 & $\mathrm{U}$ & 330 & 330 & $\mathrm{U}$ & 330 & 330 & $\mathrm{U}$ & 330 \\
\hline
\end{tabular}

\begin{tabular}{|c|c|c|c|}
\hline Attachment & 1 & Sheet No. & 90 of 113 \\
\hline Originator & J. M. Capron & Date & $10 / 04 / 07$ \\
\hline Checked & T. M. Blakley & Date & \\
\hline Calc. No. & 0100B-CA-V0299 & Rev. No. & 1 \\
\hline
\end{tabular}


Attachment 1. 118-B-1 Verification Sampling Results.

\begin{tabular}{|c|c|c|c|c|c|c|c|c|c|c|c|c|}
\hline \multirow[t]{2}{*}{ Constituents } & \multicolumn{3}{|c|}{$\begin{array}{c}\text { J12WL4 } \\
\text { BCL D4 } \\
\text { Sample Date 7/11/06 } \\
\end{array}$} & \multicolumn{3}{|c|}{\begin{tabular}{|c|} 
J12WL5 \\
Duplicate of J12WL4 \\
Sample Date 7/11/06 \\
\end{tabular}} & \multicolumn{3}{|c|}{$\begin{array}{c}\text { J12WL0 } \\
\text { BCL D5 } \\
\text { Sample Date } 7 / 11 / 06 \\
\end{array}$} & \multicolumn{3}{|c|}{\begin{tabular}{|c|} 
J12WL1 \\
BCL D6 \\
Sample Date 7/11/06
\end{tabular}} \\
\hline & $\mu \mathrm{g} / \mathrm{kg}$ & $\mathbf{Q}$ & PQL & $\mu \mathrm{g} / \mathrm{kg}$ & $Q$ & PQL & $\mu \mathrm{g} / \mathrm{kg}$ & $Q$ & MDL & $\mu \mathrm{g} / \mathrm{kg}$ & $Q$ & PQL \\
\hline \multicolumn{13}{|c|}{ Volatile Organic Compounds } \\
\hline 1,1,1-Trichloroethane & 5 & $\mathrm{U}$ & 5 & 5 & $\mathrm{U}$ & 5 & 5 & $\mathrm{U}$ & 5 & 5 & $\mathrm{U}$ & 5 \\
\hline 1,1,2,2-Tetrachloroethane & 5 & $\mathrm{U}$ & 5 & 5 & $\mathrm{U}$ & 5 & 5 & $\mathrm{U}$ & 5 & 5 & $\mathrm{U}$ & 5 \\
\hline 1,1,2-Trichloroethane & 5 & $\mathrm{U}$ & 5 & 5 & $\mathrm{U}$ & 5 & 5 & $\mathrm{U}$ & 5 & 5 & $\mathrm{U}$ & 5 \\
\hline 1,1-Dichloroethane & 5 & $\mathrm{U}$ & 5 & 5 & $\mathrm{U}$ & 5 & 5 & $\mathrm{U}$ & 5 & 5 & $\mathrm{U}$ & 5 \\
\hline 1,1-Dichloroethene & 5 & $\mathrm{U}$ & 5 & 5 & $\mathrm{U}$ & 5 & 5 & $\mathrm{U}$ & 5 & 5 & $\mathrm{U}$ & 5 \\
\hline 1,2-Dichloroethane & 5 & $\mathrm{U}$ & 5 & 5 & $\mathrm{U}$ & 5 & 5 & $\mathrm{U}$ & 5 & 5 & $\mathrm{U}$ & 5 \\
\hline 1,2-Dichloroethene (total) & 5 & $\mathrm{U}$ & 5 & 5 & U & 5 & 5 & $\mathrm{U}$ & 5 & 5 & $\mathrm{U}$ & 5 \\
\hline 1,2-Dichloropropane & 5 & $\mathrm{U}$ & 5 & 5 & $\mathrm{U}$ & 5 & 5 & $\mathrm{U}$ & 5 & 5 & $\mathrm{U}$ & 5 \\
\hline 2-Butanone & 10 & $\mathrm{U}$ & 10 & 10 & $\mathrm{U}$ & 10 & 10 & $\mathrm{U}$ & 10 & 10 & $\mathrm{U}$ & 10 \\
\hline 2-Hexanone & 10 & $\mathrm{U}$ & 10 & 10 & $\mathrm{U}$ & 10 & 10 & $\mathrm{U}$ & 10 & 10 & $\mathrm{U}$ & 10 \\
\hline 4-Methyl-2-pentanone & 10 & $\mathrm{U}$ & 10 & 10 & $\mathrm{U}$ & 10 & 10 & $\mathrm{U}$ & 10 & 10 & $\mathrm{U}$ & 10 \\
\hline Acetone & 10 & $\mathrm{U}$ & 10 & 10 & $\mathrm{U}$ & 10 & 10 & $\mathrm{U}$ & 10 & 20 & & 10 \\
\hline Benzene & 5 & $\mathrm{U}$ & 5 & 5 & $\mathrm{U}$ & 5 & 5 & $\mathrm{U}$ & 5 & 5 & $\mathrm{U}$ & 5 \\
\hline Bromodichloromethane & 5 & $\mathrm{U}$ & 5 & 5 & $\mathrm{U}$ & 5 & 5 & $\mathrm{U}$ & 5 & 5 & $\mathrm{U}$ & 5 \\
\hline Bromoform & 5 & $\mathrm{U}$ & 5 & 5 & $\mathrm{U}$ & 5 & 5 & $\mathrm{U}$ & 5 & 5 & $\mathrm{U}$ & 5 \\
\hline Bromomethane & 10 & $\mathrm{U}$ & 10 & 10 & $\mathrm{U}$ & 10 & 10 & $\mathrm{U}$ & 10 & 10 & $\mathrm{U}$ & 10 \\
\hline Carbon disulfide & 5 & $\mathrm{U}$ & 5 & 5 & $\mathrm{U}$ & 5 & 5 & $\mathrm{U}$ & 5 & 5 & $\mathrm{U}$ & 5 \\
\hline Carbon tetrachloride & 6 & & 5 & 5 & $\mathrm{U}$ & 5 & 5 & $\mathrm{U}$ & 5 & 4 & $\mathrm{~J}$ & 5 \\
\hline Chlorobenzene & 5 & $\mathrm{U}$ & 5 & 5 & $\mathrm{U}$ & 5 & 5 & $\mathrm{U}$ & 5 & 5 & $\mathrm{U}$ & 5 \\
\hline Chloroethane & 10 & $\mathrm{U}$ & 10 & 10 & $\mathrm{U}$ & 10 & 10 & $\mathrm{U}$ & 10 & 10 & $\mathrm{U}$ & 10 \\
\hline Chloroform & 5 & $\mathrm{U}$ & 5 & 5 & $\mathrm{U}$ & 5 & 5 & $\mathrm{U}$ & 5 & 5 & $\mathrm{U}$ & 5 \\
\hline Chloromethane & 10 & $\mathrm{U}$ & 10 & 10 & $\mathrm{U}$ & 10 & 10 & $\mathrm{U}$ & 10 & 10 & $\mathrm{U}$ & 10 \\
\hline cis-1,2-Dichloroethylene & 5 & $\mathrm{U}$ & 5 & 5 & $\mathrm{U}$ & 5 & 5 & $U$ & 5 & 5 & $\mathrm{U}$ & 5 \\
\hline cis-1,3-Dichloropropene & 5 & $\mathrm{U}$ & 5 & 5 & $\mathrm{U}$ & 5 & 5 & $\mathrm{U}$ & 5 & 5 & $\mathrm{U}$ & 5 \\
\hline Dibromochloromethane & 5 & $\mathrm{U}$ & 5 & 5 & $\mathrm{U}$ & 5 & 5 & $\mathrm{U}$ & 5 & 5 & $\mathrm{U}$ & 5 \\
\hline Ethylbenzene & 5 & $\mathrm{U}$ & 5 & 5 & $\mathrm{U}$ & 5 & 5 & $\mathrm{U}$ & 5 & 5 & U & 5 \\
\hline Methylene chloride & 12 & BJ & 5 & 11 & $\mathrm{BJ}$ & 5 & 6 & $\mathrm{BJ}$ & 5 & 15 & $\mathrm{BJ}$ & 5 \\
\hline Styrene & 5 & $\mathrm{U}$ & 5 & 5 & $\mathrm{U}$ & 5 & 5 & $\mathrm{U}$ & 5 & 5 & $\mathrm{U}$ & 5 \\
\hline Tetrachloroethene & 5 & $\mathrm{U}$ & 5 & 5 & $\mathrm{U}$ & 5 & 5 & $\mathrm{U}$ & 5 & 5 & $\mathrm{U}$ & 5 \\
\hline Toluene & 5 & $\mathrm{U}$ & 5 & 5 & $\mathrm{U}$ & 5 & 5 & $\mathrm{U}$ & 5 & 5 & $\mathrm{U}$ & 5 \\
\hline trans-1,2-Dichloroethylene & 5 & $\mathrm{U}$ & 5 & 5 & $\mathrm{U}$ & 5 & 5 & $\mathrm{U}$ & 5 & 5 & $\mathrm{U}$ & 5 \\
\hline trans-1,3-Dichloropropene & 5 & $\mathrm{U}$ & 5 & 5 & U & 5 & 5 & $\mathrm{U}$ & 5 & 5 & $\mathrm{U}$ & 5 \\
\hline Trichloroethene & 5 & $\mathrm{U}$ & 5 & 5 & $\mathrm{U}$ & 5 & 5 & $\mathrm{U}$ & 5 & 5 & $\mathrm{U}$ & 5 \\
\hline Vinyl chloride & 10 & $\mathrm{U}$ & 10 & 10 & $\mathrm{U}$ & 10 & 10 & $\mathrm{U}$ & 10 & 10 & $\mathrm{U}$ & 10 \\
\hline Xylenes (total) & 5 & $\mathrm{U}$ & 5 & 5 & $\mathrm{U}$ & 5 & 5 & $\mathrm{U}$ & 5 & 1 & $\mathrm{~J}$ & 5 \\
\hline & & & & Attachm & & & 1 & & & Sheet No. & & of 113 \\
\hline & & & & Originat & & J.M. Ca & oron & & & Date & & 104/07 \\
\hline & & & & Checked & & T. M. B & akley & & & Date & & \\
\hline & & & & Calc. Nc & & 0100B-C & $\mathrm{A}-\mathrm{V} 029$ & & & Rev. No. & & 1 \\
\hline
\end{tabular}


Attachment 1. 118-B-1 Verification Sampling Results.

\begin{tabular}{|c|c|c|c|c|c|c|c|c|c|c|c|c|}
\hline \multirow[t]{2}{*}{ Constituents } & \multicolumn{3}{|c|}{$\begin{array}{c}\text { J12WL4 } \\
\text { BCL D4 } \\
\text { Sample Date } 7 / 11 / 06\end{array}$} & \multicolumn{3}{|c|}{\begin{tabular}{|c|} 
J12WL5 \\
Duplicate of J12WL4 \\
Sample Date $7 / 11 / 06$
\end{tabular}} & \multicolumn{3}{|c|}{$\begin{array}{c}\text { J12WL0 } \\
\text { BCL D5 } \\
\text { Sample Date } 7 / 11 / 06 \\
\end{array}$} & \multicolumn{3}{|c|}{$\begin{array}{c}\text { J12WL1 } \\
\text { BCL D6 } \\
\text { Sample Date } 7 / 11 / 06\end{array}$} \\
\hline & $\mu \mathrm{g} / \mathrm{kg}$ & $\mathbf{Q}$ & PQL & $\mu \mathrm{g} / \mathrm{kg}$ & $\mathbf{Q}$ & PQL & $\mu \mathrm{g} / \mathrm{kg}$ & $Q$ & MDL & $\mu \mathrm{g} / \mathrm{kg}$ & $\mathbf{Q}$ & PQL \\
\hline \multicolumn{13}{|c|}{ Pesticides } \\
\hline Aldrin & 1.3 & UD & 1.3 & 1.3 & UD & 1.3 & 1.3 & UD & 1.3 & 1.3 & UD & 1.3 \\
\hline alpha-BHC & 1.3 & UDJ & 1.3 & 1.3 & UDJ & 1.3 & 1.3 & UDJ & 1.3 & 1.3 & UDJ & 1.3 \\
\hline alpha-Chlordane & 1.3 & UD & 1.3 & 1.3 & UD & 1.3 & 1.3 & UD & 1.3 & 1.3 & UD & 1.3 \\
\hline beta-BHC & 1.3 & UD & 1.3 & 1.3 & UD & 1.3 & 1.3 & UD & 1.3 & 1.3 & UD & 1.3 \\
\hline delta-BHC & 1.3 & UDJ & 1.3 & 1.3 & UDJ & 1.3 & 1.3 & UDJ & 1.3 & 1.3 & UDJ & 1.3 \\
\hline Dichlorodiphenyldichloroethane & 1.3 & UD & 1.3 & 1.3 & UD & 1.3 & 1.3 & UD & 1.3 & 1.3 & UD & 1.3 \\
\hline Dichlorodiphenyldichloroethylene & 1.3 & UDJ & 1.3 & 1.3 & UDJ & 1.3 & 1.3 & UDJ & 1.3 & 1.3 & UDJ & 1.3 \\
\hline Dichlorodiphenyltrichloroethane & 0.50 & JD & 1.3 & 1.3 & UD & 1.3 & 1.3 & UD & 1.3 & 1.3 & UD & 1.3 \\
\hline Dieldrin & 1.3 & UD & 1.3 & 1.3 & UD & 1.3 & 1.3 & UD & 1.3 & 1.3 & UD & 1.3 \\
\hline Endosulfan I & 1.3 & UD & 1.3 & 1.3 & UD & 1.3 & 4.9 & $\mathrm{D}$ & 1.3 & 1.3 & UD & 1.3 \\
\hline Endosulfan II & 1.3 & UD & 1.3 & 1.3 & UD & 1.3 & 1.3 & UD & 1.3 & 1.3 & UD & 1.3 \\
\hline Endosulfan sulfate & 1.3 & UD & 1.3 & 1.3 & UD & 1.3 & 1.3 & UD & 1.3 & 1.3 & UD & 1.3 \\
\hline Endrin & 1.3 & UD & 1.3 & 1.3 & UD & 1.3 & 1.3 & UD & 1.3 & 1.3 & UD & 1.3 \\
\hline Endrin aldehyde & 1.3 & UD & 1.3 & 1.3 & UD & 1.3 & 1.3 & UD & 1.3 & 1.3 & UD & 1.3 \\
\hline Endrin ketone & 1.3 & UD & 1.3 & 1.3 & UD & 1.3 & 1.3 & UD & 1.3 & 1.3 & UD & 1.3 \\
\hline gamma-BHC (Lindane) & 1.3 & UDJ & 1.3 & 1.3 & UDJ & 1.3 & 1.3 & UDJ & 1.3 & 1.3 & UDJ & 1.3 \\
\hline gamma-Chlordane & 1.3 & UD & 1.3 & 1.3 & UD & 1.3 & 1.3 & UD & 1.3 & 1.3 & UD & 1.3 \\
\hline Heptachlor & 1.3 & UD & 1.3 & 1.3 & UD & 1.3 & 0.43 & JDI & 1.3 & 1.3 & UD & 1.3 \\
\hline Heptachlor epoxide & 1.3 & UD & 1.3 & 1.3 & UD & 1.3 & 1.3 & UD & 1.3 & 1.3 & UD & 1.3 \\
\hline Methoxychlor & 1.7 & $\mathrm{D}$ & 1.3 & 1.3 & UD & 1.3 & 1.3 & UD & 1.3 & 5.3 & $\mathrm{D}$ & 1.3 \\
\hline Toxaphene & 13 & UD & 13 & 13 & UD & 13 & 13 & UD & 13 & 13 & UD & 13 \\
\hline \multicolumn{13}{|c|}{ Herbicides } \\
\hline $2,4,5-\mathrm{T}$ & 17 & $\mathrm{U}$ & 17 & 17 & $\mathrm{U}$ & 17 & 17 & $\mathrm{U}$ & 17 & 17 & $\mathrm{U}$ & 17 \\
\hline $2,4-D$ & 33 & $\mathrm{U}$ & 33 & 33 & $\mathrm{U}$ & 33 & 33 & $\mathrm{U}$ & 33 & 33 & $\mathrm{U}$ & 33 \\
\hline $2,4,5-\mathrm{TP}$ & 17 & $\mathrm{U}$ & 17 & 17 & $\mathrm{U}$ & 17 & 17 & U & 17 & 17 & $\mathrm{U}$ & 17 \\
\hline Dinoseb & 17 & $\bar{U}$ & 17 & 17 & $\mathrm{U}$ & 17 & 17 & $\mathrm{U}$ & 17 & 17 & $\mathrm{U}$ & 17 \\
\hline $2,4-D B$ & 17 & U & 17 & 9.8 & $\mathrm{~J}$ & 17 & 17 & $\mathrm{U}$ & 17 & 9.4 & $\mathrm{~J}$ & 17 \\
\hline Dalapon & 28 & $\mathrm{~J}$ & 33 & 33 & $\mathrm{U}$ & 33 & 33 & $\mathrm{U}$ & 33 & 33 & $\mathrm{U}$ & 33 \\
\hline Dicamba & 13 & $J$ & 33 & 10 & $\mathrm{~J}$ & 33 & 10 & $\mathrm{~J}$ & 33 & 33 & $\mathrm{U}$ & 33 \\
\hline Dichloroprop & 33 & $\mathrm{U}$ & 33 & 33 & $\mathrm{U}$ & 33 & 8.4 & $\mathrm{~J}$ & 33 & 33 & $\mathrm{U}$ & 33 \\
\hline Pentachlorophenol & 17 & $\mathrm{U}$ & 17 & 17 & $\mathrm{U}$ & 17 & 17 & $\mathrm{U}$ & 17 & 17 & $\mathrm{U}$ & 17 \\
\hline Picloram & 17 & UR & 17 & 17 & UR & 17 & 17 & UR & 17 & 17 & UR & 17 \\
\hline & & & & \multirow{4}{*}{\multicolumn{2}{|c|}{$\begin{array}{l}\text { Attachment } \\
\text { Originator } \\
\text { Checked } \\
\text { Calc. No. }\end{array}$}} & \multicolumn{3}{|c|}{1} & \multirow{4}{*}{\multicolumn{2}{|c|}{$\begin{array}{r}\text { Sheet No. } \\
\text { Date } \\
\text { Date } \\
\text { Rev. No. }\end{array}$}} & \multicolumn{2}{|c|}{92 of 113} \\
\hline & & & & & & \multicolumn{3}{|c|}{ J.M. Capron } & & & \multicolumn{2}{|c|}{$10 / 04 / 07$} \\
\hline & & & & & & \multicolumn{3}{|c|}{$\frac{\text { T. M. Blakley }}{0100 \text { B-CA-V02 }}$} & & & & \\
\hline & & & & & & $0100 \mathrm{~B}$ & $\mathrm{~A}-\mathrm{V} 02$ & & & & & 1 \\
\hline
\end{tabular}


Attachment 1. 118-B-1 Verification Sampling Results.

\begin{tabular}{|c|c|c|c|c|c|c|c|c|c|c|c|c|}
\hline \multirow[t]{2}{*}{ Constituents } & \multicolumn{3}{|c|}{\begin{tabular}{|c|} 
J13HY3 \\
SPA A1 \\
Sample Date 10/12/06
\end{tabular}} & \multicolumn{3}{|c|}{$\begin{array}{c}\text { J13HY4 } \\
\text { SPA A2 } \\
\text { Sample Date 10/12/06 }\end{array}$} & \multicolumn{3}{|c|}{\begin{tabular}{|c|} 
J13HY5 \\
SPA A3 \\
Sample Date 10/12/06 \\
\end{tabular}} & \multicolumn{3}{|c|}{\begin{tabular}{|c|} 
J13HY6 \\
SPA A4 \\
Sample Date 10/12/06 \\
\end{tabular}} \\
\hline & $\mu \mathrm{g} / \mathrm{kg}$ & $Q$ & PQL & $\mu \mathrm{g} / \mathrm{kg}$ & $Q$ & PQL & $\mu \mathrm{g} / \mathrm{kg}$ & $Q$ & PQL & $\mu \mathrm{g} / \mathrm{kg}$ & $Q$ & $\mathrm{PQL}$ \\
\hline \multicolumn{13}{|c|}{ Polychlorinated Biphenyls } \\
\hline Aroclor-1016 & 14 & U & 14 & 13 & $\mathrm{U}$ & 13 & 13 & $\mathrm{U}$ & 13 & 13 & $\mathrm{U}$ & 13 \\
\hline Aroclor-1221 & 14 & $\mathrm{U}$ & 14 & 13 & $\mathrm{U}$ & 13 & 13 & $\mathrm{U}$ & 13 & 13 & $\mathrm{U}$ & 13 \\
\hline Aroclor- 1232 & 14 & $\mathrm{U}$ & 14 & 13 & $\mathrm{U}$ & 13 & 13 & $\mathrm{U}$ & 13 & 13 & $\mathrm{U}$ & 13 \\
\hline Aroclor- 1242 & 14 & $\mathrm{U}$ & 14 & 13 & U & 13 & 13 & $U$ & 13 & 13 & $\mathrm{U}$ & 13 \\
\hline Aroclor-1248 & 14 & $\mathrm{U}$ & 14 & 13 & $\mathrm{U}$ & 13 & 13 & $\mathrm{U}$ & 13 & 13 & $\mathrm{U}$ & 13 \\
\hline Aroclor-1254 & 14 & $\mathrm{U}$ & 14 & 13 & $\mathrm{U}$ & 13 & 13 & $\mathrm{U}$ & 13 & 4.1 & $\mathrm{~J}$ & 13 \\
\hline Aroclor -1260 & 14 & $\mathrm{U}$ & 14 & 13 & $\mathrm{U}$ & 13 & 13 & $\mathrm{U}$ & 13 & 13 & $\mathrm{U}$ & 13 \\
\hline \multicolumn{13}{|c|}{ Semivolatile Organic Compounds } \\
\hline 1,2,4-Trichlorobenzene & 330 & UJ & 330 & 330 & UJ & 330 & 330 & $\overline{U J}$ & 330 & 330 & UJ & 330 \\
\hline 1,2-Dichlorobenzene & 330 & $\mathrm{U}$ & 330 & 330 & $\mathrm{U}$ & 330 & 330 & $\mathrm{U}$ & 330 & 330 & U & 330 \\
\hline 1,3-Dichlorobenzene & 330 & $\mathrm{U}$ & 330 & 330 & $\mathrm{U}$ & 330 & 330 & $\mathrm{U}$ & 330 & 330 & $\mathrm{U}$ & 330 \\
\hline 1,4-Dichlorobenzene & 330 & $\mathrm{U}$ & 330 & 330 & U & 330 & 330 & $\mathrm{U}$ & 330 & 330 & $\mathrm{U}$ & 330 \\
\hline 2,4,5-Trichlorophenol & 830 & $\mathrm{U}$ & 830 & 830 & $U$ & 830 & 830 & $\mathrm{U}$ & 830 & 830 & $\mathrm{U}$ & 830 \\
\hline 2,4,6-Trichlorophenol & 330 & $\mathrm{U}$ & 330 & 330 & $\mathrm{U}$ & 330 & 330 & $\mathrm{U}$ & 330 & 330 & $\mathrm{U}$ & 330 \\
\hline 2,4-Dichlorophenol & 330 & $\mathrm{U}$ & 330 & 330 & $\mathrm{U}$ & 330 & 330 & $\mathrm{U}$ & 330 & 330 & $\mathrm{U}$ & 330 \\
\hline 2,4-Dimethylphenol & 330 & UJ & 330 & 330 & UJ & 330 & 330 & UJ & 330 & 330 & UJ & 330 \\
\hline 2,4-Dinitrophenol & 830 & $\mathrm{U}$ & 830 & 830 & $\mathrm{U}$ & 830 & 830 & U & 830 & 830 & $\mathrm{U}$ & 830 \\
\hline 2,4-Dinitrotoluene & 330 & $\mathrm{U}$ & 330 & 330 & $\mathrm{U}$ & 330 & 330 & $\mathrm{U}$ & 330 & 330 & $\mathrm{U}$ & 330 \\
\hline 2,6-Dinitrotoluene & 330 & $\mathrm{U}$ & 330 & 330 & $\mathrm{U}$ & 330 & 330 & $\mathrm{U}$ & 330 & 330 & $\mathrm{U}$ & 330 \\
\hline 2-Chloronaphthalene & 330 & $\mathrm{U}$ & 330 & 330 & $\mathrm{U}$ & 330 & 330 & $\mathrm{U}$ & 330 & 330 & $\mathrm{U}$ & 330 \\
\hline 2-Chlorophenol & 330 & $\mathrm{U}$ & 330 & 330 & $\mathrm{U}$ & 330 & 330 & $\mathrm{U}$ & 330 & 330 & $\mathrm{U}$ & 330 \\
\hline 2-Methylnaphthalene & 330 & UJ & 330 & 330 & UJ & 330 & 330 & UJ & 330 & 330 & UJ & 330 \\
\hline 2-Methylphenol (cresol, o-) & 330 & $\mathrm{U}$ & 330 & 330 & $\mathrm{U}$ & 330 & 330 & $\mathrm{U}$ & 330 & 330 & $\mathrm{U}$ & 330 \\
\hline 2-Nitroaniline & 830 & $\mathrm{U}$ & 830 & 830 & $\mathrm{U}$ & 830 & 830 & $\mathrm{U}$ & 830 & 830 & $\mathrm{U}$ & 830 \\
\hline 2-Nitrophenol & 330 & $\mathrm{U}$ & 330 & 330 & $\mathrm{U}$ & 330 & 330 & $\mathrm{U}$ & 330 & 330 & $\mathrm{U}$ & 330 \\
\hline 3,3'-Dichlorobenzidine & 330 & $\mathrm{U}$ & 330 & 330 & $\mathrm{U}$ & 330 & 330 & $\mathrm{U}$ & 330 & 330 & $\mathrm{U}$ & 330 \\
\hline 3-Nitroaniline & 830 & $\mathrm{U}$ & 830 & 830 & $\mathrm{U}$ & 830 & 830 & $\mathrm{U}$ & 830 & 830 & $\mathrm{U}$ & 830 \\
\hline 4,6-Dinitro-2-methylphenol & 830 & $\mathrm{U}$ & 830 & 830 & $\mathrm{U}$ & 830 & 830 & $\mathrm{U}$ & 830 & 830 & $\mathrm{U}$ & 830 \\
\hline 4-Bromophenyl-phenylether & 330 & $\mathrm{U}$ & 330 & 330 & $\mathrm{U}$ & 330 & 330 & $\mathrm{U}$ & 330 & 330 & $\mathrm{U}$ & 330 \\
\hline 4-Chloro-3-methylphenol & 330 & $\mathrm{U}$ & 330 & 330 & $\mathrm{U}$ & 330 & 330 & $\mathrm{U}$ & 330 & 330 & $\mathrm{U}$ & 330 \\
\hline 4-Chloroaniline & 330 & $\mathrm{U}$ & 330 & 330 & $\mathrm{U}$ & 330 & 330 & $\mathrm{U}$ & 330 & 330 & $U$ & 330 \\
\hline 4-Chlorophenyl-phenylether & 330 & $\mathrm{U}$ & 330 & 330 & $\mathrm{U}$ & 330 & 330 & $\mathrm{U}$ & 330 & 330 & $\mathrm{U}$ & 330 \\
\hline 4-Methylphenol (p-cresol) & 330 & $\mathrm{U}$ & 330 & 330 & $\bar{U}$ & 330 & 330 & $\bar{U}$ & 330 & 330 & $\mathrm{U}$ & 330 \\
\hline 4-Nitroaniline & 830 & $\mathrm{U}$ & 830 & 830 & $\mathrm{U}$ & 830 & 830 & $\mathrm{U}$ & 830 & 830 & $\mathrm{U}$ & 830 \\
\hline 4-Nitrophenol & 830 & $\mathrm{U}$ & 830 & 830 & $\mathrm{U}$ & 830 & 830 & $\mathrm{U}$ & 830 & 830 & $\mathrm{U}$ & 830 \\
\hline Acenaphthene & 330 & $\mathrm{U}$ & 330 & 330 & $\mathrm{U}$ & 330 & 330 & $\mathrm{U}$ & 330 & 330 & $\mathrm{U}$ & 330 \\
\hline Acenaphthylene & 330 & $\mathrm{U}$ & 330 & 330 & $\mathrm{U}$ & 330 & 330 & $\mathrm{U}$ & 330 & 330 & $\mathrm{U}$ & 330 \\
\hline Anthracene & 330 & $\mathrm{U}$ & 330 & 330 & $\mathrm{U}$ & 330 & 330 & $\mathrm{U}$ & 330 & 330 & $\mathrm{U}$ & 330 \\
\hline Benzo(a)anthracene & 330 & $\mathrm{U}$ & 330 & 330 & $\mathrm{U}$ & 330 & 330 & $\mathrm{U}$ & 330 & 330 & $\mathrm{U}$ & 330 \\
\hline Benzo(a)pyrene & 330 & $\mathrm{U}$ & 330 & 330 & $\mathrm{U}$ & 330 & 330 & U & 330 & 330 & $\mathrm{U}$ & 330 \\
\hline Benzo(b)fluoranthene & 330 & $\mathrm{U}$ & 330 & 330 & $\mathrm{U}$ & 330 & 330 & $\mathrm{U}$ & 330 & 330 & $\mathrm{U}$ & $330^{\circ}$ \\
\hline Benzo $(\mathrm{g}, \mathrm{h}, \mathrm{i})$ perylene & 330 & $\mathrm{U}$ & 330 & 330 & $\mathrm{U}$ & 330 & 330 & $\mathrm{U}$ & 330 & 330 & $\mathrm{U}$ & 330 \\
\hline Benzo(k)fluoranthene & 330 & $\mathrm{U}$ & 330 & 330 & $\mathrm{U}$ & 330 & 330 & $\mathrm{U}$ & 330 & 330 & $\mathrm{U}$ & 330 \\
\hline bis(2-Chloro-1-methylethyl)ether & 330 & $\mathrm{U}$ & 330 & 330 & $\mathrm{U}$ & 330 & 330 & $\mathrm{U}$ & 330 & 330 & $\mathrm{U}$ & 330 \\
\hline bis(2-Chloroethoxy)methane & 330 & $\mathrm{U}$ & 330 & 330 & U & 330 & 330 & $\mathrm{U}$ & 330 & 330 & $\mathrm{U}$ & 330 \\
\hline bis(2-Chloroethyl)ether & 330 & $\mathrm{U}$ & 330 & 330 & U & 330 & 330 & $\mathrm{U}$ & 330 & 330 & $\mathrm{U}$ & 330 \\
\hline bis(2-Ethylhexyl)phthalate & 37 & $\mathrm{JB}$ & 330 & 31 & JB & 330 & 43 & $\mathrm{JB}$ & 330 & 31 & $\mathrm{JB}$ & 330 \\
\hline
\end{tabular}

Attachment

Originator

Checked

Calc. No.

\begin{tabular}{l}
\hline J.M. Capron \\
\hline T. M. Blakley \\
\hline 0100B-CA-V0299 \\
\hline
\end{tabular}

Sheet No. 93 of 113

Date $10 / 04 / 07$

Date

Rev. No. 1 
Attachment 1. 118-B-1 Verification Sampling Results.

\begin{tabular}{|c|c|c|c|c|c|c|c|c|c|c|c|c|}
\hline \multirow[t]{2}{*}{ Constituents } & \multicolumn{3}{|c|}{$\begin{array}{c}\text { J13HY3 } \\
\text { SPA A1 } \\
\text { Sample Date } 10 / 12 / 06\end{array}$} & \multicolumn{3}{|c|}{\begin{tabular}{|c|}
$\mathrm{J13HY4}$ \\
SPA A2 \\
Sample Date 10/12/06
\end{tabular}} & \multicolumn{3}{|c|}{$\begin{array}{c}\text { J13HY5 } \\
\text { SPA A3 } \\
\text { Sample Date 10/12/06 }\end{array}$} & \multicolumn{3}{|c|}{\begin{tabular}{|c|} 
J13HY6 \\
SPA A4 \\
Sample Date 10/12/06
\end{tabular}} \\
\hline & $\mu \mathrm{g} / \mathrm{kg}$ & $Q$ & PQL & $\mu \mathrm{g} / \mathrm{kg}$ & $Q$ & PQL & $\mu \mathrm{g} / \mathrm{kg}$ & $\mathrm{Q}$ & PQL & $\mu \mathrm{g} / \mathrm{kg}$ & $\mathbf{Q}$ & PQL \\
\hline \multicolumn{13}{|c|}{ Semivolatile Organic Compounds (continued) } \\
\hline Butylbenzylphthalate & 330 & $\mathrm{U}$ & 330 & 330 & $\mathrm{U}$ & 330 & 330 & $\bar{U}$ & 330 & 330 & $\bar{U}$ & 330 \\
\hline Carbazole & 330 & $\bar{U}$ & 330 & 330 & $\bar{U}$ & 330 & 330 & $\mathrm{U}$ & 330 & 330 & $\bar{U}$ & 330 \\
\hline Chrysene & 330 & $\mathrm{U}$ & 330 & 330 & $\mathrm{U}$ & 330 & 330 & $\mathrm{U}$ & 330 & 330 & $\mathrm{U}$ & 330 \\
\hline Di-n-butylphthalate & 330 & $\mathrm{U}$ & 330 & 40 & $\mathrm{~J}$ & 330 & 330 & $\mathrm{U}$ & 330 & 330 & $\bar{U}$ & 330 \\
\hline Di-n-octylphthalate & 330 & $\mathrm{U}$ & 330 & 330 & $\mathrm{U}$ & 330 & 330 & $U$ & 330 & 330 & $\bar{U}$ & 330 \\
\hline Dibenz $(\mathrm{a}, \mathrm{h})$ anthracene & 330 & U & 330 & 330 & $U$ & 330 & 330 & $\mathrm{U}$ & 330 & 330 & $\mathrm{U}$ & 330 \\
\hline Dibenzofuran & 330 & U & 330 & 330 & $\mathrm{U}$ & 330 & 330 & $\mathrm{U}$ & 330 & 330 & $\mathrm{U}$ & 330 \\
\hline \begin{tabular}{|l} 
Diethylphthalate \\
\end{tabular} & 330 & $U$ & 330 & 330 & $\mathrm{U}$ & 330 & 330 & $\mathrm{U}$ & 330 & 330 & $\mathrm{U}$ & 330 \\
\hline Dimethylphthalate & 330 & $U$ & 330 & 330 & $\mathrm{U}$ & 330 & 330 & $\mathrm{U}$ & 330 & 330 & $\mathrm{U}$ & 330 \\
\hline Fluoranthene & 330 & $\mathrm{U}$ & 330 & 330 & $\mathrm{U}$ & 330 & 330 & $\mathrm{U}$ & 330 & 330 & $\mathrm{U}$ & 330 \\
\hline Fluorene & 330 & $\mathrm{U}$ & 330 & 330 & $\mathrm{U}$ & 330 & 330 & $\mathrm{U}$ & 330 & 330 & $\mathrm{U}$ & 330 \\
\hline Hexachlorobenzene & 330 & $\mathrm{U}$ & 330 & 330 & $\mathrm{U}$ & 330 & 330 & $U$ & 330 & 330 & $\mathrm{U}$ & 330 \\
\hline Hexachlorobutadiene & 330 & $\mathrm{U}$ & 330 & 330 & $\mathrm{U}$ & 330 & 330 & $\mathrm{U}$ & 330 & 330 & $\mathrm{U}$ & 330 \\
\hline Hexachlorocyclopentadiene & 330 & $\mathrm{U}$ & 330 & 330 & $\mathrm{U}$ & 330 & 330 & $\mathrm{U}$ & 330 & 330 & $\mathrm{U}$ & 330 \\
\hline Hexachloroethane & 330 & $\mathrm{U}$ & 330 & 330 & $\mathrm{U}$ & 330 & 330 & U & 330 & 330 & $\mathrm{U}$ & 330 \\
\hline Indeno(1,2,3-cd)pyrene & 330 & $\mathrm{U}$ & 330 & 330 & $\mathrm{U}$ & 330 & 330 & $U$ & 330 & 330 & $\mathrm{U}$ & 330 \\
\hline Isophorone & 330 & UJ & 330 & 330 & UJ & 330 & 330 & UJ & 330 & 330 & UJ & 330 \\
\hline N-Nitroso-di-n-dipropylamine & 330 & $\mathrm{U}$ & 330 & 330 & $\mathrm{U}$ & 330 & 330 & $U$ & 330 & 330 & $\mathrm{U}$ & 330 \\
\hline N-Nitrosodiphenylamine & 330 & $\mathrm{U}$ & 330 & 330 & $\mathrm{U}$ & 330 & 330 & U & 330 & 330 & $\mathrm{U}$ & 330 \\
\hline Naphthalene & 330 & $\mathrm{U}$ & 330 & 330 & $\mathrm{U}$ & 330 & 330 & 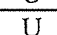 & 330 & 330 & $\mathrm{U}$ & 330 \\
\hline \begin{tabular}{|l} 
Nitrobenzene \\
\end{tabular} & 330 & UJ & 330 & 330 & UJ & 330 & 330 & UJ & 330 & 330 & UJ & 330 \\
\hline Pentachlorophenol & 830 & $\bar{U}$ & 830 & 830 & $\mathrm{U}$ & 830 & 830 & $\mathrm{U}$ & 830 & 830 & $\mathrm{U}$ & 830 \\
\hline Phenanthrene & 330 & $\bar{U}$ & 330 & 330 & $\mathrm{U}$ & 330 & 330 & $\bar{U}$ & 330 & 330 & $\mathrm{U}$ & 330 \\
\hline Phenol & 330 & $\mathrm{U}$ & 330 & 330 & $\mathrm{U}$ & 330 & 330 & $\mathrm{U}$ & 330 & 330 & $\mathrm{U}$ & 330 \\
\hline Pyrene & 330 & $\mathrm{U}$ & 330 & 330 & $\mathrm{U}$ & 330 & 330 & $\mathrm{U}$ & 330 & 330 & $\mathrm{U}$ & 330 \\
\hline
\end{tabular}

\begin{tabular}{ll} 
Attachment & $\frac{1}{\text { Originator }}$ \\
Checked & $\frac{1}{\text { J.M. Capron }}$ \\
Calc. No. & O100B-Clakley \\
\cline { 2 - 2 }
\end{tabular}

Sheet No. $\quad 94$ of 113

Date 10/04/07

Date

Rev. No. 1 
Attachment 1. 118-B-1 Verification Sampling Results.

\begin{tabular}{|c|c|c|c|c|c|c|c|c|c|c|c|c|}
\hline \multirow[t]{2}{*}{ Constituents } & \multicolumn{3}{|c|}{$\begin{array}{c}\text { J13HY3 } \\
\text { SPA A1 } \\
\text { Sample Date } 10 / 12 / 06\end{array}$} & \multicolumn{3}{|c|}{\begin{tabular}{|c|} 
J13HY4 \\
SPA A2 \\
Sample Date 10/12/06 \\
\end{tabular}} & \multicolumn{3}{|c|}{$\begin{array}{c}\text { J13HY5 } \\
\text { SPA A3 } \\
\text { Sample Date 10/12/06 } \\
\end{array}$} & \multicolumn{3}{|c|}{\begin{tabular}{|c|} 
J13HY6 \\
SPA A4 \\
Sample Date 10/12/06 \\
\end{tabular}} \\
\hline & $\mu \mathrm{g} / \mathrm{kg}$ & $\mathbf{Q}$ & PQL & $\mu \mathrm{g} / \mathrm{kg}$ & $Q$ & PQL & $\mu \mathrm{g} / \mathrm{kg}$ & $\mathbf{Q}$ & PQL & $\mu \mathrm{g} / \mathrm{kg}$ & Q & PQL \\
\hline \multicolumn{13}{|c|}{ Volatile Organic Compounds } \\
\hline 1,1,1-Trichloroethane & 5 & UJ & 5 & 5 & $\mathrm{UJ}$ & 5 & 5 & UJ & 5 & 5 & UJ & 5 \\
\hline 1,1,2,2-Tetrachloroethane & 5 & UJ & 5 & 5 & UJ & 5 & 5 & $\overline{\mathrm{UJ}}$ & 5 & 5 & UJ & 5 \\
\hline 1,1,2-Trichloroethane & 5 & UJ & 5 & 5 & UJ & 5 & 5 & UJ & 5 & 5 & UJ & 5 \\
\hline 1,1-Dichloroethane & 5 & UJ & 5 & 5 & UJ & 5 & 5 & $\overline{\mathrm{UJ}}$ & 5 & 5 & UJ & 5 \\
\hline 1,1-Dichloroethene & 5 & UJ & 5 & 5 & UJ & 5 & 5 & UJ & 5 & 5 & UJ & 5 \\
\hline 1,2-Dichloroethane & 5 & UJ & 5 & 5 & UJ & 5 & 5 & UJ & 5 & 5 & UJ & 5 \\
\hline 1,2-Dichloroethene (total) & 5 & UJ & 5 & 5 & UJ & 5 & 5 & $\mathrm{UJ}$ & 5 & 5 & $\mathrm{UJ}$ & 5 \\
\hline 1,2-Dichloropropane & 5 & UJ & 5 & 5 & UJ & 5 & 5 & UJ & 5 & 5 & UJ & 5 \\
\hline 2-Butanone & 10 & UJ & 10 & 10 & UJ & 10 & 10 & UJ & 10 & 10 & UJ & 10 \\
\hline 2-Hexanone & 10 & UJ & 10 & 10 & UJ & 10 & 10 & UJ & 10 & 10 & UJ & 10 \\
\hline 4-Methyl-2-pentanone & 10 & UJ & 10 & 10 & UJ & 10 & 10 & UJ & 10 & 10 & UJ & 10 \\
\hline Acetone & 10 & UJ & 10 & 10 & UJ & 10 & 10 & $\mathrm{UJ}$ & 10 & 10 & UJ & 10 \\
\hline Benzene & 5 & UJ & 5 & 5 & UJ & 5 & 5 & $\mathrm{UJ}$ & 5 & 5 & UJ & 5 \\
\hline Bromodichloromethane & 5 & UJ & 5 & 5 & UJ & 5 & 5 & UJ & 5 & 5 & UJ & 5 \\
\hline Bromoform & 5 & UJ & 5 & 5 & UJ & 5 & 5 & UJ & 5 & 5 & $\mathrm{UJ}$ & 5 \\
\hline Bromomethane & 10 & UJ & 10 & 10 & UJ & 10 & 10 & $\mathrm{UJ}$ & 10 & 10 & $\mathrm{UJ}$ & 10 \\
\hline Carbon disulfide & 5 & UJ & 5 & 5 & UJ & 5 & 5 & $\mathrm{UJ}$ & 5 & 5 & UJ & 5 \\
\hline Carbon tetrachloride & 5 & UJ & 5 & 5 & UJ & 5 & 5 & UJ & 5 & 5 & UJ & 5 \\
\hline Chlorobenzene & 5 & UJ & 5 & 5 & UJ & 5 & 5 & UJ & 5 & 5 & UJ & 5 \\
\hline Chloroethane & 10 & UJ & 10 & 10 & UI & 10 & 10 & UJ & 10 & 10 & UJ & 10 \\
\hline Chloroform & 5 & $\mathrm{UJ}$ & 5 & 5 & UJ & 5 & 5 & UJ & 5 & 5 & UJ & 5 \\
\hline Chloromethane & 10 & UJ & 10 & 10 & UJ & 10 & 10 & UJ & 10 & 10 & UJ & 10 \\
\hline cis-1,2-Dichloroethylene & 5 & UJ & 5 & 5 & UJ & 5 & 5 & UJ & 5 & 5 & UJ & 5 \\
\hline cis-1,3-Dichloropropene & 5 & UJ & 5 & 5 & UJ & 5 & 5 & UJ & 5 & 5 & UJ & 5 \\
\hline Dibromochloromethane & 5 & UJ & 5 & 5 & UJ & 5 & 5 & UJ & 5 & 5 & UJ & 5 \\
\hline Ethylbenzene & 5 & UJ & 5 & 5 & UJ & 5 & 5 & UJ & 5 & 5 & UJ & 5 \\
\hline Methylene chloride & 6 & BJ & 5 & 14 & $\mathrm{BJ}$ & 5 & 13 & $\mathrm{BJ}$ & 5 & 12 & $\mathrm{BJ}$ & 5 \\
\hline Styrene & 5 & UJ & 5 & 5 & UJ & 5 & 5 & UJ & 5 & 5 & UJ & 5 \\
\hline Tetrachloroethene & 5 & UJ & 5 & 5 & UJ & 5 & 5 & UJ & 5 & 5 & UJ & 5 \\
\hline Toluene & 5 & UJ & 5 & 5 & UJ & 5 & 5 & UJ & 5 & 5 & UJ & 5 \\
\hline trans-1,2-Dichloroethylene & 5 & UJ & 5 & 5 & UJ & 5 & 5 & UJ & 5 & 5 & UJ & 5 \\
\hline trans-1,3-Dichloropropene & 5 & UJ & 5 & 5 & UJ & 5 & 5 & UJ & 5 & 5 & UJ & 5 \\
\hline Trichloroethene & 5 & UJ & 5 & 5 & UI & 5 & 5 & UJ & 5 & 5 & UJ & 5 \\
\hline Vinyl chloride & 10 & UJ & 10 & 10 & UJ & 10 & 10 & UJ & 10 & 10 & UJ & 10 \\
\hline Xylenes (total) & 5 & UJ & 5 & 5 & UJ & 5 & 5 & UJ & 5 & 5 & UJ & 5 \\
\hline & & & & Attachm & & & 1 & & & Sheet No. & 95 & of 113 \\
\hline & & & & Originat & & J.M.Ca & pron & & & Date & & $04 / 07$ \\
\hline & & & & Checked & & T.M. B & lakley & & & Date & & \\
\hline & & & & Calc. No & & $0100 \mathrm{~B}-$ & $\mathrm{A}-\mathrm{V} 029$ & & & Rev. No & & 1 \\
\hline
\end{tabular}


Attachment 1. 118-B-1 Verification Sampling Results.

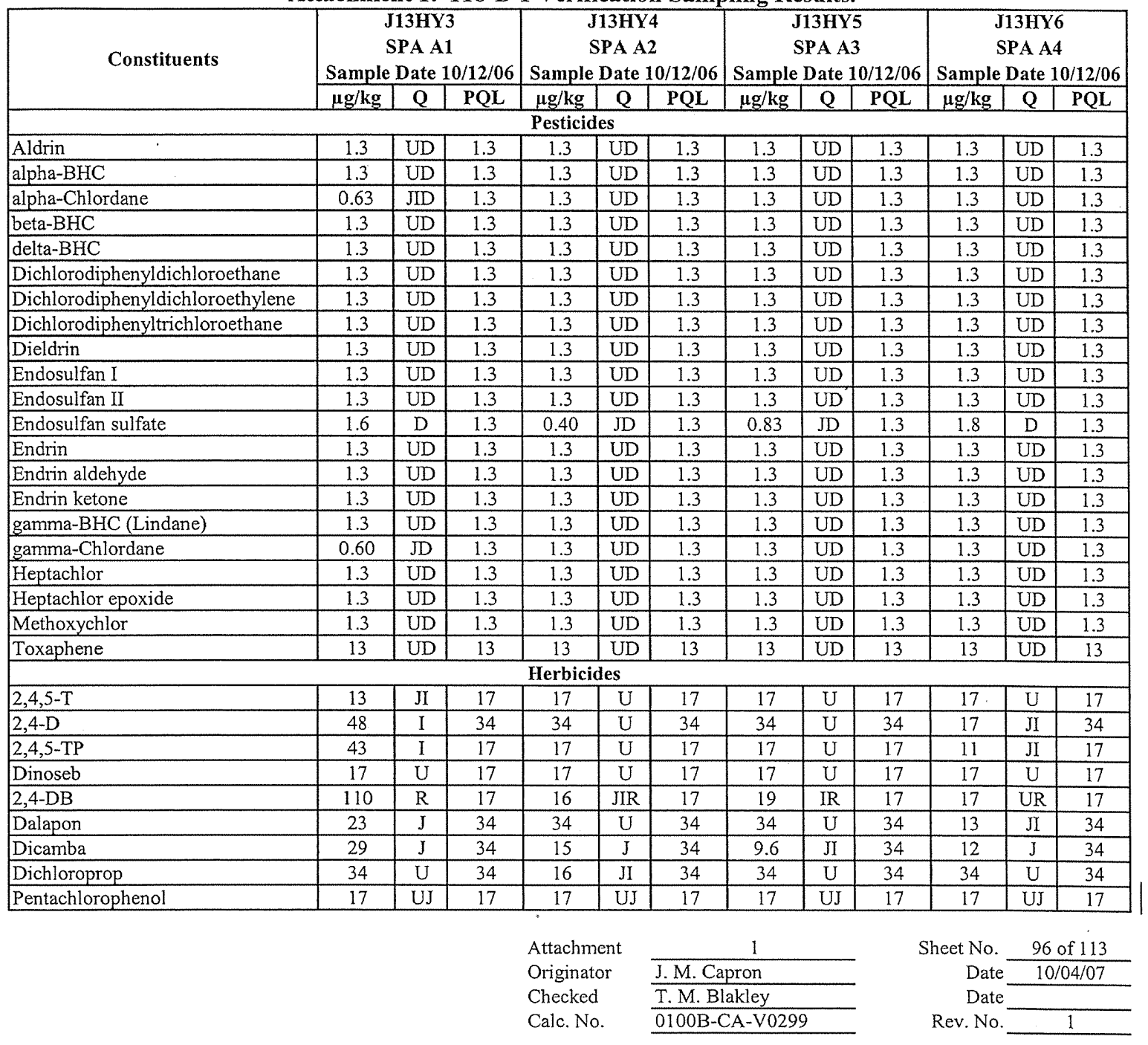


Attachment 1. 118-B-1 Verification Sampling Results.

\begin{tabular}{|c|c|c|c|c|c|c|c|c|c|c|c|c|}
\hline \multirow[t]{2}{*}{ Constituents } & \multicolumn{3}{|c|}{\begin{tabular}{|c|} 
J13HY7 \\
SPA B5 \\
Sample Date 10/12/06 \\
\end{tabular}} & \multicolumn{3}{|c|}{\begin{tabular}{|c|} 
J13J09 \\
Duplicate of J13HY7 \\
Sample Date 10/12/06 \\
\end{tabular}} & \multicolumn{3}{|c|}{\begin{tabular}{|c|} 
J13J10 \\
Split of J13HY7 \\
Sample Date $10 / 12 / 06$ \\
\end{tabular}} & \multicolumn{3}{|c|}{\begin{tabular}{|c|} 
J13HY8 \\
SPA B6 \\
Sample Date 10/17/06
\end{tabular}} \\
\hline & $\mu \mathrm{g} / \mathrm{kg}$ & $\mathrm{Q}$ & PQL & $\mu \mathrm{g} / \mathrm{kg}$ & $Q$ & PQL & $\mu \mathrm{g} / \mathrm{kg}$ & $\mathbf{Q}$ & PQL & $\mu \mathrm{g} / \mathrm{kg}$ & $\mathbf{Q}$ & PQL \\
\hline \multicolumn{13}{|c|}{ Polychlorinated Biphenyls } \\
\hline Aroclor-1016 & 13 & $\mathrm{U}$ & 13 & 13 & $\mathrm{U}$ & 13 & 17 & $\mathrm{U}$ & 17 & 14 & $\mathrm{U}$ & 14 \\
\hline Aroclor-1221 & 13 & $\mathrm{U}$ & 13 & 13 & $U$ & 13 & 17 & $\mathrm{U}$ & 17 & 14 & $\mathrm{U}$ & 14 \\
\hline Aroclor-1232 & 13 & $\mathrm{U}$ & 13 & 13 & $\mathrm{U}$ & 13 & 17 & $\mathrm{U}$ & 17 & 14 & $\mathrm{U}$ & 14 \\
\hline Aroclor-1242 & 13 & $\mathrm{U}$ & 13 & 13 & $\mathrm{U}$ & 13 & 17 & $\mathrm{U}$ & 17 & 14 & $\mathrm{U}$ & 14 \\
\hline Aroclor-1248 & 13 & $\mathrm{U}$ & 13 & 13 & $\mathrm{U}$ & 13 & 17 & $\mathrm{U}$ & 17 & 14 & $\mathrm{U}$ & 14 \\
\hline Aroclor-1254 & 13 & $\mathrm{U}$ & 13 & 13 & $\mathrm{U}$ & 13 & 17 & $\mathrm{U}$ & 17 & 14 & $\mathrm{U}$ & 14 \\
\hline Aroclor- 1260 & 13 & $\mathrm{U}$ & 13 & 13 & $\mathrm{U}$ & 13 & 17 & $\mathrm{U}$ & 17 & 14 & $\mathrm{U}$ & 14 \\
\hline \multicolumn{13}{|c|}{ Semivolatile Organic Compounds } \\
\hline 1,2,4-Trichlorobenzene & 330 & UJ & 330 & 330 & UJ & 330 & 330 & $\mathrm{U}$ & 330 & 340 & $\mathrm{U}$ & 340 \\
\hline 1,2-Dichlorobenzene & 330 & $\mathrm{U}$ & 330 & 330 & $\mathrm{U}$ & 330 & 330 & $\mathrm{U}$ & 330 & 340 & $\mathrm{U}$ & 340 \\
\hline 1,3-Dichlorobenzene & 330 & $\mathrm{U}$ & 330 & 330 & $\mathrm{U}$ & 330 & 330 & U & 330 & 340 & $\mathrm{U}$ & 340 \\
\hline 1,4-Dichlorobenzene & 330 & $\mathrm{U}$ & 330 & 330 & $\mathrm{U}$ & 330 & 330 & $\mathrm{U}$ & 330 & 340 & $\mathrm{U}$ & 340 \\
\hline 2,4,5-Trichlorophenol & 830 & $\mathrm{U}$ & 830 & 830 & $\mathrm{U}$ & 830 & 330 & $\mathrm{U}$ & 330 & 850 & $\mathrm{U}$ & 850 \\
\hline 2,4,6-Trichlorophenol & 330 & $\mathrm{U}$ & 330 & 330 & $\mathrm{U}$ & 330 & 330 & $\mathrm{U}$ & 330 & 340 & $\mathrm{U}$ & 340 \\
\hline 2,4-Dichlorophenol & 330 & $\mathrm{U}$ & 330 & 330 & $\mathrm{U}$ & 330 & 330 & $\mathrm{U}$ & 330 & 340 & $\mathrm{U}$ & 340 \\
\hline 2,4-Dimethylphenol & 330 & UJ & 330 & 330 & UJ & 330 & 330 & $\mathrm{U}$ & 330 & 340 & $\mathrm{U}$ & 340 \\
\hline 2,4-Dinitrophenol & 830 & $\mathrm{U}$ & 830 & 830 & $\mathrm{U}$ & 830 & 1600 & $\mathrm{U}$ & 1600 & 850 & $\mathrm{U}$ & 850 \\
\hline 2,4-Dinitrotoluene & 330 & $\mathrm{U}$ & 330 & 330 & $\mathrm{U}$ & 330 & 330 & $\mathrm{U}$ & 330 & 340 & $\mathrm{U}$ & 340 \\
\hline 2,6-Dinitrotoluene & 330 & $\mathrm{U}$ & 330 & 330 & $\mathrm{U}$ & 330 & 330 & $\mathrm{U}$ & 330 & 340 & $\mathrm{U}$ & 340 \\
\hline 2-Chloronaphthalene & 330 & $\mathrm{U}$ & 330 & 330 & $\mathrm{U}$ & 330 & 330 & $\mathrm{U}$ & 330 & 340 & $\mathrm{U}$ & 340 \\
\hline 2-Chlorophenol & 330 & $\mathrm{U}$ & 330 & 330 & $\mathrm{U}$ & 330 & 330 & U & 330 & 340 & $\mathrm{U}$ & 340 \\
\hline 2-Methylnaphthalene & 330 & UJ & 330 & 330 & UJ & 330 & 330 & $\mathrm{U}$ & 330 & 340 & $\mathrm{U}$ & 340 \\
\hline 2-Methylphenol (cresol, o-) & 330 & $\mathrm{U}$ & 330 & 330 & $\mathrm{U}$ & 330 & 330 & $\mathrm{U}$ & 330 & 340 & $\mathrm{U}$ & 340 \\
\hline 2-Nitroaniline & 830 & $\mathrm{U}$ & 830 & 830 & $\mathrm{U}$ & 830 & 1600 & $\mathrm{U}$ & 1600 & 850 & $\mathrm{U}$ & 850 \\
\hline 2-Nitrophenol & 330 & $\mathrm{U}$ & 330 & 330 & $\mathrm{U}$ & 330 & 330 & $\mathrm{U}$ & 330 & 340 & $\mathrm{U}$ & 340 \\
\hline 3,3'-Dichlorobenzidine & 330 & $\mathrm{U}$ & 330 & 330 & $\mathrm{U}$ & 330 & 1600 & $\mathrm{U}$ & 1600 & 340 & $\mathrm{U}$ & 340 \\
\hline 3-Nitroaniline & 830 & $\mathrm{U}$ & 830 & 830 & $\mathrm{U}$ & 830 & 1600 & $\mathrm{U}$ & 1600 & 850 & $U$ & 850 \\
\hline 4,6-Dinitro-2-methylphenol & 830 & $\mathrm{U}$ & 830 & 830 & $\mathrm{U}$ & 830 & 1600 & $\mathrm{U}$ & 1600 & 850 & $\mathrm{U}$ & 850 \\
\hline 4-Bromophenyl-phenylether & 330 & $\mathrm{U}$ & 330 & 330 & $\mathrm{U}$ & 330 & 330 & $\mathrm{U}$ & 330 & 340 & $\mathrm{U}$ & 340 \\
\hline 4-Chloro-3-methylphenol & 330 & $\mathrm{U}$ & 330 & 330 & $\mathrm{U}$ & 330 & 330 & $\mathrm{U}$ & 330 & 340 & $\mathrm{U}$ & 340 \\
\hline 4-Chloroaniline & 330 & $\mathrm{U}$ & 330 & 330 & $\mathrm{U}$ & 330 & 330 & $\mathrm{U}$ & 330 & 340 & $\mathrm{U}$ & 340 \\
\hline 4-Chlorophenyl-phenylether & 330 & $\mathrm{U}$ & 330 & 330 & $\mathrm{U}$ & 330 & 330 & $\mathrm{U}$ & 330 & 340 & $\mathrm{U}$ & 340 \\
\hline 4-Methylphenol (p-cresol) & 330 & $\mathrm{U}$ & 330 & 330 & $\mathrm{U}$ & 330 & 670 & $\mathrm{U}$ & 670 & 340 & $\mathrm{U}$ & 340 \\
\hline 4-Nitroaniline & 830 & $\mathrm{U}$ & 830 & 830 & $\mathrm{U}$ & 830 & 1600 & $\mathrm{U}$ & 1600 & 850 & $\mathrm{U}$ & 850 \\
\hline 4-Nitrophenol & 830 & $U$ & 830 & 830 & $\mathrm{U}$ & 830 & 1600 & $\mathrm{U}$ & 1600 & 850 & $\mathrm{U}$ & 850 \\
\hline Acenaphthene & 330 & $\mathrm{U}$ & 330 & 330 & $\mathrm{U}$ & 330 & 330 & $\mathrm{U}$ & 330 & 340 & $\mathrm{U}$ & 340 \\
\hline Acenaphthylene & 330 & $\mathrm{U}$ & 330 & 330 & $\mathrm{U}$ & 330 & 330 & $\mathrm{U}$ & 330 & 340 & $\mathrm{U}$ & 340 \\
\hline Anthracene & 330 & $\mathrm{U}$ & 330 & 330 & $\mathrm{U}$ & 330 & 330 & $\mathrm{U}$ & 330 & 340 & $\mathrm{U}$ & 340 \\
\hline Benzo(a)anthracene & 330 & $\mathrm{U}$ & 330 & 330 & $\mathrm{U}$ & 330 & 330 & $\mathrm{U}$ & 330 & 340 & $\mathrm{U}$ & 340 \\
\hline Benzo(a)pyrene & 330 & U & 330 & 330 & $\mathrm{U}$ & 330 & 330 & $\mathrm{U}$ & 330 & 340 & $\mathrm{U}$ & 340 \\
\hline Benzo(b)fluoranthene & 330 & $\mathrm{U}$ & 330 & 330 & $\mathrm{U}$ & 330 & 330 & $\mathrm{U}$ & 330 & 340 & $\mathrm{U}$ & 340 \\
\hline Benzo(g,h,i)perylene & 330 & $\mathrm{U}$ & 330 & 330 & $\mathrm{U}$ & 330 & 330 & $\mathrm{U}$ & 330 & 340 & $\mathrm{U}$ & 340 \\
\hline Benzo(k)fluoranthene & 330 & $\mathrm{U}$ & 330 & 330 & $\mathrm{U}$ & 330 & 330 & $\mathrm{U}$ & 330 & 340 & $\mathrm{U}$ & 340 \\
\hline bis(2-Chloro-1-methylethyl)ether & 330 & $\mathrm{U}$ & 330 & 330 & $\mathrm{U}$ & 330 & 330 & $\mathrm{U}$ & 330 & 340 & $\mathrm{U}$ & 340 \\
\hline bis(2-Chloroethoxy)methane & 330 & $\mathrm{U}$ & 330 & 330 & $\mathrm{U}$ & 330 & 330 & U & 330 & 340 & $\mathrm{U}$ & 340 \\
\hline bis(2-Chloroethyl)ether & 330 & $\mathrm{U}$ & 330 & 330 & $\mathrm{U}$ & 330 & 330 & $\mathrm{U}$ & 330 & 340 & $\mathrm{U}$ & 340 \\
\hline bis(2-Ethylhexyl)phthalate & 24 & $\mathrm{JB}$ & 330 & 27 & $\mathrm{JB}$ & 330 & 330 & $\mathrm{U}$ & 330 & 340 & $\mathrm{U}$ & 340 \\
\hline
\end{tabular}

Attachment Originator

Checked

Calc. No.

$\frac{\frac{1}{\text { J. M. Capron }}}{\frac{\text { T. M. Blakley }}{\text { 0100B-CA-V0299 }}}$

Sheet No. 97 of 113

Date 10/04/07

Date

Rev. No. $\frac{1}{1}$ 
Attachment 1. 118-B-1 Verification Sampling Results.

\begin{tabular}{|c|c|c|c|c|c|c|c|c|c|c|c|c|}
\hline \multirow[t]{2}{*}{ Constituents } & \multicolumn{3}{|c|}{$\begin{array}{c}\text { J13HY7 } \\
\text { SPA B5 } \\
\text { Sample Date 10/12/06 }\end{array}$} & \multicolumn{3}{|c|}{$\begin{array}{c}\text { J13J09 } \\
\text { Duplicate of J13HY7 } \\
\text { Sample Date } 10 / 12 / 06\end{array}$} & \multicolumn{3}{|c|}{$\begin{array}{c}\mathrm{J13J10} \\
\text { Split of J13HY7 } \\
\text { Sample Date 10/12/06 }\end{array}$} & \multicolumn{3}{|c|}{$\begin{array}{c}\text { J13HY8 } \\
\text { SPA B6 } \\
\text { Sample Date 10/17/06 }\end{array}$} \\
\hline & $\mu \mathrm{g} / \mathrm{kg}$ & $\mathbf{Q}$ & PQL & $\mu \mathrm{g} / \mathrm{kg}$ & $\mathrm{Q}$ & PQL & $\mu \mathrm{g} / \mathrm{kg}$ & $\mathbf{Q}$ & PQL & $\mu \mathrm{g} / \mathrm{kg}$ & $\mathbf{Q}$ & PQL \\
\hline \multicolumn{13}{|c|}{ Semivolatile Organic Compounds (continued) } \\
\hline Butylbenzylphthalate & 330 & $\mathrm{U}$ & 330 & 330 & $\mathrm{U}$ & 330 & 330 & $U$ & 330 & 340 & $\mathrm{U}$ & 340 \\
\hline Carbazole & 330 & $\mathrm{U}$ & 330 & 330 & $\mathrm{U}$ & 330 & 330 & $\mathrm{U}$ & 330 & 340 & $\mathrm{U}$ & 340 \\
\hline Chrysene & 330 & $\mathrm{U}$ & 330 & 330 & $\mathrm{U}$ & 330 & 330 & $\mathrm{U}$ & 330 & 340 & $\mathrm{U}$ & 340 \\
\hline Di-n-butylphthalate & 330 & $\mathrm{U}$ & 330 & 30 & $\mathrm{~J}$ & 330 & 330 & $\mathrm{U}$ & 330 & 28 & JB & 340 \\
\hline Di-n-octylphthalate & 330 & $\mathrm{U}$ & 330 & 330 & $\mathrm{U}$ & 330 & 330 & $\mathrm{U}$ & 330 & 340 & $U$ & 340 \\
\hline Dibenz $(\mathrm{a}, \mathrm{h})$ anthracene & 330 & $\mathrm{U}$ & 330 & 330 & $\mathrm{U}$ & 330 & 330 & $\mathrm{U}$ & 330 & 340 & $\mathrm{U}$ & 340 \\
\hline Dibenzofuran & 330 & $\mathrm{U}$ & 330 & 330 & $\mathrm{U}$ & 330 & 330 & $\mathrm{U}$ & 330 & 340 & $\mathrm{U}$ & 340 \\
\hline Diethylphthalate & 330 & $\mathrm{U}$ & 330 & 330 & $\mathrm{U}$ & 330 & 330 & $\mathrm{U}$ & 330 & 340 & $U$ & 340 \\
\hline Dimethylphthalate & 330 & $\mathrm{U}$ & 330 & 330 & $U$ & 330 & 330 & $\mathrm{U}$ & 330 & 340 & $\mathrm{U}$ & 340 \\
\hline Fluoranthene & 330 & $\mathrm{U}$ & 330 & 330 & $\mathrm{U}$ & 330 & 330 & $\mathrm{U}$ & 330 & 340 & $\mathrm{U}$ & 340 \\
\hline Fluorene & 330 & $U$ & 330 & 330 & $\mathrm{U}$ & 330 & 330 & $\mathrm{U}$ & 330 & 340 & $\mathrm{U}$ & 340 \\
\hline Hexachlorobenzene & 330 & $\mathrm{U}$ & 330 & 330 & $\mathrm{U}$ & 330 & 330 & $\mathrm{U}$ & 330 & 340 & $U$ & 340 \\
\hline Hexachlorobutadiene & 330 & $U$ & 330 & 330 & $\mathrm{U}$ & 330 & 330 & $\mathrm{U}$ & 330 & 340 & $\mathrm{U}$ & 340 \\
\hline Hexachlorocyclopentadiene & 330 & $\mathrm{U}$ & 330 & 330 & $\mathrm{U}$ & 330 & 1600 & $\mathrm{U}$ & 1600 & 340 & $\mathrm{U}$ & 340 \\
\hline Hexachloroethane & 330 & $\mathrm{U}$ & 330 & 330 & $\mathrm{U}$ & 330 & 330 & $\mathrm{U}$ & 330 & 340 & $\mathrm{U}$ & 340 \\
\hline Indeno(1,2,3-cd)pyrene & 330 & $\mathrm{U}$ & 330 & 330 & $\mathrm{U}$ & 330 & 330 & $\mathrm{U}$ & 330 & 340 & $\mathrm{U}$ & 340 \\
\hline Isophorone & 330 & UJ & 330 & 330 & $\mathrm{UJ}$ & 330 & 330 & $\mathrm{U}$ & 330 & 340 & $\mathrm{U}$ & 340 \\
\hline N-Nitroso-di-n-dipropylannine & 330 & $\mathrm{U}$ & 330 & 330 & $\mathrm{U}$ & 330 & 330 & $\mathrm{U}$ & 330 & 340 & $\mathrm{U}$ & 340 \\
\hline N-Nitrosodiphenylamine & 330 & $\mathrm{U}$ & 330 & 330 & $\mathrm{U}$ & 330 & 330 & $\mathrm{U}$ & 330 & 340 & $U$ & 340 \\
\hline Naphthalene & 330 & $\mathrm{U}$ & 330 & 330 & $\mathrm{U}$ & 330 & 330 & $\mathrm{U}$ & 330 & 340 & $\mathrm{U}$ & 340 \\
\hline Nitrobenzene & 330 & UJ & 330 & 330 & $\mathrm{UJ}$ & 330 & 330 & $\mathrm{U}$ & 330 & 340 & $\mathrm{U}$ & 340 \\
\hline Pentachlorophenol & 830 & $\mathrm{U}$ & 830 & 830 & $\mathrm{U}$ & 830 & 1600 & $\mathrm{U}$ & 1600 & 850 & $\mathrm{U}$ & 850 \\
\hline Phenanthrene & 330 & $\mathrm{U}$ & 330 & 330 & $\mathrm{U}$ & 330 & 330 & $\mathrm{U}$ & 330 & 340 & $\mathrm{U}$ & 340 \\
\hline Phenol & 330 & U & 330 & 330 & $\mathrm{U}$ & 330 & 330 & $\mathrm{U}$ & 330 & 340 & $\mathrm{U}$ & 340 \\
\hline Pyrene & 330 & $\mathrm{U}$ & 330 & 330 & $\mathrm{U}$ & 330 & 330 & U & 330 & 340 & $\mathrm{U}$ & 340 \\
\hline & & & & \multirow{2}{*}{\multicolumn{2}{|c|}{$\begin{array}{l}\text { Attachment } \\
\text { Originator }\end{array}$}} & \multicolumn{3}{|c|}{1} & \multicolumn{2}{|c|}{ Sheet No. } & \multicolumn{2}{|c|}{98 of 113} \\
\hline & & & & & & \multicolumn{3}{|c|}{ J. M. Capron } & & Date & \multicolumn{2}{|c|}{$10 / 04 / 07$} \\
\hline & & & & \multicolumn{2}{|c|}{ Checked } & \multicolumn{3}{|c|}{ T. M. Blakley } & & \multirow{2}{*}{$\begin{array}{l}\text { Date } \\
\text { Rev. No. }\end{array}$} & & \\
\hline & & & & Calc. N & & $0100 \mathrm{~B}-\mathrm{C}$ & $\mathrm{A}-\mathrm{V} 029$ & & & & & 1 \\
\hline
\end{tabular}


Attachment 1. 118-B-1 Verification Sampling Results.

\begin{tabular}{|c|c|c|c|c|c|c|c|c|c|c|c|c|}
\hline \multirow[t]{2}{*}{ Constituents } & \multicolumn{3}{|c|}{$\begin{array}{c}\text { J13HY7 } \\
\text { SPA B5 } \\
\text { Sample Date 10/12/06 }\end{array}$} & \multicolumn{3}{|c|}{$\begin{array}{c}\text { J13J09 } \\
\text { Duplicate of J13HY7 } \\
\text { Sample Date 10/12/06 } \\
\end{array}$} & \multicolumn{3}{|c|}{$\begin{array}{c}\mathbf{J 1 3 J 1 0} \\
\text { Split of J13HY7 } \\
\text { Sample Date 10/12/06 }\end{array}$} & \multicolumn{3}{|c|}{\begin{tabular}{|c|} 
J13HY8 \\
SPA B6 \\
Sample Date $10 / 17 / 06$ \\
\end{tabular}} \\
\hline & $\mu \mathrm{g} / \mathrm{kg}$ & $Q$ & $\mathrm{PQL}$ & $\mu \mathrm{g} / \mathrm{kg}$ & $\mathbf{Q}$ & PQL & $\mu \mathrm{g} / \mathrm{kg}$ & $Q$ & PQL & $\mu \mathrm{g} / \mathrm{kg}$ & $Q$ & PQL \\
\hline \multicolumn{13}{|c|}{ Volatile Organic Compounds } \\
\hline 1,1,1-Trichloroethane & 5 & UJ & 5 & 5 & UJ & 5 & 5.0 & $\mathrm{U}$ & 5.0 & 5 & $\mathrm{UJ}$ & 5 \\
\hline 1,1,2,2-Tetrachloroethane & 5 & UJ & 5 & 5 & UJ & 5 & 5.0 & U & 5.0 & 5 & $\mathrm{UJ}$ & 5 \\
\hline 1,1,2-Trichloroethane & 5 & UJ & 5 & 5 & UJ & 5 & 5.0 & $U$ & 5.0 & 5 & UJ & 5 \\
\hline 1,1-Dichloroethane & 5 & UJ & 5 & 5 & UJ & 5 & 5.0 & $\mathrm{U}$ & 5.0 & 5 & UJ & $\frac{5}{5}$ \\
\hline 1,1-Dichloroethene & 5 & $\mathrm{UJ}$ & 5 & 5 & UJ & 5 & 5.0 & $\mathrm{U}$ & 5.0 & 5 & UJ & 5 \\
\hline 1,2-Dichloroethane & 5 & $\mathrm{UJ}$ & 5 & 5 & UJ & 5 & 5.0 & $\mathrm{U}$ & 5.0 & 5 & UJ & 5 \\
\hline 1,2-Dichloroethene (total) & 5 & $\mathrm{UJ}$ & 5 & 5 & $\mathrm{UJ}$ & 5 & 10 & $\mathrm{U}$ & 10 & 5 & UJ & 5 \\
\hline 1,2-Dichloropropane & 5 & UJ & 5 & 5 & $\mathrm{UJ}$ & 5 & 5.0 & $\mathrm{U}$ & 5.0 & 5 & UJ & 5 \\
\hline 2-Butanone & 10 & UJ & 10 & 10 & $\mathrm{UJ}$ & 10 & 10 & $\mathrm{U}$ & 10 & 10 & UJ & 10 \\
\hline 2-Hexanone & 10 & UJ & 10 & 10 & UJ & 10 & 20 & $\mathrm{U}$ & 20 & 10 & UJ & 10 \\
\hline 4-Methyl-2-pentanone & 10 & UJ & 10 & 10 & UJ & 10 & 10 & $\mathrm{U}$ & 10 & 10 & UJ & 10 \\
\hline Acetone & 10 & UJ & 10 & 10 & UJ & 10 & 10 & JBN & 20 & 10 & UJ & 10 \\
\hline Benzene & 5 & UJ & 5 & 5 & UJ & 5 & 5.0 & $\mathrm{U}$ & 5.0 & 5 & UJ & 5 \\
\hline Bromodichloromethane & 5 & UJ & 5 & 5 & UJ & 5 & 5.0 & $\mathrm{U}$ & 5.0 & 5 & UJ & 5 \\
\hline Bromoform & 5 & UJ & 5 & 5 & UJ & 5 & 5.0 & $\mathrm{U}$ & 5.0 & 5 & UJ & 5 \\
\hline Bromomethane & 10 & UJ & 10 & 10 & UJ & 10 & 10 & UNJ & 10 & 10 & UJ & 10 \\
\hline Carbon disulfide & 5 & UJ & 5 & 5 & UJ & 5 & 5.0 & $\mathrm{U}$ & 5.0 & 5 & UJ & 5 \\
\hline Carbon tetrachloride & 5 & UJ & 5 & 5 & UJ & 5 & 5.0 & $\mathrm{U}$ & 5.0 & 5 & UJ & 5 \\
\hline Chlorobenzene & 5 & UJ & 5 & 5 & UJ & 5 & 5.0 & $\mathrm{U}$ & 5.0 & 5 & UJ & 5 \\
\hline Chloroethane & 10 & UJ & 10 & 10 & UJ & 10 & 10 & $\mathrm{U}$ & 10 & 10 & UJ & 10 \\
\hline Chloroform & 5 & $\mathrm{UJ}$ & 5 & 5 & UJ & 5 & 5.0 & $\mathrm{U}$ & 5.0 & 5 & UJ & 5 \\
\hline Chloromethane & 10 & UJ & 10 & 10 & UJ & 10 & 10 & $\mathrm{U}$ & 10 & 10 & UJ & 10 \\
\hline cis-1,2-Dichloroethylene & 5 & UJ & 5 & 5 & UJ & 5 & & & & 5 & UJ & 5 \\
\hline cis-1,3-Dichloropropene & 5 & UJ & 5 & 5 & UJ & 5 & 5.0 & $\mathrm{U}$ & 5.0 & 5 & UJ & 5 \\
\hline Dibromochloromethane & 5 & UJ & 5 & 5 & UJ & 5 & 5.0 & $\mathrm{U}$ & 5.0 & 5 & UJ & 5 \\
\hline Ethylbenzene & 5 & UJ & 5 & 5 & UJ & 5 & 5.0 & $\mathrm{U}$ & 5.0 & 5 & UJ & 5 \\
\hline Methylene chloride & 10 & $\mathrm{BJ}$ & 5 & 12 & $\mathrm{BJ}$ & 5 & 5.0 & $\mathrm{U}$ & 5.0 & 18 & $\mathrm{BJ}$ & 5 \\
\hline Styrene & 5 & UJ & 5 & 5 & UJ & 5 & 5.0 & $\mathrm{U}$ & 5.0 & 5 & UJ & 5 \\
\hline Tetrachloroethene & 5 & UJ & 5 & 5 & UJ & 5 & 5.0 & $\mathrm{U}$ & 5.0 & 5 & $\mathrm{UJ}$ & 5 \\
\hline Toluene & 5 & UJ & 5 & 5 & UJ & 5 & 0.36 & $\mathrm{JB}$ & 5.0 & 5 & $\mathrm{UJ}$ & 5 \\
\hline trans-1,2-Dichloroethylene & 5 & UJ & 5 & 5 & $\mathrm{UJ}$ & 5 & & & & 5 & UJ & 5 \\
\hline trans-1,3-Dichloropropene & 5 & UJ & 5 & 5 & UJ & 5 & 5.0 & $\mathrm{U}$ & 5.0 & 5 & UJ & 5 \\
\hline Trichloroethene & 5 & UJ & 5 & 5 & UJ & 5 & 1.0 & $\mathrm{~J}$ & 5.0 & 5 & $\mathrm{UJ}$ & 5 \\
\hline Vinyl chloride & 10 & UJ & 10 & 10 & $\mathrm{UJ}$ & 10 & 5.0 & $\mathrm{U}$ & 5.0 & 10 & $\mathrm{UJ}$ & 10 \\
\hline Xylenes (total) & 5 & UJ & 5 & 5 & $\mathrm{UJ}$ & 5 & 10 & $\mathrm{U}$ & 10 & 5 & UJ & 5 \\
\hline & & & & Attachm & & & 1 & & & Sheet No. & & of 113 \\
\hline & & & & Origina & & J.M.Ca & pron & & & Date & & $04 / 07$ \\
\hline & & & & Checke & & T.M. Bl & akley & & & Date & & \\
\hline & & & & Calc. $N$ & & $0100 \mathrm{~B}-\mathrm{C}$ & A-V029 & & & Rev. No. & & 1 \\
\hline
\end{tabular}


Attachment 1. 118-B-1 Verification Sampling Results.

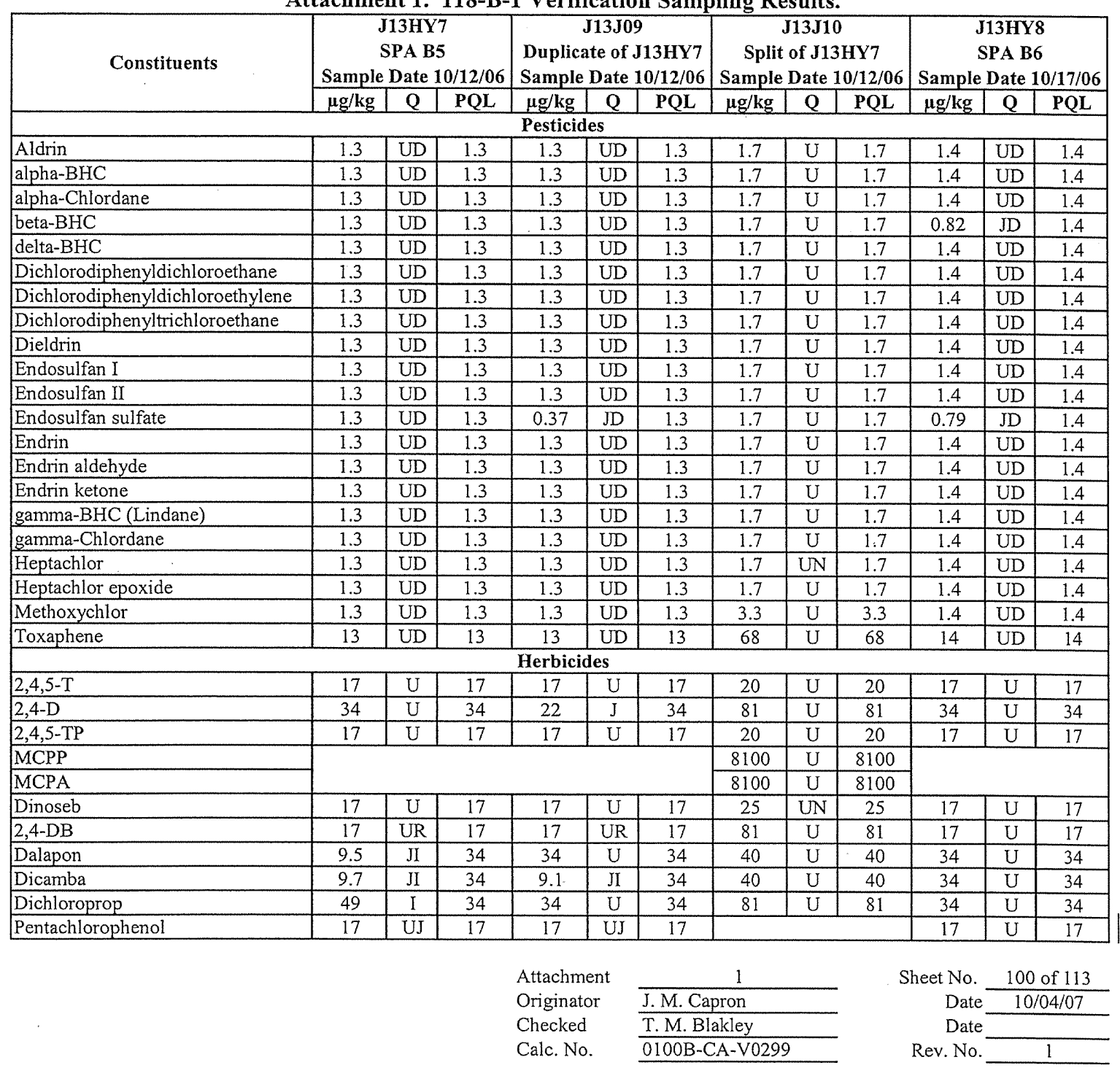


Attachment 1. 118-B-1 Verification Sampling Results.

\begin{tabular}{|c|c|c|c|c|c|c|c|c|c|c|c|c|}
\hline \multirow[t]{2}{*}{ Constituents } & \multicolumn{3}{|c|}{\begin{tabular}{|c|} 
J13HY9 \\
SPA B7 \\
Sample Date $10 / 17 / 06$
\end{tabular}} & \multicolumn{3}{|c|}{\begin{tabular}{|c|} 
J13J00 \\
SPA B8 \\
Sample Date $10 / 17 / 06$
\end{tabular}} & \multicolumn{3}{|c|}{\begin{tabular}{|c|} 
J13J01 \\
SPA C9 \\
Sample Date 10/17/06 \\
\end{tabular}} & \multicolumn{3}{|c|}{$\begin{array}{c}\text { J13J02 } \\
\text { SPA C10 } \\
\text { Sample Date } 10 / 18 / 06 \\
\end{array}$} \\
\hline & $\mu \mathrm{g} / \mathrm{kg}$ & $Q$ & PQL & $\mu \mathrm{g} / \mathrm{kg}$ & Q & PQL & $\mu \mathrm{g} / \mathrm{kg}$ & $\mathrm{Q}$ & PQL & $\mu \mathrm{g} / \mathrm{kg}$ & $\mathrm{Q}$ & PQL \\
\hline \multicolumn{13}{|c|}{ Polychlorinated Biphenyls } \\
\hline Aroclor-1016 & 14 & $\mathrm{U}$ & 14 & 14 & $\mathrm{U}$ & 14 & 14 & $\mathrm{U}$ & 14 & 14 & $\mathrm{U}$ & 14 \\
\hline Aroclor-1221 & 14 & $\mathrm{U}$ & 14 & 14 & $\mathrm{U}$ & 14 & 14 & $\mathrm{U}$ & 14 & 14 & $\mathrm{U}$ & 14 \\
\hline Aroclor-1232 & 14. & $\mathrm{U}$ & 14 & 14 & $\mathrm{U}$ & 14 & 14 & $\mathrm{U}$ & 14 & 14 & $U$ & 14 \\
\hline Aroclor-1242 & 14 & $\mathrm{U}$ & 14 & 14 & $\mathrm{U}$ & 14 & 14 & $\mathrm{U}$ & 14 & 14 & $\mathrm{U}$ & 14 \\
\hline Aroclor-1248 & 14 & $\mathrm{U}$ & 14 & 14 & $\mathrm{U}$ & 14 & 14 & $\mathrm{U}$ & 14 & 14 & $\mathrm{U}$ & 14 \\
\hline Aroclor-1254 & 14 & $\mathrm{U}$ & 14 & 14 & $\mathrm{U}$ & 14 & 14 & $\mathrm{U}$ & 14 & 14 & $\mathrm{U}$ & 14 \\
\hline Aroclor-1260 & 14 & $\mathrm{U}$ & 14 & 14 & $\mathrm{U}$ & 14 & 14 & $\mathrm{U}$ & 14 & 14 & $\mathrm{U}$ & 14 \\
\hline \multicolumn{13}{|c|}{ Semivolatile Organic Compounds } \\
\hline 1,2,4-Trichlorobenzene & 340 & U & 340 & 340 & $\mathrm{U}$ & 340 & 360 & $\mathrm{U}$ & 360 & 350 & $\mathrm{U}$ & 350 \\
\hline 1,2-Dichlorobenzene & 340 & $\mathrm{U}$ & 340 & 340 & $\mathrm{U}$ & 340 & 360 & U & 360 & 350 & $\mathrm{U}$ & 350 \\
\hline 1,3-Dichlorobenzene & 340 & $\mathrm{U}$ & 340 & 340 & U & 340 & 360 & $\mathrm{U}$ & 360 & 350 & $\mathrm{U}$ & 350 \\
\hline 1,4-Dichlorobenzene & 340 & $\mathrm{U}$ & 340 & 340 & $\mathrm{U}$ & 340 & 360 & $\mathrm{U}$ & 360 & 350 & $\mathrm{U}$ & 350 \\
\hline 2,4,5-Trichlorophenol & 860 & $\mathrm{U}$ & 860 & 850 & $\mathrm{U}$ & 850 & 890 & $\bar{U}$ & 890 & 860 & $\mathrm{U}$ & 860 \\
\hline 2,4,6-Trichlorophenol & 340 & $\mathrm{U}$ & 340 & 340 & $\mathrm{U}$ & 340 & 360 & $\mathrm{U}$ & 360 & 350 & $\mathrm{U}$ & 350 \\
\hline 2,4-Dichlorophenol & 340 & $\mathrm{U}$ & 340 & 340 & U & 340 & 360 & $\mathrm{U}$ & 360 & 350 & $\mathrm{U}$ & 350 \\
\hline 2,4-Dimethylphenol & 340 & $\mathrm{U}$ & 340 & 340 & $\mathrm{U}$ & 340 & 360 & $\mathrm{U}$ & 360 & 350 & $\mathrm{U}$ & 350 \\
\hline 2,4-Dinitrophenol & 860 & $\mathrm{U}$ & 860 & 850 & $\mathrm{U}$ & 850 & 890 & $\mathrm{U}$ & 890 & 860 & $\mathrm{U}$ & 860 \\
\hline 2,4-Dinitrotoluene & 340 & $\mathrm{U}$ & 340 & 340 & $\mathrm{U}$ & 340 & 360 & $\mathrm{U}$ & 360 & 350 & $\mathrm{U}$ & 350 \\
\hline 2,6-Dinitrotoluene & 340 & $\mathrm{U}$ & 340 & 340 & $\mathrm{U}$ & 340 & 360 & $\mathrm{U}$ & 360 & 350 & $\bar{U}$ & 350 \\
\hline 2-Chloronaphthalene & 340 & $\mathrm{U}$ & 340 & 340 & $\mathrm{U}$ & 340 & 360 & $U$ & 360 & 350 & $\mathrm{U}$ & 350 \\
\hline 2-Chlorophenol & 340 & $\mathrm{U}$ & 340 & 340 & $\mathrm{U}$ & 340 & 360 & $\mathrm{U}$ & 360 & 350 & $\mathrm{U}$ & 350 \\
\hline 2-Methylnaphthalene & 340 & $\mathrm{U}$ & 340 & 340 & $\mathrm{U}$ & 340 & 360 & $\mathrm{U}$ & 360 & 350 & $\mathrm{U}$ & 350 \\
\hline 2-Methylphenol (cresol, o-) & 340 & $\mathrm{U}$ & 340 & 340 & $\mathrm{U}$ & 340 & 360 & U & 360 & 350 & $\mathrm{U}$ & 350 \\
\hline 2-Nitroaniline & 860 & $\mathrm{U}$ & 860 & 850 & $\mathrm{U}$ & 850 & 890 & $\mathrm{U}$ & 890 & 860 & $\bar{U}$ & 860 \\
\hline 2-Nitrophenol & 340 & $\mathrm{U}$ & 340 & 340 & $U$ & 340 & 360 & $\mathrm{U}$ & 360 & 350 & $\mathrm{U}$ & 350 \\
\hline 3,3'-Dichlorobenzidine & 340 & $\mathrm{U}$ & 340 & 340 & $\mathrm{U}$ & 340 & 360 & $\mathrm{U}$ & 360 & 350 & $\mathrm{U}$ & 350 \\
\hline 3-Nitroaniline & 860 & $\mathrm{U}$ & 860 & 850 & $\mathrm{U}$ & 850 & 890 & $\mathrm{U}$ & 890 & 860 & $\mathrm{U}$ & 860 \\
\hline 4,6-Dinitro-2-methylphenol & 860 & U & 860 & 850 & $\mathrm{U}$ & 850 & 890 & $\mathrm{U}$ & 890 & 860 & $\mathrm{U}$ & 860 \\
\hline 4-Bromophenyl-phenylether & 340 & $\mathrm{U}$ & 340 & 340 & $\mathrm{U}$ & 340 & 360 & $\bar{U}$ & 360 & 350 & $\bar{U}$ & 350 \\
\hline 4-Chloro-3-methylphenol & 340 & U & 340 & 340 & $\mathrm{U}$ & 340 & 360 & $\mathrm{U}$ & 360 & 350 & $\mathrm{U}$ & 350 \\
\hline 4-Chloroaniline & 340 & $\mathrm{U}$ & 340 & 340 & $\mathrm{U}$ & 340 & 360 & $\mathrm{U}$ & 360 & 350 & 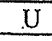 & 350 \\
\hline 4-Chlorophenyl-phenylether & 340 & $\mathrm{U}$ & 340 & 340 & $\mathrm{U}$ & 340 & 360 & $\mathrm{U}$ & 360 & 350 & $\mathrm{U}$ & 350 \\
\hline 4-Methylphenol (p-cresol) & 340 & $\mathrm{U}$ & 340 & 340 & $\mathrm{U}$ & 340 & 360 & $\mathrm{U}$ & 360 & 350 & $\mathrm{U}$ & 350 \\
\hline 4-Nitroaniline & 860 & $\mathrm{U}$ & 860 & 850 & $\mathrm{U}$ & 850 & 890 & $\mathrm{U}$ & 890 & 860 & $\mathrm{U}$ & 860 \\
\hline 4-Nitrophenol & 860 & $\mathrm{U}$ & 860 & 850 & $\bar{U}$ & 850 & 890 & $\mathrm{U}$ & 890 & 860 & $\mathrm{U}$ & 860 \\
\hline Acenaphthene & 340 & $\mathrm{U}$ & 340 & 340 & $\mathrm{U}$ & 340 & 360 & $\mathrm{U}$ & 360 & 350 & $\mathrm{U}$ & 350 \\
\hline Acenaphthylene & 340 & $\mathrm{U}$ & 340 & 340 & $\mathrm{U}$ & 340 & 360 & $\mathrm{U}$ & 360 & 350 & $\mathrm{U}$ & 350 \\
\hline Anthracene & 340 & $\mathrm{U}$ & 340 & 340 & $\mathrm{U}$ & 340 & 360 & $\mathrm{U}$ & 360 & 350 & $\mathrm{U}$ & 350 \\
\hline Benzo(a)anthracene & 340 & $\mathrm{U}$ & 340 & 340 & $\mathrm{U}$ & 340 & 360 & $\mathrm{U}$ & 360 & 350 & $\mathrm{U}$ & 350 \\
\hline Benzo(a)pyrene & 340 & $\mathrm{U}$ & 340 & 340 & $\mathrm{U}$ & 340 & 360 & $\mathrm{U}$ & 360 & 350 & $\mathrm{U}$ & 350 \\
\hline Benzo(b)fluoranthene & 340 & $\mathrm{U}$ & 340 & 340 & $\mathrm{U}$ & 340 & 360 & $\mathrm{U}$ & 360 & 350 & $\mathrm{U}$ & 350 \\
\hline Benzo(g,h,i)perylene & 340 & $\mathrm{U}$ & 340 & 340 & $\mathrm{U}$ & 340 & 360 & $\mathrm{U}$ & 360 & 350 & $\mathrm{U}$ & 350 \\
\hline Benzo(k)fluoranthene & 340 & U & 340 & 340 & $\mathrm{U}$ & 340 & 360 & $\mathrm{U}$ & 360 & 350 & $\mathrm{U}$ & 350 \\
\hline bis(2-Chloro-1-methylethyl)ether & 340 & $\mathrm{U}$ & 340 & 340 & $\mathrm{U}$ & 340 & 360 & $\mathrm{U}$ & 360 & 350 & $\mathrm{U}$ & 350 \\
\hline bis(2-Chloroethoxy)methane & 340 & $\mathrm{U}$ & 340 & 340 & $\mathrm{U}$ & 340 & 360 & $\mathrm{U}$ & 360 & 350 & $\mathrm{U}$ & 350 \\
\hline bis(2-Chloroethyl)ether & 340 & $\mathrm{U}$ & 340 & 340 & U & 340 & 360 & $\mathrm{U}$ & 360 & 350 & $\mathrm{U}$ & 350 \\
\hline bis(2-Ethylhexyl)phthalate & 20 & $\mathrm{JB}$ & 340 & 22 & $\mathrm{JB}$ & 340 & 37 & $\mathrm{JB}$ & 360 & 18 & $\mathrm{JB}$ & 350 \\
\hline & & & & Attachr & & & 1 & & & leet No. & 10 & of 113 \\
\hline & & & & Origina & & J.M. Ce & pron & & & Date & & $107 / 07$ \\
\hline & & & & Checke & & T.M.B & akley & & & & & \\
\hline & & & & Calc. $\mathrm{N}$ & & $0100 \mathrm{~B}-1$ & $\mathrm{CA}-\mathrm{V} 02$ & & & Rev. No & & 0 \\
\hline
\end{tabular}


Attachment 1. 118-B-1 Verification Sampling Results.

\begin{tabular}{|c|c|c|c|c|c|c|c|c|c|c|c|c|}
\hline \multirow[t]{2}{*}{ Constituents } & \multicolumn{3}{|c|}{$\begin{array}{c}\text { J13HY9 } \\
\text { SPA B7 } \\
\text { Sample Date 10/17/06 }\end{array}$} & \multicolumn{3}{|c|}{$\begin{array}{c}\text { J13J00 } \\
\text { SPA B8 } \\
\text { Sample Date 10/17/06 }\end{array}$} & \multicolumn{3}{|c|}{$\begin{array}{c}\text { J13J01 } \\
\text { SPA C9 } \\
\text { Sample Date 10/17/06 }\end{array}$} & \multicolumn{3}{|c|}{$\begin{array}{c}\text { J13J02 } \\
\text { SPA C10 } \\
\text { Sample Date 10/18/06 }\end{array}$} \\
\hline & $\mu \mathrm{g} / \mathrm{kg}$ & $Q$ & PQL & $\mu \mathrm{g} / \mathrm{kg}$ & $Q$ & PQL & $\mu \mathrm{g} / \mathrm{kg}$ & $Q$ & PQL & $\mu \mathrm{g} / \mathrm{kg}$ & $Q$ & PQL \\
\hline \multicolumn{13}{|c|}{ Semivolatile Organic Compounds (continued) } \\
\hline Butylbenzylphthalate & 340 & $\mathrm{U}$ & 340 & 340 & $U$ & 340 & 360 & $\mathrm{U}$ & 360 & 350 & $\mathrm{U}$ & 350 \\
\hline Carbazole & 340 & $\bar{U}$ & 340 & 340 & $\mathrm{U}$ & 340 & 360 & $U$ & 360 & 350 & 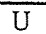 & 350 \\
\hline Chrysene & 340 & $\mathrm{U}$ & 340 & 340 & $\mathrm{U}$ & 340 & 360 & $\mathrm{U}$ & 360 & 350 & $\mathrm{U}$ & 350 \\
\hline Di-n-butylphthalate & 28 & JB & 340 & 30 & JB & 340 & 22 & $\mathrm{JB}$ & 360 & 46 & $\mathrm{JB}$ & 350 \\
\hline Di-n-octylphthalate & 340 & $\bar{U}$ & 340 & 340 & $\mathrm{U}$ & 340 & 360 & $\bar{U}$ & 360 & 350 & $\mathrm{U}$ & 350 \\
\hline Dibenz(a,h)anthracene & 340 & $U$ & 340 & 340 & $\mathrm{U}$ & 340 & 360 & $\mathrm{U}$ & 360 & 350 & $\mathrm{U}$ & 350 \\
\hline Dibenzofuran & 340 & $\bar{U}$ & 340 & 340 & $\mathrm{U}$ & 340 & 360 & $\mathrm{U}$ & 360 & 350 & $\mathrm{U}$ & 350 \\
\hline Diethylphthalate & 340 & $\mathrm{U}$ & 340 & 340 & $\bar{U}$ & 340 & 360 & $\mathrm{U}$ & 360 & 350 & $\mathrm{U}$ & 350 \\
\hline Dimethylphthalate & 340 & $\mathrm{U}$ & 340 & 340 & $\mathrm{U}$ & 340 & 360 & U & 360 & 350 & U & 350 \\
\hline Fluoranthene & 340 & $U$ & 340 & 340 & $\mathrm{U}$ & 340 & 360 & $\mathrm{U}$ & 360 & 350 & $\mathrm{U}$ & 350 \\
\hline Fluorene & 340 & $\mathrm{U}$ & 340 & 340 & $\mathrm{U}$ & 340 & 360 & $\mathrm{U}$ & 360 & 350 & $\mathrm{U}$ & 350 \\
\hline Hexachlorobenzene & 340 & $\mathrm{U}$ & 340 & 340 & $\mathrm{U}$ & 340 & 360 & $\mathrm{U}$ & 360 & 350 & $\mathrm{U}$ & 350 \\
\hline Hexachlorobutadiene & 340 & U & 340 & 340 & $\mathrm{U}$ & 340 & 360 & $\mathrm{U}$ & 360 & 350 & $\mathrm{U}$ & 350 \\
\hline Hexachlorocyclopentadiene & 340 & $\mathrm{U}$ & 340 & 340 & $\mathrm{U}$ & 340 & 360 & $\mathrm{U}$ & 360 & 350 & $U$ & 350 \\
\hline Hexachloroethane & 340 & $U$ & 340 & 340 & $U$ & 340 & 360 & $\mathrm{U}$ & 360 & 350 & $\mathrm{U}$ & 350 \\
\hline Indeno( $(1,2,3-c d)$ pyrene & 340 & $\mathrm{U}$ & 340 & 340 & $\mathrm{U}$ & 340 & 360 & $U$ & 360 & 350 & $\bar{U}$ & 350 \\
\hline Isophorone & 340 & $\mathrm{U}$ & 340 & 340 & $\mathrm{U}$ & 340 & 360 & $\mathrm{U}$ & 360 & 350 & $\mathrm{U}$ & 350 \\
\hline N-Nitroso-di-n-dipropylamine & 340 & $\mathrm{U}$ & 340 & 340 & $\mathrm{U}$ & 340 & 360 & $\mathrm{U}$ & 360 & 350 & $\bar{U}$ & 350 \\
\hline N-Nitrosodiphenylamine & 340 & $\mathrm{U}$ & 340 & 340 & $\mathrm{U}$ & 340 & 360 & $\mathrm{U}$ & 360 & 350 & $\mathrm{U}$ & 350 \\
\hline Naphthalene & 340 & $\mathrm{U}$ & 340 & 340 & $\mathrm{U}$ & 340 & 360 & $\mathrm{U}$ & 360 & 350 & $\mathrm{U}$ & 350 \\
\hline Nitrobenzene & 340 & $\mathrm{U}$ & 340 & 340 & $\mathrm{U}$ & 340 & 360 & $\mathrm{U}$ & 360 & 350 & $U$ & 350 \\
\hline Pentachlorophenol & 860 & $\mathrm{U}$ & 860 & 850 & $\mathrm{U}$ & 850 & 890 & $U$ & 890 & 860 & $\mathrm{U}$ & 860 \\
\hline Phenanthrene & 340 & $\mathrm{U}$ & 340 & 340 & $\mathrm{U}$ & 340 & 360 & $\mathrm{U}$ & 360 & 350 & $\mathrm{U}$ & 350 \\
\hline Phenol & 340 & $\mathrm{U}$ & 340 & 340 & $U$ & 340 & 360 & U & 360 & 350 & $\mathrm{U}$ & 350 \\
\hline Pyrene & 340 & $\mathrm{U}$ & 340 & 340 & $\mathrm{U}$ & 340 & 360 & $U$ & 360 & 350 & $\mathrm{U}$ & 350 \\
\hline & & & & Attach & & & 1 & & & th & & of 113 \\
\hline & . & & & Origin & & J.M.Ca & pron & & & & & $107 / 07$ \\
\hline & & & & Checke & & T.M. BI & lakley & & & & & \\
\hline & & & & Calc. $\mathrm{N}$ & & $0100 \mathrm{~B}$ & $\mathrm{CA}-\mathrm{V} 0$ & & & ev. $\mathrm{N}$ & & 0 \\
\hline
\end{tabular}


Attachment 1. 118-B-1 Verification Sampling Results.

\begin{tabular}{|c|c|c|c|c|c|c|c|c|c|c|c|c|}
\hline \multirow[t]{2}{*}{ Constituents } & \multicolumn{3}{|c|}{\begin{tabular}{|c|} 
J13HY9 \\
SPA B7 \\
Sample Date $10 / 17 / 06$ \\
\end{tabular}} & \multicolumn{3}{|c|}{\begin{tabular}{|c|} 
J13J00 \\
SPA B8 \\
Sample Date 10/17/06 \\
\end{tabular}} & \multicolumn{3}{|c|}{\begin{tabular}{|c|} 
J13J01 \\
SPA C9 \\
Sample Date 10/17/06 \\
\end{tabular}} & \multicolumn{3}{|c|}{\begin{tabular}{|c|} 
J13J02 \\
SPA C10 \\
Sample Date 10/18/06
\end{tabular}} \\
\hline & $\mu \mathrm{g} / \mathrm{kg}$ & $\mathbf{Q}$ & PQL & $\mu \mathrm{g} / \mathrm{kg}$ & $\mathbf{Q}$ & PQL & $\mu \mathrm{g} / \mathrm{kg}$ & $\mathbf{Q}$ & PQL & $\mu \mathrm{g} / \mathrm{kg}$ & $\mathbf{Q}$ & $\mathrm{PQL}$ \\
\hline \multicolumn{13}{|c|}{ Volatile Organic Compounds } \\
\hline 1,1,1-Trichloroethane & 5 & UJ & 5 & 5 & $\mathrm{UJ}$ & 5 & 5 & UJ & 5 & 5 & $\mathrm{U}$ & 5 \\
\hline 1,1,2,2-Tetrachloroethane & 5 & UJ & 5 & 5 & UJ & 5 & 5 & UJ & 5 & 5 & $\mathrm{U}$ & 5 \\
\hline 1,1,2-Trichloroethane & 5 & UJ & 5 & 5 & UJ & 5 & 5 & UJ & 5 & 5 & $\overline{\mathrm{U}}$ & 5 \\
\hline 1,1-Dichloroethane & 5 & $\mathrm{UJ}$ & 5 & 5 & $\mathrm{UJ}$ & 5 & 5 & UJ & 5 & 5 & $\mathrm{U}$ & 5 \\
\hline 1,1-Dichloroethene & 5 & UJ & 5 & 5 & $\mathrm{UJ}$ & 5 & 5 & UJ & 5 & 5 & $\mathrm{U}$ & 5 \\
\hline 1,2-Dichloroethane & 5 & $\mathrm{UJ}$ & 5 & 5 & $\mathrm{UJ}$ & 5 & 5 & UJ & 5 & 5 & $\mathrm{U}$ & 5 \\
\hline 1,2-Dichloroethene (total) & 5 & UJ & 5 & 5 & UJ & 5 & 5 & UJ & 5 & 5 & $\mathrm{U}$ & 5 \\
\hline 1,2-Dichloropropane & 5 & UJ & 5 & 5 & UJ & 5 & 5 & UJ & 5 & 5 & $\mathrm{U}$ & 5 \\
\hline 2-Butanone & 10 & UJ & 10 & 10 & UJ & 10 & 10 & UJ & 10 & 10 & $\mathrm{U}$ & 10 \\
\hline 2-Hexanone & 10 & UJ & 10 & 10 & $\mathrm{UJ}$ & 10 & 10 & $\mathrm{UJ}$ & 10 & 10 & $\mathrm{U}$ & 10 \\
\hline 4-Methyl-2-pentanone & 10 & UJ & 10 & 10 & UJ & 10 & 10 & UJ & 10 & 10 & $\mathrm{U}$ & 10 \\
\hline Acetone & 10 & UJ & 10 & 10 & $\mathrm{UJ}$ & 10 & 10 & UJ & 10 & 10 & $\mathrm{U}$ & 10 \\
\hline Benzene & 5 & $\mathrm{UJ}$ & 5 & 5 & UJ & 5 & 5 & $\mathrm{UJ}$ & 5 & 5 & $\mathrm{U}$ & 5 \\
\hline Bromodichloromethane & 5 & $\mathrm{UJ}$ & 5 & 5 & UJ & 5 & 5 & UJ & 5 & 5 & $\mathrm{U}$ & 5 \\
\hline Bromoform & 5 & $\mathrm{UJ}$ & 5 & 5 & $\mathrm{UJ}$ & 5 & 5 & UJ & 5 & 5 & $\mathrm{U}$ & 5 \\
\hline Bromomethane & 10 & $\mathrm{UJ}$ & 10 & 10 & UJ & 10 & 10 & UJ & 10 & 10 & $\mathrm{U}$ & 10 \\
\hline Carbon disulfide & 5 & $\mathrm{UJ}$ & 5 & 5 & UJ & 5 & 5 & UJ & 5 & 5 & $\mathrm{U}$ & 5 \\
\hline Carbon tetrachloride & 5 & UJ & 5 & 5 & UJ & 5 & 5 & UJ & 5 & 5 & $\mathrm{U}$ & 5 \\
\hline Chlorobenzene & 5 & UJ & 5 & 5 & UJ & 5 & 5 & UJ & 5 & 5 & $\mathrm{U}$ & 5 \\
\hline Chloroethane & 10 & UJ & 10 & 10 & UJ & 10 & 10 & UJ & 10 & 10 & $\mathrm{U}$ & 10 \\
\hline Chloroform & 5 & UJ & 5 & 5 & UJ & 5 & 5 & UJ & 5 & 5 & $\mathrm{U}$ & 5 \\
\hline Chloromethane & 10 & $\mathrm{UJ}$ & 10 & 10 & UJ & 10 & 10 & UJ & 10 & 10 & U & 10 \\
\hline cis-1,2-Dichloroethylene & 5 & UJ & 5 & 5 & $\mathrm{UJ}$ & 5 & 5 & UJ & 5 & 5 & $\mathrm{U}$ & 5 \\
\hline cis-1,3-Dichloropropene & 5 & $\mathrm{UJ}$ & 5 & 5 & UJ & 5 & 5 & UJ & 5 & 5 & $\mathrm{U}$ & 5 \\
\hline Dibromochloromethane & 5 & UJ & 5 & 5 & $\mathrm{UJ}$ & 5 & 5 & UJ & 5 & 5 & U & 5 \\
\hline Ethylbenzene & 5 & UJ & 5 & 5 & UJ & 5 & 5 & UJ & 5 & 5 & U & 5 \\
\hline Methylene chloride & 15 & $\mathrm{BJ}$ & 5 & 14 & $\mathrm{BJ}$ & 5 & 17 & $\mathrm{BJ}$ & 5 & 11 & $\mathrm{~B}$ & 5 \\
\hline Styrene & 5 & UJ & 5 & 5 & UJ & 5 & 5 & UJ & 5 & 5 & $\bar{U}$ & 5 \\
\hline Tetrachloroethene & 5 & UJ & 5 & 5 & UJ & 5 & 5 & UJ & 5 & 5 & $\mathrm{U}$ & 5 \\
\hline Toluene & 5 & UJ & 5 & 5 & UJ & 5 & 5 & $\mathrm{UJ}$ & 5 & 5 & $\mathrm{U}$ & 5 \\
\hline trans-1,2-Dichloroethylene & 5 & UJ & 5 & 5 & UJ & 5 & 5 & UJ & 5 & 5 & U & 5 \\
\hline trans-1,3-Dichloropropene & 5 & $\mathrm{UJ}$ & 5 & 5 & UJ & 5 & 5 & UJ & 5 & 5 & $\mathrm{U}$ & 5 \\
\hline Trichloroethene & 5 & UJ & 5 & 5 & UJ & 5 & 5 & $\mathrm{UJ}$ & 5 & 2 & $\mathrm{~J}$ & 5 \\
\hline Vinyl chloride & 10 & UJ & 10 & 10 & $\overline{\mathrm{UJ}}$ & 10 & 10 & UJ & 10 & 10 & $\mathrm{U}$ & 10 \\
\hline Xylenes (total) & 5 & UJ & 5 & 5 & UJ & 5 & 5 & UJ & 5 & 5 & $\mathrm{U}$ & 5 \\
\hline & & & & Attachm & & & 1 & & & Sheet No. & 10 & of 113 \\
\hline & & & & Originat & & J.M. Ca & pron & & & Date & & $04 / 07$ \\
\hline & & & & Checked & & T.M. B & akley & & & Date & & \\
\hline & & & & Calc. Nc & & $0100 \mathrm{~B}-1$ & $\mathrm{~A}-\mathrm{V} 029$ & & & Rev. No & & $\mathrm{l}$ \\
\hline
\end{tabular}


Attachment 1. 118-B-1 Verification Sampling Results.

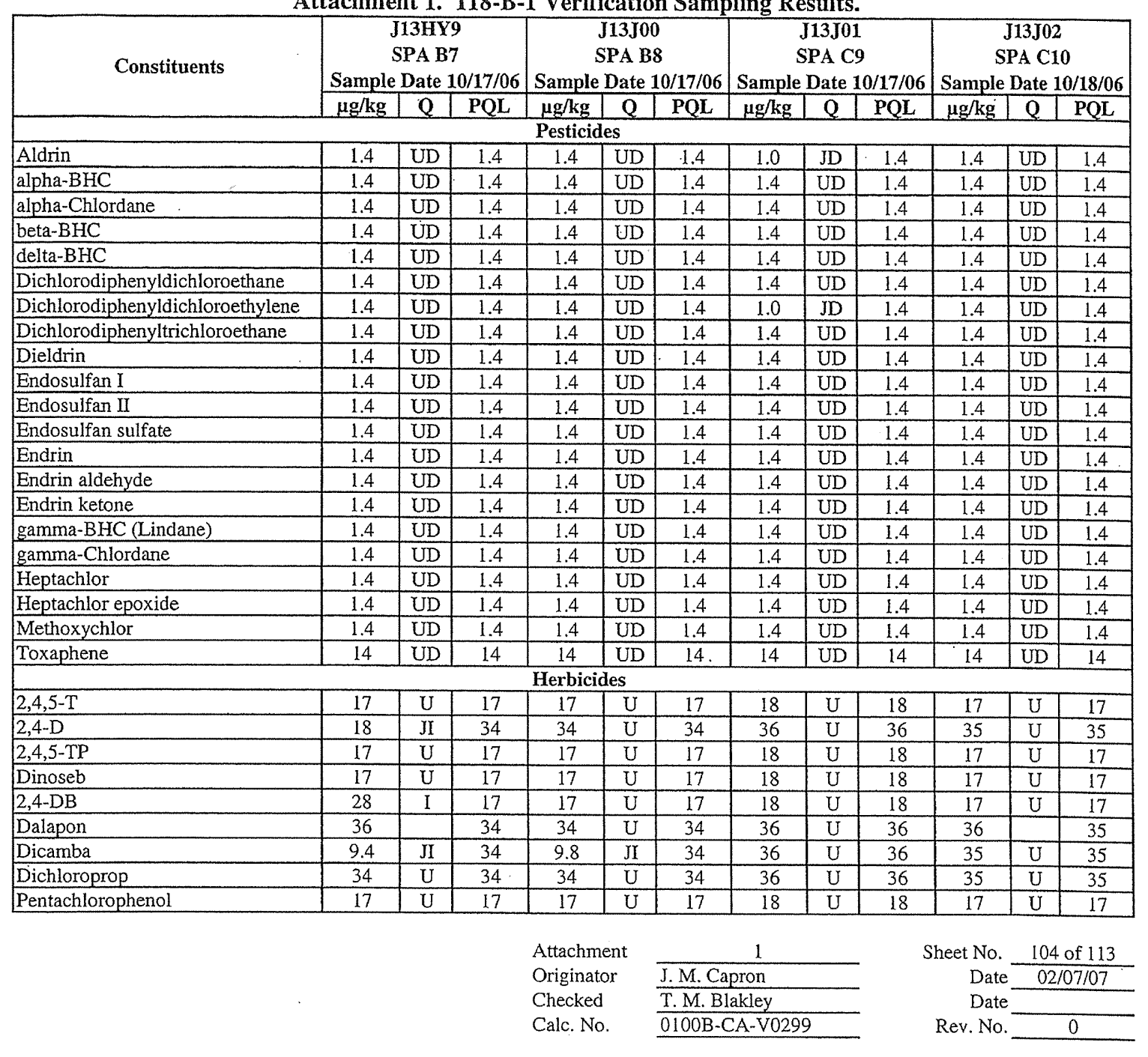


Attachment 1. 118-B-1 Verification Sampling Results.

\begin{tabular}{|c|c|c|c|c|c|c|c|c|c|c|c|c|}
\hline \multirow[t]{2}{*}{ Constituents } & \multicolumn{3}{|c|}{$\begin{array}{c}\text { J13J03 } \\
\text { SPA C1 } \\
\text { Sample Date 10/18/06 }\end{array}$} & \multicolumn{3}{|c|}{\begin{tabular}{|c|} 
J13J04 \\
SPA C2 \\
Sample Date 10/18/06 \\
\end{tabular}} & \multicolumn{3}{|c|}{\begin{tabular}{c|c|} 
J13J05 \\
SPA D3 \\
Sample Date 10/18/06 \\
\end{tabular}} & \multicolumn{3}{|c|}{$\begin{array}{c}\text { J13J06 } \\
\text { SPA D4 } \\
\text { Sample Date 10/19/06 } \\
\end{array}$} \\
\hline & $\mu \mathrm{g} / \mathrm{kg}$ & $Q$ & $\mathrm{PQL}$ & $\mu \mathrm{g} / \mathrm{kg}$ & $Q$ & PQL & $\mu \mathrm{g} / \mathrm{kg}$ & $Q$ & PQL & $\mu \mathrm{g} / \mathrm{kg}$ & Q & $\mathrm{PQL}$ \\
\hline \multicolumn{13}{|c|}{ Polychlorinated Biphenyls } \\
\hline Aroclor-1016 & 14 & $\mathrm{U}$ & 14 & 14 & $\mathrm{U}$ & 14 & 14 & $\mathrm{U}$ & 14 & 14 & $\mathrm{U}$ & 14 \\
\hline Aroclor- 1221 & 14 & $\mathrm{U}$ & 14 & 14 & $\mathrm{U}$ & 14 & 14 & $\mathrm{U}$ & 14 & 14 & $\mathrm{U}$ & 14 \\
\hline Aroclor- 1232 & 14 & U & 14 & 14 & $\mathrm{U}$ & 14 & 14 & $\mathrm{U}$ & 14 & 14 & $\mathrm{U}$ & 14 \\
\hline Aroclor- 1242 & 14 & $\mathrm{U}$ & 14 & 14 & $\mathrm{U}$ & 14 & 47 & & 14 & 14 & $\mathrm{U}$ & 14 \\
\hline Aroclor- 1248 & 14 & $U$ & 14 & 14 & $\mathrm{U}$ & 14 & 14 & U & 14 & 14 & $\mathrm{U}$ & 14 \\
\hline Aroclor- 1254 & 14 & U & 14 & 14 & $\mathrm{U}$ & 14 & 170 & & 14 & 27 & & 14 \\
\hline Aroclor- 1260 & 14 & $U$ & 14 & 14 & $\mathrm{U}$ & 14 & 56 & & 14 & 12 & $\mathrm{~J}$ & 14 \\
\hline \multicolumn{13}{|c|}{ Semivolatile Organic Compounds } \\
\hline 1,2,4-Trichlorobenzene & 350 & $\mathrm{U}$ & 350 & 340 & $\mathrm{U}$ & 340 & 340 & $\mathrm{U}$ & 340 & 360 & $\mathrm{U}$ & 360 \\
\hline 1,2-Dichlorobenzene & 350 & $\mathrm{U}$ & 350 & 340 & $\mathrm{U}$ & 340 & 340 & $\mathrm{U}$ & 340 & 360 & $\mathrm{U}$ & 360 \\
\hline 1,3-Dichlorobenzene & 350 & $\mathrm{U}$ & 350 & 340 & $\mathrm{U}$ & 340 & 340 & $\mathrm{U}$ & 340 & 360 & $\mathrm{U}$ & 360 \\
\hline 1,4-Dichlorobenzene & 350 & $\mathrm{U}$ & 350 & 340 & $\mathrm{U}$ & 340 & 340 & $\bar{U}$ & 340 & 360 & $\mathrm{U}$ & 360 \\
\hline 2,4,5-Trichlorophenol & 870 & 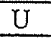 & 870 & 860 & $\mathrm{U}$ & 860 & 860 & $\mathrm{U}$ & 860 & 900 & $\mathrm{U}$ & 900 \\
\hline 2,4,6-Trichlorophenol & 350 & $\mathrm{U}$ & 350 & 340 & $\mathrm{U}$ & 340 & 340 & $\mathrm{U}$ & 340 & 360 & $\mathrm{U}$ & 360 \\
\hline 2,4-Dichlorophenol & 350 & $\mathrm{U}$ & 350 & 340 & $\mathrm{U}$ & 340 & 340 & $\mathrm{U}$ & 340 & 360 & $\mathrm{U}$ & 360 \\
\hline 2,4-Dimethylphenol & 350 & $\mathrm{U}$ & 350 & 340 & $\mathrm{U}$ & 340 & 340 & $U$ & 340 & 360 & $\mathrm{U}$ & 360 \\
\hline 2,4-Dinitrophenol & 870 & $\mathrm{U}$ & 870 & 860 & $\mathrm{U}$ & 860 & 860 & $\mathrm{U}$ & 860 & 900 & $\mathrm{U}$ & 900 \\
\hline 2,4-Dinitrotoluene & 350 & $\mathrm{U}$ & 350 & 340 & $\mathrm{U}$ & 340 & 340 & $\mathrm{U}$ & 340 & 360 & $\mathrm{U}$ & 360 \\
\hline 2,6-Dinitrotoluene & 350 & $\mathrm{U}$ & 350 & 340 & $\mathrm{U}$ & 340 & 340 & $\mathrm{U}$ & 340 & 360 & $\mathrm{U}$ & 360 \\
\hline 2-Chloronaphthalene & 350 & $\mathrm{U}$ & 350 & 340 & $\mathrm{U}$ & 340 & 340 & $\mathrm{U}$ & 340 & 360 & $\mathrm{U}$ & 360 \\
\hline 2-Chlorophenol & 350 & $\mathrm{U}$ & 350 & 340 & $\mathrm{U}$ & 340 & 340 & $\mathrm{U}$ & 340 & 360 & $\mathrm{U}$ & 360 \\
\hline 2-Methylnaphthalene & 350 & $\mathrm{U}$ & 350 & 340 & $\mathrm{U}$ & 340 & 340 & $\mathrm{U}$ & 340 & 360 & $\mathrm{U}$ & 360 \\
\hline 2-Methylphenol (cresol, o-) & 350 & $\mathrm{U}$ & 350 & 340 & $\mathrm{U}$ & 340 & 340 & U & 340 & 360 & $\mathrm{U}$ & 360 \\
\hline 2-Nitroaniline & 870 & $\mathrm{U}$ & 870 & 860 & $\mathrm{U}$ & 860 & 860 & $\mathrm{U}$ & 860 & 900 & $\mathrm{U}$ & 900 \\
\hline 2-Nitrophenol & 350 & $\mathrm{U}$ & 350 & 340 & $\mathrm{U}$ & 340 & 340 & $\mathrm{U}$ & 340 & 360 & $\mathrm{U}$ & 360 \\
\hline 3,3'-Dichlorobenzidine & 350 & $\mathrm{U}$ & 350 & 340 & $\mathrm{U}$ & 340 & 340 & U & 340 & 360 & $\mathrm{U}$ & 360 \\
\hline 3-Nitroaniline & 870 & $\mathrm{U}$ & 870 & 860 & $\mathrm{U}$ & 860 & 860 & $\mathrm{U}$ & 860 & 900 & $\mathrm{U}$ & 900 \\
\hline 4,6-Dinitro-2-methylphenol & 870 & $\mathrm{U}$ & 870 & 860 & U & 860 & 860 & $\mathrm{U}$ & 860 & 900 & $\mathrm{U}$ & 900 \\
\hline 4-Bromophenyl-phenylether & 350 & $\mathrm{U}$ & 350 & 340 & $\mathrm{U}$ & 340 & 340 & $\mathrm{U}$ & 340 & 360 & $\mathrm{U}$ & 360 \\
\hline 4-Chloro-3-methylphenol & 350 & $\mathrm{U}$ & 350 & 340 & $\mathrm{U}$ & 340 & 340 & $\mathrm{U}$ & 340 & 360. & $\mathrm{U}$ & 360 \\
\hline 4-Chloroaniline & 350 & $\mathrm{U}$ & 350 & 340 & $\mathrm{U}$ & 340 & 340 & $\mathrm{U}$ & 340 & 360 & $U$ & 360 \\
\hline 4-Chlorophenyl-phenylether & 350 & U & 350 & 340 & $\mathrm{U}$ & 340 & 340 & $\mathrm{U}$ & 340 & 360 & $\mathrm{U}$ & 360 \\
\hline 4-Methylphenol (p-cresol) & 350 & $\mathrm{U}$ & 350 & 340 & $\mathrm{U}$ & 340 & 340 & $\mathrm{U}$ & 340 & 360 & $\mathrm{U}$ & 360 \\
\hline 4-Nitroaniline & 870 & $\mathrm{U}$ & 870 & 860 & $\mathrm{U}$ & 860 & 860 & $\mathrm{U}$ & 860 & 900 & $\mathrm{U}$ & 900 \\
\hline 4-Nitrophenol & 870 & $\mathrm{U}$ & 870 & 860 & $\mathrm{U}$ & 860 & 860 & $\mathrm{U}$ & 860 & 900 & $\mathrm{U}$ & 900 \\
\hline Acenaphthene & 350 & $\mathrm{U}$ & 350 & 340 & $\mathrm{U}$ & 340 & 340 & $\mathrm{U}$ & 340 & 360 & U & 360 \\
\hline Acenaphthylene & 350 & $\mathrm{U}$ & 350 & 340 & $\mathrm{U}$ & 340 & 340 & $\mathrm{U}$ & 340 & 360 & $\mathrm{U}$ & 360 \\
\hline Anthracene & 350 & $\mathrm{U}$ & 350 & 340 & $\mathrm{U}$ & 340 & 340 & $\mathrm{U}$ & 340 & 360 & $\mathrm{U}$ & 360 \\
\hline Benzo(a)anthracene & 350 & $\mathrm{U}$ & 350 & 340 & $\mathrm{U}$ & 340 & 340 & $\mathrm{U}$ & 340 & 360 & $\mathrm{U}$ & 360 \\
\hline Benzo(a)pyrene & 350 & $\mathrm{U}$ & 350 & 340 & $\mathrm{U}$ & 340 & 340 & $\mathrm{U}$ & 340 & 360 & U & 360 \\
\hline Benzo(b)fluoranthene & 350 & $\mathrm{U}$ & 350 & 340 & $\mathrm{U}$ & 340 & 340 & $\mathrm{U}$ & 340 & 360 & $\mathrm{U}$ & 360 \\
\hline Benzo(g,h,i)perylene & 350 & $U$ & 350 & 340 & $\mathrm{U}$ & 340 & 340 & U & 340 & 360 & $\mathrm{U}$ & 360 \\
\hline Benzo(k)fluoranthene & 350 & $\mathrm{U}$ & 350 & 340 & $\mathrm{U}$ & 340 & 340 & $U$ & 340 & 360 & $\mathrm{U}$ & 360 \\
\hline bis(2-Chloro-1-methylethyl)ether & 350 & $\mathrm{U}$ & 350 & 340 & $\mathrm{U}$ & 340 & 340 & $\mathrm{U}$ & 340 & 360 & $\mathrm{U}$ & 360 \\
\hline bis(2-Chloroethoxy)methane & 350 & $\mathrm{U}$ & 350 & 340 & $\mathrm{U}$ & 340 & 340 & $\mathrm{U}$ & 340 & 360 & $\mathrm{U}$ & 360 \\
\hline bis(2-Chloroethyl)ether & 350 & $\mathrm{U}$ & 350 & 340 & $\mathrm{U}$ & 340 & 340 & $\mathrm{U}$ & 340 & 360 & $\mathrm{U}$ & 360 \\
\hline bis(2-Ethylhexyl)phthalate & 350 & $\mathrm{U}$ & 350 & 24 & $\mathrm{JB}$ & 340 & 340 & $\mathrm{U}$ & 340 & 330 & $\mathrm{U}$ & 360 \\
\hline
\end{tabular}

Attachment Originator

Checked

Calc. No.

$\frac{1}{\frac{1}{\text { J.M. Capron }}} \frac{}{\text { T. M. Blakley }}$

Sheet No 105 of 113
Date 02/07/07

Date

Rev. No. $\frac{0}{0}$ 
Attachment 1. 118-B-1 Verification Sampling Results.

\begin{tabular}{|c|c|c|c|c|c|c|c|c|c|c|c|c|}
\hline \multirow[t]{2}{*}{ Constituents } & \multicolumn{3}{|c|}{$\begin{array}{c}\text { J13J03 } \\
\text { SPA C1 } \\
\text { Sample Date 10/18/06 }\end{array}$} & \multicolumn{3}{|c|}{$\begin{array}{c}\text { J13J04 } \\
\text { SPA C2 } \\
\text { Sample Date 10/18/06 }\end{array}$} & \multicolumn{3}{|c|}{$\begin{array}{c}\text { J13J05 } \\
\text { SPA D3 } \\
\text { Sample Date 10/18/06 } \\
\end{array}$} & \multicolumn{3}{|c|}{$\begin{array}{c}\text { J13J06 } \\
\text { SPA D4 } \\
\text { Sample Date 10/19/06 }\end{array}$} \\
\hline & $\mu \mathrm{g} / \mathrm{kg}$ & $Q$ & PQL & $\mu \mathrm{g} / \mathrm{kg}$ & $Q$ & PQL & $\mu \mathrm{g} / \mathrm{kg}$ & $Q$ & $\mathrm{PQL}$ & $\mu \mathrm{g} / \mathrm{kg}$ & $Q$ & PQL \\
\hline \multicolumn{13}{|c|}{ Semivolatile Organic Compounds (continued) } \\
\hline Butylbenzylphthalate & 350 & $\bar{U}$ & 350 & 340 & $\mathrm{U}$ & 340 & 340 & $\mathrm{U}$ & 340 & 360 & $\mathrm{U}$ & 360 \\
\hline Carbazole & 350 & $\mathrm{U}$ & 350 & 340 & $\mathrm{U}$ & 340 & 340 & $\mathrm{U}$ & 340 & 360 & $\mathrm{U}$ & 360 \\
\hline Chrysene & 350 & $\mathrm{U}$ & 350 & 340 & $\mathrm{U}$ & 340 & 340 & $\mathrm{U}$ & 340 & 360 & $\mathrm{U}$ & 360 \\
\hline Di-n-butylphthalate & 35 & JB & 350 & 42 & $\mathrm{JB}$ & 340 & 33 & JB & 340 & 360 & $\mathrm{U}$ & 360 \\
\hline Di-n-octylphthalate & 350 & $\mathrm{U}$ & 350 & 340 & $\mathrm{U}$ & 340 & 340 & $\mathrm{U}$ & 340 & 360 & $\mathrm{U}$ & 360 \\
\hline Dibenz $(a, h)$ anthracene & 350 & $\mathrm{U}$ & 350 & 340 & $\mathrm{U}$ & 340 & 340 & $\mathrm{U}$ & 340 & 360 & $\mathrm{U}$ & 360 \\
\hline Dibenzofuran & 350 & $\mathrm{U}$ & 350 & 340 & $\mathrm{U}$ & 340 & 340 & $\mathrm{U}$ & 340 & 360 & $\mathrm{U}$ & 360 \\
\hline Diethylphthalate & 350 & $\mathrm{U}$ & 350 & 340 & $\mathrm{U}$ & 340 & 340 & $\mathrm{U}$ & 340 & 360 & $\mathrm{U}$ & 360 \\
\hline Dimethylphthalate & 350 & $\mathrm{U}$ & 350 & 340 & $\mathrm{U}$ & .340 & 340 & $\mathrm{U}$ & 340 & 360 & $\mathrm{U}$ & 360 \\
\hline Fluoranthene & 350 & $\mathrm{U}$ & 350 & 340 & $U$ & 340 & 340 & $\mathrm{U}$ & 340 & 360 & $\mathrm{U}$ & 360 \\
\hline Fluorene & 350 & $\mathrm{U}$ & 350 & 340 & $\mathrm{U}$ & 340 & 340 & $\mathrm{U}$ & 340 & 360 & $\mathrm{U}$ & 360 \\
\hline Hexachlorobenzene & 350 & $\mathrm{U}$ & 350 & 340 & $\mathrm{U}$ & 340 & 340 & $\mathrm{U}$ & 340 & 360 & $\mathrm{U}$ & 360 \\
\hline Hexachlorobutadiene & 350 & $\mathrm{U}$ & 350 & 340 & $\mathrm{U}$ & 340 & 340 & $\mathrm{U}$ & 340 & 360 & $\mathrm{U}$ & 360 \\
\hline Hexachlorocyclopentadiene & 350 & $\mathrm{U}$ & 350 & 340 & $\mathrm{U}$ & 340 & 340 & $\mathrm{U}$ & 340 & 360 & $\mathrm{U}$ & 360 \\
\hline Hexachloroethane & 350 & $\mathrm{U}$ & 350 & 340 & $\mathrm{U}$ & 340 & 340 & $\mathrm{U}$ & 340 & 330 & $\mathrm{U}$ & 360 \\
\hline Indeno $(1,2,3-c d)$ pyrene & 350 & U & 350 & 340 & $\mathrm{U}$ & 340 & 340 & $\mathrm{U}$ & 340 & 360 & $\mathrm{U}$ & 360 \\
\hline Isophorone & 350 & $\bar{U}$ & 350 & 340 & $\mathrm{U}$ & 340 & 340 & $\mathrm{U}$ & 340 & 360 & $\mathrm{U}$ & 360 \\
\hline $\mathrm{N}$-Nitroso-di-n-dipropylamine & 350 & $\mathrm{U}$ & 350 & 340 & $\mathrm{U}$ & 340 & 340 & $\mathrm{U}$ & 340 & 360 & $\mathrm{U}$ & 360 \\
\hline $\mathrm{N}$-Nitrosodiphenylamine & 350 & $\mathrm{U}$ & 350 & 340 & $\mathrm{U}$ & 340 & 340 & $\mathrm{U}$ & 340 & 360 & $\mathrm{U}$ & 360 \\
\hline Naphthalene & 350 & $\mathrm{U}$ & 350 & 340 & $\mathrm{U}$ & 340 & 340 & $\mathrm{U}$ & 340 & 360 & U & 360 \\
\hline Nitrobenzene & 350 & $\mathrm{U}$ & 350 & 340 & $\mathrm{U}$ & 340 & 340 & U & 340 & 360 & $\mathrm{U}$ & 360 \\
\hline Pentachlorophenol & 870 & $\mathrm{U}$ & 870 & 860 & $\mathrm{U}$ & 860 & 860 & $\mathrm{U}$ & 860 & 900 & $\mathrm{U}$ & 900 \\
\hline Phenanthrene. & 350 & $U$ & 350 & 340 & $\mathrm{U}$ & 340 & 340 & $\mathrm{U}$ & 340 & 360 & $\mathrm{U}$ & 360 \\
\hline Phenol & 350 & $\mathrm{U}$ & 350 & 340 & $\mathrm{U}$ & 340 & 340 & U & 340 & 360 & $\mathrm{U}$ & 360 \\
\hline Pyrene & 350 & $\mathrm{U}$ & 350 & 340 & $\mathrm{U}$ & 340 & 340 & $\mathrm{U}$ & 340 & 360 & $\mathrm{U}$ & 360 \\
\hline & & & & Attachm & & & 1 & & & Sheet No. & & of 113 \\
\hline & & & & Originat & & J.M.Ca & pron & & & Date & & $97 / 07$ \\
\hline & & & & Checkec & & T. M. Bl & akley & & & Date & & \\
\hline & & & & Calc. N & & $0100 \mathrm{~B}-\mathrm{C}$ & $\mathrm{A}-\mathrm{V} 029$ & & & Rev. No. & & 0 \\
\hline
\end{tabular}


Attachment 1. 118-B-1 Verification Sampling Results.

\begin{tabular}{|c|c|c|c|c|c|c|c|c|c|c|c|c|}
\hline \multirow[t]{2}{*}{ Constituents } & \multicolumn{3}{|c|}{$\begin{array}{c}\text { J13J03 } \\
\text { SPA C1 } \\
\text { Sample Date 10/18/06 }\end{array}$} & \multicolumn{3}{|c|}{$\begin{array}{c}\text { J13J04 } \\
\text { SPA C2 } \\
\text { Sample Date 10/18/06 } \\
\end{array}$} & \multicolumn{3}{|c|}{\begin{tabular}{|c|} 
J13J05 \\
SPA D3 \\
Sample Date 10/18/06 \\
\end{tabular}} & \multicolumn{3}{|c|}{$\begin{array}{c}\text { J13J06 } \\
\text { SPA D4 } \\
\text { Sample Date 10/19/06 }\end{array}$} \\
\hline & $\mu \mathrm{g} / \mathrm{kg}$ & $Q$ & PQL & $\mu \mathrm{g} / \mathrm{kg}$ & $Q$ & $\mathrm{PQL}$ & $\mu \mathrm{g} / \mathrm{kg}$ & $\mathbf{Q}$ & $\mathrm{PQL}$ & $\mu \mathrm{g} / \mathrm{kg}$ & $\mathbf{Q}$ & PQL \\
\hline \multicolumn{13}{|c|}{ Volatile Organic Compounds } \\
\hline 1,1,1-Trichloroethane & 5 & $\mathrm{U}$ & 5 & 5 & $\mathrm{U}$ & 5 & 5 & $\mathrm{U}$ & 5 & 5 & $\mathrm{U}$ & 5 \\
\hline 1,1,2,2-Tetrachloroethane & 5 & $\mathrm{U}$ & 5 & 5 & U & 5 & 5 & $\mathrm{U}$ & 5 & 5 & $\mathrm{U}$ & 5 \\
\hline 1,1,2-Trichloroethane & 5 & $\mathrm{U}$ & 5 & 5 & $\mathrm{U}$ & 5 & 5 & $U$ & 5 & 5 & $\mathrm{U}$ & 5 \\
\hline 1,1-Dichloroethane & 5 & $\mathrm{U}$ & 5 & 5 & $\mathrm{U}$ & 5 & 5 & $\mathrm{U}$ & 5 & 5 & $\mathrm{U}$ & 5 \\
\hline 1,1-Dichloroethene & 5 & $\mathrm{U}$ & 5 & 5 & $\mathrm{U}$ & 5 & 5 & $\mathrm{U}$ & 5 & 5 & $\mathrm{U}$ & 5 \\
\hline 1,2-Dichloroethane & 5 & $\mathrm{U}$ & 5 & 5 & $\mathrm{U}$ & 5 & 5 & $\mathrm{U}$ & 5 & 5 & $\mathrm{U}$ & 5 \\
\hline 1,2-Dichloroethene (total) & 5 & $\mathrm{U}$ & 5 & 5 & $\mathrm{U}$ & 5 & 5 & $\bar{U}$ & 5 & 5 & $\mathrm{U}$ & 5 \\
\hline 1,2-Dichloropropane & 5 & $\mathrm{U}$ & 5 & 5 & $\mathrm{U}$ & 5 & 5 & $\mathrm{U}$ & 5 & 5 & $\mathrm{U}$ & 5 \\
\hline 2-Butanone & 10 & $\mathrm{U}$ & 10 & 10 & $\mathrm{U}$ & 10 & 10 & $\mathrm{U}$ & 10 & 10 & $\bar{U}$ & 10 \\
\hline 2-Hexanone & 10 & $\mathrm{U}$ & 10 & 10 & $\mathrm{U}$ & 10 & 10 & $U$ & 10 & 10 & $\mathrm{U}$ & 10 \\
\hline 4-Methyl-2-pentanone & 10 & $\mathrm{U}$ & 10 & 10 & $\mathrm{U}$ & 10 & 10 & $\mathrm{U}$ & 10 & 10 & $\mathrm{U}$ & 10 \\
\hline Acetone & 10 & $\mathrm{U}$ & 10 & 10 & $\mathrm{U}$ & 10 & 10 & $\mathrm{U}$ & 10 & 10 & $\mathrm{U}$ & 10 \\
\hline Benzene & 5 & $\mathrm{U}$ & 5 & 5 & $\mathrm{U}$ & 5 & 5 & $\mathrm{U}$ & 5 & 5 & $\mathrm{U}$ & 5 \\
\hline Bromodichloromethane & 5 & $\mathrm{U}$ & 5 & 5 & $\mathrm{U}$ & 5 & 5 & $\mathrm{U}$ & 5 & 5 & $\overline{\mathrm{U}}$ & 5 \\
\hline Bromoform & 5 & $\mathrm{U}$ & 5 & 5 & U & 5 & 5 & $\mathrm{U}$ & 5 & 5 & $\mathrm{U}$ & 5 \\
\hline Bromomethane & 10 & $\mathrm{U}$ & 10 & 10 & $\mathrm{U}$ & 10 & 10 & $\mathrm{U}$ & 10 & 10 & $\mathrm{U}$ & 10 \\
\hline Carbon disulfide & 5 & $\mathrm{U}$ & 5 & 5 & $\mathrm{U}$ & 5 & 5 & $\mathrm{U}$ & 5 & 5 & $\bar{U}$ & 5 \\
\hline Carbon tetrachloride & 5 & $\mathrm{U}$ & 5 & 5 & $\mathrm{U}$ & 5 & 5 & $U$ & 5 & 5 & $\mathrm{U}$ & 5 \\
\hline Chlorobenzene & 5 & $\mathrm{U}$ & 5 & 5 & $\mathrm{U}$ & 5 & 5 & U & 5 & 5 & $\mathrm{U}$ & 5 \\
\hline Chloroethane & 10 & $\mathrm{U}$ & 10 & 10 & $U$ & 10 & 10 & $\mathrm{U}$ & 10 & 10 & $\mathrm{U}$ & 10 \\
\hline Chloroform & 5 & $\mathrm{U}$ & 5 & 5 & $\mathrm{U}$ & 5 & 5 & $U$ & 5 & 5 & $\mathrm{U}$ & 5 \\
\hline Chloromethane & 10 & $\mathrm{U}$ & 10 & 10 & $\mathrm{U}$ & 10 & 10 & $\mathrm{U}$ & 10 & 10 & $\mathrm{U}$ & 10 \\
\hline cis-1,2-Dichloroethylene & 5 & $\mathrm{U}$ & 5 & 5 & $\mathrm{U}$ & 5 & 5 & $\mathrm{U}$ & 5 & 5 & $\mathrm{U}$ & 5 \\
\hline cis-1,3-Dichloropropene & 5 & $\mathrm{U}$ & 5 & 5 & $\mathrm{U}$ & 5 & 5 & $\mathrm{U}$ & 5 & 5 & $\mathrm{U}$ & 5 \\
\hline Dibromochloromethane & 5 & $\mathrm{U}$ & 5 & 5 & $\mathrm{U}$ & 5 & 5 & $\mathrm{U}$ & 5 & 5 & U & 5 \\
\hline Ethylbenzene & 5 & $\mathrm{U}$ & 5 & 5 & $\mathrm{U}$ & 5 & 5 & $\mathrm{U}$ & 5 & 5 & $\mathrm{U}$ & 5 \\
\hline Methylene chloride & 9 & $\mathrm{~B}$ & 5 & 11 & $\mathrm{~B}$ & 5 & 12 & $\mathrm{~B}$ & 5 & 16 & $\mathrm{U}$ & 5 \\
\hline Styrene & 5 & $\mathrm{U}$ & 5 & 5 & U & 5 & 5 & $\mathrm{U}$ & 5 & 5 & $\mathrm{U}$ & 5 \\
\hline Tetrachloroethene & 5 & $\mathrm{U}$ & 5 & 5 & $U$ & 5 & 5 & $\mathrm{U}$ & 5 & 5 & $U$ & 5 \\
\hline Toluene & 5 & $\mathrm{U}$ & 5 & 5 & U & 5 & 5 & $\mathrm{U}$ & 5 & 5 & $\mathrm{U}$ & 5 \\
\hline trans-1,2-Dichloroethylene & 5 & $\mathrm{U}$ & 5 & 5 & $\mathrm{U}$ & 5 & 5 & $\mathrm{U}$ & 5 & 5 & $\mathrm{U}$ & 5 \\
\hline trans-1,3-Dichloropropene & 5 & $\mathrm{U}$ & 5 & 5 & $\mathrm{U}$ & 5 & 5 & $\mathrm{U}$ & 5 & 5 & $\mathrm{U}$ & 5 \\
\hline \begin{tabular}{|l|} 
Trichloroethene \\
\end{tabular} & 5 & $\mathrm{U}$ & 5 & 5 & $\mathrm{U}$ & 5 & 5 & $\mathrm{U}$ & 5 & 5 & $\mathrm{U}$ & 5 \\
\hline \begin{tabular}{|l} 
Vinyl chloride \\
\end{tabular} & 10 & $\mathrm{U}$ & 10 & 10 & $\mathrm{U}$ & 10 & 10 & $\mathrm{U}$ & 10 & 10 & $\mathrm{U}$ & 10 \\
\hline Xylenes (total) & 5 & $\mathrm{U}$ & 5 & 5 & $\mathrm{U}$ & 5 & 5 & $\mathrm{U}$ & 5 & 5 & $U$ & 5 \\
\hline
\end{tabular}

Attachment

Originator

Checked

Calc. No.

$\frac{1}{\frac{1}{\text { J. M. Capron }}} \frac{}{\text { T. M. Blakley }}$

Sheet No. $\quad 107$ of 113

Date 02/07/07

Date

Rev. No.

0 
Attachment 1. 118-B-1 Verification Sampling Results.

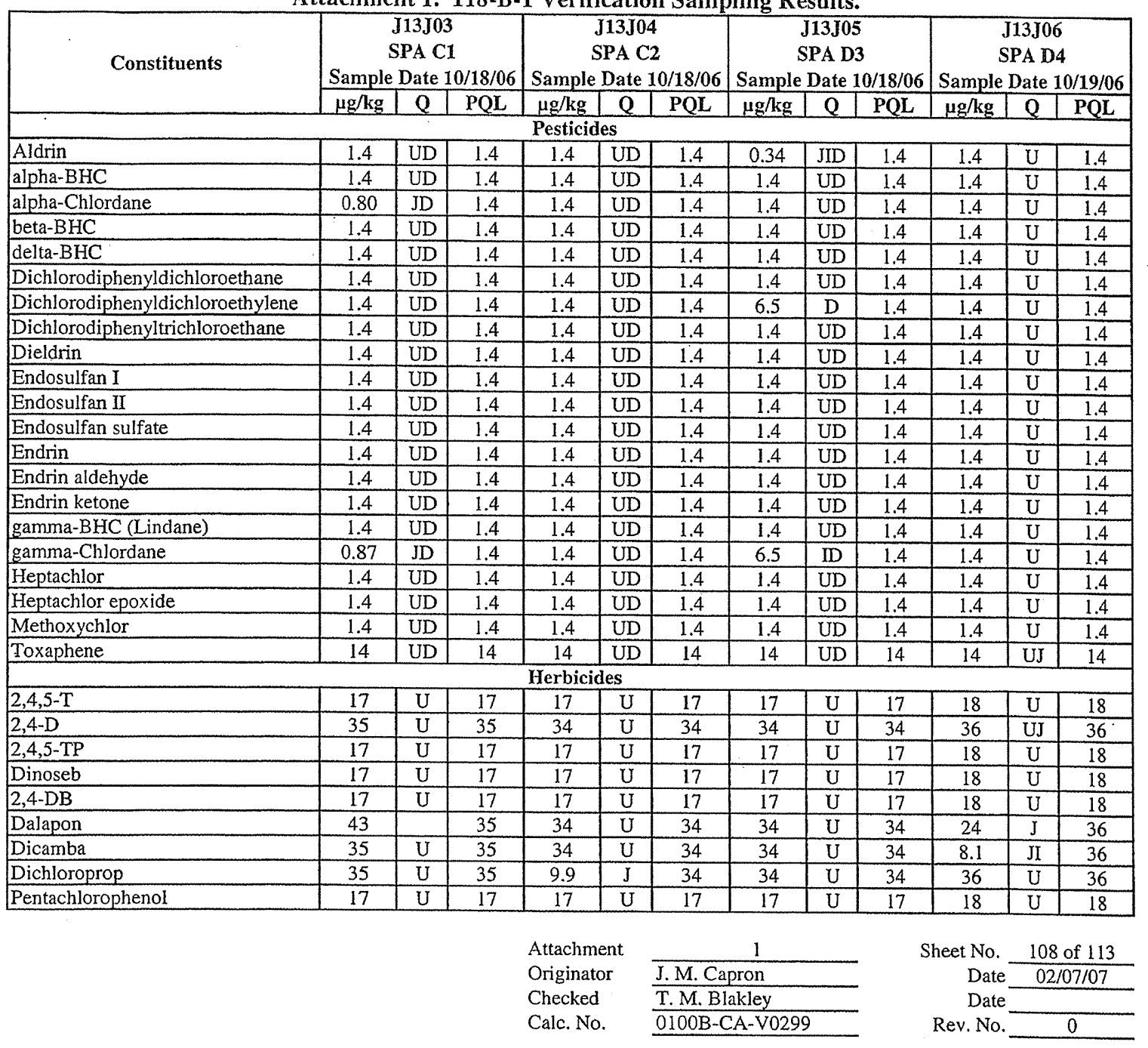


Attachment 1. 118-B-1 Verification Sampling Results.

\begin{tabular}{|c|c|c|c|c|c|c|c|c|c|c|c|c|}
\hline \multirow[t]{2}{*}{ Constituents } & \multicolumn{3}{|c|}{$\begin{array}{c}\text { J13J07 } \\
\text { SPA D5 } \\
\text { Sample Date } 10 / 23 / 06\end{array}$} & \multicolumn{3}{|c|}{$\begin{array}{c}\text { J13J08 } \\
\text { SPA D6 } \\
\text { Sample Date 10/23/06 }\end{array}$} & \multicolumn{3}{|c|}{$\begin{array}{c}\text { J12R24 } \\
\text { FS-1 } \\
\text { Sample Date } 7 / 10 / 06\end{array}$} & \multicolumn{3}{|c|}{\begin{tabular}{|c|} 
J12R33 \\
FS-10 \\
Sample Date 12/7/06 \\
\end{tabular}} \\
\hline & $\mu \mathrm{g} / \mathrm{kg}$ & $Q$ & PQL & $\mu \mathrm{g} / \mathrm{kg}$ & $\mathbf{Q}$ & PQL & $\mu \mathrm{g} / \mathrm{kg}$ & $\mathbf{Q}$ & PQL & $\mu \mathrm{g} / \mathrm{kg}$ & $\mathbf{Q}$ & $\mathbf{P Q L}$ \\
\hline \multicolumn{13}{|c|}{ Polychlorinated Biphenyls } \\
\hline Aroclor-1016 & 14 & $\mathrm{U}$ & 14 & 14 & U & 14 & 13 & $\mathrm{U}$ & 13 & 14 & UJ & 14 \\
\hline Aroclor- 1221 & 14 & $\mathrm{U}$ & 14 & 14 & $U$ & 14 & 13 & U & 13 & 14 & $\mathrm{U}$ & 14 \\
\hline Aroclor- 1232 & 14 & $\mathrm{U}$ & 14 & 14 & $\mathrm{U}$ & 14 & 13 & $\mathrm{U}$ & 13 & 14 & U & 14 \\
\hline Aroclor-1242 & 14 & $\mathrm{U}$ & 14 & 14 & $\mathrm{U}$ & 14 & 13 & $\mathrm{U}$ & 13 & 14 & $\mathrm{U}$ & 14 \\
\hline Aroclor-1248 & 14 & $\mathrm{U}$ & 14 & 14 & $\mathrm{U}$ & 14 & 13 & $\mathrm{U}$ & 13 & 14 & $\mathrm{U}$ & 14 \\
\hline Aroclor -1254 & 14 & $\mathrm{U}$ & 14 & 14 & $\mathrm{U}$ & 14 & 13 & $\mathrm{U}$ & 13 & 14 & $\mathrm{U}$ & 14 \\
\hline Aroclor -1260 & 14 & $\mathrm{U}$ & 14 & 14 & $\mathrm{U}$ & 14 & 13 & $\mathrm{U}$ & 13 & 14 & UJ & 14 \\
\hline \multicolumn{13}{|c|}{ Semivolatile Organic Compounds } \\
\hline 1,2,4-Trichlorobenzene & 340 & $\mathrm{U}$ & 340 & 350 & $U$ & 350 & 330 & $\mathrm{U}$ & 330 & 360 & UJ & 360 \\
\hline 1,2-Dichlorobenzene & 340 & $\mathrm{U}$ & 340 & 350 & $\mathrm{U}$ & 350 & 330 & $\mathrm{U}$ & 330 & 360 & $\mathrm{U}$ & 360 \\
\hline 1,3-Dichlorobenzene & 340 & $\mathrm{U}$ & 340 & 350 & $U$ & 350 & 330 & U & 330 & 360 & $\mathrm{U}$ & 360 \\
\hline 1,4-Dichlorobenzene & 340 & $\mathrm{U}$ & 340 & 350 & $U$ & 350 & 330 & $\mathrm{U}$ & 330 & 360 & $\mathrm{U}$ & 360 \\
\hline 2,4,5-Trichlorophenol & 860 & $\mathrm{U}$ & 860 & 870 & $\mathrm{U}$ & 870 & 830 & $\mathrm{U}$ & 830 & 900 & $\mathrm{U}$ & 900 \\
\hline 2,4,6-Trichlorophenol & 340 & $\mathrm{U}$ & 340 & 350 & $\mathrm{U}$ & 350 & 330 & $\mathrm{U}$ & 330 & 360 & $\mathrm{U}$ & 360 \\
\hline 2,4-Dichlorophenol & 340 & $\mathrm{U}$ & 340 & 350 & U & 350 & 330 & $\mathrm{U}$ & 330 & 360 & $\mathrm{UJ}$ & 360 \\
\hline 2,4-Dimethylphenol & 340 & $\mathrm{U}$ & 340 & 350 & $\mathrm{U}$ & 350 & 330 & $\mathrm{U}$ & 330 & 360 & UJ & 360 \\
\hline 2,4-Dinitrophenol & 860 & $\mathrm{U}$ & 860 & 870 & $\mathrm{U}$ & 870 & 830 & $\mathrm{U}$ & 830 & 900 & $\mathrm{U}$ & 900 \\
\hline 2,4-Dinitrotoluene & 340 & $\mathrm{U}$ & 340 & 350 & $\mathrm{U}$ & 350 & 330 & $\mathrm{U}$ & 330 & 360 & $\mathrm{U}$ & 360 \\
\hline 2,6-Dinitrotoluene & 340 & $\mathrm{U}$ & 340 & 350 & $\mathrm{U}$ & 350 & 330 & $\mathrm{U}$ & 330 & 360 & $\mathrm{U}$ & 360 \\
\hline 2-Chloronaphthalene & 340 & $\mathrm{U}$ & 340 & 350 & U & 350 & 330 & $\mathrm{U}$ & 330 & 360 & $\mathrm{U}$ & 360 \\
\hline 2-Chlorophenol & 340 & $\mathrm{U}$ & 340 & 350 & $\mathrm{U}$ & 350 & 330 & $U$ & 330 & 360 & $\mathrm{U}$ & 360 \\
\hline 2-Methylnaphthalene & 340 & U & 340 & 350 & $\mathrm{U}$ & 350 & 330 & U & 330 & 360 & UJ & 360 \\
\hline 2-Methylphenol (cresol, o-) & 340 & $\mathrm{U}$ & 340 & 350 & $\mathrm{U}$ & 350 & 330 & $\mathrm{U}$ & 330 & 360 & $U$ & 360 \\
\hline 2-Nitroaniline & 860 & $\mathrm{U}$ & 860 & 870 & $\bar{U}$ & 870 & 830 & $\mathrm{U}$ & 830 & 900 & $\mathrm{U}$ & 900 \\
\hline 2-Nitrophenol & 340 & $\mathrm{U}$ & 340 & 350 & $\mathrm{U}$ & 350 & 330 & $\mathrm{U}$ & 330 & 360 & $\mathrm{UJ}$ & 360 \\
\hline 3,3'-Dichlorobenzidine & 340 & $\mathrm{U}$ & 340 & 350 & $\mathrm{U}$ & 350 & 330 & $\mathrm{U}$ & 330 & 360 & $\mathrm{U}$ & 360 \\
\hline 3-Nitroaniline & 860 & $\mathrm{U}$ & 860 & 870 & $\mathrm{U}$ & 870 & 830 & $\mathrm{U}$ & 830 & 900 & $U$ & 900 \\
\hline 4,6-Dinitro-2-methylphenol & 860 & $\mathrm{U}$ & 860 & 870 & $\mathrm{U}$ & 870 & 830 & $\mathrm{U}$ & 830 & 900 & $\bar{U}$ & 900 \\
\hline 4-Bromophenyl-phenylether & 340 & $\mathrm{U}$ & 340 & 350 & $\mathrm{U}$ & 350 & 330 & $\mathrm{U}$ & 330 & 360 & $\mathrm{U}$ & 360 \\
\hline 4-Chloro-3-methylphenol & 340 & $\mathrm{U}$ & 340 & 350 & $\mathrm{U}$ & 350 & 330 & $\mathrm{U}$ & 330 & 360 & UJ & 360 \\
\hline 4-Chloroaniline & 340 & $\mathrm{U}$ & 340 & 350 & $\mathrm{U}$ & 350 & 330 & $\mathrm{U}$ & 330 & 360 & U & 360 \\
\hline 4-Chlorophenyl-phenylether & 340 & $\mathrm{U}$ & 340 & 350 & $\mathrm{U}$ & 350 & 330 & $\mathrm{U}$ & 330 & 360 & $\mathrm{U}$ & 360 \\
\hline 4-Methylphenol (p-cresol) & 340 & $\mathrm{U}$ & 340 & 350 & $\mathrm{U}$ & 350 & 330 & $\mathrm{U}$ & 330 & 360 & U & 360 \\
\hline 4-Nitroaniline & 860 & $\mathrm{U}$ & 860 & 870 & $\mathrm{U}$ & 870 & 830 & $\mathrm{U}$ & 830 & 900 & UR & 900 \\
\hline 4-Nitrophenol & 860 & $\mathrm{U}$ & 860 & 870 & $\mathrm{U}$ & 870 & 830 & $\mathrm{U}$ & 830 & 900 & $\mathrm{U}$ & 900 \\
\hline Acenaphthene & 340 & U & 340 & 350 & $\mathrm{U}$ & 350 & 330 & $\mathrm{U}$ & 330 & 360 & $\mathrm{U}$ & 360 \\
\hline Acenaphthylene & 340 & $\mathrm{U}$ & 340 & 350 & $\mathrm{U}$ & 350 & 330 & $\mathrm{U}$ & 330 & 360 & $\mathrm{U}$ & 360 \\
\hline Anthracene & 340 & U & 340 & 350 & $\mathrm{U}$ & 350 & 330 & $\mathrm{U}$ & 330 & 360 & $\mathrm{U}$ & 360 \\
\hline Benzo(a)anthracene & 340 & U & 340 & 350 & U & 350 & 330 & U & 330 & 360 & $\mathrm{U}$ & 360 \\
\hline Benzo(a)pyrene & 340 & $\mathrm{U}$ & 340 & 350 & $\mathrm{U}$ & 350 & 330 & $\mathrm{U}$ & 330 & 360 & $\bar{U}$ & 360 \\
\hline Benzo(b)fluoranthene & 340 & $\mathrm{U}$ & 340 & 350 & $\mathrm{U}$ & 350 & 330 & $\mathrm{U}$ & 330 & 360 & $\mathrm{U}$ & 360 \\
\hline Benzo(g,h,i)perylene & 340 & $\mathrm{U}$ & 340 & 350 & $\mathrm{U}$ & 350 & 330 & $\mathrm{U}$ & 330 & 360 & $\mathrm{U}$ & 360 \\
\hline Benzo(k)fluoranthene & 340 & $\mathrm{U}$ & 340 & 350 & $\mathrm{U}$ & 350 & 330 & $\mathrm{U}$ & 330 & 360 & $\mathrm{U}$ & 360 \\
\hline bis(2-Chloro-1-methylethyl)ether & 340 & $\mathrm{U}$ & 340 & 350 & $\mathrm{U}$ & 350 & 330 & $\mathrm{U}$ & 330 & 360 & $\mathrm{U}$ & 360 \\
\hline bis(2-Chloroethoxy)methane & 340 & $\mathrm{U}$ & 340 & 350 & $\mathrm{U}$ & 350 & 330 & $\mathrm{U}$ & 330 & 360 & $\mathrm{U}$ & 360 \\
\hline bis(2-Chloroethyl)ether & 340 & $\mathrm{U}$ & 340 & 350 & $\mathrm{U}$ & 350 & 330 & $\mathrm{U}$ & 330 & 360 & $\mathrm{U}$ & 360 \\
\hline bis(2-Ethylhexyl)phthalate & 42 & $\mathrm{JB}$ & 340 & 31 & $\mathrm{JB}$ & 350 & 26 & $\mathrm{JB}$ & 330 & 38 & JB & 360 \\
\hline
\end{tabular}

Attachment

Originator

Checked

Calc. No.

$\frac{1}{\frac{1}{\text { J.M. Capron }}}$

Sheet No. 109 of 113 Date $\frac{10 / 04 / 07}{107}$ Date

Rev. No. 
Attachment 1. 118-B-1 Verification Sampling Results.

\begin{tabular}{|c|c|c|c|c|c|c|c|c|c|c|c|c|}
\hline \multirow[t]{2}{*}{ Constituents } & \multicolumn{3}{|c|}{$\begin{array}{c}\text { J13J07 } \\
\text { SPA D5 } \\
\text { Sample Date 10/23/06 } \\
\end{array}$} & \multicolumn{3}{|c|}{$\begin{array}{c}\text { J13J08 } \\
\text { SPA D6 } \\
\text { Sample Date 10/23/06 } \\
\end{array}$} & \multicolumn{3}{|c|}{$\begin{array}{c}\text { J12R24 } \\
\text { FS-1 } \\
\text { Sample Date } 7 / 10 / 06 \\
\end{array}$} & \multicolumn{3}{|c|}{\begin{tabular}{|c|} 
J12R33 \\
FS-10 \\
Sample Date 12/7/06
\end{tabular}} \\
\hline & $\mu \mathrm{g} / \mathrm{kg}$ & $\mathbf{Q}$ & PQL & $\mu \mathrm{g} / \mathrm{kg}$ & $\mathbf{Q}$ & PQL & $\mu \mathrm{g} / \mathrm{kg}$ & $\mathbf{Q}$ & PQL & $\mu \mathrm{g} / \mathrm{kg}$ & $\mathbf{Q}$ & PQL \\
\hline \multicolumn{13}{|c|}{ Semivolatile Organic Compounds (continued) } \\
\hline Butylbenzylphthalate & 340 & U & 340 & 350 & U & 350 & 330 & $\mathrm{U}$ & 330 & 360 & $\mathrm{U}$ & 360 \\
\hline Carbazole & 340 & $\mathrm{U}$ & 340 & 350 & $\mathrm{U}$ & 350 & 330 & $\mathrm{U}$ & 330 & 360 & $\mathrm{U}$ & 360 \\
\hline Chrysene & 340 & $\mathrm{U}$ & 340 & 350 & $U$ & 350 & 330 & $U$ & 330 & 360 & $\mathrm{U}$ & 360 \\
\hline Di-n-butylphthalate & 34 & JB & 340 & 350 & $\mathrm{U}$ & 350 & 330 & $\mathrm{U}$ & 330 & 360 & $\mathrm{U}$ & 360 \\
\hline Di-n-octylphthalate & 340 & $\mathrm{U}$ & 340 & 350 & $\mathrm{U}$ & 350 & 330 & $\mathrm{U}$ & 330 & 360 & $\mathrm{U}$ & 360 \\
\hline Diben $z(a, h)$ anthracene & 340 & $\mathrm{U}$ & 340 & 350 & $\mathrm{U}$ & 350 & 330 & $\mathrm{U}$ & 330 & 360 & $\mathrm{U}$ & 360 \\
\hline Dibenzofuran & 340 & $\mathrm{U}$ & 340 & 350 & $\mathrm{U}$ & 350 & 330 & $\mathrm{U}$ & 330 & 360 & $\mathrm{U}$ & 360 \\
\hline Diethylphthalate & 340 & $\mathrm{U}$ & 340 & 350 & $\mathrm{U}$ & 350 & 330 & $\mathrm{U}$ & 330 & 360 & $\mathrm{U}$ & 360 \\
\hline Dimethylphthalate & 340 & $\mathrm{U}$ & 340 & 350 & $\mathrm{U}$ & 350 & 330 & $\mathrm{U}$ & 330 & 360 & $\mathrm{U}$ & 360 \\
\hline Fluoranthene & 340 & $\mathrm{U}$ & 340 & 350 & $\mathrm{U}$ & 350 & 330 & $\mathrm{U}$ & 330 & 360 & $\mathrm{U}$ & 360 \\
\hline Fluorene & 340 & $\mathrm{U}$ & 340 & 350 & $\mathrm{U}$ & 350 & 330 & $\mathrm{U}$ & 330 & 360 & $\mathrm{U}$ & 360 \\
\hline Hexachlorobenzene & 340 & $\mathrm{U}$ & 340 & 350 & U & 350 & 330 & $\mathrm{U}$ & 330 & 360 & $\mathrm{U}$ & 360 \\
\hline Hexachlorobutadiene & 340 & $\mathrm{U}$ & 340 & 350 & $\mathrm{U}$ & 350 & 330 & $\mathrm{U}$ & 330 & 360 & $\mathrm{U}$ & 360 \\
\hline Hexachlorocyclopentadiene & 340 & $\mathrm{U}$ & 340 & 350 & $\mathrm{U}$ & 350 & 330 & $\mathrm{U}$ & 330 & 360 & $\mathrm{U}$ & 360 \\
\hline Hexachloroethane & 340 & $\mathrm{U}$ & 340 & 350 & $\mathrm{U}$ & 350 & 330 & $\mathrm{U}$ & 330 & 360 & $\mathrm{U}$ & 360 \\
\hline Indeno(1,2,3-cd)pyrene & 340 & $\mathrm{U}$ & 340 & 350 & $\mathrm{U}$ & 350 & 330 & $\mathrm{U}$ & 330 & 360 & $\mathrm{U}$ & 360 \\
\hline Isophorone & 340 & $\mathrm{U}$ & 340 & 350 & $\mathrm{U}$ & 350 & 330 & $\mathrm{U}$ & 330 & 360 & $\mathrm{U}$ & 360 \\
\hline N-Nitroso-di-n-dipropylamine & 340 & $\mathrm{U}$ & 340 & 350 & $\mathrm{U}$ & 350 & 330 & U & 330 & 360 & $\mathrm{U}$ & 360 \\
\hline N-Nitrosodiphenylamine & 340 & $\mathrm{U}$ & 340 & 350 & $\mathrm{U}$ & 350 & 330 & $\mathrm{U}$ & 330 & 360 & $\mathrm{UJ}$ & 360 \\
\hline Naphthalene & 340 & $\mathrm{U}$ & 340 & 350 & $\mathrm{U}$ & 350 & 330 & $\mathrm{U}$ & 330 & 360 & $\mathrm{U}$ & 360 \\
\hline Nitrobenzene & 340 & $\mathrm{U}$ & 340 & 350 & $\mathrm{U}$ & 350 & 330 & $\mathrm{U}$ & 330 & 360 & UJ & 360 \\
\hline Pentachlorophenol & 860 & $\mathrm{U}$ & 860 & 870 & $\mathrm{U}$ & 870 & 830 & $\mathrm{U}$ & 830 & 900 & $\mathrm{U}$ & 900 \\
\hline Phenanthrene & 340 & $\mathrm{U}$ & 340 & 350 & $\mathrm{U}$ & 350 & 330 & $\mathrm{U}$ & 330 & 360 & $\mathrm{U}$ & 360 \\
\hline Phenol & 340 & $\mathrm{U}$ & 340 & 350 & $\mathrm{U}$ & 350 & 330 & $\mathrm{U}$ & 330 & 360 & $\mathrm{U}$ & 360 \\
\hline Pyrene & 340 & $\mathrm{U}$ & 340 & 350 & $\mathrm{U}$ & 350 & 330 & $\mathrm{U}$ & 330 & 360 & $\mathrm{U}$ & 360 \\
\hline & & & & Attachm & & & 1 & & & theet No. & 110 & of 113 \\
\hline & & & & Originat & & J.M. Car & oron & & & Date & & $04 / 07$ \\
\hline & & & & Checkec & & T.M. Bl & akley & & & Date & & \\
\hline & & & & Calc. No & & $0100 \mathrm{~B}-\mathrm{C}$ & $\mathrm{A}-\mathrm{V} 029 \mathrm{~S}$ & & & Rev. No. & & 1 \\
\hline
\end{tabular}


Attachment 1. 118-B-1 Verification Sampling Results.

\begin{tabular}{|c|c|c|c|c|c|c|c|c|c|c|c|c|}
\hline \multirow[t]{2}{*}{ Constituents } & \multicolumn{3}{|c|}{$\begin{array}{c}\text { J13J07 } \\
\text { SPA D5 } \\
\text { Sample Date 10/23/06 } \\
\end{array}$} & \multicolumn{3}{|c|}{\begin{tabular}{|c|} 
J13J08 \\
SPA D6 \\
Sample Date 10/23/06 \\
\end{tabular}} & \multicolumn{3}{|c|}{$\begin{array}{c}\text { J12R24 } \\
\text { FS-1 } \\
\text { Sample Date 7/10/06 }\end{array}$} & \multicolumn{3}{|c|}{\begin{tabular}{|c|} 
J12R33 \\
FS-10 \\
Sample Date 12/7/06
\end{tabular}} \\
\hline & $\mu \mathrm{g} / \mathrm{kg}$ & $\mathbf{Q}$ & PQL & $\mu \mathrm{g} / \mathrm{kg}$ & $Q$ & PQL & $\mu \mathrm{g} / \mathrm{kg}$ & $\mathbf{Q}$ & PQL & $\mu \mathrm{g} / \mathrm{kg}$ & $Q$ & PQL \\
\hline \multicolumn{13}{|c|}{ Volatile Organic Compounds } \\
\hline 1,1,1-Trichloroethane & 5 & $\mathrm{U}$ & 5 & 5 & $\bar{U}$ & 5 & 5 & $\bar{U}$ & 5 & 5 & $\mathrm{U}$ & 5 \\
\hline 1,1,2,2-Tetrachloroethane & 5 & $\mathrm{U}$ & 5 & 5 & $\bar{U}$ & 5 & 5 & $\mathrm{U}$ & 5 & 5 & $\mathrm{UJ}$ & 5 \\
\hline 1,1,2-Trichloroethane & 5 & $\mathrm{U}$ & 5 & 5 & $\mathrm{U}$ & 5 & 5 & $\mathrm{U}$ & 5 & 5 & UJ & 5 \\
\hline 1,1-Dichloroethane & 5 & $\mathrm{U}$ & 5 & 5 & $\bar{U}$ & 5 & 5 & $\bar{U}$ & 5 & 5 & $U$ & 5 \\
\hline 1,1-Dichloroethene & 5 & $\mathrm{U}$ & 5 & 5 & $\overline{\mathrm{U}}$ & 5 & 5 & $\bar{U}$ & 5 & 5 & $\mathrm{U}$ & 5 \\
\hline 1,2-Dichloroethane & 5 & $\mathrm{U}$ & 5 & 5 & $\mathrm{U}$ & 5 & 5 & $\mathrm{U}$ & 5 & 5 & $\mathrm{U}$ & 5 \\
\hline 1,2-Dichloroethene (total) & 5 & $\mathrm{U}$ & 5 & 5 & $\mathrm{U}$ & 5 & 5 & $U$ & 5 & 5 & $\mathrm{U}$ & 5 \\
\hline 1,2-Dichloropropane & 5 & $\mathrm{U}$ & 5 & 5 & $\mathrm{U}$ & 5 & 5 & $\mathrm{U}$ & 5 & 5 & $U$ & 5 \\
\hline 2-Butanone & 10 & $\mathrm{U}$ & 10 & 10 & $\mathrm{U}$ & 10 & 10 & $\mathrm{U}$ & 10 & 10 & $\mathrm{U}$ & 10 \\
\hline 2-Hexanone & 10 & $\mathrm{U}$ & 10 & 10 & $\mathrm{U}$ & 10 & 10 & $\mathrm{U}$ & 10 & 10 & $\mathrm{U}$ & 10 \\
\hline 4-Methyl-2-pentanone & 10 & $\mathrm{U}$ & 10 & 10 & $\mathrm{U}$ & 10 & 10 & $\mathrm{U}$ & 10 & 10 & UJ & 10 \\
\hline Acetone & 10 & $\mathrm{U}$ & 10 & 10 & $\mathrm{U}$ & 10 & 99 & & 10 & 39 & $\mathrm{~B}$ & 10 \\
\hline Benzene & 5 & $\mathrm{U}$ & 5 & 5 & $\mathrm{U}$ & 5 & 5 & $\mathrm{U}$ & 5 & 5 & $\mathrm{U}$ & 5 \\
\hline Bromodichloromethane & 5 & $\mathrm{U}$ & 5 & 5 & $\mathrm{U}$ & 5 & 5 & U & 5 & 5 & $\mathrm{U}$ & 5 \\
\hline Bromoform & 5 & $\mathrm{U}$ & 5 & 5 & $\mathrm{U}$ & 5 & 5 & U & 5 & 5 & UJ & 5 \\
\hline Bromomethane & 10 & $\mathrm{U}$ & 10 & 10 & $\mathrm{U}$ & 10 & 10 & $\mathrm{U}$ & 10 & 10 & $\mathrm{U}$ & 10 \\
\hline Carbon disulfide & 5 & $\mathrm{U}$ & 5 & 5 & $U$ & 5 & 5 & $\mathrm{U}$ & 5 & 5 & $\mathrm{U}$ & 5 \\
\hline Carbon tetrachloride & 5 & U & 5 & 5 & $\mathrm{U}$ & 5 & 5 & $U$ & 5 & 5 & $U$ & 5 \\
\hline Chlorobenzene & 5 & $\mathrm{U}$ & 5 & 5 & U & 5 & 5 & U & 5 & 5 & $\mathrm{U}$ & 5 \\
\hline Chloroethane & 10 & $U$ & 10 & 10 & $\mathrm{U}$ & 10 & 10 & $\mathrm{U}$ & 10 & 10 & $\mathrm{U}$ & 10 \\
\hline Chloroform & 5 & $\bar{U}$ & 5 & 5 & $\mathrm{U}$ & 5 & 5 & $\mathrm{U}$ & 5 & 5 & $\mathrm{U}$ & 5 \\
\hline Chloromethane & 10 & $\mathrm{U}$ & 10 & 10 & $\mathrm{U}$ & 10 & 10 & $\mathrm{U}$ & 10 & 10 & $\mathrm{U}$ & 10 \\
\hline cis-1,2-Dichloroethylene & 5 & $\mathrm{U}$ & 5 & 5 & $\mathrm{U}$ & 5 & 5 & $\mathrm{U}$ & 5 & 5 & $\mathrm{U}$ & 5 \\
\hline cis-1,3-Dichloropropene & 5 & $\mathrm{U}$ & 5 & 5 & $\mathrm{U}$ & 5 & 5 & $\mathrm{U}$ & 5 & 5 & U & 5 \\
\hline Dibromochloromethane & 5 & $\mathrm{U}$ & 5 & 5 & $\mathrm{U}$ & 5 & 5 & $\mathrm{U}$ & 5 & 5 & UJ & 5 \\
\hline Ethylbenzene & 5 & $\mathrm{U}$ & 5 & 5 & $\mathrm{U}$ & 5 & 5 & $\mathrm{U}$ & 5 & 5 & $\mathrm{U}$ & 5 \\
\hline Methylene chloride & 11 & $\mathrm{~B}$ & 5 & 9 & $\mathrm{~B}$ & 5 & 16 & $\mathrm{BJ}$ & 5 & 14 & $\mathrm{~B}$ & 5 \\
\hline Styrene & 5 & $\mathrm{U}$ & 5 & 5 & $\mathrm{U}$ & 5 & 5 & $\mathrm{U}$ & 5 & 5 & $\mathrm{U}$ & 5 \\
\hline Tetrachloroethene & 5 & $\mathrm{U}$ & 5 & 5 & $\mathrm{U}$ & 5 & 5 & $\mathrm{U}$ & 5 & 5 & $\mathrm{U}$ & 5 \\
\hline Toluene & 5 & $\mathrm{U}$ & 5 & 5 & $\mathrm{U}$ & 5 & 5 & $\mathrm{U}$ & 5 & 5 & $\mathrm{U}$ & 5 \\
\hline trans-1,2-Dichloroethylene & 5 & $\mathrm{U}$ & 5 & 5 & $\mathrm{U}$ & 5 & 5 & $\mathrm{U}$ & 5 & 5 & $\mathrm{U}$ & 5 \\
\hline trans-1,3-Dichloropropene & 5 & $\mathrm{U}$ & 5 & 5 & $\mathrm{U}$ & 5 & 5 & $\mathrm{U}$ & 5 & 5 & UJ & 5 \\
\hline Trichloroethene & 5 & $\mathrm{U}$ & 5 & 5 & $\mathrm{U}$ & 5 & 5 & $\mathrm{U}$ & 5 & 5 & $\mathrm{U}$ & 5 \\
\hline Vinyl chloride & 10 & $\mathrm{U}$ & 10 & 10 & $\mathrm{U}$ & 10 & 10 & $\mathrm{U}$ & 10 & 10 & $\mathrm{U}$ & 10 \\
\hline Xylenes (total) & 5 & $\mathrm{U}$ & 5 & 5 & $\mathrm{U}$ & 5 & 5 & $\mathrm{U}$ & 5 & 5 & $\mathrm{U}$ & 5 \\
\hline & & & & Attachm & & & 1 & & & Sheet No. & & 1 of 113 \\
\hline & & & & Originat & & J.M. Ca & pron & & - & Date & & $0 / 04 / 07$ \\
\hline & & & & Checked & & $\overline{\text { T.M. Bl }}$ & akley & & & Date & & \\
\hline & & & & Calc. No & & $\overline{0100 \mathrm{~B}-\mathrm{C}}$ & A-V029 & & & Rev. No. & & 1 \\
\hline
\end{tabular}


Attachment 1. 118-B-1 Verification Sampling Results.

\begin{tabular}{|c|c|c|c|c|c|c|c|c|c|}
\hline \multirow[t]{2}{*}{ Constituents } & \multicolumn{3}{|c|}{\begin{tabular}{|c|} 
J13J07 \\
SPA D5 \\
Sample Date $10 / 23 / 06$
\end{tabular}} & \multicolumn{3}{|c|}{$\begin{array}{c}\text { J13J08 } \\
\text { SPA D6 } \\
\text { Sample Date 10/23/06 }\end{array}$} & \multicolumn{3}{|c|}{$\begin{array}{c}\text { J12R24 } \\
\text { FS-1 } \\
\text { Sample Date 7/10/06 }\end{array}$} \\
\hline & $\mu \mathrm{g} / \mathrm{kg}$ & $Q$ & PQL & $\mu \mathrm{g} / \mathrm{kg}$ & $\mathbf{Q}$ & PQL & $\mu \mathrm{g} / \mathrm{kg}$ & $\mathbf{Q}$ & PQL \\
\hline \multicolumn{10}{|c|}{ Pesticides } \\
\hline Aldrin & 1.4 & UD & 1.4 & 1.4 & UD & 1.4 & 1.3 & UDJ & 1.3 \\
\hline alpha-BHC & 1.4 & UD & 1.4 & 1.4 & UD & 1.4 & 1.3 & UDJ & 1.3 \\
\hline alpha-Chlordane & 1.4 & UD & 1.4 & 1.4 & UD & 1.4 & 1.3 & UD & 1.3 \\
\hline beta-BHC & 1.4 & UD & 1.4 & 1.4 & UD & 1.4 & 1.3 & UD & 1.3 \\
\hline delta-BHC & 1.4 & UD & 1.4 & 1.4 & UD & 1.4 & 1.3 & UDJ & 1.3 \\
\hline Dichlorodiphenyldichloroethane & 1.4 & UD & 1.4 & 1.4 & UD & 1.4 & 1.3 & UD & 1.3 \\
\hline Dichlorodiphenyldichloroethylene & 1.4 & UD & 1.4 & 1.4 & UD & 1.4 & 1.3 & UDJ & 1.3 \\
\hline Dichlorodiphenyltrichloroethane & 1.4 & UD & 1.4 & 1.4 & UD & 1.4 & 1.3 & UD & 1.3 \\
\hline Dieldrin & 1.4 & UD & 1.4 & 1.4 & UD & 1.4 & 1.3 & UD & 1.3 \\
\hline Endosulfan I & 1.4 & UD & 1.4 & 1.4 & UD & 1.4 & 1.3 & UD & 1.3 \\
\hline Endosulfan II & 1.4 & UD & 1.4 & 1.4 & UD & 1.4 & 1.3 & UD & 1.3 \\
\hline Endosulfan sulfate & 0.79 & $\mathrm{JD}$ & 1.4 & 0.73 & $\mathrm{JD}$ & 1.4 & 1.3 & UDJ & 1.3 \\
\hline Endrin & 1.4 & UD & 1.4 & 1.4 & UD & 1.4 & 1.3 & UDJ & 1.3 \\
\hline Endrin aldehyde & 1.4 & UD & 1.4 & 1.4 & UD & 1.4 & 1.3 & UDJ & 1.3 \\
\hline Endrin ketone & 1.4 & UD & 1.4 & 1.4 & UD & 1.4 & 1.3 & UD & 1.3 \\
\hline gamma-BHC (Lindane) & 1.4 & UD & 1.4 & 1.4 & UD & 1.4 & 1.3 & UDJ & 1.3 \\
\hline gamma-Chlordane & 1.4 & UD & 1.4 & 1.4 & UD & 1.4 & 1.3 & UD & 1.3 \\
\hline Heptachlor & 1.4 & UD & 1.4 & 1.4 & UD & 1.4 & 1.3 & UDJ & 1.3 \\
\hline Heptachlor epoxide & 1.4 & UD & 1.4 & 1.4 & UD & 1.4 & 1.3 & $\mathrm{UD}$ & 1.3 \\
\hline Methoxychlor & 1.7 & $\mathrm{D}$ & 1.4 & 1.4 & UD & 1.4 & 4.7 & $\mathrm{D}$ & 1.3 \\
\hline Toxaphene & 14 & UD & 14 & 14 & UD & 14 & 13 & UD & 13 \\
\hline \multicolumn{10}{|c|}{ Herbicides } \\
\hline $2,4,5-\mathrm{T}$ & 17 & $\mathrm{U}$ & 17 & 17 & $\mathrm{U}$ & 17 & 17 & UJ & 17 \\
\hline $2,4-\mathrm{D}$ & 34 & $\mathrm{U}$ & 34 & 13 & $\mathrm{~J}$ & 35 & 33 & UJ & 33 \\
\hline $2,4,5-\mathrm{TP}$ & 11 & $\mathrm{~J}$ & 17 & 17 & $\mathrm{U}$ & 17 & 17 & $U$ & 17 \\
\hline Dinoseb & 17 & $\mathrm{U}$ & 17 & 17 & $\mathrm{U}$ & 17 & 17 & $\mathrm{U}$ & 17 \\
\hline $2,4-\mathrm{DB}$ & 17 & $\mathrm{U}$ & 17 & 17 & U & 17 & 17 & UJ & 17 \\
\hline Dalapon & 34 & $\mathrm{U}$ & 34 & 35 & $\mathrm{U}$ & 35 & 33 & $U$ & 33 \\
\hline Dicamba & 7.9 & $\mathrm{~J}$ & 34 & 27 & $\mathrm{~J}$ & 35 & 9.1 & $\mathrm{~J}$ & 33 \\
\hline Dichloroprop & 34 & $\mathrm{U}$ & 34 & 35 & $\mathrm{U}$ & 35 & 33 & $\mathrm{UJ}$ & 33 \\
\hline Pentachlorophenol & 4.0 & $\mathrm{~J}$ & 17 & 17 & $\mathrm{U}$ & 17 & 17 & $\mathrm{U}$ & 17 \\
\hline & \multirow{4}{*}{\multicolumn{2}{|c|}{$\begin{array}{l}\text { Attachment } \\
\text { Originator } \\
\text { Checked } \\
\text { Calc. No. }\end{array}$}} & \multicolumn{3}{|c|}{1} & \multirow{4}{*}{\multicolumn{2}{|c|}{$\begin{array}{r}\text { Sheet No. } \\
\text { Date } \\
\text { Date } \\
\text { Rev. No. }\end{array}$}} & \multicolumn{2}{|c|}{112 of 113} \\
\hline & & & \multicolumn{3}{|c|}{ J.M. Capron } & & & \multicolumn{2}{|c|}{$10 / 04 / 07$} \\
\hline & & & \multicolumn{3}{|c|}{ T.M. Blakley } & & & & \\
\hline & & & $0100 \mathrm{~B}-\mathrm{C}$ & $\mathrm{A}-\mathrm{V} 0299$ & & & & & 1 \\
\hline
\end{tabular}


Attachment 1. 118-B-1 Verification Sampling Results.

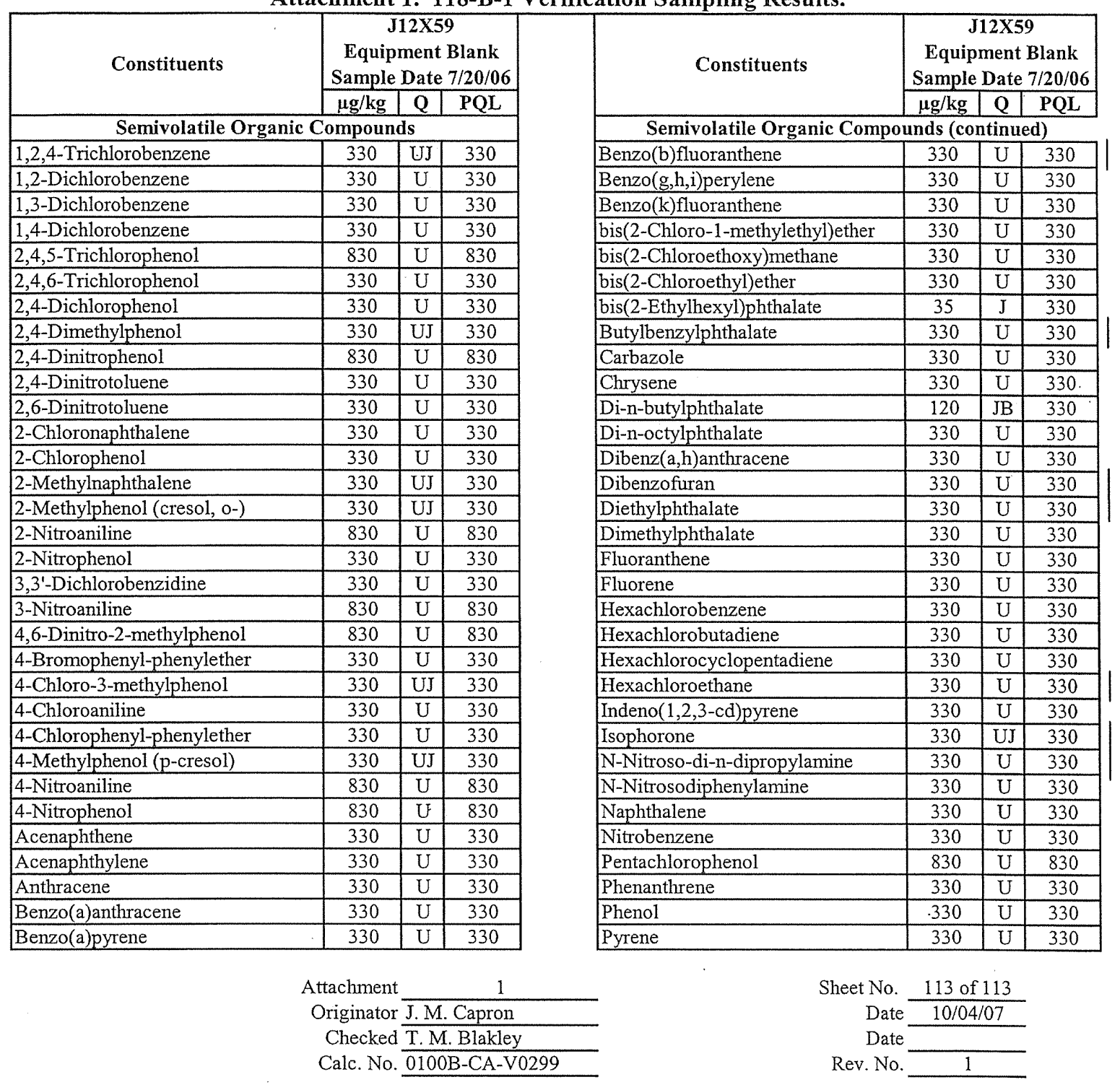




\section{CALCULATION COVER SHEET}

Project Title: $100-\mathrm{B} / \mathrm{C}$ Field Remediation

Job No. 14655

Area: $100-B / C$

Discipline: Environmental

${ }^{*}$ Calculation No: 0100B-CA-V0300

Subject: 118-B-1 Burial Ground Hazard Quotient and Carcinogenic Risk Calculations

Computer Program: Excel

Program No: Excel 2003

The attached calculations have been generated to document compliance with established cleanup levels. These calculations should be used in conjunction with other relevant documents in the administrative record.

Committed Calculation $\otimes \quad$ Preliminary $\square \quad$ Superseded $\square \quad$ Voided

\begin{tabular}{|c|c|c|c|c|c|c|}
\hline Rev. & Sheet Numbers & Originator & Ghecker & Reviewer & Approval & Date \\
\hline 0 & $\begin{array}{c}\text { Cover }=1 \\
\text { Sheets }=4 \\
\text { Total }=5\end{array}$ & J. M. Capron & S. W. Clark & N/A & D. N. Strom & $2 / 8 / 07$ \\
\hline 1 & $\begin{array}{c}\text { Cover }=1 \\
\text { Sheets }=4 \\
\text { Total }=5\end{array}$ & J.M. Capron & T.M. Blakley & N/A & D. N. Strom & $10 / 4 / 07$ \\
\hline 2 & $\begin{array}{c}\text { Cover }=1 \\
\text { Sheets }=4 \\
\text { Total }=5\end{array}$ & $\begin{array}{l}\text { J.M. Capron } \\
0,13\end{array}$ & $\begin{array}{c}\text { S.W. Clark } \\
\text { \&.w. exan }\end{array}$ & $\mathrm{N} / \mathrm{A}$ & sevista & $12-3-07$ \\
\hline & & & & & & \\
\hline & & & & & & \\
\hline & & & & & & \\
\hline & & & & & & \\
\hline
\end{tabular}

SUMMARY OF REVISION

\begin{tabular}{|c|l|}
\hline 1 & $\begin{array}{l}\text { Revision to include additional focused sample data. Cover page replaced. Sheet } 1 \text { revised to reference revised UCL calculation. } \\
\text { Sheet } 2 \text { revised to remove identification of specific focused samples. Sheet } 3 \text { updated with higher mercury value; cumulative } \\
\text { calculations on Sheet } 4 \text { and summary statement on Sheet } 2 \text { revised accordingly. }\end{array}$ \\
\hline 2 & $\begin{array}{l}\text { Revised Sheet } 3 \text { to use updated RAG values for trichloroethene; there is no impact to the summed hazard quotient or excess } \\
\text { carcinogenic risk values. Replaced cover page. }\end{array}$ \\
\hline WCH-DE-0 & $805 / 08 / 2007)$ \\
*Obtain Calc. No. from Document Control and Form from Intranet
\end{tabular}




\section{PURPOSE:}

Provide documentation to support the calculation of the hazard quotient (HQ) and excess carcinogenic risk values for the 118-B-1 burial ground remediation verification sampling results. In accordance with the remedial action goals (RAGs) in the remedial design report/remedial action work plan (RDR/RAWP) (DOE-RL 2005), the following criteria must be met:

1) An HQ of $<1.0$ for all individual noncarcinogens

2) A cumulative HQ of $<1.0$ for noncarcinogens

3) An excess carcinogenic risk of $<1 \times 10^{-6}$ for individual carcinogens

4) A cumulative excess carcinogenic risk of $<1 \times 10^{-5}$ for carcinogens.

\section{GIVEN/REFERENCES:}

1) DOE-RL, 2005, Remedial Design Report/Remedial Action Work Plan for the 100 Areas, DOE/RL-96-17, Rev. 5, U.S. Department of Energy, Richland Operations Office, Richland, Washington.

2) EPA, 1994, Guidance Manual for the Integrated Exposure Uptake Biokinetic Model for Lead in Children, EPA/540/R-93/081, Publication No. 9285.7-15-1, U.S. Environmental Protection Agency, Washington, D.C.

3) WAC 173-340, "Model Toxics Control Act - Cleanup," Washington Administrative Code, 1996.

4) WCH, 2007, 118-B-1 Burial Ground Cleanup Verification 95\% UCL Calculations, Calculation No. 0100B-CA-V0299, Rev. 1. Washington Closure Hanford, Richland, Washington.

\section{SOLUTION:}

1) Calculate an HQ for each noncarcinogenic constituent detected above background and compare to the individual HQ of $<1.0$ (DOE-RL 2005).

2) Sum the HQs and compare to the cumulative HQ criterion of $<1.0$.

3) Calculate an excess carcinogenic risk value for each carcinogenic constituent detected above background and compare to the individual excess carcinogenic risk criterion of $<1 \times 10^{-6}$ (DOE-RL 2005).

4) Sum the excess carcinogenic risk values and compare to the cumulative excess carcinogenic risk criterion of $<1 \times 10^{-5}$. 
Washington Closure Hanford CALCULATION SHEET

\begin{tabular}{|c|c|c|c|c|c|c|c|}
\hline Originator: & J.M. Capron GARC & Date: & $10 / 04 / 07$ & Calc. No.: & 0100B-CA-V0300 & Rev: & 1 \\
\hline Project: & 100-B/C Field Remediation & Job No: & 14655 & Checked: & T. M. Blakley $2 x \rho$ & Date: & $10 / 04 / 07$ \\
\hline
\end{tabular}

\section{METHODOLOGY:}

Hazard quotient and carcinogenic risk calculations were performed for the 118-B-1 burial ground using the highest statistical value from all sampling areas or individual detections in focused samples for each analyte detected above background. Of the contaminants of concern (COCs) and contaminants of potential concern (COPCs) for the site, boron and molybdenum require the HQ calculations because they were detected and Washington State or Hanford Site background values are not available. Barium, copper, lead, mercury, and selenium are included because they were quantified above their respective Hanford Site background values. Hexavalent chromium and multiple organic compounds (as identified in Table 1) are included because they were detected by laboratory analysis and cannot be attributed to natural occurrence. Arsenic was detected above the Hanford Site background value but below the WAC 173-340 Method A cleanup level. Due to the intent of Method A cleanup values and the allowance to use such values for arsenic (DOE-RL 2005), arsenic has been excluded from the Method B individual analyte and cumulative risk requirements. All other site nonradionuclide COCs and COPCs were not detected or were detected below background levels. An example of the HQ and risk calculations is presented below:

1) For example, the statistical value for boron is $24.5 \mathrm{mg} / \mathrm{kg}$, divided by the noncarcinogenic RAG value of $16,000 \mathrm{mg} / \mathrm{kg}$ (calculated in accordance with the noncarcinogenic toxics effects formula in WAC 173-340-740[3]), is $1.5 \times 10^{-3}$. Comparing this value, and all other individual values, to the requirement of $<1.0$, this criterion is met.

2) After the HQ calculations are completed for the appropriate analytes, the cumulative HQ is obtained by summing the individual values. (To avoid errors due to intermediate rounding, the individual HQ values prior to rounding are used for this calculation.) The sum of the HQ values is $\underline{8.8} \times 10^{-1}$. Comparing this value to the requirement of $<1.0$, this criterion is met.

3) To calculate the excess carcinogenic risk, the $95 \%$ upper confidence limit or maximum value is divided by the carcinogenic RAG value, then multiplied by $1 \times 10^{-6}$. For example, the maximum statistical value for hexavalent chromium is $0.29 \mathrm{mg} / \mathrm{kg}$; divided by $2.1 \mathrm{mg} / \mathrm{kg}$ and multiplied as indicated is $1.4 \times 10^{-7}$. Comparing this value, and all other individual values, to the requirement of $<1 \times 10^{-6}$, this criterion is met.

4) After these calculations are completed for the carcinogenic analytes, the cumulative excess carcinogenic risk is obtained by summing the individual values. (To avoid errors due to intermediate rounding, the individual values prior to rounding are used for this calculation.) The sum of the excess carcinogenic risk values is $8.0 \times 10^{-7}$. Comparing this value to the requirement of $<1 \times 10^{-5}$, this criterion is met.

\section{RESULTS:}

1) List individual noncarcinogens and corresponding $\mathrm{HQs}>1.0$ : None

2) List the cumulative noncarcinogenic $\mathrm{HQ}>1.0$ : None

3) List individual carcinogens and corresponding excess cancer risk $>1 \times 10^{-6}$ : None

4) List the cumulative excess cancer risk for carcinogens $>1 \times 10^{-5}$ : None. 
Washington Closure Hanford

CALCULATION SHEET

Originator: J. M. Capron $0 \% \mathrm{NC}$

\begin{tabular}{l|l|l|l|l|l} 
Project: & $100-\mathrm{B} / \mathrm{C}$ Field Remediation & Job No: & 14655 & Checked: & S. W. Clark $\mathrm{S} / \mathrm{C}$ \\
\hline
\end{tabular}

Subject: 118 -B-1 Burial Ground Hazard Quotient and Carcinogenic Risk Calculations

Date: $1 / 2 / 59 / 07$

Sheet No. $3^{6}$ of 4

1

7

Table 1 shows the results of the calculations for the 118-B-1 burial ground.

Table 1. Hazard Quotient and Excess Cancer Risk Results for the 118-B-1 Burial Ground. (2 Pages)

\begin{tabular}{|c|c|c|c|c|c|}
\hline $\begin{array}{l}\text { Contaminants of Concern/ } \\
\text { Contaminants of Potential Concern }\end{array}$ & $\begin{array}{c}\text { Maximum or } \\
\text { Statistical Value } \\
(\mathrm{mg} / \mathrm{kg})\end{array}$ & $\begin{array}{c}\text { Noncarcinogen } \\
\mathbf{R A G}^{\mathbf{b}} \\
(\mathrm{mg} / \mathrm{kg})\end{array}$ & $\begin{array}{l}\text { Hazard } \\
\text { Quotient }\end{array}$ & $\begin{array}{c}\text { Carcinogen } \\
\text { RAG }^{\mathrm{b}} \\
(\mathrm{mg} / \mathrm{kg})\end{array}$ & $\begin{array}{l}\text { Carcinogen } \\
\quad \text { Risk }\end{array}$ \\
\hline \multicolumn{6}{|c|}{ Metals } \\
\hline Barium & 261 & 5,600 & $4.7 \mathrm{E}-02$ & - & -- \\
\hline Boron & 24.5 & 16,000 & $1.5 \mathrm{E}-03$ & - & -- \\
\hline Chromium, hexavalent ${ }^{\mathrm{c}}$ & 0.29 & 240 & $1.2 \mathrm{E}-03$ & 2.1 & $1.4 \mathrm{E}-07$ \\
\hline Copper & 28.0 & 2,960 & $9.5 \mathrm{E}-03$ & - & - \\
\hline Lead $^{\mathrm{d}}$ & 34.3 & 353 & $9.7 \mathrm{E}-02$ & - & -- \\
\hline Mercury & 14.5 & 24 & $6.0 \mathrm{E}-01$ & - & - \\
\hline Molybdenum & 2.1 & 400 & $5.3 \mathrm{E}-03$ & - & -- \\
\hline Selenium & 1.0 & 400 & $2.5 \mathrm{E}-03$ & $\ldots$ & - \\
\hline \multicolumn{6}{|c|}{ Semivolatiles } \\
\hline Bis(2-ethylhexyl) phthalate & 0.41 & 1,600 & $2.6 \mathrm{E}-04$ & 71.4 & $5.7 \mathrm{E}-09$ \\
\hline Butylbenzylphthalate & 0.024 & 16,000 & $1.5 \mathrm{E}-06$ & -- & -- \\
\hline Diethylphthalate & 0.025 & 64,000 & $3.9 \mathrm{E}-07$ & $\ldots$ & -- \\
\hline Di-n-butylphthalate & 0.16 & 8,000 & $2.0 \mathrm{E}-05$ & - & - \\
\hline Methylnaphthalene; 2- & 0.026 & 320 & $8.1 \mathrm{E}-05$ & - & $-\cdots$ \\
\hline Naphthalene & 0.019 & 1,600 & $1.2 \mathrm{E}-05$ & - & - \\
\hline Phenol & 0.17 & 24,000 & $7.1 \mathrm{E}-06$ & - & -- \\
\hline \multicolumn{6}{|l|}{ Pesticides } \\
\hline Aldrin & 0.0010 & 2.40 & $4.2 \mathrm{E}-04$ & 0.0588 & $1.7 \mathrm{E}-08$ \\
\hline BHC, beta- & 0.0078 & $\cdots$ & -- & 0.556 & $1.4 \mathrm{E}-08$ \\
\hline Chlordane (alpha and gamma) & 0.0185 & 40 & $4.6 \mathrm{E}-04$ & 2.86 & $6.5 \mathrm{E}-09$ \\
\hline DDD, 4,4'- & 0.0019 & - & - & 4.17 & $4.6 \mathrm{E}-10$ \\
\hline DDE, 4,4'- & 0.016 & - & - & 2.94 & $5.4 \mathrm{E}-09$ \\
\hline DDT, $4,4^{\prime}-$ & 0.0016 & 40 & $4.0 \mathrm{E}-05$ & 2.94 & $5.4 \mathrm{E}-10$ \\
\hline Dieldrin & 0.0038 & 4 & $9.5 \mathrm{E}-04$ & 0.0625 & $6.1 \mathrm{E}-08$ \\
\hline Endosulfan (I, II, sulfate) & 0.0077 & 480 & 1.6E-05 & - & - \\
\hline Endrin (and ketone, aldehyde) & 0.0148 & 24 & $6.2 \mathrm{E}-04$ & -- & - \\
\hline Heptachlor & 0.00043 & 40 & $1.1 \mathrm{E}-05$ & 0.222 & $1.9 \mathrm{E}-09$ \\
\hline Methoxychlor & 0.0091 & 400 & $2.3 \mathrm{E}-05$ & -- & $\ldots$ \\
\hline \multicolumn{6}{|l|}{ Polychlorinated Biphenyls } \\
\hline Aroclor-1242 & 0.047 & $\sim$ & -- & 0.5 & $9.4 \mathrm{E}-08$ \\
\hline Aroclor- 1254 & 0.17 & 1.6 & $1.1 \mathrm{E}-01$ & 0.5 & $3.4 \mathrm{E}-07$ \\
\hline Aroclor- 1260 & 0.056 & -- & - & 0.5 & $1.1 \mathrm{E}-07$ \\
\hline \multicolumn{6}{|l|}{ Volatile Organic Compounds } \\
\hline Acetone & 0.099 & 72,000 & $1.4 \mathrm{E}-06$ & - & -- \\
\hline Carbon tetrachloride & 0.016 & 56 & $2.9 \mathrm{E}-04$ & 7.7 & $2.1 \mathrm{E}-09$ \\
\hline Methyl-2-pentanone; 4- & 0.011 & 6,400 & $1.7 \mathrm{E}-06$ & - & - \\
\hline Methylene chloride & 0.019 & 4,800 & $4.0 \mathrm{E}-06$ & 133 & $1.4 \mathrm{E}-10$ \\
\hline Trichloroethene & 0.002 & 24 & $8.3 \mathrm{E}-05$ & 2.5 & $8.0 \mathrm{E}-10$ \\
\hline Toluene & 0.002 & 6,400 & $3.1 \mathrm{E}-07$ & - & -- \\
\hline Xylenes (total) & 0.001 & 16,000 & $6.3 \mathrm{E}-08$ & $\ldots$ & - \\
\hline
\end{tabular}


Washington Closure Hanford CALCULATION SHEET

\begin{tabular}{|c|c|c|c|c|c|c|c|}
\hline Originator: & J.M. Capron Gyim C. & Date: & $10 / 04 / 07$ & Calc. No.: & 0100B-CA-V0300 & Rev.: & 1 \\
\hline Project: & 100-B/C Field Remediation & Job No: & 14655 & Checked: & T. M. Blakley $2 \mathrm{~m} B$ & Date: & $10 / 04 / 07$ \\
\hline Subject: & \multicolumn{5}{|c|}{ 118-B-1 Burial Ground Hazard Quotient and Carcinogenic Risk Calculations } & \multicolumn{2}{|c|}{ Sheet No. 4 of 4} \\
\hline
\end{tabular}

4

7

8

9

11

12

13

14

Table 1. Hazard Quotient and Excess Cancer Risk Results for the 118-B-1 Burial Ground. (2 Pages)

\begin{tabular}{|c|c|c|c|c|c|}
\hline $\begin{array}{l}\text { Contaminants of Concern/ } \\
\text { Contaminants of Potential Concern }\end{array}$ & $\begin{array}{c}\text { Maximum or } \\
\text { Statistical Value } \\
(\mathrm{mg} / \mathrm{kg})\end{array}$ & $\begin{array}{c}\text { Noncarcinogen } \\
\text { RAG }^{\mathbf{b}} \\
(\mathrm{mg} / \mathrm{kg})\end{array}$ & $\begin{array}{l}\text { Hazard } \\
\text { Quotient }\end{array}$ & $\begin{array}{c}\text { Carcinogen } \\
\text { RAG }^{\mathbf{b}} \\
(\mathrm{mg} / \mathrm{kg})\end{array}$ & $\begin{array}{l}\text { Carcinogen } \\
\text { Risk }\end{array}$ \\
\hline \multicolumn{6}{|c|}{ Herbrafes } \\
\hline $2,4-D$ & 0.062 & 800 & $7.8 \mathrm{E}-05$ & - & - \\
\hline 2,4-DB & 0.087 & 640 & $1.4 \mathrm{E}-04$ & $\ldots$ & - \\
\hline $2,4,5-\mathrm{T}$ & 0.013 & 800 & $1.6 \mathrm{E}-05$ & - & -- \\
\hline $2,4,5-\mathrm{TP}$ & 0.043 & 640 & $6.7 \mathrm{E}-05$ & - & - \\
\hline Dalapon & 0.048 & 2,400 & $2.0 \mathrm{E}-05$ & - & - \\
\hline Dicamba & 0.023 & 2,400 & $9.6 \mathrm{E}-06$ & - & - \\
\hline Dichloroprop & 0.21 & - & - & -- & - \\
\hline Pentachlorophenol & 0.016 & 2,400 & $6.7 \mathrm{E}-06$ & 8.33 & $1.9 \mathrm{E}-09$ \\
\hline Picloram & 0.014 & 5,600 & $2.5 \mathrm{E}-06$ & - & - \\
\hline \multicolumn{6}{|c|}{ polals } \\
\hline \multicolumn{3}{|c|}{ Curmulative Hazard Quotient: } & $8.8 \mathrm{E}-01$ & & \\
\hline \multicolumn{5}{|l|}{ Cumulative Excess Cancer Risk: } & 8.0E-07 \\
\hline
\end{tabular}

Notes:

${ }^{a}=$ From WCH (2007).

${ }^{b}=$ Value obtained from Washington Administrative Code (WAC) 173-340-740(3), Method B, 1996, unless otherwise noted.

${ }^{c}=$ Value for the carcinogen RAG calculated based on the inhalation exposure pathway (WAC) 173-340-750(3), 1996

$\stackrel{d}{a}$ Value for the noncarcinogen RAG obtained from EPA (1994).

-. = not applicable

$\mathrm{RAG}=$ remedial action goal

\section{CONCLUSION:}

This calculation demonstrates that the 118-B-1 burial ground meets the requirements for hazard quotient and excess carcinogenic risk as identified in the RDR/RAWP (DOE-RL 2005). 


\section{CALCULATION COVER SHEET}

Project Title 118-B-1 Burial Ground Cleanup Verification Job No.

Area $100 \mathrm{~B} / \mathrm{C}$ Area

Discipline Environmental

$*$ Calc. No. 0100B-CA-V0301

Subject

118-B-1 Burial Ground Cleanup Verification RESRAD Calculation Brief for

Overburden, Below Cleanup Level (BCL), and Staging Pile Area Soil

\section{Computer Program RESRAD Program No. Version 6.3}

The attached calculations have been generated to document compliance with established cleanup levels.

These calculations should be used in conjunction with other relevant documents in the administrative record.

Committed Calculation $\otimes \quad$ Preliminary $\square \quad$ Superseded $\square$ Voided

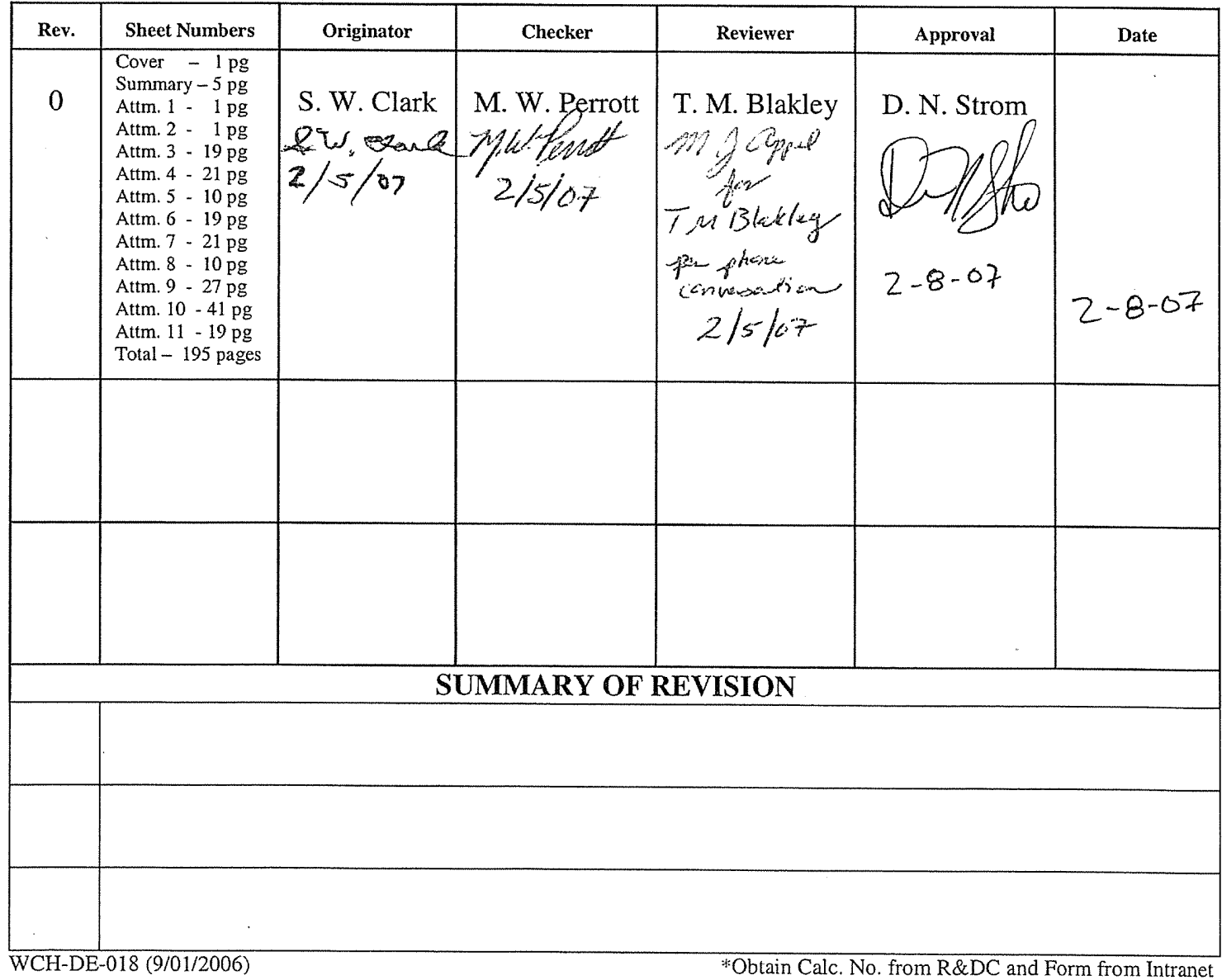




\begin{tabular}{|c|c|c|c|c|c|c|c|}
\hline Originator: & S. W. Clark & Date: & $2 / 5 / 03$ & Calc. No.: & 0100B-CA-V0301 & Rev.: & 0 \\
\hline Project: & 118-B-1 Burial Ground Cleanup & Job No: & 14655 & Checked: & M. W.Penott $\%$ gul & Date: & $2 / 5 / 07$ \\
\hline Subject: & \multicolumn{5}{|c|}{$\begin{array}{l}\text { 118-B-1 Burial Ground Cleanup Verification RESRAD Calculation Brief for Overburden, } \\
\text { Below Cleanup Level (BCL), and Staging Pile Area Soil }\end{array}$} & \multicolumn{2}{|c|}{ Sheet No. 1 of 5} \\
\hline
\end{tabular}

1

2

3

\section{PURPOSE:}

Calculate the soil and groundwater concentrations, dose, and risk contributions from remaining radionuclide contaminants in overburden soil, below cleanup level (BCL) soil, and soil beneath staging pile areas at the 118-B-1 Burial Ground over a period of 1,000 years.

\section{GIVEN/REFERENCES:}

1) Cleanup verification data from 118-B-1 Burial Ground 95\% UCL Calculation, Calculation No. 0100B-CA-V0299, Rev. 0, Washington Closure Hanford, Richland, Washington.

2) Remedial Design Report/Remedial Action Work Plan for the 100 Area (RDR/RAWP), DOE/RL-96-17, Rev. 5, U.S. Department of Energy, Richland Operations Office, Richland, Washington.

3) Radioactive and nonradioactive contaminants of potential concern (COPCs) from the 100 Area Burial Grounds Remedial Action Sampling and Analysis Plan, DOE/RL-2001-35, Rev. 0, U.S. Department of Energy, Richland Operations Office, Richland, Washington. For the purpose of these RESRAD calculations, the radioactive COPCs are americium-241, carbon-14, cesium-137, cobalt-60, europium-152, europium-154, europium-155, nickel-63, plutonium-238, plutonium-239/240, plutonium-241, silver-108m, strontium- 90 , tritium (H-3), uranium-233/234, uranium-235, and uranium-238. The nonradionuclide COPCs are cadmium, hexavalent chromium, mercury, PCBs, SVOAs, TPH, and VOAs.

4) RESidual RADioactivity (RESRAD) computer code, version 6.3, to calculate compliance with residual radioactivity guidelines, developed for the U.S. Department of Energy by the Environmental Assessment Division of Argonne National Laboratory, Argonne, Illinois.

5) Sample design data from the 118-B-1 Burial Ground Sampling Plan, Calculation No. 0100B-CA-V0284, Rev. 0, Washington Closure Hanford, Richland, Washington..

6) Groundwater elevation from Hanford Groundwater Monitoring for Fiscal Year 2005, PNNL-15670, Pacific Northwest National Laboratory, Richland, Washington.

\section{SOLUTION:}

1) Separate RESRAD runs were performed for the 118-B-1 Burial Ground overburden (OB) soil, for the below cleanup level (BCL) soil, and for the staging pile area (SPA). Table 1 shows the elevations (NAVD88) and thickness of each soil horizon. The OB/BCL/SPA soil was all considered to be in the shallow zone (between the ground surface and $4.6 \mathrm{~m}$ [15 ft] deep). Attachment 1 shows representative dimensions of soil horizons and contaminant pathways considered for dose, risk, and groundwater protection modeling. Attachment 2 shows a schematic of the 118-B-1 Burial Ground area with the pieces making up the individual units labeled. Input factors for each run are shown in the "Summary" section of the RESRAD "Mixture Sums and Single Radionuclide Guidelines" printouts in the Attachments to this Calculation Summary. 


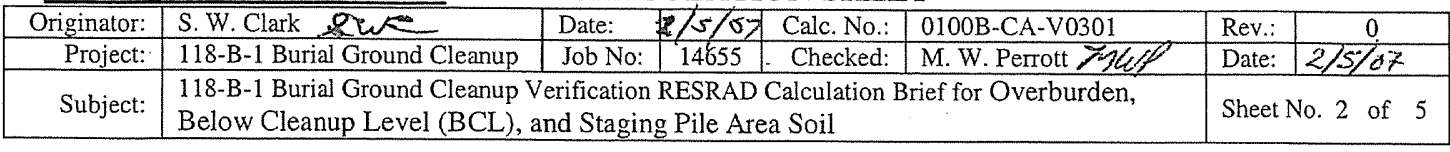

\begin{tabular}{|l|c|c|c|}
\hline \multicolumn{1}{|c|}{ Table 1. Waste Site Dimensions for RESRAD Modeling } \\
\hline \multicolumn{4}{|c|}{ Contaminated Zone Dimensions } \\
\hline \multicolumn{1}{|c|}{ Overburden } & BCL & $\begin{array}{c}\text { Staging Pile } \\
\text { Area }\end{array}$ \\
\hline Cover Depth & 0 & 0 & 0 \\
\hline Area of Contaminated Zone (CZ) & 23,032 & 20,022 & 27,883 \\
\hline Length Parallel to Aquifer Flow & 180 & 146 & 210 \\
\hline \multicolumn{4}{|c|}{ Elevations of Vadose Zone Horizons } \\
\hline Elevation: Surface & 144.5 & 144.5 & 144.5 \\
\hline Elevation: Groundwater & 121.8 & 121.8 & 121.8 \\
\hline Thickness: Contaminated Zone & 4.6 & 4.6 & 4.6 \\
\hline Thickness: Uncontaminated Unsaturated Zone & 18.1 & 18.1 & 18.1 \\
\hline BCL= Below Cleanup Levels Soil & & & \\
\hline
\end{tabular}

2) The cleanup verification data set for the 118-B-1 Burial Ground OB/BCL/SPA soil is presented in Table 2. The year where the peak dose (or concentration) occurs from each individual radionuclide contaminant of concern (COC) and layer is determined by a preliminary run. This year is then added for all horizons for the final RESRAD runs. For the direct exposure pathway (i.e. soil ingestion and inhalation and external radiation), the peak year occurred at year zero (year 2007) for all COCs. For the water pathways (i.e. drinking water and food ingestion) the peak year was year 30 for tritium. The 30 -year time period was added to all RESRAD runs. RESRAD evaluations were performed for the 118-B-1 Burial Ground $\mathrm{OB} / \mathrm{BCL} / \mathrm{SPA}$ soils assuming that the residual contamination levels from the shallow zone sample data sets extend to a maximum of $4.6 \mathrm{~m}(15 \mathrm{ft})$ below the ground surface (Attachment 1).

\section{METHODOLOGY:}

1) Runs of RESRAD version 6.3 were completed for the 118-B-1 Burial Ground OB/BCL/SPA soils using the radionuclide concentrations shown in Table 2. RESRAD numerical output reports for dose, risk, and concentration are presented in the Attachments to this calculation summary.

\begin{tabular}{|c|c|c|c|}
\hline \multicolumn{4}{|c|}{ Table 2. Cleanup Verification Data Set ${ }^{\text {a }}(2$ pages $)$} \\
\hline COCs & Overburden & $\mathrm{BCL}$ & Staging Pile Area \\
\hline \multicolumn{4}{|c|}{ Radionuclide Activity ( $\mathrm{CCi} / \mathrm{g}$ ) } \\
\hline Americium-241 & -- & -- & 0.061 \\
\hline Carbon-14 & -- & -- & 1.6 \\
\hline Cesium-137 & $O(<B G)$ & 0.159 & 0.57 \\
\hline Cobalt- 60 & -- & 0.05 & 0.028 \\
\hline Europium-152 & 0.07 & 0.068 & 0.20 \\
\hline
\end{tabular}


CVP-2007-00006

Rev. 0

Washington Closure Hanford CALCULATION SHEET

\begin{tabular}{|c|c|c|c|c|c|c|}
\hline Originator: & S. W. Clark & Date: & $2 / s / 07$ Calc. No.: & $0100 \mathrm{~B}-\mathrm{CA}-\mathrm{V} 0301$ & Rev.: & 0 \\
\hline Project: & 118-B-1 Burial Ground Cleanup & Job No: & Checked: & M. W. Perrott ØuSP & Date: & $2 / 5 / 17$ \\
\hline Subject: & \multicolumn{4}{|c|}{$\begin{array}{l}\text { 118-B-1 Burial Ground Cleanup Verification RESRAD Calculation Brief for Overburden, } \\
\text { Below Cleanup Level (BCL), and Staging Pile Area Soil }\end{array}$} & \multicolumn{2}{|c|}{ Sheet No. 3 of 5} \\
\hline
\end{tabular}

\begin{tabular}{|c|c|c|c|}
\hline \multicolumn{4}{|c|}{ Table 2. Cleanup Verification Data Set ${ }^{a}$ ( 2 pages) } \\
\hline COCs & Overburden & BCL & Staging Pile Area \\
\hline \multicolumn{4}{|c|}{ Radionuclide Activity ( $p$ Ci/g) } \\
\hline Europium-154 & -- & -- & -- \\
\hline Europium-155 & - & -- & -- \\
\hline Nickel-63 & - & $\cdots$ & 5.05 \\
\hline Plutonium-238 & -- & $\ldots$ & -- \\
\hline Plutonium-239 & - & -- & 0.103 \\
\hline Plutonium-240 & -- & -- & 0.022 \\
\hline Plutonium-241 & - & -- & $\ldots$ \\
\hline Silver $(\mathrm{Ag}-108 \mathrm{~m})$ & -- & -- & -- \\
\hline Strontium-90 & $0(<\mathrm{BG})$ & 0.120 & 0.236 \\
\hline Tritium (H-3) & -- & - & 14.6 \\
\hline Uranium-233/234 & $\mathrm{O}(<\mathrm{BG})$ & $0(<B G)$ & $0(<\mathrm{BG})$ \\
\hline Uranium-235 & $0(<\mathrm{BG})$ & $0(<B G)$ & $0(<\mathrm{BG})$ \\
\hline Uranium-238 & $0(<\mathrm{BG})$ & $0(<B G)$ & $0(<\mathrm{BG})$ \\
\hline \multicolumn{4}{|c|}{$\begin{array}{l}-=\text { Not evaluated by laboratory analysis or not detected (for all samples in the data set). } \\
\mathrm{BCL}=\text { Below Cleanup Levels } \\
\mathrm{COCs}=\text { Contaminants of Concern }\end{array}$} \\
\hline \multicolumn{4}{|c|}{$\begin{array}{l}\text { a Soil concentration values are from 118-B-1 Burial Ground 95\% UCL Calculation, Calculation No. } \\
\text { O100B-CA-V0299, Rev. O, Washington Closure Hanford, Richland, Washington. Refer to the } 95 \% \text { UCL } \\
\text { for nonradionuclide concentrations. Nonradionuclides are not evaluated in this RESRAD calculation. }\end{array}$} \\
\hline
\end{tabular}

3

4

5

6

7

\section{8}

9

10

11

12

\section{RESULTS:}

\section{1) Radionuclide "All Pathways" Dose Rate}

The "all pathways" (maximum) dose rates are shown in Table 3. The maximum all pathways dose rate from the 118-B-1 Burial Ground overburden soil is $0.309 \mathrm{mrem} / \mathrm{yr}$ at year zero (2007). For the below cleanup level (BCL) soil the total maximum all-pathways dose rate is 1.70 $\mathrm{mrem} / \mathrm{yr}$ at year zero (2007). For the staging pile area the total maximum all-pathways dose rate is $6.96 \mathrm{mrem} / \mathrm{yr}$ at year zero (2007).

\begin{tabular}{|c|c|c|c|c|c|c|c|c|c|c|}
\hline \multicolumn{11}{|c|}{ Table 3. All Pathways Dose Rate (mrem/yr) } \\
\hline \multirow{2}{*}{$\begin{array}{c}\text { RESRAD } \\
\text { Run }\end{array}$} & \multirow{2}{*}{$\begin{array}{c}\text { Vadose Zone } \\
\text { Horizons }\end{array}$} & \multicolumn{9}{|c|}{ "All Pathways" Dose Contributions in mrem/yr at Each Time Slice (yr) } \\
\hline & & 0 & 1 & 3 & 10 & 11 & 30 & 100 & 300 & 1000 \\
\hline $\mathrm{OB}$ & Shallow Zone & $3.09 \mathrm{E}-01$ & $2.93 \mathrm{E}-01$ & $2.64 \mathrm{E}-01$ & $1.83 \mathrm{E}-01$ & $1.74 \mathrm{E}-01$ & $6.48 \mathrm{E}-02$ & $1.69 \mathrm{E}-03$ & $5.10 \mathrm{E}-08$ & $1.21 \mathrm{E}-16$ \\
\hline $\mathrm{BCL}$ & Shallow Zone & $1.70 \mathrm{E}+00$ & $1.60 \mathrm{E}+00$ & $1.43 \mathrm{E}+00$ & $1.02 \mathrm{E}+00$ & $9.74 \mathrm{E}-01$ & $5.09 \mathrm{E}-01$ & $8.37 \mathrm{E}-02$ & $7.06 \mathrm{E}-04$ & $4.46 \mathrm{E}-11$ \\
\hline SPA & Shallow Zone & $6.96 \mathrm{E}+00$ & $4.38 \mathrm{E}+00$ & $3.35 \mathrm{E}+00$ & $2.60 \mathrm{E}+00$ & $2.52 \mathrm{E}+00$ & $1.58 \mathrm{E}+00$ & $3.22 \mathrm{E}-01$ & $7.50 \mathrm{E}-02$ & $5.46 \mathrm{E}-02$ \\
\hline
\end{tabular}




\begin{tabular}{|c|c|c|c|c|c|c|}
\hline Originator: & S. W. Clark & $E / 5 / 02$ & Calc. No.: & 0100B-CA-V0301 & Rev.: & 0 \\
\hline Project: & 118-B-1 Burial Ground Cleanup & Job No: 14655 & Checked: & M. W. Perrott $Z$ Mich & Date: & Jat \\
\hline Subject: & \multicolumn{4}{|c|}{$\begin{array}{l}\text { 118-B-1 Burial Ground Cleanup Verification RESRAD Calculation Brief for Overburden, } \\
\text { Below Cleanup Level (BCL), and Staging Pile Area Soil }\end{array}$} & \multicolumn{2}{|c|}{ Sheet No. 4 of 5} \\
\hline
\end{tabular}

\section{$1 \quad$ 2) Radionuclide Excess Lifetime Cancer Risk}

2 The radionuclide excess lifetime cancer risk (ELCR) results are shown in Table 4. The 3 maximum ELCR for the overburden (OB) soil is $3.65 \times 10^{-6}$ at year zero (2007). The maximum

4 ELCR for the below cleanup level (BCL) soil is $1.98 \times 10^{-5}$ at year zero (2007). For the staging 5 pile area (SPA) the total maximum all-pathways ELRC is $5.82 \times 10^{-5}$ at year zero (2007).

6

\begin{tabular}{|c|c|c|c|c|c|c|c|c|c|c|}
\hline \multirow{2}{*}{$\begin{array}{c}\text { RESRAD } \\
\text { Run } \\
\end{array}$} & \multirow{2}{*}{$\begin{array}{c}\text { Vadose Zone } \\
\text { Horizons }\end{array}$} & \multicolumn{9}{|c|}{ Excess Cancer Risk at Each Time Slice (yr) } \\
\hline & & 0 & 1 & 3 & 10 & 11 & 30 & 100 & 300 & 1000 \\
\hline $\mathrm{OB}$ & Shallow Zone & $3.65 \mathrm{E}-06$ & $3.46 \mathrm{E}-06$ & $3.12 \mathrm{E}-06$ & $2.17 \mathrm{E}-06$ & $2.06 \mathrm{E}-06$ & $7.65 \mathrm{E}-07$ & $2.00 \mathrm{E}-08$ & $6.03 \mathrm{E}-13$ & $8.14 \mathrm{E}-22$ \\
\hline $\mathrm{BCL}$ & Shallow Zone & $1.98 \mathrm{E}-05$ & $1.89 \mathrm{E}-05$ & $1.74 \mathrm{E}-05$ & $1.33 \mathrm{E}-05$ & $1.29 \mathrm{E}-05$ & $7.24 \mathrm{E}-06$ & $1.24 \mathrm{E}-06$ & $1.06 \mathrm{E}-08$ & $6.86 \mathrm{E}-16$ \\
\hline SPA & Shallow Zone & 5.82E-05 & $5.10 \mathrm{E}-05$ & 4.67E-05 & $3.81 \mathrm{E}-05$ & $3.69 \mathrm{E}-05$ & $2.19 \mathrm{E}-05$ & $4.27 \mathrm{E}-06$ & $3.10 \mathrm{E}-07$ & $1.09 \mathrm{E}-07$ \\
\hline $\begin{array}{l}\mathrm{OB}=\mathrm{Ove} \\
\mathrm{BCL}=\mathrm{Be} \\
\mathrm{SPA}=\mathrm{Sta}\end{array}$ & $\begin{array}{l}\text { len } \\
\text { Cleanup Levels } \\
\text { Pile Area }\end{array}$ & & & & & & & & & \\
\hline
\end{tabular}

8 3) Radionuclide Groundwater Protection

9 The radionuclide concentrations in groundwater calculated by the RESRAD model are 10 summarized in Table 5. Among the radionuclide COCs only tritium is calculated to reach 11 groundwater in the 1,000 years of the RESRAD model run. Tritium is predicted to reach 12 groundwater at a concentration below the remedial action goal (RAG). Therefore, no calculation 13 for comparison to drinking water standards was necessary. 14

\section{Table 5. Predicted Groundwater (Well Water/Drinking Water) Concentrations (2 pages)}

\begin{tabular}{|c|c|c|c|c|c|c|c|c|c|c|c|}
\hline \multirow{2}{*}{$\begin{array}{l}\text { Radio- } \\
\text { nuclide }\end{array}$} & \multirow{2}{*}{$\begin{array}{c}\text { Vadose Zone } \\
\text { Horizon }\end{array}$} & \multicolumn{9}{|c|}{ Ground water Concentrations in $\mathrm{pCi} / \mathrm{L}$ at Each Time Slice (yr) } & \multirow{2}{*}{$\begin{array}{l}\mathrm{RAG}, \\
\mathrm{pCi} / \mathrm{L}\end{array}$} \\
\hline & & 0 & 1 & 3 & 10 & 11 & 30 & 100 & 300 & 1000 & \\
\hline \multirow{3}{*}{ Tritium $(\mathrm{H}-3)$} & $\mathrm{OB}$ & 0 & 0 & 0 & 0 & 0 & 0 & 0 & 0 & 0 & \multirow{3}{*}{20,000} \\
\hline & $\mathrm{BCL}$ & 0 & 0 & 0 & 0 & 0 & 0 & 0 & 0 & 0 & \\
\hline & SPA & 0 & 0 & 0 & 0 & 0 & 1440 & 0 & 0 & 0 & \\
\hline
\end{tabular}

15

16

17

18

19

20

21

\section{CONCLUSIONS:}

- The "all pathways" (maximum) dose rates are shown in Table 3. The maximum all pathways dose rate for the $118-\mathrm{B}-1$ Burial Ground overburden (OB) soil is $0.309 \mathrm{mrem} / \mathrm{yr}$ at year zero (2007). The maximum all-pathways dose rate for the below cleanup level (BCL) soil is 1.70 $\mathrm{mrem} / \mathrm{yr}$ at year zero (2007). The maximum all-pathways dose rate for the staging pile area is $6.96 \mathrm{mrem} / \mathrm{yr}$ at year zero (2007).

- None of the site COCs are projected to exceed remedial action goals (RAGs). 


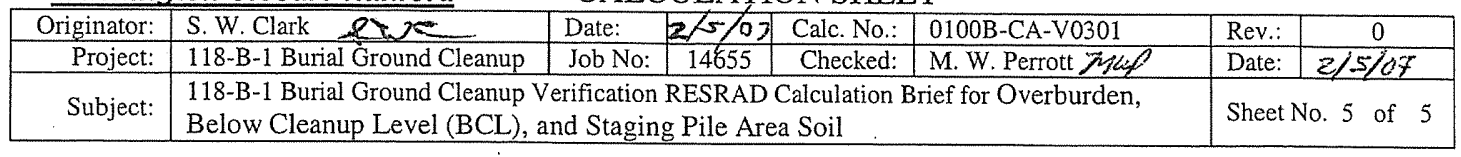

- The radionuclide excess lifetime cancer risk (ELCR) results are shown in Table 4. The maximum ELCR for the overburden (OB) soil is $3.65 \times 10^{-6}$ at year zero (2007). The maximum ELCR for the below cleanup level (BCL) soil is $1.98 \times 10^{-5}$ at year zero (2007). The maximum all-pathways ELRC for the staging pile area (SPA) is $5.82 \times 10^{-5}$ at year zero (2007).

- Among the radionuclide COCs only tritium is calculated to reach groundwater in the 1,000 years of the RESRAD model run. Tritium is predicted to reach groundwater at concentrations below the RAGs.

\section{ATTACHMENTS:}

1. Graphic showing 118-B-1 Cleanup Verification Model (1 page)

2. Schematic of the 118-B-1 Burial Ground Remediation Areas (1 page)

3. RESRAD Output: 118-B-1 Overburden Radionuclides - Mixture Sums and Single Radionuclide Guidelines (19 pages)

4. RESRAD Output: 118-B-1 Overburden Radionuclides - Intake Quantities and Health Risk Factors (21 pages)

5. RESRAD Output: 118-B-1 Overburden Radionuclides - Concentration of Radionuclides, (10 Pages)

6. RESRAD Output: 118-B-1 Below Cleanup Level Radionuclides - Mixture Sums and Single Radionuclide Guidelines (19 pages)

7. RESRAD Output: 118-B-1 Below Cleanup Level Radionuclides - Intake Quantities and Health Risk Factors (21 pages)

8. RESRAD Output: 118-B-1 Below Cleanup Level Radionuclides - Concentration of Radionuclides (10 pages)

9. RESRAD Output: 118-B-1 Staging Pile Area Radionuclides - Mixture Sums and Single Radionuclide Guidelines (27 pages)

10. RESRAD Output: 118-B-1 Staging Pile Area Radionuclides - Intake Quantities and Health Risk Factors (41 pages)

11. RESRAD Output: 118-B-1 Staging Pile Area Radionuclides - Concentration of Radionuclides (19 pages) 
118-B-1 Burial Ground OB/BCL/SPA Cleanup Verification Model

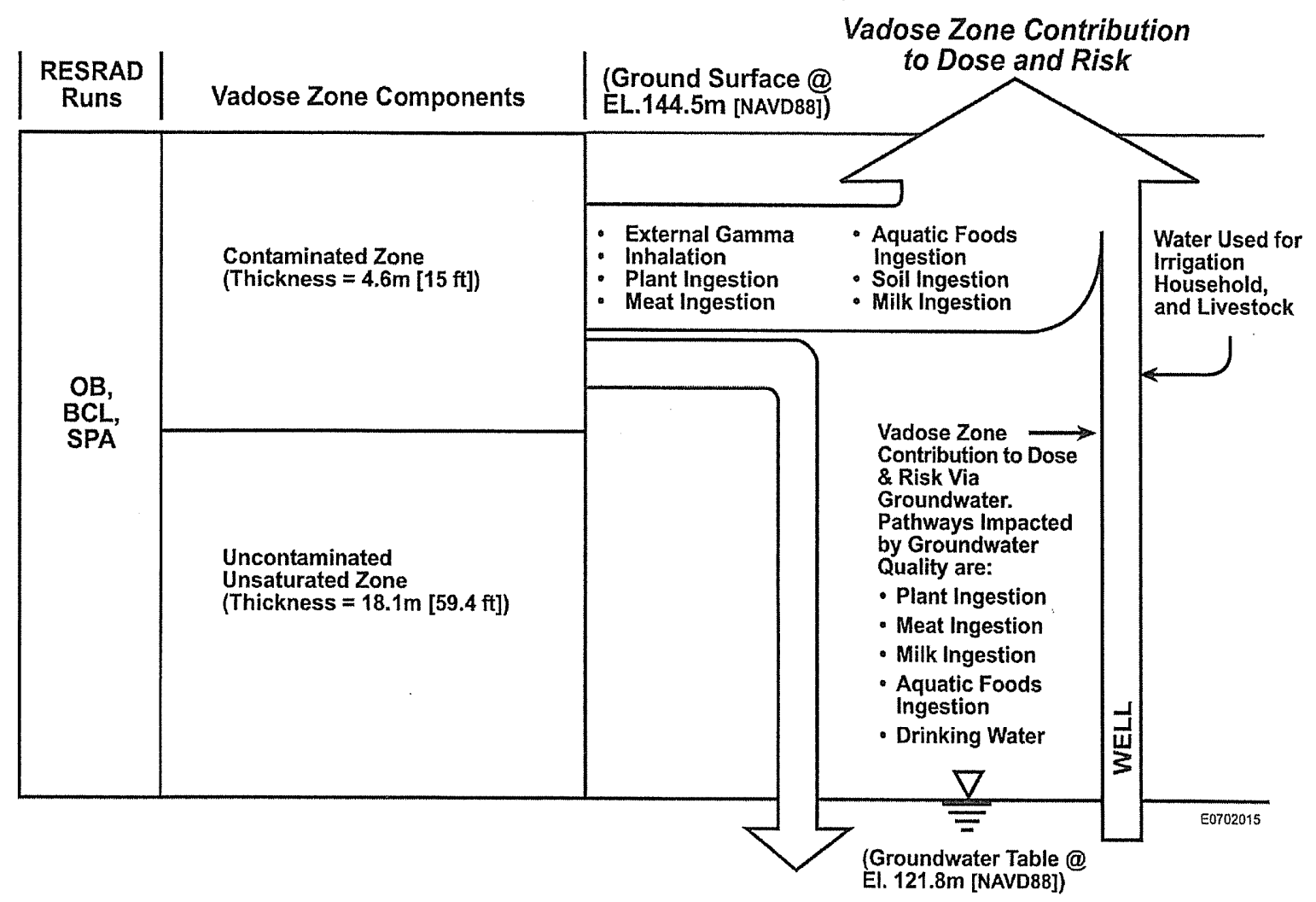

Vadose Zone Contribution to Groundwater Contamination 


\section{ATTACHMENT 2}

\section{Schematic of the 118-B-1 Burial Ground Remediation Areas Sampled for Cleanup} Verification

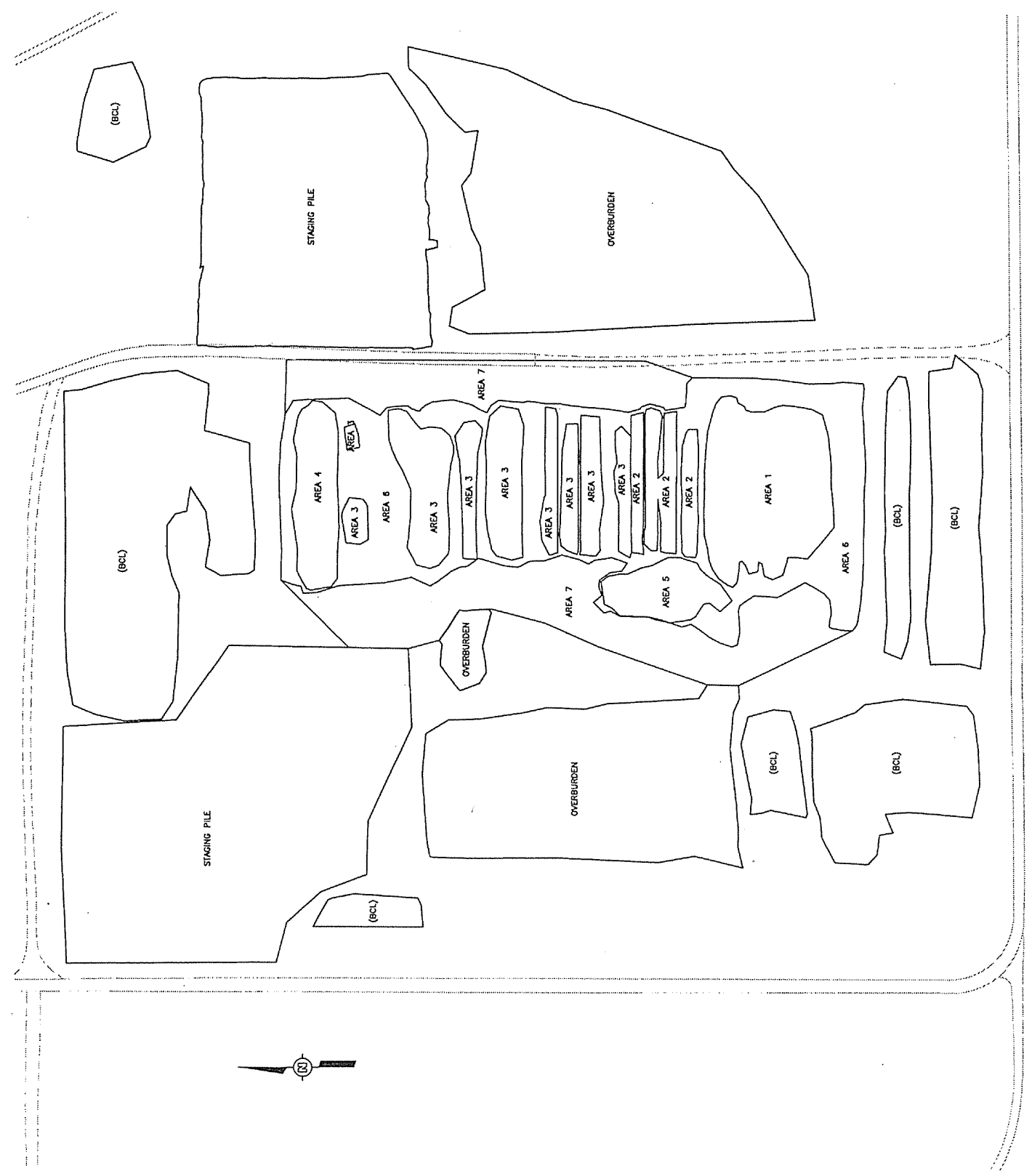

Attachment

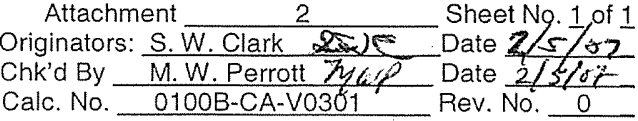


CVP-2007-00006

Rev. 0

ATTACHMENT 3

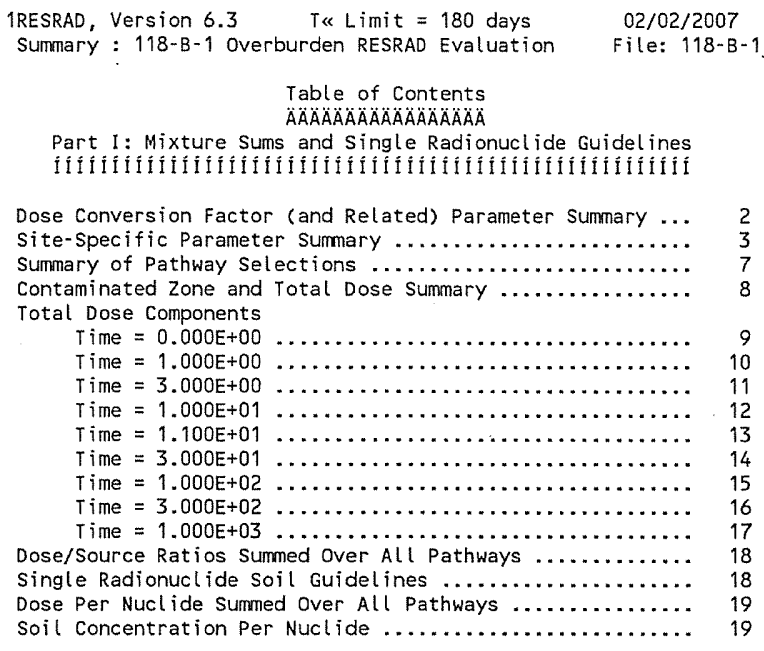

\begin{tabular}{|c|c|}
\hline hment & Sheet No. 1 \\
\hline Wr. Main & \\
\hline M.W. Perrott Mase & te 215107 \\
\hline
\end{tabular}




\section{ATTACHMENT 3}

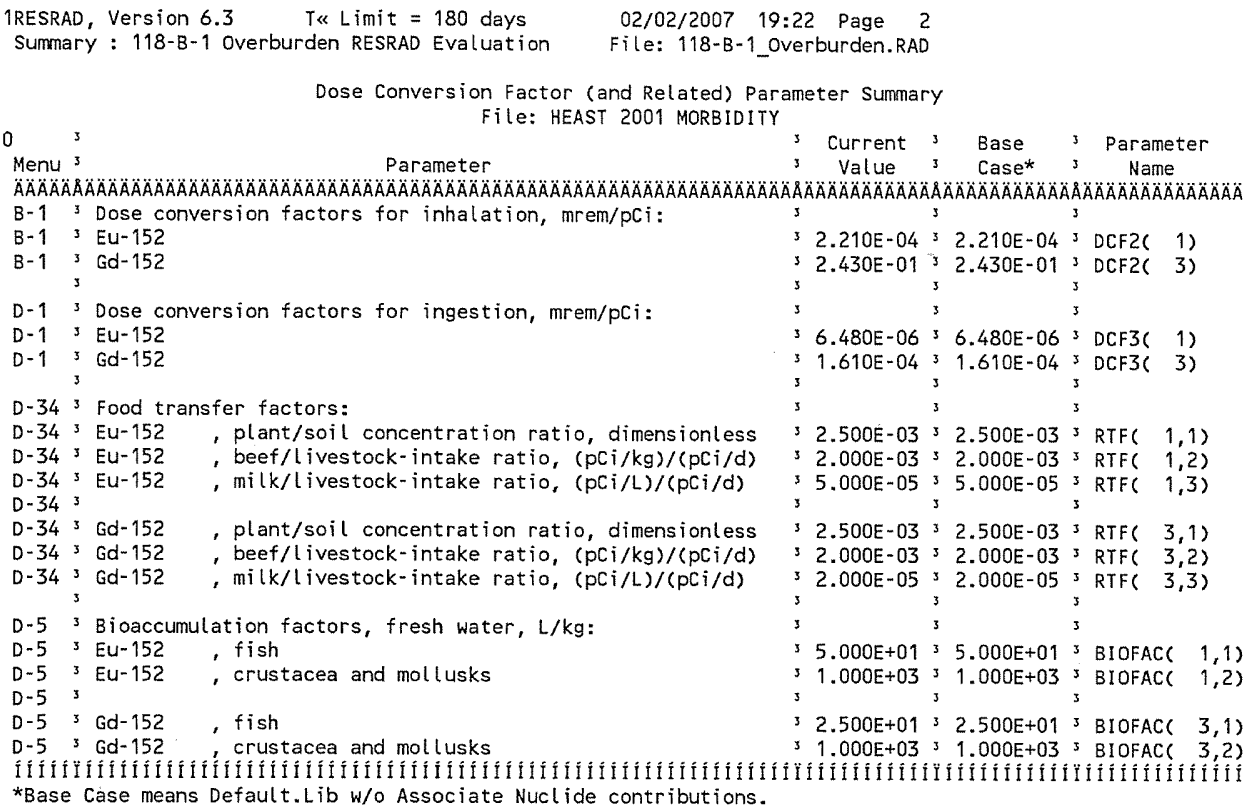

\begin{tabular}{|c|c|}
\hline Attachment & Sheet No. 2 of 19 \\
\hline Originators: S.W. Clark & Date \\
\hline M. W. Perrott & Date \\
\hline Calc. No. $\quad 0100 \mathrm{~B}-\mathrm{CA}-\mathrm{V} 0301$ & Rev. No. 0 \\
\hline
\end{tabular}




\section{ATTACHMENT 3}

1RESRAD, Version $6.3 \quad$ T\& Limit $=180$ days
Summary : $118-8-1$ Overburden RESRAD Evaluation

\section{0}

A A a rameter

A AAAAAAAAOAAAAAAAAAA

R011' 3 Thick

RO11 Basic radiation dose limit (mrem/yr)

R011 Time since placement of material (yr)

R011 'Times for calculations $(y r)$

R011 3 Times for calculations (yr)

R011' Times for calculations (yr)

R011 3 Times for calculations (yr)

R011 Times for calculations (yr)

R011 3 Times for calculations (yr)

R011 ' Times for calculations (yr)

R011 3 Times for calculations $(y r)$

R011' Times for calculations (yr)

R012 I Initial principal radionuclide $(p C i / g)$ : Eu-152

R012 Concentration in groundwater (pCi/L): Eu-152

R013 3 Cover depth (m)

R013 3 Density of cover material $(\mathrm{g} / \mathrm{cm} * * 3)$

R013 3 Cover depth erosion rate $(\mathrm{m} / \mathrm{yr})$

R013 S Density of contaminated zone $\left(\mathrm{g} / \mathrm{cm}^{* * 3}\right)$

R013 3 Contaminated zone erosion rate $(\mathrm{m} / \mathrm{Yr})$

R013 s Contaminated zone total porosity

R013 3 Contaminated zone field capacity

R013 ' Contaminated zone hydraul ic conductivity ( $\mathrm{m} / \mathrm{yr}$ )

R013 s Contaminated zone b parameter

R013 Average annual wind speed $(\mathrm{m} / \mathrm{sec})$

R013 s Humidity in air $\left(\mathrm{g} / \mathrm{m}^{\star *} 3\right)$

R013 ' Evapotranspiration coefficient

R013 3 Precipitation $(\mathrm{m} / \mathrm{yr})$

R013' Irrigation (m/yr)

R013 I Irrigation mode

R013 's Runoff coefficient

R013 3 Watershed area for nearby stream or pond $\left(m^{* *}\right)$

R013 5 Accuracy for water/soil computations

R014 3 Density of saturated zone $\left(\mathrm{g} / \mathrm{cm}^{* * 3}\right)$

R014' Saturated zone total porosity

R014 '3 Saturated zone effective porosity

$\mathrm{R} 14^{\mathrm{H}}$ Saturated zone field capacity

R014 's Saturated zone hydraulic conductivity ( $\mathrm{m} / \mathrm{yr}$ )

R014'3 Saturated zone hydraul ic gradient

R014 3 Saturated zone b parameter

R014 3 Water table drop rate $(m / y r)$

R014' Well pump intake depth (In below water table)

R014 3 Model: Nondispersion (ND) or Mass-Balance (MB)

R014 3 Well pumping rate $\left(m^{\star *} 3 / y r\right)$

R015 3 Number of unsaturated zone strata
02/02/2007 19:22 Page 3 Site-Specific Parameter Summary
User Used by RESRAD 3 Parameter Input s Default s (If different from user input) s Name

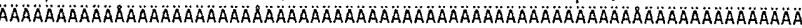
$2.303 E+04,1.000 E+04$ $3.600 \mathrm{E}+00,2.000 \mathrm{E}+00$ $31.800 \mathrm{E}+02 ; 1.000 \mathrm{E}+02$

$34.000 \mathrm{E}+0033.000 \mathrm{E}+01$

$30.000 E+00 ; 0.000 E+00$

$31.000 E+00 \div 1.000 E+00$

$33.000 E+00$ \& $3.000 E+00$ \&

$31.000 E+01$ \& $1.000 E+013$

$1.00 \mathrm{E}+01 ; 3.000 \mathrm{E}+01$

.

$1.000 E+02^{3}$

$1.000 E+0233.000 E+02$

$3.000 E+02=1.000 E+03$

$31.000 E+03=0.000 E+00$

3. not used $30.000 \mathrm{E}+00^{3}$

$7.000 E-0230.000 E+00$

not used $30.000 E+00^{3}$

3

$30.000 E+0030.000 E+003$

3 not used $31.500 E+00$

not used 3 1.000E-03

$31.600 E+00=1.500 E+00$

s $0.000 \mathrm{E}+00 \div 1.000 \mathrm{E}-03$

3 4.000E-013 4.000E-01

$31.500 \mathrm{E}-0132.000 \mathrm{E}-01$

2.500E+02 $31.000 \mathrm{E}+01$

$\checkmark 4.050 E+003.5 .300 E+00$

$33.400 E+00 \div 2.000 E+00$

3 not used $38.000 E+00$

$9.100 E-0135.000$ E-01

$1.600 \mathrm{E}-01>1.000 \mathrm{E}+00$

7.600E-01 3 2.000E-01

overhead 's overhead

$2.000 E-01$ s $2.000 E-01$

1.000 E

$1.000 \mathrm{E}-03 \times 1.000 \mathrm{E}-03$

$1.600 E+00 \div 1.500 E+00$

4.000E-01 $3.000 E-01$

2.500E-01 2.000E-01

$1.500 \mathrm{E}-01^{3} 2.000 \mathrm{E}-01$

$5.530 \mathrm{E}+03$ 3 $1.000 \mathrm{E}+02$

ก.250E-03 $3.000 E-02$

O. USEd s $5.300 \mathrm{E}$

$4.600 E+00>1.000 E+01$

ND 3 ND

$2.500 E+02 ; 2.500 E+02$

3 s 1
-..

-.. 3 THICKO

s LCZPA

$\ldots$ BRD

-. 3 T 11

$3 T(4)$

$\cdots+3(5)$

.. 3 T $(6)$

ग $T(7)$

3 Tर 9 (

$3 T(10)$

-..

... $\quad 3$ W1( 1$)$

... 3 COVERO

.. 3 DENSCV

3 VCV

-.. 3 DENSCZ

${ }^{3} \mathrm{VCZ}$

-- 3 TPCZ

3 FCCZ

- 3 HCCZ

- $\quad$ BCZ

$\cdots$

... 2 S EVAPTR

... $\quad$ S PRECIP

$3 \mathrm{R}$

3 IDITCH

3 RUNOFF

-.. 3 WAREA

-.. 3 EPS

.. 3 DENSAQ

-.. $\quad$ 3 TPSZ

$+\cdots$

3 FCSZ

-.- 3 HCSZ

a. 3 HGWT

-.. 3 BSZ

..- 3 DWIBWT

-.
-.. 3 VWT
Attachment

Originators: S.W. Clark

3

Sheet No. $\underline{3}$ of 19

Chk'd By M.W. Perrott

Calc. No. 0100B-CA-V0301
Date

Rev. No. 0 


\section{ATTACHMENT 3}

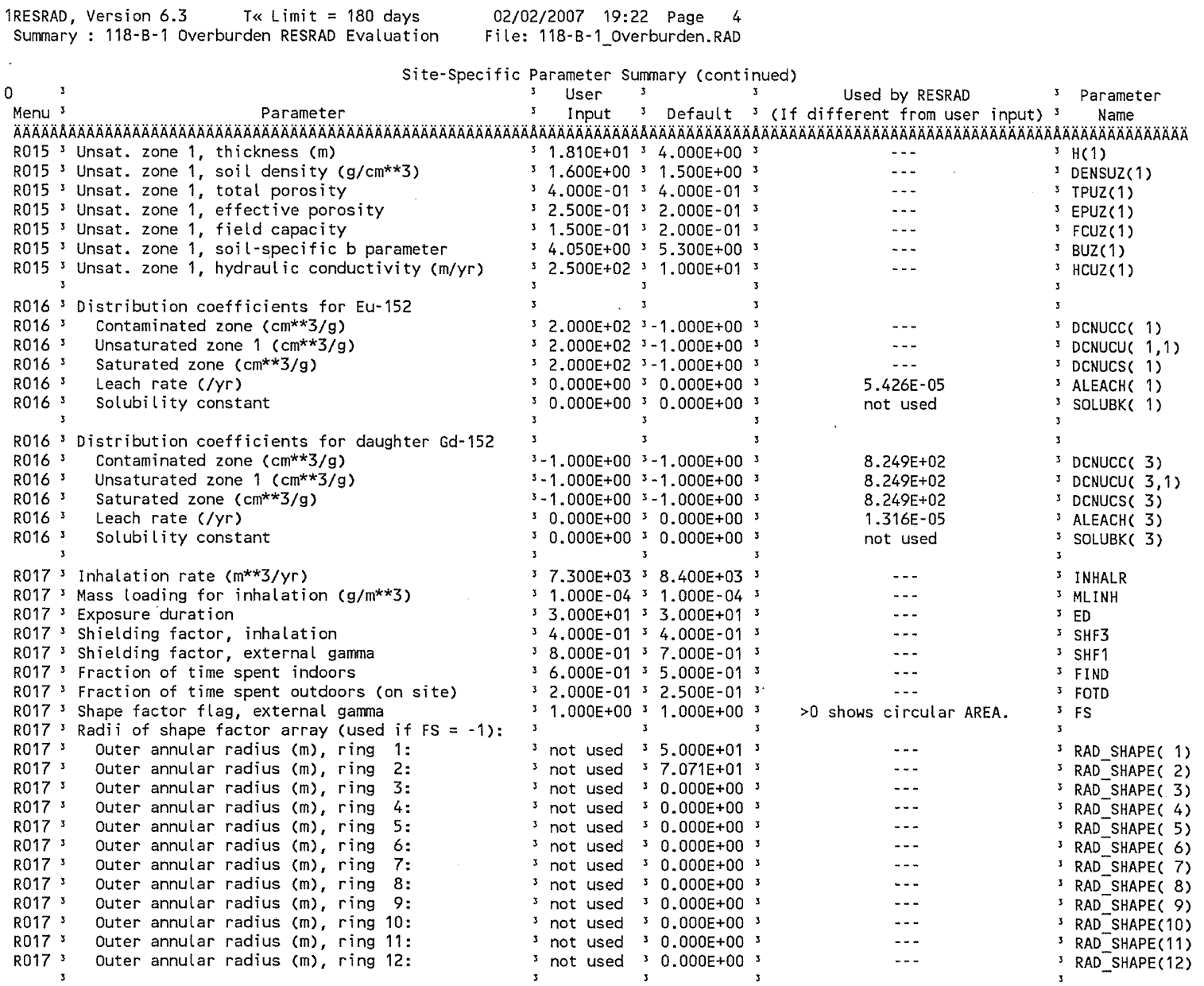

Attachment

3 Sheet No. $\underline{4}$ of 19 Originators: $S$. W. Clark Date

Chk'd By M.W. Perrott Rev. No. 0 


\section{ATTACHMENT 3} 1RESRAD, Version $6.3 \quad$ T\& Limit $=180$ days
Summary : $118-B-1$ Overburden RESRAD Evaluation
File: 118-B-1_Overburden. RAD

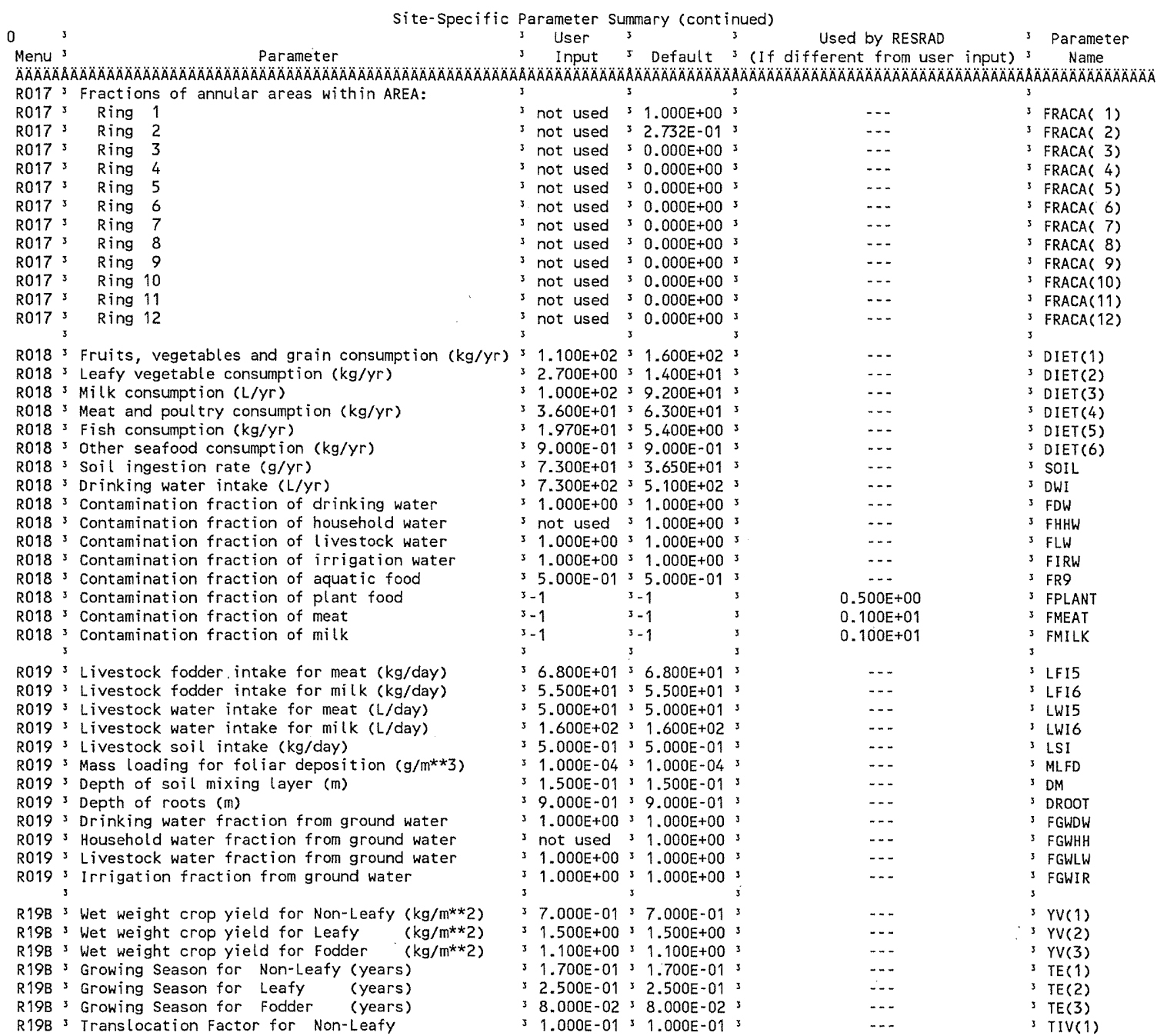

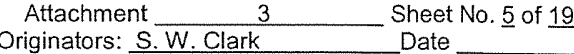
Chk'd By M.W. Perrott Date

Calc. No 0100B-CA-V0301

Rev. No. 0 


\section{ATTACHMENT 3}

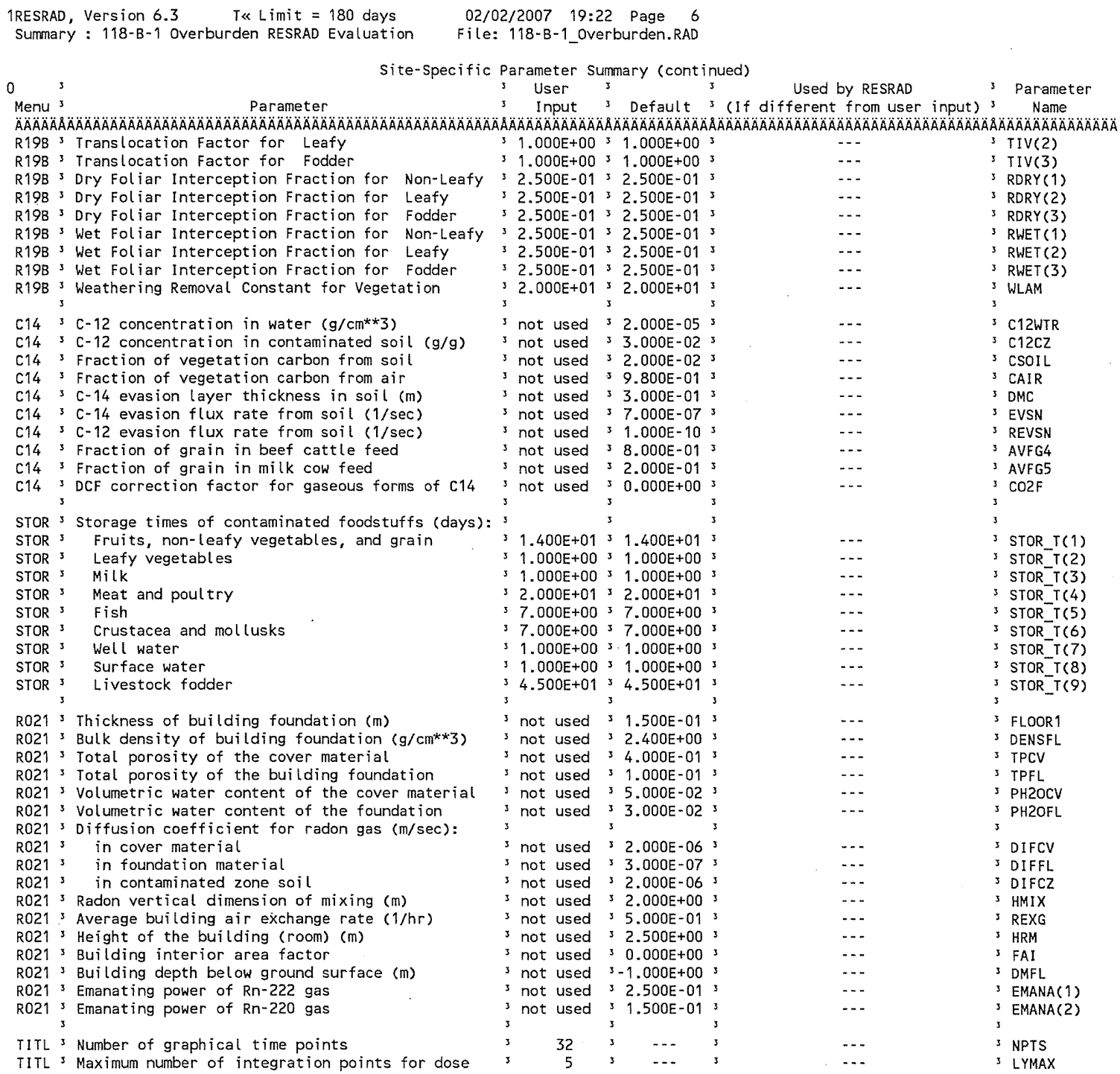

Attachment

3

Sheet No. 6 of 19

Originators: $\frac{S . \text { W. Clark }}{\text { M.W. Perro }}$
Chk'd By Date

M. W. Perrott
- Date

Calc. No. 0100B-CA-V0301 


\section{ATTACHMENT 3}

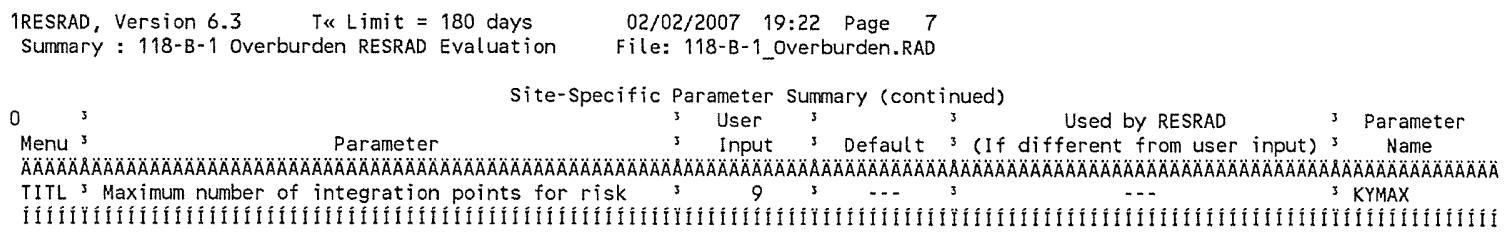

Summary of Pathway Selections

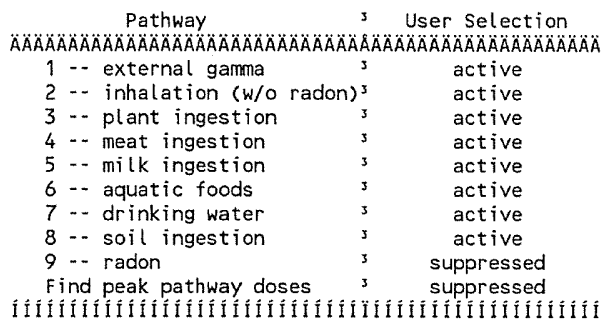

Attachment Originators: S.W. Clark

3 Sheet No. $\underline{7}$ of 19 Chk'd By M.W. Perrott Date

Calc. No. 0100B-CA-V0301
Date Rev. No. 0 


\section{ATTACHMENT 3}

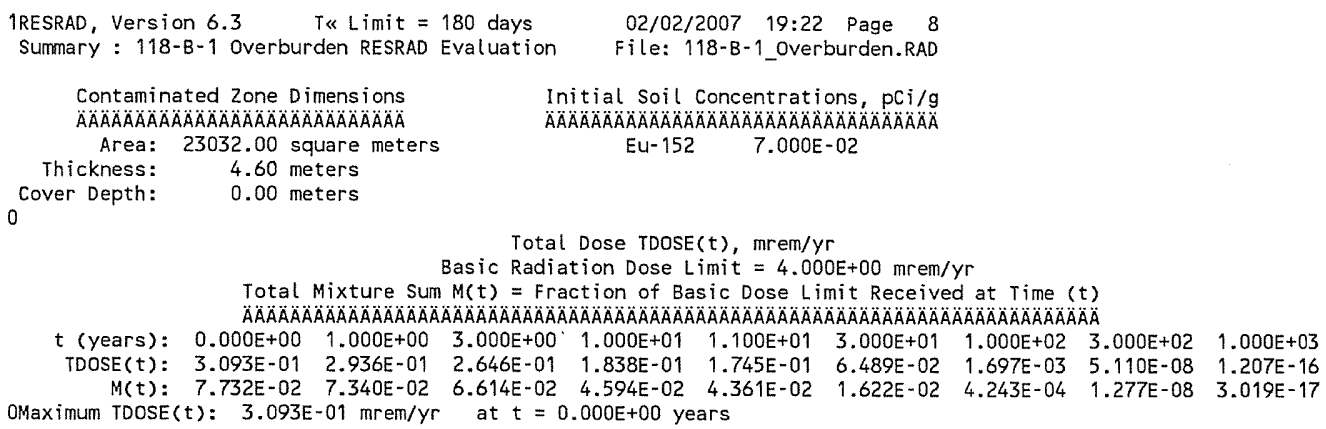

$\begin{array}{ll}\text { Attachment } \frac{3}{\text { W. Clark }} & \text { Sheet No. } \underline{8} \text { of } \underline{19} \\ \text { Originators: } \frac{\text { S.W. }}{\text { M.W. Perrott }} & \text { Date } \\ \text { Chk'd By } & \text { Rev. No. } 0 \\ \text { Calc. No. } & 0100 \text { B-CA-V0301 }\end{array}$




\section{ATTACHMENT 3}

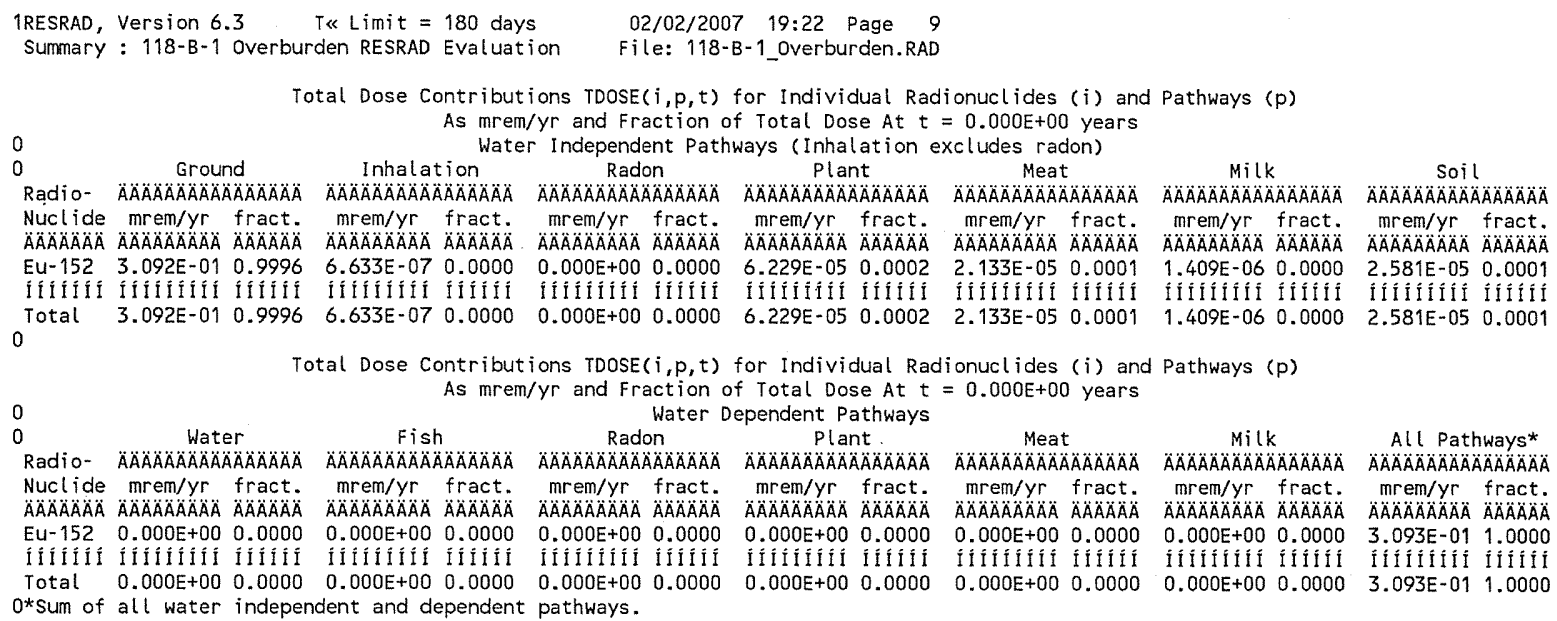




\section{ATTACHMENT 3}

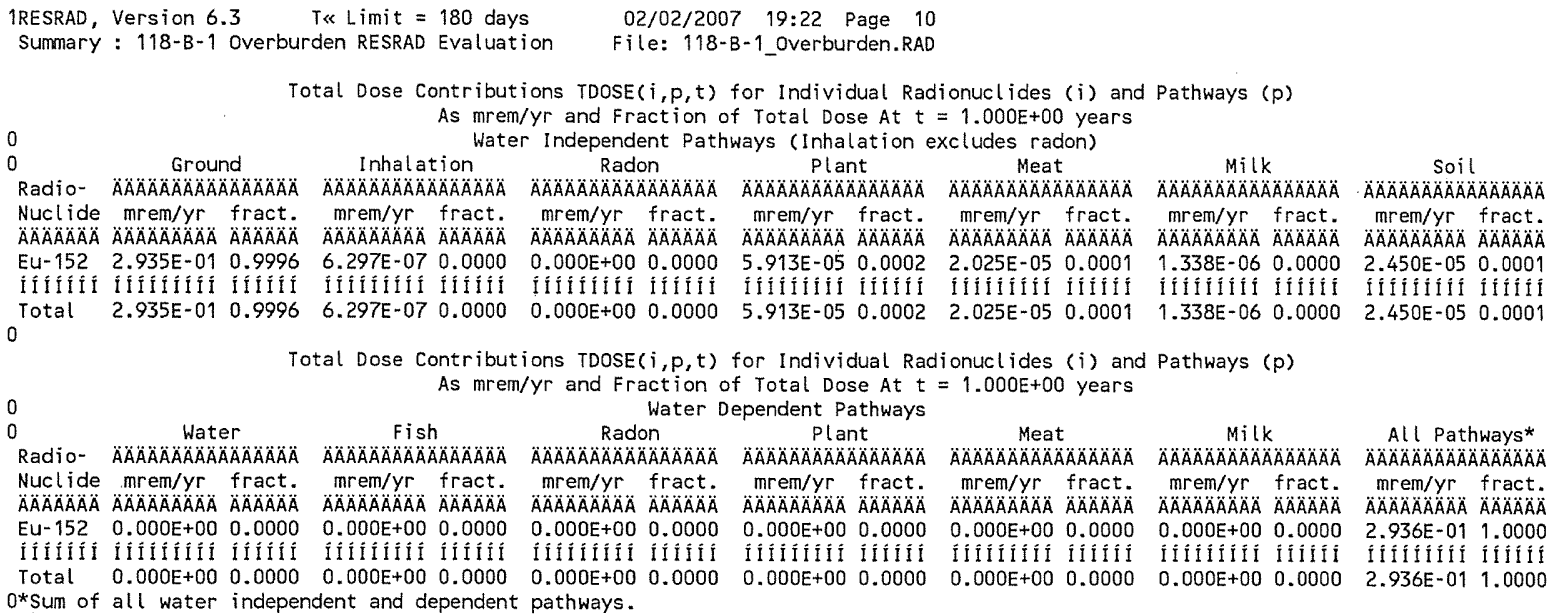




\section{ATTACHMENT 3}

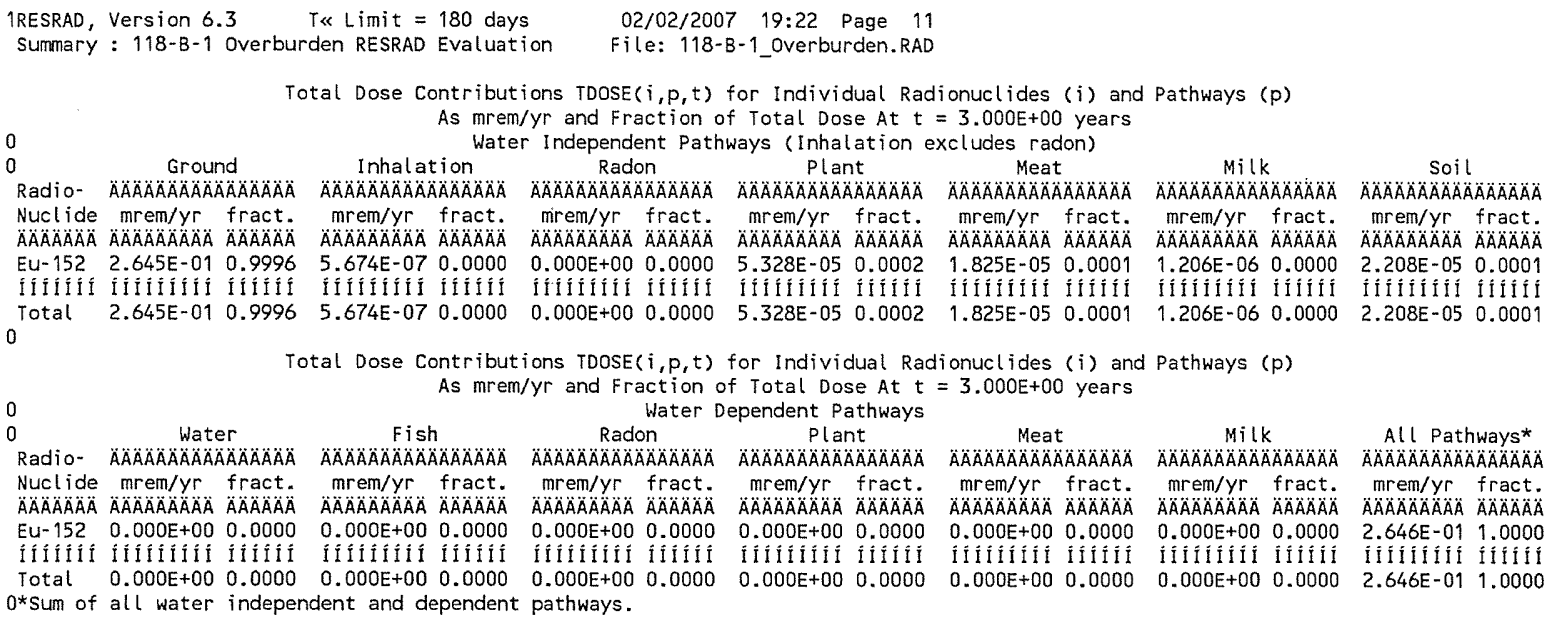

\begin{tabular}{|c|c|}
\hline Attachment & Sheet No. 11 of 19 \\
\hline Originators: S.W. Clark & Date \\
\hline Chk'd By M. W. Perrott & Date \\
\hline Calc. No. $\quad 0100 \mathrm{~B}-\mathrm{CA}-\mathrm{V} 0301$ & Rev. No. \\
\hline
\end{tabular}




\section{ATTACHMENT 3}

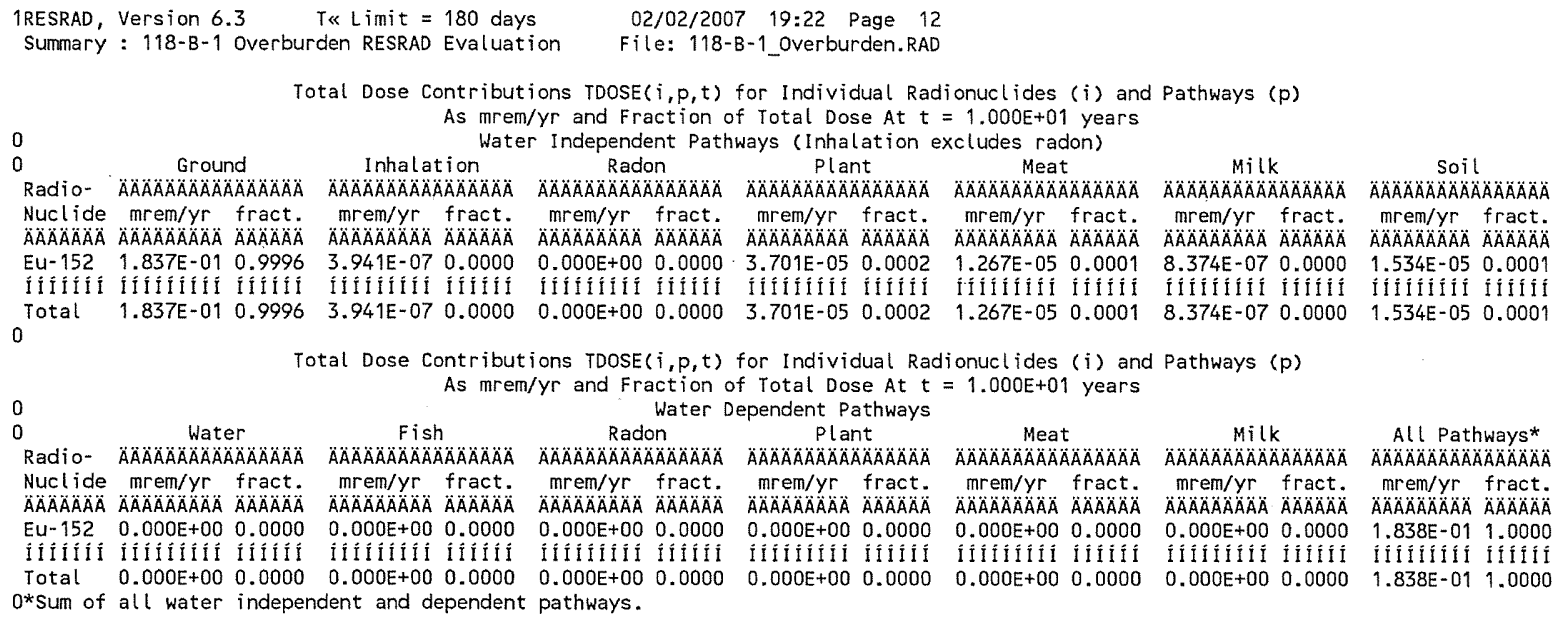

\begin{tabular}{ll} 
Attachment $\frac{3}{\text { O.W.Clark }}$ & Sheet No. 12 of 19 \\
Originators: & Date \\
Chk'd By & Date \\
Calc. No. Nerrott & Rev. No. 0 \\
\hline 0100 B-CA-V0301 & Rev
\end{tabular}


CVP-2007-00006

Rev. 0

\section{ATTACHMENT 3}

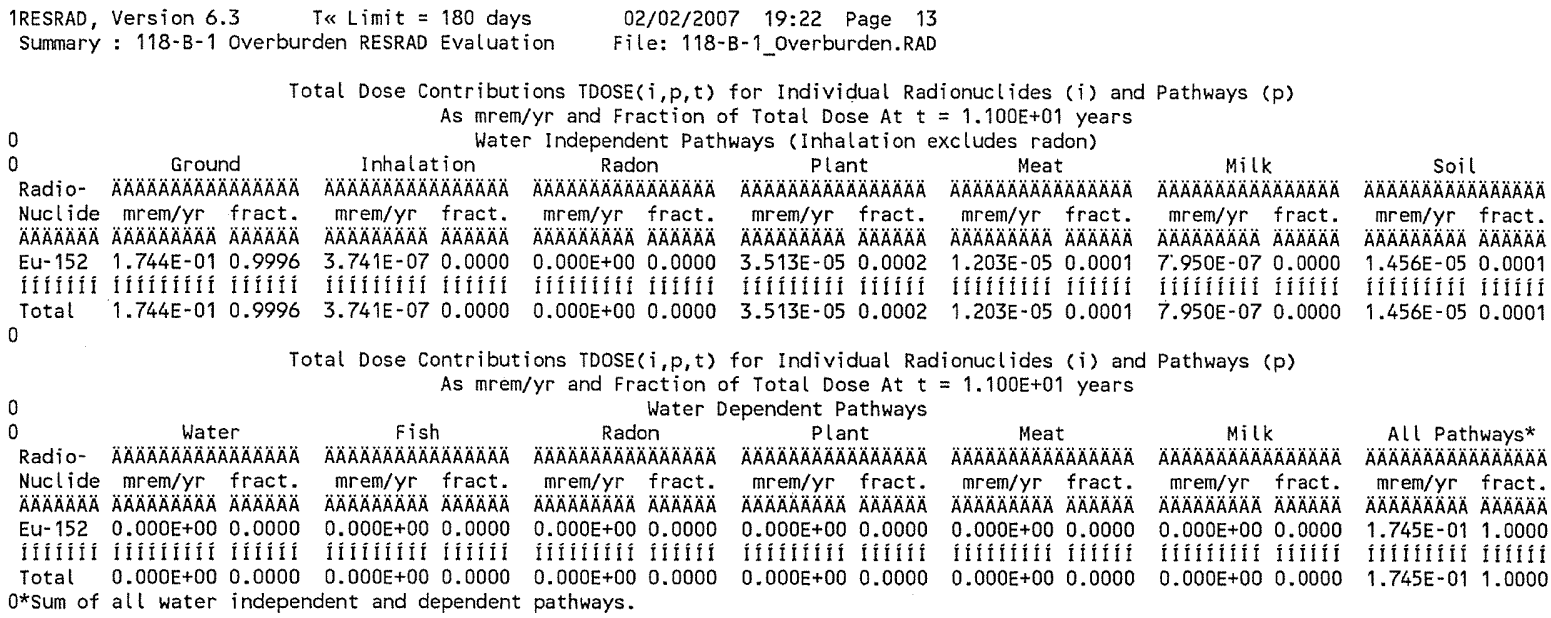




\section{ATTACHMENT 3}

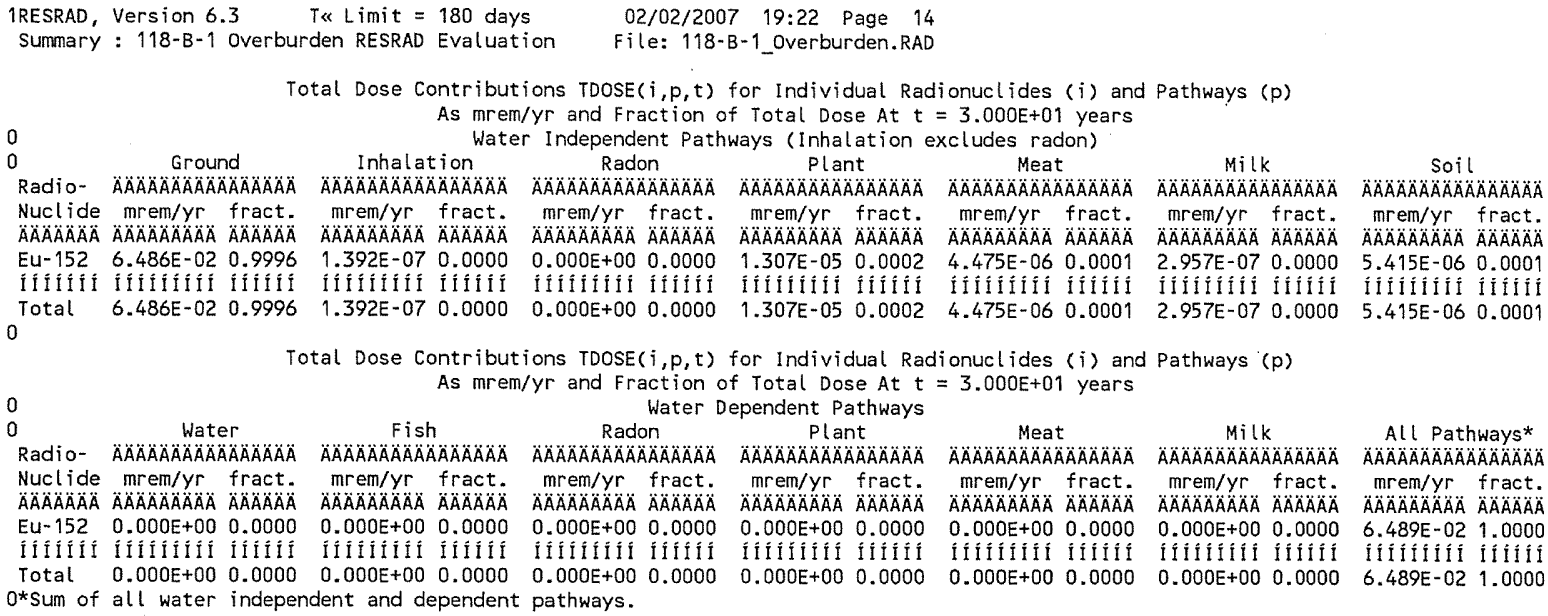




\section{ATTACHMENT 3}

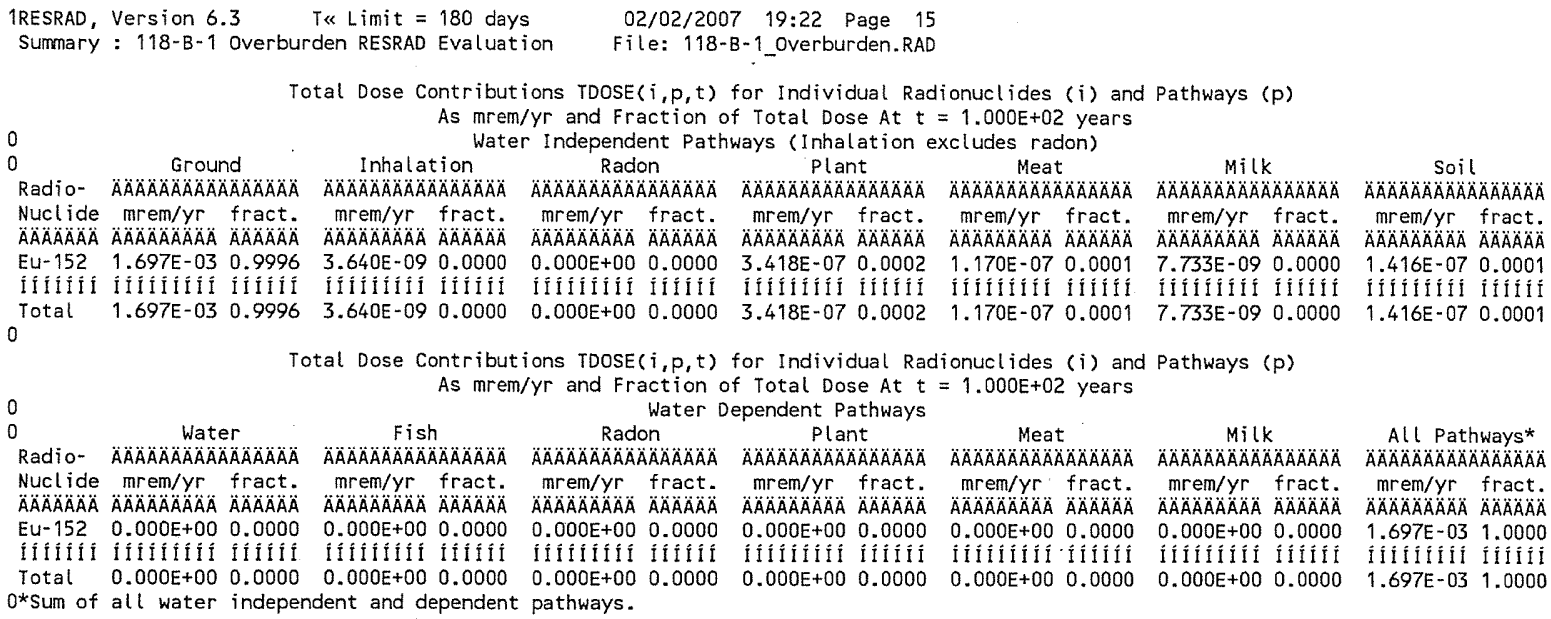




\section{ATTACHMENT 3}

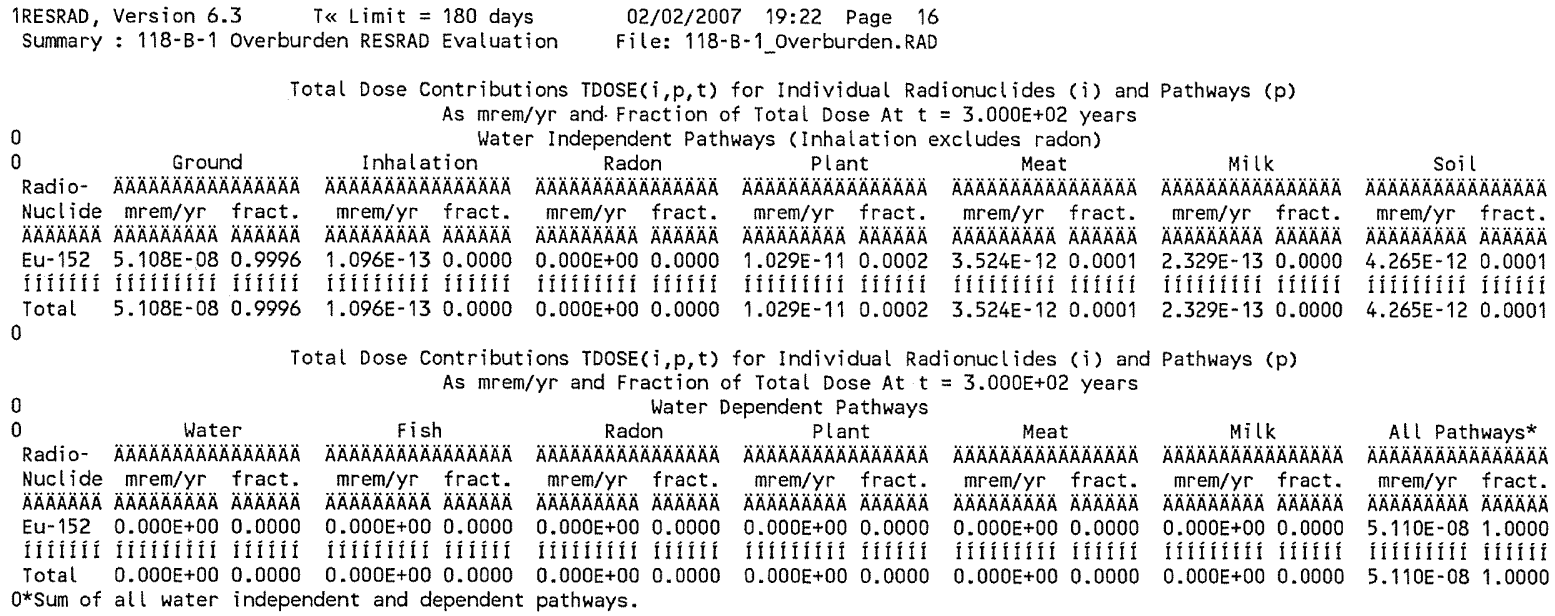

\begin{tabular}{|c|c|}
\hline Attachment & Sheet No. 16 of 19 \\
\hline Originators: S. W. Clark & Date \\
\hline Chk'd By M.W. Perrott & Date \\
\hline $0100 \mathrm{~B}-\mathrm{CA}-\mathrm{V} 0301$ & Rev. No. 0 \\
\hline
\end{tabular}




\section{ATTACHMENT 3}

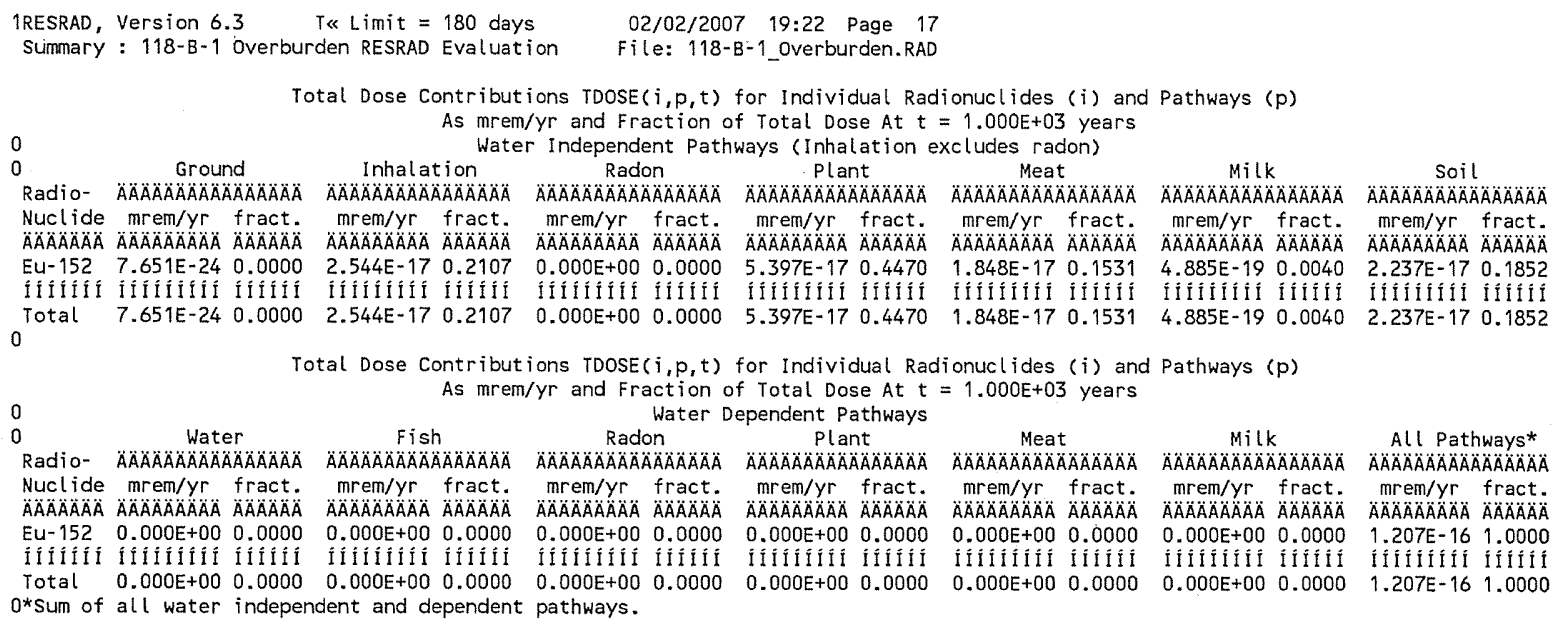

\begin{tabular}{|c|c|}
\hline Attachment & Sheet No. 17 of 19 \\
\hline Originators: S.W. Clark & Date \\
\hline Chk'd By M. W. Perrott & Date \\
\hline Calc. No. 0100B-CA-V0301 & Rev. No. 0 \\
\hline
\end{tabular}


CVP-2007-00006

Rev. 0

\title{
ATTACHMENT 3
}

\begin{abstract}
IRESRAD, Version 6.3 T« Limit $=180$ days $\quad 02 / 02 / 2007 \quad 19: 22$. Page 18 Summary : 118-B-1 Overburden RESRAD Evaluation File: 118-B-1_overburden.RAD
\end{abstract}

Dose/Source Ratios Summed Over All Pathways Parent and Progeny Principal Radionuclide Contributions Indicated

$\operatorname{DSR}(j, t)$ At Time in Years (mrem/yr)/(pCi/g)

(i) (j) Fraction $0.000 E+001.000 E+00 \quad 3.000 E+001.000 E+011.100 E+013.000 E+011.000 E+023.000 E+021.000 E+03$

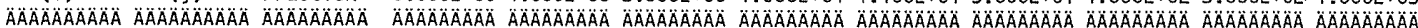

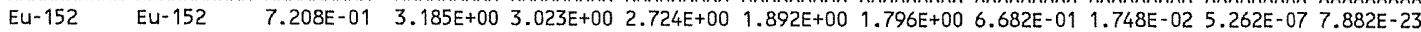

OEU-152 EU-152 2.792E-01 $1.234 E+001.171 E+001.055 E+00 \quad 7.330 E-016.958 E-012.588 E-016.769 E-032.038 E-073.053 E-23$

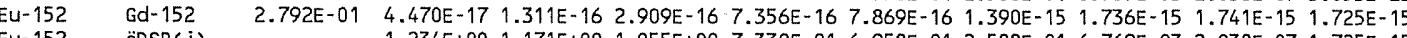

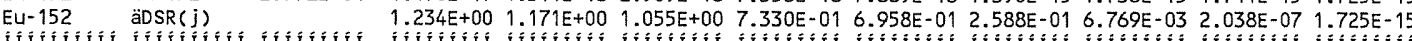

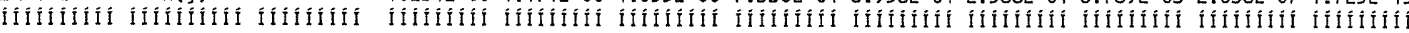
0

Single Radionuclide Soil Guidelines $G(i, t)$ in $p c i / g$

\begin{tabular}{|c|c|c|c|c|c|c|c|c|c|}
\hline $\begin{array}{c}\text { (i) } \\
\triangle A B A A A A A A\end{array}$ & $t=\begin{array}{l}0.000 E+00 \\
A A A A A A A A A A A\end{array}$ & $\begin{array}{l}1.000 E+00 \\
A A A A A B A A A A\end{array}$ & $\begin{array}{l}3.000 E+00 \\
A A B A O A A A A A\end{array}$ & $\begin{array}{l}1.000 E+01 \\
\triangle A A B A A B A O A\end{array}$ & $\begin{array}{l}1.100 E+01 \\
A B A A A B A D A A\end{array}$ & $\begin{array}{l}3.000 E+01 \\
\triangle A A A A O A O A O A\end{array}$ & $\begin{array}{l}1.000 E+02 \\
A \cap A O A O A A O A A\end{array}$ & $\begin{array}{l}3.000 E+02 \\
\triangle A O A O A A O A O A\end{array}$ & $\begin{array}{l}1.000 E+03 \\
A A O A A A A A A B\end{array}$ \\
\hline$=$ & $9.053 E-01$ & $9.537 \mathrm{E}-01$ & $1.058 \mathrm{E}+00$ & $1.524 \mathrm{E}+00$ & $1.605 E+00$ & $4.315 E+00$ & $1.650 E+02$ & $5.479 E+06$ & $* 1.765 \mathrm{E}+14$ \\
\hline If & 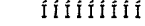 & IIIIIIIII & filtílití & fiflifififi & 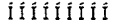 & IIIIIIIII & 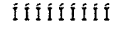 & 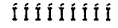 & \\
\hline
\end{tabular}
*At specific activity limit

Summed Dose/Source Ratios DSR( $i, t)$ in (mrem/yr) $/(\mathrm{pC} i / g)$

and single Radionuclide Soil Guidelines $G(i, t)$ in $\mathrm{pci} / \mathrm{g}$

at $\operatorname{tmin}=$ time of minimum single radionuclide soil guidel ine

and at tmax $=$ time of maximum total dose $=0.000 \mathrm{E}+00$ years

ONuclide Initial tmin DSR(i,tmin) $G(i, t \min ) \operatorname{DSR}(i, t \operatorname{tmax}) G(i, t \max )$

(i) $\quad(\mathrm{pC} i / g)$

(years)

$(\mathrm{pC} i / g)$
$(\mathrm{P})$

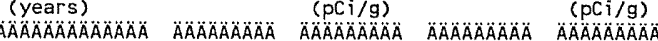

$\begin{array}{lllllll}\mathrm{Eu}-152 & 7.000 \mathrm{E}-02 & 0.000 \mathrm{E}+00 & 4.418 \mathrm{E}+00 & 9.053 \mathrm{E}-01 & 4.418 \mathrm{E}+00 & 9.053 \mathrm{E}-01\end{array}$

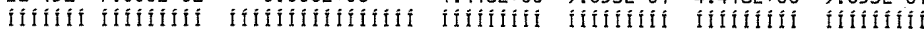

\begin{tabular}{|c|c|}
\hline Attachment & Sheet No. 18 of \\
\hline Driginators: S.W. Clark & Date \\
\hline Chk'd By M. W. Perrott & Date \\
\hline 0100B-CA-V0301 & Rev. No. 0 \\
\hline
\end{tabular}




\section{ATTACHMENT 3}

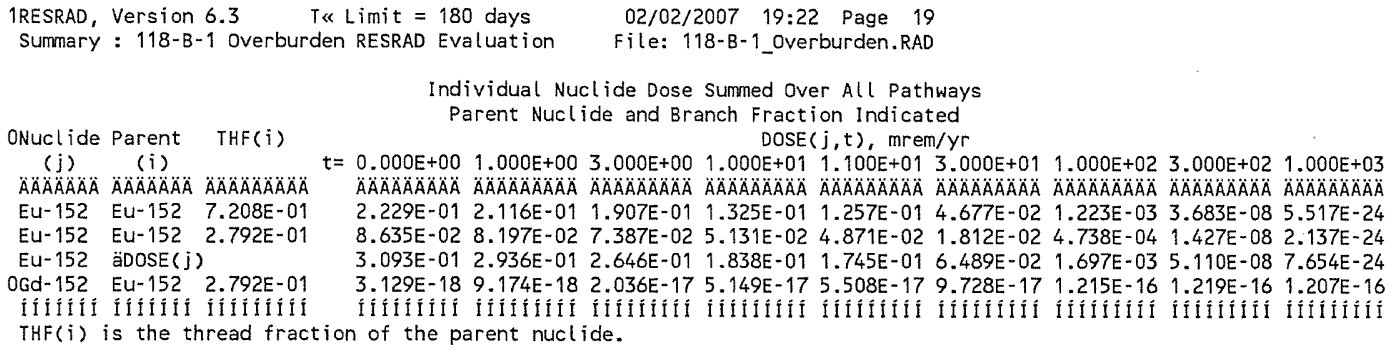
THF(i) is the thread fraction of the parent nuclide.

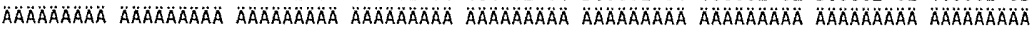

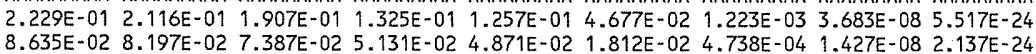

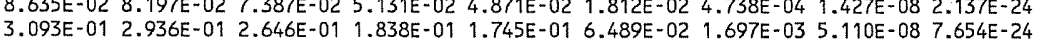

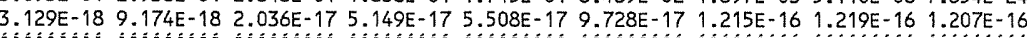

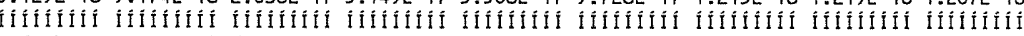

Individual Nuclide Soil Concentration

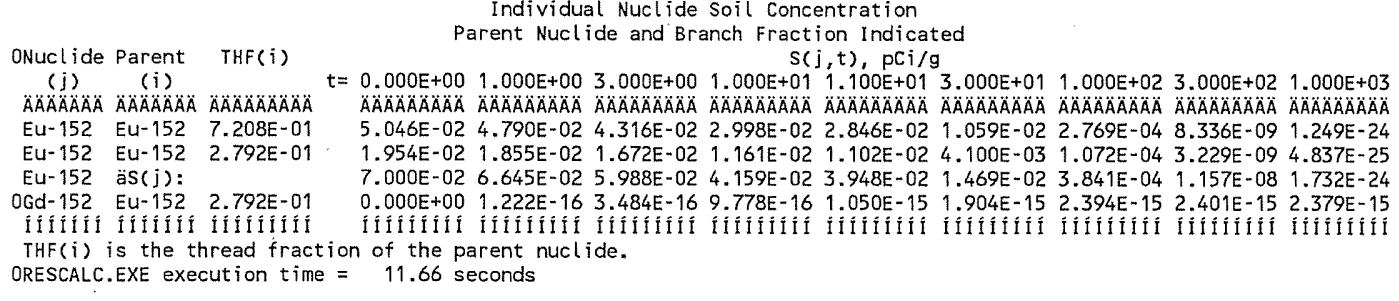

Attachment Originators: S. W. Clark 3 Sheet No. $\underline{19}$ of $\underline{19}$ Chk'd By M.W. Perrott Date

Calc. No. 0100B-CA-V0301 Date 


\section{ATTACHMENT 4}

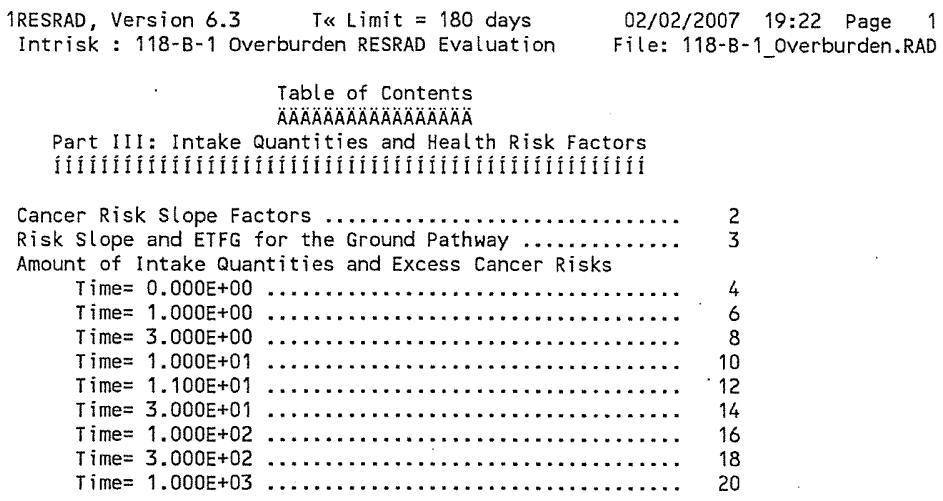

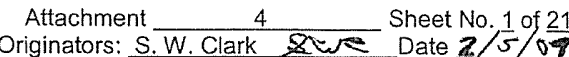

Chk'd By M.W. Perrott 'Mcef Date $2 / 5 / 67$

Calc. No. 0100 B-CA-V0301 Rev. No. _ 0 


\section{ATTACHMENT 4}

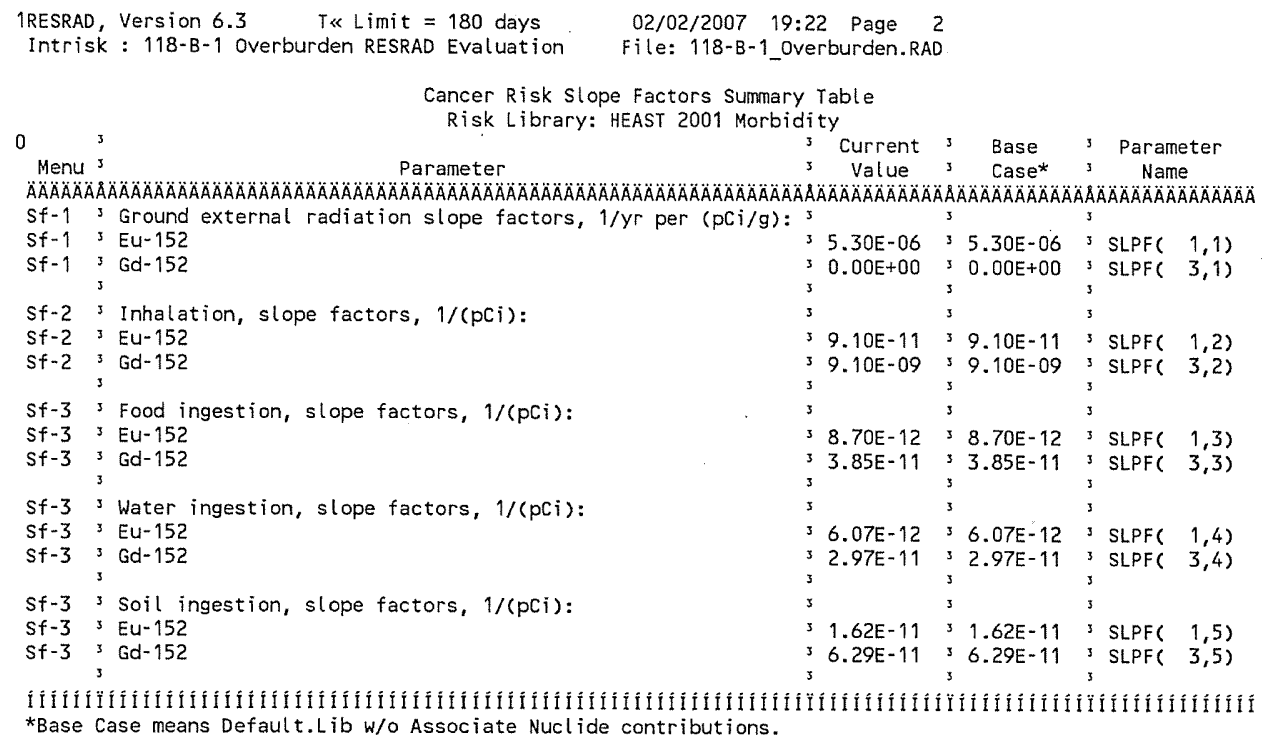

Attachment 
CVP-2007-00006

Rev. 0

\title{
ATTACHMENT 4
}

\begin{abstract}
1RESRAD, Version $6.3 \quad$ T\& Limit $=180$ days
Intrisk : $118-B-1$ Overburden RESRAD Evaluation
ONuclide Slope(i)*

Risk Slope and Environmental Transport Factors for the Ground Pathway

ETFG( $i, t$ ) At Time in Years (dimensionless)

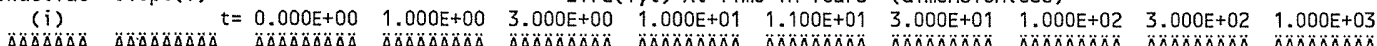

AAAAAAAAÄ ÄÄÄÄÄÄA A

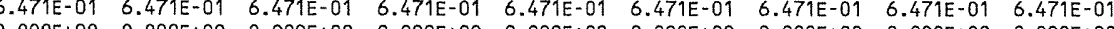

$\begin{array}{lllllllllll}\text { Gd-1 } & 0.000 E+00 & 0.000 E+00 & 0.000 E+00 & 0.000 E+00 & 0.000 E+00 & 0.000 E+00 & 0.000 E+00 & 0.000 E+00 & 0.000 E+00\end{array}$

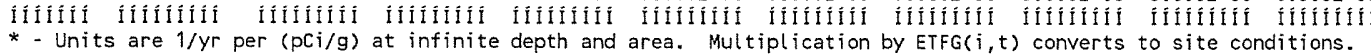

\begin{tabular}{|c|c|}
\hline Attachment & Sheet No. 3 of 21 \\
\hline Originators: S. W. Clark & Date \\
\hline Chk'd By M. W. Perrott & Date \\
\hline $0100 B-C A-V 0301$ & Rev. No. 0 \\
\hline
\end{tabular}




\section{ATTACHMENT 4}

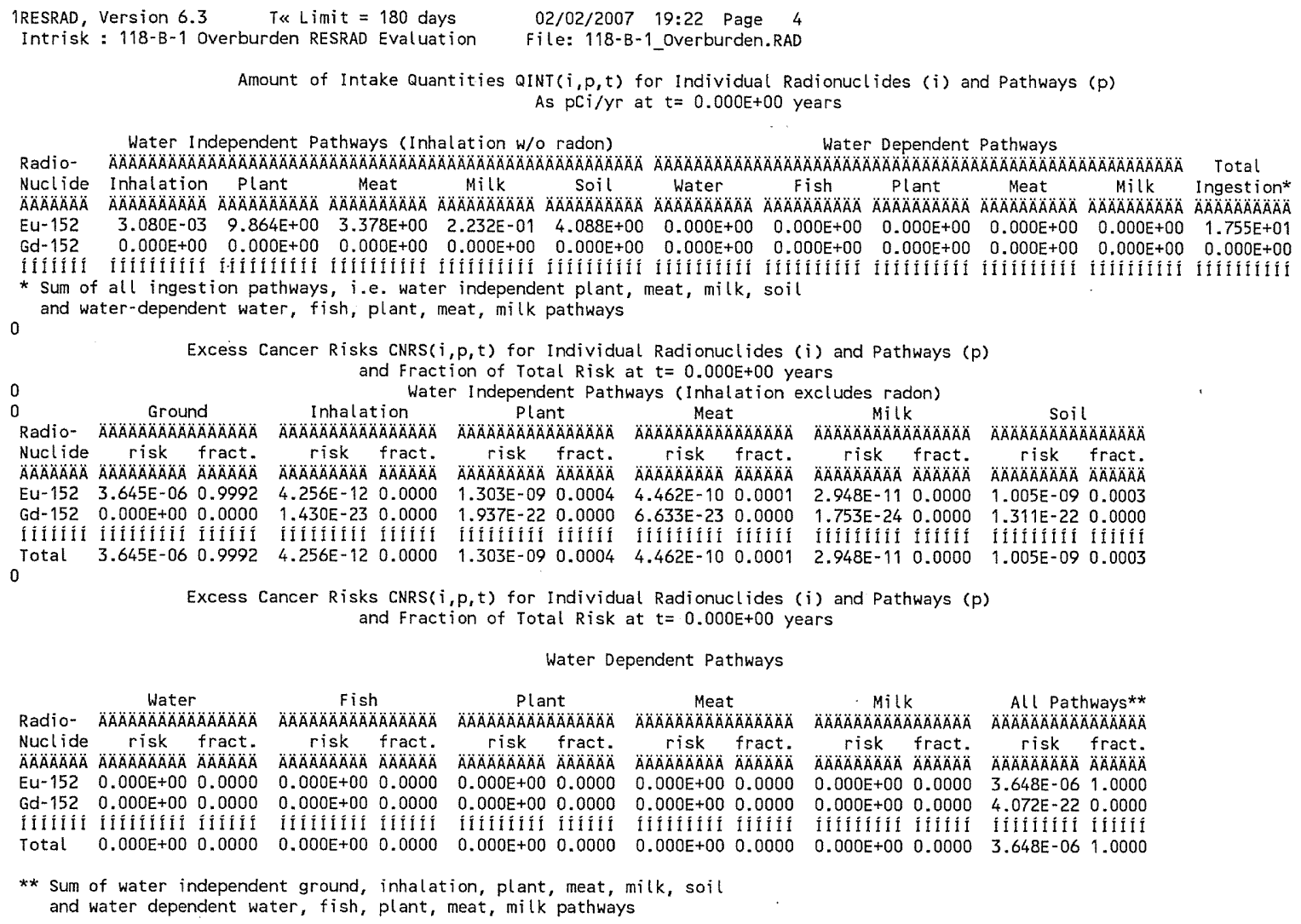


CVP-2007-00006

Rev. 0

\section{ATTACHMENT 4}

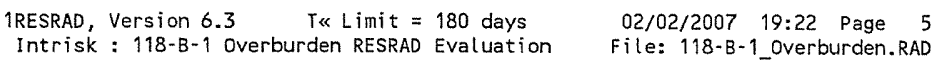

Total Excess Cancer Risk CNRSI $(i, p, t) * * *$ for Initially Existent Radionuclides $(i)$ and Pathways $(p)$ and Fraction of Total Risk at $t=0.000 E+00$ years

Water Dependent Pathways

\begin{tabular}{|c|c|c|c|c|c|c|c|}
\hline & 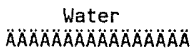 & 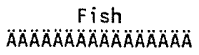 & $\begin{array}{c}\text { Radon } \\
\text { ÄÄÄAAAAAAAAOAOÄ }\end{array}$ & 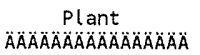 & $\begin{array}{c}\text { Meat } \\
\text { AAAAAAAAAAAAAAAAAÄ }\end{array}$ & $\begin{array}{c}M i l k \\
\text { AOAAAAAOAOAOAOAOAOA }\end{array}$ & $\begin{array}{c}\text { All pathways } \\
\text { AAAAAAAAAAAAAAAOA }\end{array}$ \\
\hline $\begin{array}{l}\text { Nuclide } \\
\text { ÄÄÄÄÄÄ }\end{array}$ & $\begin{array}{r}\text { risk fract. } \\
\triangle A A A A A B A A O A O A O A O A\end{array}$ & 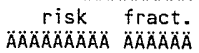 & 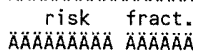 & $\begin{aligned} & r i s k \text { fract. } \\
& \text { AAAAAAAAAO AOAAAÄÄ }\end{aligned}$ & $\begin{array}{r}\text { risk fract. } \\
\text { ÄÄÄÄÄÄÄÄ ÄÄÄÄ̈̈ }\end{array}$ & 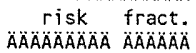 & 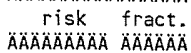 \\
\hline Eu-152 & $0.000 E+00 \quad 0.0000$ & $0.000 E+00 \quad 0.0000$ & $0.000 E+00 \quad 0.0000$ & $0.000 E+00 \quad 0.0000$ & $0.000 E+00 \quad 0.0000$ & $0.000 E+00 \quad 0.0000$ & -061.0000 \\
\hline & & IIIIIIIII IIIIII & İiIIIIII ÎIIIII & 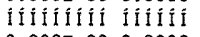 & 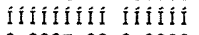 & 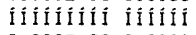 & IIf Itî́n \\
\hline al & $0.000 E+00 \quad 0.0000$ & $0.000 E+00 \quad 0.0000$ & $0.000 E+00 \quad 0.0000$ & $0.000 \mathrm{E}+00 \quad 0.0000$ & $0.000 E+00 \quad 0.0000$ & $0.000 E+00 \quad 0.0000$ & $3.648 \mathrm{E}-06 \quad 1.0000$ \\
\hline
\end{tabular}

$* * * \operatorname{CNRS} I(i, p, t)$ includes contribution from decay daughter radionuclides

Attachment

Originators: S.W. Clark

4

Sheet No. $\underline{5}$ of $\underline{21}$

Chk'd By M.W. Perrott Date

Calc. No. 0100B-CA-V0301

Rev. No. 


\section{ATTACHMENT 4}

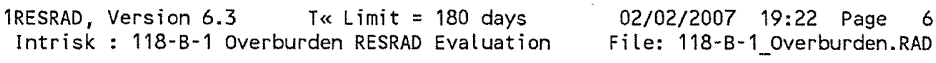

Water Dependent Pathways

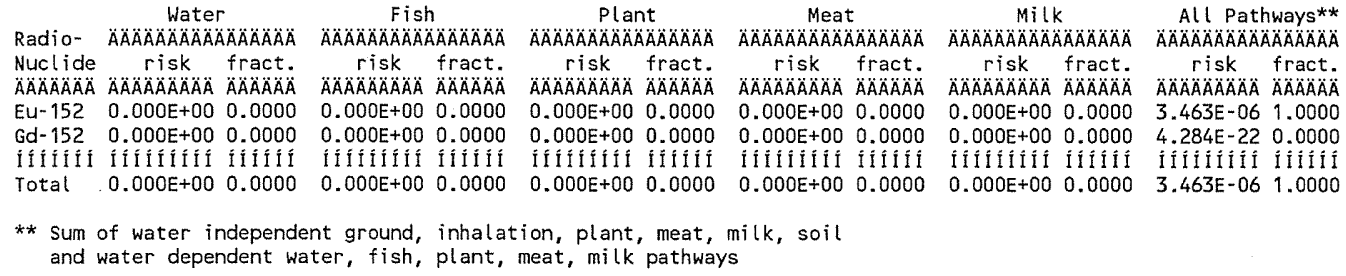

\begin{tabular}{ll} 
Attachment $\frac{4}{\text { On. Clark }}$ & Sheet No. $\underline{6}$ of $\underline{21}$ \\
Originators: & Date \\
Chk'd By & Date \\
Calc. No. W. Perrott & Rev. No. $\quad 0$ \\
\hline
\end{tabular}


CVP-2007-00006

Rev. 0

\section{ATTACHMENT 4}

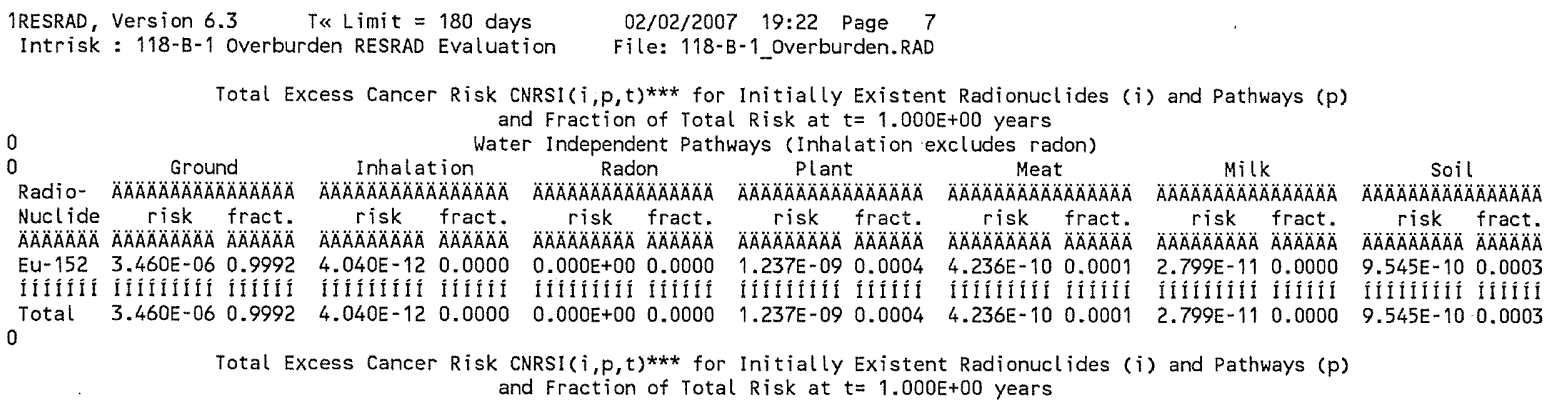

Water Dependent Pathways

\begin{tabular}{|c|c|c|c|c|c|c|c|}
\hline Radio- & $\begin{array}{c}\text { Water } \\
A A A A A A A A A A A B A B\end{array}$ & $\begin{array}{c}F i s h \\
\text { AAAAAAAAAAAAAOAOÄ }\end{array}$ & $\begin{array}{c}\text { Radon } \\
\text { AAAAAAAAAOAAAAAA }\end{array}$ & 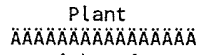 & $\begin{array}{c}\text { Meat } \\
\text { AAOAAAAOAOAOAOAOAOAOA }\end{array}$ & 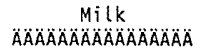 & 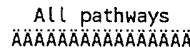 \\
\hline $\begin{array}{l}\text { Nucl ide } \\
\text { ÄAAAAAAA }\end{array}$ & 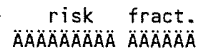 & $\begin{array}{cc}\text { risk fract. } \\
\text { AAAAAAAA AAAAAA }\end{array}$ & $\begin{array}{c}\text { risk fract. } \\
\text { ÁAAAAAAAA AAAAAA }\end{array}$ & $\begin{array}{r}\text { risk fract. } \\
\text { ÄAAAÄAAAA AAÄÄÄ }\end{array}$ & $\begin{array}{r}\text { risk fract. } \\
\text { ÁAAAAAAAAA AAÄÄÄA }\end{array}$ & $\begin{array}{r}\text { risk fract. } \\
\text { AAAAAAAAA AAAAAA }\end{array}$ & $\begin{array}{r}\text { risk fract. } \\
\text { AAAAAAAAA AAAAAAA }\end{array}$ \\
\hline Eu- 152 & $0.000 E+000.0000$ & $0.000 E+00 \quad 0.0000$ & $0.000 E+00 \quad 0.0000$ & $0.000 E+00 \quad 0.0000$ & $0.000 E+00 \quad 0.0000$ & $0.000 E+00 \quad 0.0000$ & -061.0000 \\
\hline & & IIIIIIIIII IIIIIII & 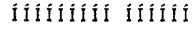 & IIIIIIIIII IIIIIII & IIIIAIIIII IIIIIÍ & 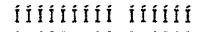 & IIIIIIIII IfIIII \\
\hline Total & $0.000 \mathrm{E}+000.0000$ & $0.000 E+00 \quad 0.0000$ & $0.000 E+00 \quad 0.0000$ & $0.000 E+00 \quad 0.0000$ & $0.000 E+00 \quad 0.0000$ & $0.000 E+00 \quad 0.0000$ & $3.463 \mathrm{E}-06 \quad 1.0000$ \\
\hline
\end{tabular}

$* * * \operatorname{CNRSI}(i, p, t)$ includes contribution from decay daughter radionucl ides

Attachment

Sheet No. 7 of 21

Originators: S. W. Clark Date

Chk'd By M.W. Perrott Date

Calc. No. 0100B-CA-V0301 Rev. No. 0 


\section{ATTACHMENT 4}

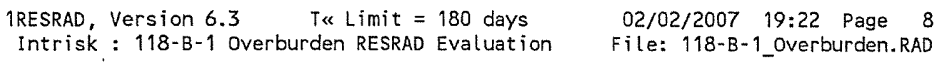

Water Dependent Pathways

Water
Radio-

and water dependent water, fish, plant, meat, milk pathways

\begin{tabular}{|c|c|}
\hline Attachment & Sheet No. 8 of 21 \\
\hline $\begin{array}{l}\text { Attachment } \\
\text { riqinators: S. W. Clark }\end{array}$ & Date \\
\hline M.W. Perrott & Date \\
\hline Calc. No. $0100 \mathrm{~B}-\mathrm{CA}-\mathrm{V} 0301$ & Rev. No. 0 \\
\hline
\end{tabular}




\section{ATTACHMENT 4}

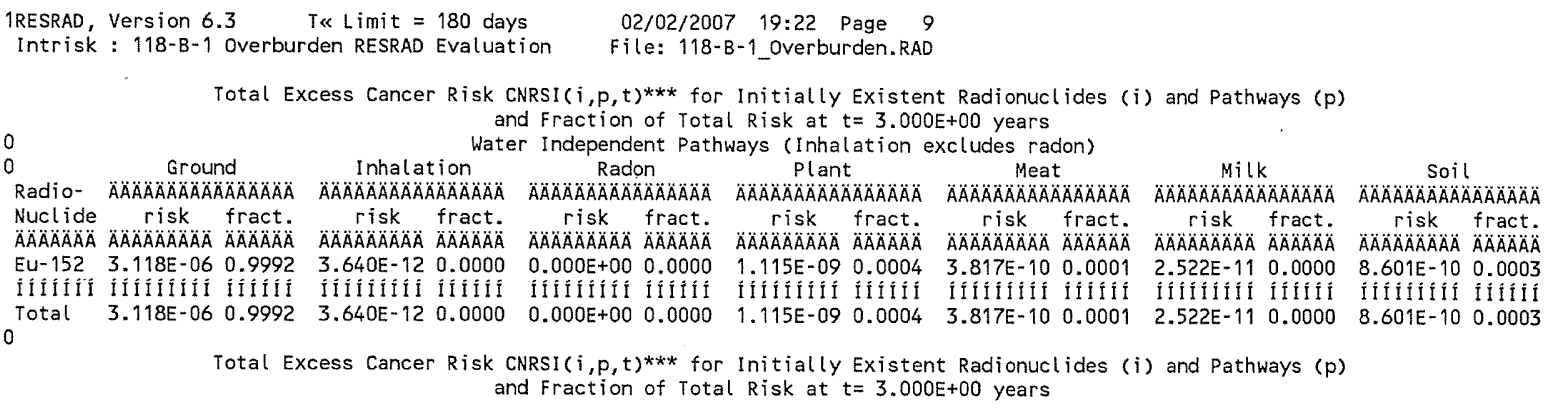

Total Excess Cancer Risk CNRSI $(i, p, t) * * *$ for Initially Existent Radionuclides $(i)$ and Pathways $(p)$ and Fraction of Total Risk at $t=3.000 E+00$ years

Water Dependent Pathways

\begin{tabular}{|c|c|c|c|c|c|c|c|}
\hline Radio- & 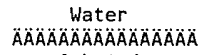 & 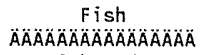 & $\begin{array}{c}\text { Radon } \\
\text { ÄAAAAAAAAAAAAAA }\end{array}$ & $\begin{array}{c}\text { Plant } \\
\text { ÄAAAAAAAAAAAAOAOAOA }\end{array}$ & $\begin{array}{c}\text { Meat } \\
\text { AAAAAAAAAAAAAAAOAOA }\end{array}$ & 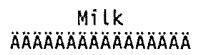 & $\begin{array}{c}\text { All pathways } \\
\text { AAAAAAAAAAAAAAAAAA }\end{array}$ \\
\hline $\begin{array}{l}\text { Nuclide } \\
\text { ÄÄÄÄÄÄ }\end{array}$ & $\begin{array}{l}\text { risk fract. } \\
\text { AAAAAAAOAO AAAAAAA }\end{array}$ & $\begin{array}{r}\text { risk fract. } \\
\text { AAAAAAOAAOA } \triangle A A O A A A A\end{array}$ & $\begin{array}{r}\text { risk fract. } \\
\triangle A A A A B A A A B A A A B A A\end{array}$ & $\begin{array}{l}\text { risk fract. } \\
\text { AAAAAAAAAO AAAAAAA }\end{array}$ & $\begin{array}{r}\text { risk fract. } \\
\text { AAAAAAAAAOA AOAAAOÄ }\end{array}$ & $\begin{array}{r}\text { risk fract. } \\
\text { AAAAAAAAA AOAAAAOA }\end{array}$ & 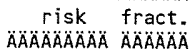 \\
\hline$E u-152$ & $0.000 E+00 \quad 0.0000$ & $0.000 E+00 \quad 0.0000$ & $0.000 E+00 \quad 0.0000$ & $0.000 \mathrm{E}+00 \quad 0.0000$ & $0.000 E+00 \quad 0.0000$ & $0.000 E+00 \quad 0.0000$ & -061.0000 \\
\hline IfIifíf & Iffifitio & IIII fiIIII & ílítilitil & 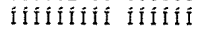 & IIIIIIIII IIIIII & 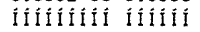 & 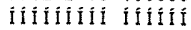 \\
\hline Total & $0.000 E+00 \quad 0.0000$ & $0.000 E+00 \quad 0.0000$ & $0.000 E+00 \quad 0.0000$ & $0.000 E+00 \quad 0.0000$ & $0.000 E+00 \quad 0.0000$ & $0.000 E+00 \quad 0.0000$ & $3.120 E-06 \quad 1.0000$ \\
\hline
\end{tabular}

***CNRSI $(i, p, t)$ includes contribution from decay daughter radionuclides

Attachment

Originators: S.W. Clark

4

Sheet No. $\underline{9}$ of $\underline{21}$

Chk'd By M.W. Perrott

Date

Calc. No. $0100 \mathrm{~B}-\mathrm{CA}-\mathrm{V} 0301$

Rev. No. 0 


\section{ATTACHMENT 4}

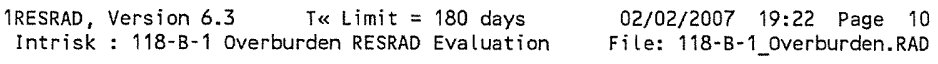

Excess Cancer Risks CNRS $(i, p, t)$ for Individual Radionuclides (i) and Pathways $(p)$ and Fraction of Total Risk at $t=1.000 E+01$ years

Water Dependent Pathways

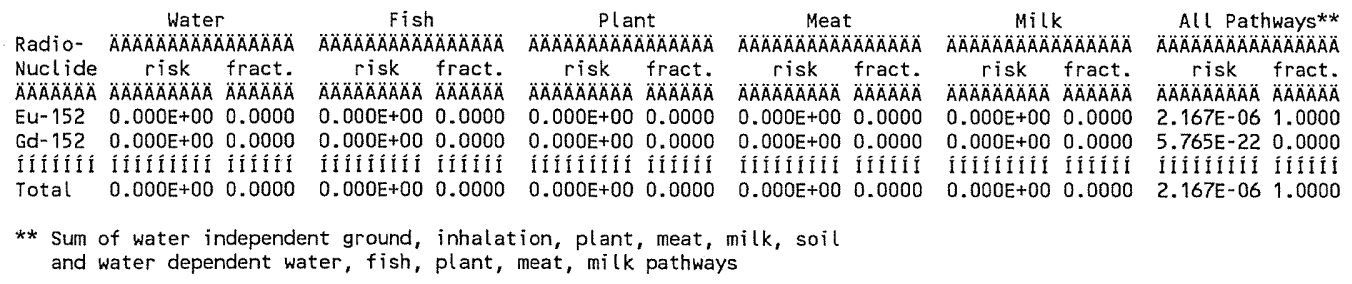

and water dependent water, fish, plant, meat, milk pathways

\begin{tabular}{|c|c|c|}
\hline \multirow{3}{*}{\multicolumn{3}{|c|}{$\begin{array}{l}\text { Attachment } \frac{4}{\text { Originators: }} \text { S.W. Clark } \\
\text { Date }\end{array}$}} \\
\hline & & \\
\hline & & \\
\hline Calc & $0100 B-C A-V 0301$ & Rev. No. 0 \\
\hline
\end{tabular}




\section{ATTACHMENT 4}

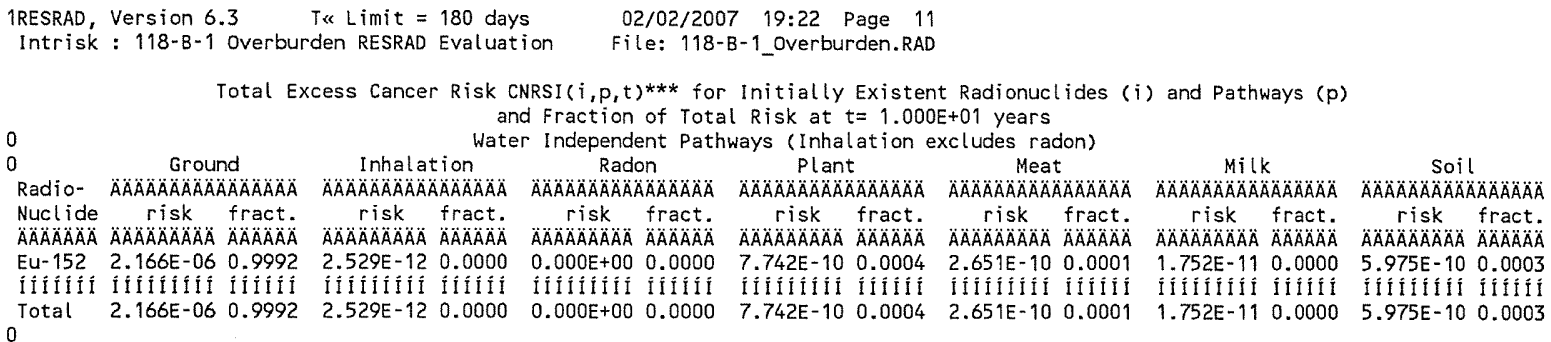

Water Dependent Pathways

\begin{tabular}{|c|c|c|c|c|c|c|c|}
\hline Rad & 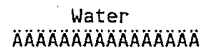 & $\begin{array}{c}F i s h \\
\triangle A A A B A A A O A A B A A A O A A\end{array}$ & $\begin{array}{c}\text { Radon } \\
\triangle A A A A B A O A O A O A O A O A O A O A\end{array}$ & 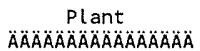 & 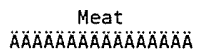 & 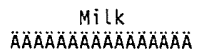 & 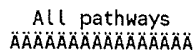 \\
\hline $\begin{array}{l}\text { Nuclide } \\
\dddot{A A O A O A O A O A}\end{array}$ & $\begin{array}{l}\text { risk fract. } \\
\text { AAAAAAAAA AAAAAA }\end{array}$ & $\begin{array}{rr}r i s k & \text { fract. } \\
\text { AAAAAAAAA ÄAAAAAA }\end{array}$ & $\begin{array}{l}\text { risk fract. } \\
\text { AAAAAAAAAOA AAAAAAOA }\end{array}$ & $\begin{array}{r}\text { risk fract } \\
\text { AAAAAAAAO AAAAAA }\end{array}$ & $\begin{array}{r}\text { risk fract. } \\
\text { AAAAAAAAAA AAAAAAA }\end{array}$ & $\begin{array}{r}\text { risk fract. } \\
\text { AAAAAAAAA ABÄÄÄ }\end{array}$ & $\begin{array}{r}\text { risk fract. } \\
\text { AAAAAOAAOA AAAAÄÄ }\end{array}$ \\
\hline Eu- 152 & $0.000 E+00 \quad 0.0000$ & $0.000 E+00 \quad 0.0000$ & $0.000 E+00 \quad 0.0000$ & $0.000 E+00 \quad 0.0000$ & $0.000 E+00 \quad 0.0000$ & $0.000 E+00 \quad 0.0000$ & $2.167 \mathrm{E}-06 \quad 1.0000$ \\
\hline$a l$ & $0.000 E+00 \quad 0.0000$ & $0.000 E+00 \quad 0.0000$ & $0.000 E+00 \quad 0.0000$ & $0.000 E+00 \quad 0.0000$ & $0.000 E+00 \quad 0.0000$ & $0.000 E+00 \quad 0.0000$ & $2.167 \mathrm{E}-06 \quad 1.0000$ \\
\hline
\end{tabular}

$* * *$ CNRSI $(i, p, t)$ includes contribution from decay daughter radionuclides

\begin{tabular}{|c|c|}
\hline Attachment & Sheet No. 11 of 21 \\
\hline Originators: S.W. Clark & Date \\
\hline M. W. Perrott & Date \\
\hline Calc. No. 0100B-CA-V0301 & Rev. No. \\
\hline
\end{tabular}




\title{
ATTACHMENT 4
}

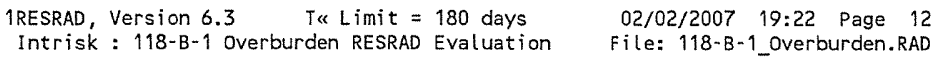
and water-dependent water, fish, plant, meat, milk pathways

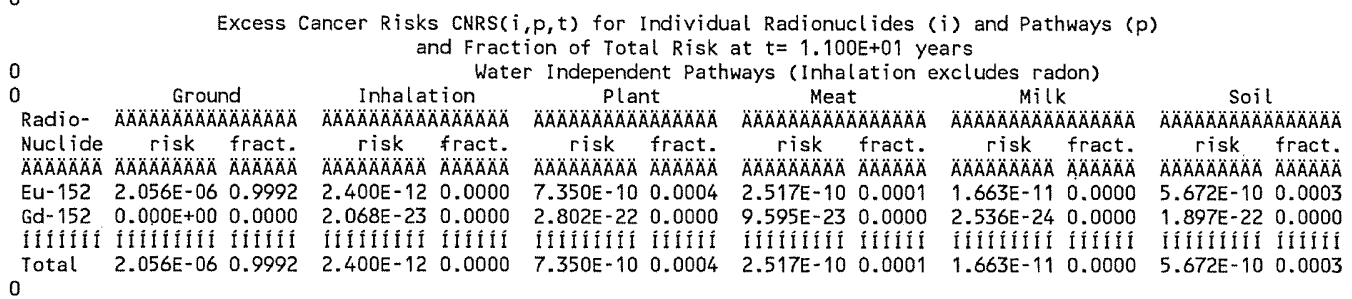
$0^{\top}$ Excess Cancer Risks CNRS $(i, p, t)$ for Individual Radionuclides $(i)$ and Pathways $(p)$
and Fraction of Total Risk at $t=1.100 E+01$ years

Water Dependent Pathways

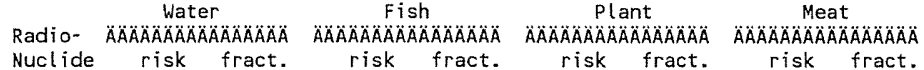
Water
Radio-
Auclide Water
Radio-
Auclide

Meat Nuclide risk fract. risk fract. risk fract. risk fract.

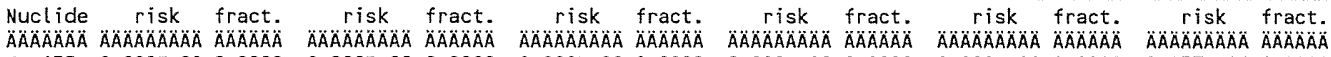

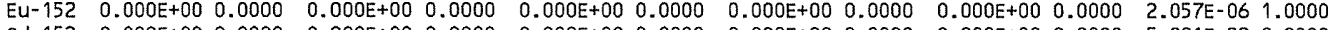
$\begin{array}{lllllllllllllll}G d-152 & 0.000 E+00 & 0.0000 & 0.000 E+00 & 0.0000 & 0.000 E+00 & 0.0000 & 0.000 E+00 & 0.0000 & 0.000 E+00 & 0.0000 & 5.891 E-22 & 0.0000\end{array}$

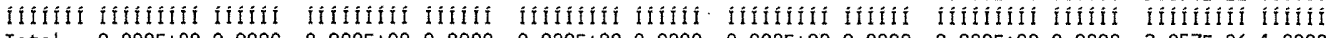
$\begin{array}{llllllllllllllllllllll}\text { Tatal } & 0.000 E+00 & 0.0000 & 0.000 E+00 & 0.0000 & 0.000 E+00 & 0.0000 & 0.000 E+00 & 0.0000 & 0.000 E+00 & 0.0000 & 2.057 E-06 & 1.0000\end{array}$

** Sum of water independent ground, inhalation, plant, meat, milk, soil

and water dependent water, fish, plant, meat, milk pathways
\end{abstract}

\begin{tabular}{|c|c|}
\hline Attachment & Sheet No. 12 of 21 \\
\hline Originators: S.W. Clark & Date \\
\hline M. W. Perrott & Date \\
\hline $0100 B-C A-V 0301$ & Rev. No. \\
\hline
\end{tabular}




\section{ATTACHMENT 4}

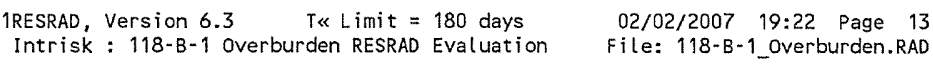

Total Excess Cancer Risk CNRSI $(i, p, t) * * *$ for Initially Existent Radionuclides ( $i$ ) and Pathways ( $p$ ) and Fraction of Total Risk at $t=1.100 E+01$ years

Water Dependent Pathways

\begin{tabular}{|c|c|c|c|c|c|c|c|}
\hline 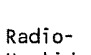 & $\begin{array}{c}\text { Water } \\
\triangle A \cap A A A O A A A O A A A A O A A O A\end{array}$ & 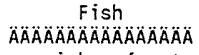 & 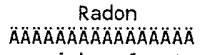 & 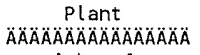 & 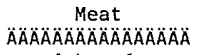 & $\begin{array}{c}M i l k \\
\triangle A O A A O A A O A O A A A A O A O A O A\end{array}$ & 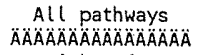 \\
\hline $\begin{array}{l}\text { Nucl ide } \\
\triangle A A A B A B A B\end{array}$ & $\begin{array}{ll}\text { risk fract. } \\
\triangle A A B A O A O A O A O A B\end{array}$ & $\begin{array}{r}\text { risk fract. } \\
\text { AAAAAAAOAOA AOAAOAOA }\end{array}$ & $\begin{array}{r}\text { risk fract. } \\
\text { AAAAAAAAA AOAAAA }\end{array}$ & $\begin{array}{r}\text { risk fract. } \\
\text { AAAOAOAAAO } A O A O A O A B\end{array}$ & $\begin{array}{r}\text { risk fract. } \\
\text { AAAOAOAOAOA }\end{array}$ & 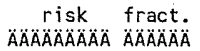 & $\begin{array}{l}\text { risk fract. } \\
\triangle A A A A B A \text { AAAAAAA }\end{array}$ \\
\hline Eu -152 & $0.000 E+00 \quad 0.0000$ & $0.000 E+00 \quad 0.0000$ & $0.000 \mathrm{E}+00 \quad 0.0000$ & $0.000 E+00 \quad 0.0000$ & $0.000 \mathrm{E}+00 \quad 0.0000$ & $0.000 E+00 \quad 0.0000$ & FAM AI \\
\hline fififif & 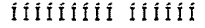 & 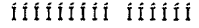 & 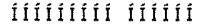 & Iilílilifí & 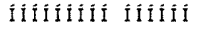 & ifitilifi fififi & Ifififitílití \\
\hline To & $0.000 E+00 \quad 0.0000$ & $0.000 E+00 \quad 0.0000$ & $0.000 E+00 \quad 0.0000$ & $0.000 E+00 \quad 0.0000$ & $0.000 E+00 \quad 0.0000$ & $0.000 E+00 \quad 0.0000$ & $2.057 \mathrm{E}-06 \quad 1.000$ \\
\hline
\end{tabular}

$* * * \operatorname{CNRSI}(i, p, t)$ includes contribution from decay daughter radionuclides

Attachment $4 \quad$ Sheet No. 13 of $\underline{21}$ Chk'd By. $\frac{\text { S.W. Clark }}{\text { M.W. Perrott }}$ Date

Calc. No. $0100 \mathrm{~B}-\mathrm{CA}-\mathrm{V} 0301$ 


\section{ATTACHMENT 4}

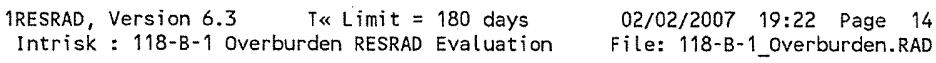

Water Dependent Pathways

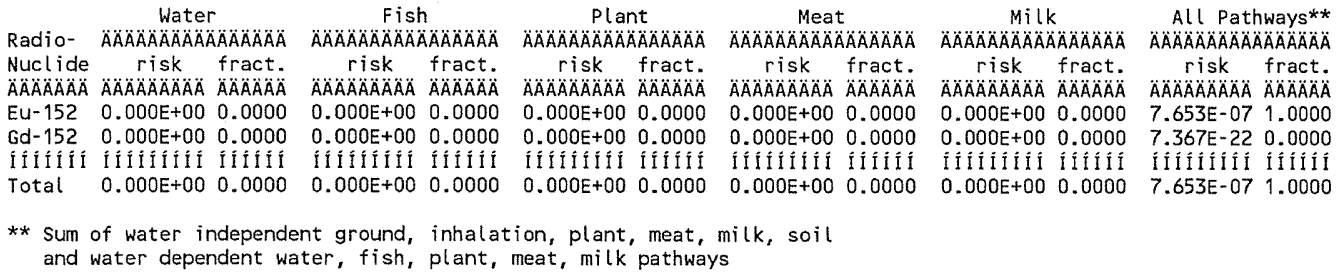

** Sum of water independent ground, inhalation, plant, meat, milk, soil

and water dependent water, fish, plant, meat, milk pathways

\begin{tabular}{|c|c|c|}
\hline Attachment & 4 & Sheet No. 1 \\
\hline Originators: & S. W. Clark & Date \\
\hline Chk'd By & M. W. Perrott & Date \\
\hline Calc. No. & $0100 B-C A-V 0301$ & Rev. No. \\
\hline
\end{tabular}




\section{ATTACHMENT 4}

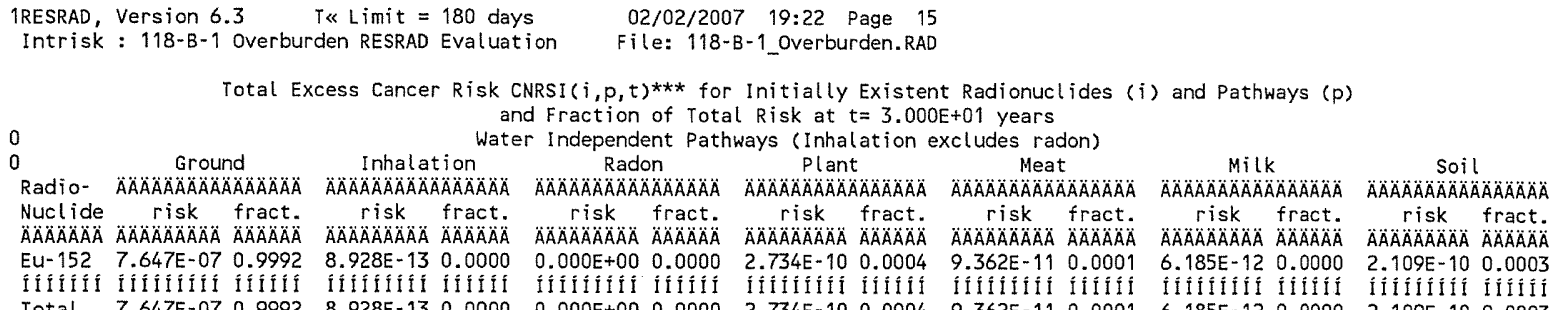

\begin{tabular}{|c|c|}
\hline Attachment & Sheet No. 15 of 21 \\
\hline Originators: S.W. Clark & Date \\
\hline Chk'd By M. W. Perrott & Date \\
\hline Calc. No. $0100 \mathrm{~B}-\mathrm{CA}-\mathrm{V} 0301$ & Rev. No. \\
\hline
\end{tabular}




\section{ATTACHMENT 4}

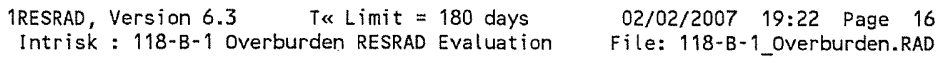

Water Dependent Pathways

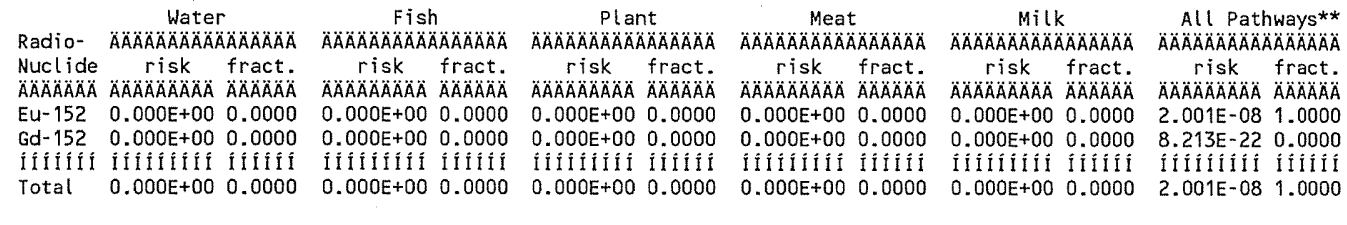

** Sum of water independent ground, inhalation, plant, meat, milk, soil

and water dependent water, fish, plant, meat, milk pathways

\begin{tabular}{|c|c|c|}
\hline \multirow{2}{*}{\multicolumn{3}{|c|}{$\begin{array}{ll}\text { Attachment } & 4 \\
\text { Originators: S.W. Clark } & \text { Sheet No. } 16 \text { of } \underline{21} \\
\text { Date }\end{array}$}} \\
\hline & & \\
\hline Chk'd By & M.W. Perrott & Date \\
\hline Calc. No. & $0100 B-C A-V 0301$ & Rev. No. \\
\hline
\end{tabular}


CVP-2007-00006

Rev. 0

\section{ATTACHMENT 4}

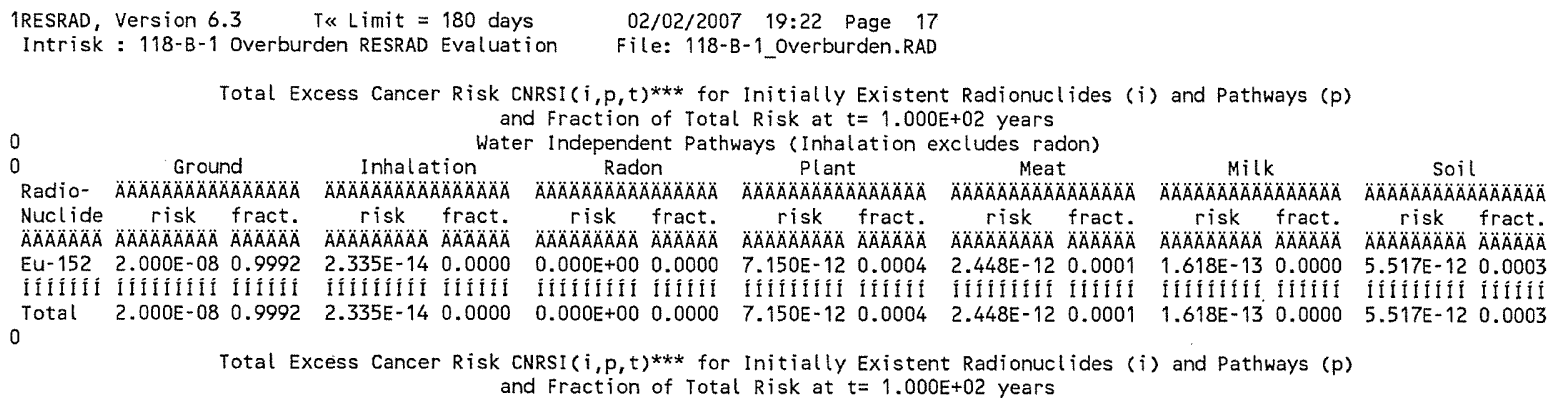

Water Dependent Pathways

Water
Radio-

***CNRSI $(i, p, t)$ includes contribution from decay daughter radionuclides

Attachment

Originators: S.W. Clark

Sheet No. $\underline{17}$ of $\underline{21}$

Chk'd By M.W. Perrott Date

Calc. No. 0100B-CA-V0301

Rev. No. 


\section{ATTACHMENT 4}

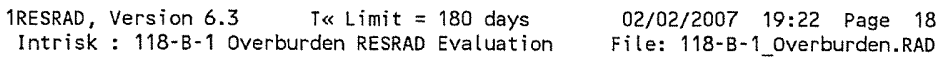
and water-dependent water, fish, plant, meat, milk pathways

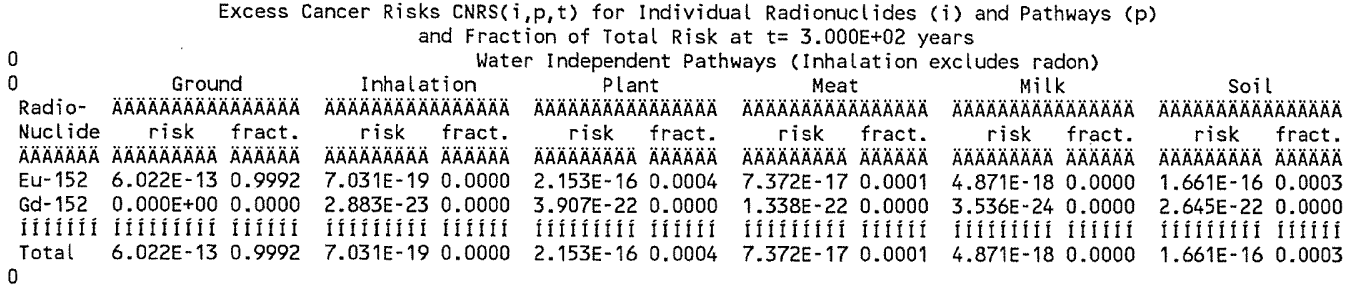

Excess Cancer Risks CNRS $(i, p, t)$ for Individual Radionuclides (i) and Pathways $(p)$ and Fraction of Total Risk at $t=3.000 E+02$ years

Water Dependent Pathways

\begin{tabular}{|c|c|c|c|c|c|c|c|c|c|c|}
\hline Radio- & $\begin{array}{r}\text { Wate } \\
\triangle A \cap A B A A A B A\end{array}$ & $\stackrel{r}{A}$ & $\begin{array}{r}F i s h \\
\text { AOAAAAAAA }\end{array}$ & 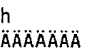 & $\begin{array}{r}P l a r \\
A A A A A A A A A I\end{array}$ & $\begin{array}{l}n t \\
\text { ÄÄÄÄÄÄÄ }\end{array}$ & $\begin{array}{r}\text { Mea } \\
\text { ÄÄÄAÄÄÄ }\end{array}$ & 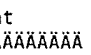 & 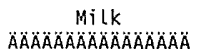 & $\begin{array}{r}\text { All Pathways** } \\
\text { ÄÄÄAAAAAAOAAAOAAA }\end{array}$ \\
\hline $\begin{array}{l}\text { Nuclide } \\
\ddot{A A O A A} \ddot{A} \ddot{A} \ddot{A}\end{array}$ & $\begin{array}{c}\text { risk } \\
\triangle A \cap A O A ̈ A O A O A O A\end{array}$ & $\begin{array}{l}\text { fract. } \\
\dddot{A} \ddot{A} \ddot{A} A \ddot{A} \bar{A}\end{array}$ & 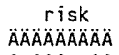 & $\begin{array}{l}\text { fract. } \\
\triangle A B A B A B A\end{array}$ & $\begin{array}{c}\text { risk } \\
\text { AिAAAAAAAA }\end{array}$ & 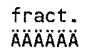 & $\begin{array}{c}\text { risk } \\
\text { AAAAAAAAA }\end{array}$ & $\begin{array}{l}\text { fract. } \\
\text { AAAAAAÄ }\end{array}$ & $\begin{array}{c}\text { risk fract. } \\
\text { ÄAAAAOAOAO AAAOABÄ }\end{array}$ & 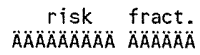 \\
\hline Eu-152 & $0.000 E+00$ & 0.0000 & $0.000 E+00$ & 0.0000 & $0.000 E+00$ & 0.0000 & $0.000 E+00$ & 0.0000 & $0.000 E+00 \quad 0.0000$ & $6.026 \mathrm{E}-13 \quad 1.0000$ \\
\hline Gd-152 & $0.000 E+00$ & 0.0000 & $0.000 \mathrm{E}+00$ & 0.0000 & $0.000 E+00$ & 0.0000 & $0.000 \mathrm{E}+00$ & 0.0000 & $0.000 E+00 \quad 0.0000$ & $8.214 \mathrm{E}-22 \quad 0.0000$ \\
\hline Ilitififíl & Iflílílít & İílífí & IIIIIYîYi & IIIfIII & 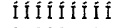 & 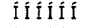 & IIfIfIfII & IIIIIII & 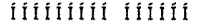 & 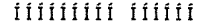 \\
\hline Total & $0.000 E+00$ & 0.0000 & $0.000 E \div 00$ & 0.0000 & $0.000 E+00$ & 0.0000 & $0.000 E+00$ & 0.0000 & $0.000 E+00 \quad 0.0000$ & $6.026 \mathrm{E}-13 \quad 1.0000$ \\
\hline
\end{tabular}
Attachment $\frac{4}{\text { Originators: S.W. Clark }}$ Sheet No. 18 of $\underline{21}$ Chk'd By M.W.Perrott Date

Calc. No. $0100 \mathrm{~B}-\mathrm{CA}-\mathrm{V} 0301$

Rev. No. 0 
CVP-2007-00006

Rev. 0

\section{ATTACHMENT 4}

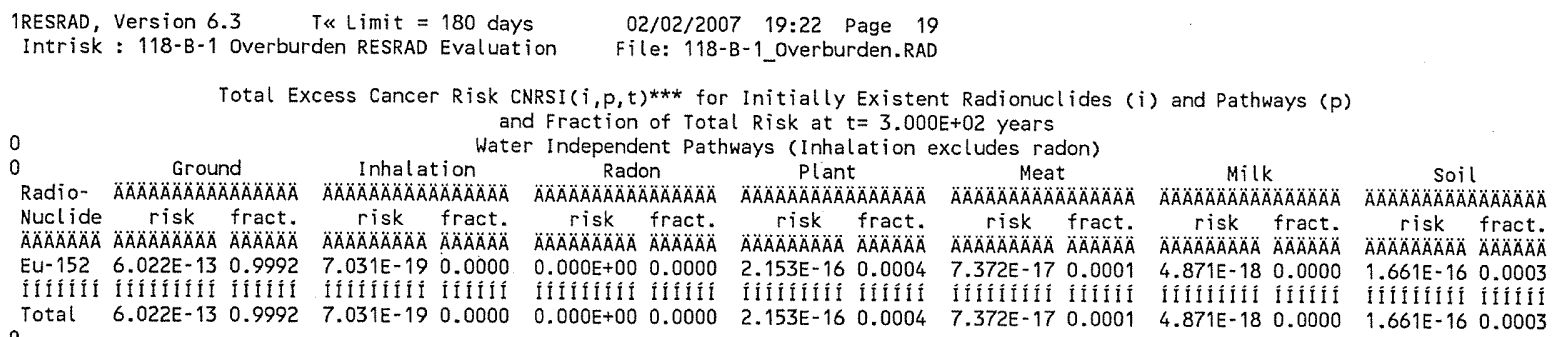

Water Dependent Pathways

\begin{tabular}{|c|c|c|c|c|c|c|c|c|c|c|c|c|}
\hline & 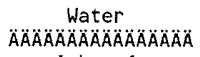 & $\begin{array}{c}F i s h \\
\triangle A \cap A A A A B A A A B A A B A B\end{array}$ & 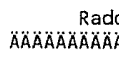 & On & 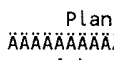 & 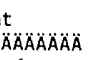 & $\begin{array}{r}\text { Meat } \\
\text { ĀÄÄAAAAOA }\end{array}$ & $\triangle A \cap A \cap A A B A$ & $\begin{array}{r}M i l \\
\text { A ÄAAAAAÄA }\end{array}$ & $\triangle A A A A A O A$ & $\begin{array}{l}\text { AlL pat } \\
A A B A B A A B A\end{array}$ & $\begin{array}{l}\text { hways } \\
\triangle A A A O A A O A B\end{array}$ \\
\hline $\begin{array}{l}\text { Nuclide } \\
\text { ÄÄAAAAÄ }\end{array}$ & $\begin{array}{c}\text { risk fract. } \\
\text { ÄAAAAAAA ÄÄÄÄ }\end{array}$ & $\begin{array}{c}\text { risk fract. } \\
\text { ÄAAAAAAAA AAAAAA }\end{array}$ & $\begin{array}{c}\text { risk } \\
\text { AAAAAAAAA }\end{array}$ & $\begin{array}{l}\text { fract. } \\
\because A B A A B A B\end{array}$ & $\begin{array}{c}\text { risk } \\
\text { ÄÄÄÄÄÄÄ }\end{array}$ & $\begin{array}{l}\text { fract. } \\
\text { ÄÄÄÄÄ }\end{array}$ & $\begin{array}{c}\text { risk } \\
A ̈ A \cap A B A A A A B\end{array}$ & $\begin{array}{l}\text { fract. } \\
\triangle A B A A O A B A\end{array}$ & $\begin{array}{c}\text { risk } \\
\text { AAOAAOAOAOAOA }\end{array}$ & $\begin{array}{l}\text { fract. } \\
\text { ÄAAAAA }\end{array}$ & $\begin{array}{c}\text { risk } \\
\text { AAAAAAAAAB }\end{array}$ & $\begin{array}{l}\text { fract. } \\
A A A A A B A\end{array}$ \\
\hline & $0.000 E+00 \quad 0.0000$ & $0.000 E+00 \quad 0.0000$ & $0.000 E+00$ & 0.0000 & $0.000 E+00$ & 0.0000 & $0.000 E+00$ & 0.0000 & $0.000 E+00$ & 0.0000 & $6.026 E-13$ & 0 \\
\hline II & IIIIIIII İIIIII & IIIIIIIII IIIIII & ilifíifit & ifififi & Iilílítín & Ifffíf & IIIIIIIf & î & II i & if & & î́î́ \\
\hline & $000 E+00 \quad 0.0000$ & $0.000 E+00 \quad 0.0000$ & $0.000 \mathrm{E}+00$ & 0.0000 & $0.000 E+00$ & 0.0000 & $0.000 E+00$ & 0.0000 & $0.000 E+00$ & 0.0000 & $6.026 E-13$ & 1.0000 \\
\hline
\end{tabular}

***CNRSI $(i, p, t)$ includes contribution from decay daughter radionuclides

\begin{tabular}{|c|c|}
\hline Attachment & Sheet No. 1 \\
\hline Originators: S.W. Clark & Date \\
\hline M.W. Perrott & Date \\
\hline $0100 B-C A-V 0301$ & Rev. No. \\
\hline
\end{tabular}




\section{ATTACHMENT 4}

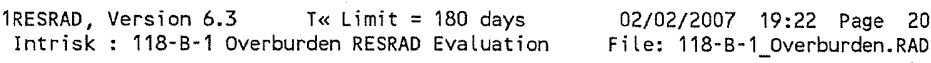

Water Dependent Pathways

\begin{tabular}{|c|c|c|c|c|c|c|}
\hline Radio- & $\begin{array}{c}\text { Water } \\
\text { AAAAAAAAOAAOAOAAÄA }\end{array}$ & $\begin{array}{c}F i s h \\
\text { ÄÄÄAAAAAOAAOAOAOÄ }\end{array}$ & 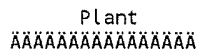 & $\begin{array}{c}\text { Meat } \\
\text { ÄÄÄAOAOAAAAAAAOAOÄA }\end{array}$ & 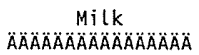 & 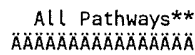 \\
\hline $\begin{array}{l}\text { Nucl ide } \\
\text { AAAAAAA }\end{array}$ & 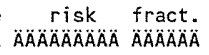 & 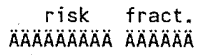 & $\begin{array}{cc}\text { risk fract. } \\
\text { ÄAAAAAAAA ÄÄAAA }\end{array}$ & $\begin{array}{r}\text { risk fract. } \\
\text { ÄAAAAAAAAOA AAAAOAOÄ }\end{array}$ & $\begin{array}{r}\text { risk fract. } \\
\text { ÄÄÄÄÄÄA ÄÄÄÄ }\end{array}$ & $\begin{array}{r}\text { risk fract. } \\
\text { AAAAAAAAAAO ÄAAAAAA }\end{array}$ \\
\hline Eu- 152 & $0.000 E+00 \quad 0.0000$ & $0.000 E+00 \quad 0.0000$ & $0.000 \mathrm{E}+00 \quad 0.0000$ & $0.000 E+00 \quad 0.0000$ & $0.000 E+00 \quad 0.0000$ & $7.559 \mathrm{E}-29 \quad 0.0000$ \\
\hline$G d-152$ & $0.000 E+00 \quad 0.0000$ & $0.000 E+00 \quad 0.0000$ & $0.000 E+00 \quad 0.0000$ & $0.000 E+00 \quad 0.0000$ & $0.000 E+00 \quad 0.0000$ & $8.138 \mathrm{E}-22 \quad 1.0000$ \\
\hline IIIfifít & 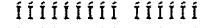 & 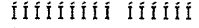 & 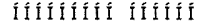 & IIIIIIIII IIIIII & 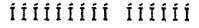 & 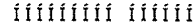 \\
\hline Total & $0.000 \mathrm{E}+00 \quad 0.0000$ & $0.000 E+00 \quad 0.0000$ & $0.000 E+00 \quad 0.0000$ & $0.000 E+00 \quad 0.0000$ & $0.000 E+00 \quad 0.0000$ & $8.138 E-221.0000$ \\
\hline
\end{tabular}

and water dependent water, fish, plant, meat, milk pathways

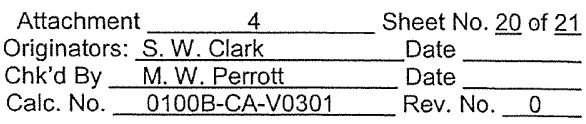




\section{ATTACHMENT 4}

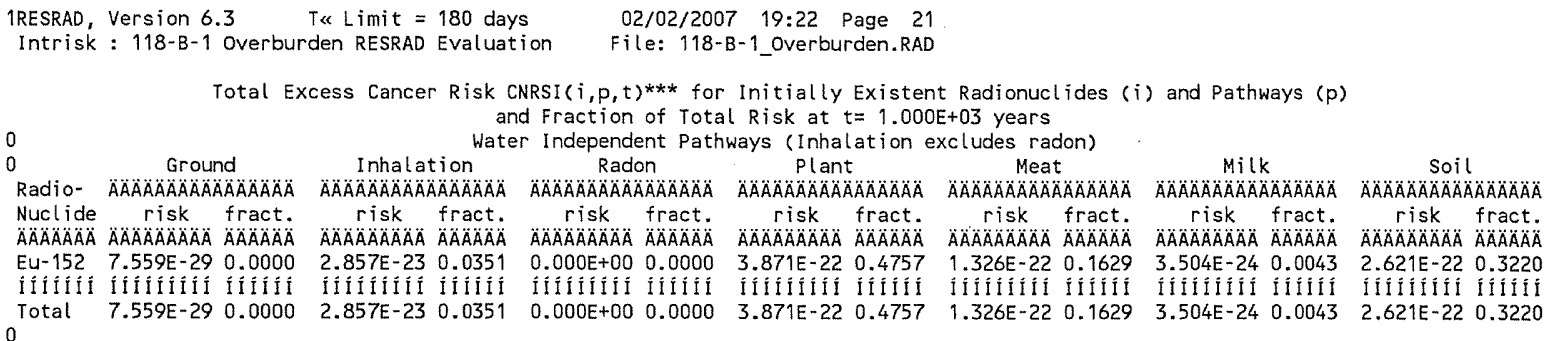

Total Excess Cancer Risk CNRSI $(i, p, t) * * *$ for Initially Existent Radionuclides $(i)$ and Pathways $(p)$ and Fraction of Total Risk at $t=1.000 E+03$ years

Water Dependent Pathways

\begin{tabular}{|c|c|c|c|c|c|c|c|}
\hline Radio- & 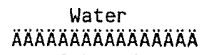 & $\begin{array}{c}F i s h \\
\triangle A \cap A B A A A B A A A O A O A O A O\end{array}$ & 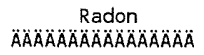 & $\begin{array}{c}\text { Plant } \\
\text { ÄAAAAAAAAAAAAAAA }\end{array}$ & 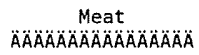 & $\begin{array}{c}\text { Milk } \\
\text { AAAAAAAAAAOAAAAA }\end{array}$ & $\begin{array}{c}\text { All pathways } \\
\text { ALAAAAAAAAAAAAAAA }\end{array}$ \\
\hline $\begin{array}{l}\text { Nuclide } \\
\text { AAAAAAAA }\end{array}$ & $\begin{array}{r}\text { risk fract. } \\
\text { AAAAAOAOAOA AOAAAAAOA }\end{array}$ & $\begin{array}{r}\text { risk fract. } \\
\text { AAAÄÄÄÄÄ ÄÄÄÄÄ }\end{array}$ & 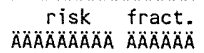 & $\begin{array}{r}\text { risk fract. } \\
\text { ÄÄÄÄÄÄ ÄÄÄÄA }\end{array}$ & 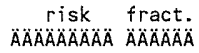 & $\begin{array}{r}\text { risk fract. } \\
\triangle A B A O A B A O A O A\end{array}$ & 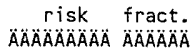 \\
\hline Eu-152 & $0.000 E+00 \quad 0.0000$ & $0.000 E+00 \quad 0.0000$ & $0.000 E+00 \quad 0.0000$ & $0.000 E+00 \quad 0.0000$ & $0.000 E+00 \quad 0.0000$ & $0.000 E+00 \quad 0.0000$ & $8.138 \mathrm{E}-22 \quad 1.0000$ \\
\hline Total & $0.000 E+00 \quad 0.0000$ & $0.000 E+00 \quad 0.0000$ & $0.000 E+00 \quad 0.0000$ & $0.000 E+00 \quad 0.0000$ & $0.000 E+00 \quad 0.0000$ & $0.000 E+00 \quad 0.0000$ & $8.138 E-22 \quad 1.0000$ \\
\hline
\end{tabular}

$* * *$ CNRSI $(i, p, t)$ includes contribution from decay daughter radionuclides

\begin{tabular}{|c|c|}
\hline Attachment & Sheet No. 21 of 21 \\
\hline Originators: S.W. Clark & Date \\
\hline Chk'd By M.W. Perrott & Date \\
\hline $0100 B-C A-V 0301$ & Rev. No. 0 \\
\hline
\end{tabular}




\section{ATTACHMENT 5}

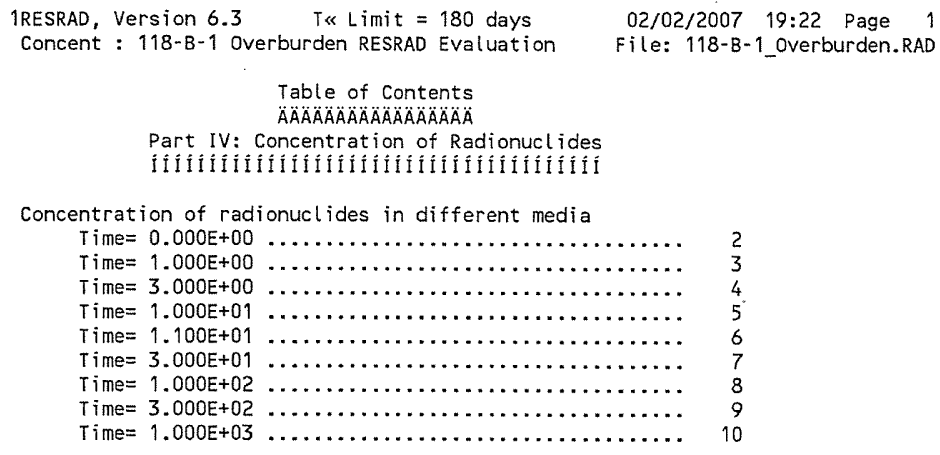

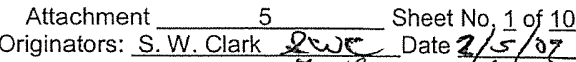
Chk'd By M.W. Perrott Mlef Date $2 / 5 / 02$ Calc. No. $0100 \mathrm{~B}-\mathrm{CA}-\mathrm{V} 0301$ Rev. No. 0 
CVP-2007-00006

Rev. 0

\section{ATTACHMENT 5}

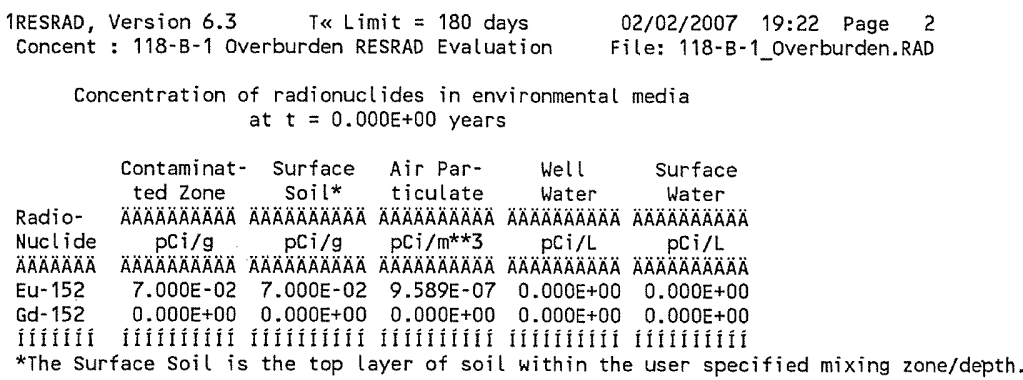

Concentrations in the media occurring in pathways that are suppressed are calculated using the current input parameters, i.e. using parameters appearing in the input screen when the pathways are active.

Concentration of radionuclides in foodstuff media at $t=0.000 \mathrm{E}+00$ years*

\begin{tabular}{|c|c|c|c|c|c|c|c|c|c|}
\hline & $\begin{array}{c}\text { Drinking } \\
\text { Water }\end{array}$ & $\begin{array}{l}\text { Nonleafy } \\
\text { Vegetable }\end{array}$ & $\begin{array}{c}\text { Leafy } \\
\text { Vegetable }\end{array}$ & $\begin{array}{l}\text { Fodder } \\
\text { Meat }\end{array}$ & $\begin{array}{l}\text { Fodder } \\
\text { Milk }\end{array}$ & Meat & Milk & Fish & Crustacea \\
\hline io- & $\triangle A A A A A A B A A B$ & $\triangle A \cap A \cap A \cap A \cap A \cap A$ & 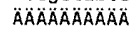 & $\triangle A \cap A ̈ A \cap A A A A$ & $\triangle A A A A A A A A B$ & $\triangle A A A A O A A A A A A$ & $\triangle \triangle A A A A A O A A O A$ & $\triangle A O A O A A O A O A O A$ & $\triangle A A A A A A A A$ \\
\hline Nuclide & $\mathrm{pCi} / \mathrm{L}$ & $\mathrm{pCi} / \mathrm{kg}$ & $p c i / k g$ & $\mathrm{pCi} / \mathrm{kg}$ & $\mathrm{pCi} / \mathrm{kg}$ & $\mathrm{pCi} / \mathrm{kg}$ & $\mathrm{pCi} / \mathrm{L}$ & $\mathrm{pCi} / \mathrm{kg}$ & $\mathrm{pCi} / \mathrm{kg}$ \\
\hline ÄÄÄĀÄA & $A A A A A O A A A A B A$ & 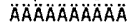 & 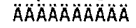 & 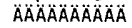 & $\triangle A A A A A A A A A B$ & $\triangle A A A A A A A A A A A$ & $\triangle A A A A A A A A A A$ & $\triangle A A A A A A A A A A O A$ & AAAAAAAA \\
\hline Eu- 152 & $0.000 \mathrm{E}+00$ & $1.751 E-01$ & $1.753 \mathrm{E}-01$ & $1.753 \mathrm{E}-01$ & 1.753E-01 & $9.384 E-02$ & 2. 2. 232E-03 & $0.000 E+00$ & $0.000 E+00$ \\
\hline Gd-152 & $0.000 E+00$ & $0.000 \mathrm{E}+00$ & $0.000 E+00$ & $0.000 E+00$ & $0.000 E+00$ & $0.000 \mathrm{E}+00$ & $0.000 E+00$ & $0.000 E+00$ & $0.000 E+00$ \\
\hline IIIIIIII İ & IIIIIII & ÍIXIIIIIII & IfIfífílí & İIIIIIIIIII & 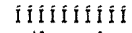 & 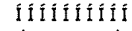 & IfIIfIIII & EIfifífíf & IfII \\
\hline - & & & & & & & & & \\
\hline
\end{tabular}

For livestock fodder, consumption time is $t$ minus meat or milk storage time.

Concentrations in the media occurring in pathways that are suppressed are calculated using the current input parameters $i . e$. using parameters appearing in the input screen when the pathways are active.

$\begin{array}{ll}\text { Attachment } \frac{5}{\text { W. Clark }} & \text { Sheet No. } 2 \text { of } 10 \\ \text { Originators: } \frac{\text { S.W. }}{\text { W.W. Perrott }} & \text { Date } \\ \text { Chk'd By } & \text { Rev. No. _ } 0 \\ \text { Calc. No. } \quad \text { O100B-CA-V0301 } & \text { Rev. }\end{array}$


CVP-2007-00006

Rev. 0

\section{ATTACHMENT 5}

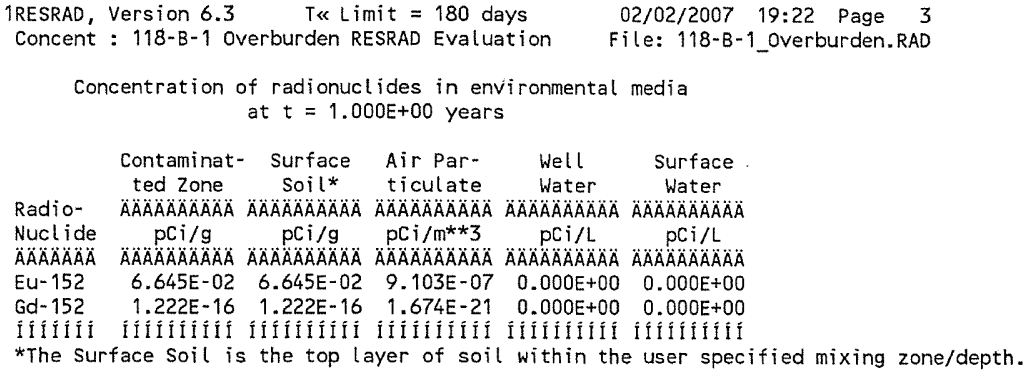

Concentrations in the media occurring in pathways that are suppressed are calculated using the current input parameters, i.e. using parameters appearing in the input screen when the pathways are active.

Concentration of radionuclides in foodstuff media at $t=1.000 E+00$ years*

\begin{tabular}{|c|c|c|c|c|c|c|c|c|c|}
\hline & $\begin{array}{c}\text { Drinking } \\
\text { Water }\end{array}$ & $\begin{array}{l}\text { Nonleafy } \\
\text { Vegetable }\end{array}$ & $\begin{array}{c}\text { Leafy } \\
\text { Vegetable }\end{array}$ & $\begin{array}{l}\text { Fodder } \\
\text { Meat }\end{array}$ & $\begin{array}{l}\text { Fodder } \\
\text { Milk }\end{array}$ & Meat & Milk & Fish & Crustacea \\
\hline & $A A A B A A$ & $\triangle A \not A ̈ A$ & $\triangle A A A A A A A A A B A ̈$ & $\triangle A O A A A A A A A A$ & 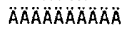 & $\triangle A A A A A A A A A B A$ & $\triangle A O A A A A A A A B A ̈$ & ÄÄÄ & $\triangle A ̈ A$ \\
\hline & $\mathrm{pCi} / \mathrm{L}$ & & $\mathrm{pCi}$ & $\mathrm{pCi} / \mathrm{kg}$ & $\mathrm{pCi} / \mathrm{kg}$ & & $\mathrm{pC} i / \mathrm{L}$ & & \\
\hline & $\triangle A \cap A \cap A B A A A A A A$ & $\ddot{A} \ddot{A} A \dot{A}$ & $\ddot{A} \ddot{A} A \ddot{A} A$ & $\ddot{A} \ddot{A} \ddot{A} A$ & $\dddot{A} A A$ & & $\ddot{A} A \ddot{A}$ & $\ddot{A} A \ddot{A}$ & \\
\hline & 0.000 & 1.6 & -01 & & & & & 0.0 & \\
\hline & 0.0 & & & 2.8 & & & & 0. & \\
\hline & & & & & & & & & \\
\hline & & & & & & & & & \\
\hline
\end{tabular}

For livestock fodder, consumption time is t minus meat or milk storage time.

Concentrations in the media occurring in pathways that are suppressed are calculated using the current input parameters, i.e. using parameters appearing in the input screen when the pathways are active.

\begin{tabular}{|c|c|}
\hline Attachment & Sheet No. 3 of 10 \\
\hline Originators: s. W. Clark & Date \\
\hline Chk'd By M.W.Perrott & Date \\
\hline Calc. No. $\quad 0100 \mathrm{~B}-\mathrm{CA}-\mathrm{V} 0301$ & Rev. No. 0 \\
\hline
\end{tabular}


CVP-2007-00006

Rev. 0

\section{ATTACHMENT 5}

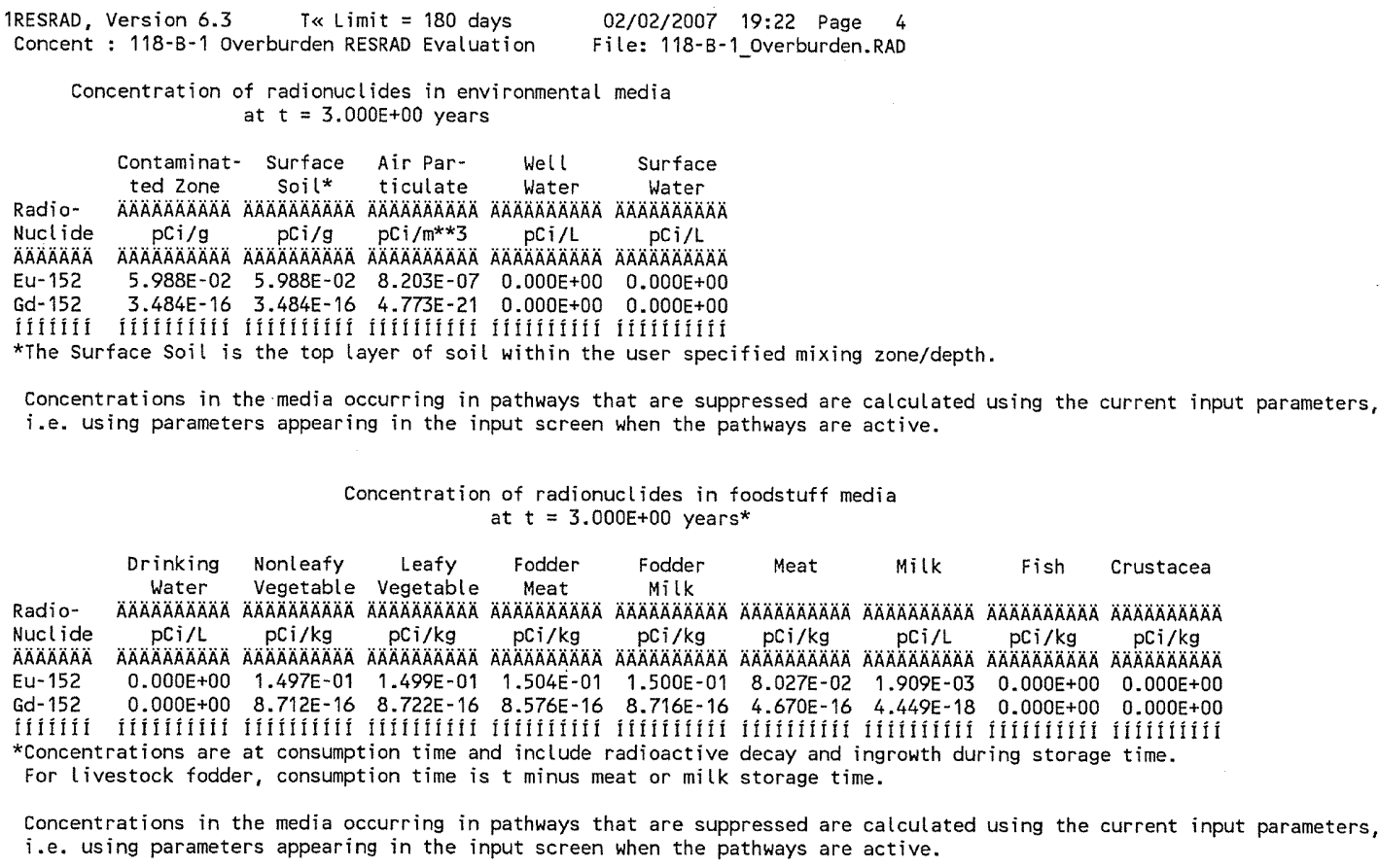

Concentrations in the media occurring in pathways that are suppressed are calculated using the current input parameters, i.e. using parameters appearing in the input screen when the pathways are active. 


\section{ATTACHMENT 5}

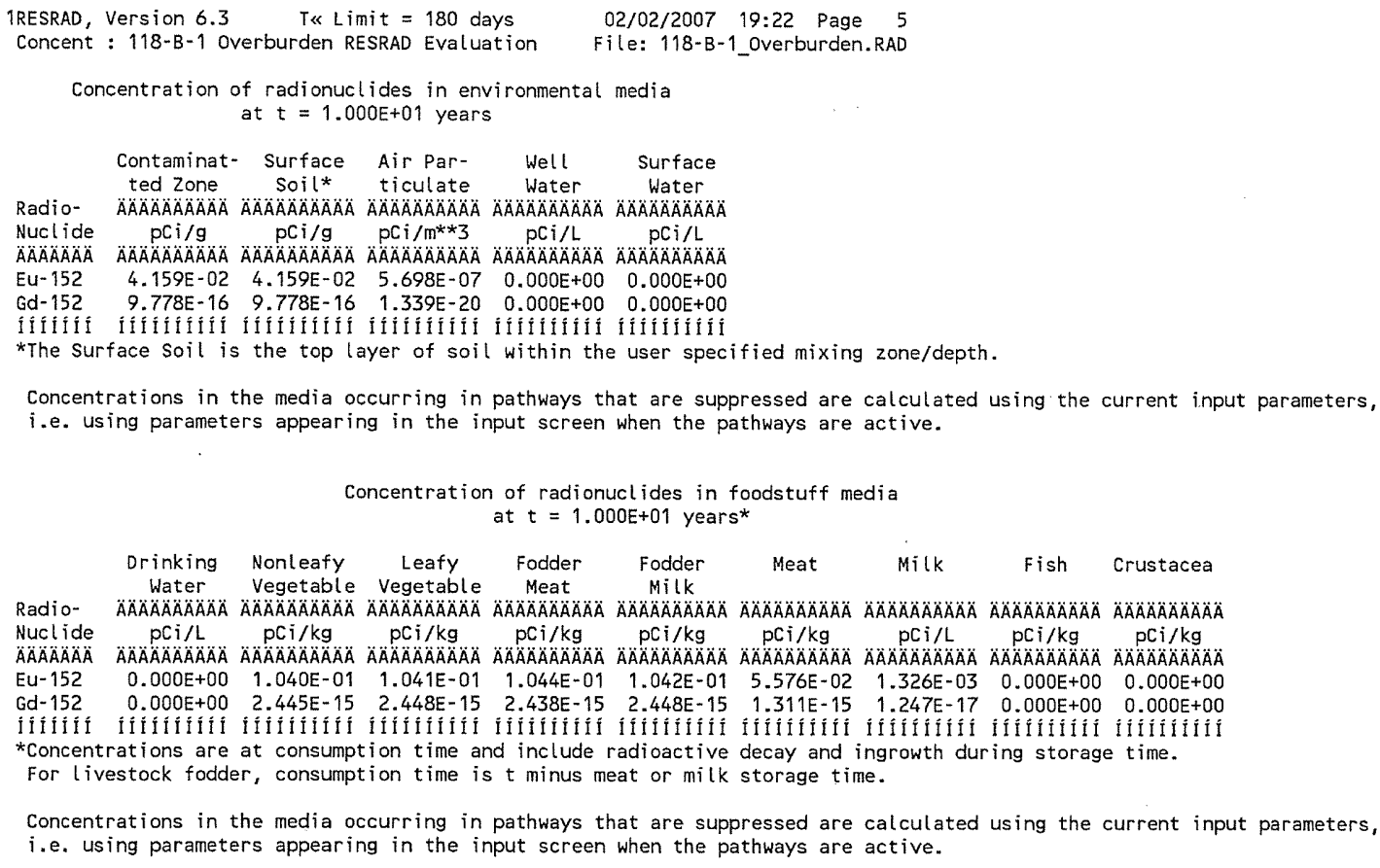

Concentrations in the media occurring in pathways that are suppressed are calculated using the current input parameters, i.e. using parameters appearing in the input screen when the pathways are active.

Attachment

\begin{tabular}{|c|c|}
\hline Attachment & Sheet No. 5 of 10 \\
\hline Originators: S.W. Clark & Date \\
\hline M. W. Perrott & Date \\
\hline $0100 \mathrm{~B}-\mathrm{CA}-\mathrm{V} 0301$ & No. \\
\hline
\end{tabular}


CVP-2007-00006

Rev. 0

\section{ATTACHMENT 5}

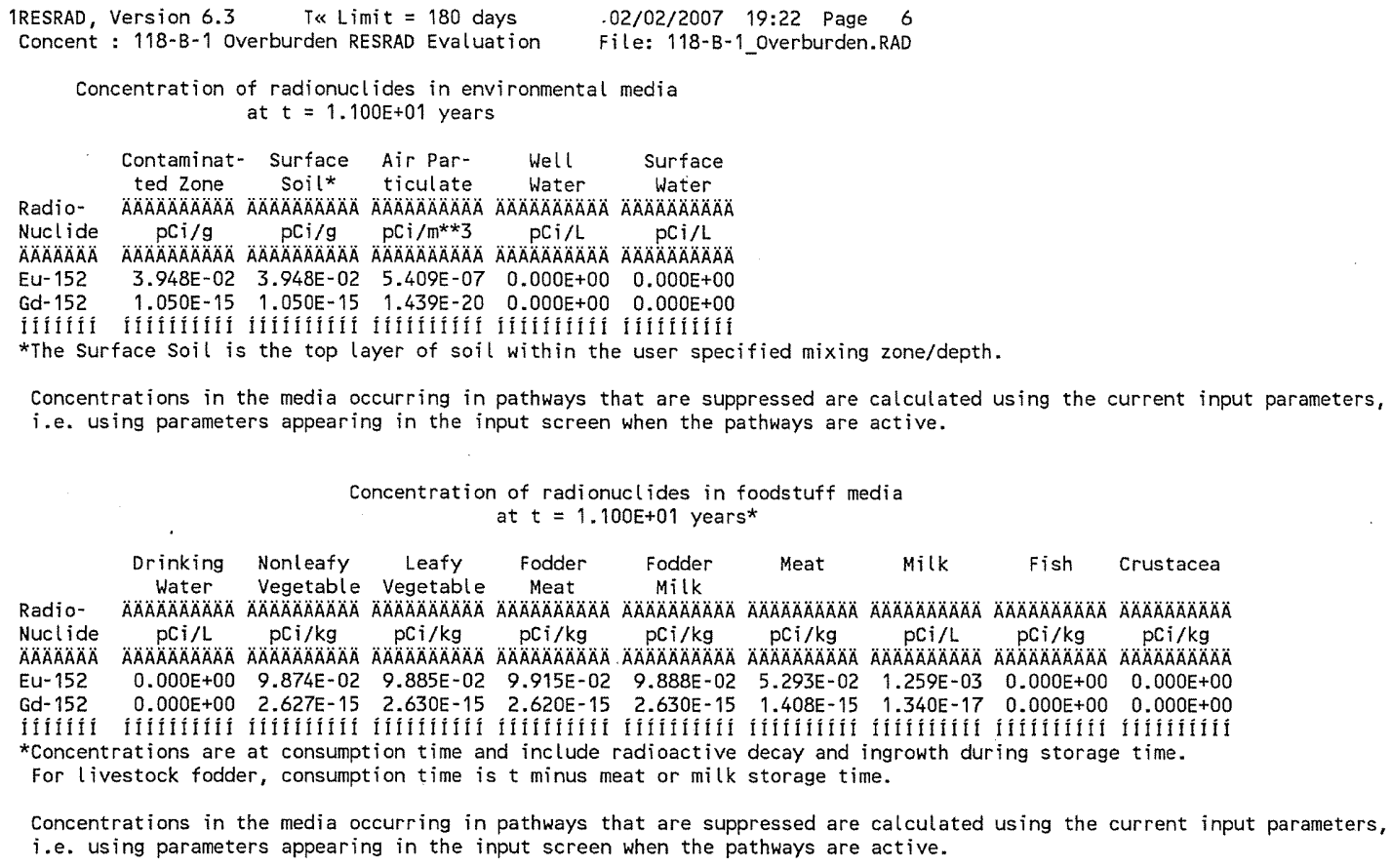

Concentrations in the media occurring in pathways that are suppressed are calculated using the current input parameters, i.e. using parameters appearing in the input screen when the pathways are active.

Concentration of radionuclides in foodstuff media at $t=1.100 \mathrm{E}+01$ years*

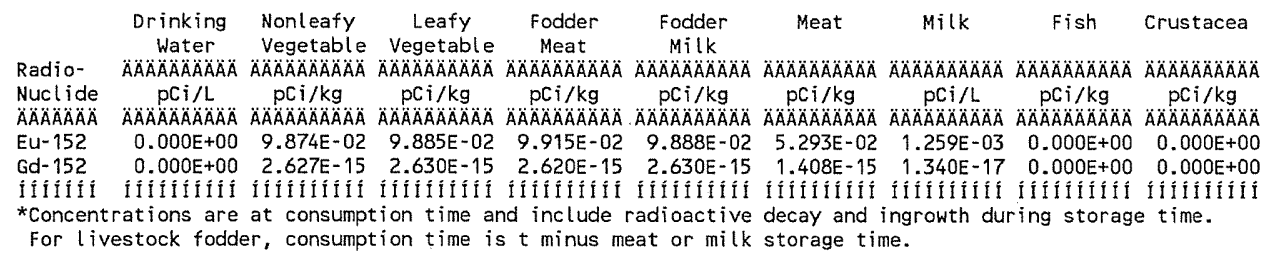

For livestock fodder, consumption time is $t$ minus meat or milk storage time.

Concentrations in the media occurring in pathways that are suppressed are calculated using the current input parameters, i.e. using parameters appearing in the input screen when the pathways are active.

$\begin{array}{ll}\text { Attachment } \frac{5}{\text { W. Clark }} & \text { Sheet No. } \underline{6} \text { of } 10 \\ \text { Originators: } & \text { Date } \\ \text { Chk'd By } & \text { Date W. Perrott } \\ \text { Calc. No. } & \text { Rev. No. } 0100 \text { B-CA-V0301 }\end{array}$


CVP-2007-00006

Rev. 0

\section{ATTACHMENT 5}

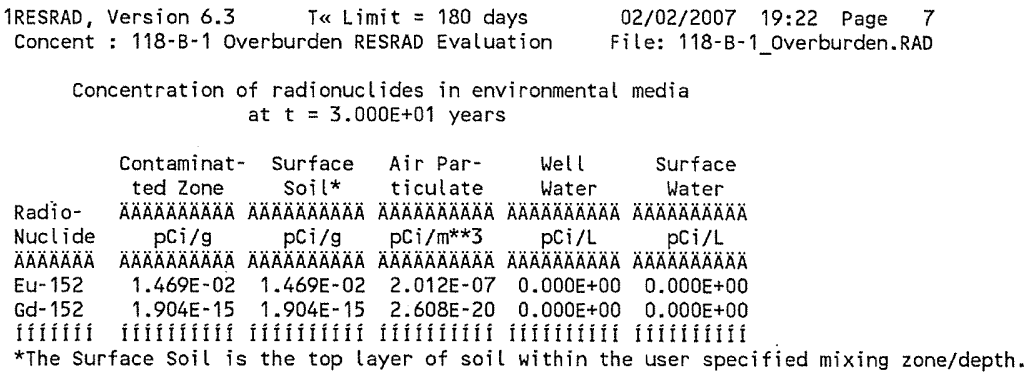

Concentrations in the media occurring in pathways that are suppressed are calculated using the current input parameters, i.e. using parameters appearing in the input screen when the pathways are active. Concentration of radionuclides in foodstuff media
at $t=3.000 E+01$ years*

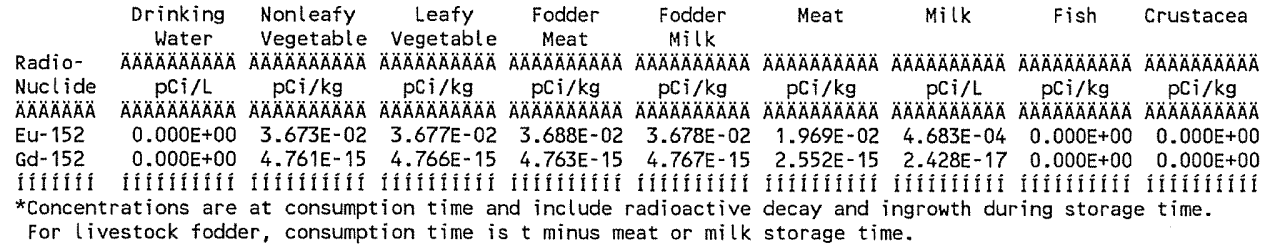

For livestock fodder, consumption time is t minus meat or milk storage time.

Concentrations in the media occurring in pathways that are suppressed are calculated using the current input parameters, i.e. using parameters appearing in the input screen when the pathways are active.

\begin{tabular}{|c|c|}
\hline \multirow{2}{*}{\multicolumn{2}{|c|}{$\begin{array}{cc}\text { Attachment } & 5 \\
\text { Originators: Sheet No. } \mathrm{I} \text {. W. Clark } 10\end{array}$}} \\
\hline & \\
\hline Chk'd By M. W. Perrott & Date \\
\hline 0100B-CA-V0301 & Rev. No. \\
\hline
\end{tabular}


CVP-2007-00006

Rev. 0

\section{ATTACHMENT 5}

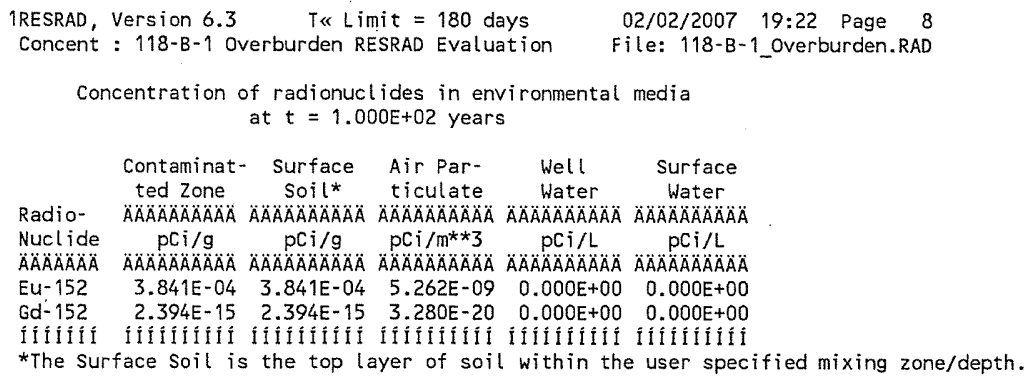

Concentrations in the media occurring in pathways that are suppressed are calculated using the current input parameters, i.e. using parameters appearing in the input'screen when the pathways are active.

Concentration of radionuclides in foodstuff media at $\mathrm{t}=1.000 \mathrm{E}+02$ years*

\begin{tabular}{|c|c|c|c|c|c|c|c|c|c|}
\hline & $\begin{array}{c}\text { Drinking } \\
\text { Water }\end{array}$ & $\begin{array}{l}\text { Nonleafy } \\
\text { Vegetable }\end{array}$ & $\begin{array}{c}\text { Leafy } \\
\text { Vegetable }\end{array}$ & $\begin{array}{l}\text { Fodder } \\
\text { Meat }\end{array}$ & $\begin{array}{l}\text { Fodder } \\
\text { Milk }\end{array}$ & Meat & Milk & Fish & Crustacea \\
\hline (2) & $\triangle \triangle A \cap A B A A B A O A$ & 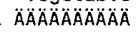 & $\ddot{A} \ddot{A} \ddot{A} \ddot{A} \ddot{A} \ddot{A} \ddot{A} \ddot{A} \ddot{A}$ & $\triangle A \cap A ̈ A ̈ A \cap A \cap A$ & ÄÄÄÄÄÄÄ & $\triangle \triangle A A A B A B A O A A O A$ & $\triangle A A A A A A A A B A$ & $\triangle A A A A A A A A A B A$ & $\triangle A A A A A A A A A A$ \\
\hline ulide & $\mathrm{pCi} / \mathrm{L}$ & $\mathrm{pCi} / \mathrm{kg}$ & $\mathrm{pCi} / \mathrm{kg}$ & $\mathrm{pCi} / \mathrm{kg}$ & $\mathrm{pCi} / \mathrm{kg}$ & $\mathrm{pCi} / \mathrm{kg}$ & $\mathrm{pCi} / \mathrm{L}$ & $\mathrm{pCi} / \mathrm{kg}$ & $\mathrm{pCi} / \mathrm{kg}$ \\
\hline ÄÄÄÄÄÄ & $\triangle A O A A A A A A A O A$ & - AAAAAAAAAÄÄ & $\triangle A A A A B A A A A O A B$ & $\triangle A A A A A O A A A A$ & $\triangle A A A A A A A A A B A$ & 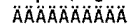 & $\triangle A A A A A A A A B A$ & AAAAAAAAAAÄÄ & $\triangle A \cap A A A B A A A A A B$ \\
\hline$u-152$ & $0.000 E+00$ & $9.606 \mathrm{E}-04$ & $9.616 \mathrm{E}-04$ & $9.645 E-04$ & $9.619 E-04$ & $5.149 \mathrm{E}-04$ & $1.225 \mathrm{E}-05$ & $0.000 E+00$ & $0.000 E+00$ \\
\hline & $0.000 E+00$ & $5.987 E-15$ & $5.993 E-15$ & $5.994 E-15$ & $5.994 E-15$ & $3.209 E-15$ & $3.053 E-17$ & $0.000 E+00$ & $0.000 E+00$ \\
\hline$\because \neq$ & & & 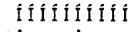 & & & & & & İíí \\
\hline & & & & & & & & & \\
\hline
\end{tabular}

For livestock fodder, consumption time is $t$ minus meat or milk storage time.

Concentrations in the media occurring in pathways that are suppressed are calculated using the current input parameters, i.e. using parameters appearing in the input screen when the pathways are active.

Attachment

$\begin{array}{ll}\text { Attachment } \frac{5}{\text { W. Clark }} & \text { Sheet No. } 8 \text { of } 10 \\ \text { Originators: } \frac{\text { S.W. }}{\text { M.W. Perrott }} & \text { Date } \\ \text { Chk'd By } & \text { Rev. No. } \quad 0 \\ \text { Calc. No. } & \text { O100B-CA-V0301 }\end{array}$


CVP-2007-00006

Rev. 0

\section{ATTACHMENT 5}

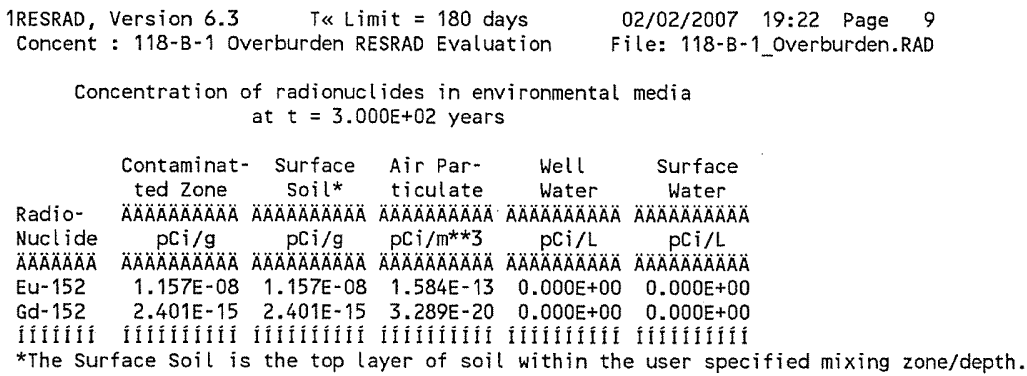

Concentrations in the media occurring in pathways that are suppressed are calculated using the current input parameters, i.e. Using parameters appearing in the input screen when the pathways are active.

Concentration of radionuclides in foodstuff media at $\mathrm{t}=3.000 \mathrm{E}+02$ years*

\begin{tabular}{|c|c|c|c|c|c|c|c|c|c|}
\hline & $\begin{array}{c}\text { Drinking } \\
\text { Water }\end{array}$ & $\begin{array}{l}\text { Nonl eafy } \\
\text { Vegetable }\end{array}$ & $\begin{array}{c}\text { Leafy } \\
\text { Vegetable }\end{array}$ & $\begin{array}{l}\text { Fodder } \\
\text { Meat }\end{array}$ & $\begin{array}{l}\text { Fodder } \\
\text { Milk }\end{array}$ & Meat & Milk & Fish & Crustacea \\
\hline Radio- & 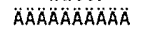 & 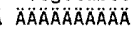 & 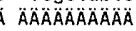 & 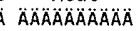 & 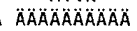 & A & A ÄÄÄÄÄÄÄÄÄ & ¿ & ÄÄÄÄÄÄÄÄ \\
\hline Nuclide & $\mathrm{pCi} / \mathrm{L}$ & $\mathrm{pCi} / \mathrm{kg}$ & $\mathrm{pCi} / \mathrm{kg}$ & $\mathrm{pCi} / \mathrm{kg}$ & $\mathrm{pCi} / \mathrm{kg}$ & $\mathrm{pCi} / \mathrm{kg}$ & $\mathrm{pCi} / \mathrm{L}$ & $\mathrm{pCi} / \mathrm{kg}$ & $\mathrm{pCi} / \mathrm{kg}$ \\
\hline$A A A A A A ̈ A ̈$ & AAAAAAAAAAAAÄ & 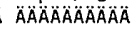 & 1 AAAAAAAAAAAOA & $\triangle A O A A A A A A A A$ & ÄAAAAAAAOA & 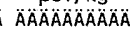 & 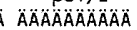 & $\triangle A A A O A O A B A A A B A$ & 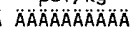 \\
\hline Eu-152 & $0.000 E+00$ & $2.892 \mathrm{E}-08$ & $2.895 E-08$ & $8 \quad 2.904 E-08$ & $2.896 \mathrm{E}-08$ & $3 \quad 1.550 \mathrm{E}-08$ & $3 \quad 3.688 \mathrm{E}-10$ & $0.000 E+00$ & $0.000 E+00$ \\
\hline$G d-152$ & $0.000 E+00$ & $6.004 E-15$ & $6.011 \mathrm{E}-15$ & $6.012 E-15$ & $6.012 \mathrm{E}-15$ & ; $3.218 E-15$ & $53.062 \mathrm{E}-17$ & $0.000 E+00$ & $0.000 E+00$ \\
\hline II I I I I I I & IIIIIIIIII & LIIIIIIIII & 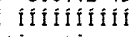 & IIIIIIIIIIIín & IIIIIIIIIII & UIIIIIIIII & Línitíni & 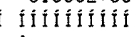 & 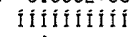 \\
\hline $\begin{array}{l}\text { * Concent } \\
\text { For liv }\end{array}$ & $\begin{array}{l}\text { rations are } \\
\text { estock fodde }\end{array}$ & & $\begin{array}{l}\text { tion time ar } \\
\text { tion time is }\end{array}$ & & $\begin{array}{l}\text { radioactive } \\
\text { neat or milk }\end{array}$ & $\begin{array}{l}\text { decay and } \\
\text { storage tin }\end{array}$ & $\begin{array}{l}\text { ingrowth dur } \\
\text { ime. }\end{array}$ & uring storag & \\
\hline
\end{tabular}
i.e. using parameters appearing in the input screen when the pathways are active.

Attachment

5 
CVP-2007-00006

Rev. 0

\section{ATTACHMENT 5}

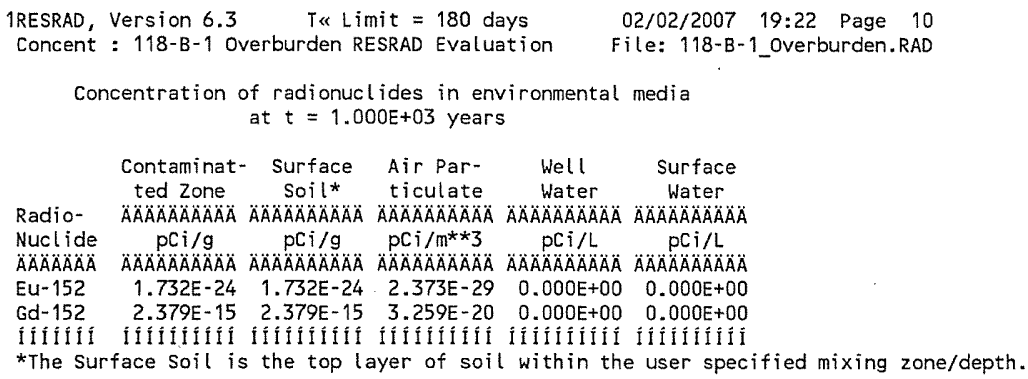

Concentrations in the media occurring in pathways that are suppressed are calculated using the current input parameters, i.e. using parameters appearing in the input screen when the pathways are active.

Concentration of radionuclides in foodstuff media at $\mathrm{t}=1.000 \mathrm{E}+03$ years*

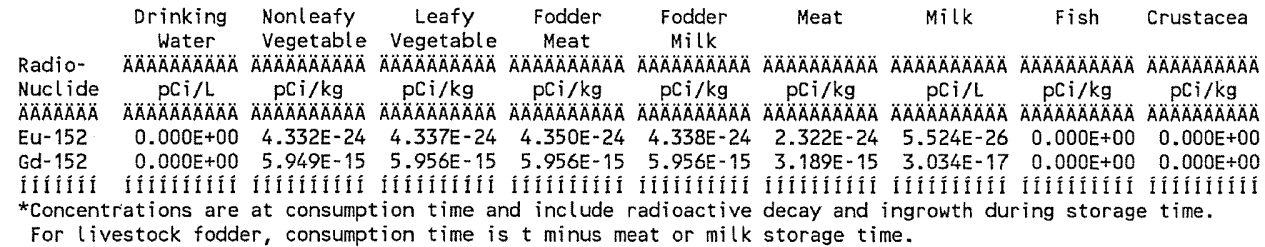

For livestock fodder, consumption time is $t$ minus meat or milk storage time.

Concentrations in the media occurring in pathways that are suppressed are calculated using the current input parameters, i.e. using parameters appearing in the input screen when the pathways are active.

\begin{tabular}{|c|c|}
\hline Attachment & Sheet No. 10 of 10 \\
\hline Originators: S.W.Clark & Date \\
\hline Chk'd By M. W. Perrott & Date \\
\hline 0100B-CA-V0301 & Rev. No. 0 \\
\hline
\end{tabular}


CVP-2007-00006

Rev. 0

\section{ATTACHMENT 6}

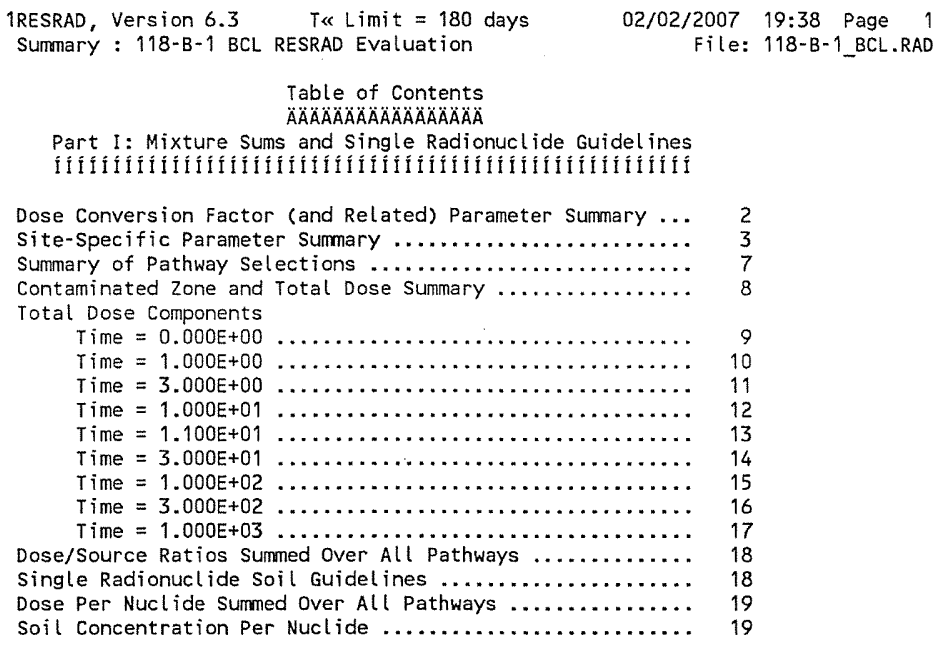

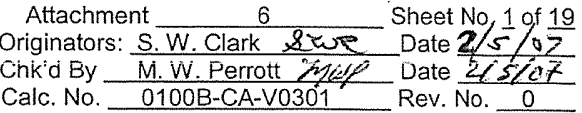




\section{ATTACHMENT 6}

$\begin{array}{rrr}\text { IRESRAD, Version } 6.3 & \text { T« Limit }=180 \text { days } & \text { 02/02/2007 } 19: 38 \text { Page } 2 \\ \text { Summary : } 118-B-1 \text { BCL RESRAD Evaluation } & \text { File: } 118-\mathrm{B}-1 \text { BCL.RAD }\end{array}$

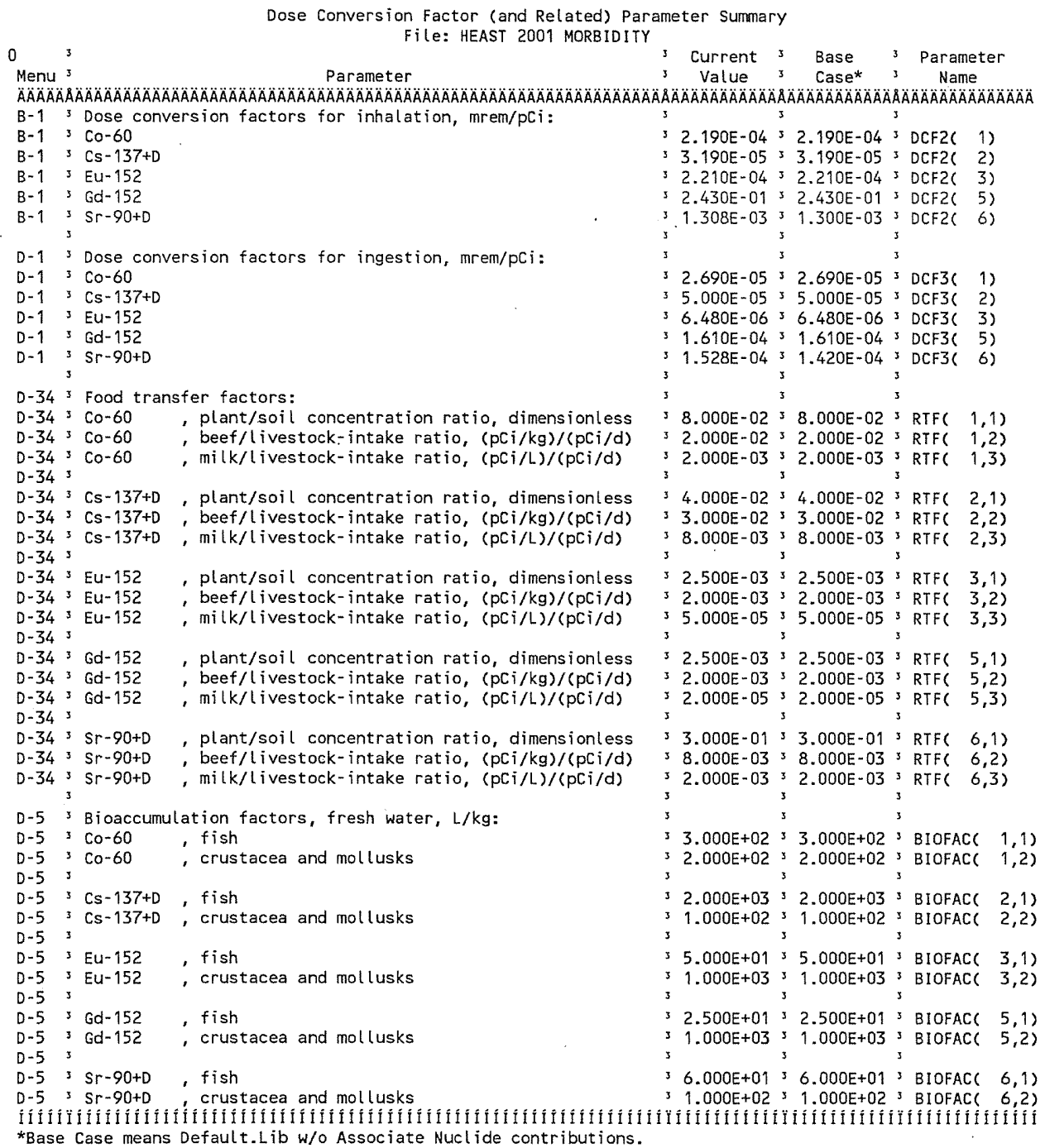

\begin{tabular}{|c|c|}
\hline Attachment & Sheet No. 2 of 19 \\
\hline Originators: S. W. Clark & Date \\
\hline Chk'd By M.W. Perrott & Date \\
\hline 0100B-CA-V0301 & Rev. No. 0 \\
\hline
\end{tabular}


CVP-2007-00006

Rev. 0

\section{ATTACHMENT 6}

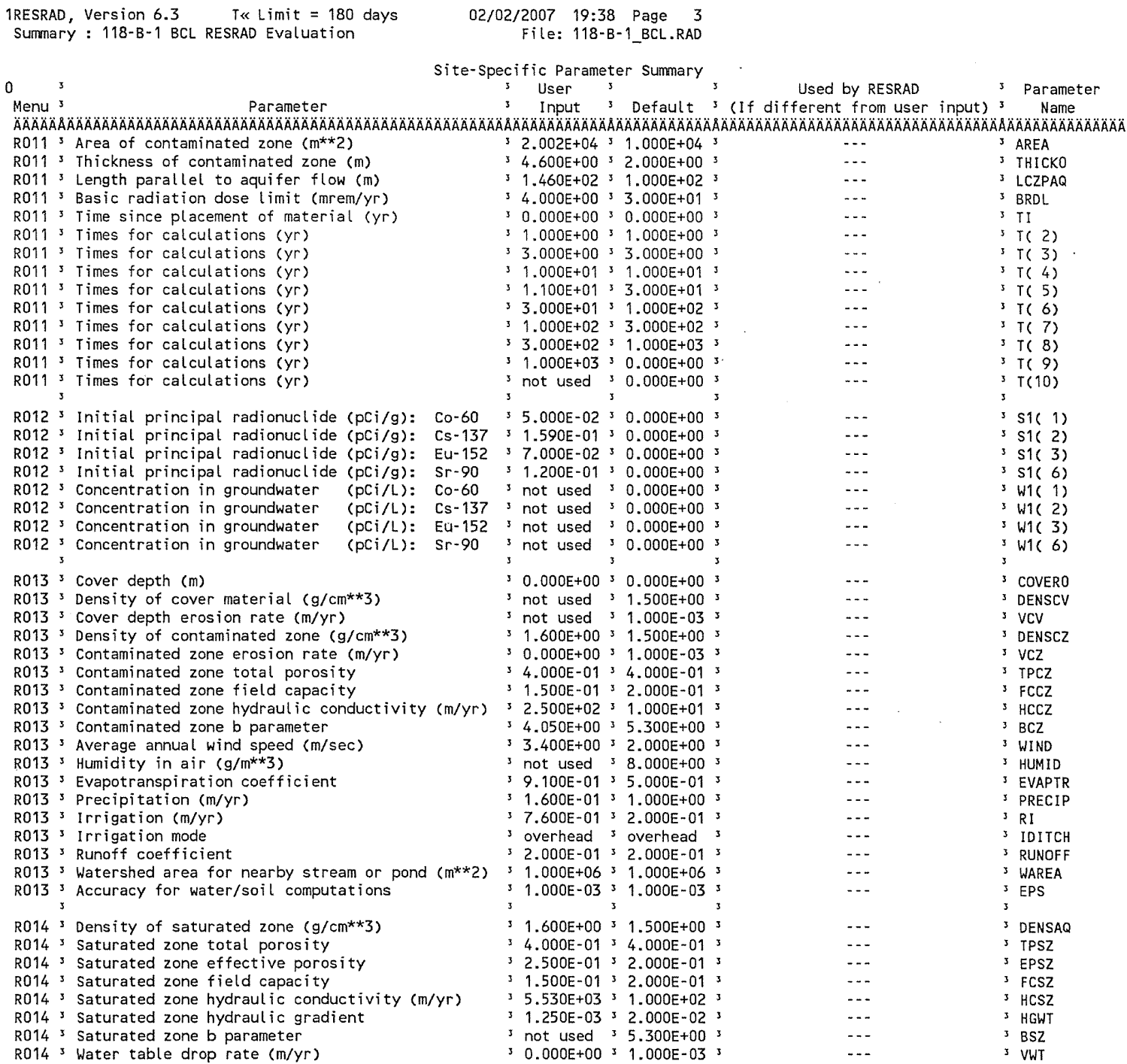

Attachment Sheet No. $\underline{3}$ of 19 6 Date Chk'd By M.W. Perrott Date Calc. No. $0100 B-C A-V 0301 \quad$ Rev. No. 0 
CVP-2007-00006

Rev. 0

\section{ATTACHMENT 6}

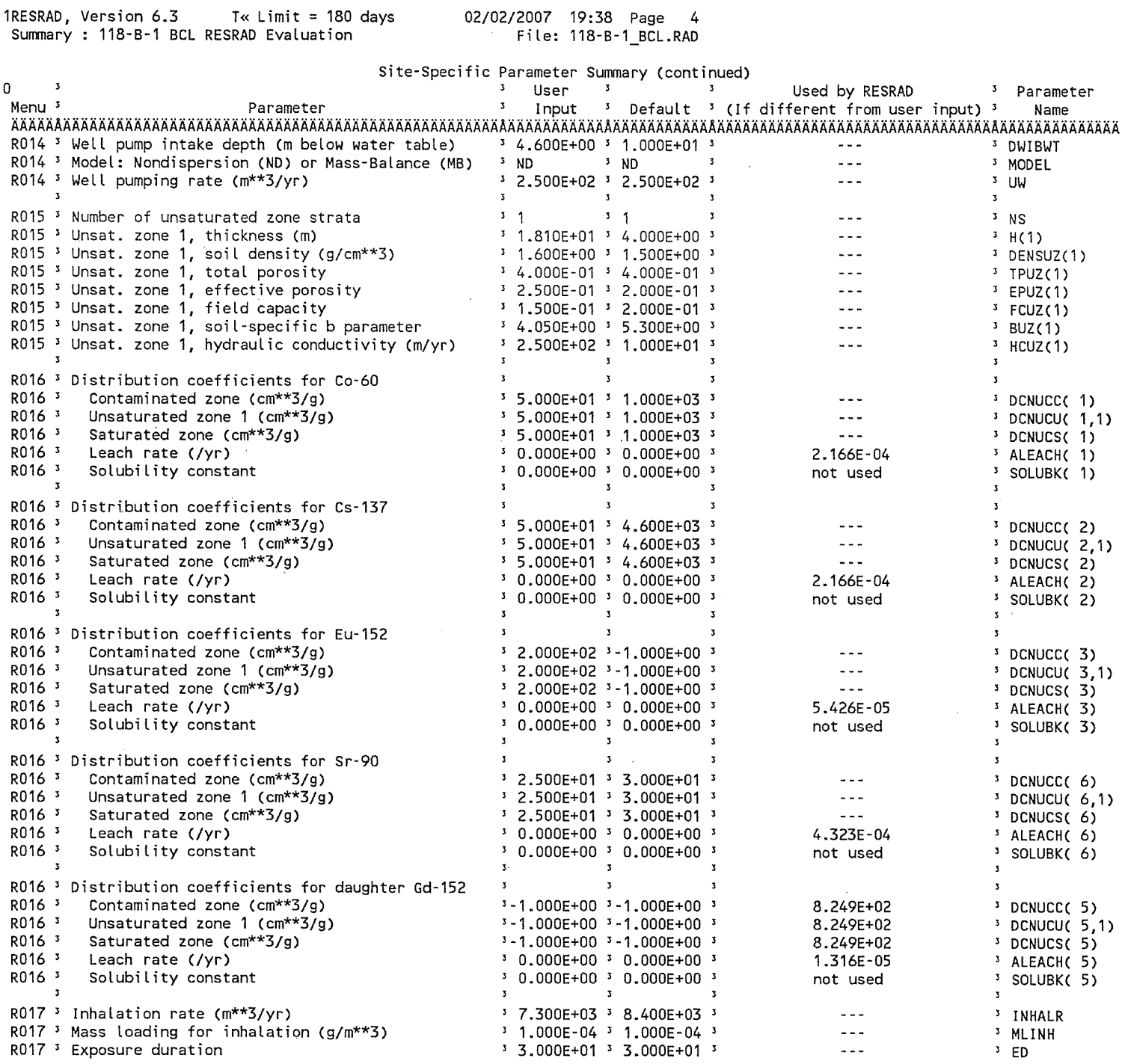

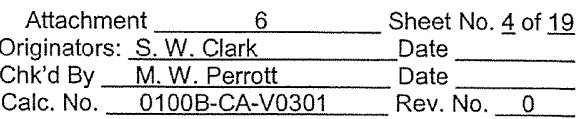




\section{ATTACHMENT 6}

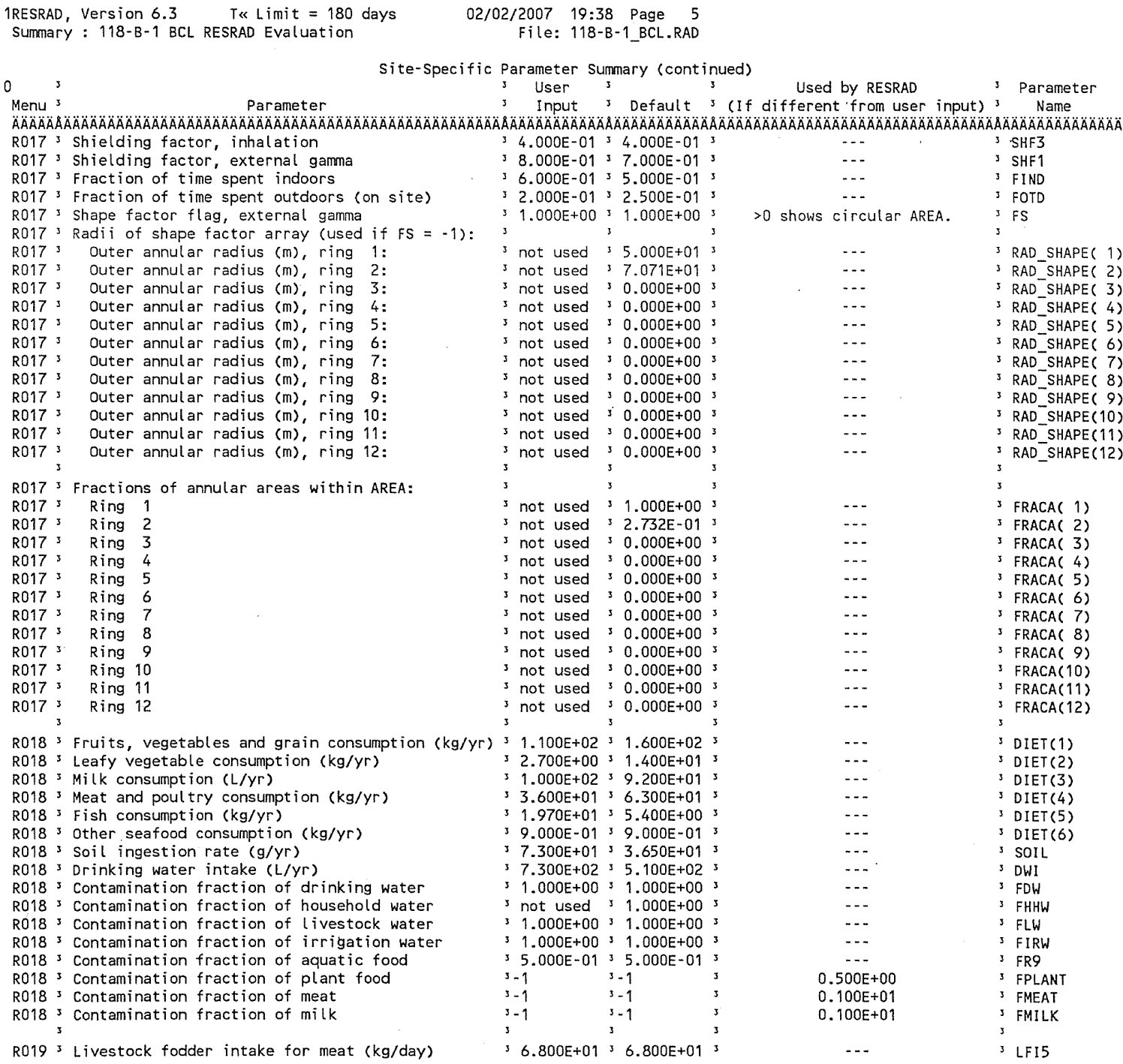




\section{ATTACHMENT 6}

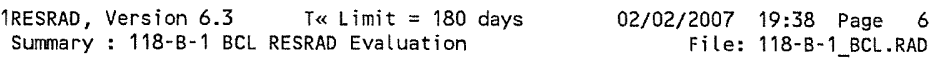

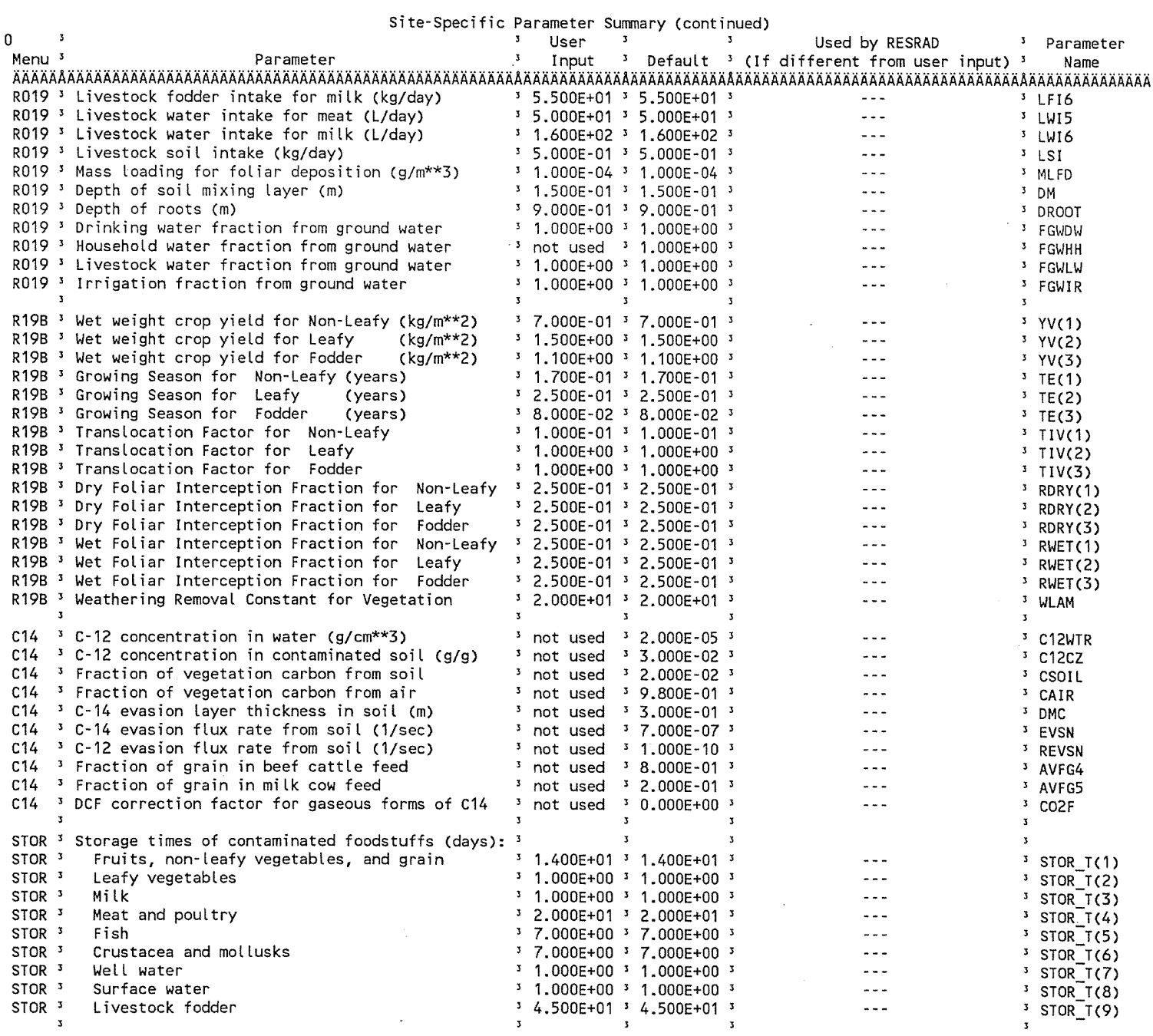

Attachment 


\section{ATTACHMENT 6}

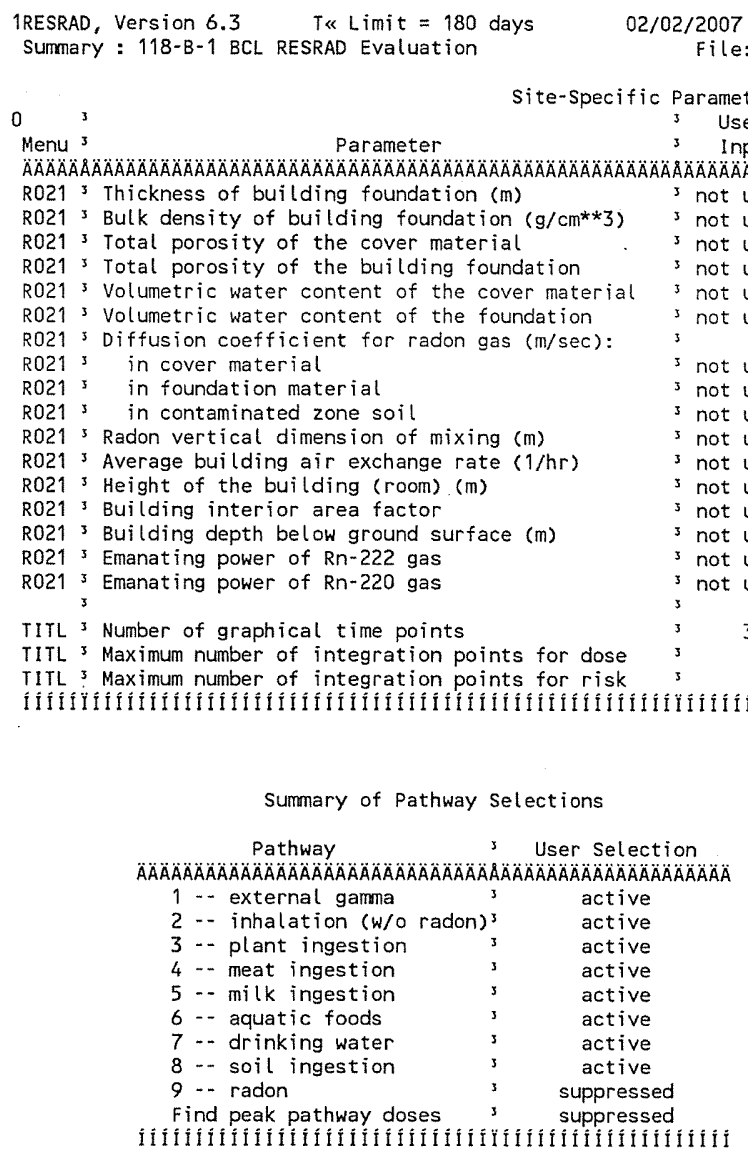

rameter Summary (continued)

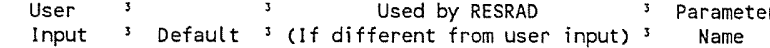

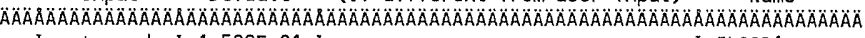

not used $2.400 E+00$ s DENSFL

not used $34.000 E-0133$...

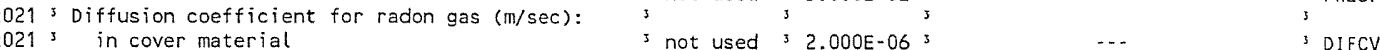

not used $32.000 E+00$

not used 3 5.000E-01

not used $32.500 E+00$

not used $30.000 E+00$

not used $s-1.000 E+00$

not used $32.500 \mathrm{E}-01$

not used $31.500 E-013$

$32 \quad 32 \quad \ldots 35$

TITL ${ }^{3}$ Number of graphical time points

TITL ${ }^{3}$ Maximum number of integration points for dose

$\begin{array}{rrrr}5 & 3 & \cdots & 3 \\ 2 & 3 & \cdots & 3\end{array}$

$\begin{array}{lll}\cdots & \text { DIFCV } \\ \cdots & 3 \text { DIFFL } \\ -.- & 3\end{array}$

$\begin{array}{ll}-.- & 3 \text { DIFFL } \\ \ldots & 3 \text { DIFCZ }\end{array}$

$\begin{array}{ll}\ldots & 3 \text { DIFCZ } \\ \ldots & 3 \text { HMIX }\end{array}$

$\begin{array}{ll}-.- & \text { HMIX } \\ \ldots & 3\end{array}$

...

...

FAI

-..

...

-..

$\begin{array}{ll}3 .- & 3 \text { LYMAX } \\ \ldots & 3 \text { KYMAX }\end{array}$

$\begin{array}{ll}\text { Attachment } \frac{6}{\text { W. Clark }} & \text { Sheet No. } I \text { of } 19 \\ \text { Originators: } \frac{\text { S.W. }}{\text { M. W. Perrott }} & \text { Date } \\ \text { Chk'd By } & \text { Date } \\ \text { Calc. No. } & \text { Rev. No. } 0\end{array}$




\section{ATTACHMENT 6}

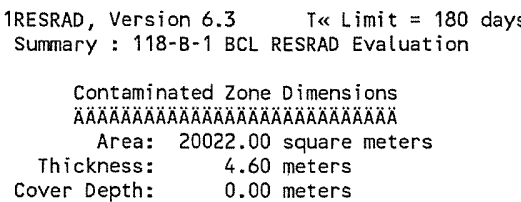

Total Dose TDOSE( $t$ ), mrem/yr

02/02/2007 19:38 Page 8

File: 118-B-1_BCL.RAD

Initial soil concentrations, $\mathrm{pCi} / \mathrm{g}$

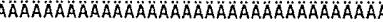

CO-60 5.000E-02

Cs-137 1.590E-01

Cs-137 $7.590 \mathrm{C}$

$\begin{array}{ll}\text { EU-152 } & 7.000 E-02 \\ S P-90 & 1.200 E-01\end{array}$

Basic Radiation Dose Limit $=4.000$

Total Mixture Sum $M(t)=$ Fraction of Basic Dose Limit Received at Time $(t)$

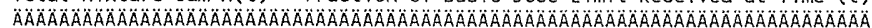

$t$ (years) : $\quad \begin{array}{lllllllllll}0.000 E+00 & 1.000 E+00 & 3.000 E+00 & 1.000 E+01 & 1.100 E+01 & 3.000 E+01 & 1.000 E+02 & 3.000 E+02 & 1.000 E+03\end{array}$

$\operatorname{TDOSE}(t): \begin{array}{lllllllll}1.697 \mathrm{E}+00 & 1.599 \mathrm{E}+00 & 1.428 \mathrm{E}+00 & 1.017 \mathrm{E}+00 & 9.743 \mathrm{E}-01 & 5.084 \mathrm{E}-01 & 8.370 \mathrm{E}-02 & 7.061 \mathrm{E}-04 & 4.458 \mathrm{E}-11\end{array}$

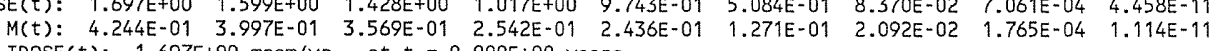
OMaximum TDOSE $(t): 1.697 \mathrm{E}+00 \mathrm{mrem} / \mathrm{yr}$ at $t=0.000 E+00$ years

Attachment

6 Sheet No. $\underline{8}$ of 19

S.W. Clark Date

Chk'd By M.W. Perrott

Date

Calc. No. 0100B-CA-V0301

Rev. No. 
CVP-2007-00006

Rev. 0

\section{ATTACHMENT 6}

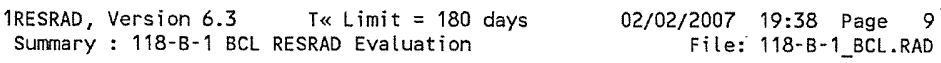

Attachment

$6 \quad$ Sheet No. $\underline{9}$ of 19 
CVP-2007-00006

Rev. 0

\section{ATTACHMENT 6}

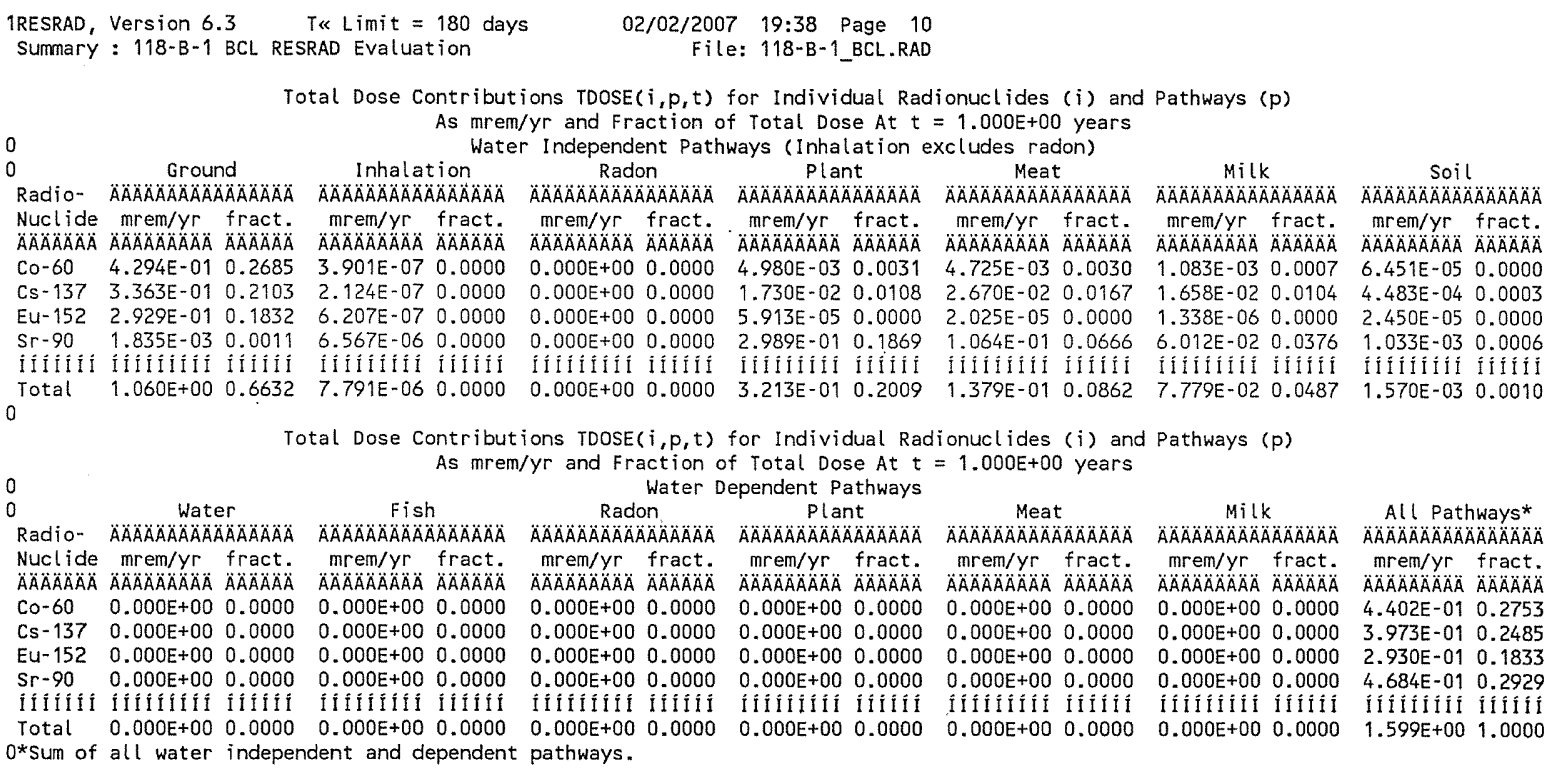

Attachment 


\section{ATTACHMENT 6}

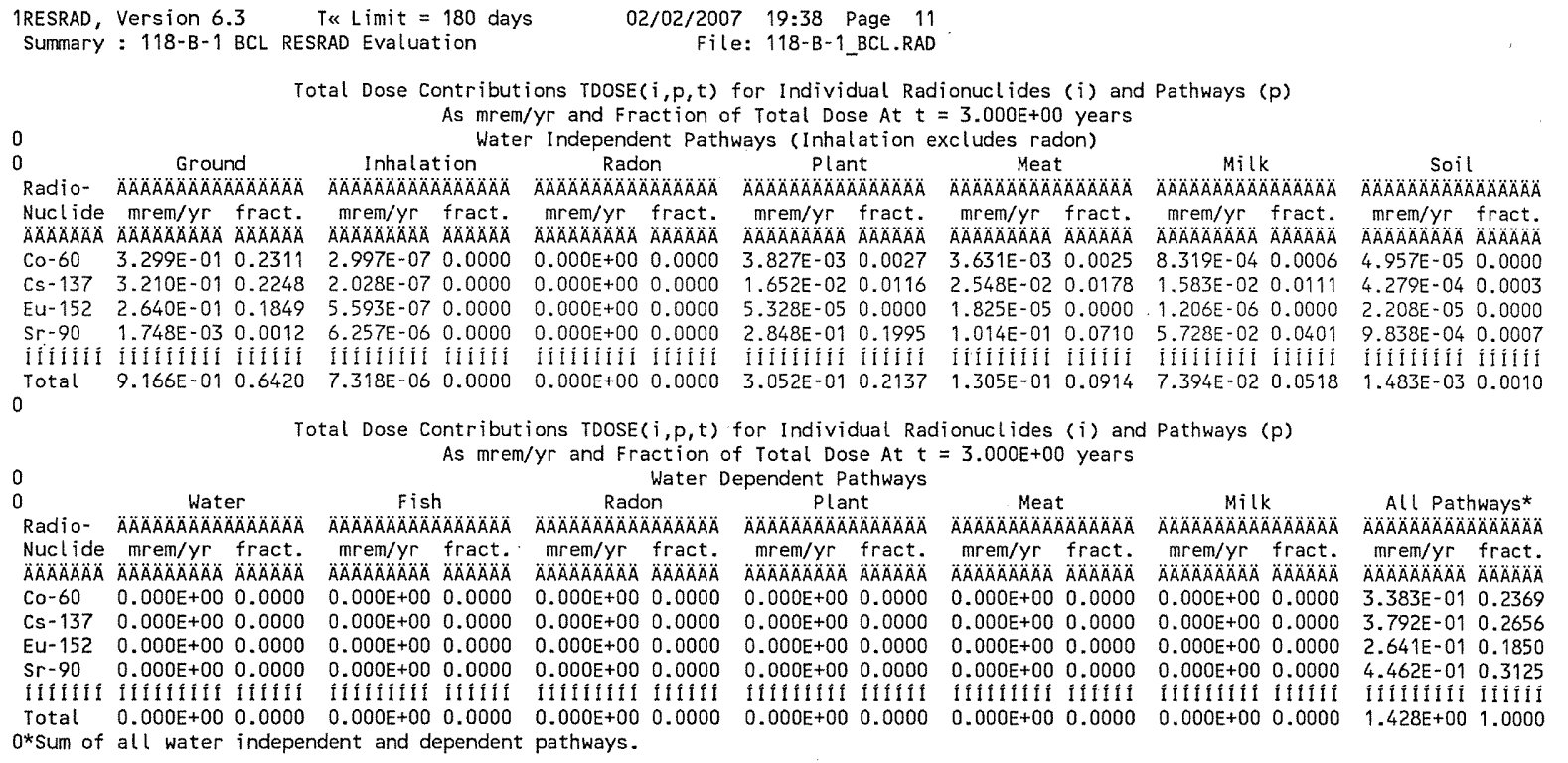

Total Dose Contributions $\operatorname{TDOSE}(i, p, t)$ for Individual Radionuclides (i) and Pathways $(p)$

As mrem/yr and Fraction of Total Dose At $t=3.000 E+00$ years

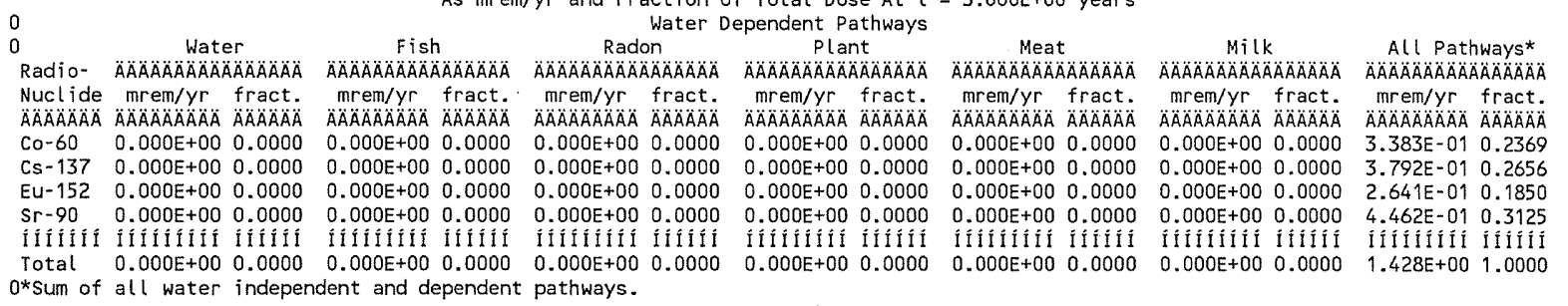

Attachment

6

Sheet No. 11 of 19

Originators: S.W. Clark Date

Chk'd By M.W. Perrott

Date

Calc. No. 0100B-CA-V0301

Rev. No. 0




\section{ATTACHMENT 6}

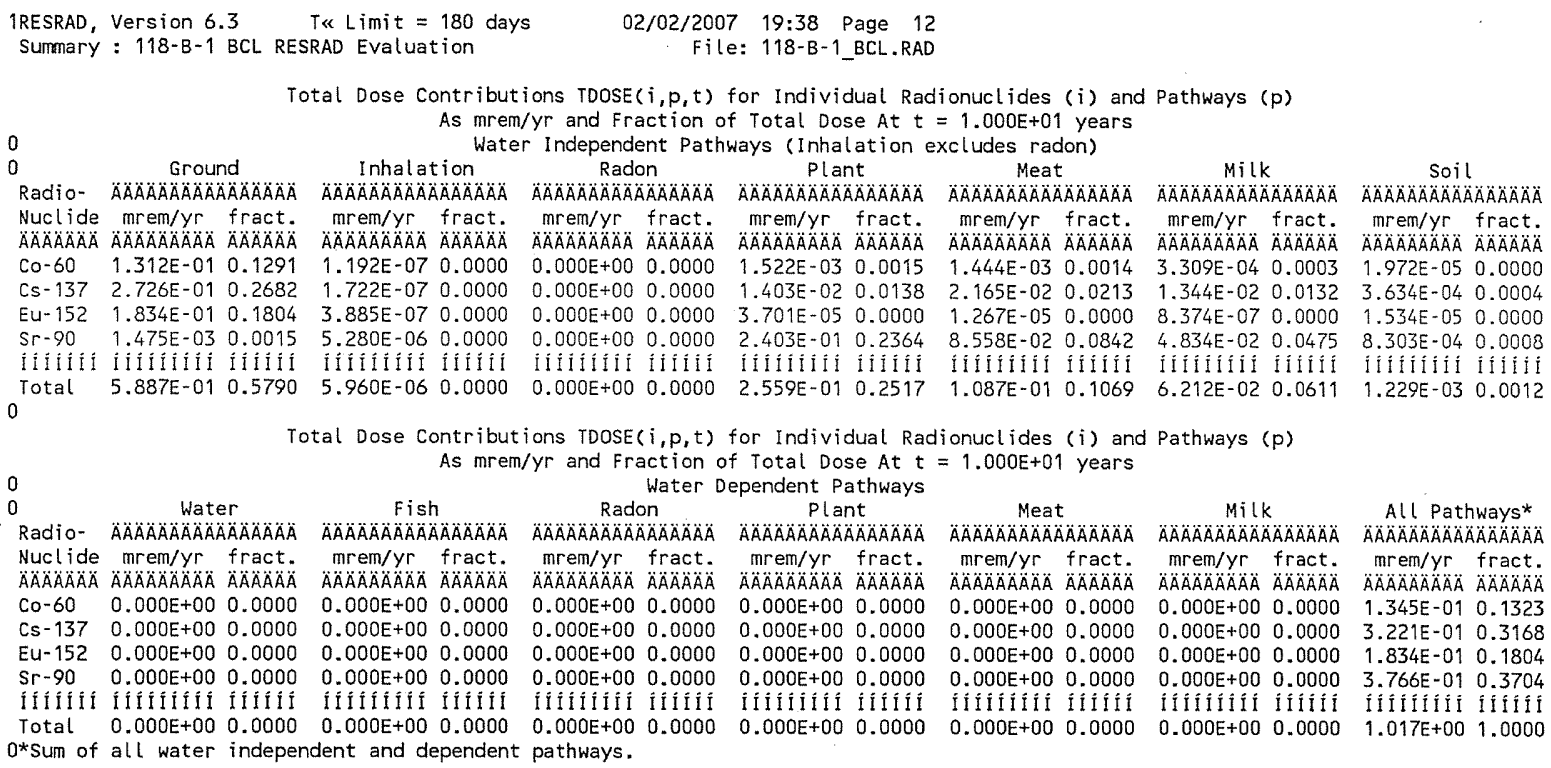

Total Dose Contributions $\operatorname{TDOSE}(i, p, t)$ for Individual Radionuclides (i) and Pathways $(p)$

As $\mathrm{mrem} / \mathrm{yr}$ and Fraction of Total Dose At $t=1.000 \mathrm{E}+01$ years

water Independent Pathways (Inhalation excludes radon)

0 Inhalation Water Independent Pathways (Inhalation excludes radon)
Radon Meat

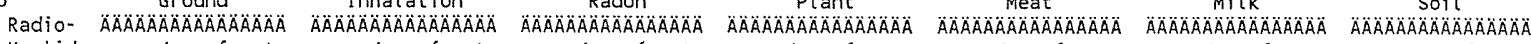
Nuclide $m r e m / y r$ fract. mrem/yr fract. mrem/yr fract. mrem/yr fract. mrem/yr fract. mrem/yr fract. mrem/yr fract.

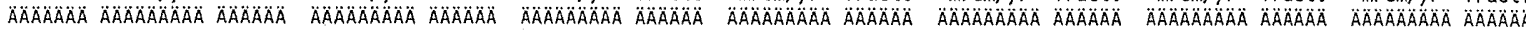

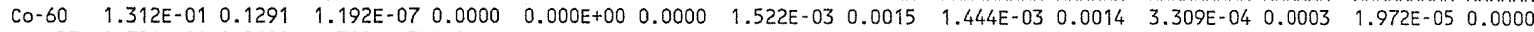

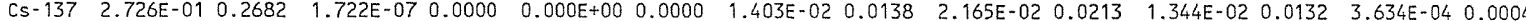

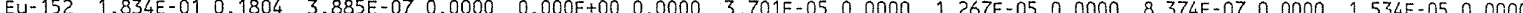

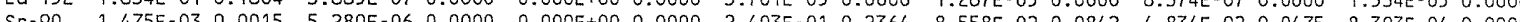
Sr-

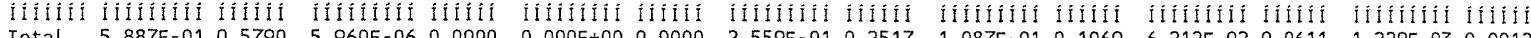

Total Dose Contributions $\operatorname{TDOSE}(i, p, t)$ for Individual Radionuclides (i) and Pathways ( $p$ ) As mrem/yr and Fraction of Total Dose At $t=1.000 E+01$ years

0 Water Dependent Pathways

Water Fish Radon Plant Meat Mathways*

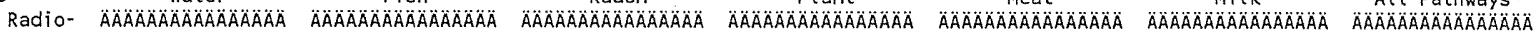
Nuclide mrem/yr fract. mrem/yr fract. mrem/yr fract. mrem/yr fract. mrem/yr fract. mrem/yr fract. mrem/yr fract.

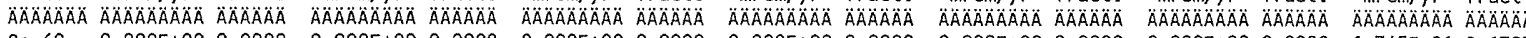
$\begin{array}{lllllllllllllllllll}C 0-60 & 0.000 E+00 & 0.0000 & 0.000 E+00 & 0.0000 & 0.000 E+00 & 0.0000 & 0.000 E+00 & 0.0000 & 0.000 E+00 & 0.0000 & 0.000 E+00 & 0.0000 & 1.345 E-01 & 0.1323\end{array}$

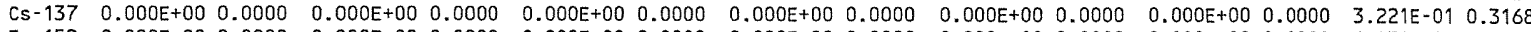
$\begin{array}{lllllllllllllllll}\text { Eu-152 } & 0.000 E+00 & 0.0000 & 0.000 E+00 & 0.0000 & 0.000 E+00 & 0.0000 & 0.000 E+00 & 0.0000 & 0.000 E+00 & 0.0000 & 0.000 E+00 & 0.0000 & 1.834 E-01 & 0.1804\end{array}$ $\begin{array}{lllllllllllllllllll}\mathrm{Sr}-90 & 0.000 \mathrm{E}+00 & 0.0000 & 0.000 \mathrm{E}+00 & 0.0000 & 0.000 \mathrm{E}+00 & 0.0000 & 0.000 \mathrm{E}+00 & 0.0000 & 0.000 \mathrm{E}+00 & 0.0000 & 0.000 \mathrm{E}+00 & 0.0000 & 3.766 \mathrm{E}-01 & 0.3704\end{array}$

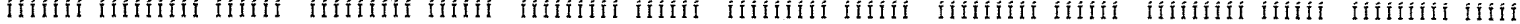

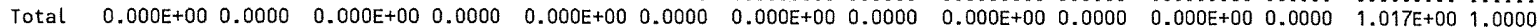
$0 *$ Sum of all water independent and dependent pathways.

\begin{tabular}{|c|c|}
\hline Attachment & Sheet No. 12 of 19 \\
\hline Originators: S. W. Clark & Date \\
\hline Chk'd By M. W. Perrott & Date \\
\hline $0100 B-C A-V 0301$ & Rev. No. \\
\hline
\end{tabular}




\section{ATTACHMENT 6}

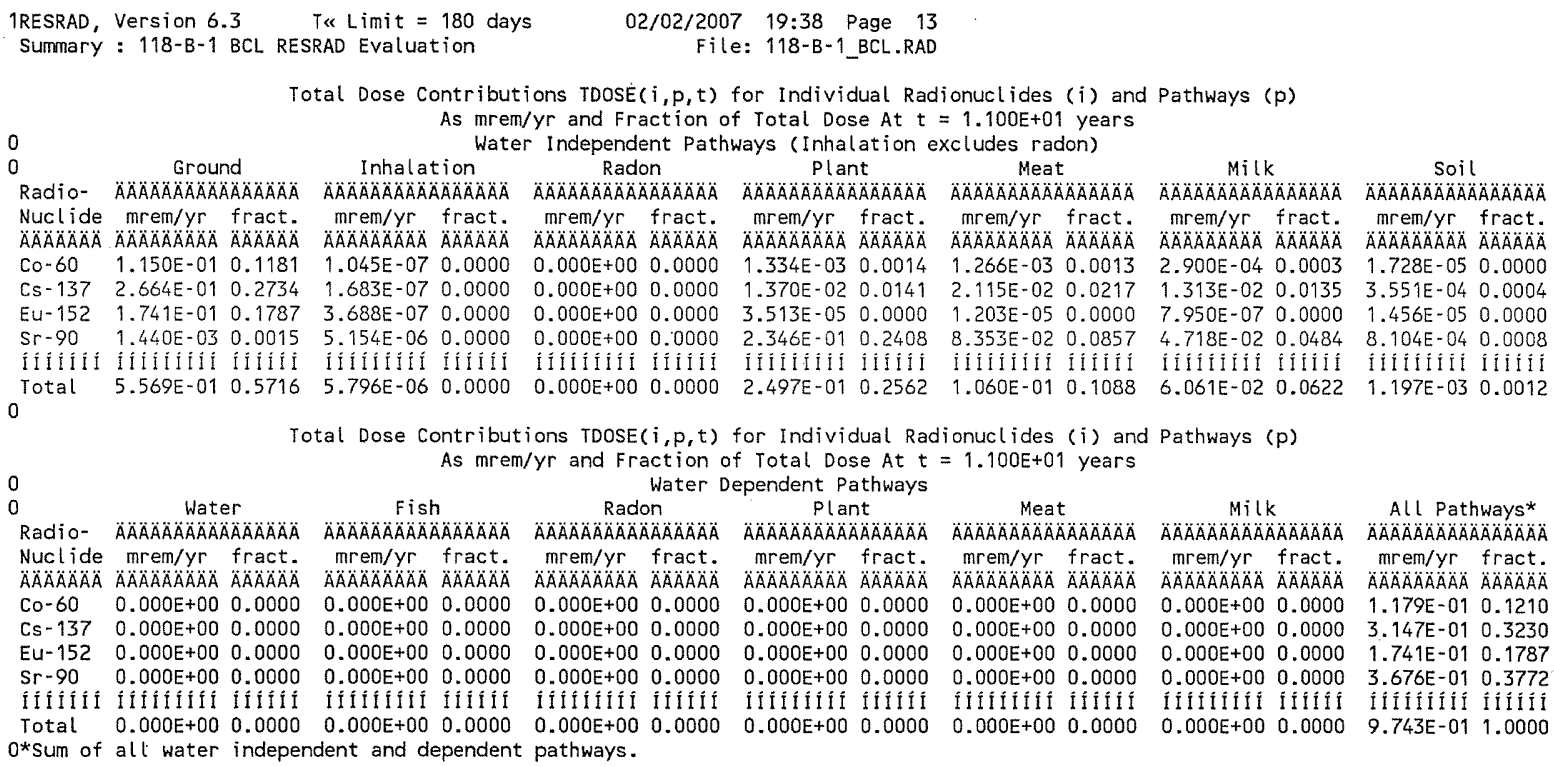

Total Dose Contributions $\operatorname{TDOSE}(i, p, t)$ for Individual Radionuclides (i) and Pathways ( $p$ ) As $\mathrm{mrem} / \mathrm{yr}$ and Fraction of Total Dose At $t=1.100 \mathrm{E}+01$ years

Total Dose Contributions $\operatorname{TDOSE}(i, p, t)$ for Individual Radionuclides (i) and Pathways $(p)$

As mrem/yr and Fraction of Total Dose At $t=1.100 \mathrm{E}+01$ years

0 Water Dependent Pathways

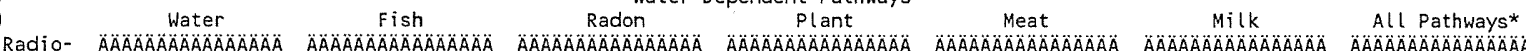
Nuclide mrem/yr fract mren

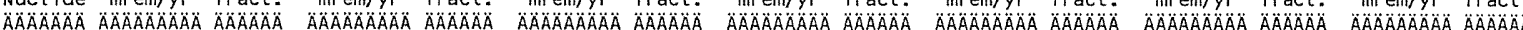

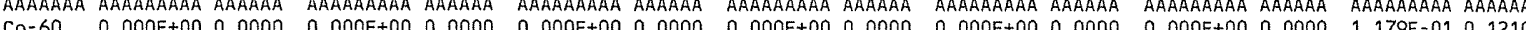
$\begin{array}{lllllllllllllll}C 0-60 & 0.000 E+00 & 0.0000 & 0.000 E+00 & 0.0000 & 0.000 E+00 & 0.0000 & 0.000 E+00 & 0.0000 & 0.000 E+00 & 0.0000 & 0.000 E+00 & 0.0000 & 1.179 E-01 & 0.1210\end{array}$

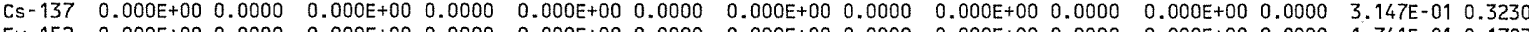
$\begin{array}{llllllllllllllll}\text { Eu-152 } & 0.000 E+00 & 0.0000 & 0.000 E+00 & 0.0000 & 0.000 E+00 & 0.0000 & 0.000 E+00 & 0.0000 & 0.000 E+00 & 0.0000 & 0.000 E+00 & 0.0000 & 1.741 E-01 & 0.1787\end{array}$ $\begin{array}{llllllllllllllll}\mathrm{Sr}-90 & 0.000 \mathrm{E}+00 & 0.0000 & 0.000 \mathrm{E}+00 & 0.0000 & 0.000 \mathrm{E}+00 & 0.0000 & 0.000 \mathrm{E}+00 & 0.0000 & 0.000 \mathrm{E}+00 & 0.0000 & 0.000 \mathrm{E}+00 & 0.0000 & 3.676 \mathrm{E}-01 & 0.3772\end{array}$

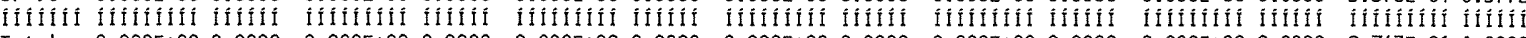
$\begin{array}{llllllllllllllllllllllllllll}\text { Total } & 0.000 E+00 & 0.0000 & 0.000 E+00 & 0.0000 & 0.000 E+00 & 0.0000 & 0.000 E+00 & 0.0000 & 0.000 E+00 & 0.0000 & 0.000 E+00 & 0.0000 & 9.743 E-01 & 1.0000\end{array}$ $0 *$ Sum of all water independent and dependent pathways.

\begin{tabular}{|c|c|}
\hline Attachment & Sheet No. 13 of 19 \\
\hline Originators: S. W. Clark & Date \\
\hline M. W. Perrott & Date \\
\hline $0100 \mathrm{~B}-\mathrm{CA}-\mathrm{V} 0301$ & Rev. No. 0 \\
\hline
\end{tabular}




\section{ATTACHMENT 6}

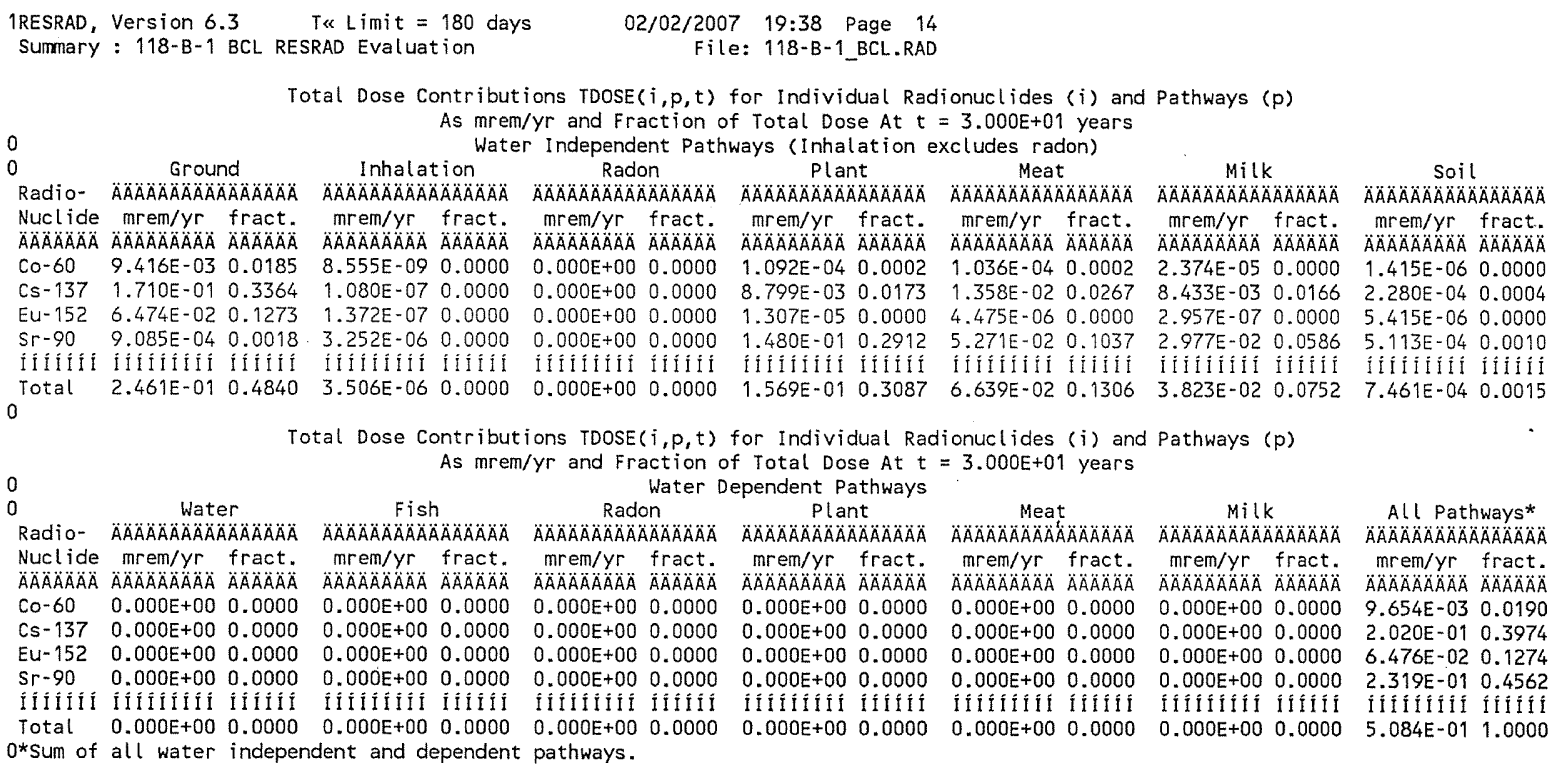

Total Dose Contributions $\operatorname{TOOSE}(i, p, t)$ for Individual Radionuclides $(i)$ and Pathways $(p)$ As mrem/yr and Fraction of Total Dose At $t=3.000 E+01$ years

0 Water Independent Pathways (Inhalation excludes radon)
Inhalation Plant Meat Meat Milk SOit Radio- ÄÄÄÄÄÄÄÄÄÄÄA A Nuclide $m r e m / y r$ fract. mrem/yr fract. mrem/yr fract. mrem/yr fract. mrem/yr fract. mrem/yr fract. mrem/yr fract.

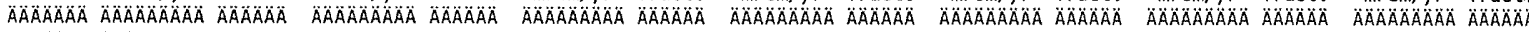
$\begin{array}{lllllllllllllllll}\mathrm{C} 0-60 & 9.416 \mathrm{E}-03 & 0.0185 & 8.555 \mathrm{E}-09 & 0.0000 & 0.000 \mathrm{E}+00 & 0.0000 & 1.092 \mathrm{E}-04 & 0.0002 & 1.036 \mathrm{E}-04 & 0.0002 & 2.374 \mathrm{E}-05 & 0.0000 & 1.415 \mathrm{E}-06 & 0.0000\end{array}$ $\begin{array}{lllllllllllllll}\text { CS-137 } & 1.710 E-01 & 0.3364 & 1.080 E-07 & 0.0000 & 0.000 E+00 & 0.0000 & 8.799 E-03 & 0.0173 & 1.358 E-02 & 0.0267 & 8.433 E-03 & 0.0166 & 2.280 E-04 & 0.0004\end{array}$

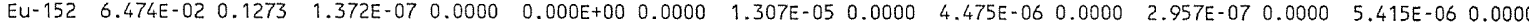

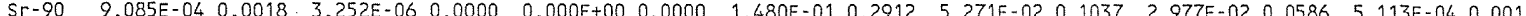
Ifíli

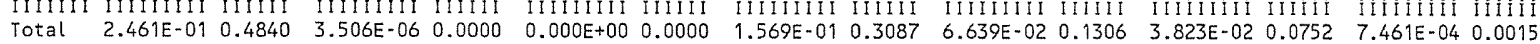
0 Total Dose Contributions TOOSE $(i, p, t)$ for Individual Radionuclides (i) and Pathways ( $p$ ) As $\mathrm{mrem} / \mathrm{yr}$ and Fraction of Total Dose At $t=3.000 \mathrm{E}+01$ years 0 Water Fish Wadon Dependent Pathways Plant Meat Milk All Pathways* (n) mrem/yr fract. mrem/yr fract. mrem/yr fract. mrem/yr fract. mrem/yr fract.

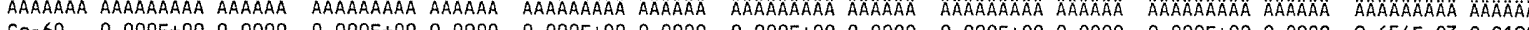

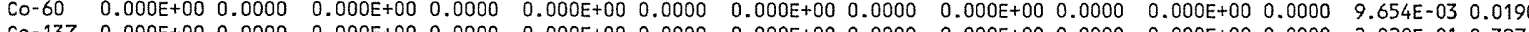

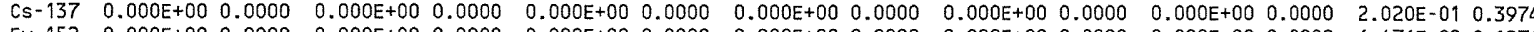
$\begin{array}{lllllllllllllll}E L-152 & 0.000 E+00 & 0.0000 & 0.000 E+00 & 0.0000 & 0.000 E+00 & 0.0000 & 0.000 E+00 & 0.0000 & 0.000 E+00 & 0.0000 & 0.000 E+00 & 0.0000 & 6.476 E-02 & 0.1274\end{array}$ $\begin{array}{lllllllllllllll}\mathrm{Sr}-90 & 0.000 \mathrm{E}+00 & 0.0000 & 0.000 \mathrm{E}+00 & 0.0000 & 0.000 \mathrm{E}+00 & 0.0000 & 0.000 \mathrm{E}+00 & 0.0000 & 0.000 \mathrm{E}+00 & 0.0000 & 0.000 \mathrm{E}+00 & 0.0000 & 2.319 \mathrm{E}-01 & 0.4562\end{array}$

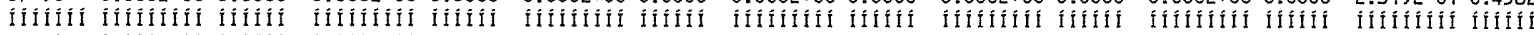

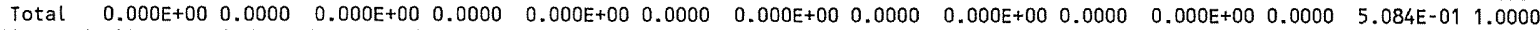
0 *Sum of all water independent and dependent pathways.

\begin{tabular}{|c|c|c|}
\hline & Sheet $N$ \\
\hline \multicolumn{2}{|c|}{$\begin{array}{c}\text { Attachment } \frac{6}{\text { Originators: S. W. Clark }} \\
\text { Sris }\end{array}$} & Date \\
\hline Chk & M.W. Perrott & Date \\
\hline No. & $0100 B-C A-V 0301$ & Rev. No. \\
\hline
\end{tabular}




\section{ATTACHMENT 6}

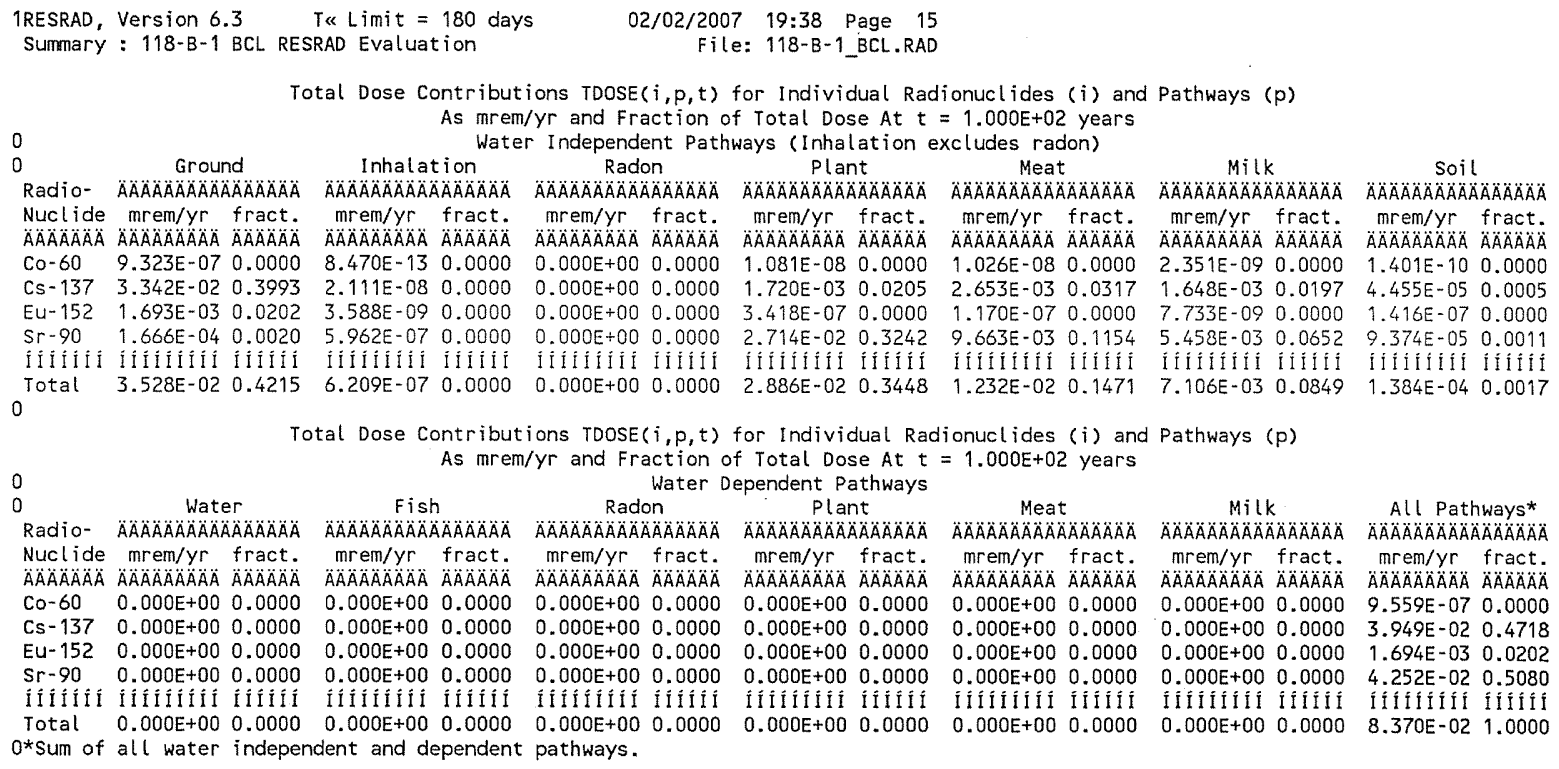

Total Dose Contributions $\operatorname{TDOSE}(i, p, t)$ for Individual Radionuclides (i) and Pathways $(p)$

As mrem/yr and Fraction of Total Dose At $t=1.000 E+02$ years Water Independent Pathways (Inhalation excludes radon)

0

Ground Inhalation Radon Meat

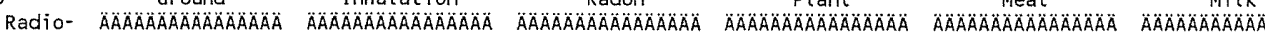
Nuclide mrem/yr fract mrem/yr fract mrem/yr fract mrem/yr fract mrem/yr fract mrem/yr fract. ÄÄÄ

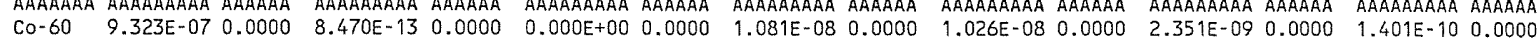

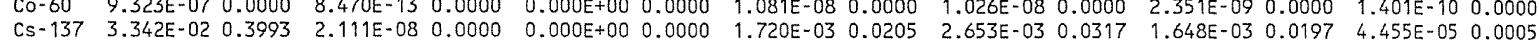

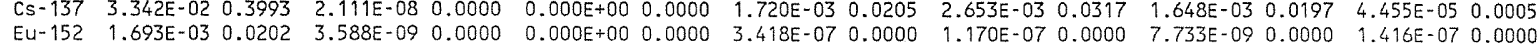
$\begin{array}{llllllllllllllllllll}\mathrm{Sr}-90 & 1.666 \mathrm{E}-04 & 0.0020 & 5.962 \mathrm{E}-07 & 0.0000 & 0.000 \mathrm{E}+00 & 0.0000 & 2.714 \mathrm{E}-02 & 0.3242 & 9.663 \mathrm{E}-03 & 0.1154 & 5.458 \mathrm{E}-03 & 0.0652 & 9.374 \mathrm{E}-05 & 0.0011\end{array}$

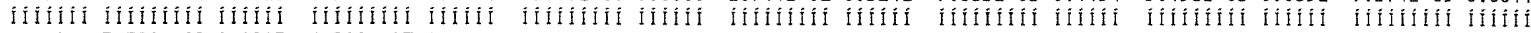

Total Dose Contributions $\operatorname{TDOSE}(i, p, t)$ for Individual Radionuclides (i) and Pathways ( $p$ ) As mrem/yr and Fraction of Total Dose At $t=1.000 E+02$ years

\begin{tabular}{|c|c|c|c|c|c|c|c|c|c|c|c|}
\hline 0 & & & & & & & & & & & \\
\hline 0 & Wat & & Fist & & Rad & & Plar & & Meat & Milk & All Pathways* \\
\hline Radio- & $A \cap A A A A A A A$ & $\triangle A O A A A A A ̈ A ̈$ & ÄÄÄÄÄÄÄ & $A A A A A A A ̈$ & AAAAAAAAAA & 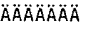 & 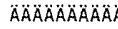 & $\triangle A O A O A Z A Z A ̈$ & 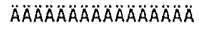 & 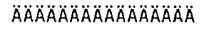 & 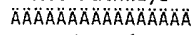 \\
\hline $\begin{array}{l}\text { Nuclide } \\
\text { ÄAAÄAAÄ }\end{array}$ & $\begin{array}{l}m r e m / y r \\
A \cap A ̈ A ̈ A B A A A B\end{array}$ & $\begin{array}{l}\text { fract. } \\
\text { AAAAAAA }\end{array}$ & $\begin{array}{c}m r e m / y r \\
\triangle A \cap A ̈ A ̈ A ̈ A B A B\end{array}$ & $\begin{array}{l}\text { fract. } \\
\qquad A \cap A ̈ A \cap A ̈ A\end{array}$ & $\begin{array}{c}m r e m / y r \\
A R A A A A A A\end{array}$ & 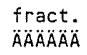 & $\begin{array}{c}m r e m / y r \\
A M A A A A A B\end{array}$ & $\begin{array}{l}\text { fract. } \\
\qquad A \cap A B A A B A\end{array}$ & $\begin{array}{rr}m r e m / y r & \text { fract. } \\
\text { AAAAAAAA AAAAAA }\end{array}$ & 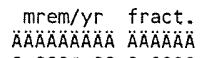 & 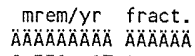 \\
\hline $\mathrm{Co}-60$ & $0.000 E+00$ & 0.0000 & $0.000 E+00$ & 0.0000 & $0.000 \mathrm{E}+00$ & 0.0000 & $0.000 E+00$ & 0.0000 & $0.000 E+00 \quad 0.0000$ & $0.000 E+00 \quad 0.0000$ & $9.559 E-070.00$ \\
\hline Cs -137 & $0.000 \mathrm{E}+00$ & 0.0000 & $0.000 E+00$ & 0.0000 & $0.000 E+00$ & 0.0000 & $0.000 E+00$ & 0.0000 & $00 E+00 \quad 0.0000$ & $0.000 E+00 \quad 0.0000$ & -02 \\
\hline Eu-152 & $0.000 E+00$ & 0.0000 & $0.000 E+00$ & 0.0000 & $0.000 E+00$ & 0.0000 & $0.000 E+00$ & 0.0000 & $0.000 E+00 \quad 0.0000$ & $0.000 E+00 \quad 0.0000$ & $1.694 \mathrm{E}-03 \quad 0.02$ \\
\hline & & & 0.0 & 0.0000 & $E+00$ & 0.0000 & 0.0 & 10 & $0.000 \mathrm{E}+000.0$ & $0.000 E+00 \quad 0.0000$ & $4.252 \mathrm{E}-02 \quad 0.50$ \\
\hline Ififiti & ifíi & IIIIIII & II Í I & I I I I I i & IÍí & IIIIIII & IIII & i & İ Î́ & Iíîlî́t & \\
\hline Total & $0.000 E+00$ & 0.0000 & $0.000 E+00$ & 0.0000 & $0.000 E+00$ & 0.0000 & $0.000 E+00$ & 0.0000 & $0.000 E+00 \quad 0.0000$ & $0.000 E+00 \quad 0.0000$ & $8.370 E-02 \quad 1.000$ \\
\hline
\end{tabular}

Attachment 6 Sheet No. 15 of 19 Originators: S.W. Clark Date Chk'd By M.W. Perrott Date Caic. No. 0100 B-CA-V0301 Rev. No. 0 


\section{ATTACHMENT 6}

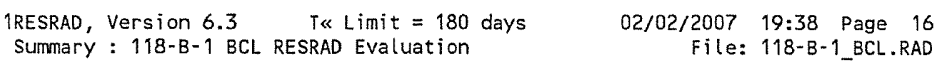

Total Dose Contributions $\operatorname{TDOSE}(i, p, t)$ for Individual Radionuclides (i) and Pathways $(p)$

As mrem/yr and Fraction of Total Dose At $t=3.000 E+02$ years

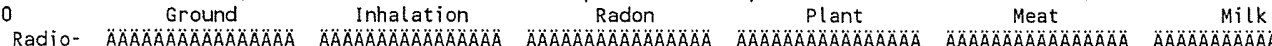
Water Independent Pathways (Inhalation excludes radon)

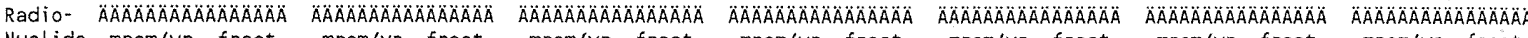
Nuclide m r fract mrem/y fract. mrem/yr fract. mrem/yr fract. mrem/yr fract. mrem/yr fract.

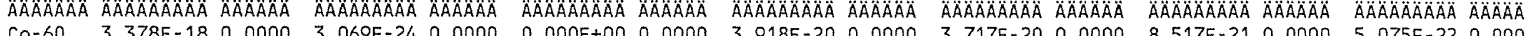

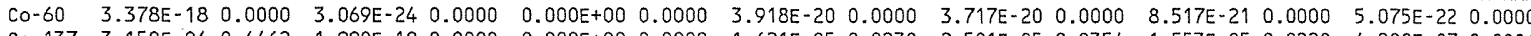

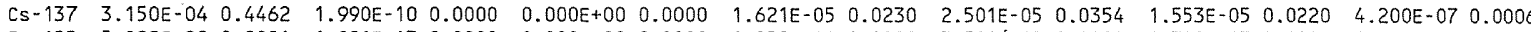

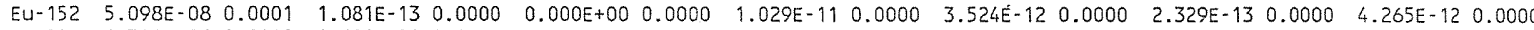

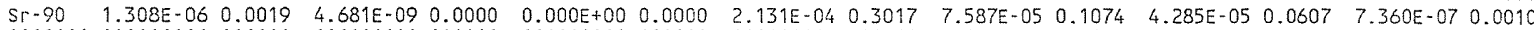

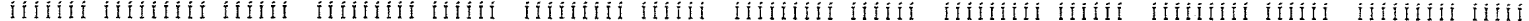

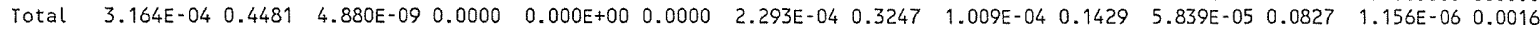

Total Dose Contributions TDOSE $(i, p, t)$ for Individual Radionuclides (i) and Pathways ( $p$ )

As mrem/yr and Fraction of Total Dose At $t=3.000 E+02$ years Water Dependent Pathways

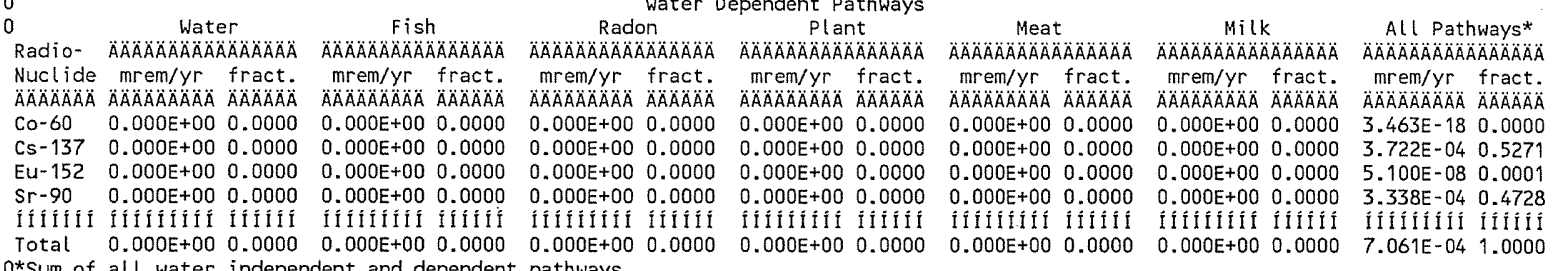
$0 *$ Sum of all water independent and dependent pathways.

\begin{tabular}{|c|c|}
\hline \multirow{2}{*}{\multicolumn{2}{|c|}{$\begin{array}{cc}\text { Attachment } & 6 \\
\text { Originators: S.W. Clark } & \text { Sheet No. } 16 \text { of } 19 \\
\text { Date }\end{array}$}} \\
\hline & \\
\hline M. W. Perrott & Date \\
\hline 0100B-CA-V0301 & Rev. No. \\
\hline
\end{tabular}


CVP-2007-00006

Rev. 0

\section{ATTACHMENT 6}

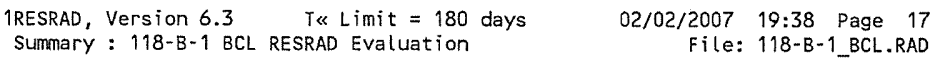

Total Dose Contributions $\operatorname{TDOSE}(i, p, t)$ for Individual Radionuclides $(i)$ and Pathways $(p)$

As mrem/yr and Fraction of Total Dose At $t=1.000 E+03$ years

Water Independent Pathways (Inhalation excludes radon)

$$
\text { Inhalation Radon Mlant Meat }
$$

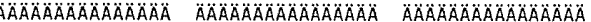

Meat

$M i l k$

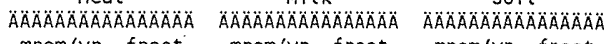
mrem/yr fract. mrem/yr fract. mrem/yr fract. mrem/yr fract. mrem/yr fract. mrem/yr fract.

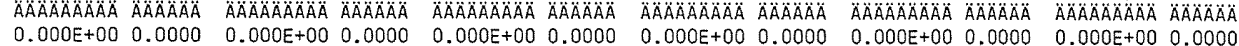
$\begin{array}{llllllllllll}0.000 E+00 & 0.0000 & 0.000 E+00 & 0.0000 & 0.000 E+00 & 0.0000 & 0.000 E+00 & 0.0000 & 0.000 E+00 & 0.0000 & 0.000 E+00 & 0.0000 \\ 1.618 E-17 & 0.0000 & 0.000 E+00 & 0.0000 & 1.318 E-12 & 0.0296 & 2.033 E-12 & 0.0456 & 1.263 E-12 & 0.0283 & 3.414 E-14 & 0.0008\end{array}$ $\begin{array}{llllllllllllll}2.507 E-17 & 0.0000 & 0.000 E+00 & 0.0000 & 5.397 E-17 & 0.0000 & 1.848 E-17 & 0.0000 & 4.885 E-19 & 0.0000 & 2.237 E-17 & 0.0000\end{array}$ $\begin{array}{lllllllllllll}2.007 E-16 & 0.0000 & 0.000 E+00 & 0.0000 & 9.137 E-12 & 0.2050 & 3.253 E-12 & 0.0730 & 1.838 E-12 & 0.0412 & 3.156 E-14 & 0.0007\end{array}$

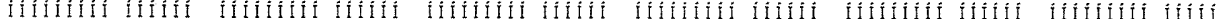
$2.420 \mathrm{E}-16 \quad 0.0000 \quad 0.000 \mathrm{E}+000.0000 \quad 1.045 \mathrm{E}-110.2345 \quad 5.287 \mathrm{E}-120.1186 \quad 3.101 \mathrm{E}-120.0696 \quad 6.573 \mathrm{E}-14$ IIII

Total Dose Contributions $\operatorname{TDOSE}(i, p, t)$ for Individual Radionuclides (i) and Pathways ( $p$ )

As mrem/yr and Fraction of Total Dose At $t=1.000 E+03$ years

0 Water Dependent Pathways

\begin{tabular}{|c|c|c|c|c|c|c|c|}
\hline & & & & & & & \\
\hline & $\begin{array}{l}\text { Water } \\
\triangle A A B A A B\end{array}$ & $\begin{array}{l}\text { Fish } \\
A B A O A O A\end{array}$ & $\begin{array}{l}\text { Radon } \\
A A B A A A B\end{array}$ & $\begin{array}{l}P \operatorname{lant} \\
\end{array}$ & $\begin{array}{l}\text { Meat } \\
A A B A A\end{array}$ & Milk & $\begin{array}{l}\text { All Pathways* } \\
\text { AL }\end{array}$ \\
\hline $\begin{array}{l}\text { Nuclide } \\
\triangle A B A A O A O A B\end{array}$ & 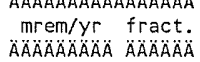 & 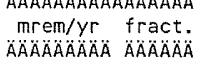 & 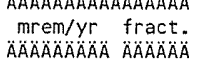 & 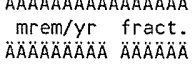 & 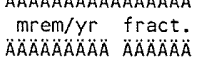 & 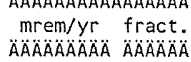 & 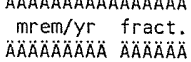 \\
\hline Co- 60 & $0.000 E+00 \quad 0.0000$ & $0.000 E+00 \quad 0.0000$ & $0.000 E+00 \quad 0.0000$ & $0.000 \mathrm{E}+00 \quad 0.0000$ & $0.000 E+00 \quad 0.0000$ & $0.000 E+00 \quad 0.0000$ & $+00 \quad 0.0000$ \\
\hline Cs -137 & $0.000 E+00 \quad 0.0000$ & $0.000 E+00 \quad 0.0000$ & $0.000 E+00 \quad 0.0000$ & $0.000 E+00 \quad 0.0000$ & $0.000 E+00 \quad 0.0000$ & $0.000 E+00 \quad 0.0000$ & 110.8 \\
\hline Eu-152 & $0.000 E+00 \quad 0.0000$ & $0.000 E+00 \quad 0.0000$ & $0.000 E+00 \quad 0.0000$ & $E+00 \quad 0.0000$ & $00 E+00 \quad 0.0000$ & $0.000 E+00 \quad 0.0000$ & 160.0000 \\
\hline $\mathrm{sr}$ & $0.000 E+00 \quad 0.0000$ & $0.000 E+00 \quad 0.0000$ & $E+00 \quad 0.0000$ & $0.000 E+00 \quad 0.0000$ & $0.000 E+00 \quad 0.0$ & $E+00 \quad 0.0$ & \\
\hline Ílífî́n & 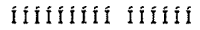 & 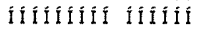 & IIIIIIIIII & 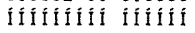 & IIIIIIIII IIÍ & 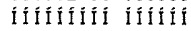 & III ÎIIIII \\
\hline t & $0.000 E+00 \quad 0.0000$ & $0.000 E+00 \quad 0.0000$ & $0.000 E+00 \quad 0.0000$ & $0.000 E+00 \quad 0.0000$ & $0.000 E+00 \quad 0.0000$ & $0.000 E+00 \quad 0.0000$ & $4.458 E-111.0000$ \\
\hline
\end{tabular}

0 *Sum of all water independent and dependent pathways.

Attachment Sheet No. 17 of 19 Originators: S.W. Clark Date

Chk'd By M.W. Perrott Date

Calc. No. 0100B-CA-V0301

Rev. No. 


\section{ATTACHMENT 6}

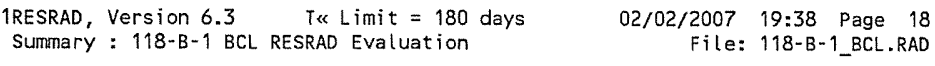

\begin{tabular}{|c|c|c|}
\hline \multirow{2}{*}{\multicolumn{3}{|c|}{ 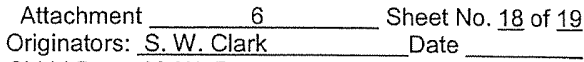 }} \\
\hline & & \\
\hline Chk'd By & M. W. Perrott & Date \\
\hline Calc. No. & 0100B-CA-V0301 & Rev \\
\hline
\end{tabular}




\section{ATTACHMENT 6}

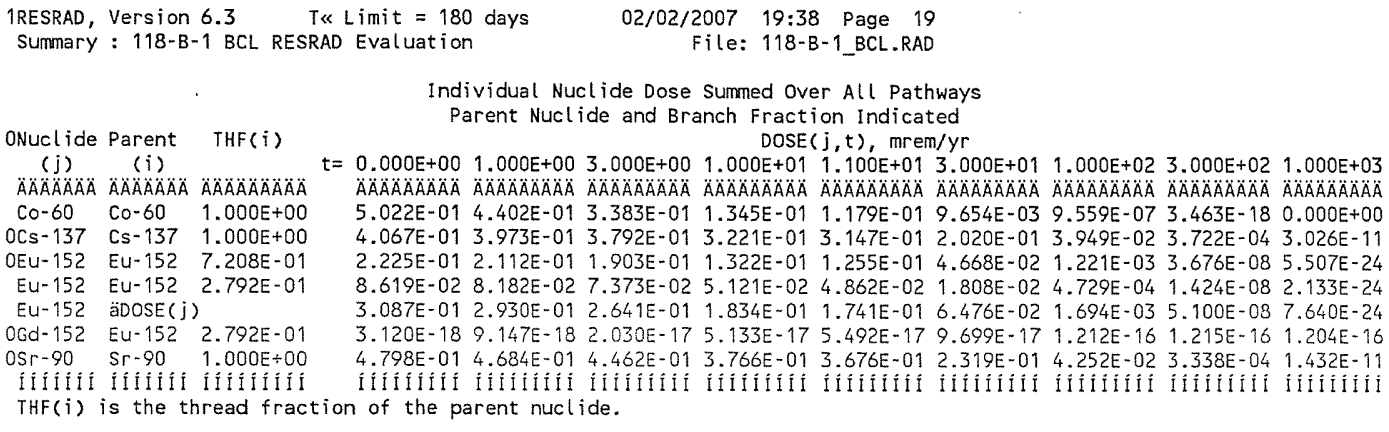

THF(i) is the thread fraction of the parent nuclide.

$t=0.000 E+00 \quad 1.000 E+00 \quad 3.000 E+00 \quad 1.000 E+011.100 E+01 \quad 3.000 E+01 \quad 1.000 E+02 \quad 3.000 E+02 \quad 1.000 E+03$

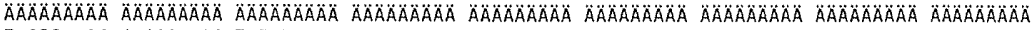

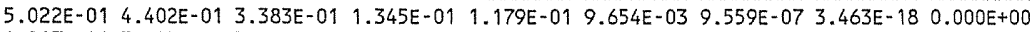

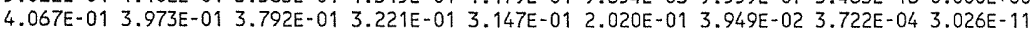

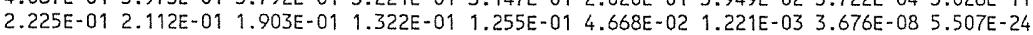

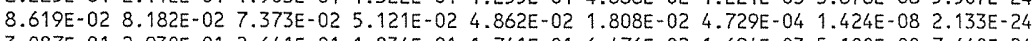

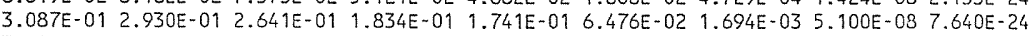
$3.120 E-18 \quad 9.147 E-18 \quad 2.030 E-17 \quad 5.133 E-17 \quad 5.492 E-17 \quad 9.699 E-17 \quad 1.212 E-16 \quad 1.215 E-16 \quad 1.204 E-16$ 4.798E-01 4.684E-01 4.462E-01 3.766E-01 3.676E-01 2.319E-01 4.252E-02 3.338E-04 $1.432 E-11$

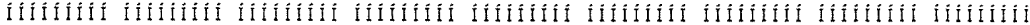

Individual Nuclide Soil Concentration Parent Nuclide and Branch Fraction Indicated $S(j, t), p C i / g$

ONuclide Parent THF(i) (j) (i) AAAAAAAA AAAOÄÄÄA ÄÄÄÄÄÄÄÄA CO-60 CO-60 $1.000 E+00$ OCs-137 Cs-137 1.000E+00 OEU-152 EU-152 7.208E-01 Eu-152 Eu-152 2.792E-0 Eu-152 äS(j): OGd-152 Eu-152 2.792E-01 $0 \mathrm{Sr}-90 \quad \mathrm{Sr}-90 \quad 1.000 \mathrm{E}+00$

IIIIIII ÎIIIIII İî́ílíi

THF (i) is the thread fraction ORESCALC.EXE execution time = $t=0.000 E+00 \quad 1.000 E+00 \quad 3.000 E+001.000 E+011.100 E+013.000 E+01 \quad 1.000 E+02 \quad 3.000 E+02 \quad 1.000 E+03$

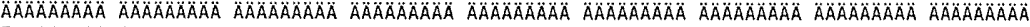
5.000E-02 4.383E-02 3.368E-02 $1.339 \mathrm{E}-02 \quad 1.174 \mathrm{E}-02 \quad 9.612 \mathrm{E}-04 \quad 9.517 \mathrm{E}-08 \quad 3.448 \mathrm{E}-19 \quad 0.000 \mathrm{E}+00$

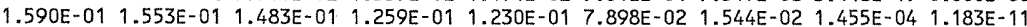

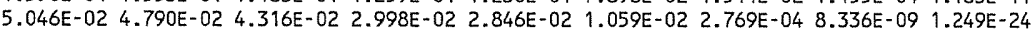

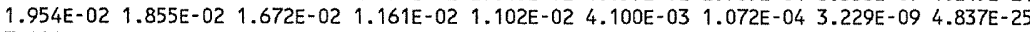
7.000E-02 $6.645 \mathrm{E}-02 \quad 5.988 \mathrm{E}-02 \quad 4.159 \mathrm{E}-02 \quad 3.948 \mathrm{E}-02 \quad 1.469 \mathrm{E}-02 \quad 3.841 \mathrm{E}-04 \quad 1.157 \mathrm{E}-08$ 1.732E-24

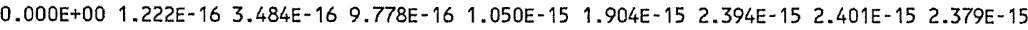
1.200E-01 1.171E-01 1.116E-01 9.417E-02 9.192E-02 5.800E-02 1.063E-02 8.348E-05 3.580E-12

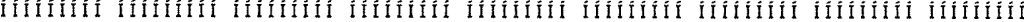
the parent nuclide. 9.00 seconds

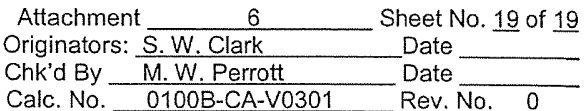




\section{ATTACHMENT 7}

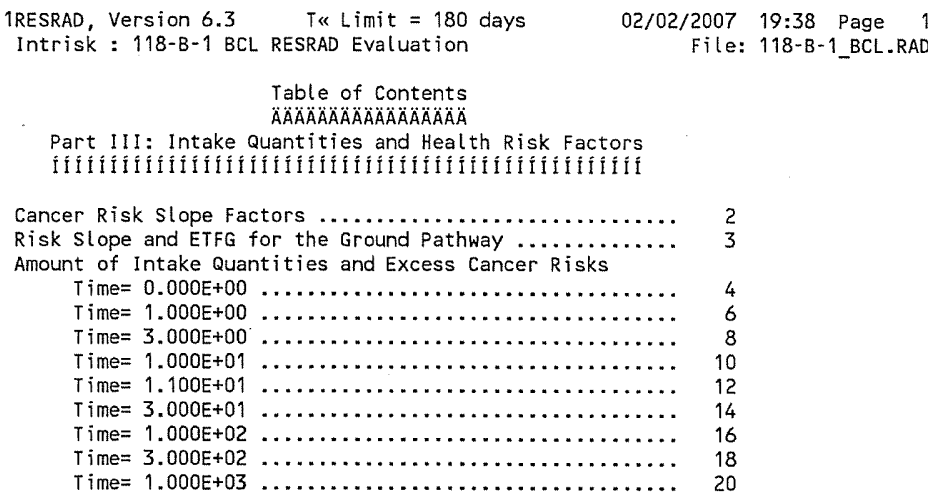

Attachment

Sheet No. 1 of 21

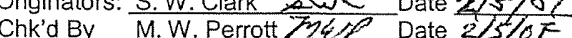

Calc. No. $0100 \mathrm{~B}-\mathrm{CA}-\mathrm{V} 0301 \quad$ Rev. No. 0 


\section{ATTACHMENT 7}

1RESRAD, Version $6.3 \quad T \ll$ Limit $=180$ days Intrisk : 118-B-1 BCL RESRAD Evaluation
02/02/2007 19:38 Page 2
Cancer Risk Slope Factors Summary Table
Risk Library: HEAST 2001 Morbidity

$0{ }^{3}$

Parameter

Current ' Base ' Parameter

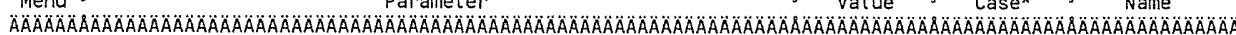
$\mathrm{Sf}-1{ }^{3}$ Ground external radiation slope factors, $1 / \mathrm{yr}$ per $(\mathrm{pC} \mathrm{i} / \mathrm{g}) \mathrm{:}^{3}$ Sf -1 S Co-60

$\mathrm{Sf}-1$; $\mathrm{Cs}-137+\mathrm{D}$

Sf-1 3 Eu-152

Sf-1 $3 \mathrm{Gd}-152$

Sf-1 s $\mathrm{Sr}-90+\mathrm{D}$

Sf-2 Inhalation, slope factors, $1 /(\mathrm{pC} i)$ :

$\mathrm{Sf}-2$ ? $\mathrm{Co}-60$

Sf-2 3 CS $-137+D$

$\mathrm{Sf}-2 \mathrm{~S}$ EU-152

Sf -2 s Gd-152

$S f-2$ s $S r-90+D$

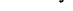

Sf $-33^{3}$ Food ingestion, slope factors, $1 /(p c i)$ :

$\mathrm{Sf}-3 \rightarrow \mathrm{Co}-60$

$\mathrm{Sf}-3 \quad 3 \mathrm{Cs}-137+D$

$\mathrm{Sf}-3$ 3 Eu-152

$\mathrm{Sf}-3 \quad 3 \mathrm{Gd}-152$

$\mathrm{Sf}-3$ ? $\mathrm{Sr}-90+\mathrm{D}$

$\mathrm{Sf}-3$ 3 Water ingestion, slope factors, 1/(pCi):

$\mathrm{Sf}-3$ > Co-60

Sf $-3 \quad 3$ Cs $-137+D$

$\mathrm{Sf}-3$ s Eu-152

Sf $-3 \quad 3$ Gd -152

$\mathrm{Sf}-3$ ? $\mathrm{Sr}-90+\mathrm{D}$

sf -3 , soil ingestion, slope factors, $1 /(\mathrm{pCi})$ :

$\mathrm{Sf}-3$ 3 Co-60

$\mathrm{Sf}-3 \quad 3 \mathrm{Cs}-137+0$

$\mathrm{Sf}-3$ ? Eu-152

$S f-3$ s $G d-15$

$\mathrm{Sf}-3$ s $\mathrm{Sr}-9 \mathrm{OO+D}$

3 3

$1.24 \mathrm{E}-05$ 3 $1.24 \mathrm{E}-053 \operatorname{SLPF}(1,1)$

2.55E-06 $5.32 E-10$ SLPF( 2,1)

5.30E-06 $3.30 E-06$ SLPF( 3,1)

$0.00 E+00 \div 0.00 E+00$ SLPF 5,1$)$

$1.96 \mathrm{E}-08 \div 4.82 \mathrm{E}-10$ \&SLPF( 6,1$)$

$3.58 \mathrm{E}-1133.58 \mathrm{E}-11$ 3 $\operatorname{SLPFC}(1,2)$

$31.19 E-11>1.19 E-113 \operatorname{SLPFC} 2,2)$

$9.10 E-1139.10 E-11 ; \operatorname{SLPF}(3,2)$

$39.10 E-09$ 3 $9.10 E-09$ S SLPF 5,2$)$

$\leqslant 1.13 E-10 ; 1.05 E-10 \div \operatorname{SLPF}(6,2)$

$32.23 \mathrm{E}-11,2.23 \mathrm{E}-113$ SLPF( 1,3$)$

$33.74 \mathrm{E}-1133.74 \mathrm{E}-11$ 3 SLPF( 2,3$)$

$38.70 E-12,8.70 E-123 \operatorname{SLPF}(3,3)$

$3.85 E-11,3.85 E-11$ S SLPF $(5,3)$

$39.53 E-1136.88 E-11$ 3 $\operatorname{SLPF}(6,3)$

3 1.57E-11 3 1.57E-11 3 SLPF( 1,4$)$

6.07E-1

7.40E-11 5.59E-11 3 SLPF( 6,4)

3

$4.03 E-11 ; 4.03 E-11 ; \operatorname{SLPF}(1,5)$

$34.33 E-1134.33 E-113$ SLPF( 2,5$)$

$1.62 E-113,1.62 E-11$ S SLPF $(3,5)$

- $36.29 E-113$ SLPF $(5,5)$

3 $1.44 \mathrm{E}-1039.18 \mathrm{E}-11$ S $\operatorname{SLPF}(6,5)$

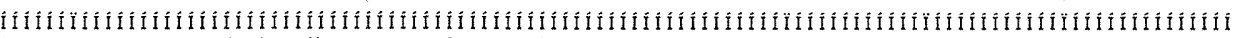

*Base Case means Default.Lib w/o Associate Nuclide contributions.

Attachment

Originators: S. W. Clark

7

Sheet No. $\underline{2}$ of $\underline{21}$

Chk'd By M. W. Perrott

Calc. No. 0100B-CA-V0301

Rev. No. 
CVP-2007-00006

Rev. 0

\section{ATTACHMENT 7}

\begin{tabular}{|c|c|c|c|c|c|c|c|c|c|c|}
\hline $\begin{array}{l}\text { 1RESRAD } \\
\text { Intrisk }\end{array}$ & $\begin{array}{l}\text { Version } 6.3 \\
: 118-\mathrm{B}-1 \mathrm{BCL}\end{array}$ & $\begin{array}{r}T \ll L i \\
L \text { RESRAD E }\end{array}$ & $\begin{array}{l}\text { nit }=180 \text { da } \\
\text { valuation }\end{array}$ & & $\begin{array}{r}\text { 02/02/2007 } \\
\text { File: }\end{array}$ & $\begin{array}{l}19: 38 \text { Page } \\
118-B-1 \_B C L\end{array}$ & $\begin{array}{r}3 \\
. R A D\end{array}$ & & & \\
\hline ONuclide & Slope(i)* & Risk & & $\begin{array}{r}\text { Imental } \\
\text { ETFG( }\end{array}$ & $(i, t)$ At $T i n$ & $\begin{array}{l}\text { Factors for } \\
\text { ne in Years }\end{array}$ & $\begin{array}{l}\text { the Ground } \\
\text { (dimension }\end{array}$ & $\begin{array}{l}\text { Pathway } \\
\text { (ess) }\end{array}$ & & \\
\hline & & $0.000 E+00$ & $1.000 \mathrm{E}+00$ & $3.000 \mathrm{E}+00$ & $1.000 \mathrm{E}+01$ & $1.100 E+01$ & $3.000 E+01$ & $1.000 E+02$ & $3.000 E+02$ & $1.000 E+03$ \\
\hline$\triangle \triangle A ̈ A \cap A \cap A ̈ A ̈$ & $\triangle A A A B A A A A A B A$ & $\triangle \triangle A O A O A O A O A O A O A$ & 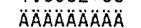 & 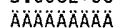 & 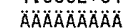 & 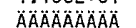 & $\triangle A A B A A A A O A$ & $\triangle \triangle A O A O A O A A O A B$ & $\triangle A A A B A A A A A$ & 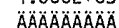 \\
\hline $8 a-137 m$ & $2.690 \mathrm{E}-06$ & $6.421 \mathrm{E}-01$ & $6.421 E-01$ & $6.421 E-01$ & $6.421 \mathrm{E}-01$ & $6.421 \mathrm{E}-01$ & $6.421 \mathrm{E}-01$ & $6.421 \mathrm{E}-01$ & $6.421 \mathrm{E}-01$ & $6.421 E-01$ \\
\hline Co- 60 & $1.240 \mathrm{E}-05$ & $6.448 \mathrm{E}-01$ & $6.448 \mathrm{E}-01$ & $6.448 \mathrm{E}-01$ & $6.448 \mathrm{E}-01$ & $6.448 \mathrm{E}-01$ & $6.448 \mathrm{E}-01$ & $6.448 \mathrm{E}-01$ & $6.448 E-01$ & $6.448 E-01$ \\
\hline Cs $=137$ & $5.320 E-10$ & $6.512 E-01$ & $6.512 \mathrm{E}-01$ & $6.512 \mathrm{E}-01$ & $6.512 E-01$ & $6.512 E-01$ & $6.512 \mathrm{E}-01$ & $6.512 E-01$ & $6.512 E-01$ & $6.512 \mathrm{E}-01$ \\
\hline Eu-152 & $5.300 \mathrm{E}-06$ & $6.458 \mathrm{E}-01$ & $6.458 \mathrm{E}-01$ & $6.458 E-01$ & $6.458 \mathrm{E}-01$ & $6.458 \mathrm{E}-01$ & $6.458 \mathrm{E}-01$ & $6.458 \mathrm{E}-01$ & $6.458 \mathrm{E}-01$ & $6.458 \mathrm{E}-01$ \\
\hline$G d-152$ & $0.000 \mathrm{E}+00$ & $0.000 E+00$ & $0.000 E+00$ & $0.000 E+00$ & $0.000 E+00$ & $0.000 E+00$ & $0.000 E+00$ & $0.000 E+00$ & $0.000 \mathrm{E}+00$ & $0.000 E+00$ \\
\hline $5 r-90$ & $4.820 \mathrm{E}-10$ & $6.523 \mathrm{E}-01$ & $6.523 E-01$ & $6.523 E-01$ & $6.523 \mathrm{E}-01$ & $6.523 \mathrm{E}-01$ & $6.523 \mathrm{E}-01$ & $6.523 E-01$ & $6.523 \mathrm{E}-01$ & $6.523 E-01$ \\
\hline$\gamma-90$ & $1.910 \mathrm{E}-08$ & $6.439 \mathrm{E}-01$ & $6.439 \mathrm{E}-01$ & $6.439 E-01$ & $6.439 \mathrm{E}-01$ & $6.439 E-01$ & $6.439 E-01$ & $6.439 E-01$ & $6.439 E-01$ & $6.439 E-01$ \\
\hline Iifíîlí & IIIIIIIIII & IAinitiol & IIIIIIII & IIIIIIIfí & Difitifíl & IIIIIIAI & IIIIIIII & IIIIIIII & IIIIIIII & IIIIIIII \\
\hline
\end{tabular}

Attachment Sheet No. 3 of $\underline{21}$ Originators: S. W. Clark Date

Chk'd By M.W. Perrott Date

Calc. No. 0100B-CA-V0301

Rev. No. 
CVP-2007-00006

Rev. 0

\section{ATTACHMENT 7}

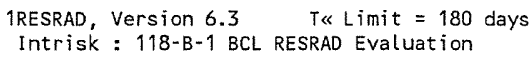

Excess Cancer Risks CNRS $(i, p, t)$ for Individual Radionuclides (i) and Pathways $(p)$ and Fraction of Total Risk at $t=0.000 E+00$ years

Water Dependent Pathways

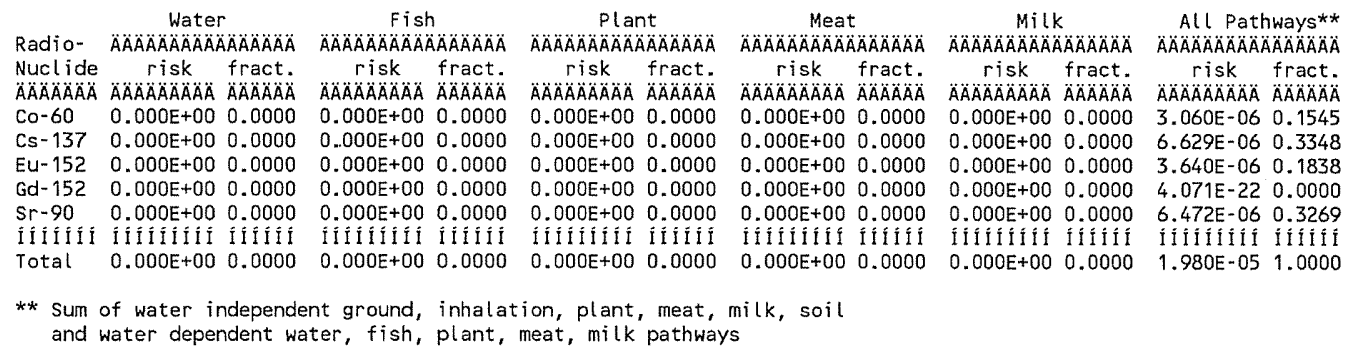

Sum of water independent ground, inhalation, plant, meat, milk
and water dependent water, fish, plant, meat, milk pathways

$\begin{array}{ll}\text { Attachment } \frac{7}{\text { S.W. Wlark }} & \text { Sheet No. } 4 \text { of } 21 \\ \text { Originators: } & \text { Date } \\ \text { Chk'd By } & \text { Date } \\ \text { Calc. No. Perrott } & \text { Rev. No. } \quad \text { O100B-CA-V0301 }\end{array}$




\section{ATTACHMENT 7}

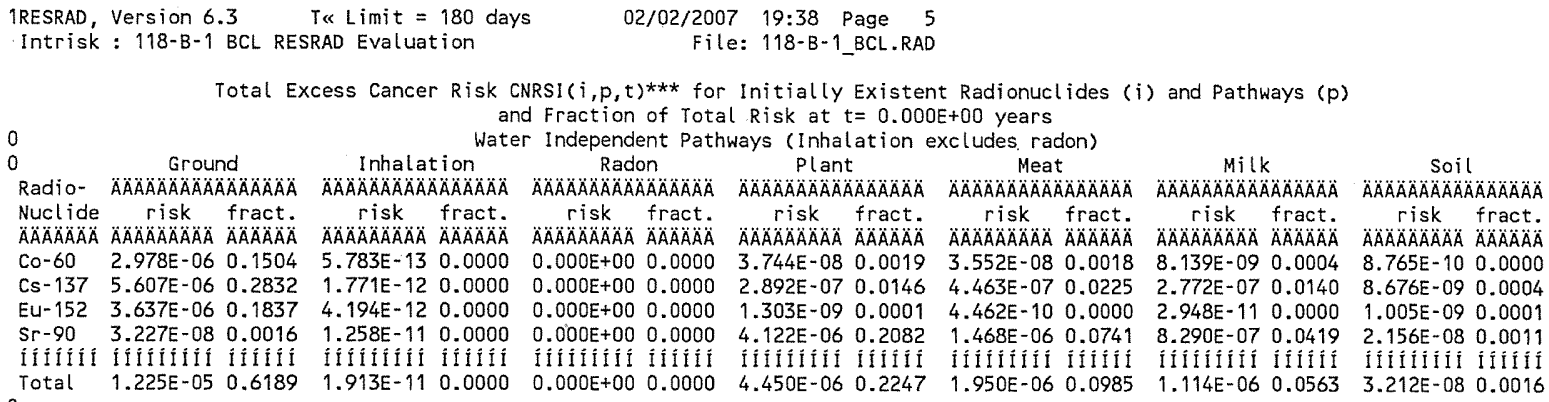

Total Excess Cancer Risk CNRSI $(i, p, t) * * *$ for Initially Existent Radionuclides $(i)$ and Pathways $(p)$ and Fraction of rotal Risk at $t=0.000 E+00$ years

Water Dependent Pathways

\begin{tabular}{|c|c|c|c|c|c|c|c|c|c|c|c|c|c|}
\hline & 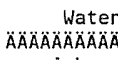 & 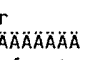 & $\begin{array}{r}\text { Fish } \\
\text { AÄAAAAAAAA }\end{array}$ & $\triangle A \cap A ̈ A ̈ A ̈ A ̈ A ̈$ & $\begin{array}{r}\text { Rado } \\
\triangle A \cap A A A A A B A B A\end{array}$ & on & $\begin{array}{r}P \operatorname{lan} t \\
A \cap A A A B A B A B A\end{array}$ & ${ }_{A}^{t} \triangle A A A O A B A$ & $\begin{array}{r}\text { Meat } \\
\text { ÄÄÄÄÄÄÄÄ }\end{array}$ & $\triangle A \cap A ̈ A ̈ A ̈$ & 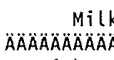 & KäÄÄÄÄÄ & $\begin{array}{c}\text { All pathways } \\
\text { AAAAAAAAAAAAAAAAAAA }\end{array}$ \\
\hline $\begin{array}{l}\text { Jclide } \\
\text { ÄÄÄÄÄA }\end{array}$ & $\begin{aligned} r i s k \\
A \cap A O A O A O A\end{aligned}$ & $\begin{array}{l}\text { fract. } \\
\triangle A B A O A O A\end{array}$ & $\begin{array}{c}\text { risk } \\
\text { AAAAAAAAAOA }\end{array}$ & $\begin{array}{l}\text { fract. } \\
\ddot{A} \ddot{A} A \ddot{A} A \ddot{A} A\end{array}$ & 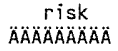 & $\begin{array}{l}\text { fract. } \\
A Q A A O A B A\end{array}$ & $\begin{array}{c}\text { risk } \\
\triangle A \cap A ̈ A B A B A B A\end{array}$ & $\begin{array}{l}\text { fract. } \\
\triangle A B A A A B\end{array}$ & $\begin{array}{l}\text { risk } \\
A B A O A O A B A\end{array}$ & & $\begin{array}{c}\text { risk } \\
\triangle A A B A B A B A O A\end{array}$ & & $\begin{array}{l}\text { risk fract. } \\
\text { AAAAAAA } \triangle A A B A A A\end{array}$ \\
\hline-60 & $0.000 E+00$ & 0.0000 & $0.000 E+00$ & 0.0000 & $0.000 \mathrm{E}+00$ & 0.0000 & $0.000 E+00$ & 0.0000 & $E+00$ & 0.0000 & $0.000 \mathrm{E}+00$ & 0.0 & 060 \\
\hline & 0.0 & 0. & +00 & 0. & & & 0. & & & & & & \\
\hline $1-152$ & $0.000 E+00$ & 0.0000 & $0.000 E+00$ & 0.0000 & 0.0 & 0. & 0. & 0 & 0 & & -00 & 10 & \\
\hline & 0.00 & 0. & $E+00$ & 0.0 & & 0.00 & 0.0 & 0.0 & & & 0 & & \\
\hline & & & & & & & & & & & & & \\
\hline & & 00 & & 300 & & 000 & 0 & 000 & & & & & \\
\hline
\end{tabular}

$* * * \operatorname{CNRSI}(i, p, t)$ includes contribution from decay daughter radionuclides

\begin{tabular}{|c|c|}
\hline \multirow[t]{2}{*}{ Attachment $\frac{7}{7}$} & \multirow{2}{*}{$\begin{array}{l}\text { Sheet No. } 5 \text { of } 21 \\
\text { Date }\end{array}$} \\
\hline & \\
\hline M.W. Perrott & \\
\hline $0100 \mathrm{~B}-\mathrm{CA}-1$ & $\mathrm{ReV}$ \\
\hline
\end{tabular}




\section{ATTACHMENT 7}

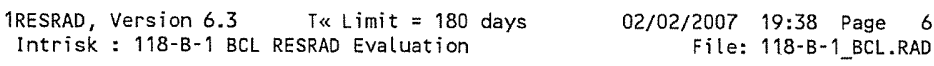

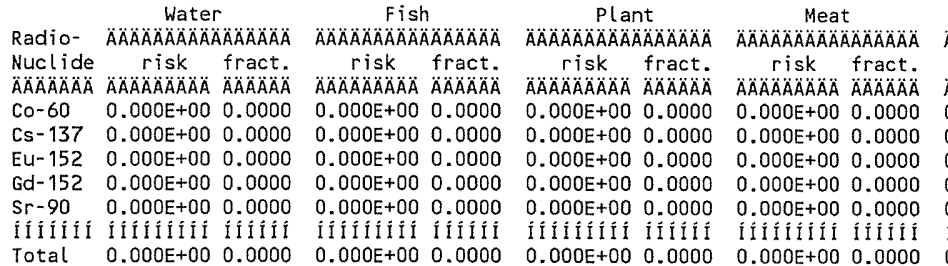

Total $0.000 \mathrm{E}+000.000$

$0.000 \mathrm{E}+000.00000 .000 \mathrm{E}+000.00000 .000 \mathrm{E}+000.0000$

** Sum of water independent ground, inhatation, plant, meat, milk, soil and water dependent water, fish, plant, meat, milk pathways

Attachment Milk All Pathways** risk risk fract. risk fract. ÄÄÄÄÄÄA ÄÄÄÄ ÄÄÄÄÄA ÄÄÄÄÄ $\begin{array}{llllll}0.000 E+00 & 0.0000 & 2.682 E-06 & 0.1417\end{array}$ $\begin{array}{llll}0.000 E+00 & 0.0000 & 6.476 E-06 & 0.3421\end{array}$ $\begin{array}{llll}0.000 E+00 & 0.0000 & 3.456 E-06 & 0.1825\end{array}$ $\begin{array}{llll}0.000 E+00 & 0.0000 & 4.282 E-22 & 0.0000\end{array}$ $0.000 E+00 \quad 0.0000 \quad 6.317 E-06 \quad 0.3337$

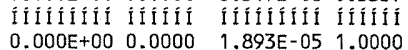

$\begin{array}{ll}\text { Attachment } \frac{7}{1} & \text { Sheet No. } \underline{6} \text { of } \underline{21} \\ \text { Originators: } \frac{\text { S.W. Clark }}{\text { M.W. Perrott }} & \text { Date } \\ \text { Chk'd By } & \text { Date } \\ \text { Calc. No. } & \text { Rev. No. } \underline{0100 \text { B-CA-V0301 }}\end{array}$




\section{ATTACHMENT 7}

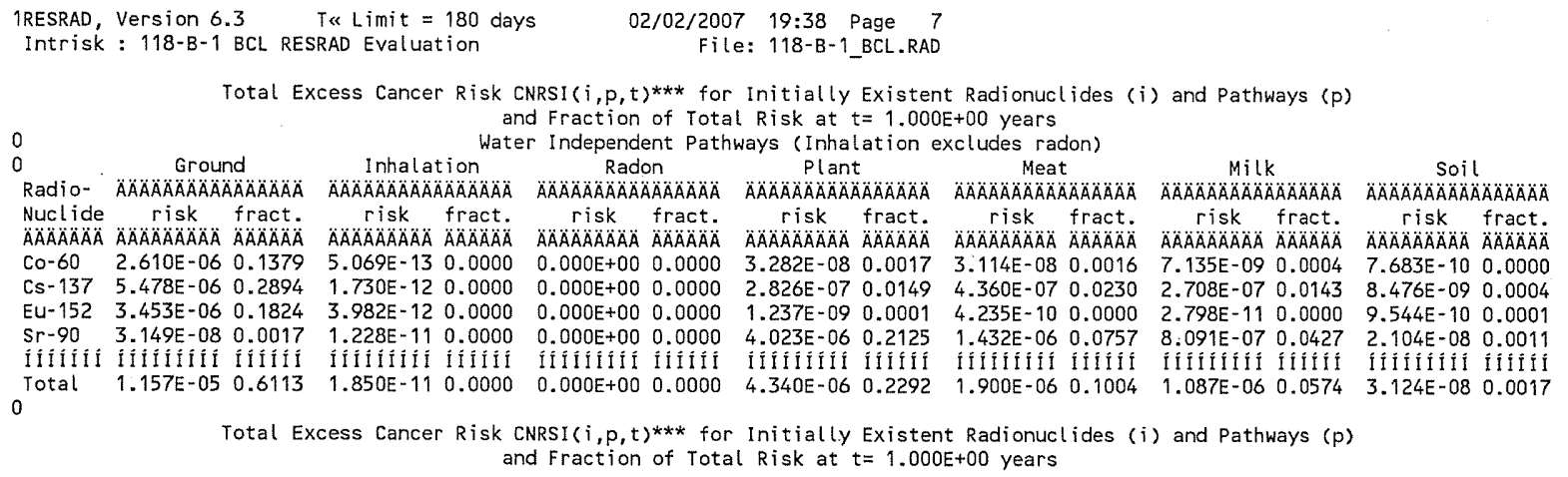

Total Excess Cancer Risk CNRSI $(i, p, t) * * *$ for Initially Existent Radionuclides ( $i$ ) and Pathways ( $p$ ) and Fraction of Total Risk at $t=1.000 E+00$ years

\begin{tabular}{|c|c|c|c|c|c|c|c|c|c|c|c|c|}
\hline \\
\hline & Ground & Inhala & tion & Rado & & $P \operatorname{lan} t$ & & Meat & & Mill & & Soil \\
\hline Radio- & 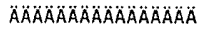 & $\triangle A \triangle A A A A A B A B$ & $\triangle A A A A A O A A B$ & $\triangle A \cap A \triangle A O A \triangle A B A A B$ & $\triangle A \cap A A A O A B$ & $\triangle A \triangle A O A A A A A A A$ & $\ddot{A} \ddot{A} \ddot{A} A \ddot{A} \ddot{A} \ddot{A}$ & $\triangle A \triangle A A A A B A A A A$ & 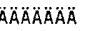 & $\triangle \triangle A \triangle A \cap A ̈ A A A A$ & $\triangle \triangle A \triangle A \triangle A \cap A ̈ A ̈$ & 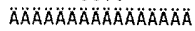 \\
\hline $\begin{array}{l}\text { Nuclide } \\
\text { ÄÄÄAAAAA }\end{array}$ & $\begin{array}{ll}\text { risk } & \text { fract. } \\
\triangle A A B A O A B & A A B A O A A \\
\end{array}$ & $\begin{array}{c}\text { risk } \\
A A B A A A B A\end{array}$ & $\begin{array}{l}\text { fract. } \\
A B A B A B A\end{array}$ & $\begin{array}{c}\text { risk } \\
\text { AAAAAAAAABA }\end{array}$ & $\begin{array}{l}\text { fract. } \\
\triangle A A A A A B A\end{array}$ & $\begin{array}{c}\text { risk } \\
\text { ĀAAAÄÄÄÄÄ }\end{array}$ & $\begin{array}{l}\text { fract. } \\
\ddot{A} \ddot{A} A \ddot{A} \ddot{A} A\end{array}$ & $\begin{array}{c}\text { risk } \\
\triangle A A A O A ̈ A ̈ A O A O A ̈\end{array}$ & 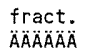 & $\begin{array}{l}\text { risk } \\
\triangle A ̈ A O A O A O A\end{array}$ & 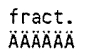 & 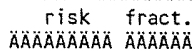 \\
\hline Co- 60 & $2.610 E-06 \quad 0.1379$ & $5.069 E-13$ & 0.0000 & $0.000 E+00$ & 0.0000 & $3.282 E-08$ & 0.0017 & $3.114 E-08$ & 0.0016 & $7.135 \mathrm{E}-09$ & 0.0004 & $\begin{array}{ll}-10 & 0.0000\end{array}$ \\
\hline Cs & $-06 \quad 0.2894$ & 1.7 & 00 & $E+00$ & 0. & & & & 0.0 & 38E-07 & 0.0 & .090 .0004 \\
\hline Eu- & $3.453 E-06 \quad 0.1824$ & $3.982 \mathrm{E}-12$ & 0.0000 & $0.000 E+00$ & 000 & 09 & 0 & 0 & 0 & 2. & & -100 \\
\hline $\mathrm{Sr}^{-}$ & $3.149 \mathrm{E}-08 \quad 0.0017$ & & 0.0000 & $E+00$ & 0.0000 & $E-06$ & 0.2125 & 06 & 0.0 & 8. & 0. & -080.0011 \\
\hline Iif & I ÎI & ifi & IIIIII & LIIIIIIIII & IIIIIII & IIIIIIIIYII & IIIfín & IIIfífilí & ÎIIIIII & 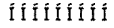 & IIIIII & 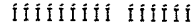 \\
\hline Tot & $1.157 E-05 \quad 0.6113$ & EE-11 & 0.0000 & $0.000 E+00$ & 0.0000 & $4.340 E-06$ & 0.2292 & $1.900 E-06$ & 0.1004 & $1.087 E-06$ & 0.0574 & $3.124 E-08 \quad 0.0017$ \\
\hline
\end{tabular}

Total Excess Cancer Risk CNRSI $(i, p, t)^{* * *}$ for Initially Existent Radionuclides $(i)$ and Pathways $(p)$ and Fraction of Total Risk at $t=1.000 E+00$ years

Water Dependent Pathways

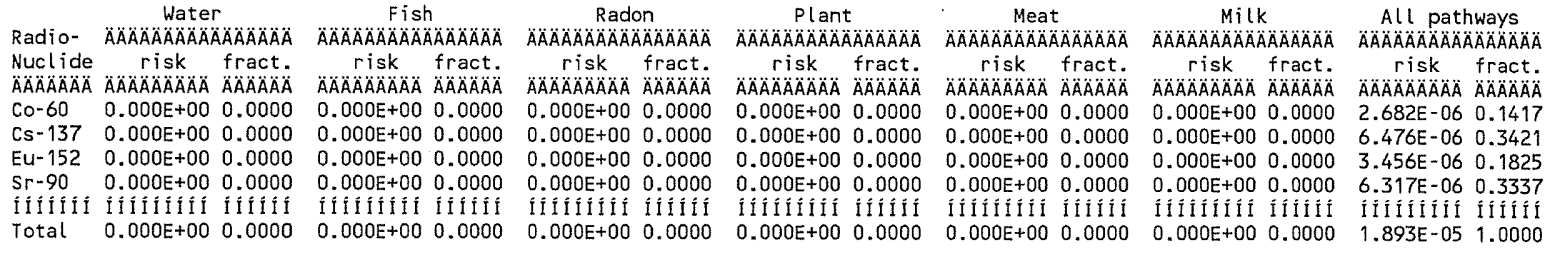

$* * * \operatorname{CNRSI}(i, p, t)$ includes contribution from decay daughter radionuclides

Attachment

$7 \quad$ Sheet No. $\underline{7}$ of $\underline{21}$ Originators: S.W. Clark Date

Chk'd By M.W. Perrott Date

Calc. No. 0100B-CA-V0301

Rev. No, 0 


\title{
ATTACHMENT 7
}

\author{
1RESRAD, Version $6.3 \quad$ T« Limit $=180$ days \\ 02/02/2007 19:38 Page 8 \\ Intrisk : 118-B-1 BCL RESRAD Evaluation \\ File: $118-B-1$ - BCL.RAD
}

Amount of Intake Quantities QINT( $i, p, t)$ for Individual Radionuclides (i) and Pathways $(p)$

As $\mathrm{pCi} / \mathrm{yr}$ at $\mathrm{t}=3.000 \mathrm{E}+00$ years

\begin{tabular}{|c|c|c|c|c|c|c|c|c|c|c|c|}
\hline \multirow{4}{*}{$\begin{array}{l}\text { Radio- } \\
\text { Nuclide } \\
\ddot{A} \ddot{A} A \ddot{A} \ddot{A} \ddot{A} \ddot{A}\end{array}$} & \multicolumn{5}{|c|}{ Water Independent Pathways (Inhalation w/o radon) } & \multicolumn{5}{|c|}{ Water Dependent Pathways } & \multirow{4}{*}{$\begin{array}{c}\text { Total } \\
\text { Ingest jon* } \\
\text { AOAAAAOAAÄÄA }\end{array}$} \\
\hline & $\triangle A Q A A A A A A A A$ & $\triangle A A A A A A A A A B A$ & $\triangle A A A A A A A A A A$ & $A A A A A A A A A A$ & $\triangle A O A A A A A A A A O A A$ & $\triangle A ̈ A$ & $\triangle \triangle A A A A A A A A O$ & $\triangle A A A A A A O A A A B A$ & ÄA AOAAAAAAAAAA & $A A A A A A$ & \\
\hline & Inhalation & Plant & Meat & Milk & Soil & Water & Fish & Plant & Meat & Milk & \\
\hline & 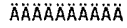 & ÄAOAAAAOAOÄ & A AAAAAAAÄÄ & $\triangle A A A A A A B A B A ̈$ & 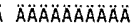 & $\triangle A O A A A A B A A A$ & ÄÄÄAAAAAAOA & $\triangle A O A O A O A A A O A O A$ & 1 ÄÄÄAAAOAOÄ & $\triangle A A A A A A A A A A$ & \\
\hline 0.60 & $1.461 E-03$ & $1.518 E+02$ & $1.440 E+02$ & $3.301 E+01$ & $1.967 E+00$ & $0.000 E+00$ & $0.000 E+00$ & 0 & $E+00$ & $=00$ & ++02 \\
\hline Cs -137 & $6.431 \mathrm{E}-03$ & $3.342 E+02$ & $5.156 E+02$ & $3.203 E+02$ & $8.658 E+00$ & $E+00$ & $0.000 E+00$ & $0.000 E+00$ & $E+00$ & $E+00$ & T. \\
\hline Eu- 152 & 2.5 & $8.438 E+00$ & $2.890 E+00$ & 1.905 & $3.497 E+00$ & 0.0 & $0.000 E+00$ & 0. & +00 & +00 & $=+C$ \\
\hline Gd- 152 & 1.5 & 4.9 & 1.68 & 4.4 & $2.035 E-14$ & +0.0 & $0.000 E+00$ & 0. & +00 & +00 & $=-14$ \\
\hline $\mathrm{Sr}_{-}$ & $4.840 \mathrm{E}-03$ & 1.88 & 6.71 & 3.7 & $6.517 E+00$ & $0.000 E+00$ & $0 E+00$ & 0.0 & +00 & $+\infty 0$ & $=+03$ \\
\hline Iíîlíl & & IIII & & & & & $f=$ & & & & \\
\hline
\end{tabular}

Excess Cancer Risks CNRS $(i, p, t)$ for Individual Radionuclides ( $i$ ) and Pathways ( $p$ ) and Fraction of Total Risk at $t=3.000 E+00$ years

0 Water Independent Pathways (Inhalation excludes radon)
Inhalation Plant Meat
Milk
Soil

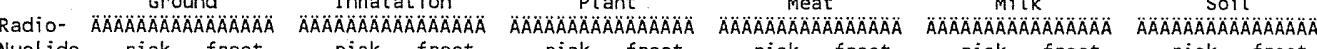
Nuclide risk fract. risk fract. risk fract. risk fract. risk fract. risk fract.

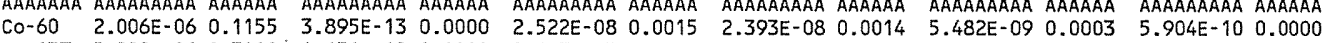
$\begin{array}{lllllllllllll}C s-137 & 5.228 E-06 & 0.3009 & 1.651 E-12 & 0.0000 & 2.697 E-07 & 0.0155 & 4.161 E-07 & 0.0240 & 2.585 E-07 & 0.0149 & 8.090 E-09 & 0.0005\end{array}$

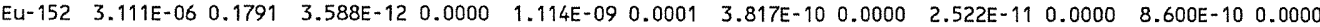

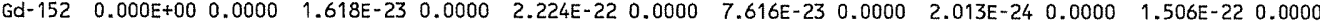
$\begin{array}{lllllllllllll}\mathrm{Sr}-90 & 3.000 \mathrm{E}-08 & 0.0017 & 1.170 \mathrm{E}-11 & 0.0000 & 3.833 \mathrm{E}-06 & 0.2206 & 1.365 \mathrm{E}-06 & 0.0785 & 7.709 \mathrm{E}-07 & 0.0444 & 2.005 \mathrm{E}-08 & 0.0012\end{array}$

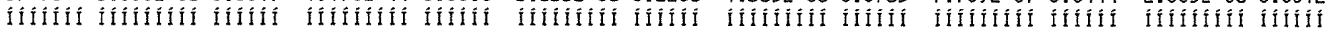

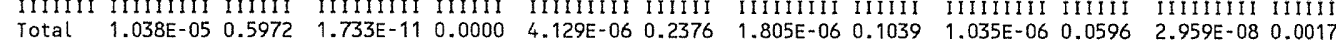

Excess Cancer Risks CNRS $(i, p, t)$ for Individual Radionuclides (i) and Pathways $(p)$ and Fraction of Total Risk at $t=3.000 E+00$ years

Water Dependent Pathways

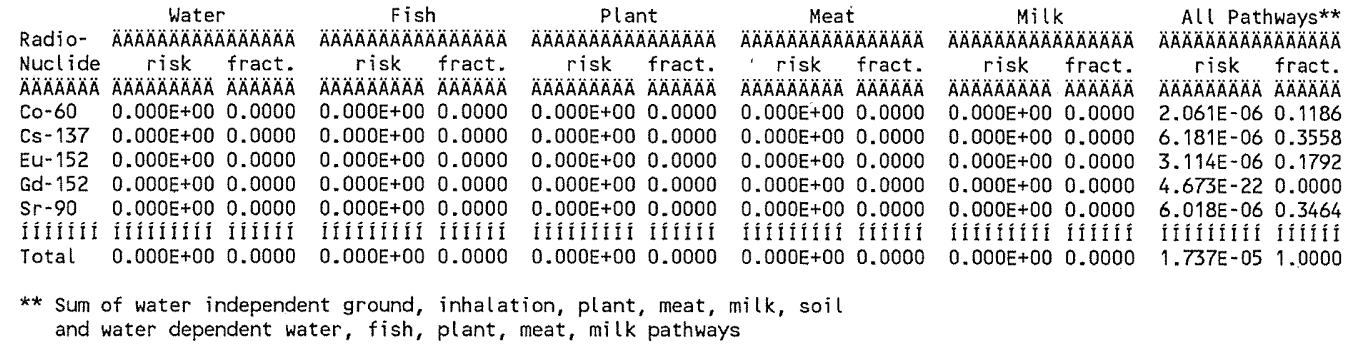

Attachment

Originators: S.W. Clark

7 Sheet No. $\underline{8}$ of $\underline{21}$

Chk'd By M.W. Perrott Date

Calc. No. 0100B-CA-V0301 Rev. No. 0 


\section{ATTACHMENT 7}

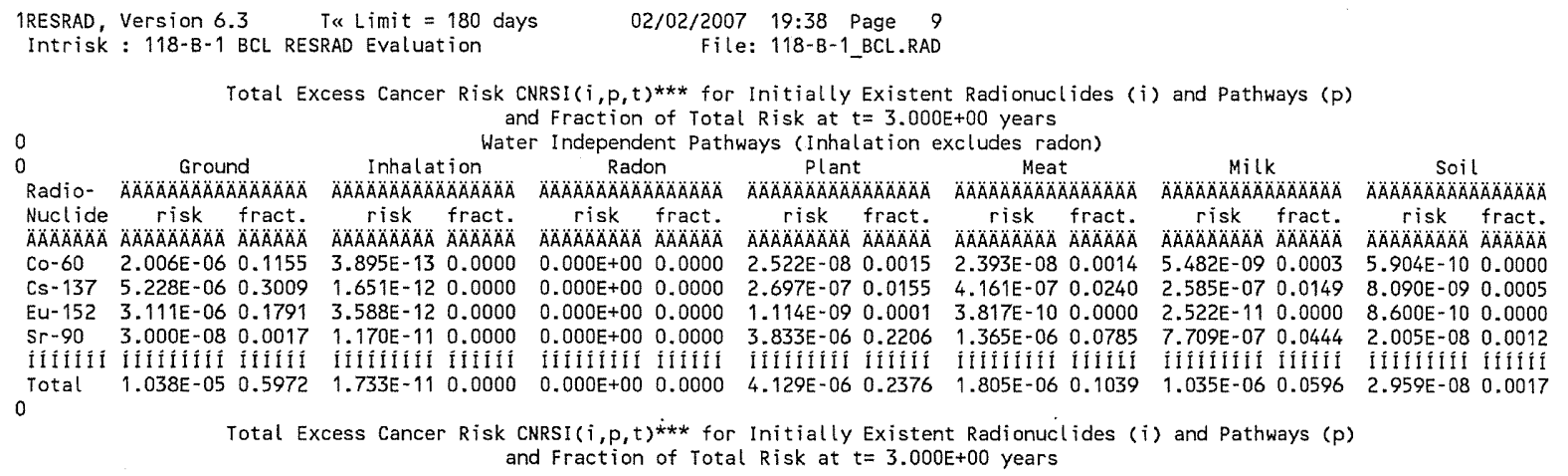

Total Excess Cancer Risk CNRSI $(i, p, t)^{* * *}$ for Initially Existent Radionuclides $(i)$ and Pathways $(p)$ and Fraction of Total Risk at $t=3.000 E+00$ years

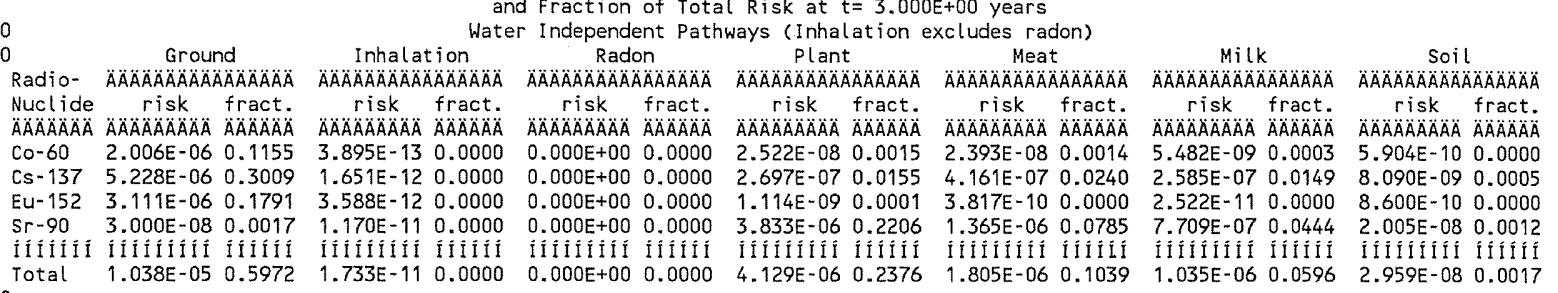

Total Excess Cancer Risk CNRSI $(i, p, t)^{* * * *}$ for Initially Existent Radionuclides ( $i$ ) and Pathways $(p)$ and Fraction of Total Risk at $t=3.000 E+00$ years

Water Dependent Pathways

\begin{tabular}{|c|c|c|c|c|c|c|c|c|c|c|c|c|c|}
\hline & $\begin{array}{r}\text { Wate } \\
\text { ÄAAAAAAAOÄ }\end{array}$ & 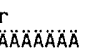 & $\begin{array}{r}F i s \\
\triangle \triangle A \cap A \cap A ̈ A \cap A ̈ A ̈\end{array}$ & ÄÄÄÄÄA & $\begin{array}{r}R a d \\
\text { AAÄAAAAÄÄ }\end{array}$ & On & $\begin{array}{r}\text { Plan } \\
\text { AAAAAAAAAA }\end{array}$ & t & $\begin{array}{r}\text { Mea } \\
\text { AAAAAAAAAÄ }\end{array}$ & 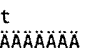 & $\begin{array}{r}M i l \\
\text { AAAAOAAAAÄL }\end{array}$ & 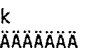 & $\begin{array}{c}\text { All pathways } \\
\text { AAAAAAAAAAAAAAAAAA }\end{array}$ \\
\hline $\begin{array}{l}\text { Nuclide } \\
\text { AAAAAAA }\end{array}$ & $\begin{array}{c}\text { risk } \\
\text { ÄAÄAAAA }\end{array}$ & fract. & 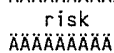 & $\begin{array}{l}\text { fract. } \\
\text { ÄÄÄÄÄA }\end{array}$ & $\begin{array}{c}\text { risk } \\
\text { AAAÄAOAOAOÄ }\end{array}$ & $\begin{array}{l}\text { fract. } \\
\ddot{A} \ddot{A} \ddot{A} \ddot{A} \ddot{A} A\end{array}$ & $\begin{array}{c}\text { risk } \\
\triangle A \cap A B A A A B A\end{array}$ & $\begin{array}{l}\text { fract. } \\
\triangle A O A O A B A ̈ A\end{array}$ & $\begin{array}{c}r i s k \\
A B A B A B A B A\end{array}$ & $\begin{array}{l}\text { fract. } \\
\triangle A A A A A A A B\end{array}$ & $\begin{array}{c}r i s k \\
\triangle A A A O A B A O A O A\end{array}$ & $\begin{array}{l}\text { fract. } \\
\triangle A \cap A ̈ A ̈ A ̈ A\end{array}$ & 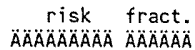 \\
\hline Co- 60 & $0.000 E+00$ & 0.0000 & $0.000 E+00$ & 0.0000 & $0.000 E+00$ & 0.0000 & $0.000 E+00$ & 0.0000 & $0.000 E+00$ & 0.0000 & $0.000 E+00$ & 0.0000 & $2.061 \mathrm{E}-06 \quad 0.1186$ \\
\hline Cs -137 & $0.000 E+00$ & 0.0000 & $0.000 E+00$ & 0.0000 & $0.000 E+00$ & 0.0000 & $0.000 E+00$ & 0.0000 & $0.000 E+00$ & 0.0000 & $0.000 E+00$ & 0.0000 & $6.181 E-06 \quad 0.3558$ \\
\hline Eu-152 & $0.000 E+00$ & 0.0000 & $0.000 E+00$ & 0.0000 & $0.000 E+00$ & 0.0000 & $0.000 E+00$ & 0.0000 & $0.000 E+00$ & 0.0000 & $0.000 E+00$ & 0.0000 & $3.114 \mathrm{E}-06 \quad 0.1792$ \\
\hline $\mathrm{Sr}-90$ & $0.000 E+00$ & 0.0000 & $0.000 E+00$ & 0.0000 & $0.000 E+00$ & 0.0000 & $0.000 E+00$ & 0.0000 & $0.000 E+00$ & 0.0000 & $0.000 E+00$ & 0.0000 & 0.3464 \\
\hline Ifififi & 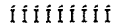 & Iifíníf & 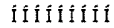 & filfíli & IIfIIII & IIfííl & IIIfIIII & IIIfÍ & IIIIIIII & IIIIII & IIIIIIIII & IIIIII & 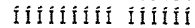 \\
\hline Total & $0.000 E+00$ & 0.0000 & $0.000 E+00$ & 0.0000 & $0.000 E+00$ & 0.0000 & $0.000 \mathrm{E}+00$ & 0.0000 & $0.000 E+00$ & 0.0000 & $0.000 E+00$ & 0.0000 & $1.737 E-05 \quad 1.0000$ \\
\hline
\end{tabular}

$* * * \operatorname{CNRSI}(i, p, t)$ includes contribution from decay daughter radionuclides

Attachment Sheet No. 9 of 21 Originators: $S$. W. Clark Date

Chk'd By M.W. Perrott Date

Calc. No. 0100B-CA-V0301 Rev. No. 0 


\section{ATTACHMENT 7}

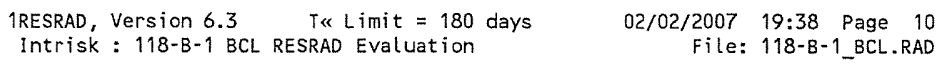

Water Dependent Pathways

\begin{tabular}{|c|c|c|c|c|c|c|}
\hline Radio- & $\begin{array}{c}\text { Water } \\
\text { AAAAAAAAAAAAAAAAA }\end{array}$ & 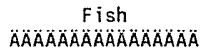 & $\begin{array}{c}\text { Plant } \\
\text { ÄAAAAAAAAAAAAAOA }\end{array}$ & $\begin{array}{c}\text { Meat } \\
\text { ÄÄAAAAAAAAOAOÄÄ }\end{array}$ & $\begin{array}{c}\text { Milk } \\
\text { ÄÄÄAAAAAAAOAOAOA }\end{array}$ & $\begin{array}{r}\text { All Pathways** } \\
\text { AAAAAAAAAAAAAAAAA }\end{array}$ \\
\hline $\begin{array}{l}\text { Nuclide } \\
\triangle A B A A A O A O A B\end{array}$ & 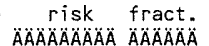 & 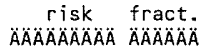 & $\begin{array}{r}\text { risk fract. } \\
\triangle A A A A B A O A A \text { AOAAAOAB }\end{array}$ & 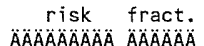 & 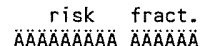 & $\begin{aligned} \text { risk fract. } & \text { f } \\
\triangle A A A A B A B A & \end{aligned}$ \\
\hline Co-60 & $0.000 E+00 \quad 0.0000$ & $0.000 E+00 \quad 0.0000$ & $0.000 E+00 \quad 0.0000$ & $\begin{array}{lll}0.000 E+00 & 0.0000\end{array}$ & $0.000 E+00 \quad 0.0000$ & $8.197 E-070.0616$ \\
\hline Cs -137 & $0.000 E+00 \quad 0.0000$ & $0.000 E+00 \quad 0.0000$ & $0.000 E+00 \quad 0.0000$ & $0.000 E+00 \quad 0.0000$ & $0.000 E+00 \quad 0.0000$ & $5.250 \mathrm{E}-06 \quad 0.3944$ \\
\hline Eu-152 & $0.000 E+00 \quad 0.0000$ & $0.000 \mathrm{E}+00 \quad 0.0000$ & $0.000 E+00 \quad 0.0000$ & $0.000 \mathrm{E}+00 \quad 0.0000$ & $0.000 E+00 \quad 0.0000$ & $2.163 \mathrm{E}-06 \quad 0.1625$ \\
\hline Gd-152 & $0.000 E+00 \quad 0.0000$ & $0.000 E+00 \quad 0.0000$ & $0.000 E+00 \quad 0.0000$ & $0.000 E+00 \quad 0.0000$ & $0.000 E+00 \quad 0.0000$ & $5.762 E-22 \quad 0.0000$ \\
\hline$s r-90$ & $0.000 E+00 \quad 0.0000$ & $0.000 E+00 \quad 0.0000$ & $0.000 E+00 \quad 0.0000$ & $0.000 E+00 \quad 0.0000$ & $0.000 E+00 \quad 0.0000$ & $5.079 E-06 \quad 0.3816$ \\
\hline IIIIIII & IIIIIIIII IIIII & 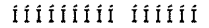 & 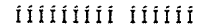 & 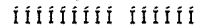 & IIIIIIIIII IIIIII & 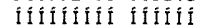 \\
\hline Total & $0.000 E+00 \quad 0.0000$ & $0.000 E+00 \quad 0.0000$ & $0.000 E+00 \quad 0.0000$ & $0.000 E+00 \quad 0.0000$ & $0.000 E+00 \quad 0.0000$ & $1.331 E-05 \quad 1.0000$ \\
\hline
\end{tabular}

and water dependent water, fish, plant, meat, milk pathways

\begin{tabular}{|c|c|}
\hline Attachment & Sheet No \\
\hline Originators: S.W. Clark & Date \\
\hline M. W. Perrott & Date \\
\hline $0100 B-C A-V 0301$ & Rev. No. \\
\hline
\end{tabular}




\section{ATTACHMENT 7}

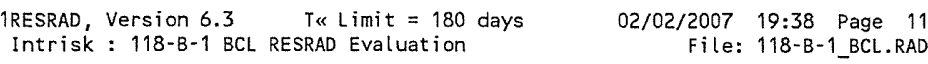

Total Excess Cancer Risk CNRSI $(i, p, t)^{* * *}$ for Initially Existent Radionuclides $(i)$ and Pathways $(p)$

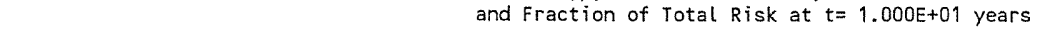

Total Excess Cancer Risk CNRSI $(i, p, t) * * *$ for Initially Existent Radionuclides ( $i$ ) and Pathways $(p)$ and Fraction of Total Risk at $t=1.000 E+01$ years

Water Dependent Pathways

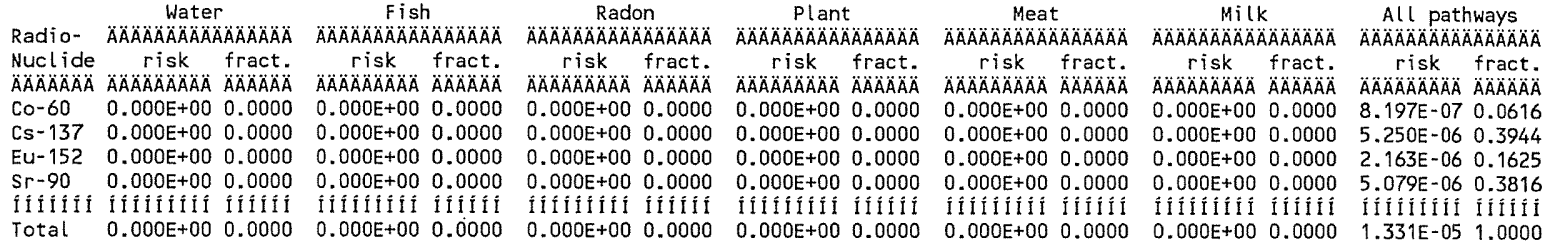

***CNRSI $(i, p, t)$ includes contribution from decay daughter radionuclides

$\begin{array}{ll}\text { Attachment } \frac{7}{\text { S.W. Clark }} & \text { Sheet No. } 11 \text { of } 21 \\ \text { Originators: } & \text { Date } \\ \text { Chk'd By } & \text { Date } \\ \text { Calc. No. W. Perrott } & \text { Rev. No. } 0\end{array}$


CVP-2007-00006

Rev. 0

\section{ATTACHMENT 7}

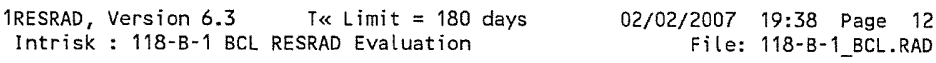

Excess Cancer Risks CNRS( $i, p, t)$ for Individual Radionuclides ( $i$ ) and Pathways $(p)$ and Fraction of Totat Risk at $\mathrm{t}=1.100 \mathrm{E}+01$ years

Water Dependent Pathways

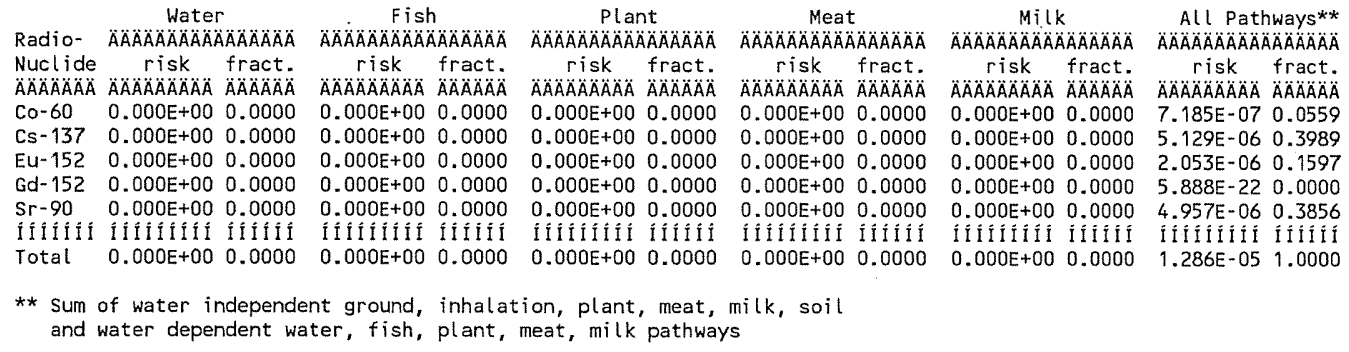

and water dependent water, fish, plant, meat, milk pathways

\begin{tabular}{|c|c|c|}
\hline \multirow{2}{*}{\multicolumn{3}{|c|}{$\begin{array}{cc}\text { Attachment } & 7 \\
\text { Originators: S.W. Clark } & \text { Sheet No. } 12 \text { of } 21 \\
\text { Date }\end{array}$}} \\
\hline & & \\
\hline Chk & M. W. Perrott & Date \\
\hline & 0100B-CA-V0301 & 0 \\
\hline
\end{tabular}




\section{ATTACHMENT 7}

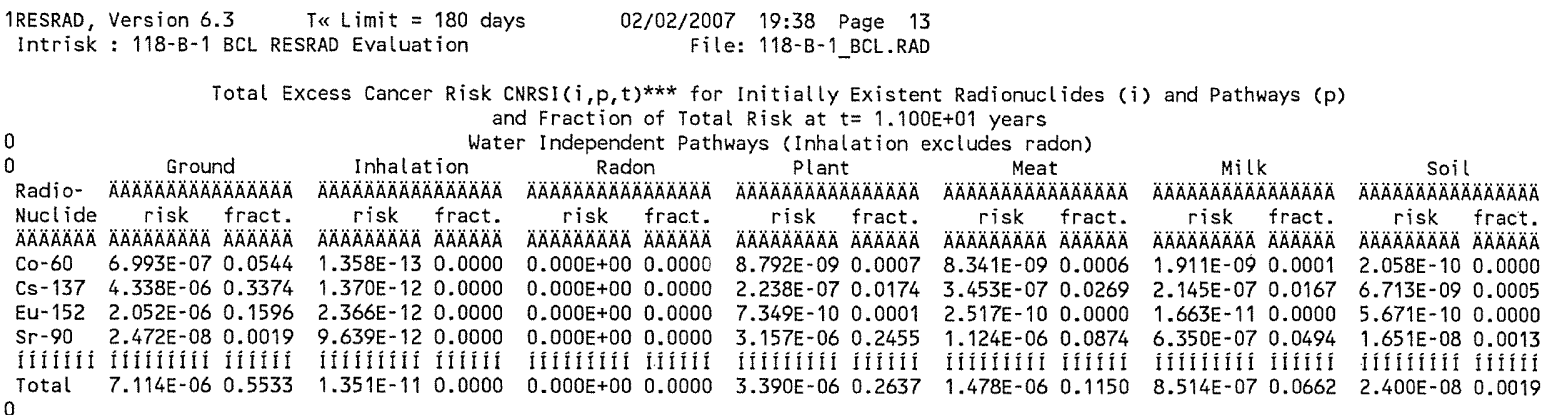

Total Excess Cancer Risk CNRSI $(i, p, t)^{* * *}$ for Initially Existent Radionuclides $(i)$ and Pathways $(p)$

and Fraction of Total Risk at $t=1.100 E+01$ years

0 Water Independent Pathways (Inhalation excludes radon)

0 Ground Inhalation Radon Plant Po Milk

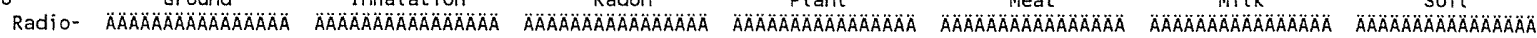
Nuclide risk fract. risk fract. risk fract. risk fract. risk fract. risk fract. risk fract.

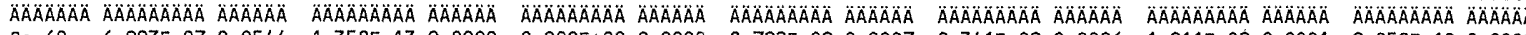
$\begin{array}{lllllllllllllll}\text { Co-60 } & 6.993 \mathrm{E}-07 & 0.0544 & 1.358 \mathrm{E}-13 & 0.0000 & 0.000 \mathrm{E}+00 & 0.0000 & 8.792 \mathrm{E}-09 & 0.0007 & 8.341 \mathrm{E}-09 & 0.0006 & 1.911 \mathrm{E}-09 & 0.0001 & 2.058 \mathrm{E}-10 & 0.0000\end{array}$

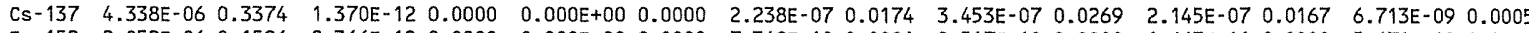

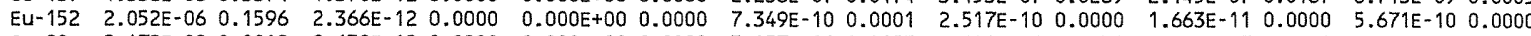

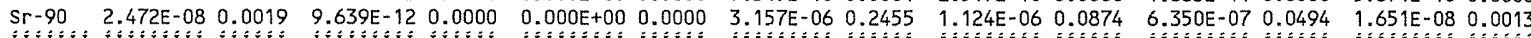

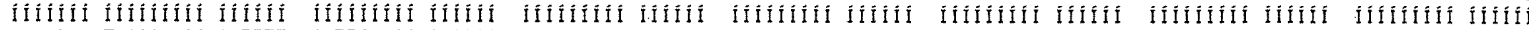
0

Total Excess Cancer Risk CNRSI $(i, p, t) * * *$ for Initially Existent Radionuclides ( $i)$ and Pathways $(p)$ and Fraction of Total Risk at $t=1.100 E+01$ years

Water Dependent Pathways

\begin{tabular}{|c|c|c|c|c|c|c|c|c|c|c|c|c|c|}
\hline \multirow{2}{*}{$\begin{array}{l}\text { Radio- } \\
\text { Nuclide } \\
\ddot{A B A M A A A ̈ A ̈}\end{array}$} & $\begin{array}{c}\text { Water } \\
\text { ÄÄÄÄÄÄÄÄÄÄÄÄ }\end{array}$ & \multicolumn{2}{|c|}{ 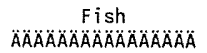 } & \multicolumn{2}{|c|}{$\begin{array}{c}\text { Radon } \\
\text { ÄÄÄÄÄÄÄÄÄÄÄÄ }\end{array}$} & \multicolumn{2}{|c|}{$\begin{array}{c}\text { Plant } \\
\text { AAAAAAAAAAAAAAA }\end{array}$} & \multicolumn{2}{|c|}{$\begin{array}{c}\text { Meat } \\
\text { ÄAAAAAAAAAAAAAÄ }\end{array}$} & \multicolumn{2}{|c|}{$\begin{array}{c}\text { Milk } \\
\text { AAAAAAAAAAAAAAAAAAA }\end{array}$} & \multicolumn{2}{|c|}{$\begin{array}{c}\text { All pathways } \\
\text { AAAAAAAAAAAAAAAAAA }\end{array}$} \\
\hline & 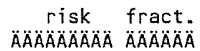 & $\begin{array}{c}r i s k \\
\triangle A A A O A B A O A A\end{array}$ & $\begin{array}{l}\text { fract. } \\
\dddot{A} \ddot{A} \ddot{A} A \ddot{A} \bar{A}\end{array}$ & $\begin{array}{c}r i s k \\
\triangle A A A A B A O A A A B A\end{array}$ & $\begin{array}{l}\text { fract. } \\
\because A A A A A B A\end{array}$ & $\begin{array}{c}r i s k \\
\triangle A B A O A B A B A A A\end{array}$ & 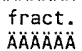 & $\begin{array}{c}r i s k \\
A A A A B A A A A B\end{array}$ & $\begin{array}{l}\text { fract. } \\
\because A O A B A ̈ A ̈ A\end{array}$ & $\begin{array}{c}\text { risk } \\
\triangle A \cap A B A B A B A ̈\end{array}$ & $\begin{array}{l}\text { fract. } \\
\text { AAAAAAB }\end{array}$ & $\begin{array}{c}\text { risk } \\
\text { AAAAAAAAAA }\end{array}$ & $\begin{array}{l}\text { fract. } \\
\text { ÄÄAAÄ }\end{array}$ \\
\hline Co- 60 & $0.000 E+00 \quad 0.0000$ & $0.000 \mathrm{E}+00$ & 0.0000 & $0.000 E+00$ & 0.0000 & $0.000 E+00$ & 0.0000 & $0.000 E+00$ & 0.0000 & $0.000 \mathrm{E}+00$ & 0.0000 & & AARA \\
\hline & $0.000 E+000$. & $.000 E+00$ & 0.0 & 0.0 & & 0.0 & & $+\infty$ & & .00 & & & \\
\hline 152 & $0.000 E+00 \quad 0.0000$ & $0.000 \mathrm{E}+00$ & 0.0000 & $0.000 E+00$ & 0.0000 & $0.000 \mathrm{E}+00$ & 0 & $0.000 E+00$ & 0 & $E+\infty$ & bo & & \\
\hline $\mathrm{Sr}-90$ & $0.000 E+00 \quad 0.0000$ & $0.000 E+00$ & 0.0000 & $0.000 E+00$ & 0.0000 & $0.000 \mathrm{E}+00$ & 0.0000 & $0.000 E+00$ & 0 & $O E+00$ & 000 & & \\
\hline$\hat{I} \hat{I} \mathbf{I}$ & IIII & & & & & & & & & & & & \\
\hline Total & $0.000 E+00 \quad 0.0000$ & $D O E+00$ & 0.0000 & $0.000 E+00$ & 0.0000 & $0.000 E+00$ & 0.0000 & 0. & 0.0000 & $0.000 E+00$ & 0.0000 & $6 \mathrm{E}-05$ & \\
\hline
\end{tabular}

***CNRSI $(i, p, t)$ includes contribution from decay daughter radionuclides

$\begin{array}{ll}\text { Attachment } \frac{7}{\text { S.W. Clark }} & \text { Sheet No. } 13 \text { of } 21 \\ \text { Originators: } & \text { Date } \\ \text { Chk'd By } & \text { Date } \\ \text { Calc. No. N. Perrott } & \text { Rev. No. } 0\end{array}$


CVP-2007-00006

Rev. 0

\section{ATTACHMENT 7}

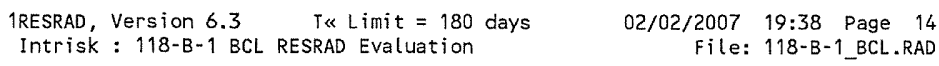

Water

Fish

Plant

Meat

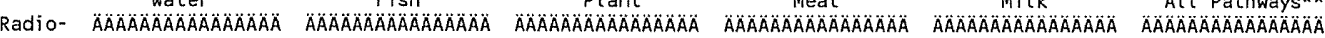
Nuclide risk fract. risk fract. risk fract. risk fract. risk fract. risk fract.

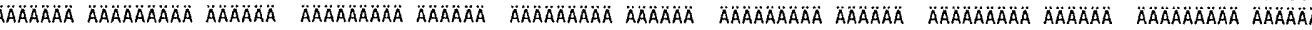
$\begin{array}{llllllllllllll}\mathrm{C} 0-60 & 0.000 \mathrm{E}+00 & 0.0000 & 0.000 \mathrm{E}+00 & 0.0000 & 0.000 \mathrm{E}+00 & 0.0000 & 0.000 \mathrm{E}+00 & 0.0000 & 0.000 \mathrm{E}+00 & 0.0000 & 5.882 \mathrm{E}-08 & 0.0081\end{array}$ $\begin{array}{lllllllllllll}\text { Cs }-137 & 0.000 E+00 & 0.0000 & 0.000 E+00 & 0.0000 & 0.000 E+00 & 0.0000 & 0.000 E+00 & 0.0000 & 0.000 E+00 & 0.0000 & 3.293 E-06 & 0.4546\end{array}$ $\begin{array}{lllllllllllll}\text { EU-152 } & 0.000 E+00 & 0.0000 & 0.000 E+00 & 0.0000 & 0.000 E+00 & 0.0000 & 0.000 E+00 & 0.0000 & 0.000 E+00 & 0.0000 & 7.637 E-07 & 0.1054\end{array}$ $\begin{array}{llllllllllllll}G d-152 & 0.000 E+00 & 0.0000 & 0.000 E+00 & 0.0000 & 0.000 E+00 & 0.0000 & 0.000 E+00 & 0.0000 & 0.000 E+00 & 0.0000 & 7.364 E-22 & 0.0000\end{array}$

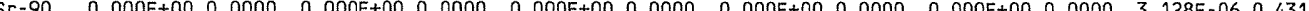

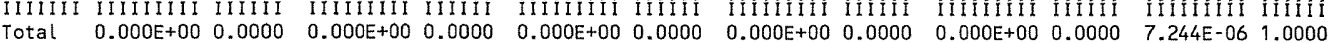

** Sum of water independent ground, inhalation, plant, meat, milk, soil and water dependent water, fish, plant, meat, milk pathways

\begin{tabular}{|c|c|}
\hline Attachment & Sheet No. 14 of 21 \\
\hline Originators: S. W. Clark & Date \\
\hline M. W. Perrott & Date \\
\hline Calc. No. $\quad$ 0100B-CA-V0301 & Rev. No. \\
\hline
\end{tabular}




\section{ATTACHMENT 7}

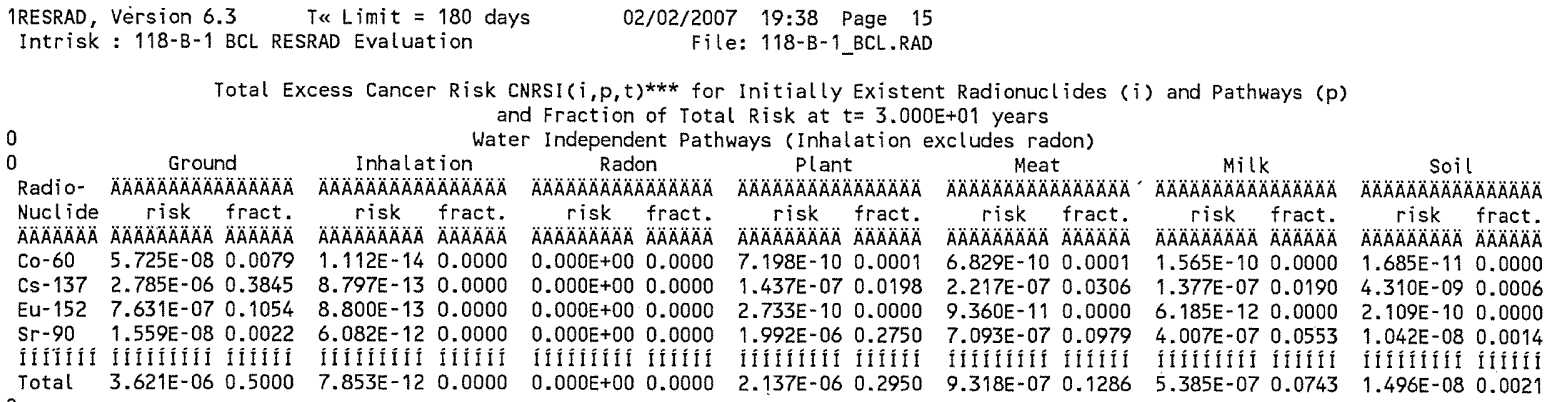

Total Excess Cancer Risk CNRSI $(i, p, t) * * *$ for Initially Existent Radionuclides ( $i$ ) and Pathways ( $p$ ) and Fraction of Total Risk at $t=3.000 E+01$ years

Water Dependent Pathways

\begin{tabular}{|c|c|c|c|c|c|c|c|c|c|c|c|c|c|}
\hline & $\begin{array}{l}\text { Water } \\
\triangle A \cap A B A B A\end{array}$ & $\stackrel{r}{r} \dot{A}$ & $\begin{array}{l}\text { Fish } \\
A B A B A\end{array}$ & & $\begin{array}{l}\text { Rado } \\
\qquad A B A A B\end{array}$ & & 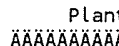 & & $\begin{array}{l}\text { Meat } \\
\text { Mea }\end{array}$ & & Milk & & $\begin{array}{l}\text { All pathways } \\
\text { ALAAAAAAAAABA }\end{array}$ \\
\hline & $r$ isk & & $A A R A$ & $A A$ & GAAAR & & AAAAA & AAA & AAAAA & & AAAA & & \\
\hline & $\triangle A B A A B A$ & & $\ddot{A} \ddot{A}$ & $\ddot{\theta}$ & & & $A \cap A ̈$ & & $\ddot{A A A ̈}$ & & & & \\
\hline & $0.000 E+00$ & 0. & +00 & 0.00 & 0. & 10 & 00 & 0 & .00 & 0 & +00 & & 08 \\
\hline & 0.0 & 0. & +00 & 0. & 0 & 0. & 0 & 0 & +00 & 0 & -00 & & 36 \\
\hline & 0.0 & & & & & & & & 0 & & -00 & & 07 \\
\hline & 0.0 & 0. & 0.0 & 0. & 0.0 & 10 & & & & & & & \\
\hline İIYîî & & & & & & Ififí & & & & & 111 & & 11 \\
\hline ot & $0.000 E+00$ & 0.0000 & $0.000 E+00$ & 0.0000 & $0.000 E+00$ & 0000 & $0.000 E+00$ & 000 & $0.000 E+00$ & 0000 & $0.000 E+00$ & .0000 & 06 \\
\hline
\end{tabular}

***CNRSI $(i, p, t)$ includes contribution from decay daughter radionuclides

\begin{tabular}{|c|c|}
\hline Attachment & Sheet No. 15 of 21 \\
\hline Originators: S.W. Clark & Date \\
\hline Chk'd By M.W. Perrott & Date \\
\hline $0100 \mathrm{~B}-\mathrm{CA}-\mathrm{V} 0301$ & Rev. No. 0 \\
\hline
\end{tabular}




\section{ATTACHMENT 7}

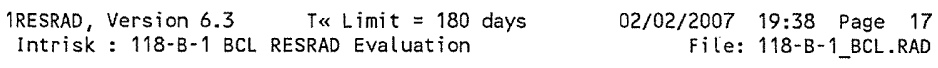

Total Excess Cancer Risk CNRSI $(i, p, t) * * *$ for Initially Existent Radionuclides $(i)$ and Pathways $(p)$ and Fraction of Total Risk at $t=1.000 E+02$ years Water Independent Pathways (Inhalation excludes radon)

0 Ground Inhalation Plant An

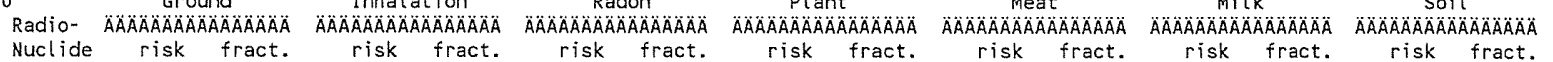

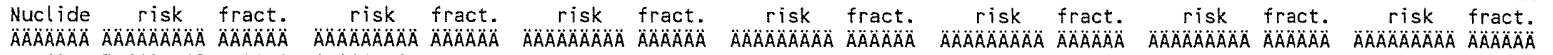

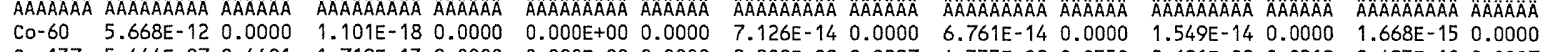

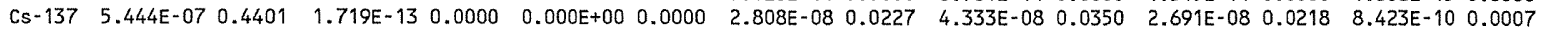

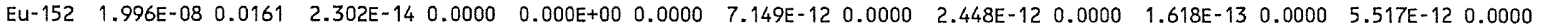
$\begin{array}{llllllllllllllllllllll}\mathrm{Sr}-90 & 2.859 \mathrm{E}-09 & 0.0023 & 1.115 \mathrm{E}-12 & 0.0000 & 0.000 \mathrm{E}+00 & 0.0000 & 3.652 \mathrm{E}-07 & 0.2952 & 1.300 \mathrm{E}-07 & 0.1051 & 7.345 \mathrm{E}-08 & 0.0594 & 1.910 \mathrm{E}-09 & 0.0015\end{array}$

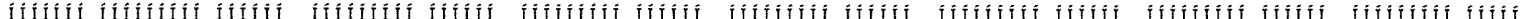

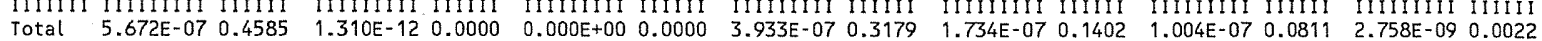

Total Excess Cancer Risk CNRSI $(i, p, t) * * *$ for Initially Existent Radionuclides (i) and Pathways (p) and Fraction of Total Risk at $t=1.000 E+02$ years

Water Dependent Pathways

\begin{tabular}{|c|c|c|c|c|c|c|c|c|c|c|c|c|c|}
\hline Radio- & $\begin{array}{r}\text { Water } \\
\text { AMAAAAAAAAA }\end{array}$ & $\triangle A \cap A O A O A B A B$ & $\begin{array}{r}F i s h \\
\triangle A O A A A B A B A B\end{array}$ & AÄÄĀĀÄÄ & $\begin{array}{r}\text { Rado } \\
\text { ÄÄAAAÄÄÄÁ }\end{array}$ & $\triangle A \triangle A \triangle A O A O A$ & $\begin{array}{r}P L a n \\
\triangle A A A A A A A A B A\end{array}$ & A & $\begin{array}{r}\text { Meat } \\
\text { ÄÄÄÄÄÄÄA }\end{array}$ & AAAAAAA & $\begin{array}{r}M i l \\
\text { AAÄÄÄÄÄÄA }\end{array}$ & $\triangle A \triangle A \cap A \cap A$ & $\begin{array}{c}\text { All pathways } \\
\text { AAAAAAAAAAAAAAAAA }\end{array}$ \\
\hline $\begin{array}{l}\text { Nuclide } \\
\triangle A A A B A A A B\end{array}$ & $\begin{array}{l}\text { risk } \\
\text { ANAAAAAB }\end{array}$ & $\begin{array}{l}\text { fract. } \\
A B A A B A\end{array}$ & $\begin{array}{c}\text { risk } \\
\triangle A A B A A A B A\end{array}$ & 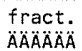 & $\begin{array}{c}r i s k \\
\triangle A B A B A B A O A B\end{array}$ & $\begin{array}{l}\text { fract. } \\
\text { ÄÄÄÄÄ }\end{array}$ & $\begin{array}{c}r i s k \\
\triangle A B A B A B A A B\end{array}$ & $\begin{array}{l}\text { fract. } \\
\triangle A A A A A B A\end{array}$ & $\begin{array}{c}\text { risk } \\
\text { AिAÄAAAOAOÄA }\end{array}$ & $\begin{array}{l}\text { fract. } \\
\text { AAAAAAA }\end{array}$ & $\begin{array}{c}\text { risk } \\
\triangle A A A A B A A B A A\end{array}$ & 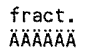 & 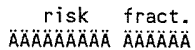 \\
\hline Co- 60 & $0.000 E+00$ & 0.0000 & $0.000 E+00$ & 0.0000 & $0.000 E+00$ & 0.0000 & $0.000 E+00$ & 0.0000 & $0.000 \mathrm{E}+00$ & 0.0000 & $0.000 \mathrm{E}+00$ & 0.0000 & $5.824 \mathrm{E}-120.0000$ \\
\hline $\mathrm{Cs}-137$ & $0.000 E+00$ & 0.0000 & $0.000 E+00$ & 0.0000 & $0.000 E+00$ & 0.0000 & $0.000 E+00$ & 0.0000 & $0.000 E+00$ & 0.0000 & $0.000 E+00$ & 0.0000 & $6.436 E-07 \quad 0.5203$ \\
\hline Eu-152 & $0.000 E \div 00$ & 0.0000 & $0.000 E+00$ & 0.0000 & $0.000 E+00$ & 0.0000 & $0.000 E+00$ & 0.0000 & $0.000 E+00$ & 0.0000 & $0.000 E+00$ & 0.0000 & $08 \quad 0.0161$ \\
\hline $\mathrm{sr}-90$ & $0.000 E+00$ & 0.0000 & $0.000 E+00$ & 0.0000 & $0.000 E+00$ & 0.0000 & $0.000 E+00$ & 0.0000 & $0.000 E+00$ & 0.0000 & $0.000 E+00$ & 0.0000 & 070.4636 \\
\hline IIIfíîl & Î́ílítít & IIIIIII & IItítiliti & I I I I I I I I & Iftítítín & IIIII & Ifififit & IIIIfí & IIIIIIIII & LIIIII & IIIIIIIIII & IIIIII & 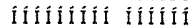 \\
\hline Total & $0.000 \mathrm{E} \div 00$ & 0.0000 & $0.000 E+00$ & 0.0000 & $0.000 \mathrm{E}+00$ & 0.0000 & $0.000 E+00$ & 0.0000 & $0.000 E+00$ & 0.0000 & $0.000 \mathrm{E}+00$ & 0.0000 & $1.237 \mathrm{E}-06 \quad 1.0000$ \\
\hline
\end{tabular}

$* * *$ CNRSI $(i, p, t)$ includes contribution from decay daughter radionuclides

\begin{tabular}{|c|c|c|}
\hline \multirow{2}{*}{\multicolumn{3}{|c|}{ 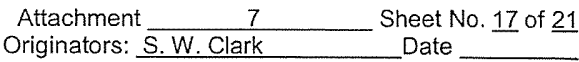 }} \\
\hline & & \\
\hline Chk'd By & M. W. Periott & Date \\
\hline Calc. No. & 0100B-CA-V0301 & Rev. No. 0 \\
\hline
\end{tabular}




\section{ATTACHMENT 7}

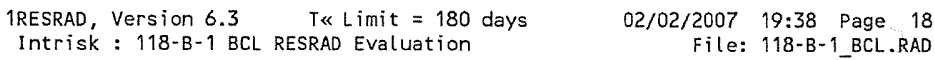

Amount of Intake Quantities QINT( $i, p, t)$ for Individual Radionuclides (i) and Pathways ( $p$ )

As $\mathrm{pCi} / \mathrm{yr}$ at $\mathrm{t}=3.000 \mathrm{E}+02$ years

\begin{tabular}{|c|c|c|c|c|c|c|c|c|c|c|c|}
\hline \multirow{4}{*}{$\begin{array}{l}\text { Radio- } \\
\text { Nucl ide } \\
\text { ÄÄÄAAÄA }\end{array}$} & \multicolumn{5}{|c|}{ Water Independent Pathways (Inhalation w/o radon) } & \multirow{2}{*}{\multicolumn{5}{|c|}{$\begin{array}{l}\text { Water Dependent Pathways } \\
\end{array}$}} & \multirow{4}{*}{$\begin{array}{c}\text { Total } \\
\text { Ingestion* }\end{array}$} \\
\hline & $\triangle A B A A A A B A A$ & $\triangle A A B A A A A A A B$ & $\triangle A A A A A A A A A A O A$ & $\triangle A A A A A A A A A A A B$ & $\triangle A O A A A A A A A A A A$ & & & & & $\triangle A A A A A A A O A A$ & \\
\hline & Inhalation & Plant & Meat & Milk & Soil & Water & Fish & Plant & Meat & & \\
\hline & $\triangle A O A A O A O A O A ̈ A$ & $\triangle A O A O A O A A A O A ̈$ & 1 AAAAAAAAAAOA & AAAAAAAAAAAÄÄ & $1 \triangle A A A A A A A A A B A$ & A & $\triangle A A A A A A A A B A B$ & 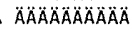 & $\triangle A \cap A ̈ A ̈ A ̈ A ̈ A ̈ A ̈ A$ & $\triangle A \cap A A A A A A \cap$ & \\
\hline $\operatorname{co-} 60$ & $1.496 E-20$ & $1.554 E-15$ & $1.475 E-15$ & $3.379 E-16$ & $2.014 \mathrm{E}-17$ & $0.000 E+00$ & $0.000 E+00$ & $0.000 E+00$ & $0.000 E+00$ & $0.000 \mathrm{E}+00$ & $3.387 E-15$ \\
\hline Cs -137 & $6.311 \mathrm{E}-06$ & $3.280 \mathrm{E}-01$ & $5.061 \mathrm{E}-01$ & $3.143 \mathrm{E}-01$ & $8.497 \mathrm{E}-03$ & $0.000 E+00$ & $0.000 \mathrm{E}+00$ & $0.000 E+00$ & $0.000 E+00$ & $0.000 E+00$ & $E+00$ \\
\hline Eu-152 & $5.016 E-10$ & $1.630 \mathrm{E}-06$ & $5.581 E-07$ & $3.688 \mathrm{E}-08$ & $6.754 \mathrm{E}-07$ & $0.000 E+00$ & $0.000 E+00$ & $0.000 E+00$ & $0.000 E+00$ & $E+\infty$ & $=-06$ \\
\hline Gd-152 & $1.041 \mathrm{E}-16$ & $3.383 E-13$ & $1.159 E-13$ & $3.062 E-15$ & $1.402 E-13$ & 0.00 & $0.000 \mathrm{E}+00$ & $0.000 E+00$ & $0.000 E+00$ & $0.000 E+00$ & $E-13$ \\
\hline $\mathrm{Sr}-90$ & $3.621 \mathrm{E}-06$ & $1.411 E+00$ & $5.025 \mathrm{E}-01$ & $2.839 \mathrm{E}-01$ & $4.875 \mathrm{E}-03$ & $0.000 E+00$ & $0.000 E+00$ & $0.000 E+00$ & $0.000 E+00$ & 0.0 & $E+00$ \\
\hline ÎIIIIII & İítilitít & 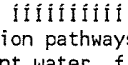 & II Î́ & IIIIIIIIIII & & 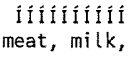 & IIIIIIIIII & 8 & & & \\
\hline
\end{tabular}

0

Excess Cancer Risks CNRS( $i, p, t)$ for Individual Radionuclides (i) and Pathways $(p)$ and Fraction of Total Risk at $t=3.000 E+02$ years Pathways (Inhalation excludes radon) Ground Plant

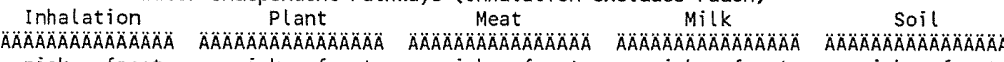
MilK

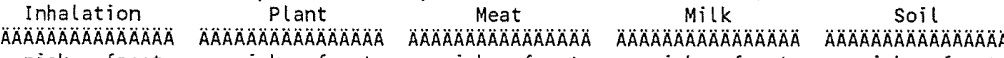
risk fract. risk fract. risk fract. risk fract. risk fract.

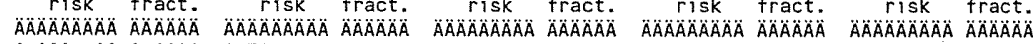
$\begin{array}{lllllll} & \end{array}$ $\begin{array}{llllllllll}0.000 E+00 & 0.0000 & 2.582 E-25 & 0.0000 & 2.449 E-25 & 0.0000 & 5.613 E-26 & 0.0000 & 6.044 E-27 & 0.0000 \\ 1.621 E-15 & 0.0000 & 2.647 E-10 & 0.0250 & 4.084 E-10 & 0.0386 & 2.537 E-10 & 0.0240 & 7.939 E-12 & 0.0008\end{array}$ $\begin{array}{llllllllll}6.930 E-19 & 0.0000 & 2.153 E-16 & 0.0000 & 7.371 E-17 & 0.0000 & 4.870 E-18 & 0.0000 & 1.661 E-16 & 0.0000\end{array}$ $\begin{array}{llllllllll}2.842 E-23 & 0.0000 & 3.907 E-22 & 0.0000 & 1.338 E-22 & 0.0000 & 3.536 E-24 & 0.0000 & 2.645 E-22 & 0.0000\end{array}$ $8.754 E-15 \quad 0.0000 \quad 2.867 E-090.2713 \quad 1.021 E-090.0966 \quad 5.767 E-100.0546 \quad 1.500 E-110.0014$

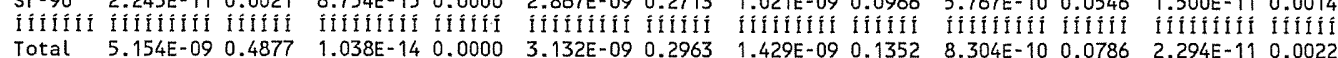
0

Excess Cancer Risks CNRS $(i, p, t)$ for Individual Radionuclides (i) and Pathways $(p)$ and Fraction of Total Risk at $t=3.000 E+02$ years

Water Dependent Pathways

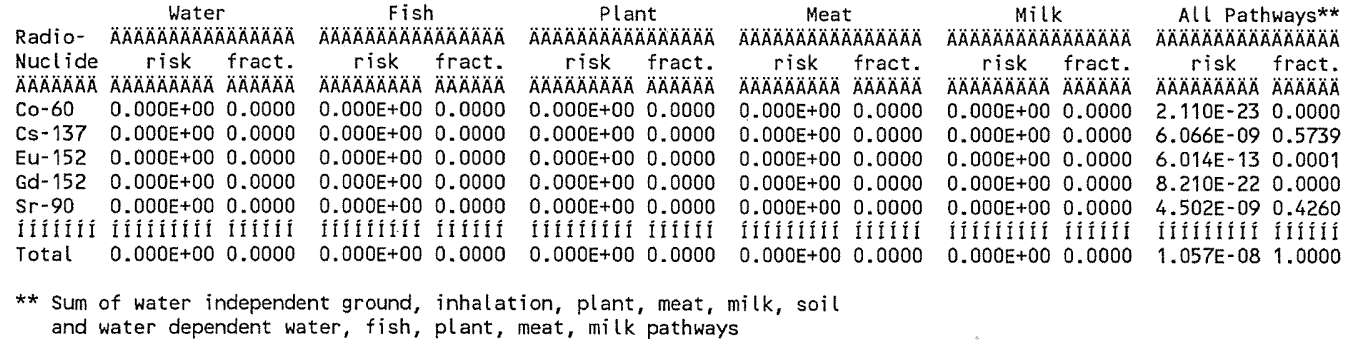

and water dependent water, fish, plant, meat, milk pathways

Attachment

Originators: S.W. Clark

7

Sheet No. 18 of $\underline{21}$

Chk'd By

Calc. No. $0100 \mathrm{~B}-\mathrm{CA}-\mathrm{V} 0301$

Date

Rev. No. $\quad 0$ 


\section{ATTACHMENT 7}

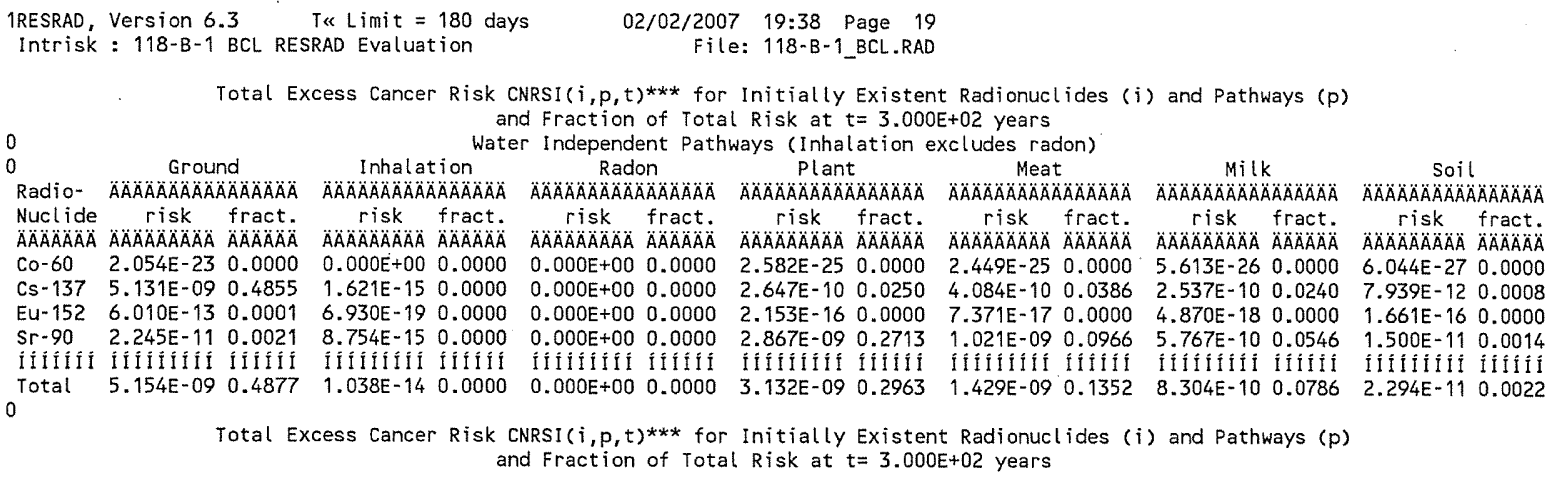

Total Excess Cancer Risk CNRSI $(i, p, t)^{* * *}$ for Initially Existent Radionuclides ( $i$ ) and Pathways ( $p$ ) and Fraction of Total Risk at $t=3.000 E+02$ years

Total Excess Cancer Risk CNRSI $(i, p, t) * * *$ for Initially Existent Radionuclides $(i)$ and Pathways $(p)$ and Fraction of Total Risk at $t=3.000 E+02$ years

Water Dependent Pathways

\begin{tabular}{|c|c|c|c|c|c|c|c|}
\hline & $\begin{array}{c}\text { Water } \\
\text { AOAOAOAOAOAOAOABÄ }\end{array}$ & $\begin{array}{c}\text { Fish } \\
\triangle A O A O A B A B A B A B A A A\end{array}$ & $\begin{array}{c}\text { Radon } \\
\text { AAAAAAAAAAAAAAOA }\end{array}$ & $\begin{array}{c}\text { Plant } \\
\text { AAAAAAAAAAAAOAOA }\end{array}$ & $\begin{array}{c}\text { Meat } \\
\text { AOAAAAAAAAAAAAAÄ }\end{array}$ & $\begin{array}{c}\text { MilK } \\
\text { AOAAAAAAAAAAAA }\end{array}$ & $\begin{array}{l}\text { All pathways } \\
\text { AAAAAAAAAAOAAAA }\end{array}$ \\
\hline Nuclide & risk fract. & risk fract. & risk fract. & $\begin{array}{l}\text { risk fract. } \\
\text { risk }\end{array}$ & $\begin{array}{l}\text { risk fract. } \\
\text { risk }\end{array}$ & $\begin{array}{l}\text { Aisk fract. } \\
\text { riskAAAAAAAAA }\end{array}$ & $\begin{array}{l}\text { PASk fract. } \\
\text { risk }\end{array}$ \\
\hline$\triangle A A ̈ A ̈ A A ̈ A$ & $\triangle \triangle A A A A A A A A B A A A A A B$ & $\triangle A A A A A A A A A A A A A A A A$ & $\triangle A A A A A A A A A$ AAAAAAA & 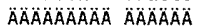 & 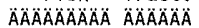 & $\triangle A A A A A A A O A O A A A A B A B A$ & 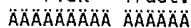 \\
\hline $\mathrm{Co}-60$ & $0.000 E+00 \quad 0.0000$ & $0.000 E+00 \quad 0.0000$ & $0.000 E+00 \quad 0.0000$ & $0.000 E+00 \quad 0.0000$ & $0.000 E+00 \quad 0.0000$ & $0.000 E+00 \quad 0.0000$ & $2.110 E-23$ \\
\hline Cs -137 & $0.000 \mathrm{E}+00 \quad 0.0000$ & $0.000 \mathrm{E}+00 \quad 0.0000$ & $0.000 \mathrm{E}+00 \quad 0.0000$ & $0.000 E+00 \quad 0.0000$ & $0.000 E+00 \quad 0.0000$ & $0.000 E+00 \quad 0.0000$ & $6.066 E-09 \quad 0.5739$ \\
\hline Eu-152 & $0.000 E+00 \quad 0.0000$ & $0.000 E+00 \quad 0.0000$ & $0.000 E+00 \quad 0.0000$ & $0.000 E+00 \quad 0.0000$ & $0.000 E+00 \quad 0.0000$ & $0.000 E+00 \quad 0.0000$ & $6.014 E-13 \quad 0.0001$ \\
\hline$S r-90$ & $0.000 E+00 \quad 0.0000$ & $0.000 E+00 \quad 0.0000$ & $0.000 E+00 \quad 0.0000$ & $0.000 E+00 \quad 0.0000$ & $0.000 E+00 \quad 0.0000$ & $0.000 E+00 \quad 0.0000$ & 4. \\
\hline IIIIYíñ & 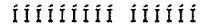 & 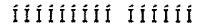 & IIIAIIII IIIIII & IIIIIIII IIIII & 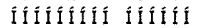 & 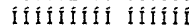 & 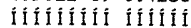 \\
\hline Total & $0.000 E+00 \quad 0.0000$ & $0.000 E+00 \quad 0.0000$ & $0.000 E+00 \quad 0.0000$ & $0.000 E+00 \quad 0.0000$ & $0.000 E+00 \quad 0.0000$ & $0.000 E+00 \quad 0.0000$ & $1.057 \mathrm{E}-08 \quad 1.0000$ \\
\hline
\end{tabular}

***CNRSI $(i, p, t)$ includes contribution from decay daughter radionuclides

\begin{tabular}{|c|c|}
\hline Attachment & Sheet No. \\
\hline Originators: S. W. Clark & Date \\
\hline Chk'd By M. W. Perrott & Date \\
\hline Calc. No. $\quad 0100 \mathrm{~B}-\mathrm{CA}-\mathrm{V} 0301$ & Rev. No. \\
\hline
\end{tabular}


CVP-2007-00006

Rev. 0

\section{ATTACHMENT 7}

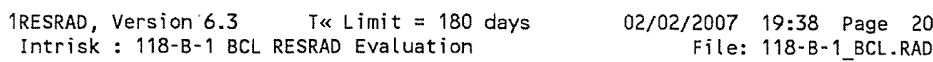

Amount of Intake Quantities QINT $i, p, t$ ) for Individual Radionuclides (i) and Pathways ( $P$ )

As $\mathrm{pCi} / \mathrm{yr}$ at $t=1.000 \mathrm{E}+03$ years

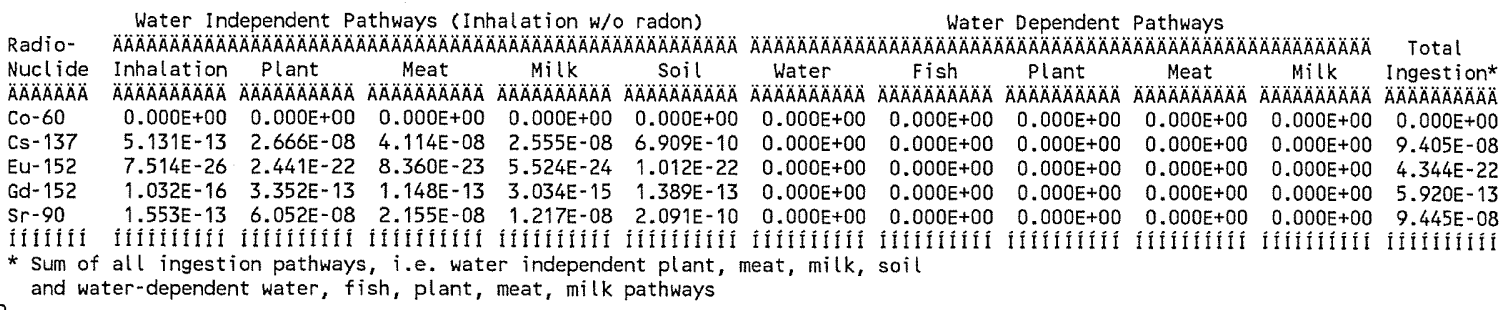

Water Dependent Pathways

\begin{tabular}{|c|c|c|c|c|c|c|c|c|c|c|c|c|}
\hline \multirow{2}{*}{$\begin{array}{l}\text { Radio- } \\
\text { Nucl ide } \\
\text { ÂAÄAAAAÄ }\end{array}$} & \multicolumn{2}{|c|}{ 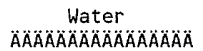 } & \multicolumn{2}{|c|}{ 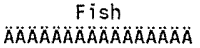 } & \multicolumn{2}{|c|}{ 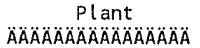 } & \multicolumn{2}{|c|}{$\begin{array}{c}\text { Meat } \\
\triangle A O A O A A O A O A O A O A O A O A O A ̈ A ̈\end{array}$} & \multicolumn{2}{|c|}{ 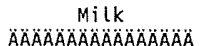 } & \multicolumn{2}{|c|}{$\begin{array}{r}\text { All Pathways** } \\
\text { AAAAAAAAAAAAAAAAAA }\end{array}$} \\
\hline & $\begin{array}{c}\text { risk } \\
\text { AAAAAAAAAOA }\end{array}$ & $\begin{array}{l}\text { fract. } \\
\triangle A A B A B A B A\end{array}$ & $\begin{array}{c}\text { risk } \\
\triangle A A A A A A A B\end{array}$ & $\begin{array}{l}\text { fract. } \\
A A A A A B A\end{array}$ & $\begin{array}{c}\text { risk } \\
\text { AAAAAAOAAABA }\end{array}$ & $\begin{array}{l}\text { fract. } \\
A Q A B A B A\end{array}$ & $\begin{array}{c}r i s k \\
\text { ÄÄÄÄÄÄÄ }\end{array}$ & $\begin{array}{l}\text { fract. } \\
A B A ̈ A B A B\end{array}$ & $\begin{array}{c}\text { risk } \\
\text { AAAAAAAOAOA }\end{array}$ & fract: & $\begin{array}{c}\text { risk } \\
\text { AAAAAAAAA }\end{array}$ & \\
\hline Co-60 & $0.000 E+00$ & 0.0000 & $0.000 E+00$ & 0.0000 & $0.000 E+00$ & 0.0000 & $0.000 E+00$ & 0.0000 & $0.000 E+00$ & 0.0000 & $0.000 \mathrm{E}+00$ & \\
\hline 77 & $0.000 E+00$ & 0.0 & $0 E+00$ & 0.0 & $\mathrm{EE}+00$ & 00 & $=+00$ & 0 & $\approx+00$ & 10 & & \\
\hline 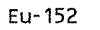 & $0.000 E+00$ & 0.0000 & $0.000 E+00$ & 0.0 & $0.000 E+00$ & 0.0000 & $0.000 E+00$ & 0. & $O E+00$ & 0 & -29 & \\
\hline$G d-152$ & $0.000 E+00$ & 0.0000 & $0.000 E+00$ & 0.0000 & $0.000 E+00$ & 0.0000 & $0.000 E+00$ & 0.0 & $E+00$ & bo & 22 & 0. \\
\hline & 0.00 & & & & & & & & & & & \\
\hline & & & & & & & & & & & & \\
\hline Total & $0.000 E+00$ & 0.0000 & $0.000 E+00$ & 0.0000 & $0.000 E+00$ & 0.0000 & $0.000 E+00$ & 0.0000 & $0.000 E+00$ & 0.0000 & $6.863 E-16$ & 1. \\
\hline
\end{tabular}

and water dependent water, fish, plant, meat, milk pathways

Attachment

Originators: S.W. Clark

Chk'd By M.W. Perrott

Calc. No. $\frac{\text { M.W. Perrott }}{0100 \mathrm{~B}-\mathrm{CA}-\mathrm{V} 0301}$
Sheet No. 20 of 21

Date

Date

Rev. No. $\mathrm{O}$ 


\section{ATTACHMENT 7}

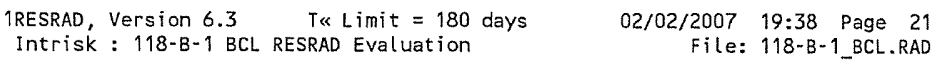

Total Excess Cancer Risk CNRSI $(i, p, t) * * *$ for Initially Existent Radionuclides ( $i$ ) and Pathways ( $p$ ) and Fraction of Total Risk at $t=1.000 E+03$ years

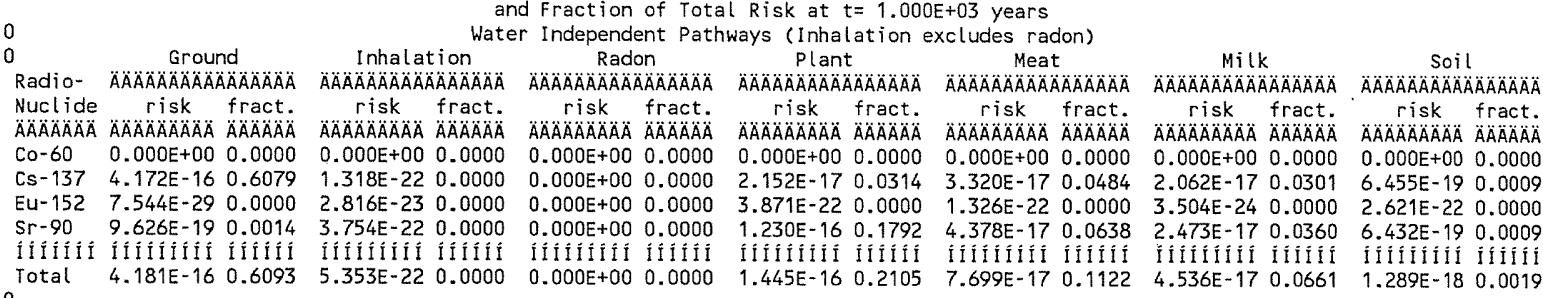

Total Excess Cancer Risk CNRSI $(i, p, t) * * *$ for Initially Existent Radionuclides ( $i$ ) and Pathways ( $p$ ) and Fraction of Total Risk at $\mathrm{t}=1.000 \mathrm{E}+03$ years

Water Dependent Pathways

\begin{tabular}{|c|c|c|c|c|c|c|c|c|c|c|c|c|c|c|}
\hline \multirow{2}{*}{$\begin{array}{l}\text { Radio- } \\
\text { Nuclide } \\
\because A B A B A A B\end{array}$} & \multicolumn{2}{|c|}{$\begin{array}{c}\text { Water } \\
\triangle A A A A O A A O A O A O A O A O A O A O A O A\end{array}$} & \multicolumn{2}{|c|}{ 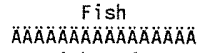 } & \multicolumn{2}{|c|}{ 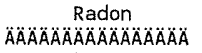 } & \multicolumn{2}{|c|}{$\begin{array}{c}\text { Plant } \\
\text { ALAAAOAOAOAAAAAAAAAOA }\end{array}$} & \multicolumn{2}{|c|}{$\begin{array}{c}\text { Meat } \\
\triangle A A A A A A A A A A A A A B A\end{array}$} & \multicolumn{2}{|c|}{$\begin{array}{c}M i L K \\
\text { ÄAAAAAAAAAAAAAAAA }\end{array}$} & \multicolumn{2}{|c|}{$\begin{array}{c}\text { All pathways } \\
\text { AAOAAAAAAAAAAAAAA }\end{array}$} \\
\hline & isk & $\begin{array}{l}\text { fract. } \\
\triangle A A A O A B A\end{array}$ & $\begin{array}{l}\text { risk } \\
\triangle A B A O A B A\end{array}$ & $\begin{array}{l}\text { fract. } \\
\triangle A B A A A A B A\end{array}$ & $\begin{array}{c}r i s k \\
\triangle A A A O A A O A O A B\end{array}$ & $\begin{array}{l}\text { fract. } \\
\triangle A O A O A B A ̈\end{array}$ & $\begin{array}{c}\text { risk } \\
\text { ÄAAÄAAAOAAÄ }\end{array}$ & 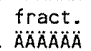 & $\begin{array}{c}r i s k \\
\triangle A A B A B A O A O A B\end{array}$ & 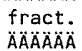 & $\begin{array}{c}\text { risk } \\
\triangle A \cap A O A B A B A\end{array}$ & $\begin{array}{l}\text { fract. } \\
\triangle A B A ̈ A B A A\end{array}$ & $\begin{array}{c}\text { risk } \\
\triangle A \cap A B A A B A B\end{array}$ & $\begin{array}{l}\text { fract. } \\
\text { ÄAAAAÄ }\end{array}$ \\
\hline Co- 60 & $0.000 E+00$ & 0.0000 & $0.000 E+00$ & 0.0000 & $0.000 E+00$ & 0.0000 & $0.000 E+00$ & 0.0000 & $0.000 E+00$ & 0.0000 & $0.000 \mathrm{E}+00$ & 00 & $00 E+00$ & .00 \\
\hline Cs -137 & $0.000 E+00$ & 0.0000 & $0.000 E+00$ & 0.0000 & $0.000 E+00$ & 0.0000 & 0. & 0 & $0.000 \mathrm{E}+00$ & 0.0000 & $0.000 E+00$ & 0.0000 & -16 & .71 \\
\hline Eu- 152 & $0.000 E+00$ & 0.0000 & $0.000 E+00$ & 0.0000 & $0.000 E+00$ & 0.0000 & $0.000 \mathrm{E}+00$ & 100 & $0.000 E+00$ & 00 & $0.000 E+00$ & 00 & & 0 \\
\hline $\mathrm{Sr}-90$ & $0.000 E+00$ & 0.0000 & $0.000 \mathrm{E}+00$ & 0.0000 & $0.000 E+00$ & 0.0000 & $0.000 E+00$ & 0. & $E+00$ & 0 & 0 . & 0.0000 & 1. & 0.28 \\
\hline ifí & IfII & & & & & II I & II I & IIIIII & 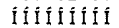 & IIIIII & IUIIISISt & IIIIIII & IIIIIIIII & IIIII \\
\hline Total & $0.000 E+00$ & 0.0000 & $0.000 E+00$ & 0.0000 & $0.000 E+00$ & 0.0000 & $0.000 E+00$ & 0.0000 & $0.000 E+00$ & 0.0000 & $0.000 E+00$ & 0.0000 & $6.863 \mathrm{E}$ & 10 \\
\hline
\end{tabular}

$* * * \operatorname{CNRSI}(i, p, t)$ includes contribution from decay daughter radionuclides

Attachment

Originators: S.W. Clark

Chk'd By M.W. Perrott

Calc. No. 0100B-CA-V0301
Sheet No. 21 of $\underline{21}$

Date

Rev. No. 0 


\section{ATTACHMENT 8}

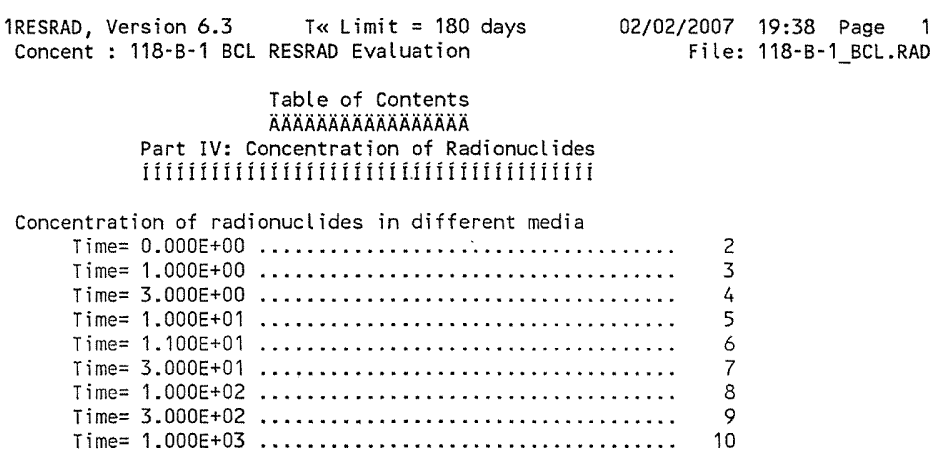

Attachment Sheet No. 1 of 10 Originators: S.W. Clark RWC Date $2 / 5 \%$ Chk'd By M.W.Perrott ryzfp Date $2 / 5 / d z$ Calc. No. 0100 B-CA-V0301 Rev. No. 0 
CVP-2007-00006

Rev. 0

\section{ATTACHMENT 8}

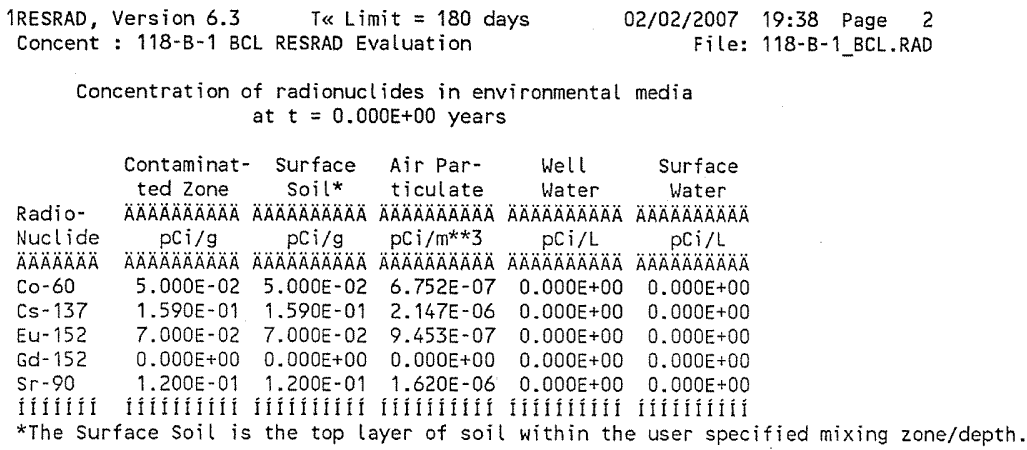

Concentrations in the media occurring in pathways that are suppressed are calculated using the current input parameters, $i$.e. Using parameters appearing in the input screen when the pathways are active.

Concentration of radionuclides in foodstuff media

$$
\text { at } t=0.000 E+00 \text { years* }
$$

\begin{tabular}{|c|c|c|c|c|c|c|c|c|c|}
\hline & $\begin{array}{c}\text { Drinking } \\
\text { Water }\end{array}$ & $\begin{array}{l}\text { Nonleafy } \\
\text { Vegetable }\end{array}$ & $\begin{array}{c}\text { Leafy } \\
\text { Vegetable }\end{array}$ & $\begin{array}{l}\text { Fodder } \\
\text { Meat }\end{array}$ & $\begin{array}{l}\text { Fodder } \\
\text { Milk }\end{array}$ & Meat & Milk & Fish & Crustacea \\
\hline Radio- & $\triangle A A \cap A \cap A O A A O A O A$ & $\triangle A A A A A A A A A A A$ & $\triangle A \triangle A \cap A \cap A \cap A \cap A \cap A A$ & $\triangle A \cap A \cap A \cap A \cap A \cap A \cap A ̈ A$ & $\triangle A A A A A A A A A$ & $\triangle A A A A A A A A B A$ & $\triangle A A A A A A A A A A$ & $\triangle A A O A A A A A A A B$ & $\triangle \triangle A \cap A \cap A \cap A ̈ A \cap A$ \\
\hline Nuclide & $p C i / L$ & $\mathrm{pCi} / \mathrm{kg}$ & $\mathrm{pCi} / \mathrm{kg}$ & $p c i / k g$ & $\mathrm{pCi} / \mathrm{kg}$ & $\mathrm{pCi} / \mathrm{kg}$ & $\mathrm{pCi} / \mathrm{L}$ & $\mathrm{pCi} / \mathrm{kg}$ & $\begin{array}{r}\mathrm{pCi} / \mathrm{kg} \\
A A A A A B A\end{array}$ \\
\hline $\begin{array}{l}\triangle A A A A A A A \\
C O-60\end{array}$ & ÄÄÄÄÄÄA & AAÄAAAAAAAAA & $\triangle A \cap A B A A B A A A B A$ & 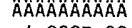 & $\triangle A \cap A ̈ A \cap A \cap A ̈$ & $\triangle A A A A A A A$ & $\triangle A A \cap A \cap A \cap A ̈$ & $\triangle A O A A A A A A$ & $\begin{array}{r}A A A A A A A A B \\
0.000 E+C\end{array}$ \\
\hline Co- 60 & $0.000 \mathrm{E}+00$ & $4.000 E+00$ & $4.000 \mathrm{E}+00$ & $4.000 E+00$ & $4.000 E+00$ & $5.940 \mathrm{E}+00$ & $4.900 E-01$ & $0.000 E+00$ & $0.000 E+C$ \\
\hline Cs -137 & $0.000 \mathrm{E}+00$ & $6.360 E+00$ & $6.361 \mathrm{E}+00$ & $6.361 E+00$ & $6.361 \mathrm{E}+00$ & $1.536 E+01$ & $3.435 E+00$ & $0.000 E+00$ & $0.000 \mathrm{E}+$ \\
\hline Eu-152 & $0.000 E+00$ & $1.751 \mathrm{E}-01$ & $1.752 E-01$ & $1.753 \mathrm{E}-01$ & 1.753E-01 & $9.384 E-02$ & $2.232 E-03$ & $0.000 E+00$ & $0.000 E+C$ \\
\hline Gd -152 & $0.000 \mathrm{E}+00$ & $0.000 E+00$ & $0.000 E+00$ & $0.000 E+00$ & $0.000 E+00$ & $0.000 E+00$ & $0.000 \mathrm{E}+00$ & $0.000 E+00$ & $0.000 \mathrm{E}+\mathrm{C}$ \\
\hline$s r-90$ & $0.000 E+00$ & $3.600 \mathrm{E}+01$ & $3.600 \mathrm{E}+01$ & $3.600 E+01$ & $3.600 E+01$ & $2.006 E+01$ & $4.080 E+00$ & $0.000 \mathrm{E}+00$ & $0.000 E+$ \\
\hline II I I I I I I & IIIfifilifil & If I I & $60^{\prime}$ & If Î́ & If & $\{\%$ & & fiftifíti & fifit \\
\hline
\end{tabular}

Concentrations in the media occurring in pathways that are suppressed are calculated using the current input parameters, i.e. using parameters appearing in the input screen when the pathways are active.

\begin{tabular}{|c|c|}
\hline Attachment & Sheet No. 2 of 10 \\
\hline Originators: S.W. Clark & Date \\
\hline M. W. Perrott & Date \\
\hline 0100B-CA-V0301 & Rev. No. \\
\hline
\end{tabular}


CVP-2007-00006

Rev. 0

\section{ATTACHMENT 8}

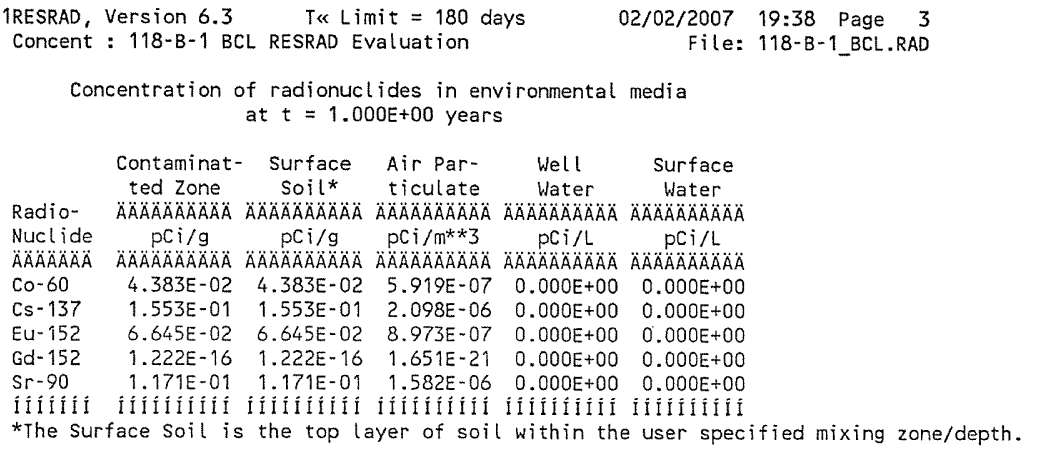

Concentrations in the media occurring in pathways that are suppressed are calculated using the current input parameters, i.e. using parameters appearing in the input screen when the pathways are active.

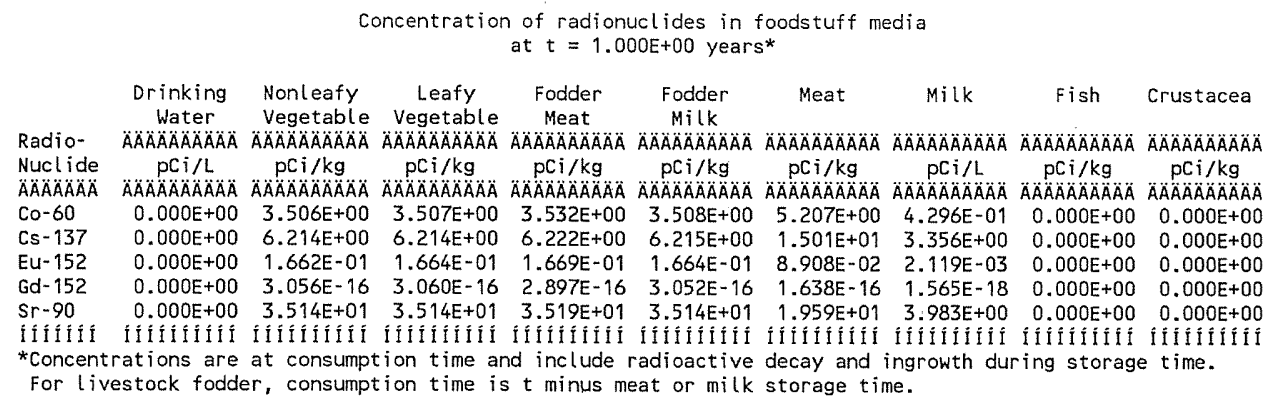

Concentrations in the media occurring in pathways that are suppressed are calculated using the current input parameters, i.e. using parameters appearing in the input screen when the pathways are active.

$\begin{array}{ll}\text { Attachment } \frac{8}{1} & \text { Sheet No. } 3 \text { of } 10 \\ \text { Originators: } \frac{8 . \text { W. Clark }}{\text { M. W. Perrott }} & \text { Date } \\ \text { Chk'd By } & \text { Date } \\ \text { Calc. No. } & \text { Rev. No. } \quad \text { No0B-CA-V0301 }\end{array}$


CVP-2007-00006

Rev. 0

\section{ATTACHMENT 8}

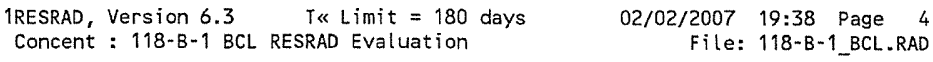

Concentration of radionuclides in environmental media

at $\mathrm{t}=3.000 \mathrm{E}+00$ years

\begin{tabular}{|c|c|c|c|c|c|}
\hline & $\begin{array}{c}\text { Conta } \\
\text { ted }\end{array}$ & $\begin{array}{l}\text { Surface } \\
\text { Soil* }\end{array}$ & $\begin{array}{l}\text { Air Par } \\
\text { ticulate }\end{array}$ & $\begin{array}{l}\text { Well } \\
\text { Water }\end{array}$ & $\mathrm{ce}$ \\
\hline & $\triangle A A A B A A A A A A A$ & 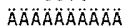 & & $\triangle \triangle A \triangle A \cap A B A O A$ & \\
\hline & & & & & \\
\hline & $\triangle A A A O A$ & $A A A A$ & $\ddot{A A A ̈}$ & $\triangle A A A$ & $\ddot{A} A ̈ A$ \\
\hline & & & & & 0. \\
\hline & & & & & +00 \\
\hline & & & & & $+\infty$ \\
\hline & & & & & \\
\hline & $E-01$ & $6 E-01$ & -06 & $30 E+00$ & 0000 \\
\hline
\end{tabular}

$\begin{array}{llllll}\mathrm{Sr}-90 & 1.116 \mathrm{E}-01 & 1.116 \mathrm{E}-01 & 1.507 \mathrm{E}-06 & 0.000 \mathrm{E}+00 & 0.000 \mathrm{E}+00\end{array}$

* The Surface soil is the top layer of soil within the user specified mixing zone/depth.

Concentrations in the media occurring in pathways that are suppressed are calculated using the current input parameters, i.e. using parameters appearing in the input screen when the pathways are active.

Concentration of radionuclides in foodstuff media at $t=3.000 \mathrm{E}+00$ year ${ }^{*}$

\begin{tabular}{|c|c|c|c|c|c|c|c|c|c|}
\hline & $\begin{array}{c}\text { Drinking } \\
\text { Water }\end{array}$ & $\begin{array}{l}\text { Nonleafy } \\
\text { Vegetable }\end{array}$ & $\begin{array}{c}\text { Leafy } \\
\text { Vegetable }\end{array}$ & $\begin{array}{l}\text { Fodder } \\
\text { Meat }\end{array}$ & $\begin{array}{l}\text { Fodder } \\
\text { Milk }\end{array}$ & Meat & Milk & Fish & rustacea \\
\hline & 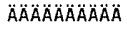 & $A \cap A B A$ & $\triangle \triangle A A A A A A O A A A O A$ & I AAAAAAAAAAAOAOA & AAAAAAAAAAAÄ & $\triangle A O A O A A$ & AAAAÄ & AAAAAAAOA & i \\
\hline & & & & & & & & & \\
\hline & $A O A ̈ A$ & $\ddot{A A O A}$ & 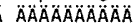 & $\ddot{A}$ & & & & & \\
\hline & & & & & & & & & \\
\hline & & & & & & & & & \\
\hline & & & & & & & & & \\
\hline & & & & & & & & & 0. \\
\hline & & 3 & 3 & 3. & & & & 0. & 0. \\
\hline & & & & & & & & & \\
\hline
\end{tabular}

For Livestock fodder, consumption time is t minus meat or milk storage time.

Concentrations in the media occurring in pathways that are suppressed are calculated using the current input parameters, $i$.e. using parameters appearing in the input screen when the pathways are active. 
CVP-2007-00006

Rev. 0

\section{ATTACHMENT 8}

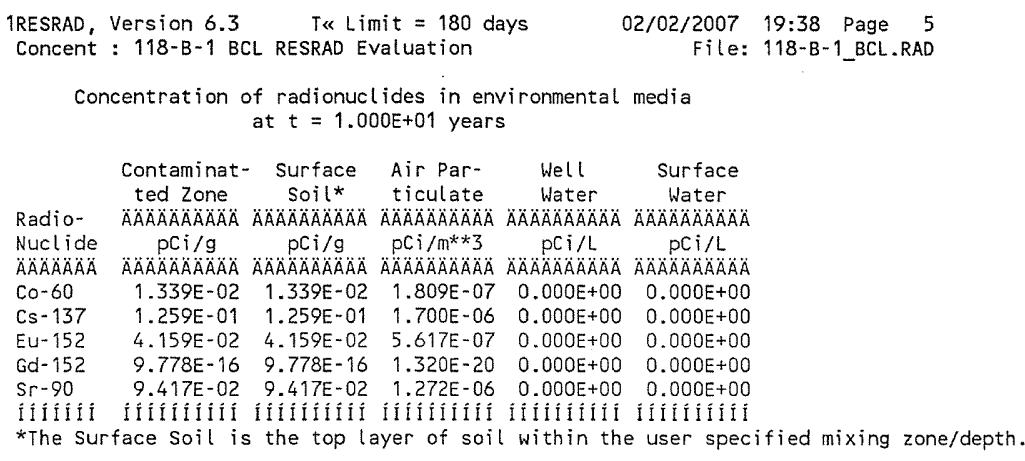

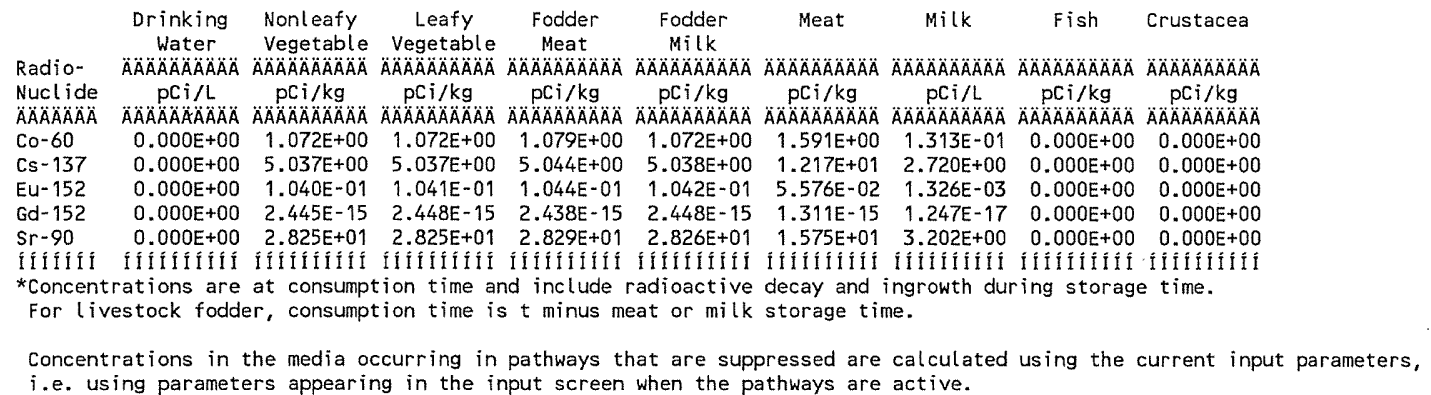
i.e. using parameters appearing in the input screen when the pathways are active.

$\begin{array}{ll}\text { Attachment } \frac{8}{2} & \text { Sheet No. } \underline{5} \text { of } \underline{10} \\ \text { Originators: } \frac{\text { S. W. Clark }}{\text { M. W. Perrott }} & \text { Date } \\ \text { Chk'd By } & \text { Date } \\ \text { Calc. No. } & \text { Rev. No. } 0\end{array}$


CVP-2007-00006

Rev. 0

\section{ATTACHMENT 8}

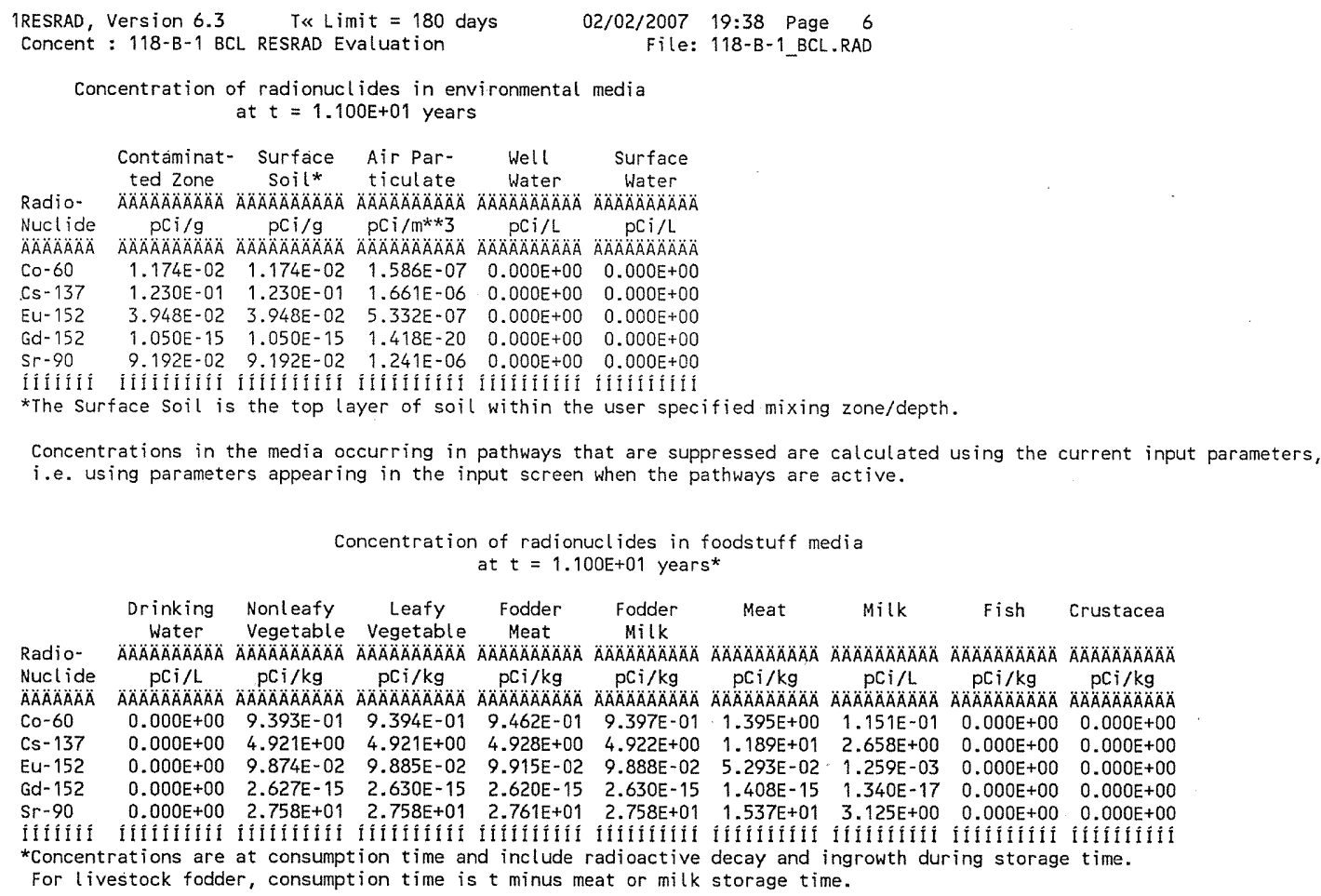

Concentrations in the media occurring in pathways that are suppressed are calculated using the current input parameters, i.e. using parameters appearing in the input screen when the pathways are active.

Attachment

8 Sheet No. $\underline{6}$ of $\underline{10}$ Originators: $S$. W. Clark

Chk'd By M.W. Perrott Date

Calc. No. 0100B-CA-V0301

Date

Rev. No. 0 
CVP-2007-00006

Rev. 0

\section{ATTACHIMENT 8}

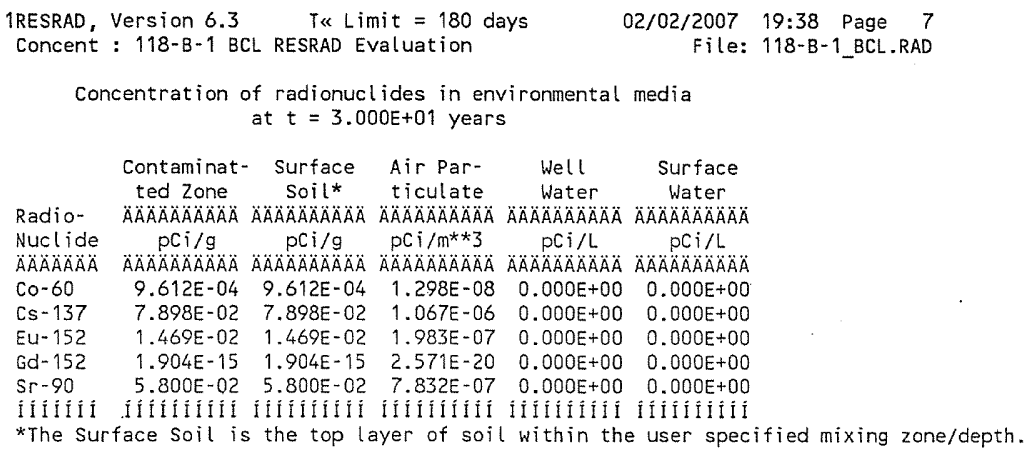

\begin{tabular}{|c|c|c|c|c|c|c|c|c|c|}
\hline & $\begin{array}{c}\text { Drinking } \\
\text { Water }\end{array}$ & $\begin{array}{l}\text { Nonleafy } \\
\text { Vegetable }\end{array}$ & $\begin{array}{c}\text { Leafy } \\
\text { Vegetable }\end{array}$ & $\begin{array}{l}\text { Fodder } \\
\text { Meat }\end{array}$ & $\begin{array}{l}\text { Fodder } \\
\text { Milk }\end{array}$ & Meat & Milk & Fish & Crustacea \\
\hline dio- & $\triangle A \cap A A A A A A O A O A$ & ÄÄÄÄAिÄÄÄÄ & A & $\triangle A A A A A A A A A A$ & $\triangle A O A A A A A A A O A$ & $\triangle A A A A A A A A A$ & AAAAAAAÄÄÄ & $\triangle A B A A A O A A A B$ & $\triangle \triangle A O A ̈ A ̈ A ̈ A ̈ A ̈ A ̈ A$ \\
\hline Nucl ide & $p C i / L$ & $\mathrm{pci} / \mathrm{kg}$ & $\mathrm{pci} / \mathrm{kg}$ & $\mathrm{pCi} / \mathrm{kg}$ & $\mathrm{pCi} / \mathrm{kg}$ & $\mathrm{pCi} / \mathrm{kg}$ & $\mathrm{pCi} / \mathrm{L}$ & $\mathrm{pCi} / \mathrm{kg}$ & $\mathrm{pCi} / \mathrm{kg}$ \\
\hline ÄÄÄÄÄ & AAAAAAAAAÄ & ÄÄÄAAAOÄ & AAAAAAAAAÄ & A ÄÄÄÄÄÄ & $\triangle A A A O A A A A O A$ & 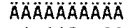 & 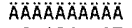 & $\triangle A A A A A A A A A B A$ & $\triangle A A A A A A B A A A A$ \\
\hline Co-60 & $0.000 E+00$ & $7.690 \mathrm{E}-02$ & $7.690 \mathrm{E}-02$ & $7.746 \mathrm{E}-02$ & $7.693 \mathrm{E}-02$ & $1.142 E-01$ & $9.421 E-03$ & $0.000 E+00$ & $0.000 E+00$ \\
\hline Cs -137 & $0.000 E+00$ & $3.159 E+00$ & $3.160 E+00$ & $3.164 E+00$ & $3.160 E+00$ & $7.631 E+00$ & $1.706 E+00$ & $0.000 E+00$ & $0.000 E+00$ \\
\hline Eu-152 & $0.000 E+00$ & $3.673 E-02$ & $3.677 E-02$ & $3.688 E-02$ & $3.678 E-02$ & $1.969 \mathrm{E}-02$ & $4.683 E-04$ & $0.000 E+00$ & $0.000 E+00$ \\
\hline$G d-152$ & $0.000 E+00$ & $4.761 \mathrm{E}-15$ & $4.766 E-15$ & $4.763 E-15$ & $4.766 E-15$ & $2.552 E-15$ & $2.428 E-17$ & $0.000 E+00$ & $0.000 E+00$ \\
\hline $\begin{array}{l}s r-90 \\
\text { IIIIII }\end{array}$ & $\begin{array}{r}0.000 E+00 \\
\text { IIIIIIIIII }\end{array}$ & $\begin{array}{l}1.740 E+01 \\
\text { IIIIIIIII }\end{array}$ & $\begin{array}{r}1.740 E+01 \\
\text { Itififiti }\end{array}$ & $\begin{array}{r}1.742 E+01 \\
\text { IIIIIIIIA }\end{array}$ & $\begin{array}{r}1.740 E+01 \\
\text { IIIIIIIIII }\end{array}$ & $\begin{array}{r}9.698 E+00 \\
\text { IIIIIIIIII }\end{array}$ & $\begin{array}{l}1.972 E+00 \\
\text { IIIIIIIIII }\end{array}$ & $\begin{array}{r}0.000 E+00 \\
\text { IIIIIIIII }\end{array}$ & $\begin{array}{r}0.000 E+00 \\
\text { IIIIIIIIII }\end{array}$ \\
\hline-1 & 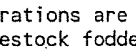 & & $-a_{-1}$ & & & & & & \\
\hline
\end{tabular}

For livestock fodder, consumption time is $t$ minus meat or milk storage time.

Concentrations in the media occurring in pathways that are suppressed are calculated using the current input parameters, i.e. using parameters appearing in the input screen when the pathways are active.

$\begin{array}{ll}\text { Attachment } \frac{8}{\text { S. Clark }} & \text { Sheet No. } 7 \text { of } 10 \\ \text { Originators: } \frac{\text { S.W. }}{\text { M.W. Perrott }} & \text { Date } \\ \text { Chk'd By } & \text { Rev. No. } \quad 0 \\ \text { Calc. No. } & \text { R100B-CA-V0301 }\end{array}$


CVP-2007-00006

Rev. 0

\section{ATTACHMENT 8}

$\begin{array}{rrr}\text { 1RESRAD, Version } 6.3 & \text { T\& Limit }=180 \text { days } & \text { 02/02/2007 } 19: 38 \text { Page } 8 \\ \text { Concent }: 118-\mathrm{B}-1 \mathrm{BCL} \text { RESRAD Evaluation } & \text { File: } 118-\mathrm{B}-1 \text { BCL. RAD }\end{array}$

Concentration of radionuclides in environmental media

at $t=1.000 E+02$ years

Contaminat- Surface Air Par- Well surface

ted Zone Soil* ticulate Water Water

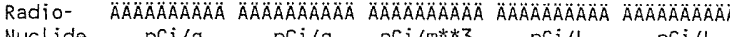

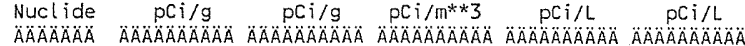

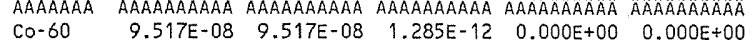

$\begin{array}{llllll}\text { CS-137 } & 1.544 \mathrm{E}-02 & 1.544 \mathrm{E}-02 & 2.085 \mathrm{E}-07 & 0.000 \mathrm{E}+00 & 0.000 \mathrm{E}+00\end{array}$

$\begin{array}{llllll}\text { Eu-152 } & 3.841 \mathrm{E}-04 & 3.841 \mathrm{E}-04 & 5.187 \mathrm{E}-09 & 0.000 \mathrm{E}+00 & 0.000 \mathrm{E}+00\end{array}$

$\begin{array}{llllll}\text { Gd-152 } & 2.394 E-15 & 2.394 E-15 & 3.233 E-20 & 0.000 E+00 & 0.000 E+00\end{array}$

$\begin{array}{llllll}\mathrm{Sr}-90 & 1.063 \mathrm{E}-02 & 1.063 \mathrm{E}-02 & 1.436 \mathrm{E}-07 & 0.000 \mathrm{E}+00 & 0.000 \mathrm{E}+00\end{array}$

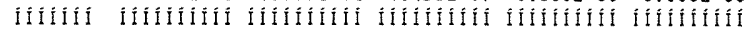

*The surface Soil is the top layer of soil within the user specified mixing zone/depth.

Concentrations in the media occurring in pathways that are suppressed are calculated using the current input parameters, i.e. using parameters appearing in the input screen when the pathways are active.

Concentration of radionuclides in foodstuff media

at $\mathrm{t}=1.000 \mathrm{E}+02$ years*

\begin{tabular}{|c|c|c|c|c|c|c|c|c|c|}
\hline & $\begin{array}{c}\text { Drinking } \\
\text { Water }\end{array}$ & $\begin{array}{l}\text { Nonleafy } \\
\text { Vegetable }\end{array}$ & $\begin{array}{c}\text { Leafy } \\
\text { Vegetable }\end{array}$ & $\begin{array}{l}\text { Fodder } \\
\text { Meat }\end{array}$ & $\begin{array}{l}\text { Fodder } \\
\text { Milk }\end{array}$ & Meat & Milk & Fish & Crustacea \\
\hline & $\triangle A B A A A A A A A$ & & & $\triangle A \cap A \cap A ̈ A O A O A O A ̈ A$ & ÄAAÄÄÄÄÄÄ & $\triangle A O A$ & $\triangle A \cap A ̈ A ̈ A ̈ A ̈ A ̈$ & ÄÄÄ & \\
\hline & & & & & & & & & \\
\hline & $A A A A$ & $A A A$ & $A A A B$ & $A O A A$ & ÄÄÄ & $\because A \ddot{A}$ & $\ddot{A} A ̈ A ̈$ & $A \ddot{A}$ & \\
\hline & 0.0 & & & & & & & & \\
\hline & 0.0 & & & & & & & & \\
\hline & & & & & & & & & 0. \\
\hline & 0.0 & & & & & & & & \\
\hline $\mathrm{Sr}-$ & 0.0 & 00 & 3.1 & $3:$ & 3.1 & & 3. & 00 & 0. \\
\hline & & & & & & & & & \\
\hline
\end{tabular}

Concentrations in the media occurring in pathways that are suppressed are calculated using the current input parameters, i.e. using parameters appearing in the input screen when the pathways are active.

$\begin{array}{ll}\text { Attachment } \frac{8}{\text { W. Clark }} & \text { Sheet No. } \underline{8} \text { of } 10 \\ \text { Originators: } \frac{\text { S.W. }}{\text { M.W. Perrott }} \text { Date } \\ \text { Chk'd By Date } \\ \text { Calc. No. } \\ \text { O100B-CA-V0301 Rev. No. o }\end{array}$


CVP-2007-00006

Rev. 0

\section{ATTACHMENT 8}

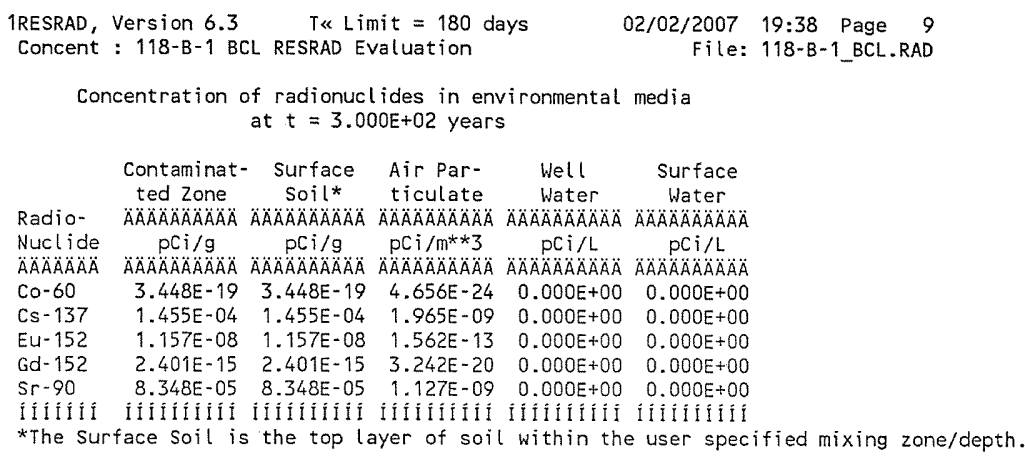

Concentrations in the media occurring in pathways that are suppressed are calculated using the current input parameters, $i$.e. using parameters appearing in the input screen when the pathways are active. 
CVP-2007-00006

Rev. 0

\section{ATTACHMENT 8}

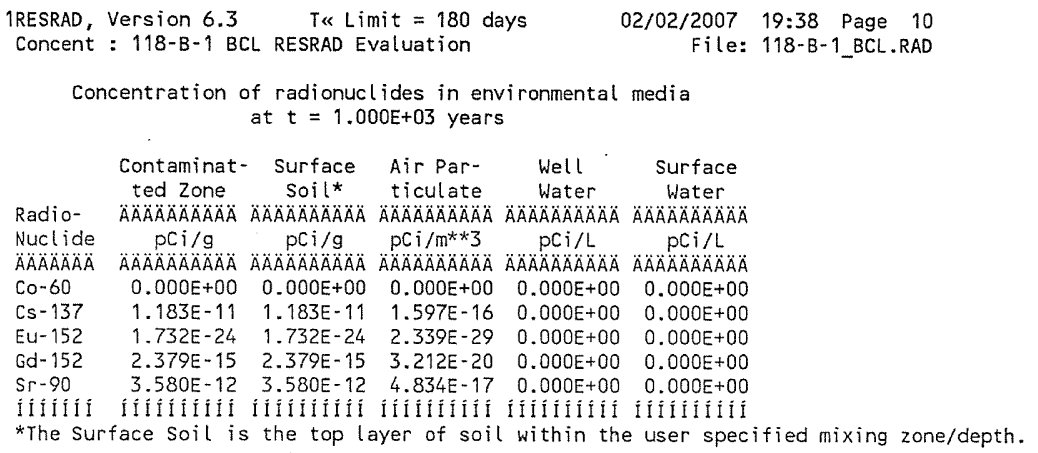

Concentrations in the media occurring in pathways that are suppressed are calculated using the current input parameters, i.e. using parameters appearing in the input screen when the pathways are active.

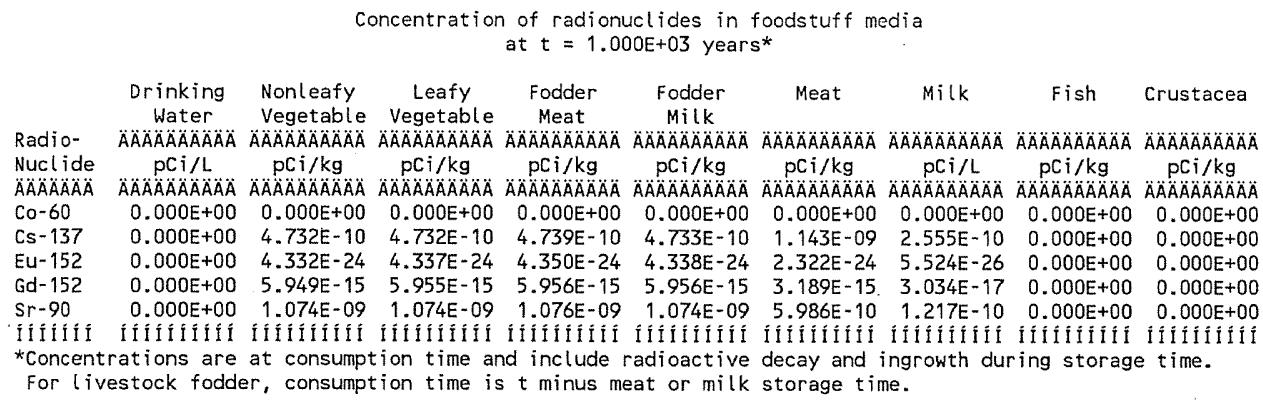

Concentrations in the media occurring in pathways that are suppressed are calculated using the current input parameters, i.e. using parameters appearing in the input screen when the pathways are active.

$\begin{array}{ll}\text { Attachment } \frac{8}{\text { S.W. Clark }} & \text { Sheet No. } 10 \text { of } 10 \\ \text { Originators: } & \text { Date } \\ \text { Chk'd By } & \text { Date } \\ \text { Calc. No. W. Perrott } & \text { Rev. No. } \quad 0\end{array}$




\section{ATTACHMENT 9}

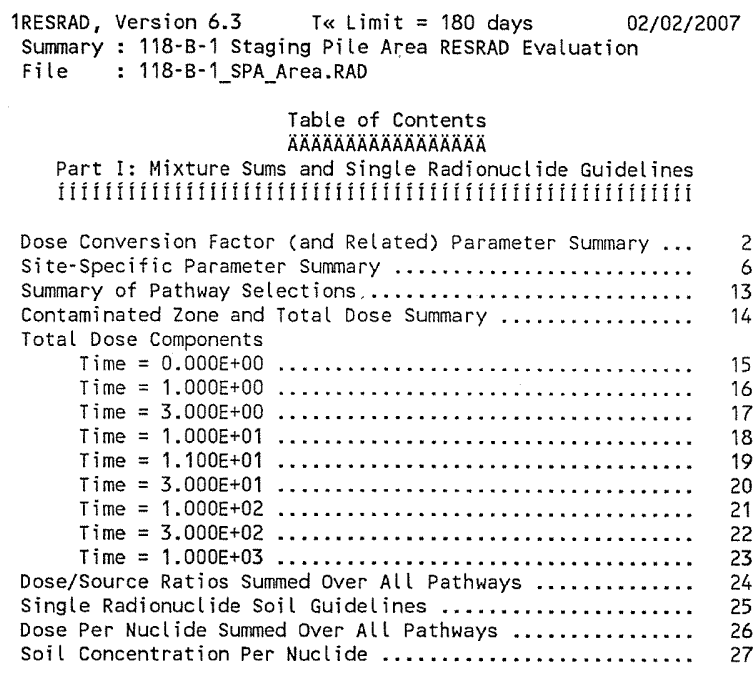

Attachment 9 Sheet No. 1 of 27 Originators: S.W. Clark SEJe Date $2 / 5 / 67$ Chk'd By M.W. Perrott-yicif Date $2 / 5 / 62$ Calc. No. 0100B-CA-V0301 Rev. No. 


\section{ATTACHMENT 9}

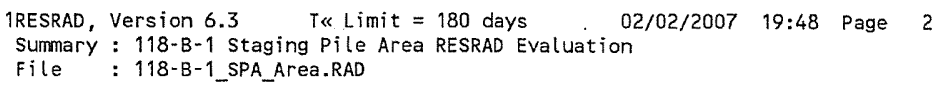

$36.724 E+00^{3} 6.700 E+000^{3}$ DCF2( 1$)$ $34.440 E-0134.440 E-013$ DCF2( 2$)$ $32.090 E-0632.090 E-06$ \& DCF2( 3$)$

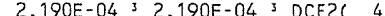
5 $3.190 \mathrm{E}-05$; DCFZ( 5$)$ 3.210E-

(2.430E$6.290 E-0636.290 E-06$ DCF2( 10$)$ $35.400 E-0135.400 E-01^{3}$ DCF2 (11) $1.280 E+00$ 3 $1.280 E+00$ DCF2( 12$)$ $4.290 E-0134.290 E-013$ DCF2( 13) $3.290 E-013$ 3 $4.290 E-01$ 's DCF2( 14$)$ $5.078 E-0334.770 E-03$ 3 DCF2( 16) $1.308 E-03$ ' $1.300 E-03$ ' DCF2( 17$)$ $1.640 E+0031.640 E+00$ ' DCF2( 20$)$ $1.350 E-01^{3} 1.350 E-01^{3}$ DCF2( 21$)$ $1.230 E-0131.230 E-013$ DCF2( 22) $1.250 E-0131.250 E-013$ DCF2( 23)

$1.480 E-02^{3} 1.410 E-02^{3}$ DCF3( 1) $3.640 \mathrm{E}-03 \times 3.640 \mathrm{E}-03$ DCF3( 2) $2.090 \mathrm{E}-06^{3}$ 2.090E-06 ${ }^{3}$ DCF3( 3) $2.690 \mathrm{E}-05^{3} 2.690 \mathrm{E}-05^{3} \mathrm{DCF} 3(4)$ 3 5.000E-05 3 5.000E-05 3 DCF3( 5)

$6.480 E-06^{3} 6.480 E-063$ DCF3( 6$)$

$1.610 E-04331.610 E-043$ DCF3( 8$)$

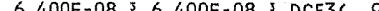

$5.7700-07$ ? $5.770 \mathrm{E}-07$ ' DCF3

$34.44 E-03$ 3 $4.440 E-03$ \& DCF3 (11)

O 1.060 E 02 DCF3( 12$)$

$3.540 E-03$ 3 $3.540 E-03$ DCF3( 13) $3.540 E-03$ 3 $3.540 E-03$ ' DCF3( 14) $1.442 E-03$ 3 $1.440 E-03$ ? DCF3( 16) $38.086 E-04$ 3 $3.960 E-04$ 3 DCF3( 18$)$ $4.027 E-03$ $3.730 E-03$ ' $2.730 E-03$ s DCF3( 20$)$ $32.890 E-04$ ' $2.890 E-03$ 'S DCF3( 20$)$ $2.673 E-04$ S $2.660 E-04$ DCF3( 22$)$

$32.500 E-03 ; 2.500 E-033$ RTFC 1,1$)$

$2.000 E-0532.000 E-053 \operatorname{RTF}(1,2)$

$32.000 E-05 \div 2.000 E-05>\operatorname{RTF}(1,3)$

Attachment 


\section{ATTACHMENT 9}

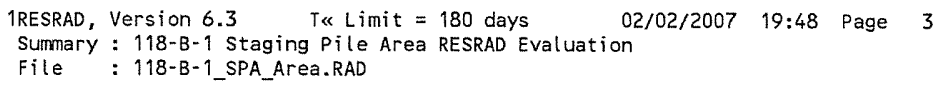

plant/soil concentration ratio, dimensionless beef/livestock-intake ratio, ( $\mathrm{pCi} / \mathrm{kg}) /(\mathrm{pCi} / \mathrm{d})$ - milk/livestock-intake ratio, $(\mathrm{pCi} / \mathrm{L}) /(\mathrm{pCi} / \mathrm{d})$

- plant/soil concentration ratio, dimensionless , beef/livestock-intake ratio, $(\mathrm{pCi} / \mathrm{kg}) /(\mathrm{pCi} / \mathrm{d})$ 3.500E+00 $35.500 E+00 \div \operatorname{RTF}(3,1)$ $3.100 E-0233.100 E-023 \operatorname{RTF}(3,2)$ $31.200 E-02 \div 1.200 E-02 \div \operatorname{RTF}(3,3)$

$8.000 E-02 \times 8.000 E-023 \operatorname{RTF}(4,1)$ $2.000 E-0232.000 E-023 \operatorname{RTF}(4,2)$ $32.000 E-03 \div 2.000 E-03 ; \operatorname{RTF}(4,3)$

4.000E-02 3 4.000E-02 3 RTF( 5,1$)$

3.000E-02 $33.000 E-02 \div \operatorname{RTF}(5,2)$ 8.000E-03 $3.000 \mathrm{E}-03 \div \operatorname{RTF}(5,3)$ $2.500 E-033^{3} 2.500 E-03^{3} \operatorname{RTF}(6,1)$ 2.000 -03 $32.000 E-03$ RTF( 6,2$)$ $35.000 E-0535.000 E-05 ? \operatorname{RTF}(6,3)$ $32.500 \mathrm{E}-03$ 3 $2.500 \mathrm{E}-03$ 3 RTFC 8,1$)$ $32.000 E-0332.000 E-033$ RTF 8 , 2) $32.000 E-0532.000 E-053 \operatorname{RTF}(8,3)$ $2.000 \mathrm{E}-05,2.000 \mathrm{E}-05, \operatorname{RTF}(8,3)$ $34.800 E+0034.800 E+003 \operatorname{RTF}(9,1)$ $31.200 E-02 ; 1.200 E-02 ; \operatorname{RTF}(9,2)$ $31.000 E-02 \times 1.000 E-02 \times \operatorname{RTF}(9,3)$ $35.000 E-02 \div 5.000 E-023 \operatorname{RTF}(10,1)$ $35.000 E-03 \div 5.000 E-03 \div \operatorname{RTF}(10,2)$ $32.000 E-02=2.000 E-023 \operatorname{RTF}(10,3)$ 2.000E-02 $32.000 E-023$ RTF( 11,1$)$ $31.000 E-0331.000 E-03=\operatorname{RTF}(11,2)$ 5.000E-06?5.000E-06 $3 \operatorname{RTF}(11,3)$ $31.000 E-02,1.000 E-02, \operatorname{RTF}(12,1)$ 3 5.000E-03 $35.000 E-03$ \& RTFC 12,3 3 5.000E-06'5.000E-06' $\operatorname{RTF}(12,3)$

$31.000 E-03 ; 1.000 E-03 ; \operatorname{RTF}(13,1)$ $31.000 E-0431.000 E-043 \operatorname{RTF}(13,2)$ $1.000 E-0631.000 E-06 ; \operatorname{RTF}(13,3)$ 31.0 1.000 E 0331.000 E-03 $3 \operatorname{RTF}(14,1)$ $31.000 E-0431.000 E-043$ RTF $(14,2)$ $31.000 E-0631.000 E-063 \operatorname{RTF}(14,3)$

$\begin{array}{ll}\text { Attachment } \frac{9}{9} & \text { Sheet No. } 3 \text { of } 27 \\ \text { Originators: } \frac{\text { S. W. Clark }}{\text { M. W. Perrott }} & \text { Date } \\ \text { Chk'd By } & \text { Date } \\ \text { Calc. No. } & \text { Rev. No. } \frac{0}{0100 \text { B-CA-V0301 }}\end{array}$




\section{ATTACHMENT 9}

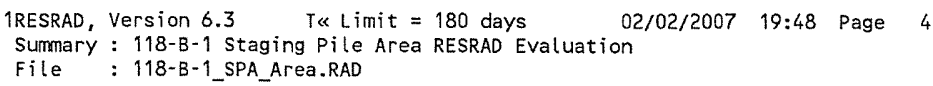




\section{ATTACHMENT 9}

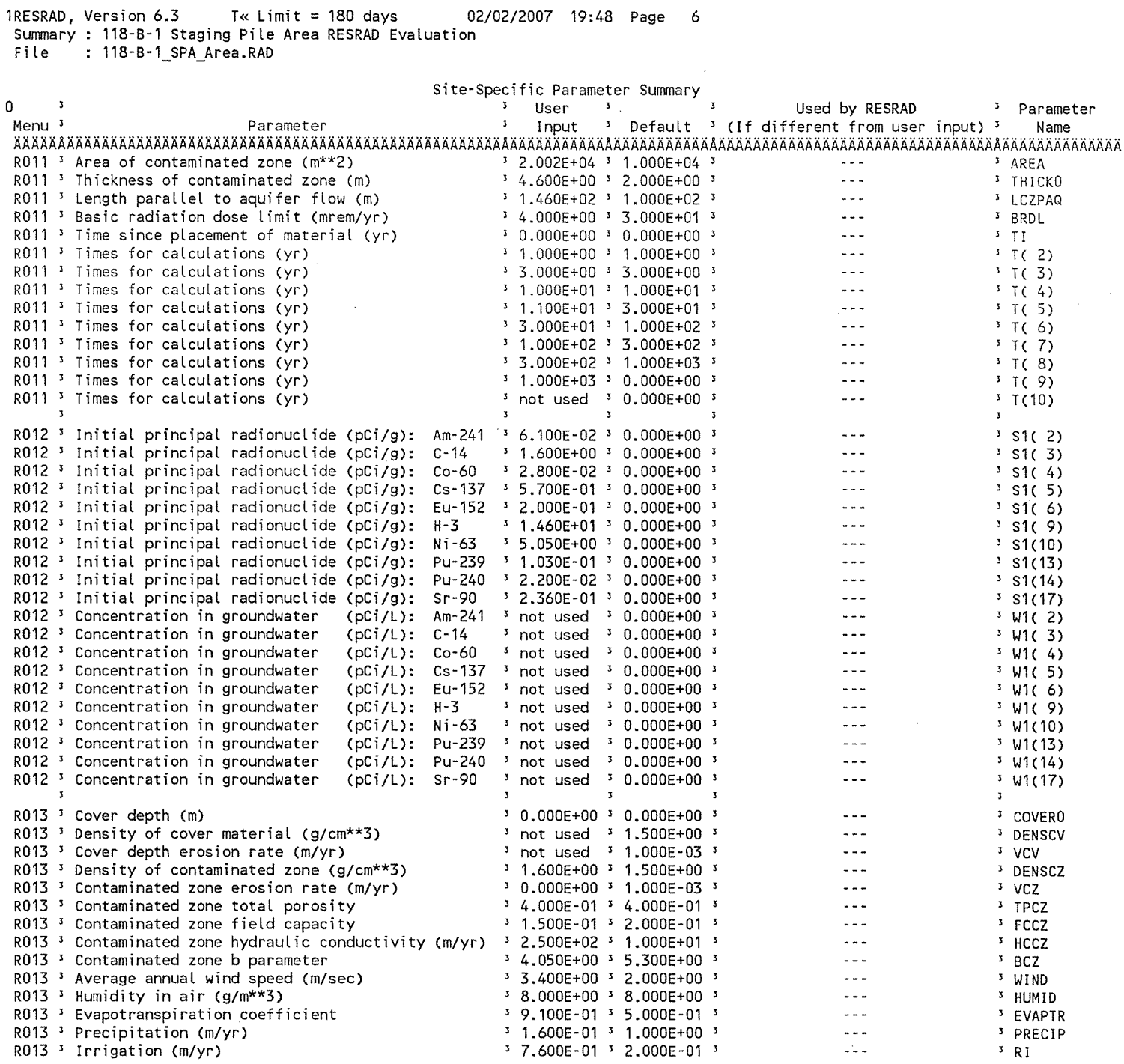

Attachment Originators: S. W. Clark Chk'd By M.W. Perrott Calc. No. 0100B-CA-V0301
Sheet No. $\underline{6}$ of $\underline{27}$ Date

Rev. No. 0 


\section{ATTACHMENT 9}

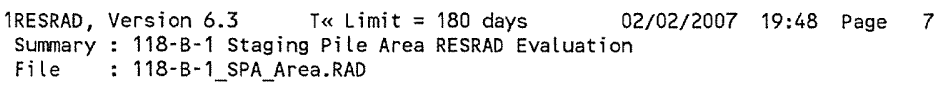

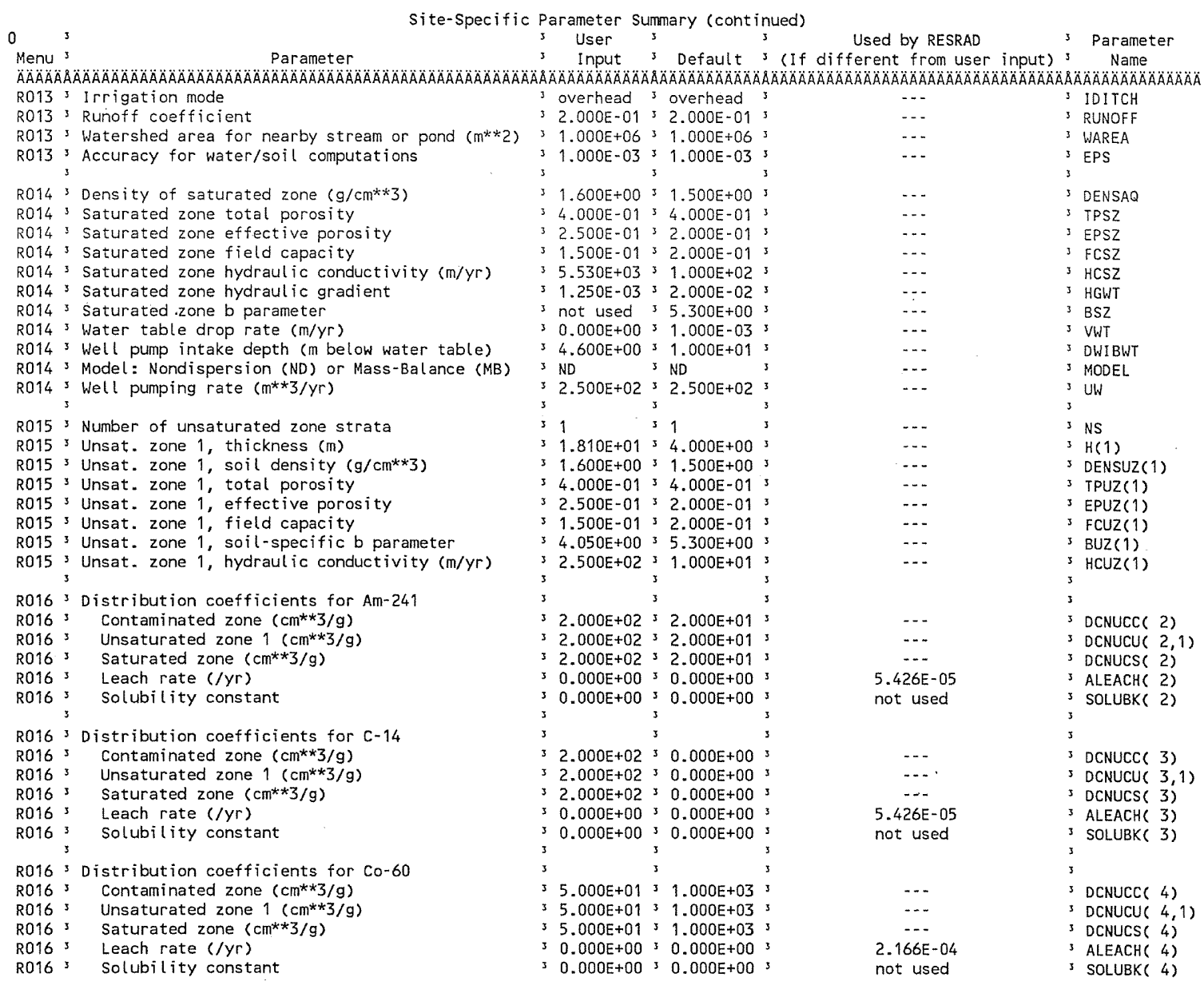




\section{ATTACHMENT 9}

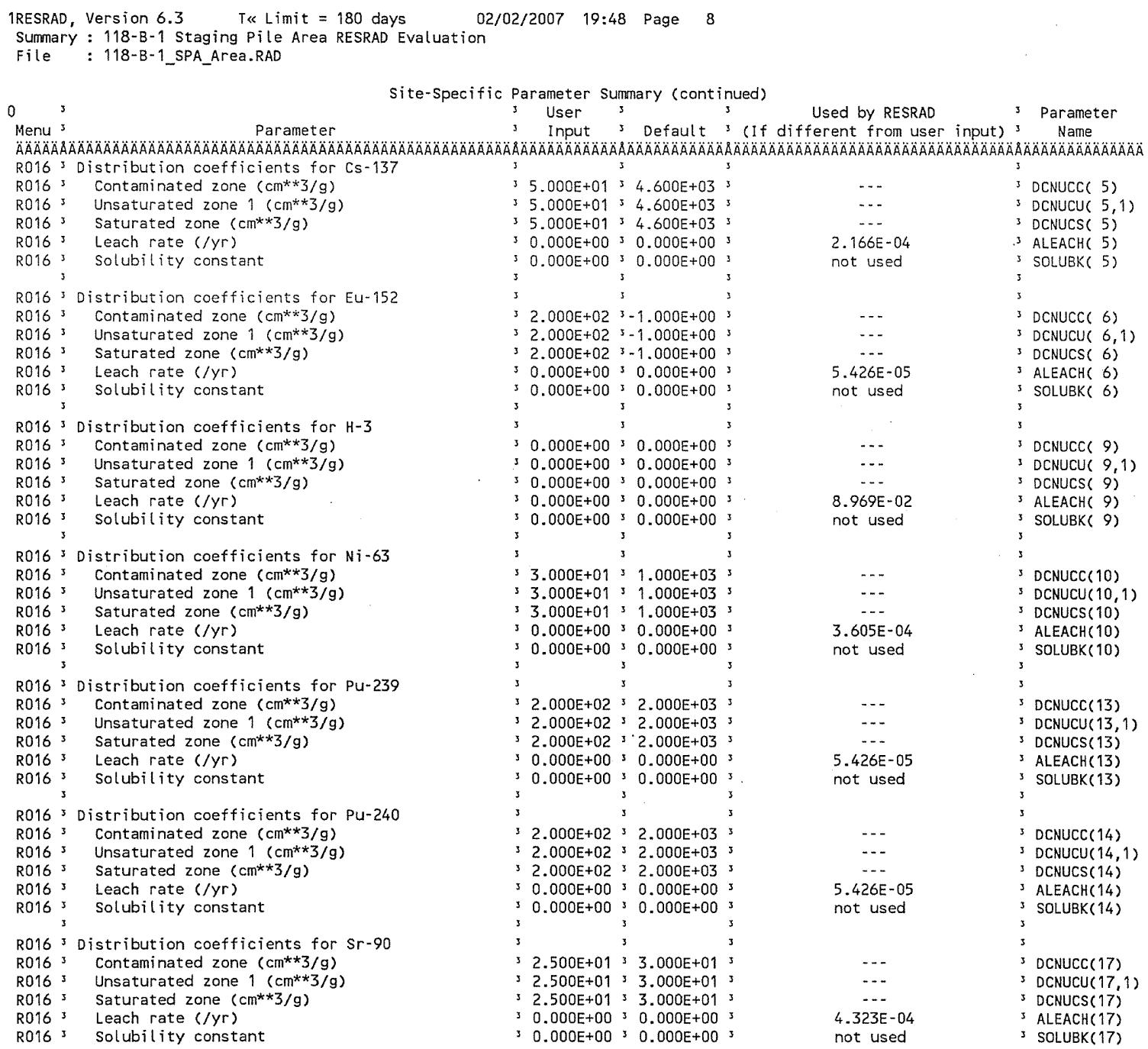




\section{ATTACHMENT 9}

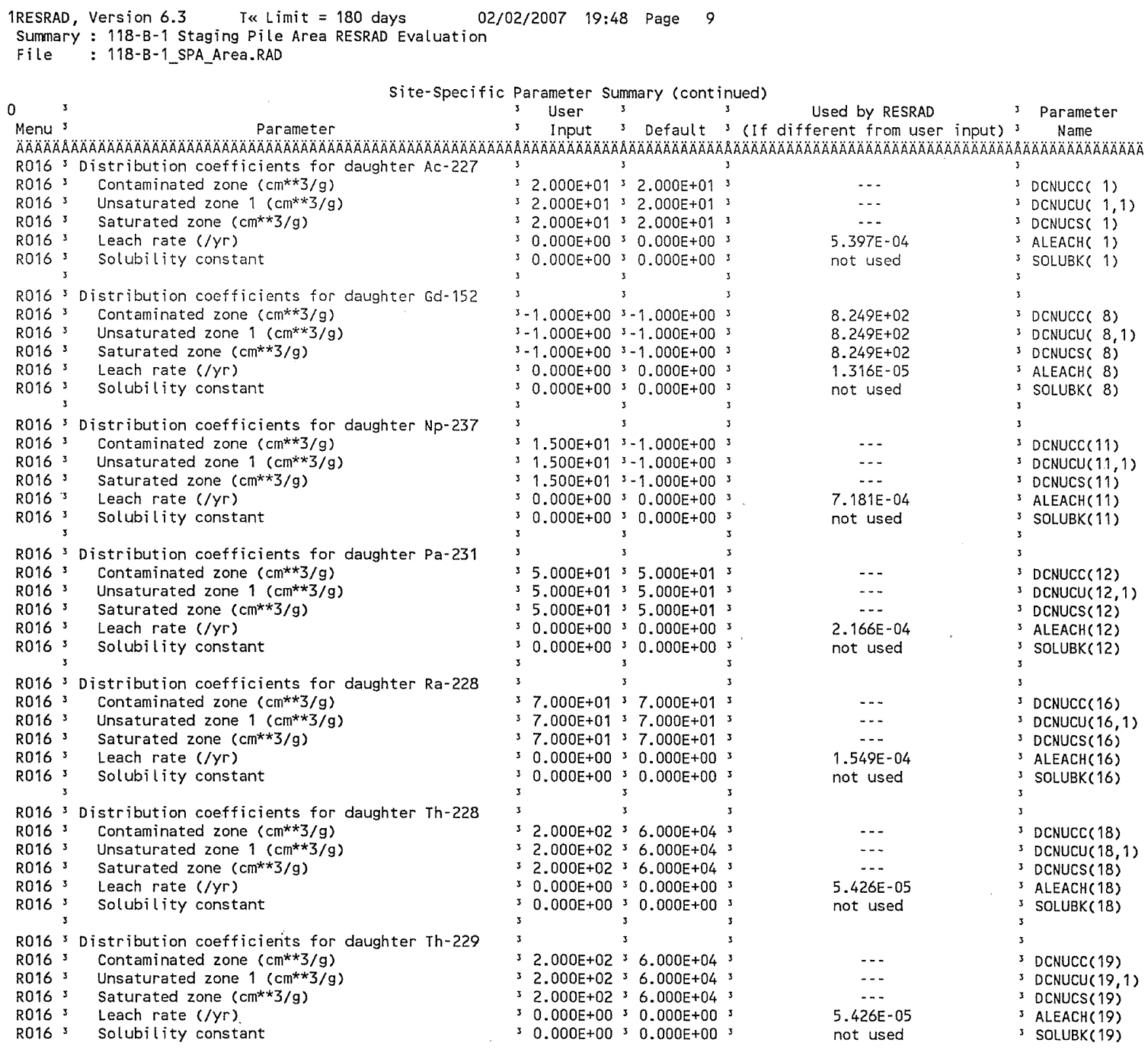

\begin{tabular}{|c|c|}
\hline Attachment & Sheet No. 9 of 27 \\
\hline Originators: S. W. Clark & Date \\
\hline Chk'd By M. W. Perrott & Date \\
\hline Calc. No. $0100 \mathrm{~B}-\mathrm{CA}-\mathrm{V} 0301$ & Rev. No. $\quad 0$ \\
\hline
\end{tabular}




\section{ATTACHMENT 9}

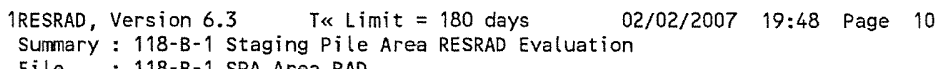

$\ldots$
$\ldots$.
$5.426 E-05$
not used
$\ldots$
$\ldots$

DCNUCC (20)

DCNUCU $(20,1)$

DCNUCS $(20)$

ALEACH (20)

SOLUBK (20)

DCNUCC (21)

3 DCNUCS (21)

5.119E-03 3 ALEACH(21)

not used 3 SOLUBK(21)

-..

... $\quad 3$ DCNUCU $(22,1)$

$119 E-03$

not used

-.. $\quad 3$ DCNUCC (23)

-.. 3 DCNUCU $(23,1)$

3 DCNUCS (23)

5.119E-03 I ALEACH(23)

not used 3 SOLUBK(23)

... 3 INHALR

3 MLINH

-.. 3 ED

-. 3 SHF3

-. SHF1

-.-

$>0$ shows circular AREA. $\quad 3_{3}$ FS

\begin{tabular}{|c|c|}
\hline$\cdots$ & S RAD_SHAPE ( 1) \\
\hline & ${ }^{3}$ RAD_SHAPE ( 2) \\
\hline & $=$ RAD_SHAPE (3) \\
\hline & ${ }^{3}$ RAD_SHAPE ( 4) \\
\hline & 3 RAD_SHAPE ( 5) \\
\hline & 3 RAD_SHAPE( 6) \\
\hline & ${ }^{3}$ RAD_SHAPE ( 7) \\
\hline & ${ }^{3}$ RAD_SHAPE ( 8) \\
\hline & 3 RAD_SHAPE (9) \\
\hline & ${ }^{3}$ RAD_SHAPE $(10)$ \\
\hline & 3 RAD_SHAPE $(11$ \\
\hline & ${ }^{3}$ RAD SHAPE $(1$ \\
\hline
\end{tabular}

Attachment Sheet No. 10 of 27

Chk'd By M.W Perrott

Calc. No. $0100 \mathrm{~B}-\mathrm{CA}-\mathrm{V} 0301 \quad$ Rev. No. 0 


\section{ATTACHMENT 9}

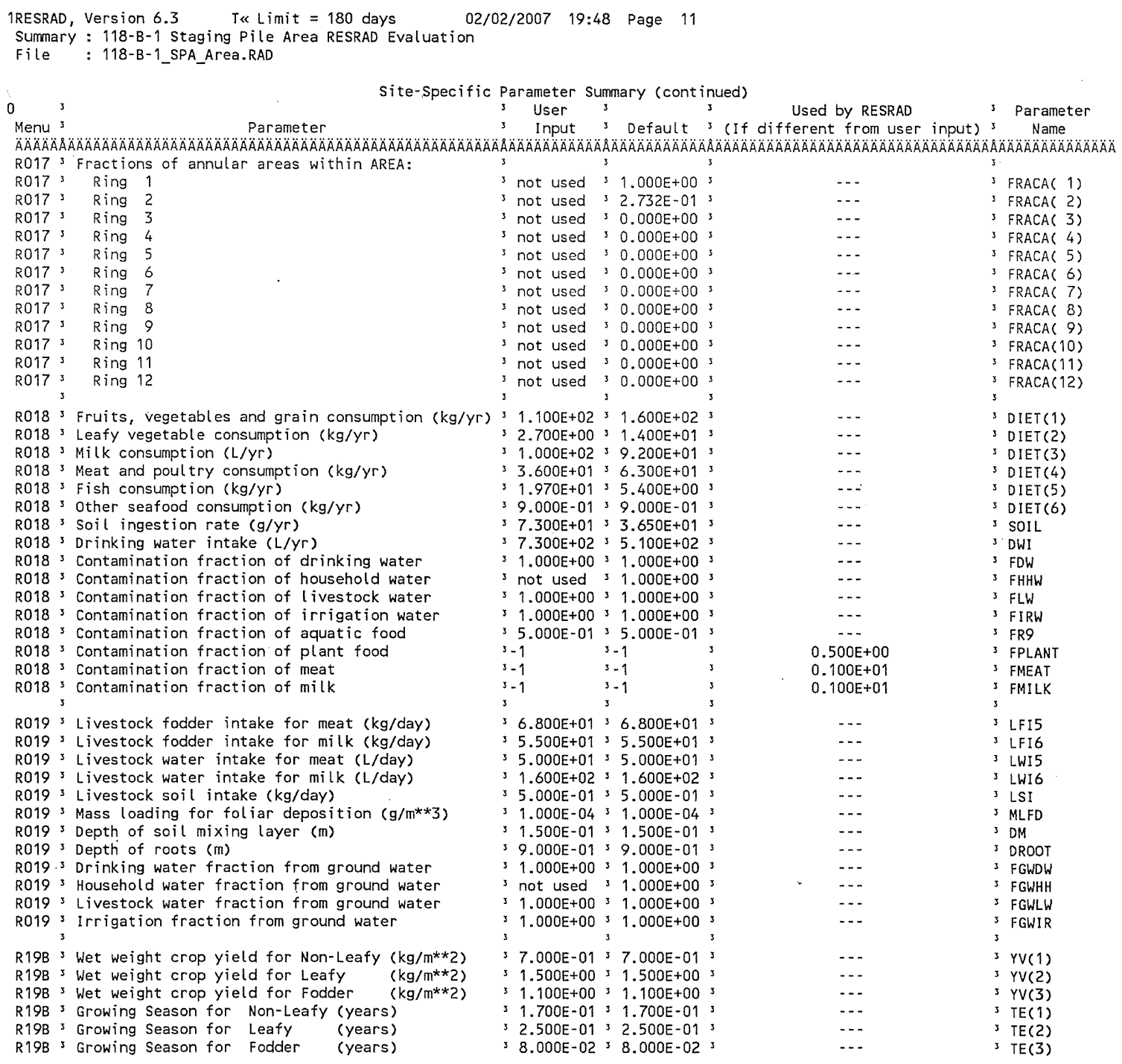

\begin{tabular}{|c|c|}
\hline Attachment & Sheet No. 11 of 27 \\
\hline Originators: S.W. Clark & Date \\
\hline M. W. Perrott & Date \\
\hline 0100B-CA-V0301 & Rev. No. \\
\hline
\end{tabular}




\section{ATTACHMENT 9}

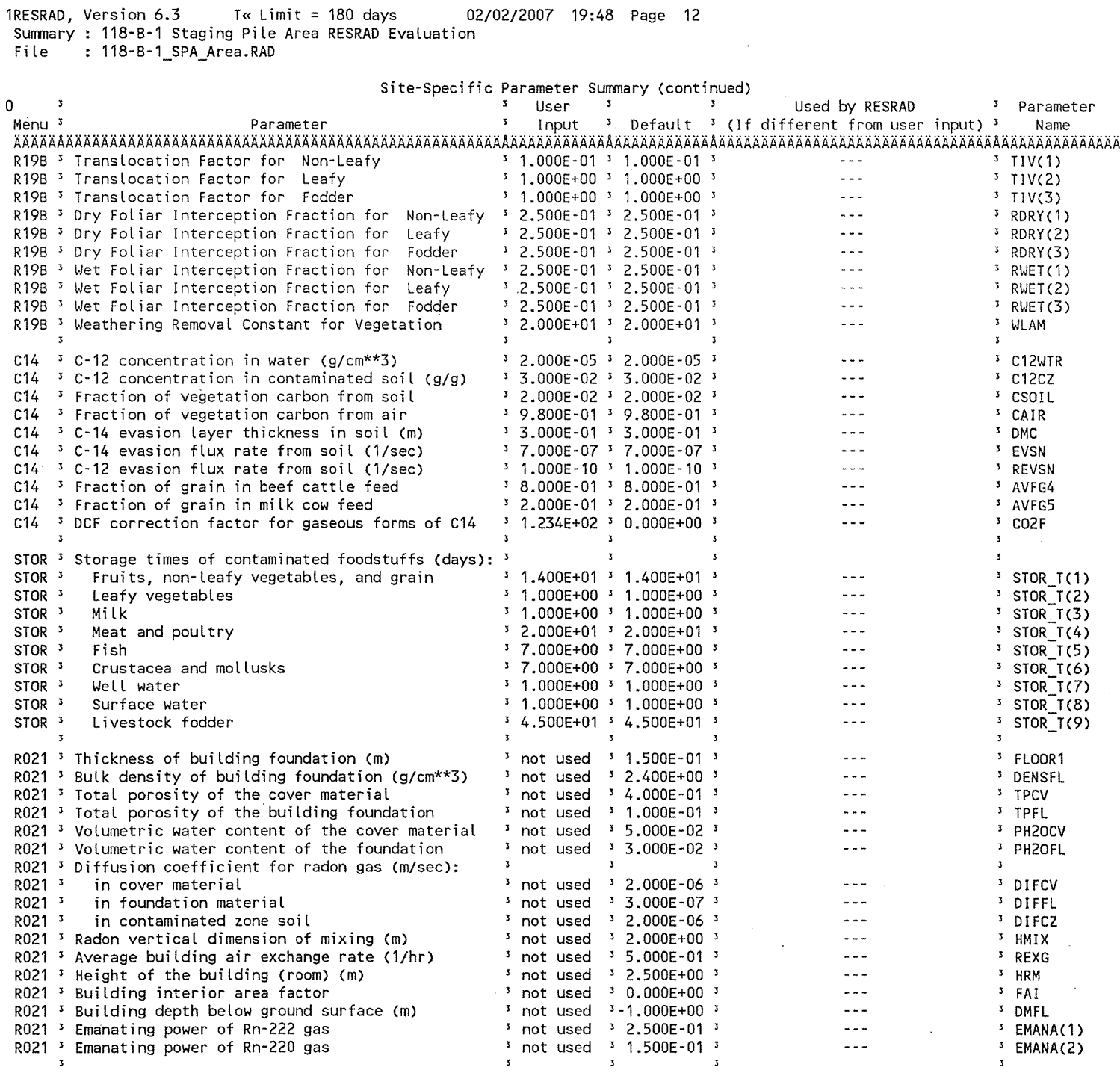

\begin{tabular}{|c|c|c|}
\hline \multicolumn{2}{|c|}{ Attachment $\quad 9$} & \multirow{2}{*}{$\begin{array}{l}\text { Sheet No. } 12 \text { of } 27 \\
\text { Date }\end{array}$} \\
\hline Originators: & S. W. Clark & \\
\hline Chk'd By & M. W. Perrott & Date \\
\hline Calc. No. & $0100 \mathrm{~B}-\mathrm{CA}-\mathrm{V} 0301$ & Rev. No. 0 \\
\hline
\end{tabular}


CVP-2007-00006

Rev. 0

\section{ATTACHMENT 9}

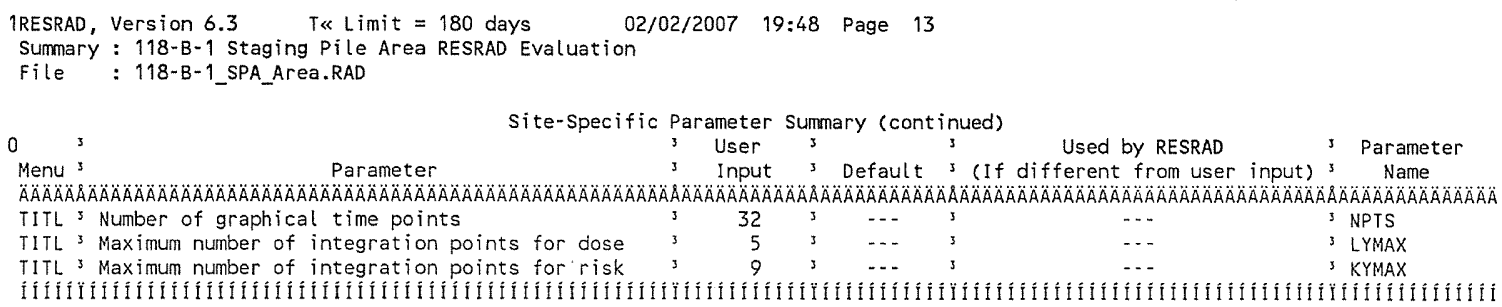




\section{ATTACHMENT 9}

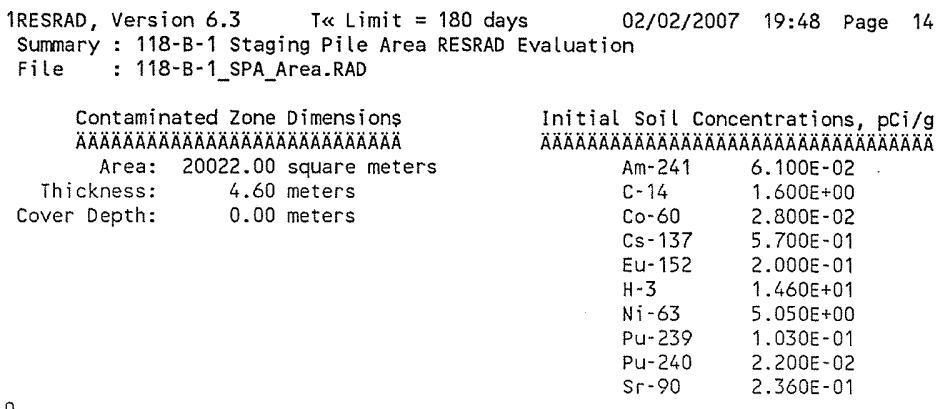

Total Dose $\operatorname{TDOSE}(t), \mathrm{mrem} / \mathrm{yr}$

Basic Radiation Dose Limit $=4.000 E+00 \mathrm{mrem} / \mathrm{yr}$
Total Mixture Sum $M(t)=$ Fraction of Basic Dose Limit Received at Time $(t)$

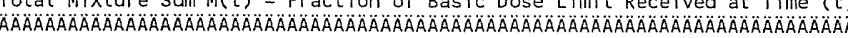

$t$ (years): $\begin{array}{lllllllll}0.000 E+00 & 1.000 E+00 & 3.000 E+00 & 1.000 E+01 & 1.100 E+01 & 3.000 E+01 & 1.000 E+02 & 3.000 E+02 & 1.000 E+03\end{array}$

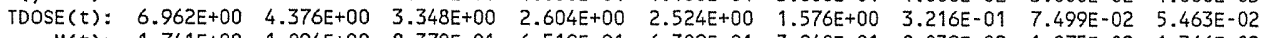
$M(t): \quad \begin{array}{lllllllll}1.741 E+00 & 1.094 E+00 & 8.370 E-01 & 6.510 E-01 & 6.309 E-01 & 3.940 E-01 & 8.039 E-02 & 1.875 E-02 & 1.366 E-02\end{array}$ OMaximum $\operatorname{TDOSE}(t): 6.962 E+00 \mathrm{mrem} / \mathrm{yr}$ at $t=0.000 E+00$ years

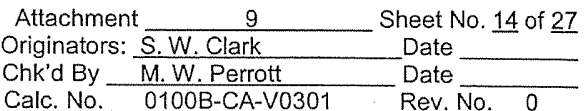




\section{ATTACHMENT 9}

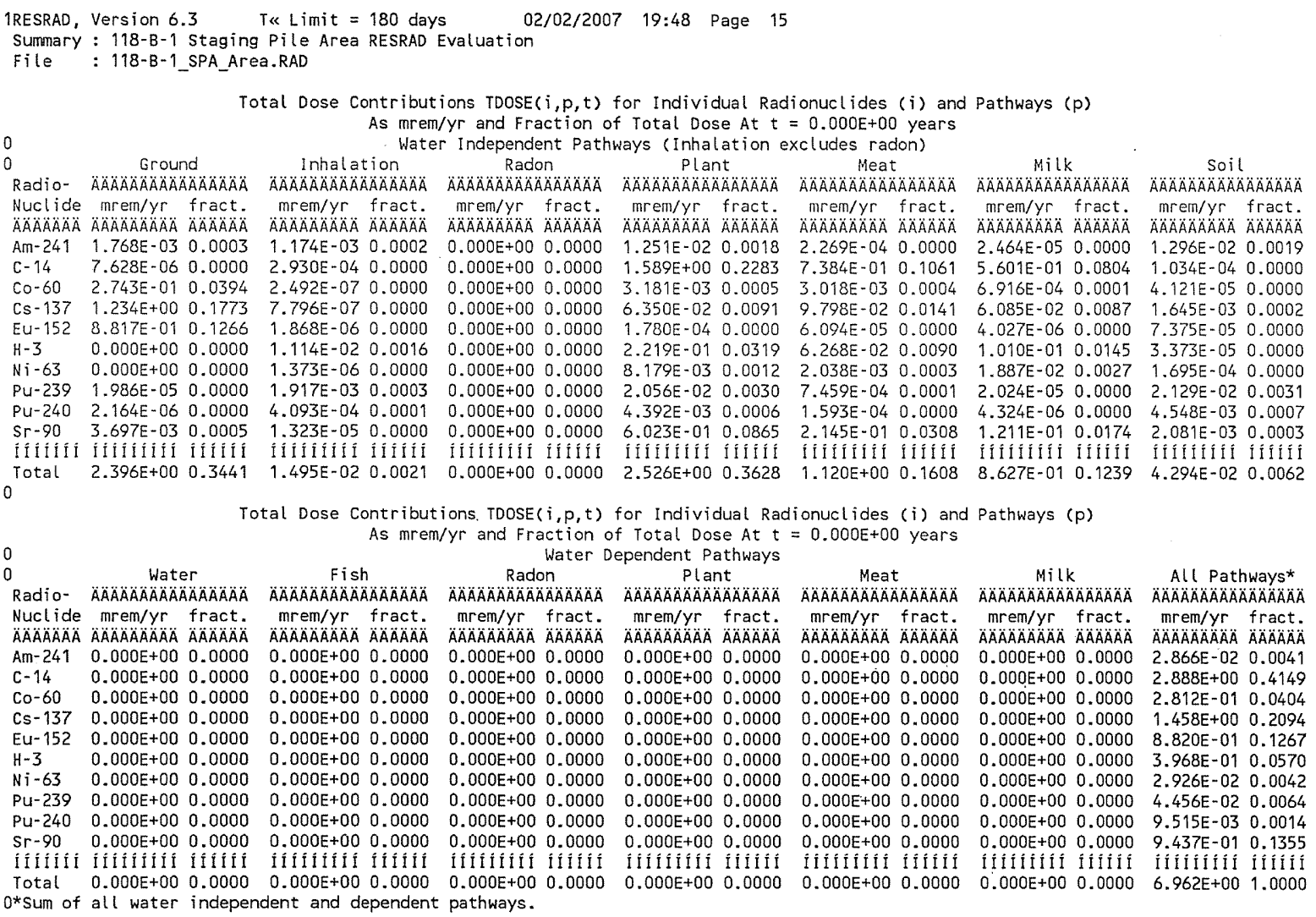

$\begin{array}{ll}\text { Attachment } \frac{9}{\text { S.W.Clark }} & \text { Sheet No. } 15 \text { of } \underline{27} \\ \text { Originators: } & \text { Date } \\ \text { Chk'd By } & \text { Date } \\ \text { Calc. No. Perrott } & \text { Rev. No. N } \quad \text { O100B-CA-V0301 }\end{array}$




\section{ATTACHMENT 9}

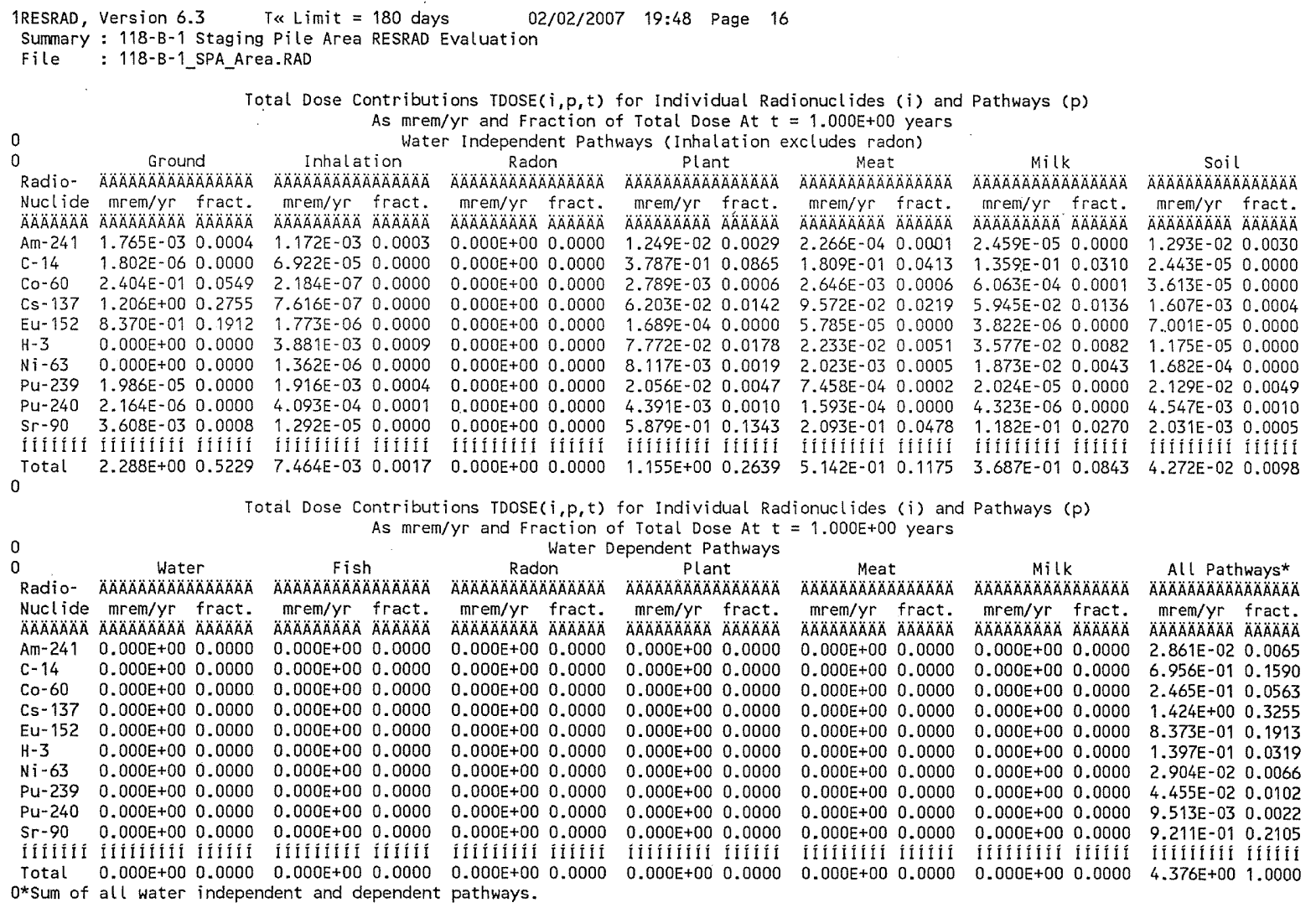

Milk SOil

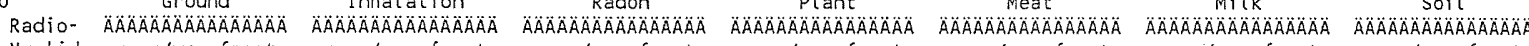
Nuclide $m r e m / y r$ fract. mrem/yr fract. mrem/yr fract. mrem/yr fract. mrem/yr fract. mrem/yr fract. mrem/yr fract.

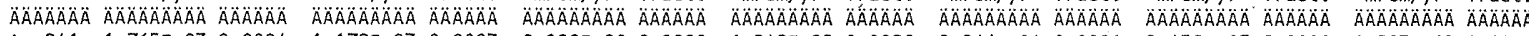

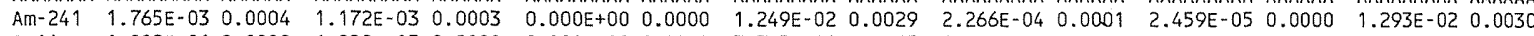

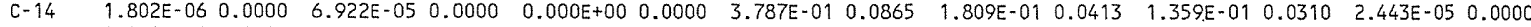
$\begin{array}{lllllllllllllll}C 0-60 & 2.404 E-01 & 0.0549 & 2.184 E-07 & 0.0000 & 0.000 E+00 & 0.0000 & 2.789 E-03 & 0.0006 & 2.646 E-03 & 0.0006 & 6.063 E-04 & 0.0001 & 3.613 E-05 & 0.0000\end{array}$

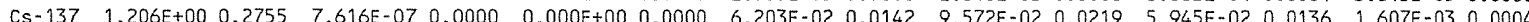

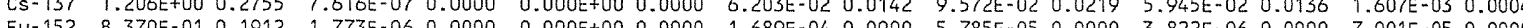

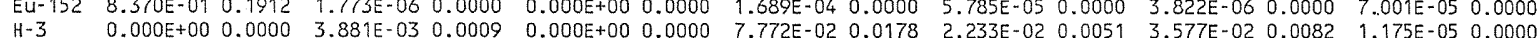

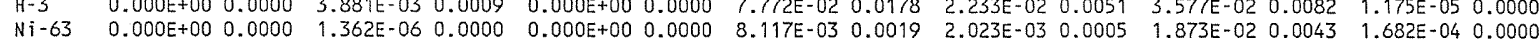

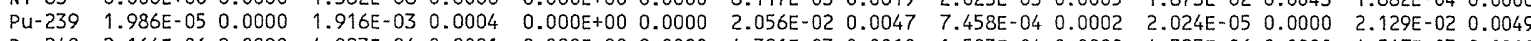

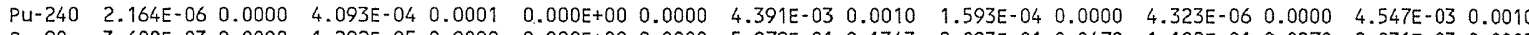
$\begin{array}{lllllllllllllll}\mathrm{Sr}-90 & 3.608 \mathrm{E}-03 & 0.0008 & 1.292 \mathrm{E}-05 & 0.0000 & 0.000 \mathrm{E}+00 & 0.0000 & 5.879 \mathrm{E}-01 & 0.1343 & 2.093 \mathrm{E}-01 & 0.0478 & 1.182 \mathrm{E}-01 & 0.0270 & 2.031 \mathrm{E}-03 & 0.0005\end{array}$

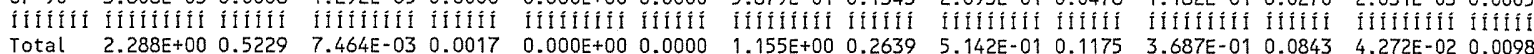
Total $2.288 \mathrm{E}+00 \quad 0.5229$

Total Dose Contributions $\operatorname{TDOSE}(i, p, t)$ for Individual Radionuclides (i) and Pathways ( $p$ ) As mrem/yr and Fraction of Total Dose At $t=1.000 E+00$ years

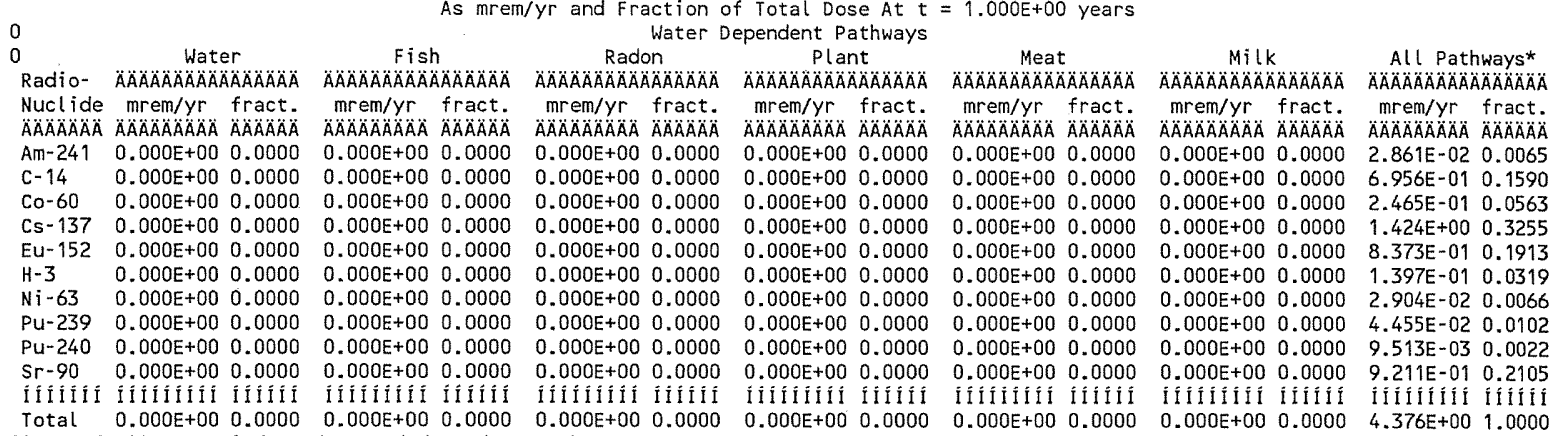
0 *Sum of all water independent and dependent pathways.

\begin{tabular}{|c|c|}
\hline Attachment & Sheet No. 16 of 27 \\
\hline Originators: S.W. Clark & Date \\
\hline Chk'd By M.W. Perrott & Date \\
\hline $0100 B-C A-V 0301$ & Rev. No. $\quad 0$ \\
\hline
\end{tabular}




\section{ATTACHMENT 9}

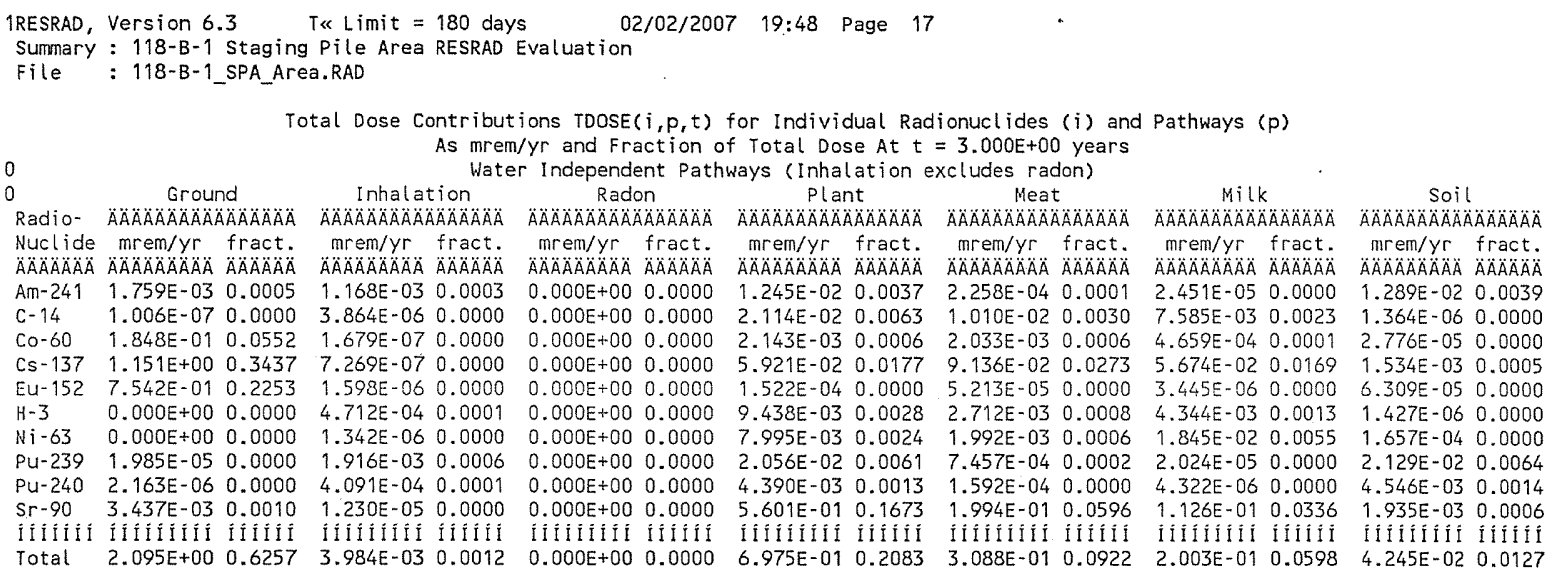

\begin{tabular}{|c|c|}
\hline Attachment & Sheet No. 17 of 27 \\
\hline Originators: S.W. Clark & Date \\
\hline Chk'd By M. W. Perrott & Date \\
\hline Calc. No. $0100 \mathrm{~B}-\mathrm{CA}-\mathrm{V} 0301$ & Rev. No. \\
\hline
\end{tabular}




\section{ATTACHMENT 9}

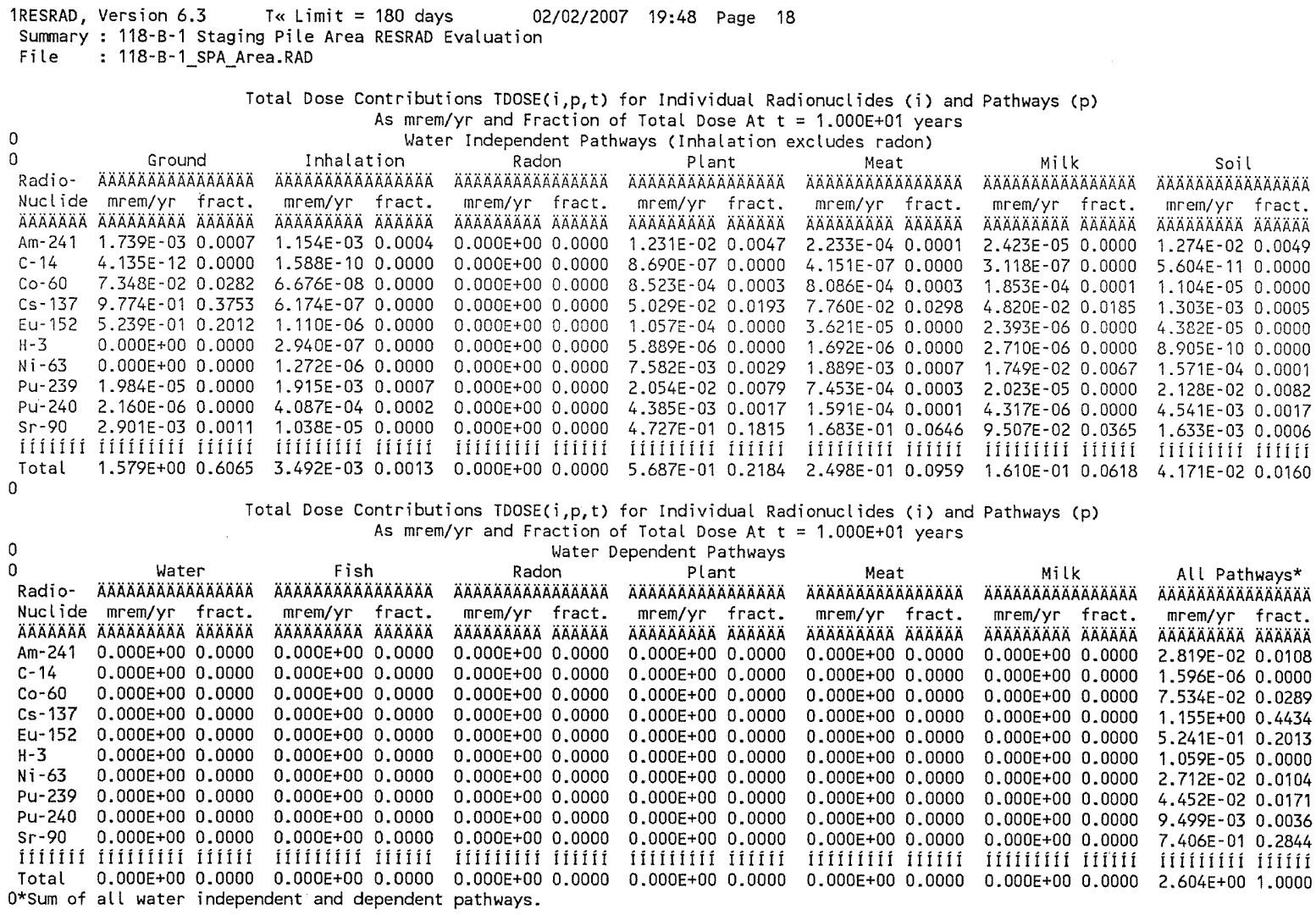

\begin{tabular}{|c|c|}
\hline Attachment & Sheet No. 18 of 27 \\
\hline Originators: S.W. Clark & Date \\
\hline M. W. Perrott & Date \\
\hline 0100B-CA-V0301 & Rev. No. \\
\hline
\end{tabular}




\section{ATTACHMENT 9}

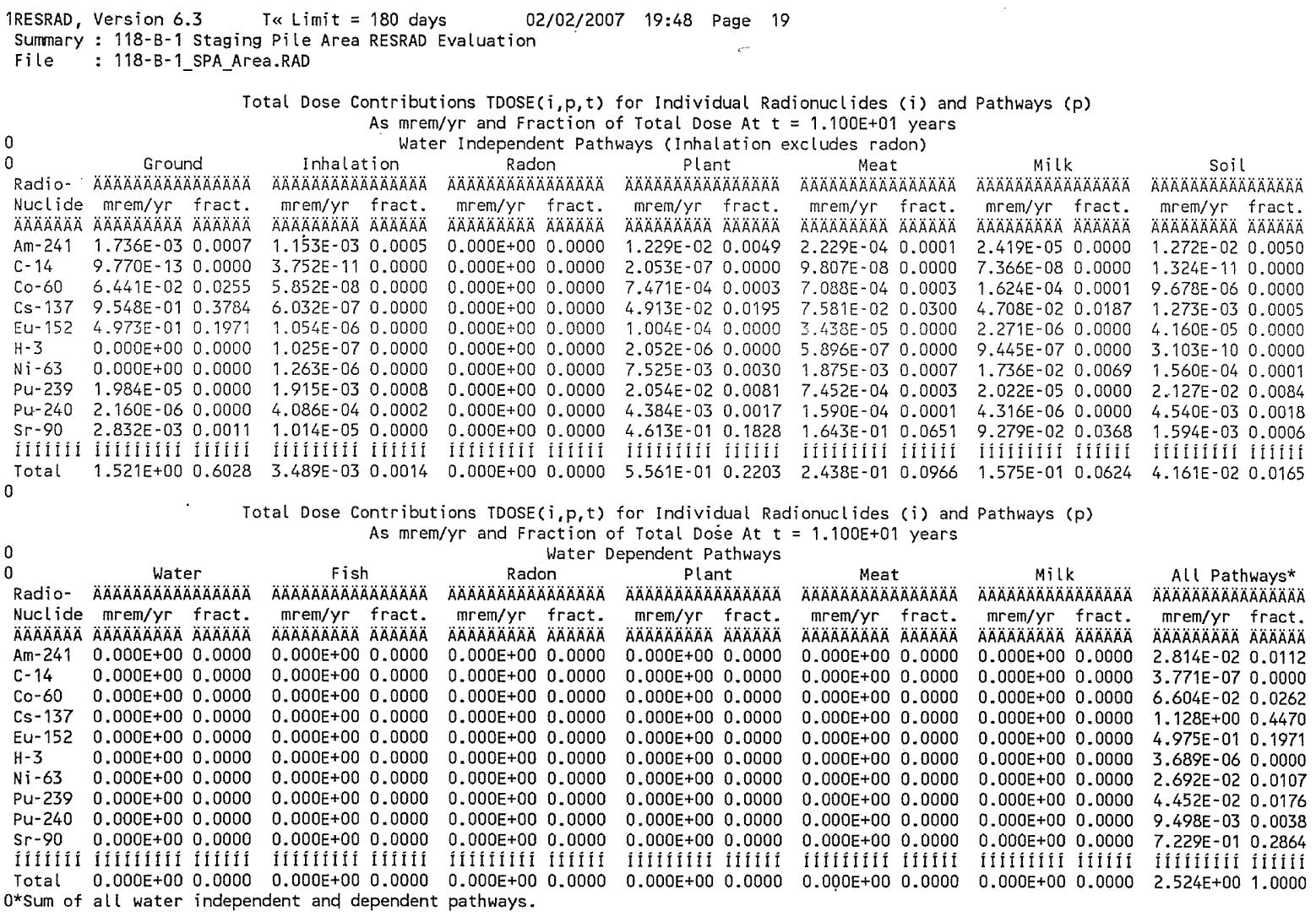

\begin{tabular}{ll} 
Attachment $\frac{9}{2}$ & Sheet No. 19 of 27 \\
Originators: $\frac{\text { S.W. Clark }}{\text { M.W. Perrott }}$ & Date \\
Chk'd By & Date \\
Calc. No. No. & Rev. No. $\quad 0$ \\
\hline O100B-CA-V0301 & Rev.
\end{tabular}


CVP-2007-00006

Rev. 0

\section{ATTACHMENT 9}

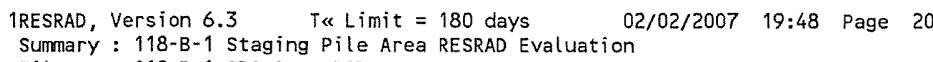

Attachment 9 


\section{ATTACHMENT 9}

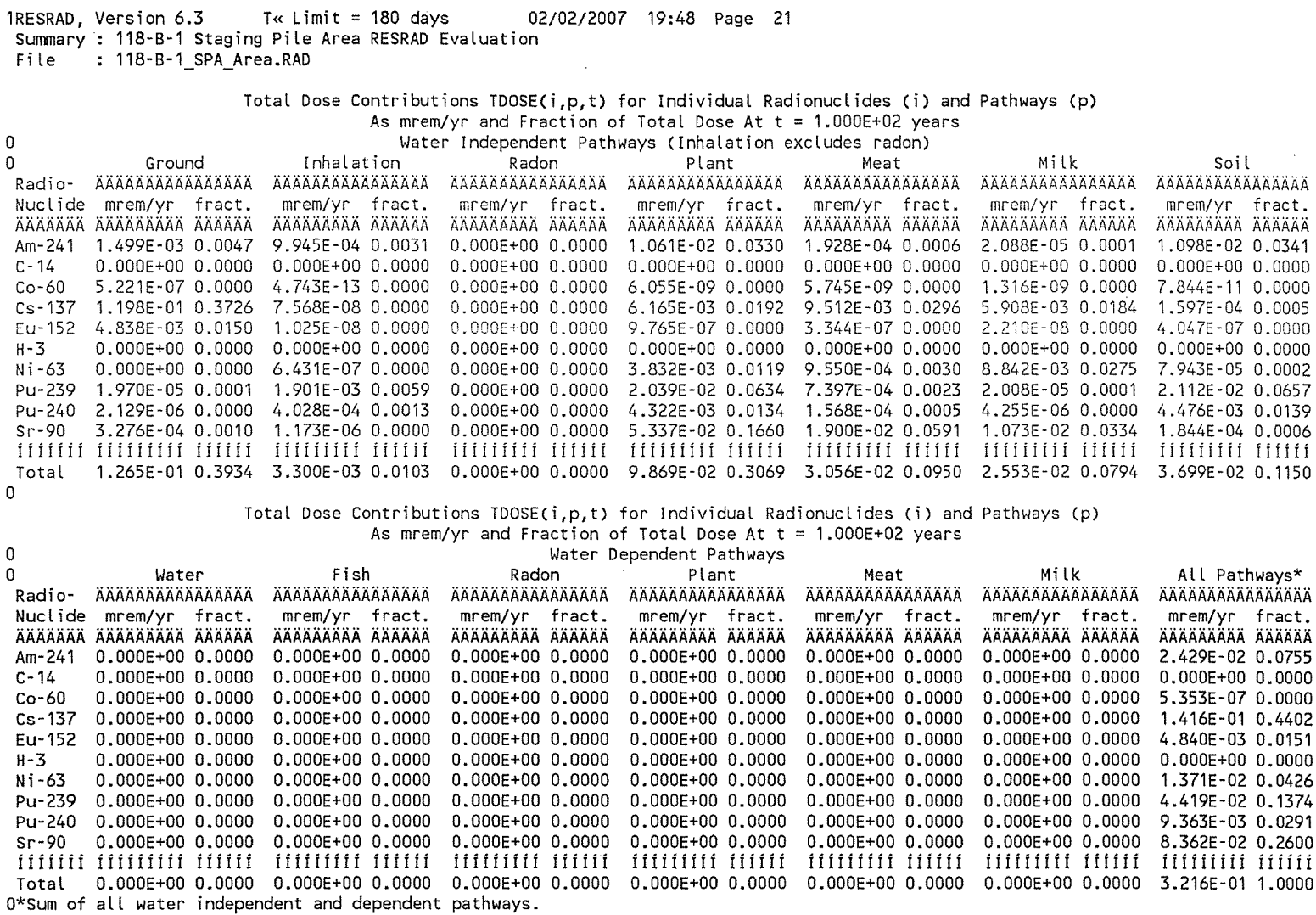

$\begin{array}{ll}\text { Attachment } \frac{9}{\text { S.W. Clark }} & \text { Sheet No. } 21 \text { of } 27 \\ \text { Originators: } & \text { Date } \\ \text { Chk'd By } & \text { Date } \\ \text { Calc. N. No. Perrott } & \text { Rev. No. } \quad 0\end{array}$




\section{ATTACHMENT 9}

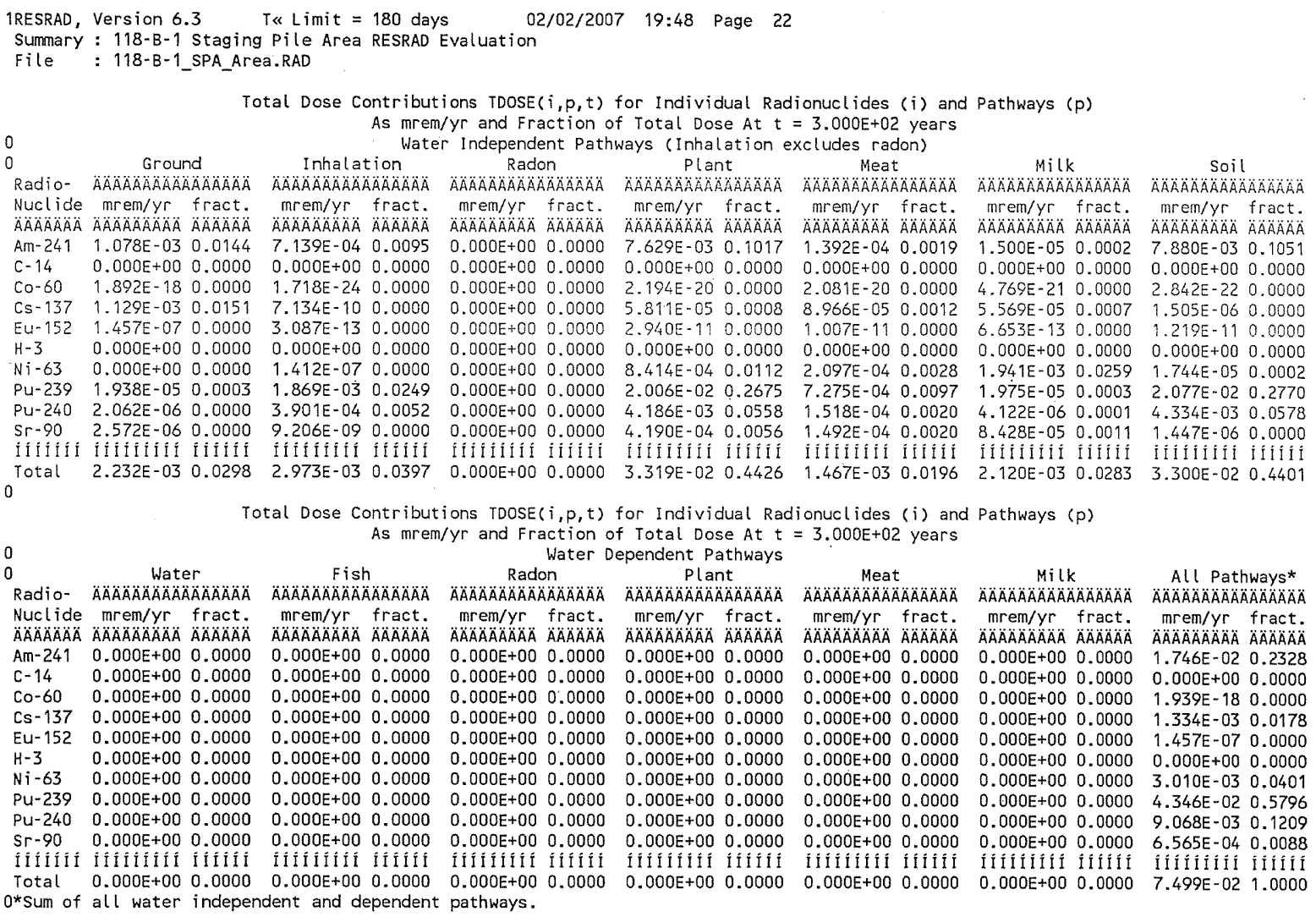

\begin{tabular}{|c|c|}
\hline Attachment & Sheet No. \\
\hline Originators: S.W. Clark & Date \\
\hline M. W. Perrott & Date \\
\hline $0100 B-C A-V 0301$ & Rev. No. 0 \\
\hline
\end{tabular}




\section{ATTACHMENT 9}

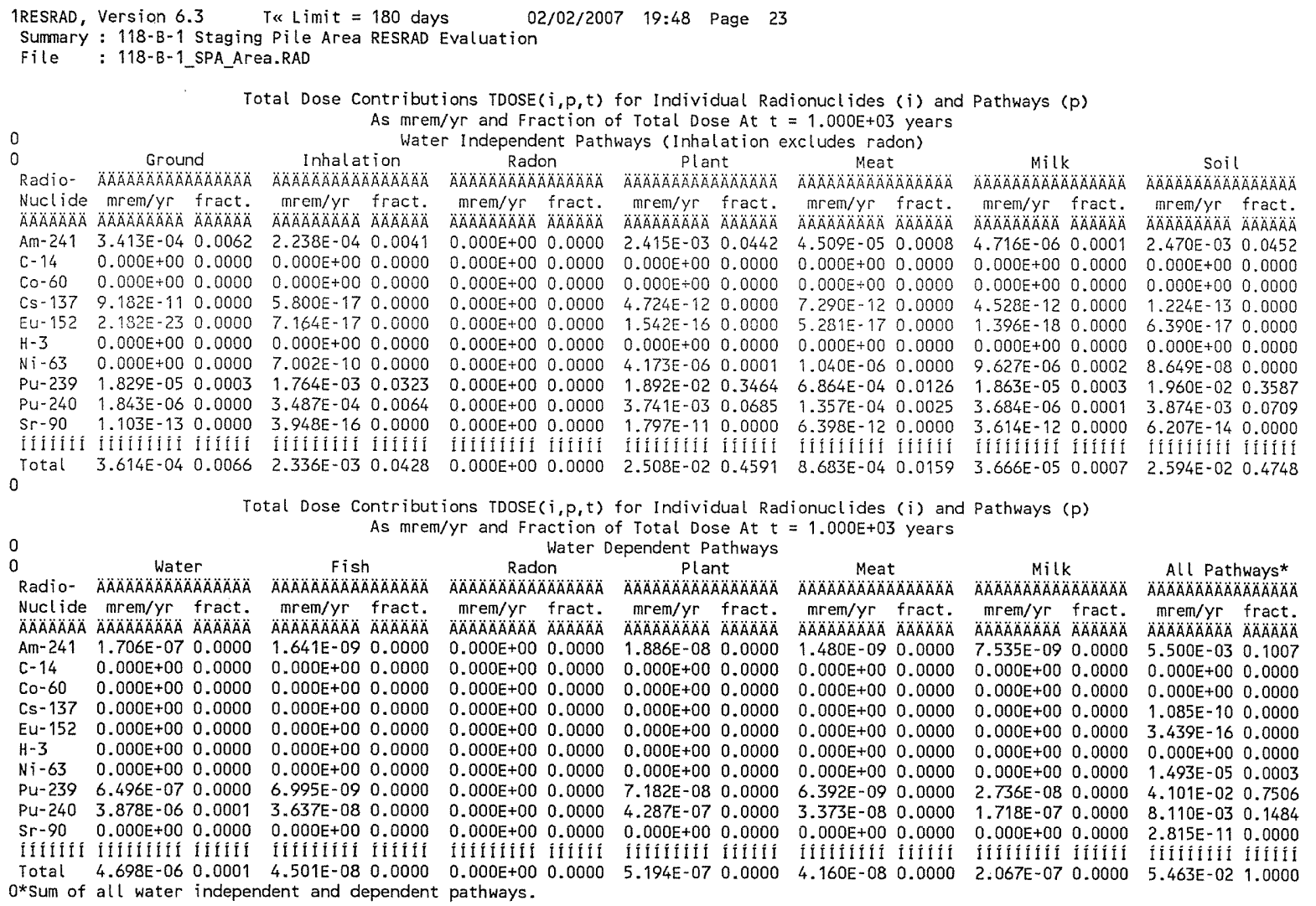

Total Dose Contributions $\operatorname{TDOSE}(i, p, t)$ for Individual Radionuclides (i) and Pathways ( $p$ )

As mrem/yr and Fraction of Total Dose At $t=1.000 E+03$ years

\begin{tabular}{|c|c|c|c|c|c|c|c|c|c|c|c|c|c|}
\hline & & & & & & & & & & & & & \\
\hline 0 & Wate & & Fish & & Rad & & Plan & & Meat & & Mill & & All Pathways \\
\hline adio- & $\ddot{A} \ddot{A}$ & $A \ddot{A}$ & & & & $\ddot{A} A \ddot{A} A \ddot{A}$ & $\triangle A B A A$ & $\triangle A ̈ A ̈ A ̈ A ̈$ & $\triangle A A A B$ & & $\triangle A A A A$ & $\triangle A O A ̈ A ̈$ & 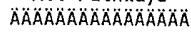 \\
\hline $\begin{array}{l}\text { Nuclide } \\
\text { ÄÄÄÄÄÄ }\end{array}$ & & $\begin{array}{l}\text { fract. } \\
A A A A B A B A\end{array}$ & $\begin{array}{c}m r e m / y r \\
A A A A A A A A B\end{array}$ & $\begin{array}{l}\text { fract. } \\
A A A A A B A\end{array}$ & A & $\begin{array}{l}\text { fract. } \\
\ddot{A A} \ddot{A} \ddot{A} \ddot{A}\end{array}$ & $\triangle A A A A A A A A A B$ & 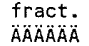 & $\begin{array}{r}m r e \\
\triangle A B A M\end{array}$ & $\begin{array}{l}\text { trá } \\
\ddot{A} \ddot{A} \ddot{A}\end{array}$ & $A ̈ A \ddot{A}$ & & $\begin{array}{ll}\Gamma & f r a \\
\triangle A A & A A B A\end{array}$ \\
\hline$A m=241$ & $1.706 \mathrm{E}-07$ & 0.0000 & $1.641 E-09$ & 0.0000 & $0.000 E+00$ & 0.0000 & $1.886 E-08$ & 0.0000 & $1.480 \mathrm{E}-09$ & 0.0000 & $7.535 \mathrm{E}-09$ & 0.0000 & $\begin{array}{cl}E-03 & 0.1007\end{array}$ \\
\hline$c-14$ & $0.000 E+00$ & 0.0000 & $0.000 E+00$ & 0.0000 & $0.000 E+00$ & 0.0000 & $0.000 E+00$ & 0.0000 & $O E+00$ & 0.0 & $O E+00$ & & $=00 \quad 0.0000$ \\
\hline $\mathrm{Co}-60$ & $0.000 E+00$ & 0.0000 & $0.000 E+00$ & 0.0000 & $0.000 E+00$ & 0.0000 & $O E+00$ & 0.0000 & $0 E+00$ & 0.0 & $E+00$ & 0 & 000 \\
\hline Cs -137 & $0.000 E+00$ & 0.0000 & $E+D O$ & 0.0000 & $0.000 E+00$ & 0.0000 & $E+00$ & 0.0000 & $=00$ & 0.0000 & $O E+00$ & 0.0000 & $\begin{array}{ll}-10 & 0.0000\end{array}$ \\
\hline Eu-152 & 0.00 & & +00 & & $+\infty$ & & & & & & $O E+00$ & 0.0000 & -160.0000 \\
\hline $\mathrm{H}-3$ & $E+00$ & 0.0000 & $0.000 E+00$ & 0.0000 & $0.000 E+00$ & 0.0000 & $E+00$ & 0. & .00 & 0. & & 10 & \\
\hline $\mathrm{Ni}-6$ & 0.00 & 0.0000 & $E+00$ & 0.0000 & $0.000 \mathrm{E}+00$ & 0.0000 & $0.000 E+00$ & 0.0000 & $+\infty$ & 0.0000 & $0 E+00$ & 0.0000 & $-05 \quad 0.0003$ \\
\hline Pu-239 & & & $E-09$ & & $E+\infty 0$ & & $E-08$ & & & 0. & -08 & bo & -020.7506 \\
\hline Pu- 240 & 3.8 & 0.0001 & $E-08$ & 0 . & $E+\infty O$ & 0.0 & $=-07$ & & & & & & \\
\hline & 0.00 & 0.0000 & $E+\infty$ & 0.0000 & $0.000 E+00$ & 0.0000 & $E+\infty$ & 0.0 & $E+\infty$ & 0.0 & $O E+\infty O$ & 0.0000 & $\begin{array}{ll}-11 & 0.0\end{array}$ \\
\hline & & & & & & & & & & & IIIIIII & IIIIIII & İII IIIIII \\
\hline Tota & $4.698 \mathrm{E}-06$ & 0.0001 & $4.501 E-08$ & 0.0000 & $+\infty$ & 0.0000 & $5.194 E-07$ & 0.0000 & 4.160E-08 & 0.0000 & $2.067 E-07$ & 0.0000 & $5.463 \mathrm{E}-021.0000$ \\
\hline
\end{tabular}
$0 *$ Sum of all water independent and dependent pathways.

$\begin{array}{ll}\text { Attachment } \frac{9}{9 . \text { W. Clark }} & \text { Sheet No. } 23 \text { of } 27 \\ \text { Originators: } \frac{\text { S. W.te }}{\text { M. W. Perrott }} & \text { Date } \\ \text { Chk'd By } & \text { Rev. No. } 0 \\ \text { Calc. No. } & 0100 \text { B-CA-V0301 }\end{array}$




\section{ATTACHMENT 9}

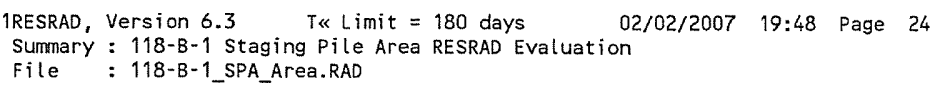

\begin{tabular}{|c|c|c|}
\hline & Sheet No. 24 of 27 \\
\hline \multicolumn{2}{|c|}{$\begin{array}{c}\text { Attachment } \frac{9}{\text { Originators: }} \text { S.W. Clark } \\
\text { Oring }\end{array}$} & Date \\
\hline Chk'd By & M.W. Perrott & Date \\
\hline No. & $0100 B-C A-V 0301$ & No. \\
\hline
\end{tabular}


CVP-2007-00006

Rev. 0

\section{ATTACHMENT 9}

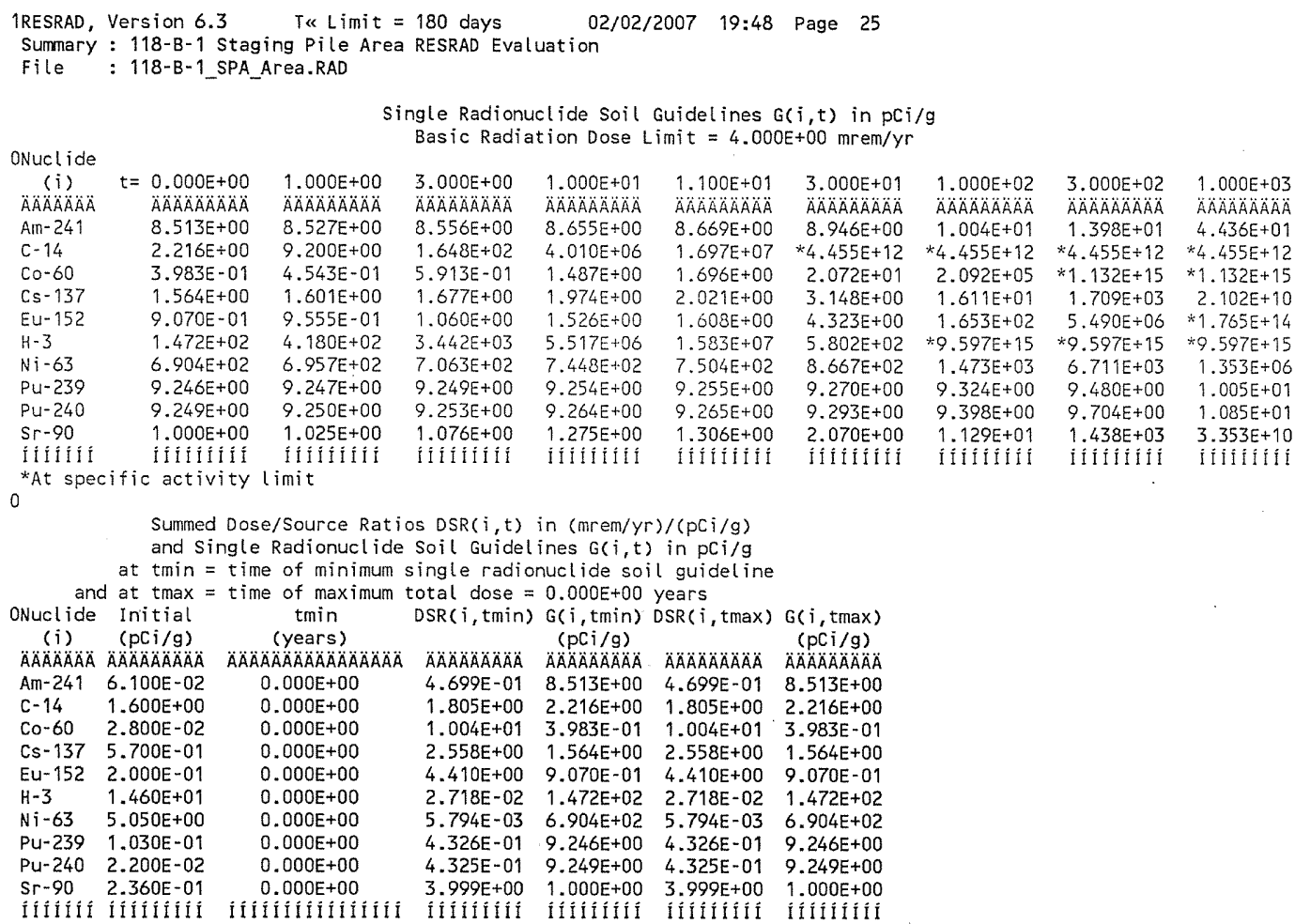$$
\text { Attachment }
$$$$
9
$$

ott

Chk'd By $\frac{\text { M.W. Perrott }}{\text { Calc. No. }}$
0100B-CA-V0301 Sheet No. 25 of 27 Date Date Rev. No. 0 
CVP-2007-00006

Rev. 0

\title{
ATTACHMENT 9
}

\begin{abstract}
1RESRAD, Version 6.3 Te Limit $=180$ days 02/02/2007 19:48 Page 26
Summary : 118-B-1 Staging Pile Area RESRAD Evaluation

file : 118-B-1_SPA_Area.RAD

Individual Nuclide Dose Sumned Over All Pathways

Parent Nucl ide and Branch Fraction Indicated

ONuclide Parent THF(i) $\quad t=0.000 E+001,000 E+003.000 E+001.000 E+01, i, t), \mathrm{mrem} / \mathrm{yr}$

(j) (i) $\quad t=0.000 E+001.000 E+003.000 E+001.000 E+011100 E+013.000 E+011.000 E+023.000 E+021.000 E+03$

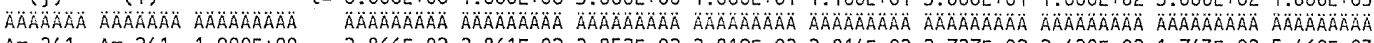

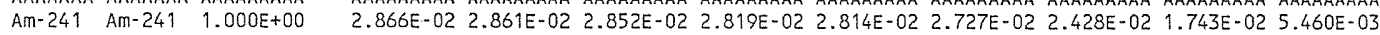

ONP-237 Am-241 1.000E+00 5.826E-08 1.822E-07 4.299E-07 1.288E-06 1.409E-06 3.661E-06 1.111E-05 2.628E-05 3.938E-05

OU-233 Am-241 $1.000 \mathrm{E}+00 \quad 1.656 \mathrm{E}-15 \quad 9.564 \mathrm{E}-15 \quad 4.528 \mathrm{E}-14 \quad 3.739 \mathrm{E}-13 \quad 4.461 \mathrm{E}-13 \quad 2.935 \mathrm{E}-12 \quad 2.664 \mathrm{E}-11 \quad 1.491 \mathrm{E}-10 \quad 1.999 \mathrm{E}-07$

OTh-229 Am-241 1.000E+DO $5.761 E-19 \quad 8.322 E-18 \quad 9.547 E-17 \quad 2.483 E-15 \quad 3.254 E-15 \quad 5.838 E-14 \quad 1.831 E-12 \quad 3.434 E-11 \quad 1.044 E-09$

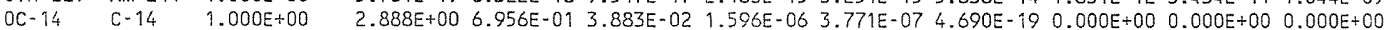

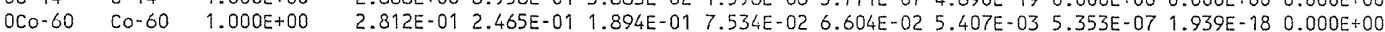

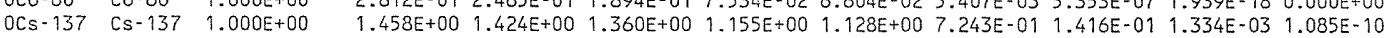

OEU-152 EU-152 7.208E-01 6.357E-01 6.035E-01 5.438E-01 3.778E-01 3.586E-01 1.334E-01 3.488E-03 1.050E-07 1.573E-23

EU-152 EU-152 2.792E-01 2.463E-01 2.338E-01 2.106E-01 1.463E-01 1.389E-01 5.166E-02 1.351E-03 4.069E-08 6.094E-24

8.820E-01 8.373E-01 7.545E-01 5.241E-01 4.975E-01 1.850E-01 4.840E-03 1.457E-07 2.183E-23

OGd-152 EU-152 $2.792 \mathrm{E}-01 \quad 8.913 \mathrm{E}-18 \quad 2.613 \mathrm{E}-17 \quad 5.800 \mathrm{E}-17 \quad 1.467 \mathrm{E}-16 \quad 1.569 \mathrm{E}-16 \quad 2.771 \mathrm{E}-16 \quad 3.462 \mathrm{E}-16 \quad 3.471 \mathrm{E}-16 \quad 3.439 \mathrm{E}-16$

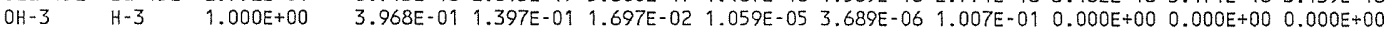

ONi-63 Ni-63 1.000E+00 2.926E-02 2.904E-02 2.860E-02 2.712E-02 2.692E-02 $2.331 E-02 \quad 1.371 E-02 \quad 3.010 E-03 \quad 1.493 E-05$

OPU-239 PU-239 $1.000 E+00 \quad 4.456 E-02 \quad 4.455 E-02 \quad 4.455 E-02 \quad 4.452 E-02 \quad 4.452 E-02 \quad 4.445 E-02 \quad 4.419 E-02 \quad 4.346 E-02 \quad 4.101 E-02$

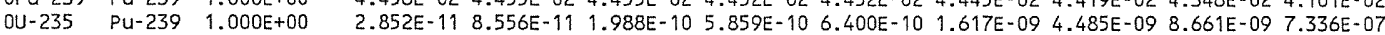

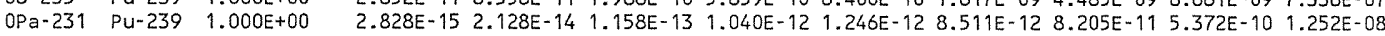

OAC-227 PU-239 $1.000 E+00 \quad 1.672 E-17 \quad 2.220 E-16 \quad 2.368 E-15 \quad 5.630 E-14 \quad 7.312 E-14 \quad 1.138 E-12 \quad 2.395 E-112.273 E-10 \quad 3.042 E-08$

OPU-240 PU-240 4.950E-08 4.710E-10 4.709E-10 4.708E-10 4.702E-10 4.701E-10 4.687E-10 4.635E-10 4.489E-10 4.012E-10

Pu-240 Pu-240 1.000E+00 9.515E-03 9.513E-03 9.510E-03 9.499E-03 9.498E-03 9.469E-03 9.363E-03 9.068E-03 8.105E-03

PU-240 äDOSE(j) $\quad 9.515 E-03 \quad 9.513 E-03 \quad 9.510 E-03 \quad 9.499 E-03 \quad 9.498 E-03 \quad 9.469 E-03 \quad 9.363 E-03 \quad 9.068 E-03 \quad 8.105 E-03$

OU-236 PU-240 $1.000 \mathrm{E}+00 \quad 2.260 \mathrm{E}-11 \quad 6.898 \mathrm{E}-11 \quad 1.611 \mathrm{E}-10 \quad 4.761 \mathrm{E}-10 \quad 5.202 \mathrm{E}-10 \quad 1.314 \mathrm{E}-09 \quad 3.635 \mathrm{E}-096.950 \mathrm{E}-09 \quad 4.556 \mathrm{E}-06$

$0 T h-232$ PU-240 $1.000 E+002.240 E-211.509 E-207.812 E-206.039 E-19$ B.

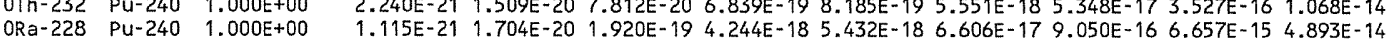

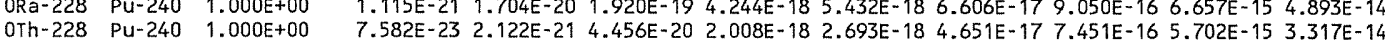

$\begin{array}{llllllllllll}0 \mathrm{Th}-228 & \mathrm{Pu}-240 & 1.000 \mathrm{E}+00 & 7.582 \mathrm{E}-23 & 2.122 \mathrm{E}-21 & 4.456 \mathrm{E}-20 & 2.008 \mathrm{E}-18 & 2.693 \mathrm{E}-18 & 4.651 \mathrm{E}-17 & 7.451 \mathrm{E}-16 & 5.702 \mathrm{E}-15 & 3.317 \mathrm{E}-14 \\ \mathrm{OSr}-90 & \mathrm{Sr}-90 & 1.000 \mathrm{E}+00 & 9.437 \mathrm{E}-01 & 9.211 \mathrm{E}-01 & 8.775 \mathrm{E}-01 & 7.406 \mathrm{E}-01 & 7.229 \mathrm{E}-01 & 4.561 \mathrm{E}-01 & 8.362 \mathrm{E}-02 & 6.565 \mathrm{E}-04 & 2.815 \mathrm{E}-11\end{array}$

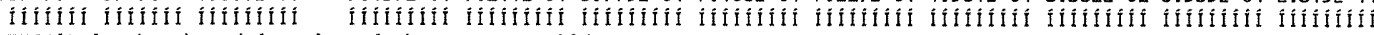

THF(i) is the thread fraction of the parent nuclide.

\begin{tabular}{|c|c|}
\hline Attachment & Sheet No. 26 of 27 \\
\hline Originators: S.W. Clark & Date \\
\hline M. W. Perrott & Date \\
\hline 0100B-CA-V0301 & Rev. No. \\
\hline
\end{tabular}




\section{ATTACHMENT 9}

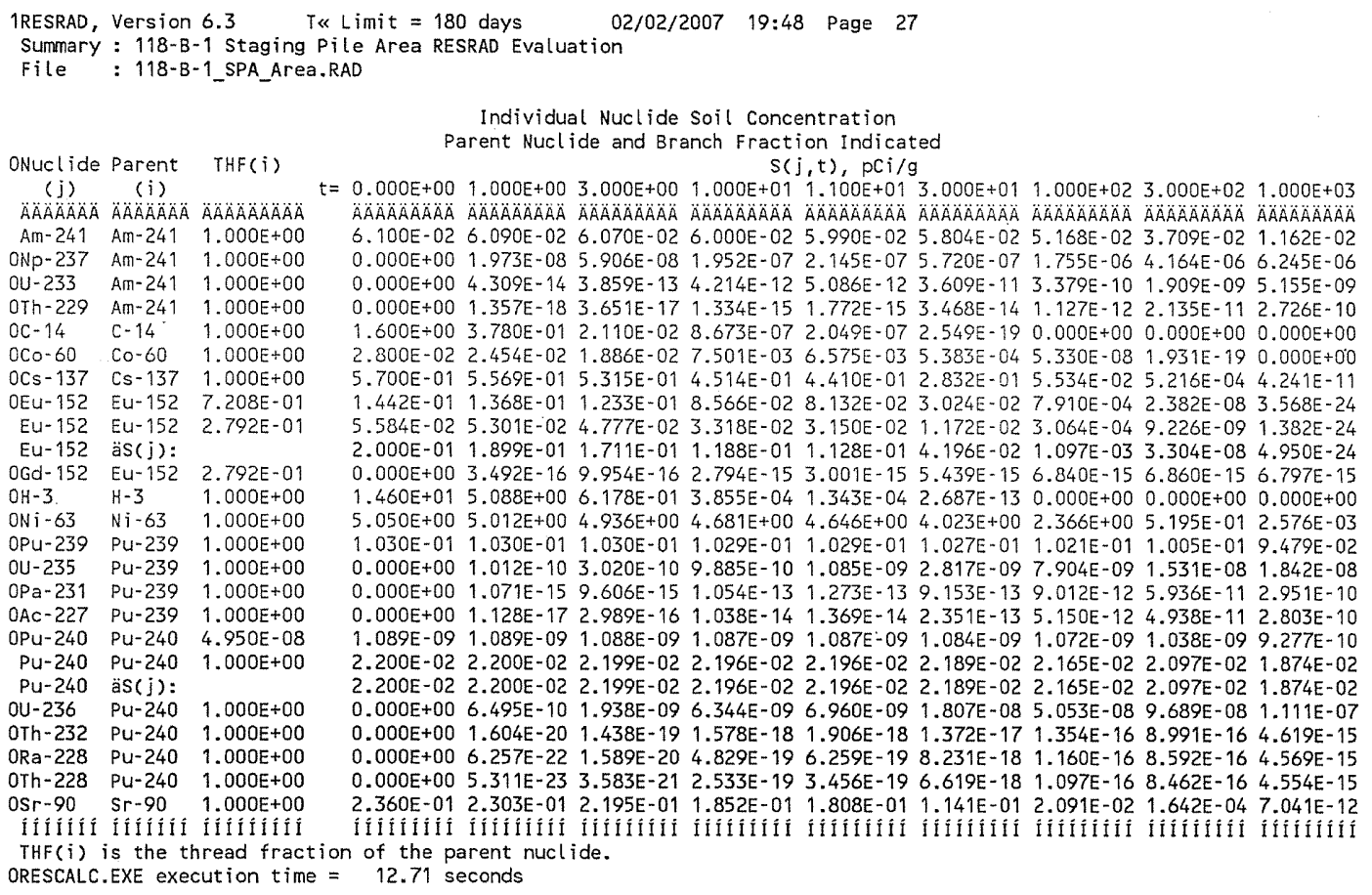

Attachment 9 Sheet No. 27 of 27 Originators: $\frac{S . \text { W. Clark }}{\text { M.W. Perrott }}$ Date 


\section{ATTACHMENT 10}

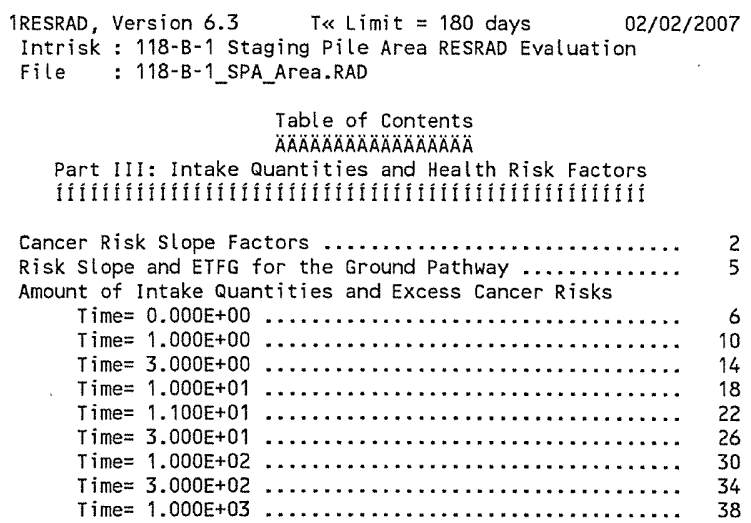




\section{ATTACHMENT 10}

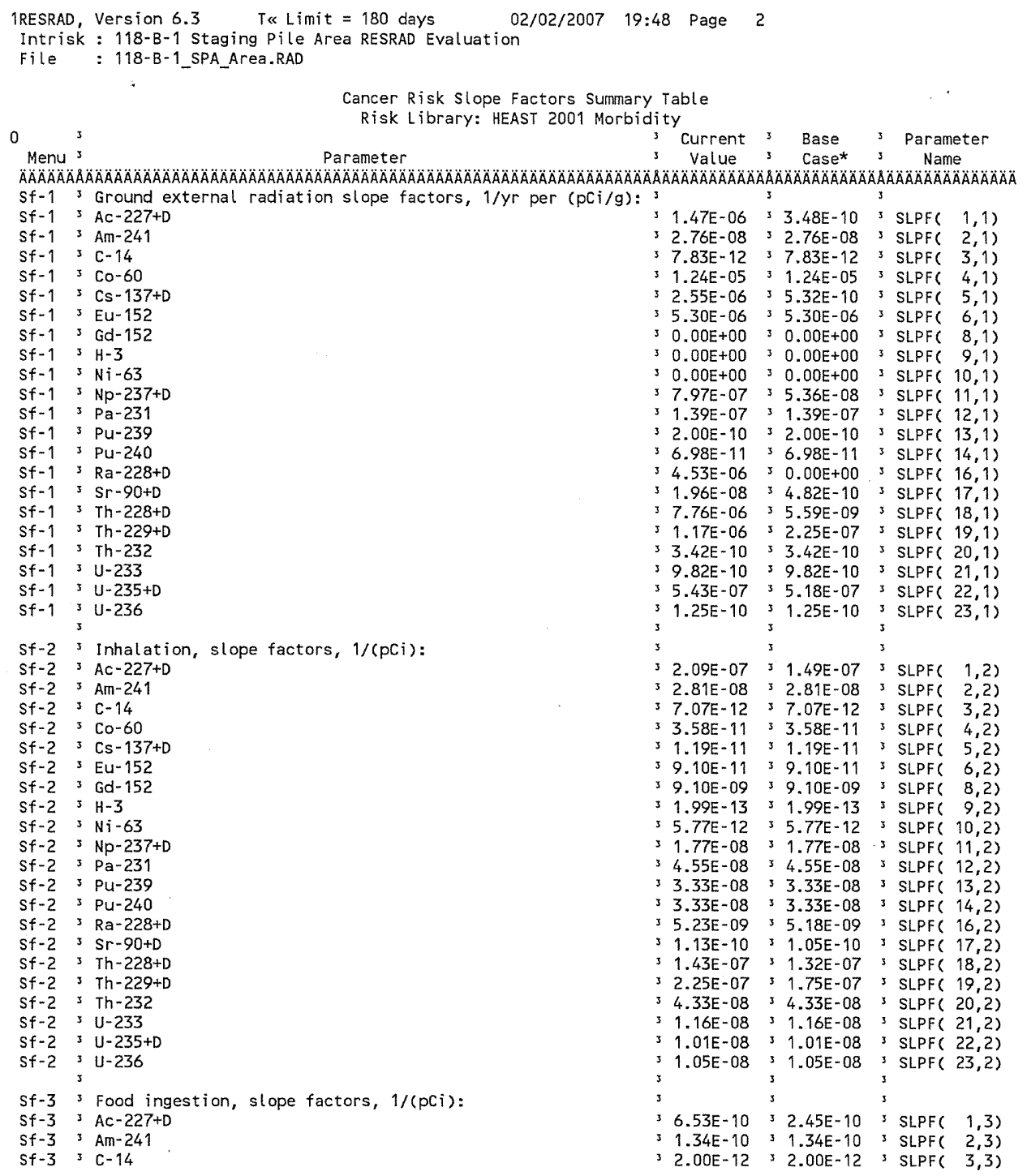

\begin{tabular}{|c|c|}
\hline Attachment & Sheet No. 2 of 41 \\
\hline Originators: S. W. Clark & Date \\
\hline M. W. Perrott & Date \\
\hline 0100B-CA-V0301 & Rev. No. $\quad 0$ \\
\hline
\end{tabular}




\section{ATTACHMENT 10}

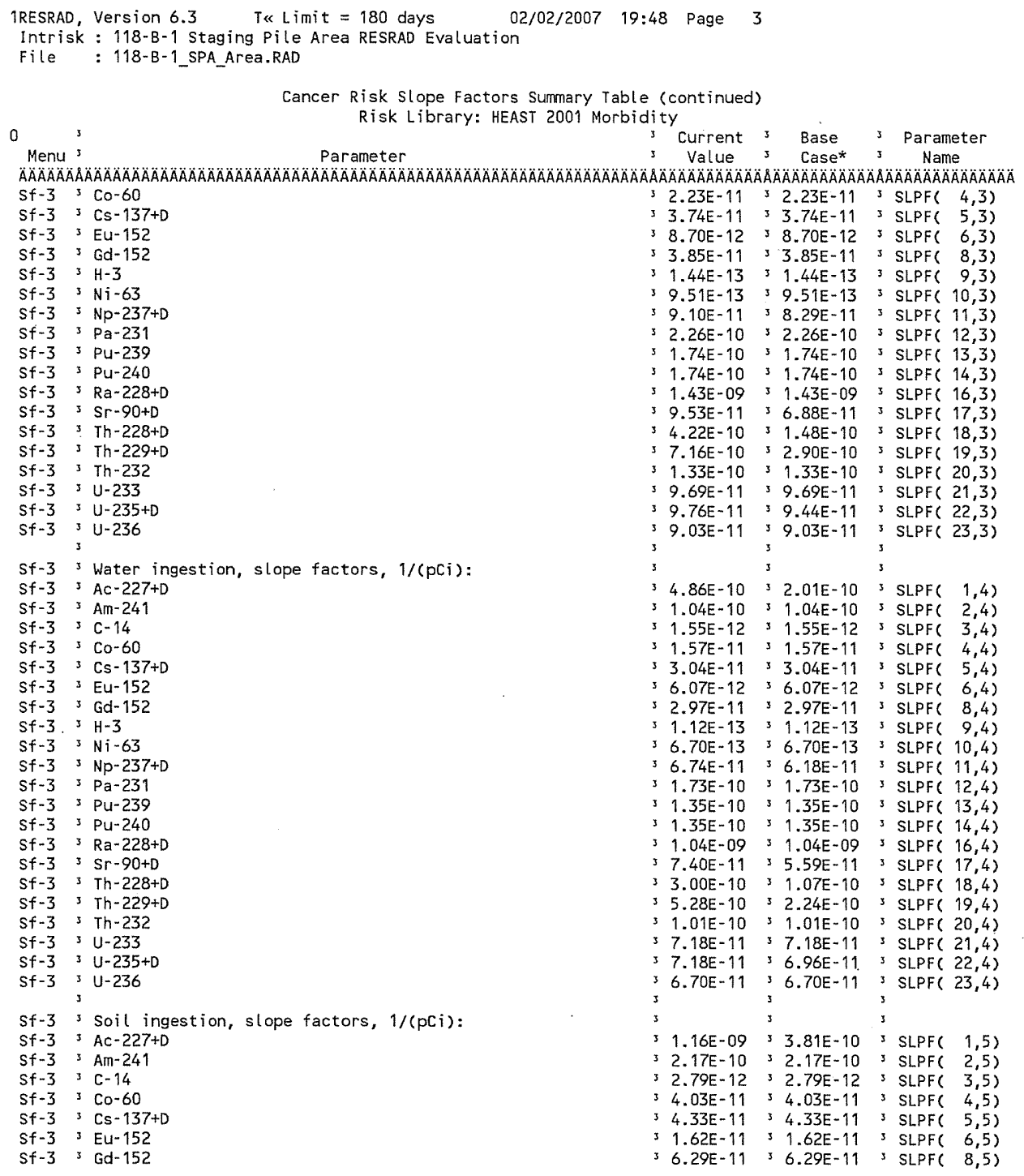

Attachment 
CVP-2007-00006

Rev. 0

\section{ATTACHMENT 10}

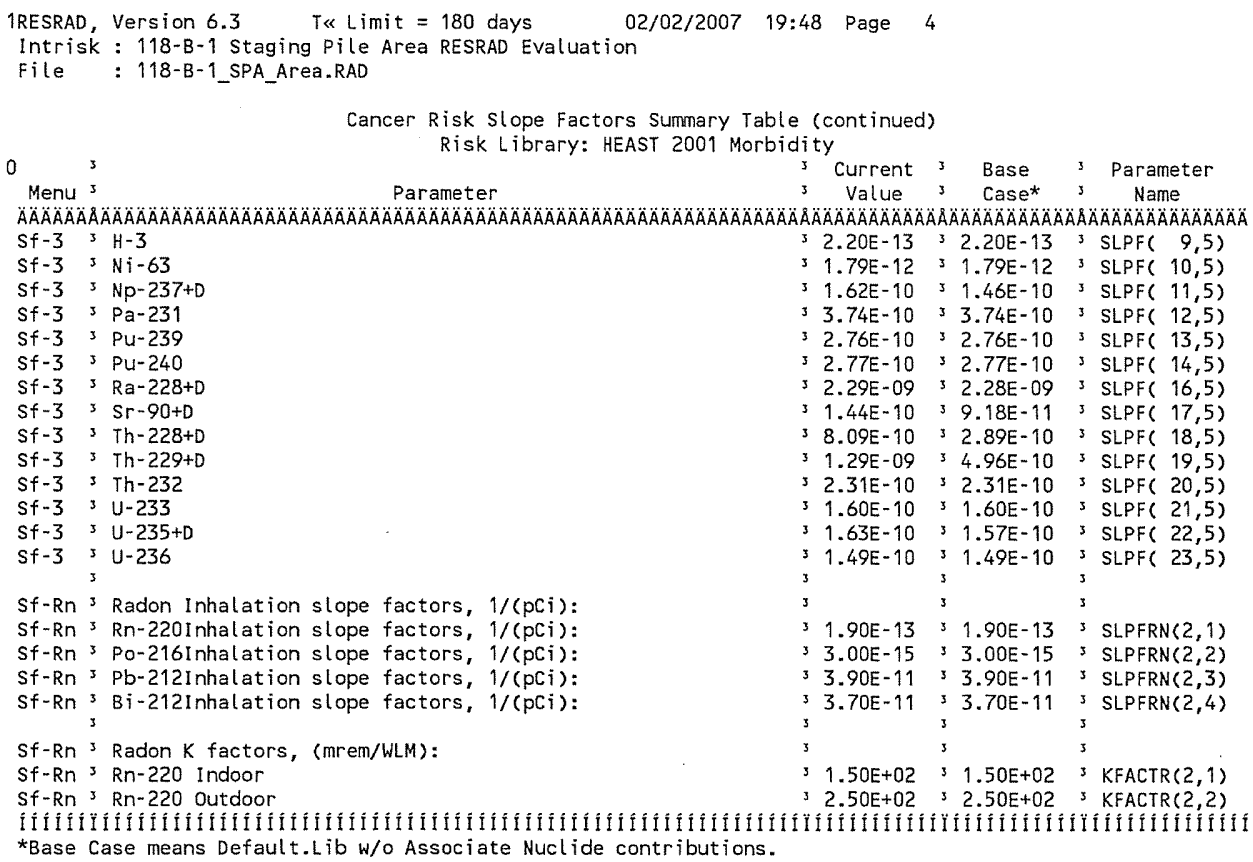

$\begin{array}{ll}\text { Attachment } \frac{10}{\text { S.W. Clark }} & \text { Sheet No. } 4 \text { of } 41 \\ \text { Originators: } \frac{\text { S.W. }}{\text { M. W. Perrott }} & \text { Date } \\ \text { Chk'd By } & \text { Rev. No. } \quad 0 \\ \text { Calc. No. } & 0100 \text { B-CA-V0301 }\end{array}$




\section{ATTACHMENT 10}

\begin{tabular}{|c|c|c|c|c|c|c|c|c|c|c|}
\hline $\begin{array}{l}\text { 1RESRAD, } \\
\text { Intrisk } \\
\text { File }\end{array}$ & $\begin{array}{l}\text { Version } 6.3 \\
: 118-\mathrm{B}-1 \mathrm{~S} \\
: 118-\mathrm{B}-1 \text { _Sf }\end{array}$ & $\begin{array}{l}\text { ng } \mathrm{Pil} \\
\text { rea. RA }\end{array}$ & ESRA & E & $2 / 2007$ & 48 & 5 & & & \\
\hline & Slope $i$ & & & $E$ & & & & & & \\
\hline & & 0 & $+\infty 0$ & $000 E+00$ & & $1.100 E+01$ & $3.000 E+01$ & $1.000 E+02$ & $.000 E+02$ & $.000 E+$ \\
\hline$\ddot{A} A \ddot{A} \ddot{A}$ & $\triangle A \cap A A A O A O A$ & 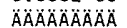 & 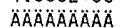 & 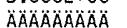 & 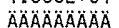 & AAAAOAAAAA & $\triangle A \triangle A O A O A O A O A$ & 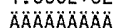 & $\triangle \triangle A O A B A B A$ & \\
\hline & & $6.546 \mathrm{E}-01$ & & .01 & & & E-01 & $=-01$ & -01 & \\
\hline & & & & & & & & & & \\
\hline$A C-$ & $4.530 \mathrm{E}-06$ & $6.453 \mathrm{E}-01$ & $6.453 \mathrm{E}-01$ & $3 E-01$ & $3 E-01$ & $6.453 \mathrm{E}-01$ & $6.453 \mathrm{E}-01$ & $6.453 \mathrm{E}-01$ & $6.453 \mathrm{E}-01$ & $453 E-01$ \\
\hline Am- & -08 & $36 E-01$ & -01 & $E-01$ & E-01 & 6.636E-01 & $6.636 \mathrm{E}-01$ & $6.636 \mathrm{E}-01$ & $6.636 \mathrm{E}-01$ & $6.636 \mathrm{E}-01$ \\
\hline & & & & & & & & & & \\
\hline $\mathrm{Ba}$ - & & & & & & $6.421 E-01$ & & & & $E-01$ \\
\hline & -07 & $1 E-01$ & & & & $6.481 E-01$ & & & $481 E-01$ & $E-01$ \\
\hline & & & & & & E-01 & & & & \\
\hline & & & & & & & & & & \\
\hline$c-1$ & -12 & $6 E-01$ & & & -01 & & & -01 & -01 & -01 \\
\hline 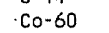 & & & & & & E & & & & \\
\hline & & & & & & & & & & \\
\hline u & & & & & & & & & 01 & -01 \\
\hline $\mathrm{Fr}$ & & & & & & 1 & & & & \\
\hline & & & & & & r & & & & \\
\hline Gd & +00 & $E+\infty$ & & & $+\infty$ & bo & & 00 & -00 & $+\infty$ \\
\hline $\mathrm{H}-3$ & & 01 & & & -01 & & & & & \\
\hline & & & & & & & & & & \\
\hline & & $3 E-01$ & & & & & & 01 & & \\
\hline $\mathrm{Pa}-$ & .07 & 01 & & & & & & & & \\
\hline & & & & & & & & & & \\
\hline & & 01 & & & 01 & 01 & & & & -01 \\
\hline & & & & & & & & & & \\
\hline & & & & & & & & & & \\
\hline & & -01 & & & 01 & 01 & & & & \\
\hline & & & & & & & & & & \\
\hline Po- & & & & & & & & & & \\
\hline & & & & & & & & & & \\
\hline & & & & & & & & & & \\
\hline & & & & & & & & & & \\
\hline & & & & & & & & & & \\
\hline & & & & & & & & & & \\
\hline & & & & & & & & & & \\
\hline & & & & & & & & & & \\
\hline & & & & & & & & & & \\
\hline & & & & & & & & & & \\
\hline & & & & & & & & & & \\
\hline & & & & & & & & & & \\
\hline & & & & & & & & & & \\
\hline & & & & & & & & & & \\
\hline & & & & & & & & & & \\
\hline & & & & & & & & & & \\
\hline & & & & & & & & & & \\
\hline & & & & & & & & & & -01 \\
\hline & & & & & & & & & & \\
\hline & & & & & & & & & & E-01 \\
\hline & & & & & & & & & & SE-01 \\
\hline & & & & & & & & & & \\
\hline 2 & & 6. & & & & & & & & BE-01 \\
\hline & & & & & & 6. & & & & \\
\hline & & & & & 1111 & ÎIIIIIIIIII & & IIIIIIIIII & İfílífín & 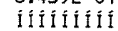 \\
\hline
\end{tabular}

* - Units are $1 / y r$ per $(\mathrm{pCi} / \mathrm{g})$ at infinite depth and area. Multiplication by $E$ TfG(i,t) converts to site conditions.

\begin{tabular}{|c|c|}
\hline Attachment & Sheet No. $\underline{5}$ of 41 \\
\hline Originators: S. W. Clark & Date \\
\hline M. W. Perrott & Date \\
\hline Calc. No. $0100 \mathrm{~B}-\mathrm{CA}-\mathrm{V} 0301$ & Rev. No. 0 \\
\hline
\end{tabular}


CVP-2007-00006

Rev. 0

\section{ATTACHMENT 10}

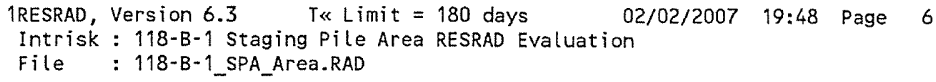

Water Independent Pathways (Inhalation w/o radon)

Water Dependent Pathways

\begin{tabular}{|c|c|c|c|c|c|c|c|c|c|c|c|}
\hline & & & & & & & & & & & \\
\hline & tion & Plant & Meat & Milk & Soil & Water & Fish & Plant & Meat & & Inge \\
\hline & & 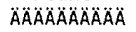 & AAAAAAAAAAÄÄ & $\triangle A A O A O A O A ̈ A ̈$ & AAAAAAAAAAAA & $\triangle A \cap A \cap A B A A A A$ & $\triangle A \cap A B A A A A A A B A$ & $\triangle A \triangle A A A A A A A A A$ & $\triangle A \cap A A A A A A A B$ & $\triangle A A A A A A A A A A$ & $\triangle A B A A$ \\
\hline & $0.000 E+00$ & $0.000 E+00$ & $0.000 E+00$ & $0.000 E+00$ & $0.000 \mathrm{E}+00$ & $0.000 \mathrm{E}+00$ & $0.000 E+00$ & & & & \\
\hline & & $0 E+00$ & $240 E-02$ & $.774 E-03$ & $.562 E+00$ & & $D O O E+00$ & OOE +00 & $O E+O O$ & $+\infty 0$ & \\
\hline & & $=+06$ & $E+05$ & $E+05$ & $E+01$ & $0.000 E+00$ & $0.000 E+00$ & $000 E+00$ & $D O E+00$ & $E+00$ & \\
\hline & & & & & & -00 & 00 & 00 & +00 & & \\
\hline & & & & +03 & & & & & & & \\
\hline & & & +00 & & +01 & & & & & & \\
\hline & & & $+\infty 0$ & $+\infty$ & & & & & & & +00 \\
\hline & & & & +06 & & & & & & & \\
\hline & & & & & & & & & +00 & +00 & \\
\hline & & $E+\infty$ & $+\infty 0$ & $=+\infty$ & & & & & & & $+\infty 0$ \\
\hline & & +00 & +00 & +00 & & & & & & & \\
\hline & & & & & & & & & +00 & & \\
\hline & & +00 & 4. & & & & & & & +00 & +00 \\
\hline & & +00 & +00 & $+\infty$ & & & & & & & \\
\hline & & & & $E+02$ & & & & & & & +03 \\
\hline & & & & & & & & & +00 & $+\infty$ & $+\infty 0$ \\
\hline & & & & $+\infty 0$ & & & & & & & \\
\hline & +00 & $0.000 E+00$ & $0.000 E+00$ & $0.000 E+00$ & $0.000 E+00$ & -00 & 0.00 & & & & +00 \\
\hline & & & & & & & $0.000 E+00$ & $0.000 E+00$ & $E+00$ & $\mathrm{BE}+00$ & $0 E+00$ \\
\hline & & & & & & & & & & & \\
\hline & & 00 & 0 & 0. & & $F \rightarrow 0$ & & & & & \\
\hline & & & & & & & & & & & \\
\hline
\end{tabular}

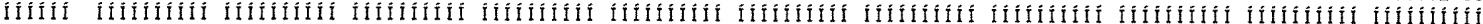

* Sum of all ingestion pathways, i.e. water independent plant, meat, milk, soil

Amount of Intake Quantities QINTS(irn, $i, t)$ and QINTOW(irn, $i, t)$ for Inhalation of Radon and its Decay Products as $\mathrm{pCi} / \mathrm{yr}$ at $\mathrm{t}=0.000 \mathrm{E}+00$ years Radionuclides

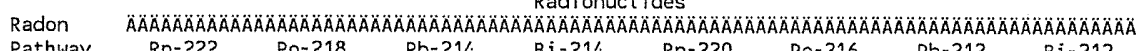

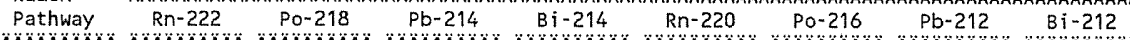

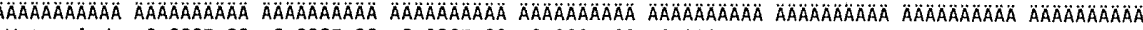

$\begin{array}{lllllllll}\text { Water }-i n d . & 0.000 \mathrm{E}+00 & 0.000 \mathrm{E}+00 & 0.000 \mathrm{E}+00 & 0.000 \mathrm{E}+00 & 0.000 \mathrm{E}+00 & 0.000 \mathrm{E}+00 & 0.000 \mathrm{E}+00 & 0.000 \mathrm{E}+00\end{array}$

$\begin{array}{lllllllll}\text { Water-dep. } & 0.000 E+00 & 0.000 E+00 & 0.000 E+00 & 0.000 E+00 & 0.000 E+00 & 0.000 E+00 & 0.000 E+00 & 0.000 E+00\end{array}$

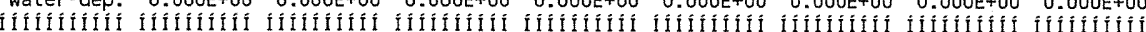

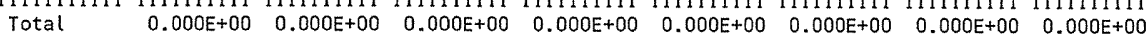

Water-ind. = Water-independent Water-dep. $==$ Water-dependent

\begin{tabular}{|c|c|}
\hline \multirow{2}{*}{\multicolumn{2}{|c|}{ Sheet No. $\underline{6}$ of $\underline{41}$}} \\
\hline & \\
\hline M. W. Perrott & Date \\
\hline $0100 \mathrm{~B}-\mathrm{CA}-\mathrm{V} 0$ & Rev. I \\
\hline
\end{tabular}




\section{ATTACHMENT 10}

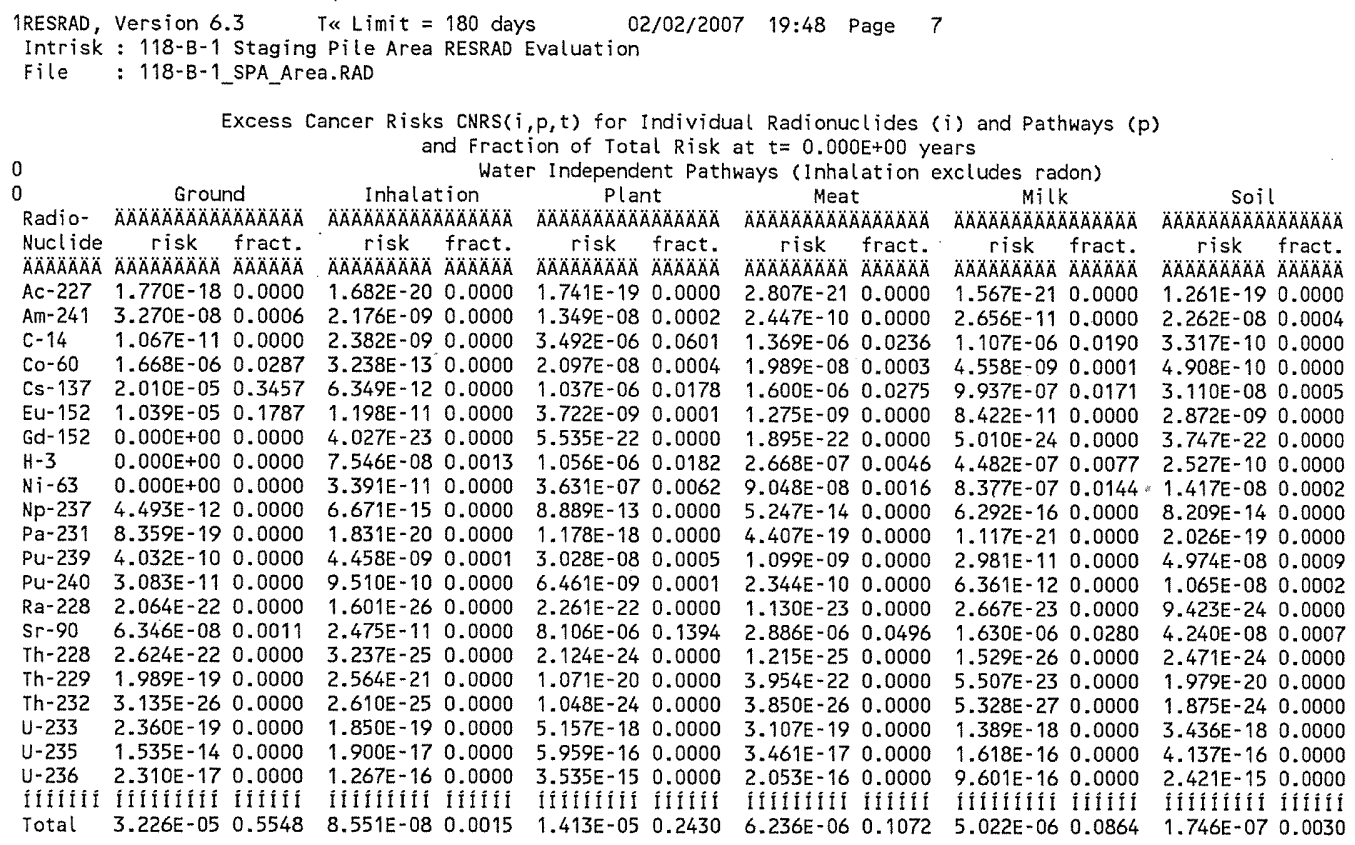

\begin{tabular}{|c|c|}
\hline Attachment & Sheet No. 7 of 41 \\
\hline Originators: S.W. Clark & Date \\
\hline Chk'd By M.W. Perrott & Date \\
\hline $0100 B-C A-V 0301$ & Rev. No. 0 \\
\hline
\end{tabular}




\section{ATTACHMENT 10}

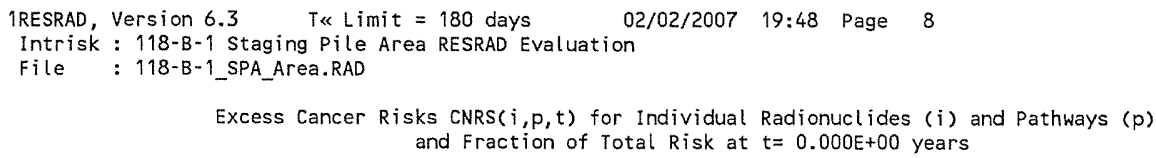

Water Dependent Pathways

\begin{tabular}{|c|c|c|c|c|c|c|c|c|c|c|c|c|}
\hline & $\begin{array}{r}\text { Water } \\
A \cap A O A O A O A B A\end{array}$ & 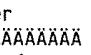 & 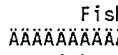 & 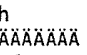 & 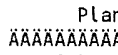 & ñ & $\begin{array}{r}\text { Meat } \\
\triangle A B A A A B A A A B A\end{array}$ & t & 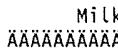 & $\triangle A A A O A O A ̈ A$ & $\begin{array}{r}\text { All Path } \\
\text { ÄAAAAAAA }\end{array}$ & 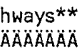 \\
\hline $\begin{array}{l}\text { Uclide } \\
A A A A O A\end{array}$ & $\begin{array}{c}\text { risk } \\
\text { ÄÄÄÄÄA }\end{array}$ & $\begin{array}{l}\text { fract. } \\
\text { ÄAÄÄÄ }\end{array}$ & $\begin{array}{c}r i s k \\
A \cap A A A B A B A O A\end{array}$ & $\begin{array}{l}\text { fract. } \\
\triangle A A A B A A A\end{array}$ & $\begin{array}{c}\text { risk } \\
\triangle A \cap A B A B A B A\end{array}$ & $\begin{array}{l}\text { fract. } \\
\triangle A O A B A B A B\end{array}$ & $\begin{array}{c}\text { risk } \\
\triangle A \cap A O A B A B A O A B\end{array}$ & $\begin{array}{l}\text { fract. } \\
\text {AAAAAAAAAB}\end{array}$ & $\begin{array}{c}r i s k \\
\triangle A A A B A A A B\end{array}$ & $\begin{array}{l}\text { fract. } \\
A A A B A A B\end{array}$ & $\begin{array}{c}r i s k \\
A \cap A B A B A A A\end{array}$ & $\begin{array}{l}\text { fract. } \\
\ddot{A} \ddot{A} \ddot{A} \ddot{A} \ddot{A}\end{array}$ \\
\hline$c-227$ & $0.000 E+00$ & & $0.000 E+00$ & 0.0000 & $0.000 E+00$ & 0.0000 & $0.000 E+00$ & 0.0000 & $0.000 E \div 00$ & 0.0000 & & \\
\hline 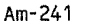 & $0 O E+00$ & 0.0000 & $O E+00$ & 0.0000 & $.000 E+00$ & 0.0000 & $0.000 E+00$ & 0.0000 & $0.000 E+00$ & 0.0000 & $7.126 \mathrm{E}-08$ & \\
\hline & & & & & $O E+O O$ & 0.0000 & $0 E+00$ & 00 & +00 & & & \\
\hline & & 0.0 & +00 & & $E+00$ & & $E+\infty$ & & & & & \\
\hline & $E E+00$ & 0.0 & 0.0 & & $E+00$ & 0.0000 & $0 E+00$ & & 00 & & & \\
\hline & 10 & & 00 & & 100 & & $E+00$ & & & & & \\
\hline & & & & & & & & & & & & \\
\hline & 1.4 & & & & & & & & & & & \\
\hline & 10 & & 00 & & $+\infty 0$ & & +00 & & & & & \\
\hline & & & & & & & +00 & & & & & \\
\hline & & & & & & 0 & $E+00$ & 0. & +00 & & & 100 \\
\hline & 0 & & & & & & & & & & & \\
\hline & .0 & & 00 & & $+\infty 0$ & & $=+\infty$ & & & & & \\
\hline & 0.0 & & 0.0 & & +00 & 100 & $E+\infty$ & 100 & +00 & & & 00 \\
\hline & 0 & & & & & & & & & & & \\
\hline & 0 & & 0.0 & & 00 & & $E+00$ & & 00 & & & \\
\hline$T h-229$ & $0.000 E+00$ & 0.0 & $E+00$ & & +00 & & $E+00$ & & $+\infty 0$ & & & 300 \\
\hline & & & & & & & & & & & & \\
\hline & 00 & & +00 & & & & & & & & & \\
\hline$U-2$ & $0.000 E+00$ & 0.0000 & $0.000 E+00$ & 0. & $E+\infty$ & 100 & $E+\infty$ & 0 & $E+\infty$ & & 1. & 000 \\
\hline & & & & & & & & & & & & \\
\hline & & & & & & & & & & & & \\
\hline te & $472 E-07$ & 0.0025 & $=-10$ & 0.0000 & & & & & & & & DOS \\
\hline
\end{tabular}

** Sum of water independent ground, inhalation, plant, meat, milk, soil

0

and water dependent water, fish, plant, meat, milk pathways

Excess Cancer Risks CNRS9(irn, $i, t)$ and CNRSPW(irn, $i, t)$ for Inhalation of

Radon and its Decay Products at $t=0.000 E+00$ years

$0 \quad$ Radionuclides

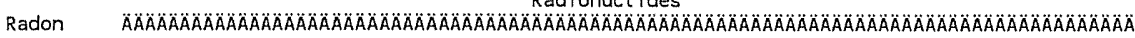

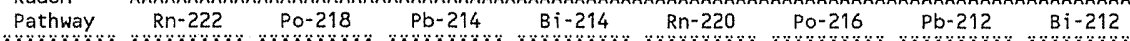

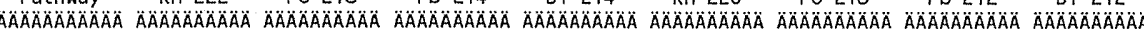

$\begin{array}{lllllllll}\text { Water-ind. } & 0.000 \mathrm{E}+00 & 0.000 \mathrm{E}+00 & 0.000 \mathrm{E}+00 & 0.000 \mathrm{E}+00 & 0.000 \mathrm{E}+00 & 0.000 \mathrm{E}+00 & 0.000 \mathrm{E}+00 & 0.000 \mathrm{E}+00\end{array}$

Water-dep. $\quad \begin{array}{lllllllll}0.000 E+00 & 0.000 E+00 & 0.000 E+00 & 0.000 E+00 & 0.000 E+00 & 0.000 E+00 & 0.000 E+00 & 0.000 E+00\end{array}$

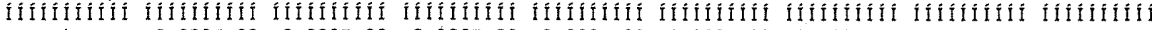

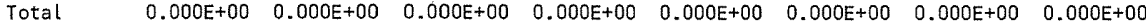

Water-ind. =" Water-independent Water-dep. == Water-dependent

$\begin{array}{ll}\text { Attachment } \frac{10}{\text { S.W. Clark }} & \text { Sheet No. } \underline{8} \text { of } 41 \\ \text { Originators: } & \text { Date } \\ \text { Chk'd By } & \text { Date } \\ \text { Calc. No. W. Perrott } & \text { Rev. No. } \quad 0\end{array}$




\section{ATTACHMENT 10}

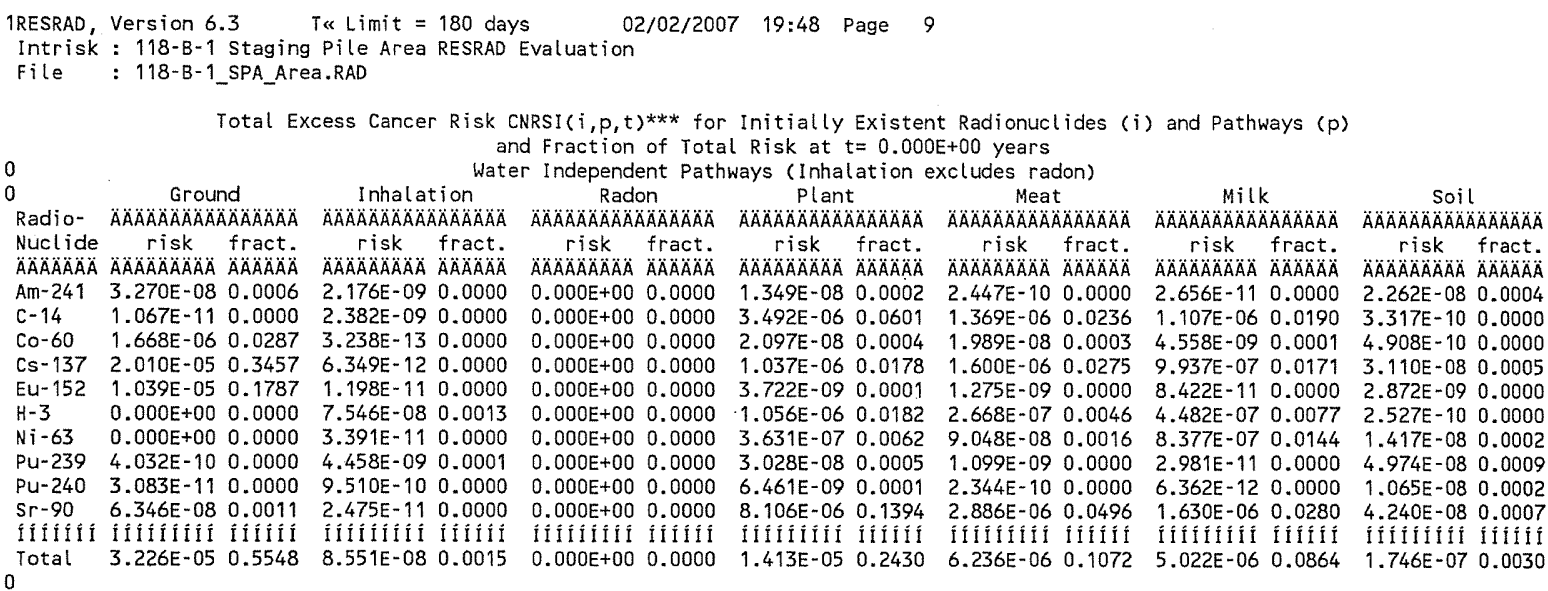

Total Excess Cancer Risk CNRSI $(i, p, t) * * *$ for Initially Existent Radionuclides $(i)$ and Pathways $(p)$ and Fraction of Total Risk at $t=0.000 E+00$ years

Water Dependent Pathways

\begin{tabular}{|c|c|c|c|c|c|c|c|c|c|c|c|c|c|}
\hline & $\begin{array}{r}\text { Wate } \\
\triangle A A A O A A O A O A O A\end{array}$ & A & $\begin{array}{r}F i s \\
\triangle A \triangle A A A O A A O A B\end{array}$ & ÄÄÄÄÄ̈ & $\begin{array}{r}\text { Radc } \\
\triangle A A A A A A A A A A A\end{array}$ & $\triangle A A A A B A A B$ & $\begin{array}{r}P l a n \\
\triangle A A A O A A A O A O A ̈\end{array}$ & It & $\begin{array}{r}\text { Meat } \\
\triangle A O A B A A O A B A B A\end{array}$ & $t$ & $\begin{array}{r}M i L K \\
\triangle A A A A B A O A O A O A B\end{array}$ & $\begin{array}{l}k \\
\ddot{A} A \cap A \cap A \cap A ̈ A ̈\end{array}$ & 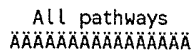 \\
\hline $\begin{array}{l}\text { Nuclide } \\
\text { ÄAAAAAAA }\end{array}$ & $\begin{array}{c}\text { risk } \\
\text { ÄAAAAAAAAA }\end{array}$ & $\begin{array}{l}\text { fract. } \\
\text { ÄAAAAAA }\end{array}$ & $\begin{array}{c}\text { risk } \\
\text { AAAAAAAAAAB }\end{array}$ & 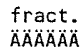 & $\begin{array}{c}\text { risk } \\
\text { ÄÄÄÄÄÄA }\end{array}$ & $\begin{array}{l}\text { fract. } \\
\text { ÄÄAAAÁ }\end{array}$ & $\begin{array}{c}\text { risk } \\
\text { ÄAAAÄÄAAÄ }\end{array}$ & $\begin{array}{l}\text { fract. } \\
\triangle A B A A A B A\end{array}$ & $\begin{array}{c}\text { risk } \\
\text { AAAAAOAAAAÄA }\end{array}$ & $\begin{array}{l}\text { fract. } \\
\triangle A \cap A ̈ A ̈ A ̈\end{array}$ & $\begin{array}{c}\text { risk } \\
\text { AAAAAAOAOAOA }\end{array}$ & $\begin{array}{l}\text { fract. } \\
A A A ̈ A O A\end{array}$ & $\begin{array}{l}\text { risk fract. } \\
\text { AAAAAAAAAOA AOAOAOAOA }\end{array}$ \\
\hline$n-241$ & $0.000 E+00$ & 0.0000 & $0.000 E+00$ & 0.0000 & $0.000 E+00$ & 0.0000 & $0.000 \mathrm{E}+00$ & 0.0000 & $0.000 \mathrm{E}+00$ & 0.0000 & $0.000 \mathrm{E}+00$ & .0000 & 7.12 \\
\hline C & $0.000 \mathrm{E}+00$ & 0.0000 & $0.000 \mathrm{E}+00$ & 0.0000 & $.000 E+00$ & 0.0000 & $0.000 E+00$ & 0.0000 & $0.000 E+00$ & 200 & $0.000 E+00$ & 000 & 06 \\
\hline Co- 60 & $0.000 E+00$ & 0.0000 & $0.000 E+00$ & 0.0000 & $0.000 E+00$ & 0.0000 & $0.000 E+00$ & 0.0000 & $0.000 E+00$ & 0.0000 & $0.000 \mathrm{E}+00$ & 0.0000 & .0295 \\
\hline Cs -137 & $0.000 E+00$ & 0.0000 & $0.000 E+00$ & 0.0000 & $.000 E+00$ & 0.0000 & $0.000 \mathrm{E}+00$ & 0.0000 & $0.000 \mathrm{E}+00$ & 100 & $0.000 E+00$ & 00 & 887 \\
\hline Eu- 152 & $0.000 E+00$ & 0.0000 & $0.000 \mathrm{E}+00$ & 0.0000 & $.000 E+00$ & 0.0000 & $0.000 E+00$ & 0.0000 & $E+\infty$ & 0. & $E+00$ & 00 & 05 \\
\hline$H-3$ & $1.4-201$ & 0.0025 & $1.455 \mathrm{E}$ & 0.0000 & $0.000 E+00$ & 0.0000 & $5.538 \mathrm{E}-08$ & 0.0010 & $9.041 E-09$ & 0.0002 & $2.869 E-08$ & 0.0005 & -060.0359 \\
\hline $\mathrm{Ni}-63$ & $0.000 E+00$ & 0.0000 & $0.000 E+00$ & 0.0000 & $0.000 E+00$ & 0.0000 & $0.000 E+00$ & 0.0 & +00 & & +00 & & 06 \\
\hline Pu- 239 & $0.000 E+00$ & 0.0000 & $0.000 E+00$ & 0.0000 & $.000 E+00$ & 0.0000 & $0.000 E+00$ & 0.0000 & $0.000 E+00$ & 0.0000 & $0.000 E+00$ & 0.0000 & -08 \\
\hline Pu-240 & $0.000 \mathrm{E}+00$ & 0.0000 & $0.000 E+00$ & 0.0000 & $0.000 E+00$ & 0.0000 & $0.000 \mathrm{E}+00$ & 0.0000 & $0.000 E+00$ & 0.0000 & $0.000 E+00$ & 0.0000 & $1.833 \mathrm{E}-08 \quad 0.0003$ \\
\hline & & & & & & & & & & & 0 & 0.0000 & 0.2189 \\
\hline & & & & & & & & & & & & & \\
\hline - & $1.472 E-07$ & 0.0025 & $1.455 E-10$ & 0.0000 & $0.000 E+00$ & 0.0000 & $5.538 \mathrm{E}-08$ & 0.0010 & $9.041 \mathrm{E}-09$ & 0.0002 & $2.869 E-08$ & 0.0005 & $5.815 \mathrm{E}-05 \quad 1.0000$ \\
\hline
\end{tabular}

***CNRSI $(i, p, t)$ includes contribution from decay daughter radionuclides

$\begin{array}{ll}\text { Attachment } \frac{10}{\text { S.W.Clark }} & \text { Sheet No. } \underline{9} \text { of } 41 \\ \text { Originators: } & \text { Date } \\ \text { Chk'd By } & \text { Date } \\ \text { Calc. No. Perrott } & \text { Rev. No. } 0\end{array}$


CVP-2007-00006

Rev. 0

\section{ATTACHMENT 10}

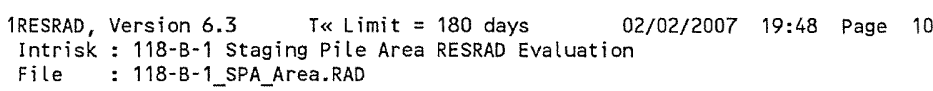

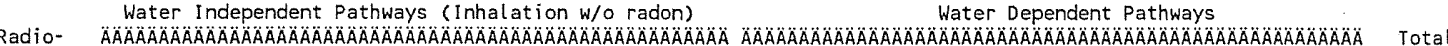

Water Dependent Pathways

Nuclide Inhalation Plant Meat Milk Soil Water Fish Plant Meat Milk Ingestion*

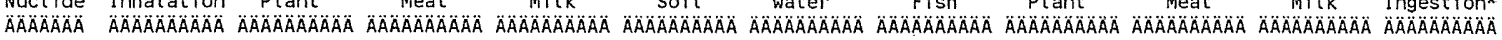

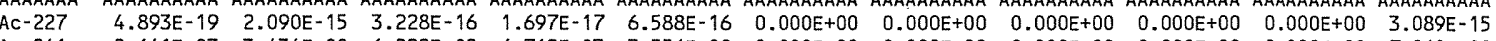
$\begin{array}{llllllllllll}A m-241 & 2.641 E-03 & 3.434 E+00 & 6.229 E-02 & 6.762 E-03 & 3.556 E+00 & 0.000 E+00 & 0.000 E+00 & 0.000 E+00 & 0.000 E+00 & 0.000 E+00 & 7.060 E+00\end{array}$ $\begin{array}{llllllllllll}\mathrm{C}-14 & 6.256 \mathrm{E}+01 & 3.423 \mathrm{E}+05 & 1.635 \mathrm{E}+05 & 1.228 \mathrm{E}+05 & 2.208 \mathrm{E}+01 & 0.000 \mathrm{E}+00 & 0.000 \mathrm{E}+00 & 0.000 \mathrm{E}+00 & 0.000 E+00 & 0.000 \mathrm{E}+00 & 6.287 \mathrm{E}+05\end{array}$

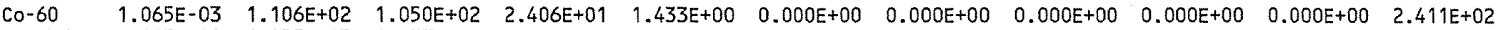
$\begin{array}{llllllllllll}\text { Cs-137 } & 2.415 E-02 & 1.255 E+03 & 1.937 E+03 & 1.203 E+03 & 3.252 E+01 & 0.000 E+00 & 0.000 E+00 & 0.000 E+00 & 0.000 E+00 & 0.000 E+00 & 4.427 E+03\end{array}$ $\begin{array}{llllllllllll}E L-152 & 8.235 E-03 & 2.675 E+01 & 9.162 E+00 & 6.054 E-01 & 1.109 E+01 & 0.000 E+00 & 0.000 E+00 & 0.000 E+00 & 0.000 E+00 & 0.000 E+00 & 4.761 E+01\end{array}$

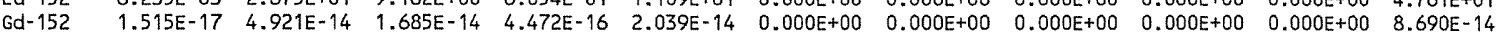

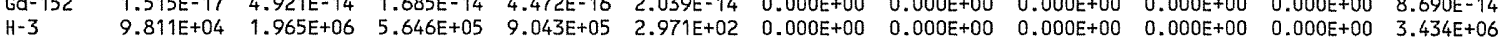

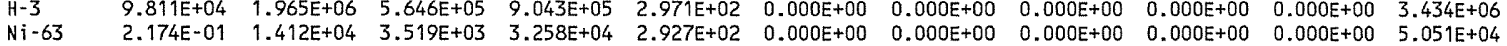

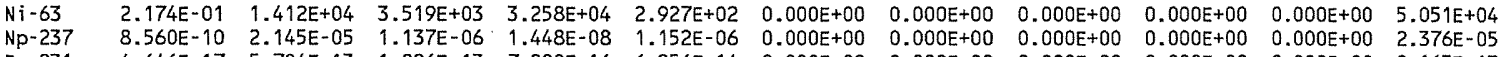
$\begin{array}{llllllllllll}\mathrm{Pa}-231 & 4.646 \mathrm{E}-17 & 5.704 \mathrm{E}-13 & 1.826 \mathrm{E}-13 & 7.280 \mathrm{E}-16 & 6.256 \mathrm{E}-14 & 0.000 \mathrm{E}+00 & 0.000 \mathrm{E}+00 & 0.000 \mathrm{E}+00 & 0.000 \mathrm{E}+00 & 0.000 \mathrm{E}+00 & 8.163 \mathrm{E}-13\end{array}$ $\begin{array}{llllllllllll}\text { Pu-239 } & 4.467 E-03 & 5.808 E+00 & 2.107 E-01 & 5.718 E-03 & 6.015 E+00 & 0.000 E+00 & 0.000 E+00 & 0.000 E+00 & 0.000 E+00 & 0.000 E+00 & 1.204 E+01\end{array}$ $\begin{array}{llllllllllll}\mathrm{PU}-240 & 9.541 \mathrm{E}-04 & 1.240 \mathrm{E}+00 & 4.500 \mathrm{E}-02 & 1.221 \mathrm{E}-03 & 1.285 \mathrm{E}+00 & 0.000 \mathrm{E}+00 & 0.000 \mathrm{E}+00 & 0.000 \mathrm{E}+00 & 0.000 \mathrm{E}+00 & 0.000 \mathrm{E}+00 & 2.571 \mathrm{E}+00\end{array}$ $\begin{array}{llllllllllll}R a-228 & 2.714 E-23 & 1.259 E-18 & 4.376 E-20 & 1.233 E-19 & 3.654 E-20 & 0.000 E+00 & 0.000 E+00 & 0.000 E+00 & 0.000 E+00 & 0.000 E+00 & 1.462 E-18\end{array}$ $\begin{array}{llllllllllll}\mathrm{Sr}-90 & 9.991 \mathrm{E}-03 & 3.894 \mathrm{E}+03 & 1.387 \mathrm{E}+03 & 7.832 \mathrm{E}+02 & 1.345 \mathrm{E}+01 & 0.000 \mathrm{E}+00 & 0.000 \mathrm{E}+00 & 0.000 \mathrm{E}+00 & 0.000 \mathrm{E}+00 & 0.000 \mathrm{E}+00 & 6.077 \mathrm{E}+03\end{array}$

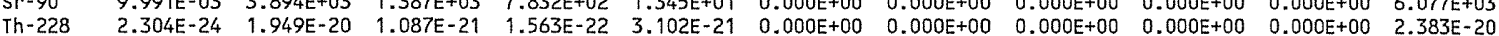

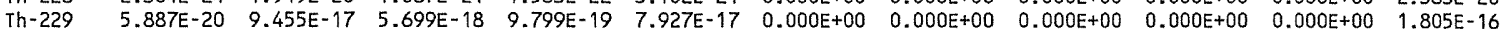
$\begin{array}{llllllllllll}\text { Th-232 } & 6.956 \mathrm{E}-22 & 1.002 \mathrm{E}-18 & 4.395 \mathrm{E}-20 & 7.865 \mathrm{E}-21 & 9.365 \mathrm{E}-19 & 0.000 \mathrm{E}+00 & 0.000 \mathrm{E}+00 & 0.000 \mathrm{E}+00 & 0.000 \mathrm{E}+00 & 0.000 \mathrm{E}+00 & 1.991 \mathrm{E}-18\end{array}$

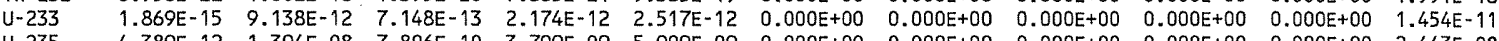
$\begin{array}{llllllllllll}\text { U-235 } & 4.389 \mathrm{E}-12 & 1.394 \mathrm{E}-08 & 7.806 \mathrm{E}-10 & 3.799 \mathrm{E}-09 & 5.909 \mathrm{E}-09 & 0.000 \mathrm{E}+00 & 0.000 \mathrm{E}+00 & 0.000 \mathrm{E}+00 & 0.000 \mathrm{E}+00 & 0.000 \mathrm{E}+00 & 2.443 \mathrm{E}-08\end{array}$ $\begin{array}{llllllllllll}\text { U-236 } & 2.817 \mathrm{E}-11 & 8.949 \mathrm{E}-08 & 5.011 \mathrm{E}-09 & 2.439 \mathrm{E}-08 & 3.793 \mathrm{E}-08 & 0.000 \mathrm{E}+00 & 0.000 \mathrm{E}+00 & 0.000 \mathrm{E}+00 & 0.000 \mathrm{E}+00 & 0.000 \mathrm{E}+00 & 1.568 \mathrm{E}-07\end{array}$

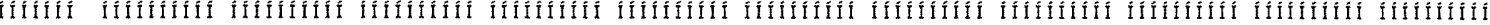

* Sum of all ingestion pathways, i.e. water independent plant, meat, milk, soil and water-dependent water, fish, plant, meat, milk pathways

Amount of Intake Quantities QINTg(irn, $i, t)$ and GINTOW(irn, $i, t)$ for Inhalation of

0 Radon and its Decay Products as $\mathrm{pCi} / \mathrm{yr}$ at $\mathrm{t}=1.000 \mathrm{E}+00$ years Radionuclides

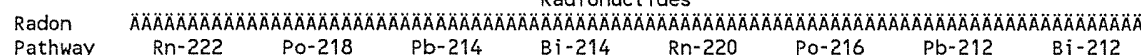

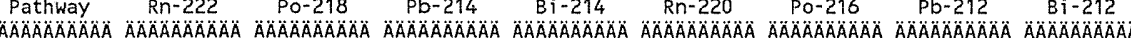

Water-ind $0.000 \mathrm{E}+000.000 \mathrm{E}+00 \quad 0.000 \mathrm{E}+00 \quad 0.000 \mathrm{E}+00 \quad 0.000 \mathrm{E}+00 \quad 0.000 \mathrm{E}+000.000 \mathrm{E}+00 \quad 0.000 \mathrm{E}+00$ $\begin{array}{lllllllll}\text { Water-dep. } 0.000 E+00 & 0.000 E+00 & 0.000 E+00 & 0.000 E+00 & 0.000 E+00 & 0.000 E+00 & 0.000 E+00 & 0.000 E+00\end{array}$

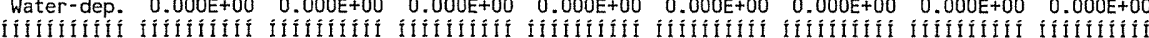

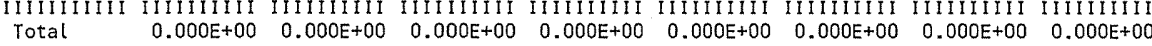

Water-ind. = Water-independent Water-dep. $=$ Water-dependent

\begin{tabular}{|c|c|c|}
\hline \multirow{2}{*}{\multicolumn{3}{|c|}{$\begin{array}{lll}\text { Attachment } & 10 & \text { Sheet No. } 10 \text { of } 41 \\
\text { Originators: S.W. Clark } & \text { Date }\end{array}$}} \\
\hline & & \\
\hline Chk'd By & M. W. Perrott & Date \\
\hline Calc. No. & $0100 \mathrm{~B}-\mathrm{CA}$ & Rev. No. \\
\hline
\end{tabular}




\section{ATTACHMENT 10}

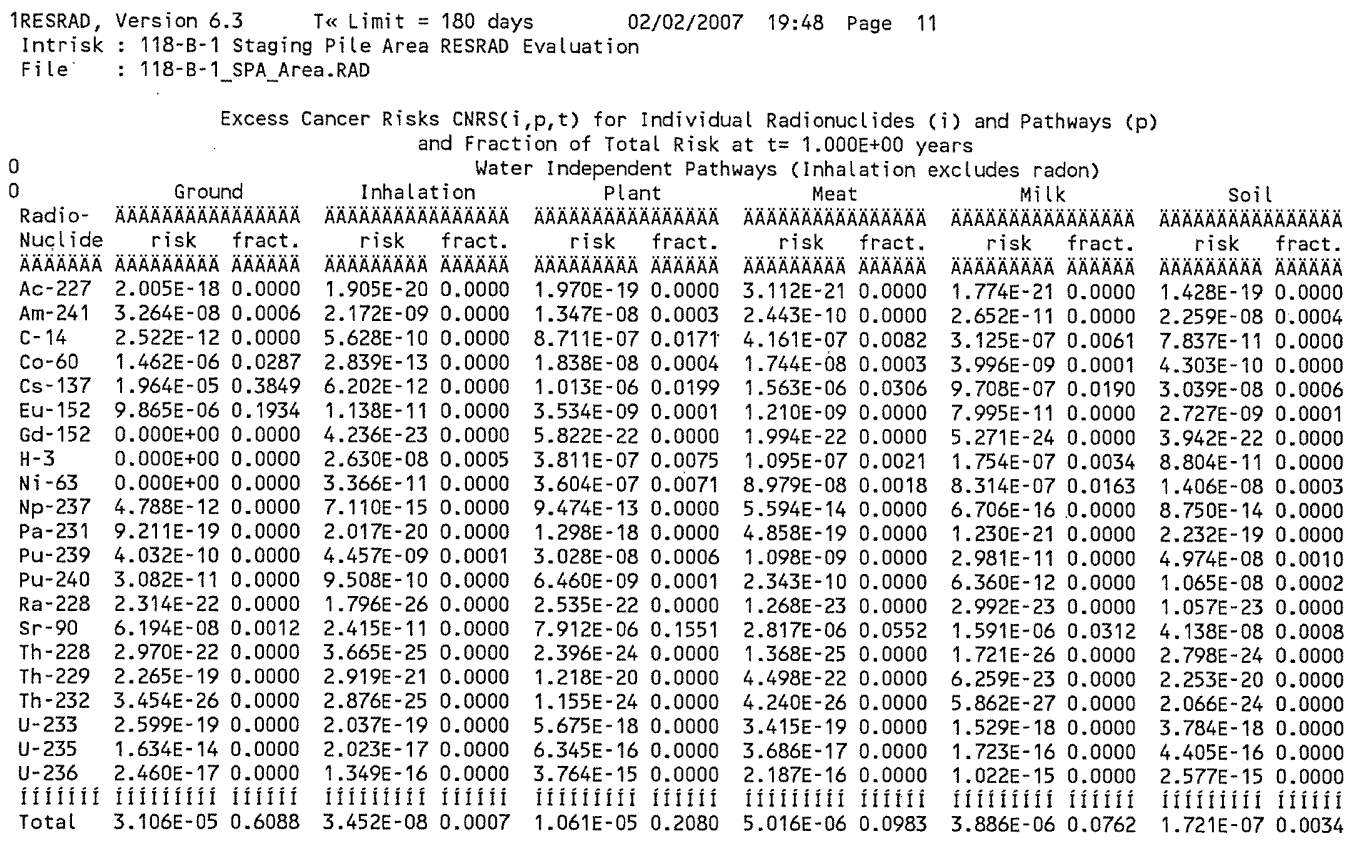

\begin{tabular}{|c|c|c|}
\hline & Sheet No \\
\hline \multicolumn{3}{|c|}{$\begin{array}{ll}\text { Attachment } & 10 \\
\text { Originators: S. W. Clark }\end{array}$} \\
\hline Chk'd By & M. W. Perrott & Date \\
\hline Calc. No. & $0100 B-C A-V 0301$ & Rev. No. \\
\hline
\end{tabular}




\section{ATTACHMENT 10}

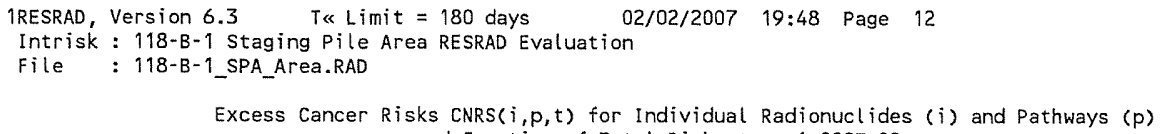

Water Dependent Pathways

\begin{tabular}{|c|c|c|c|c|c|c|c|c|c|c|c|c|}
\hline \multirow[b]{2}{*}{$\begin{array}{l}\text { Nucl ide } \\
\text { AAAAAAAAAA }\end{array}$} & \multicolumn{2}{|c|}{ 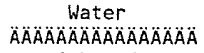 } & \multicolumn{2}{|c|}{ 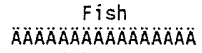 } & \multicolumn{2}{|c|}{ 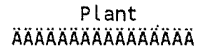 } & \multicolumn{2}{|c|}{$\begin{array}{c}\text { Meat } \\
\triangle A A A A A A A A B A A A A A A\end{array}$} & \multicolumn{2}{|c|}{ 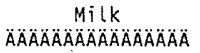 } & \multicolumn{2}{|c|}{ 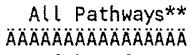 } \\
\hline & ÄÄÄÄ & $\overparen{A} A \ddot{A} \ddot{A} \ddot{A}$ & $\begin{array}{c}\text { risk } \\
A A A A A B A B A\end{array}$ & $\triangle A O A$ & 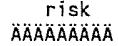 & $\begin{array}{l}\text { fract. } \\
\triangle A A A A A B A\end{array}$ & $\begin{array}{c}\text { risk } \\
A A A A A A A A\end{array}$ & 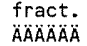 & $\begin{array}{c}\text { risk } \\
\text { AAAAAAAAAA }\end{array}$ & $\begin{array}{l}\text { fract. } \\
\text { ÄAAÄÄ }\end{array}$ & $\begin{array}{c}\text { risk } \\
\text { AAAAAAAA }\end{array}$ & $\begin{array}{l}\text { fract. } \\
\text { AAAAAAB }\end{array}$ \\
\hline$A c-227$ & $0.000 E+00$ & 0.0000 & $E+\infty$ & & & & & & & & & \\
\hline & & & $+\infty$ & & & & & & $O E+O D$ & & & \\
\hline & & & & & & & & & & & & \\
\hline & & & & & & & & & & & & \\
\hline & & & & & & & & & & & & \\
\hline & & & & & & & & & & & & \\
\hline & & & & & & & & & & & & \\
\hline & & & & & & & & & & & & \\
\hline & & & & & & & & & & & & \\
\hline & & & & & & & & & & & & \\
\hline & & & & & & & & & & & & \\
\hline & & & & & & & & & & & & \\
\hline & & & & & & & & & & & & \\
\hline & & & & & & & .00 & & & & & \\
\hline & & & & & & & & & & & & \\
\hline & & & & & & & & & & & & \\
\hline & 0.0 & & $+\infty 0$ & & & & $+\infty$ & & & & & \\
\hline & & & & & & & & & & & & \\
\hline & & & & & & & & & & & & \\
\hline कos & $0.000 E+00$ & 0.0 & $.000 E+00$ & 0.0 & 0 & 0. & 00 & & 00 & & 14 & \\
\hline & & & & & & & & & & & & \\
\hline & & & & & & & & & & & & \\
\hline Tota & $1.464 \mathrm{E}-07$ & 0.0029 & $1.448 E-10$ & 0.0000 & -08 & 0.0011 & 9.06 & 0. & $E-08$ & 0.0 & $5.102 E-05$ & 1. \\
\hline
\end{tabular}

** Sum of water independent ground, inhalation, plant, meat, milk, soil

0 and water dependent water, fish, plant, meat, milk pathways

Excess Cancer Risks CNRS9(irn, $i, t)$ and CNRSPW(irn, $i, t)$ for Inhalation of

Radionuclides

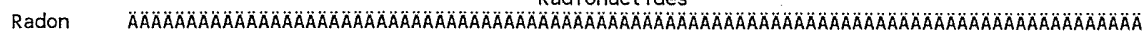

$\begin{array}{llllllll}\text { Pathway Rn-222 } & \text { Po-218 } & \text { Pb-214 } & \text { Bi }-214 & \text { Rn-220 } & \text { Po-216 } & \text { Pb-212 } & B i-212\end{array}$

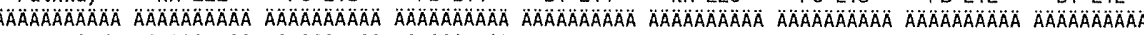

$\begin{array}{lllllllll}\text { Water }- \text { ind. } & 0.000 \mathrm{E}+00 & 0.000 \mathrm{E}+00 & 0.000 \mathrm{E}+00 & 0.000 \mathrm{E}+00 & 0.000 \mathrm{E}+00 & 0.000 \mathrm{E}+00 & 0.000 \mathrm{E}+00 & 0.000 \mathrm{E}+00\end{array}$

$\begin{array}{llllllllll}\text { Water-dep. } \quad 0.000 E+00 & 0.000 E+00 & 0.000 E+00 & 0.000 E+00 & 0.000 E+00 & 0.000 E+00 & 0.000 E+00 & 0.000 E+00\end{array}$

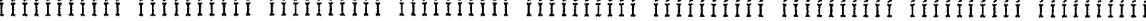

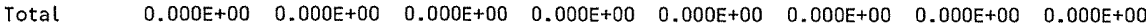

Water-ind. = = Water-independent Water-dep. == Water-dependent

\begin{tabular}{|c|c|c|}
\hline \multicolumn{2}{|c|}{ Attachment $\quad 10$} & \multirow{2}{*}{$\begin{array}{l}\text { Sheet No. } 12 \text { of } 41 \\
\text { Date }\end{array}$} \\
\hline Originators: & S. W. Clark & \\
\hline Chk'd By & M. W. Perrott & Date \\
\hline Calc. No. & $0100 \mathrm{~B}-\mathrm{CA}-\mathrm{V} 0301$ & Rev. No. \\
\hline
\end{tabular}




\section{ATTACHMENT 10}

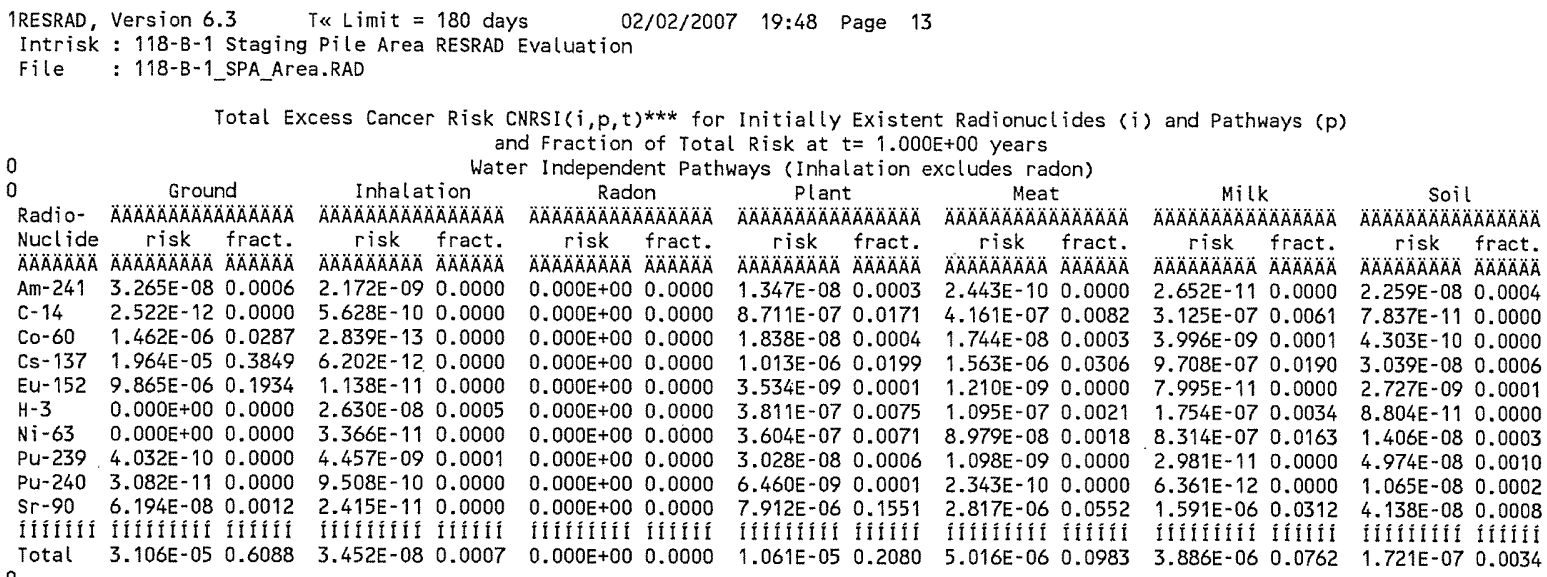

Total Excess Cancer Risk CNRSI $(i, p, t) * * *$ for Initially Existent Radionuclides $(i)$ and Pathways $(p)$ and Fraction of Total Risk at $t=1.000 E+00$ years

Water Dependent Pathways

\begin{tabular}{|c|c|c|c|c|c|c|c|c|c|c|c|c|c|c|}
\hline \multirow{2}{*}{$\begin{array}{l}\text { Radio- } \\
\text { Nuclide } \\
\dddot{A} A \ddot{A} A \ddot{A B A ̈}\end{array}$} & \multicolumn{2}{|c|}{ 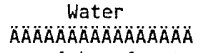 } & \multicolumn{2}{|c|}{ 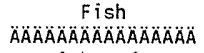 } & \multicolumn{2}{|c|}{ 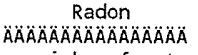 } & \multicolumn{2}{|c|}{ 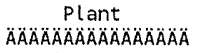 } & \multicolumn{2}{|c|}{$\begin{array}{c}\text { Meat } \\
\text { AAAAAAAAAAAAOAAAAÄÄ }\end{array}$} & \multicolumn{2}{|c|}{ 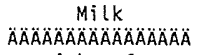 } & \multicolumn{2}{|c|}{ 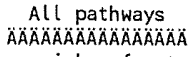 } \\
\hline & $\begin{array}{c}\text { risk } \\
\text { A AAAAAAÄ }\end{array}$ & $\begin{array}{l}\text { fract. } \\
\triangle A A A A A A B\end{array}$ & $\begin{array}{c}\text { risk } \\
A A A A A A B A\end{array}$ & $\begin{array}{l}\text { fract. } \\
A A B A A A B A\end{array}$ & $\begin{array}{c}r i s k \\
A \cap A A A B A O A O A\end{array}$ & $\begin{array}{l}\text { fract. } \\
A A A A A B A B\end{array}$ & $\begin{array}{c}\text { risk } \\
\text { AAAAAAAAAOA }\end{array}$ & $\begin{array}{l}\text { fract. } \\
\triangle A A A A A B A\end{array}$ & $\begin{array}{c}r i s k \\
A A A A A B A A B A\end{array}$ & $\begin{array}{l}\text { fract. } \\
A A A A B A B\end{array}$ & $\begin{array}{c}\text { risk } \\
\text { AAAAAAAAAA }\end{array}$ & $\begin{array}{l}\text { fract. } \\
A \cap A B A O A B\end{array}$ & $\begin{array}{c}\text { risk } \\
\triangle A A B A O A A A B A\end{array}$ & fract. \\
\hline An-241 & $0.000 \mathrm{E}+00$ & 0.0000 & $0.000 E+00$ & 0.0000 & $0.000 E+00$ & 0.0000 & $0.000 \mathrm{E}+00$ & 0.0000 & $0.000 \mathrm{E}+00$ & 0.0000 & $0.000 \mathrm{E}+00$ & 0.0000 & $7.115 \mathrm{E}-08$ & 0.001 \\
\hline$c-1$ & $0.000 \mathrm{E}+00$ & 0 & $E+00$ & 0 & $0 E+00$ & 100 & $0 E+00$ & 0 & $00 E+00$ & 0000 & $0.000 E+00$ & 000 & & 0071 \\
\hline & $E+00$ & & 00 & & 00 & & & & +00 & 00 & $.000 E+00$ & & & \\
\hline & & & & & & & 0 & & 00 & כo & & & & \\
\hline & $0.000 E+00$ & & 00 & & $E+\infty$ & & 10 & & 00 & & $=00$ & & & \\
\hline $\mathrm{H}-3$ & $1.464 \mathrm{E}-07$ & 0 & 0 & 0. & $=+\infty$ & & & & & & 2 & & & \\
\hline$N_{1}$ & $0.000 E+00$ & 0.0 & +00 & 0.0 & $0.000 E+00$ & 0.0 & 0.0 & 0. & 00 & 0 & .00 & & & \\
\hline & 0.0 & & & & 0 & & 0. & 0 & $0.000 E+00$ & 00 & $0.000 E+00$ & 00 & 8 & 0.00 \\
\hline & $0.000 E+00$ & & 00 & & $E+00$ & & & & & & & & & \\
\hline & & 0.0000 & & & & & & & & & & & & \\
\hline & & & & & & & & & IIfififin & & IIIIIIIIII & ifi & $101+1+2$ & \\
\hline Tot & $1.464 \mathrm{E}-07$ & 0.0029 & $48 E-10$ & 0.0 & $.000 E+00$ & 0.0000 & $.520 \mathrm{E}-08$ & 0.0011 & $9.069 E-09$ & 0.0002 & $2.862 E-08$ & 0.0006 & $5.102 E-05$ & \\
\hline
\end{tabular}

$* * *$ CNRSI $(i, p, t)$ includes contribution from decay daughter radionuclides

\begin{tabular}{|c|c|}
\hline Attachment & Sheet No. \\
\hline Originators: S. W. Clark & Date \\
\hline Chk'd By M.W. Perrott & Date \\
\hline Calc. No. $\quad 0100$ B-CA-V0301 & Rev. No. \\
\hline
\end{tabular}




\section{ATTACHMENT 10}

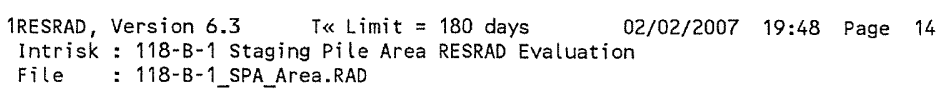




\section{ATTACHMENT 10}

\begin{tabular}{|c|c|c|c|c|c|c|c|c|c|c|}
\hline \multirow[t]{2}{*}{$\begin{array}{l}\text { 1RESRAD, } \\
\text { Intrisk } \\
\text { File }\end{array}$} & $\begin{array}{l}\text { Version } 6 \\
: 118-\mathrm{B}-1 \\
: 118-\mathrm{B}-1\end{array}$ & $\begin{array}{l}-5 \\
\text { Stagin } \\
\text { SPA_Ar }\end{array}$ & \multicolumn{3}{|c|}{$\begin{array}{l}\text { T« Limit }=180 \text { days } \\
\text { Pile Area RESRAD Evaluation } \\
\text { a.RAD }\end{array}$} & $02 / 02 / 20$ & $19: 48$ & age 15 & \multicolumn{2}{|c|}{15} \\
\hline & \\
\hline & Groun & & Inhalat & tion & Pla & ant & Mea & & Milk & Soil \\
\hline Radio- & $\triangle A A A A A A A A B A$ & $\triangle A ̈ A ̈ A ̈ A ̈ A ̈$ & $\triangle A A A A A A A A$ & $\triangle A A A A A B$ & $\triangle A A A A A A A A A$ & $\triangle A A A A A O A O A$ & $\triangle A A A A A A O A O A B A$ & $\triangle A O A O A O A O A ̈ A ̈$ & 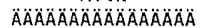 & $\triangle A \cap A B A A A A A A B A O A O A A A$ \\
\hline $\begin{array}{l}\text { Nuclide } \\
\text { ÄAÄAAAOÄ }\end{array}$ & risk & $\begin{array}{l}\text { fract. } \\
\ddot{A} A \ddot{A} \ddot{A} \ddot{A} \ddot{A}\end{array}$ & $\begin{array}{l}\text { risk } \\
\triangle A ̈ A ̈ A ̈ A ̈ A ̈ A ̈\end{array}$ & $\begin{array}{l}\text { fract. } \\
\ddot{A} \ddot{A} \ddot{A} A \ddot{A} \ddot{A}\end{array}$ & $\begin{array}{l}\text { risk } \\
M A B A O A B A B\end{array}$ & $\begin{array}{l}\text { fract. } \\
\triangle A B A O A B A ̈\end{array}$ & $\begin{array}{l}\text { risk } \\
\triangle A O A O A O A B A\end{array}$ & $\begin{array}{l}\text { fract. } \\
\triangle A B A A B A B A\end{array}$ & $\begin{array}{l}\text { risk fract. } \\
\text { ÄABÄÄ AAAÄÄÄ }\end{array}$ & $\begin{array}{r}\text { risk fract. } \\
\text { AAAAAAAAA AAAAAA }\end{array}$ \\
\hline$A C-227$ & $2.539 E-18$ & 0.0000 & $2.413 \mathrm{E}-20$ & 0.0000 & $2.494 \mathrm{E}-19$ & 0.0000 & $3.786 \mathrm{E}-21$ & 10.0000 & $2.246 E-210.0000$ & $1.808 E-190.0000$ \\
\hline$A m-241$ & $3.254 \mathrm{E}-08$ & 0.0007 & $2.165 \mathrm{E}-09$ & 0.0000 & -08 & 30.0003 & $5 E-10$ & 00.0000 & $E-110.0000$ & $2.251 \mathrm{E}-08 \quad 0.0005$ \\
\hline$c-14$ & 1.4 & 0.0000 & 3.16 & 0.0000 & -08 & 80.0010 & $3 E-08$ & 80.0005 & .080 .0004 & -120.0000 \\
\hline $\mathrm{Co}-60$ & & 0.0240 & 13 & 0.0000 & -08 & 80.0003 & 1.3 & 80.0003 & -090.0 & 100.0000 \\
\hline Cs - & 1.8 & 0.4010 & 2 & 0.0000 & -07 & 70.0207 & $2 E-06$ & 60.0319 & $E-07 \quad 0.0198$ & -080.0006 \\
\hline Eu-1 & 8.8 & 0.1902 & 1. & 0.0000 & & & $E-09$ & 90.0000 & $\begin{array}{ll}-11 & 0.0000\end{array}$ & $2.457 \mathrm{E}-09 \quad 0.0001$ \\
\hline Gd-1 & $E+00$ & 0.0000 & 4.6 & 0.0000 & -22 & 00 & $E-22$ & 20.0000 & 240.0000 & $-22 \quad 0.0000$ \\
\hline $\mathrm{H}-3$ & +00 & 0.0000 & 3.1 & 0.0001 & -08 & 80.0010 & 1.3 & 80.0003 & $.08 \quad 0.0005$ & -110.0000 \\
\hline $\mathrm{Ni}-6$ & & 0.0000 & 11 & & & & $4=08$ & 80.0019 & $-07 \quad 0.0175$ & $=-080.0003$ \\
\hline $\mathrm{Np}-2$ & 5 & 0.0000 & 7.9 & 0.0000 & 12 & 20. & $B E-14$ & 40.0000 & 160.0 & -140.0000 \\
\hline $\mathrm{Pa}-$ & 1.1 & 0.0000 & -20 & 0.0000 & -18 & 30.0000 & $5.844 E-19$ & 90.0000 & -210.0000 & -190.0000 \\
\hline $\mathrm{Pu}-2$ & 10 & 0.0000 & -09 & 0.0001 & & & $1.098 \mathrm{E}-09$ & 90.0000 & $2.981 E-11 \quad 0.0000$ & $4.973 E-08 \quad 0.0011$ \\
\hline $\mathrm{Pu}-2$ & 3.0 & 0.0000 & 10 & 0.0 & 6 & & 2. & 00.0000 & 120.0000 & $.08 \quad 0.0002$ \\
\hline Ra- & & 0.0000 & -26 & 0.0000 & -22 & 20.0000 & $1.578 E-23$ & 30.0000 & -230.0000 & -230.0000 \\
\hline $\mathrm{sr}-\mathrm{s}$ & & 0.0013 & -11 & 0.0 & & & $2.684 \mathrm{E}-06$ & 60.0574 & $\begin{array}{lll}1.516 \mathrm{E}-06 & 0.0324\end{array}$ & $\begin{array}{lll}3.943 \mathrm{E}-08 & 0.0008\end{array}$ \\
\hline Th-228 & $3.757 E-22$ & 0.0000 & 4.63 & 0.0000 & -24 & & $3 E-25$ & 50.0000 & $=-260.0000$ & $E-24 \quad 0.0000$ \\
\hline Th-? & 2.8 & 0.0000 & 3.73 & 0.0000 & $E-20$ & 0.0000 & $5.749 E-22$ & 20.0000 & $7.988 \mathrm{E}-23 \quad 0.0000$ & $2.884 \mathrm{E}-20 \quad 0.0000$ \\
\hline Th-232 & $4.153 E-26$ & 0.0000 & $3.458 E-25$ & 0.0000 & $1.388 \mathrm{E}-24$ & +0.0000 & $5.091 \mathrm{E}-26$ & 60.0000 & 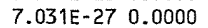 & $2.484 \mathrm{E}-240.0000$ \\
\hline$u-233$ & $3.121 \mathrm{E}-19$ & 0.0000 & $2.447 \mathrm{E}-19$ & 0.0000 & $6.804 E-18$ & & $4.086 E-19$ & 90.0000 & $1.834 E-18 \quad 0.0000$ & $4.544 E-18 \quad 0.0000$ \\
\hline$U-23$ & & & 2.2 & & -16 & 60.0 & $4.133 \mathrm{E}-17$ & 70.0000 & 160.0 & $=-16 \quad 0.0000$ \\
\hline & & & & & & 50.0000 & & 0.00 & 150.0 & $2.888 \mathrm{E}-15 \quad 0.0000$ \\
\hline & & & & & & & IIIfítín & I IfIIIfi & 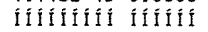 & 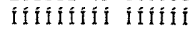 \\
\hline Total & $2.885 E-05$ & 0.6172 & $1.087 \mathrm{E}-08$ & 0.0002 & $1 E-06$ & 60.1930 & $4.317 \mathrm{E}-06$ & 60.0923 & $\begin{array}{lll}3.303 E-06 & 0.0707\end{array}$ & $1.680 E-07 \quad 0.0036$ \\
\hline
\end{tabular}

\begin{tabular}{|c|c|c|}
\hline \multicolumn{2}{|c|}{ Attachment $\quad 10$} & Sheet No. 15 of 41 \\
\hline Originators & S. W. Clark & Date. \\
\hline d By & M. W. Perrott & Date \\
\hline Calc. No. & 0100B-CA-V0301 & Rev. No. 0 \\
\hline
\end{tabular}




\section{ATTACHMENT 10}

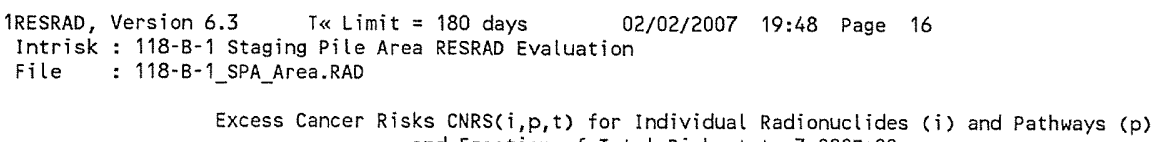

Water Dependent Pathways

\begin{tabular}{|c|c|c|c|c|c|c|c|c|c|c|c|c|}
\hline & \multicolumn{2}{|c|}{ Water } & \multicolumn{2}{|c|}{ Fish } & \multicolumn{2}{|c|}{ Plant } & \multicolumn{2}{|c|}{ Meat } & \multicolumn{2}{|c|}{ Milk } & \multicolumn{2}{|c|}{$\begin{array}{l}\text { All Pathways** } \\
A A A A A A A A A A A A A A A\end{array}$} \\
\hline $\begin{array}{l}\text { Nuclide } \\
\qquad A \cap A B A A A\end{array}$ & $\begin{array}{c}\text { risk } \\
\triangle A \cap A A B A A B A\end{array}$ & $\begin{array}{l}\text { fract. } \\
A R A B A A B A\end{array}$ & $\begin{array}{c}\text { risk } \\
\triangle A A A A B A A A A A\end{array}$ & $\begin{array}{l}\text { fract. } \\
\ddot{A} \ddot{A} A \ddot{A} \ddot{A} \ddot{A}\end{array}$ & 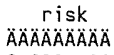 & 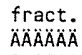 & 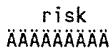 & $\begin{array}{l}\text { fract. } \\
\text { ArAAAAAA }\end{array}$ & $\begin{array}{c}r i s k \\
A \cap A ̈ A B A A A A B\end{array}$ & $\begin{array}{l}\text { fract. } \\
\ddot{A} \ddot{A} A \ddot{A} \ddot{A} A\end{array}$ & $\begin{array}{c}\text { risk } \\
\triangle A \cap A B A B A A A\end{array}$ & $\begin{array}{l}\text { fract. } \\
\text { ÄAAAAAA }\end{array}$ \\
\hline & $0.000 E+00$ & 0 . & $.000 \mathrm{E}+00$ & 1000 & $.000 E+00$ & 0.0000 & $.000 E+00$ & 0.0000 & $0.000 E+00$ & 0.0000 & $3.000 E-18$ & 0.00 \\
\hline & & & $00 F+00$ & & $O E+00$ & & $E+00$ & & $O E+O O$ & & & \\
\hline & & & 0.0 & 0.0000 & $E+00$ & 000 & $E+00$ & & & & & \\
\hline & $E+00$ & & 0.0 & 0.0000 & $000 E+00$ & 0.0000 & $O E+0 O$ & & $O E+O D$ & & & 0.0247 \\
\hline & -00 & & $+\infty 0$ & 0.0 & +00 & 0 & $+\infty 0$ & & $E+00$ & & & \\
\hline & & & & & $=00$ & & & & & & & \\
\hline & 0.0 & & & & 00 & & $+\infty$ & & 00 & & 21 & \\
\hline $4-3$ & 77 & & 6. & & -07 & & & & & & & \\
\hline & & & +00 & & $:+00$ & & +00 & & 00 & & & \\
\hline & & & & & $=+\infty$ & & +00 & & -00 & & & \\
\hline & $+\infty 0$ & & 0. & & +00 & & & & & & & \\
\hline & 100 & & .0 & 0. & +00 & & 00 & & & & & \\
\hline & & & 0.0 & & 00 & & 00 & & +00 & & & \\
\hline & 100 & & & & & & & & $E+00$ & 100 & -22 & 000 \\
\hline & .00 & & 0. & & +00 & & $E+\infty 0$ & & & & & \\
\hline Th- & $.000 E+00$ & 0.0 & $0.000 E+00$ & 0.0 & $E+00$ & & $=00$ & & +00 & & & \\
\hline & & & & & & & $E+\infty$ & & $0.000 E+00$ & 00 & $E-19$ & 0.0000 \\
\hline & 100 & & +00 & & 0 & & & & & & & \\
\hline$u-23$ & $.000 E+00$ & 0.0 & $.000 E+00$ & & & & & & & & & \\
\hline & & & & & $0.000 E+00$ & & +00 & & $0.000 \mathrm{E}+00$ & 00 & $E-14$ & 0.0000 \\
\hline & & & & & & & & & & & & \\
\hline & & & & & & & & & & & & \\
\hline Tat & $.570 E-07$ & .012 & $.502 E-10$ & 0.0000 & -07 & 33 & $E-08$ & 0.0009 & $1.285 E-07$ & 28 & 0. & 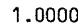 \\
\hline
\end{tabular}

** Sum of water independent ground, inhalation, plant, meat, milk, soil

0

and water dependent water, fish, plant, meat, milk pathways

Excess Cancer Risks CNRSP(irn, $i, t)$ and CNRSPW(irn, $i, t)$ for Inhalation of Radon and $i$ ts Decay Products at $t=3.000 E+00$ years Radionucl ides

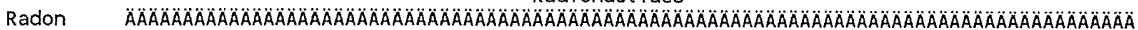
Pathway Rn-222 Po-218 $\quad$ Pb-214 Bi-214 $\quad$ Rn-220 Po-216 Pb-212 Bi-212

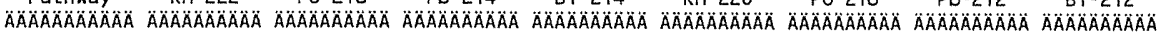

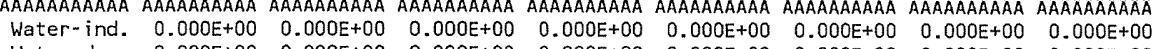
$\begin{array}{lllllllll}\text { Water-dep: } & 0.000 E+00 & 0.000 E+00 & 0.000 E+00 & 0.000 E+00 & 0.000 E+00 & 0.000 E+00 & 0.000 E+00 & 0.000 E+00\end{array}$

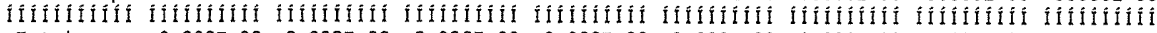

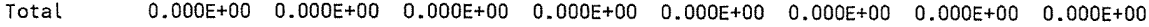

Water-ind. = water-independent Water-dep. == Water-dependent

\begin{tabular}{|c|c|}
\hline Attachment & Sheet No. 16 of 41 \\
\hline Originators: S. W. Clark & Date \\
\hline Chk'd By M. W. Perrott & Date \\
\hline Calc. No. $0100 \mathrm{~B}-\mathrm{CA}-\mathrm{V} 0301$ & Rev. No. $\quad 0$ \\
\hline
\end{tabular}




\section{ATTACHMENT 10}

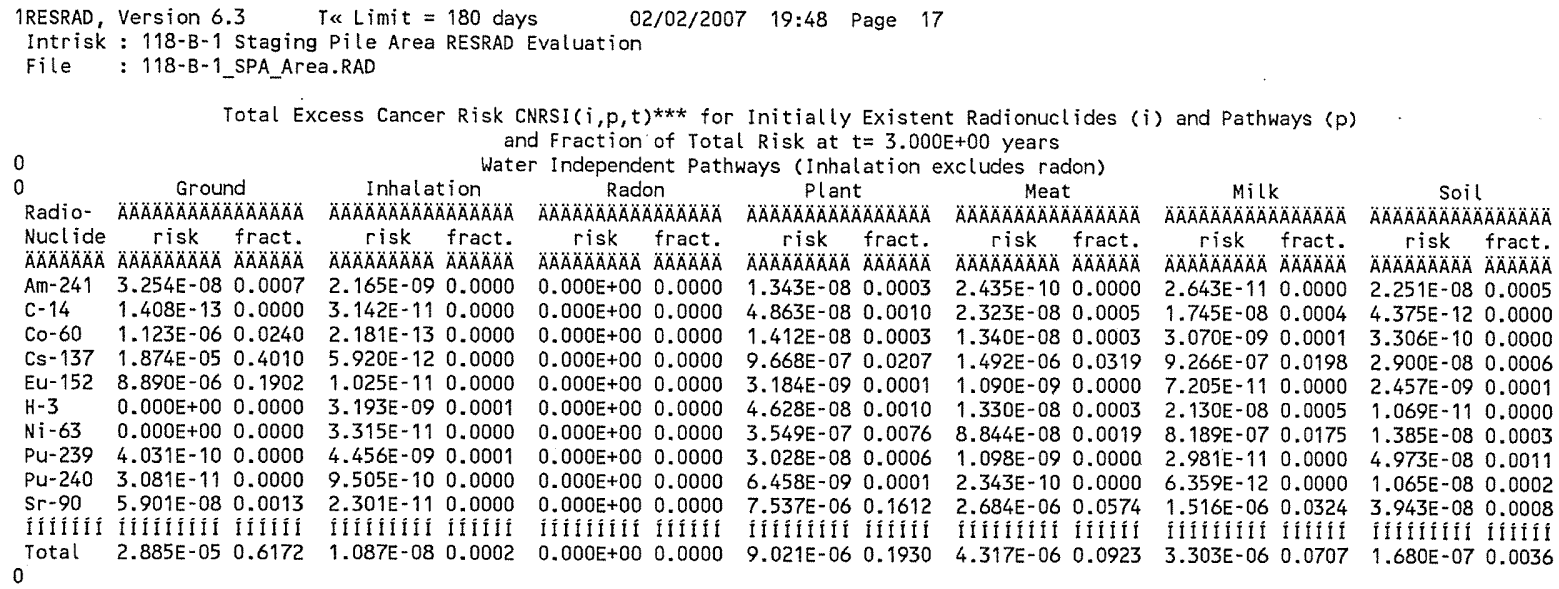

Total Excess Cancer Risk CNRSI $(i, p, t) * * *$ for Initially Existent Radionuclides (i) and Pathways ( $p$ ) and Fraction of Total Risk at $t=3.000 E+00$ years

Water Dependent Pathways

\begin{tabular}{|c|c|c|c|c|c|c|c|c|c|c|c|c|c|c|}
\hline & \multicolumn{2}{|c|}{ Water } & \multicolumn{2}{|c|}{$\begin{array}{c}\text { Fish } \\
\text { ĀAAAAAAAAAAAAA }\end{array}$} & \multicolumn{2}{|c|}{$\begin{array}{c}\text { Radon } \\
\text { AAAAAAAAAAAAAAAOA }\end{array}$} & \multicolumn{2}{|c|}{$\begin{array}{c}P l \text { ant } \\
\text { ÄAAAAAAAAAAAAAAOA }\end{array}$} & \multicolumn{2}{|c|}{$\begin{array}{c}\text { Meat } \\
\text { AAAAAAAAAAAAAAAOA }\end{array}$} & \multicolumn{2}{|c|}{$\begin{array}{c}M i l k \\
\text { AOAAAAAAAAAAAAAAAOA }\end{array}$} & \multicolumn{2}{|c|}{$\begin{array}{c}\text { All pathways } \\
A A A A A A A A A A A A A A B A\end{array}$} \\
\hline $\begin{array}{l}\text { uclide } \\
A B A A B A B A\end{array}$ & $\begin{array}{c}\text { risk } \\
A A A A A A A A\end{array}$ & $\begin{array}{l}\text { fract. } \\
\triangle A A A A A A\end{array}$ & $\begin{array}{c}r i s k \\
\triangle A A A A A B A A B\end{array}$ & $\begin{array}{l}\text { fract. } \\
\triangle A B A A B A ̈\end{array}$ & $\begin{array}{c}\text { risk } \\
A A B A A O A A A\end{array}$ & 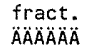 & risk & $\begin{array}{l}\text { fract. } \\
\mathscr{A} \ddot{A} A B A O A B A\end{array}$ & $\begin{array}{c}\text { risk } \\
\triangle A A A A A A A A A\end{array}$ & $\begin{array}{l}\text { fract. } \\
\overparen{A A B A O A O A}\end{array}$ & $\begin{array}{c}\text { risk } \\
\triangle A A A B A A B A A\end{array}$ & $\begin{array}{l}\text { fract. } \\
\text { AAABAAA }\end{array}$ & $\begin{array}{c}\text { risk } \\
A A A A A A B A\end{array}$ & $\begin{array}{l}\text { fract. } \\
A B A B A B A\end{array}$ \\
\hline$m-241$ & $0.000 E+00$ & 0.0000 & $0.000 E+00$ & 0.0000 & $0.000 E+00$ & & & & 0.00 & 0.0 & $0.000 E+00$ & 0.0000 & $1 E-08$ & 15 \\
\hline C- 14 & $0.000 \mathrm{E}+00$ & 0.0000 & $0.000 \mathrm{E}+00$ & 0000 & $0.000 E+00$ & 0.0000 & 0. & & $1 O E+00$ & 0.0000 & $0.000 E+00$ & 0.0000 & & 0.0019 \\
\hline 0.60 & $0.000 E+00$ & 0.0 & $O E+0 O$ & 00 & $0.000 E+00$ & 0.0000 & $E+00$ & & $O E+00$ & 000 & $0.000 E+00$ & 0.0000 & 1.1 & 0.0247 \\
\hline & $0.000 E+00$ & 0. & $10 E+00$ & 000 & $0.000 E+00$ & 0.0000 & 0 . & 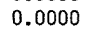 & 30 & 00 & & & & \\
\hline Eu-152 & & & & & 0.00 & 0.0000 & 0. & 0 & 00 & 000 & $0.000 E+00$ & 100 & 8.8 & 0.1903 \\
\hline$H-3$ & .57 & & 10 & & $0.000 E+00$ & 100 & & & & & $1.285 \mathrm{E}-07$ & 028 & -06 & 0.0248 \\
\hline 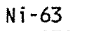 & $0.000 E+00$ & 0.0000 & $0.000 E+00$ & 00 & 0.00 & 0. & $E+00$ & & $+\infty$ & 00 & 10 & 00 & & \\
\hline Pu-239 & $0.000 E+00$ & 0.0000 & $0.000 E+00$ & 0.0000 & $0.000 E+00$ & 0.0000 & $0.000 E+00$ & 0.0000 & 0.0 & 0.0000 & $0.000 E+00$ & 0. & & 018 \\
\hline & $0.000 \mathrm{E}+00$ & & & & 0.00 & 0. & 0.00 & 0.0 & $0.000 E+00$ & 0.0000 & $0.000 E+00$ & 0.0000 & $E-08$ & 0.0004 \\
\hline & & & $E+00$ & & 0.00 & & & & & & & & & \\
\hline & & & fíflifiti & & IIIIIIII & Ififif & Ififítili & 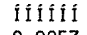 & & & & & & \\
\hline otal & $6.570 \mathrm{E}-07$ & 0.0141 & $6.502 E-10$ & 0.0000 & $0.000 E+00$ & 0.0000 & $2.479 E-07$ & 0.0053 & $4.081 E-08$ & 0.0009 & $1.285 E-07$ & 0.0028 & $4.674 E-05$ & 1.0000 \\
\hline
\end{tabular}

$* * *$ CNRSI $(i, p, t)$ includes contribution from decay daughter radionuclides

\begin{tabular}{|c|c|}
\hline Attachment & Sheet No. 17 of 41 \\
\hline Originators: S. W. Clark & Date \\
\hline Chk'd By M.W. Perrott & Date \\
\hline Calc. No. $\quad 0100 B-C A-V 0301$ & Rev. No. \\
\hline
\end{tabular}




\section{ATTACHMENT 10}

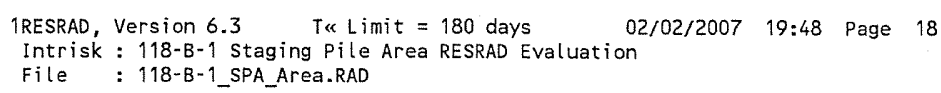

Water-ind. = = Water-independent Water-dep. == Water-dependent

\begin{tabular}{|c|c|}
\hline Attachment & Sheet No. 18 of 41 \\
\hline Originators: S. W. Clark & Date \\
\hline Chk'd By M. W. Perrott & Date \\
\hline Calc. No. 0100B-CA-V0301 & Rev. No. 0 \\
\hline
\end{tabular}




\section{ATTACHMENT 10}

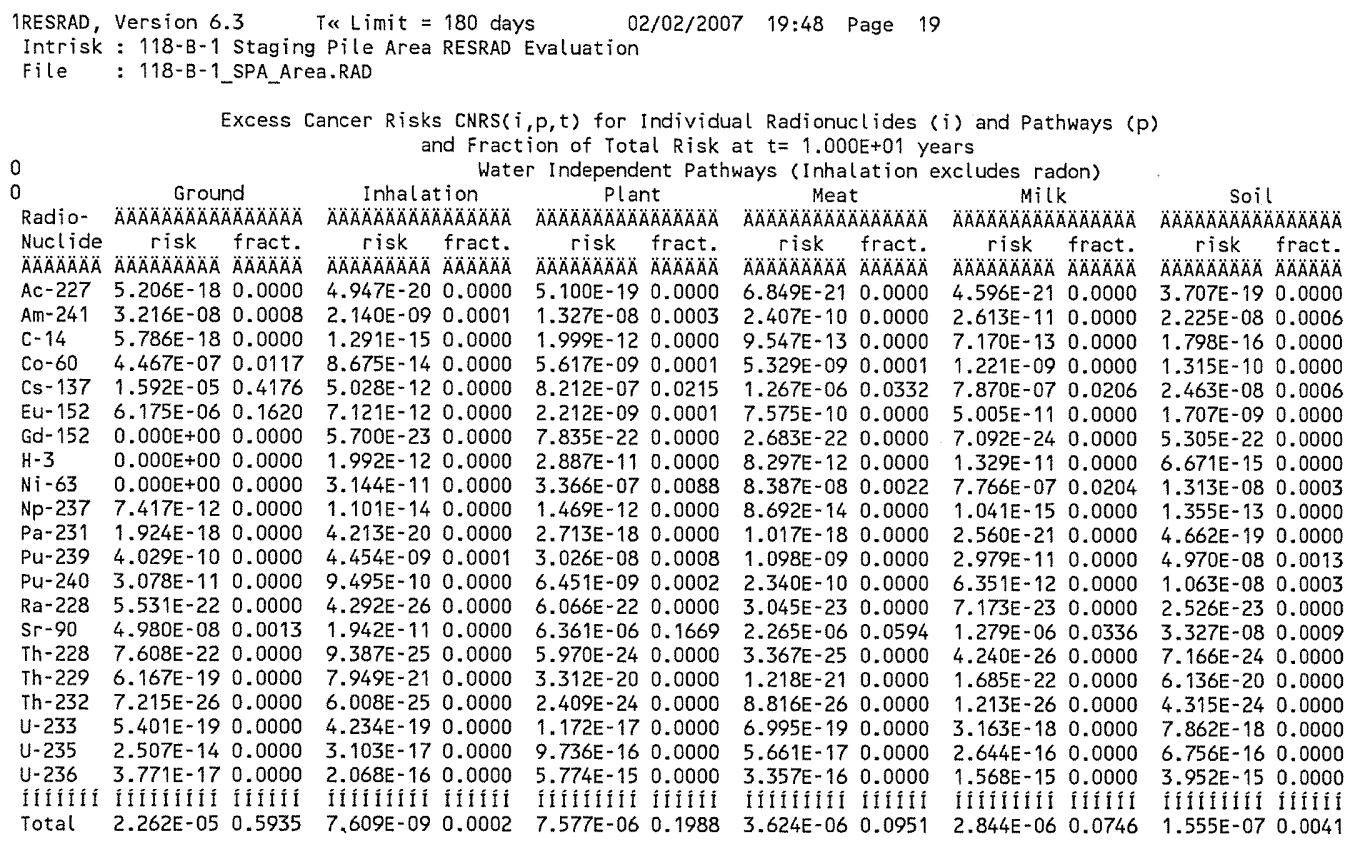

\begin{tabular}{|c|c|c|}
\hline Attachment & 10 & Sheet No. 19 of 41 \\
\hline Originators: & S. W. Clark & Date \\
\hline $\mathrm{Chk}^{2} \mathrm{~d} \mathrm{By}$ & M. W. Perrott & Date \\
\hline Calc. No. & $0100 B-C A-V 0301$ & Rev. No. \\
\hline
\end{tabular}




\section{ATTACHMENT 10}

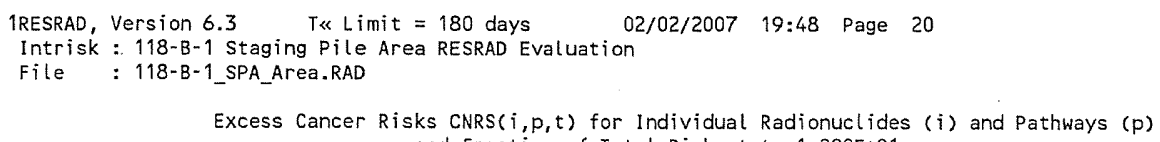

Water Dependent Pathways

\begin{tabular}{|c|c|c|c|c|c|c|c|c|c|c|c|c|}
\hline & \multicolumn{2}{|c|}{ Water } & \multicolumn{2}{|c|}{ Fish } & \multicolumn{2}{|c|}{ Plant } & \multicolumn{2}{|c|}{ Meat } & \multicolumn{2}{|l|}{ Milk } & \multicolumn{2}{|c|}{$\begin{array}{r}\text { All Pathways** } \\
\text { AAAAAAAAAAAAAAAAA }\end{array}$} \\
\hline $\begin{array}{l}\text { Uclide } \\
\text { ACAÄÄÄA }\end{array}$ & $\begin{array}{c}\text { risk } \\
\triangle A ̈ A ̈ A ̈ A ̈ A ̈ A\end{array}$ & $\begin{array}{l}\text { fract. } \\
\qquad A \cap A B A B A\end{array}$ & $\begin{array}{c}\text { risk } \\
A \cap A B A A A A A\end{array}$ & $\begin{array}{l}\text { fract: } \\
\text { ÁAAAAA }\end{array}$ & $\begin{array}{c}r i s k \\
A \because A O A O A O A O A\end{array}$ & $\begin{array}{l}\text { fract. } \\
\text { ÄÄÄÄÄ }\end{array}$ & $\begin{array}{c}\text { risk } \\
\text { ÄÄÄÄÄÄÄÄ }\end{array}$ & $\ddot{A}$ & $\begin{array}{c}\text { risk } \\
\triangle A A A A A A A A A A\end{array}$ & $\begin{array}{l}\text { fra } \\
\stackrel{A}{ } \mathrm{~A} A ̈\end{array}$ & $\begin{array}{c}\text { risk } \\
\text { ĀAAAAAAAÄ }\end{array}$ & $\begin{array}{l}\text { fract. } \\
\triangle A A A A A B A\end{array}$ \\
\hline$c-227$ & $500 E+00$ & 0000 & $000 E+00$ & 0000 & $0.000 E+00$ & 000 & $0.000 E+00$ & 0.0000 & $0.000 E+00$ & 0.0000 & $6.148 E-18$ & 0.0000 \\
\hline & & & & & & & & & $E+00$ & & & \\
\hline-14 & & & & & & & & & $E+\infty O$ & & & \\
\hline & $E+00$ & & $\mathrm{DE}+0 \mathrm{O}$ & & $000 E+00$ & 0.0000 & $.000 E+00$ & & $0.000 E+00$ & & .07 & 120 \\
\hline & 00 & & 0 & & 0 & & $O E+0 O$ & & $E+00$ & & & \\
\hline & & & & & 10 & & +00 & & & & & \\
\hline & & & & & & 000 & $0 E+00$ & & $E+\infty 0$ & & & 000 \\
\hline-3 & 07 & & 10 & & & & 08 & & .07 & & & \\
\hline & & & & & & & +00 & & -00 & & & \\
\hline & & & & & & 000 & $E+00$ & & +00 & & & 00 \\
\hline & +00 & & & & & & .00 & & & & & \\
\hline & 00 & & jo & & & & +00 & & +00 & & & \\
\hline & +00 & & o & & 00 & 0. & $=+00$ & & +00 & & & \\
\hline & & & & & & & & & & & & \\
\hline & +00 & & 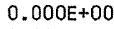 & & 0 & & $+\infty$ & & $E+\infty$ & & & \\
\hline & $E+00$ & 0.0 & $=+00$ & 0.0 & $0.000 \mathrm{E}+00$ & 000 & $O E+00$ & & $E+00$ & & & \\
\hline & & & & & & & & & & & & \\
\hline & 0.00 & & & & 10 & & 0 & & & & & \\
\hline 2 & $0.000 E+00$ & 0.0 & $.000 E+00$ & 0.0 & $0.000 E+00$ & 0.0 & $0.000 E+00$ & & $E+00$ & & 2.4 & \\
\hline & & & & & & & & & & & & \\
\hline & 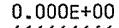 & & & & & & & & & & & \\
\hline & & & & & & & & & & & & \\
\hline ot & $70015=0$ & 07 & $.792 E-10$ & 0.0000 & $2.958 E-07$ & 0.0 & $E-08$ & 12 & $-0, \quad-2$ & 40 & $=-03$ & 1.0000 \\
\hline
\end{tabular}

** Sum of water independent ground, inhalation, plant, meat, milk, soil

0 and water dependent water, fish, plant, meat, milk pathways Excess Cancer Risks CNRS9(irn, $i, t$ ) and CNRSPW(irn, $i, t)$ for Inhalation of Radon and its Decay Products at $t=1.000 E+01$ years

0 Radionuclides

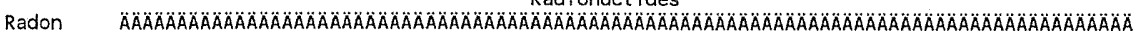

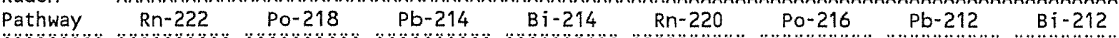

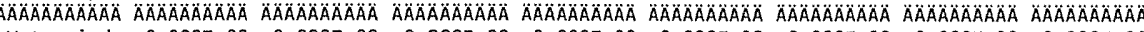
$\begin{array}{llllllllll}\text { Water }- \text { ind. } & 0.000 \mathrm{E}+00 & 0.000 \mathrm{E}+00 & 0.000 \mathrm{E}+00 & 0.000 \mathrm{E}+00 & 0.000 \mathrm{E}+00 & 0.000 \mathrm{E}+00 & 0.000 \mathrm{E}+00 & 0.000 \mathrm{E}+00\end{array}$ $\begin{array}{lllllllll}\text { Water-dep. } & 0.000 E+00 & 0.000 E+00 & 0.000 E+00 & 0.000 E+00 & 0.000 E+00 & 0.000 E+00 & 0.000 E+00 & 0.000 E+00\end{array}$

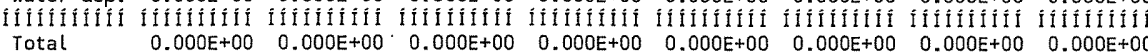

Water-ind. $==$ Water-independent Water-dep. $==$ Water-dependent

\begin{tabular}{|c|c|}
\hline Attachment & Sheet No. 20 of 41 \\
\hline Originators: S.W. Clark & Date \\
\hline M.W. Perrott & Date \\
\hline $0100 B-C A-V 0301$ & Rev. No. 0 \\
\hline
\end{tabular}




\section{ATTACHMENT 10}

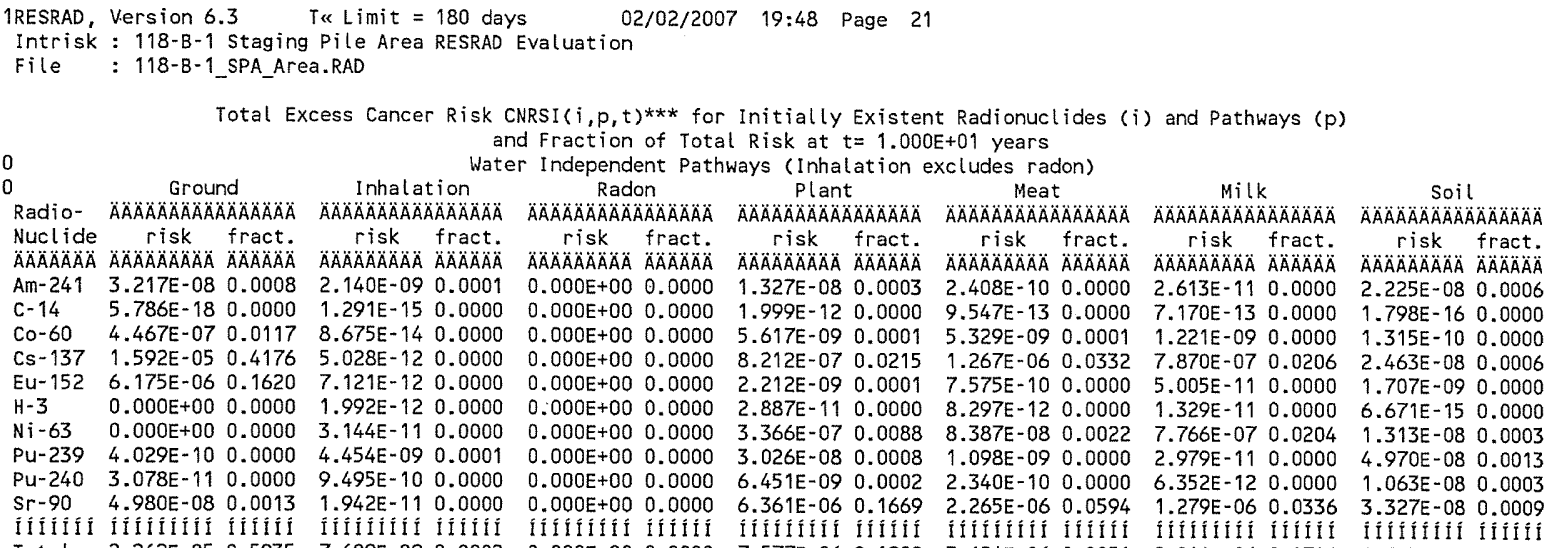

Total Excess Cancer Risk CNRSI $(i, p, t) * * *$ for Initially Existent Radionuclides (i) and Pathways ( $p$ ) and Fraction of Total Risk at $t=1.000 E+01$ years

Water Dependent Pathways

\begin{tabular}{|c|c|c|c|c|c|c|c|c|c|c|c|c|c|c|}
\hline & \multicolumn{2}{|c|}{ Water } & \multicolumn{2}{|c|}{ Fish } & 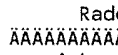 & IO & 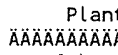 & I & Meat & ${ }^{t}$ & $\begin{array}{r}M i L K \\
\text { AAAAAAAAAA }\end{array}$ & $\begin{array}{l}k \\
K A A A A A A B A\end{array}$ & \multicolumn{2}{|c|}{$\begin{array}{c}\text { All pathways } \\
\text { ÄÄÄAAAAAAAAAAAAAAA }\end{array}$} \\
\hline $\begin{array}{l}\text { Nuclide } \\
\text { ÄAAAAAAAA }\end{array}$ & $\begin{array}{c}r i s k \\
\triangle A A A A B A O A O A B A\end{array}$ & $\begin{array}{l}\text { fract. } \\
\triangle A A A A A A B\end{array}$ & $\begin{array}{c}r i s k \\
\triangle A A B A O A B A B A\end{array}$ & $\begin{array}{l}\text { fract. } \\
\triangle A O A A B A A\end{array}$ & $\begin{array}{c}r i s k \\
\triangle A B A O A O A B A B A\end{array}$ & $\begin{array}{l}\text { fract. } \\
\because A O A B A B A B\end{array}$ & $\begin{array}{c}r i s k \\
\triangle A A A B A B A O A A\end{array}$ & 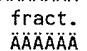 & $\begin{array}{c}r i s k \\
A B A A B A A A B\end{array}$ & 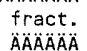 & $\begin{array}{r}\text { risk } \\
\triangle A A B A A A B A\end{array}$ & $\begin{array}{l}\text { fract. } \\
\triangle A A A B A\end{array}$ & $\begin{array}{c}\text { risk } \\
\text { AAAAAAAOA }\end{array}$ & fract. \\
\hline$A m-241$ & $0.000 E+00$ & 0.0000 & $0.000 \mathrm{E}+00$ & 0.0000 & $0.000 E+00$ & 0.0000 & $0.000 \mathrm{E}+00$ & 0.0000 & $0.000 E+00$ & 0.0000 & $0.000 \mathrm{E}+00$ & 0.0000 & $7.010 E-08$ & 0.0018 \\
\hline C -14 & $0.000 E+00$ & 0.0000 & $0.000 E+00$ & 0.0000 & $0.000 E+00$ & 0.0000 & $0.000 E+00$ & 0.0000 & $0.000 E+00$ & 0.0000 & $0.000 E+00$ & 0.0000 & $3.672 E-12$ & 0.0000 \\
\hline $\mathrm{Co}-60$ & $0.000 E+00$ & 0.0000 & $0.000 \mathrm{E}+00$ & 0.0000 & $0.000 E+00$ & 0.0000 & $0.000 E+00$ & 0.0000 & $0.000 E+00$ & 0.0000 & $0.000 E+00$ & 0.0000 & $4.590 \mathrm{E}-07$ & 0.0120 \\
\hline & $0.000 \mathrm{E}+00$ & 0.0000 & $0.000 \mathrm{E}+00$ & 0.0000 & $0.000 E+00$ & 0.0000 & $0.000 E+00$ & 0.0000 & $0.000 E+00$ & 0. & $00 \mathrm{E}+00$ & 00 & & 937 \\
\hline Eu & $0.000 E+00$ & 0.0000 & 0.0 & 0.0000 & $0.000 E+00$ & 0.0000 & $0.000 E+00$ & 0.0000 & $0.000 E+00$ & 0.0000 & $30 E+00$ & 0.0000 & -06 & 0.1621 \\
\hline$H-3$ & $7.901 E-07$ & 0.0207 & $7.792 \mathrm{E}-10$ & 0.0000 & $0.000 E+00$ & 0.0000 & $2.958 E-07$ & 0.0078 & -08 & & -07 & & -06 & 0.0338 \\
\hline $\mathrm{Ni}-63$ & $0.000 \mathrm{E}+00$ & 0.0000 & $0.000 \mathrm{E}+00$ & 0.0000 & $0.000 E+00$ & 0.0000 & $0.000 E+00$ & 0.0000 & $0.000 E+00$ & 000 & $0.000 \mathrm{E}+00$ & 000 & & 17 \\
\hline Pu-239 & $0.000 E+00$ & 0.0000 & $0.000 \mathrm{E}+00$ & 0.0000 & $0.000 \mathrm{E}+00$ & 0.0000 & $0.000 E+00$ & 0.0000 & $0.000 E+00$ & 0.0000 & $0.000 E+00$ & 0.0000 & $8.595 E-08$ & 0.0023 \\
\hline Pu- $2<0$ & $0.000 \mathrm{E}+00$ & 0.0000 & $0.000 \mathrm{E}+00$ & 0.0000 & $.000 E+00$ & 0.0000 & $0.000 E \div 00$ & 0.0000 & $0.000 E+00$ & 0.0000 & $0.000 \mathrm{E}+00$ & 0.0000 & $1.831 E-08$ & 0.0005 \\
\hline & $0.000 E+00$ & 0.0000 & $0.000 E+00$ & 0.0000 & $0.000 E+00$ & 0.0000 & $0.000 \mathrm{E}+00$ & 0.0000 & 0.000 & & & & & \\
\hline IIIIIIII & litilifíl & İ́líí & IIIIIIIIII & IIfíín & fiffífín & II I I íf & 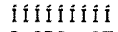 & IIIIII Í & litilitílín & IIII & iffitifit & Iffítí & IIIIIIIIIII & IIIIIII \\
\hline Tota & $.901 E-07$ & 0.0207 & $7.792 \mathrm{E}-10$ & 0.0000 & $.000 E+00$ & 0.0000 & $2.958 E-07$ & 0.0078 & $4.753 E-08$ & 0.0012 & $1.529 E-07$ & 0.0040 & $3.812 E-05$ & 1.0000 \\
\hline
\end{tabular}

$* * *$ CNRSI $(i, p, t)$ includes contribution from decay daughter radionuclides

\begin{tabular}{|c|c|c|}
\hline Attachment & 10 & Sheet No. 21 of 41 \\
\hline Originators: & S.W. Clark & Date \\
\hline Chk'd By & M.W. Perrott & Date \\
\hline Calc. No. & $0100 B-C A-V 0301$ & Rev. No. 0 \\
\hline
\end{tabular}




\section{ATTACHMENT 10}

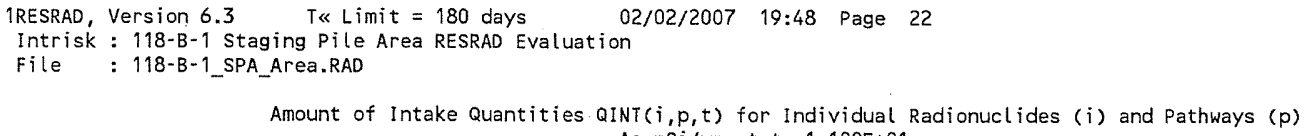

Water Independent Pathways (Inhalation w/o radon)

Water Dependent Pathways

\begin{tabular}{|c|c|c|c|c|c|c|c|c|c|c|c|}
\hline & & & & & & & & & & & \\
\hline & 11 & Plant & Meat & Milk & Soil & Water & Fish & Plant & Meat & Milk & Ingestion \\
\hline & & & & & & $\triangle \triangle A \cap A \cap A \cap A \triangle A \cap A \cap A$ & $\triangle A \cap A \cap A A A A A A B$ & $\triangle A \triangle A \cap A A A A A A B$ & $\triangle A \triangle A A B A A B$ & & \\
\hline & $5.938 E-16$ & $1.993 E-12$ & $5.288 E-14$ & $1.785 E-14$ & & $0.000 E+00$ & $0 E+00$ & $0.000 \mathrm{E}+00$ & $E+\infty$ & & \\
\hline & 7 & $3 E+00$ & -02 & & $\mathrm{BE}+00$ & $0.000 E+00$ & $0.000 E+00$ & $0.000 E+00$ & $0.000 E+00$ & $00 E+00$ & \\
\hline & & & & & & $E+00$ & $=+\infty$ & $E+00$ & $E+00$ & $E+00$ & \\
\hline & & & & & & $+\infty$ & & & & & \\
\hline & & & & & +01 & 00 & & & +00 & $E+\infty$ & \\
\hline & & 01 & & & 00 & & & & $E+00$ & & \\
\hline & & & & & & & & & & & \\
\hline & & & & +01 & & & & & & & \\
\hline & & & & & & & & & & & \\
\hline & & & & & & & & & & & \\
\hline & & & & & & 00 & & & & & \\
\hline & & & & & & & & & $+\infty$ & +00 & \\
\hline & & & & & & 00 & & & & 00 & \\
\hline & & & & & & $=00$ & & & +00 & $+\infty$ & -15 \\
\hline & & & & & & & & & $E+00$ & $E+00$ & +03 \\
\hline & -20 & 7 & 2.2 & & & & & & & $=+00$ & \\
\hline & $=-17$ & -13 & -15 & $5.464 E-16$ & 1.0 & & & & & $+\infty$ & -13 \\
\hline & & & & & & $E+00$ & $0.000 E+00$ & $0.000 E+00$ & $0.000 E+00$ & $0.000 E+00$ & $E-16$ \\
\hline & -13 & -10 & & & & & & & $E+00$ & & $E-0$ \\
\hline & $=-11$ & 1.5 & -09 & & & +00 & & & +00 & $E+00$ & \\
\hline & 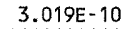 & & & & & +00 & $B E+00$ & UET & & $E+0$ & 0 \\
\hline & & & & & & & & & & & \\
\hline
\end{tabular}

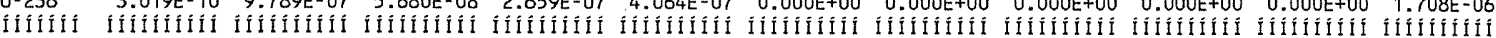

* Sum of all ingestion pathways, i.e. water independent plant, meat, milk, soil

Amount of Intake Quantities QINTP(irn, $i, t)$ and QINTOW(irn, $i, t)$ for Inhalation of Radon and its Decay. Products as $p C i / y r$ at $t=1.100 E+01$ year Radi onucl ides

\begin{tabular}{|c|c|c|c|c|c|c|c|c|}
\hline adon & $A A A A A A A$ & $7 \pi$ & חת & 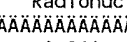 & ÄÄÄÄÄÄÄÄÁA & $\triangle A O A A O A A A A O A A O A$ & $\triangle A \triangle A \cap A \cap A A A A A$ & 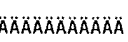 \\
\hline thway & $R n-222$ & $P o-218$ & $\mathrm{~Pb}-214$ & $\mathrm{Bi}-214$ & $R n-220$ & Po-216 & $\mathrm{Pb}-212$ & $\mathrm{Bi}-212$ \\
\hline 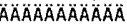 & $\triangle A A A A B A A A B A$ & $\triangle A A A A A O A O A A O A$ & $\triangle A A A A O A A A A B$ & $\triangle A A A A A A A A A B A$ & $\triangle A A A A A A A A A B A ̈$ & $\triangle A \cap A \cap A A A A A A B A$ & $\triangle A \cap A \cap A A \cap A A A A A B$ & $\triangle A A A ̈ A B$ \\
\hline Water-ind. & $0.000 E+00$ & $0.000 E+00$ & $0.000 E+00$ & $0.000 E+00$ & $0.000 \mathrm{E}+00$ & $0.000 \mathrm{E}+00$ & $0.000 E+00$ & $0.000 \mathrm{E}$ \\
\hline Water-dep. & $0.000 \mathrm{E}+00$ & $0.000 \mathrm{E}+00$ & $0.000 E+00$ & $0.000 E+00$ & $0.000 \mathrm{E}+00$ & $0.000 E+00$ & $0.000 E+00$ & 0. \\
\hline & & 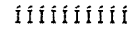 & MUIAIIIA & fiflifilit & intiotio & 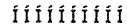 & IIIffiti & Ififi \\
\hline Total & $0.000 E+00$ & $0.000 E+00$ & $0.000 E+00$ & $0.000 E+00$ & $0.000 E+00$ & $0.000 E+00$ & $0.000 E+00$ & $0.000 \mathrm{E}+$ \\
\hline
\end{tabular}

Water-ind. = Water-independent Water-dep. = Water-dependent

\begin{tabular}{|c|c|c|}
\hline \multirow{2}{*}{\multicolumn{3}{|c|}{$\begin{array}{ll}\text { Attachment } & 10 \\
\text { Originators: S.W. Clark } & \text { Sheet No. } 22 \text { of } 41 \\
\text { Date }\end{array}$}} \\
\hline & & \\
\hline $\mathrm{Chk}^{\prime} \mathrm{d} \mathrm{By}$ & M.W. Perrott & Date \\
\hline Calc. No. & 0100B-CA-V0301 & Rev. No. 0 \\
\hline
\end{tabular}




\section{ATTACHMENT 10}

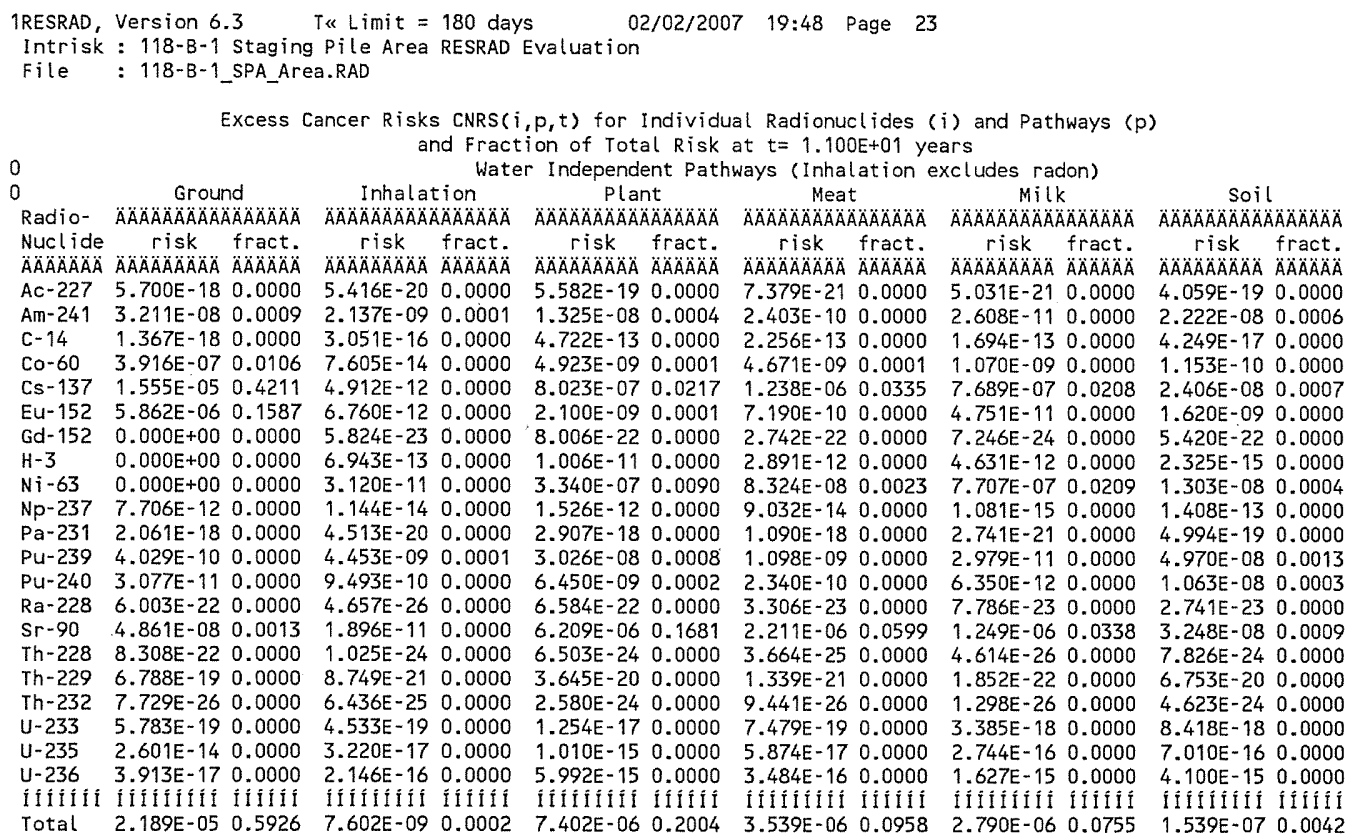

\begin{tabular}{|c|c|}
\hline Attachment & Sheet No \\
\hline Originators: S.W. Clark & Date \\
\hline M. W. Perrott & Date \\
\hline $0100 B-C A-V 0301$ & Rev, No. \\
\hline
\end{tabular}




\section{ATTACHMENT 10}

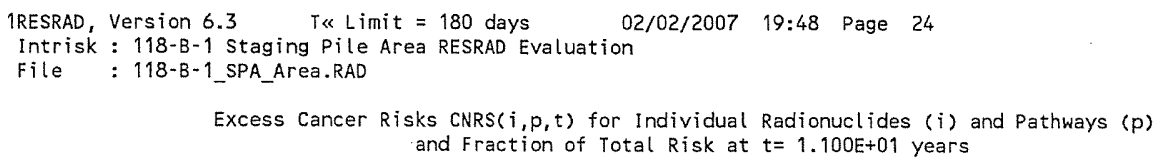

Water Dependent Pathways

\begin{tabular}{|c|c|c|c|c|c|c|c|c|c|c|c|c|}
\hline & \multicolumn{2}{|l|}{ Water } & \multicolumn{2}{|c|}{ Fish } & \multicolumn{2}{|c|}{$P l$ ant } & \multicolumn{2}{|c|}{ Meat } & \multicolumn{2}{|c|}{ Milk } & \multicolumn{2}{|c|}{ All Pathways** } \\
\hline $\begin{array}{l}\text { UClide } \\
\dot{A} \ddot{A} \ddot{A} \ddot{A} A \vec{A}\end{array}$ & 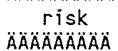 & $\begin{array}{l}\text { fract. } \\
\qquad A \ddot{A} \ddot{A} \ddot{A} \ddot{A}\end{array}$ & $\begin{array}{c}r i s k \\
\triangle A A A A A B A\end{array}$ & 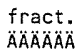 & $\begin{array}{r}r i s k \\
\because A \cap A B A B A B A\end{array}$ & 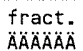 & $\begin{array}{r}r i s k \\
\triangle A B A A A B A B A\end{array}$ & $\begin{array}{l}\text { fract. } \\
\because A A A B A B A\end{array}$ & $\begin{array}{c}r i s k \\
\triangle A A B A B A B A A B A\end{array}$ & $\begin{array}{l}\text { fract. } \\
\triangle A A A A A B A\end{array}$ & $\begin{array}{c}\text { risk } \\
\text { AAAAAAAAAAA }\end{array}$ & $\begin{array}{l}\text { fract. } \\
\text { ÄAAAAOÄA }\end{array}$ \\
\hline$c-227$ & $0.000 E+00$ & 0.0000 & $0.000 E+00$ & 0.0000 & $0.000 E+00$ & 0.0000 & $0.000 E+00$ & 0.0000 & $0.000 E+00$ & 0.0000 & & 0.0000 \\
\hline & $0 E+00$ & & $O E+O O$ & & $E+00$ & & $O E+\infty O$ & & $0.000 E+00$ & & $97 E-08$ & \\
\hline & & & $+\infty$ & & & & & & 00 & & & \\
\hline & & & $+\infty$ & & & & & & $E+00$ & & & \\
\hline & $E+00$ & & $E+\infty$ & & & & & & $E+00$ & & & \\
\hline & & & & & & & +00 & & +00 & & & \\
\hline & & & & & & & & & & & & \\
\hline$H-3$ & & & & & & & & & & & & 313 \\
\hline & & & +00 & & +00 & & & & +00 & & & \\
\hline & & & +00 & & & & & & & & & \\
\hline & & & & & & & & & +00 & & & 000 \\
\hline & 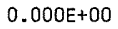 & & +00 & & & & & & & & & 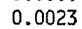 \\
\hline & 10 & 0.0 & +00 & & & & & & & & & 5 \\
\hline & & & & & & & & & +00 & & & 0 \\
\hline & & & & & & & & & & & & \\
\hline & & & & & jo & & & & 100 & & & \\
\hline & & & $+\infty$ & & $0.000 \mathrm{E}+00$ & & $+\infty$ & & $+\infty 0$ & & 19 & 000 \\
\hline & & & & & & & & & & & & \\
\hline & & & +00 & & 0 & & & & & & & \\
\hline 235 & $.000 E+00$ & 0.0 & $.000 E+00$ & 0.0 & $0.000 \mathrm{E}+00$ & 0.0 & $=+00$ & & +00 & & 2. & 0 \\
\hline & & & & & & & & & & & & \\
\hline & & & & & & & & & & & & \\
\hline Tota & $.051 E-07$ & 0.0191 & $6.989 E-10$ & 0.0000 & $2.670 \mathrm{E}-07$ & 0.0072 & $4.444 E-08$ & 0.0012 & $E-07$ & 0.0038 & $E-05$ & 1.0 \\
\hline
\end{tabular}

** Sum of water independent ground, inhalation, plant, meat, milk, soil

0 Excess Cancer Risks CNRS9 $(i r n, i, t)$ and CNRS9W(irn, $i, t)$ for Inhalation of Radon and its Decay Products at $t=1.100 \mathrm{E}+01$ years

\begin{tabular}{|c|c|c|c|c|c|c|c|c|}
\hline Radon & ÄÄÂAAAAAÄ & ÄAAAAAAAAAAA & $A A A A A A A A A A A$ & $\begin{array}{r}\text { RadionUC } \\
A A A A A B A B A\end{array}$ & $\begin{array}{l}\text { lides } \\
A A A A A B A B\end{array}$ & & & \\
\hline Pathway & $R n-222$ & Po-218 & $\mathrm{Pb}-214$ & $\mathrm{Bi}-214$ & $\mathrm{Rn}-220$ & Po-216 & $\mathrm{Pb}-212$ & $B i-212$ \\
\hline ÄÄÄÄÄÄÄ & ÄÄÄÄÄÄÄÄÄ & A ÄÄÄÄÄÄ & ÄÄÄÄÄÄÄ & AÄÄÄÄÄÄA & I $\triangle A \cap A A A A A B$ & 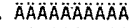 & 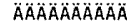 & $\triangle A A A A A A A A A$ \\
\hline Water-ind. & $0.000 E+00$ & $0.000 E+00$ & $0.000 E+00$ & $0.000 E+00$ & $0.000 E+00$ & $0.000 E+00$ & $0.000 \mathrm{E}+00$ & $0.000 E+00$ \\
\hline Wat & $0.000 E+00$ & $0.000 E+00$ & $0.000 E+00$ & $\begin{array}{r}0.000 E+00 \\
0\end{array}$ & $0.000 E+00$ & $0.000 E+00$ & $0.000 E+00$ & $0.000 E+00$ \\
\hline & İ $\hat{I} \hat{I}$ & ifi & Ifífítiliti & fifififitif & 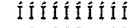 & IIIIIIIIII & II I I I I I I I I I I I & IIIfílííl \\
\hline Tote & $0.000 E+00$ & $0.000 \mathrm{E}+00$ & $0.000 E+00$ & $0.000 \mathrm{E}+00$ & $0.000 E+00$ & $0.000 E+00$ & $0.000 E+00$ & $0.000 E+00$ \\
\hline
\end{tabular}

Water - ind, $==$ Water-independent Water-dep. $==$ Water-dependent

\begin{tabular}{|c|c|}
\hline Attachment & Sheet No. 24 of 41 \\
\hline Originators: S.W. Clark & Date \\
\hline M. W. Perrott & Date \\
\hline $0100 B-C A-V 0301$ & Rev. No. 0 \\
\hline
\end{tabular}


CVP-2007-00006

Rev. 0

\section{ATTACHMENT 10}

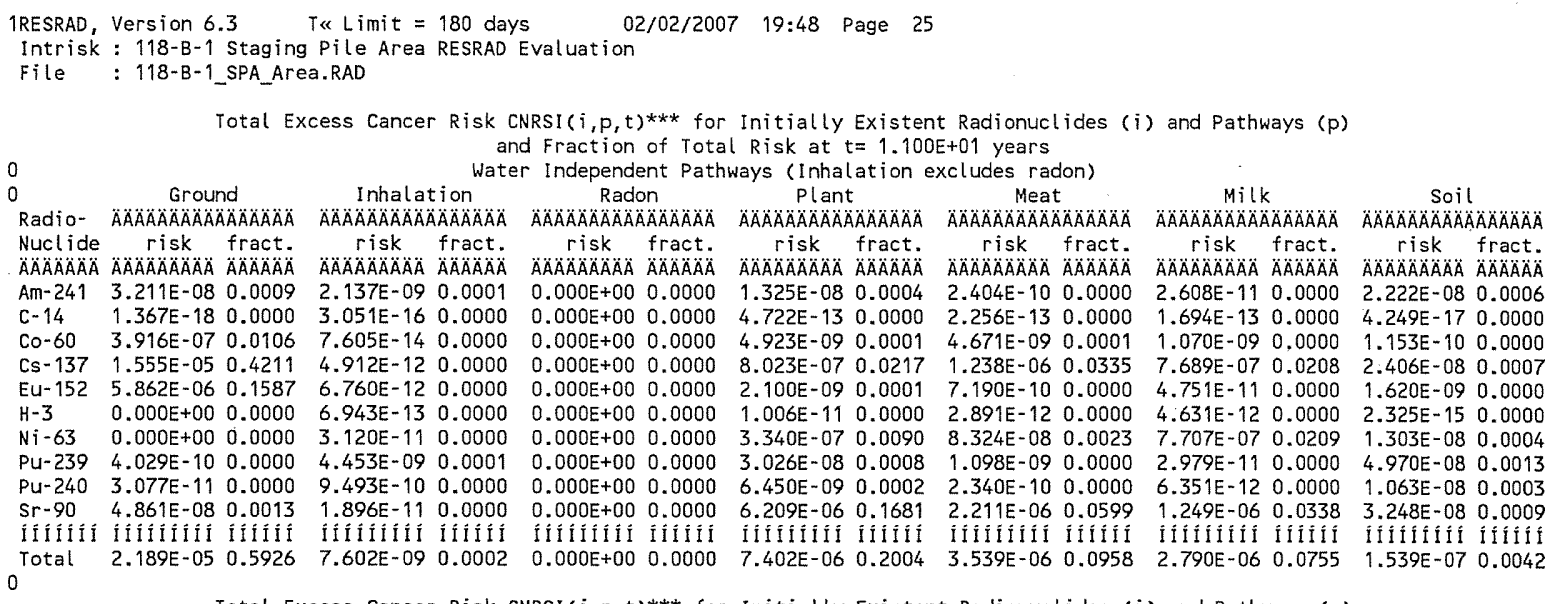

Total Excess Cancer Risk CNRSI $(i, p, t) * * *$ for Initially Existent Radionuclides $(i)$ and Pathways $(p)$ and Fraction of Total Risk at $t=1.100 E+01$ years

Water Dependent Pathways

\begin{tabular}{|c|c|c|c|c|c|c|c|c|c|c|c|c|c|c|}
\hline \multirow{2}{*}{$\begin{array}{l}\text { Radio- } \\
\text { Nucl ide } \\
\text { ÄÄÄÄÄA }\end{array}$} & \multicolumn{2}{|c|}{ Water } & \multicolumn{2}{|c|}{ Fish } & \multicolumn{2}{|c|}{ Radon } & \multicolumn{2}{|c|}{$P$ lant } & \multicolumn{2}{|c|}{ Meat } & \multicolumn{2}{|c|}{ Milk } & \multicolumn{2}{|c|}{$\begin{array}{c}\text { All pathways } \\
\text { AAAAAAAAAAAAAAAAA }\end{array}$} \\
\hline & $\begin{array}{c}\text { risk } \\
\text { ÄÄÄAAAÄ }\end{array}$ & $\begin{array}{l}\text { fract. } \\
\text { ÄÄÄÄÄA }\end{array}$ & $\begin{array}{c}\text { risk } \\
\text { ÄAAAAAAAÄA }\end{array}$ & 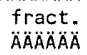 & $\begin{array}{c}\text { risk } \\
\text { ÄAAAAAAAAOA }\end{array}$ & $\begin{array}{l}\text { fract. } \\
\text { AAAAAAAA }\end{array}$ & $\begin{array}{c}\text { risk } \\
\text { AAAAAAAAAÄ }\end{array}$ & $\begin{array}{l}\text { fract. } \\
A A B A A A B A\end{array}$ & $\begin{array}{c}\text { risk } \\
\text { ÄAAAAAAAÄA }\end{array}$ & $\begin{array}{l}\text { fract. } \\
\text { ÄAAAAAA }\end{array}$ & $\begin{array}{c}\text { risk } \\
\text { AAAAAAAAAA }\end{array}$ & 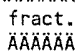 & $\begin{array}{c}\text { risk } \\
A B A A O A A B A ̈ A\end{array}$ & $\begin{array}{l}\text { fract. } \\
\triangle A B A O A B A B\end{array}$ \\
\hline$A m-241$ & $0.000 E+00$ & 0.0000 & $0.000 E+00$ & 0.0000 & $0.000 E+00$ & 0.0000 & $0.000 \mathrm{E}+00$ & 0.0000 & $0.000 \mathrm{E}+00$ & 0.0000 & $0.000 \mathrm{E}+00$ & 0.0000 & ARAAAAARA & AMA \\
\hline & $O E+0 O$ & 0.0000 & $100 E+00$ & 0.0000 & $0.000 E+00$ & 0.0000 & $O E+00$ & 000 & $0.000 E+00$ & & $O E+00$ & & & \\
\hline Co- 60 & $0.000 E+00$ & 0.0000 & $0.000 E+00$ & 0.0000 & $0.000 E+00$ & 0.0000 & $0.000 E+00$ & 0.0000 & $0.000 \mathrm{E}+00$ & 0.0000 & $0.000 E+00$ & 000 & 07 & 01 \\
\hline Cs -137 & $0.000 E+00$ & 0.0000 & $0.000 E+00$ & 0.0000 & $0.000 E+00$ & 0.0000 & $0.000 E+00$ & 0.0 & $0.000 E+00$ & & $E+00$ & & 1. & 0.497 \\
\hline & $0.000 E+00$ & 0.0000 & $0.000 E+00$ & 0.0000 & $0.000 E+00$ & 0.0000 & 0.0 & 0. & 00 & 10 & 00 & & & \\
\hline$H-3$ & & 91 & -10 & 0.0000 & $0.000 E+00$ & 0.0000 & -07 & 0.0072 & -08 & 0. & -07 & 0. & 1. & y \\
\hline $\mathrm{Ni}-6$ & $0.000 E+00$ & 0.0 & $0.000 E+00$ & & $E+00$ & & $\mathrm{DE}+00$ & & $E+00$ & & 0 & & & \\
\hline Pu-239 & $0.000 E+00$ & 0.0000 & $0.000 E+00$ & 0.0000 & $0.000 E+00$ & 0.0 & $0.000 E+00$ & 00 & $0.000 E \div 00$ & jo & $E+00$ & 00 & & \\
\hline Pu-240 & $0.000 E+00$ & 0.0000 & $0.000 E+00$ & 0.0000 & $0.000 E+00$ & 0.0000 & $0.000 \mathrm{E}+00$ & 0.0000 & $0.000 E+00$ & 0.0 & $0.000 E+00$ & 0.0000 & $30 E-08$ & .000 \\
\hline & & & & & & & & & & & & & & \\
\hline & & & & & & & & & & & & & & \\
\hline Total & $7.051 \mathrm{E}-07$ & 0.0191 & $6.989 E-10$ & 0.0000 & $0.000 E+00$ & 0.0000 & 2.67 & 0.0072 & 4.44 & 0.0012 & -07 & 0.0038 & $4 E-05$ & 1.00 \\
\hline
\end{tabular}

***CNRSI $(i, p, t)$ includes contribution from decay daughter radionuclides

\begin{tabular}{|c|c|}
\hline Attachment & Sheet No. 25 of 41 \\
\hline Originators: S.W. Clark & Date \\
\hline M. W. Perrott & Date \\
\hline $0100 \mathrm{~B}-\mathrm{CA}-\mathrm{V} 0301$ & Rev. No. \\
\hline
\end{tabular}




\section{ATTACHMENT 10}

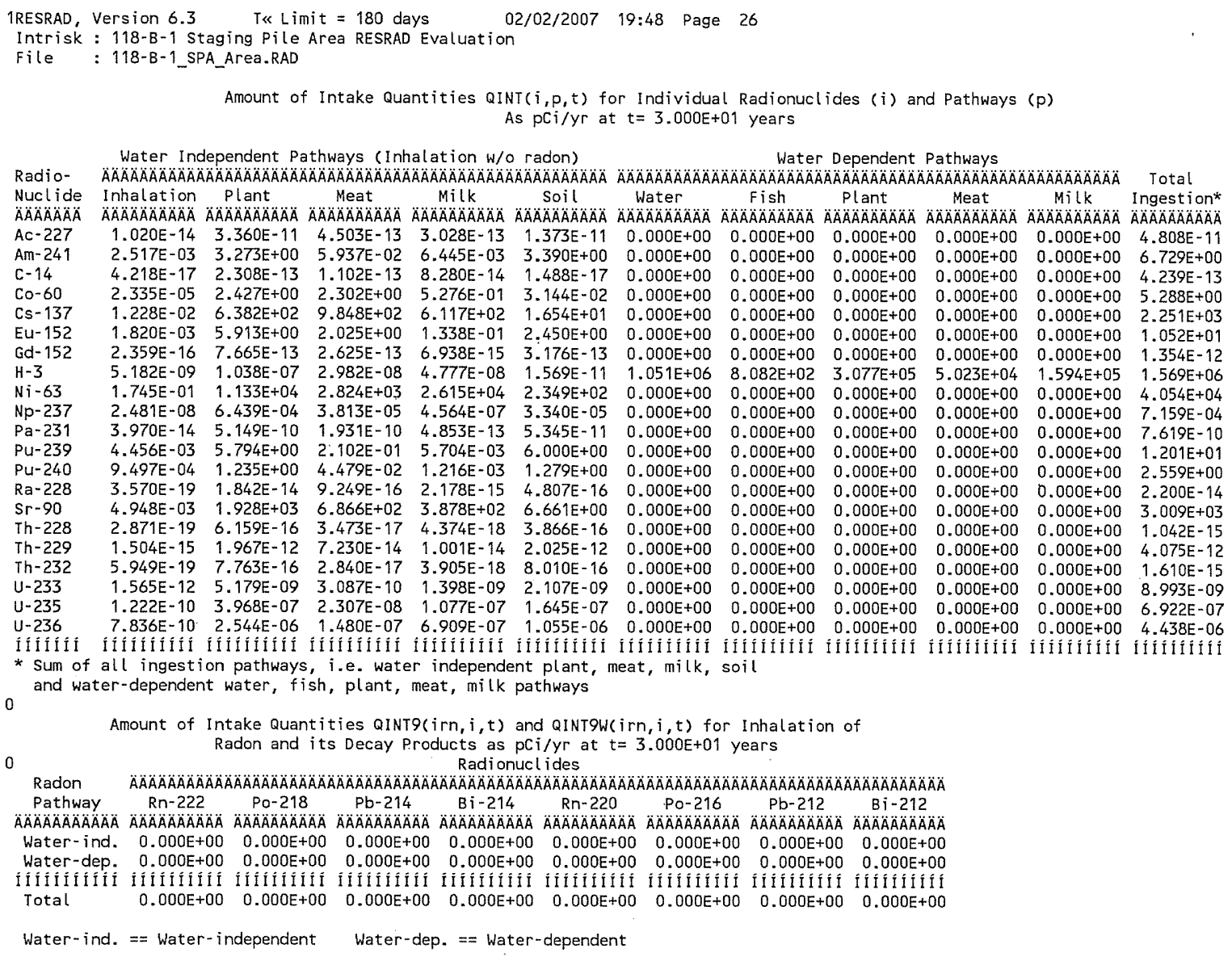

\begin{tabular}{|c|c|c|}
\hline \multirow{2}{*}{\multicolumn{3}{|c|}{ 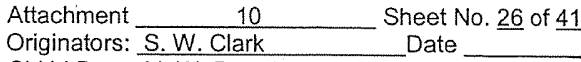 }} \\
\hline & & \\
\hline Chk'd By & M. W. Perrott & Date \\
\hline Calc. No. & 0100B-CA-V0301 & Rev. $\bar{T}$ \\
\hline
\end{tabular}




\section{ATTACHMENT 10}

\begin{tabular}{|c|c|c|c|c|c|c|c|c|c|c|}
\hline \multirow[t]{2}{*}{$\begin{array}{l}\text { 1RESRAD, } \\
\text { Intrisk } \\
\text { File }\end{array}$} & $\begin{array}{l}\text { Version } 6 \\
: 118-\mathrm{B}-1 \\
: 118-\mathrm{B}-1\end{array}$ & $\begin{array}{l}\text { 1 Stagi } \\
1 \_ \text {SPA_A }\end{array}$ & $\begin{array}{l}\text { T\& Limit }= \\
\text { Pile Area } \\
\text { a.RAD }\end{array}$ & $\begin{array}{l}180 \text { day } \\
\text { RESRAD }\end{array}$ & 's Evaluation & $32 / 02 / 200$ & $719: 48$ & Page 27 & & \\
\hline & \multicolumn{2}{|r|}{ Excess } & \multicolumn{7}{|c|}{$\begin{array}{c}\text { Cancer Risks CNRS }(i, p, t) \text { for Individual Radionuclides }(i) \text { and Pathways }(p) \\
\text { and Fraction of Total Risk at } t=3.000 E+01 \text { years }\end{array}$} & \\
\hline & ur & and & Inhat & tion & Pla & ant & Mea & & Milk & Soil \\
\hline & $\triangle O A B$ & $\triangle A O A A A A A ̈$ & $A B ̈ A$ & $A \ddot{A A ̈ A ̈}$ & ÄÄÄÄÄÄ & $\triangle A O A A A O A A A$ & $\triangle A O A ̈ A ̈$ & AAAAAA & $\triangle A A A A A A A A A$ & 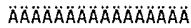 \\
\hline 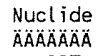 & Kä & 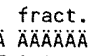 & $\begin{array}{c}\text { risk } \\
\triangle A A A B A B A A B A\end{array}$ & $\begin{array}{l}\text { fract. } \\
\ddot{A} \ddot{A B A ̈ A ̈ A ̈}\end{array}$ & $\begin{array}{c}\text { risk } \\
\text { ÄAAAÄAAAA }\end{array}$ & $\begin{array}{l}\text { fract. } \\
\text { A AAAAAAOA }\end{array}$ & $\begin{array}{c}\text { risk } \\
\text { AAAAAAAAÄ }\end{array}$ & $\begin{array}{l}\text { fract. } \\
\text { A ÄÄÄÄA }\end{array}$ & $\begin{array}{l}\text { risk fract. } \\
\text { ÄAAÄÄÄÄ ÄÄÄÄÄ }\end{array}$ & 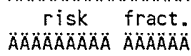 \\
\hline$A C-227$ & $E-17$ & 70.0000 & & & & 80.0000 & $2.207 \mathrm{E}-20$ & 0.0000 & $1.895 \mathrm{E}-20 \quad 0.0000$ & $1.533 E-18 \quad 0.0000$ \\
\hline & & & & & & & -10 & & -110. & $.08 \quad 0.0010$ \\
\hline$c-1$ & & 0.0000 & & 0.0000 & & 0.0000 & -25 & 0.0000 & -250.0000 & $-29 \quad 0.0000$ \\
\hline Co & & 0.0015 & & & & & $E-10$ & 0.0000 & $\begin{array}{ll}-11 & 0.0000\end{array}$ & -120.0000 \\
\hline & 6 & 0.4 & & & & & & & 070. & 080. \\
\hline & 2. & 60.0 & & 0.0000 & & 0. & 2. & 0.0000 & 110.0 & 100.0000 \\
\hline Gd & $E+00$ & 0.0000 & & 0.0000 & 1.0 & 10.0000 & $=-22$ & 20.0000 & -240.0000 & -220.0000 \\
\hline$H-3$ & 0 & 0.0 & & & & & & & 210. & 240 \\
\hline & & 0.0 & & & & & & 33 & 770.0 & $08 \quad 0.0005$ \\
\hline & & 0.0 & & 0.0 & & 20. & -13 & 30.0000 & $=-150.0000$ & -130.0000 \\
\hline & & & & & & & & & 210. & 180 \\
\hline & & & & & & & 1. & 90 & 110. & 0 \\
\hline & & 0.0 & -10 & 0.0000 & & 90.0003 & $=-10$ & 0.0000 & $\begin{array}{ll}-12 & 0.0\end{array}$ & $08 \quad 0.0005$ \\
\hline & & & & & & & & & 220. & \\
\hline & & & & & & & & & & \\
\hline & 2.9 & 0.0000 & $E-24$ & 0.0000 & 2.2 & 30.0000 & 1.2 & 40.0000 & -250.0 & -230.0000 \\
\hline & & & & & & & $E-21$ & 10.0 & -220.0 & 190. \\
\hline & & & 1.7 & 0.0 & & & 2.5 & 50.0 & $\begin{array}{ll}-26 & 0.0\end{array}$ & \\
\hline$u-2$ & & 30.0 & $1 . z^{2}-x-x$ & 0.00 & 3.3 & & $1.8-200-10$ & 30.0 & -180.0 & -170.0000 \\
\hline & & & & & & & & & 60.0000 & $1.160 E-15 \quad 0.0000$ \\
\hline & & & & & & & & & & \\
\hline & & & & & & & & & i i i & Î ÎIIIII \\
\hline Total & $1.226 \mathrm{E}-05$ & 0.5591 & $7.508 \mathrm{E}-09$ & 0.0003 & 4.773E-06 & 60.2176 & $2.264 E-06$ & 60.1032 & $1.949 E-06 \quad 0.0889$ & $1.296 E-07 \quad 0.0059$ \\
\hline
\end{tabular}

\begin{tabular}{|c|c|}
\hline Attachment & Sheet No, 27 of 41 \\
\hline Originators: S. W. Clark & Date \\
\hline M. W. Perrott & Date \\
\hline 0100B-CA-V0301 & Rev. No. 0 \\
\hline
\end{tabular}




\section{ATTACHMENT 10}

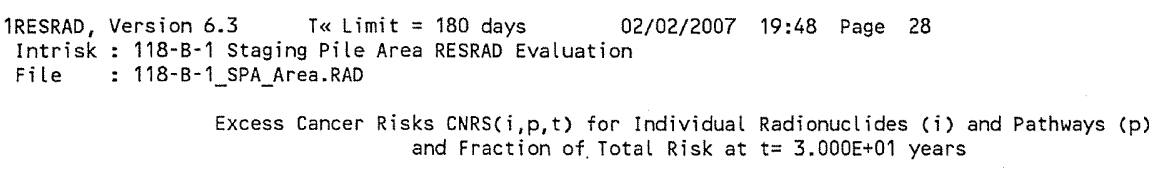

Water Dependent Pathways

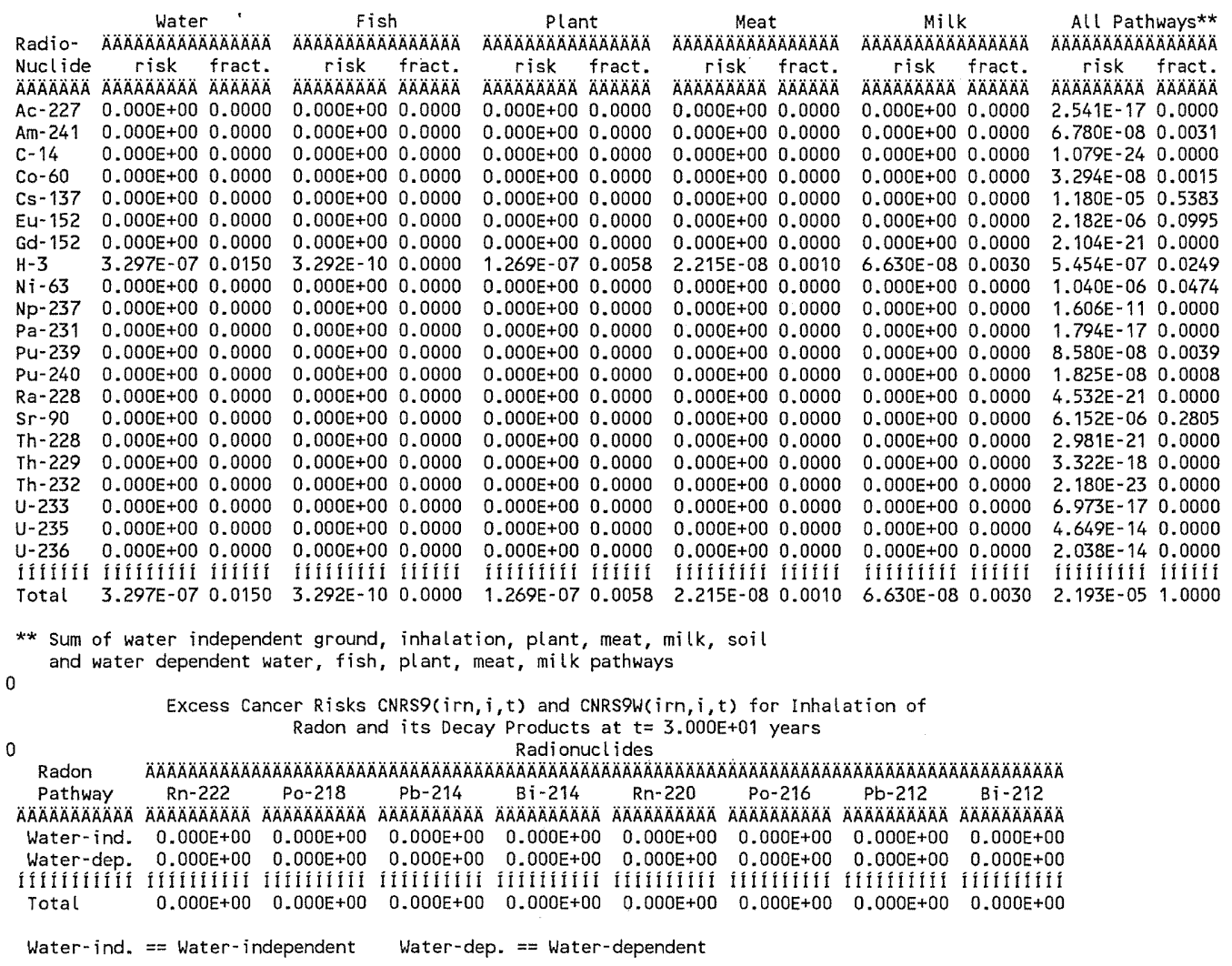

\begin{tabular}{|c|c|c|}
\hline \multirow{2}{*}{\multicolumn{3}{|c|}{ 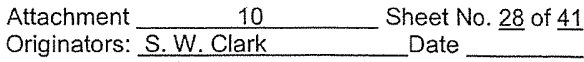 }} \\
\hline & & \\
\hline Chk'd By & M. W. Perrott & Date \\
\hline Calc. No & 0100B-CA-V0301 & Rev. No. \\
\hline
\end{tabular}




\section{ATTACHMENT 10}

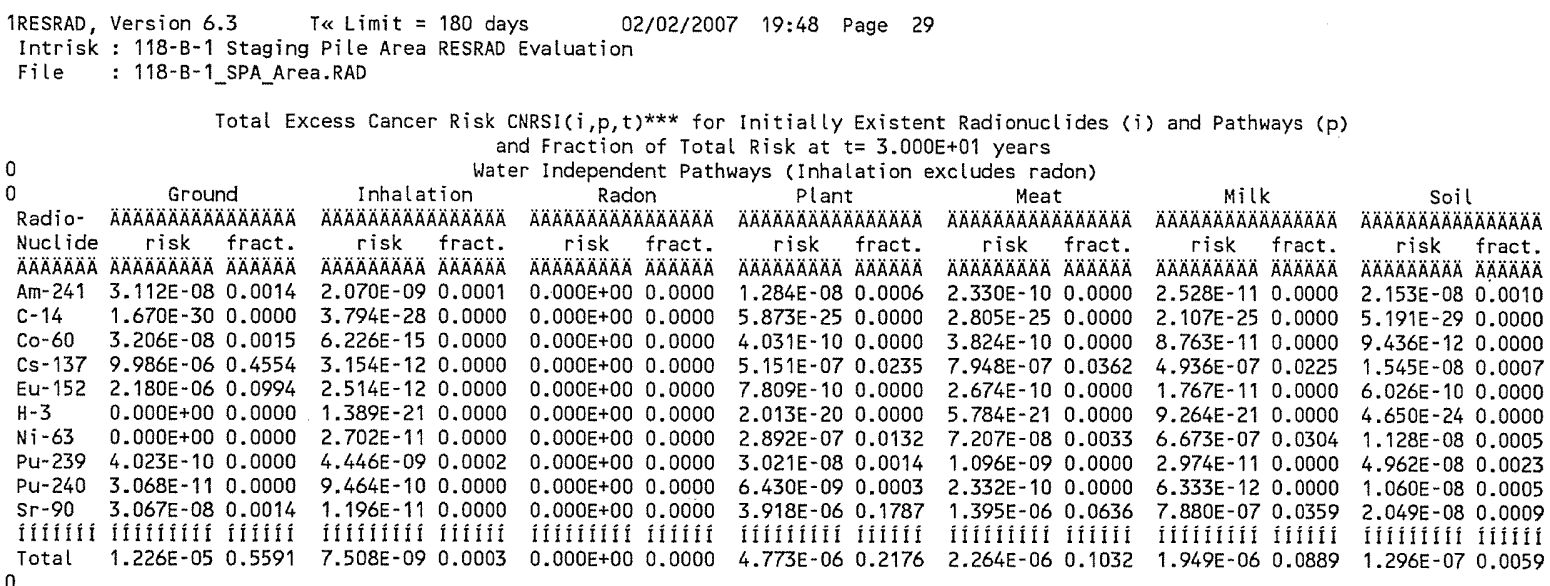

Total Excess Cancer Risk CNRSI $(i, p, t)^{* * *}$ for Initially Existent Radionuclides (i) and Pathways (p)

and Fraction of Total Risk at $\mathrm{t}=3.000 \mathrm{E}+01$ years

Water Dependent Pathways

\begin{tabular}{|c|c|c|c|c|c|c|c|c|c|c|c|c|c|c|}
\hline \multirow{2}{*}{ 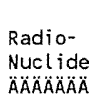 } & \multicolumn{2}{|c|}{$\begin{array}{c}\text { Water } \\
\text { AAAAAAAAAAAAAAA }\end{array}$} & \multicolumn{2}{|c|}{$\begin{array}{c}F i s h \\
\text { AAAAAAAAAAAAAAAA }\end{array}$} & \multicolumn{2}{|c|}{$\begin{array}{c}\text { Radon } \\
\text { ĀAAAAAAAAAAAAAOA }\end{array}$} & \multicolumn{2}{|c|}{$\begin{array}{c}\text { Plant } \\
\text { ÄAAAAAAAAAAAAAOAOA }\end{array}$} & \multicolumn{2}{|c|}{$\begin{array}{c}\text { Meat } \\
\text { ÄAAAAAAAAAAAAAAAÄ }\end{array}$} & \multicolumn{2}{|c|}{$\begin{array}{c}M i l k \\
\text { A } \\
\text { MAAAAAAOAAAAAAAA }\end{array}$} & \multicolumn{2}{|c|}{$\begin{array}{c}\text { All pathways } \\
\text { AAAOAAOAAAAAAAAAAA }\end{array}$} \\
\hline & 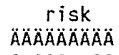 & 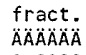 & $\begin{array}{c}\text { risk } \\
A A A B A B A B A\end{array}$ & $\begin{array}{l}\text { fract. } \\
\dddot{A} A \ddot{A} \ddot{A} \ddot{A} \ddot{A}\end{array}$ & $\begin{array}{c}\text { risk } \\
\triangle A \cap A B A B A A A B A\end{array}$ & $\begin{array}{l}\text { fract. } \\
\triangle A B A A A O A\end{array}$ & $\begin{array}{c}\text { risk } \\
\triangle A A A B A A A A B\end{array}$ & 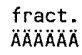 & $\begin{array}{c}\text { risk } \\
\text { ĀÄÄÄÄÄÄ }\end{array}$ & fract. & $\begin{array}{c}r i s k \\
\text { ÄAAAAAAOAA }\end{array}$ & $\begin{array}{l}\text { fract. } \\
\text { ÄÄÄÄÄA }\end{array}$ & $\begin{array}{c}\text { risk } \\
A \cap A A A A A A B A\end{array}$ & $\begin{array}{l}\text { fract. } \\
A A A A A B A\end{array}$ \\
\hline$A m-241$ & $0.000 E+00$ & 0.0000 & $0.000 E+00$ & 0.0000 & $.000 E+00$ & 0.0000 & $0.000 E+00$ & 0.0000 & $0.000 E+00$ & 000 & $0.000 E+0$ & 30 & . & 031 \\
\hline & & & & & +00 & 00 & $0.000 E+00$ & 0.0000 & $0.000 E+00$ & & +00 & & & \\
\hline Co- 60 & $0.000 E+00$ & 0.0000 & $0.000 E+00$ & 0.0000 & $0.000 \mathrm{E}+00$ & 0.0000 & $0.000 E+00$ & 0.0000 & $E+00$ & 000 & $D O E+00$ & & & 0015 \\
\hline & $0.000 E+00$ & 0.0 & 0.0 & 0.8 & 0.0 & 0 & 0.0 & & 00 & & & & & \\
\hline Eu- & $0.000 E+00$ & 0.0 & 0.0 & 0. & +00 & 0 & +00 & 0 & 0 & 30 & 0 & & & \\
\hline$H-3$ & & & 3.2 & 100 & 00 & 000 & -07 & 0.0058 & -08 & 0.0 & 0 & 30 & & 249 \\
\hline & 0.000 & & 0.0 & 0.0 & 0.000 & & 0.0 & & 0 & & & & & \\
\hline Pu-239 & $0.000 E+00$ & 0.0000 & 0.000 & 0. & .00 & 0 & 0.000 & 0 & 0.00 & 00 & $0.000 \mathrm{E}$ & & & \\
\hline Pu-240 & $0.000 E+00$ & 0.0000 & $0.000 E+00$ & 0.0000 & $0.000 E+00$ & 0.0000 & $0.000 E+00$ & 0.0000 & $0.000 \mathrm{E}+00$ & 0.0000 & $0.000 \mathrm{E}+00$ & 0.0000 & 1.8 & 0.0008 \\
\hline & $0.000 E+00$ & 0.0000 & 0.00 & 0.0000 & $0.000 E+00$ & 0.0000 & & 0.0000 & $0.000 \mathrm{E}+00$ & 0.0000 & & & & 0.2805 \\
\hline & & & & & & & & & & & & & & \\
\hline Tolat & $E-07$ & 0.0150 & $3.292 E-10$ & 0.0000 & $0.000 \mathrm{E}+00$ & 0.0000 & $1.269 \mathrm{E}-07$ & 0.0058 & $2.215 E-08$ & 0.0010 & $0.63 U E=08$ & 0.0030 & $2.193 E-05$ & 1.0000 \\
\hline
\end{tabular}

***CNRSI $(i, p, t)$ includes contribution from decay daughter radionuclides

\begin{tabular}{|c|c|c|}
\hline Attachmen & 10 & Sheet No. 29 of 41 \\
\hline Originators & S. W. Clark & Date \\
\hline Chk'd By & M. W. Perrott & Date \\
\hline Calc. No. & $0100 \mathrm{~B}-\mathrm{CA}-\mathrm{V} 0301$ & Rev. No. \\
\hline
\end{tabular}




\section{ATTACHMENT 10}

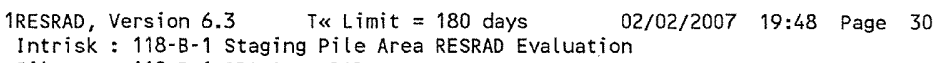

0

Amount of Intake Quantities QINT9(irn, i, t) and QINTOW(irn, $i, t)$ for Inhalation of Radon and its Decay Products as $\mathrm{pCi} / \mathrm{yr}$ at $t=1.000 \mathrm{E}+02$ years Radionuclides

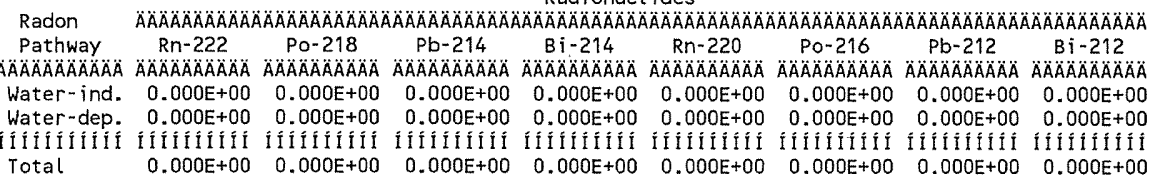

Water-ind. == Water-independent Water-dep. $==$ Water-dependent

\begin{tabular}{|c|c|}
\hline Attachment & Sheet No. 30 of 41 \\
\hline Originators: S.W. Clark & Date \\
\hline M. W. Perrott & Date \\
\hline $0100 B-C A-V 0301$ & Rev. No. \\
\hline
\end{tabular}


CVP-2007-00006

Rev. 0

\section{ATTACHMENT 10}

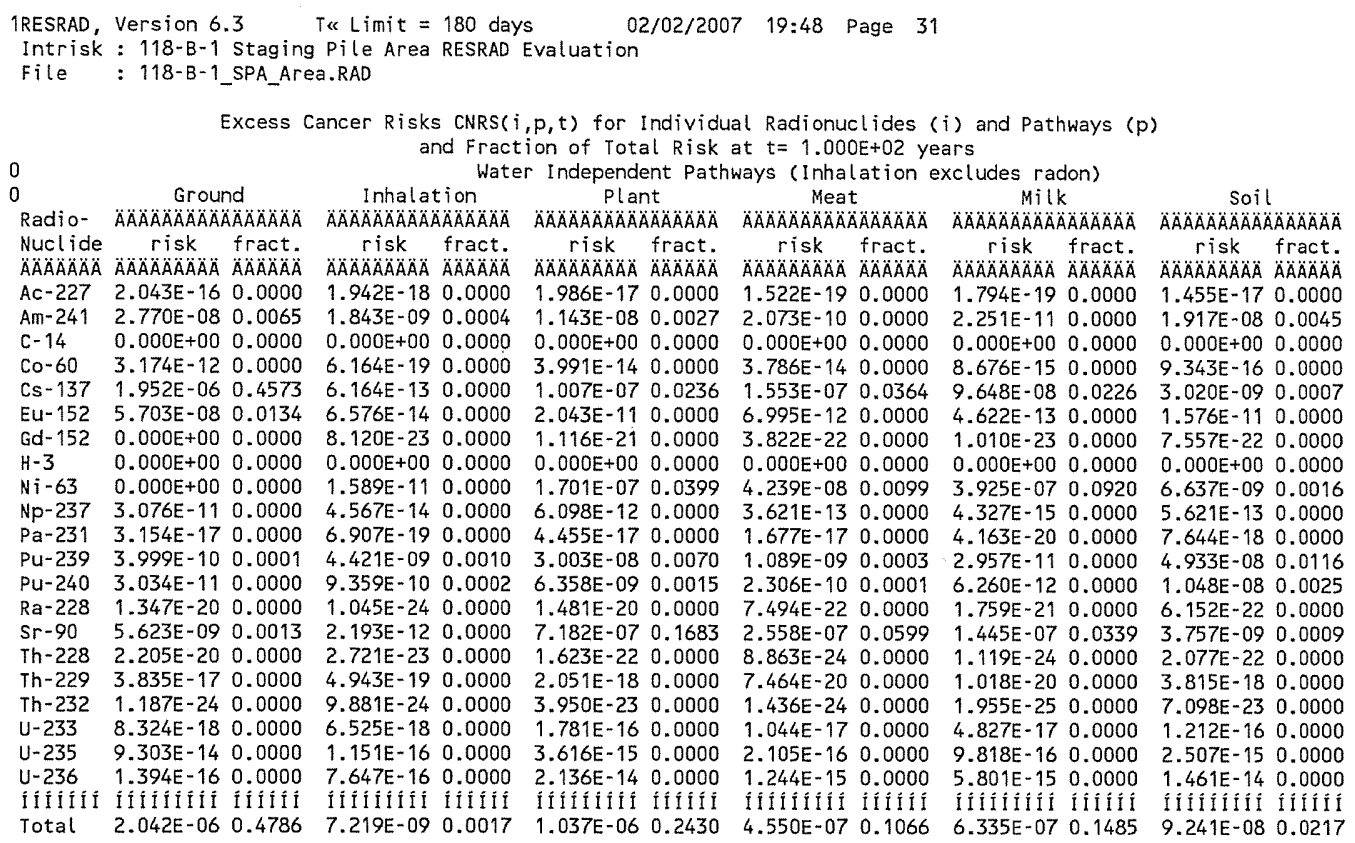

\begin{tabular}{|c|c|c|}
\hline \multicolumn{2}{|c|}{ Attachment $\quad 10$} & \multirow{2}{*}{$\begin{array}{l}\text { Sheet No. } 31 \text { of } 41 \\
\text { Date }\end{array}$} \\
\hline Originators: & S. W. Clark & \\
\hline Chk'd By & M. W. Perrott & Date \\
\hline Calc. No. & $0100 B-C A-V 0301$ & Rev. No. \\
\hline
\end{tabular}




\section{ATTACHMENT 10}

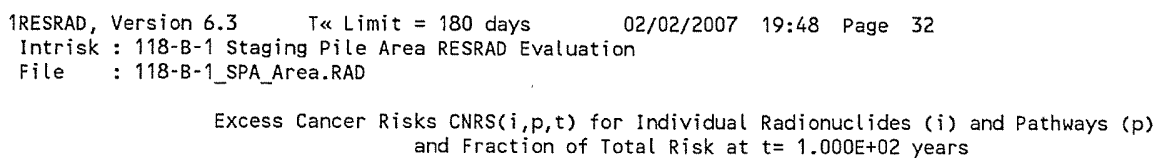

Water Dependent Pathways

Water

\begin{tabular}{|c|c|c|c|c|c|c|c|c|c|c|c|c|}
\hline & $\begin{array}{l}\text { Water } \\
A A B A A A A\end{array}$ & & $\begin{array}{r}F i s h \\
\triangle A A A O A A B\end{array}$ & $\triangle A O A A A A A B$ & $\begin{aligned} \text { Pla } & \text { A ÄÄÄÄA }\end{aligned}$ & $\because A O A ̈ A ̈$ & $\begin{array}{r}\text { Meat } \\
A O A A O A O A O A O A\end{array}$ & ÄÄ & $\begin{array}{r}\text { Mill } \\
\triangle A B A A B A A ̈\end{array}$ & 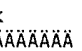 & $\begin{array}{l}\text { All Path Path } \\
A B A A A A A O A A\end{array}$ & 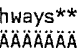 \\
\hline & isk & fract. & risk & frac & risk & act. & risk & n & risk & & $\begin{array}{l}\text { risk } \\
\text { A }\end{array}$ & \\
\hline-227 & $0.000 E+00$ & 0.0000 & $.000 E+00$ & 0000 & $\begin{array}{l}\text { AAAAAAAAA } \\
0.000 E+00\end{array}$ & $\begin{array}{l}\text { AAAA } \\
0000\end{array}$ & $\begin{array}{l}\text { AAAAAAAAA } \\
0.000 \mathrm{E}+00\end{array}$ & .0000 & $\begin{array}{l}\text { AAAAAAAAAA } \\
0.000 E+00\end{array}$ & Do & & \\
\hline & $0 E+00$ & & $O E+00$ & 000 & $D O E+00$ & 0000 & $00 E+00$ & 100 & $000 E+00$ & 60 & & \\
\hline & & & $E+00$ & & & & $O E+00$ & & $O E+00$ & 000 & & \\
\hline & $+\infty 0$ & & $E+00$ & 000 & $O E+00$ & 0.0000 & $\mathrm{OE}+00$ & 000 & $E+00$ & 1000 & & \\
\hline 7 & +00 & & $O E+00$ & 0. & $\mathrm{OE}+00$ & 0.0000 & $0.000 E+00$ & & $.000 E+00$ & & & \\
\hline & $D E+00$ & & $O E+00$ & & $E+00$ & 0 & $E+00$ & & $E+00$ & 00 & & \\
\hline & & & & & +00 & & & & .00 & & & \\
\hline & & & +00 & & $E+00$ & & $E+00$ & & $E+00$ & & & \\
\hline & $E+00$ & & $=00$ & & +00 & & $E+00$ & & $E+00$ & & & \\
\hline & & & +00 & & +00 & & $E+00$ & & $E+00$ & & & \\
\hline & & & & & & & $E+00$ & & +00 & & & \\
\hline & +00 & & +00 & & +00 & & $E+00$ & & $=+00$ & & & \\
\hline & +00 & & $E+\infty 0$ & 0. & +00 & & $E+00$ & & $E+00$ & & & \\
\hline & & & +00 & & $+\infty$ & & $E+00$ & & $E+00$ & 0. & $=-20$ & \\
\hline & +00 & & +00 & & +00 & & $E+00$ & & & & & \\
\hline & $\div 00$ & & +00 & & $\div+\infty$ & & $E+00$ & & $E+00$ & & & \\
\hline & +00 & & $\mathrm{EE}+00$ & 0.0 & $E+00$ & & $E+00$ & & $E+00$ & & E- 17 & \\
\hline & $+\infty 0$ & & & & & & $+\infty$ & & \pm+00 & & 1. & \\
\hline & & & $+\infty 0$ & & +00 & & $E+\infty$ & & $=+00$ & & & \\
\hline & $000 E+00$ & 0.00 & $.000 E+00$ & 0.0000 & $0.000 E+00$ & 0. & $0.000 E+00$ & & $E+00$ & & 13 & \\
\hline & & & & & & & & & & & & \\
\hline & & & & & & & & & & & & \\
\hline & & & $0 E+00$ & 0.0000 & +00 & & $O O E+0$ & & $E+0$ & & & \\
\hline
\end{tabular}

** sum of water independent ground, inhalation, plant, meat, milk, soil

0 and water dependent water, fish, plant, meat, milk pathways

Excess Cancer Risks CNRS $9(i r n, i, t)$ and CNRSSW(irn, $i, t)$ for Inhalation of Radon and its Decay Products at $t=1.000 \mathrm{E}+02$ years

0 Radionuclides

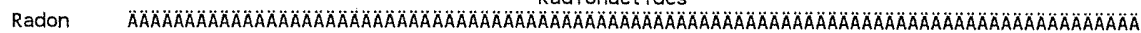
Pathway Rn-222 Po-218 Pb-214 Bi-214 Rn-220 Po-216 Pb-212 Bi-212

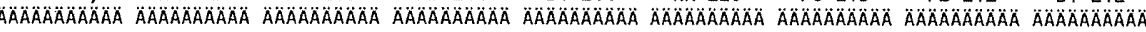

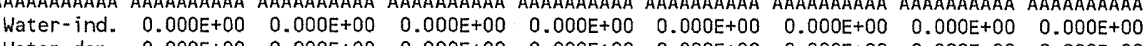

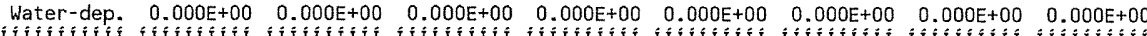

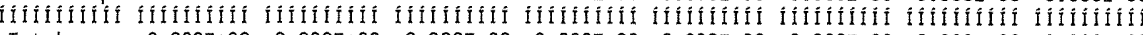

$\begin{array}{lllllllll}\text { Total } & 0.000 E+00 & 0.000 E+00 & 0.000 E+00 & 0.000 E+00 & 0.000 E+00 & 0.000 E+00 & 0.000 E+00 & 0.000 E+00\end{array}$

Water-ind. $=$ Water-independent Water-dep. $==$ Water-dependent

\begin{tabular}{|c|c|c|}
\hline \multirow{2}{*}{\multicolumn{3}{|c|}{$\begin{array}{lll}\text { Attachment } & 10 & \text { Sheet No. } 32 \text { of } 41 \\
\text { Originators: S.W. Clark } & \text { Date }\end{array}$}} \\
\hline & & \\
\hline Chk'd By & M.W. Perrott & Date \\
\hline Calc. No. & 0100B-CA-V0301 & Rev. No. \\
\hline
\end{tabular}




\section{ATTACHMENT 10}

\begin{tabular}{|c|c|c|c|c|c|c|c|c|c|c|c|}
\hline $\begin{array}{l}\text { 1RESRAD, } \\
\text { Intrisk } \\
\text { File }\end{array}$ & $\begin{array}{l}\text { Version } 6 \\
: 118-\mathrm{B}-1 \\
: 118-\mathrm{B}-1\end{array}$ & $\begin{array}{l}.3 \\
\text { Staging } \\
\text { SPA_Are }\end{array}$ & $\begin{array}{l}\text { T« Limit }= \\
\text { Pile Area } \\
\text { a.RAD }\end{array}$ & $\begin{array}{l}180 \text { day } \\
\text { RESRAD }\end{array}$ & Evaluation & $\begin{array}{l}02 / 02 / 2007 \\
n\end{array}$ & $719: 48$ & Page 33 & & & \\
\hline 0 & & Tota & 50 & $\begin{array}{r}\text { Risk } \\
\text { a } \\
\text { Wat }\end{array}$ & $\begin{array}{l}\text { NRSI }(i, p, t \\
\text { d Fractior } \\
r \text { Indepeno }\end{array}$ & $\begin{array}{l}t * * * \text { for } \\
n \text { of Total } \\
\text { dent Pathw }\end{array}$ & I & alat $i$ & $\begin{array}{l}\text { adionuclides } \\
2 \text { years } \\
\text { udes radon) }\end{array}$ & nd & \\
\hline U & Grour & & Inhalat & tion & Rac & don & Plan & & Meat & Milk & Soil \\
\hline Radi & $\triangle A A A A A A A A$ & AÄÄÄÄÄ & AAAAAAOAAAAOA & $\triangle A A A A A B$ & AAAAAAAAAA & $\triangle A \triangle A O A A A O A$ & $\triangle A A O A A A A A B A$ & $\triangle A O A O A O A O A ̈$ & 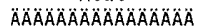 & 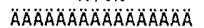 & 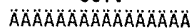 \\
\hline $\begin{array}{l}\text { Nucl ide } \\
\text { ÄÄÄÄÄA }\end{array}$ & $\begin{array}{c}r i s k \\
A A A A A A A O A B\end{array}$ & $\begin{array}{l}\text { fract. } \\
\triangle A A A A A A B\end{array}$ & $\begin{array}{c}\text { risk } \\
\triangle A B A O A O A O A O A B\end{array}$ & $\begin{array}{l}\text { fract. } \\
\triangle A A O A O A O A\end{array}$ & $\begin{array}{c}\text { risk } \\
\text { AAAÄÄABAOÄ }\end{array}$ & $\begin{array}{l}\text { fract. } \\
\ddot{A} \ddot{A} \ddot{A} \ddot{A B A ̈ A}\end{array}$ & $\begin{array}{c}\text { risk } \\
\triangle A \cap A A A A A B A\end{array}$ & $\begin{array}{l}\text { fract. } \\
\triangle A B A O A O A\end{array}$ & $\begin{aligned} \text { risk fract. } \\
\triangle A A B A O A B A B A\end{aligned}$ & $\begin{array}{l}\text { risk fract. } \\
\text { AAAAAAAAB AAAAAAA }\end{array}$ & $\begin{array}{r}\text { risk fract. } \\
\text { AAAAAAAAA AAOAAA }\end{array}$ \\
\hline$A m-241$ & $2.773 E-08$ & 0.0065 & $1.843 \mathrm{E}-09$ & 0.0004 & $0.000 E+00$ & 00.0000 & $1.144 E-08$ & 0.0027 & $2.077 \mathrm{E}-100.0000$ & $2.251 \mathrm{E}-110.0000$ & $1.917 E-080.0045$ \\
\hline$C-14$ & $0.000 E+00$ & 0.0000 & $0.000 E+00$ & 0.0000 & $0.000 E+00$ & 00.0000 & $0.000 E+00$ & 0.0000 & $0.000 \mathrm{E}+00 \quad 0.0000$ & $0.000 E+00 \quad 0.0000$ & $0.000 E+00 \quad 0.0000$ \\
\hline Co- 60 & $3.174 E-12$ & 0.0000 & $6.164 \mathrm{E}-19$ & 0.0000 & $0.000 E+0 C$ & 00.0000 & $3.991 \mathrm{E}-14$ & 0.0000 & $3.786 \mathrm{E}-140.0000$ & $8.676 \mathrm{E}-15 \quad 0.0000$ & $9.343 E-16 \quad 0.0000$ \\
\hline Cs -137 & $1.952 E-06$ & 0.4573 & $6.164 \mathrm{E}-13$ & 0.0000 & $0.000 E+00$ & 00.0000 & $1.007 \mathrm{E}-07$ & 0.0236 & $1.553 \mathrm{E}-07 \quad 0.0364$ & $E-08 \quad 0.0$ & $3.020 E-09 \quad 0.0007$ \\
\hline Eu-152 & $5.703 E-08$ & 0.0134 & $6.576 \mathrm{E}-14$ & 0.0000 & $0.000 E+00$ & 00.0000 & $2.043 E-11$ & 0.0000 & $6.995 \mathrm{E}-12 \quad 0.0000$ & $\begin{array}{lll}4.622 \mathrm{E}-13 & 0.0000\end{array}$ & $1.576 \mathrm{E}-11 \quad 0.0000$ \\
\hline $\mathrm{H}-3$ & $0.000 E+00$ & 0.0000 & $0.000 \mathrm{E}+00$ & 0.0000 & $0.000 E+00$ & 00.0000 & $0.000 E+00$ & 0.0000 & $0.000 E+00 \quad 0.0000$ & $0.000 E+00 \quad 0.0000$ & $0.000 \mathrm{E}+00 \quad 0.0000$ \\
\hline $\mathrm{Ni}-63$ & $0.000 E+00$ & 0.0000 & $1.589 E-11$ & 0.0000 & $0.000 E+00$ & 00.0000 & $1.701 \mathrm{E}-07$ & 0.0399 & $4.239 E-08 \quad 0.0099$ & $3.925 \mathrm{E}-07 \quad 0.0920$ & $6.637 \mathrm{E}-090.0016$ \\
\hline Pu-239 & $4.000 E-10$ & 0.0001 & $4.421 \mathrm{E}-09$ & 0.0010 & $0.000 E+00$ & 00.0000 & $3.003 E-08$ & 0.0070 & $1.089 \mathrm{E}-09 \quad 0.0003$ & $2.957 E-110.0000$ & $4.933 E-080.0116$ \\
\hline Pu-240 & $3.034 E-11$ & 0.0000 & $9.359 E-10$ & 0.0002 & $0.000 E+0 C$ & 00.0000 & $6.358 \mathrm{E}-09$ & 0.0015 & $2.306 E-10 \quad 0.0001$ & $6.265 E-120.0000$ & $1.048 \mathrm{E}-08 \quad 0.0025$ \\
\hline $5 r-90$ & & 0.0013 & & & $0.000 E+00$ & 00.0000 & $7.182 \mathrm{E}-07$ & 0.1683 & $2.558 E-07 \quad 0.0$ & $1.445 \mathrm{E}-070.0$ & $E-09 \quad 0.0009$ \\
\hline İIíílín & & IIIIIII & İílíilíl & & ifítítít & I Iffiff & IIIIIIIII & ÎIî́î́ & 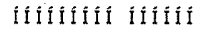 & Iffíf i î́ & 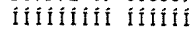 \\
\hline Total & $2.042 E-06$ & 0.4786 & $7.219 E-09$ & 0.0017 & $0.000 E+00$ & 00.0000 & $1.037 E-06$ & 0.2430 & $4.550 E-07 \quad 0.1066$ & $6.335 \mathrm{E}-07 \quad 0.1485$ & $9.241 \mathrm{E}-08 \quad 0.0217$ \\
\hline
\end{tabular}

Total Excess Cancer Risk CNRSI $(i, p, t) * \star *$ for Initially Existent Radionuclides $(i)$ and Pathways $(p)$

and Fraction of Total Risk at $t=1.000 E+02$ years

Water Dependent Pathways

\begin{tabular}{|c|c|c|c|c|c|c|c|c|c|c|c|c|c|}
\hline \multirow[b]{2}{*}{$\begin{array}{l}\text { Nuclide } \\
\text { ÄAOAOAOAOAB }\end{array}$} & \multicolumn{2}{|c|}{ 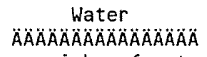 } & \multicolumn{2}{|c|}{ 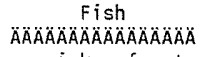 } & \multicolumn{2}{|c|}{ 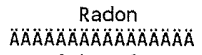 } & \multicolumn{2}{|c|}{$\begin{array}{c}\text { Plant } \\
\text { AMAAAAAAAAAAAAAAA }\end{array}$} & \multicolumn{2}{|c|}{ 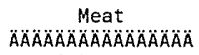 } & \multicolumn{2}{|c|}{ 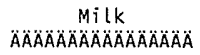 } & $\begin{array}{c}\text { All pathways } \\
\text { ALAAAAAAAAAAAAA }\end{array}$ \\
\hline & $\begin{array}{c}r i s k \\
A \cap A A A A B A B A\end{array}$ & $\begin{array}{l}\text { fract. } \\
\text { ÄÄÄÄÄ }\end{array}$ & $\begin{array}{c}\text { risk } \\
\triangle A A A O A A O A B A B\end{array}$ & 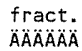 & $\begin{array}{c}\text { risk } \\
\triangle A B A A O A O A O A B\end{array}$ & $\begin{array}{l}\text { fract. } \\
\triangle A O A O A O A ̈ A\end{array}$ & $\begin{array}{c}r i s k \\
\triangle A B A O A O A O A O A O A\end{array}$ & $\begin{array}{l}\text { fract. } \\
\triangle A \cap A B A B A B A\end{array}$ & $\begin{array}{c}r i s k \\
\triangle A A B A B A A A O A\end{array}$ & $\begin{array}{l}\text { fract. } \\
\because A A A A A A A B\end{array}$ & $\begin{array}{c}r i s k \\
\triangle A B A B A B A B A B A\end{array}$ & $\begin{array}{l}\text { fract. } \\
\text { ÄÄÄÄÄ }\end{array}$ & $\begin{array}{l}\text { risk fract. } \\
\triangle A A A A B A A B A A B A A B A\end{array}$ \\
\hline$A m-241$ & $0.000 E+00$ & 0.0000 & $0.000 E+00$ & 0.0000 & $0.000 \mathrm{E}+00$ & 0.0000 & $0.000 E+00$ & 0.0000 & $0.000 E+00$ & 0.0000 & $0.000 E+00$ & 0000 & -08 \\
\hline 67 & $0.000 E+00$ & 0.0000 & $0.000 E+00$ & 0.0000 & $0.000 E+00$ & 0.0000 & $0.000 E+00$ & 0.0000 & $0.000 E+00$ & 0.0000 & $0.000 E+00$ & 0.0000 & $0.000 \mathrm{E}+00 \quad 0.0000$ \\
\hline $\mathrm{Co}-6 \mathrm{C}$ & $0.000 E+00$ & 0.0000 & $0.000 \mathrm{E}+00$ & 0.0000 & 00 & & $0 E+00$ & 00 & $E+00$ & & & & 12 \\
\hline & 0 & 0.00 & $0.000 E$ & 0.0000 & 0.000 & 0.0000 & $0.000 E+00$ & 0.0000 & $0.000 E+00$ & 0.0000 & $0.000 E+00$ & .0000 & 0 \\
\hline & $0.000 E+00$ & & $0.000 E+00$ & & 0.000 & & $0.000 E+00$ & 0.0000 & $0.000 E+00$ & 0.0000 & $0 E+00$ & 000 & $=-08$ \\
\hline$H^{-}$ & $0.000 E+00$ & 0.0000 & $0.000 E+00$ & 0.0000 & $.000 E+00$ & & +00 & 00 & $E+00$ & & 00 & & \\
\hline $\mathrm{Ni}-63$ & $0.000 E+00$ & 0.0000 & $0.000 E+00$ & 0.0000 & $0.000 E+00$ & 0.0000 & $0.000 E+00$ & 0.0000 & $0.000 E+00$ & 0.0000 & $0.000 E+00$ & 0.0000 & $E=07$ \\
\hline Pu-239 & $0.000 E+00$ & & $0.000 E+00$ & 0.0 & $0.000 E+00$ & 0.0000 & $0.000 E+00$ & 0.0000 & $0.000 \mathrm{E}+00$ & 0.0000 & $0.000 E+00$ & 0.0000 & $8.531 E-08 \quad 0.0200$ \\
\hline Pu-240 & $0.000 E+00$ & 0.0000 & 0.000 & & 0.000 & & $10 E+00$ & & $+\infty$ & & & & \\
\hline & 0.0 & 0.0000 & 0.0 & 0.0000 & 0.0 & 0 & & & +00 & & & & \\
\hline & & & & & & & III & IIIIIII & IIII & IIIIIII & IIIIIIIIII & if & $\mathrm{i}$ \\
\hline Total & $0.000 E+00$ & 0.0000 & $0.000 E+00$ & 0.0000 & $0.000 E+00$ & 0.0000 & $0.000 E+00$ & 0.0000 & $0.000 E+00$ & 0.0000 & $0.000 E+00$ & 0.0000 & $4.267 \mathrm{E}-06 \quad 1.0000$ \\
\hline
\end{tabular}

\begin{tabular}{|c|c|c|}
\hline \multirow{2}{*}{\multicolumn{3}{|c|}{$\begin{array}{ll}\text { Attachment } & \frac{10}{\text { Stacheet No. } 33 \text { of } 41} \\
\text { Originators: S. W. Clark } & \text { Date }\end{array}$}} \\
\hline & & \\
\hline Chk'd By & M. W. Perrott & Date \\
\hline Calc. No & $0100 \mathrm{~B}-\mathrm{CA}$ & Rev. No. \\
\hline
\end{tabular}


CVP-2007-00006

Rev. 0

\section{ATTACHMENT 10}

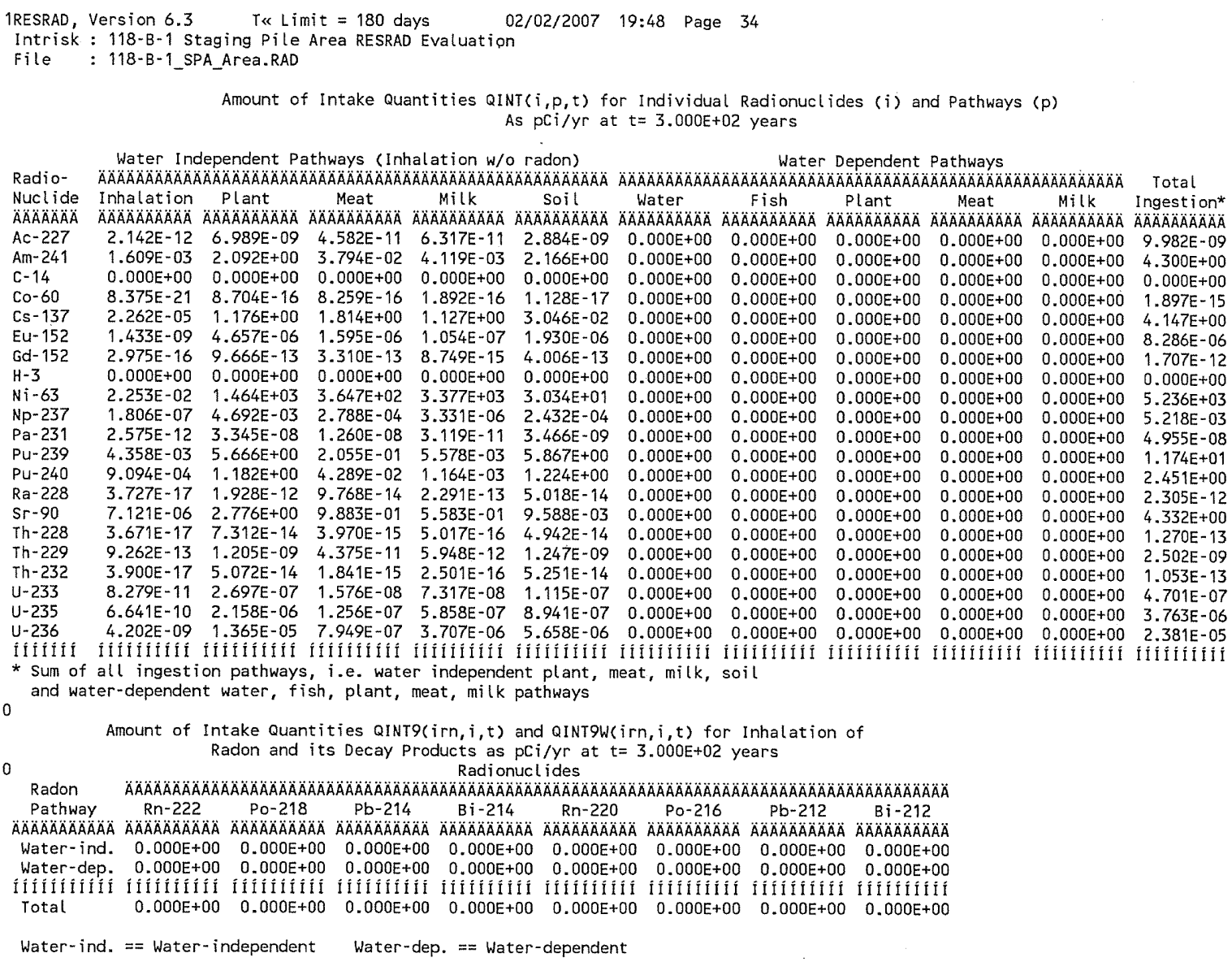

\begin{tabular}{ll} 
Attachment & \multicolumn{1}{c}{$\begin{array}{c}\text { Sheet No. } 34 \text { of } 41 \\
\text { Originators: }\end{array}$} \\
Chk' W. Clark & Date \\
Calc. No. N. Perrott & Date \\
\hline O100B-CA-V0301 & Rev. No. $\quad 0$
\end{tabular}




\section{ATTACHMENT 10}

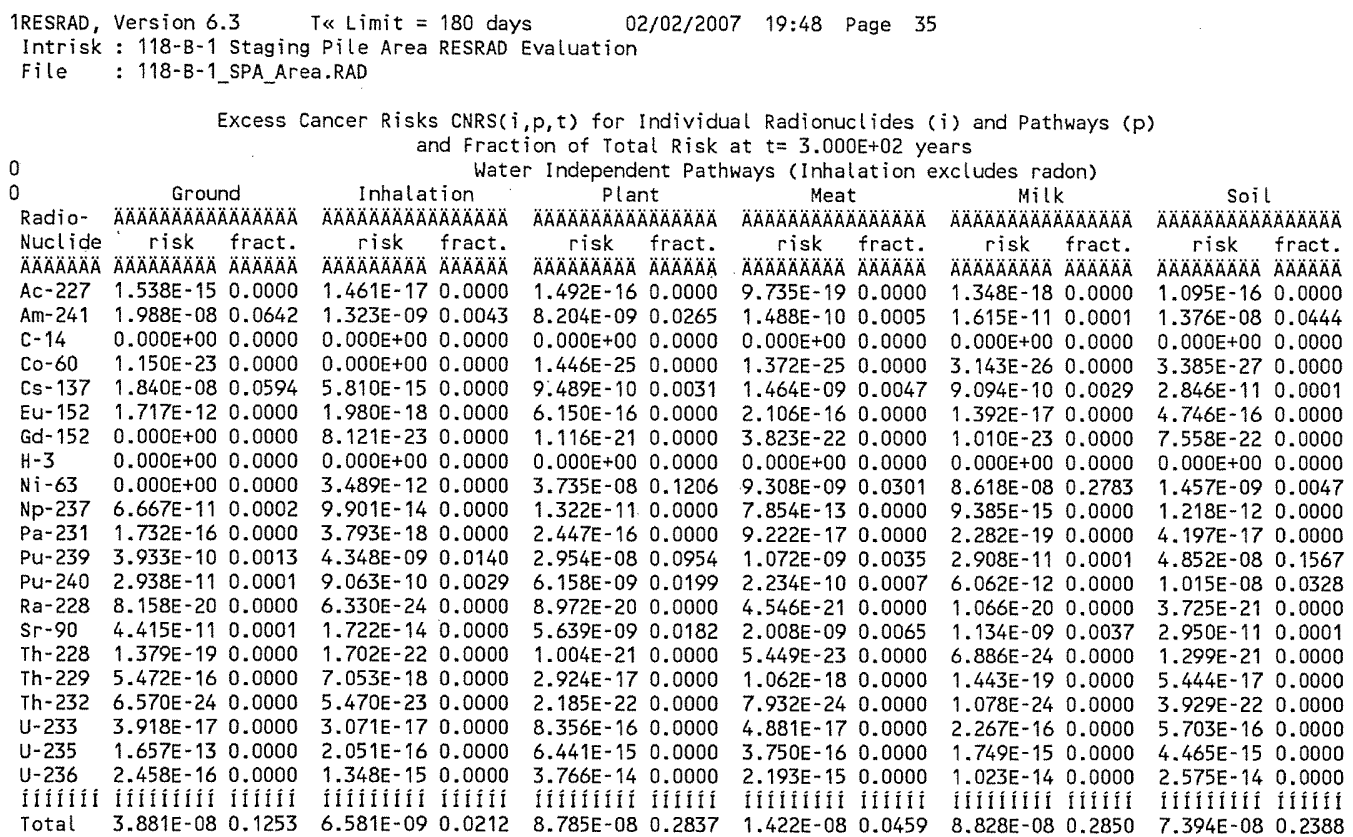

Attachment 10 


\section{ATTACHMENT 10}

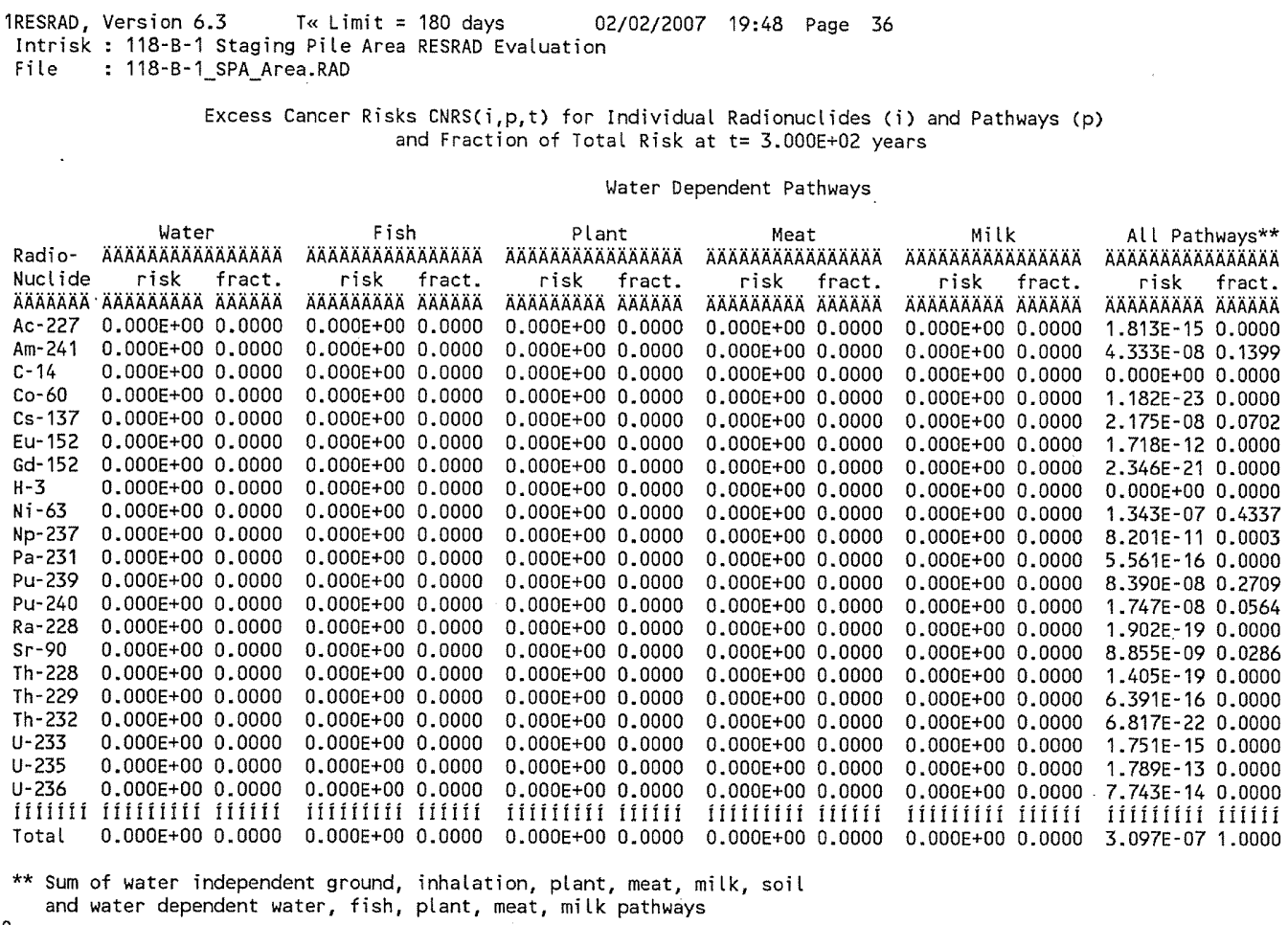

Water - ind. == Water-independent Water-dep. $==$ Water-dependent

\begin{tabular}{|c|c|}
\hline Attachment & Sheet No. 36 of 41 \\
\hline Originators: S.W. Clark & Date \\
\hline M.W. Perrott & Date \\
\hline $0100 \mathrm{~B}-\mathrm{CA}-\mathrm{V} 0301$ & Rev. No. \\
\hline
\end{tabular}




\section{ATTACHMENT 10}

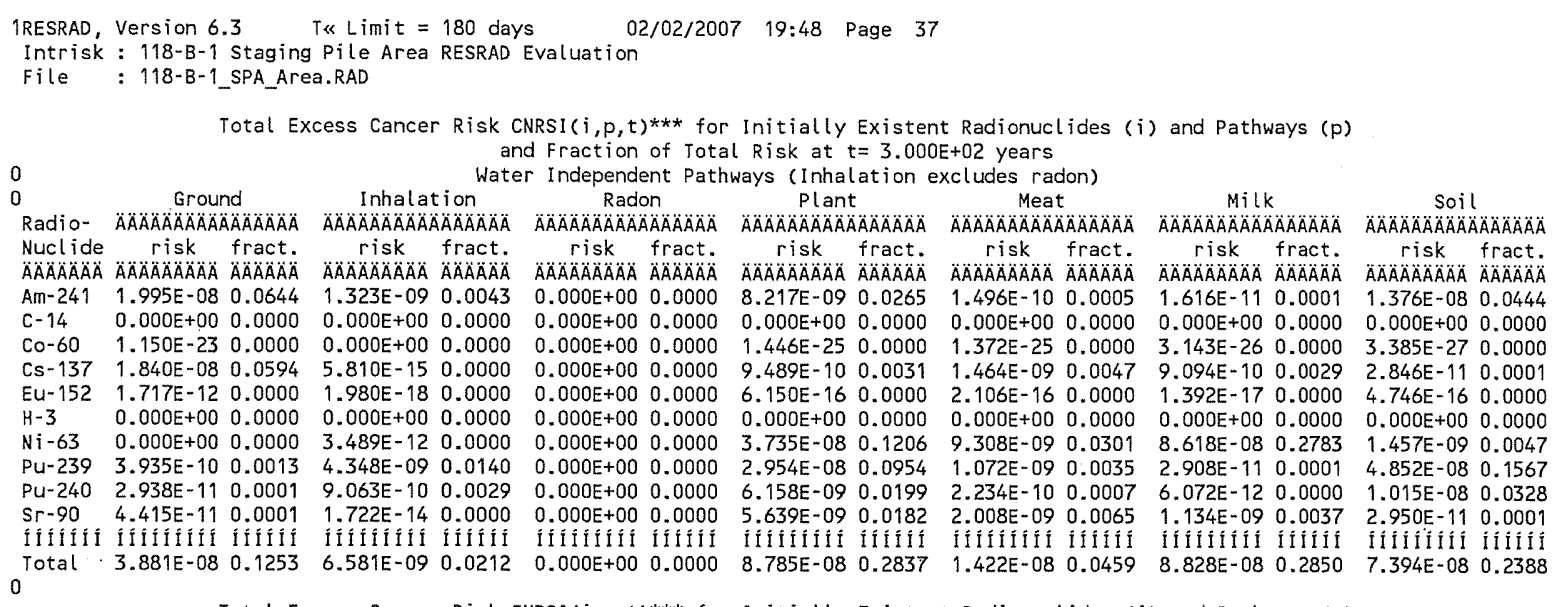

Total Excess Cancer Risk CNRSI $(i, p, t) * * *$ for Initially Existent Radionuclides $(i)$ and Pathways $(p)$ and Fraction of Total Risk at $t=3.000 E+02$ years

Water Dependent Pathways

\begin{tabular}{|c|c|c|c|c|c|c|c|c|c|c|c|c|c|}
\hline \multirow[b]{2}{*}{$\begin{array}{l}\text { Nuclide } \\
\text { ÄÄÄÄÄÄ }\end{array}$} & \multicolumn{2}{|c|}{ Water } & \multicolumn{2}{|c|}{$\begin{array}{c}F i s h \\
\text { AAAAAAAAAAAAAAAA }\end{array}$} & \multicolumn{2}{|c|}{$\begin{array}{c}\text { Radon } \\
\text { AAAAAAAAAAAAOAAOÄ }\end{array}$} & \multicolumn{2}{|c|}{$\begin{array}{c}P l a n t \\
\text { ÄÄAAAAAAAAAAAAOÄÄ }\end{array}$} & \multicolumn{2}{|c|}{$\begin{array}{c}\text { Meat } \\
\text { ÄÄAAAAOAOAOAOAOAAOAOA }\end{array}$} & \multicolumn{2}{|c|}{$\begin{array}{c}\text { MilK } \\
\text { AAAOAAAAAAAAAAOA }\end{array}$} & $\begin{array}{c}\text { All pathways } \\
\text { ALAAAAAAAAAAAAAA }\end{array}$ \\
\hline & $\begin{array}{l}\text { risk } \\
\triangle A ̈ A B A O A ̈ A\end{array}$ & $\begin{array}{l}\text { fract. } \\
\ddot{A} \ddot{A} \ddot{A} \ddot{A} \ddot{A}\end{array}$ & $\begin{array}{c}r i s k \\
\triangle A B A O A ̈ A B A ̈ A ̈ A ̈\end{array}$ & $\begin{array}{l}\text { fract. } \\
\because A A A A A B A\end{array}$ & 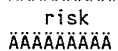 & $\begin{array}{l}\text { fract. } \\
\triangle A A A A B A B\end{array}$ & $\begin{array}{c}\text { risk } \\
\triangle A A A B A A A B A A\end{array}$ & $\begin{array}{l}\text { fract. } \\
\not A A A B A B A\end{array}$ & $\begin{array}{c}\text { risk } \\
\triangle A A B A A A A B\end{array}$ & $\begin{array}{l}\text { fract. } \\
\triangle A A A A A B A\end{array}$ & $\begin{array}{c}r i s k \\
\text { AAAAAAAA }\end{array}$ & $\begin{array}{l}\text { fract. } \\
\triangle A A B A B A\end{array}$ & $\begin{array}{l}\text { risk fract. } \\
\text { AOAAAAAA AAAAAOA }\end{array}$ \\
\hline$A m-241$ & $0.000 E+00$ & 0.0000 & $0.000 \mathrm{E}+00$ & 0.0000 & $0.000 \mathrm{E}+00$ & 0.0000 & $0.000 E+00$ & 0.0000 & $0.000 \mathrm{E}+00$ & 0.0000 & $0.000 \mathrm{E}+00$ & & -08 \\
\hline$c-14$ & $0.000 \mathrm{E}+00$ & 0.0000 & $0.000 E+00$ & 0.0000 & $0.000 E+00$ & 0.0000 & $0.000 E+00$ & 0.0000 & $0.000 E+00$ & 0.0000 & $0.000 E+00$ & 0.0000 & $0.000 E+00 \quad 0.0000$ \\
\hline $0-60$ & $0.000 E+00$ & 0.1 & $\mathrm{DE}+00$ & 0. & $10 E+00$ & 100 & $0.000 E+00$ & 00 & $E+00$ & 00 & $E+00$ & & 23 \\
\hline & 0.0 & 0.0000 & $0.000 E+00$ & 0.0000 & $0.000 E+00$ & 0.0000 & $0.000 E+00$ & 0.0000 & $0.000 E+00$ & 100 & +00 & 00 & \\
\hline Eu-152 & $0.000 \mathrm{E}+00$ & & $0.000 \mathrm{E}+00$ & 0.0000 & $0.000 \mathrm{E}+00$ & 0.0000 & $0.000 E+00$ & 0.0000 & $00 E+00$ & 0. & +00 & 100 & -12 \\
\hline$H-3$ & $0.000 E+00$ & & $E+00$ & & $0 E+00$ & 00 & $E+\infty 0$ & & 0.0 & & 10 & & 100 \\
\hline Ni -63 & $0.000 E+00$ & 0.0000 & $0.000 E+00$ & 0.0000 & $0.000 E+00$ & 0.0000 & $10 E+00$ & 00 & $+\infty$ & 0.0 & $E+\infty$ & 0.0 & -07 \\
\hline Pu-239 & $0.000 \mathrm{E}+00$ & 0.0000 & $0.000 E+00$ & 0.0000 & $0.000 E+00$ & 0.0000 & $0.000 E+00$ & 0.0000 & $0.000 E+00$ & 0.0000 & $0.000 E+00$ & 0.0000 & $E-080.2709$ \\
\hline & $0.000 E+00$ & & $0.000 E+00$ & & $.000 E+00$ & & $0.000 E+00$ & & & & 00 & & \\
\hline & 0.0 & 00 & $+\infty$ & & 0. & & $=+\infty$ & & 0. & & & & \\
\hline & Ififfiflí & & Iffififit & & IIIIIIIIII & IIIfíi & IIIIYATI & IIIIIIII & 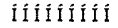 & fíîî & IIIIIIIII & IXIIIII & 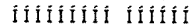 \\
\hline - & $0.000 E+00$ & 0.0000 & $0.000 E+00$ & 0.0000 & $0.000 E+00$ & 0.0000 & $0.000 E+00$ & 0.0000 & $0.000 E+00$ & 0.0000 & $0.000 \mathrm{E}+00$ & 0.0000 & $3.097 \mathrm{E}-07 \quad 1.0000$ \\
\hline
\end{tabular}

***CNRSI $(i, p, t)$ includes contribution from decay daughter radionuclides

\begin{tabular}{|c|c|}
\hline Attachment & Sheet No. 37 of 41 \\
\hline Originators: S. W. Clark & Date \\
\hline Chk'd By M.W. Perrott & Date \\
\hline Calc. No. $\quad 0100 \mathrm{~B}-\mathrm{CA}-\mathrm{V} 0301$ & Rev. No. 0 \\
\hline
\end{tabular}




\section{ATTACHMENT 10}

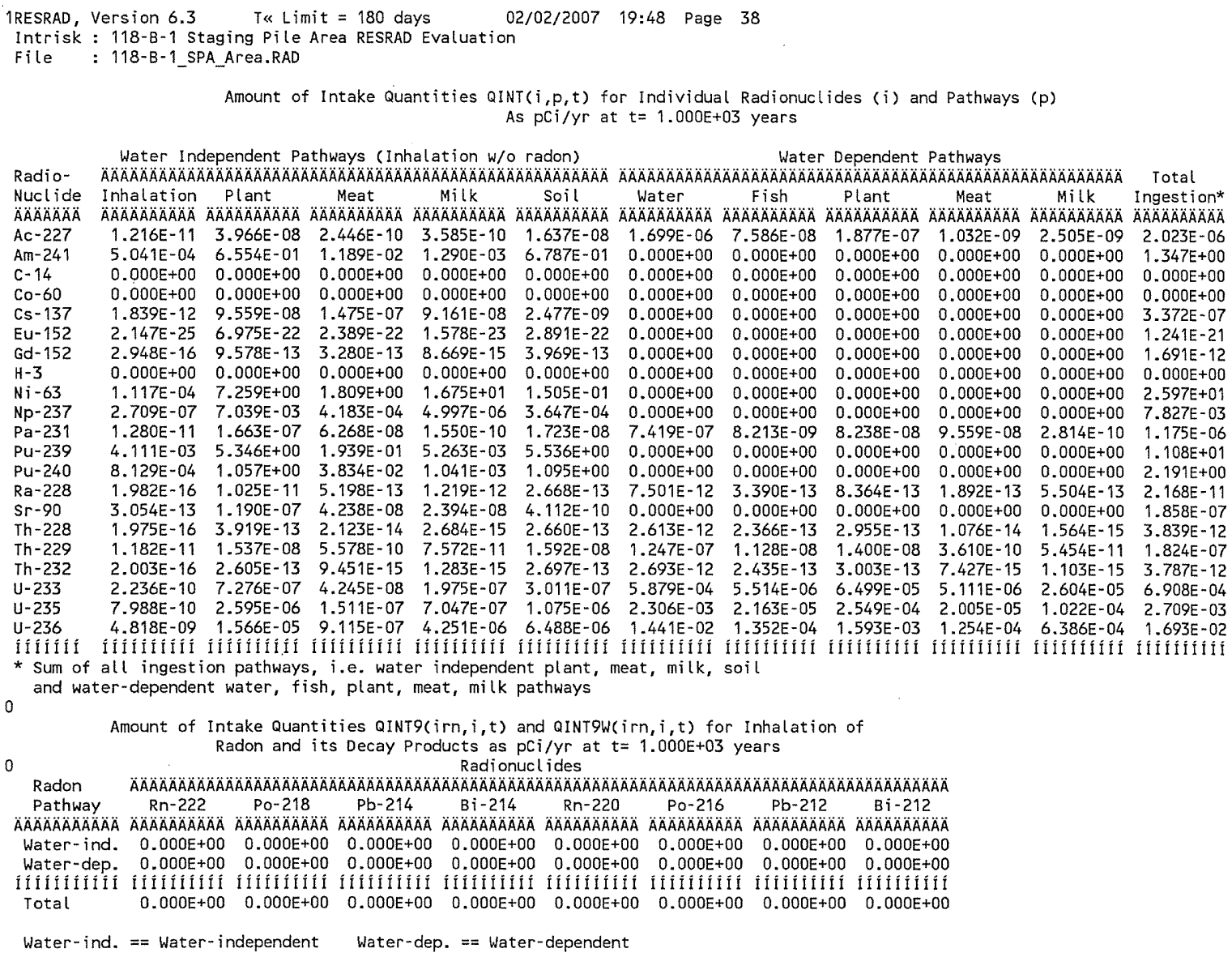

\begin{tabular}{|c|c|c|}
\hline \multirow{2}{*}{\multicolumn{3}{|c|}{ 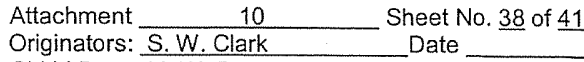 }} \\
\hline & & \\
\hline Chk'd By & M. W. Perrott & Eate \\
\hline Calc. No. & 0100B-CA-V0301 & Rev. No. \\
\hline
\end{tabular}




\section{ATTACHMENT 10}

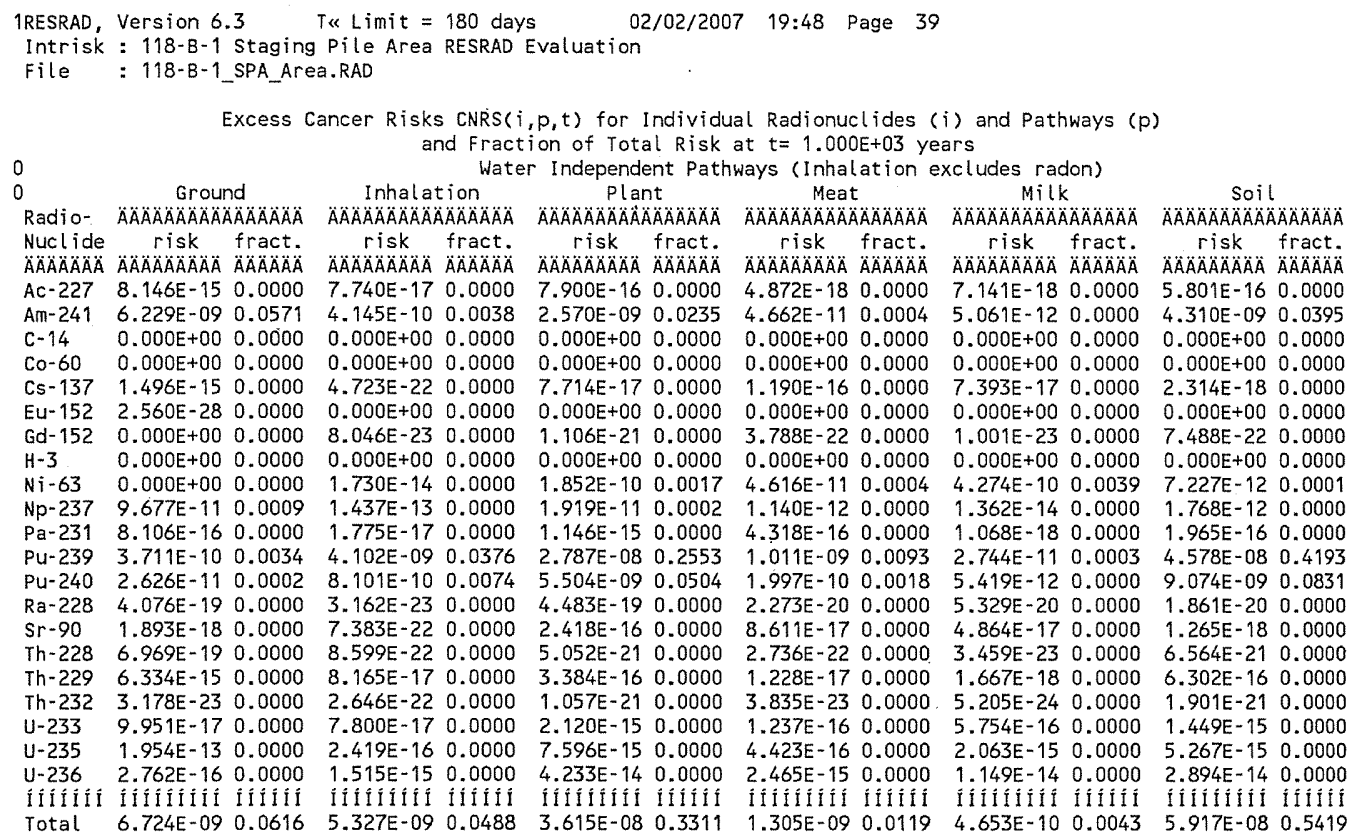

\begin{tabular}{|c|c|}
\hline Attachment & Sheet No \\
\hline Originators: S.W. Clark & Date \\
\hline M. W. Perrott & Date \\
\hline $0100 \mathrm{~B}-\mathrm{CA}-\mathrm{V} 0301$ & Rev. No. \\
\hline
\end{tabular}




\section{ATTACHMENT 10}

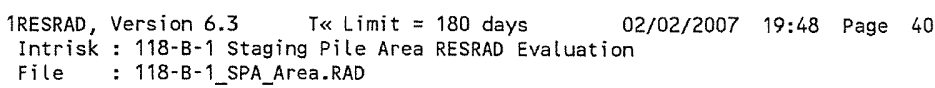
Excess Cancer Risks CNRS9(irn, $i, t)$ and CNRSPW(irn, $i, t)$ for Inhalation of Radon and $i$ ts Decay Products at $t=1.000 \mathrm{E}+03$ years

Radon Radionucl ides

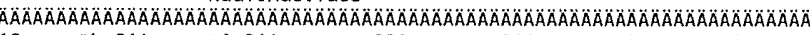

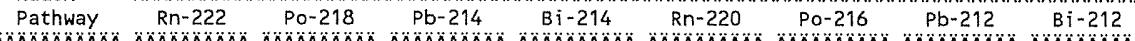

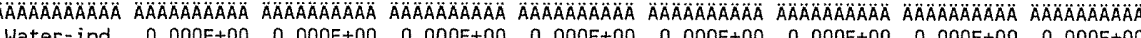
$\begin{array}{lllllllll}\text { Water-ind. } & 0.000 \mathrm{E}+00 & 0.000 \mathrm{E}+00 & 0.000 \mathrm{E}+00 & 0.000 \mathrm{E}+00 & 0.000 \mathrm{E}+00 & 0.000 \mathrm{E}+00 & 0.000 \mathrm{E}+00 & 0.000 \mathrm{E}+00\end{array}$ Water-dep. $0.000 E+00 \quad 0.000 E+00 \quad 0.000 E+00 \quad 0.000 E+00 \quad 0.000 E+00 \quad 0.000 E+00 \quad 0.000 E+00 \quad 0.000 E+00$

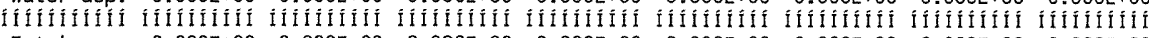

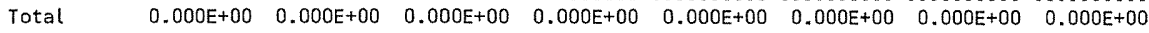

Water-ind. = Water-independent Water-dep. == Water-dependent

\begin{tabular}{|c|c|c|}
\hline Attachment & 10 & Sheet No. 40 of 41 \\
\hline Originators: & S. W. Clark & Date \\
\hline Chk'd By & M. W. Perrott & Date \\
\hline Calc. No. & $0100 B-C A-V 0301$ & Rev. No. \\
\hline
\end{tabular}




\section{ATTACHMENT 10}

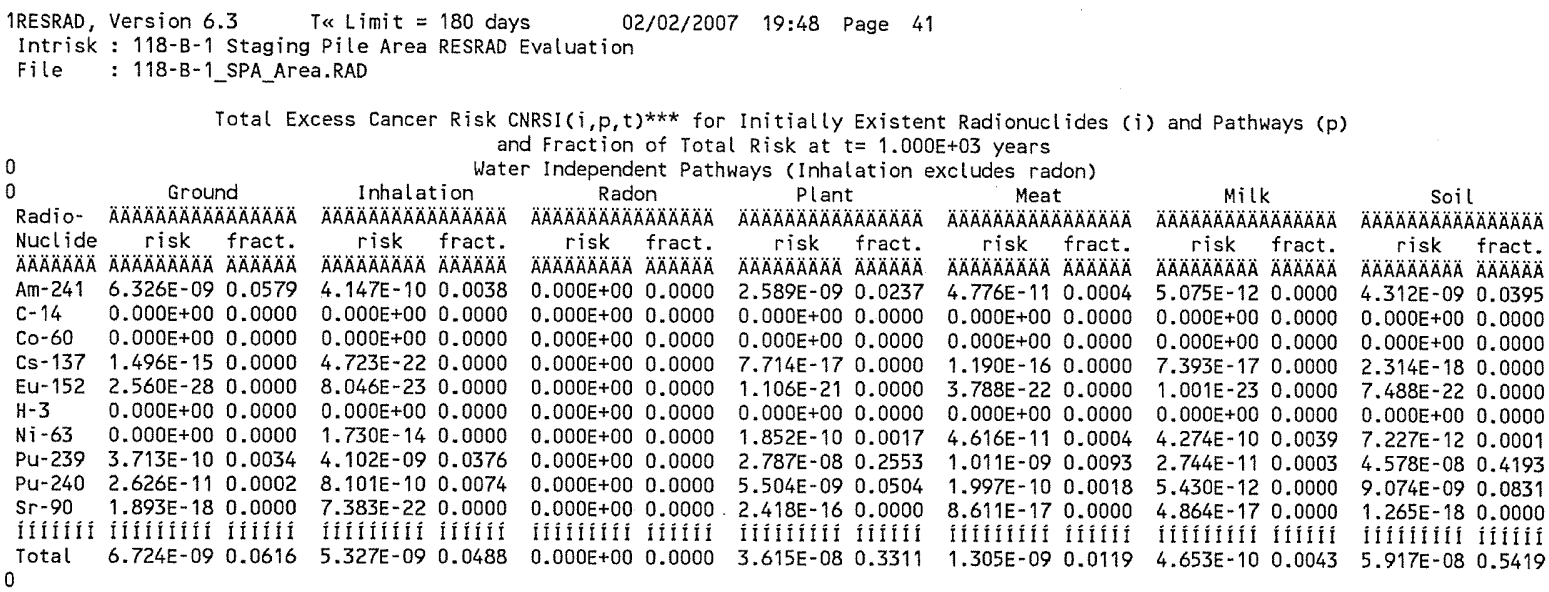

Total Excess Cancer Risk CNRSI $(i, p, t)^{* * *}$ for Initially Existent Radionuclides (i) and Pathways $(p)$ and Fraction of Total Risk at $t=1.000 E+03$ years

Water Dependent Pathways

\begin{tabular}{|c|c|c|c|c|c|c|c|c|c|c|c|c|c|}
\hline & 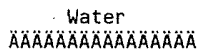 & $\begin{array}{r}F i s \\
\triangle A B A A A O A O A B A\end{array}$ & Ä & $\begin{array}{r}\text { Rad } \\
\triangle A A A A A A O A O A\end{array}$ & Ion & $\begin{array}{r}P l a n \\
\because A A A A A \cap A \cap A \cap A\end{array}$ & $\triangle A \cap A O A A A A$ & $\begin{array}{r}\text { Mea } \\
\triangle A \cap A ̈ A ̈ A ̈ A ̈ A ̈ A\end{array}$ & $\triangle A O A O A O A ̈ A ̈ A ̈$ & $\begin{array}{r}M i L \\
\triangle A A B A O A O A O A B\end{array}$ & $\triangle A O A ̈ A O A O A ̈$ & $\begin{array}{r}\text { All pat } \\
\text { AAAAAAOAAOAOAB }\end{array}$ & $\begin{array}{l}\text { hways } \\
\text { ÄAAAAAAA }\end{array}$ \\
\hline $\begin{array}{l}\text { Nuclide } \\
\text { AAAAAAAAÄA }\end{array}$ & $\begin{array}{r}\text { risk fract. } \\
\text { AAAAOAAOAOA ÄAAAAOA }\end{array}$ & $\begin{array}{c}\text { risk } \\
\text { ÄAAAAAAAA }\end{array}$ & $\begin{array}{l}\text { fract. } \\
\text { ÄÄÄÄA }\end{array}$ & $\begin{array}{c}\text { risk } \\
\triangle A A A A B A O A B A\end{array}$ & 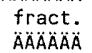 & $\begin{array}{c}\text { risk } \\
\text { AAAAAAAAAÄ }\end{array}$ & $\begin{array}{l}\text { fract. } \\
\text { ÄÄÄÄÄ }\end{array}$ & $\begin{array}{c}\text { risk } \\
\text { AAAAAAAAÄÄ }\end{array}$ & $\begin{array}{l}\text { fract. } \\
\triangle A \cap A ̈ A ̈ A ̈ A\end{array}$ & $\begin{array}{c}\text { risk } \\
A A A B A A A O A O A\end{array}$ & $\begin{array}{l}\text { fract. } \\
\text { ÄÄÄÄÄA }\end{array}$ & $\begin{array}{c}r i s K \\
\triangle A A B A A O A B A B\end{array}$ & $\begin{array}{l}\text { fract. } \\
\because A \cap A B A B A\end{array}$ \\
\hline$A m-241$ & $1.312 \mathrm{E}-120.0000$ & $1.684 E-14$ & 0.0000 & $0.000 E+00$ & 0.0000 & $1.957 \mathrm{E}-13$ & 0.0000 & $1.538 E-14$ & 0.0000 & $7.831 \mathrm{E}-14$ & 0.0000 & $1.370 \mathrm{E}-08$ & 0.1254 \\
\hline$c-14$ & $0.000 E+00 \quad 0.0000$ & $0.000 E+00$ & 0.0000 & $0.000 \mathrm{E}+00$ & 0.0000 & $0.000 \mathrm{E}+00$ & 0.0000 & $0.000 E+00$ & 0.0000 & $0.000 E+00$ & 0.0000 & $0.000 E+00$ & 0000 \\
\hline Co- 60 & $0.000 E+00 \quad 0.0000$ & $0.000 E+00$ & 0.0000 & $0.000 E+00$ & 0.0000 & $0.000 E+00$ & 0.0000 & $0.000 E+00$ & 0.0000 & $0.000 E+00$ & 0.0000 & $0.000 E+00$ & 0.0000 \\
\hline Cs -137 & $0.000 E+00 \quad 0.0000$ & $0.000 E+00$ & 0.0000 & $0.000 \mathrm{E}+00$ & 0.0000 & $0.000 \mathrm{E}+00$ & 0.0000 & $0.000 E+00$ & 0.0000 & $0.000 E+00$ & 000 & 1 & .0000 \\
\hline Eu-152 & $0.000 E+00 \quad 0.0000$ & $0.000 E+00$ & 0.0000 & $0.000 E+00$ & 0.0000 & $0.000 \mathrm{E}+00$ & 0.0000 & $0.000 E+00$ & 0.0000 & $0.000 \mathrm{E}+00$ & 00 & 2. & 0.0000 \\
\hline$H-3$ & $0.000 E+00 \quad 0.0000$ & $0.000 E+00$ & 0.0000 & $0.000 E+00$ & 0.0000 & $0.000 E+00$ & 0.0000 & $0.000 E+00$ & 0.0000 & $0.000 E+00$ & 0.0000 & $0.000 \mathrm{E}+00$ & 0.0000 \\
\hline $\mathrm{Ni}-63$ & $0.000 E+000.0000$ & $0.000 E+00$ & 0.0000 & $0.000 E+00$ & 0.0000 & $0.000 E+00$ & 0.0000 & $0.000 E+00$ & 0.0000 & $0.000 \mathrm{E}+00$ & 00 & & 00,1 \\
\hline Pu-239 & $5.027 \mathrm{E}-12 \quad 0.0000$ & $6.534 E-14$ & 0.0000 & $0.000 E+00$ & 0.0000 & $7.555 E-13$ & 0.0000 & $5.979 E-14$ & 0.0000 & $3.011 E-13$ & 0.0000 & $7.917 \mathrm{E}-08$ & 0.7251 \\
\hline Pu-240 & $2.911 E-110.0003$ & $E-13$ & 0.0000 & $0.000 E+00$ & 0.0000 & $4.338 E-12$ & 0.0000 & $3.413 E-13$ & 0.0000 & $1.739 E-12$ & 0.0000 & $1.566 \mathrm{E}-08$ & 0.1434 \\
\hline & $0.000 E+00 \quad 0.0000$ & 0.00 & 0.0000 & $0.000 \mathrm{E}+00$ & 0.0000 & 0.0 & 0.0000 & $0.000 \mathrm{E}$ & .0000 & & & & \\
\hline Ififtit & fifitifl & IIIIIIIIII & IIIIIII & IIIIIIIII & IIIIIII & IIfIIIIf & IIfítín & IIIIIIIII & IIIIII & IIIIIIIISI & & & 111 \\
\hline Total & $3.545 \mathrm{E}-110.0003$ & $4.502 E-13$ & 0.0000 & $0.000 E+00$ & 0.0000 & $5.289 \mathrm{E}-12$ & 0.0000 & $4.164 E-13$ & 0.0000 & $2.118 E-12$ & 0.0000 & $1.092 \mathrm{E}-07$ & 1.0000 \\
\hline
\end{tabular}

\begin{tabular}{|c|c|c|}
\hline \multirow{2}{*}{\multicolumn{3}{|c|}{ 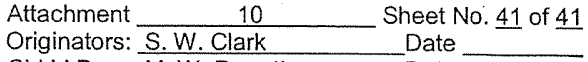 }} \\
\hline & & \\
\hline Chk'd By & M.W. Perrott & Date \\
\hline Calc. No. & 0100B-CA-V0301 & Rev. No. \\
\hline
\end{tabular}




\section{ATTACHMENT 11}

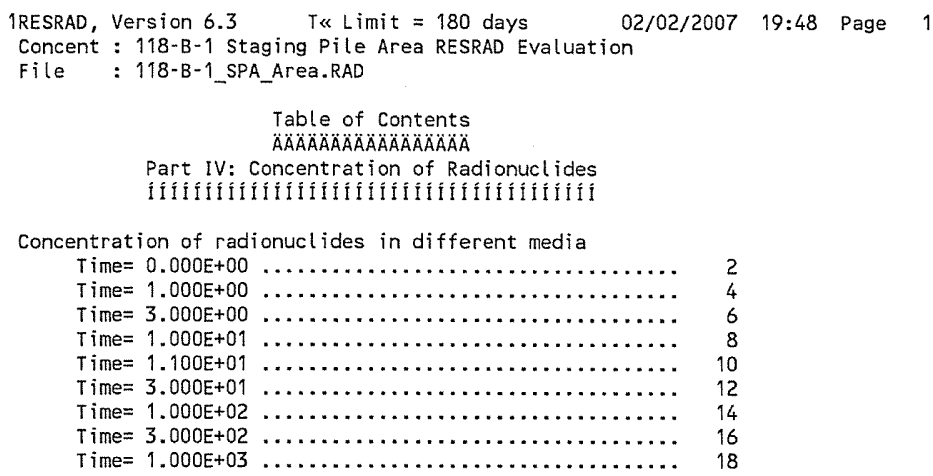


CVP-2007-00006

Rev. 0

\section{ATTACHMENT 11}

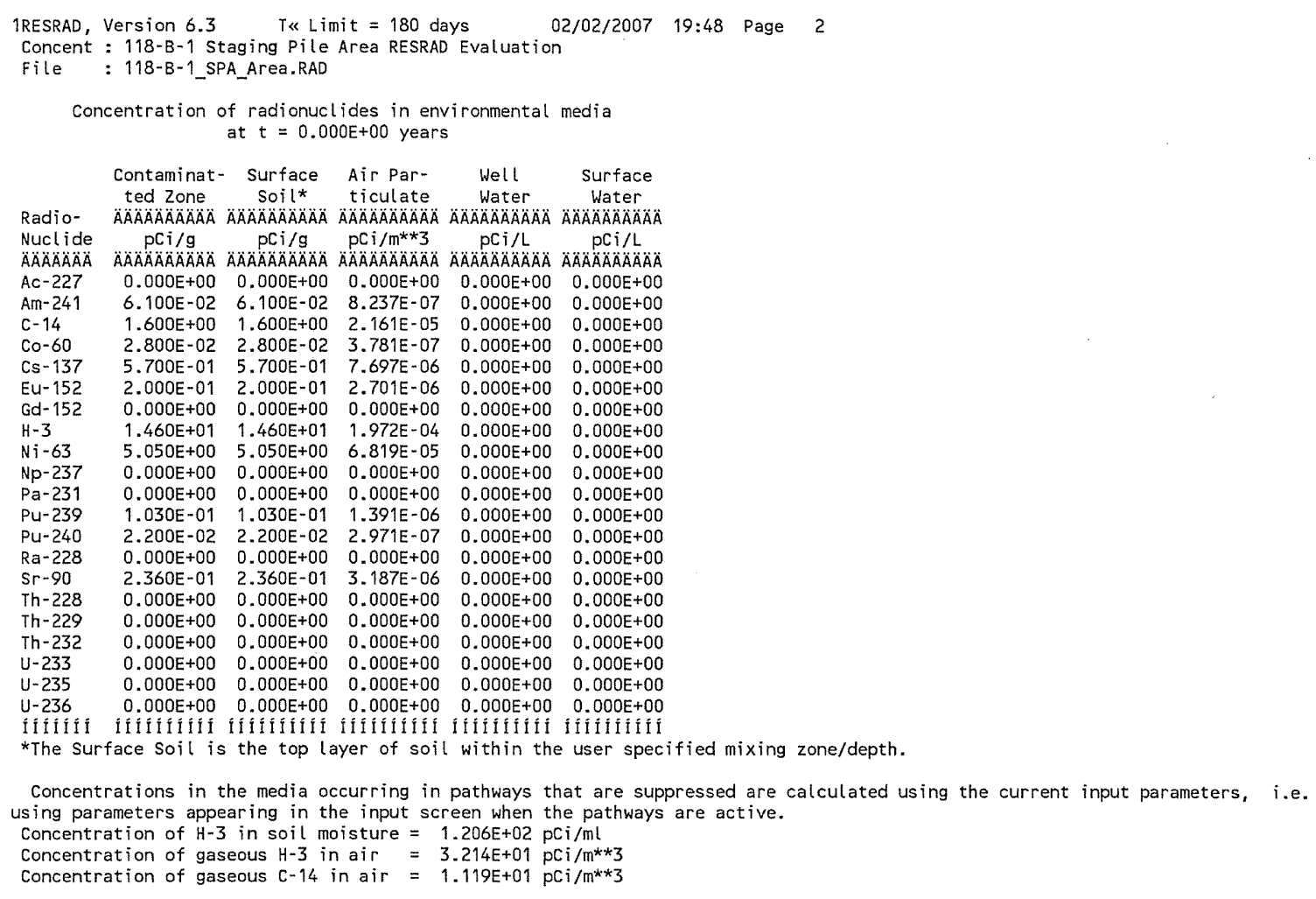

\begin{tabular}{|c|c|}
\hline Attachment & Sheet No. 2 of 19 \\
\hline Originators: S.W. Clark & Date \\
\hline Chk'd By M.W. Perrott & Date \\
\hline $0100 B-C A-V 0301$ & Rev. No. 0 \\
\hline
\end{tabular}


CVP-2007-00006

Rev. 0

\section{ATTACHMENT 11}

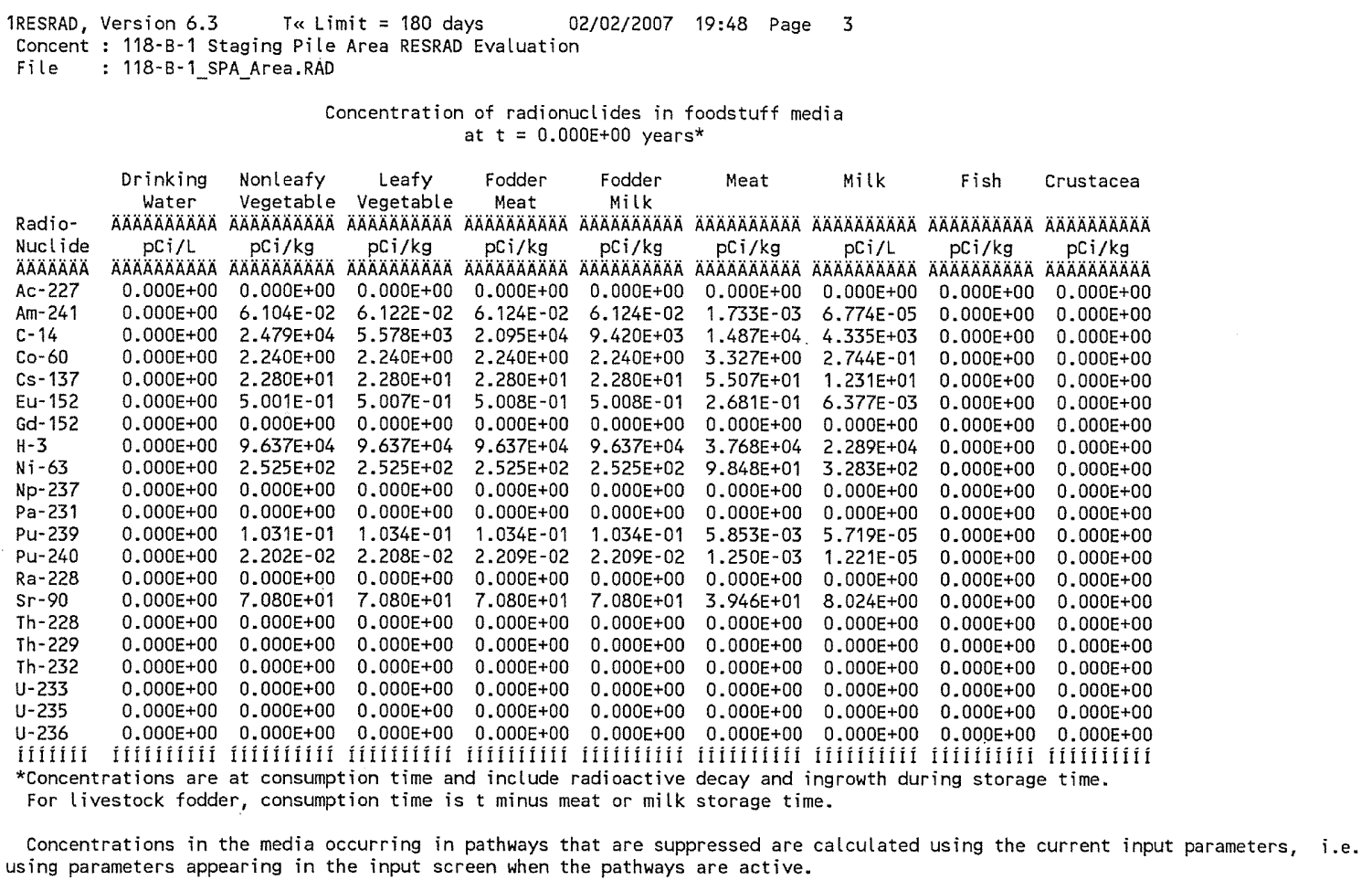
using parameters appearing in the input screen when the pathways are active.

$\begin{array}{ll}\text { Attachment } \frac{11}{\text { S.W.Clark }} & \text { Sheet No. } 3 \text { of } 19 \\ \text { Originators: } & \text { Date } \\ \text { Chk'd By } & \text { Date } \\ \text { Calc. No. Perrott } & \text { Rev. No. } \quad \text { O100B-CA-V0301 }\end{array}$


CVP-2007-00006

Rev. 0

\section{ATTACHMENT 11}

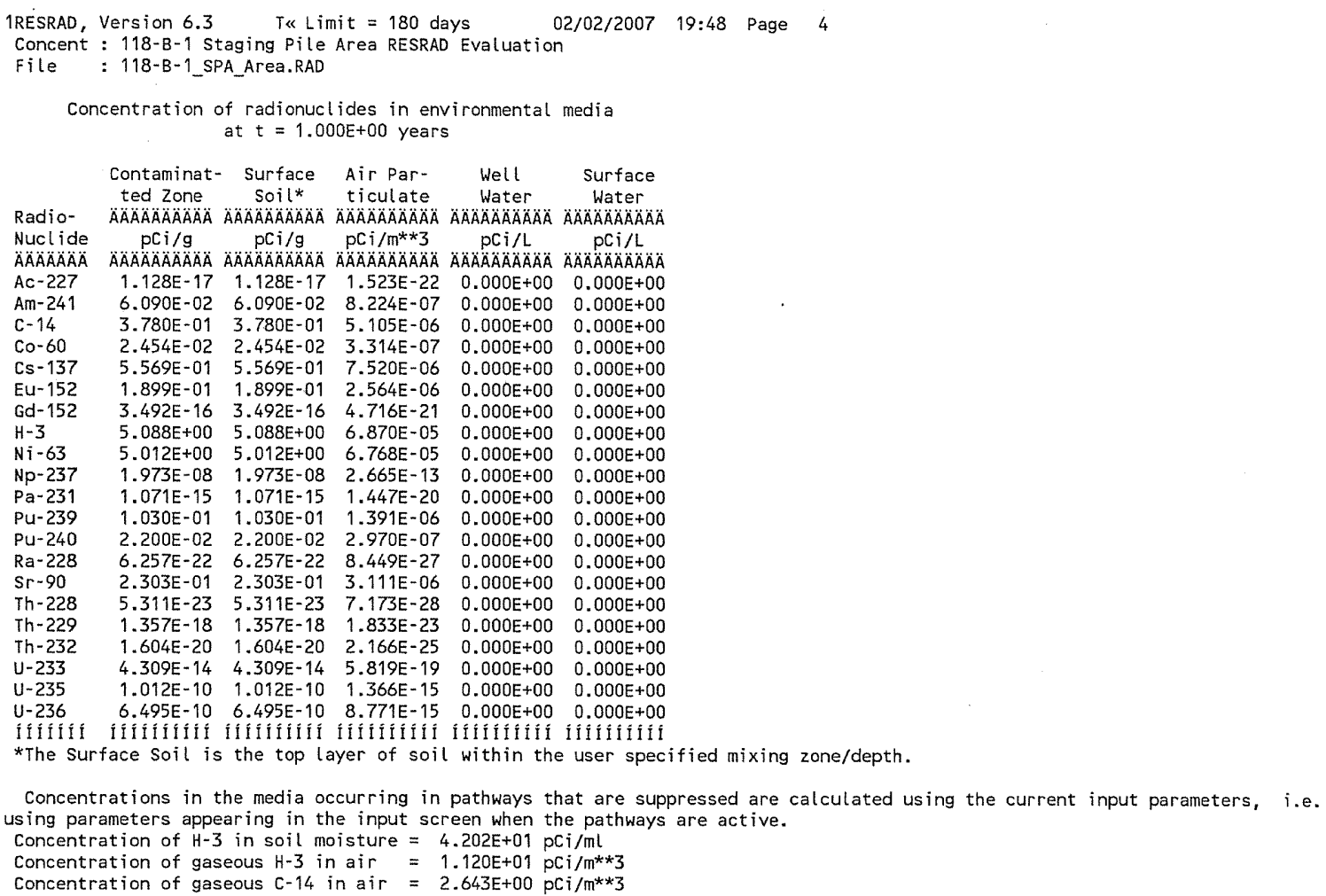

$\begin{array}{ll}\text { Attachment } \frac{11}{\text { S.W. Clark }} & \text { Sheet No. } 4 \text { of } 19 \\ \text { Originators: } & \text { Date } \\ \text { Chk'd By } & \text { Date } \\ \text { Calc. No. Perrott } & \text { Rev. No. } \quad \text { O100B-CA-V0301 }\end{array}$




\section{ATTACHMENT 11}

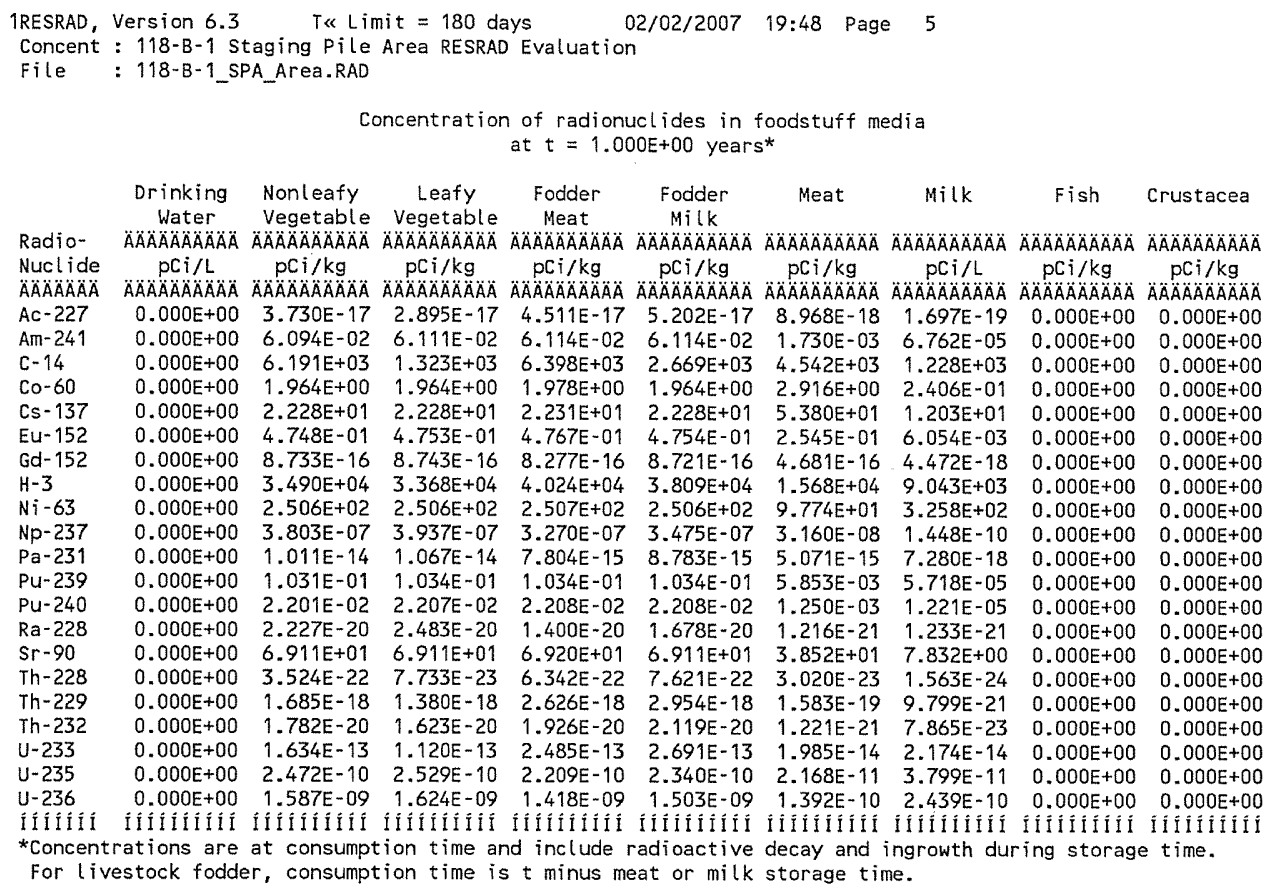

Concentrations in the media occurring in pathways that are suppressed are calculated using the current input parameters, i.e. using parameters appearing in the input screen when the pathways are active.

\begin{tabular}{ll} 
Attachment $\frac{11}{\text { S.W. Clark }}$ & Sheet No. $\underline{5}$ of $\underline{19}$ \\
Originators: & Date \\
Chk'd By & Date \\
Calc. No. Perrott & Rev. No. $\quad 0$ \\
\hline O100B-CA-V0301 & Rev.
\end{tabular}




\section{ATTACHMENT 11}

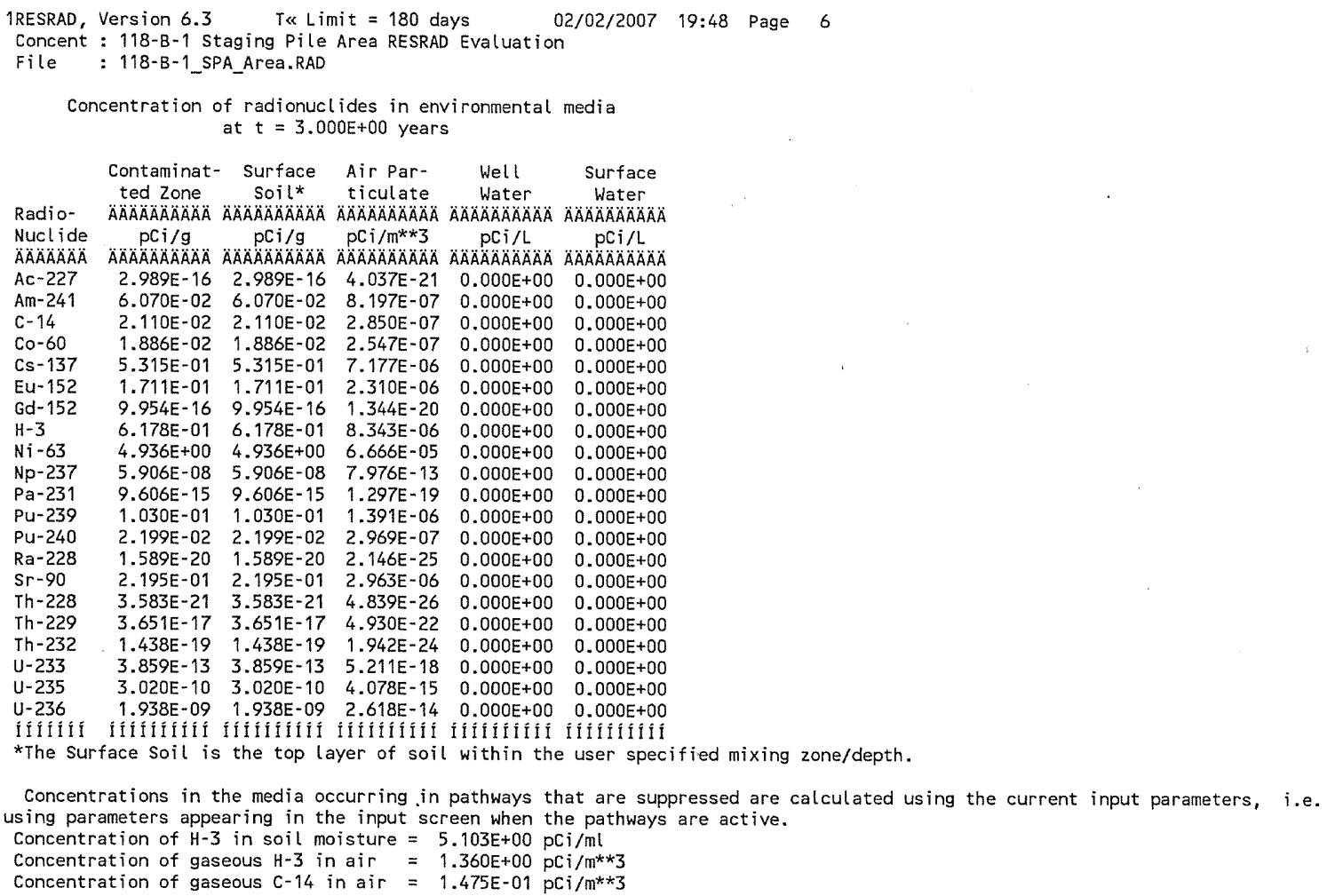

$\begin{array}{ll}\text { Attachment } & \frac{11}{\text { Sheet No. } \underline{6} \text { of } 19} \\ \text { Originators: } \frac{\text { S. W. Clark }}{\text { M.W. Perrott }} & \text { Date } \\ \text { Chk'd By } & \text { Date } \\ \text { Calc. No. } & \text { Rev. No. } 0100 \text { B-CA-V0301 }\end{array}$




\section{ATTACHMENT 11}

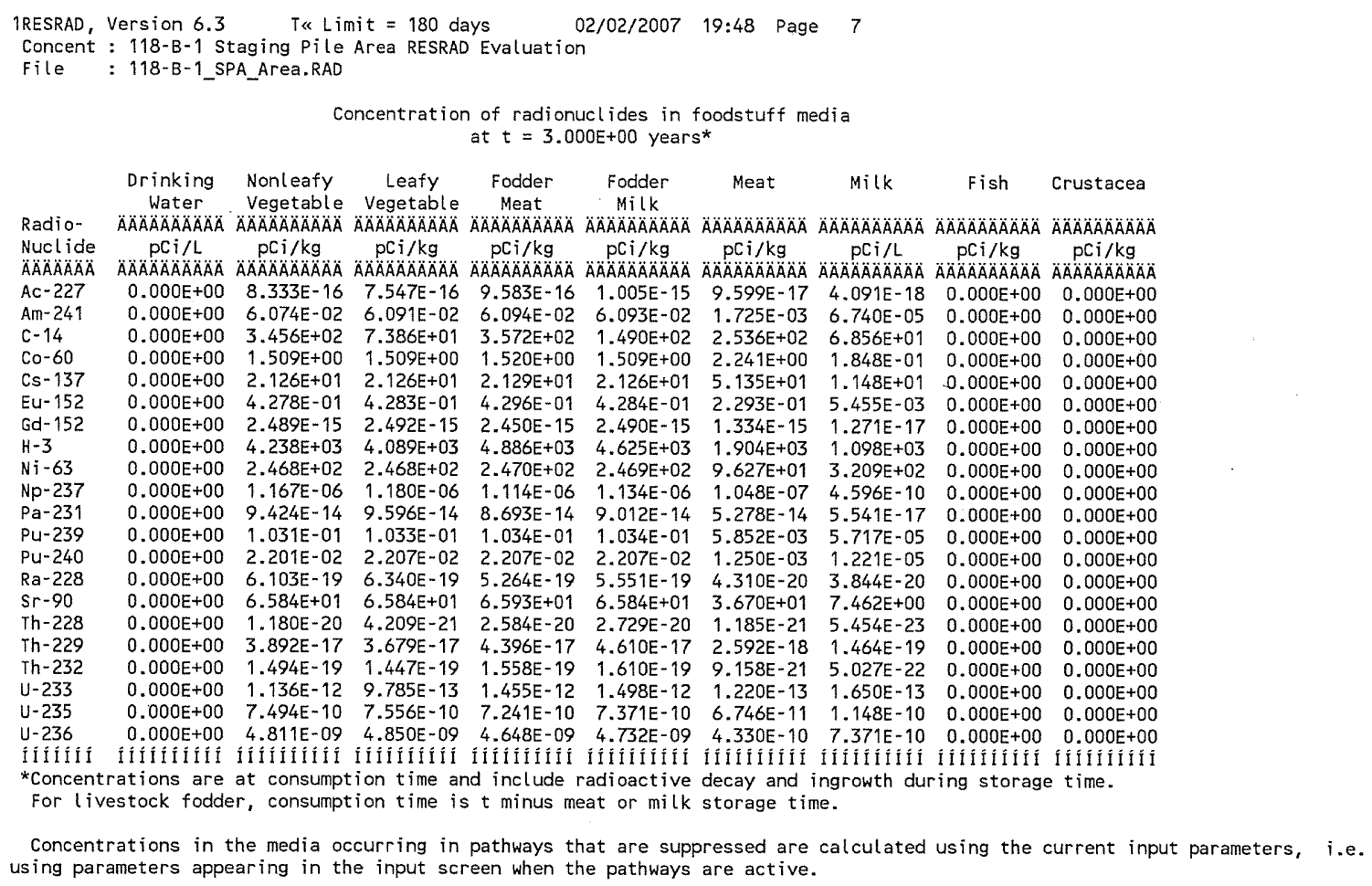

using parameters appearing in the input screen when the pathways are active.

\begin{tabular}{|c|c|}
\hline Attachment & Sheet No. 7 of 19 \\
\hline Originators: S. W. Clark & Date \\
\hline M. W. Perrott & Date \\
\hline 0100B-CA-V0301 & Rev. No. 0 \\
\hline
\end{tabular}




\section{ATTACHMENT 11}

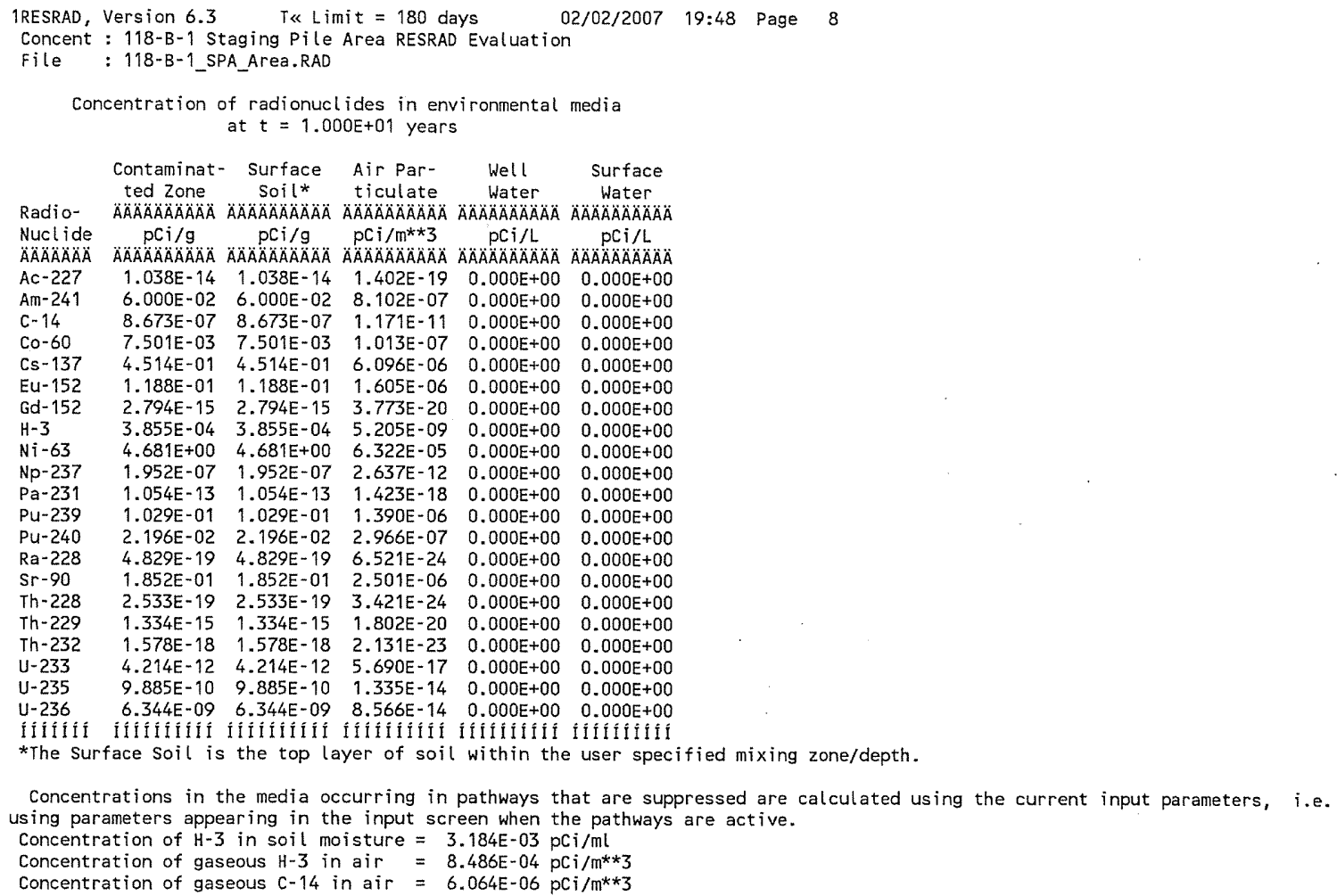

\begin{tabular}{|c|c|}
\hline Attachment & Sheet No. 8 of 19 \\
\hline Originators: S.W. Clark & Date \\
\hline Chk'd By M. W. Perrott & Date \\
\hline Calc. No. $\quad 0100 \mathrm{~B}-\mathrm{CA}-\mathrm{V} 0301$ & Rev. No. \\
\hline
\end{tabular}


CVP-2007-00006

Rev. 0

\section{ATTACHMENT 11}

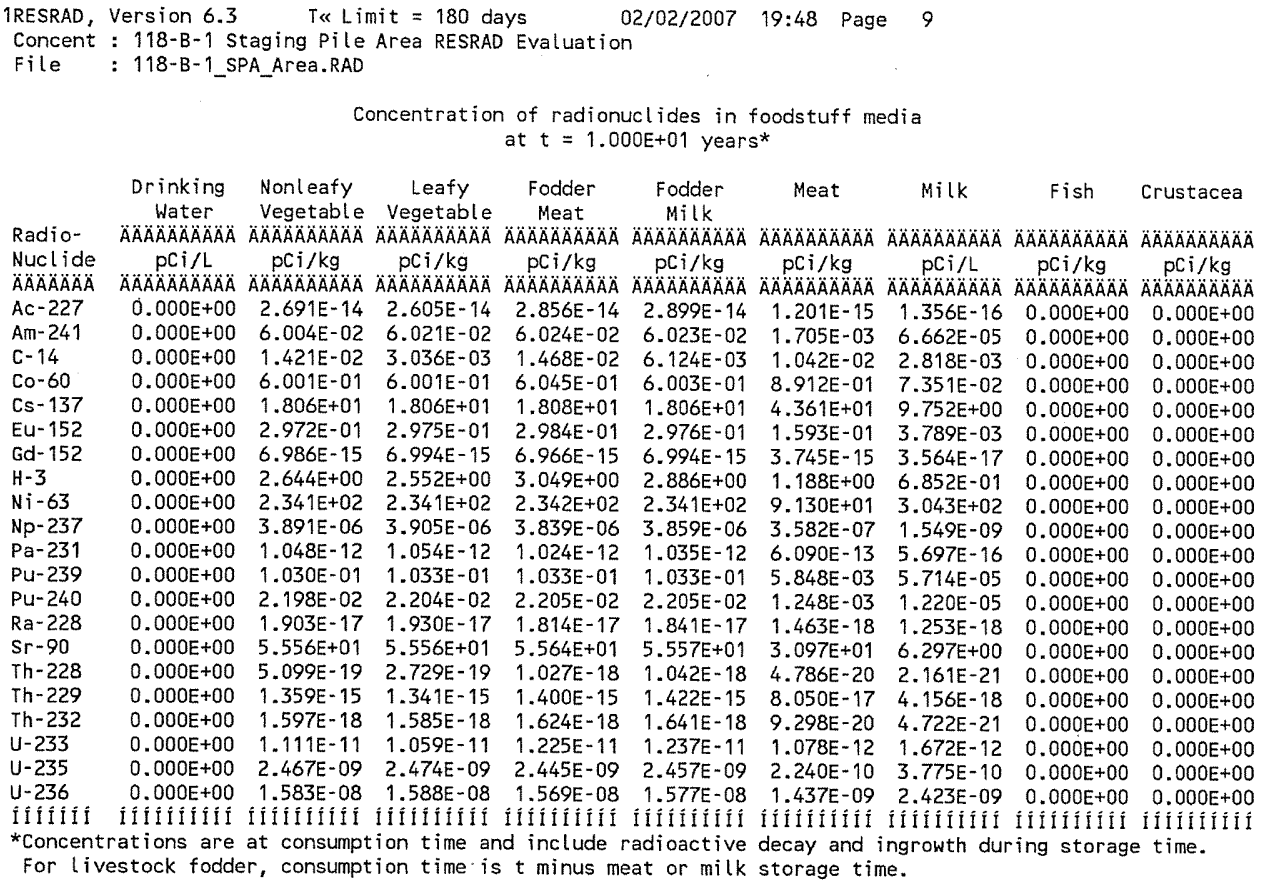

Concentrations in the media occurring in pathways that are suppressed are calculated using the current input parameters, i.e. using parameters appearing in the input screen when the pathways are active.

\begin{tabular}{|c|c|}
\hline Attachment & Sheet No. 9 of 19 \\
\hline Originators: S. W. Clark & Date \\
\hline Chk'd By M. W. Perrott & Date \\
\hline $0100 B-C A-V 0301$ & - Rev. No. \\
\hline
\end{tabular}


CVP-2007-00006

Rev. 0

\section{ATTACHMENT 11}

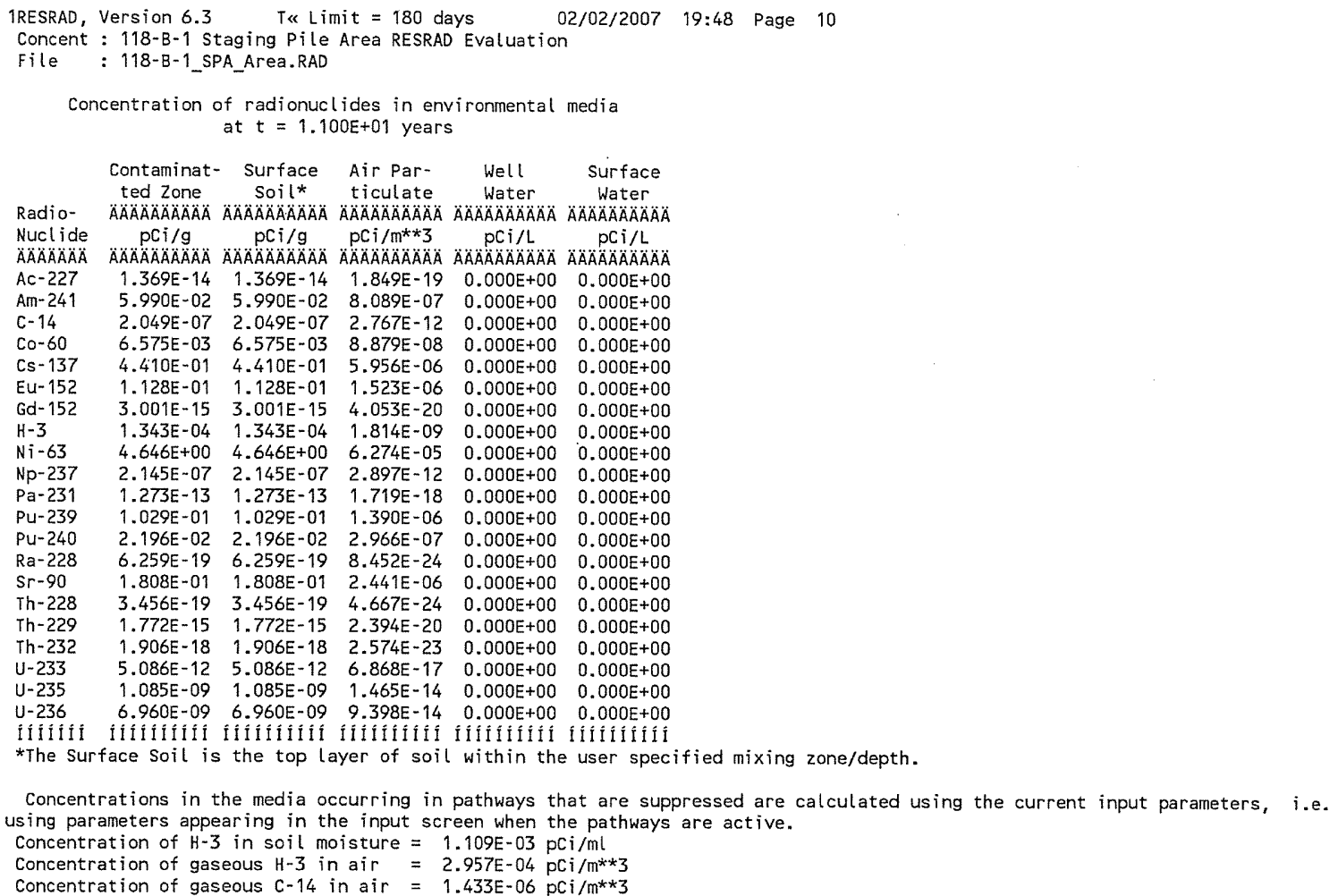

\begin{tabular}{ll} 
Attachment & \multicolumn{1}{c}{$\begin{array}{c}\text { Sheet No. } 10 \text { of } 19 \\
\text { Originators: }\end{array}$} \\
Chk'd By. Clark & Date \\
Calc. No. & Date \\
C100B-CA-V0301 & Rev. No. N 0
\end{tabular}




\section{ATTACHMENT 11}

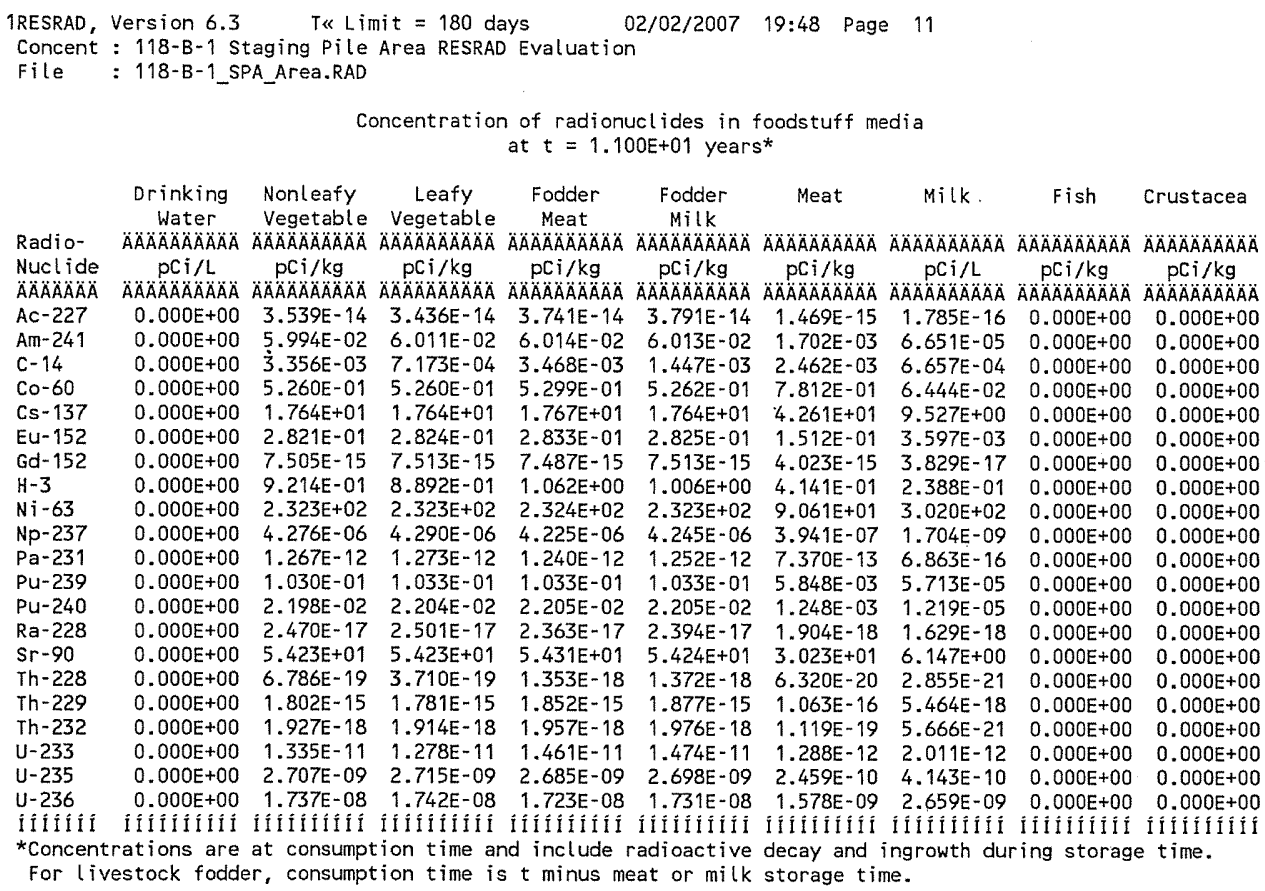

Concentrations in the media-occurring in pathways that are suppressed are calculated using the current input parameters, i.e. using parameters appearing in the input screen when the pathways are active.

\begin{tabular}{|c|c|}
\hline Attachment & Sheet No. 11 of 19 \\
\hline Originators: S. W. Clark & Date \\
\hline Chk'd By M. W. Perrott & Date \\
\hline 0100B-CA-V0301 & Rev. No. 0 \\
\hline
\end{tabular}


CVP-2007-00006

Rev. 0

\section{ATTACHMENT 11}

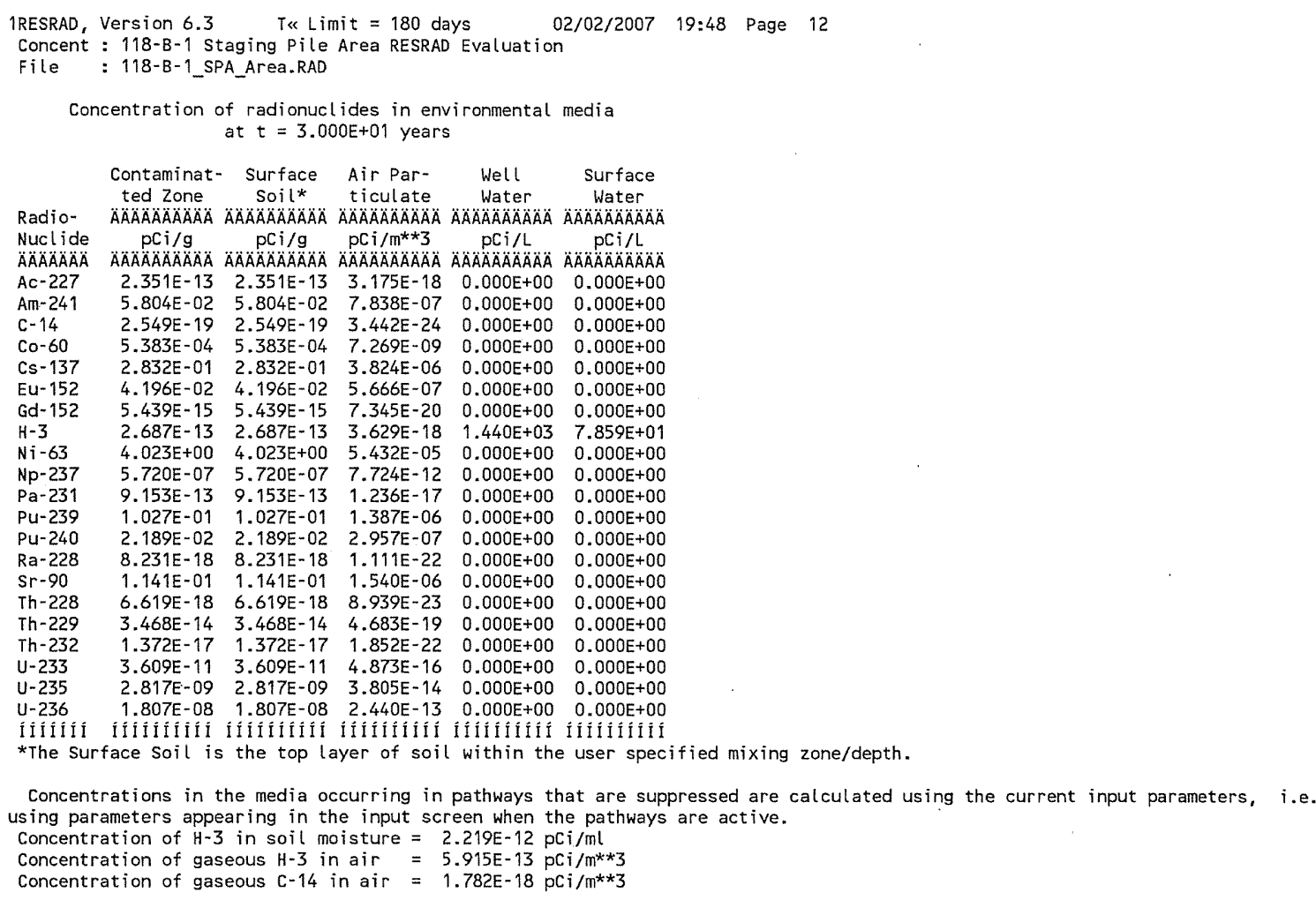

\begin{tabular}{|c|c|}
\hline Attachment & Sheet No. 12 of 19 \\
\hline Originators: S.W. Clark & Date \\
\hline Chk'd By M.W. Perrott & Date \\
\hline Calc. No. $0100 \mathrm{~B}-\mathrm{CA}-\mathrm{V} 0301$ & Rev. No. 0 \\
\hline
\end{tabular}


CVP-2007-00006

Rev. 0

\section{ATTACHMENT 11}

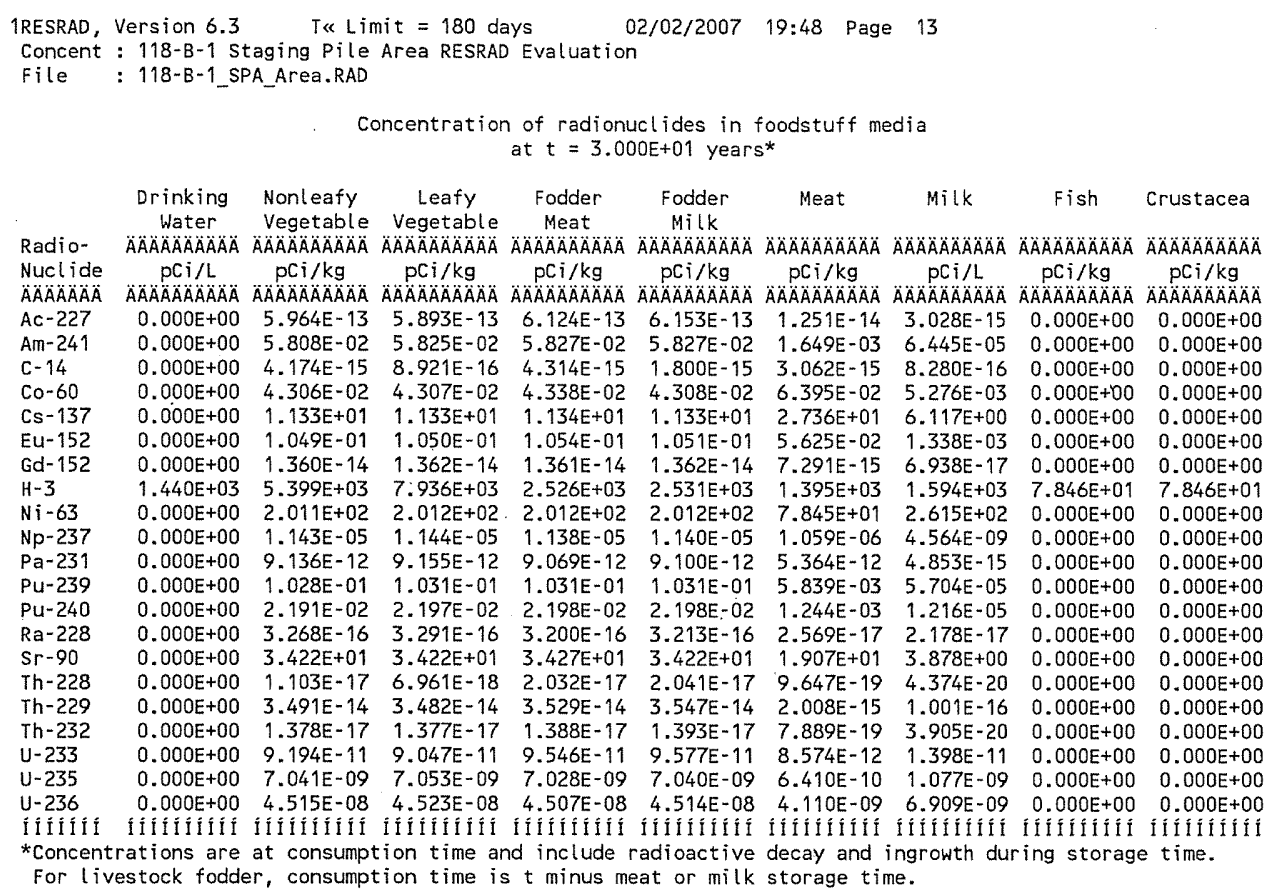

For livestock fodder, consumption time is $t$ minus meat or milk storage time.

Concentrations in the media occurring in pathways that are suppressed are calculated using the current input parameters, i.e. using parameters appearing in the input screen when the pathways are active.

\begin{tabular}{|c|c|c|}
\hline Attachment & 11 & Sheet No. \\
\hline Originators & S.W. Clark & Date \\
\hline Chk'd By & M. W. Perrott & Date \\
\hline Calc. No. & $0100 B-C A-V 0301$ & Rev. No. \\
\hline
\end{tabular}




\section{ATTACHMENT 11}

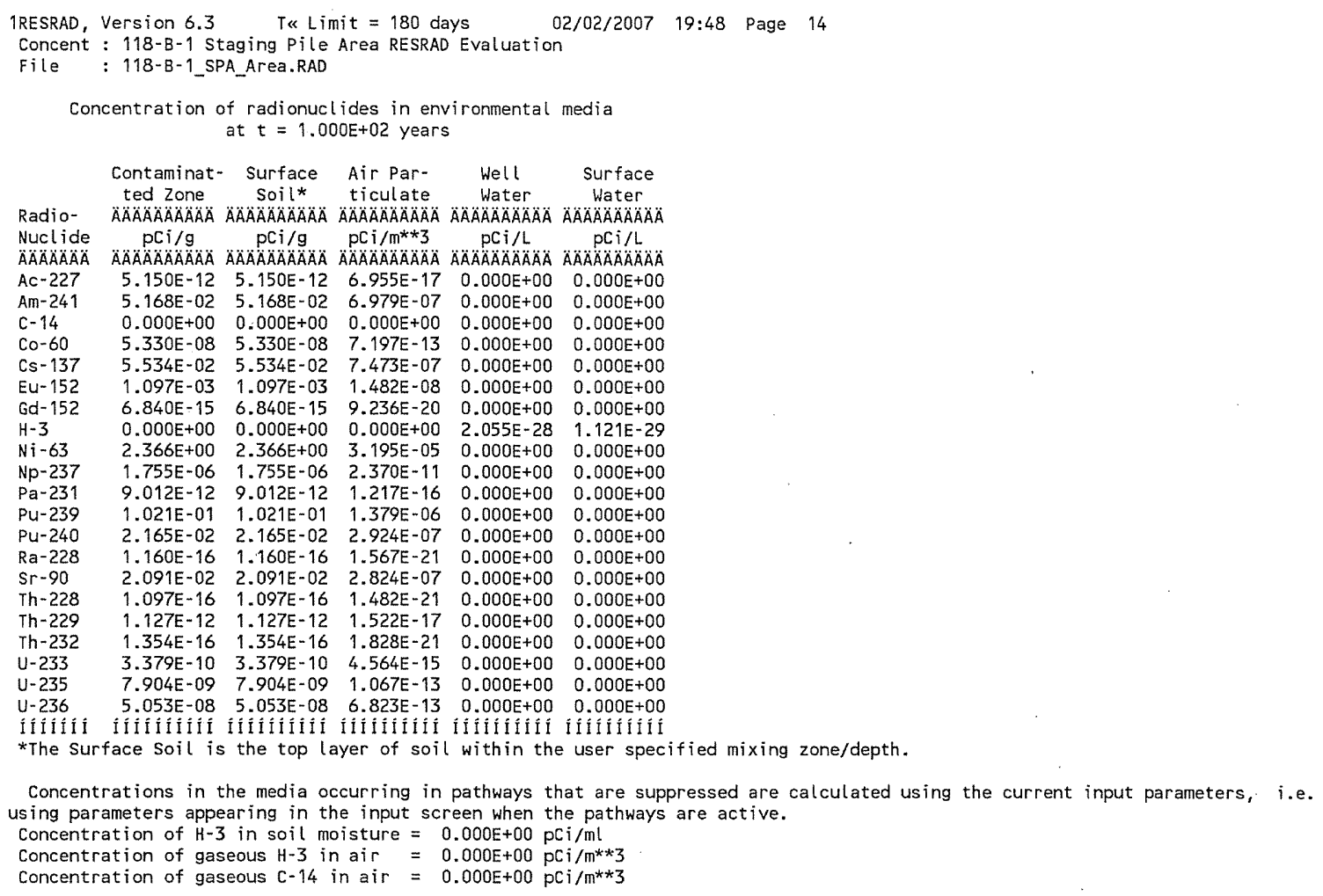

\begin{tabular}{|c|c|}
\hline Attachment & Sheet No. 14 of 19 \\
\hline Originators: S.W. Clark & Date \\
\hline M.W. Perrott & Date \\
\hline 0100B-CA-V0301 & Rev. No. 0 \\
\hline
\end{tabular}


CVP-2007-00006

Rev. 0

\section{ATTACHMENT 11}

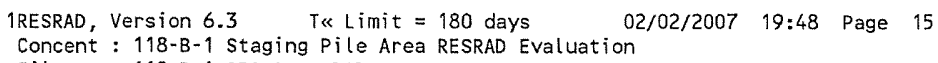

Concentrations in the media occurring in pathways that are suppressed are calculated using the current input parameters, i.e. using parameters appearing in the input screen when the pathways are active.

\begin{tabular}{|c|c|c|}
\hline \multirow{2}{*}{\multicolumn{3}{|c|}{ 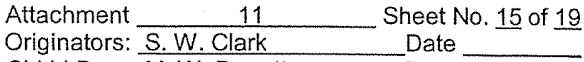 }} \\
\hline & & \\
\hline Chk'd By & M. W. Perrott & Date \\
\hline Calc. No. & $0100 B-C A-V 0301$ & Rev. No. \\
\hline
\end{tabular}


CVP-2007-00006

Rev. 0

\section{ATTACHMENT 11}

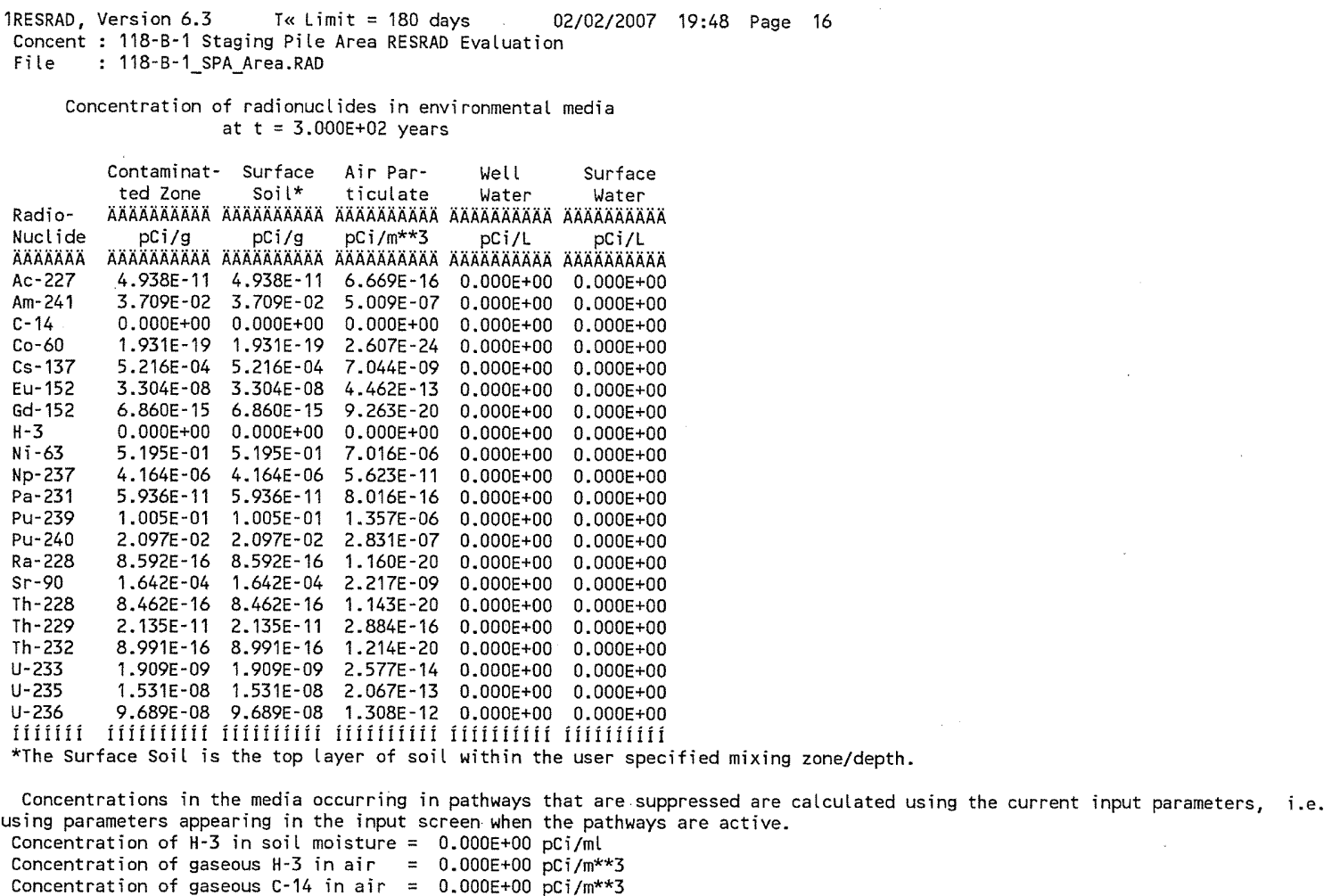

\begin{tabular}{|c|c|}
\hline Attachment & Sheet No. 16 of 19 \\
\hline Originators: S.W. Clark & Date \\
\hline Chk'd By M.W.Perrott & Date \\
\hline Calc. No. $0100 \mathrm{~B}-\mathrm{CA}-\mathrm{V} 0301$ & Rev. No. \\
\hline
\end{tabular}


CVP-2007-00006

Rev. 0

\section{ATTACHMENT 11}

\begin{tabular}{|c|c|c|c|c|c|c|c|c|c|}
\hline $\begin{array}{l}\text { 1RESRAD, } \\
\text { Concent } \\
\text { File }\end{array}$ & $\begin{array}{l}\text { Version } 6.3 \\
: 118-\mathrm{B}-1 \mathrm{St} \\
: 118-\mathrm{B}-1 \text { SP }\end{array}$ & $\begin{array}{l}\text { T«Lin } \\
\text { ttaging Pile } \\
\text { PA_Area.RAD }\end{array}$ & $\begin{array}{l}\text { mit }=180 \text { day } \\
\text { Area RESRAD }\end{array}$ & ays Evaluation & $\begin{array}{l}02 / 02 / 2007 \\
\text { on }\end{array}$ & $19: 48 \quad \mathrm{~Pa}$ & 17 & \multirow[b]{3}{*}{ Fish } & \multirow[b]{3}{*}{ Crustacea } \\
\hline & \multicolumn{7}{|c|}{$\begin{array}{c}\text { Concentration of radionuclides in foodstuff media } \\
\text { at } t=3.000 E+02 \text { years* }\end{array}$} & & \\
\hline & $\begin{array}{l}\text { Drinking } \\
\text { Water }\end{array}$ & $\begin{array}{l}\text { Nonleafy } \\
\text { Vegetable }\end{array}$ & $\begin{array}{l}\text { Leafy } \\
\text { Vegetable }\end{array}$ & $\begin{array}{l}\text { Fodder } \\
\text { Meat }\end{array}$ & $\begin{array}{l}\text { Fodder } \\
\text { Milk }\end{array}$ & Meat & Milk & & \\
\hline Radio- & $\triangle A \cap A \cap A \cap A \cap A \cap A B$ & 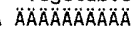 & 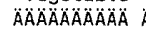 & $\triangle \triangle A A B A A A B A \cap A$ & 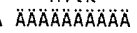 & 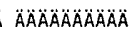 & ÄÄÄĂĀAÄÄ & ÄĀÄÄAAAÄA & $\triangle \triangle A A A A A A A A B$ \\
\hline Nucl ide & $\mathrm{pCi} / \mathrm{L}$ & $\mathrm{pCi} / \mathrm{kg}$ & $\mathrm{pCi} / \mathrm{kg}$ & $\mathrm{pCi} / \mathrm{kg}$ & $\mathrm{pCi} / \mathrm{kg}$ & $\mathrm{pCi} / \mathrm{kg}$ & $\mathrm{pCi} / \mathrm{L}$ & $\mathrm{pCi} / \mathrm{kg}$ & $\mathrm{pCi} / \mathrm{kg}$ \\
\hline$\triangle A O A A A B A ̈$ & $\triangle A A A A A A A O A A A$ & 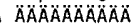 & $\triangle A A A A A A O A A O A O A$ & $\triangle A A A A A A A A A O A$ & 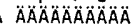 & 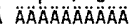 & $\triangle A A A A A B A O A B A$ & AAAAAAAAAAAAOA & $\triangle A A A A A A A A A A B A$ \\
\hline$A C-227$ & $0.000 \mathrm{E}+00$ & $1.240 E-10$ & $1.237 E-10$ & $1.254 E-10$ & $1.254 \mathrm{E}-10$ & $1.273 E-12$ & $6.317 E-13$ & $0.000 E+00$ & $0.000 E+00$ \\
\hline$A m-24$ & $0.000 \mathrm{E}+00$ & $3.712 E-02$ & $3.723 E-02$ & $3.724 \mathrm{E}-02$ & $3.724 \mathrm{E}-02$ & ? $1.054 \mathrm{E}-03$ & 4.119E-05 & $0.000 E+00$ & $0.000 E+00$ \\
\hline$c-14$ & $0.000 E+00$ & $0.000 E+00$ & $0.000 E+00$ & $0.000 E+00$ & $0.000 E+00$ & $0.000 E+00$ & $0.000 E+00$ & $0.000 E+00$ & $0.000 E+00$ \\
\hline Co- 60 & $0.000 E+00$ & $1.545 E-17$ & $1.545 \mathrm{E}-17$ & $1.556 \mathrm{E}-17$ & $1.545 \mathrm{E}-17$ & $2.294 \mathrm{E}-17$ & $1.892 \mathrm{E}-18$ & $0.000 E+00$ & $0.000 E+00$ \\
\hline$C s-13$ & $0.000 E+00$ & $2.087 \mathrm{E}-02$ & $2.087 \mathrm{E}-02$ & $2.089 E-02$ & $2.087 E-02$ & ? $5.039 \mathrm{E}-02$ & $1.127 E-02$ & $0.000 E+00$ & $0.000 E+00$ \\
\hline Eu- & $0.000 E+00$ & $8.263 E-08$ & $8.273 E-08$ & -08 & 8.2 & $3 \quad 4.430 E-08$ & $1.054 \mathrm{E}-09$ & $0.000 \mathrm{E}+00$ & $0.000 \mathrm{E}+00$ \\
\hline Gd- & 0.00 & $1.715 E-14$ & $1.717 E-14$ & $1.718 E-14$ & $1.718 \mathrm{E}-14$ & $9.195 \mathrm{E}-15$ & $8.749 \mathrm{E}-17$ & $0.000 E+00$ & $10 E+00$ \\
\hline $\mathrm{H}-3$ & +00 & $0.000 E+00$ & $0.000 E+00$ & $0.000 E+00$ & $0.000 E+00$ & $0.000 E+00$ & $0.000 E+00$ & $0.000 E+00$ & $0.000 E+00$ \\
\hline $\mathrm{Ni}-6$ & $0.000 E+00$ & $2.598 E+01$ & $2.598 \mathrm{E}+01$ & & 2.5 & $1.013 \mathrm{E}+01$ & $3.377 \mathrm{E}+01$ & $0.000 \mathrm{E}+00$ & $O O E+00$ \\
\hline$N p-23$ & $0.000 E+00$ & $8.327 E-05$ & -05 & 8.3 & 8.3 & $7.743 \mathrm{E}-06$ & $E-08$ & $D E+00$ & $\mathrm{DE}+00$ \\
\hline $\mathrm{Pa}-$ & $0.000 E+00$ & $5.935 E-10$ & $5.938 E-10$ & $5.934 E-10$ & $5.935 E-10$ & $3.501 E-10$ & $3.119 E-13$ & $0.000 E+00$ & $0.000 E+00$ \\
\hline Pu- & 0.00 & $1.005 E-01$ & 1.0 & -01 & 1.00 & 1) $5.709 E-03$ & $5.578 \mathrm{E}-05$ & $0.000 \mathrm{E}+00$ & $30 E+00$ \\
\hline Pu-24 & $0.000 E+00$ & 2.09 & 2.10 & 2.1 & 2.1 & 1.19 & $=-05$ & $E+00$ & $O E+0 O$ \\
\hline $\mathrm{Ra}-2$ & $0.000 E+00$ & $3.421 E-14$ & $3.436 E-14$ & $3.385 E-14$ & $3.386 \mathrm{E}-14$ & $+2.713 \mathrm{E}-15$ & $2.291 \mathrm{E}-15$ & $0.000 E+00$ & $0.000 E+00$ \\
\hline $\mathrm{Sr}-90$ & $0.000 E+00$ & $4.926 \mathrm{E}-02$ & $4.926 \mathrm{E}-02$ & $4.932 \mathrm{E}-02$ & $4.926 \mathrm{E}-02$ & $2.745 \mathrm{E}-02$ & $5.583 \mathrm{E}-03$ & $0.000 E+00$ & $0.000 E+00$ \\
\hline Th-22 & $0.000 E+00$ & $1.308 E-15$ & $8.824 E-16$ & $2.300 E-15$ & 2.30 & $51.103 E-16$ & $5.017 E-18$ & $0.000 E+00$ & $0.000 E+00$ \\
\hline Th- & $0.000 E+00$ & 2. $138 \mathrm{E}-11$ & $2.143 E-11$ & $2.146 \mathrm{E}-11$ & $2.147 \mathrm{E}-11$ & $1.215 E-12$ & $5.948 \mathrm{E}-14$ & $0.000 E+00$ & $0.000 E+00$ \\
\hline Th-232 & $0.000 E+00$ & $9.000 E-16$ & $9.023 E-16$ & $9.032 E-16$ & $9.034 E-16$ & $5 \quad 5.114 E-17$ & $2.501 E-18$ & $0.000 E+00$ & $0.000 E+00$ \\
\hline$U-233$ & $0.000 E+00$ & 4.786E-09 & $4.779 E-09$ & 4.820E-09 & $4.821 E-09$ & $4.377 \mathrm{E}-10$ & $7.317 E-10$ & $0.000 E+00$ & $0.000 E+00$ \\
\hline$U-235$ & $0.000 E+00$ & $3.829 E-08$ & $3.833 E-08$ & $3.834 \mathrm{E}-08$ & $3 \quad 3.834 \mathrm{E}-08$ & $3 \quad 3.489 E-09$ & $5.858 \mathrm{E}-09$ & $0.000 \mathrm{E}+00$ & $0.000 \mathrm{E}+00$ \\
\hline & $0.000 E+00$ & $2.423 \mathrm{E}-07$ & $2.426 \mathrm{E}-07$ & $2.426 E-07$ & $2.426 \mathrm{E}-07$ & $2.208 E-08$ & $3.707 \mathrm{E}-08$ & $0.000 \mathrm{E}+00$ & $0.000 E+00$ \\
\hline & & & & & & & & & î́i îi \\
\hline & 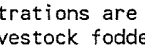 & & & & & & & & \\
\hline
\end{tabular}

Concentrations in the media occurring in pathways that are suppressed are calculated using the current input parameters, i.e. using parameters appearing in the input screen when the pathways are active.

\begin{tabular}{|c|c|}
\hline Attachment & Sheet No. 17 of 19 \\
\hline Originators: S.W. Clark & Date \\
\hline Chk'd By M.W. Perrott & Date \\
\hline Calc. No. $0100 \mathrm{~B}-\mathrm{CA}-\mathrm{V} 0301$ & Rev. No. \\
\hline
\end{tabular}


CVP-2007-00006

Rev. 0

\section{ATTACHMENT 11}

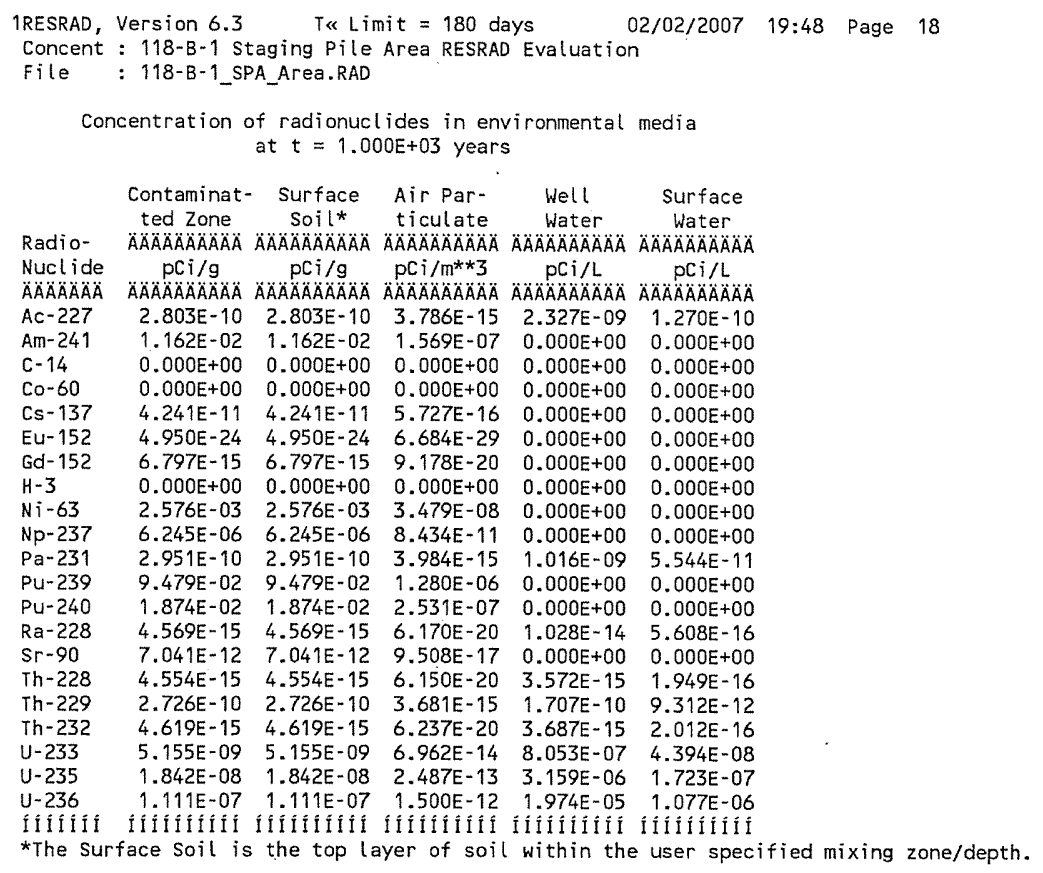

Concentrations in the media occurring in pathways that are suppressed are calculated using the current input parameters, $i . e$. using parameters appearing in the input screen when the pathways are active.

Concentration of $\mathrm{H}-3$ in soil moisture $=0.000 \mathrm{E}+00 \mathrm{pCi} / \mathrm{mL}$

Concentration of gaseous $\mathrm{H}-3$ in air $=0.000 \mathrm{E}+00 \mathrm{pCi} / \mathrm{m}^{* * 3}$

Concentration of gaseous $\mathrm{C}-14$ in air $=0.000 \mathrm{E}+00 \mathrm{pCi} / \mathrm{m}^{2 * 3}$

\begin{tabular}{|c|c|c|}
\hline Attachment & 11 & Sheet No. 18 of 19 \\
\hline Originators: & S. W. Clark & Date \\
\hline Chk'd By & M. W. Perrott & Date \\
\hline Calc. No. & $0100 B-C A-V 0301$ & Rev. No. 0 \\
\hline
\end{tabular}




\section{ATTACHMENT 11}

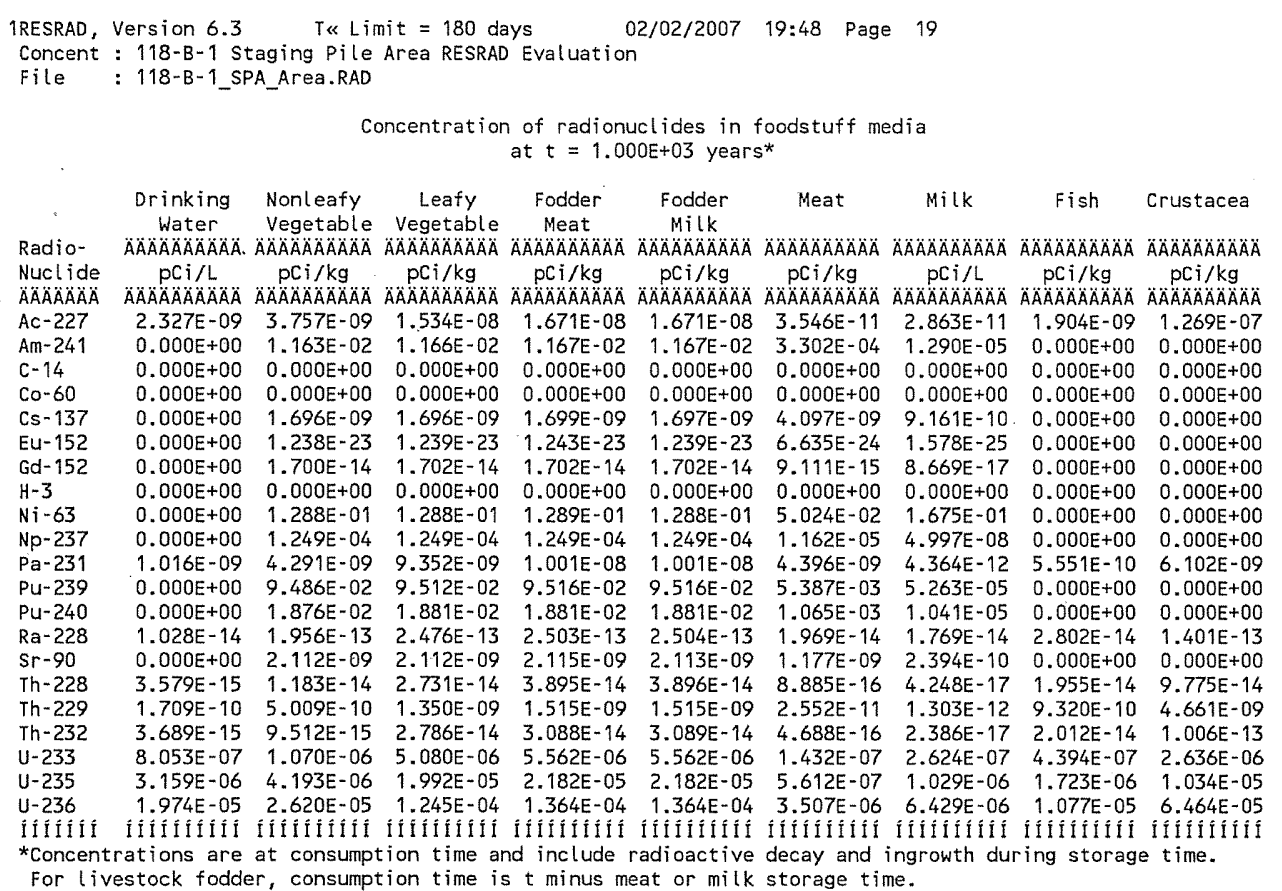

For livestock fodder, consumption time is $t$ minus meat or milk storage time.

Concentrations in the media occurring in pathways that are suppressed are calculated using the current input parameters, i.e. using parameters appearing in the input screen when the pathways are active.

\begin{tabular}{|c|c|c|}
\hline \multirow{2}{*}{\multicolumn{3}{|c|}{$\begin{array}{ll}\text { Attachment } & 11 \\
\text { Originators: S.W. Slark } & \text { Sheet No. } 19 \text { of } 19 \\
\text { Date }\end{array}$}} \\
\hline & & \\
\hline Chk'd By & M. W. Perrott & Date \\
\hline Calc. No. & 0100B-CA-V0301 & Rev. No. \\
\hline
\end{tabular}


CVP-2007-00006

Rev. 0

Acrobat 8.0

\section{CALCULATION COVER SHEET}

Project Title: Field Remediation

Job No. 14655

Area: $\quad 100-\mathrm{BC}$

Discipline: Environmental

${ }^{*}$ Calculation No: 0100B-CA-V0308

Subject: $\quad$ 118-B-1 Combined Areas Cleanup Verification RESRAD Calculation Brief

Computer Program: RESRAD

Program No: Version 6.3

The attached calculations have been generated to document compliance with established cleanup levels. These calculations should be used in conjunction with other relevant documents in the administrative record.

Committed Calculation $\bigotimes$

Preliminary $\square$

Superseded $\square$

Voided

\begin{tabular}{|c|c|c|c|c|c|c|}
\hline Rev. & Sheet Numbers & Originator & Checker & Reviewer & Approval & Date \\
\hline 0 & $\begin{array}{l}\text { Cover }-1 \mathrm{pg} \\
\text { Summary }-5 \mathrm{pg} \\
\text { Attm. } 1-1 \mathrm{pg} \\
\text { Attm. } 2-1 \mathrm{pg} \\
\text { Attm. } 3-20 \mathrm{pg} \\
\text { Attm. } 4-21 \mathrm{pg} \\
\text { Attm. } 5-10 \mathrm{pg} \\
\text { Total }-59 \text { pages }\end{array}$ & $\begin{array}{l}\text { Xu el en } \\
\text { S. W. Clark }\end{array}$ & $\begin{array}{l}\text { We Peetf } \\
\text { M. W. Perrott }\end{array}$ & N/A & J.M. Capron & $10 / 6 / 07$ \\
\hline & & & & & & \\
\hline & & & & & & \\
\hline & & & & & & \\
\hline
\end{tabular}

SUMMARY OF REVISION

\begin{tabular}{|l|l|}
\hline & \\
\hline & \\
\hline & \\
& \\
\hline
\end{tabular}

WCH-DE-018 (05/08/2007)

*Obtain Calc. No. from Document Control and Form from Intranet 
CVP-2007-00006

Rev. 0

Washington Closure Hanford

CALCULATION SHEET

\begin{tabular}{|c|c|c|c|c|c|c|c|}
\hline igin & S. W. Clark & Date: & $10 / 3 / 67$ & Calc. No.: & $0100 \mathrm{~B}-\mathrm{CA}$ & Rev.: & 0 \\
\hline & 118-B-1 Burial Ground Cleanup & Job No: & $14655^{\circ}$ & Checked: & M. W. Perrott $M \mathbb{N}$ & Date: & $\sigma 7$ \\
\hline & \multicolumn{5}{|c|}{ 118-B-1 Burial Ground Cleanup Verification RESRAD Calculation Brief } & \multicolumn{2}{|c|}{ Sheet No. 1 of 5} \\
\hline
\end{tabular}

\section{PURPOSE:}

Calculate the soil and groundwater concentrations, dose, and risk contributions from remaining radionuclide contaminants in the combined remediated 118-B-1 Burial Ground areas over a period of 1,000 years.

\section{GIVEN/REFERENCES:}

1) Cleanup verification data from the 118-B-1 Burial Ground Cleanup Verification $95 \%$ UCL Calculation, Calculation No. 0100B-CA-V0299, Rev. 0, Washington Closure Hanford, Richland, Washington.

2) Remedial Design Report/Remedial Action Work Plan for the 100 Area (RDR/RAWP), DOE/RL-96-17, Rev. 5, U.S. Department of Energy, Richland Operations Office, Richland, Washington.

3) Radioactive and nonradioactive contaminants of concern from the 100 Area Burial Grounds Remedial Action Sampling and Analysis Plan, DOE/RL-2001-35, Rev. 0, U.S. Department of Energy, Richland Operations Office, Richland, Washington and from the Site Specific Instruction for Closeout Approach for 118-B-1, SSI-100-BC-09, CCN 130128, Rev. 0, Washington Closure Hanford, Richland, Washington. For the purpose of these RESRAD calculations, the radioactive contaminants of potential concern (COPCs) are americium-241, carbon-14, cesium-137, cobalt-60, europium-152, europium-154, europium-155, nickel-63, plutonium-238, plutonium-239/240, plutonium-241, silver- $108 \mathrm{~m}$, strontium- 90 , tritium (H-3), uranium-233/234, uranium-235, and uranium-238. The nonradionuclide COPCs include cadmium, hexavalent chromium, lead, mercury, PCBs, SVOAs, TPH, VOAs, herbicides and pesticides. The full suite of detected nonradionuclide COPCs are identified in the 118-B-1 95\% UCL Calculation; attainment of nonradionuclide RAGs will be discussed in the Cleanup Verification Package (CVP).

4) RESidual RADioactivity (RESRAD) computer code, version 6.3, to calculate compliance with residual radioactivity guidelines, developed for the U.S. Department of Energy by the Environmental Assessment Division of Argonne National Laboratory, Argonne, Illinois.

5) RESRAD evaluation of 118-B-1 overburden (OB) soil, below cleanup level (BCL) soil, and soil beneath staging pile areas (SPA) was previously performed in the $118-B-1$ Burial Ground Cleanup Verification RESRAD Calculation Brieffor Overburden, Below Cleanup Level (BCL), and Staging Pile Area Soil, Calculation No. 0100B-CA-V0301, Rev. 0, Washington Closure Hanford, Richland, Washington.

6) Sample design data from the 118-B-1 Shallow Zone, Overburden, BCL, and Staging Pile Sampling Plan, Calculation No. 0100B-CA-V0284, Rev. 0, Washington Closure Hanford, Richland, Washington.

7) Groundwater elevation from Hanford Groundwater Monitoring for Fiscal Year 2006, PNNL-16346, Pacific Northwest National Laboratory, Richland, Washington.

\section{SOLUTION:}

1) Table 1 shows the representative dimensions of soil horizons and contaminant pathways considered for dose, risk, and groundwater protection. The average surrounding grade 


\begin{tabular}{|c|c|c|c|c|c|c|}
\hline Originator: & S. W. Clark & $10 / 3 / 07$ & Calc. No.: & $0100 \mathrm{~B}-\mathrm{CA}-\mathrm{V} 0308$ & Rev.: & 0 \\
\hline Project: & 118-B-1 Burial Ground Cleanup & Job No: 14655 & Checked: & M. W. Perrott $7 / D$ & Date: & $10 / 3 / 07$ \\
\hline Subject: & \multicolumn{4}{|c|}{ 118-B-1 Burial Ground Cleanup Verification RESRAD Calculation Brief } & \multicolumn{2}{|c|}{ Sheet No. 2 of 5} \\
\hline
\end{tabular}

1 elevation and the maximum excavation depth are used to represent the burial ground excavation. Attachment 1 shows elevations (NAVD88) and thickness of each soil horizon. Attachment 2 shows a schematic of the 118-B-1 Burial Ground area with the pieces making up the individual units labeled.

2) Separate cleanup verification sampling was performed for each of the 118-B-1 Burial Ground Areas 1, 2, 3, 4, 5, 6, and 7. The maximums of the statistical and focused sample values from the individual Burial Ground Areas, shown in Table 2, were used to perform a RESRAD run for the combined areas. This is more conservative than performing RESRAD evaluations for the individual areas, so RESRAD evaluations for the individual areas were not performed.

3) All sources of tritium in the 118-B-1 Burial Ground have been removed and disposed. Sampling of test pits and boreholes in the vadose zone beneath 118-B-1 shows residual tritium soil contamination. RESRAD modeling predicts that residual tritium will continue to exceed groundwater protection criteria for approximately 140 years. Measurements at a downgradient monitoring well have remained above the maximum contaminant level (MCL) for tritium in drinking water since 1998. An Explanation of Significant Difference for the Interim Action Record of Decision for the 100-BC-1, 100-BC-2, 100-DR-1, 100-DR-2, 100-FR-2, 100-HR-2, and 100-KR-2 Operable Units (100 Area Burial Grounds) (ESD, October 2007) is in the approval process based on the balancing factors evaluation, which showed that additional institutional controls will be required to ensure protection of groundwater and the Columbia River. In summary, institutional controls include prohibition of irrigation at 118-B-1 to minimize further mobilization of residual tritium-contamination to groundwater and the Columbia River. Statistical and focused sample concentrations of tritium reported in the 118-B-1 95\% UCL calculation were not evaluated for groundwater and river protection (see Table 2) because institutional controls will be implemented per the October 2007 ESD to allow attenuation of residual concentrations of tritium that exist in deep zone soil.

4) To be conservative, the shallow zone RESRAD evaluation for the 118-B-1 combined areas was performed assuming that residual contamination levels extend uniformly from the surface to groundwater. Carbon- 14 and strontium- 90 in the pathways affected by plant ingestion caused the total all pathways dose to be greater than $15 \mathrm{mrem} / \mathrm{yr}$. Because the 
CVP-2007-00006

Rev. 0

Washington Closure Hanford

CALCULATION SHEET

\begin{tabular}{|c|c|c|c|c|c|c|}
\hline Origine & DUts & Date: & Calc. No.: & 010 & Rev.: & U \\
\hline & 118-B-1 Burial Ground Cleanup & Job No: & Checked: & M. W. Perrott THCOP & Date: & $10 / 3 / 07$ \\
\hline & \multicolumn{6}{|c|}{ 118-B-1 Burial Ground Cleanup Verification RESRAD Calculation Brief } \\
\hline
\end{tabular}

1 elevated residual carbon-14 and strontium-90 concentrations are deeper than the root

2 penetration zone $(2.5 \mathrm{~m}[8 \mathrm{ft}])$, and not accessible to plants, the RESRAD plant ingestion

3 input parameters for "contaminated fractions" for the combined areas are set equal to zero.

4

5) The year where the peak dose (or concentration) occurs from each individual radionuclide $\mathrm{COC}$ and layer is determined by a preliminary run. This year is then added for all horizons for the final RESRAD runs. For the direct exposure pathways (i.e. soil ingestion, inhalation, and external radiation), the peak year occurred at year zero (year 2007) for all COCs. For the water pathways (i.e. drinking water and food ingestion) the peak year occurred at year 7 for cobalt-60 and at year 43 for cesium-137 and strontium-90. The 7 and 43 -year time periods were added to all RESRAD runs.

\section{METHODOLOGY:}

1) Runs of RESRAD version 6.3 were completed for the 118-B-1 Burial Ground combined areas using the radionuclide concentrations shown in Table 2. RESRAD numerical output reports for dose, risk, and concentration for the shallow zone evaluation are presented in the Attachments to this calculation summary.

19

\begin{tabular}{|c|c|c|c|c|c|c|c|c|c|}
\hline \multicolumn{10}{|c|}{ Table 2. Cleanup Verification Data Set ${ }^{a}$ (2 Pages) } \\
\hline COPCs & Area 1 & Area 2 & Area 3 & Area 4 & Area 5 & Area 6 & Area 7 & $\begin{array}{l}\text { Focused } \\
\text { Samples } \\
\end{array}$ & $\begin{array}{c}\text { Combined } \\
\text { Areas }\end{array}$ \\
\hline \multicolumn{10}{|c|}{ Radionuclide Activity $(\mathrm{pCi} / \mathrm{g}$ ) } \\
\hline Am-241 & -- & - & -- & - & -- & -- & $\ldots$ & $\ldots$ & - \\
\hline C-14 & 1.93 & - & -- & -- &.- & $\ldots$ & -- & - & 1.93 \\
\hline Cs- 137 & 0.201 & 0.163 & 0.776 & 0.345 & 2.40 & 0.150 & 0.517 & 0.353 & 0.776 \\
\hline Co-60 & 0.039 & - & -- & & -- & - & $-\cdots$ & 0.155 & 0.155 \\
\hline Eu-152 & 0.695 & - & - & - & 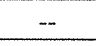 & - & 0.098 & $\cdots$ & 0.695 \\
\hline Eu-154 & - & $\cdots$ & - & $\ldots$ & -- & - & 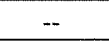 & $\cdots$ & - \\
\hline Eu-155 & -- & - & -. & $\ldots$ & - & -- & -- & - & - \\
\hline $\mathrm{Ni}-63$ & & - & - & & -- & & -- & - & - \\
\hline $\mathrm{Pu}-238$ & -- & - & - & - & - & - & -- & -- & -- \\
\hline $\mathrm{Pu}-239 / 240$ & -- & -- & - & $\cdots$ & $\ldots$ & - & - & - & - \\
\hline $\mathrm{Pu}-241$ & - & -- & - & - & - & -- & -- & -- & - \\
\hline Silver $(\mathrm{Ag}-108 \mathrm{~m})$ & - & - & - & - & - & - & -- & - & - \\
\hline Sr-90 & 0.125 & 0.172 & 2.56 & - & 1.89 & - & -- & -- & 2.56 \\
\hline Tritium $(\mathrm{H}-3)$ & \multicolumn{9}{|c|}{$\begin{array}{l}\text { Not evaluated for groundwater and river protection based on the balancing factors evaluation } \\
\text { documented in the October } 2007 \text { ESD, which showed that additional institutional controls will be } \\
\text { required to ensure protection of groundwater and the Columbia River. In summary, institutional } \\
\text { controls include prohibition of irrigation at } 118-\mathrm{B}-1 \text { to minimize further mobilization of residual } \\
\text { tritium-contamination to groundwater and the Columbia River. Additionally, the ESD states that } \\
\text { RESRAD modeling in the soil may be conducted without accounting for the irrigation rate of } \\
0.76 \mathrm{~m} / \mathrm{yr} \text { ( } 30 \mathrm{in} / \mathrm{yr} \text { ) for tritium at the } 118-\mathrm{B}-1 \text { waste site. }\end{array}$} \\
\hline $\mathrm{U}-233 / 234$ & $0(<B G)$ & - & - & -- & - & $0(<\mathrm{BG})$ & $0(<\mathrm{BG})$ & $0(<B G)$ & $0(<\mathrm{BG})$ \\
\hline
\end{tabular}


CVP-2007-00006

Rev. 0

Washington Closure Hanford

CALCULATION SHEET

\begin{tabular}{|c|c|c|c|c|c|c|}
\hline Ori & S. W. Clark & Dat & Calc. No & 0100 & Rev.: & 0 \\
\hline & 1 Ground Cleanup & Job No: & Checked: & M. W. Perrott Tha & Date: & $10 / 3 / 07$ \\
\hline Sul & \multicolumn{6}{|c|}{ 118-B-1 Burial Ground Cleanup Verification RESRAD Calculation Brief } \\
\hline
\end{tabular}

\begin{tabular}{|c|c|c|c|c|c|c|c|c|c|}
\hline \multicolumn{8}{|c|}{ Table 2. Cleanup Verification Data Set ${ }^{\text {a }}$ (2 Pages) } \\
\hline CoPCs & Area 1 & Area 2 & Area 3 & Area 4 & Area 5 & Area 6 & Area 7 & $\begin{array}{c}\text { Focused } \\
\text { Samples }\end{array}$ & $\begin{array}{c}\text { Combined } \\
\text { Areas }\end{array}$ \\
\hline $\mathrm{U}-235$ & $0(<\mathrm{BG})$ & -- & - & - & - & -- & - & - & $0(<\mathrm{BG})$ \\
\hline $\mathrm{U}-238$ & $0(<\mathrm{BG})$ & -- & -- & -- & -- & $0(<\mathrm{BG})$ & $0(<\mathrm{BG})$ & $0(<\mathrm{BG})$ & $0(<\mathrm{BG})$ \\
\hline
\end{tabular}

-- = Not evaluated by laboratory analysis or not detected (for all samples in the data set).

${ }^{a}$ Soil concentration values are from 118-B-1 Burial Ground Cleamup Verification 95\% UCL. Calculation, Calculation No. 0100B-CA-V0299, Rev. 0, Washington Closure Hanford, Richland, Washington. Refer to the $95 \%$ UCL for nonradionuclide concentrations. Nonradionuclides are not evaluated in this RESRAD calculation.

1

2

3

4

5 The maximum all pathways dose rate, shown in Table 3, for the 118-B-1 Burial Ground

6 combined areas is $13.0 \mathrm{mrem} / \mathrm{yr}$ at year zero (2007).

10 The maximum radionuclide excess lifetime cancer risk (ELCR), shown in Table 4, for the

11 118-B-1 Burial Ground combined areas is $1.38 \times 10^{-4}$ at year zero (2007).

12

13

14

15 The radionuclide concentrations in groundwater calculated by the RESRAD model are

16 summarized in Table 5. Among the radionuclide COCs, cesium-137, cobalt-60, and

17 strontium-90 are calculated to reach groundwater in the 1,000 years of the RESRAD model run.

18 All COCs are predicted to reach groundwater at concentrations significantly below their RAGs.

19

Table 5. Predicted Groundwater (Well Water/Drinking Water) Concentrations (2 pages)

\begin{tabular}{|c|c|c|c|c|c|c|c|c|c|c|c|}
\hline \multirow{2}{*}{$\begin{array}{l}\text { Radio- } \\
\text { nuclides }\end{array}$} & \multirow{2}{*}{$\begin{array}{c}\text { Vadose Zone } \\
\text { Horizon }\end{array}$} & \multicolumn{9}{|c|}{ Groundwater Concentrations in $\mathrm{pCi} / \mathrm{L}$ at Each Time Slice (yr) } & \multirow{2}{*}{$\begin{array}{l}\text { RAGs, } \\
\mathrm{pCi} / \mathrm{L}\end{array}$} \\
\hline & & 0 & 1 & 3 & 7 & 11 & 43 & 100 & 300 & 1000 & \\
\hline Cs- 137 & Comb. Areas & 0 & 7.52E-04 & $2.16 \mathrm{E}-03$ & 4.60E-03 & $6.58 \mathrm{E}-03$ & 1.23E-02 & $7.66 \mathrm{E}-03$ & $2.26 \mathrm{E}-04$ & 0 & 60 \\
\hline Co-60 & Comb. Areas & 0 & $1.35 \mathrm{E}-04$ & $3.12 \mathrm{E}-04$ & $4.30 \mathrm{E}-04$ & $3.99 \mathrm{E}-04$ & $2.32 \mathrm{E}-05$ & $3.00 \mathrm{E}-08$ & $3.40 \mathrm{E}-19$ & $7.11 \mathrm{E}-11$ & 100 \\
\hline Sr-90 & Comb. Areas & 0 & $9.85 \mathrm{E}-03$ & $2.82 \mathrm{E}-02$ & $6.00 \mathrm{E}-02$ & $8.56 \mathrm{E}-02$ & $1.56 \mathrm{E}-01$ & $9.35 \mathrm{E}-02$ & $2.40 \mathrm{E}-03$ & $3.03 \mathrm{E}-10$ & 8 \\
\hline
\end{tabular}

RAGs $=$ Remedial action goals from the 100 Area RDR/RAWP

20 
CVP-2007-00006

Rev. 0

Washington Closure Hanford

CALCULATION SHEET

\begin{tabular}{|c|c|c|c|c|c|c|}
\hline Oris & Dewe & $10 \%$ & No & $0100 \mathrm{~B}-\mathrm{C}$ & Rev.: & 0 \\
\hline & 118-B-1 Burial Ground Cleanup & Job No: 14655 & Checked: & M. W. Perrott IMle/f & Date: & $10 / 3107$ \\
\hline Subjec & \multicolumn{6}{|c|}{ 118-B-1 Burial Ground Cleanup Verification RESRAD Calculation Brief } \\
\hline
\end{tabular}

\section{CONCLUSIONS:}

- The "all pathways" (maximum) dose rates are shown in Table 3. The maximum all pathways dose rate from the 118-B-1 Burial Ground excavated combined areas is $13.0 \mathrm{mrem} / \mathrm{yr}$ at year zero (2007).

- The dominant pathway for the dose rate is direct external exposure (external gamma).

- The primary radionuclide contributing to the direct exposure pathway is europium- 152 .

- None of the site COCs that were evaluated are projected to exceed remedial action goals (RAGs).

- The radionuclide excess lifetime cancer risk (ELCR) results are shown in Table 4. The maximum ELCR from the 118-B-1 Burial Ground areas combined areas $1.38 \times 10^{-4}$ at year zero (2007).

- The radionuclide concentrations in groundwater calculated by the RESRAD model are summarized in Table 5. Among the radionuclide COCs, cesium-137, cobalt-60, and strontium-90 are calculated to reach groundwater in the 1,000 years of the RESRAD model evaluation. All COCs are predicted to reach groundwater at concentrations significantly below their RAGs.

\section{ATTACHMENTS:}

1. Graphic showing 118-B-1 Cleanup Verification Model (1 pages)

2. Schematic of the 118-B-1 Burial Ground Remediation Areas (1 page)

3. RESRAD Output: 118-B-1 Combined Areas Radionuclides - Mixture Sums and Single Radionuclide Guidelines (20 pages)

4. RESRAD Output: 118-B-1 Combined Areas Radionuclides - Intake Quantities and Health Risk Factors (21 pages)

5. RESRAD Output: 118-B-1 Combined Areas Radionuclides - Concentration of Radionuclides (10 pages) 


\section{ATTACHMENT 1}
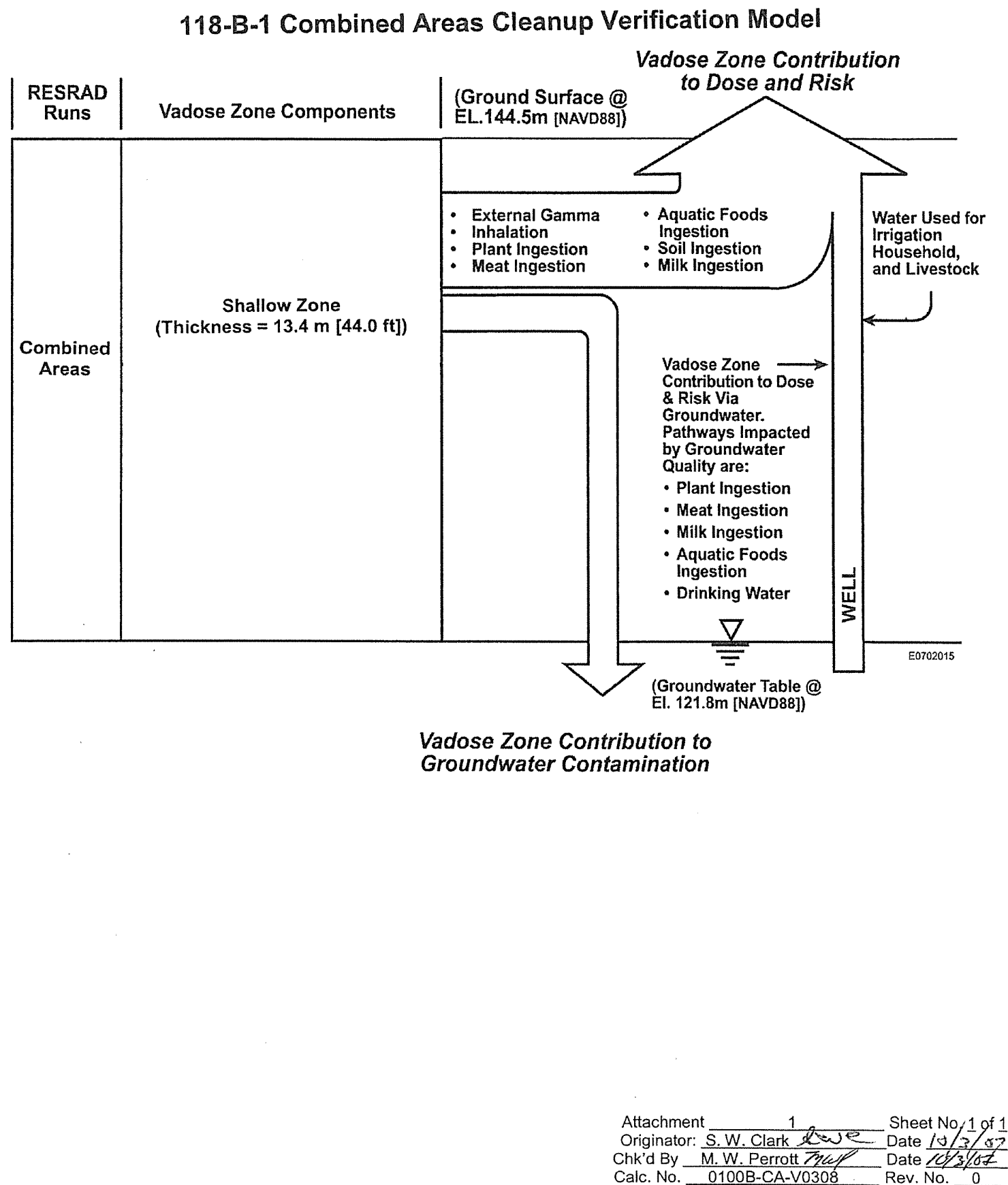
ATTACHMENT 2

Schematic of the 118-B-1 Burial Ground Remediation Areas Sampled for Cleanup Verification
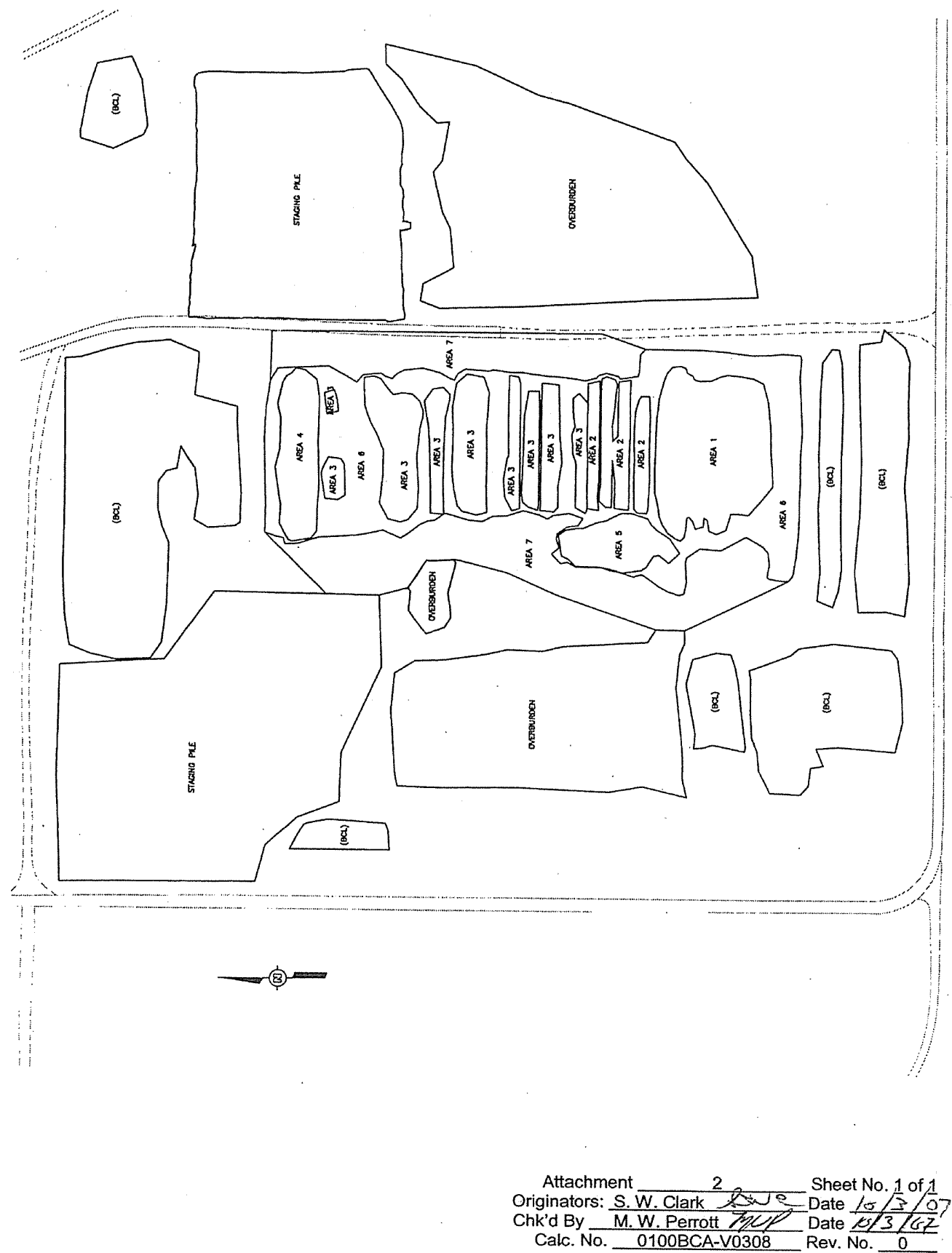


\section{ATTACHMENT 3}

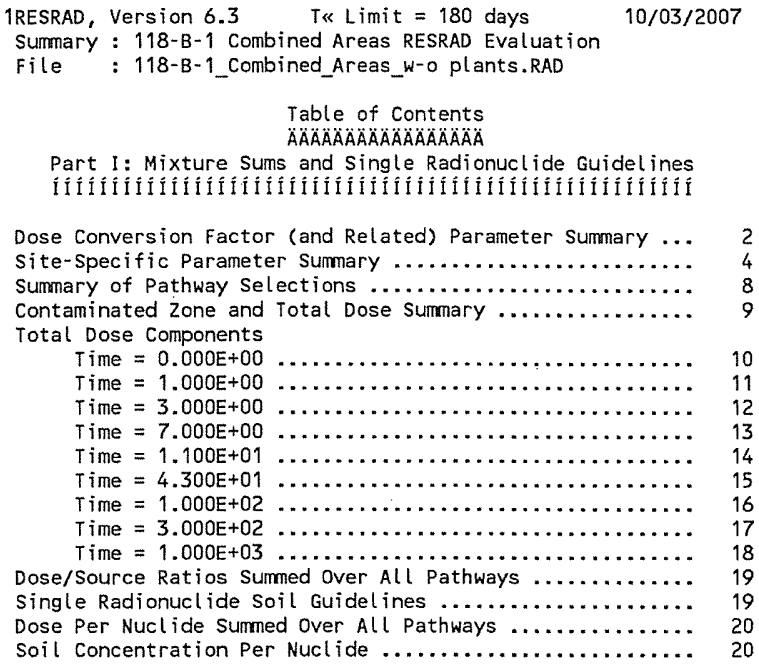


CVP-2007-00006

Rev. 0

\section{ATTACHMENT 3}

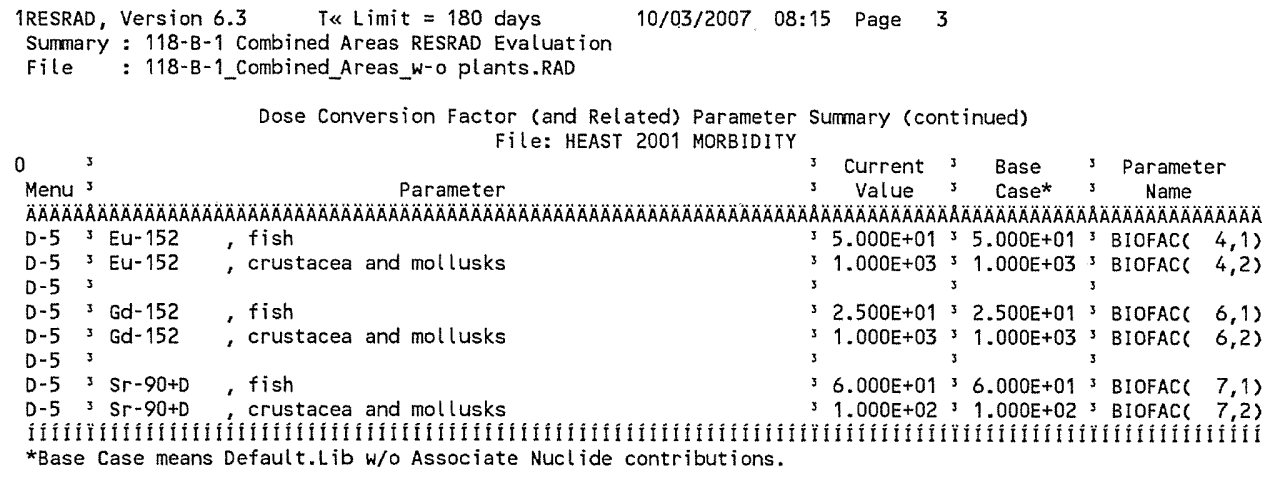




\section{ATTACHMENT 3}

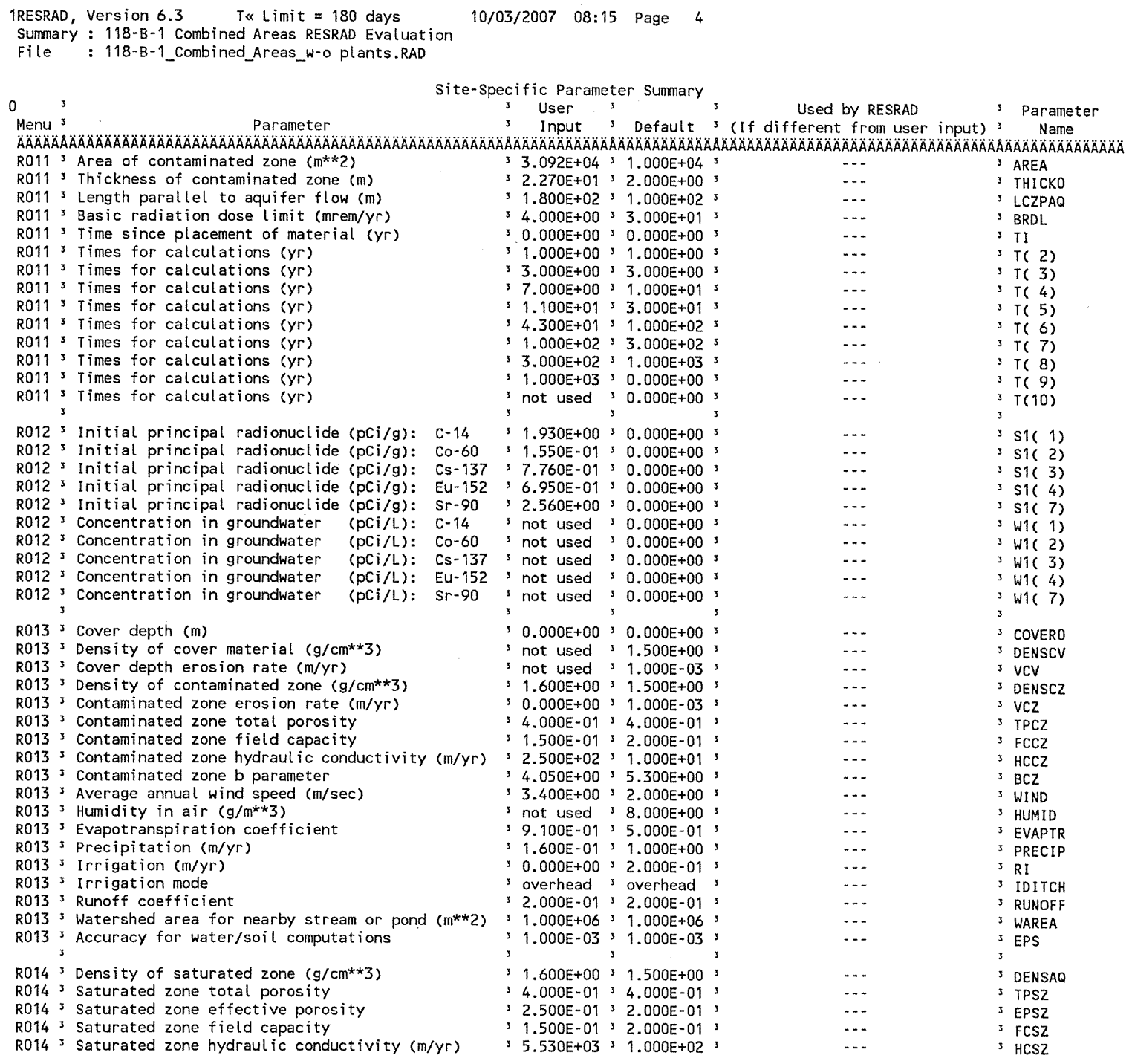


CVP-2007-00006

Rev. 0

\section{ATTACHMENT 3}

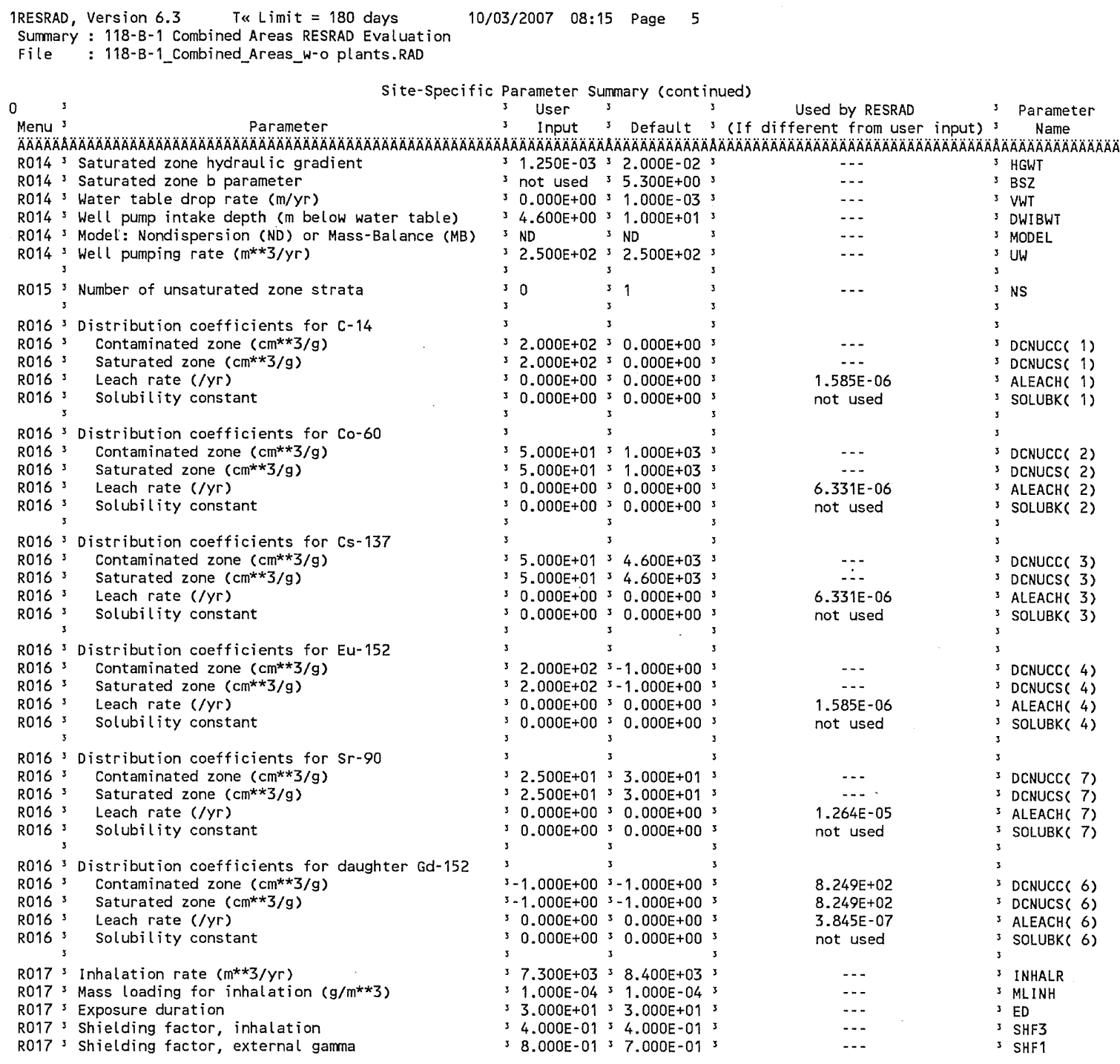

Attachment 
CVP-2007-00006

Rev. 0

\section{ATTACHMENT 3}

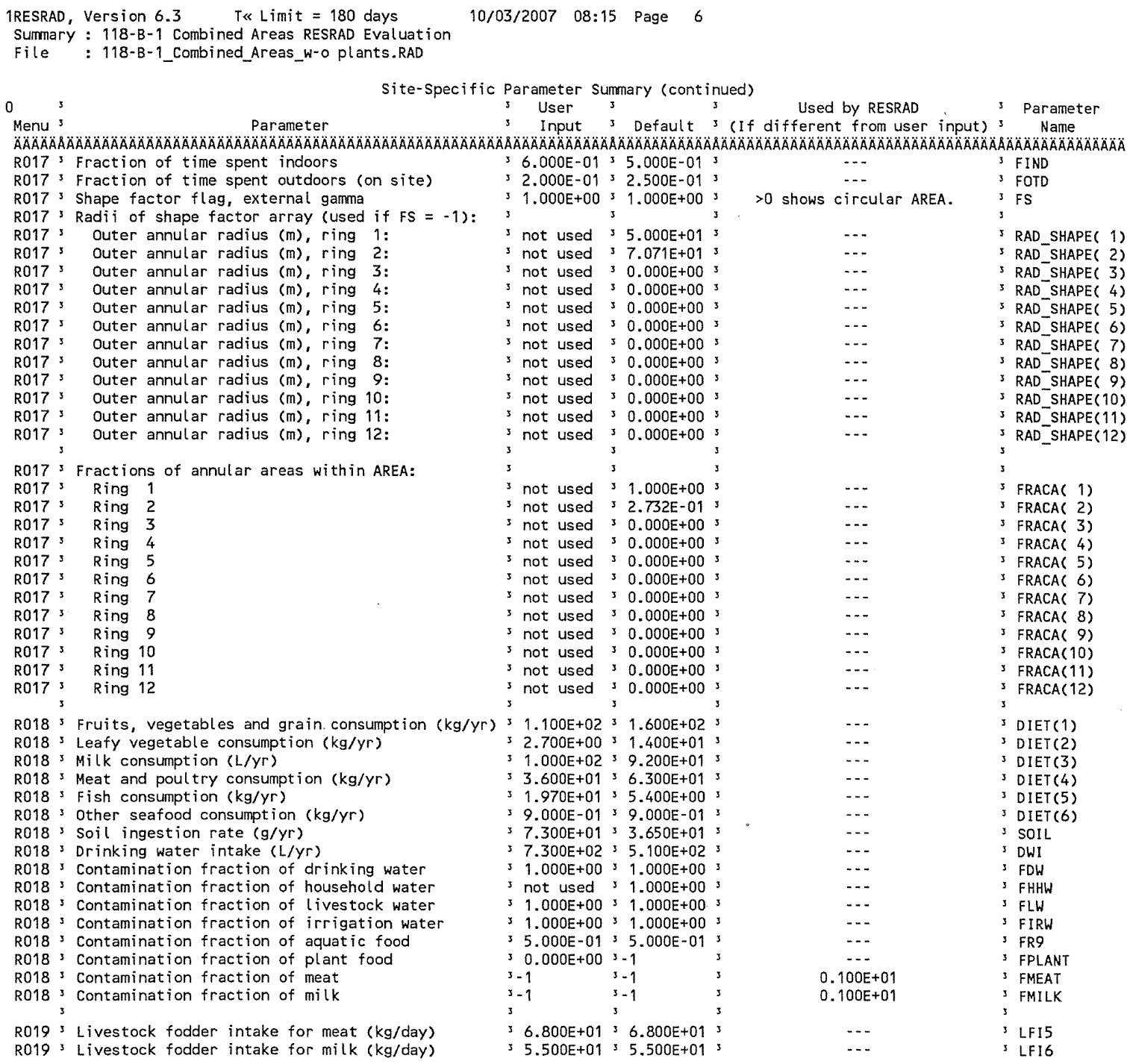

Attachment 
CVP-2007-00006

Rev. 0

\section{ATTACHMENT 3}

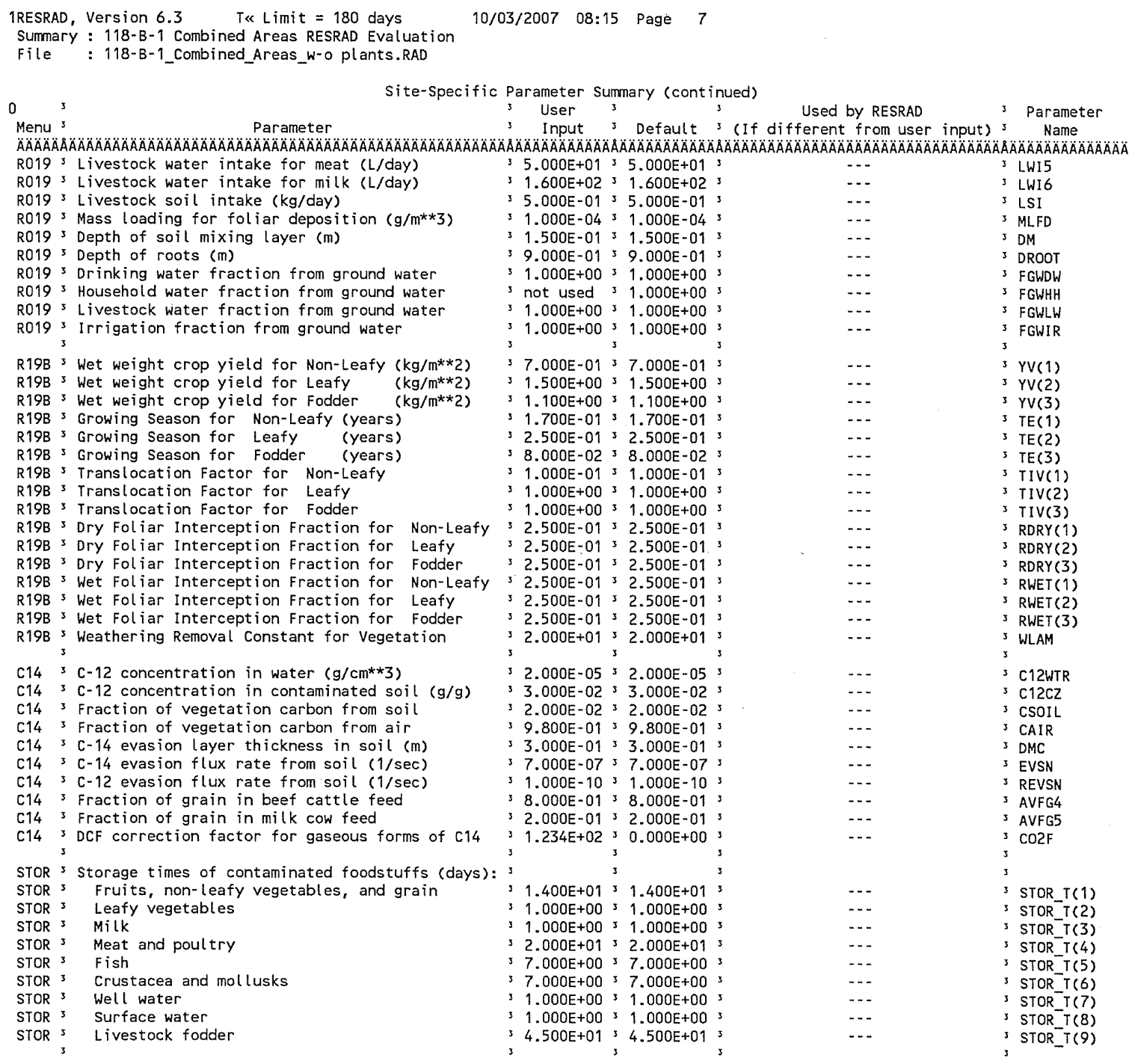

\begin{tabular}{|c|c|}
\hline chment & Sheet No. $\underline{7}$ of $\underline{2}$ \\
\hline Originator: $\mathrm{S} . \mathrm{W}$. Clark & Date \\
\hline M.W. Perrott & Date \\
\hline $0100 B-C A-V 0308$ & Rev. No. \\
\hline
\end{tabular}


CVP-2007-00006

Rev. 0

\section{ATTACHMENT 3}

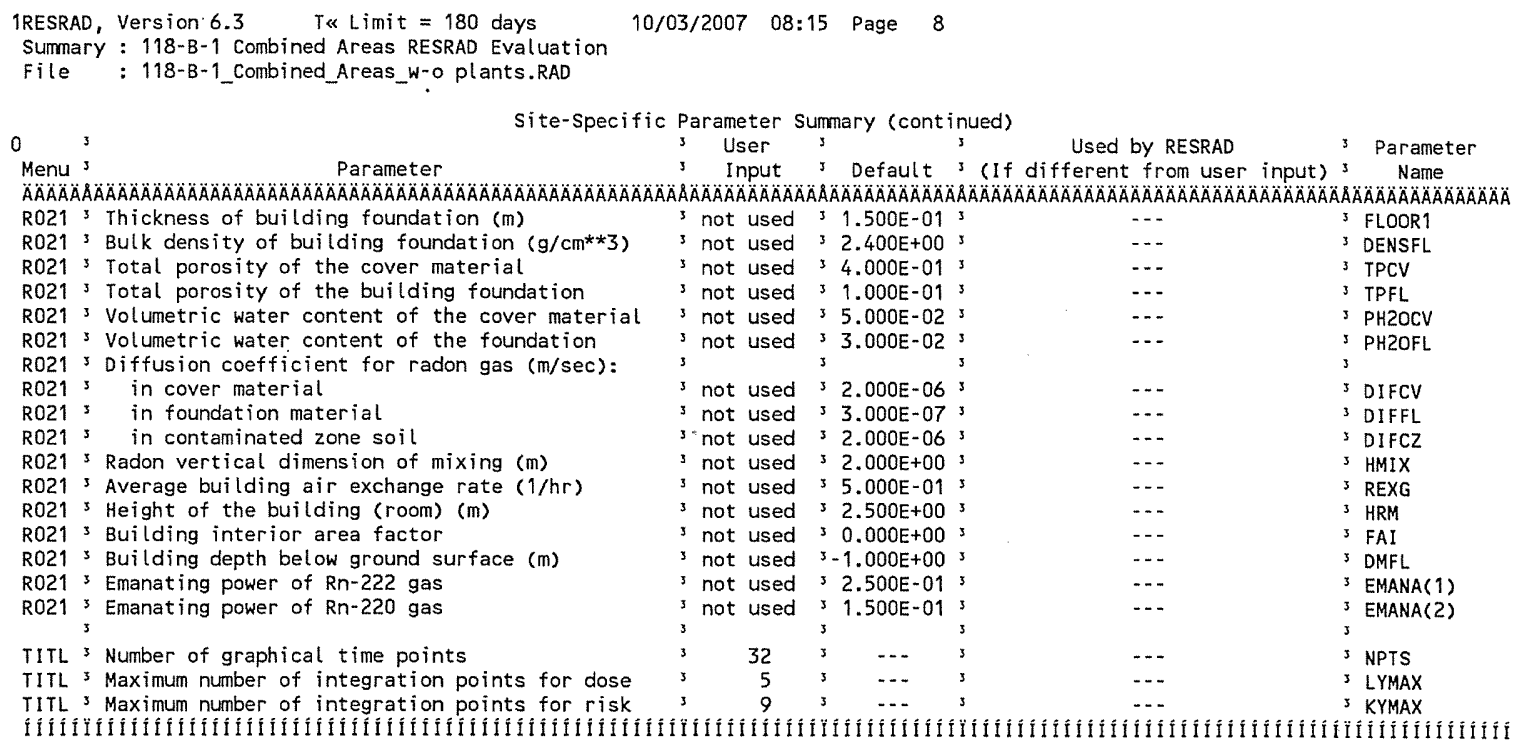

Summary of Pathway Selections

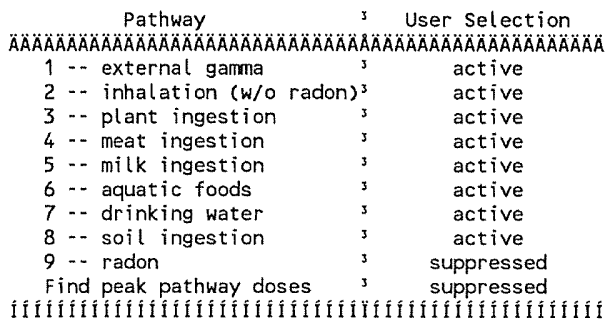

\begin{tabular}{ll} 
Attachment $\frac{3}{1}$ & Sheet No. $\underline{8}$ of $\underline{20}$ \\
Originator: S.W. Clark & Date \\
Chk'd By & Date \\
Calc. N. Perrott & Rev. No. 0 \\
\hline O100B-CA-V0308 & Rev No
\end{tabular}




\section{ATTACHMENT 3}

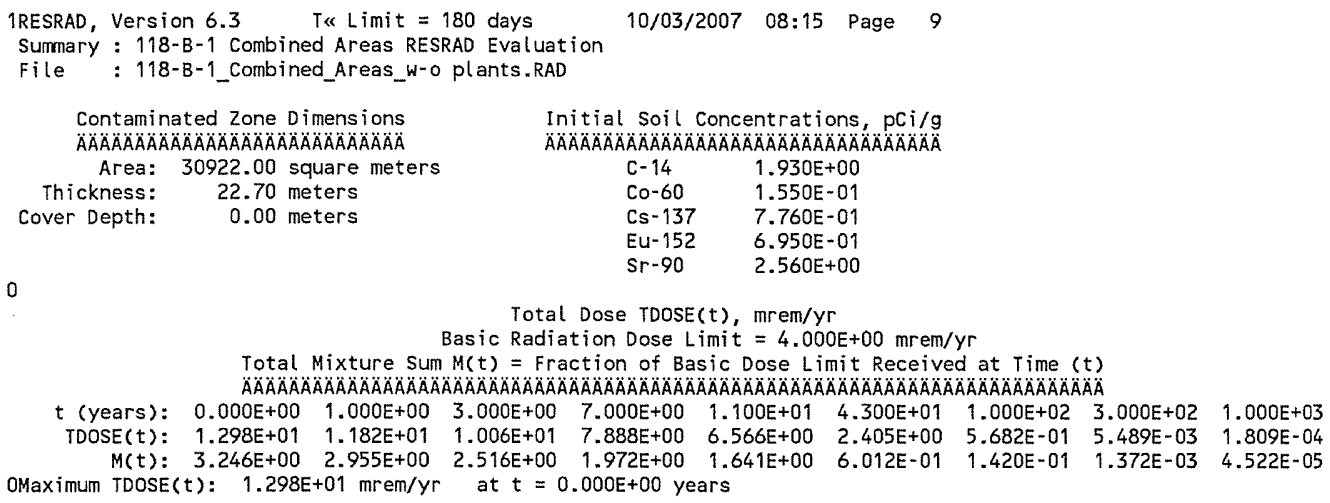

0

Total Dose TDOSE(t), mrem/yr

Basic Radiation Dose Limit $=4.000 \mathrm{E}+00 \mathrm{mrem} / \mathrm{yr}$

Total Mixture Sum $M(t)=$ Fraction of Basic Dose Limit Received at Time $(t)$

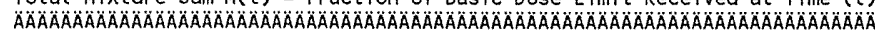

$t$ (years): $\quad \begin{array}{llllllllll}0.000 E+00 & 1.000 E+00 & 3.000 E+00 & 7.000 E+00 & 1.100 E+01 & 4.300 E+01 & 1.000 E+02 & 3.000 E+02 & 1.000 E+03\end{array}$

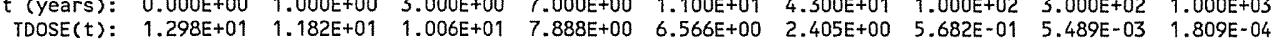

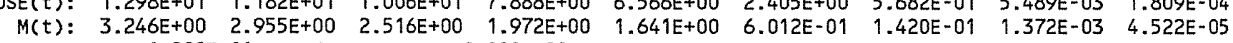
OMaximum TDOSE $(t): 1.298 E+01 \mathrm{mrem} / \mathrm{yr}$ at $t=0.000 E+00$ years

Attachment

3 Sheet No. 9 of $\underline{20}$ Originator: S.W. Clark Date

Chk'd By M.W. Perrott Date

Calc. No. 0100B-CA-V0308

Rev. No. 0 
CVP-2007-00006

Rev. 0

\section{ATTACHMENT 3}

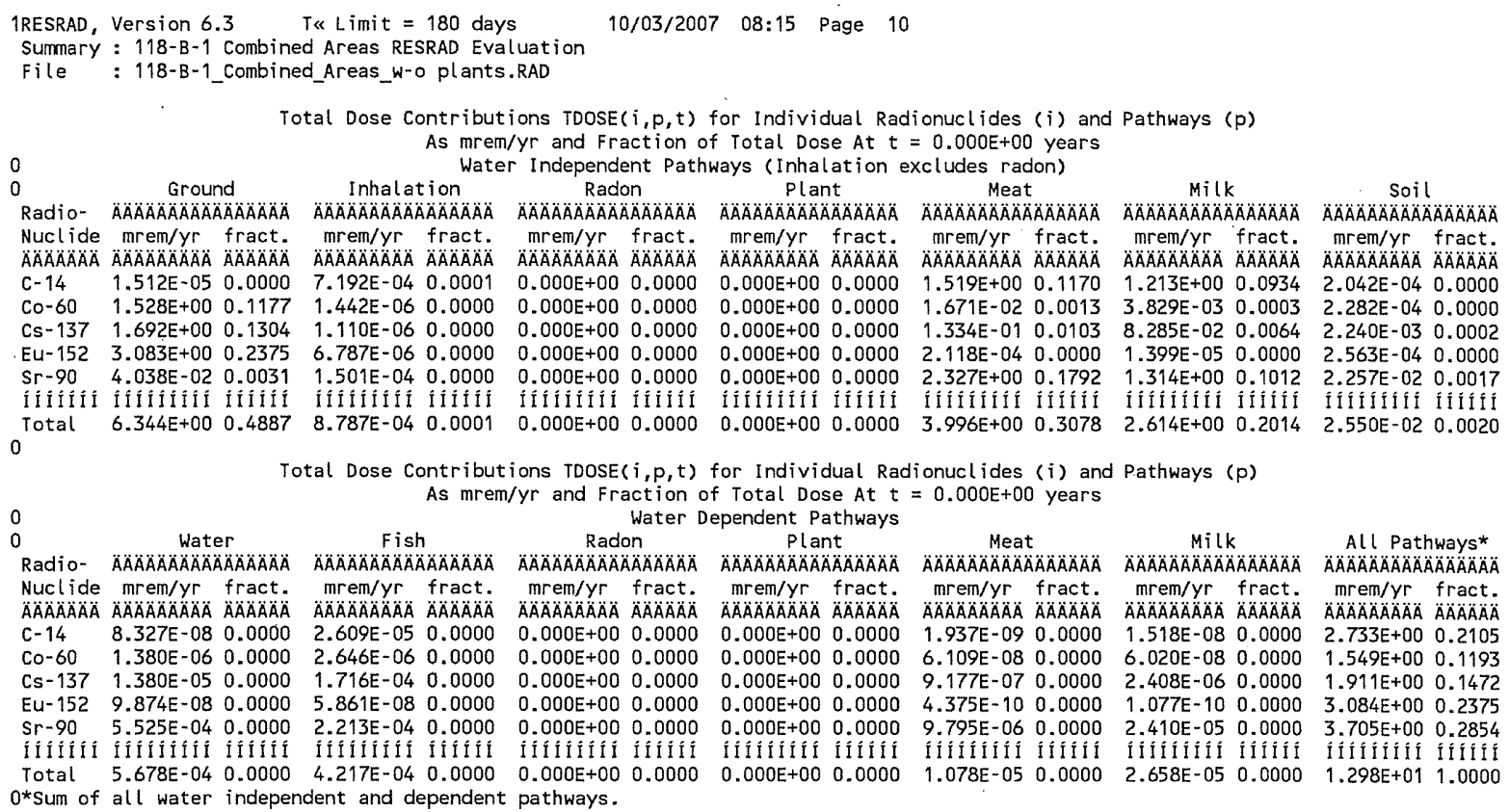

\begin{tabular}{|c|c|}
\hline Attachment & Sheet No. 10 of 20 \\
\hline Originator: S.W. Clark & Date \\
\hline Chk'd By M.W. Perrott & Date \\
\hline 0100B-CA-V0308 & Rev. No. $\quad 0$ \\
\hline
\end{tabular}


CVP-2007-00006

Rev. 0

\section{ATTACHMENT 3}

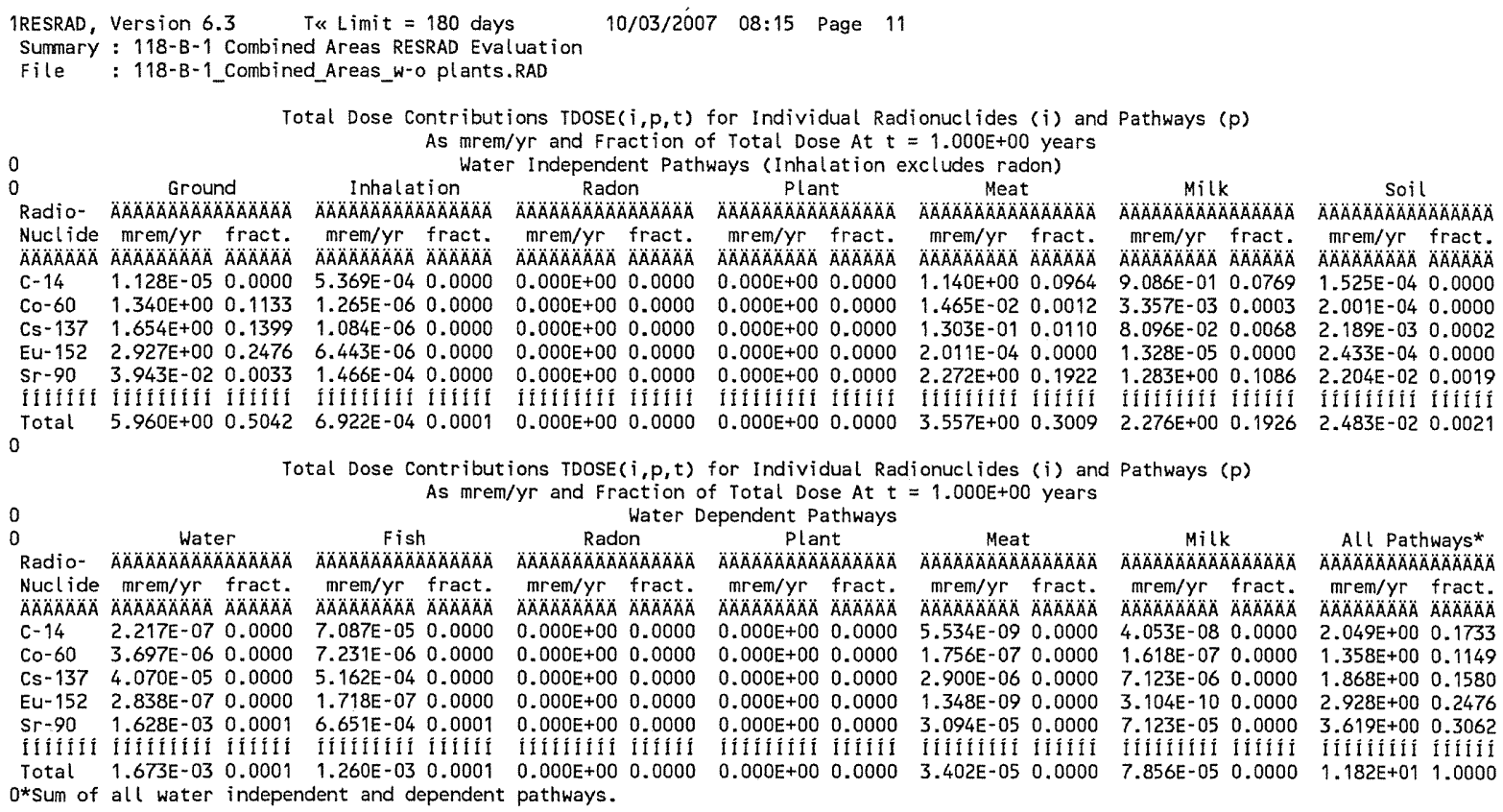

$\begin{array}{ll}\text { Attachment } \frac{3}{\text { O.W. Clark }} & \text { Sheet No. } 11 \text { of } \underline{20} \\ \text { Originator: } \frac{\text { S.W. }}{\text { M.W. Perrott }} & \text { Date } \\ \text { Chk'd By } & \text { Rev. No. } \quad 0 \\ \text { Calc. No. } \frac{0100 \text { B-CA-V0308 }}{0 . \text { Rev. No }} & \end{array}$


CVP-2007-00006

Rev. 0

\section{ATTACHMENT 3}

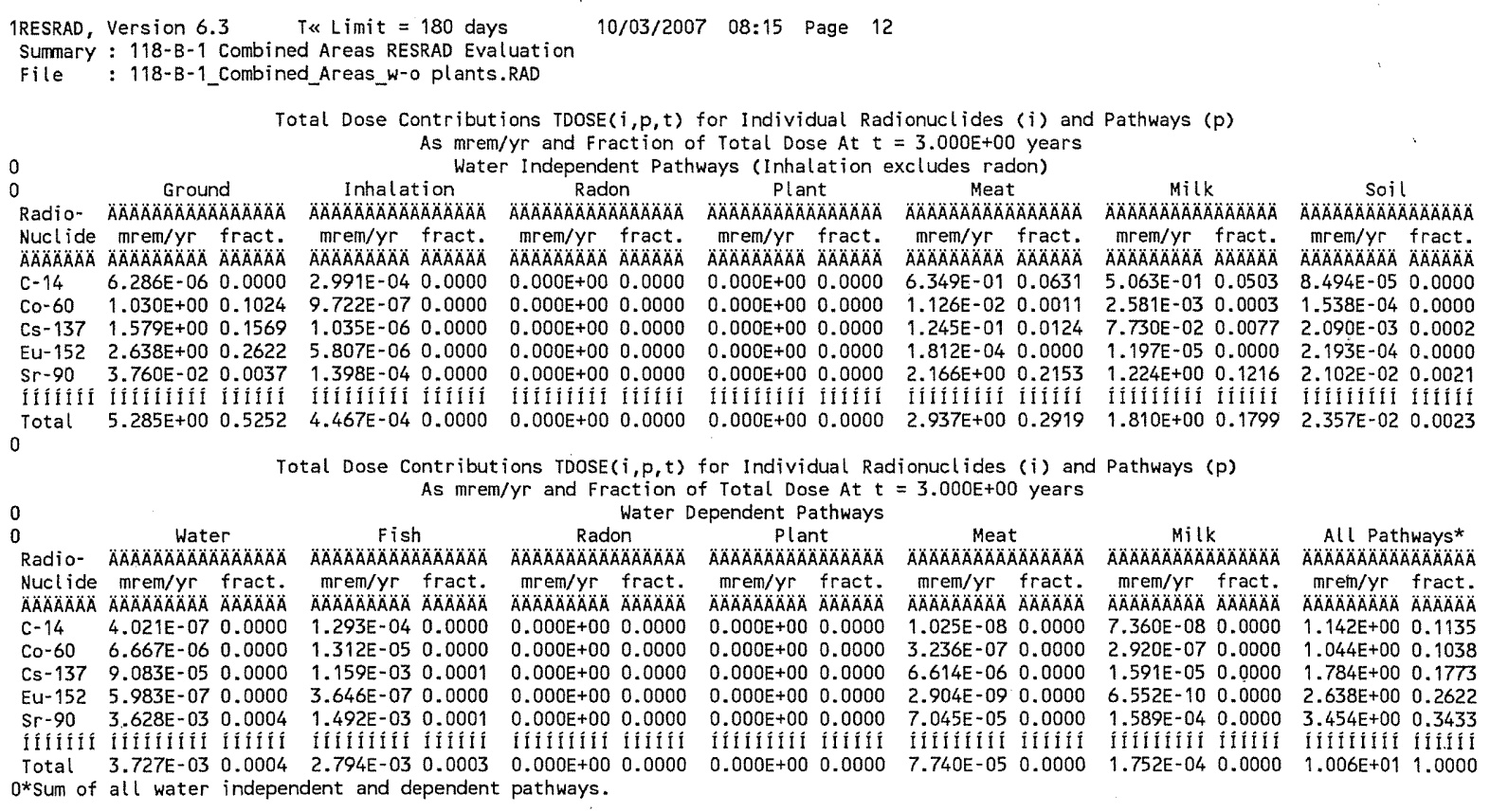

Attachment

3 Sheet No. 12 of 20

Originator: $\frac{\text { S.W. Clark }}{\text { M.W. Perrott }}$ Date

Calc. No. 0100B-CA-V0308

Rev. No. 0 


\section{ATTACHMENT 3}

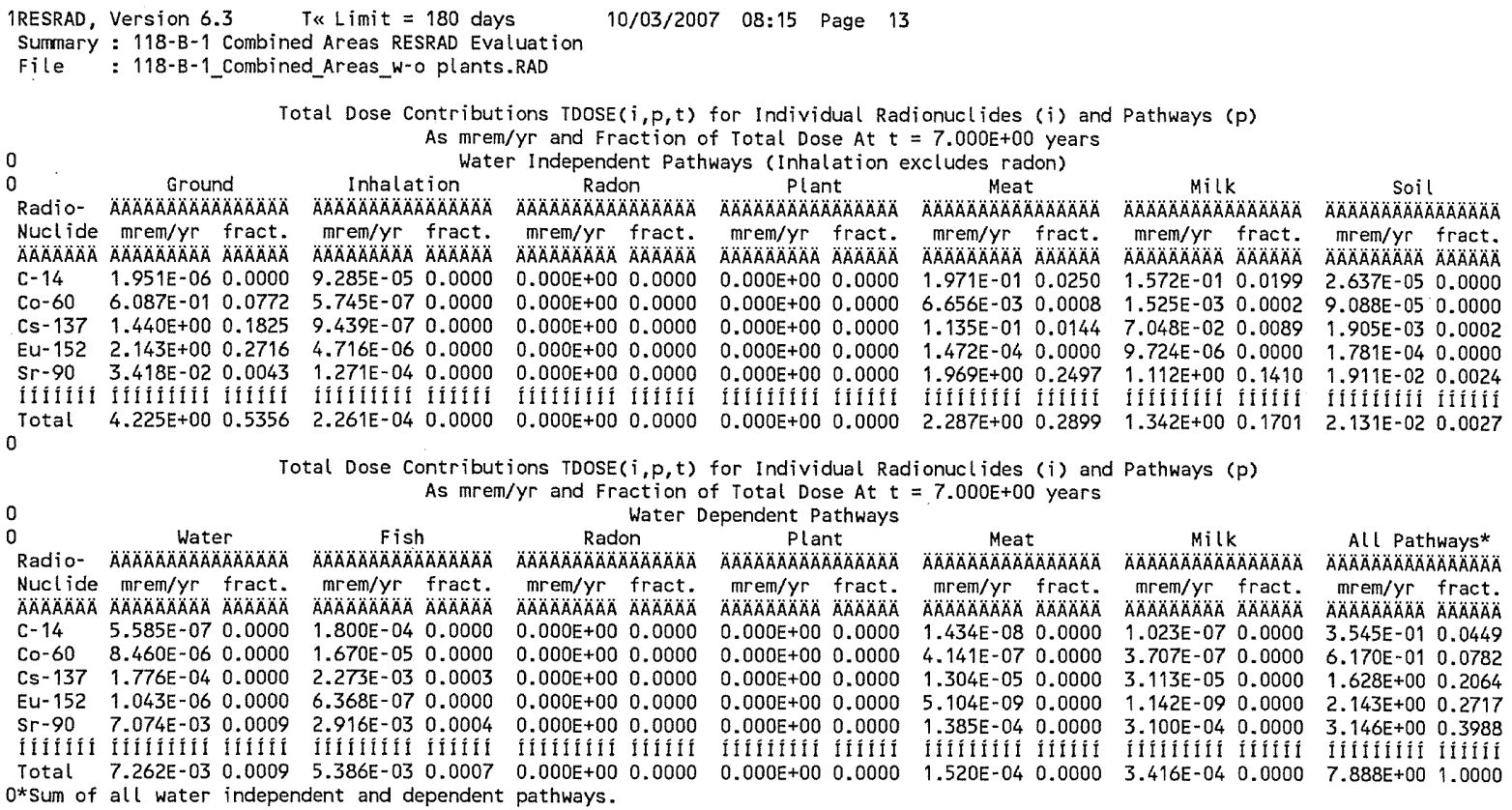

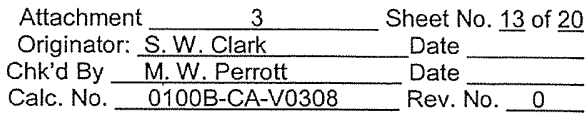




\section{ATTACHMENT 3}

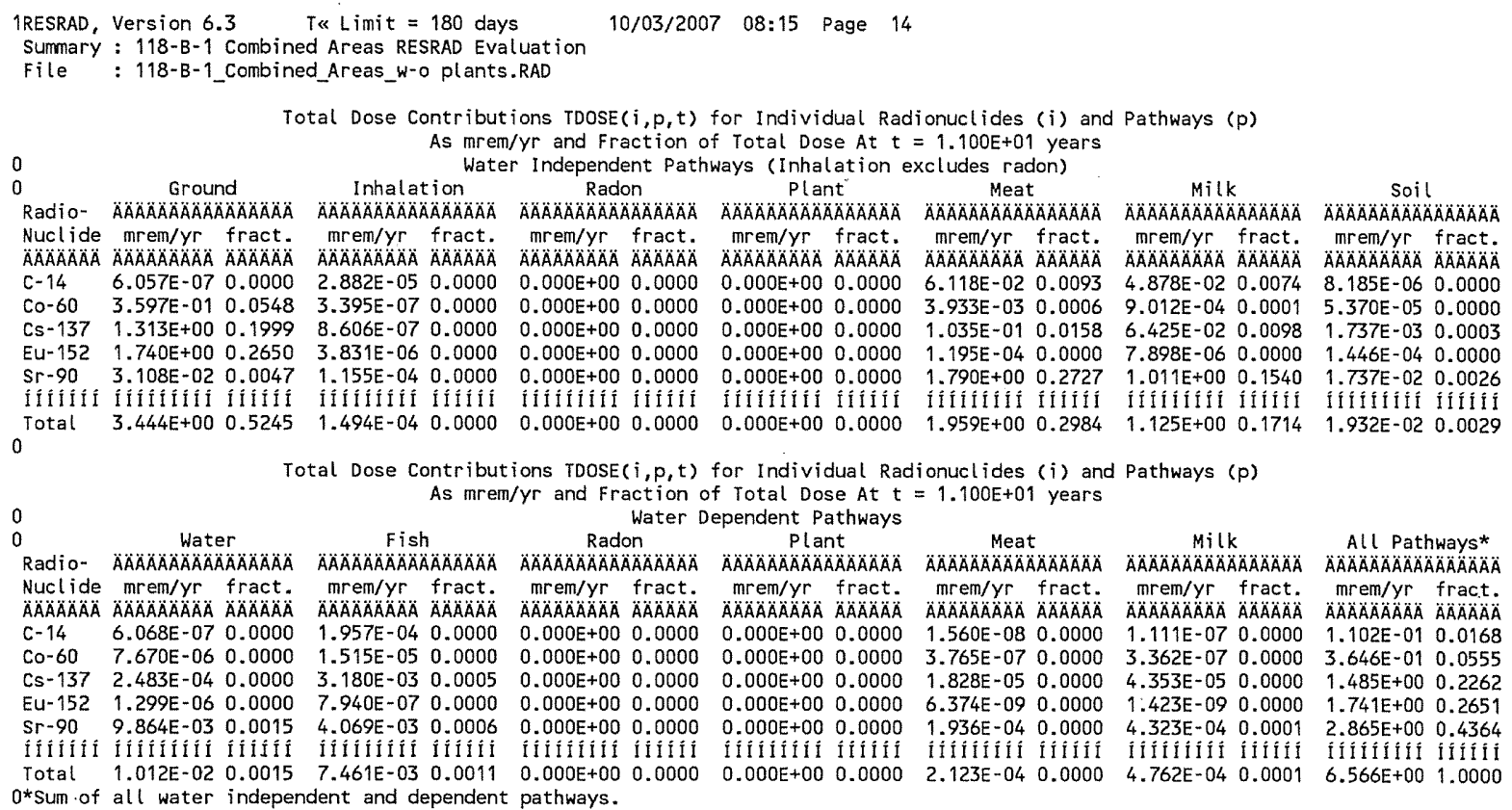

\begin{tabular}{|c|c|}
\hline Attachment & She \\
\hline Originator: S.W. Clark & Date \\
\hline Chk'd By M.W. Perrott & Date \\
\hline Calc. No. $\quad 0100 \mathrm{~B}-\mathrm{CA}-\mathrm{V} 0308$ & Rev. No. 0 \\
\hline
\end{tabular}




\section{ATTACHMENT 3}

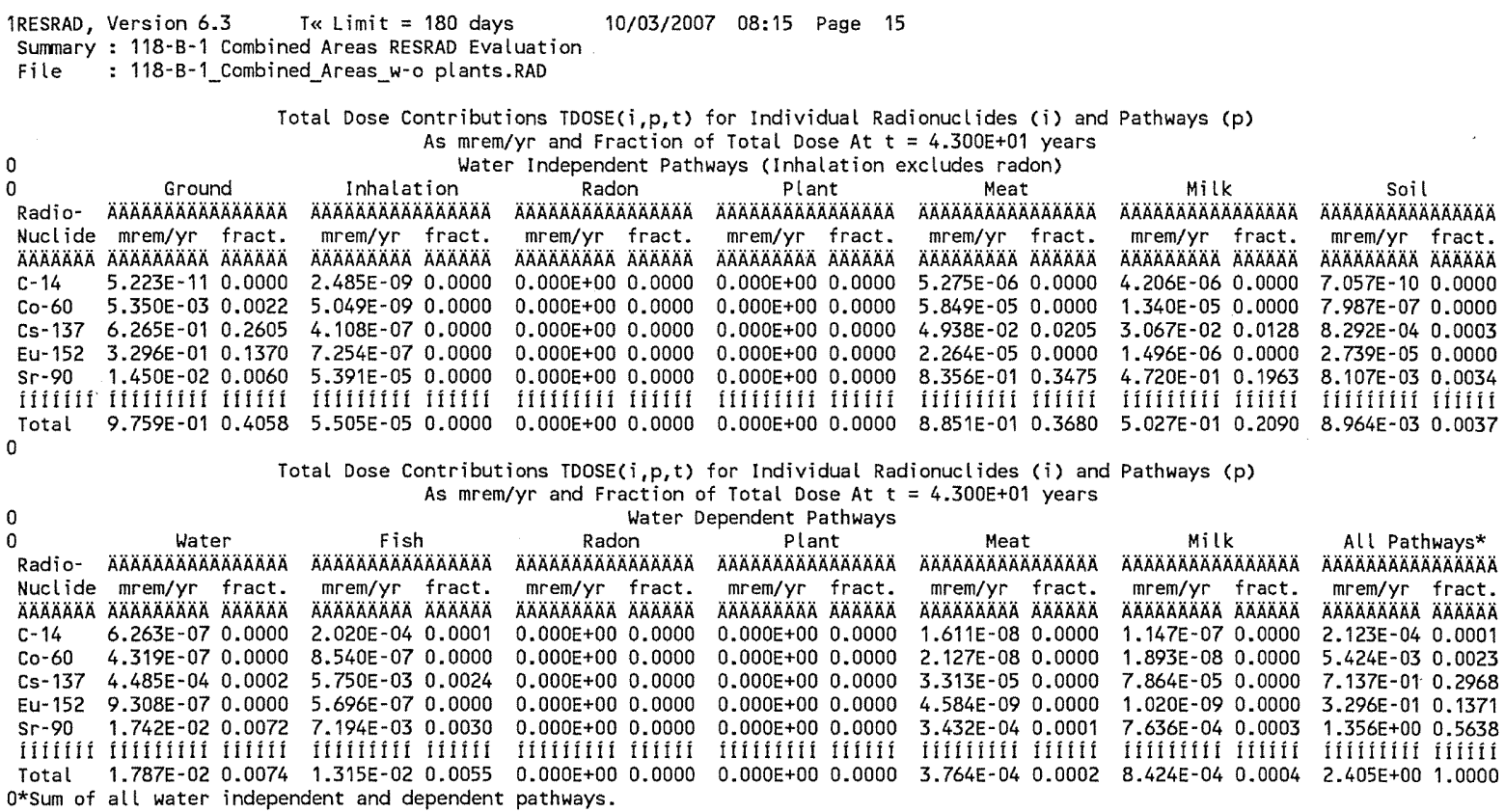

\begin{tabular}{|c|c|}
\hline Attachment & Sheet No. 15 of 20 \\
\hline Originator: S.W. Clark & Date \\
\hline M. W. Perrott & Date \\
\hline $0100 B-C A-V 0308$ & Rev. No. 0 \\
\hline
\end{tabular}




\section{ATTACHMENT 3}

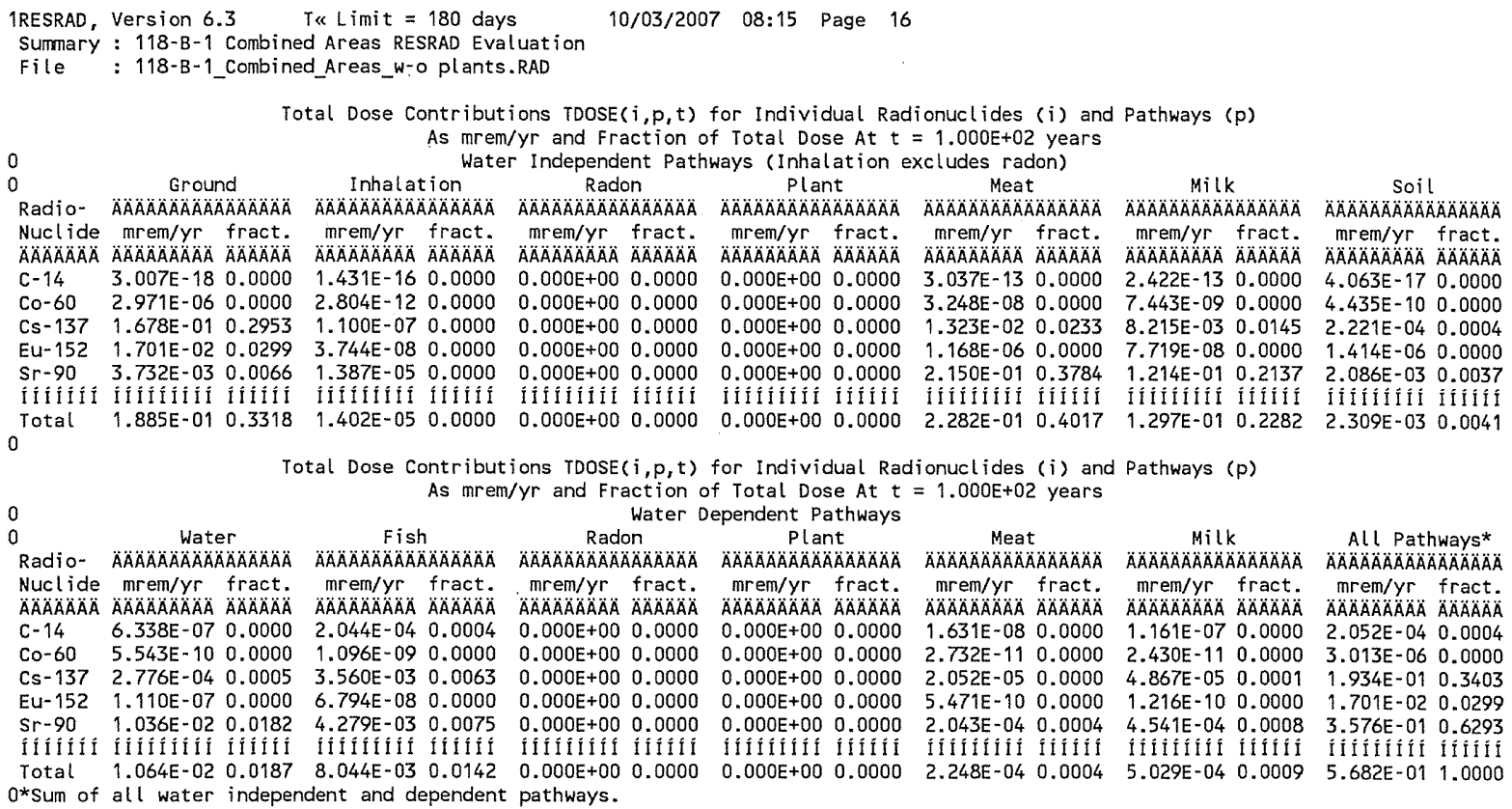

$\begin{array}{ll}\text { Attachment } \frac{3}{\text { S.W. Clark }} & \text { Sheet No. } 16 \text { of } 20 \\ \text { Originator: } & \text { Date } \\ \text { Chk'd By } & \text { Date } \\ \text { Calc. No. Perrott } & \text { Rev. No. }\end{array}$


CVP-2007-00006

Rev. 0

\section{ATTACHMENT 3}

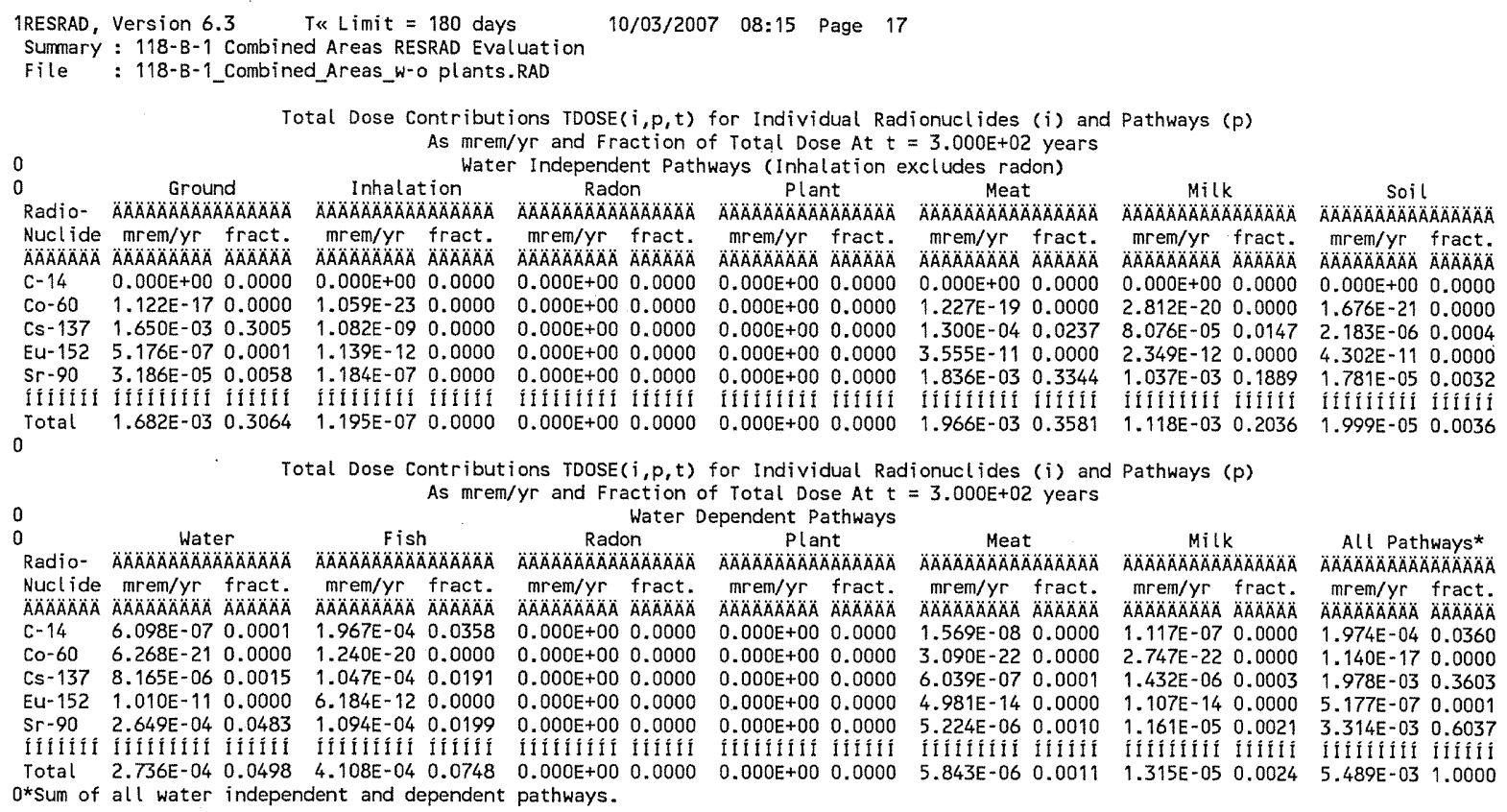




\section{ATTACHMENT 3}

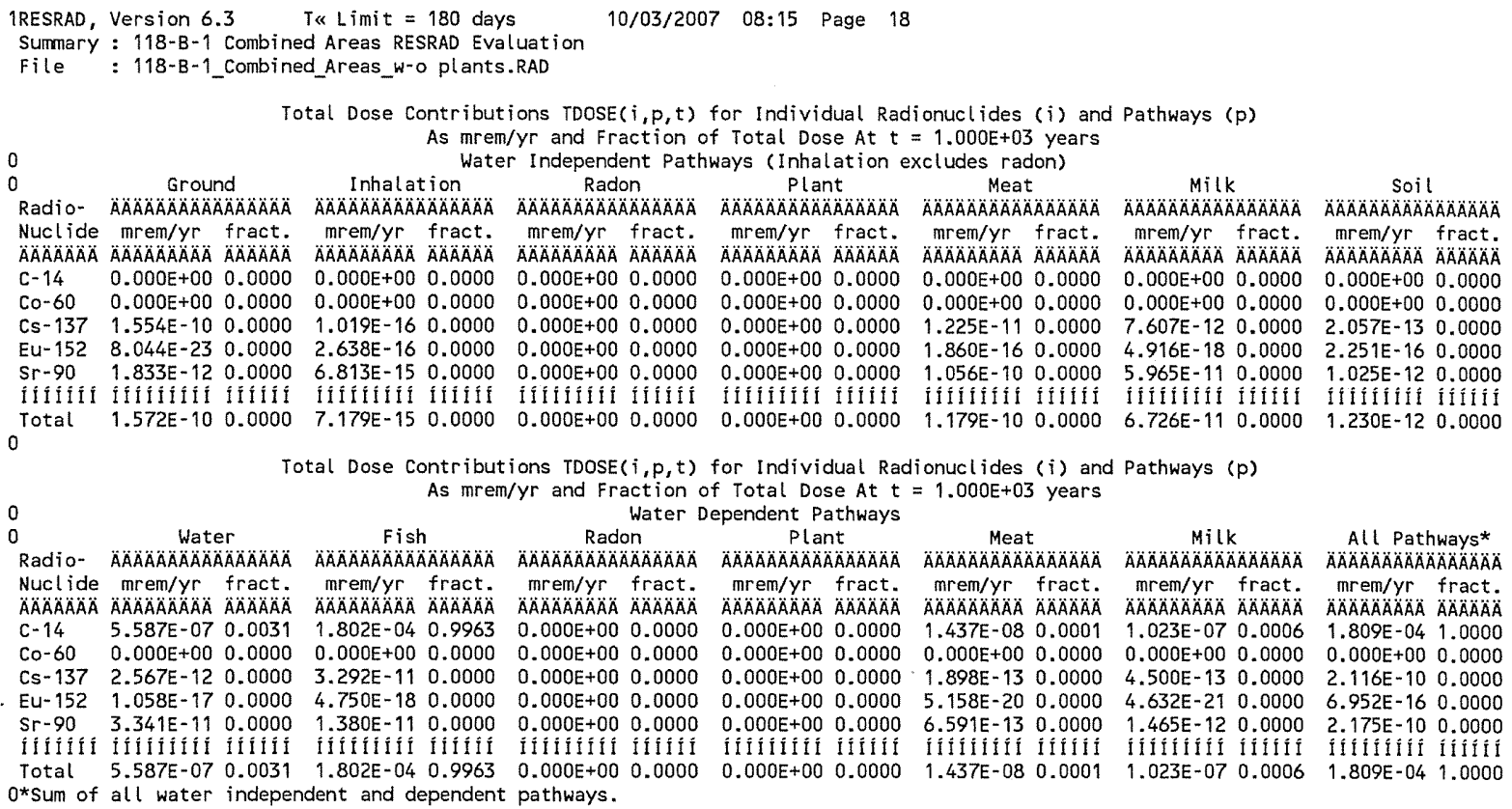

\begin{tabular}{|c|c|}
\hline Attachment & Sheet No. 18 of 20 \\
\hline Originator: S.W. Clark & Date \\
\hline M.W. Perrott & Date \\
\hline 0100B-CA-V0308 & Rev. No. \\
\hline
\end{tabular}


CVP-2007-00006

Rev. 0

\section{ATTACHMENT 3}

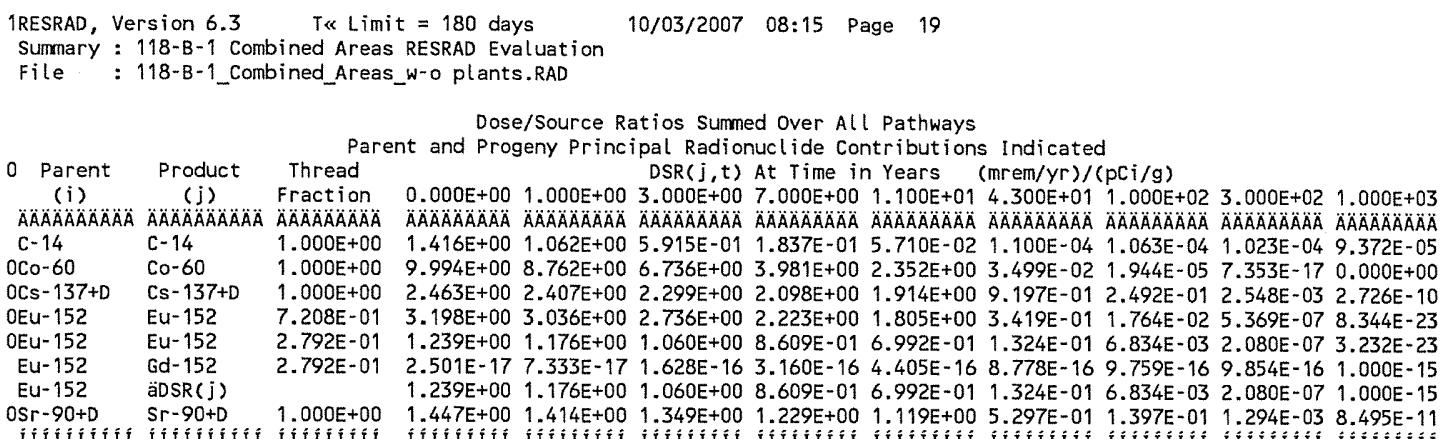

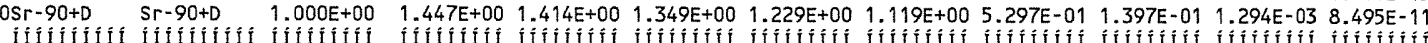
The DSR includes contributions from associated (half-life ó 180 days) daughters.

\begin{tabular}{|c|c|c|c|c|c|c|}
\hline \multicolumn{7}{|c|}{ Basic Radiation Dose Limit $=4.000 \mathrm{E}+00 \mathrm{mrem} /$} \\
\hline ONucl ide & & & & & & \\
\hline (i) & $t=0.000 E+00$ & $1.000 E+00$ & $3.000 E+00$ & $7.000 E+00$ & $1.100 E+01$ & $4.300 \mathrm{E}$ \\
\hline A A A A ÄÄA & A ÄAÄÄÄÄÄ & $\triangle A Q A A A A A A O A$ & 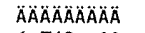 & 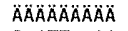 & $\triangle A \cap A ̈ A ̈ A ̈ A ̈ A ̈ A ̈ A$ & \\
\hline$c-14$ & $2.824 E+00$ & $3.768 E+00$ & $6.762 E+00$ & $2.177 E+01$ & $7.006 \mathrm{E}+01$ & \\
\hline $\cos 60$ & $4.003 E-01$ & $5 E-01$ & $E-01$ & $+\infty$ & DOE +00 & \\
\hline 37 & $1.624 E+00$ & $1.662 \mathrm{E}+00$ & $0 E+00$ & 00 & 00 & \\
\hline & $9.015 E-01$ & $9.496 E-01$ & 00 & 00 & & \\
\hline $\mathrm{Sr}-90$ & $2.764 E+00$ & 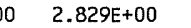 & & & & \\
\hline ifi & iffififi & If i & & If & II & \\
\hline & vity & : limi & & & & \\
\hline & & & & & & \\
\hline & $\begin{array}{l}\text { med Do } \\
\text { singl }\end{array}$ & & & & & \\
\hline & a & & & & & \\
\hline & d a & & & & & \\
\hline uclide & Initial & $\operatorname{tmi}$ & $\operatorname{DSR}(i, t \min )$ & & $\operatorname{DSR}(i, t \operatorname{tmax})$ & \\
\hline & $(\mathrm{pCi} / \mathrm{g})$ & & & & & \\
\hline ÄÄÄ & $\triangle A A A O A O A A A ̈$ & ÄÄÄÄÄ & & ÄAAAAAAAAAÄ & $A ̈ A ̈ A ̈$ & $\triangle A$ \\
\hline$c-14$ & $1.930 E+00$ & 0.000 & 1. & & +00 & \\
\hline$C_{0}$ & 1. & 0.000 & & 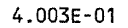 & $E+\infty$ & -01 \\
\hline Cs -137 & $7.760 \mathrm{E}-01$ & $0.000 E+00$ & $2.463 E+00$ & $=+\infty$ & $2.463 E+00$ & $1.624 \mathrm{E}+00$ \\
\hline Eu-152 & $6.950 E-01$ & $0.000 E+00$ & & 9.0 & $4.437 E+00$ & \\
\hline $\mathrm{Sr}^{-}$ & $2.560 \mathrm{E}$ & 0.00 & 1.4 & $E+00$ & $1.447 E+00$ & 2.76 \\
\hline & İilíílíl & fillílílílín & 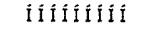 & 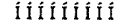 & Ifitilitît & filfitif \\
\hline
\end{tabular}

\begin{tabular}{|c|c|c|c|c|c|c|}
\hline $3.000 E+00$ & $7.000 E+00$ & $1.100 E+01$ & 4.300E+01 & $1.000 E+02$ & $3.000 E+02$ & $1.000 E+03$ \\
\hline AAAAAAAAAÄ & AAAAAAAAAAA & $\triangle A A A O A A A O A O A$ & $\triangle A B A A A A O A O A O A$ & $\triangle A A A A A O A A A A O A$ & 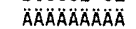 & $\triangle A \cap A A A O A A B A B$ \\
\hline $6.762 E+00$ & $2.177 \mathrm{E}+01$ & $7.006 \mathrm{E}+01$ & $3.637 E+04$ & $3.762 E+04$ & $3.910 E+04$ & $4.268 \mathrm{E}+04$ \\
\hline $5.938 \mathrm{E}-01$ & $1.005 E+00$ & $1.700 E+00$ & $1.143 E+02$ & $2.058 E+05$ & $* 1.132 E+15$ & $\star 1.132 E+15$ \\
\hline $1.740 E+00$ & $1.907 E+00$ & $2.090 E+00$ & $4.349 E+00$ & $1.605 E+01$ & $1.570 E+03$ & $1.467 E+10$ \\
\hline $1.054 E+00$ & $1.297 \mathrm{E}+00$ & $1.597 \mathrm{E}+00$ & $8.434 E+00$ & $1.634 E+02$ & $5.370 E+06$ & $* 1.765 E+14$ \\
\hline $2.965 E+00$ & $3.255 \mathrm{E}+00$ & $3.574 E+00$ & $7.552 E+00$ & $2.864 E+01$ & $3.090 E+03$ & $4.709 E+10$ \\
\hline fiffifiti & IIIIIIIIfí & IIIIIIIIII & Ififilifi & 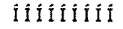 & 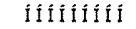 & 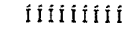 \\
\hline
\end{tabular}


CVP-2007-00006

Rev. 0

\section{ATTACHMENT 3}

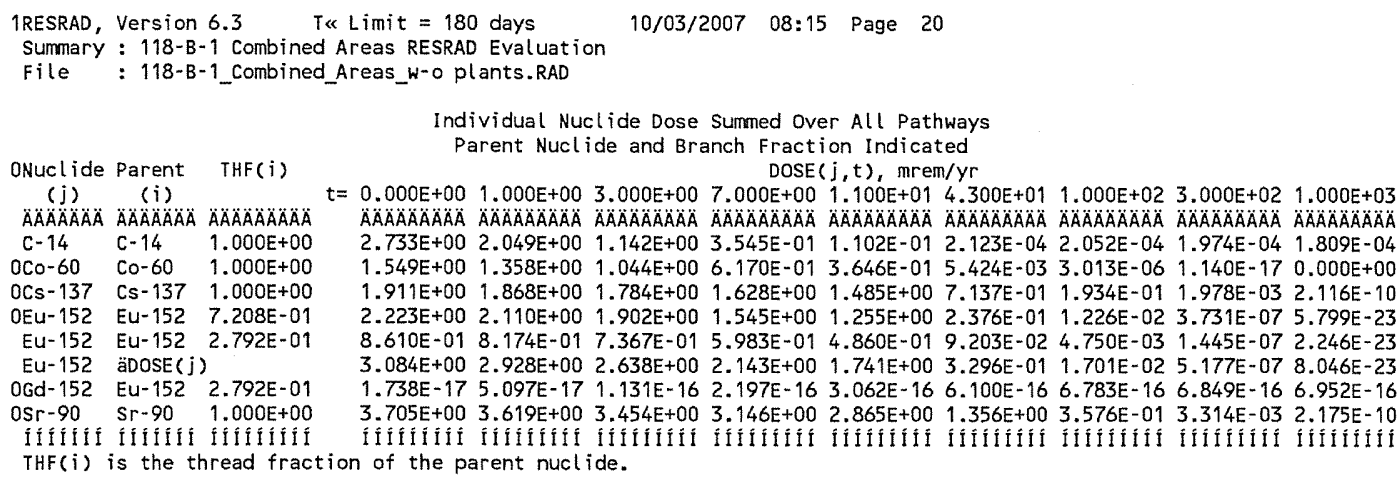

$t=0.000 E+00 \quad 1.000 E+00 \quad 3.000 E+00 \quad 7.000 E+00 \quad 1.100 E+01 \quad 4.300 E+01 \quad 1.000 E+02 \quad 3.000 E+02 \quad 1.000 E+03$

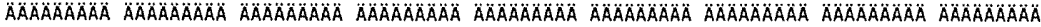
$2.733 E+00 \quad 2.049 E+001.142 E+00 \quad 3.545 E-011.102 E-01 \quad 2.123 E-04 \quad 2.052 E-04 \quad 1.974 E-04 \quad 1.809 E-04$ $1.549 \mathrm{E}+001.358 \mathrm{E}+00 \quad 1.044 \mathrm{E}+00 \quad 6.170 \mathrm{E}-01 \quad 3.646 \mathrm{E}-01 \quad 5.424 \mathrm{E}-03 \quad 3.013 \mathrm{E}-06 \quad 1.140 \mathrm{E}-170.000 \mathrm{E}+00$ $1.911 E+00 \quad 1.868 E+00 \quad 1.784 E+001.628 E+00 \quad 1.485 E+00 \quad 7.137 E-01 \quad 1.934 E-01 \quad 1.978 E-03 \quad 2.116 E-10$

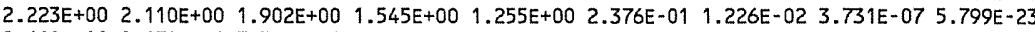
8.610E-01 8.174E-01 7.367E-01 5.983E-01 4.860E-01 9.203E-02 4.750E-03 1.445E-07 2.246E-23

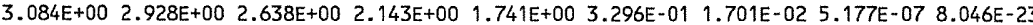
$1.738 \mathrm{E}-17 \quad 5.097 \mathrm{E}-17 \quad 1.131 \mathrm{E}-16 \quad 2.197 \mathrm{E}-16 \quad 3.062 \mathrm{E}-16 \quad 6.100 \mathrm{E}-16 \quad 6.783 \mathrm{E}-16 \quad 6.849 \mathrm{E}-16 \quad 6.952 \mathrm{E}-16$

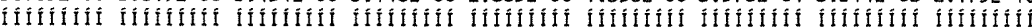

\section{Individual Nuclide Soil Concentration} Parent Nuclide and Branch Fraction Indicated

ONuclide Parent THF(i) (j) (i) $\begin{array}{lll}\text { AAAAAAA AAAAAAA AAAAAAAAA } \\ C-14 & \mathrm{C}-14 & 1.000 E+00\end{array}$ $\begin{array}{ccc}C-14 & C-14 & 1.000 E+00 \\ 0 C 0-60 & C O-60 & 1.000 E+00\end{array}$ $\begin{array}{lll}0 \mathrm{CO}-60 & \mathrm{CO}-60 & 1.000 \mathrm{E}+00 \\ \mathrm{OCs}-137 & \mathrm{Cs}-137 & 1.000 \mathrm{E}+00\end{array}$ $\begin{array}{lll}\text { OCs }-137 & \text { Cs }-137 & 1.000 E+00 \\ \text { OEU-152 } & \text { Eu-152 } & 7.208 E-01\end{array}$ $\begin{array}{ccc}\text { OEU-152 } & \text { EU-152 } & 7.208 E-01 \\ \text { EU-152 } & \text { EU-152 } & 2.792 E-01\end{array}$ Eu-152 äS(j):

OGd-152 EU-152 2.792E-01

OSr-90 Sr-90 $1.000 E+00$

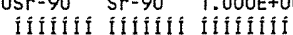
THF (i) is the thread fraction ORESCALC.EXE execution time =

$s(j, t), p C i / g$

. $7.000 E+001.100 E+014.300 E+019.000 E+023.000 E+021.000 E+03$

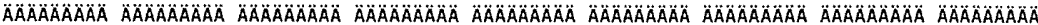

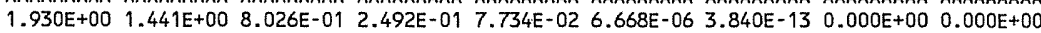

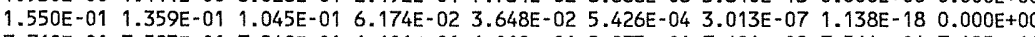
7.760E-01 7.583E-01 7.240E-01 6.601E-01 6.018E-01 2.873E-01 7.694E-02 7.564E-04 7.125E-11

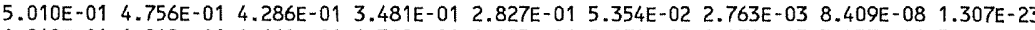
1.940E-01 1.842E-01 1.660E-01 1.348E-01 1.095E-01 2.074E-02 1.070E-03 3.257E-08 5.062E-24 6.950E-01 6.598E-01 5.946E-01 4.829E-01 3.923E-01 7.428E-02 3.834E-03 1.167E-07 1.813E-23 $0.000 E+00 \quad 1.214 E-15 \quad 3.459 E-15 \quad 7.307 E-15 \quad 1.043 E-14 \quad 2.139 E-14 \quad 2.382 E-14 \quad 2.395 E-14 \quad 2.394 E-14$ $2.560 \mathrm{E}+002.500 \mathrm{E}+002.383 \mathrm{E}+002.167 \mathrm{E}+001.970 \mathrm{E}+00$ 9 $194 \mathrm{E}-012366 \mathrm{E}-012.020 \mathrm{E}-031.162 \mathrm{E}-10$

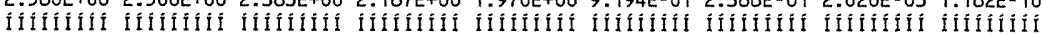
of the parent nuclide. 5.84 seconds

\begin{tabular}{|c|c|}
\hline \multicolumn{2}{|r|}{ Sheet No. 20 of } \\
\hline Originator: S.W. Clark & Date \\
\hline Chk'd By M. W. Perrott & Date \\
\hline $0100 B-C A-V 0308$ & No. \\
\hline
\end{tabular}




\section{ATTACHMENT 4}

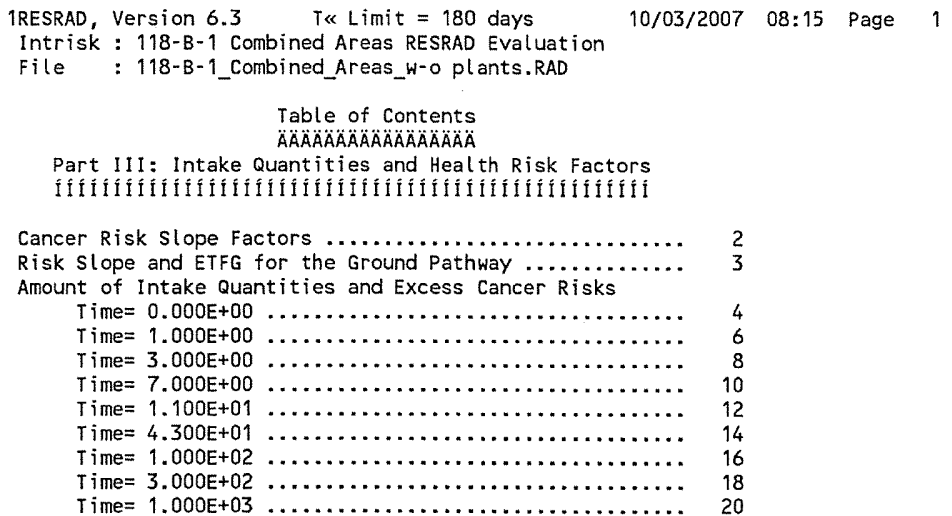


CVP-2007-00006

Rev. 0

\section{ATTACHMENT 4}

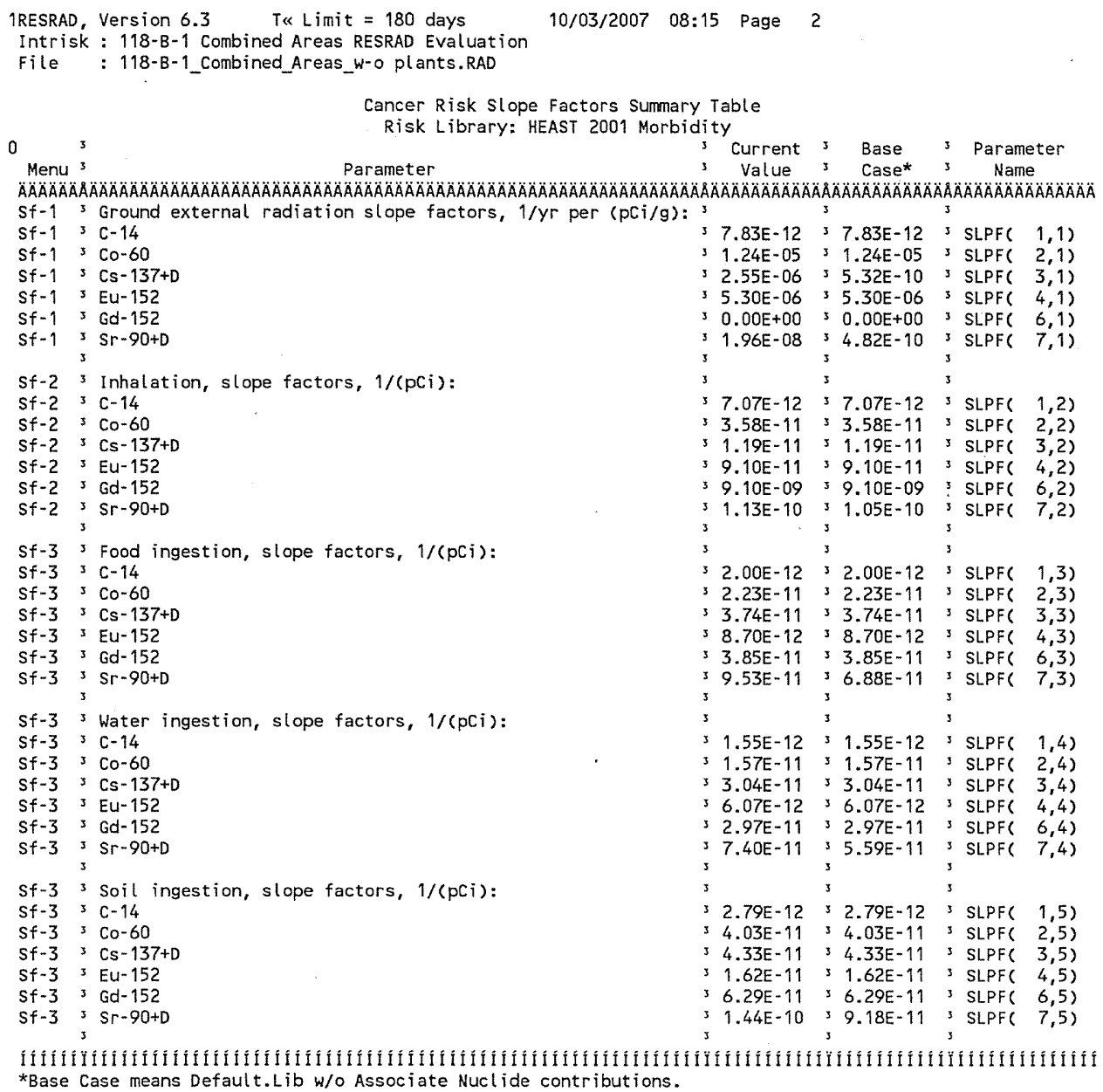

\begin{tabular}{|c|c|}
\hline Attachment & Sheet No. 2 of 21 \\
\hline Originator: S.W. Clark & Date \\
\hline Chk'd By M. W. Perrott & Date \\
\hline Calc. No. $0100 \mathrm{~B}-\mathrm{CA}-\mathrm{V} 0308$ & Rev. No. \\
\hline
\end{tabular}


CVP-2007-00006

Rev. 0

\section{ATTACHMENT 4}

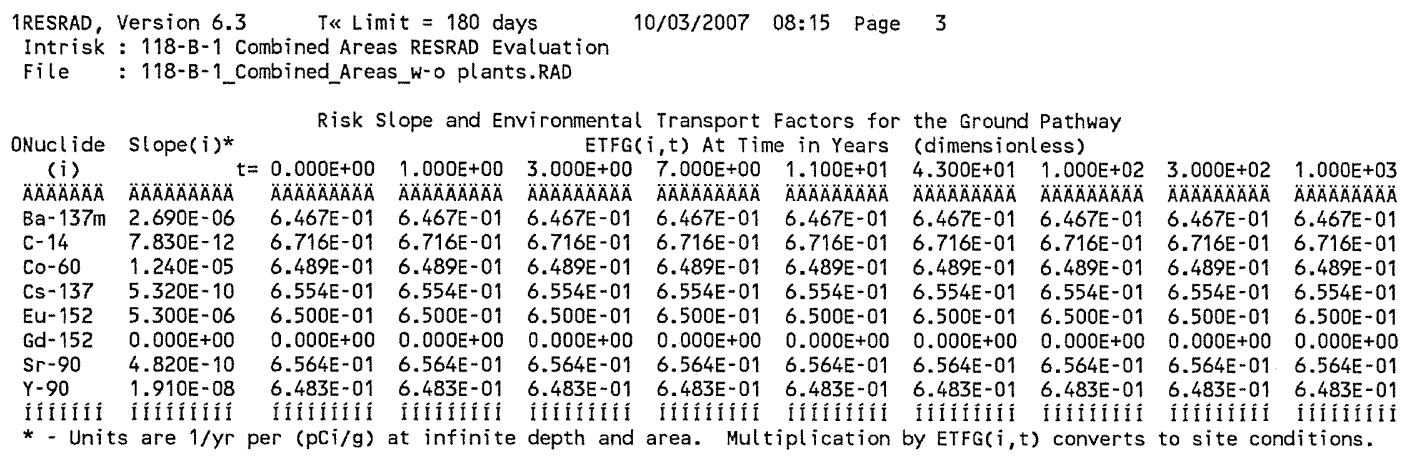

Attachment

Originator: S.W. Clark

4

Sheet No. 3 of 21

Calc. No. $\frac{\text { M.W. Perrott }}{0100 \text { B-CA-V0308 }}$ Rev. No. _ o

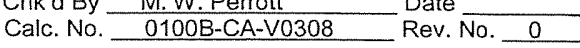

Date 


\section{ATTACHMENT 4}

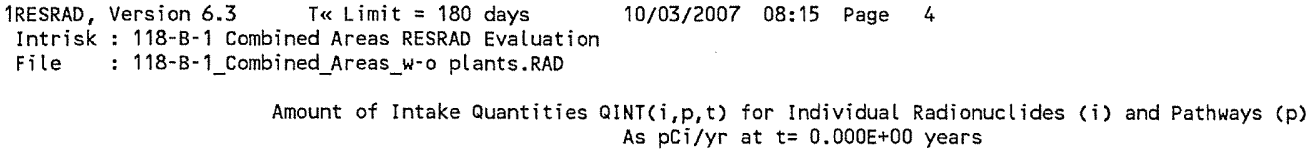

Attachment 
CVP-2007-00006

Rev. 0

\section{ATTACHMENT 4}

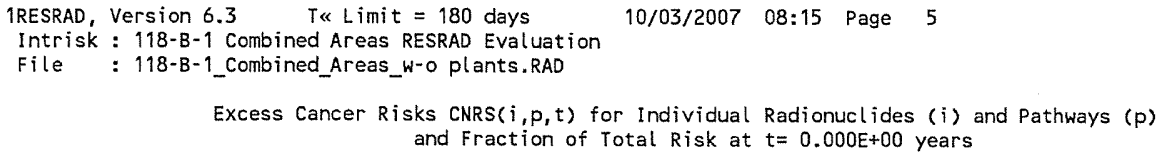

Water Dependent Pathways

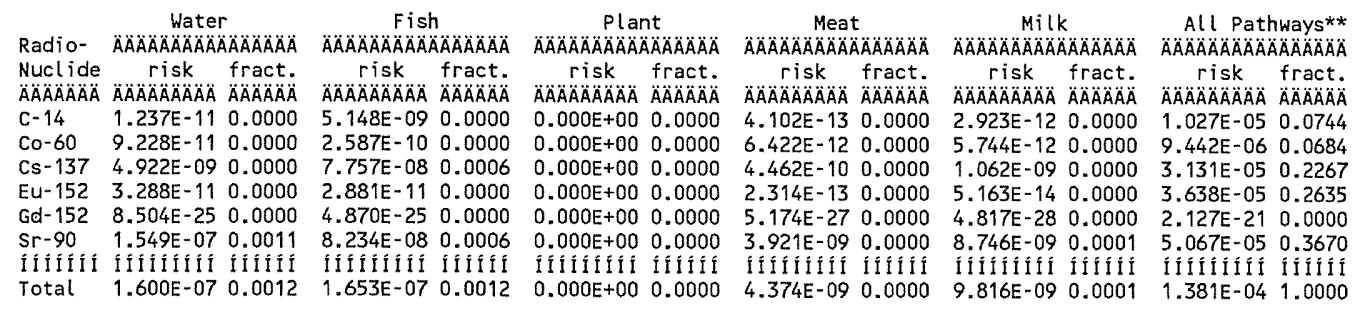

** Sum of water independent ground, inhalation, plant, meat, milk, soil

and water dependent water, fish, plant, meat, milk pathways

Total Excess Cancer Risk CNRSI $(i, p, t) * * *$ for Initially Existent Radionuclides ( $i)$ and Pathways $(p)$ and Fraction of Total Risk at $t=0.000 E+00$ years

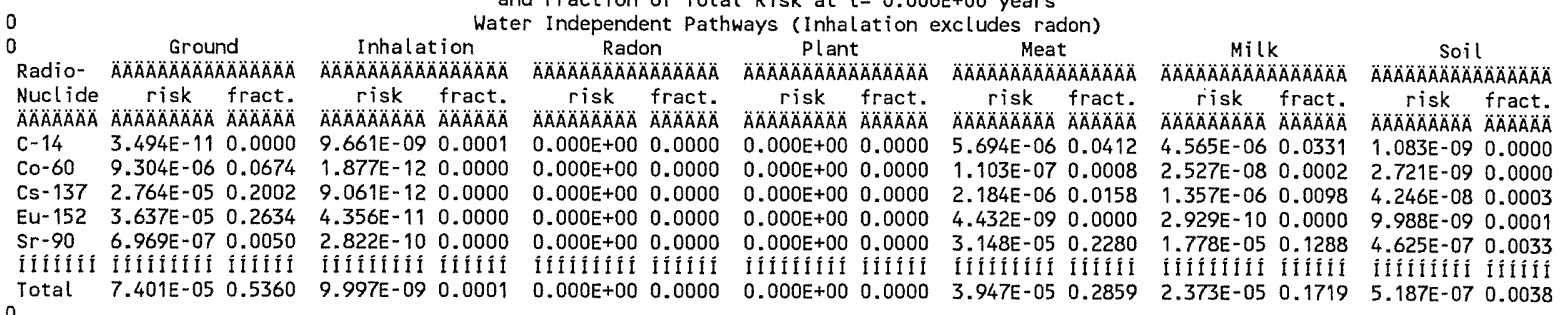
0

Total Excess Cancer Risk CNRSI $(i, p, t) * * *$ for Initially Existent Radionuclides $(i)$ and Pathways $(p)$ and Fraction of Total Risk at $t=0.000 E+00$ years

Water Dependent Pathways

\begin{tabular}{|c|c|c|c|c|c|c|c|c|c|c|c|}
\hline & $\begin{array}{c}\text { Water } \\
\text { AAAAAAAAAAAAAAAAA }\end{array}$ & 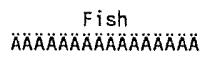 & $\begin{array}{r}\text { Rad } \\
\text { AAAAAAÄÄÄA }\end{array}$ & AAAAAAAA & $\begin{array}{r}\text { Plant } \\
\triangle A A A A A A A A B A\end{array}$ & it & $\begin{array}{r}M e a \\
\triangle A A A B A A A A A\end{array}$ & $A O A O A A B A$ & $\begin{array}{r}M i l \\
\text { ÄAAAÄÄÄA }\end{array}$ & $\ddot{A} \ddot{A} A \dddot{A} A \ddot{A} A \ddot{A}$ & $\begin{array}{c}\text { ALL pathways } \\
A A B A A B A A B A A B A A A A\end{array}$ \\
\hline $\begin{array}{l}\text { Uclide } \\
A O A A A O A B\end{array}$ & $\begin{array}{l}\text { risk fract. } \\
A B A O A O A O A A O A O A O\end{array}$ & $\begin{array}{c}\text { risk fract. } \\
A A A A A A A A O A O A A A A\end{array}$ & rist & $\begin{array}{l}\text { fract. } \\
\text { ÄAAAAOA }\end{array}$ & $\begin{array}{r}r i s k \\
A A A A A A A\end{array}$ & $\begin{array}{l}\text { fract. } \\
\triangle A ̈ A O A B A B\end{array}$ & risk & $\begin{array}{l}\text { fract. } \\
\text { AOAAAAOA }\end{array}$ & risk & & $\begin{array}{c}\text { risk } \\
\text { ÄAAAAAAOA }\end{array}$ \\
\hline & -110.0000 & & 00 & & 00 & & 13 & & & & \\
\hline & & & 00 & 0.0 & & & & & & & \\
\hline & & & 00 & 0.0 & & & & 0 & & & 050 \\
\hline & $\begin{array}{lll}-11 & 0 .\end{array}$ & & & & & & & & & & 050. \\
\hline & & & & & & & & & & & \\
\hline & & & & & líilition & IIII & & III & Ifiñîtîn & & 1111 \\
\hline tat & $1.600 \mathrm{E}-07 \quad 0.0012$ & $-07 \quad 0.0012$ & $O O O E+00$ & 0.0000 & $0.000 E+00$ & 0.0000 & $4.374 E-09$ & 0.0000 & $9.816 \mathrm{E}-09$ & 0.0001 & $31 E-041.00$ \\
\hline
\end{tabular}

$* *$ CNRSI $(i, p, t)$ includes contribution from decay daughter radionuclides

\begin{tabular}{|c|c|}
\hline Attachment & Sheet No. $\underline{5}$ of $\underline{21}$ \\
\hline Originator: $s . W$. Clark & Date \\
\hline M. W. Perrott & Date \\
\hline $0100 B-C A-V 0308$ & Rev. No. \\
\hline
\end{tabular}


CVP-2007-00006

Rev. 0

\section{ATTACHMENT 4}

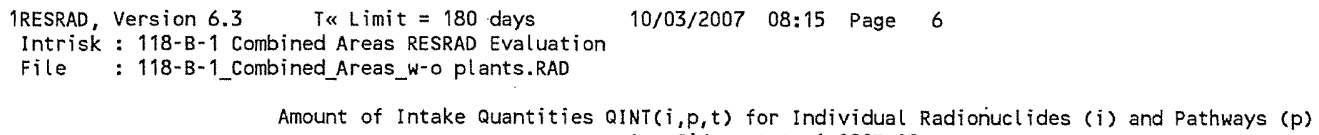

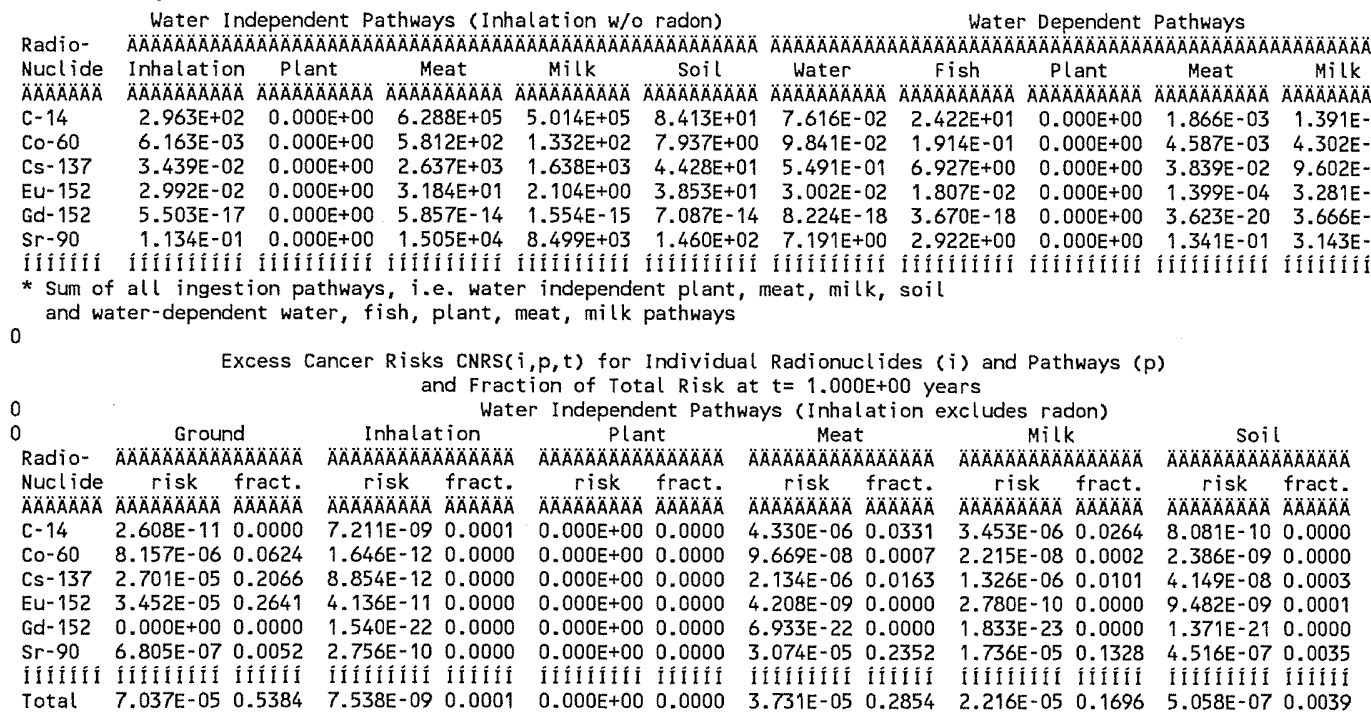

Attachment

4 Sheet No. $\underline{6}$ of $\underline{21}$ 
Rev. 0

\section{ATTACHMENT 4}

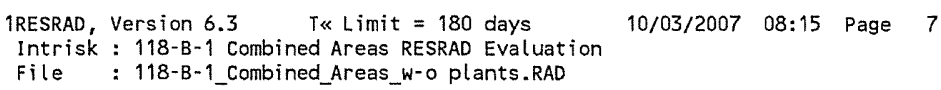

Water Dependent Pathways

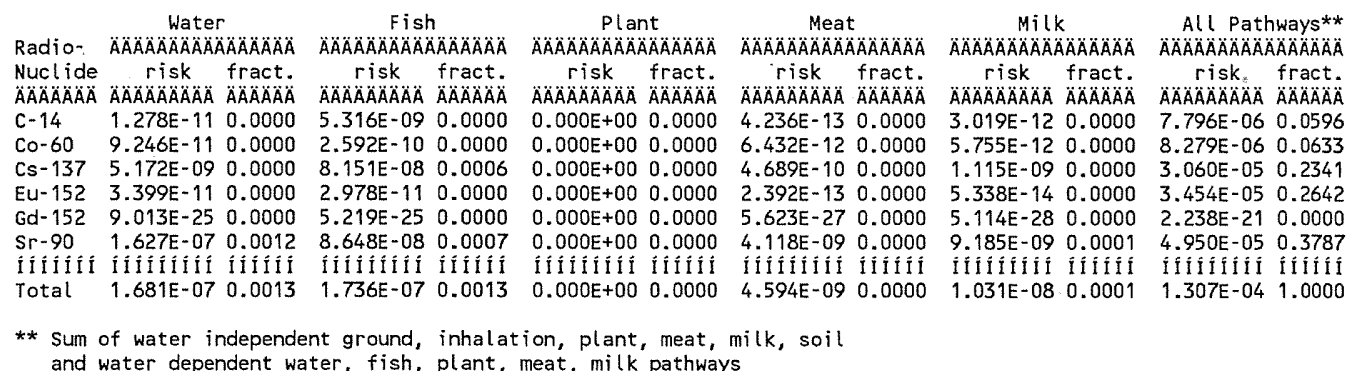

0

Total Excess Cancer Risk CNRSI $(i, p, t)^{* * *}$ for Initially Existent Radionuclides (i) and Pathways ( $p$ )

and Fraction of Total Risk at $t=1.000 E+00$ years

0 Water Independent Pathways (Inhalation excludes radon)

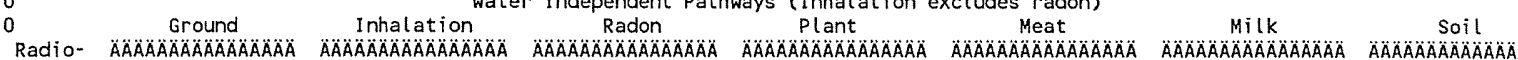

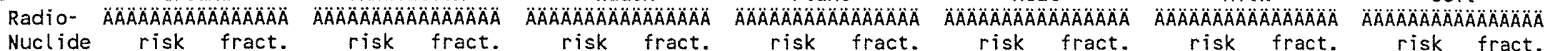

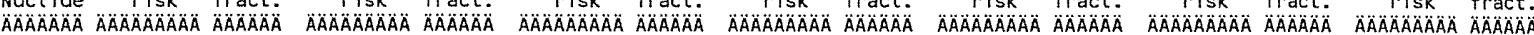

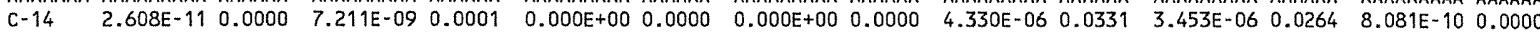

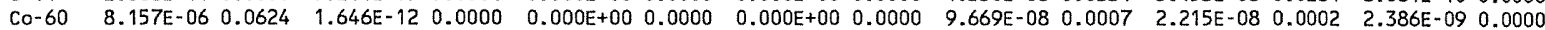
$\begin{array}{lllllllllllllll}C s-137 & 2.701 E-05 & 0.2066 & 8.854 E-12 & 0.0000 & 0.000 E+00 & 0.0000 & 0.000 E+00 & 0.0000 & 2.134 E-06 & 0.0163 & 1.326 E-06 & 0.0101 & 4.149 E-08 & 0.0003\end{array}$

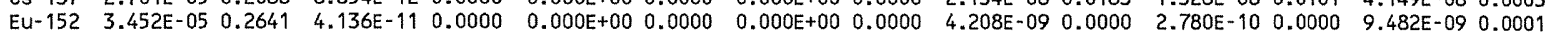

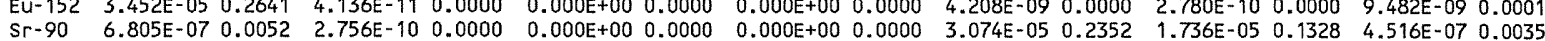

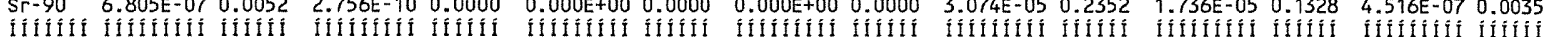

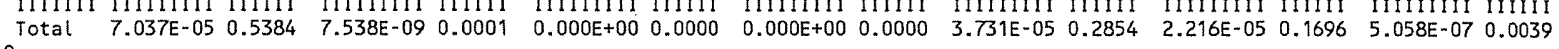
0

Total Excess Cancer Risk CNRSI $(i, p, t)^{* * *}$ for Initially Existent Radionuclides (i) and Pathways ( $p$ ) and Fraction of Total Risk at $t=1.000 E+00$ years

Water Dependent Pathways

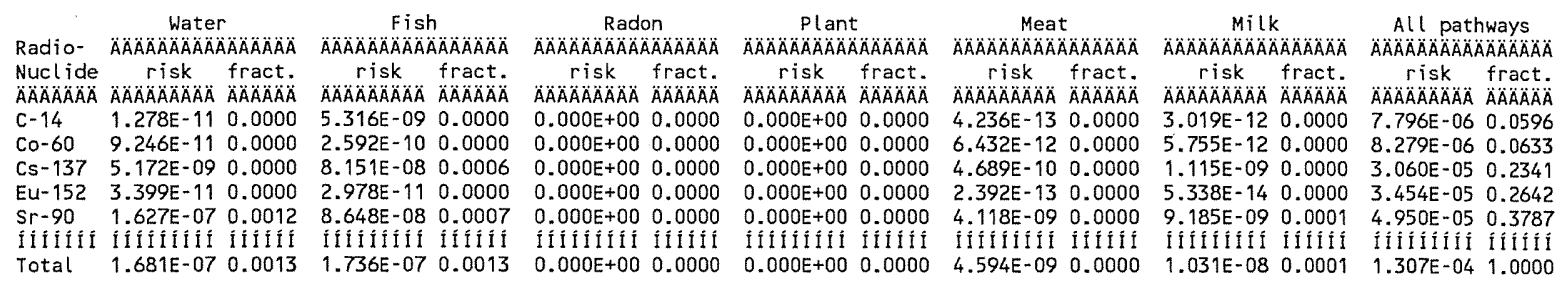

***CNRSI $(i, p, t)$ includes contribution from decay daughter radionuclides

\begin{tabular}{|c|c|}
\hline \multirow{2}{*}{\multicolumn{2}{|c|}{ Attachment $\frac{4}{\mathrm{~W} \text { Clark }}$}} \\
\hline & Date \\
\hline M. W. Perrott & Date \\
\hline $0100 B-C A-V 0308$ & Rev. No \\
\hline
\end{tabular}


CVP-2007-00006

Rev. 0

\section{ATTACHMENT 4}

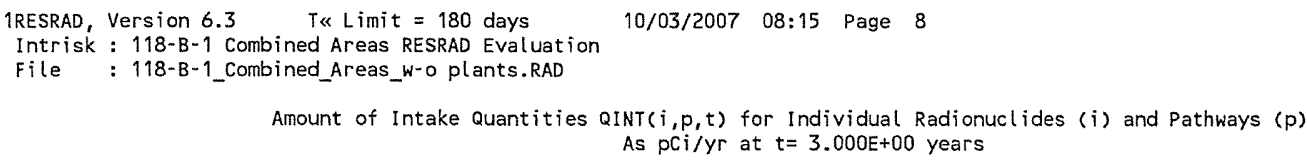


CVP-2007-00006

Rev. 0

\section{ATTACHMENT 4}

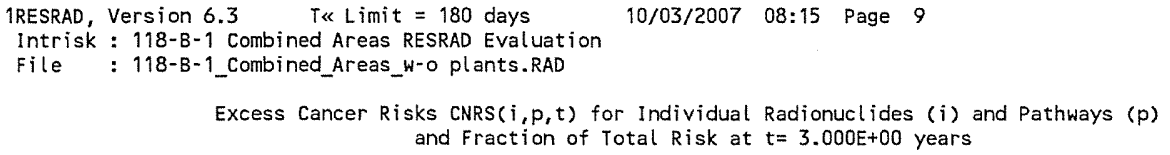

Water Dependent Pathways

\begin{tabular}{|c|c|c|c|c|c|c|c|c|c|c|c|}
\hline & $\begin{array}{r}\text { Wat } \\
\text { AAAAAAAOAAOA }\end{array}$ & 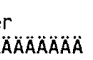 & $\begin{array}{r}F i s \\
A A A A A A A A\end{array}$ & $\triangle A \cap A \cap A \cap A ̈$ & $\begin{array}{r}\text { Plar } \\
\text { AÄÄAAAAAA }\end{array}$ & 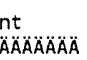 & $\begin{array}{r}\text { Mea } \\
\triangle A A B A A A A B A A A\end{array}$ & t & $\begin{array}{r}M i l \\
\triangle A \cap A O A O A O A\end{array}$ & $\widetilde{A} A \ddot{A} A \ddot{A} A \ddot{A}$ & $\begin{array}{r}\text { All Pathways** } \\
\text { AAAAAAAAAAOAOAOAAAOA }\end{array}$ \\
\hline 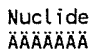 & $\begin{array}{l}\text { risk } \\
A \ddot{A B O A O A O A}\end{array}$ & $\begin{array}{l}\text { fract. } \\
\because A B A B A B A B\end{array}$ & $\begin{array}{l}\text { risk } \\
\text { ÄÄÄÄÄA }\end{array}$ & $\begin{array}{l}\text { fract. } \\
\qquad A ̈ A ̈ A B A B A\end{array}$ & $\begin{array}{c}r i s k \\
\triangle A O A O A ̈ A ̈ A ̈ A ̈\end{array}$ & $\begin{array}{l}\text { fract. } \\
\ddot{A} A \ddot{A} A ̈ A ̈ A ̈ A\end{array}$ & $\begin{array}{l}\text { risk } \\
\triangle A B A B A B A B A\end{array}$ & & risk & & 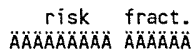 \\
\hline-14 & $1.331 \mathrm{E}-11$ & 0.0000 & & 0.0000 & $E+\infty$ & & & & & & 060 \\
\hline & & & & & & & & & & & \\
\hline & & & & & & & & & & & \\
\hline & $563 E-11$ & 0.0 & 3. & 0.0 & $+\infty$ & 0.0 & & & -14 & & 3. \\
\hline & & 0.0 & & & & & & & 8 & & \\
\hline & & & & & & & & & & & \\
\hline & & & & & & & & & & & In \\
\hline otal & UT & 5 & $F_{1}$ & 0.0016 & $E+00$ & 0. & -09 & 0.0000 & $E-08$ & 1 & -041.0000 \\
\hline
\end{tabular}

** sum of water independent ground, inhalation, plant, meat, milk, soil

0

and water dependent water, fish, plant, meat, milk pathways

Total Excess Cancer Risk CNRSI $(i, p, t)^{\star * *}$ for Initially Existent Radionuclides (i) and Pathways $(p)$ and Fraction of Total Risk at $t=3.000 \mathrm{E}+00$ years

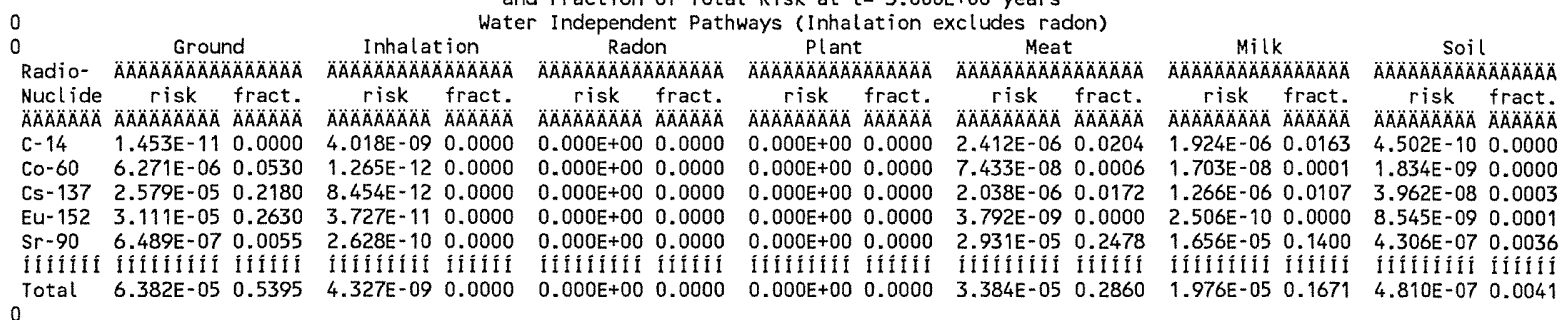

Total Excess Cancer Risk CNRSI $(i, p, t) * * *$ for Initially Existent Radionuclides (i) and Pathways ( $p$ ) and Fraction of Total Risk at $t=3.000 E+00$ years

Water Dependent Pathways

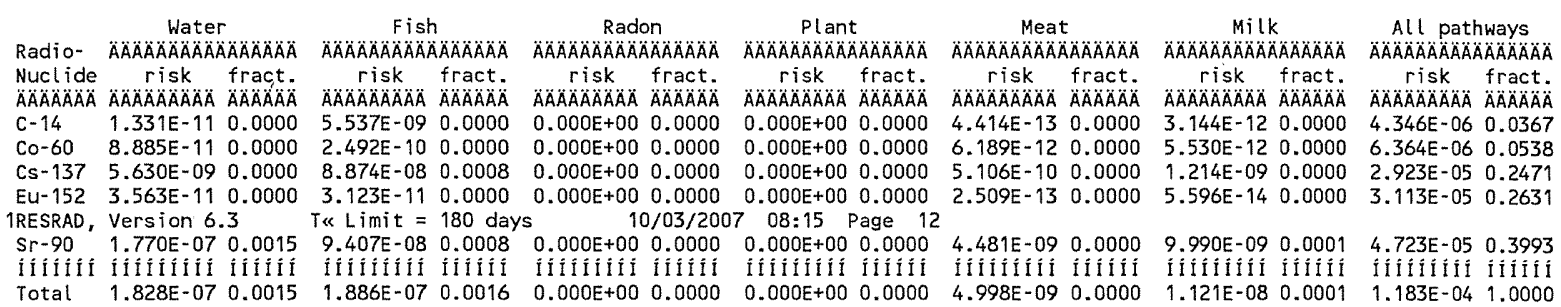

***CNRSI $(i, p, t)$ includes contribution from decay daughter radionuclides

\begin{tabular}{|c|c|}
\hline \multicolumn{2}{|l|}{ Attachment $\quad 4$} \\
\hline Originator: $S . W$. Clark & Date \\
\hline Chk'd By M.W. Perrott & Date \\
\hline $0100 B-C A-V 0308$ & Rev. No. 0 \\
\hline
\end{tabular}


CVP-2007-00006

Rev. 0

\section{ATTACHIMENT 4}

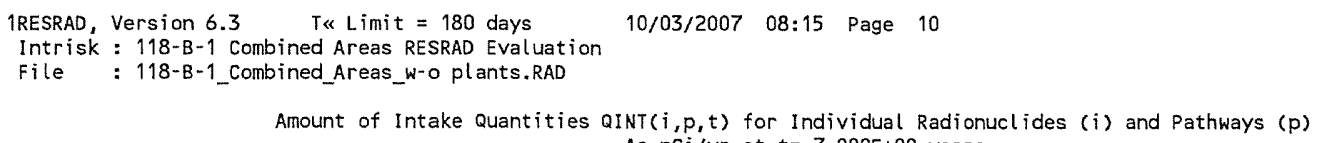

Attachment

4 Sheet No. 10 of $\underline{21}$ 
CVP-2007-00006

Rev. 0

\section{ATTACHMENT 4}

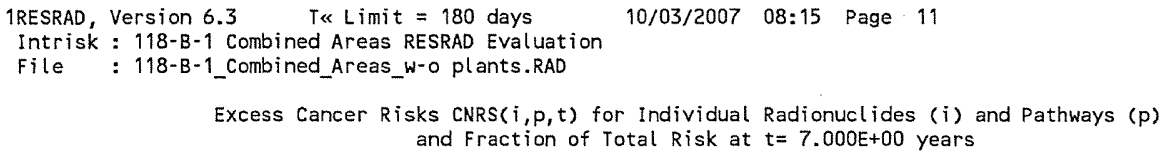

Water Dependent Pathways

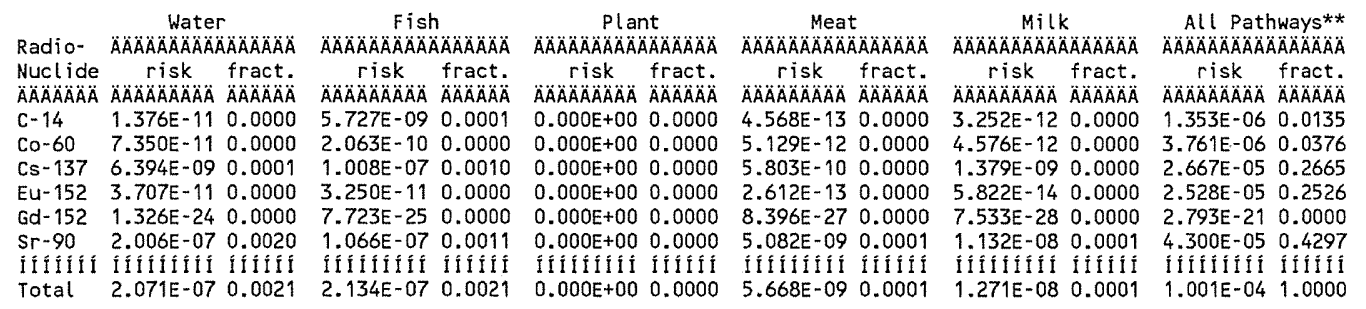

** Sum of water independent ground, inhalation, plant, meat, milk, soil

0

and water dependent water, fish, plant, meat, milk pathways

Total Excess Cancer Risk CNRSI $(i, p, t)^{* * *}$ for Initially Existent Radionuclides (i) and Pathways $(p)$ and Fraction of Total $R i s k$ at $t=7.000 E+00$ years
and

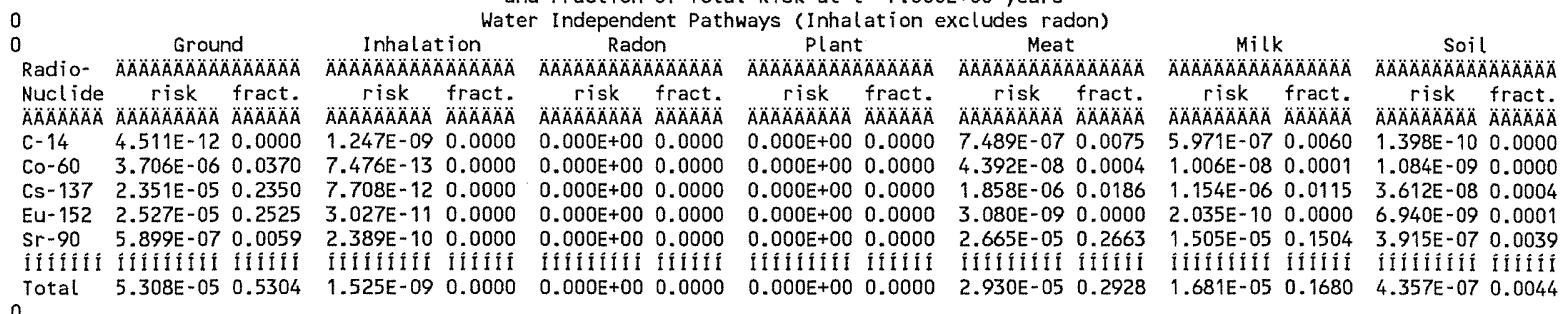

Total Excess Cancer Risk CNRSI $(i, p, t) * * *$ for Initially Existent Radionuclides $(i)$ and Pathways $(p)$ and Fraction of Total Risk at $t=7.000 E+00$ years

Water Dependent Pathways

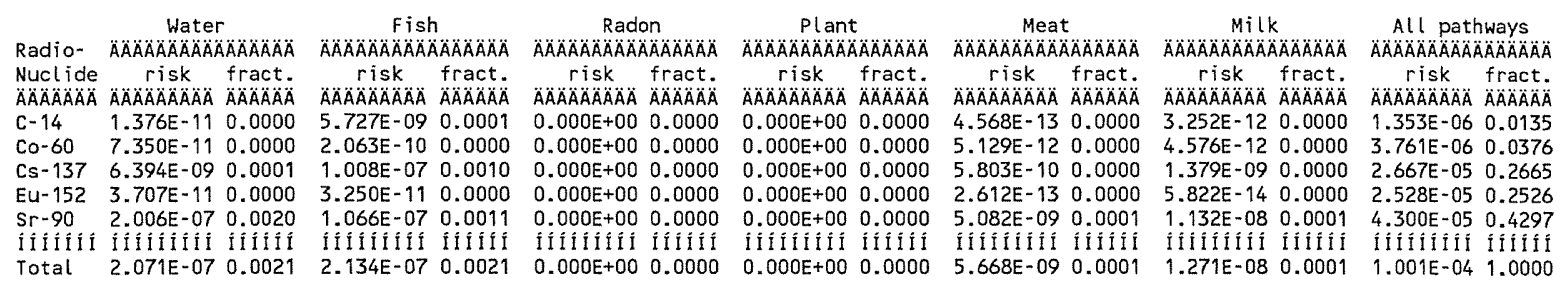

$* * * \operatorname{CNRSI}(i, p, t)$ includes contribution from decay daughter radionuclides

\begin{tabular}{|c|c|}
\hline \multirow{2}{*}{\multicolumn{2}{|c|}{ Attachment $\frac{4}{\text { S. W Clark }}$ Sheet No. 11 of 21}} \\
\hline & \\
\hline M. W. Perrott & \\
\hline $0100 B-C A-V 0308$ & Rev. No. \\
\hline
\end{tabular}


CVP-2007-00006

Rev. 0

\section{ATTACHMENT 4}

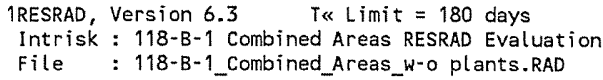

Water Independent Pathways (Inhalation w/o radon)

Water Dependent Pathways

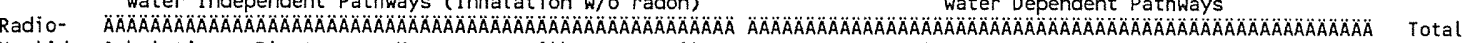
Nuclide Inhalation Plant Meat Milk soil Water Fish Plant Meat Milk Ingestion*

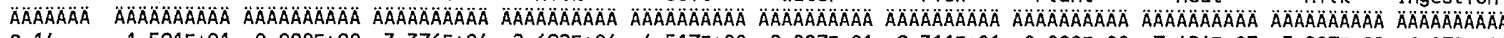
$\begin{array}{llllllllllll}\mathrm{C}-14 & 1.591 \mathrm{E}+01 & 0.000 \mathrm{E}+00 & 3.376 \mathrm{E}+04 & 2.692 \mathrm{E}+04 & 4.517 \mathrm{E}+00 & 2.887 \mathrm{E}-01 & 9.311 \mathrm{E}+01 & 0.000 \mathrm{E}+00 & 7.424 \mathrm{E}-03 & 5.287 \mathrm{E}-02 & 6.078 \mathrm{E}+04\end{array}$ $\begin{array}{llllllllllll}C 0-60 & 1.654 \mathrm{E}-03 & 0.000 E+00 & 1.560 \mathrm{E}+02 & 3.575 \mathrm{E}+01 & 2.131 \mathrm{E}+00 & 2.913 \mathrm{E}-01 & 5.754 \mathrm{E}-01 & 0.000 \mathrm{E}+00 & 1.430 \mathrm{E}-02 & 1.277 \mathrm{E}-02 & 1.948 \mathrm{E}+02\end{array}$ $\begin{array}{llllllllllll}\text { CS-137 } & 2.729 E-02 & 0.000 E+00 & 2.093 E+03 & 1.300 E+03 & 3.515 E+01 & 4.806 E+00 & 6.155 E+01 & 0.000 E+00 & 3.537 E-01 & 8.425 E-01 & 3.496 E+03\end{array}$

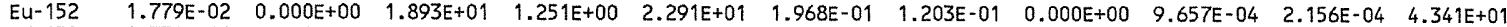

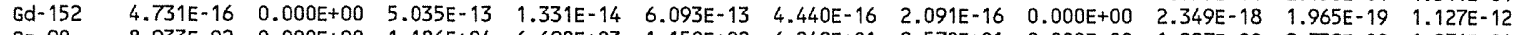
$\begin{array}{llllllllllll}\mathrm{Sr}-90 & 8.933 \mathrm{E}-02 & 0.000 \mathrm{E}+00 & 1.186 \mathrm{E}+04 & 6.698 \mathrm{E}+03 & 1.150 \mathrm{E}+02 & 6.249 \mathrm{E}+01 & 2.578 \mathrm{E}+01 & 0.000 \mathrm{E}+00 & 1.227 \mathrm{E}+00 & 2.739 \mathrm{E}+00 & 1.876 \mathrm{E}+04\end{array}$

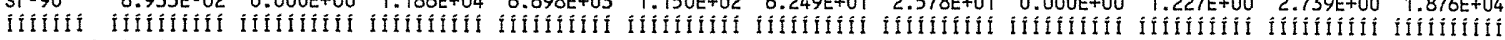
* Sum of all ingestion pathways, i.e. water independent plant, meat, milk, soil

0 and water-dependent water, fish, plant, meat, milk pathways

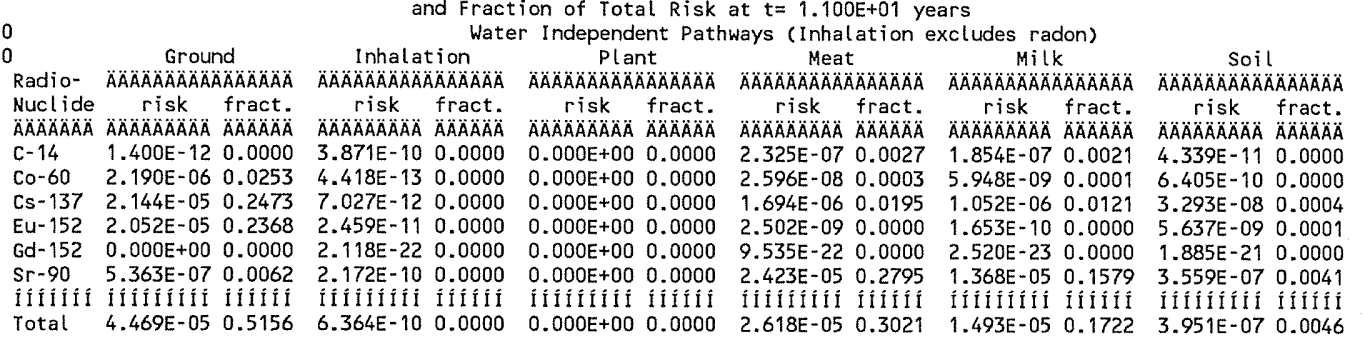

$\begin{array}{ll}\text { Attachment } \frac{4}{\text { S.W.Clark }} & \text { Sheet No. } 12 \text { of } 21 \\ \text { Originator: } & \text { Date } \\ \text { Chk'd By } & \text { Date } \\ \text { Calc. No. } \frac{\text { M. Perrott }}{0100 B-C A-V 0308} & \text { Rev. No. N }\end{array}$




\section{ATTACHMENT 4}

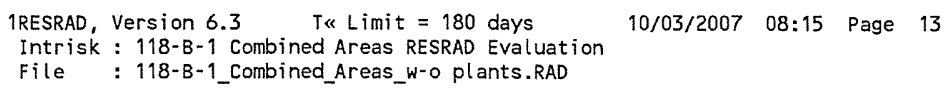

Water Dependent Pathways

\begin{tabular}{|c|c|c|c|c|c|c|c|c|c|c|c|c|}
\hline \multirow[b]{2}{*}{$\begin{array}{l}\text { Nucl ide } \\
\text { AAAAAAAAA }\end{array}$} & \multicolumn{2}{|c|}{ 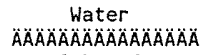 } & \multicolumn{2}{|c|}{ 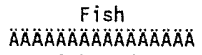 } & \multicolumn{2}{|c|}{ 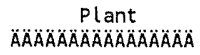 } & \multicolumn{2}{|c|}{$\begin{array}{c}\text { Meat } \\
\text { AAAAAAAAAAAAAAAA }\end{array}$} & \multicolumn{2}{|c|}{$\frac{\text { MilK }}{\text { ÄAAAAAAAAAAAAAOAOA }}$} & \multicolumn{2}{|c|}{$\begin{array}{r}\text { All Pathways** } \\
\text { AOAAAAAAAAAAAAAAAA }\end{array}$} \\
\hline & risk & $\begin{array}{l}\text { fract. } \\
\triangle A B A A A B A\end{array}$ & $\begin{array}{c}\text { risk } \\
A A O A B A B A B A\end{array}$ & & $\begin{array}{c}\text { risk } \\
\triangle A ̈ A ̈ A B A O A\end{array}$ & $\begin{array}{l}\text { fract. } \\
\text { ÄÄÄAAAOA }\end{array}$ & 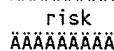 & $\begin{array}{l}\text { fract. } \\
\text { ÄAAAAÄA }\end{array}$ & risk & $\begin{array}{l}\text { fract. } \\
A A A A B A\end{array}$ & risk & \\
\hline & & & & & & & & & & & & \\
\hline & & & & & & & & & & & & \\
\hline & 6 & & & & & & & & & & & \\
\hline & & & & & & & & & & & & \\
\hline & & & & & & & & & & & & \\
\hline & & & & & & & & & & & & \\
\hline & & & & & & & & & & & & \\
\hline otal & $2.255 \mathrm{E}-07$ & 0.0026 & 21E-07 & 0.0027 & $E+00$ & 0.0000 & 6. & 0.0001 & $E=08$ & 0.0002 & -05 & 1.1 \\
\hline
\end{tabular}

** Sum of water independent ground, inhalation, plant, meat, milk, soil

and water dependent water, fish, plant, meat, milk pathways

Total Excess Cancer Risk CNRSI $(i, p, t)^{* * *}$ for Initially Existent Radionuclides (i) and Pathways $(p)$ and Fraction of Total Risk at $t=1.100 E+01$ years

G Ground Inhalation Water Independent Pathways (Inhalation excludes radon) Plant Madon Milk Soil

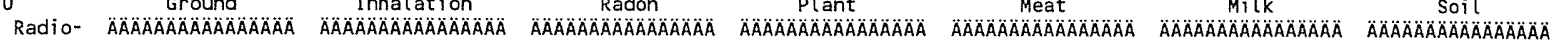
Nuclide risk fract. risk fract. risk fract. risk fract. risk fract. risk fract. risk fract.

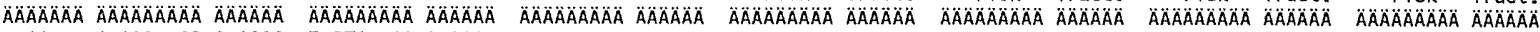

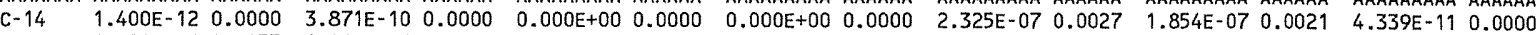

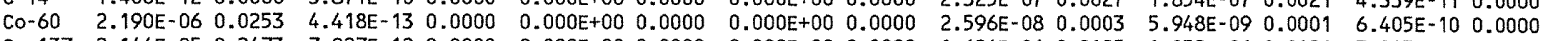

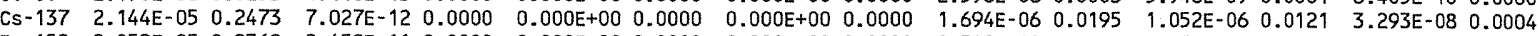
$\begin{array}{llllllllllllllllll}\text { Eu-152 } & 2.052 E-05 & 0.2368 & 2.459 E-11 & 0.0000 & 0.000 E+00 & 0.0000 & 0.000 E+00 & 0.0000 & 2.502 E-09 & 0.0000 & 1.653 E-10 & 0.0000 & 5.637 E-09 & 0.0001\end{array}$

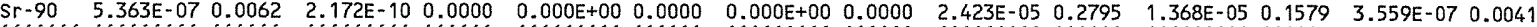

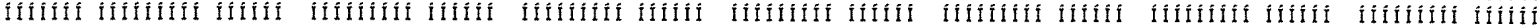
$\begin{array}{lllllllllllllllll}\text { Total } & 4.469 E-05 & 0.5156 & 6.364 E-10 & 0.0000 & 0.000 E+00 & 0.0000 & 0.000 E+00 & 0.0000 & 2.618 E-05 & 0.3021 & 1.493 E-05 & 0.1722 & 3.951 E-07 & 0.0046\end{array}$

Total Excess Cancer Risk CNRSI $(i, p, t) \star * *$ for Initially Existent Radionuclides ( $i$ ) and Pathways ( $p$ ) and Fraction of Total Risk at $t=1.100 E+01$ years

Water Dependent Pathways

\begin{tabular}{|c|c|c|c|c|c|c|c|c|c|c|c|c|c|c|}
\hline & $\begin{array}{r}\text { Wate } \\
\text { AaAAAAAAÄA }\end{array}$ & Г & $\begin{array}{r}F i s \\
\text { A }\end{array}$ & $\triangle A ̈ A ̈ A ̈ A ̈$ & $\begin{array}{r}R a d \\
\because A B A B A A B A B A\end{array}$ & $\triangle A \triangle A \cap A$ & $\begin{array}{r}P l a n \\
\triangle A A A A B A O A O A O A\end{array}$ & $\triangle A \cap A A O A O A$ & $\begin{array}{r}\text { Mea } \\
\triangle A A B A A A A A A O A\end{array}$ & $\triangle A \triangle A B A A A$ & $\begin{array}{r}M i l \\
\text { AAAAAAAAOÄA }\end{array}$ & $\triangle A A A A \cap A B$ & $\begin{array}{r}\text { All path } \\
A A B A A A A A A A\end{array}$ & 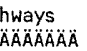 \\
\hline $\begin{array}{l}\text { Nucl ide } \\
\triangle A \triangle A B A A A\end{array}$ & $\begin{array}{c}\text { risk } \\
A A A A A A A B\end{array}$ & $\begin{array}{l}\text { fract. } \\
\text { AAAAAAAA }\end{array}$ & $\begin{array}{c}\text { risk } \\
A A A A A A B A\end{array}$ & $\begin{array}{l}\text { fract. } \\
A A A A A B A ̈\end{array}$ & $\begin{array}{c}\text { risk } \\
A A O A B A B A B A\end{array}$ & $\begin{array}{l}\text { fract. } \\
A \ddot{A} A ̈ A ̈ A ̈ A\end{array}$ & $\begin{array}{l}\text { risk } \\
A \triangle A A A O A B\end{array}$ & & $\begin{array}{c}\text { risk } \\
A A B A A A A A\end{array}$ & $\begin{array}{l}\text { fract. } \\
\ddot{A} \ddot{A} \ddot{A} A \bar{A}\end{array}$ & $\begin{array}{c}\text { risk } \\
\text { A AAAABAAÄABA }\end{array}$ & & $\begin{array}{c}\text { risk } \\
\triangle A A A A B A B A O A\end{array}$ & \\
\hline$c-14$ & $1.390 E-11$ & 0.0000 & $E-09$ & 0.0001 & $0.000 \mathrm{E}+00$ & .0000 & $0.000 E+00$ & 0.0000 & 13 & 0000 & 12 & 00 & $E-07$ & \\
\hline $0-6$ & $5.585 E-11$ & & -10 & & $E+00$ & & 0 & & & & & & -06 & 256 \\
\hline & 6.98 & U. & -07 & -2 & $E+\infty$ & 5 & $O E+O O$ & 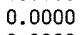 & & 0 & & 0 & 05 & \\
\hline -152 & $3.671 \mathrm{E}-11$ & 0.0000 & $=-11$ & 0.0000 & $0.000 \mathrm{E}+00$ & 0.0000 & $0.000 E+00$ & 0.0 & & & & 00 & & \\
\hline & & & & & & 0 & & & & & 1. & & & \\
\hline & & & & & & & & & & & & & & \\
\hline tal & $255 \mathrm{E}-07$ & 0.0026 & $E-07$ & .0027 & $E+\infty$ & .0000 & $O E+$ & 000 & Uy & 01 & & 02 & 86 & \\
\hline
\end{tabular}

***CNRSI $(i, p, t)$ includes contribution from decay daughter radionuclides

\begin{tabular}{|c|c|}
\hline Attachment & Sheet $N$ \\
\hline Originator: S. W. Clark & Date \\
\hline Chk'd By M.W. Perrott & Date \\
\hline Calc. No. $\quad 0100 \mathrm{~B}-\mathrm{CA}-\mathrm{V} 0308$ & Rev. No. \\
\hline
\end{tabular}


CVP-2007-00006

Rev. 0

\section{ATTACHMENT 4}

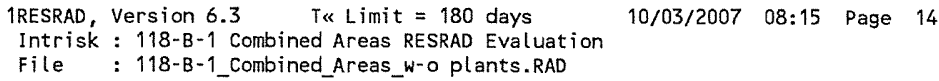

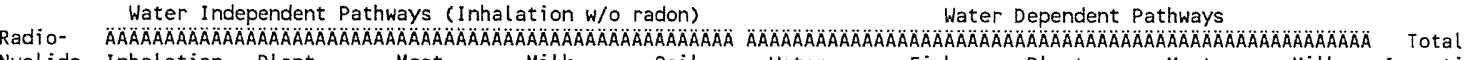

Water Dependent Pathways Nuclide Inhalation Plant Meat Milk Soil water Fish Plant Meat Milk Ingestion*

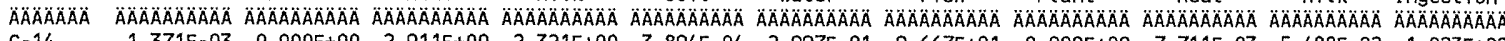
$\begin{array}{llllllllllll}\mathrm{C}-14 & 1.371 \mathrm{E}-03 & 0.000 \mathrm{E}+00 & 2.911 \mathrm{E}+00 & 2.321 \mathrm{E}+00 & 3.894 \mathrm{E}-04 & 2.997 \mathrm{E}-01 & 9.667 \mathrm{E}+01 & 0.000 \mathrm{E}+00 & 7.711 \mathrm{E}-03 & 5.488 \mathrm{E}-02 & 1.023 \mathrm{E}+02\end{array}$ $\begin{array}{llllllllllll}\mathrm{CO}-60 & 2.460 \mathrm{E}-05 & 0.000 \mathrm{E}+00 & 2.321 \mathrm{E}+00 & 5.317 \mathrm{E}-01 & 3.169 \mathrm{E}-02 & 1.694 \mathrm{E}-02 & 3.350 \mathrm{E}-02 & 0.000 \mathrm{E}+00 & 8.345 \mathrm{E}-04 & 7.427 \mathrm{E}-04 & 2.936 \mathrm{E}+00\end{array}$ $\begin{array}{llllllllllll}\text { CS-137 } & 1.303 E-02 & 0.000 E+00 & 9.990 E+02 & 6.205 E+02 & 1.678 E+01 & 8.970 E+00 & 1.150 E+02 & 0.000 E+00 & 6.627 E-01 & 1.573 E+00 & 1.763 E+03\end{array}$ $\begin{array}{llllllllllll}\text { EU-152 } & 3.369 E-03 & 0.000 E+00 & 3.585 E+00 & 2.369 E-01 & 4.338 E+00 & 1.457 E-01 & 8.918 E-02 & 0.000 E+00 & 7.177 E-04 & 1.597 E-04 & 8.396 E+00\end{array}$

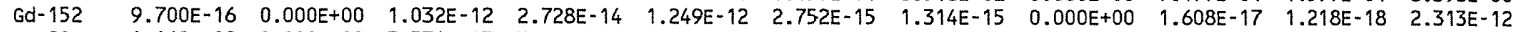

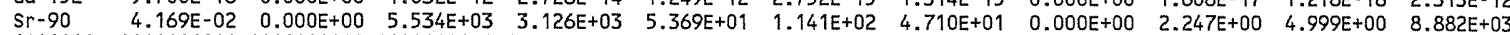

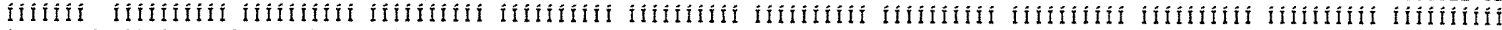
* Sum of all ingestion pathways, i.e. water independent plant, meat, milk, soil

0 and water-dependent water, fish, plant, meat, milk pathways

Excess Cancer Risks CNRS $(i, p, t)$ for Individual Radionuclides ( $i$ ) and Pathways $(p)$ and Fraction of Total Risk at $t=4.300 E+01$ years

\begin{tabular}{|c|c|c|c|c|c|c|c|c|c|c|c|}
\hline & & & & & & & VS & & & & \\
\hline & 40 & & Thisat & on & & & Meat & & $\mathrm{MiL}$ & & Soil \\
\hline & ÄÄÄÄÄÄA & ÄÄÄÄA & 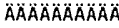 & $A \cap A ̈ A A A O A$ & $A \cap A O A A$ & $\triangle A O A O A O A Z A ̈$ & AAÄÄÄAAAÄA & $\triangle A O A A A O A ̈ A$ & AÄÄAOAAAOA & ÄÄÄAÄÄ & $\triangle A O A O A A A A O A O A ̈$ \\
\hline & & & & & & & $\ddot{A}$ & & risk & & risk \\
\hline$c-1$ & 1.2 & 0. & & & & & & & & & 5 \\
\hline & & & & & & & & & & & 20 \\
\hline & 1.0 & & & & & & & & & & \\
\hline & & & & & & & & & & & \\
\hline Gd- & & 0.0 & & & 0.0 & & & & & & 10 \\
\hline & & & & & & & & & & & \\
\hline & & & & & & & & & & & \\
\hline Total & & 0.4222 & -10 & & 0. & & & 552 & & 020 & $9 E-070$ \\
\hline
\end{tabular}

\begin{tabular}{ll} 
Attachment & \multicolumn{1}{c}{$\frac{4}{\text { Sheet No. } 14 \text { of } 21}$} \\
Originator: $\frac{\text { S.W. Clark }}{\text { M.W. Perrott }}$ & Date \\
Chk'd By & Date \\
Calc. No. & Rev. No. 0
\end{tabular}


CVP-2007-00006

Rev. 0

\section{ATTACHMENT 4}

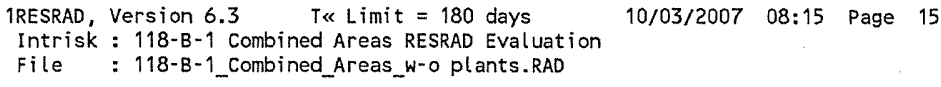

Water Dependent Pathways

\begin{tabular}{|c|c|c|c|c|c|c|}
\hline & $\begin{array}{l}\text { Water } \\
\text { ÄÄÄÄÄÄÄÄÄÄÄÄÄ }\end{array}$ & $\begin{array}{c}F i s h \\
\triangle A B A A A A A A A A A A A A\end{array}$ & $\begin{array}{c}P \text { lant } \\
A A A A A B A A A A A A A B A B\end{array}$ & $\begin{array}{c}\text { Meat } \\
\text { AAAAAAAAAAAAAAAAA }\end{array}$ & $\begin{array}{c}\text { Milk } \\
A A A A A A A A B A B A O A A A\end{array}$ & $\begin{array}{r}\text { All Pathways } \\
\text { AAAAAAAAAAAAAAA }\end{array}$ \\
\hline $\begin{array}{l}\text { JClide } \\
\text { AÄÄÄÄ }\end{array}$ & 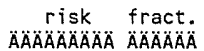 & $\begin{array}{l}\text { risk fract. } \\
\text { ÄÄÄAAA } \triangle A A O A O A O A\end{array}$ & $\begin{array}{l}\text { risk fract. } \\
\triangle A B A B A B A B A A A B A A\end{array}$ & 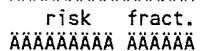 & $\begin{array}{l}\text { risk fract. } \\
\text { AAAAAABA AAAAAAA }\end{array}$ & risk fract. \\
\hline-14 & & & & & & \\
\hline & & & & & & \\
\hline & & & & & & \\
\hline & 1.6 & 10 & 00 & 130 & & \\
\hline & & & & & & \\
\hline & 2.3 & & & & & \\
\hline & & & & & & \\
\hline otal & 070. & 170.0074 & $+\infty 00$ & $8 E-09 \quad 0.0002$ & $503 E-08 \quad 0.0004$ & $.411 E-05 \quad 1.00$ \\
\hline
\end{tabular}

** Sum of water independent ground, inhalation, plant, meat, milk, soil

and water dependent water, fish, plant, meat, milk pathways

Total Excess Cancer Risk CNRSI $(i, p, t) * * *$ for Initially Existent Radionuclides (i) and Pathways $(p)$ and Fraction of Total $R$ isk at $t=4.300 \mathrm{E}+01$ years

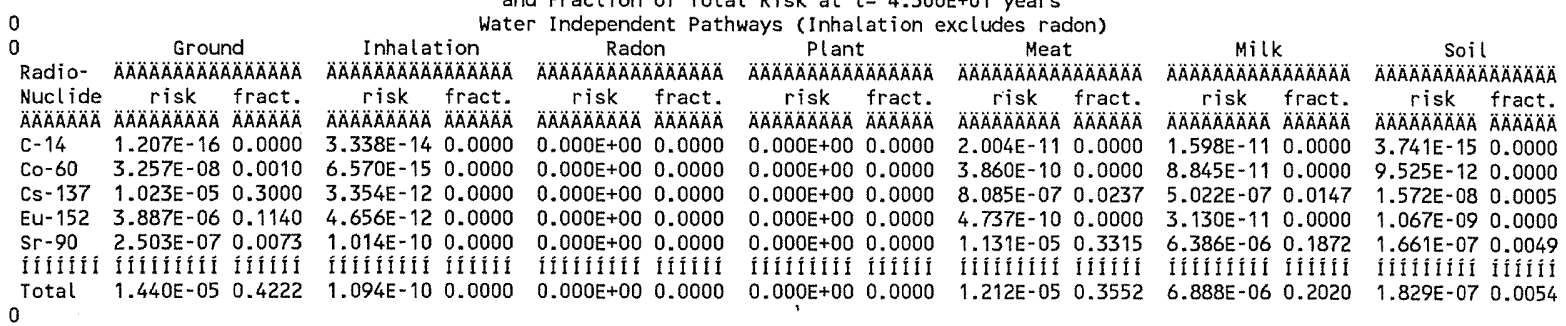

Total Excess Cancer Risk CNRSI $(i, p, t) * * *$ for Initially Existent Radionuclides ( $i)$ and Pathways ( $p$ ) and Fraction of Total Risk at $t=4.300 E+01$ years

Water Dependent Pathways

\begin{tabular}{|c|c|c|c|c|c|c|c|c|c|c|c|c|c|}
\hline & $\begin{array}{r}\text { Water } \\
\triangle A A A A A A A A A A A\end{array}$ & $\because \widetilde{A}$ & $\begin{array}{r}\text { Fish } \\
\triangle A A A \cap A A A O A B A ̈ A\end{array}$ & $\begin{array}{l}\text { h } \\
\ddot{A} \ddot{A} \ddot{A} A \ddot{A} A \ddot{A} A\end{array}$ & $\begin{array}{r}\text { Rado } \\
\text { ÄÄÄÄÄÄÄÄÄÄA }\end{array}$ & $\begin{array}{l}\text { on } \\
\ddot{A} \ddot{A} A \ddot{A} \ddot{A} \ddot{A} \ddot{A}\end{array}$ & $\begin{array}{r}\text { Plant } \\
\triangle A O A O A O A O A O A O A O A\end{array}$ & $\triangle A B A A A B A B A$ & 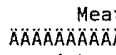 & $\triangle A A A A B A B A$ & $\begin{array}{r}M i l l \\
\triangle A A A A A ̈ A ̈ A ̈ A ̈ I\end{array}$ & ÄÄÄÄÄA & 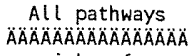 \\
\hline & & $\begin{array}{l}\text { fract. } \\
\text { ÄAAAAAAA }\end{array}$ & risk & & isk & fract. & risk & $\begin{array}{l}\text { fract. } \\
A O A A O A O A\end{array}$ & risk & fract. & risk & 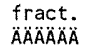 & $\begin{array}{l}\text { risk fract. } \\
\text { AAAAAAA AAAAAAOA }\end{array}$ \\
\hline & 1.3 & & & & & & & & & & & & 7 \\
\hline & & & & & & & & & & & & & 080 \\
\hline & & & & & & & & & & & & & \\
\hline & 1.65 & 0.0 & 1. & & 0 & & & & & & & & \\
\hline & & & & & & & & & & & & & \\
\hline & & & & & & & & & & & & & Iî́ ÎIIII \\
\hline & & 0.0072 & & 0.0074 & $=00$ & 0.0000 & $0.000 E+00$ & 0.0000 & $.708 E-09$ & 0.0002 & $3 E-08$ & 0.0004 & $3.411 \mathrm{E}-05 \quad 1.0000$ \\
\hline
\end{tabular}

***CNRSI $(i, p, t)$ includes contribution from decay daughter radionuclides

\begin{tabular}{|c|c|}
\hline Attachment & Sheet No. 15 of 21 \\
\hline Originator: S.W. Clark & Date \\
\hline Chk'd By M.W. Perrott & Date \\
\hline Calc. No. $\quad 0100 \mathrm{~B}-\mathrm{CA}-\mathrm{V} 0308$ & Rev. No. \\
\hline
\end{tabular}


CVP-2007-00006

Rev. 0

\section{ATTACHMENT 4}

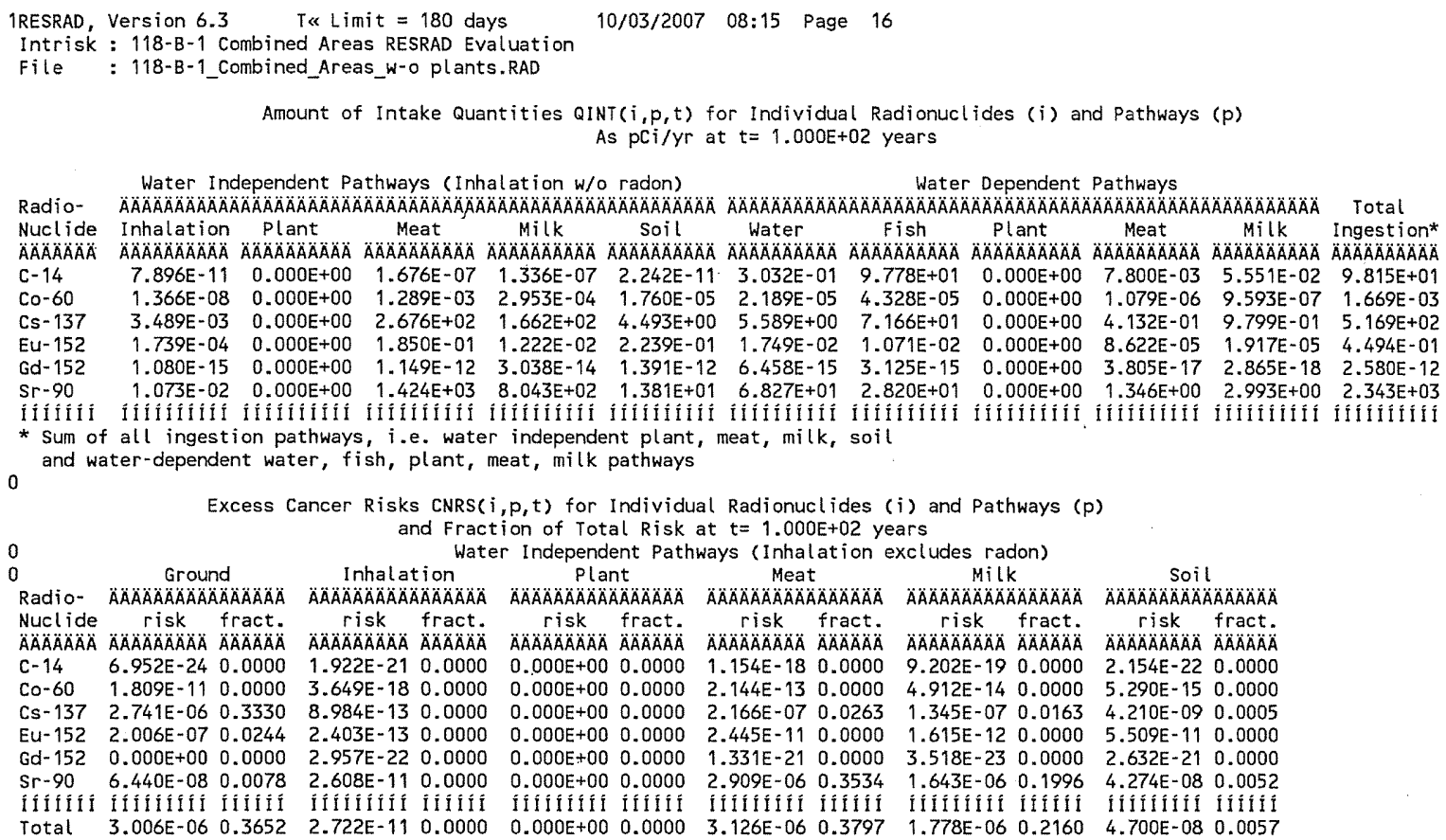

\begin{tabular}{|c|c|}
\hline Attachment & Sheet No. 16 of 21 \\
\hline Originator: S. W. Clark & Date \\
\hline Chk'd By M.W.Perrott & Date \\
\hline 0100B-CA-V0308 & Rev. No. 0 \\
\hline
\end{tabular}




\section{ATTACHMENT 4}

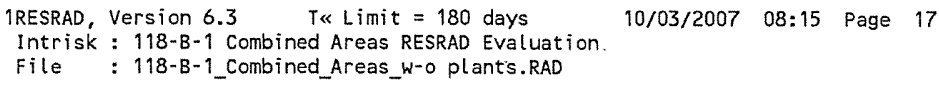

Water Dependent Pathways

\begin{tabular}{|c|c|c|c|c|c|c|c|c|c|c|}
\hline & $\begin{array}{c}\text { Water } \\
\text { AAAAOAAAAAAAAAOÄA }\end{array}$ & $\begin{array}{c}\text { Fish } \\
\triangle A A A A A A A A A A A A O A B\end{array}$ & $\begin{array}{r}P l a r \\
A B A A A A A O A O A\end{array}$ & $\begin{array}{l}\text { nt } \\
A B A O A O A O A O A\end{array}$ & $\begin{array}{r}M e a \\
\triangle A A A A A A A O A O A\end{array}$ & $\triangle A A A A A A A$ & 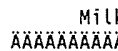 & $\triangle A O A A A O A$ & $\begin{array}{rl}A l l & P a \\
A C H A O A & \end{array}$ & 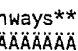 \\
\hline & $\begin{array}{l}\text { risk fract. } \\
A B A A A A B A A A B A B A\end{array}$ & 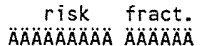 & $\begin{array}{c}\text { risk } \\
\text { ÄAAÄABAA }\end{array}$ & $\begin{array}{l}\text { fract. } \\
\triangle A O A O A B A\end{array}$ & risk & $\begin{array}{l}\text { fract. } \\
\text { ÄAOAOAOÄ }\end{array}$ & $\begin{array}{l}\text { risk } \\
\text { AAAABA }\end{array}$ & fract. & $\begin{array}{l}\text { risk } \\
\text { A }\end{array}$ & fract. \\
\hline & 110. & & & & & & & & & \\
\hline & & & & & & & & & & \\
\hline & & & & & & & & & & \\
\hline & 20. & & & & & & & & & \\
\hline & 240. & & & & & & & & & \\
\hline & & & & & & & & & & \\
\hline & & & & & & & & & & \\
\hline & & 070 & & 0.0 & & & & 170 & & \\
\hline
\end{tabular}

** Sum of water independent ground, inhalation, plant, meat, milk, soil

0

and water dependent water, fish, plant, meat, milk pathways

Total Excess Cancer Risk CNRSI $(i, p, t) * * *$ for Initially Existent Radionuclides (i) and Pathways ( $p$ ) and Fraction of Total $R i s k$ at $t=1.000 E+02$ years

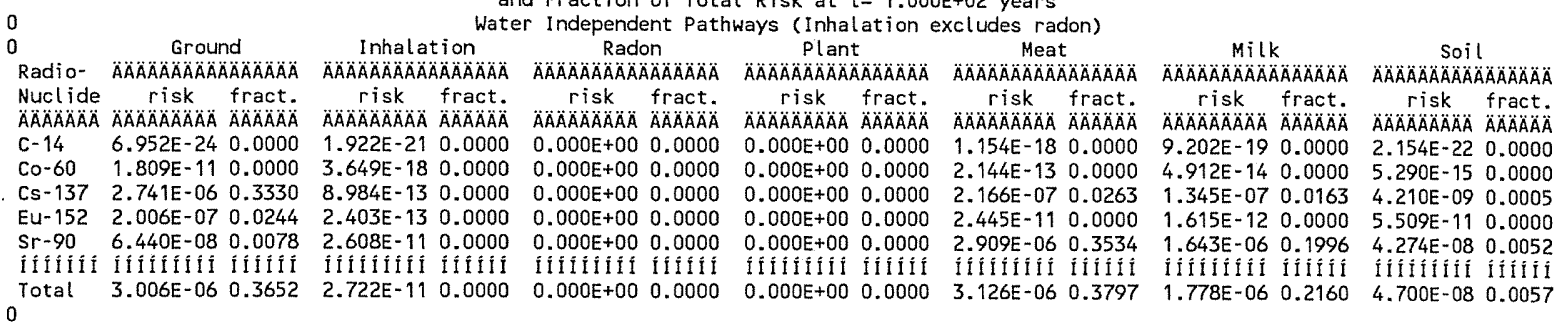

Total Excess Cancer Risk CNRSI $(i, p, t)^{* * *}$ for Initially Existent Radionuclides (i) and Pathways $(p)$ and Fraction of Total Risk at $t=1.000 E+02$ years

Water Dependent Pathways

\begin{tabular}{|c|c|c|c|c|c|c|c|}
\hline & $\begin{array}{c}\text { water } \\
\text { AAAAAAAAAAAAAOAOAOÄ }\end{array}$ & $\begin{array}{c}\text { Fish } \\
\text { ÄAAAAAAAAAAAOAZÄÄ }\end{array}$ & 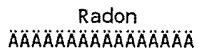 & $\begin{array}{c}\text { Plant } \\
\text { ALAAAAAAOAAAAAAAAA }\end{array}$ & 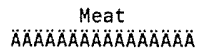 & 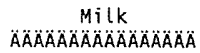 & 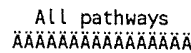 \\
\hline $\begin{array}{l}\text { Uclide } \\
A A A A B A B\end{array}$ & 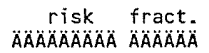 & $\begin{array}{r}\text { risk fract. } \\
\text { AAAÄÄÄÄ ÄÄÄÄÄA }\end{array}$ & 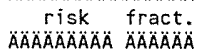 & $\begin{array}{l}\text { risk fract. } \\
\text { AAAAAAAA AAAAAAOA }\end{array}$ & $\begin{array}{l}\text { risk fract. } \\
\text { AAAAAAOA AAOAAÄÄ }\end{array}$ & $\begin{array}{l}\text { risk fract. } \\
\triangle A B A B A O A O A A A A B A\end{array}$ & 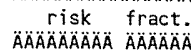 \\
\hline$=-14$ & $\begin{array}{ll}-11 & 0.0000\end{array}$ & 090.0007 & 000.0 & & & & \\
\hline & & & 00.0 & $=+\infty \quad 0$ & 160 & 160. & \\
\hline & & & & & & 100 & 06 \\
\hline & 120. & 120. & 00 & 000 & & & \\
\hline & 1. & 080. & & & & & \\
\hline & & & & & 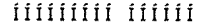 & 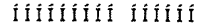 & \\
\hline 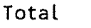 & $1.268 \mathrm{E}-07 \quad 0.0154$ & $1.367 E-070.0166$ & $0.000 E+00 \quad 0.0000$ & $0.000 E+00 \quad 0.0000$ & $3.493 E-09 \quad 0.0004$ & $7.825 \mathrm{E}-09 \quad 0.0010$ & $8.231 \mathrm{E}-06 \quad 1.0000$ \\
\hline
\end{tabular}

***CNRSI $(i, p, t)$ includes contribution from decay daughter radionuclides

\begin{tabular}{|c|c|}
\hline Attachment & Sheet No. 17 of 21 \\
\hline Originator: S.W. Clark & Date \\
\hline M. W. Perrott & Date \\
\hline $0100 B-C A-V 0308$ & Rev. No. \\
\hline
\end{tabular}


CVP-2007-00006

Rev. 0

\section{ATTACHMENT 4}

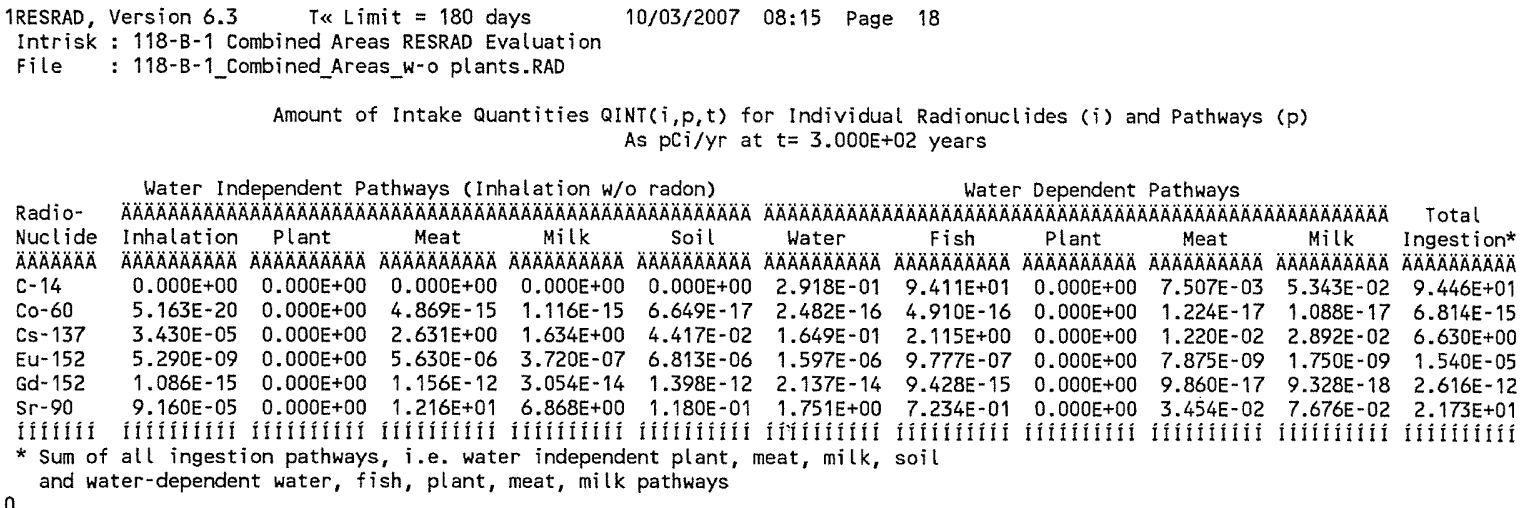

Water Independent Pathways (Inhalation w/o radon)

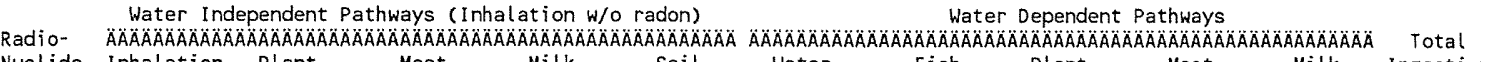
Nuclide Inhalation Plant Meat Milk Soil water Fish Plant Meat Milk Ingestion*

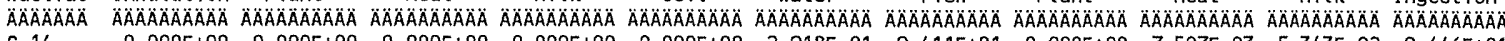

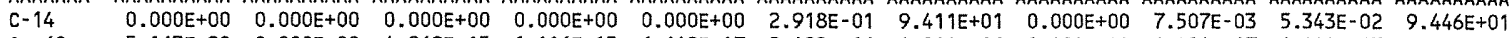

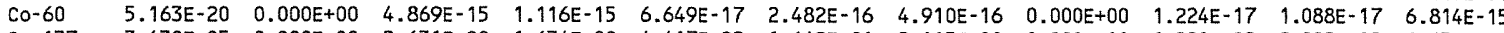

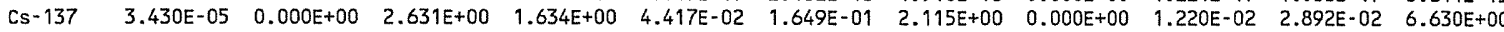
$\begin{array}{llllllllllll}\text { EU-152 } & 5.290 E-09 & 0.000 E+00 & 5.630 E-06 & 3.720 E-07 & 6.813 E-06 & 1.597 E-06 & 9.777 E-07 & 0.000 E+00 & 7.875 E-09 & 1.750 E-09 & 1.540 E-05\end{array}$

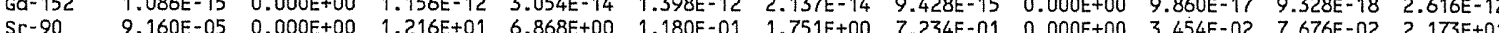

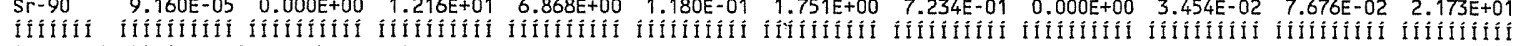
* Sum of all ingestion pathways, i.e. water independent plant, meat, milk, soil and water-dependent water, fish, plant, meat, milk pathways

0

Excess Cancer Risks CNRS $(i, p, t)$ for Individual Radionuclides (i) and Pathways $(p)$ and Fraction of Total Risk at $t=3.000 E+02$ years Water Independent Pathways (Inhalation excludes radon)

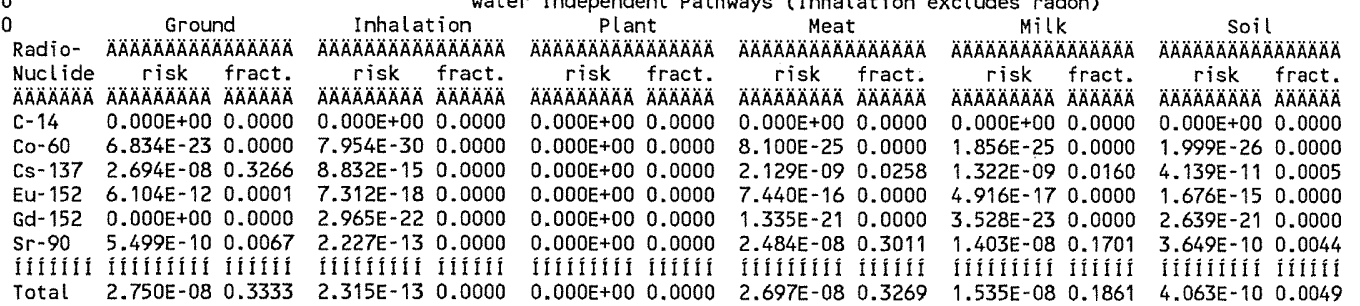

Attachment 4 Sheet No. 18 of 21 
CVP-2007-00006

Rev. 0

\section{ATTACHMENT 4}

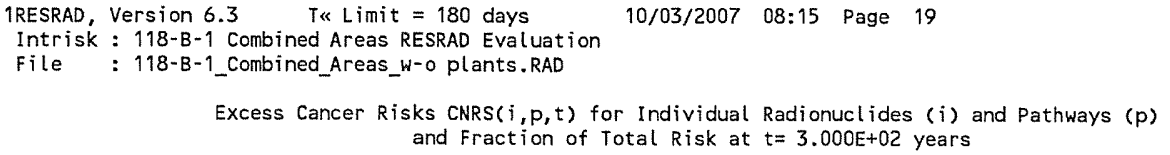

Water Dependent Pathways

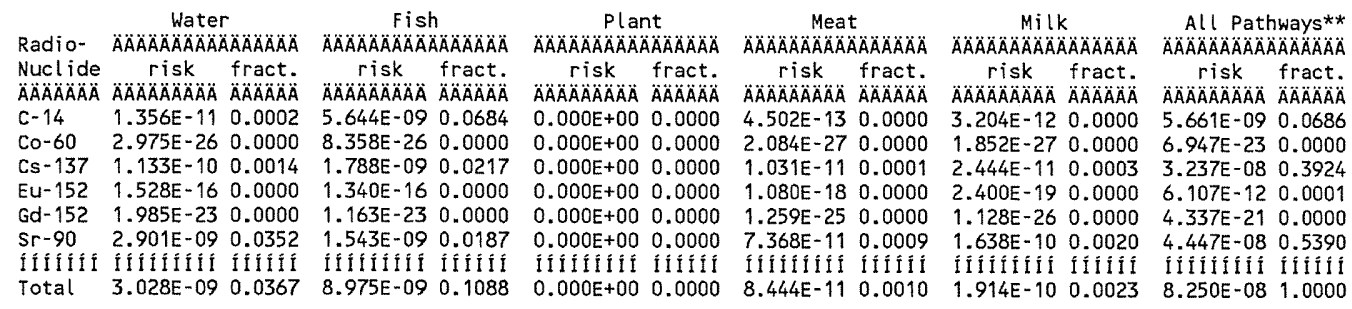

** Sum of water independent ground, inhalation, plant, meat, milk, soil

0 and water dependent water, fish, plant, meat, milk pathways

Total Excess Cancer Risk CNRSI $(i, p, t)^{* * *}$ for Initially Existent Radionuclides $(i)$ and Pathways $(p)$ and Fraction of Total $R i s k$ at $t=3.000 E+02$ years
and

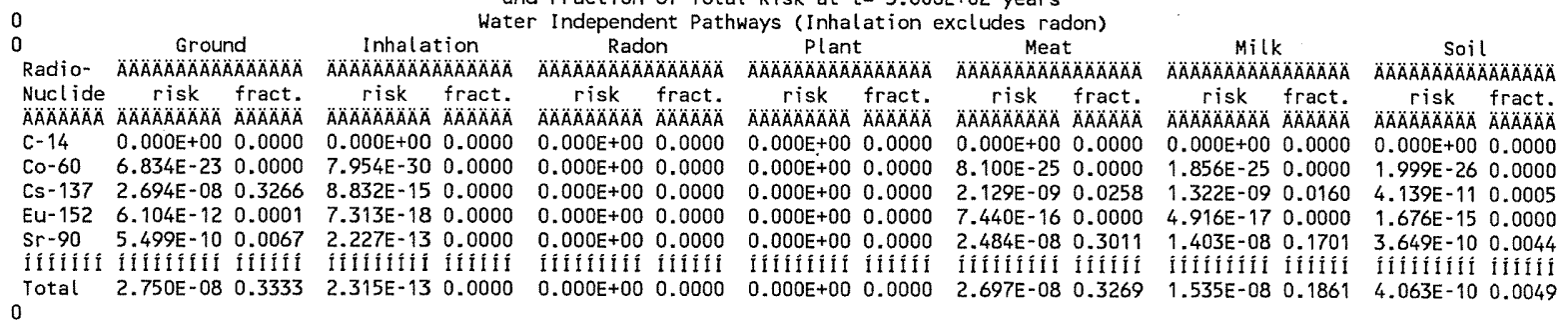

Total Excess Cancer Risk CNRSI $(i, p, t)^{* * *}$ for Initially Existent Radionuclides (i) and Pathways ( $p$ ) and Fraction of Total Risk at $t=3.000 E+02$ years

Water Dependent Pathways

\begin{tabular}{|c|c|c|c|c|c|c|c|c|c|c|c|c|c|}
\hline & $\begin{array}{r}\text { Water } \\
\text { ÄAAAAOAAAAA }\end{array}$ & 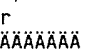 & $\begin{array}{r}\text { Fish } \\
\text { AAAAAAAAÁ }\end{array}$ & 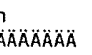 & $\begin{array}{r}\text { Rado } \\
\text { ÄÄAAAAAAÄA }\end{array}$ & on & $\begin{array}{r}\text { Plant } \\
\text { ÄÄAAAAAAAA }\end{array}$ & T & $\begin{array}{r}\text { Meat } \\
\text { ÄÄAAAAAOAOA }\end{array}$ & AOAAAAA & $\begin{array}{r}\text { MilK } \\
\text { AAAAAAAAOÄ }\end{array}$ & $\triangle A ̈ A \cap A \cap A ̈ A ̈$ & $\begin{array}{c}\text { All pathways } \\
\text { ALAAAAAAAAAAAAAAA }\end{array}$ \\
\hline de & risk & $\begin{array}{l}\text { fract. } \\
\not A B A A B A B\end{array}$ & $\begin{array}{r}r i s k \\
\triangle A A A B A B A B A\end{array}$ & $\begin{array}{l}\text { fract. } \\
\not A B A B A B A\end{array}$ & risk & fract. & risk & $\begin{array}{l}\text { fract. } \\
\triangle A A B A A A A\end{array}$ & risk & & $\begin{array}{c}\text { risk } \\
A A B A A A B\end{array}$ & & $\begin{array}{cc}r i s k & f r \\
A B A A A B A B & A B A\end{array}$ \\
\hline 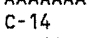 & 1. & & 5.6 & 0.0 & & & & & & & & & $A B$ \\
\hline & & & & & & & & & & & & & 230 \\
\hline & & & & & & & & & & & & & \\
\hline & 1.5 & 0. & 1.3 & & 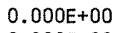 & & 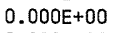 & & & & & & \\
\hline & & & & & & & & & & & & & \\
\hline & & & & & & & & & & & & filifí & IIIIIIIIII İ \\
\hline rotal & -09 & 0.0367 & 8. & 0.1088 & $0.000 E+00$ & 0.0000 & $0.000 E+00$ & 0.0000 & $8.444 E-11$ & 0.0010 & $1.914 E-10$ & 0.0023 & $8.250 \mathrm{E}-08 \quad 1.00$ \\
\hline
\end{tabular}

***CNRSI $(i, p, t)$ includes contribution from decay daughter radionucl ides

\begin{tabular}{|c|c|}
\hline Attachment & Sheet No. 10 \\
\hline Originator: S.W. Clark & Date \\
\hline M. W. Perrott & Date \\
\hline 0100B-CA-V0308 & $\operatorname{Rev}$ \\
\hline
\end{tabular}




\section{ATTACHMENT 4}

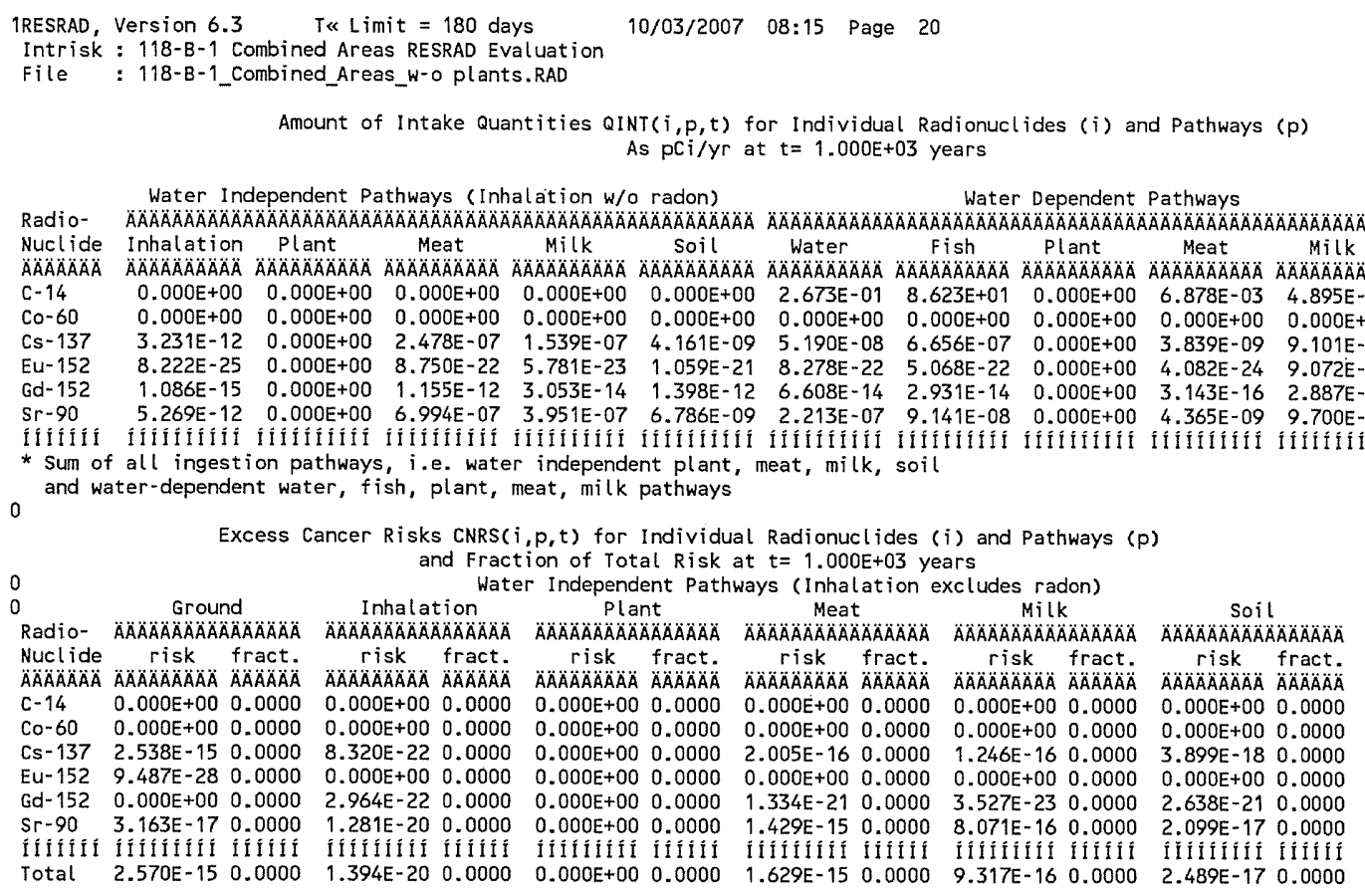

\begin{tabular}{ll} 
Attachment & \multicolumn{1}{c}{$\begin{array}{c}\text { Sheet No. } 20 \text { of } 21 \\
\text { Originator: }\end{array}$} \\
Ch.W. W. Clark & Date \\
Chk'd By & Date \\
Calc. No. Perrott & Rev. No. $\quad 0$ \\
\hline
\end{tabular}




\section{ATTACHMENT 4}

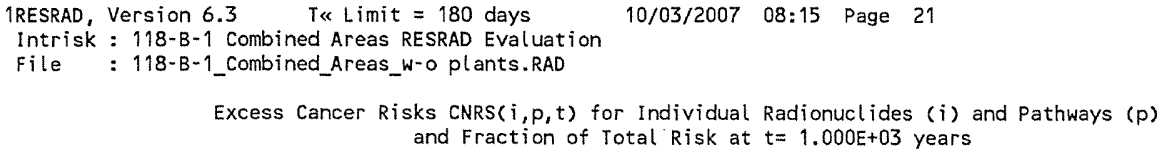

Water Dependent Pathways

\begin{tabular}{|c|c|c|c|c|c|c|c|c|c|c|c|c|}
\hline & \multicolumn{2}{|c|}{ Water } & \multicolumn{2}{|c|}{ Fish } & \multicolumn{2}{|c|}{ Plant } & \multicolumn{2}{|c|}{$\begin{array}{c}\text { Meat } \\
\text { AAAAAAAAAAAAAAAÄ }\end{array}$} & \multicolumn{2}{|c|}{$\begin{array}{c}M i l k \\
\text { AAAAAAAAAAAAAAAA }\end{array}$} & \multicolumn{2}{|c|}{$\begin{array}{r}\text { All Pathways** } \\
\text { AAAAAOAAAAAAAAAAA }\end{array}$} \\
\hline 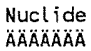 & $\begin{array}{l}\text { risk } \\
A B A A B A B\end{array}$ & $\begin{array}{l}\text { fract. } \\
\text { ÄÄÄAOA }\end{array}$ & $\begin{array}{l}\text { Isk } \\
A B A A O A B\end{array}$ & $\begin{array}{l}\text { fra } \\
\ddot{A} \ddot{A} A ̈\end{array}$ & $\begin{array}{l}\text { risk } \\
\triangle A ̈ A A A B A\end{array}$ & & $\begin{array}{l}\text { risk } \\
A B A A B A B A\end{array}$ & & $\begin{array}{c}r i s k \\
A A B A B A B A\end{array}$ & & risk & \\
\hline 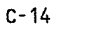 & & & & & & & & & & & & \\
\hline & & & & & & & & & & & & \\
\hline & & & & & & & & & & & & \\
\hline & 0.0 & & & 0.0 & & & & & & & & \\
\hline & & & & & & & & & & & & \\
\hline & & & & & & & & & & & & \\
\hline & & & & & & & & & & & & \\
\hline & & & & 0 & +00 & & $5-1$ & & $=-16$ & 0.0006 & $5.181 \mathrm{E}-09$ & 1.000 \\
\hline
\end{tabular}

** sum of water independent ground, inhalation, plant, meat, milk, soil

and water dependent water, fish, plant, meat, milk pathways

Total Excess Cancer Risk CNRSI $(i, p, t) * * *$ for Initially Existent Radionuclides (i) and Pathways ( $p$ ) and Fraction of Total $R$ isk at $t=1.000 E+03$ years

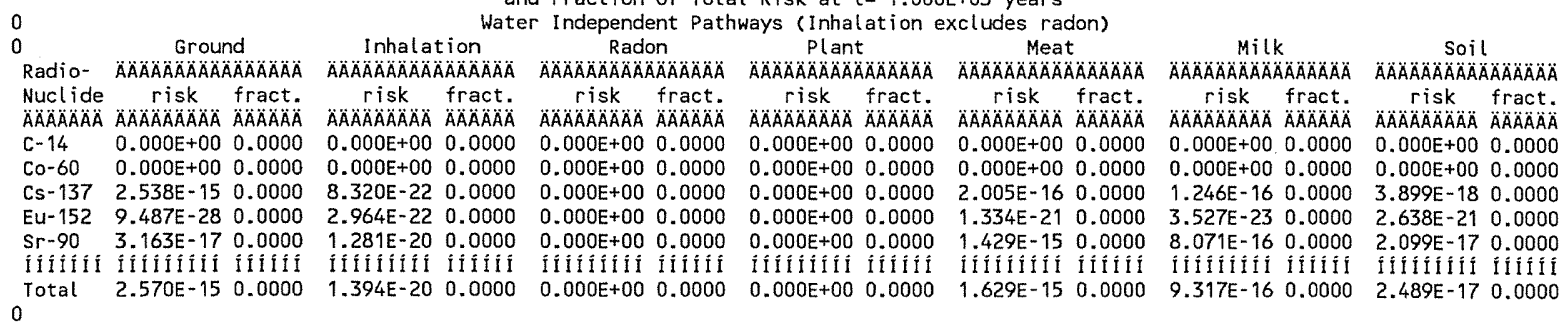

Total Excess Cancer Risk CNRSI $(i, p, t) * * *$ for Initially Existent Radionuclides ( $i)$ and Pathways ( $p$ ) and Fraction of Total Risk at $t=1.000 E+03$ years

Water Dependent Pathways

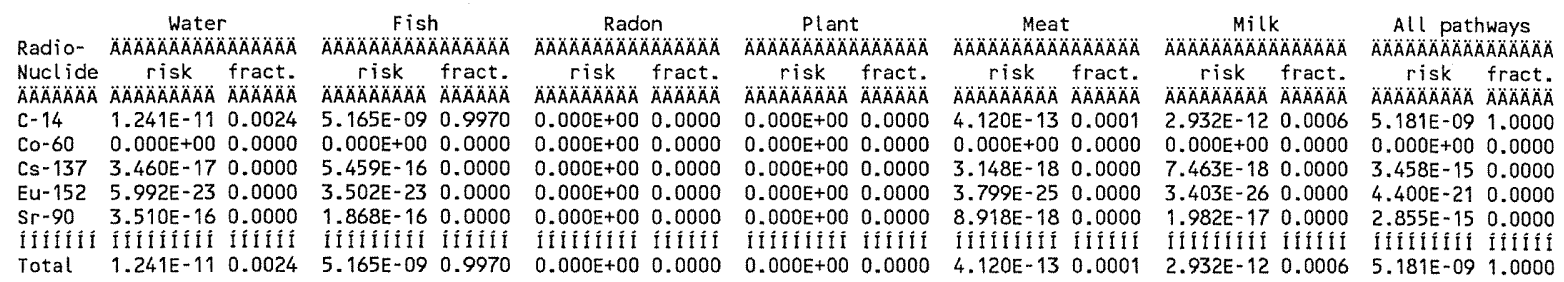

***CNRSI $(i, p, t)$ includes contribution from decay daughter radionuclides

\begin{tabular}{|c|c|}
\hline \multirow{2}{*}{\multicolumn{2}{|c|}{$\begin{array}{l}\text { Attachment } \frac{4}{\text { Originator: S.W. Clark }} \quad \text { Sheet No. } 21 \text { of } \underline{21} \\
\text { Date }\end{array}$}} \\
\hline & \\
\hline Chk'd By M.W. Perrott & Date \\
\hline $0100 B-C A-V 0308$ & Rev. No. \\
\hline
\end{tabular}




\section{ATTACHMENT 5}

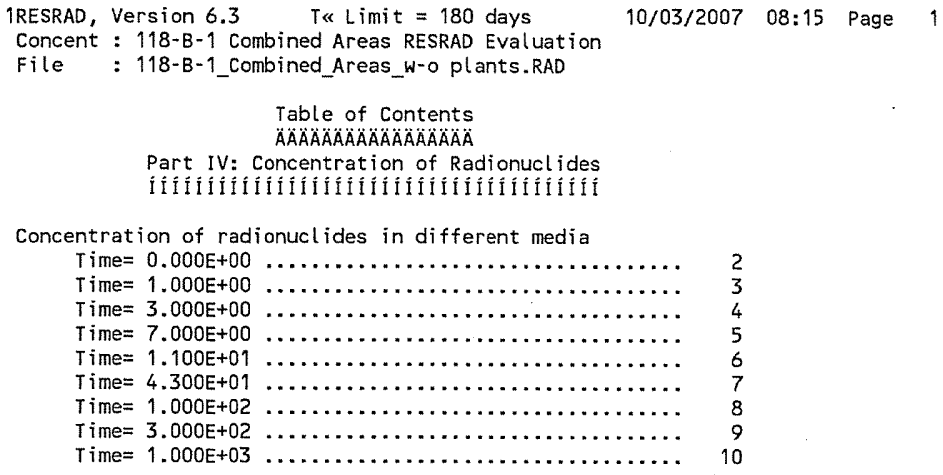

Attachment $\frac{5}{5}$ Sheet No. 1 of 10 Originator: S.W. Clark Br.e Date $/ 0 / 9 / 07$ Chk'd By M.W. Perrott Ticef Date 10/3/0 Calc. No. 0100B-CA-V0308 Rev. No. 0 
CVP-2007-00006

Rev. 0

\section{ATTACHMENT 5}

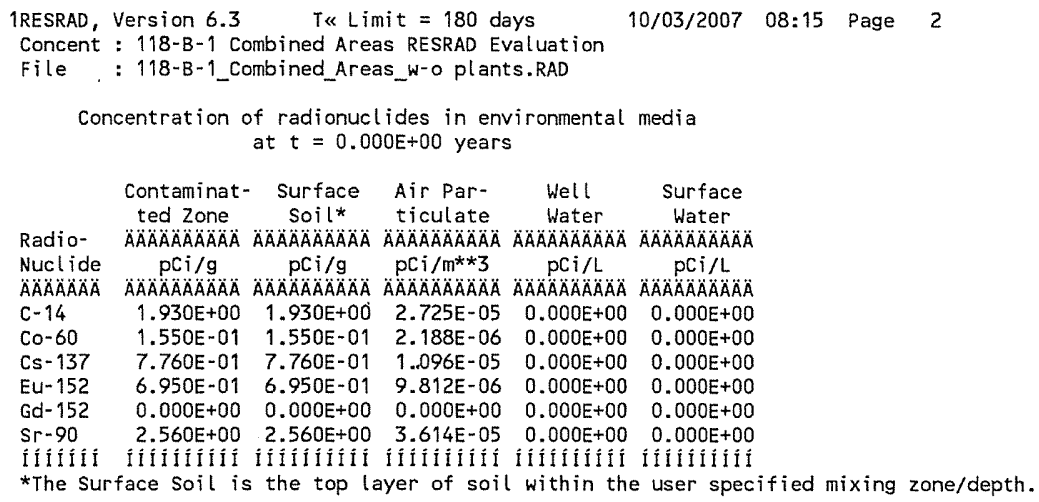

Concentrations in the media occurring in pathways that are suppressed are calculated using the current input parameters, i.e. using parameters appearing in the input screen when the pathways are active.

Concentration of gaseous $\mathrm{C}-14$ in air $=1.677 \mathrm{E}+01 \mathrm{pCi} / \mathrm{m}^{* * 3}$

\begin{tabular}{|c|c|c|c|c|c|c|c|c|c|}
\hline \multicolumn{10}{|c|}{$\begin{array}{c}\text { Concentration of radionuclides in foodstuff media } \\
\text { at } t=0.000 E+00 \text { years* }\end{array}$} \\
\hline & $\begin{array}{l}\text { Drinking } \\
\text { Water }\end{array}$ & $\begin{array}{l}\text { Nonleafy } \\
\text { Vegetable }\end{array}$ & $\begin{array}{l}\text { Leafy } \\
\text { Vegetable }\end{array}$ & $\begin{array}{l}\text { Fodder } \\
\text { Meat }\end{array}$ & $\begin{array}{l}\text { Fodder } \\
\text { Milk }\end{array}$ & Meat & Milk & Fish & Crustacea \\
\hline 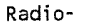 & $\triangle A A A A A A A A A A A$ & 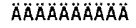 & 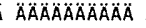 & AAAAAAAAAÄÄ & AAAAAAAAAAAAA & 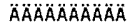 & $\triangle A A O A A A A O A A A ̈$ & $\triangle A O A O A A O A O A O A ̈ A ̈$ & $\triangle A A A A A A A B A O A$ \\
\hline & & & & & & $\mathrm{pCi} / \mathrm{kg}$ & & & \\
\hline & $\triangle A A A A A A A A O A O A ̈$ & $\triangle A A A A A A A$ & $\triangle A A A A A O A$ & $\ddot{A} A$ & $A A A B$ & $\triangle A A A A$ & $\triangle A A A A A A$ & $\triangle A A B A$ & $A A B A$ \\
\hline$c-14$ & $0.000 E+00$ & $3.704 E+04$ & $8.333 E+03$ & $3.129 \mathrm{E}+04$ & $1.407 E+04$ & $2.222 E+04$ & $6.475 E+03$ & $0.000 E+00$ & $0.000 \mathrm{E}+00$ \\
\hline Co- 60 & $0.000 E+00$ & $1.240 E+01$ & $1.240 E+01$ & $1.240 E+01$ & $1.240 E+01$ & $1.841 E+01$ & $1.519 \mathrm{E}+00$ & $0.000 E+00$ & $0.000 E+00$ \\
\hline & 0.00 & 3.10 & 3.10 & & & & & +00 & $+\infty$ \\
\hline $\mathrm{Eu}-$ & 0.00 & $1.738 E+00$ & $1.740 E+00$ & 1.7 & 1.740 & $9.317 E-01$ & $2.216 E-02$ & $0.000 E+00$ & $0.000 E+00$ \\
\hline Gd- 152 & $0.000 E+00$ & $0.000 E+00$ & $0.000 E+00$ & $0.000 E+00$ & 0.000 & $0.000 E+00$ & $0.000 E+00$ & $0.000 E+00$ & $0.000 E+00$ \\
\hline & $0.000 E+00$ & $7.680 \mathrm{E}+02$ & $7.680 \mathrm{E}+02$ & $7.680 E+02$ & $7.680 \mathrm{~B}$ & & 8. $704 E+01$ & $0.000 E+00$ & $0.000 \mathrm{E}+00$ \\
\hline$f t$ & $f ; F^{\prime}$ & if & $n$ time & 5 & $k$ & 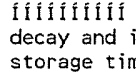 & $f \neq=0$ & & Ifif \\
\hline
\end{tabular}

Concentrations in the media occurring in pathways that are suppressed are calculated using the current input parameters, i.e. using parameters appearing in the input screen when the pathways are active.

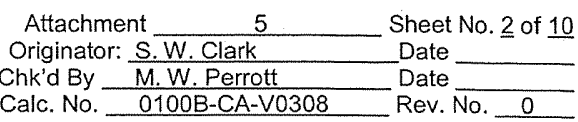




\section{ATTACHMENT 5}

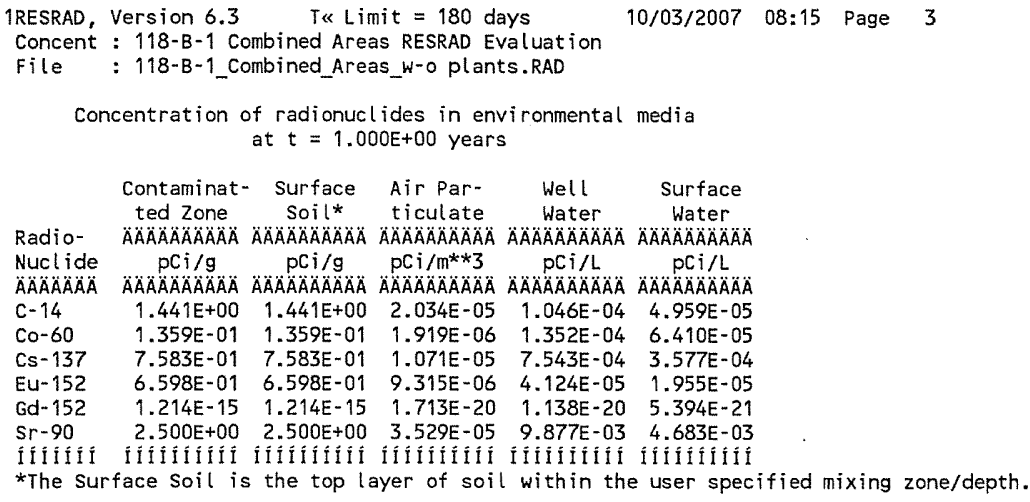

Concentrations in the media occurring in pathways that are suppressed are calculated using the current input parameters, i.e. using parameters appearing in the input screen when the pathways are active. Concentration of gaseous $\mathrm{C}-14$ in air $=1.252 \mathrm{E}+01 \mathrm{pCi} / \mathrm{m}^{* * 3}$

Concentration of radionuclides in foodstuff media at $t=1.000 \mathrm{E}+00$ years*

Drinking Nonleafy Leafy Fodder Fodder Meat Milk Fish Crustacea Water vegetable vegetable Meat Milk

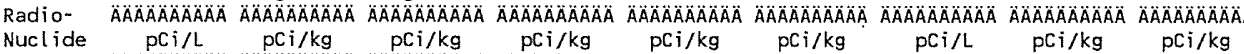

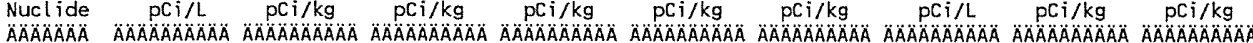

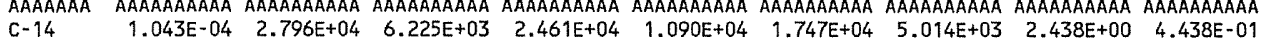
$\begin{array}{llllllllllll}\mathrm{C} 0-60 & 1.348 \mathrm{E}-04 & 1.087 \mathrm{E}+01 & 1.087 \mathrm{E}+01 & 1.095 \mathrm{E}+01 & 1.088 \mathrm{E}+01 & 1.615 \mathrm{E}+01 & 1.332 \mathrm{E}+00 & 1.886 \mathrm{E}-02 & 1.257 \mathrm{E}-02\end{array}$ $\begin{array}{lllllllllll}\mathrm{Cs}-137 & 7.522 \mathrm{E}-04 & 3.033 \mathrm{E}+01 & 3.033 \mathrm{E}+01 & 3.037 \mathrm{E}+01 & 3.034 \mathrm{E}+01 & 7.326 \mathrm{E}+01 & 1.638 \mathrm{E}+01 & 7.016 \mathrm{E}-01 & 3.508 \mathrm{E}-02\end{array}$ $\begin{array}{llllllllll}\text { EU-152 } & 4.112 E-05 & 1.650 E+00 & 1.652 E+00 & 1.657 E+00 & 1.652 E+00 & 8.845 E-01 & 2.104 E-02 & 9.589 E-04 & 1.918 E-02\end{array}$ $\begin{array}{llllllllll}\text { Gd-152 } & 1.127 E-20 & 3.035 E-15 & 3.038 E-15 & 2.876 E-15 & 3.031 E-15 & 1.627 E-15 & 1.554 E-17 & 1.424 E-19 & 5.038 E-18\end{array}$

$\begin{array}{llllllllll}\mathrm{Sr}-90 & 9.850 \mathrm{E}-03 & 7.499 \mathrm{E}+02 & 7.499 \mathrm{E}+02 & 7.509 \mathrm{E}+02 & 7.500 \mathrm{E}+02 & 4.180 \mathrm{E}+02 & 8.500 \mathrm{E}+01 & 2.756 \mathrm{E}-01 & 4.594 \mathrm{E}-01\end{array}$

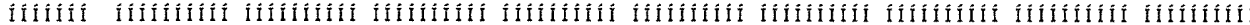

*Concentrations are at consumption time and include radioactive decay and ingrowth during storage time.

For livestock fodder, consumption time is $t$ minus meat or milk storage time.

Concentrations in the media occurring in pathways that are suppressed are calculated using the current input parameters, i.e. using parameters appearing in the input screen when the pathways are active.

$\begin{array}{ll}\text { Attachment } \frac{5}{1} & \text { Sheet No. } \underline{3} \text { of } \underline{10} \\ \text { Originator: } \frac{\mathrm{S} . \text { W. Clark }}{\text { D.W. Perrott }} & \text { Date } \\ \text { Chk'd By } & \text { Date } \\ \text { Calc. No. } & \text { Rev. No. } 0\end{array}$


CVP-2007-00006

Rev. 0

\section{ATTACHMENT 5}

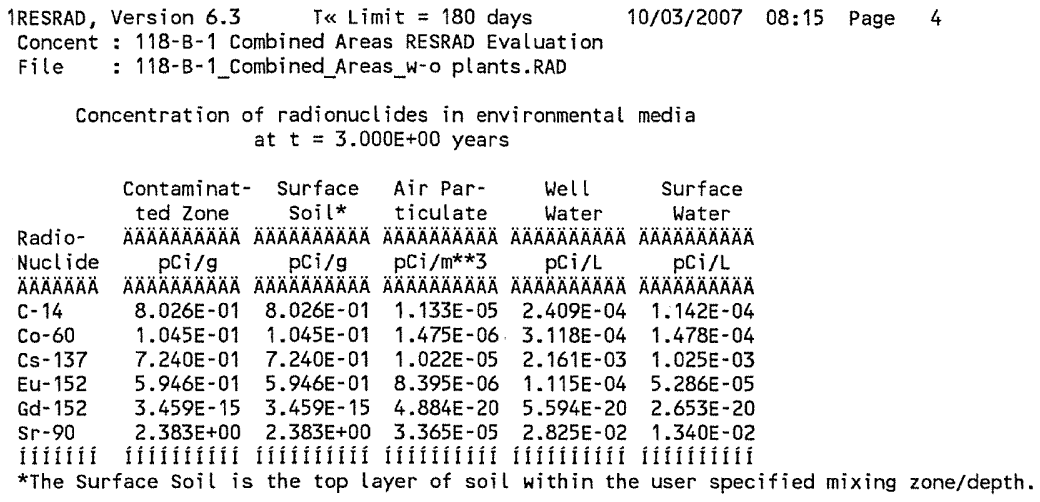

Concentrations in the media occurring in pathways that are suppressed are calculated using the current input parameters, i.e. using parameters appearing in the input screen when the pathways are active.

Concentration of gaseous $\mathrm{C}-14$ in air $=6.974 \mathrm{E}+00 \mathrm{pCi} / \mathrm{m}^{* * 3}$

Concentration of radionuclides in foodstuff media at $t=3.000 E+00$ years*

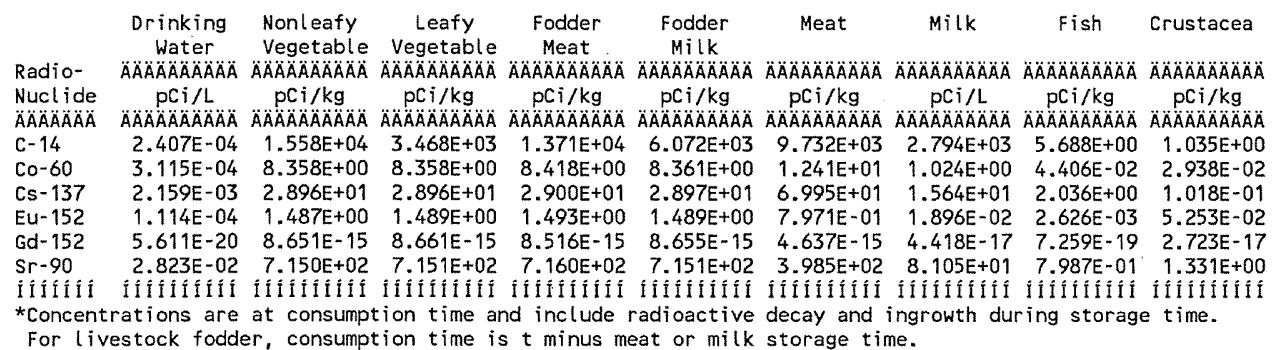

For livestock fodder, consumption time is $t$ minus meat or milk storage time.

Concentrations in the media occurring in pathways that are suppressed are calculated using the current input parameters, i.e. using parameters appearing in the input screen when the pathways are active.

\begin{tabular}{|c|c|}
\hline Attachment & Sheet No. 4 of 10 \\
\hline Originator: S.W. Clark & Date \\
\hline Chk'd By M.W. Perrott & Date \\
\hline $0100 \mathrm{~B}-\mathrm{CA}-\mathrm{V} 0308$ & Rev. No. 0 \\
\hline
\end{tabular}


CVP-2007-00006

Rev. 0

\section{ATTACHMENT 5}

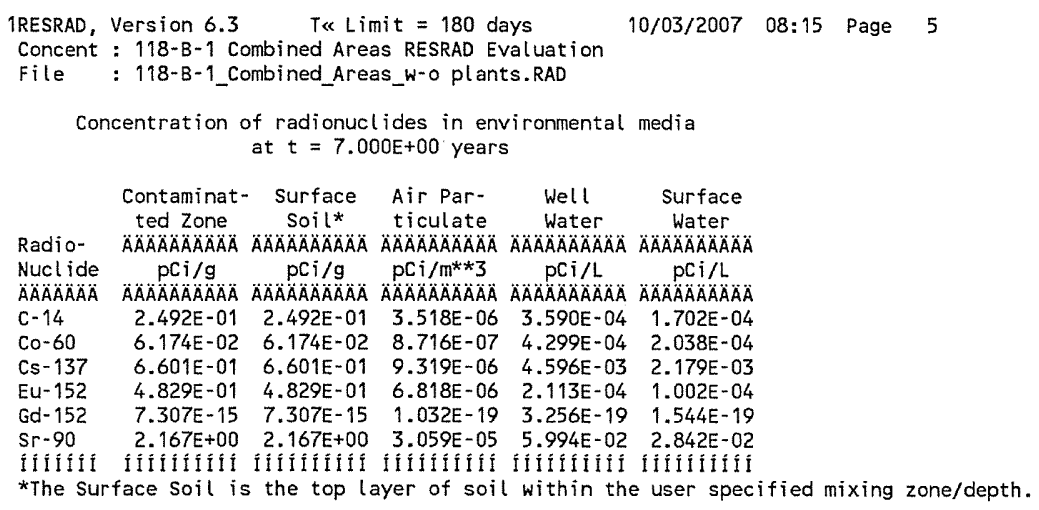

Concentrations in the media occurring in pathways that are suppressed are calculated using the current input parameters, i.e. using parameters appearing in the input screen when the pathways are active.

Concentration of gaseous $\mathrm{C}-14$ in air $=2.165 \mathrm{E}+00 \mathrm{pCi} / \mathrm{m}^{\star *} 3$

\section{Concentration of radionuclides in foodstuff media} at $\mathrm{t}=7.000 \mathrm{E}+00$ years*

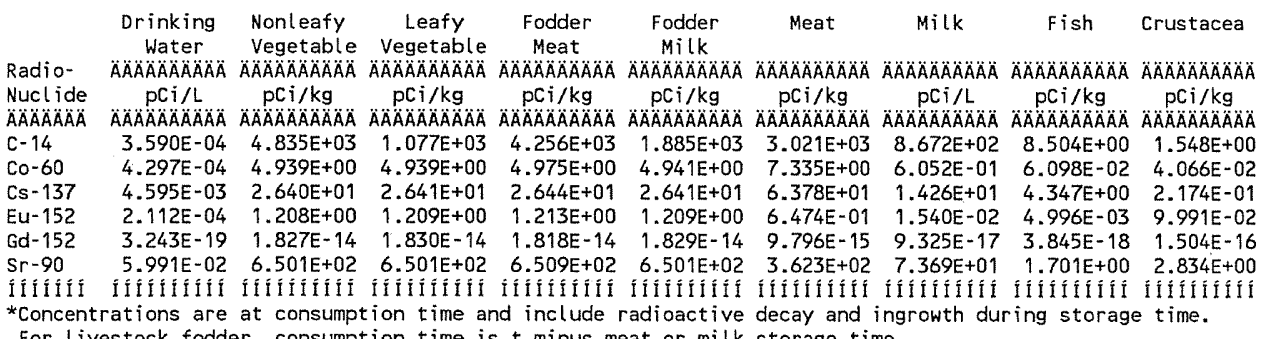

* Concentrations are at consumption time and include radioactive decay and ingrowth during storage time.

Concentrations in the media occurring in pathways that are suppressed are calculated using the current input parameters, i.e. using parameters appearing in the input screen when the pathways are active.

$\begin{array}{ll}\text { Attachment } \frac{5}{\text { W. Clark }} & \text { Sheet No. } \underline{5} \text { of } 10 \\ \text { Originator: } \frac{\text { S. W. }}{\text { M.W. Perrott }} & \text { Date } \\ \text { Chk'd By } & \text { Date } \\ \text { Calc. No. } 0100 B-C A-V 0308 & \text { Rev. No. } \quad 0\end{array}$


CVP-2007-00006

Rev. 0

\section{ATTACHMENT 5}

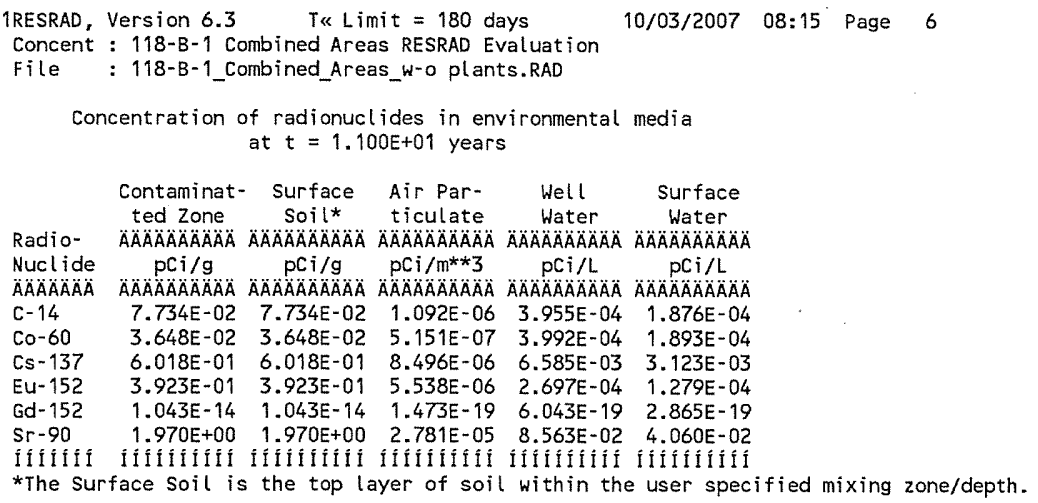

Concentrations in the media occurring in pathways that are suppressed are calculated using the current input parameters, i.e. Using parameters appearing in the input screen when the pathways are active.

Concentration of gaseous $\mathrm{C}-14$ in air $=6.720 \mathrm{E}-01 \mathrm{pCi} / \mathrm{m}^{* * 3}$

Concentration of radionuclides in foodstuff media at $t=1.100 E+01$ years*

\begin{tabular}{|c|c|c|c|c|c|c|c|c|c|}
\hline & $\begin{array}{c}\text { Drinking } \\
\text { Water }\end{array}$ & $\begin{array}{l}\text { Nonleafy } \\
\text { Vegetable }\end{array}$ & $\begin{array}{c}\text { Leafy } \\
\text { Vegetable }\end{array}$ & $\begin{array}{l}\text { Fodder } \\
\text { Meat }\end{array}$ & $\begin{array}{l}\text { Fodder } \\
\text { Milk }\end{array}$ & Meat & Milk & Fish & Crustacea \\
\hline dio & $\triangle A \cap A \cap A \cap A \cap A \cap A \cap$ & 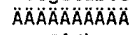 & $\triangle A A A A A A A A A A A A$ & $\triangle A A A A A A A A A A A B$ & $\triangle \triangle A A O A A A A A A O A A A$ & 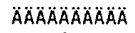 & 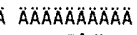 & $\triangle A A A B A A A A A A A ̈$ & 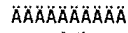 \\
\hline $\begin{array}{l}U c \text { lide } \\
A \ddot{A B A O A O A}\end{array}$ & $\begin{array}{c}p C i / L \\
\triangle A B A A A B A A\end{array}$ & $\begin{array}{r}\mathrm{pCi} / \mathrm{kg} \\
\triangle A B A A B A\end{array}$ & $\begin{array}{c}p C i / k g \\
A A A A A A A A A\end{array}$ & $\begin{array}{c}\mathrm{pCi} / \mathrm{kg} \\
A \bar{A} \mathrm{ABAAA}\end{array}$ & $\begin{array}{r}p C i / k g \\
A A A A A A A\end{array}$ & $\mathrm{pCi} / \mathrm{kg}$ & $\mathrm{pCi} / \mathrm{L}$ & $\mathrm{pCi} / \mathrm{kg}$ & prikr \\
\hline 14 & $\begin{array}{r}\text { AAAAAAAAAAA } \\
3.955 \mathrm{E}-04\end{array}$ & $\begin{array}{r}\text { AAAA } \\
1.5\end{array}$ & $\begin{array}{l}\text { AAAA } \\
3.3\end{array}$ & AAA & AAA & & $\begin{array}{l}A A \\
0 ?\end{array}$ & $A A$ & AAA \\
\hline $0-60$ & $3.991 \mathrm{E}-04$ & $2.919 E+00$ & $2.919 E+00$ & $2.940 E+00$ & $2.920 E+00$ & 4.3 & $=-01$ & -02 & E-02 \\
\hline & $6.584 E-03$ & $2.407 E+01$ & $2.407 E+01$ & $2.411 \mathrm{E}+01$ & $2.408 E+01$ & $E+01$ & $E+01$ & +00 & $E-01$ \\
\hline Eu-152 & $2.696 \mathrm{E}-04$ & 9.80 & 9.8 & -01 & 9.8 & 5.2 & 1. & .03 & $=-01$ \\
\hline Gd -15 & $6.082 E-19$ & $2.609 E-14$ & $=-14$ & $2.603 E-14$ & $2.612 E-14$ & $1.399 E-14$ & 1.33 & $E-18$ & $E-16$ \\
\hline$S r$ & $8.561 E-02$ & $5.910 \varepsilon+02$ & $5.910 E+02$ & $5.918 \mathrm{E}+02$ & $E+02$ & +02 & +01 & $E+00$ & $E+00$ \\
\hline fiflifif & & & & & & & & & \\
\hline Samones & 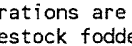 & & & & & & & & me \\
\hline
\end{tabular}

Concentrations in the media occurring in pathways that are suppressed are calculated using the current input parameters, $i$.e. using parameters appearing in the input screen when the pathways are active.

\begin{tabular}{|c|c|}
\hline Attachment & Sheet No. 6 of 10 \\
\hline Originator: S.W. Clark & Date \\
\hline M. W. Perrott & Date \\
\hline $0100 B-C A-V 0308$ & Rev. No. \\
\hline
\end{tabular}


CVP-2007-00006

Rev. 0

\section{ATTACHMENT 5}

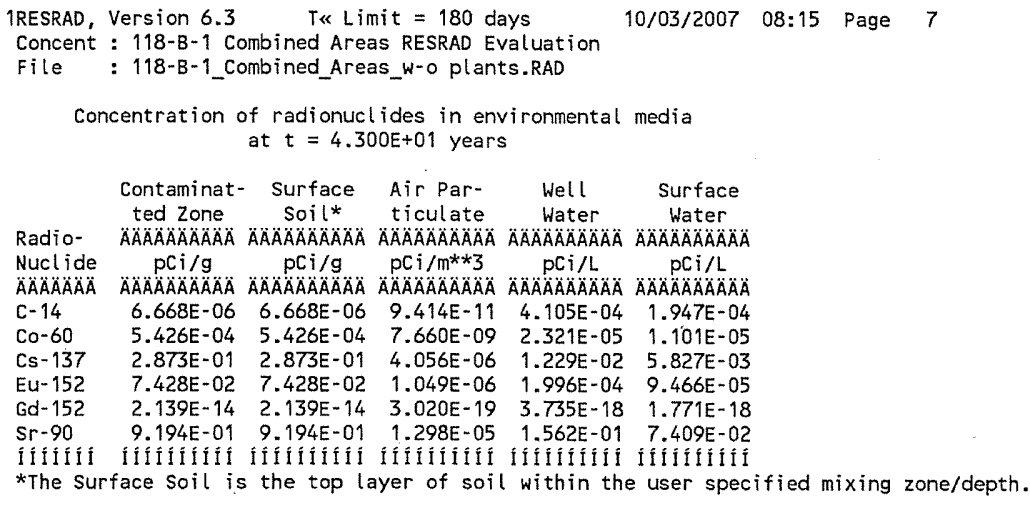

Concentrations in the media occurring in pathways that are suppressed are calculated using the current input parameters, i.e. using parameters appearing in the input screen when the pathways are active.

Concentration of gaseous $\mathrm{C}-14$ in air $=5.794 \mathrm{E}-05 \mathrm{pCi} / \mathrm{m}^{* * 3}$

Concentration of radionuclides in foodstuff media at $\mathrm{t}=4.300 \mathrm{E}+01$ years*

Drinking Nonleafy Leafy Fodder Fodder Meat Milk Fish Crustacea

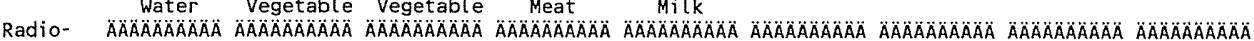
Nuclide $\mathrm{pCi} / \mathrm{L} p \mathrm{pi} / \mathrm{kg} \mathrm{pCi} / \mathrm{kg} \mathrm{pCi} / \mathrm{kg} p \mathrm{pCi} / \mathrm{kg} p \mathrm{pCi} / \mathrm{kg} \quad \mathrm{pCi} / \mathrm{L} \quad \mathrm{pCi} / \mathrm{kg} \mathrm{pCi} / \mathrm{kg}$

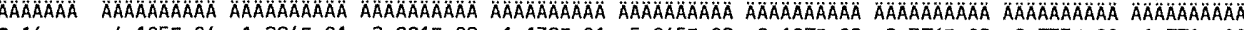
$\begin{array}{llllllllll}\mathrm{C}-14 & 4.105 \mathrm{E}-04 & 1.294 \mathrm{E}-01 & 2.881 \mathrm{E}-02 & 1.139 \mathrm{E}-01 & 5.045 \mathrm{E}-02 & 8.107 \mathrm{E}-02 & 2.376 \mathrm{E}-02 & 9.733 \mathrm{E}+00 & 1.771 \mathrm{E}+00\end{array}$ $\begin{array}{llllllllll}C 0-60 & 2.321 E-05 & 4.341 \mathrm{E}-02 & 4.341 \mathrm{E}-02 & 4.372 \mathrm{E}-02 & 4.342 \mathrm{E}-02 & 6.448 \mathrm{E}-02 & 5.325 \mathrm{E}-03 & 3.300 \mathrm{E}-03 & 2.200 \mathrm{E}-03\end{array}$

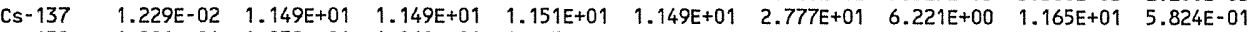
$\begin{array}{lllllllllll}\text { Eu-152 } & 1.996 \mathrm{E}-04 & 1.858 \mathrm{E}-01 & 1.860 \mathrm{E}-01 & 1.865 \mathrm{E}-01 & 1.860 \mathrm{E}-01 & 9.960 \mathrm{E}-02 & 2.370 \mathrm{E}-03 & 4.731 \mathrm{E}-03 & 9.462 \mathrm{E}-02\end{array}$

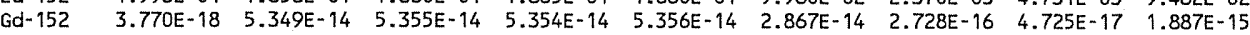
$\begin{array}{llllllllll}\mathrm{Sr}-90 & 1.562 \mathrm{E}-01 & 2.758 \mathrm{E}+02 & 2.758 \mathrm{E}+02 & 2.762 \mathrm{E}+02 & 2.758 \mathrm{E}+02 & 1.538 \mathrm{E}+02 & 3.131 \mathrm{E}+01 & 4.443 \mathrm{E}+00 & 7.405 \mathrm{E}+00\end{array}$

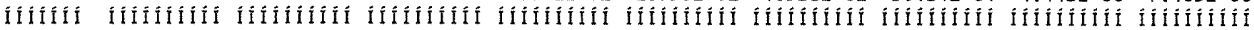
* Concentrations are at consumption time and include radioactive decay and ingrowth during storage time. For livestock fodder, consumption time is $t$ minus meat or milk storage time.

Concentrations in the media occurring in pathways that are suppressed are calculated using the current input parameters, i.e. using parameters appearing in the input screen when the pathways are active.

\begin{tabular}{|c|c|}
\hline \multirow{2}{*}{\multicolumn{2}{|c|}{ 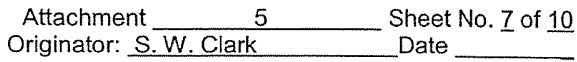 }} \\
\hline & \\
\hline Chk'd By M. W. Perrott & Date \\
\hline $0100 \mathrm{~B}-\mathrm{CA}$ & Rev. \\
\hline
\end{tabular}


CVP-2007-00006

Rev. 0

\section{ATTACHMENT 5}

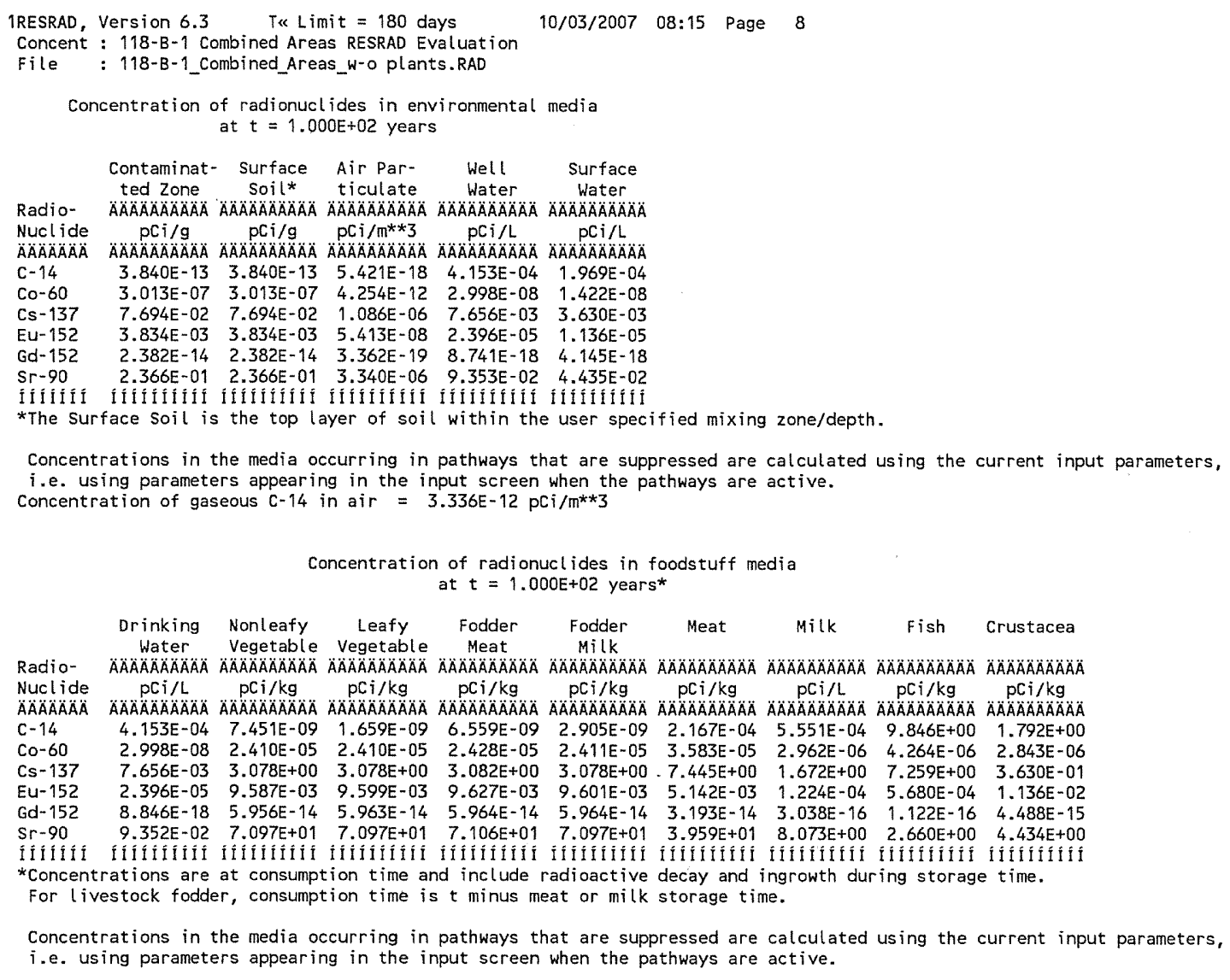

$i . e$. using parameters appearing in the input screen when the pathways are active.

\begin{tabular}{|c|c|c|}
\hline \multirow{2}{*}{\multicolumn{2}{|c|}{$\begin{array}{l}\text { Attachment } \frac{5}{\text { Originator: S. W. Clark }} \\
\text { Oris }\end{array}$}} & Sheet No \\
\hline & & Date \\
\hline Chk'd By & U. W. Perrott & Date \\
\hline Calc. No. & $0100 B-C A-V 0308$ & Rev \\
\hline
\end{tabular}


CVP-2007-00006

Rev. 0

\section{ATTACHMENT 5}

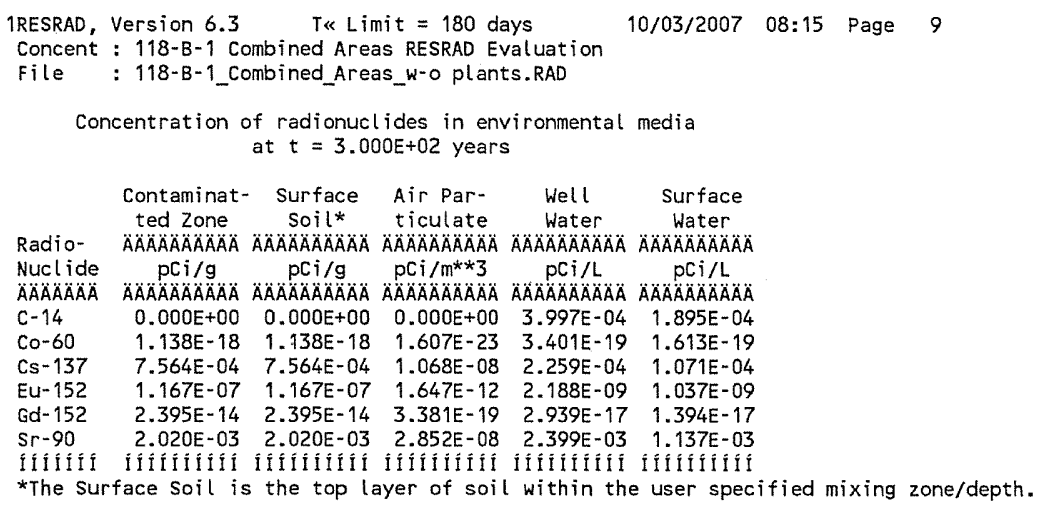

Concentrations in the media occurring in pathways that are suppressed are calculated using the current input parameters, i.e. using parameters appearing in the input screen when the pathways are active. Concentration of gaseous $\mathrm{C}-14$ in air $=0.000 \mathrm{E}+00 \mathrm{pCi} / \mathrm{m}^{* * 3}$

\section{Concentration of radionuclides in foodstuff media} at $\mathrm{t}=3.000 \mathrm{E}+02$ years*

\begin{tabular}{|c|c|c|c|c|c|c|c|c|c|}
\hline & $\begin{array}{c}\text { Drinking } \\
\text { Water }\end{array}$ & $\begin{array}{l}\text { Nonleafy } \\
\text { Vegetable }\end{array}$ & $\begin{array}{c}\text { Leafy } \\
\text { Vegetable }\end{array}$ & $\begin{array}{l}\text { Fodder } \\
\text { Meat }\end{array}$ & $\begin{array}{l}\text { Fodder } \\
\text { Milk }\end{array}$ & Meat & Milk & Fish & Crustacea \\
\hline Radio- & $\triangle A \cap A ̈ A ̈ A ̈ A ̈ A A A O A ̈$ & $\triangle A O A A A A A A A A A$ & $\triangle \triangle A O A A A A B A O A O A O A$ & $\triangle A O A O A A A A A A A B$ & $\triangle A A B A B A A B A B$ & 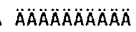 & ÄÄÄÄÄÄÄÄ & $\triangle A A \cap A A A A B A ̈ A ̈ A ̈$ & $\triangle A A A \cap A O A A A A A ̈$ \\
\hline Nuclide & $\mathrm{pCi} / \mathrm{L}$ & $\mathrm{pCi} / \mathrm{kg}$ & $\mathrm{pCi} / \mathrm{kg}$ & $\mathrm{pCi} / \mathrm{kg}$ & $\mathrm{pCi} / \mathrm{kg}$ & $\mathrm{pCi} / \mathrm{kg}$ & $\mathrm{pCi} / \mathrm{L}$ & $\mathrm{pCi} / \mathrm{kg}$ & $\mathrm{pCi} / \mathrm{kg}$ \\
\hline$\triangle A A A A A A O A ̈$ & $\triangle A A O A A O A A A A A B A ̈$ & $\triangle A A A O A A A A A A A O A$ & 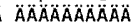 & $\triangle A A A A A A A A A A A A$ & $1 \triangle A B A A A A A A A A A$ & $1 \triangle A A A A A A A A A A O A$ & $\triangle A A A A A A A A A A A$ & $\triangle \triangle A A A A A A A A A A B$ & $\triangle A A A A A A A A A B A$ \\
\hline$c-14$ & $3.997 \mathrm{E}-04$ & $0.000 E+00$ & $0.000 E+00$ & $0.000 E+00$ & $0.000 E+00$ & $2.085 E-04$ & $5.343 \mathrm{E}-04$ & $9.476 \mathrm{E}+00$ & $1.725 E+00$ \\
\hline Co- 60 & $3.401 E-19$ & $9.108 E-17$ & $9.108 E-17$ & $9.174 E-17$ & $9.112 \mathrm{E}-17$ & $1.356 \mathrm{E}-16$ & $1.127 E-17$ & $4.837 \mathrm{E}-17$ & $3.225 \mathrm{E}-17$ \\
\hline Cs -137 & $2.259 E-04$ & $3.026 \mathrm{E}-02$ & $3.026 \mathrm{E}-02$ & $3.030 E-02$ & $3.026 \mathrm{E}-02$ & $7.341 \mathrm{E}-02$ & $1.663 E-02$ & ? $2.142 E-01$ & $1.071 E-02$ \\
\hline Eu- 152 & $2.188 \mathrm{E}-09$ & $2.917 E-07$ & $2.921 \mathrm{E}-07$ & $2.929 \mathrm{E}-07$ & $2.922 E-07$ & $7.566 E-07$ & $3.737 E-09$ & $5.187 E-08$ & $1.037 E-06$ \\
\hline $6 d-152$ & $2.927 \mathrm{E}-17$ & $5.989 E-14$ & $5.996 E-14$ & $5.996 \mathrm{E}-14$. & $5.996 \mathrm{E}-14$ & $3.210 \mathrm{E}-14$ & $3.055 E-16$ & $3.385 \mathrm{E}-16$ & $1.354 E-14$ \\
\hline$s r-90$ & $2.399 \mathrm{E}-03$ & $6.060 \mathrm{E}-01$ & $6.060 E-01$ & $6.068 \mathrm{E}-01$ & $6.060 E-01$ & $3.387 E-01$ & $6.944 E-02$ & $6.824 \mathrm{E}-02$ & $1.137 \mathrm{E}-01$ \\
\hline & IIIIIIIIIIII & 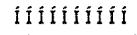 & 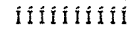 & 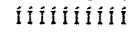 & IIIIIIIIIII & IIIIIIIílín & İîîî́î́ & IIIIIIIIIIIII & Î́î́îIIIIII \\
\hline *Concer & ations are & at & ion time an & nd include & radioactive & decay and & ingrowth dur & ring storag & e time. \\
\hline
\end{tabular}
i.e. using parameters appearing in the input screen when the pathways are active.

$\begin{array}{ll}\begin{array}{c}\text { Attachment } \\ \text { Originator: } \frac{5}{\text { S.W. Clark }}\end{array} & \begin{array}{c}\text { Sheet No. } 9 \text { of } 10 \\ \text { Date }\end{array} \\ \text { Chk'd By } & \text { Date } \\ \text { Calc. No. Perrott } & \text { Rev. No. } \quad 0\end{array}$


CVP-2007-00006

Rev. 0

\section{ATTACHMENT 5}

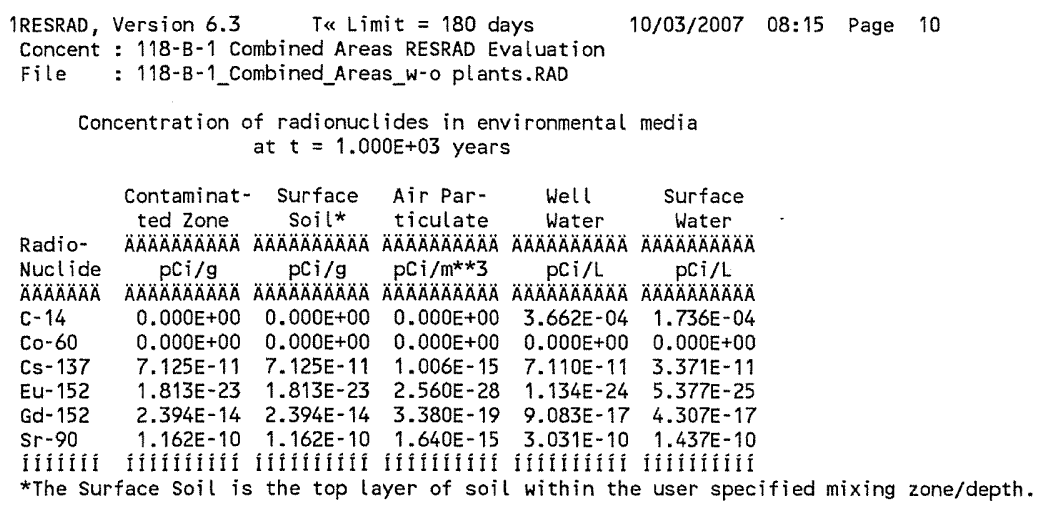

Concentrations in the media occurring in pathways that are suppressed are calculated using the current input parameters, i.e. using parameters appearing in the input screen when the pathways are active. Concentration of gaseous $\mathrm{C}-14$ in air $=0.000 \mathrm{E}+00 \mathrm{pCi} / \mathrm{m}^{* * 3}$

Concentration of radionuclides in foodstuff media at $\mathrm{t}=1.000 \mathrm{E}+03$ years*

\begin{tabular}{|c|c|c|c|c|c|c|c|c|c|}
\hline & $\begin{array}{c}\text { Drinking } \\
\text { Water }\end{array}$ & $\begin{array}{l}\text { Nonleafy } \\
\text { Vegetable }\end{array}$ & $\begin{array}{c}\text { Leafy } \\
\text { Vegetable }\end{array}$ & $\begin{array}{l}\text { Fodder } \\
\text { Meat }\end{array}$ & $\begin{array}{l}\text { Fodder } \\
\text { Milk }\end{array}$ & Meat & Milk & Fish & Crustacea \\
\hline Raulo- & $\triangle A B A O A A A O A O A ̈ A$ & $\triangle A A B A A A A A A B A B$ & $\triangle A A A A A A A A B A B A$ & $\triangle A O A O A O A O A Z A ̈ A ̈$ & $\triangle \triangle A A A A A A A A A B$ & $\triangle A A A O A A A A A B A$ & $\triangle A A A A A A A A A O A B$ & $\triangle \triangle A A A A A O A A A O A$ & AOAOAAAOAOAOA \\
\hline & $\mathrm{pCi} / \mathrm{L}$ & $\mathrm{pCi} / \mathrm{kg}$ & $\mathrm{pCi} / \mathrm{kg}$ & $\mathrm{pCi} / \mathrm{kg}$ & $\mathrm{pCi} / \mathrm{kg}$ & $\mathrm{pCi} / \mathrm{kg}$ & $\mathrm{pCi} / \mathrm{L}$ & $\mathrm{pCi} / \mathrm{kg}$ & $\mathrm{pCi} / \mathrm{kg}$ \\
\hline$\triangle A A O A O A ̈ A$ & $\triangle A A A A A A A A A A O A$ & 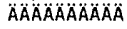 & $\triangle A A A A A A A A O A A$ & 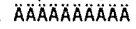 & $\triangle A A A A A B A O A A A A$ & 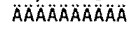 & A AAAAAAAAAÄÄ & $\triangle A A A A A A A A A A B$ & A AAAAAAAAAAÄ \\
\hline$c-14$ & $3.662 \mathrm{E}-04$ & $0.000 \mathrm{E}+00$ & $0 \quad 0.000 E+00$ & $0.000 \mathrm{E}+00$ & $0.000 E+00$ & $1.911 \mathrm{E}-04$ & $4.895 E-04$ & $8.682 E+00$ & $1.580 E+00$ \\
\hline $\mathrm{Co}-60$ & $0.000 \mathrm{E}+00$ & $0.000 E+00$ & $0 \quad 0.000 E+00$ & $0.000 E+00$ & $0.000 E+00$ & $0.000 E+00$ & $0.000 \mathrm{E}+00$ & $0.000 E+00$ & $0.000 E+00$ \\
\hline $5-137 \quad$ & $7.110 \mathrm{E}-11$ & $2.850 E-09$ & $9 \cdot 2.850 E-09$ & $2.854 \mathrm{E}-09$ & $2.850 E-09$ & $E-09$ & $1.630 \mathrm{E}-09$ & 6.7 & $3.371 \mathrm{E}-09$ \\
\hline $\mathrm{Eu}-152$ & $1.134 \mathrm{E}-24$ & $4.534 \mathrm{E}-23$ & $34.539 E-23$ & $4.553 E-23$ & $34.541 \mathrm{E}-23$ & $2.442 E-23$ & $5.872 E-25$ & ; $2.689 E-23$ & $5.377 \mathrm{E}-22$ \\
\hline Gd-152 & $9.052 \mathrm{E}-17$ & $5.987 E-14$ & $4 \quad 5.994 \mathrm{E}-14$ & $5.995 E-14$ & $+\quad 5.995 E-14$ & $3.210 E-14$ & $3.056 \mathrm{E}-16$ & $5 \quad 1.053 E-15$ & $4.210 E-14$ \\
\hline $\mathrm{sr}-90$ & $3.031 \mathrm{E}-10$ & $3.486 E-08$ & $8 \quad 3.486 E-08$ & $3.490 \mathrm{E}-08$ & $3 \quad 3.486 E-08$ & $1.955 E-08$ & $34.048 E-09$ & 9 $8.624 E-09$ & $1.437 E-08$ \\
\hline $\cos x$ & Ififififif & IIfIf & 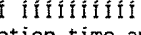 & iflifififif & LIIIIIIIII & I IIIIIIIIIII & I Iifififili & ifi i & filifiliti \\
\hline
\end{tabular}

For livestock fodder, consumption time is $t$ minus meat or milk storage time.

Concentrations in the media occurring in pathways that are suppressed are calculated using the current input parameters, i.e. using parameters appearing in the input screen when the pathways are active.

\begin{tabular}{|c|c|}
\hline Attachment & Sheet No. 10 of 10 \\
\hline Originator: S.W. Clark & Date \\
\hline Chk'd By M.W. Perrott & Date \\
\hline 0100B-CA-V0308 & Rev. No. $\quad 0$ \\
\hline
\end{tabular}




\section{CALCULATION COVER SHEET}

Project Title: Field Remediation

Job No. 14655

Area: $\quad 100-\mathrm{BC}$

Discipline: Environmental

*Calculation No: 0100B-CA-V0309

118-B-1 Burial Ground Combined Areas Comparison to Drinking Water Standards Subject: (MCL) Calculation Brief

Computer Program: Excel

Program No: Excel 2003

The attached calculations have been generated to document compliance with established cleanup levels. These calculations should be used in conjunction with other relevant documents in the administrative record.

Committed Calculation $\bigotimes$

Preliminary

Superseded $\square$

Voided

\begin{tabular}{|c|c|c|c|c|c|c|}
\hline Rev. & Sheet Numbers & Originator & Checker & Reviewer & Approval & Date \\
\hline 0 & $\begin{array}{l}\text { Cover }-1 \mathrm{pg} \\
\text { Calculations }-3 \mathrm{pg} \\
\text { Total }-4 \mathrm{pages}\end{array}$ & $\begin{array}{c}\text { S. W Clark } \\
\text { S. W. }\end{array}$ & $\begin{array}{l}\text { Morifuete } \\
\text { M.W.Perrott }\end{array}$ & N/A & J. M. Capron & $10 / 6 / 07$ \\
\hline & & & & & & \\
\hline & & & & & & \\
\hline & & & & & & \\
\hline & & & & & & \\
\hline
\end{tabular}

SUMMARY OF REVISION

\begin{tabular}{|l|l|}
\hline & \\
\hline & \\
\hline & \\
\hline & \\
\hline & \\
\hline
\end{tabular}




\section{CALCULATION SHEET}

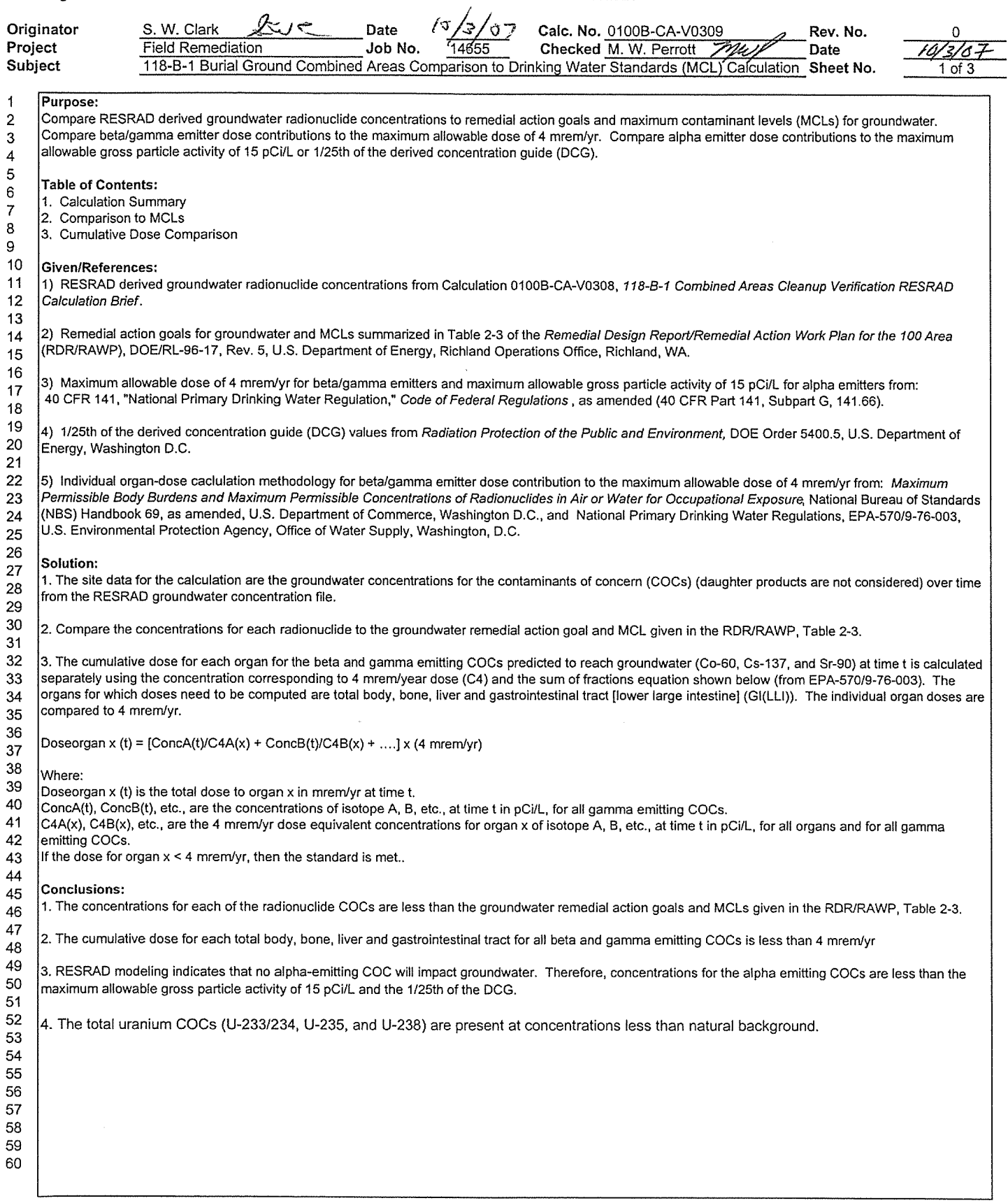




\section{Washington Closure Hanford}

\section{CALCULATION SHEET}

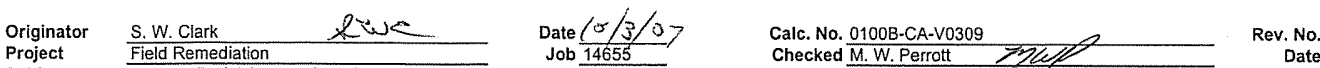

$\frac{\text { Field Remediation }}{118-B-1 \text { Burial Ground Combined Areas Comparison to Drinking Water Standards (MCL) Calculation }}$

Date

Subject

Sheet No. $\frac{1 C Z \text { Z } 37}{2 \text { of } 3}$

1 Table of Comparisons of the Maximum Concentration of Each Radionuclide to its Groundwater MCL

2 Data derived from RESRAD modelling, units are $\mathrm{PCi} / \mathrm{L}$.

\begin{tabular}{|c|c|c|c|c|c|c|c|c|c|c|c|c|c|}
\hline Radionuclide & $\frac{\text { Time fyear }}{0}$ & 1 & 3 & 7 & 11 & 43 & 135 & 300 & 1000 & MCL & $\begin{array}{l}\text { Exceeds } \\
\text { MCL? }\end{array}$ & $\begin{array}{l}\text { Peak } \\
\text { Conc. }\end{array}$ & $\begin{array}{c}\text { Year of Peak } \\
\text { Conc. }\end{array}$ \\
\hline & & & & & & & & & & & & & \\
\hline $\mathrm{Co}-60$ & 0 & $1.35 \mathrm{E}-04$ & $3.12 \mathrm{E}-04$ & $4.30 \mathrm{E}-04$ & $3.99 \mathrm{E}-04$ & $2.32 \mathrm{E}-05$ & $3.00 E-08$ & $3.40 E-19$ & $7.11 \mathrm{E}-11$ & 100 & No & $4.30 \mathrm{E}-04$ & 7 \\
\hline Cs- 137 & 0 & $7.52 \mathrm{E}-04$ & $2.16 \mathrm{E}-03$ & $4.60 \mathrm{E}-03$ & $6.58 \mathrm{E}-03$ & $1.23 \mathrm{E}-02$ & $7.66 \mathrm{E}-03$ & $2.26 \mathrm{E}-04$ & 0 & 60 & No & $1.23 \mathrm{E}-02$ & 43 \\
\hline & & & & & & & & & & & & & \\
\hline Sr-90 & 0 & $9.85 \mathrm{E}-03$ & $2.82 \mathrm{E}-02$ & $6.00 \mathrm{E}-02$ & $8.56 \mathrm{E}-02$ & $1.56 \mathrm{E}-01$ & $9.35 \mathrm{E}-02$ & $2.40 E-03$ & $3.03 E-10$ & 8 & No & $1.56 E-01$ & 43 \\
\hline
\end{tabular}




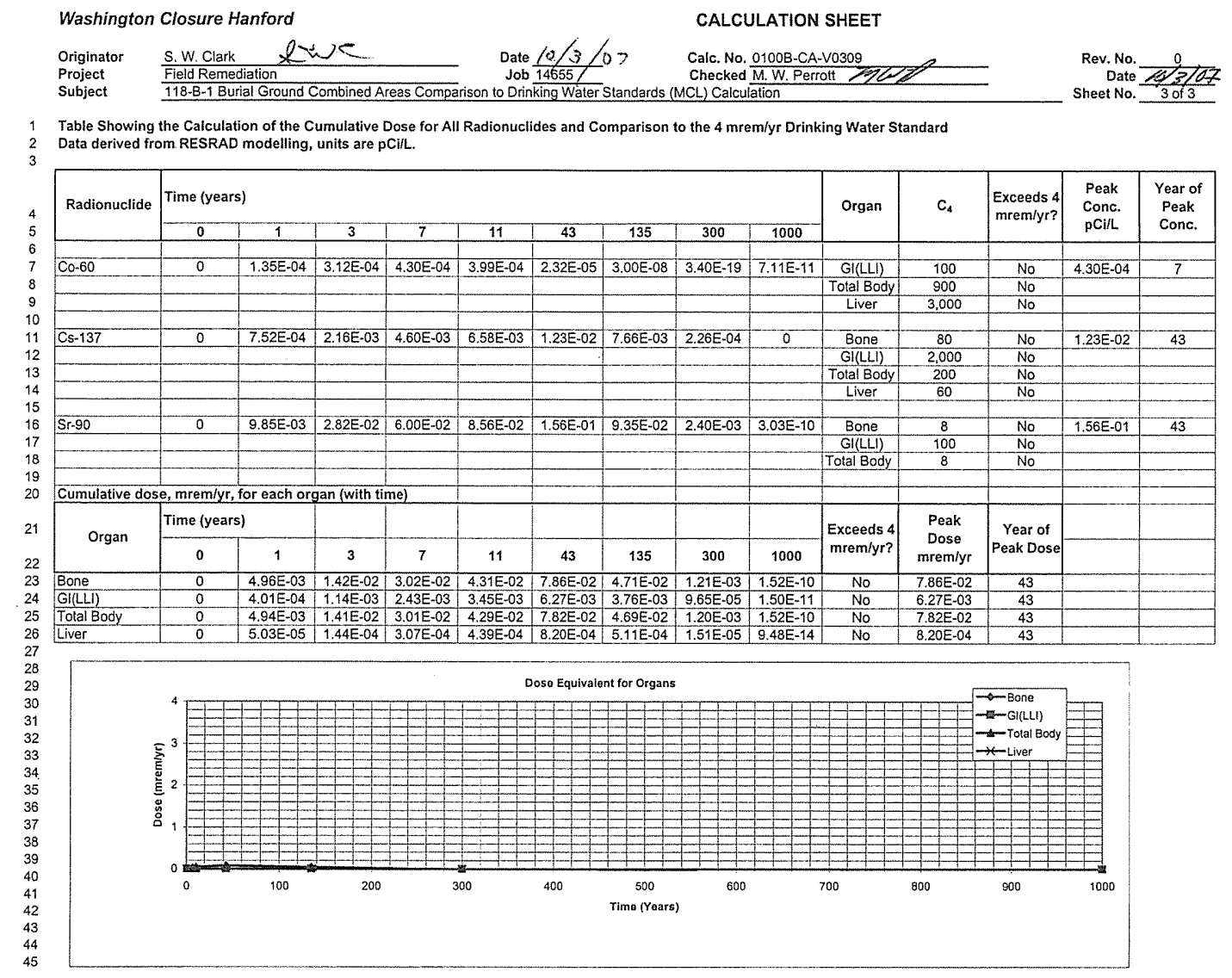

118-B-1 MCL (0100B-CA-V0309)

Cumulative Dose Comparison 
CVP-2007-00006

Rev. 0

APPENDIX D

TRITIUM PLUME CHARACTERIZATION SAMPLING RESULTS 
CVP-2007-00006

Rev. 0

D-ii 


\section{Tritium Plume Characterization Sampling Results}

Residual tritium contamination was detected in multiple statistical soil verification samples collected from Areas 1, 2, and 3 of the 118-B-1 Burial Ground remediation footprint, as well as in focused soil verification samples associated with caches of tritium-contaminated wastes. Because verification samples are collected from the exposed soil surface, and the surfaces sampled had all been exposed for a significant length of time prior to sampling, it was postulated that tritium contamination in the strata sampled may have been reduced by evaporative loss after completion of excavation activities. Therefore, additional investigation was undertaken to characterize residual tritium contamination in the deep vadose zone underlying the burial ground remediation footprint.

Initially, discrete samples were collected at each of the four composite sampling nodes associated with the highest reported detection of tritium, in Area 1. Based on the results of these samples, the sampling node associated with tritium contamination was isolated. A test pit was then excavated at this location, and soil samples collected for tritium analysis at approximately $1 \mathrm{~m}$ depth intervals. Results of these samples showed that tritium concentrations in the uppermost stratum were significantly lower than those in lower strata, confirming loss to evaporative transport at exposed surfaces.

Additional excavations to collect vertical profile data were then performed in Areas 2 and 3 . Due to the frozen ground conditions at the time of this investigation, sampling nodes associated with tritium contamination could not be isolated, and test pits and associated sampling-at-depth were completed at all sampling nodes associated with composite verification samples with elevated detections of tritium. Following review of all test pit data, a characterization borehole was completed in Area 1, Node A2-3, at the location of the highest observed deep vadose zone tritium contamination levels.

Test pit locations are shown in Figure D-1. Analytical results provided in Table D-1, and summarized in Figures D-2 and D-3. 


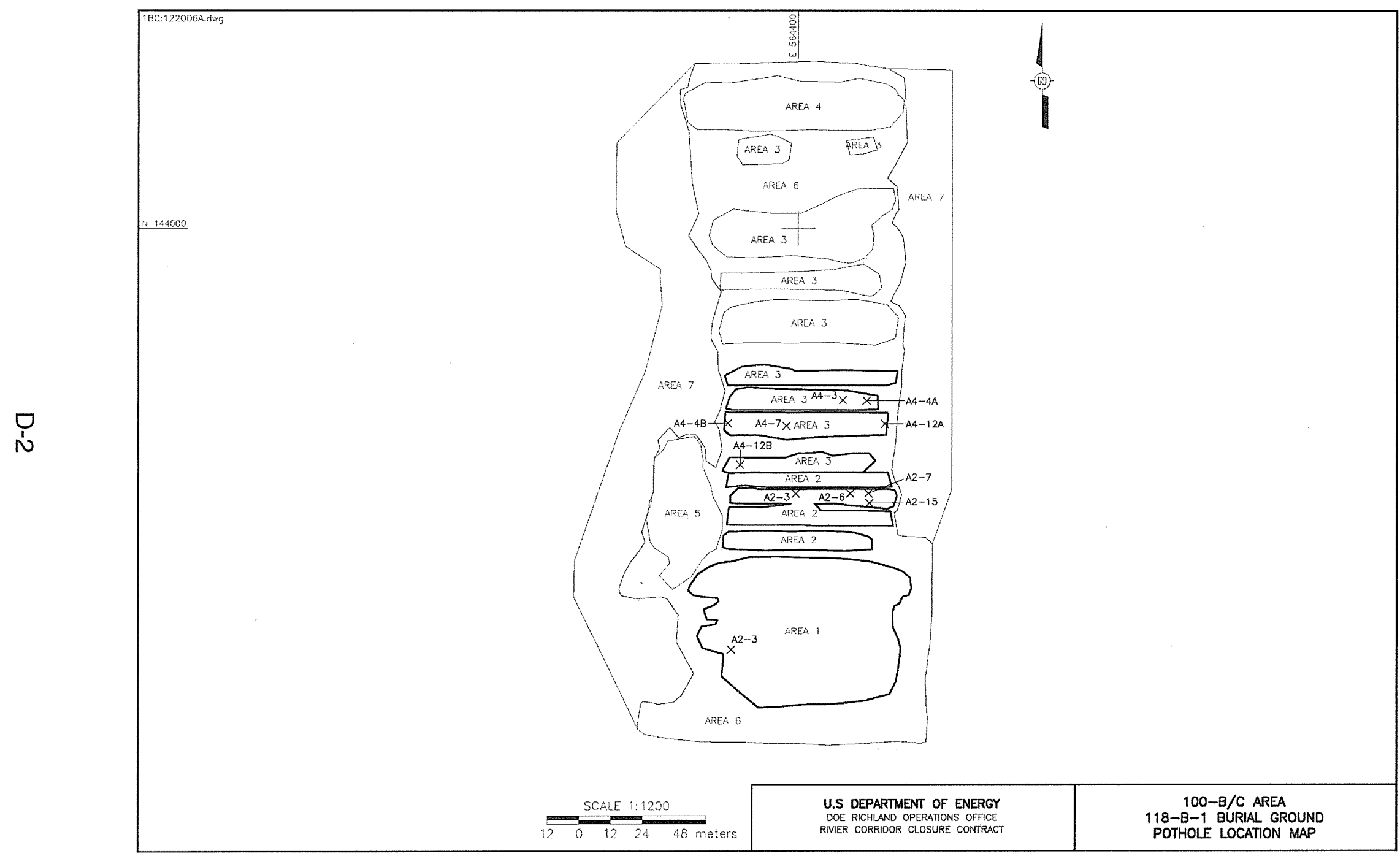

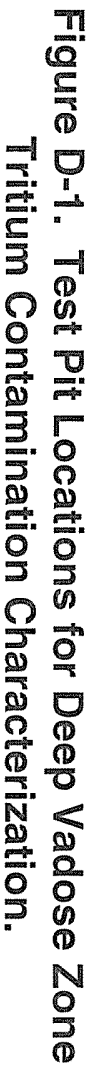

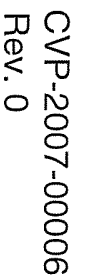


CVP-2007-00006

Rev. 0

Table D-1. Tritium Results for Characterization Potholes and Borehole. (2 Pages)

\begin{tabular}{|c|c|c|c|c|c|}
\hline \multirow{2}{*}{ Sample Location } & \multirow{2}{*}{$\begin{array}{c}\text { Sample } \\
\text { Number }\end{array}$} & Sample & \multicolumn{3}{|c|}{ Tritium } \\
\cline { 6 - 6 } & Date & pCi/g & Q & MDA \\
\hline Area 2, A2-7, 3' bef & J13VL1 & $12 / 12 / 06$ & 306 & & 4.4 \\
\hline Area 2, A2-7, 6' bef & J13VL2 & $12 / 12 / 06$ & 3370 & & 5.9 \\
\hline Area 2, A2-7, 9' bef & J13VL3 & $12 / 12 / 06$ & 19700 & & 15 \\
\hline Area 2, A2-7, 22' bef & J13VL4 & $12 / 12 / 06$ & 6350 & & 7.8 \\
\hline
\end{tabular}

Excavation floor at Area 2, A2-7 was approximately $14 \mathrm{ft}$ bgs

\begin{tabular}{|c|c|c|c|c|c|}
\hline Area 2, A2-15, 3' bef & J13VL5 & $12 / 12 / 06$ & 10300 & & 9.8 \\
\hline Area 2, A2-15, 6' bef & J13VL6 & $12 / 12 / 06$ & 11600 & & 11 \\
\hline Area 2, A2-15, 9' bef & J13VL7 & $12 / 12 / 06$ & 24700 & & 17 \\
\hline Area 2, A2-15, 22' bef & J13VL8 & $12 / 12 / 06$ & 16200 & & 13 \\
\hline
\end{tabular}

Excavation floor at Area 2, A2-15 was approximately $12 \mathrm{ft}$ bgs \begin{tabular}{|l|l|l|l|l|l|}
\hline Area 3, A4-3, 3' bef & J13V22 & $12 / 8 / 2006$ & 15.8 & & 3.0
\end{tabular} \begin{tabular}{|l|l|l|l|l|l}
\hline Area 3, A4-3, 6' bef & J13V23 & $12 / 8 / 2006$ & 48.4 & & 2.7
\end{tabular} \begin{tabular}{|l|l|l|l|l|l}
\hline Area 3, A4-3, 9' bef & J13V24 & $12 / 8 / 2006$ & 36.6 & & 2.7
\end{tabular} \begin{tabular}{|l|l|l|l|l|l|}
\hline Area 3, A4-3, 24' bef & J13V25 & $12 / 8 / 2006$ & 6.16 & & 2.7 \\
\hline
\end{tabular}

Excavation floor at Area 3, A4-3 was approximately $8 \mathrm{ft}$ bgs

\begin{tabular}{|c|c|c|c|c|c|}
\hline Area 3, A4-4A, 3' bef & J13V18 & $12 / 8 / 2006$ & -5.48 & $\mathrm{U}$ & 2.7 \\
\hline Area 3, A4-4A, 6' bef & $\mathrm{J} 13 \mathrm{~V} 19$ & $12 / 8 / 2006$ & -6.44 & $\mathrm{U}$ & 2.8 \\
\hline Area 3, A4-4A, 9' bef & $\mathrm{J} 13 \mathrm{~V} 20$ & $12 / 8 / 2006$ & -6.64 & $\mathrm{U}$ & 2.5 \\
\hline Area 3, A4-4A, 25' bef & $\mathrm{J} 13 \mathrm{~V} 21$ & $12 / 8 / 2006$ & -5.76 & $\mathrm{U}$ & 2.7 \\
\hline
\end{tabular}

Excavation floor at Area 3, A4-4A was approximately $5 \mathrm{ft}$ bgs

\begin{tabular}{|c|c|c|c|c|c|c|}
\hline Area 3, A4-4B, 3' bef & $\mathrm{J} 13 \mathrm{~V} 26$ & $12 / 8 / 2006$ & 1.58 & $\mathrm{U}$ & 1.9 \\
\hline Area 3, A4-4B, 6' bef & $\mathrm{J} 13 \mathrm{~V} 27$ & $12 / 8 / 2006$ & 1.46 & $\mathrm{U}$ & 1.9 \\
\hline Area 3, A4-4B, 9' bef & $\mathrm{J} 13 \mathrm{~V} 28$ & $12 / 8 / 2006$ & 1.72 & $\mathrm{U}$ & 1.9 \\
\hline Area 3, A4-4B, 24' bef & $\mathrm{J} 13 \mathrm{~V} 29$ & $12 / 8 / 2006$ & 1.13 & $\mathrm{U}$ & 1.9 \\
\hline \multicolumn{6}{|c|}{ Excavation floor at Area 3, A4-4B was approximately $8 \mathrm{ft}$ bgs } \\
\hline Area 3, A4-7, 3' bef & $\mathrm{J} 13 \mathrm{~V} 30$ & $12 / 8 / 2006$ & 704 & & 6.2 \\
\hline Area 3, A4-7, 6' bef & $\mathrm{J} 13 \mathrm{~V} 31$ & $12 / 8 / 2006$ & 4310 & & 17 \\
\hline Area 3, A4-7, 9' bef & $\mathrm{J} 13 \mathrm{~V} 32$ & $12 / 8 / 2006$ & 2230 & & 11 \\
\hline Area 3, A4-7, 20' bef & $\mathrm{J} 13 \mathrm{~V} 33$ & $12 / 8 / 2006$ & 484 & & 4.3 \\
\hline \multicolumn{6}{|c|}{ Excavation floor at Area 3, A4-7 was approximately $20 \mathrm{ft}$ bgs } \\
\hline Area 3, A4-12A, 3' bef & $\mathrm{J} 13 \mathrm{~V} 14$ & $12 / 8 / 2006$ & 0 & $\mathrm{U}$ & 2.6 \\
\hline Area 3, A4-12A, 6' bef & $\mathrm{J} 13 \mathrm{~V} 15$ & $12 / 8 / 2006$ & -4.18 & $\mathrm{U}$ & 2.5 \\
\hline Area 3, A4-12A, 9' bef & $\mathrm{J} 13 \mathrm{~V} 16$ & $12 / 8 / 2006$ & -3.84 & $\mathrm{U}$ & 2.5 \\
\hline Area 3, A4-12A, 24' bef & $\mathrm{J} 13 \mathrm{~V} 17$ & $12 / 8 / 2006$ & 60.0 & & 2.5 \\
\hline
\end{tabular}

Excavation floor at Area 3, A4-12A was approximately $15 \mathrm{ft}$ bgs

\begin{tabular}{|c|c|c|c|c|c|}
\hline Area 3, A4-12B, 3' bef & $\mathrm{J} 13 \mathrm{~V} 34$ & $12 / 8 / 2006$ & 0.123 & $\mathrm{U}$ & 2.1 \\
\hline Area 3, A4-12B, 6' bef & $\mathrm{J} 13 \mathrm{~V} 35$ & $12 / 8 / 2006$ & 1.34 & $\mathrm{U}$ & 2.0 \\
\hline Area 3, A4-12B, 9' bef & $\mathrm{J} 13 \mathrm{~V} 36$ & $12 / 8 / 2006$ & 1.15 & $\mathrm{U}$ & 2.1 \\
\hline Area 3, A4-12B, 20' bef & $\mathrm{J} 13 \mathrm{VL} 0$ & $12 / 8 / 2006$ & 2.98 & & 2.0 \\
\hline
\end{tabular}

Excavation floor at Area $3, \mathrm{~A} 4-12 \mathrm{~B}$ was approximately $15 \mathrm{ft}$ bgs

bef $=$ below excavation floor

acronym $=$ bef

bgs $=$ below ground surface

$\mathrm{MDA}=$ minimum detectable activity

$\mathrm{Q}=$ qualifier

$\mathrm{U}=$ undetected 
Figure D-2. Results of Test Pit Sampling for Deep Vadose Zone Tritium Contamination Characterization.

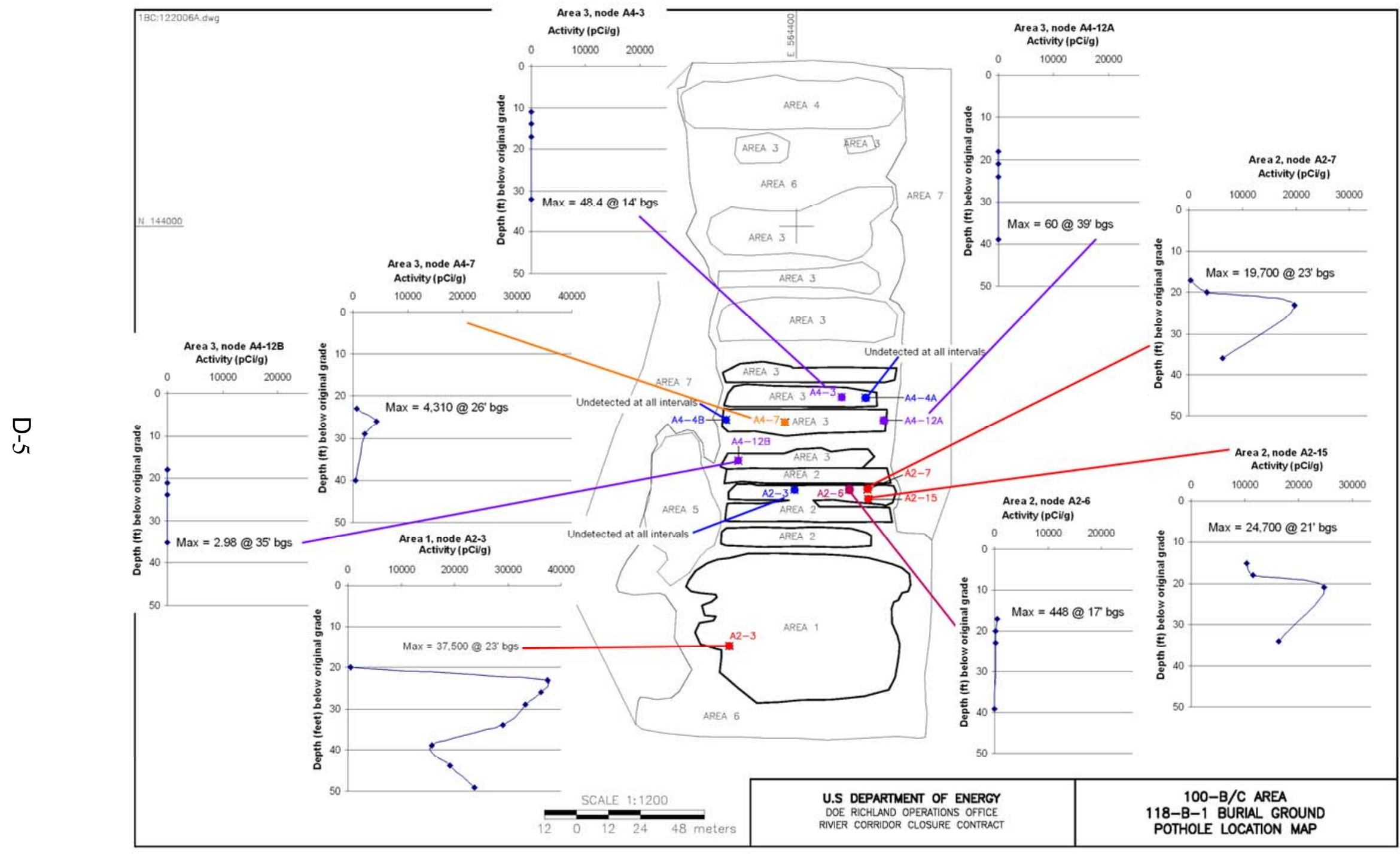


Figure D-3. Results of Borehole Sampling for Deep Vadose Zone Tritium Contamination Characterization at Area 1, Node A2-3.

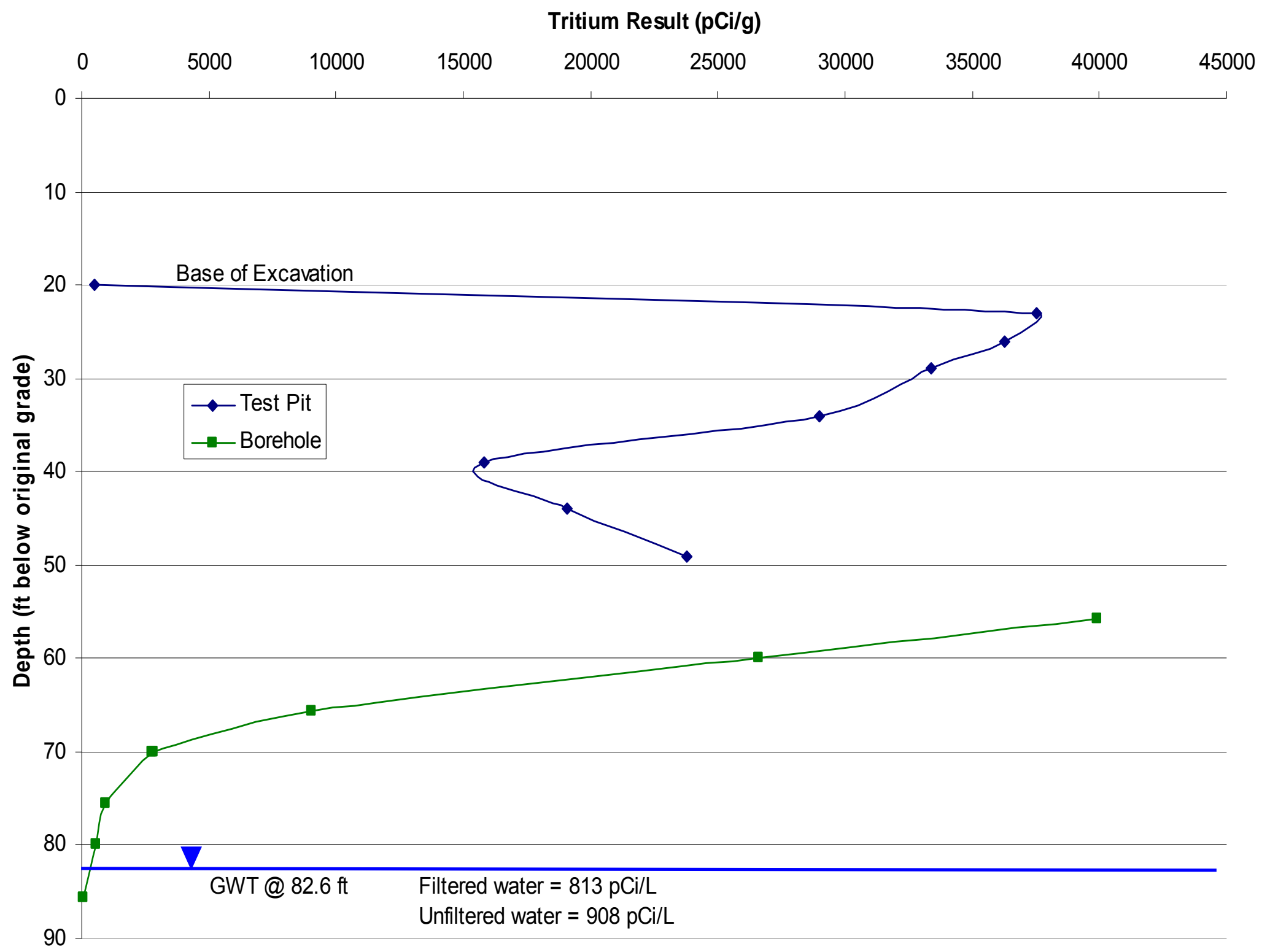


CVP-2007-00006

Rev. 0

\section{APPENDIX E}

\section{DATA QUALITY ASSESSMENT}


CVP-2007-00006

Rev. 0

E-ii 


\section{DATA QUALITY ASSESSMENT FOR THE 118-B-1 BURIAL GROUND}

\section{C1.0 OVERVIEW}

The data quality assessment (DQA) completes the data life cycle (i.e., planning, implementation, and assessment) that was initiated by the data quality objectives process. The DQA includes a review of the field logbook information (WCH 2007a, 2007b, 2007c, $2007 \mathrm{~d}, 2007 \mathrm{e}$ ) to verify sample location, date, and time. It also involves a scientific and statistical evaluation of the data to determine if they are of the right type, quality, and quantity to support their intended use for closeout decisions (EPA 2000).

This DQA was performed in accordance with data quality objectives found in the 100 Area Burial Grounds Remedial Action Sampling and Analysis Plan (Burial Ground SAP) (DOE-RL 2001). The DQA is based on the guidelines presented in Guidance for Data Quality Assessment (EPA 2000). Statistical tests used in this DQA were performed as specified in the SAP and the Remedial Design Report/Remedial Action Work Plan for the 100 Area (RDR/RAWP) (DOE-RL 2005).

Prior to performing statistical tests, the field logbooks (WCH 2007a, 2007b, 2007c, 2007d, 2007e), the Site Specific Instruction for Close-out Approach for 118-B-1 (WCH 2006), and the sample analytical data were evaluated. The site-specific verification sampling design was developed to provide a more comprehensive sampling strategy than the general design provided by the Burial Ground SAP based on grouping of waste forms encountered during 118-B-1 Burial Ground remediation. All samples were collected per the sample design. A portion of the cleanup verification sample analytical data was validated for compliance requirements (DOE-RL 2005). An evaluation is performed to determine if the laboratory carried out all steps required by the SAP and the laboratory contract governing the conduct of analysis and reporting of the data. Third-party data validation, in accordance with validation procedures specified in Data Validation Procedure for Chemical Analysis (BHI 2000a) and in Data Validation Procedure for Radiochemical Analysis (BHI 2000b), is performed as part of data evaluation. After validation and data evaluation, the appropriate statistical analyses are performed on the analytical data (Appendix $\mathrm{C}$ ) to determine statistical values, as appropriate, for each contaminant. The cleanup verification sample analytical data are stored in the Environmental Restoration (ENRE) project-specific database prior to being submitted for inclusion in the Hanford Environmental Information System (HEIS) database and are also summarized in the $95 \%$ upper confidence limit calculation in Appendix C. 


\section{C1.1 LABORATORY QUALITY MEASURES}

All verification samples are subject to laboratory-specific quality assurance (QA) requirements, including instrument procurement, maintenance, calibration, and operation. Additional laboratory quality control (QC) checks are performed, as appropriate, for the analytical method at a rate of 1 per sample delivery group (SDG), or 1 per 20 samples, whichever is more frequent. Laboratory internal QC checks include the following:

- Laboratory Contamination. Each analytical batch contains a laboratory (method) blank (material of similar composition as the samples with known/minimal concentrations of the analytes of interest) carried through the complete analytical process. The method blank is used to evaluate samples for false-positive results due to contamination at the laboratory.

- Analytical Accuracy. For most analyses, a known quantity of representative analytes of interest are added to a separate aliquot of a sample from the analytical batch, known as the matrix spike/matrix spike duplicate (MS/MSD). The recovery percentages of the added MS are used to evaluate analytical accuracy. For analyses not amenable to MS techniques (e.g., gamma energy analysis) or where analytical recovery is corrected via internal standards (e.g., alpha spectral analyses), accuracy is evaluated from recovery of the QC reference sample (i.e., laboratory control sample [LCS] or blank spike sample).

- Analytical Precision. Separate aliquots removed from the same sample container are analyzed for each analytical batch, and referred to as the laboratory duplicate or replicate. The replicate sample results (evaluated by relative percent differences [RPDs]) are used to assess the analytical precision achieved at the laboratory. However, natural heterogeneities often found in soil matrix samples also play a significant part in the RPD result.

- QC Reference Samples. A QC reference sample is prepared from an independent standard at a concentration other than that used for calibration, but within the calibration range. Reference samples provide an independent check on analytical technique and methodology.

Laboratories are also subject to periodic and random assessments of the laboratory performance, systems, and overall program. These assessments are performed by the Washington Closure Hanford QA group to ensure that the laboratories are performing within laboratory contract requirements.

\section{C1.2 DATA VALIDATION}

The 118-B-1 Burial Ground is the largest burial ground at 100-B/C, and the closeout approach included a sample design with multiple decision units. Verification sample 
data collected at the 118-B-1 waste site were provided by the laboratories in 28 SDGs, and 13 separate SDGs for asbestos samples. This DQA addresses the SDGs by the decision units as they were designed in the Site Specific Instruction for Close-out Approach for 118-B-1 (WCH 2006). As the 118-B-1 waste site closeout includes multiple decision units, samples from several decision units may be included in any one SDG. Unless otherwise noted, deficiencies listed below are specific to the individual SDG, but apply to all samples within that SDG.

After sampling was completed, all of the fixed laboratory data from SDGs K0494 and K0601 were submitted for third-party Level $\mathrm{C}$ validation. Level $\mathrm{C}$ validation procedures are specified in Data Validation Procedure for Radiochemical Analysis (BHI 2000b) and Data Validation Procedure for Chemical Analysis (BHI 2000a).

Level C validation procedures were used to review and qualify the data for the following parameters:

- Sample holding times

- Method blanks

- MS/MSD recovery

- Surrogate recovery

- Sample replicates (duplicates)

- Laboratory control sample results

- Data package completeness

- Achievement of required detection limits (RDLs) or contract required quantitation limits.

Data qualified as rejected (i.e., "R" flagged) indicate that the associated analytical result is tainted by a major deficiency in the quality of the data. Rejected data are unsuitable for decision-making purposes. Data qualified as estimated (i.e., "J" flagged) indicate that the data is estimated, but may be used for decision-making purposes. Data qualified as undetected (i.e., "U" flagged) indicate the analyte was analyzed for but not detected. For nonradionuclides, nondetected results are reported at the practical quantitation limit (PQL). For radionuclides, nondetected results are reported at the actual value obtained from analysis (positive or negative but less than the minimum detected activity [MDA]), except for limited analyses where no value can be calculated and the analytes are reported nondetected at the MDA. All other validated results are considered accurate within the standard errors associated with the methods.

The adequacy of laboratory QA/QC was evaluated for precision, accuracy, completeness, and RDLs pursuant to the SAP (DOE-RL 2001). The organization performing the data validation reported that, of the data given formal validation, the laboratory met the standards for performance for precision $( \pm 30 \%)$, accuracy $( \pm 30 \%)$, and completeness (>90\%). 


\section{C1.2.1 Remediation Footprint}

For Areas 1 through 7, representing Trenches 2-21 and Trench 23, the area between the targeted trenches, and the surface of the haul roads between the burial ground and the staging pile area, verification sample data was provided in ten SDGs: SDG K0486, SDG J00085, SDG J00097, SDG K0491, SDG J00087, SDG K0493, SDG K0494, SDG K0638, SDG K0749, and SDG K0551. SDG K0601 was submitted for third-party validation. Verification sample data for bulk asbestos was provided in four SDGs: SDG 06I-3787-01, SDG 06I-3786-01, SDG 06I-3912-01, and SDG 06I-3936-01. Major deficiencies were identified in the analytical data set, rendering some data unacceptable for decision-making purposes. Major and minor deficiencies are discussed below.

\section{SDG K0486}

This SDG comprises 4 field samples, three collected from Area 1 (J12X44, J12X45, J12X48), and one focused field sample collected from the Cell 4 pothole (J12XB3). These samples were analyzed for ICP metals, mercury, hexavalent chromium, total petroleum hydrocarbons (TPH), volatile organic compounds (VOCs), semivolatile organic compounds (SVOCs), polychlorinated biphenyls (PCBs), and by alpha spectroscopy, beta counting, gamma spectroscopy, and liquid scintillation counting, as directed in the sample design ( $\mathrm{WCH} 2006)$. One field duplicate pair is included in this SDG (J12X44/J12X48). A major deficiency was found in SDG K0486. Major and minor deficiencies are as follows:

In the ICP metals analysis, the LCS recovery for silicon is below the acceptance criteria at $5.6 \%$. Associated sample results for silicon are likely biased low. However, the MS recovery for silicon is $182.5 \%$, showing conflicting QC results. As the QC results are significantly different and the LCS recovery is less than $10 \%$, the silicon sample results for SDG K0486 are qualified as "rejected", and flagged with an "R". Silicon is not a COPC for the 118-B-1 waste site, and the rejected silicon data do not impact completeness of the verification sampling data.

In addition, the MS recoveries for five ICP metals (aluminum, iron, manganese, silicon, and antimony) are out of acceptance criteria. For most of these analytes, the spiking concentration is insignificant compared to the native concentration in the sample from which the MS was prepared. For these analytes, the deficiency in the MS is a reflection of the analytical variability of the native concentration rather than a measure of the recovery from the sample. To confirm quantitation, post digestion spikes and serial dilutions were prepared, and the results for the four analytes are in the range of $96.6 \%$ and $102.8 \%$. Antimony and silicon did not have mismatched spike and native concentrations in the original MS. The original MS recovery for antimony is $60 \%$. Sample results for antimony are considered estimated and flagged "J". The data are useable for decision-making purposes. Silicon sample results for SDG K0486 are qualified as "rejected", and flagged with an "R", as the MS and LCS recoveries conflict significantly. 
The analytes barium, calcium, and potassium are reported in the $\mathrm{MB}$ at concentrations that are below the contract-required quantitation limits (CRQLs), however, the sample results are greater than 5 times the MB concentration. The data are useable for decision-making purposes.

The relative percent difference (RPD) value for boron is above the laboratory acceptance criteria at $87.8 \%$. The duplicate pair is near the detection limit, analysis of RPDs is not considered to be useful in the precision determination. The data are usable for decision-making purposes.

In the VOC analysis, sixteen MS recoveries are above the acceptance criteria. The MS for acetone is $358 \%$, and the MSD recovery is $308 \%$. The 1,2-dichloroethane MS and MSD are $149 \%$ and $145 \%$, respectively. The MS and MSD for 2 -butanone are $289 \%$ and $234 \%$, respectively. The carbon tetrachloride MS recovery is $142 \%$ and MSD is $141 \%$. The MS recovery is $143 \%$ for bromodichloromethane, and is $136 \%$ for 1,2-dichloropropane. For dibromochloromethane, the MS is 139\% and the MSD is $131 \%$. For trans-1,3-dichloropropene the MS is $146 \%$ and the MSD is $133 \%$. The 2-hexanone MS and MSD are $237 \%$ and $204 \%$, respectively. Field sample data for these analystes may contain high bias. Sample data detected results are considered estimated and flagged "J". However, high biased data and/or estimated data are useable for decision making purposes.

The LCS recovery for 2-butanone exceeded the acceptance criteria at $214 \%$. The data are useable for decision making purposes.

In the SVOC analysis, the MS recoveries for nitrobenzene is $47 \%$, for isophorone is $59 \%$, for 2,4 -dimethylphenol is $45 \%$, for 4 -chloro-3-methylphenol is $55 \%$, for 2-methylnaphthalene is $56 \%$, and for 1,2,4-trichlorobenzene is $53 \%$. Each of these MS recoveries is out of acceptance criteria. Sample results for these SVOCs are considered estimated and flagged "J". The data are useable for decision-making purposes.

The common laboratory contaminants bis(2-ethylhexyl)phthalate and di-n-butylphthalate are detected in the MB at concentrations less than the CRQL. The data are not otherwise affected and are useable for decision-making purposes.

In the radionuclides, the RPD calculated for total strontium in the laboratory duplicate pair is above the acceptance criteria of $30 \%$, at $160 \%$. The data are considered estimated and flagged "J". Elevated RPDs in environmental soil samples are attributed to natural heterogeneities in the soil matrix from which the sample and duplicate are prepared. The data are useable for decision-making purposes. 


\section{SDG J00085}

This SDG comprises one field sample (J12X49), a split of sample J12X44 from SDG K0486. This sample was analyzed at Lionville Laboratory, Inc., with the analyses including ICP metals, mercury, hexavalent chromium, VOC, SVOC, PCBs, and gamma spectroscopy. No major deficiencies were found in SDG J00085. Minor deficiencies are as follows:

In the ICP metals analysis, the analytes calcium, silicon, and zinc are reported in the $\mathrm{MB}$ at concentrations that are below the CRQLs, however, the sample results are greater than 5 times the MB concentration, and the data are useable for decisionmaking purposes.

In addition, the MS recoveries for seven ICP metals (aluminum, cadmium, calcium, copper, iron, antimony, and silicon) are out of acceptance criteria. For aluminum and iron, the spiking concentration is insignificant compared to the native concentration in the sample from which the MS was prepared. For these analytes, the deficiency in the MS is a reflection of the analytical variability of the native concentration rather than a measure of the recovery from the sample. Antimony and cadmium have low MS recoveries, at $50 \%$ and $32 \%$, respectively. These analytes are considered estimated and flagged "J". The calcium, copper, and silicon MS recoveries are high, indicating the sample data may be biased high. High biased or estimated data for SDG J00085 are useable for decision-making purposes.

In the VOC analysis, four MS recoveries are above the acceptance criteria. The MS for bromomethane is $162 \%$, and the MSD recovery is $158 \%$. The MS and MSD for tetrachloroethane are $155 \%$ and $153 \%$, respectively. Field sample data for these analystes may contain high bias. Sample data detected results are considered estimated and flagged "J". However, high biased data and/or estimated data are useable for decision-making purposes.

The LCS recovery for bromomethane is above the acceptance criteria, at $154 \%$. The data are useable for decision-making purposes.

In the SVOC analysis, the LCS recovery is below the acceptance criteria for 2,4-dinitrophenol, with a recovery of 0\%. All 2,4-dinitrophenol data for SDG J00085 are rejected and flagged "R." It should be noted that this analyte has been rejected on a technical basis. There were no associated analytes detected in the samples, such as the related mononitrophenols: 2-nitrophenol and 4-nitrophenol. Solvents such as this are not typically found individually without other detections. There are no known Hanford Site processes that would have resulted exclusively in this analyte. Therefore, there is no reason to assume that 2,4-dinitrophenol was present in the samples. The LCS recovery for 4,6-dinitro-2-methylphenol is $20 \%$, also below the acceptance criteria. All other QC results for 4,6-dinitro-2-methylphenol are acceptable, however, the data may be considered estimated. The 4,6-dinitro-2-methylphenol data are useable for decision-making purposes. 
In the gamma spectroscopy analysis, there was insufficient material to perform a duplicate analysis. The sample was recounted on a different detector to provide a laboratory duplicate. The RPDs calculated for europium-154 and europium-155 in the laboratory duplicate pair, are above the acceptance criteria at $51.1 \%$ and $79 \%$, respectively. In the alpha spectroscopy analysis, the RPD calculated for plutonium-239/240 in the laboratory duplicate pair is above the acceptance criteria at $200 \%$. In the liquid scintillation counting analysis, the RPDs calculated for carbon- 14 and nickel-63 in the laboratory duplicate pair are above the acceptance criteria at $45.2 \%$ and $96.7 \%$, respectively. Each of the samples results are below their MDAs, therefore, analysis of RPDs is not considered to be useful in the precision determination. The data are useable for decision-making purposes.

In the gas proportional counting analysis, the RPD calculated for total strontium in the laboratory duplicate pair is above the acceptance criteria at $170.5 \%$. The primary sample is detected with a result close to the MDA, and the duplicate is non-detected. When one of the two samples is non-detected or when the duplicate pair is near the detection limit, analysis of RPDs is not considered to be useful in the precision determination. The data are useable for decision-making purposes.

In the hexavalent chromium analysis, the MS recovery is below the acceptance criterion with a result of $42 \%$. The data are considered estimated and flagged "J", but are useable for decision-making purposes.

\section{SDG J00097}

This SDG comprises two samples (J12X49-A and J12WWO-A) that were submitted for additional radiochemical analysis. The original SDG for sample J12X49 was SDG J00085, the original SDG for sample J12WW0 was SDG J00084. For this SDG, these samples were analyzed by alpha spectroscopy and liquid scintillation counting. No major deficiencies were found in SDG J00097. Minor deficiencies are as follows:

In the alpha spectroscopy analysis, the sample J12X49-A laboratory duplicate RPDs calculated for plutonium-238, plutonium-239/240, americium-241, uranium-235, and uranium-238 are above the acceptance criteria at $200 \%, 90.5 \%, 82.0,42.7 \%$, and $35.8 \%$, respectively. The sample J12WWO-A laboratory duplicate RPDs calculated for uranium-235 and uranium-238 are above the acceptance criteria at $58.2 \%$ and $41.5 \%$, respectively. For these analytes, either one of the two samples is non-detected or the duplicate pair is near the detection limit, and analysis of RPDs is not considered to be useful in the precision determination. The data are useable for decision-making purposes.

Also in the alpha spectroscopy analysis, uranium-234 and uranium-238 are reported in the MB. The uranium-234 and uranium-238 analytical results for samples J12X49-A and J12WW0-A are biased high, and detected sample results are considered estimated and flagged "J". High biased data are useable for decision-making purposes. 


\section{SDG K0491}

This SDG comprises 12 field samples, two collected from Area 1 (J12X46, J12X47), four collected from Area 2 (J12X50-J12X53), five (J12X54-J12X58) collected from Area 3, and an equipment blank (J12X59). These samples were analyzed for ICP metals, mercury, hexavalent chromium, TPH, VOCs, SVOCs, PCBs, pesticides, herbicides, and by alpha spectroscopy, beta counting, gamma spectroscopy, and liquid scintillation counting. One field duplicate pair is included in this SDG (J12X56/J12X58). A major deficiency was found in SDG K0491. Major and minor deficiencies are as follows:

In the ICP metals analysis, the LCS recovery for silicon is below the acceptance criteria at $5.6 \%$. Associated sample results for silicon are likely biased low. However, the MS recovery for silicon is $148.0 \%$, showing conflicting quality control (QC) results. As the QC results are significantly different and the LCS recovery is less than $10 \%$, the silicon sample results for SDG K0491 are qualified as "rejected", and flagged with an "R." Silicon is not a COPC for the 118-B-1 waste site, and the rejected silicon data do not impact completeness of the verification sampling data.

The MS recoveries for five ICP metals (aluminum, iron, manganese, antimony, and silicon) are out of acceptance criteria. For most of these analytes, the spiking concentration is insignificant compared to the native concentration in the sample from which the MS was prepared. For these analytes, the deficiency in the MS is a reflection of the analytical variability of the native concentration rather than a measure of the recovery from the sample. To confirm quantitation, post digestion spikes and serial dilutions were prepared, and the results for the analytes are in the range of $88.9 \%$ and $101.9 \%$. Antimony and silicon did not have mismatched spike and native concentrations in the original MS. The original MS recovery for antimony is $51.4 \%$, and sample results are considered estimated and flagged "J". The original MS recovery for silicon is $148.0 \%$ indicating a potential high bias to the sample results. The data are useable for decision-making purposes.

The analytes barium, calcium, and potassium are reported in the $\mathrm{MB}$ at concentrations that are below the CRQLs, however, the sample results are greater than 5 times the MB concentration, and the data are useable for decision-making purposes.

In the SVOC analysis, nine MS recoveries are below the acceptance criteria. The MS for 2,4-dimethylphenol is $44 \%$, and the MSD recovery is $49 \%$. The 1,2,4-trichlorobenzene MS and MSD are $51 \%$ and $57 \%$, respectively. The isophorone MS recovery is $58 \%$, the 4-chloro-3-methylphenol MS is $55 \%$, and the 2-methylnaphthalene MS is 56\%. The MSD recovery is 59\% for 2-methylphenol and 4-methylphenol. The data are considered estimated and flagged "J". Estimated data are useable for decision-making purposes. 
No LCS recoveries results are reported for the SVOC. Due to an extraction error, no spike was added to the LCS sample. The data are not otherwise affected and are useable for decision-making purposes.

The common laboratory contaminants di-n-butylphthalate is detected in the MB at concentrations less than the CRQL. The data are not otherwise affected and are useable for decision-making purposes.

In the hexavalent chromium analysis, the sample holding time was missed for samples J12X50 through J12X53, which were taken from 118-B-1 Area 2. The samples were collected on July 20, 2006, and analyzed on September 1, 2006. Because the holding time was exceeded by less that twice the limit, the data are useable for decision-making purposes. The sample results for hexavalent chromium in SDG K0491 are considered estimated and flagged "J".

In the VOC analysis, three MS recoveries are above the acceptance criteria. The 1,2-dichloroethane MSD recovery is $142 \%$, the cis-1,3-dichloropropene MSD is $132 \%$, and the trans-1,3-dichloropropene MSD is $142 \%$. Also, the LCS recovery for 2-butanone exceeded the acceptance criteria at $214 \%$. Field sample data for these analytes may contain high bias. All sample results for these analytes were reported as nondetected. The data are useable for decision-making purposes.

Toluene and 4-methyl-2-pentanone are detected in the MB at concentrations less than the CRQL. The data are not otherwise affected and are useable for decision-making purposes.

In the chlorinated pesticides analysis, one surrogate recovery for the MSD is above the acceptance criteria with a value of $119 \%$. However, the secondary criterion for surrogate recoveries is met, as there is no more than one outlier. The data are useable for decision-making purposes.

\section{SDG K0493}

This SDG comprises nine field samples, five collected from Area 4 (J12X61 - J12X65), and four collected from Area $6(\mathrm{~J} 12 \mathrm{XC2}-\mathrm{J} 12 \mathrm{XC5})$. These samples were analyzed for ICP metals, mercury, hexavalent chromium, TPH, VOCs, SVOCs, PCBs, pesticides, herbicides, and by alpha spectroscopy, beta counting, gamma spectroscopy, and liquid scintillation counting. One field duplicate pair is included in this SDG (J12X61/J12X65). No major deficiencies were found in SDG K0493. Minor deficiencies are as follows:

All of the picloram data in SDG K0493 may be considered estimated due to lack of a MS, MSD, or LCS analysis for the analyte. The data are considered estimated and flagged "J". The data are acceptable for decision-making purposes.

In the pesticide analysis, the surrogate recoveries for samples J12X62, J12X63, $\mathrm{J} 12 \mathrm{X} 64, \mathrm{~J} 12 \mathrm{X} 65, \mathrm{~J} 12 \mathrm{XC} 4$, and the MS and MSD samples for J12X61 are outside the 
initial criterion, with high results. These samples do not meet the secondary criterion for surrogate recoveries, as there is more than one outlier for each sample. The results for these samples are considered estimated and flagged "J". Samples J12X61, J12XC2, J12XC3, and J12XC5 have one surrogate outside the acceptance criteria, also with high results. All pesticide data are acceptable for decision-making purposes.

The MS and MSD results for each of the pesticides reported in SDG K0493 are high, with all results exceeding the acceptance criteria except the MS and MSD for 4,4'-DDE and the MS and MSD for methoxyclor. The high MS and MSD results indicate that the sample results may be biased high, and the detected sample results for the pesticides in SDG K0493 (excluding 4,4'-DDE) are flagged "J". The analytes with detected results that may be biased high are beta-BHC in sample J12X64, and gamma-chlordane and endosulfan sulfate in sample J12X65. The data are useable for decision-making purposes.

The LCS recovery for aldrin is outside the acceptance criteria at $55 \%$, indicating the sample results may be biased low. However, the LSC recovery for aldrin is within the QC limits for aldrin in soil (34\% - 132\%) assigned in the Contract Laboratory Program Statement of Work for Organics Analysis (EPA 2006). The data are acceptable for decision-making purposes.

All of the toxaphene data in SDG K0493 are considered estimated due to lack of a MS, MSD, or LCS analysis for the analyte, and flagged "J". The data are acceptable for decision-making purposes.

In the VOC analysis, $13 \mathrm{MS}$ recoveries are outside the acceptance criteria. The MS for bromomethane is $234 \%$, and the MSD recovery is $232 \%$. The 2-butanone MS and MSD are $294 \%$ and $301 \%$, respectively. The MS and MSD for trichloroethene are $146 \%$ and $147 \%$, respectively. The dibromochloromethane MS recovery is $148 \%$ and MSD is $154 \%$. For bromoform, the MS is $143 \%$ and the MSD is $152 \%$. The 2 -hexanone MSD is $210 \%$. The high MS and MSD results indicate that the sample results may be biased high, however, the sample results for these VOC analytes in SDG K0493 are reported as nondetected. The data are useable for decision-making purposes.

LCS recoveries for three VOCs are outside the acceptance criteria. The bromomethane LCS recovery is $241 \%$, the 2 -butanone LCS recovery is $223 \%$, and the dibromochloromethane LCS recovery is $131 \%$. The laboratory discussed the bromomethane recovery in the data package narrative, noting that the instrument response factor was elevated for bromomethane, causing a high method blank recovery. The results for 2-butanone and dibromochloromethane are all reported as nondetected. The data are useable for decision-making purposes.

In the SVOC analysis, three MS recoveries are outside the acceptance criteria. The MS recovery for $1,2,4$-trichlorobenzene is $59 \%$, and the MSD recovery is $57 \%$. The 2-methylnaphthalene MSD is $59 \%$. The data are considered estimated and flagged "J". The data are useable for decision-making purposes. 
The common laboratory contaminants bis(2-ethylhexyl)phthalate and di-n-butylphthalate were detected in the MB at concentrations less than the CRQL. The target compound phenol was detected in the MB at levels less than the CRQL. The data are not otherwise affected and are useable for decision-making purposes.

The analytes calcium and zinc are reported in the $\mathrm{MB}$ at concentrations that are below the CRQLs. However, the sample results are greater than 5 times the MB concentration, and the data are useable for decision-making purposes.

In addition, the MS recoveries for four ICP metals (aluminum, iron, manganese, and silicon) are out of acceptance criteria. For most of these analytes, the spiking concentration is insignificant compared to the native concentration in the sample from which the MS was prepared. For these analytes, the deficiency in the MS is a reflection of the analytical variability of the native concentration rather than a measure of the recovery from the sample. To confirm quantitation, post digestion spikes and serial dilutions were prepared, and the results for the analytes are in the range of $97.0 \%$ to $107.7 \%$. Silicon did not have mismatched spike and native concentrations in the original MS. The original MS recovery for silicon is $152.4 \%$. The data are useable for decision-making purposes.

Also, in the ICP metals analysis, the LCS recovery for silicon is below the acceptance criteria at $33.0 \%$. Silicon is not a COPC for the 118-B-1 waste site.

The RPDs calculated for silicon in the laboratory duplicate pair (sample J12X61, and J12X61 [duplicate]), are above the acceptance criteria with the value of $32.8 \%$. Elevated RPDs in environmental soil samples are attributed to heterogeneities in the sample matrix and not to deficiencies in the analytical methodologies. However, because there are multiple QC issues with the silicon samples, the silicon results for SDG K0493 are considered estimated and flagged "J". The data are useable for decision-making purposes.

In the alpha spectroscopy analysis, the RPDs calculated for uranium-233/234 and uranium-238 in the laboratory duplicate pair are above the acceptance criteria at $41 \%$ and $34 \%$, respectively. Each sample result is just above the detection limit. When the duplicate pair is near the detection limit, analysis of RPDs is not considered to be useful in the precision determination. The data are useable for decision-making purposes.

\section{SDG J00087}

This SDG comprises two split samples: sample J12X60 is a split of sample J12X56 from SDG K0491, and sample J12X66 is a split of sample J12X61 from SDG K0493. This SDG was analyzed for ICP metals, mercury, hexavalent chromium, TPH, VOCs, SVOCs, pesticides, PCBs, herbicides, and by alpha spectroscopy, beta counting, gamma spectroscopy, and liquid scintillation counting. No major deficiencies were found in SDG J00087. Minor deficiencies are as follows: 
In the VOC analysis, the bromomethane LCS recovery is above the acceptance criteria with a value of $154 \%$, suggesting a high bias. The sample results for bromomethane in SDG J00087 were reported as non-detected. The data are useable for decision-making purposes.

Also in the VOC analysis, the MS and MSD recoveries for toluene are $75 \%$ and $142 \%$, respectively. The MS and MSD for tetrachloroethene are both $153 \%$. These MS and MSD recoveries are out of acceptance criteria, with high results suggesting a high bias. The sample analytical results are all below the detection limits. The data are useable for decision-making purposes.

In the SVOC analysis, the MS and MSD recoveries for 2,4-dinitrophenol are $13 \%$ and $18 \%$, respectively. The 2,4-dinitrophenol sample results are considered estimated, and flagged "J". The data are useable for decision-making purposes.

In the PCB analysis, the surrogate recovery for sample J12X66 is above the acceptance criteria with a value of $196 \%$. The surrogate recovery for the MB and one of two MS surrogates are above the acceptance criteria with values of $178 \%$ and $212 \%$ respectively. The surrogate recoveries suggest a high bias for the PCB samples, however, the PCB sample results are all reported as non-detected. The data are useable for decision-making purposes.

In the herbicide analysis, the dinoseb LCS recovery is below the acceptance criteria with a value of $37 \%$. The MS and MSD for dinoseb are also below the acceptance criteria, at $46 \%$ and $76 \%$ respectively. The dinoseb sample results in SDG J00087 may be considered estimated. The data are useable for decision-making purposes.

The analytes calcium and silicon are reported in the MB at concentrations that are below the CRQLs, however, the sample results are greater than 5 times the MB concentration. The data are useable for decision-making purposes.

The MS recoveries for eight ICP metals (aluminum, cadmium, manganese, molybdenum, silicon, silver, vanadium, and antimony) are out of acceptance criteria. For aluminum and manganese, the spiking concentration is insignificant compared to the native concentration in the sample from which the MS was prepared. For these analytes, the deficiency in the MS is a reflection of the analytical variability of the native concentration rather than a measure of the recovery from the sample. Antimony, cadmium, molybdenum, silicon, and silver have low MS recoveries, at $58 \%, 36 \%, 50 \%$, $33 \%$, and $37 \%$, respectively. These analytes are considered estimated, and flagged "J". The vanadium MS recovery is high, indicating the sample data may be biased high. All ICP metals data for SDG J00087 are useable for decision-making purposes.

The RPD value for mercury is above the laboratory acceptance criteria at $43 \%$. Elevated RPDs are attributed to natural heterogeneity of the sample matrixes. The data are usable for decision-making purposes. 
In the TPH analysis, the LCS recovery is outside the acceptance criteria at $57 \%$ and $51 \%$, and the MS recovery is outside the acceptance criteria at $45 \%$. The sample results are considered estimated and flagged "J". The data are useable for decisionmaking purposes.

The RPD calculated for TPH in the laboratory duplicate pair is above the acceptance criterion at $200 \%$. Each of the samples results are below the PQL. When the duplicate pair is near the detection limit, analysis of RPDs is not considered to be useful in the precision determination. The data are useable for decision-making purposes.

In the liquid scintillation counting analysis, the RPD calculated for carbon-14, nickel-63, and tritium in the laboratory duplicate pair is above the acceptance criteria at $69.7 \%$, $71.9 \%$, and $39.2 \%$, respectively. For tritium, the primary sample is detected just above the detection limit, and the duplicate sample is reported as below the detection limit. For carbon-14, each of the samples results is below the detection limit. The nickel-63 results are both just above the detection limit. When the duplicate pair is near the detection limit, analysis of RPDs is not considered to be useful in the precision determination. The data are useable for decision-making purposes.

The radionuclide nickel-63 is reported in the MB at a concentration similar to the MDA. Nickel-63 in sample J12X66 is reported below the detection limit. Sample J12X60 has a nickel-63 result similar to the result reported for the MB. The MB nickel-63 result indicates that the J12X60 nickel-63 result may be biased high. The J12X60 nickel-63 result is considered estimated and flagged " $\mathrm{J}$ ". The data are useable for decisionmaking purposes.

\section{SDG K0638}

This SDG comprises four field samples collected from Area 5 (J13V10-J13V13) and one focused sample (J12R33), also from Area 5. These samples were analyzed for ICP metals, mercury, hexavalent chromium, TPH, VOCs, SVOCs, PCBs, and by beta counting, gamma spectroscopy, and liquid scintillation counting. No major deficiencies were found in SDG K0594. Minor deficiencies are as follows:

In the VOC analysis, seven MS recoveries are outside the acceptance criteria. The MS and MSD recoveries for 1,1,2,2-tetrachloroethane are $66 \%$ and $40 \%$, respectively. The dibromochloromethane, 1,1,2-trichloroethane, trans-1,3-dichloropropene, bromoform, and 4-methyl-2-pentanone MSD recoveries are $66 \%, 64 \%, 65 \%, 50 \%$, and $36 \%$, respectively. The sample results for these analytes are considered estimated and flagged "J". The data are useable for decision-making purposes.

Also in the VOC analysis, one surrogate recovery for the MSD is outside the acceptance criteria with a value of $60 \%$. However, the secondary criterion for surrogate recoveries is met, as there is no more than one outlier. The data are useable for decision-making purposes. 
The LCS recovery for 1,1,2,2-tetrachloroethane is outside the acceptance criteria at $137 \%$. The high LCS recovery may indicate a high bias for the analyte in samples, however, all sample results are below the PQL. The data are useable for decisionmaking purposes.

The common laboratory contaminants methylene chloride and acetone are detected in the MB at concentrations less than the CRQL. The data are not otherwise affected and are useable for decision-making purposes.

In the SVOC analysis, nine MS recoveries are outside the acceptance criteria. The MS for nitrobenzene is $46 \%$, and the MSD recovery is $45 \%$. The MS for 2-nitrophenol is $46 \%$, and the MSD recovery is $43 \%$. The MS for $1,2,4$-trichlorobenzene is $41 \%$, and the MSD recovery is $40 \%$. The MS for 2,4-dichlorophenol is $48 \%$, and the MSD recovery is $44 \%$. The MS for 2,4 -dimethylphenol is $42 \%$, and the MSD recovery is $40 \%$. The 4-chloro-3-methylphenol MS and MSD are $58 \%$ and $49 \%$, respectively. The MS for 2-methylnaphthalene is $52 \%$, and the MSD recovery is $45 \%$. The isophorone MSD recovery is $56 \%$, and the n-nitrosodiphenylamine (1) MSD is $48 \%$. The sample results for these analytes are considered estimated and flagged "J". The data are useable for decision-making purposes.

Also in the SVOC analysis, one surrogate recovery for the sample J12R33 is outside the acceptance criteria with a value of $140 \%$. However, the secondary criterion for surrogate recoveries is met, as there is no more than one outlier. The data are useable for decision-making purposes.

The LCS recoveries for 2-nitrophenol, 2,4-dimethylphenol, 4-chloro-3-methylphenol, and 2-methylnapthalene are outside the acceptance criteria at $45 \%, 39 \%, 56 \%$, and $54 \%$, respectively. The LCS recovery for 2,4-dichlorophenol and 1,2,4-trichlorobenzene are both $48 \%$. The sample results for these analytes are considered estimated and flagged "J". The data are useable for decision-making purposes.

The LCS recovery for 4-nitroaniline is $1 \%$, therefore, the 4-nitroaniline results for SDG K0638 are rejected and flagged "R". It should be noted that this analyte has been rejected on a technical basis. There were no associated analytes detected in the samples, such as the related mononitroanaline 2-nitroanaline. Solvents such as this are not typically found individually without other detections. There are no known Hanford Site processes that would have resulted exclusively in this analyte. Therefore, there is no reason to assume that 4-nitroanaline was present in the samples. The data are not useable for decision-making purposes.

The common laboratory contaminants bis(2-ethylhexyl)phthalate and di-n-butylphthalate were detected in the MB at concentrations less than the CRQL. The data are not otherwise affected and are useable for decision-making purposes. 
In the PCB analysis, one surrogate recovery for the sample J13V12 is outside the acceptance criteria with a value of $127 \%$. However, the secondary criterion for surrogate recoveries is met, as there is no more than one outlier. The data are useable for decision-making purposes.

No MS or MSD recoveries results are reported for aroclor-1016 and aroclor-1260. The laboratory sample discrepancy report stated that no matrix spike was added to these samples. The sample results for these analytes are considered estimated and flagged "J". The data are useable for decision-making purposes.

The analytes barium, calcium, iron, magnesium, molybdenum, silver, sodium, silicon, and zinc are reported in the MB at concentrations that are just above the PQLs. The sample results are greater than 5 times the MB concentration, with the exception of silver and molybdenum, which may be biased high. The sample results for silver and molybdenum are considered estimated and flagged "J". The data are useable for decision-making purposes.

In addition, the MS recoveries for five ICP metals (aluminum, calcium, iron, manganese, and antimony) are out of acceptance criteria. For aluminum, iron, and manganese, the spiking concentration is insignificant compared to the native concentration in the sample from which the MS was prepared. For these analytes, the deficiency in the MS is a reflection of the analytical variability of the native concentration rather than a measure of the recovery from the sample. To confirm quantitation, post digestion spikes and serial dilutions were prepared for all five analytes, and the results for the analytes are in the range of $95.4 \%$ and $99.2 \%$. Antimony and calcium did not have mismatched spike and native concentrations in the original MS. The original MS recovery for calcium is $146.8 \%$, indicating a high bias in the samples. The original MS recovery for antimony is $48 \%$, and the sample results for antimony are considered estimated, and flagged with a "J". The data are useable for decision-making purposes.

Also in the ICP metals analysis, the LCS recovery for silicon is below the acceptance criteria at $14.2 \%$. Associated sample results for silicon are considered estimated and flagged "J". Silicon is not a COPC for the 118-B-1 waste site. The data are usable for decision-making purposes.

The RPD values for mercury and lead are outside the laboratory acceptance criteria at $35.3 \%$ and $30.3 \%$, respectively. Elevated RPDs are attributed to natural heterogeneity of the sample matrixes. The data are usable for decision-making purposes. 
CVP-2007-00006

Rev. 0

\section{SDG K0749}

This SDG comprises one field sample collected from Area 5 (J14JH6). Sample J14JH6 was a resample taken from Area $5 \mathrm{~A} 3$, following the removal of material at the location. This sample was analyzed by beta counting, gamma spectroscopy, and liquid scintillation counting. No major or minor deficiencies were found in SDG K0749.

\section{SDG K0494}

This SDG comprises four field samples collected from Area 7 (J12XC6 - J12XC9). These samples were analyzed for ICP metals, mercury, hexavalent chromium, VOC, SVOC, PCBs, pesticides, herbicides, and by alpha spectroscopy, beta counting, gamma spectroscopy, and liquid scintillation counting. SDG K0494 was submitted for formal third-party validation. No major deficiencies were found in SDG K0494. Minor deficiencies are as follows:

In the SVOC analysis, the MS and MSD recoveries for 1,2,4-trichlorobenzene are $57 \%$ and $59 \%$, both out of acceptance criteria. Third-party validation qualified all results for these analytes in the SDG K0494 as estimated and flagged "J". Estimated data are useable for decision-making purposes.

The RPD for the 2,4-dinitrophenol MS and MSD is outside the acceptance criteria at $65 \%$. Third party validation qualified all 2,4-dinitrophenol field sample results as estimated and flagged "J". Estimated results are useable for decision-making purposes.

The common laboratory contaminants bis(2-ethylhexyl)phthalate and di-n-butylphthalate were detected in the $\mathrm{MB}$ at $70 \mathrm{ug} / \mathrm{kg}$ and $51 \mathrm{ug} / \mathrm{kg}$, respectively; both concentrations are less than the CRQL. The target compound phenol is detected in the MB at $34 \mathrm{ug} / \mathrm{kg}$, less than the CRQL. Third-party validation raised the reported values for phenol, bis(2-ethylhexyl)phthalate, and di-n-butylphthalate in the SDG K0494 field samples to the required quantitation limit of $330 \mu \mathrm{g} / \mathrm{kg}$ and qualified them as undetected and flagged "U". Butylbenzylphthalate was also detected in the MB, at $35 \mathrm{ug} / \mathrm{kg}$, however, butylbenzylphthalate was not detected in any of the field samples. The data are not affected and are useable for decision-making purposes.

No matrix spike analysis was performed for tritium. Third party validation qualified all tritium results as estimated and flagged "J". The data are useable for decision-making purposes.

The analyte hexavalent chromium was detected just above the detection limit in the laboratory duplicate result for sample J12XC6. In the initial analysis for the sample, hexavalent chromium was non-detected. The laboratory reported an RPD value for these results of $36.6 \%$. However, when the duplicate pair is near the detection limit, analysis of RPDs is not considered to be useful in the precision determination. The data are useable for decision-making purposes. 
In the VOC analysis, $10 \mathrm{MS}$ recoveries are outside the acceptance criteria. The laboratory sample discrepancy report stated that the bromomethane response factor was running high during analysis and the percent difference for bromomethane is greater than in-house limits, causing the percent recovery for the matrix spike to run high. Bromomethane was not detected in any field samples from SDG K0494, therefore, there is no impact to the data. The MS and MSD recovery for bromomethane are $295 \%$ and $307 \%$, respectively. The 2 -butanone MS and MSD are $237 \%$ and $227 \%$, respectively. The MS and MSD for bromodichloromethane are $145 \%$ and $146 \%$, respectively. The dibromochloromethane MS is $168 \%$ and the MSD is $167 \%$. The bromoform MS and MSD are $164 \%$ and $160 \%$, respectively. The data are useable for decision-making purposes.

The LCS recoveries for bromomethane, 2-butanone, and dibromochloromethane are outside the acceptance criteria at $241 \%, 223 \%$, and $131 \%$, respectively. The data are useable for decision-making purposes.

In the pesticide analysis, third-party validation assigned a "J" flag to all aldrin results in SDG K0494, as the LCS recovery is outside QC limits at $55 \%$. The data are useable for decision-making purposes.

An MS recovery for dieldrin is outside acceptance criteria at $134 \%$. Third-party validation qualified the dieldrin sample result for sample J12XC6 as an estimate and flagged "J". Estimated data are useable for decision-making purposes.

In the pesticides analysis, all surrogate recoveries for SDG K0494 samples are above the acceptance criteria. All detected pesticide results for the samples are flagged "J" by third-party validation. The data are useable for decision-making purposes.

In the herbicides analysis, the RPD for the MS and MSD recoveries for dicamba $(76 \%)$, 2,4,5-T (36\%), and 2,4-DB (112\%) are outside the acceptance criteria. Third-party validation qualified the results as estimated and flagged "J". The data are useable for decision making purposes.

In the herbicides analysis, two MSD recoveries are below the acceptance criteria. The MSD for dicamba is $18 \%$ and for $2,4-\mathrm{B}$ is $13 \%$. All dicamba and $2,4-\mathrm{B}$ results for the samples were flagged "J" by third-party validation. The data are useable for decisionmaking purposes.

All of the toxaphene and picloram data in SDG K0494 were qualified by third-party validation as estimated with "J" flags, due to lack of a MS, MSD, or LCS analysis for the analyte. Estimated, or "J"-flagged, data are acceptable for decision-making purposes.

The analytes calcium and zinc are reported in the MB at concentrations that are just above the PQLs, however, the sample results are greater than 5 times the MB concentration, and the data are useable for decision-making purposes. 
The MS recoveries for four ICP metals (aluminum, iron, manganese, antimony and silicon) are out of acceptance criteria. For aluminum, iron, and manganese, the spiking concentration is insignificant compared to the native concentration in the sample from which the MS was prepared. For these analytes, the deficiency in the MS is a reflection of the analytical variability of the native concentration rather than a measure of the recovery from the sample. To confirm quantitation, post digestion spikes and serial dilutions were prepared for all five analytes, and the results for the analytes are in the range of $98.1 \%$ and $113.6 \%$. Antimony and silicon did not have mismatched spike and native concentrations in the original MS. The original MS recovery for silicon is $422.8 \%$, indicating the sample results may be biased high. The original MS recovery for antimony is $50.2 \%$. All antimony results for the samples are flagged "J" by third-party validation. Estimated, or "J"-flagged, data are considered acceptable for the intended use of the data. The data are useable for decision-making purposes.

Also, in the ICP metals analysis, the LCS recovery for silicon is below the acceptance criteria at $33.0 \%$. Associated sample results for silicon are likely biased low. All silicon results for the samples are flagged "J" by third-party validation. Estimated, or "J"flagged, data are considered acceptable for the intended use of the data. The data are useable for decision-making purposes.

Finally, the RPD values for molybdenum and nickel are above the laboratory acceptance criteria at $52.6 \%$ and $40.7 \%$, respectively. Elevated RPDs are attributed to natural heterogeneity of the sample matrixes. The data are usable for decision-making purposes.

\section{SDG K0551}

This SDG comprises eight field samples (J13D51 - J13D58) collected from Sampling Areas 6 and 7. These samples were analyzed for TPH only. No major or minor deficiencies were found in SDG K0551.

\section{Bulk Asbestos SDGs}

Bulk asbestos samples were collected from Areas 1, 6, and 7, and reported in four SDGs. SDG 06I-3787-01 comprises three field samples taken from Area 1. SDG 06I3786-01 comprises two field samples taken from Area 1 (in addition to one sample from the below cleanup level [BCL] material stockpiles). SDG 06I-3912-01 comprises four samples taken from Area 6. SDG 06I-3936-01 comprises four samples taken from Area 7. No major or minor deficiencies were found in SDG 06I-3787-01, SDG 06I-378601, SDG 06I-3912-01, or SDG 06I-3936-01.

\section{C1.2.2 Overburden and BCL Stockpiles}

For the overburden and $B C L$ stockpiles, verification sample data was provided in six SDGs: SDG K0471, SDG K0484, SDG K0474, SDG K0491, SDG J00084, and 
SDG J00097. Verification sample data for bulk asbestos was provided in four SDGs: SDG 06I-3564-01, SDG 06I-3658-01, SDG 06I-3634-01, and SDG 06I-3786-01. Major deficiencies were identified in the analytical data set, rendering some data unacceptable for decision-making purposes. Major and minor deficiencies are discussed below.

\section{SDG K0471}

This SDG comprises 16 field samples, seven collected from the 118-B-1 overburden stockpiles (J12WKO - J12WK6), and nine collected from the BCL stockpiles (J12WK7 J12WK9 and J12WL0 - J12WL5). These samples were analyzed for ICP metals, mercury, hexavalent chromium, TPH, VOCs, SVOCs, PCBs, pesticides, herbicides, and by alpha spectroscopy, beta counting, gamma spectroscopy, and liquid scintillation counting. One field duplicate pair is included in this SDG (J12WL4/J12WL5). One major deficiency was found in SDG K0471. Major and minor deficiencies are as follows:

In the herbicide analysis, eight MS recoveries are below the acceptance criteria. The dicamba MS and MSD are $0 \%$ and $22 \%$, respectively. The picloram MS and MSD are $3 \%$ and $10 \%$, respectively. The dichloroprop, 2,4-D, and 2,4,5-T MSDs are $28 \%, 23 \%$, and $25 \%$, respectively. The 2,4-DB MS is $25 \%$. The LCS for picloram is below EPA Contract Laboratory Program Quality Control criteria with a recovery of $53 \%$. As the picloram MS, MSD, and BS are all outside the criteria, the picloram sample results are rejected for the SDG K0471; and are flagged "R". All other herbicide data are useable for decision-making purposes.

Also in the herbicides analysis, surrogate recoveries for sample J12WK0, J12WK1, and J12WL2 are below the acceptance criteria, with results of $35 \%, 16 \%$, and $39 \%$ respectively. All herbicide results for the samples are considered estimated and flagged "J". The data are useable for decision-making purposes.

In the SVOC analysis, seven MS recoveries are below the acceptance criteria. The MS and MSD recovery for 2,4-dimethylphenol are both $46 \%$. The 1,2,4-trichlorobenzene MS and MSD are $52 \%$ and $51 \%$, respectively. The MS and MSD for 2-methylnaphthalene are $57 \%$ and $58 \%$, respectively. The isophorone MS is $59 \%$. The sample results for these SVOCs are considered estimated and flagged "J". Estimated data are useable for decision-making purposes.

The LCS recovery for 2,4-dinitrophenol is below the acceptance criteria at $3 \%$. The sample results for 2,4-dinitrophenol are rejected and flagged " $R$ ". It should be noted that this analyte has been rejected on a technical basis. There were no associated analytes detected in the samples, such as the related mononitrophenols: 2-nitrophenol and 4-nitrophenol. Solvents such as this are not typically found individually without other detections. There are no known Hanford Site processes that would have resulted exclusively in this analyte. Therefore, there is no reason to assume that 2,4dinitrophenol was present in the samples. 
The common laboratory contaminants bis(2-ethylhexyl)phthalate is detected in the MB at concentrations less than the CRQL. The data are not otherwise affected and are useable for decision-making purposes.

In the VOC analysis, methylene chloride is reported in the MB at a concentration of $11 \mathrm{ug} / \mathrm{kg}$, above the PQL of $10 \mathrm{ug} / \mathrm{kg}$. The sample results are reported at concentrations similar to the PQL, with a range of $3-18 \mathrm{ug} / \mathrm{kg}$. The sample results for methylene chloride are likely biased high, and are considered estimated and flagged "J". Estimated data are useable for decision-making purposes.

In the chlorinated pesticides analysis, surrogate recoveries for five samples are outside the acceptance criteria. However, the secondary criterion for surrogate recoveries is met in four samples, as there is no more than one outlier. Sample J12WK4 recoveries are outside the acceptance criteria for both surrogates, and all pesticide results for the sample are flagged "J". The data are useable for decision-making purposes.

Five MS recoveries in the pesticides analysis are below the acceptance criteria. The alpha-BHC MS and MSD are $58 \%$ and $23 \%$, respectively. The gammabenzenehexachloride, delta-benzenehexachloride, and 4,4'-dichlorodiphenyldichloroethylene MSDs are $26 \%, 30 \%$, and $57 \%$, respectively. The sample results for these analytes are considered estimated and flagged "J". The data are useable for decision-making purposes.

The analytes calcium, iron, sodium, and silicon are reported in the MB at concentrations that are just above the PQLs, however, the sample results are greater than 5 times the $\mathrm{MB}$ concentration, and the data are useable for decision-making purposes.

In addition, the MS recoveries for five ICP metals (aluminum, iron, manganese, antimony, and silicon) are out of acceptance criteria. For aluminum, iron, and manganese, the spiking concentration is insignificant compared to the native concentration in the sample from which the MS was prepared. For these analytes, the deficiency in the MS is a reflection of the analytical variability of the native concentration rather than a measure of the recovery from the sample. To confirm quantitation, post digestion spikes and serial dilutions were prepared for all five analytes, and the results for the analytes are in the range of $90.4 \%$ and $103.7 \%$. Antimony and silicon did not have mismatched spike and native concentrations in the original MS. The original MS recovery for antimony is $38 \%$ and for silicon is $57 \%$. The sample results for antimony and silicon in SDG K0471 are considered estimated and flagged "J". Estimated data are useable for decision-making purposes.

Also, in the ICP metals analysis, the LCS recovery for silicon is below the acceptance criterion at $51.1 \%$. Associated sample results for silicon are likely biased low. Silicon is not a COPC for the 118-B-1 waste site. The data are useable for decision-making purposes. 
The analyte hexavalent chromium was detected just above the detection limit in the initial result for sample J11VL2. In the laboratory duplicate for the sample, hexavalent chromium is non-detected. The laboratory reported an RPD value for these results of $54.5 \%$. However, when the duplicate pair is near the detection limit, analysis of RPDs is not considered to be useful in the precision determination. The data are useable for decision-making purposes.

In the gamma spectroscopy analysis, the RPD calculated for cesium-137 in the laboratory duplicate pair is above the acceptance criteria at $56.0 \%$. The primary sample is detected just above the detection limit, and the duplicate sample is reported as below the detection limit. When the duplicate pair is near the detection limit, analysis of RPDs is not considered to be useful in the precision determination. The data are useable for decision-making purposes:

\section{SDG K0484}

This SDG comprises 5 field samples collected from the 118-B-1 overburden stockpiles (J12WM7 - J12WM9 and J12WN0 - J12WN1). These samples were analyzed for ICP metals, mercury, hexavalent chromium, TPH, VOCs, SVOCs, PCBs, pesticides, herbicides, and by alpha spectroscopy, beta counting, gamma spectroscopy, and liquid scintillation counting. No major deficiencies were found in SDG K0471. Minor deficiencies are as follows:

In the VOC analysis, five MS recoveries are above the acceptance criteria. The MS and MSD recovery for 1,1,2,2-tetrachloroethene are $153 \%$ and $182 \%$, respectively. The MSD recoveries for dibromochloromethane, 1,1,2-trichloroethane, and trans-1,3dichloropropene are $143 \%, 139 \%$, and $131 \%$, respectively. These MS recoveries indicate a potential high bias for the sample results. However, all sample results for these analytes are reported as nondetected. The data are useable for decision-making purposes.

The LCS recoveries for 2-butanone are above the acceptance criteria at 214\% and $207 \%$, indicating a potential high bias for the data. However, the sample results for 2-butanone are reported as the PQL. The data are useable for decision-making purposes.

The toluene and 4-methyl-2-pentanone are detected in the MB at concentrations less than the CRQL, indicating that sample data may be biased high. All sample results for these analytes are similar to the PQL. The data are not otherwise affected and are useable for decision-making purposes.

For the herbicide analyses, the target compound dalapan was detected in the MB at a concentration above the PQL. All samples were reanalyzed, and dalapan was again detected in the MB at a concentration above the PQL. Both sets of analytical results are reported. All dalapan analytical results are reported as the PQL or detected below the PQL. The data are useable for decision-making purposes. 
For the herbicide analysis, the surrogate recoveries for two of the samples are below the acceptance criteria. These samples are J12WN0 and J12WN1. The herbicide data for these samples are considered estimated and flagged "J". Estimated data are useable for decision-making purposes.

The MSDs for dicamba do not meet the acceptance criteria for either the first or second sample analysis. However, the MSs for dicamba are within the acceptance criteria, and the data are useable for decision-making purposes.

Nine LCS recoveries for herbicides are below the acceptance criteria. The dicamba LCS and LCS duplicate are $22 \%$ and $17 \%$, respectively. The 2,4-D LCS and LCS duplicate are $20 \%$ and $25 \%$, respectively. The $2,4,5$-T LCS and LCS duplicate are $28 \%$ and $23 \%$, respectively. The LCS recoveries for dalaprop and dichloroprop are $6 \%$ and $10 \%$, respectively, and the LCS duplicate for pentachlorophenol is $47 \%$. The data for these analytes are considered estimated and flagged "J". The data are useable for decision-making purposes.

In the SVOC analysis, the common laboratory contaminants bis(2-ethylhexyl)phthalate and di-n-butylphthalate are detected in the MB at concentrations less than the CRQL. The data are not otherwise affected and are useable for decision-making purposes.

The LCS recovery for 1,2,4-trichlorobenzene is below the acceptance criteria with a reported result of $57 \%$. The data for 1,2,4-trichlorobenzene are considered estimated and flagged "J". The data are useable for decision-making purposes.

In the pesticides analysis, one surrogate recovery for sample J12WM9 is outside the acceptance criteria. However, the secondary criterion for surrogate recoveries is met for the sample, as there is no more than one outlier. Both surrogate recoveries for sample J12WM7, and the sample J12WM7 MS and MSD are above the acceptance criteria, indicating a potential high bias in the data. The sample results are all reported as nondetects, with the exception of methoxychlor. The detected methoxychlor results in SDG K0484 are considered estimated and flagged "J". The data are useable for decision-making purposes.

Also in the pesticides analysis, four MS recoveries are above the acceptance criteria. The MS recoveries for alpha-BHC, endosulfan I, and endosulfan II are $131 \%, 129 \%$, and $131 \%$, respectively, indicating a potential high bias in the samples for these analytes. The sample results for these analytes are reported as non-detects. The MSD for alpha-BHC is $47 \%$, however, the MS was within the acceptance criteria. The data are useable for decision-making purposes.

In the metals analysis, calcium was reported in the MB at a concentration just above the $\mathrm{PQL}$, however, the sample results are greater than 5 times the MB concentration, and the data are useable for decision-making purposes. 
The MS recoveries for six ICP metals (aluminum, iron, manganese, antimony, silicon, and vanadium) are out of acceptance criteria. For aluminum, iron, and manganese, the spiking concentration is insignificant compared to the native concentration in the sample from which the MS was prepared. For these analytes, the deficiency in the MS is a reflection of the analytical variability of the native concentration rather than a measure of the recovery from the sample. To confirm quantitation, post digestion spikes and serial dilutions were prepared for all five analytes, and the results for the analytes are in the range of $97.7 \%$ and $103.6 \%$. Antimony, silicon, and vanadium did not have mismatched spike and native concentrations in the original MS. The original MS recovery for silicon is high, at $192.2 \%$. The original MS recoveries for antimony $(41.6 \%)$ and for vanadium $(65.5 \%)$ are low. The antimony and vanadium data are considered estimated and flagged "J". Estimated data are considered acceptable for decision-making purposes.

The LCS recovery for silicon is below the acceptance criteria at $13.3 \%$. Associated sample results for silicon are likely biased low, and the sample results for silicon are considered estimates and flagged "J". Silicon is not a COPC for the 118-B-1 waste site. Estimated data are considered acceptable for decision-making purposes.

The RPD value for potassium and sodium are above the laboratory acceptance criteria at $31.1 \%$ and $115.4 \%$, respectively. Elevated RPDs are attributed to natural heterogeneity of the sample matrixes. The data are usable for decision-making purposes.

\section{SDG K0474}

This SDG comprises 11 field samples, four collected from the 118-B-1 overburden stockpiles (J12WM3 - J12WM6), and seven collected from the BCL stockpiles (J12WM0 - J12WM3 and J12WL6 - J12WL9). These samples were analyzed for ICP metals, mercury, hexavalent chromium, TPH, VOCs, SVOCs, PCBs, pesticides, herbicides, and by alpha spectroscopy, beta counting, gamma spectroscopy, and liquid scintillation counting. No major deficiencies were found in SDG K0474. Minor deficiencies are as follows:

In the VOC analysis, holding times were exceeded for samples J12WM5 and J12WM6. The holding time was also exceeded when sample J12WL7 was reanalyzed. The samples were collected on 7/12/2006, and were analyzed on 7/25/2006 through $7 / 31 / 2006$. The holding time was exceeded by less that twice the limit, and the data are considered estimated and flagged "J". The data are useable for decision-making purposes.

Also in the VOC analysis, $24 \mathrm{MS}$ recoveries are above the acceptance criteria, indicating a potential high bias in the data. The MS for acetone is $409 \%$, and the MSD recovery is $417 \%$. The MS for 1,2-dichloroethane is $143 \%$, and the MSD recovery is $153 \%$. The MS for 2-butanone is $318 \%$, and the MSD recovery is $340 \%$. The MS for carbon tetrachloride is $193 \%$, and the MSD recovery is $176 \%$. The MS for 1,2-dichloropropane is $133 \%$, and the MSD recovery is $134 \%$. The 
dibromochloromethane MS and MSD are $132 \%$ and $133 \%$, respectively. The MS for trans-1,3-dichloropropene is $137 \%$, and the MSD recovery is $134 \%$. The 4-methyl-2pentanone MS and MSD are $160 \%$ and $180 \%$, respectively. The MS for 2-hexanone is $245 \%$, and the MSD recovery is $263 \%$. The 1,1,2,2-tetrachloroethane MS recovery is $136 \%$, and the MSD is $137 \%$. The MS recovery for bromodichloromethane and cis-1,3-dichloropropene are $142 \%$ and $131 \%$, respectively. The 1,1,2-trichlorethane MSD is $131 \%$. The data for these analytes are reported as detected below the PQL or as nondetected, with the exception of carbon tetrachloride in select samples. The detected carbon tetrachloride sample results are considered estimated and flagged "J". The data are useable for decision-making purposes.

In the PCB analysis, one surrogate recovery for sample J12WM0 is above the acceptance criteria. However, the secondary criterion for surrogate recoveries is met for the sample, as there is no more than one outlier. Both surrogate recoveries for samples J12WL9 and J12WM6 are below the acceptance criteria. The reported results for these samples are considered estimated and flagged "J". The data are useable for decisionmaking purposes.

In the pesticides analysis, both surrogate recoveries for samples J12WL6, J12WM6, and J12WL9 are outside the acceptance criteria. The surrogate recoveries for J12WL6 are high, at $135 \%$ and $149 \%$, indicating a potential high bias. All pesticide results for these samples are less than the PQL. The surrogate recoveries for J12WM6 and J12WL9 are low, at $12 \%$ and $5 \%$, and $19 \%$ and $11 \%$, respectively. The pesticide results for these samples are considered estimated and flagged "J". The data are useable for decision-making purposes.

All of the toxaphene data in SDG K0474 is considered estimated with "J" flags, due to lack of a MS, MSD, or LCS analysis for the analyte. Estimated, or "J"-flagged, data are acceptable for decision-making purposes.

In the SVOC analysis, the common laboratory contaminants bis(2-ethylhexyl)phthalate and di-n-butylphthalate were detected in the MB at concentrations less than the CRQL. The data are not otherwise affected and are useable for decision-making purposes.

Also in the SVOC analysis, 37 MS recoveries are below the acceptance criteria. The laboratory sample discrepancy report cited an MS loss during extraction as the probable cause for the low recoveries. The MSD recovery, surrogate recovery, and LCS recovery for each of these analytes are within the acceptance criteria. The data are useable for decision-making purposes.

In the herbicide analysis, the surrogate recoveries for samples J12WL9, J12WM0, and J12WM5, the J12WM5 MS, the MB, and the MB duplicate are below the acceptance criteria. It appears that samples J12WL9, J12WM0, J12WM5, and J12WM0 MS were rerun, with similar or slightly improved surrogate recoveries, though still below the acceptance criteria. Herbicide analytical results for these samples are considered estimated and flagged "J". The data are useable for decision-making purposes. 
Also in the herbicide analysis, the MS recovery for dichloroprop is $6 \%$, below the acceptance criteria. The J12WMO MS was rerun, and the MS and MSD for that sample are within the criteria. The data are useable for decision-making purposes.

The LCS recoveries for six herbicides are below the acceptance criteria. The LCS and LCS duplicate for dicamba are both $18 \%$, for dichloroprop, they are both $21 \%$, and for $2,4-\mathrm{D}$ they are both $14 \%$. The $2,4,5$-TP LCS recovery is $27 \%$, with a LCS duplicate recovery of $25 \%$. The $2,4,5-\mathrm{T}$ LCS recovery is $17 \%$, with a LCS duplicate recovery of $16 \%$. The pentachlorophenol LCS recovery is $39 \%$. The results for these analytes are considered estimated and flagged "J". The data are useable for decision-making purposes.

The MS recoveries for six ICP metals (aluminum, iron, mercury, manganese, antimony, and silicon) are out of acceptance criteria. For aluminum, iron, manganese, and silicon, the spiking concentration is insignificant compared to the native concentration in the sample from which the MS was prepared. For these analytes, the deficiency in the MS is a reflection of the analytical variability of the native concentration rather than a measure of the recovery from the sample. To confirm quantitation, post digestion spikes and serial dilutions were prepared for all five analytes, and the results for the analytes are in the range of $99.3 \%$ and $107.4 \%$. Antimony and mercury did not have mismatched spike and native concentrations in the original MS. The original MS recovery for mercury is $197.8 \%$, indicating the sample results may be biased high. The sample results are reported as either the PQL or similar to the PQL. The original MS recovery for antimony is $52.6 \%$. The antimony data is considered estimated and flagged "J". All the metals data are useable for decision-making purposes.

The LCS recovery for silicon is below the acceptance criteria at $30.1 \%$. Associated sample results for silicon are likely biased low. Silicon is not a COPC for the 118-B-1 waste site. The data are useable for decision-making purposes.

In the gamma spectroscopy analysis, the RPDs calculated for cesium-137 and europium-155 in the laboratory duplicate pair are above the acceptance criteria at $50 \%$ and $47 \%$, respectively. For both analytes, the primary sample is detected just above the detection limit, and the duplicate sample is reported as below the detection limit. When the duplicate pair is near the detection limit, analysis of RPDs is not considered to be useful in the precision determination. The data are useable for decision-making purposes.

\section{SDG J00084}

This SDG comprises one field sample (J12WW0), a split of sample J12WL6 in SDG K0474. This sample was analyzed for ICP metals, mercury, hexavalent chromium, TPH, VOCs, SVOCs, PCBs, pesticides, herbicides, and by alpha spectroscopy, beta counting, gamma spectroscopy, and liquid scintillation counting. No major deficiencies were found in SDG J00084. Minor deficiencies are as follows: 
In the VOC analysis, one surrogate recovery is above the acceptance criteria with a value of $129 \%$. However, the secondary criterion for surrogate recoveries is met, as there is no more than one outlier. The data are useable for decision-making purposes.

In the SVOC analysis, the MSD recovery for 2,6-dinitrotoluene is $108 \%$, and for 2,4-dinitrotoluene, it is $113 \%$. Both of these recoveries are above the acceptance criteria indicating a potential high bias. The samples results were reported as nondetected. The data are useable for decision-making purposes.

In the PCB analysis, one surrogate recovery is above the acceptance criteria with a value of $190 \%$. The surrogate recovery for the MS and MSD are above the acceptance criteria with values of $180 \%$ and $173 \%$ respectively. The detected sample results for aroclor- 1254 and aroclor- 1260 are may be biased high, and are considered estimated and flagged "J." The data are useable for decision-making purposes.

The analytes barium, calcium, silicon and sodium are reported in the MB at concentrations that are below the CRQLs, however, the sample results are greater than 5 times the MB concentration, and the data are useable for decision-making purposes.

The MS recoveries for eleven ICP metals (aluminum, cadmium, calcium, copper, iron, magnesium, manganese, mercury, silicon, silver, and antimony) are out of acceptance criteria. For aluminum, iron, and manganese, the spiking concentration is insignificant compared to the native concentration in the sample from which the MS was prepared. For these analytes, the deficiency in the MS is a reflection of the analytical variability of the native concentration rather than a measure of the recovery from the sample. Four of the analytes that did not have mismatched spike and native concentrations in the original MS have low MS recoveries. Antimony has MS and MSD recoveries of $44 \%$ and $45 \%$, respectively. Cadmium has MS and MSD recoveries of $51 \%$ and $55 \%$, respectively. Silver has MS and MSD recoveries of $67 \%$ and $62 \%$, respectively. Silicon has a MS recovery of $44 \%$; however, the MSD is within acceptance criteria at $78 \%$. Antimony, cadmium, and silver are considered estimated and flagged "J." The remaining analytes with MS recoveries out of acceptance criteria had MS and MSD recoveries that are high, indicating the sample data may be biased high. All ICP metals data for SDG J00084 are useable for decision-making purposes.

Also, in the ICP metals analysis, the LCS recovery for silicon is above the acceptance criteria at $144 \%$. Associated sample results for silicon are likely biased high. Silicon is not a COPC for the 118-B-1 waste site. The data are useable for decision-making purposes.

In the TPH analysis, the LCS recovery is below the acceptance criteria at $54 \%$ and $53 \%$, and the MS recovery is below the acceptance criteria at $49 \%$. The sample results are considered estimated and flagged "J." The data are useable for decision-making purposes. 
In the hexavalent chromium analysis, the MS recovery is below the acceptance criteria with a result of $62 \%$. The sample results are considered estimated and flagged "J." The data are useable for decision-making purposes.

In the alpha-energy analysis for plutonium-239/240, the RPD calculated in the laboratory duplicate pair is above the acceptance criteria at $251 \%$. The primary sample is detected just above the detection limit, and the duplicate sample is reported as the PQL. When the duplicate pair is near the detection limit, analysis of RPDs is not considered to be useful in the precision determination. The data are useable for decision-making purposes.

In the liquid scintillation counting analysis, the RPDs calculated for carbon-14, nickel-63, and tritium in the laboratory duplicate pair are above the acceptance criteria at $40.5 \%$, $174.1 \%$, and $161.4 \%$, respectively. For carbon-14, the primary sample is reported as below the PQL, and the duplicate sample is detected just above the detection limit. For nickel-63 and tritium, each of the samples results is below the detection limit. When the duplicate pair is near the detection limit, analysis of RPDs is not considered to be useful in the precision determination. The data are useable for decision-making purposes.

In the gamma spectroscopy analysis, the RPDs calculated for silver-108m, cobalt-60, and europium-154 in the laboratory duplicate pair are above the acceptance criteria at $233 \%, 95.3 \%$, and $49 \%$, respectively. Each of the samples results are below the PQL. When the duplicate pair is near the detection limit, analysis of RPDs is not considered to be useful in the precision determination. The data are useable for decision-making purposes.

\section{Bulk Asbestos SDGs}

Bulk asbestos samples were collected from the overburden and BCL stockpiles, and reported in four SDGs. SDG 06I-3564-01 comprises 16 field samples taken from the overburden and BCL piles. SDG 06I-3658-01 comprises five field samples taken from the overburden piles. SDG 06I-3634-01 comprises 11 samples taken from the overburden and BCL piles. SDG 06I-3786-01 comprises one sample taken from the $B C L$ piles (in addition to two samples from Area 1). No major or minor deficiencies were found in SDG 06I-3564-01, SDG 06I-3658-01, SDG 06I-3634-01, or SDG 06I-3786-01.

\section{C1.2.3 Waste Staging/Sorting Area Footprint}

The verification sample data collected from the waste staging pile area (SPA) were provided in six SDGs: SDG K0594, SDG J00103, SDG K0595, SDG K0597, SDG K0601, and SDG K0602. Verification sample data for bulk asbestos samples were provided in five SDGs: SDG 06-A-5594, SDG 06-A-5679, SDG 06I-5661-01, SDG 06I-5717-01, and SDG 06I-5735-01. Major deficiencies were identified in the analytical data set, rendering some data unacceptable for decision-making purposes. Major and minor deficiencies are discussed below. 


\section{SDG K0594}

This SDG comprises six field samples collected from the SPA (J13HY3 - J13HY7 and J13J09). These samples were analyzed for ICP metals, mercury, hexavalent chromium, TPH, VOCs, SVOCs, PCBs, pesticides, herbicides, and by alpha spectroscopy, beta counting, gamma spectroscopy, and liquid scintillation counting. One field duplicate pair is included in this SDG (J13HY7/J13J09). A major deficiency was found in SDG K0594. Major and minor deficiencies are as follows:

In the VOC analysis, the samples were analyzed outside the required holding time. The samples were collected on 10/12/06, and were analyzed on 11/2/06. The holding time was exceeded by less that twice the limit, and the data are considered estimated and flagged "J". Estimated data are useable for decision-making purposes.

The common laboratory contaminant methylene chloride is detected in the MB at concentrations less than the CRQL. The data are not otherwise affected and are useable for decision-making purposes.

Five MS recoveries in the VOC analysis are above the acceptance criteria. The acetone MS recovery is $304 \%$. The laboratory case narrative explained that the internal standard area criteria were not met for sample J13HY3 MS, and that the analysis of associated matrix spike duplicate fulfills the reanalysis requirement. The MSD recoveries for 1,1-dichloroethane and 1,2-dichloropropane are $143 \%$ and $139 \%$, respectively. The chloroform and 1,1,1-trichloroethane MSDs are both $141 \%$. The J13HY3 MSD was inadvertently analyzed out of tune time (12 hr), according to the laboratory case narrative. The data are useable for decision-making purposes.

In the SVOC analysis, five MS recoveries are below the acceptance criteria. The MSD recoveries for nitrobenzene, isophorone, and 2-methylnaphthalene are $48 \%, 57 \%$, and $54 \%$, respectively. The 1,2,4-trichlorobenzene MS and MSD are $49 \%$ and $47 \%$, respectively. The data are considered estimated and flagged "J." Estimated data are useable for decision-making purposes.

The LCS recoveries for 2,4-dimethylphenol, 1,2,4-trichlorobenzene, and 2 -methylnaphthalene are below the acceptance criteria at $48 \%, 53 \%$, and $59 \%$, respectively. The data are considered estimated and flagged "J." The data are useable for decision-making purposes.

The common laboratory contaminants bis(2-ethylhexyl)phthalate was detected in the $\mathrm{MB}$ at concentrations less than the CRQL. The data are not otherwise affected and are useable for decision-making purposes.

In the herbicides analysis, five MS recoveries are below the acceptance criteria. The MS recoveries for dichloroprop, 2,4-D, and 2,4,5-T are 29\%, 25\%, and $28 \%$, respectively. The 2,4-DB MS and MSD are both $0 \%$. The 2,4-DB sample results in SDG K0594 are rejected and flagged "R." In soil, the compound 2,4-DB has a half-life 
of approximately seven days, and is broken down into 2,4-D. The 2,4-D analytical results were reported as non-detected, indicating the 2,4-DB sample concentrations are below the RAG concentrations. The rejected results for 2,4-DB do not impact completeness of the verification sampling data.

The LCS recovery for pentachlorophenol is below the acceptance criteria at $47 \%$. The data are considered estimated and flagged "J." The data are useable for decisionmaking purposes.

In the pesticides analysis, six surrogate recoveries for five samples are above the acceptance criteria. However, the secondary criterion for surrogate recoveries is met for four of the five samples, as there is no more than one outlier for the samples. Both surrogate recoveries for sample J13HY5 exceeded the acceptance criteria. One pesticide is detected for sample J13HY5, however, it is detected below the CRQL, and therefore is already flagged "J" and considered estimated. The data are useable for decision-making purposes.

Also in the pesticides analysis, six MS recoveries are above the acceptance criteria, indicating a potential high bias. The aldrin MS recovery is $121 \%$. The Endosulfan I MS recovery is $125 \%$. The MS and MSD recovery for $4,4^{\prime}$-DDE are $138 \%$ and $126 \%$, respectively. The 4,4'-DDD MS and MSD are $153 \%$ and $147 \%$, respectively. The sample results for these analytes are reported as nondetected. The data are useable for decision-making purposes.

The LCS recovery for 4,4'-DDE is above the acceptance criterion at $125 \%$. The high MB may indicate a high bias for 4,4'-DDE in samples, however, all sample results for 4,4'DDE are below the PQL. The data are useable for decision-making purposes.

The analytes calcium, chromium, potassium, and sodium were reported in the $\mathrm{MB}$ at concentrations that are just above the PQLs, however, the sample results are greater than 5 times the MB concentration, and the data are useable for decision-making purposes.

The MS recoveries for four ICP metals (aluminum, iron, manganese, and silicon) are out of acceptance criteria. For aluminum, iron, and manganese, the spiking concentration is insignificant compared to the native concentration in the sample from which the MS was prepared. For these analytes, the deficiency in the MS is a reflection of the analytical variability of the native concentration rather than a measure of the recovery from the sample. To confirm quantitation, post digestion spikes and serial dilutions were prepared for all five analytes, and the results for the analytes are in the range of $87.6 \%$ and $99.4 \%$. Silicon did not have mismatched spike and native concentrations in the original MS. The original MS recovery for silicon is $210.1 \%$, indicating a potential high bias. Silicon is not a COPC for the 118-B-1 waste site. The data are useable for decision-making purposes.

Also in the ICP metals analysis, the LCS recovery for silicon is below the acceptance criterion at $41.5 \%$. Associated sample results for silicon are likely biased low. Silicon is 
not a COPC for the 118-B-1 waste site. The data are useable for decision-making purposes.

The RPD value for molybdenum is above the laboratory acceptance criteria at $36.0 \%$. When the duplicate pair is near the detection limit, analysis of RPDs is not considered to be useful in the precision determination. The data are usable for decision-making purposes.

In the TPH analysis, the initial result is reported as non-detected for sample J13HY3. In the laboratory duplicate for the sample, TPH were detected just above the detection limit. The laboratory reported an RPD value for these results of $44.3 \%$. However, when the duplicate pair is near the detection limit, analysis of RPDs is not considered to be useful in the precision determination. The data are useable for decision-making purposes.

\section{SDG J00103}

This SDG comprises one field sample (J13J10), a split of sample J13HY7. This sample was analyzed for ICP metals, mercury, hexavalent chromium, TPH, VOCs, SVOCs, PCBs, and by alpha spectroscopy, beta counting, gamma spectroscopy, and liquid scintillation counting. No major deficiencies were found in SDG J00103. Minor deficiencies are as follows:

In the VOC analysis, acetone and toluene are detected in the MB at concentrations less than the CRQL. The data are not affected and are useable for decision-making purposes.

The bromomethane LCS recovery is below the acceptance criteria with a value of $43 \%$. The MS and MSD recoveries for bromomethane are below the acceptance criteria, at $31 \%$ and $28 \%$, respectively. The bromomethane sample results for SDG J00103 are considered estimated and flagged "J." The data are useable for decision-making purposes.

The MS and MSD recoveries for acetone are out of acceptance criteria, at $158 \%$ and $217 \%$, respectively, indicating a potential high bias. The sample analytical result was detected at a concentration less than the PQL. The data are useable for decisionmaking purposes.

In the pesticide analysis, the heptachlor LCS recovery is above the acceptance criterion with a value of $984 \%$. The MS and MSD for heptachlor are also above the acceptance criteria, at $177 \%$ and $150 \%$, respectively. These QC results indicate a potential high bias, however, the heptachlor data is reported as non-detected. The data are useable for decision-making purposes.

In the herbicide analysis, the dinoseb LCS recovery is below the acceptance criterion with a value of $69 \%$. The MS and MSD for dinoseb are also out of acceptance criteria, 
at $105 \%$ and $84 \%$, respectively. The data may be considered estimated. The data are useable for decision-making purposes.

The analytes boron, calcium, selenium, silicon, sodium, and zinc are reported in the MB at concentrations that are below the CRQLs. The sample results are greater than 5 times the MB concentration, with the exception of selenium. The selenium sample results for SDG J00103 may be biased high, and are considered estimated with a "J" flag. Estimated data are useable for decision-making purposes.

In addition, the MS recoveries for eight ICP metals (aluminum, calcium, iron, manganese, lead, silicon, silver, and antimony) are out of acceptance criteria. For aluminum and iron, the spiking concentration is insignificant compared to the native concentration in the sample from which the MS was prepared. For these analytes, the deficiency in the MS is a reflection of the analytical variability of the native concentration rather than a measure of the recovery from the sample. Three of the analytes that did not have mismatched spike and native concentrations in the original MS has low MS recoveries. Antimony has MS and MSD recoveries of $40 \%$ and $41 \%$, respectively. Silicon has MS and MSD recoveries of $42 \%$ and $34 \%$, respectively. Silver has a MS recovery of $74 \%$; however, the MSD is within acceptance criteria at $75 \%$. These analytes are considered estimated and flagged "J." The remaining analytes with MS recoveries out of acceptance criteria had MS and MSD recoveries that are high, indicating the sample data may be biased high. All ICP metals data for SDG J00103 are useable for decision-making purposes.

In the alpha-energy analysis for uranium-235, uranium-238, plutonium-238 and plutonium-239/240, the RPDs calculated in the laboratory duplicate pair are above the acceptance criteria at $200 \%, 38.9 \%, 326 \%$ and $77.4 \%$, respectively. The uranium-235 and plutonium-238 results are both below the detection limit. The plutonium-239/240 results are both detected just above the detection limit. The uranium-238 primary result is below the detection limit with the duplicate result just above the detection limit. When the duplicate pair is near the detection limit, analysis of RPDs is not considered to be useful in the precision determination. The data are useable for decision-making purposes.

In the liquid scintillation counting analysis, the RPDs calculated for plutonium-241, carbon-14, and nickel-63 in the laboratory duplicate pair are above the acceptance criteria at $124.6,738.9 \%$, and $43.6 \%$, respectively. For plutonium- 241 and carbon- 14 , each of the samples results is below the detection limit. The nickel-63 results were both detected slightly above the detection limit. When the duplicate pair is near the detection limit, analysis of RPDs is not considered to be useful in the precision determination. The data are useable for decision-making purposes.

In the gamma spectroscopy analysis, there was insufficient material to perform a duplicate analysis. The sample was recounted on a different detector to provide a laboratory duplicate. The RPDs calculated for silver-108m, cobalt-60, europium-152, and europium-154 in the laboratory duplicate pair, are above the acceptance criteria at $158.9 \%, 69.8 \%, 209.8$, and $1295.8 \%$, respectively. Each of the samples results are 
below the PQL. When the duplicate pair is near the detection limit, analysis of RPDs is not considered to be useful in the precision determination. The data are useable for decision-making purposes.

The radionuclide americium-241 was reported in the MB at a concentration similar to the MDL. Sample J13J10 has an americium-241 result that is less than five times greater than the result reported for the MB, indicating that the sample may be biased high. The sample result is considered estimated and flagged "J." The data are useable for decision-making purposes.

\section{SDG K0595}

This SDG comprises four field samples collected from the SPA (J13HY8, J13HY9, J13J00, and J13J01). These samples were analyzed for ICP metals, mercury, hexavalent chromium, TPH, VOCs, SVOCs, PCBs, pesticides, herbicides, and by alpha spectroscopy, beta counting, gamma spectroscopy, and liquid scintillation counting. No major deficiencies were found in SDG K0595. Minor deficiencies are as follows:

In the VOC analysis, the samples were analyzed outside the required holding time. The samples were collected on 10/17/06, and were analyzed on 11/3/06. The holding time was exceeded by less that twice the limit, and the data are considered estimated and flagged "J." Estimated data are useable for decision-making purposes.

The common laboratory contaminants methylene chloride is detected in the MB at concentrations less than the CRQL. The data are not otherwise affected and are useable for decision-making purposes.

Also in the VOC analysis, $12 \mathrm{MS}$ recoveries are above the acceptance criteria. The acetone MS and MSD recoveries are 523\% and 504\%, respectively. The MS and MSD recoveries for 2-butanone are $310 \%$ and $289 \%$, respectively. The 4-methyl-2-pentanone MS and MSD recoveries are $186 \%$ and $179 \%$, respectively. The 2-hexanone MS and MSD recoveries are $257 \%$ and $237 \%$, respectively. The MS and MSD recoveries for 1,1,2,2-tetrachloroethane are $145 \%$ and $137 \%$, respectively. The chloromethane and 1,2-dichloropropane MSDs are $181 \%$ and $131 \%$, respectively. These recoveries indicate a potential high bias, however, the sample results are reported as nondetected. The data are useable for decision-making purposes.

In the metals analysis, the analytes beryllium, calcium, chromium, iron, magnesium, sodium, and zinc are reported in the MB at concentrations that are just above the PQLs, however, the sample results are greater than 5 times the MB concentration, and the data are useable for decision-making purposes. Molybdenum is reported in the MB at $0.18 \mathrm{mg} / \mathrm{kg}$, above the PQL of $0.10 \mathrm{mg} / \mathrm{kg}$. Molybdenum sample results for $\mathrm{J} 13 \mathrm{HY} 8$, $\mathrm{J} 13 \mathrm{HY} 9, \mathrm{~J} 13 \mathrm{~J} 00$, and $\mathrm{J} 13 \mathrm{~J} 01$ are $0.37,0.34,0.34$, and $0.42 \mathrm{mg} / \mathrm{kg}$, respectively. The detected MB results indicate that the sample results may be biased high. The molybdenum sample results for these samples are considered estimated and flagged "J." The data are useable for decision-making purposes. 
In addition, the MS recoveries for four ICP metals (aluminum, iron, manganese, and silicon) are out of acceptance criteria. For aluminum, iron, and manganese, the spiking concentration is insignificant compared to the native concentration in the sample from which the MS was prepared. For these analytes, the deficiency in the MS is a reflection of the analytical variability of the native concentration rather than a measure of the recovery from the sample. To confirm quantitation, post digestion spikes and serial dilutions were prepared for all five analytes, and the results for the analytes are in the range of $91.6 \%$ and $105 \%$. Silicon did not have mismatched spike and native concentrations in the original MS. The original MS recovery for silicon is $49.6 \%$. The silicon sample results are considered estimated and flagged "J." The data are useable for decision-making purposes.

Also in the ICP metals analysis, the LCS recovery for silicon is below the acceptance criteria at $52.5 \%$. Associated sample results for silicon are likely biased low. The silicon sample results are considered estimated and flagged "J." Silicon is not a COPC for the 118-B-1 waste site.

The analyte hexavalent chromium is detected just above the detection limit in the laboratory duplicate result for sample J13HY8. In the initial result for the sample, hexavalent chromium was non-detected. The laboratory reported an RPD value for these results of $69.5 \%$. However, when the duplicate pair is near the detection limit, analysis of RPDs is not considered to be useful in the precision determination. The data are useable for decision-making purposes.

In the SVOC analysis, the common laboratory contaminants bis(2-ethylhexyl)phthalate and di-n-butylphthalate were detected in the MB at concentrations less than the CRQL. The data are not otherwise affected and are useable for decision-making purposes.

In the alpha spectroscopy analysis, the RPD calculated for uranium-238 in the laboratory duplicate pair is above the acceptance criteria at $40 \%$. Each sample result is just above the detection limit. When the duplicate pair is near the detection limit, analysis of RPDs is not considered to be useful in the precision determination. The data are useable for decision-making purposes.

\section{SDG K0597}

This SDG comprises four field samples collected from the SPA (J13J02 - J13J05). These samples were analyzed for ICP metals, mercury, hexavalent chromium, TPH, VOCs, SVOCs, PCBs, pesticides, herbicides, and by alpha spectroscopy, beta counting, gamma spectroscopy, and liquid scintillation counting. No major deficiencies were found in SDG K0597. Minor deficiencies are as follows:

In the VOC analysis, the common laboratory contaminant methylene chloride was detected in the MB at concentrations less than the CRQL. The data are not affected and are useable for decision-making purposes. 
MS recoveries for two VOC analyses are above the acceptance criteria. The MS and MSD recoveries for acetone are $485 \%$ and $415 \%$, respectively. The 2-butanone MS and MSD recoveries are $267 \%$ and $236 \%$, respectively. All other sample results are below the CRQL. According to the laboratory sample discrepancy report, the MS and MSD samples for acetone and 2-butanone may have been double-spiked. The data are useable for decision-making purposes.

In the SVOC analysis, the common laboratory contaminants bis(2-ethylhexyl)phthalate and di-n-butylphthalate were detected in the MB at concentrations less than the CRQL. The data are not otherwise affected and are useable for decision-making purposes.

For sample J13J04, four SVOC surrogate recoveries were above the acceptance criteria, indicating a potential high bias. All SVOC results for sample J13J04 are below the CRQL. The laboratory sample discrepancy report stated that the sample may have been concentrated, with the observation that the sample caps didn't fit tightly, as a probable cause for the high surrogate recoveries. The data are useable for decisionmaking purposes.

The analytes beryllium, calcium, chromium, iron, magnesium, molybdenum, sodium, and zinc are reported in the MB at concentrations that are just above the PQLs. The sample results are greater than 5 times the MB concentration, with the exception of molybdenum, which may be biased high. The molybdenum sample results are considered estimated and flagged "J." The data are useable for decision-making purposes.

In addition, the MS recoveries for five ICP metals (aluminum, iron, manganese, antimony, and silicon) are out of acceptance criteria. For aluminum, iron, and manganese, the spiking concentration is insignificant compared to the native concentration in the sample from which the MS was prepared. For these analytes, the deficiency in the MS is a reflection of the analytical variability of the native concentration rather than a measure of the recovery from the sample. To confirm quantitation, post digestion spikes and serial dilutions were prepared for all five analytes, and the results for the analytes are in the range of $95.4 \%$ and $103.4 \%$. Antimony and silicon did not have mismatched spike and native concentrations in the original MS. The original MS recovery for silicon is $206.5 \%$, indicating a potential high bias. The original MS recovery for antimony is $57 \%$, and the antimony sample results are considered estimated and flagged "J." Estimated data are useable for decision-making purposes.

Also, in the ICP metals analysis, the LCS recovery for silicon is below the acceptance criteria at $52.5 \%$. Associated sample results for silicon are likely biased low. Silicon is not a COPC for the 118-B-1 waste site. The data are useable for decision-making purposes.

In the alpha-spectroscopy analysis, the RPD calculated for uranium-233/234 in the laboratory duplicate pair is above the acceptance criteria at $37 \%$. Each sample result is 
just above the detection limit. When the duplicate pair is near the detection limit, analysis of RPDs is not considered to be useful in the precision determination. The data are useable for decision-making purposes.

\section{SDG K0601}

This SDG comprises one field sample from the SPA (J13J06). This sample was analyzed for ICP metals, mercury, hexavalent chromium, TPH, VOCs, SVOCs, PCBs, pesticides, herbicides, and by alpha spectroscopy, beta counting, gamma spectroscopy, and liquid scintillation counting. SDG K0601 was submitted for third-party validation. No major deficiencies were found in SDG K0601. Minor deficiencies are as follows:

In the VOC analysis, methylene chloride is reported in the method blank at a concentration less than the CRQL. Third-party validation qualified all methylene chloride results in the SDG K0601 as undetected and flagged "U." The data are not otherwise affected and are useable for decision-making purposes.

Also in the VOC analysis, the MS recoveries for acetone and 2-butanone are above the acceptance criteria. The acetone MS and MSD recoveries are $377 \%$ and $318 \%$, respectively. The 2-butanone MS recovery is $205 \%$. The laboratory sample discrepancy report stated that the matrix spike may have inadvertently been doubled. All associated sample results are below the CRQL. The data are useable for decisionmaking purposes.

In the SVOC analysis, hexachloroethane and the common laboratory contaminant bis(2-ethylhexyl)phthalate were detected in the MB at concentrations less than the CRQL. Third-party validation qualified all hexachloroethane and bis(2-ethylhexyl)phthalate results in the SDG K0601 as undetected and flagged "U". The data are not otherwise affected and are useable for decision-making purposes.

In the herbicide analysis, the MS recovery for dalapon is below the acceptance criteria at $23 \%$, and the RPD for dalapon is below the acceptance criteria at $58 \%$. Third-party validation qualified all dalapon results in the SDG K0601 as estimated and flagged "J." The data are useable for decision-making purposes.

The RPD for 2,4-D is below the acceptance criterion at $36 \%$. Third-party validation qualified all 2,4-D results in the SDG K0601 as estimated and flagged "J." The data are useable for decision-making purposes.

All of the toxaphene data in SDG K0601 was qualified by third-party validation as estimated with "J" flags, due to lack of a MS, MSD, or LCS analysis for the analyte. Estimated, or "J"-flagged, data are acceptable for decision-making purposes.

In the metals analysis, the MS recoveries for six ICP metals (aluminum, calcium, iron, manganese, antimony, and silicon) are out of acceptance criteria. For aluminum, iron, and manganese, the spiking concentration is insignificant compared to the native 
concentration in the sample from which the MS was prepared. For these analytes, the deficiency in the MS is a reflection of the analytical variability of the native concentration rather than a measure of the recovery from the sample. To confirm quantitation, post digestion spikes and serial dilutions were prepared for all five analytes, and the results for the analytes are in the range of $102.8 \%$ and $108.8 \%$. Antimony, silicon, and calcium did not have mismatched spike and native concentrations in the original MS. The original MS recoveries for antimony, silicon, and calcium are $52.6 \%, 145.6 \%$, and $64.7 \%$, respectively. Third party validation qualified all calcium, antimony, and silicon results in the SDG K0601 as estimated and flagged "J." Estimated, or "J"-flagged, data are considered acceptable for the intended use of the data. The data are useable for decision-making purposes.

The analytes aluminum, beryllium, calcium, chromium, lead, silicon, and zinc were reported in the MB at concentrations that are just above the PQLs. The sample results are greater than 5 times the MB concentration. The data are useable for decisionmaking purposes.

Also, in the ICP metals analysis, the LCS recovery for silicon is below the acceptance criterion at $31.2 \%$. Associated sample results for silicon are likely biased low. Silicon is not a COPC for the 118-B-1 waste site.

The RPD values for arsenic (39.3\%) and molybdenum (42.4\%) are above the laboratory acceptance criteria. When the duplicate pair is near the detection limit, analysis of RPDs is not considered to be useful in the precision determination. However, the RPD value for nickel is $90.1 \%$, and all nickel results in SDG K0601 were qualified as estimated and flagged "J" by third-party validation. The data are usable for decisionmaking purposes.

In the liquid scintillation counting analysis, the MS for tritium was not performed. All tritium results in SDG K0601 were qualified as estimated and flagged "J" by third-party validation. The data are usable for decision-making purposes.

\section{SDG K0602}

This SDG comprises two field samples from the SPA (J13J07 and J13J08). These samples were analyzed for ICP metals, mercury, hexavalent chromium, TPH, VOCs, SVOCs, PCBs, pesticides, herbicides, and by alpha spectroscopy, beta counting, gamma spectroscopy, and liquid scintillation counting. No major deficiencies were found in SDG K0602. Minor deficiencies are as follows:

In the VOC analysis, methylene chloride is reported in the method blank at a concentration less than the CRQL. The data are not otherwise affected and are useable for decision-making purposes.

Also in the VOC analysis, five MS recoveries are outside the acceptance criteria. The MS recovery for 1,1,2,2-tetrachloroethane is $63 \%$, the MSD recovery is within the 
acceptance criteria. The acetone, 2-butanone, 4-methyl-2-pentanone, and 2-hexanone MSD recoveries are $538 \%, 301 \%, 174 \%$, and $243 \%$, respectively. These recoveries indicate a potential high bias. However, all associated sample results are below the CRQLs. The data are useable for decision-making purposes.

In the SVOC analysis, the common laboratory contaminants bis(2-ethylhexyl)phthalate and di-n-butylphthalate are detected in the MB at a concentration less than the CRQL. The data are not affected and are useable for decision-making purposes.

In the herbicide analysis, the surrogate recovery for sample $\mathrm{J} 13 \mathrm{~J} 07$ is above the acceptance criteria. All sample results are undetected or detected below the CRQL, and, therefore, the detected results are already flagged "J" and considered estimated. The data are useable for decision-making purposes.

In the pesticide analysis, both surrogate recoveries for sample J13J08 are above the acceptance criteria. All sample results are undetected or detected below the CRQL, and therefore the detected results are already flagged " $\mathrm{J}$ " and considered estimated. The data are useable for decision-making purposes.

In the metals analysis, the MS recoveries for six ICP metals (aluminum, calcium, iron, manganese, antimony, and silicon) are out of acceptance criteria. For aluminum, iron, manganese, and silicon, the spiking concentration is insignificant compared to the native concentration in the sample from which the MS was prepared. For these analytes, the deficiency in the MS is a reflection of the analytical variability of the native concentration rather than a measure of the recovery from the sample. To confirm quantitation, post digestion spikes and serial dilutions were prepared for all five analytes, and the results for the analytes are in the range of $95.9 \%$ and $99.6 \%$. Antimony and calcium did not have mismatched spike and native concentrations in the original MS. The original MS recovery for antimony is $51.9 \%$. The original MS recovery for calcium is $70.2 \%$. The antimony and calcium sample results are considered estimated and flagged "J." The data are useable for decision-making purposes.

The analytes aluminum, beryllium, calcium, chromium, lead, sodium, silicon, and zinc were reported in the MB at concentrations that are slightly above the PQLs. The sample results are greater than 5 times the MB concentration. The data are useable for decision-making purposes.

Also in the ICP metals analysis, the LCS recovery for silicon is below the acceptance criterion at $31.2 \%$. Associated sample results for silicon are likely biased low. Silicon is not a COPC for the 118-B-1 waste site. The data are useable for decision-making purposes.

Finally, the RPD value for arsenic is above the laboratory acceptance criterion, with a result of $32.6 \%$. When the duplicate pair is near the detection limit, analysis of RPDs is not considered to be useful in the precision determination. The data are usable for decision-making purposes. 


\section{SDG K0679}

This SDG comprises one field sample (J14BH3) collected from the SPA. This sample was analyzed for tritium only. No major or minor deficiencies were found in SDG K0679.

\section{Bulk Asbestos SDGs}

Bulk asbestos samples were collected from the SPA, and reported in five SDGs. SDG 06-A-5594 comprises six field samples, SDG 06-A-5679 comprises four field samples, SDG 06I-5661-01 comprises four samples, SDG 06I-5717-01 comprises one sample, and SDG 06I-5735-01 comprises two samples. No major or minor deficiencies were found in SDG 06-A-5594, SDG 06-A-5679, SDG 06I-5661-01, SDG 06I-5717-01 or SDG 06I-5735-01.

\section{C1.2.3 Focused Samples}

Focused samples were taken from the 118-B-1 waste site and verification sample data are provided in six SDGs: SDG K0470, SDG K0623, SDG K0638, SDG K0486, SDG K0783, and SDG K0853. The deficiencies for SDG K0638 and K0486 are discussed in the DQA section covering the Area 1 through 7 subunits. The deficiencies for SDG K0470, SDG K0623, SDG K0730, and SDG K0853 are discussed below.

\section{SDG K0470}

This SDG comprises nine focused field samples (J12R24 - J12R29, J12R31, J12R32, and J12R34). These samples were analyzed for ICP metals, mercury, hexavalent chromium, TPH, VOCs, SVOCs, PCBs, pesticides, herbicides, and by gamma spectroscopy. No major deficiencies were found in SDG K0594. Minor deficiencies are as follows:

In the pesticides analysis, 13 of $40 \mathrm{MS}$ recoveries are below the acceptance criteria. The MS and MSD recovery for alpha-benzenehexachloride are $10 \%$ and $43 \%$, respectively. The MS and MSD recovery for gamma-benzenehexachloride (lindane) are $13 \%$ and $44 \%$, respectively. The MS and MSD recovery for delta-benzenehexachloride are $20 \%$ and $38 \%$, respectively. The heptachlor MS is $56 \%$. The aldrin MS is $31 \%$. The MS and MSD recovery for 4,4'-DDE are $48 \%$ and $55 \%$, respectively. The endrin MS, endrin aldehyde MS, and endosulfan sulfate MS are $36 \%, 22 \%$, and $49 \%$, respectively. The sample results for these analytes are considered estimated and flagged "J." The data are useable for decision-making purposes.

In the VOC analysis, methylene chloride is reported in the method blank at a concentration of $11 \mathrm{ug} / \mathrm{kg}$, above the PQL of $10 \mathrm{ug} / \mathrm{kg}$. The J12R24 sample result is reported at $16 \mathrm{ug} / \mathrm{kg}$, slightly above the PQL. The sample result for methylene chloride 
is likely biased high, and is considered estimated with a "J" flag. Estimated data are useable for decision-making purposes.

In the SVOC analysis, the common laboratory contaminant bis(2-ethylhexyl)phthalate is detected in the MB at a concentration less than the CRQL. The data are not otherwise affected and are useable for decision-making purposes.

In the herbicides analysis, six of $20 \mathrm{MS}$ recoveries are below the acceptance criteria. The MS and MSD recovery for picloram are $16 \%$ and $29 \%$, respectively. The MS recovery for dichloroprop, 2,4-D, 2,4,5-T, and 2,4-DB MS are $21 \%, 22 \%, 22 \%$, and $27 \%$, respectively. The sample results for these analytes are considered estimated and flagged "J." The data are useable for decision-making purposes.

The LCS for picloram is below acceptance criteria with a recovery of $33 \%$. The sample results for these analytes are considered estimated and flagged "J." The data are useable for decision-making purposes.

In the metals analysis, the MS recoveries for six ICP metals (aluminum, calcium, iron, manganese, antimony, and silicon) are out of acceptance criteria. For aluminum, calcium, iron, manganese, and silicon, the spiking concentration is insignificant compared to the native concentration in the sample from which the MS was prepared. For these analytes, the deficiency in the MS is a reflection of the analytical variability of the native concentration rather than a measure of the recovery from the sample. To confirm quantitation, post digestion spikes and serial dilutions were prepared for all five analytes, and the results for the analytes are in the range of $95.9 \%$ and $99.6 \%$. Antimony did not have mismatched spike and native concentrations in the original MS. The original MS recovery for antimony is $40.6 \%$, and the antimony data are considered estimated and flagged "J." Estimated data are useable for decision-making purposes.

Also in the ICP metals analysis, the LCS recovery for silicon is below the acceptance criteria at $57.0 \%$. Associated sample results for silicon are likely biased low. The data are useable for decision making purposes. Silicon is not a COPC for the 118-B-1 waste site.

The RPD values for boron and sodium are above the laboratory acceptance criteria, both with an RPD of $31.7 \%$. When the duplicate pair is near the detection limit, analysis of RPDs is not considered to be useful in the precision determination. The data are usable for decision-making purposes.

Metals analysis was added to SDG K0470 for samples J12R34 and J12R28. The MS recoveries for eight ICP metals (aluminum, calcium, iron, magnesium, manganese, lead, antimony, and silicon) are out of acceptance criteria. For aluminum, calcium, iron, manganese, and silicon, the spiking concentration is insignificant compared to the native concentration in the sample from which the MS was prepared. For these analytes, the deficiency in the MS is a reflection of the analytical variability of the native concentration rather than a measure of the recovery from the sample. To confirm quantitation, post digestion spikes and serial dilutions were prepared for all five 
analytes, and the results for the analytes are in the range of $95.9 \%$ and $99.6 \%$. Antimony, magnesium, and lead did not have mismatched spike and native concentrations in the original MS. The original MS recovery for magnesium was high, with a result of $131.8 \%$. The original MS recovery for lead and antimony are $73.9 \%$ and $43.3 \%$, respectively. The sample results for lead and antimony for samples J12R34 and J12R28 are considered estimated and flagged "J." The data are useable for decisionmaking purposes.

Also in the ICP metals analysis for samples J12R34 and J12R28, the LCS recovery for silicon is below the acceptance criteria at $38.9 \%$. Associated sample results for silicon are likely biased low. Silicon is not a COPC for the 118-B-1 waste site. The data are useable for decision-making purposes.

The analytes calcium, cadmium, chromium, potassium, magnesium, molybdenum, sodium, silicon, and vanadium are reported in the MB for samples J12R34 and J12R28 at concentrations that are just above the PQLs. The sample results are greater than 5 times the MB concentration, with the exception of cadmium and molybdenum in sample J12R28. The cadmium result was reported as a nondetect, and the molybdenum was reported at a concentration just above the PQL. The molybdenum MB result indicates the sample J12R28 molybdenum result may be biased high. The sample result is considered estimated and flagged "J." Estimated data are useable for decision-making purposes.

Finally, the RPD values for boron, cadmium, chromium, molybdenum, and lead for samples J12R34 and J12R28 are above the laboratory acceptance criteria, with RPDs of $31.6 \%, 64.8 \%, 43.4 \%, 101.8 \%$, and $31.0 \%$, respectively. When the duplicate pair is near the detection limit, analysis of RPDs is not considered to be useful in the precision determination. The data are usable for decision-making purposes.

\section{SDG K0623}

This SDG comprises three field samples (J13P84, J13P85, and J13NX4). These samples were analyzed for ICP metals only. No major deficiencies were found in SDG K0623. Minor deficiencies are as follows:

In the metals analysis, the MS recoveries for five ICP metals (aluminum, iron, manganese, antimony, and silicon) are out of acceptance criteria. For aluminum, iron, and manganese, the spiking concentration is insignificant compared to the native concentration in the sample from which the MS was prepared. For these analytes, the deficiency in the MS is a reflection of the analytical variability of the native concentration rather than a measure of the recovery from the sample. To confirm quantitation, post digestion spikes and serial dilutions were prepared for all five analytes, and the results for the analytes are in the range of 98.8 and $105.7 \%$. Antimony and silicon did not have mismatched spike and native concentrations in the original MS. The original MS recovery for silicon is $174.6 \%$ indicating a potential high bias. The original MS recovery 
for antimony is $58.7 \%$. The sample results for antimony are considered estimated and flagged "J." Estimated data are useable for decision-making purposes.

The analytes barium, calcium, potassium, sodium, silicon, and zinc are reported in the $\mathrm{MB}$ at concentrations that are just above the PQLs. The sample results are greater than 5 times the MB concentration. The data are useable for decision-making purposes.

Also, in the ICP metals analysis, the LCS recovery for silicon is below the acceptance criteria at $17.3 \%$. Silicon is not a COPC for the 118-B-1 waste site. The data are usable for decision-making purposes.

Finally, the RPD value for arsenic is above the laboratory acceptance criteria, with a result of $56.2 \%$. When the duplicate pair is near the detection limit, analysis of RPDs is not considered to be useful in the precision determination. The data are usable for decision-making purposes.

\section{SDG K0730}

This SDG comprises three field samples (J14L38, J13L39, and J14JJ3). These samples were analyzed for ICP metals, by alpha spectroscopy, gamma spectroscopy, and liquid scintillation counting. Major and minor deficiencies were found in SDG K0730, as follows:

In the metals analysis, the MS recoveries for five ICP metals (aluminum, iron, mercury, antimony, and silicon) are out of acceptance criteria. For aluminum, iron, and mercury, the spiking concentration is insignificant compared to the native concentration in the sample from which the MS was prepared. For these analytes, the deficiency in the MS is a reflection of the analytical variability of the native concentration rather than a measure of the recovery from the sample. To confirm quantitation, post digestion spikes and serial dilutions were prepared for all five analytes, and the results for the analytes are in the range of 80.4 and $120.0 \%$. Antimony and silicon did not have mismatched spike and native concentrations in the original MS. The original MS recoveries for silicon and antimony are $3.7 \%$ and $49.3 \%$, respectively. Also, the LCS recovery for silicon is below the acceptance criteria at $53.4 \%$. As the MS recovery is below $10 \%$ and the LCS recover did not meet the acceptance criteria, the sample results for silicon for SDG K0783 are qualified as rejected, and flagged with an "R." The sample results for antimony are considered estimated and flagged "J." Estimated data are useable for decision-making purposes.

The analytes aluminum, barium, calcium, chromium, iron, magnesium, manganese, silicon, and zinc are reported in the MB at concentrations that are just above the PQLs. The sample results are greater than 5 times the MB concentration. The data are useable for decision-making purposes.

Finally, the RPD value for silicon is above the laboratory acceptance criteria, with a result of $32.6 \%$. The silicon sample results for SDG K0783 are qualified as rejected, 
and flagged with an "R," as multiple QC criteria are not met for the analyte. Silicon is not a COPC for the 118-B-1 waste site, and the rejected silicon data do not impact completeness of the verification sampling data.

\section{SDG K0853}

This SDG comprises two field samples (J14Y75 and J14Y76). These samples were analyzed by alpha spectroscopy, gamma spectroscopy, and liquid scintillation counting. No major deficiencies were found in SDG K0853. Minor deficiencies are as follows:

The MDA for plutonium-241 for sample J14Y75 is greater than the required MDA of $15 \mathrm{pCi} / \mathrm{g}$. The results for sample J14Y75 are reported as below the detection limit. The J14Y75 sample results for plutonium-241 are considered estimated and flagged "J." Estimated data are useable for decision-making purposes.

\section{SDG K0783}

This SDG comprises one focused field soil sample (J152M1). The sample was analyzed for ICP metals, mercury, and tritium by liquid scintillation counting. A major deficiency was found in SDG K0783. Major and minor deficiencies are as follows:

In the ICP metals analysis, the LCS recovery for silicon is below the acceptance criteria at less than $1 \%$. As the LCS recovery is less than $10 \%$, the silicon sample results for SDG K0783 are qualified as "rejected", and flagged with an "R." Silicon is not a COPC for the 118-B-1 waste site, and the rejected silicon data do not impact completeness of the verification sampling data.

The analytes barium, calcium, magnesium, sodium, and silicon are reported in the MB at concentrations that are below the CRQLs. However, the sample results are greater than 5 times the MB concentration, and the data are useable for decision-making purposes.

In addition, the MS recoveries for six metals (aluminum, iron, manganese, mercury, antimony, and silicon) are out of acceptance criteria. For most of these analytes, the spiking concentration is insignificant compared to the native concentration in the sample from which the MS was prepared. For these analytes, the deficiency in the MS is a reflection of the analytical variability of the native concentration rather than a measure of the recovery from the sample. To confirm quantitation, post digestion spikes and serial dilutions were prepared, and the results for the analytes are in the range of $88.1 \%$ to $100.7 \%$. Antimony had a native concentrations in the original MS below the detection limit. The original MS recovery for antimony is $43.4 \%$. The antimony results in SDG K0783 are considered estimated and flagged "J." The data are useable for decisionmaking purposes.

The RPDs calculated for mercury and nickel in the laboratory duplicate pair (sample J152M1, and J152M1 [duplicate]), are above the acceptance criteria with the value of 
$55.2 \%$ and $33.7 \%$, respectivley. Elevated RPDs in environmental soil samples are attributed to heterogeneities in the sample matrix and not to deficiencies in the analytical methodologies. The data are useable for decision-making purposes.

\section{C.1.3 FIELD QUALITY ASSURANCE/QUALITY CONTROL}

RPD evaluations of main sample(s) versus the laboratory duplicate(s) are routinely performed and reported by the laboratory. Any deficiencies in those calculations are reported by SDG in the previous sections.

Field QA/QC measures are used to assess potential sources of error and cross contamination of samples that could bias results. Field QA/QC samples, listed in the field logbooks (WCH 2007a, 2007b), are summarized in Table 1. The main and QA/QC sample results are presented with the calculations in Appendix $\mathrm{C}$.

Table 1. Field Quality Assurance/Quality Control Samples.

\begin{tabular}{|c|c|c|c|}
\hline Area & $\begin{array}{c}\text { Main } \\
\text { Sample }\end{array}$ & $\begin{array}{c}\text { Duplicate } \\
\text { Sample }\end{array}$ & $\begin{array}{c}\text { Split } \\
\text { Sample }\end{array}$ \\
\hline Area 1 A1 & $\mathrm{J} 12 \mathrm{X} 44$ & $\mathrm{~J} 12 \mathrm{X} 48$ & $\mathrm{~J} 12 \mathrm{X} 49$ \\
\hline Area 3 A3 & $\mathrm{J} 12 \mathrm{X} 56$ & $\mathrm{~J} 12 \mathrm{X} 58$ & $\mathrm{~J} 12 \mathrm{X} 60$ \\
\hline Area 4 A1 & $\mathrm{J} 12 \mathrm{X} 61$ & $\mathrm{~J} 12 \mathrm{X} 65$ & $\mathrm{~J} 12 \mathrm{X} 66$ \\
\hline BCL D4 & $\mathrm{J} 12 \mathrm{WL4}$ & $\mathrm{J} 12 \mathrm{WL5}$ & -- \\
\hline BCL B8 & $\mathrm{J} 12 \mathrm{WL6}$ & -- & $\mathrm{J} 12 \mathrm{WW} 0$ \\
\hline SPA B5 & $\mathrm{J} 13 \mathrm{HY} 7$ & $\mathrm{~J} 13 \mathrm{~J} 09$ & $\mathrm{~J} 13 \mathrm{~J} 10$ \\
\hline
\end{tabular}

Field duplicate samples are collected to provide a relative measure of the degree of local heterogeneity in the sampling medium, unlike laboratory duplicates that are used to evaluate precision in the analytical process. The field duplicates are evaluated by computing the RPD of the duplicate samples for each analyte. Only analytes with values above five times the detection limits for both the main and duplicate samples are compared. The $95 \%$ upper confidence limit (UCL) calculation brief in Appendix C provides details on duplicate pair evaluation and RPD calculation. The data are suitable for the intended purpose of cleanup verification.

Split samples are collected to provide a relative measure of the variability in the sampling, sample handling, and analytical techniques used by commercial laboratories. The field main and split samples are evaluated by computing the RPD of the split samples for each analyte to determine the usability of the verification data. The U.S. Environmental Protection Agency Contract Laboratory Program duplicate sample comparison methodology, USEPA Contract Laboratory Program National Functional Guidelines for Inorganic Data Review (EPA 1994), is used as an initial test of the data from the splits. Only analytes that had values above five times the CRQL for both the 
main and split sample were compared. The 95\% UCL calculation brief in Appendix C provides details on split pair RPD calculation. The RPD acceptance criteria for projectsplit samples is $\leq 30 \%$ (less than or equal to $30 \%$ ).

Radionuclides. The RPDs calculated for cesium-137 in the Area 3 A3 field duplicate (J12X58) and the Area 3 A3 split sample (J12X60) are above the acceptance criterion $(30 \%)$ at $64 \%$ and $61 \%$, respectively. The RPD calculated for europium-152 in the Area 1 A1 duplicate (J12X48) analysis is above the acceptance criterion at $59 \%$. The RPDs calculated for potassium-40 in the Area 4 A1 (J12X61), the BCL D4 (J12WL5), and the SPA B5 (J13J09) duplicates are above the acceptance criterion (30\%) at $67 \%$, $58 \%$, and $67 \%$, respectively. The RPDs calculated for radium-226 in the BCL D4 (J12WL5) and the SPA B5 (J13J09) duplicates are above the acceptance criterion $(30 \%)$ at $56 \%$, and $42 \%$, respectively. Elevated RPDs, such as these, in the analysis of environmental soil samples, are largely attributed to heterogeneities in the soil matrix and only in small part attributed to precision and accuracy issues at the laboratory. The data are useable for decision-making purposes.

Nonradionuclides. The RPDs calculated for aluminum in the Area 4 A1 (J12X66), BCL B8 (J12WW0), and SPA B5 (J13J10) split samples are above the acceptance criterion $(30 \%)$ at $38 \%, 39 \%$, and $47 \%$, respectively. The RPD calculated for barium in the Area $4 \mathrm{~A} 1$ (J12X66) split analysis is above the acceptance criterion at $41 \%$. The RPD calculated for calcium in the Area 4 A1 (J12X66) split analysis is above the acceptance criterion at $36 \%$. The RPDs calculated for chromium in the Area $3 \mathrm{~A} 3$ (J12X60) and the SPA B5 (J13J10) splits are above the acceptance criterion (30\%) at $34 \%$ and $44 \%$, respectively. The RPD calculated for cobalt in the Area 1 A1 (J12X49) split is above the acceptance criterion (30\%) at $36 \%$. The RPD calculated for magnesium in the SPA B5 (J13J10) split sample is above the acceptance criterion $(30 \%)$ at $34 \%$. The RPDs calculated for silicon in the Area 4 A1 (J12X66), BCL B8 (J12WW0), and SPA B5 (J13J10) split samples are above the acceptance criterion $(30 \%)$ at $38 \%, 103 \%$, and $62 \%$, respectively. The RPD calculated for sodium in the Area 1 A1 (J12X49) split analysis is above the acceptance criterion at $54 \%$. The RPDs calculated for vanadium in the Area 1 A1 (J12X49) and the SPA B5 (J13J10) splits are above the acceptance criterion $(30 \%)$ at $39 \%$ and $33 \%$, respectively. Elevated RPDs, such as these, in the analysis of environmental soil samples, are largely attributed to heterogeneities in the soil matrix and only in small part attributed to precision and accuracy issues at the laboratory. The data are useable for decision-making purposes.

RPDs for the remaining radionuclides and nonradionuclide analytes are not calculated because an evaluation of the data shows the analytes are not detected in both the main and duplicate sample at more than 5 times the target detection limit (TDL). RPDs of analytes detected at low concentrations (less than five times the detection limit) are not considered to be indicative of the analytical system performance. The data are useable for decision-making purposes.

A secondary check of the data variability is used when one or both of the samples being evaluated (main and duplicate) is less than 5 times the TDL, including undetected 
analytes. In these cases, a control limit of \pm 2 times the TDL is used (Appendix C) to indicate that a visual check of the data is required by the reviewer. For the Area 3 duplicate sample (J12X58), the radium-226 results exceeded this check. The Area 4 A4 (J12X65) duplicate sample cesium-137, radium-226, radium-228, and 4,4'-DDE results exceeded this check. The Area 4 A4 split sample (J12X66) cesium-137, boron, chromium, cobalt, sodium, arochlor-1254, and 44'-DDE results exceeded this check. The BCL D4 duplicate sample (J12WL5) radium-228 result exceeded this check. The BCL B8 split sample (J12WW0) cobalt-60, sodium, aroclor-1254, aroclor-1260, and 4,4'-DDT results also exceeded this check. The SPA B5 duplicate sample (J13J09) radium-228 results exceeded this check. The SPA B5 split sample (J13J10) boron and cobalt results exceeded this check. All of these results are attributed to heterogeneities in the sample matrix from which the samples were collected. A visual inspection of all of the data is also performed. No additional major or minor deficiencies are noted. The data are useable for decision-making purposes.

\section{C1.4 SUITABILITY OF DATA}

Limited, random, or sample matrix-specific influenced batch QC issues, such as those discussed above, are a potential for any analysis. The number and types seen in these data sets are within expectations for the matrix types and analyses performed. The DQA review of the 118-B-1 verification sampling data found that the analytical results are accurate within the standard errors associated with the analytical methods, sampling, and sample handling. The DQA review for 118-B-1 waste site concludes that the reviewed data are of the right type, quality, and quantity to support the intended use. Detection limits, precision, accuracy, and sampling data group completeness were assessed to determine if any analytical results should be rejected as a result of QA and $\mathrm{QC}$ deficiencies. The analytical data were found acceptable for decision-making purposes, with the exception of the sample results reported for silicon within SDG K0486, SDG K0491, and K0730, picloram within SDG K0471, and 2,4-DB in K0594. The verification sample analytical data are stored in the ENRE project-specific database prior to being submitted for inclusion in the HEIS database. The verification sample analytical data are also summarized in the $95 \%$ UCL calculation in Appendix C.

\section{REFERENCES}

BHI, 2000a, Data Validation Procedure for Chemical Analysis, BHI-01435, Rev. 0, Bechtel Hanford, Inc., Richland, Washington.

BHI, 2000b, Data Validation Procedure for Radiochemical Analysis, BHI-01433, Rev. 0, Bechtel Hanford, Inc., Richland, Washington.

DOE-RL, 2001, 100 Area Burial Grounds Remedial Action Sampling and Analysis Plan, DOE/RL-2001-35, Rev. 0, U.S. Department of Energy, Richland Operations Office, Richland, Washington. 
DOE-RL, 2005, Remedial Design Report/Remedial Action Work Plan for the 100 Area, DOE/RL-96-17, Rev. 5, U.S. Department of Energy, Richland Operations Office, Richland, Washington.

EPA, 1994, USEPA Contract Laboratory Program National Functional Guidelines for Inorganic Data Review, EPA 540/R-94/013, U.S. Environmental Protection Agency, Washington, D.C.

EPA, 2000, Guidance for Data Quality Assessment, EPA QA/G-9, QA00 Update, U.S. Environmental Protection Agency, Office of Environmental Information, Washington, D.C.

EPA, 2006, CLP Statement of Work for Multi-Media, Multi-Concentration Organics Analysis, SOM01.1, U.S. Environmental Protection Agency, Superfund Analytical Services, Washington, D.C.

WCH, 2006, Site Specific Instruction for Close-out Approach for 118-B-1, Rev. 0, SSI No. 100-BC-09, CCN 130128, Washington Closure Hanford, Richland, Washington.

WCH, 2007a, 100B/C Burial Grounds and Remaining Sites Sampling and Field Activities, Logbook EFL-1173-8, pp. 64 \& 74, Washington Closure Hanford, Richland, Washington.

WCH, 2007b, 100B/C Burial Grounds and Remaining Sites Sampling and Field Activities, Logbook EFL-1173-10, pp. 21 - 42, Washington Closure Hanford, Richland, Washington.

WCH, 2007c, 100B/C Burial Grounds and Remaining Sites Sampling and Field Activities, Logbook EFL-1173-11, pp. 61 - 62, Washington Closure Hanford, Richland, Washington.

WCH, 2007d, 100B/C Burial Grounds and Remaining Sites Sampling and Field Activities, Logbook EFL-1173-12, P. 52, Washington Closure Hanford, Richland, Washington.

WCH, 2007e, 100B/C Burial Grounds and Remaining Sites Sampling and Field Activities, Logbook EFL-1173-13, pp. 12 - 13, Washington Closure Hanford, Richland, Washington. 


\section{DISTRIBUTION}

U.S. Department of Energy

Richland Operations Office

D. C. Smith (5)

Fluor Hanford, Inc.

J. P. Shearer (WIDS)

Washington Closure Hanford

J. M. Capron

R. A. Carlson

S. W. Clark

L. A. Dietz

L. M. Dittmer

S. L. Feaster

E. T. Feist

M. T. Hughes

W. F. Johnson

D. N. Strom

Records and Document Control DOE-RL Public Reading Room

Hanford Technical Library
A3-04

E6-35

X3-40

$\mathrm{X} 2-07$

$\mathrm{H} 4-23$

$\mathrm{H} 4-22$

$\mathrm{H} 4-23$

H4-24

H4-22

H4-23

$\mathrm{H} 4-22$

X3-40

$\mathrm{H} 4-11$

$\mathrm{H} 2-53$

P8-55 
CVP-2007-00006

Rev. 0

Distr-2 